
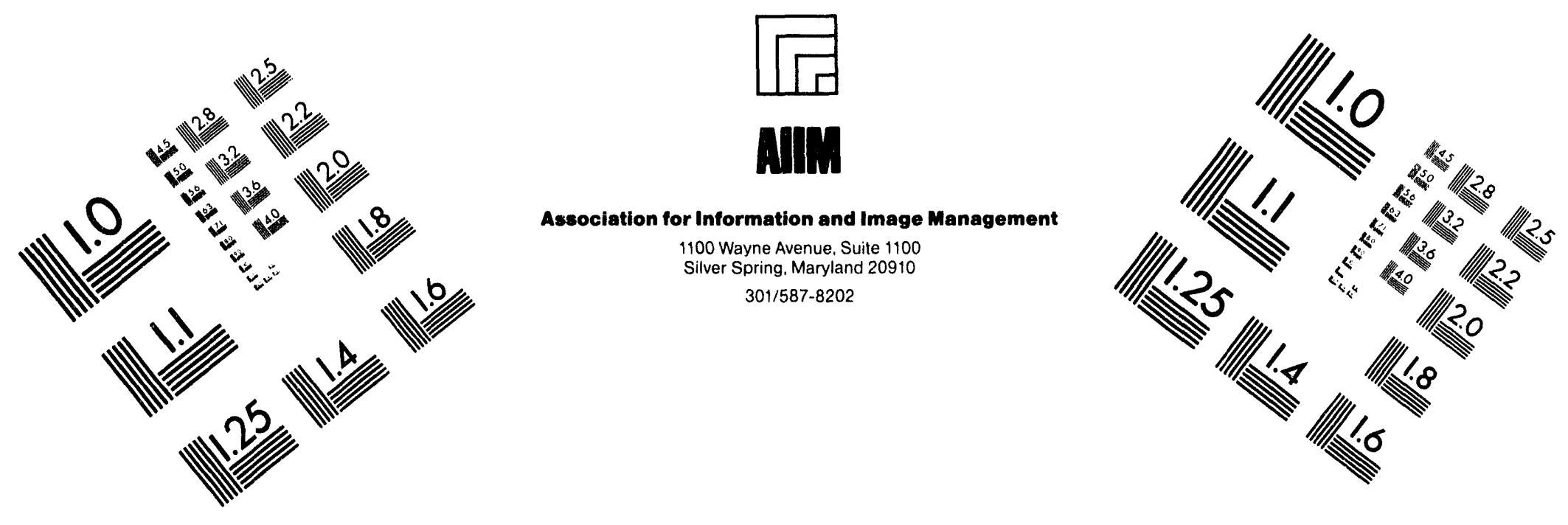

\title{
Centimeter
}

$\begin{array}{llllllllllllllll}1 & 2 & 3 & 4 & 5 & 6 & 7 & 8 & 9 & 10 & 11 & 12 & 13 & 14 & 15 & 15 m\end{array}$

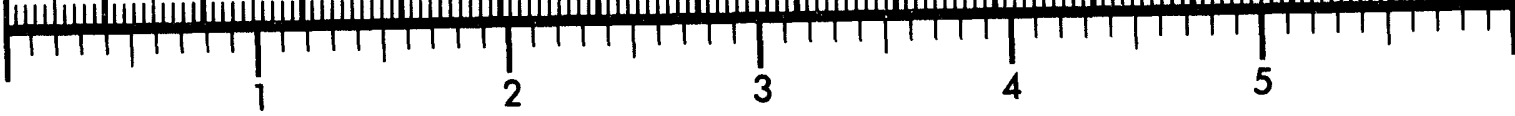
Inches
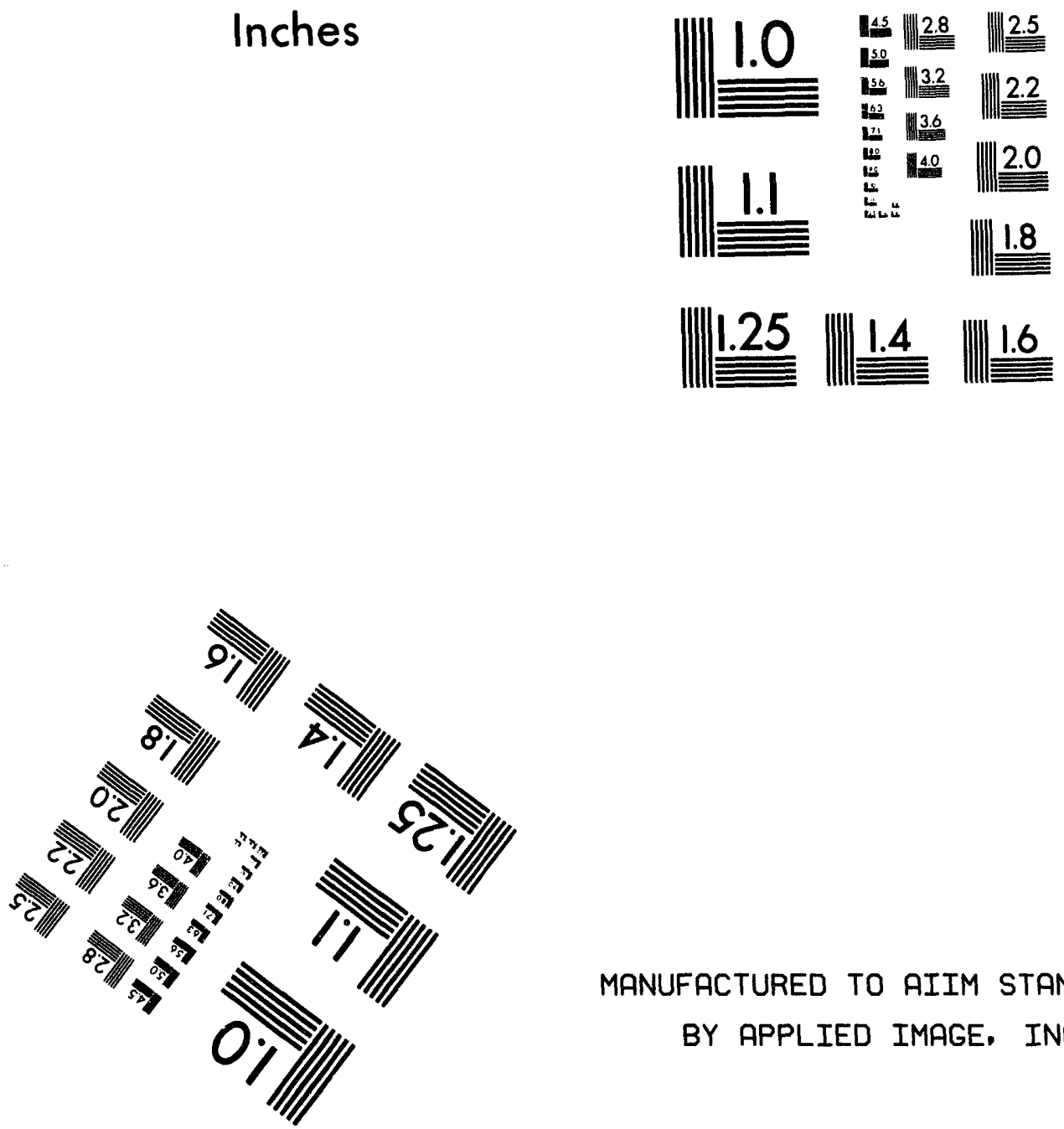

MANUFACTURED TO AIIM STANDARDS

$$
\text { BY APPLIED IMAGE, INC. }
$$

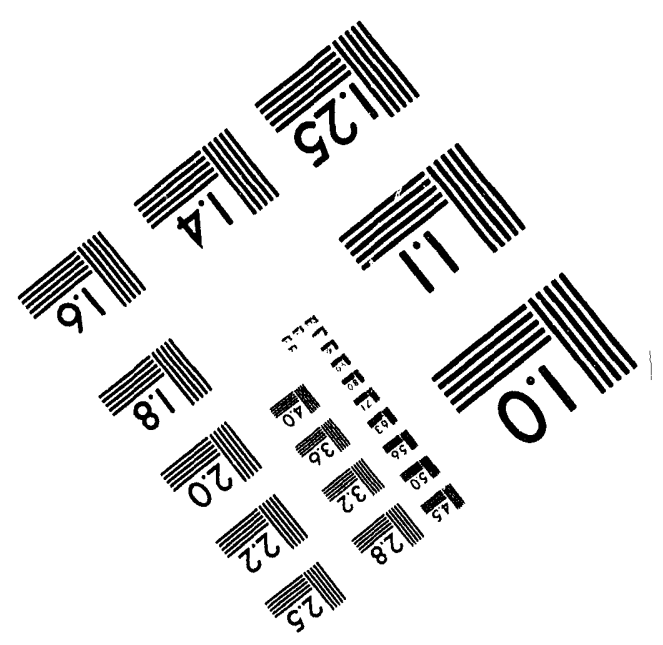



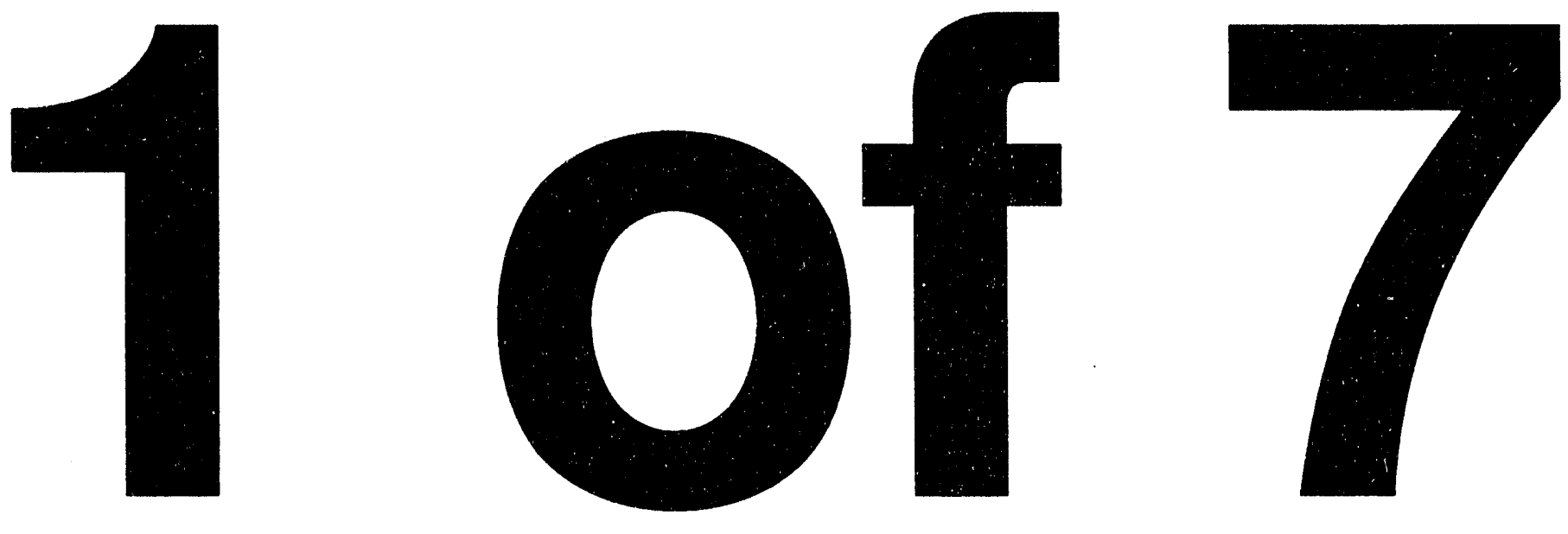


\section{Review of the Diablo Canyon Probabilistic Risk Assessment}

Manuscript Completad: September 1993

Date Published: August 1994

Prepared by

G. E. Bozoki, R. G. Fitzpatrick, M. P. Bohn', M. G. Sabek²,

M. K. Ravindra3, J. J. Johnson ${ }^{3}$

Brookthaven National Laboratory

Upton, NY 11973

\section{Prepared for}

Division of Safety Issue Resolution

Ofince of Nuclear Regulatory Research

U.S. Nuclear Regulatory Commission

Washington, DC 20555-0001

NRC FIN A3958

'Sandia National Laboratory, Albuquerque, NM

2Atomic Energy Authority, Nuclear Regulatory and

Safety Center, Cairo, Egypt

'EOE Engineering, San Francisco,, CA 


\begin{abstract}
This report details the review of the Diablo Canyon Probabilistic Risk Assessment (DCPRA). The study was performed under contract from the Probabilistic Risk Analysis Branch, Office of Nuclear Reactor Research, USNRC by Brookhaven National Laboratory. The DCPRA is a full scope Level 1 effort and although the review touched on all aspects of the PRA, the internal events and seismic events received the vast majority of the review effort. The report includes a number of independent systems analyses, sensitivity studies, importance analyses as well as conclusions on the adequacy of the DCPRA for use in the Diablo Canyon Long Term Seismic Program.
\end{abstract}




\section{TABLE OF CONTENTS}

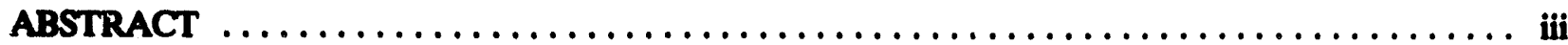

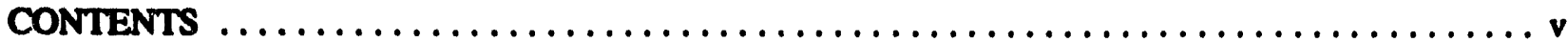

LIST OF FIGURES $\ldots \ldots \ldots \ldots \ldots \ldots \ldots \ldots \ldots \ldots \ldots \ldots \ldots \ldots \ldots \ldots \ldots \ldots \ldots$ ix

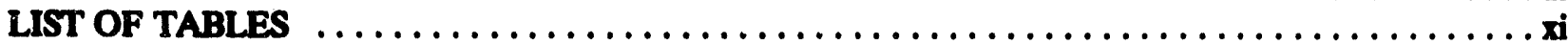

EXECUTTVE SUMMARY $\ldots \ldots \ldots \ldots \ldots \ldots \ldots \ldots \ldots \ldots \ldots \ldots \ldots \ldots \ldots \ldots \ldots \ldots \ldots \ldots$

ACKNOWLEDGEMENTS $\ldots \ldots \ldots \ldots \ldots \ldots \ldots \ldots \ldots \ldots \ldots \ldots \ldots \ldots \ldots \ldots \ldots \ldots$

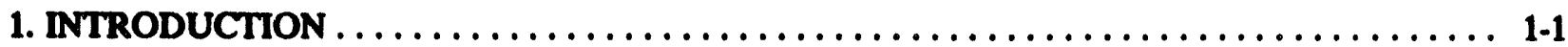

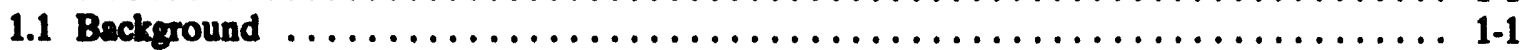

1.2 Objectives and Scope of the Review $\ldots \ldots \ldots \ldots \ldots \ldots \ldots \ldots \ldots \ldots \ldots \ldots \ldots \ldots \ldots$

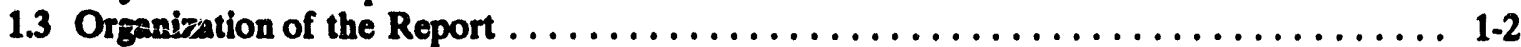

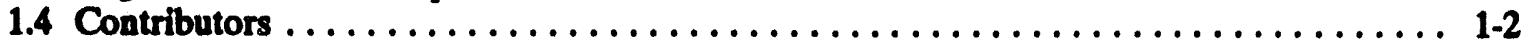

2. OVERVIEW OF THE DCPRA APPROACH AND RESULTS $\ldots \ldots \ldots \ldots \ldots \ldots \ldots \ldots \ldots .1$

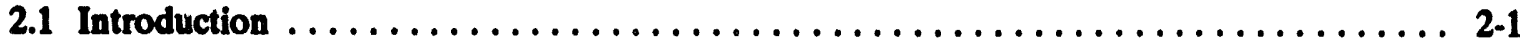

2.1.1 Background $\ldots \ldots \ldots \ldots \ldots \ldots \ldots \ldots \ldots \ldots \ldots \ldots \ldots \ldots \ldots \ldots \ldots \ldots \ldots, 2-1$

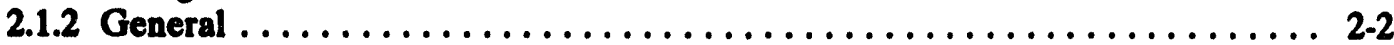

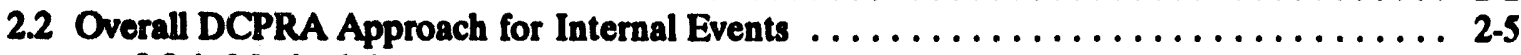

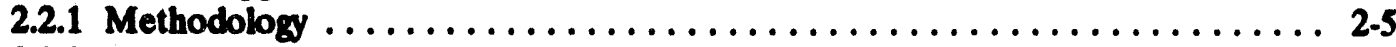

2.2.2 Advanced and Novel Features of the DCPRA ................ 2-5

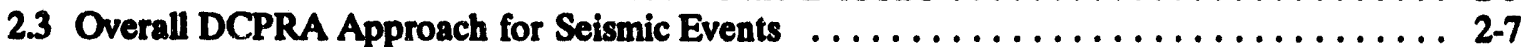

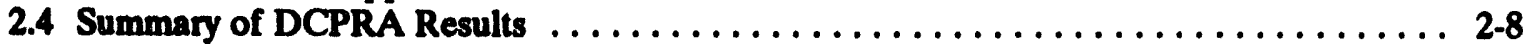

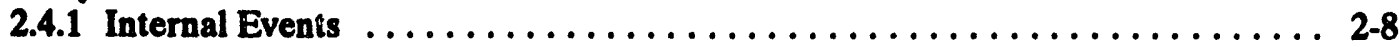

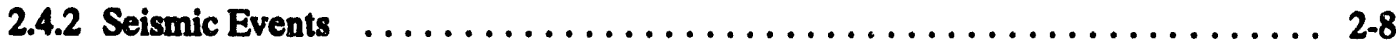

2.4.3 Other External Events ........................... 2-10

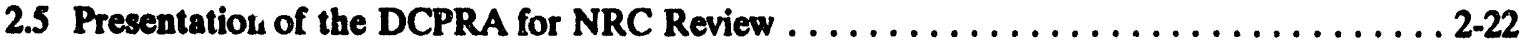

3. REVIEW OF THE INTERNAL EVENTS ANALYSIS $\ldots \ldots \ldots \ldots \ldots \ldots \ldots \ldots \ldots \ldots \ldots$.1

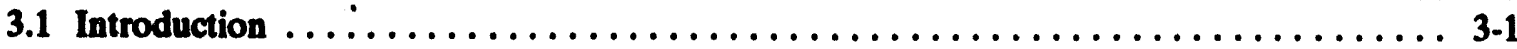

3.2 Description of the Internal Events Review Approach $\ldots \ldots \ldots \ldots \ldots \ldots \ldots \ldots \ldots$ 3-1

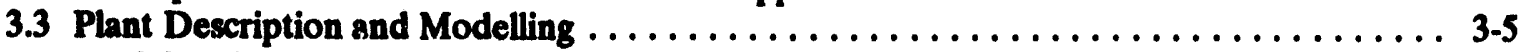

3.3.1 Safety Functions and Corresponding Frontline Systems $\ldots \ldots \ldots \ldots \ldots \ldots$. 3-5

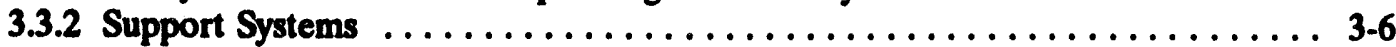

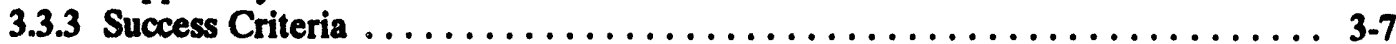

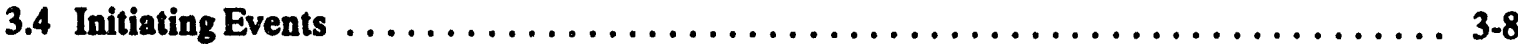

3.4.1 General . . . . . . . . . . . .

3.4.2 Initiating Event Selection in the DCPRA .................. 3-8

3.4.3 Quantification of the "Internal Initiating Events" in the DCPRA ........ 3-9

3.4.4 Review of the Initiating Event Analysis . . . . . . . . . . . . . . . 3-10

3.4.4.1 Completeness ............................ 3-10

3.4.4.2 Initiating Event Frequencies ...............

3.4.5 Selection of Generic Initiating Event Frequencies for the DCPRA . . . . . 3-17

3.4.6 References .................................... 38

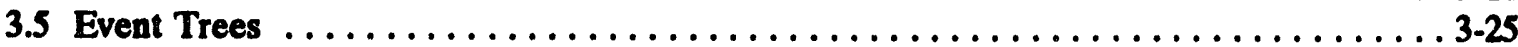

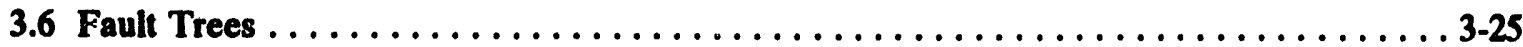

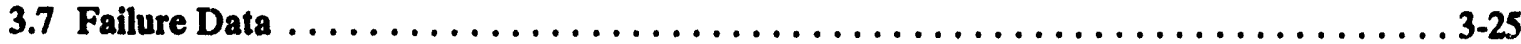

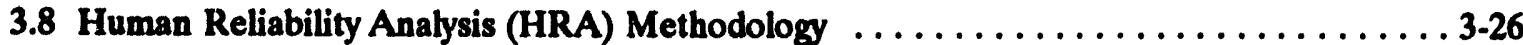

3.8.1 Scope of the HRA Methodology Review ................. 3-26

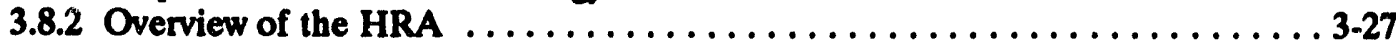

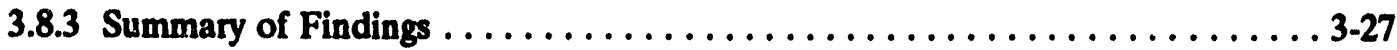

3.8.3.1 Positive Findings . . . . . . . . . . . . . . . . $\ldots \ldots \ldots \ldots$ 


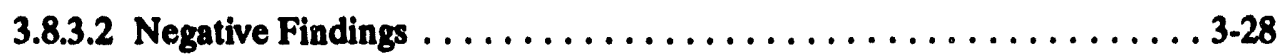

3.8.4 Discussion of each Finding Vis-A-Vis Review Guidelines $\ldots \ldots \ldots \ldots \ldots \ldots$ 3-28

3.8.4.1 HRA Team Members ...................... 3-28

3.8.4.2 Human Systems Analysis Performed . . . . . . . . . . . . . 3-29

3.8.4.3 Types of Human Task Actions Analyzed . . . . . . . . . . . 3-29

3.8.4.4 Performance Shaping Factors Evaluated . . . . . . . . . . . . . 3-29

3.8.4.5 Quantification Methods Used to Estimate Human Error Rates . . . . 3-29

3.8.4.6 Generic Human Error Data Sources . . . . . . . . . . . . . 3-30

3.8.4.7 Sensitivity Modeling Approach $\ldots \ldots \ldots \ldots \ldots \ldots \ldots \ldots \ldots \ldots 30 \ldots \ldots$

3.8.4.8 Insights Gained from the Analyses $\ldots \ldots \ldots \ldots \ldots \ldots \ldots \ldots \ldots \ldots$

3.8.4.9 Adequacy of Documentation .................... 3-30

3.8.5 Summary of the HRA Methodology Review ................. 3-31

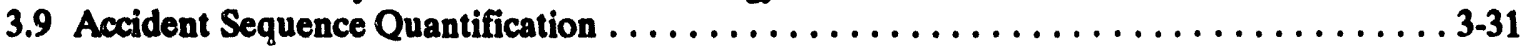

3.9.1 The Event Sequence Model of the DCPRA ................. 3-31

3.9.2 Some Technical Aspects of the Internal Event Sequence Quantification . . . . 3-32

3.9.3 Internal Event Sequence Characteristics . . . . . . . . . . . . . . . 3-35

3.9.3.1 Introductory Comments ..................... 36

3.9.3.2 Non-Seismic Core Damage Frequencies; Initiator Importances . . . . 3-36

3.9.3.3 Characteristics of Internal Event Sequences . . . . . . . . . 3-36

3.9.3.4 Comparison of the Characteristics of Certain Internal Event

Sequence Groups With Those of Other Plants .......... 3-37

3.9.3.5 Leading Non-Seismic Event Scenarios . . . . . . . . . . . . . 3-38

3.9.3.6 Special Issues of Interest to PRA ..................

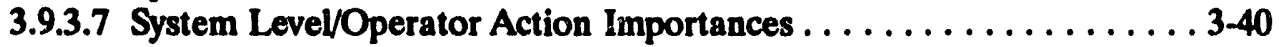

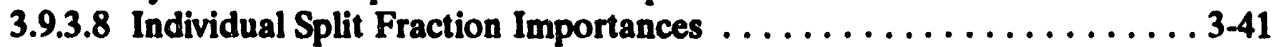

3.9.3.9 System Importances in Accident Sequences Induced by Various

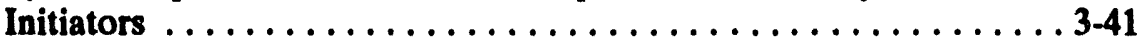

3.9.3.10 Importances of System/Top Event Split Fraction Pairs . . . . . . 3-42

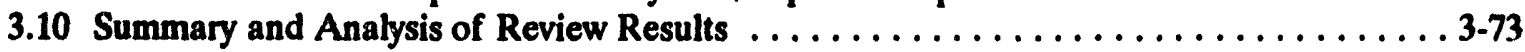

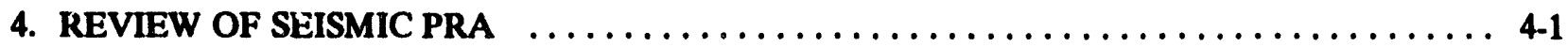

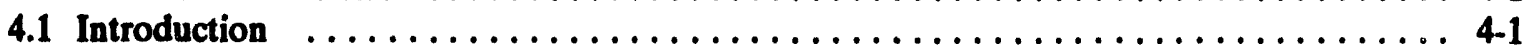

4.2 Detailed Description of DCPRA Approach Used in the Seismic Analysis . . . . . . 4-2

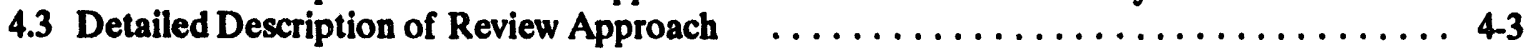

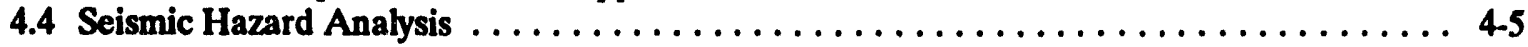

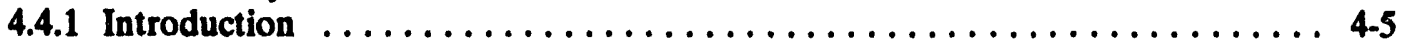

4.4.2 Seismic Hazard Analysis Methodology .................. 4-5

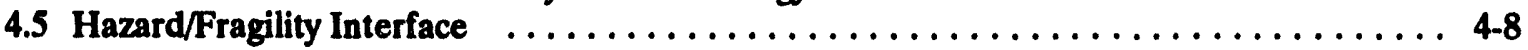

4.6 Fragility and Building Response Analysis Review $\ldots \ldots \ldots \ldots \ldots \ldots \ldots \ldots \ldots$

4.6.1 Fragility and Building Response Analysis Methodology ............ 4-8

4.6.2 Fragility Analysis Review Procedure $\ldots \ldots \ldots \ldots \ldots \ldots \ldots \ldots \ldots . \ldots$ 4-9

4.6.3 Structure Response and Fragility Review 4-10 4.6.3.1 Benchmarking of Auxiliary Building Response and Variability Study ....................................4-10

4.6.3.2 Structural Fragility Review $\ldots \ldots \ldots \ldots \ldots \ldots \ldots \ldots \ldots \ldots \ldots$ 4-10

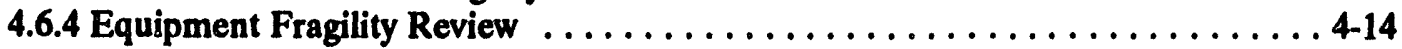

4.6.4.1 Failure Modes and Issues Reviewed . . . . . . . . . . . 4-15

4.6.4.2 Audit of Equipment Seismic Fragilities $\ldots \ldots \ldots \ldots \ldots \ldots \ldots$ 4-16

4.6.4.3 Review of Important Low Capacity Equipment $\ldots \ldots \ldots \ldots \ldots$ 4-20

4.6.4.4 Conclusions of Fragility Review ................ 4-21

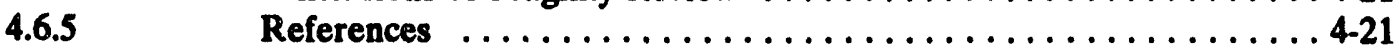

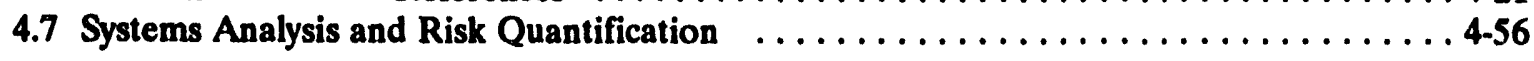




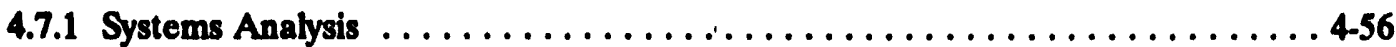

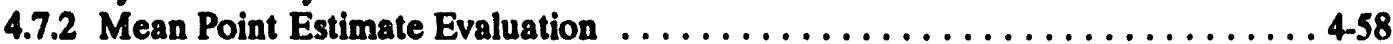

4.7.3 Uncertainty Analysis . . . . . . . . . . . . . . . . . . . . . 4-61

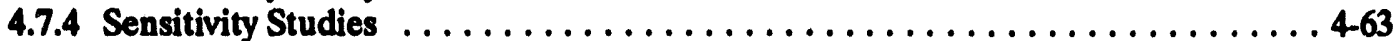

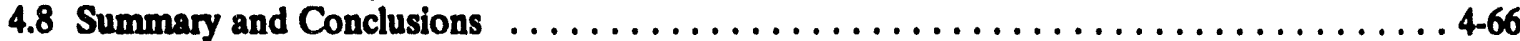

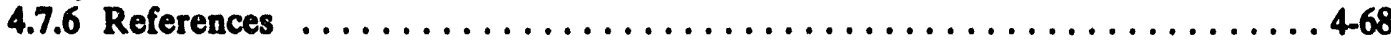

5. OTHER EXTERNAL EVENTS $\ldots \ldots \ldots \ldots \ldots \ldots \ldots \ldots \ldots \ldots \ldots \ldots \ldots \ldots \ldots \ldots \ldots$

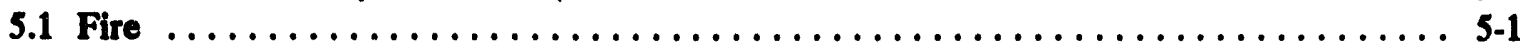

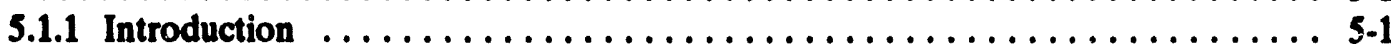

5.1.2 Methodology ................................ 5-1

5.1.3 Turbine Building Fire Scenarios ...................... 5-1

5.1.4 Requantification of Two Control Room Fire Scenarios ............. 5-2

5.1.5 Cable Spreading Room Fires ........................... 5-4

5.1.6 Summary of Analysis of Review Results .................. 5-5

5.2 Non-Seismic/Non-Fire External Events $\ldots \ldots \ldots \ldots \ldots \ldots \ldots \ldots \ldots \ldots \ldots \ldots$

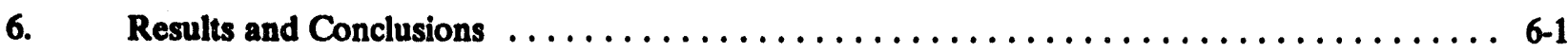

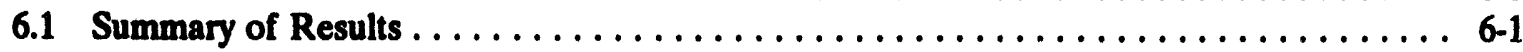

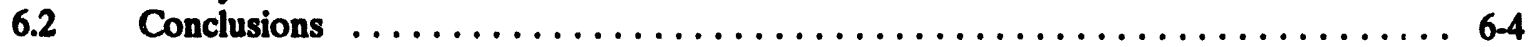

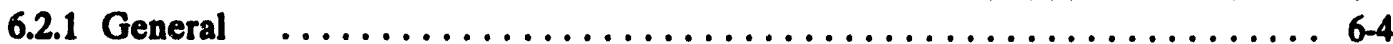

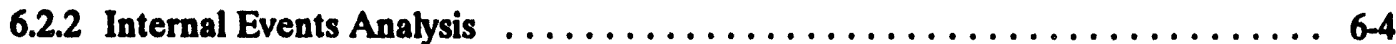

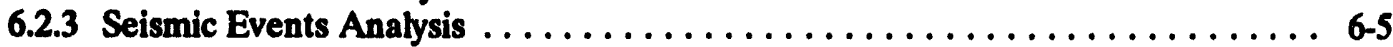

6.3 Suggested Enhancements for Future DCPRA Use $\ldots \ldots \ldots \ldots \ldots \ldots \ldots \ldots \ldots \ldots$ 6-6

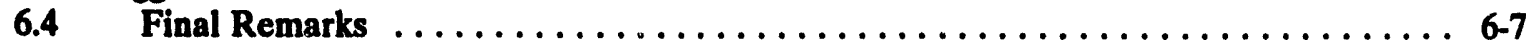

APPENDIX A

System Analyses for Selected Frontline Systems

A1: High Pressure Injection System

A2: Low Pressure Injection System

A3: Auxiliary Feedwater System

APPENDIX B System Analyses for Selected Support Systems

B1: Diesel Generator \& Diesel Fuel Transfer Systems

B2: Electrical Power Systems

B3: Auxiliary Saltwater System

B4: Component Cooling Water System

B5: Solid State Protection/Reactor Protection Systems

APPENDIX C DCPRA BASIC EVENT DATA DISTRIBUTIONS

C.1: Basic Event Definitions

C.2: Basic Event Distributions 
TABLE OF CONTENTS (continued)

APPENDIX D DCPRA DOMINANT ACCIDENT SEQUENCE MODEL

D1: Description of the Diablo Canyon Reduced Core Damage

Frequency Model

D2: Pair Importance Calculations by Conditional Split Fractions 


\section{LIST OF FIGURES}

FIGURE NO.

TITLE

PAGE NO.

2.41 Contribution of each earthquake range to the seismic core damage frequency (Figure 6-49 of DCPRA).

3.4-1 Annual exceedance frequency vs. leak rate for Westinghouse plants with four RCPs (lower bound, best estimate, upper bound) (figure is taken from Reference 20). ................ 3-20

Probability distributions for total core damage frequencies

due to various groups of initiating events. The leading

sequences of internal and external events constitute the

Dominant Sequence Model (DSM). . . . . . . . . . . . . . . . . . . . . . . 3-45

4.41 Total Aggregate Hazard Curves

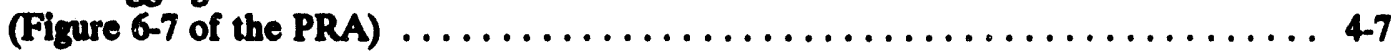

4.6-1

Configuration of the Simplified Auxiliary Building Model. . . . . . . . . . . 4-22

4.6-2

Rigid rectangular foundation geometry for the Simplified

4.6-3

Auxiliary Building Model ............................ 4-23

Comparison of median in-structure response for the Diablo

Canyon Auxiliary Building west core el. $140^{\circ}$ N-S Dir. . .................. . . .

Comparison of 84th percentile in-structure response for the Diablo Canyon Auxiliary Building

west core el. 140' N-S Dir. . ........................... 4-25

4.6-5 Comparison of median in-structure response for the Diablo

Canyon Auxiliary Building west core el. 140' E-W Dir. . . . . . . . . . . . . . 4-26

4.6-6 Comparison of 84th percentile in-structure response for the

Diablo Canyon Auxiliary Building west core el. 140' E-W Dir. . . . . . . . . . . 4-27

4.6-7 Diablo Canyon Auxiliary Building benchmark analysis

in-structure response core west el. 164' $\mathbf{N}$-S Dir. . . . . . . . . . . . . . . 4-28

4.6-8 Diablo Canyon Auxiliary Building benchmark analysis

in-structure response core west el. 164' E-W Dir. . . . . . . . . . . . . . . . . 4-29

4.6-9 Diablo Canyon Auxiliary Building benchmark analysis

in-structure response core west el. $1288^{\prime} \mathbf{N}-S$. . . . . . . . . . . . . . . 4-30

4.6-10 Diablo Canyon Auxiliary Building benchmark analysis

in-structure response core west el. $128^{\prime}$ E-W. . . . . . . . . . . . . . . . 4-31

4.6-11 Diablo Canyon Auxiliary Building benchmark analysis

in-structure response core west el. 115' $\mathrm{N}$-S. .................. 4-32

4.6-12 Diablo Canyon Auxiliary Building benchmark analysis

in-structure response core west el. 115 ' E-W. . . . . . . . . . . . . . . 43

4.6-13 Comparison of median in-structure response for the Diablo

Canyon Auxiliary Building west core el. 140' N-S Dir.

4.6-14 Comparison of 84th percentile in-structure response for

Diablo Canyon Auxiliary Building work

core el. 140' N-S Dir.

4.6-15 Comparison of Median in-structure response for the

Diablo Canyon Auxiliary Building west core

elev. 140' $E=$ W Dir. .................................4-36

4.6-16 Comparison of 84th percentile in-structure response for Diablo Canyon Auxiliary Building west core elev. 140' E-W Dir. .......................... 4-37

4.7-1 Seismic Early Frontline Tree (Figure $6-47$ of DCPRA) $\ldots \ldots \ldots \ldots \ldots \ldots \ldots .64$

4.7-2 DCPRA Electrical Support Tree (Figure 6-44 of DCPRA) $\ldots \ldots \ldots \ldots \ldots \ldots$.70

4.7-3 DCPRA Actuation and Mechanical Support Tree

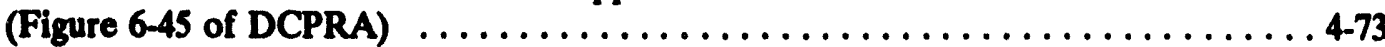




\section{LIST OF FIGURES (continued)}

FIGURE NO.

Sample Sheet of DCPRA Table 6-46

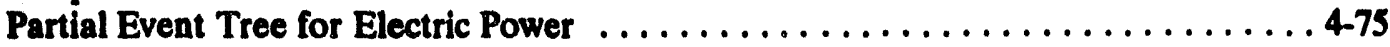

4.7-6

Simplified Logic Block Diagram for DCPRA

4.7-8

Contribution of Each Earthquake Range to the

4.7-9

Seismic Core Damage Frequency (DPD Versus Monte Carlo)

Plant Total Mean Fragility Curve ................ 4-78

Sensitivity Study on 230Kv Fragility Median

4-79 


\section{LIST OF TABLES}

TABLE NO.

TITLE

PAGE NO.

Overall DCRPA Mean CDF Estimates. $\ldots \ldots \ldots \ldots \ldots \ldots \ldots \ldots \ldots \ldots \ldots$ xix

2.1.1

2.1.2

2.4 .1

2.4 .2

2.4.3

2.4.4

2.4.5

2.4 .6

2.4.7

2.4.8

3.4.1

3.4.2a

3.4.2b

3.9.1a

3.9.1b

3.9.2a

$3.9 .2 \mathrm{~b}$

3.9.3

3.9.4

3.9.5

3.9.6

3.9.7

3.9.8

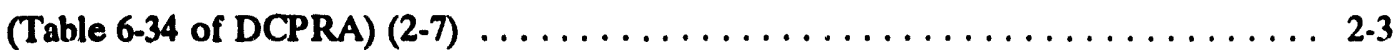

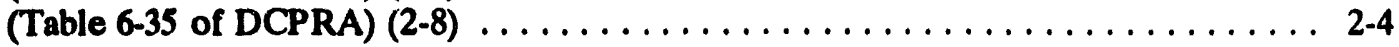

Initiating Event Categories Selected for Quantification

of the Diablo Station Risk Model

(Table 6-26 of DCPRA) (2-19) . . . . . . . . . . . . . . . . . . 2-12

Contributions of Mean Core Damage Frequency

(Table $6-55$ of DCPRA) $(2-21) \ldots \ldots \ldots \ldots \ldots \ldots \ldots \ldots \ldots \ldots \ldots \ldots \ldots \ldots \ldots \ldots \ldots .14$

Internal Initiating Event Contribution to Core Damage

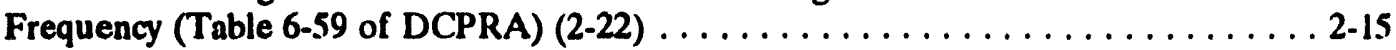

Functional Internal Event Scenarios Contributing to Core

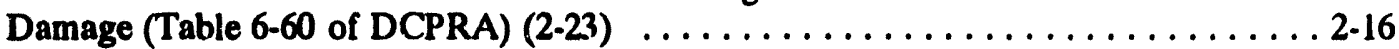

Diablo Canyon Probabilistic Risk Assessment

Summary Statistics (Table 6-54 of DCPRA) $\ldots \ldots \ldots \ldots \ldots \ldots \ldots \ldots \ldots .2-18$

Seismic Failures of Components Contributing to Core Damage

(Table 6-58 of DCPRA) ............................ 2-19

Core Damage Sequences Initiated by Fires and Floods

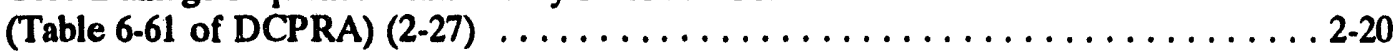

Other External Events Contribuling to Core Damage

(Table 6-62 of DCPRA) (2-28) . . . . . . . . . . . . . . . . . . . 21

Initiating Event Categories Selected for Quantification

of the Diablo Station Risk Modil

(Table $6-26$ of DCPRA) . . . . . . . . . . . 3.21

Initiating Events and Their Mean Frequencies/year

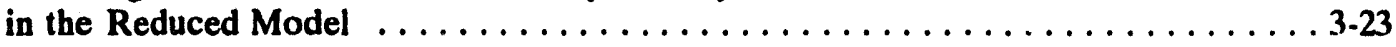

Initiating Events and Their Mean Frequencies/year

Not Included in the Reduced Model . . . . . . . . . . . . . . . . . 3-24

Initiating Event Contributions to Non-Seismic

Core Damage Frequency Dominant Sequence Model . . . . . . . . . . . . 3-46

Initiating Event Contributions to Internal Event Core

Damage Frequency Initiating Events Not Included in the

Dominant Sequence Model . . . . . . . . . . . . . . . . . . . . . . . . . 3-48

Comparison of Core Damage Frequency (CDF) Contribution by

Initiating Event Group . . ......................... 3-49

Comparison of Conditional Core Damage Probabilities by

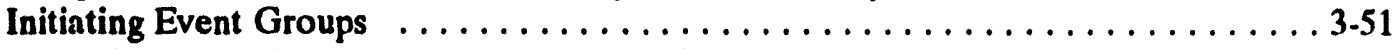

Event Scenarios Ranked According to Non-Seismic CDF

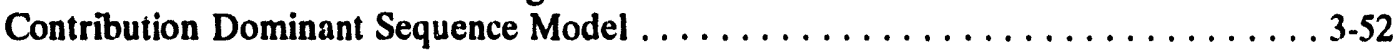

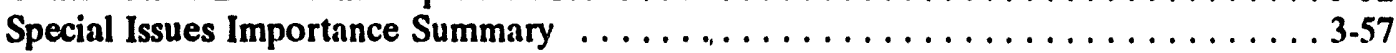

System/Operator Action Importances for Non-Seismic

Core Damage Frequency Ranking According

to System/Operator Action Importances $\ldots \ldots \ldots \ldots \ldots \ldots \ldots \ldots \ldots \ldots \ldots . \ldots \ldots$

Top Event Importances - Conditional Split Fractions

(Ranking According to Fussel-Vesely Importance) $\ldots \ldots \ldots \ldots \ldots \ldots \ldots \ldots$ 3-62

Unnormalized'System/Operator Action Importances for

Internal Event Initiators Dominant Sequence Model

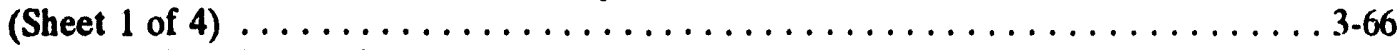

Unnormalized System/Operator Action Importances for

Fire/Flood Scenarios Dominant Sequence Model . 


\section{LIST OF TABLES}

TABLE NO.

TITLE

PAGE NO.

3.9 .9

Unnormalized Fussel-Vesely Importances of Support System

3.9.10

- Support System Pairs . . . . . . . . . . . . . . . . . . . . . . . . . . . 3-71

Unnormalized Fussel-Vesely Importances of Frontline System

- Support System Pairs . . . . . . . . . . . . . . . . . . . . . . . .

3.10 .1

Major Elements of Alternative Review Quantification .............. 3-75

3.10 .2

3.10.3

3.10.4A

Overall Internal Event CDF Estimates vs Review

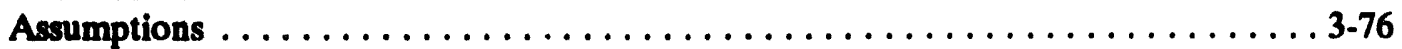

Comparison of Internal Event Contributions to Core

Damage Frequency (Ranking of Top Ten Internal Event

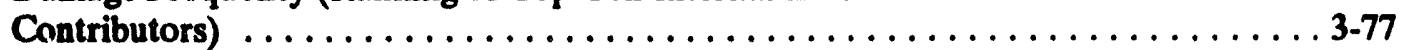

Ranking of Support Systems Importance

Internal Events-Case $4 \ldots \ldots \ldots \ldots \ldots \ldots \ldots \ldots \ldots \ldots \ldots \ldots \ldots \ldots .78$

3.10.4B Ranking of Front-Line Systems Importance

Internal Events-Case $4 \ldots \ldots \ldots \ldots \ldots \ldots \ldots \ldots \ldots \ldots \ldots \ldots \ldots . \ldots \ldots$

3.10.4C Ranking of Operator/Recovery Actions Importance

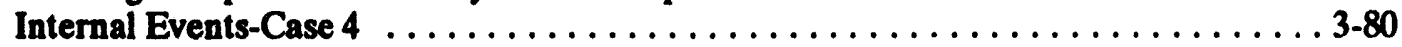

4.6.1

DCPRA Seismic Fragilities $\ldots \ldots \ldots \ldots \ldots \ldots \ldots \ldots \ldots \ldots \ldots \ldots \ldots$ 4-38

4.6.2 Earthquake Records Used to Develop Time Histories

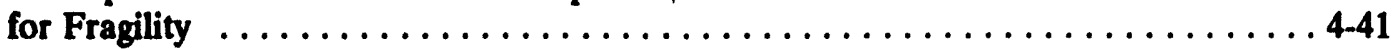

4.6.3 Fault Models Used to Generate Simulated Time Histories

For Fragility Studies . . . . . . . . . . . . .

4.6.4 Fixed Base Auxiliary Building Model Frequencies and Mass

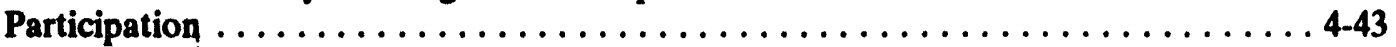

4.6.5 Foundation Rock Profile and Properties $\ldots \ldots \ldots \ldots \ldots \ldots \ldots \ldots \ldots \ldots \ldots \ldots$ 4.44

4.6.6 Building Impedance Function Embedment Factors $\ldots \ldots \ldots \ldots \ldots \ldots \ldots \ldots \ldots 4-45$

4.6.7 Impedance Function Value Comparison at $8 \mathrm{hz} \ldots \ldots \ldots \ldots \ldots \ldots \ldots \ldots$

4.6.8 Log Normal Standard Deviations for Auxiliary

4.6.9 Model Parameter Values and Scaling Factors $\ldots \ldots \ldots$

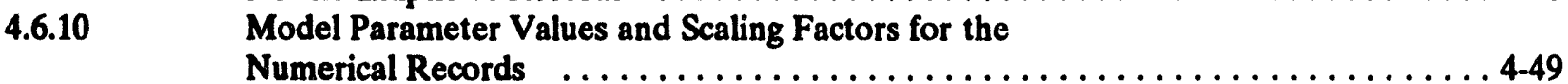

4.6.11 Comparison of PG\&E and Bechnmark Analysis Attributes . . . . . . . . . . 4-50

4.6.12 Comparison of PG\&E and Independent Analysis Attributes . . . . . . . . . 4-51

4.6.13 North-South Response Combined Variability $(B c) \ldots \ldots \ldots \ldots \ldots \ldots \ldots \ldots$ 4-52

4.6.14 East-West Response Combined Variability $(\mathrm{Bc}) \ldots \ldots \ldots \ldots \ldots \ldots \ldots \ldots \ldots$

4.6.15 North-South Response Combined Variability, Variability

Due to Ground Motion Only, and Variability Due to

SSI/Structure Uncertainties Only . . . . . . . . . . . . . . . .

4.6.16 East-West Response Combined Variability, Variability Due to

Ground Motion Only, and Variability Due to

SSI/Structure Uncertainties Only . . . . . . . . . . . . $\ldots \ldots \ldots \ldots$

4.7.1 Descriptions of Top Events on Seismic Early Frontline

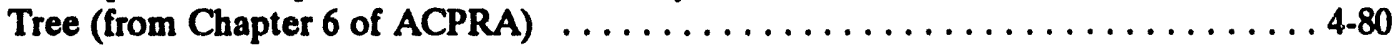

4.7.2 Definitions of Basic Events on Electric Power Support Tree . . . . . . . . 4-83

4.7.3 $\begin{aligned} & \text { Deninitions of Basic Events in Actuation and Mechanical } \\ & \text { Support Tree } \ldots \ldots \ldots \ldots \ldots \ldots \ldots \ldots \ldots \ldots \ldots \ldots \ldots \ldots \ldots \ldots\end{aligned}$ 


\section{LIST OF TABLES}

TABLE NO.

TITLE

PAGE NO.

4.7.4

4.7.5

4.7.6

4.7 .7

4.7.8

4.7-9

4.7-10

4.7-11

5.1.1

6.1.1

Boolean Expressions for Failure Events

(Derived from Table 6-43 of DCPRA) $\ldots \ldots \ldots \ldots \ldots \ldots \ldots \ldots \ldots \ldots \ldots .4 .6 \ldots$

Top Ten Point Estimate Accident Sequences . . . . . . . . . . . . . . . . . 4-88

Top Ten Point Estimate Accident Sequences - Grouped . . . . . . . . . . . . . . 4-89

Description of Logical Blocks as Provided by PG\&E . . . . . . . . . . . . 4-90

Comparison of DCPRA Seismic Core Damage Frequency

Percentiles with Monte Carlo Results . . . . . . . . . . . . . . . . . . . . 4-93

Ranking of Block Model Accident Sequences from Monte Carlo

Uncertainty Analysis . . . . . . . . . . . . . . . . . . . . . . . . . . . . . . 4-94

Accident Sequence Mean Frequency Increments for Intervals

on the Hazard Curve ............................... 4-95

Component Risk Reduction Potentials as Given in the DCPRA

and as Computed by Monte Carlo Approach . . . . . . . . . . . . . . . . . 4-96

Fire Scenario Core Damage Frequencies $\ldots \ldots \ldots \ldots \ldots \ldots \ldots \ldots \ldots \ldots \ldots$ 5-6

Overall DCPRA Mean CDF Estimates $\ldots \ldots \ldots \ldots \ldots \ldots \ldots \ldots \ldots \ldots \ldots$ 6-3 


\section{EXECUTIVE SUMMARY}

On February 23, 1984, the U.S. Nuclear Regulatory Commission (NRC) staff proposed a license condition for the Diablo Canyon Power Plant (DCPP) based in part on the Advisory Committee on Reactor Safeguards' (ACRS) letter of July 14, 1978, which recommended that "...the seismic design of Diablo Canyon be reevaluated in about 10 years taking into account applicable new information." The Commission adopted the staff recommendation and a license condition, consisting of four parts, was added to the operating license for DCPP. In part, this license condition required that the licensee (Pacific Gas and Electric Co. or PG\&E) assess the significance of conclusions drawn from the seismic reevaluation studies (as required by other parts of the license condition) utilizing a probalvilistic risk analysis and deterministic studies, as necessary, to assure the adequacy of seismic margins. In order to comply with the license condition, PG\&E developed a Long Term Seismic Program (LTSP). As a part of this LTSP, PG\&E performed a Level 1 Probabilistic Risk Assessment (PRA) including both internal and external cvents.

This report documests the results of the review of the Diablo Canyon PRA (DCPRA). Brookhaven National Laboratory (BNL) was selected by the NRC to assume lead technical responsibility for the review and the Probabilistic Risk Analysis Branch, Division of Systems Research, Office of Nuclear Reactor Research (ORES) was assigaed the programmatic responsibility for the PRA review.

The overall objective of the review was to provide assurance that the DCPRA was sufficiently complete :scope, accurate in modelling and data, and detailed such that the findings derived from it within the LTSP (and beyond) had a sound basis for acceptance.

Given that the driving force (from a regulatory perspective) for the PRA came from seismic concerns, the scope of the review was dominated by an internal events review and a seismic events review. Other external events were also reviewed bat to a lower level of effort. The internal events review concentrated on the traditional major elements of a PRA (i.e., initiating events, event trees, fault trees, data analysis and reduction, human reliability analysis, and leading accident sequences as well as independent sensitivity studies, importance analyses and requantification). The seismic events review concentrated on fragilities, hazards, structural analyses and uncertainty.

The DCPRA reflects the plant as it existed in the summer of 1988, including the changes in hardware and procedures that resulted from the early phases of the Long Term Seismic Program (LTSP). PG\&E identified the following modifications that were either completed or committed to by April 1988. These modifications were in response to issues identified by preliminary versions of the DCPRA and were included in the final DCPRA model submitted for revicw.

- Diesel Generator Fuel-Oil 'Iransfer System. Constant recirculation paths were designed to eliminate multiple pump starts. Connections for a backup portable fuel oil pump were also added.

- Charging Pump Backup Cooling. Hose connections were added to allow use of the firewater system for emergency cooling in the event of a total loss of component cooling water.

- Substation Spare Parts. Dedicated spare parts will be stored at the $230 \mathrm{kV}$ substation to allow rapid recovery of offsite power in the event of a substation failures.

- Valve Control Switch Replacement. The modification consisted of replacing threeposition valve switches with two-position valve control switches to prevent valve positions 
from changing in response to relay chatter during an earthquake.

The DCPRA relates to Unit 1 only. However, it also models interactions between the two units at the site, such as the possibility that the swing diesel generator may go to Unit 2 and the ability to crosstie auxiliary saltwater cooling capability across units.

The DCPRA was given a detailed and broad-scoped review. The basis for such a comprehensive review originated with the license condition discussed above that required a full reevaluation of the seismic risk of the plant. The seismic portion of the PRA, therefore, required special review attention. In order to provide a base from which to develop a seismic or other external event PRA model, the internal events PRA including all the systems analyses must first be formulated. Therefore, the internal event portion of the PRA was also given an indepth review. As fire-related initiating events contributed a significant fraction of the overall CDF, these too were included in the review.

The primary reason for the difference in internal event quantification presented in Table E.1 between the PG\&E and BNL results is the alternate conditional split fractions quantified for the auxiliary saltwater system and a higher initiating frequency for the LOOP event. Overall, requantification has not significantly changed insights regarding which initiating events are the major contributors to the core damage frequency.

As seen from Table E.1, the review did not propose alternative results for the seismic-induced CDF as the review found that there were no significant disagreements in the hazard and fragility estimates used in the DCPRA. In past PRA reviews, alternative estimates of these two parameters have been generally the cause of different CDF estimates. Parthy, this is the result of the interactive mode in which this review was conducted. This mode of review identified several issues and offered a number of sensitivity studies earty in the review such that the resolutions were incorporated in the final analysis or issues were shown to be not important. However, the main reason for the fewer disagreements is the very rigorous and detailed plant/site-specific analyses performed for both the hazard and fragility estimates in the DCPRA by PG\&E. Sensitivity studies in the seismic analysis were performed as part of the review to verify the DCPRA analyses and draw additional insights.

A number of the fire sequences were also requantified. The reasons for the higher fire-induced CDF estimated by the review are two fold: 1) higher estimate of frequency of the turbine deck fires; and 2) less credit to several operator actions used in the DCPRA to mitigate the fire scenarios.

From an overall perspective, (in the context of the license condition and the LTSP program) the seismic events are not dominant contributors to the CDF estimates for the Diablo Canyon plant. The overall seismic contribution in percentages varies from about 10 percent (review) to about 20 percent (DCPRA). Both the internal events and fire events contribute more to the CDF estimates than the seismic events based upon the results of this review.

A comparative analysis of the overall results of the DCPRA with those of nine other PRAs was also conducted. The estimated CDFs for the Diablo Canyon plant were found to be similar to those computed for other comparable PWRs.

The following are considered the major insights from the internal events review:

- The internal events are the major contributors to the total core damage frequency (roughly 65\%).

- The Loss of Offsite Power (LOOP) initiator is by far the greatest contributor to the 
internal event core damage frequency (roughly $1 / 3$ to $1 / 2$ ) and in the number of leading sequences. About $3 / 4$ of the LOOP-induced core damage is associated with extended unit blackout.

- Initiators representing support system (common cause) failures other than LOOP (i.e., Loss of one dc bus (L1DC), Total loss of auxiliary saltwater (LOSW), Total loss of component cooling water (LPCC), Loss of $480 \mathrm{~V}$ switchgear ventilation (LOSWV), Loss of control room ventilation (LOCV)) contribute in the range of $9-11$ percent to the internal event core damage frequency.

- Transients (other than LOOP) contribute between 25 and $\mathbf{4 0}$ percent to the internal event core damage frequency.

- The total contribution to the internal event core damage frequency of initiators belonging; to the Loss of Coolant Inventory group is small, about 5 to 10 percent.

- By examining the sequences, the following observation can be made regarding the leading individual sequences. No single internal event sequence contributes more than 3 percent to the non-seismic CDF.

- Among the operational failures of the plant, loss of feed and bleed cooling, loss of the auxiliary feed water system, and the occurrence of RCP seal LOCA are the most frequent.

- The following scenarios and their contribution to the non-seismic CDF are: Station Blackout (18\%); RCP Seal LOCA (with and without station blackout - 31\%); Primary Relief Valve Opens and Fails to Reclose (12\%); Bleed and Feed Cooling (12\%); and Pressurized Thermal Shock (4\%).

- The most important frontline systems were the auxiliary feedwcter system and primary system pressure relief.

- The most important support systems were the diesel generator systems, the $125 \mathrm{~V} \mathrm{dc}$ power systems and the auxiliary saltwater system.

- Based upon the review requantification, operator failure to actuate SSPS (upon failure to automatically actuate) went from one of the less important operator actions to the most important.

The following are considered to be the major insights from the seismic events review.

- The overall seismic CDF profile is dominated by accident sequences leading to station blackout (79\%). These may be due to loss of offsite power (LOOP) in conjunction with failures in the emergency diesel generator system, or due to failures of buildings (i.e., Turbine Building) or components (e.g., $4 \mathrm{kV}$ switchgear or $4 \mathrm{kV} / 480 \mathrm{~V}$ transformers) which can lead to station blackout with or without loss of offsite power. Of these blackout sequences, 53 percent do not directly fail the auxiliary feedwater system, but lead to reactor coolant pump seal failures, and hence, small LOCAs. Direct failures of the AFWS account for 22 percent of the station blackout sequence contributions.

- The single greatest contributor is the failure of the turbine building, followed by the loss of 230kV offsite power. There appear to be no overwhelmingly weak links in the plant.

- The seismic hazard analysis provided a reasonable probabilistic representation of the earthquake ground motions at the site considering the information developed in the LTSP program. The Hosgri fault zone was found to dominate the seismic hazard at the site. The Los Osos and San Luis Bay faults each contribute only a few percent to the wit hazard. Relative contributions to the total hazard from th witer faults is insignificant. Sensitivity studies showed the important parameters are slip rate, maximum magnitude, and ground motion attenuation. The spectral shape and uncertainties used in the fragility 
analyais are thought to be a reasonable estimate and consistent, in most part, with the detailed ground motion studies carried out in the LTSP.

- The fragility parameters for the structures and equipment were found to be reasonable. The level of effort in developing the seismic fragilities for equipment went well beyond those expended on previous seismic PRAs. A review of the identified and analyzed modes found that the appropriate failure modes have been considered.

- Based on the systems review, it appears that the sequence of scenarios and events which can be portrayed by the early front line tree represents a reasonable and complete approach to modeling the Diablo Canyon seismic scenarios.

- Using the results of the seismic PRA, the component and plant seismic margins were derived, and the margin against the 84 percent site-specific ground motion was calculated. It can be seen that all components whose failure will lead to seismic core damage have at least 40 percent margin over the site-specific ground motion. The median capacities were shown to be much higher.

- The largest contribution to the seismic-induced CDF comes from earthquakes with average spectral accelerations in the $2 \mathrm{~g}$ to $3 \mathrm{~g}$ range. This provides an important insight that for the high seismic sites, the seismic CDF estimates appear to be governed by seismic levels at or near the HCLPF value.

In conclusion, there were two primary goals associated with the review of the DCPRA. The first was to ensure that the DCPRA was sufficiently complete and accurate to provide a reasonable foundation upon which the necessary elements of the Diablo Canyon Long Term Seismic Program (LTSP) could be based. The second was to provide quality feedback, where appropriate, so that the DCPRA might become an even more useful tool in any future applications. It is believed that both goals were met. The review was sufficiently rigorous and broad enough in scope to conclude with a high degree of confidence that the DCPRA does indeed provide a reasonable foundation to support the LTSP. Feedback was also provided such that some elements of the DCPRA were modified during the review. Finally, other elements have been identified by PG\&E for future revisions as a result of the review. 
Table E.1 Overall DCRPA Mean CDF Estimates.

\begin{tabular}{|l|l|l|l|}
\hline & \multicolumn{1}{|c|}{$\begin{array}{c}\text { Initial DCPRA } \\
\text { Submittal }\end{array}$} & $\begin{array}{c}\text { Updated DCPRA } \\
\text { (Based Upon Review } \\
\text { Feedback) }\end{array}$ & $\begin{array}{c}\text { Alternative Results } \\
\text { Offered by the Review }\end{array}$ \\
\hline Internal Events & $1.3 \mathrm{E}-4(65 \%)$ & $1.3 \mathrm{E}-4(62 \%)$ & $2.1 \mathrm{E}-4(63 \%)$ \\
\hline Seismic Events & $3.7 \mathrm{E}-5(19 \%)$ & $3.7 \mathrm{E}-5(18 \%)$ & $3.7 \mathrm{E}-5(11 \%)$ \\
\hline Fire Events & $2.8 \mathrm{E}-5(14 \%)$ & $3.5 \mathrm{E}-5(17 \%)$ & $8.0 \mathrm{E}-5(24 \%)$ \\
\hline $\begin{array}{l}\text { Other External } \\
\text { Events (e.g. } \\
\text { Foods) }\end{array}$ & $6.9 \mathrm{E}-6(3 \%)$ & $6.9 \mathrm{E}-6(3 \%)$ & $6.9 \mathrm{E}-6(2 \%)$ \\
\hline Total & $2.0 \mathrm{E}-4$ & $2.1 \mathrm{E}-4$ & $3.3 \mathrm{E}-4$ \\
\hline
\end{tabular}




\section{ACKNOWLEDGEMENTS}

The authors would like to express our sincerest appreciation to Dr. Nilesh C. Chokshi, the NRC Program Manager, for his continual support and advice over the duration of this project.

The authors wish to express our thanks to Dr. Robert W. Youngblood, the original Principal Investigator for this project, for the significant help he has provided. This is especially true for this help in facilitating the extensive and sophisticated set of importance analyses documented throughout this report.

The authors wish to acknowledge Dr. Peter Kohut of BNL for his constructive collaboration on selected issues throughout the review as well as Eric Klamerus and John Lambright of the Sandia Laboratories Adverse Environment Safety Assessment Division for their insights and sensitivity calculations performed for the review of the seismic portion of the DCPRA.

Finally, we would like to express our appreciation to Florence $O^{\prime} B$ rien for an excellent job in putting this report into its final form. 


\section{INTRODUCTION}

\subsection{Background}

On Fobruary 23, 1984, the U. S. Nuclear Regulatory Commission (NRC) staff proposed a license condition for the Diablo Canyon Power Plant (DCPP) based in part on the Advisory Committee on Reactor Safeguards' (ACRS) letter of July 14, 1978, which recommended that "...the seismic design of Diablo Canyon be reevaluated in about 10 years taking into account applicable new information." The commission adopted the staff recommendation and a license condition, consisting of four parts, was added to the operating license for DCPP. In part, this license condition required that the licensee (Pacific Gas and Electric Co. or PG\&E) assess the significance of conclusions drawn from the seismic reevaluation studies (as required by other parts of the license condition) utilizing a probabilistic risk analysis and deterministic studies, as necessary, to assure the adequacy of seismic margins.

The license condition concerning the Seismic Design Bases Reevaluation Program read as follows:

"PG\&E shall develop and implement a program to reevaluate the seismic design bases used for the Diablo Canyon Nuclear Power Plant. The program shall include the following elements:

1. PG\&E shall identify, examine, and evaluate all relevant geologic and seismic data, information, and interpretations that have become available since the 1979 ASLB hearing in order to update the geology, seismology and tectonics in the region of the Diablo Canyon Nuclear Power Plant. If needed to define the earthquake potential of the region as it affects the Diablo Canyon Plant, PG\&E will also reevaluate the earlier information and acquire additional new data.

2. PG\&E shall reevaluate the magnitude of the earthquake used to determine the seismic basis of the Diablo Canyon Nuclear Plant using the information from Element 1.

3. PG\&E shall reevaluate the ground motion at the site based on the results obtained from Element 2 with full consideration of site and other relevant effects.

4. PG\&E shall assess the significance of conclusions drawn from the seismic reevaluation studies in Elements 1, 2, and 3, utilizing a probabilistic risk analysis and deterministic studies, as necessary, to assure adequacy of seismic margins."

In order to comply with the license condition, PG\&E developed a Long Term Seismic Program (LTSP) consisting of three phases. Phase I was the development of a detailed program plan to address the license condition. This plan was submitted for NRC staff review on January 30, 1985. Phase II consisted of a scoping study to refine the scope of work for Phase III and associated schedules. A report describing Phase II activities and conclusions was submitted to the NRC staff on January 30, 1986. Phase III represented the actual detailed studies and the final report was submitted in July 1988. As a part of this LTSP, PG\&E performed a Level 1 Probabilistic Risk Assessment (PRA) including both internal and external events.

This report documents the results of the review of the Diablo Canyon PRA (DCPRA). Brookhaven National Laboratory (BNL) was selected by the NRC to assume lead technical responsibility for the review and the Probabilistic Risk Analysis Branch, Division of Systems Research, Office of Nuclear Reactor Research (ORES) was assigned the programmatic responsibility for the PR.A review. 


\section{Introduction}

Probabilistic Risk Analysis Branch, Division of Systems Research, Office of Nuclear Reactor Research (ORES) was assigned the programmatic responsibility for the PRA review.

\subsection{Objectives and Scope of the Review}

The overall objective of the review was to provide assurance that the DCPRA was sufficiently complete in scope, accurate in modelling and data, and detailed stch that the findings derived from it within the LTSP (and beyond) had a sound basis for acceptance.

Given that the driving force (from a regulatory perspective) for the PRA came from seismic concerns, the scope of the revicw was dominated by an internal events review and a seismic events review. Other external events were also reviewed but to a lower level of effort. The internal event review concentrated on the traditional major elements of a PRA (i.e., initiating events, event trees, fault trees, data analysis and reduction, human reliability analysis, and leading accident sequences as well as independent sensitivity studies, importance analyses and requantification). The seismic events review concentrated on fragilities, hazards, structural analyses and uncertainty. Detailed descriptions of the review for internal and seismic events can be found in Sections 3 and 4 respectively.

\subsection{Organization of the Report}

Section 2 provides a description of the approach used by PG\&E to develop the DCPRA as well as a summary of the DCPRA results. Section 3 concentrates on the internal events review and its findings but also includes the remaining dominant non-seismic initiators in the quantification offered in Sections 3.9, 3.10 and Appendix D. Section 4 addresses the seismic-related review and its findings. Section 5 addresses the other (non-seismic) external events review with an emphasis on the fire-related portions of the DCPRA. Section 6 provides the overall summary and conclusions of the DCPRA review.

\subsection{Contributors}

The review of the DCPRA was a collegial effort involving a number of people and organizations. The NRC Program Manager charged with the overall responsibility for the review was $\mathrm{N}$. Chokshi. The BNL Principal Investigator was R. G. Fitzpatrick and the Technical Leader of the review was G. Bozoki. Also participating on the BNL review team was M. Sabek. The Technical Leader for the seismic review was M. Bohn of Sandia National Laboratory (SNL) under sub-contract to BNL. Also participating in the seismic review were M. Ravindra and J. Johnson of EQE Engineering. Other contributors from the NRC include T. Ryan (HRA methodology), A. Buslik (fire analysis) and P. Sobel (hazards). 


\section{OVERVIEW OF THE DCPRA APPROACH AND RESULTS}

\subsection{Introduction}

\subsubsection{Background}

This section of the report is intended to provide a brief synopsis of the DCPRA including a general plant description, a discussion of methodological approaches (especially novel features) and a condensed summary of the DCPRA results. Specific review results/findings are discussed throughout the remaining sections of the report and comparisons to the DCPRA results are made in those sections.

Diablo Canyon is a twin unit reactor site located on the California coast approximately midway between San Francisco and Los Angeles. Each unit employs a four-loop pressurized water reactor nuclear steam supply system (NSSS) furnished by Westinghouse Electric Corporation. The NSSS for each unit is contained within a steel-lined reinforced concrete structure that is capable of withstanding the pressure that might be developed as a result of the most severe design basis loss of coolant accident (LOCA).

The following paragraphs, extracted from the DCPRA, highlight some of the more important plant-specific features.

The vital $4 \mathrm{kV}, 480 \mathrm{~V}$, and dc systems are arranged to provide three redundancies, except for the instrument ac system which has four trains and six inverters. Emergency power for the two units is supplied by five diesel generators. One of these generators is a "swing" unit that is automatically transferred to the unit with the first need. The diesel generators have self-contained automotive-style radiators that do not require plant cooling water. For long-term operation, the fuel to the day tanks is replenished by two redundant fuel oil transfer pump trains supplied from underground fuel oil storage tanks.

Two redundant auxiliary saltwater system pumps at the intake structure pump seawater to the component cooling water heat exchangers. Three redundant component cooling water pumps are crosstied to the two component cooling water heat exchangers which in turn feed three headers. The three headers provide component cooling water to the fan coolers, reactor coolant pump seals, charging pumps, and other systems requiring heat removal. The auxiliary saltwater system and component cooling water systems may be crosstied between units if necessary.

The instrument air and nitrogen systems are classified as non-vital and for modelling purposes were assumed to fail; therefore, vital air-operated equipment that must operate is furnished with backup air bottles. These systems were analyzed to determine the amount of time that the operator could use the equipment before the backup air was exhausted. The recovery action modelled for these systems was manual operation of a valve or replenishment of the backup air.

The reactor charging system is important because it provides seal water to the reactor coolant pumps (RCPs). Loss of seal water will lead to failure of the seals and a resulting RCP seal LOCA. The charging system is dependent on component cooling water for pump cooling. However, the dependence on component cooling water may be mitigated by an emergency pump cooling system fed from the fire mains.

For completeness, Tables 2.1:1 and 2.1.2 have been included herein to provide simplified dependency tables of support systems to support systems and support systems to mainline (frontline) systems, respectively. These tables were taken directly from the DCPRA (Tables 6-34 and 6-35). 


\subsubsection{General}

The Diablo Canyon Nuclear Power Plant Unit 1 Probabilistic Risk Assessment (DCPRA) is a full scope, level 1 PRA. It utilizes a scenario-based approach to risk analysis, as any other PRA. The scenarios represent the plant and perconnel responses to any initiating event that causes the plant to depart from its otherwise normal state of operation. The scenarios are chains of events leading to a variety of end strites, including restablization of the plant or various lovels of plant damage up to and including core damage. The objective of the DCPRA was to determine the probuble frequency of having reactor core damage due to "all reasonably concetvable" accident initiating events, with an emphasis on seismically initiated scenarios. It was developed over several years by a large team of PRA specialists from Pickard, Lowe and Garrick (PLG) with the active help of a number of Pacific Gas and Electric Co. (PG\&E) personnel.

The DCPRA reflects the plant as it existed in the summer of 1988, including the changes in hardware and procedures that resulted from the prior phases of the Long Term Seismic Program (LTSP). PG\&E identified the following modifications that were either completed or committed to by April 1988. These modifications wero in resporise to iswes identified by earlierversions of the DCPRA and were included in the final DCPRA model submitted for review.

- Diesel Generator Fuel-Oil Transfer System. Constant recirculation paths were designed to eliminate multiple pump starts. Connections for a backup portable fuel oil pump were also added.

- Charging Pump Backup Cooling. Hose connections were added to allow use of the firewater system for emergency cooling in the event of a total loss of component cooling water.

- Subatation Spare Parts. Dedicated spare parts will be stored at the $230 \mathrm{kV}$ substation to allow rapid recovery of off-site power in the event of a substation failures.

- Valve Control Switch Replacemunt. The modification consisted of replacing three-position valve switches with two-position valve control switches to prevent valve positions from changing in response to relay chatter during an earthquake.

In addition to identifying a number of desiga/procedural changes from which the plant could benefit, the DCPRA has also been used to demonstrate the viability of Technical Specification changes. For example, during this review, PG\&E submitted a Technical Specification Change Request to NRC concerning the diesel generators and based upon the DCPRA. As BNL was already deeply involved in the review of the PRA and the diesel systems, NRC contracted BNL to review the PG\&E submittal. This review is documented in a BNL letter report from R. G. Fitzpatrick to N. Chokshi, dated September 11, 1989. Favorable findings were forthcoming from the review and NRC approved changes to the Diablo Canyon Technical Specifications.

The DCPRA relates to Unit 1 only. However, it also models interactions between the two units at the site, such as the possibility that the swing diesel generator may 80 to Unit 2 and the ability to crosstie auxiliary saltwater cooling capability across units. 


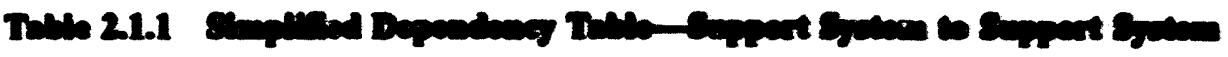

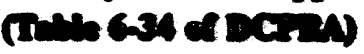

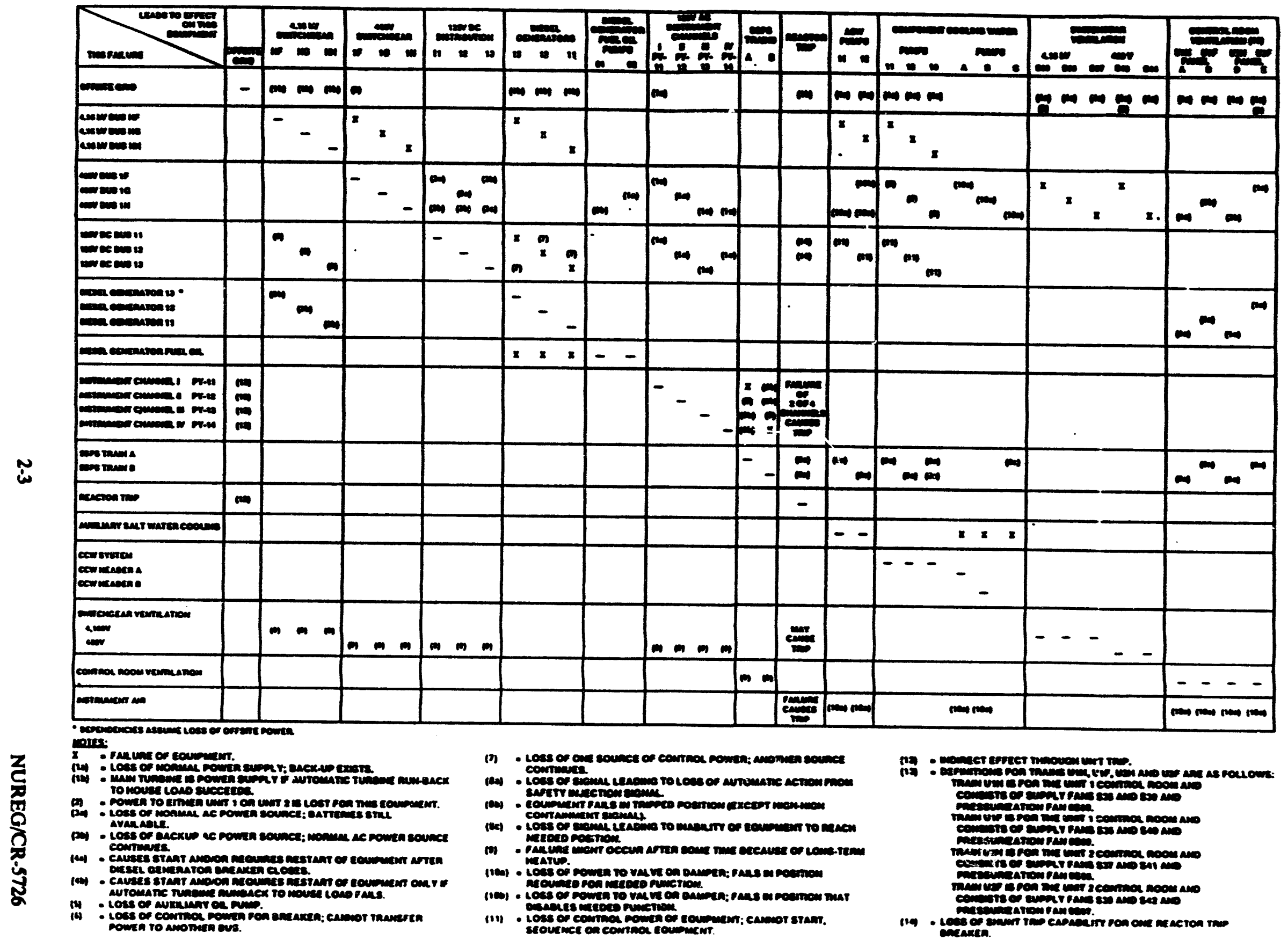


(Mntre 6.35 of DCrin)

\begin{tabular}{|c|c|c|c|c|c|c|c|c|c|c|c|c|c|c|c|c|c|c|}
\hline$=$ & 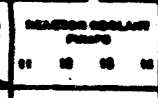 & $=-$ & $=$ & $=$ & & $=$ & $E$ & $E$ & $\because$ & $=$ & $=$ & $=$ & $=$ & $=$ & $\equiv$ & 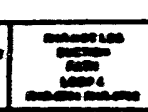 & 플 & $\equiv$ \\
\hline$-\infty$ & $\cdots$ & 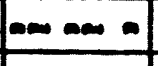 & $\cdots$ & - & & $-\infty$ & - & - & & & & & & $\ldots \ldots$ & & & $\infty$ & $\overline{-}$ \\
\hline$=$ & & & & & & & & & $\cdot$ & $\because \ldots$ & $\because$ & $1 .=$ & $1 \because .1$ & 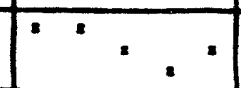 & & & & \\
\hline$E=$ & &.. & & & & & & & & & . & $\therefore$ & $\therefore$ & $\because 2.1$ & $\Xi$ & 8 & & $\Xi$ \\
\hline Ex: & $-\infty$ & $\therefore$ & $:::$ & $\approx$ & $\Xi$ & $\approx$ & $=$ & $=$ & $\therefore=$ & 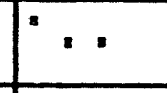 & - & $\therefore$ & 1.: & $-\infty \ldots$ & $\equiv$ & & $\infty$ & $\equiv$ \\
\hline$=$ & &.- & & & & & & & $\cdot$ & $=$ & $=$ & 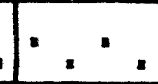 & $\because$. & 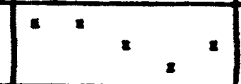 & $\Xi$ & & & \\
\hline$E=$ & $\Xi \Xi \Xi \vdots$ & $\Xi \Xi \Xi$ & & & & $\Xi$ & $:$ & $\vdots$ & & & & $\Xi \Xi \cdots$. & & & & $\approx$ & & \\
\hline$=$ & $E=z z$ & & $\vdots$ & $\equiv$ & $\approx$ & & $\Xi$ & & En & 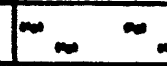 & $\approx$ & $\ldots$ & $-\infty$ & $-\infty-\approx$ & $=$ & & & : \\
\hline$=$ & & & -- & - & & & & & & & & & & & & & & \\
\hline$=$ & $\cdots$ & & & & & & & & & & $:$ & $\because=$ & & $\because: \vdots: \vdots$ & & & & \\
\hline$=$ & & $-\infty$ & & & & -1 & 2 & $\div$ & & $=$ & & tan & & $a$ & 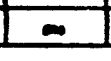 & & & \\
\hline
\end{tabular}

Mres:

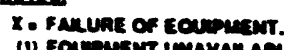

(1) Eov

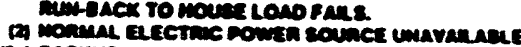

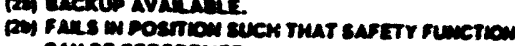

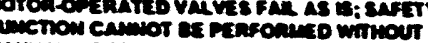

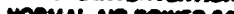

sounce unavak apere.

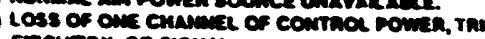
cuecurtar, on remele.

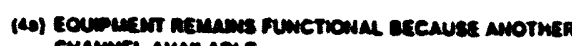

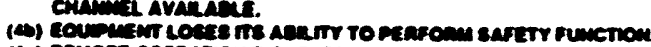

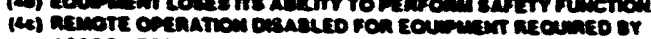

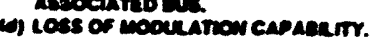

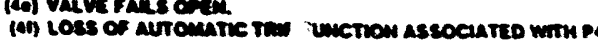

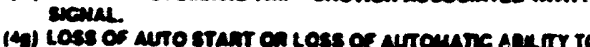
(1) cramas posinom

(3) Loss of pownth ro alocx valve.

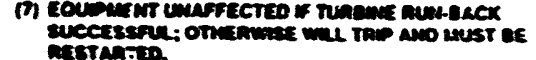

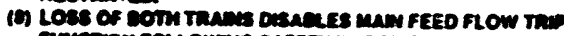

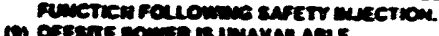

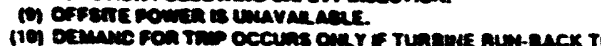
nowak lono smes

(ii) Num mon fow val ves closeso

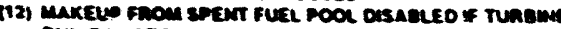

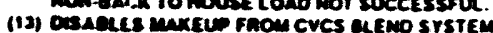




\subsection{Overall DCPRA Approach for Internal Events}

\subsubsection{Methodology}

The overall methodology of constructing the PRA model, i.e., of describing the accident scenarios due to various initiating events and rendering them amenable for quantification, is the "large event tree, small fault tree" approach. This methodology is the same as it was when introduced by PLG a decade ago, but is now incomparably refined. The refinements incorporate PLG's experience of preparing more than 20 prior PRAs. In addition, the DCPRA itself includes some other novel features compelling the reviewers to classify it as an "advanced state-of-the-art" PRA.

\subsubsection{Advanced and Novel Features of the DCPRA}

Initiating Events - In most of the previous PRAs, the number of initiating events were classified into 15-25 initiator categories. The DCPRA considers 50 initiating categories falling into six major groups such as:

1. LOCAs (9),

2. Transients (14),

3. Support System Faults (6),

4. Seismic Events (6),

5. Fire and Smoke (12), and

6. Flood, Jets and Sprays (3).

(Groups 3 through 6 represent essentially Common Cause Initiating Events.)

Extensive Application of Event Sequence Diagrams - The central element of the DCPRA is the plant event sequence model (see further explanation and a schematic diagram in Section 3.9.1) derived from so-called Event Sequence Diagrams, ESDs. The ESDs represent the flow charts of the unfolding accident scenarios in the plant. They were developed according to detailed operational analyses, which included specific steps in the Diablo Canyon emergency procedures, available control room indications and alarms and realistic training simulator experiences. The development required very strong collaboration of the PLG analysts with the licensed operators, the training personnel and the PRA team of the utility. The application of ESDs is a relatively new feature of state-of-the-art PRAs and represents a major advance in PRA methodology.

Preparation of Large Event Trees of Still Manageable Size from the ESDs - The plant event sequence model consists of large event trees. The large event trees were abstracted from the ESDs. In the ESDs, states (failure/success) of plant equipment, automatically or manually actuated "actions" or passive processes were grouped to define event tree top events. Sometimes a top event included more than one system or parts of a system. In order to keep the size of the event trees to still manageable size, the DCPRA used a modularized event tree technique. This technique provided successive event tree modules instead of super large event trees. The event tree modules facilitated the construction of the event sequence model and kept the model tractable for quantification. The technique exploited the functional and shared intersystem (support systems - support systems and support systems - frontline systems) dependences. It was developed from the realization that a) given an initiating event, various outcomes of the support system event trees (support system states) had identical impact on the frontline systems and b) numerous frontline system event tree scenarios ended up with identical final event sequences (i.e., each main tree scenario did not require its own unique long term subtree). 


\section{Overview of the DCPRA Approach and Results}

The event sequence model of the DCPRA is based upon a network of 13 event tree modules each consisting of numerous top events. These modules include:

a. Two support systom event tree modules: an electrical event tree with 21 top events and a mechanical event tree with 13 top events.

b. Seven earty frontline event troe modules, such as: general transient event tree with 14 top events, large LOCA event tree with 17 top events, steam generator tube rupture event tree with 14 main and 12 auxiliary top events, interfacing systems LOCA event tree with 17 top events, seisinic event tree with 13 top events, a transients without trip (ATWT) event tree with 14 top events, and a medium LOCA event tree with 18 top events.

c. Four long torm (LT) frontline event tree modules, such as: LT1 with 16 top events, LT2 with 5 top events, LT3 with 15 top events, and LT4 with 12 topevents.

Note that each event tree module represents a sizeable event tree and the associated computational complexity for its quantification is remarkable compared with those of earlier PRAs when a support state event tree contained only 5-6 top events and a frontline event tree had only 10-15 top events.

The modularized format of the event trees has the great advantage that it makes more efficient the quantification process and facilitates easier requantification of the entire risk model if it becomes necessary due to extensive changes. (The quantification of the event tree modules and the individual scenarios are described in Section 3.9.)

Enhanced Human Action Analysis - In order to make the risk model as realistic as possible, a large number of human actions (more than 70) were incorporated into the PRA in the following three levels: 1) below the event tree top event level, in the unavailability models of the systems (e.g., leaving a train in misalignment after test or maintenance);2) at the event tree top event level (e.g., manual initiation of a system or switchover from injection to recirculation) and in the recovery actions. The human actions themselves were subjected to very extensive analysis by means of an interactive six step process. The quantification for many of the actions was also made earthquake-level-dependent. (For more aspects of the DCPRA's human action analysis, see Section 3.8.) No previous PRA has applied human factors in such a versatile way in its risk model.

Treatment and Documentation of Internal Events - The event tree top event quantification (system models), the evaluation of accident scenarios to calculate the core damage frequency together with its uncertainty; i.e., the treatment of internal events, is highly sophisticated. Computers were used in somewhat novel ways to integrate model development and model documentation. The system analysis descriptions and presentation of the results are completely different from previous PRAs. They reflect a homogenized "assembly line" type analyzing process. Each system and subsystem model was developed in "cause table" format that displays the contributions to system unavailabilityfrom independent hardware failures, dependent (common cause) failures, testing, maintenance, inadvertent human errors, and other causes identified by each analyst. This framework facilitated detailed investigation of many system-level changes without requiring reevaluation of the full plant model. The system descriptions, given in computer software format, contain some of the most important documentation and reflect document and quality controls. 


\section{Overview of the DCPRA Approach and Results}

Defenalbility and Serutability of the Analyseg - The original submittal of the DCPRA (Chapter 6 of the LTSP final report) suffered from inadequate defensibility and scrutability of the analyses compared with other PRAs. The adequate defensibility and scrutability of the analyses is at least as important as the results. Most of the problems encountered during the review process were somehow connected with the problem of inadequate presentation/documentation. The systems poition of the documentation that was provided did contain most of the information for independent scrutiny but in a review-resistant and sometimes inaccessible format. System schematics, P\&IDs and electrical one-line drawings had to be requested for the review because the PRA presented only system reliability block diagrams. Explanations of certain analytical approaches in the system analyses were missing or found to be incomplete. In many cases significant detailed information was added to the PRA as the result of the review process; not because the results were incorrect but rather because the details were necessary for defensibility. The scrutiny of the software associated with each of the reviewed systems required the creation of additional review software. The reviewers believe that the review would have gone smoother if PG\&E had included as much detail as possible at the start of the review rather than to expend resources defending its approach afterward.

\subsection{Overall DCPRA Approach for Seismic Events}

The Seismic Events Anolysis of the DCPRA is a natural extension of the internal event analysis. In this approach, a single initiating event is considered which is the occurrence of the earthquake itself. The probability of earthquakes of different sizes is specified by a discrete family of hazard curves. Each curve is associated with a weight, normalized sio that the summation of weights equals unity.

The response of the plant to this earth quake is then identified through a front line event tree which has the major structures and safety systems as top events (see the Plant Event Sequence Miodel, Figure 3.9.1). Each of the accident sequences identified on the front line tree are related to two support trees - one mechanical support tree and one electrical suppiort tree. These support state trees are used to specify different combinations of successes or failures of the support systems for use in quantifying the accident sequences determined on the front line trees. Using the front line tree, a large number of accident sequences can be identified.

The accident sequences are in terms of basic events or groups of basic events and include both random and seismically induced failures. The seismic failure probabilities were derived from site specific fragilities, all of which utilized average spectral ground acceleration as the independent variable. Detailed building response analyses were carried out for the auxiliary building and the turbine building to determine median responses and associated variabilities. Fragilities were developed for both buildings and for components.

For the components, both structural and functional failures were considered:

a. For structural failures, the engineering factor of safety approach due to Kennedy, et al., was utilized. This approach was benchmarked, in a limited sense, with the detailed non-linear analyses of the turbine building.

b. For functional failures, seismic qualification test data were used with an assumed level of conservatism to determine the median failure probability.

For the structures, the engineering factor of safety approach was also used. The independent review of the component and structural fragilities is presented in Sections 4.6 .3 and 4.6.4. 
The accident sequences were quantified using mean values for each of the seismic component failure probabilities, and mean values for all random failure events, and then the dominant accident sequences were identified. Note that each different earthquake level yields a different accident sequence from the same branch on the event tree. From this initial point estimate quantification, a total of 791 accident sequences were identified and studied for the Diablo Canyon Seismic PRA.

Finally, the 791 sequences were used to build a simplified block model for which an uncertainty analysis using the Discrete Probability Distribution approach could be used to obtain mean core melt frequencies and uncertainty bands about the distribution of core melt frequency.

\subsection{Summary of DCPRA Results}

Table 2.4.1 (Table 6-26 of DCPRA) provides the complete list of all initiating events selected for quantification in the DCPRA. The overall DCPRA results are summarized in Table 2.4.2 (Table 6-55 of DCPRA) and show a mean total core damage frequency of $2.02 \mathrm{E}-4$. The following subsections provide a brief description of the various initiator categories.

\subsubsection{Internal Events}

In the DCPRA, internal events accounted for 63 percent of the overall CDF. Table 2.4.3 (Table 6-59 of DCPRA) provides the breakdown by individual initiating event. One readily sees that loss of off-site power (LOOP) represents the single most dominant contribution. Table 2.4.4 (Table 6-60 of DCPRA) presents the leading functional internal event scenarios leading to core damage. (BNL's review comments are provided in Section 3.)

\subsubsection{Seismic Events}

This section summarizes the results of the Diablo Canyon Probabilistic Risk Assessment for seismic events which result in core damage. The total mean core damage frequency due to seismic initiated events is 3.7E-5, contributing only 18 percent of the total (all events) core damage frequency on a mean basis, as shown in Table 2.4.5 (Table 6-54 of DCPRA). This table also shows the range of uncertainty associated with the core damage frequency. The median or 50th percentile is only $6.2 \mathrm{E}-6$, which is a factor of six less than the mean, as compared to the internal events which had the same median and mean core damage frequency. The 95 percent and 5 percent frequency for seismic events is 1.1E-4 and 9.3E-7 respectively, which gives an error factor of about 17. This is considerably higher than the error factor for total core damage frequency of about 3. However, it is not uncommon for the uncertainty of seismic events to be this high as demonstrated by the results of other nuclear power plant seismic PRAs.

The seismic risk assessment model was quantified for six discrete ranges of spectral acceleration within the range of $0.2 \mathrm{~g}$ to $4.0 \mathrm{~g}$. (Average spectral acceleration over the range 3.0 to $8.5 \mathrm{Hertz}$ was used as the independent parameter for both the hazard curves and the fragilities in an effort to reduce overall uncertainty, as discussed later in Section 4.5. The average spectral acceleration variable can be shown to be 2.34 times peak ground acceleration). The interval of each range as well as the contribution to core damage frequency of each earthquake level is shown in Figure 2.4.1 (Figure 6-49 of the DCPRA). In this figure it can easily be 
seen that most of the contribution comes form spectral accelerations between $2.0 \mathrm{~g}$ and $3.0 \mathrm{~g}$. In fact, 55 percent of the seismic core damage frequency comes from this range, and only 14 percent comes from earthquakes larger than $3.0 \mathrm{~g}$ spectral acceleration.

A total of 791 non-negligible accident sequences leading to core damage were determined from the mean point estimate evaluation of all the accident sequences resulting from the combinations of all the support states and all the branches of the seismic event tree. These were presented in an appendix (but without inclusion of complement events). No detailed discussion of the dominant scenarios was presented.

However, in response to BNL's request to identify which sequences were associated with certain "special issues" of interest to NRC, the PG\&E staff performed an importance study by sorting the point estimate model sequences into groups (defined by the "special issues" and summing the frequencies of each group to determine the relative contribution (see Section 3.9.3.6 and Table 3.9.4 for further details). The results of the seismic sequences are:

Issue 1 - Station Blackout:

$\begin{array}{lr}\text { RCP Seal LOCA Sequences } & 53 \% \\ \text { Failure of Secondary Heat Removal Sequences } & 22 \% \\ \text { Failure of PORVs to Open and Reclose } & 4 \% \\ \quad \text { Total } & 79 \%\end{array}$

Issue 2 - Failure of Reactor Trip:

$\begin{array}{lr}\text { Non-Station Blackout Sequences } & 2 \% \\ \text { Station Blackout Sequences } & 9 \% \\ \text { Total } & 11 \%\end{array}$

Issue 3 - RCP Seal LOCA:

$\begin{array}{ll}\text { Non-Station Blackout Sequences } & 5 \% \\ \text { Issue } 4 \text { - PORVs Open and Fail to Reclose } & 3 \% \\ \text { Issue 5 - Failure of Operator to Perform Feed and Bleed Cooling } & 0 \% \\ \text { Issue 6 - Pressurized Thermal Shock: Not modelled for seismic. } & \end{array}$

Note that each issue was examined separately. The contributions of each special issue group are not mutually exclusive, and should not be combined from different issues.

A component importance study was also included in the seismic portion of the DCPRA. The importance of each component to core damage was evaluated by setting the seismic failure probability of each component to zero, which gives a measure of the net reduction in core damage frequency that would occur is that component was made impervious to an earthquake. Table 2.4 .6 shows the reduced core damage frequency and the percent improvement for all the dominant components. The single greatest contributor is the turbine building, followed by the loss of $230 \mathrm{kV}$ off-site power. In addition, this table also shows the importance of key components if they were very weak and guaranteed to fail, and how much the core damage frequency would increase. 


\section{Overview of the DCPRA Approach and Results}

Based on the component importance study in the DCPRA and the "special issues" study provided later, the seismic risk profile could be determined. In summary, the overall picture is a risk profile dominated by accident sequences leading to station blackout (79\%). These may be due to loss of off-site power (LOSP) in conjunction with failures in the emergency diesel generator system, or due to failures of buildings (i.e., Turbine Building) or components (e.g., $4 \mathrm{kV}$ switchgear or $4 \mathrm{kV} / 480 \mathrm{~V}$ transformers) which can lead to station blackout with or without loss of off-site power.

Of these blackout sequences, 53 percent are those not directly failing the auxiliary feedwater system, but leading to reactor coolant pump seal cooling failures, and, hence, small LOCAs. Direct failures of the AFWS account for 22 percent of the station blackout sequence contributions. (As part of the review, the dominant sequences were determined as described in Section 4.7. Clearly, however, this is one area that was not fully documented in the DCPRA and resulted in the request for a significant additional amount of documentation.)

\subsubsection{Other External Events}

The contribution from the non-seismic external events come primarily from fire and flood scenarios arising within the plant. Altogether, they are responsible for 19 percent of the core damage frequency (16 percent fires, 3 percent floods). From the large number of potential fire and flood scenarios identified by a spatial interactions analysis, 17 were judged significant enough to warrant more thorough analysis and propagation through the full DCPRA model. The results for these fire and flood scenarios are summarized in Table 2.4.7 (Table 6-61 of DCPRA).

A number of additional external initiating events were also considered. The results are summarized in Table 2.4.8 (Table 6-62 of DCPRA). None of these events contributed substantially to the core damage frequency. All these results were reported in the DCPRA as conservative upper bound calculations, except for the hazardous chemical release for which a somewhat greater depth of study was performed. 
2 Overview of the DCPRA Approach and Results

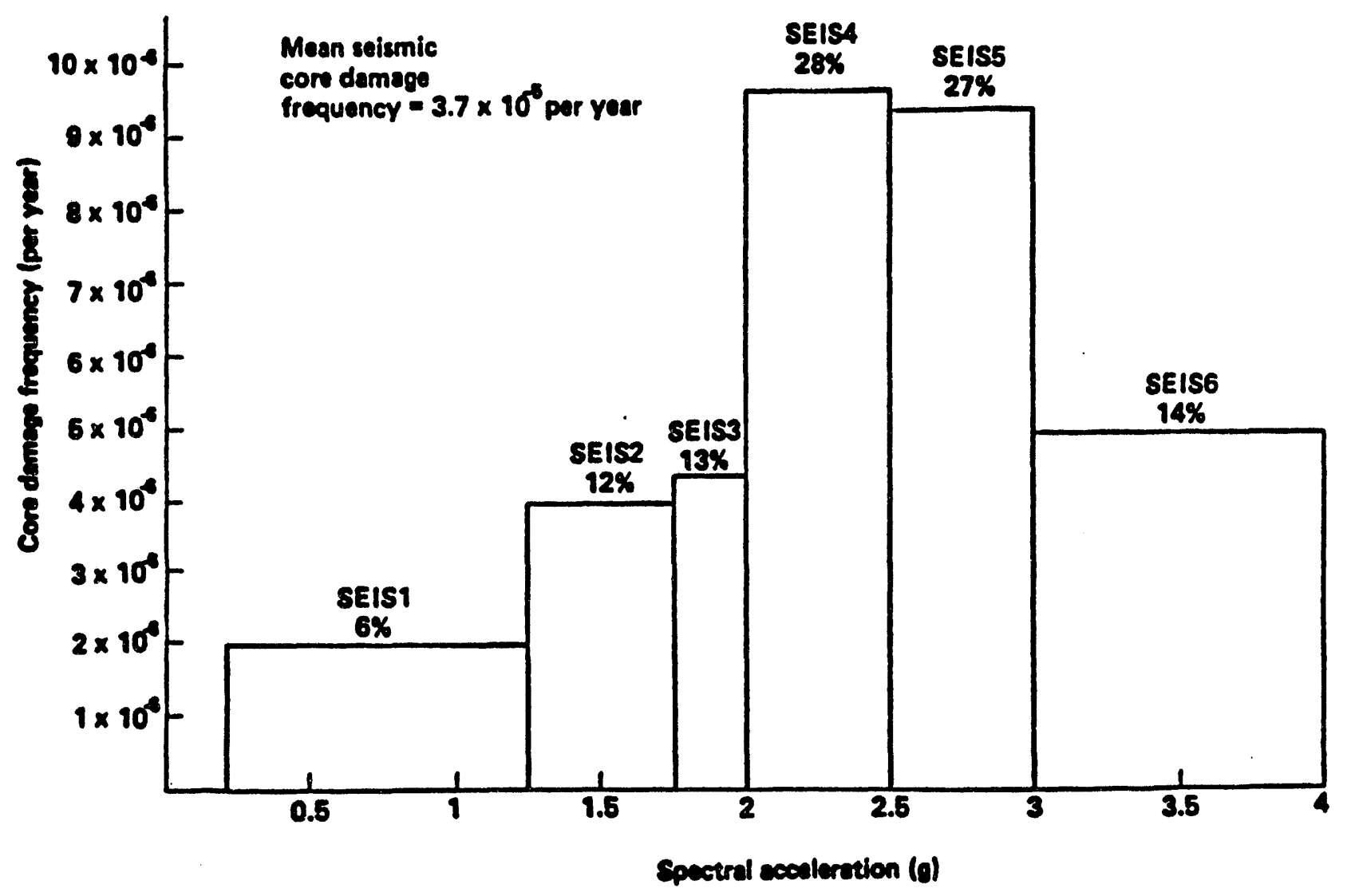

Figure 2.4.1 Contribution of Each Earthquake Range to the Seismic Core Damage Frequency (Figure 6-49 of DCPRA). 
Overview of the DCPRA Approach and Results

Table 2.4.1 Initiating Event Categories Selected for Quantification of the Diablo Station Risk Model (Table 6-26 of DCPRA)

\begin{tabular}{|c|c|c|c|}
\hline Group & & $\begin{array}{l}\text { Initiating Event Categories Selected } \\
\text { for Separate Quantification }\end{array}$ & $\begin{array}{c}\text { Code } \\
\text { Designator }\end{array}$ \\
\hline $\begin{array}{l}\text { Loss of Coolant } \\
\text { Inventory }\end{array}$ & $\begin{array}{l}1 . \\
3 . \\
3 . \\
4 . \\
5 . \\
6 . \\
6 \text {. } \\
6 \text { b. } \\
7 .\end{array}$ & $\begin{array}{l}\text { Excessive LOCA } \\
\text { Large LOCA } \\
\text { Medium LOCA } \\
\text { Small LOCA, nonisolable } \\
\text { Small LOCA, isolable } \\
\text { Interfacing systems LOCA } \\
\text { At RHR pump suction } \\
\text { At RHR pump discharge } \\
\text { Steam geherator tube rupture }\end{array}$ & $\begin{array}{l}\text { ELOCA } \\
\text { LLOCA } \\
\text { MLOCA } \\
\text { SLOCN } \\
\text { SLOCI } \\
\text { VS } \\
\text { VD } \\
\text { SGTR }\end{array}$ \\
\hline Transients & $\begin{array}{l}8 . \\
9 . \\
10 . \\
11 . \\
12 . \\
13 . \\
14 . \\
15 . \\
16 . \\
17 . \\
18 . \\
19 . \\
20 . \\
21 .\end{array}$ & $\begin{array}{l}\text { Reactor trip } \\
\text { Turbine trip } \\
\text { Loss of condenser vacuum } \\
\text { Closure of all MSIVs } \\
\text { Steam line break inside containment } \\
\text { Steam line break outside containment } \\
\text { Inadvertent safety injection } \\
\text { Main steam relief valve opening } \\
\text { Total main feedwater loss } \\
\text { (includes feedwater line break) } \\
\text { Partial main feedwater loss } \\
\text { Excessive feedwater } \\
\text { Closure of one main steam } \\
\text { isolation valve (MSIV) } \\
\text { Core power excursion } \\
\text { Loss of primary flow }\end{array}$ & $\begin{array}{l}\text { RT } \\
\text { TT } \\
\text { LCV } \\
\text { AMSIV } \\
\text { SLBI } \\
\text { SLBO } \\
\text { ISI } \\
\text { MSRV } \\
\text { TLMFW } \\
\text { PLMFW } \\
\text { EXFW } \\
\text { IMSIV } \\
\text { CPEXC } \\
\text { LOPF }\end{array}$ \\
\hline \multicolumn{4}{|l|}{$\begin{array}{l}\text { Common Cause } \\
\text { Initiating Events }\end{array}$} \\
\hline $\begin{array}{l}\text { Support System } \\
\text { Faults }\end{array}$ & $\begin{array}{l}22 . \\
23 . \\
24 . \\
25 . \\
26 . \\
27 .\end{array}$ & $\begin{array}{l}\text { Loss of offsite power } \\
\text { Loss of one DC bus } \\
\text { Total loss of auxiliary saltwater } \\
\text { Total loss of component couling water } \\
\text { Loss of } 480-\mathrm{V} \text { switchgear ventilation } \\
\text { Loss of control room ventilation }\end{array}$ & $\begin{array}{l}\text { LOSP } \\
\text { L1DC } \\
\text { LOSW } \\
\text { LOCC } \\
\text { LOSWV } \\
\text { LOCV }\end{array}$ \\
\hline Seismic Events & $\begin{array}{l}28 . \\
29 . \\
30 . \\
31 . \\
32 . \\
33 .\end{array}$ & $\begin{array}{l}0.2 \mathrm{~g} \text { to } 1.25 \mathrm{~g} \\
1.25 \mathrm{~g} \text { to } 1.7 \mathrm{~S}_{\mathrm{g}} \\
1.75 \mathrm{~g} \text { to } 2.0 \mathrm{~g} \\
2.0 \mathrm{~g} \text { to } 2.5 \mathrm{~g} \\
2.5 \mathrm{~g} \text { to } 3.0 \mathrm{~g} \\
3.0 \mathrm{~g} \text { to } 4.0 \mathrm{~g}\end{array}$ & $\begin{array}{l}\text { SEIS1 } \\
\text { SEIS2 } \\
\text { SEIS3 } \\
\text { SEIS4 } \\
\text { SEIS5 } \\
\text { SEIS6 }\end{array}$ \\
\hline
\end{tabular}


Table 2.4.1 (continued)

\begin{tabular}{ccc}
\hline Group & $\begin{array}{c}\text { Initiating Event Categories Selected } \\
\text { for Separate Quantification }\end{array}$ & $\begin{array}{c}\text { Code } \\
\text { Designator }\end{array}$ \\
\hline
\end{tabular}

Fire and Smoke

34. Loss of both motor-driven AFW pumps

FS1

35. Loss of all charging pumps and MSIV closure FS2

36. Loss of component cooling

FS3

37. Loss of control ventilation

FS4

38. Loss of auxillary saltwater

39. Loss of $4-\mathrm{kV}$ buses HF and HG

40. Loss of $4-k V$ buses $H G$ and $H H$

41. Loss of $4-\mathrm{kV}$ buses HF, HG, and HH

FS5

FS6

FS7

42. Control room fire at vertical board VB-1 CR1

43. Control room fire at vertical board VB-2 CR2

44. Control room fire at the interface of CR3 vertical boards VB-2 and VB-3.

45. Control room fire at vertical board VB-4 CR4

46. Cable spreading room fire one CS1

47. Cable spreading room fire two CS2

Flood, Jets, and Sprays (pipe breaks)
48. Loss of all auxiliary feedwater

49. Loss of both motor-driven AFW pumps

50. Loss of auxiliary saltwater
FS9

FS10

FS11 
2 Overview of the DCPRA Approach and Results

Table 2.4.2 Contributions of Mean Core Damage Frequency

(Table 6-55 of DCPRA)

Initiating Event Category

LOCAS

Interfacing system LOCAs

Steam generator tube rupture

Transients

Loss of offsite power

Loss of one DC bus

Loss of auxiliary saltwater or component cooling water

Loss of ventilation

TOTAL INTERNAL EVENTS

Seismic events

Fires

Floods, jets, and sprays

Chemical hazards

TOTAL EXTERNAL EVENTS

TOTAL
Mean Core Damage

Frequency (Per Year)

1.1-5

5.6-7

$3.0-6$

5.3-5

4.1-5

9.2-6

4.3-6

2.7-6

1.3-4

3.7-5

3.2-5

6.6-6

3.5-7

7.6-5

$2.02-4$
63

18

16

3

$<1$

37

Contribu-

tion

6

1

2

26

20

5

2

1

100 
2 Overview of the DCPRA Approach and Results

Table 2.4.3 Internal Initiating Event Contribution to Core Damage Frequency

(Table 6.59 of DCPRA)

\section{Initlating Event Category}

1. Excessive LOCA

2. Large LOCA

3. Medium LOCA

4. Small LOCA, nonsolable

5.. Small LOCA, isolable

6. Interfacing systems LOCAs

6.a. At RHR pump suction

6.b. At RHR pump discharge

7. Steam generator tube rupture

8. Reactor trip

9. Turbine trip

10. Loss of condenser vacuum

11. Closure of all MSIVs

12. Steamline break inside containment

13. Steamline break outside containment

14. Inadvertent safety injection

15. Main steam relief valve opening

16. Total MFW loss

17. Partial MFW loss

18. Excessive feedwater

19. Closure of one main steam isolation valve

20. Core power excursion

21. Loss of primary flow

22. Loss of offsite power

23. Loss of one DC bus

24. Total loss of auxiliary saltwater

25. Total loss of CCW

26. Loss of $480 \mathrm{~V}$ switchgear ventilation

27. Loss of control room ventilation

TOTAL

\author{
Core Damage \\ Frequency \\ (Per Year)
}

Contribution
(Percent)

2.7-7

0.2

2.5-6

2.0

5.8-6

4.6

9.0-7

0.7

1.7-6

1.4

5.0-7

0.4

5.8-8

$<0.1$

3.0-6

1.6-5

1.4-5

7.0-7

2.4-7

2.3-6

2.7-6

6.0-7

5.0-8

8.0-7

1.1-5

3.0-6

9.0-7

3.0-7

1.0-6

4.1-5

9.2-5

1.3-6

3.0-6

1.5-6

1.2-6

2.4

12.5

11.2

0.6

0.2

1.8

2.2

0.5

$<0.1$

0.6

8.8

2.4

0.7

0.2

0.8

32.4

7.3

1.0

2.4

1.2

1.0

1.3-4

100 
2 Overview of the DCPRA Approach and Results

Table 2.4.4 Functional Internal Event Scenarios Contributing to Core Damage (Table 6-60 of DCPRA)

\begin{tabular}{|c|c|c|c|c|c|c|}
\hline leonarlo & $\begin{array}{l}\text { Prequency } \\
\text { (Per Yeer) }\end{array}$ & $\begin{array}{c}\text { Contribution } \\
\text { (Poreent) } \\
\end{array}$ & Inlelator & Direct Fallures & $\begin{array}{l}\text { Purther } \\
\text { Recovery } \\
\text { Poestble } \\
\end{array}$ & $\begin{array}{l}\text { Dependent } \\
\text { Fellures }\end{array}$ \\
\hline 1 & $5.36-6$ & 3.1 & $\mathbf{B T}$ & $\begin{array}{l}\text { Fallure to malntain } \\
\text { hot standby }\end{array}$ & Yes & $\begin{array}{l}\text { Unspecifled } \\
\text { Operator Enors }\end{array}$ \\
\hline 2 & $4.96-6$ & 2.9 & $\mathbf{T}$ & $\begin{array}{l}\text { Falluse to maintain hot } \\
\text { standby }\end{array}$ & Yes & $\begin{array}{l}\text { Unspectfled } \\
\text { Operator Errors }\end{array}$ \\
\hline 3 & $3.53-6$ & 2.0 & PLMFW & $\begin{array}{l}\text { Fallure to maintain hot } \\
\text { standby }\end{array}$ & Yes & $\begin{array}{l}\text { Unspeciffed } \\
\text { Operator Errors }\end{array}$ \\
\hline 4 & $2.28-6$ & 1.3 & MLOCA & $\begin{array}{l}\text { Manual switchover to } \\
\text { recireulation }\end{array}$ & No & $\begin{array}{l}\text { Fathure of } \\
\text { Recirculation }\end{array}$ \\
\hline $\boldsymbol{s}$ & 2.13-6 & 1.2 & Locc & $\begin{array}{l}\text { Fall to allgn backup } \\
\text { cooling to charglng pumps } \\
\text { for RCP seal injection }\end{array}$ & No & $\begin{array}{l}\text { Component } \\
\text { Cooling Water. } \\
\text { Churging SI } \\
\text { Pumps, RCP } \\
\text { Seal LOCA }\end{array}$ \\
\hline 6 & $1.72-6$ & 1.0 & LDC & $\begin{array}{l}\text { DC power train } H \text {, } \\
\text { one AFW motor-driven } \\
\text { pumps }\end{array}$ & Yos & $\begin{array}{l}\text { Instrument } \\
\text { Channals 12, } \\
\text { 13, 14; } 2 \text { AFW } \\
\text { Pumps; Bleed } \\
\text { and Feed } \\
\text { Cooling }\end{array}$ \\
\hline 7 & $1.71-6$ & 1.0 & MLOCA & Both RHR pump trains & No & $\begin{array}{l}\text { Fallure of } \\
\text { Injection }\end{array}$ \\
\hline 8 & $1.70-6$ & 1.0 & LIDC & $\begin{array}{l}\text { 4-kV vital bus HH and } \\
\text { one AFW motor-driven } \\
\text { pumps }\end{array}$ & Yes & $\begin{array}{l}\text { Instrument } \\
\text { Channels I2, } \\
\text { 14; } 2 \text { AFW } \\
\text { Pumps; Bleed } \\
\text { and Feed } \\
\text { Cooling }\end{array}$ \\
\hline 9 & $1.40-6$ & 0.8 & EXFW & $\begin{array}{l}\text { Fallure to maintain hot } \\
\text { standby }\end{array}$ & Yes & $\begin{array}{l}\text { Unspecifled } \\
\text { Operator Errors }\end{array}$ \\
\hline 10 & $1.27-6$ & 0.7 & LLCA & Accumulators & No & $\begin{array}{l}\text { Pallure of } \\
\text { Injection }\end{array}$ \\
\hline 11 & $1.25-6$ & 0.7 & $\mathbf{R T}$ & $\begin{array}{l}\text { Instrument channel 13, } \\
\text { auxlliary foedwater, bleed } \\
\text { and foed cooling }\end{array}$ & Yes & None \\
\hline 12 & $1.25-6$ & $\begin{array}{c}0.7 \\
:\end{array}$ & $\mathbf{R T}$ & $\begin{array}{l}\text { Instrument channel II, } \\
\text { auxiliary feedwater, bleed } \\
\text { and feed cooling }\end{array}$ & Yes & None \\
\hline 13 & $1.24-6$ & 0.7 & LOOP & $\begin{array}{l}\text { Swing dlesel goes to Unit } 2 \text {, } \\
\text { euxiliary feedwater, vessel } \\
\text { intogrity falls due to PTS }\end{array}$ & No & None \\
\hline 14 & $1.16-6$ & 0.7 & $\mathbf{T T}$ & $\begin{array}{l}\text { Instrument channel 13, } \\
\text { auxiliary feedwater, bleed } \\
\text { and foed cooling }\end{array}$ & Yes & None \\
\hline
\end{tabular}


2 Overview of the DCPRA Approach and Results

Table 2.4.4 (continued)

\begin{tabular}{|c|c|c|c|c|c|c|}
\hline Seonerte & $\begin{array}{l}\text { Iroqueney } \\
\text { (Per Year) }\end{array}$ & $\begin{array}{l}\text { Contributlon } \\
\text { (Percent) }\end{array}$ & Inltiator & Direct Eallures & $\begin{array}{l}\text { Further } \\
\text { Recovery } \\
\text { Posalble }\end{array}$ & $\begin{array}{c}\text { Dependent } \\
\text { Pallures }\end{array}$ \\
\hline 13 & $1.16-6$ & 0.7 & $\mathbf{T T}$ & $\begin{array}{l}\text { Instrumont obannel I1, } \\
\text { auxdllary foedwator, bleed } \\
\text { and foed coollas }\end{array}$ & Yos & None \\
\hline 16 & $1.16-6$ & 0.7 & $\mathbf{R T}$ & $\begin{array}{l}\text { Auxillary foedwater, bloed } \\
\text { and feed coollag }\end{array}$ & Yes & None \\
\hline 17 & $1.14-6$ & 0.7 & $\mathbf{T T}$ & $\begin{array}{l}\text { Auxdliary foodwater, bleed } \\
\text { and feod coollas }\end{array}$ & Yos & None \\
\hline 18 & $1.13-6$ & 0.7 & LoOP & $\begin{array}{l}\text { Dieasl for bus HH, PORV } \\
\text { gticke open and is not } \\
\text { bolated, RHR pump trato } \\
\text { A falls, fallure to recover } \\
\text { AC bofore reeireulation } \\
\text { required }\end{array}$ & Yos & $\begin{array}{l}\text { Fathure of } \\
\text { Rectrculatloa }\end{array}$ \\
\hline
\end{tabular}


2 Overview of the DCPRA Approach and Results

Table 2.4.5 Diablo Canyon Probabilistic Riak Asseasment Summary Statiatics (Table 6.54 of DCPRA)

\begin{tabular}{|c|c|c|c|c|c|}
\hline \multirow[b]{2}{*}{ Contributor } & \multicolumn{4}{|c|}{ Core Demage Frequency } & \multirow{2}{*}{$\begin{array}{l}\text { Most Likely } \\
\text { Recurrence } \\
\text { Rate }\end{array}$} \\
\hline & $\underset{\sin }{\text { Porcentile }}$ & $\begin{array}{c}\operatorname{sosh} \\
\text { Percentlle }\end{array}$ & Mean & $\begin{array}{c}\text { Osth } \\
\text { Porcentile }\end{array}$ & \\
\hline Solemic Events & $9.3 \times 10^{-9}$ & $6.2 \times 10^{-4}(553)$ & $3.7 \times 10^{-5}(185)$ & $1.1 \times 10^{-4}$ & $\begin{array}{l}1 \text { in } 160,000 \\
\text { years }\end{array}$ \\
\hline Internal Events & $3.2 \times 10^{-1}$ & $113 \times 10^{-4}(825)$ & $1.3 \times 10^{-4}(63 \%)$ & $2.3 \times 10^{-4}$ & $\begin{array}{l}1 \text { in } 10,000 \\
\text { years }\end{array}$ \\
\hline $\begin{array}{l}\text { Orher Excomal } \\
\text { Events }\end{array}$ & $4.1 \times 10^{-6}$ & $1.5 \times 10^{-8}(13 \%)$ & $3.9 \times 10^{-5}(19 \%)$ & $1.0 \times 10^{-4}$ & $\begin{array}{l}1 \text { in } 65.000 \\
\text { jears }\end{array}$ \\
\hline TOTAL & $7.7 \times 10^{48}$ & $1.5 \times 10^{-}(100 \%)$ & $2.0 \times .10^{-}(100 \%)$ & $4.0 \times 10^{-4}$ & 1 in 7,000 years \\
\hline
\end{tabular}


2 Overview of the DCPRA Approach and Results

Table 2.4.6 Selamic Fallures of Components Contributing to Core Damage

(Table 6.58 of DCPRA)

\begin{tabular}{|c|c|c|c|c|c|}
\hline \multirow[b]{3}{*}{ Component } & \multirow[b]{3}{*}{$\begin{array}{c}\text { Does Fallure } \\
\text { Ounrantee } \\
\text { Core } \\
\text { Demese? } \\
\end{array}$} & \multicolumn{4}{|c|}{ Imporience } \\
\hline & & \multicolumn{2}{|c|}{$\begin{array}{c}\text { Impoct If Very sirons, } \\
\text { that is, if impervlous } \\
\text { to Earthqueke }\end{array}$} & \multicolumn{2}{|c|}{$\begin{array}{l}\text { Impect If Very Woak. } \\
\text { inat is, If Guerranteed } \\
\text { to Foll }\end{array}$} \\
\hline & & $\begin{array}{c}\text { Core } \\
\text { Demage } \\
\text { Erequency } \\
\end{array}$ & $\begin{array}{c}\text { Percent(1) } \\
\text { Improvement } \\
\text { In Mean } \\
\text { solemie Core } \\
\text { Demege } \\
\text { Froquency } \\
\end{array}$ & $\begin{array}{r}\text { Core } \\
\text { Demase } \\
\text { Prequency } \\
\end{array}$ & $\begin{array}{c}\text { Porceni(1) } \\
\text { Degredetlon } \\
\text { In Meen } \\
\text { solismle Core } \\
\text { Demege } \\
\text { Frequency } \\
\end{array}$ \\
\hline $\begin{array}{l}\text { Orfploal core damage } \\
\text { trequesey }\end{array}$ & - & $3.7-5$ & - & $3.7-5$ & - \\
\hline Turbloe bullding sbeerwall & Yos & 2.6-5 & 30.5 & & \\
\hline 230-kV oftalie power & No & $2.8-5$ & 24.4 & & \\
\hline Vitu DC & No & & & & \\
\hline Exenesive LOCA & Yos' & & & & \\
\hline Diesul geverator control Pasel & No & $3.3-5$ & 10.0 & & \\
\hline 4,160-kV/480V trandormers & Yos & $3.5-5$ & 5.0 & & \\
\hline steam generators & Yes & $3.5-5$ & 3.4 & & \\
\hline 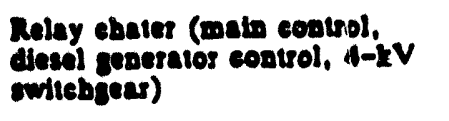 & No & $3.6-5$ & 2.4 & & \\
\hline DOP plpies and supports & No & $3.6-5$ & 2.2 & & \\
\hline Pronsurtaes PORV & No & $3.6-5$ & 0.7 & $1.4-4$ & 291 \\
\hline Strut for nurbien bulling & No & $3.7-5$ & 0.4 & & \\
\hline 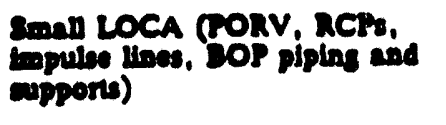 & No & & & $1.4-4$ & 291 \\
\hline 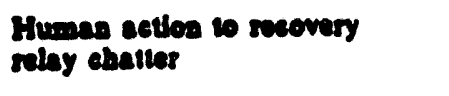 & No & & & $1.0-4$ & 173 \\
\hline 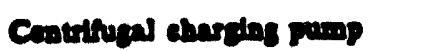 & No & & & $3.7-3$ & 0.2 \\
\hline
\end{tabular}


2 Overview of the DCPRA Approach and Results

Table 2.4.7 Core Damage Sequences Initiated by Fires and Floods (Table 6.61 of DCPRA)

\section{Initiator}

1. CS2

(Cable Spreading Room Fire)

2. CS1

(Cable Spreading Room Fire)

3. FS8 (Turbine Building Fire)

4. CR4

(Control Room Fire)

5. FS11

(Pipe Break in CCW Pump Room)

6. All Other Fires and Floods
Fire-Related Fallures

Pressurizer PORV sticks open

Auxiliary saltwater and component cooling water

Fail of all three trains of vital 4-kV switchgear

Failure of all vital $4-\mathrm{kV}$ breakers; 4-kV buses, HF, HG, and HH deenergized

CCW fails
Sequence Frequency

Independent Failures

Operators abandon control (Per Year)

$1.20-5$ room and fail to isolate PORV from hot shutdown panel. No credit for establishing recirculation from outside the control room.

Operators fail to either trip the RCPs or reestablish CCW.

Core damage assumed from resulting RCP seal LOCA.

Operators fail to trip RCPs or to reestablish vital $A C$ before core uncovery due to RCP seal LOCA.

$7.7-6$

Operators fail to align fire water cooling to charging pumps to avoid RCP seal LOCA. 
2 Overview of the DCPRA Approach and Results

Table 2.4.8 Other External Events Contributing to Core Damage

(Table 6-62 of DCPRA)

\section{Core Darnage}

Aircraft crash and falling objects

Ship impact

External flooding

Hurricane and tornado wind and missile

Hazardous chemical

Tusbine missile

External fire
Upper Bound Frequency

of Core Damage

$<10^{-8}$

$2.1 \times 10^{-8}$

$2.1 \times 10^{-8}$

$3.2 \times 10^{-7}$

$3.5 \times 10^{-7}$

$\simeq 10^{-7}$

$<10^{-8}$ 
2 Overview of the DCPRA Approach and Results

\subsection{Presentation of the DCPRA for NRC Review}

This section is provided in order to give the reader a clearer understanding of both the magnitude and breadth of the DCPRA as well as to provide a background as to why following sections in this report discuss problems associated with a lack of documentation.

The DCPRA was summarized in Chapter 6 of the LTSP final report. Chapter 6 was initially the only PRA information available for review and is approximately one inch thick (single sided). The DCPRA itself is a multi-volume report that was not submitted in full for review but rather sections of which were submitted as requested by the review. Table 2.5.1 presents the table of contents of the actual DCPRA. As can be seen, the DCPRA is a large and comprehensive document. It is expected that this table will be of benefit in providing background and perspective as selected sections of the DCPRA are referenced within the remainder of this report. 


\section{REVIEW OF THE INTERNAL EVENTS ANALYSIS}

\subsection{Introduction}

This section describes the overall review approach applied to the internal events portion of the DCPRA as well as the major findings in each of the review areas. Section 3.2 provides the details of the review strategy and bases for its selection. Section 3.3 provides the description of selected plant systems, DCPRA modelling techniques, and outlines the results of BNL's independent systems analyses. (The detailed system analyses are found in Appendices A and B.) Section 3.4 addresses initiating event selection as well as a detailed Bayesian updating of the Loss of Service Water and Loss of Component Cooling Water events performed by BNL. Section 3.5 provides an overall description of the DCPRA approach to generating event trees. Sections 3.6 and 3.7 address fault trees and the overall DCPRA data base respectively with specific review details found in Appendices A and B. Section 3.8 provides a focused review of the overall human reliability analysis (HRA) methodology. Section 3.9 presents the results of the BNL review of the accident sequence quantification including selected sensitivity and importance measure calculations as well as a comparison of the overall DCPRA results with nine other PRAs. Section 3.10 presents a summary and analysis of the internal events review results and offers some alternative sets of quantification.

\subsection{Description of the Internal Events Review Approach}

The major elements and novel features of the DCPRA are outlined in Section 2.2. Given the unprecedented size and complexity of the PRA, it was determined that a novel approach would be required for the detailed review and analysis. The review itself was divided into two phases. The first phase was termed "interactive" and was conducted while the PRA was still being developed. The goal of this phase was to both familiarize the reviewers with the PRA in order to get a head start on the formal review and to provide a potential early feedback mechanism to the DCPRA team should something questionable be detected. During this initial phase, two site visits to the Diablo Canyon plant were made for familiarization purposes and three PRA workshops (approximately one-week each) were conducted.

The second phase was to formally review the final DCPRA report. The review strategy employed had to take into account the fact that neither the NRC nor the national laboratories had in-place processing software that could directly accept the DCPRA large event tree/small iault tree model. In addition, the strategy had to accept the fact that employing a full independent requantification type of PRA review as outlined in the PRA Review Manual (NUREG/CR-3485) with the use of the large fault tree/small event tree methodology, to a level of detail commensurate with that in the DCPRA, would simply be cost-prohibitive and unnecessary.

The resulting DCPRA review strategy, therefore, called for a detailed review of selected portions of each of the major elements of the DCPRA. These elements essentially conform to the subsection headings of the remainder of Section 3. As the actual review progressed, some subjects received more attention than others according to the perceived needs by the reviewers.

BNL developed the following seven point plan as the overall review basis for the DCPRA:

1. The logic for the primary event trees will be reviewed to verify consistency and accuracy. 


\section{Review of the Internal Events Analysis}

2. Selected frontline and support systems will undergo an independent fault tree analysis to verify the DCPRA's approach to unavailability modelling (the systems will be selected based upon perceived importance). This effort will include requantification of an appropriate number of top event conditional split fractions.

3. Selected failure probabilities and initiating event frequencies will be reviewed (including the Bayesian updating process) to verify the DCPRA data analysis. Actual failure data selection will be determined by the results of item 2 above.

4. An abbreviated fault model of the entire Diablo Canyon plant will be developed by incorporating the leading accident sequences from the DCPRA.

5. Given the fault model from item 4 above, investigation will be undertaken on the impact of the findings from items 1 through 3 above as well as the performance of other analyses such as importance measures, pair-importance, and sensitivity calculations.

6. In addition to the above overall review plan, two novel aspects of the DCPRA which are: a) the approach to human reliability analysis and $b$ ) the relay chatter analysis will receive special attention.

7. The seismic portion of the PRA review will follow a similar overall methodological approach modified as necessary to account for the specifics of the seismic analysis.

In terms of item 1 above, BNL checked for any obvious errors/omissions in the DCPRA event tree structures but none were apparent. The event trees were not given a rigorously detailed review by BNL as part of the overall review process. The basis for this was that there was an extremely detailed and comprehensive methodology applied to the event tree development and, therefore, BNL believed that the review effort should concentrate resources on other areas of the PRA. The DCPRA methodology utilized event sequence diagrams (ESDs) and stressed the involvement of both PRA analysts and plant operations personnel.

The fault tree analysis portion of the review (item 2 above) was conducted as part of the systems analyses. The system documentation associated with the DCPRA provided reliability block diagrams (as opposed to actual fault trees) containing supercomponents covering large portions of the system. BNL converted these diagrams into fault trees and used the SETS computer code to solve them. This allowed BNL to display the leading cut sets for those top events so modelled. Such cut sets are not provided within the DCPRA. In addition, the fault trees had to be prepared according to the specific requirements of the $\alpha$-factor common cause failure methodology. .

The quantification of the supercomponents was supplied in algebraic equation form by PG\&E. That is, in order for BNL to supply the value block for input to the SETS code, the algebraic equation for each of the supercomponents had to be computed as well as broken down to identify its constituent parts. Each equation represented an expression that combined all the failure modes of each of the elements of the given supercomponent. BNL also checked each equation against the plant drawings, test/maintenance procedures, and Technical Specifications to verify that all major components/failure modes/unavailabilitieswere included.

In order to then verify the various split fractions associated with each fault tree, BNL had to set various elements to one or zero to define each boundary condition and then solve that version of the fault tree four times to account for the different postulated sets of system alignment. The methodology of systems analysis 
applied in the DCPRA requires that the top event split fraction (associated with a system under a given boundary condition) should reflect the notion that the system (or its portion) in question is in one of the following mutually exclusive alignments: 1) normal alignment, 2) testing alignment, 3) maintenance alignment, or 4) misalignment. Thus, the contribution to the system unavailability from a specific alignment is determined by the conditional system unavailability, given that the system is in that alignment multiplied by the fraction of time that the system spends in that alignment. The quantification/verification of the conditional split fractions in most cases provided good agreement with the PG\&E results. The difference in the majority of the cases coming from some modeling errors of minor significance and from the use of Monte Carlo techniques by PG\&E and point estimates by BNL.

The following systems/functions were subjected to detailed review/requantification:

High Pressure Injection Function

Low Pressure Injection Function

Auxiliary Feedwater System

Diesel Generator \& Diesel Fuel Transfer Systems

Electrical Power Systems (AC \& DC)

Auxiliary Saltwater System

Component Cooling Water System

Solid State Protection/Reactor Protection Systems

The review of the first three above (frontline systems) is documented in Appendix A. The review of the remainder (support systems) is documented in Appendix B.

BNL carried out the following types of analyses to verify the DCPRA data base. The DCPRA data base was derived from the PLG proprietary data base and updated using Bayesian techniques to incorporate Diablo Canyon-specific data/experience. As part of the auxiliary feedwater system review, BNL solved the derived fault trees first with the DCPRA data and then with an alternate generic data base derived from other recent PRAs. This was done to see the sensitivity of the model to the different data bases. The quantification of the conditional split fractions was in fairly close agreement; demonstrating little sensitivity to the two data bases. Had the data bases provided significantly divergent results, further review effort would have been devoted to this particular area of the review.

In terms of initiating event quantification, BNL checked all of the initiators against other industry sources. A number of the initiating event frequencies seemed somewhat low. This was attributed to the rather restrictive criteria applied by PG\&E to select some prior event samples for Bayesian updating (mainly transients). However, use of less restrictive selection criteria in sensitivity studies did not result in large variations in total core damage frequency. Additionally, BNL selected two initiators for detailed scrutiny. The loss of auxiliary saltwater (LOSW) and the loss of component cooling (LPCC) were selected for this purpose. Both of these initiators were quantified by fault tree analyses in the DCPRA and the latter initiator was basically limited to loss of the CCW pumps (thus LPCC rather than LOCC). BNL's approach was to carry out a detailed industry-wide LER-type search for all LOSW and LOCC events. BNL then screened this list for events that, due to design considerations, could not happen at Diablo Canyon and then proceeded to undertake a Bayesian updating of this data with the Diablo Canyon experience, (i.e., no events in either category). This effort yielded significantly larger initiating frequencies and, therefore, significantly larger core damage contributions from these two initiators than that presented in the DCPRA. Following meetings with 


\section{Review of the Internal Events Analysis}

the DCPRA team (Pacific Gas and Electric, et al.), PG\&E submitted new and higher values for both LOSW and LPCC. The increases were 44 percent and 47 percent respectively. Details of this portion of the review are provided in Section 3.4 and Appendices B3 and B4.

The abbreviated fault tree model (item 4 above) was originally going to be developed by BNL, however, PG\&E developed a reduced model (Dominant Sequence Model-DSM) for their own purposes and agreed to share this with BNL. The PGE model contained both internal and the non-seismic external events and therefore the BNL results based upon this model were termed "non-seismic" results. The leading sequences and the quantification associated with all of the conditional split fractions and basic event failure probabilities were provided to BNL on a floppy disk. BNL had to modify the DSM in order to convert it into a Boolean expression and then utilized this model as the basis for the quantification described in Sections 3.9, 3.10 and Appendix D (i.e., i tem 5 above). A full description of this model as modified by BNL and all input data can be found in Appendix D1.

The relay chatter analysis mentioned in item 6 above was audited by BNL in conjunction with a review meeting held with PG\&E in San Francisco. PG\&E performed the relay chatter analysis in a truly conservative fashion. For each relay that was determined to have the potential for chattering, the circuit was reviewed in detail. PG\&E applied the conservative assumption that all relays in a given circuit that could chatter would indeed chatter together in order to make/break the circuit. This assumption was uniformly applied even if it took six, seven or more relays chattering in synchronism to accomplish the event. PG\&E then looked at the consequence of the circuit failing/changing state and determined what circuits could lead to problems in the plant. At this point, the results of the relay chatter analysis were not simply input to the DCPRA model but rather those circuits identified as particularly vulnerable received hardware modifications to prevent the action.

The BNL audit of the relay chatter analysis therefore focused on methodology, scope, and completeness. BNL audited a number of the systems (e.g., auxiliary saltwater, electrical power) and concluded that a rigorous and conservative electrical circuit analysis had been performed. The seismic PRA aspects of the review are addressed in Section 4.

One of the key elements of the review process turned out to be its interactive nature. As discussed previously, the first phase of the review was termed the interactive phase, however, the formal review turned out to be even more interactive. All eight system analysis reviews listed above were documented in letter reports to the NRC Program Manager as they were accomplished. These reports were forwarded to PG\&E and meetings were held to discuss the preliminary findings. Each meeting typically covered two to three letter reports.

As with any large and complex piece of work such as the DCPRA, it is almost impossible to document every detail, assumption, success criterion, etc. Therefore, when the meetings were held, much of the open item material was found to be because of insufficient documentation. Other open items were shown to have merit with some being dismissed as having very low impact and others accepted in whole or in part as feedback into the DCPRA. 


\subsection{Plant Description and Modelling}

\subsubsection{Safety Punctions and Corresponding Frontline Systems}

The goal of this portion of the DCPRA review was to select a small number of frontline systems that supported a number of important safety functions and do a full systems analysis review including requantification of the associated conditional split fractions where possible. The safety functions/systems selected for this review were the ECCS high pressure injection function, the ECCS low pressure injection function, and secondary side heat removal via the Auxiliary Feedwater System. The complete detailed review documentation for these system analyses can be found in Appendix A.

The system analyses of the selected frontline systems encompassed validity of the fault trees, level of detail of the fault trees, support system interaction, success criteria, failure data and requantification of at least a representative number of conditional split fractions.

The results of BNL's detailed system analysis of the High Pressure Injection (HPI) function can be found in Appendix A1. BNL reviewed the high pressure injection top event logic diagrams and corresponding fault trees along with supporting drawings and other information provided by PG\&E. This information included the Technical Specifications, relevant operating and surveillance test procedures as well as fluid flow and actuation logic diagrams.

The DCPRA applied the simplifying assumption that the leak would abways occur in cold leg number one. BNL conducted an independent calculation that allowed the leak to occur in any of the four cold legs. The results of the BNL analysis verified the validity of this assumption. BNL concluded that the HPI function system analysis in the DCPRA represented a fully adequate characterization.

The results of BNL's system analysis of the Low Pressure Injection (LPI) function can be found in Appendix A2. This review covered the same scope and depth of the HPI review discussed above as well as an additional sensitivity analysis on the one-out-of-three (1/3) success criterion (flow to $1 / 3$ of the intact loops) used in the DCPRA. The sensitivity analysis was performed to see the effect of using a $2 / 3$ success criterion as had other previous PRAs. Later in the review, PG\&E provided additional documentation to support the $1 / 3$ criterion that was used. Based upon the additional information, BNL was satisfied with the $1 / 3$ criterion.

The DCPRA also used a simplifying assumption as to break location for the low pressure case. That is, it was again assumed that the break would always be in cold leg number one. When BNL recalculated the split fractions assuming the break could occur in any of the four cold legs, the use of this assumption provided (on average) higher values. The difference in applicability of the same simplifying assumption between the HPI and LPI is the result of differences in the design features of the injection headers. In the case of the RHR system (LPI), each of two header systems feeds its own two branch lines. In the case of the charging and safety injection systems (HPI), one header feeds all four branch lines. As a result of the BNL review, certain LPI function split fractions were requantified by PG\&E. In the end, the BNL audit calculations were in good agreement with those presented by PG\&E and the alternative break location assumption produced a negligible increase in CDF when substituted into the DSM. 


\section{Review of the Internal Events Analysis}

The results of BNL's detailed system analysis of the Auxiliary Feedwater System can be found in Appendix A3. BNL performed a thorough review of the top event logic diagrams and the corresponding fault trees related to the "low pressure" boundary conditions. The fault trees related to the "high pressure" boundary conditions were not reviewed as they pertained to the ATWS sequences which had no measurable impact on the CDF. During the review, BNL discovered an anomaly between split fractions AW5, AW7 and AW8. AW8 represents a more degraded set of boundary conditions and yet it had a higher quantified availability than either AW5 or AW7. In the BNL calculations, this anomaly did not appear. PG\&E responded that this was the result of the way these split fractions went through the truncation process. Recalculation of these split fractions by PG\&E on a point estimate basis came into good agreement with the BNL results. When PG\&E substituted the new values for these split fractions into their dominant sequence model, they discovered they had a siemificant effect on the CDF. (BNL calculations showed a 38 percent increase in non-seismic CDF over PG\&E's final results when BNL's values for AW5, AW7 and AW8 were used.) PG\&E therefore decided to remove some of the modelling conservatisms present in their original calculations in attaining their final results. (This is discussed in detail in Appendix A3.) The remaining numerical differences between the PG\&E and BNL calculations for the AW split fractions were attributed to the Monte Carlo vs. mean value approach used by PG\&E and BNL, respectively.

\section{3 .2 Support Systems}

The goal of this portion of the review was exactly the same as outlined in Section 3.3.1 for the frontline systems. That is, to perform a full systems analysis review including requantification of the associated conditional split fractions where possible. The support systems selected for full review encompassed all of the electrical power systems, the diesel generators and fuel oil transfer system, and the auxiliary saltwater system. The component cooling water system and the solid state protection/reactor protection systems (SSPS/RPS) were reviewed to somewhat less detail. The SSPS and RPS reviews were limited to a comparative review between the DCPRA and previous reviews performed by BNL. The complete detailed review documentation for these systems can be found in Appendix B.

The results of BNL's detailed system analysis of the diesel generator and diesel fuel transfer systems can be found in Appendix B1. The BNL review identified several inconsistencies and neglection of failures of diesel subsystems in the unavailability modelling of diesel generators in the DCPRA and the omission of the unavailabilitycontribution from Unit 2 (and swing) diesel overhauls. The combined effect of these neglections may result in underestimation of the associated top event split fractions and through them the expected core damage frequency value of Unit 1.

As an overall sensitivity study on the Diesel Generator and Diesel Fuel Transfer Systems, the BNL values for the conditional split fractions found in Tables B1.2.5 and B1.2.6 were substituted into the dominant sequence model. The overall unnormalized Fussel-Vesely importance of this class of events was 4.30E-05 (PG\&E values) and 4.12E-05 (BNL values), respectively. This demonstrates excellent agreement between BNL and PG\&E.

The results of BNL's detailed system analysis of the entire electrical power system (non-vital electric power, vital $125 \mathrm{~V} \mathrm{dc}$, vital ac, instrument ac, and Unit 2 vital ac and dc) can be found in Appendix B2. The BNL review identified several inconsistencies and potential omissions in the unavailability modelling of the Diablo Canyon electrical power systems. These resulted in several questions that were discussed with PG\&E and are highlighted in the appendix. The combined results of the identified omissions may result in a slight 
underestimation of the expected core damage frequency of Unit 1. BNL's audit calculations were in close agreement with those within the DCPRA concerning the electrical power systems.

The results of BNL's detailed system analysis of the auxiliary saltwater system (ASW) can be found in Appendix B3. As a result of the BNL system analysis review of the Auxiliary Saltwater System, PG\&E performed a number of additional calculations. One of the calculations demonstrated that under most circumstances (but not all as had originally been assumed) a single Unit 2 ASW pump could supply both Unit 1 (via the crosstie) and Unit 2 with sufficient ASW flow. This was one of the major concerns of the ASW review. PG\&E then carried out a sensitivity study to determine the impact of this finding on their overall results. PG\&E determined that the change in non-seismic CDF was not appreciable and none of the conditional split fractions in Table A3.2.3 were changed as a result. BNL also performed an independent analysis of the loss of auxiliary saltwater (LOSW) initiator frequency. This is described in detail in Section 3.4 and Appendix B3.

The BNL review of the component cooling water system (CCW) can be found in Appendix B4. This review was not as extensive as the previously described support system reviews. The review was more qualitative in nature in that none of the split fractions were requantified. BNL found a number of minor omissions/discrepancies but none were believed to be significant with respect to the overall non-seismic CDF. BNL also performed an independent analysis of the loss of CCW (LOCC) initiator frequency. This is described in detail in Section 3.4 and Appendix B4.

BNL found that the DCPRA predicted a lower SSPS unreliability for both a single channel and for system failure (both channels) than the predicted values presented by the Westinghouse Owners Group (WOG) in support of Technical Specification change requests. This work was reviewed previously by BNL. (A full set of references is supplied in Appendix B5.). BNL believes the reason for this difference is that the WOG effort represented an unprecedented detailed fault tree review of the system and therefore included more components and failure modes. As this was a comparative review, BNL did not attempt to requantify the split fractions. BNL did perform a sensitivity study to determine the impact of increasing the applicable conditional split fractions (as discussed in Appendix B5) and determined that increasing the SA split fractions (single channel failures) by a factor of 5.0 and the SB split fractions (conditional probability of the second channel failing given that the first had already failed) by a factor of 2.0 increased the overall non-seismic CDF by approximately 19 percent. (The WOG work is proprietary in nature-PG\&E is a member - and therefore no quantification is quoted. The factors of five and two used herein were simply chosen to demonstrate the effect of a full order of magnitude increase in system unavailability.) The comparative review of the RPS system analysis with the WOG study/BNL review results showed reasonable agreement and no further review effort beyond that was considered necessary.

\subsubsection{Success Criteria}

As part of the BNL system analysis reviews (3 frontline and 5 support systems) documented in Appendices $A$ and $B$, all success criteria were reviewed to determine their validity. As part of this process, two specific success criteria were questioned. The first was associated with the ECCS low pressure injection function provided by the residual heat removal (RHR) system. In some previous PRAs the success criterion for a four loop plant was taken to be injection to two out of the three intact loops with no credit for the broken loop. The DCPRA success criterion for this scenario is only one-out-of-three (1/3) intact loops requires injection. In response to a BNL question, PG\&E referenced a study that demonstrated that $1 / 3$ was sufficient for Diablo Canyon (see Appendix A2 for details). 


\section{Roviow of the Internal Events Analysis}

The other success criterion that BNL questioned dealt with the ability of a single Unit 2 auxiliary saltwater pump to supply both Unit 2 as well as Unit 1 given the loss of the Unit 1 ASW system. PG\&E supplied the results of their calculations that demonstrated a nearly equal split of the flow between the two units and acknowledged that there are certain circumstances in which one pump will not suffice. PG\&E responded with a sensitivity study that indicated that the overall effect on the non-seismic CDF was negligible. BNL's calculations showed a measurable impact on the non-seismic CDF (see Appendix B3 for details and Section 3.10 for its integrated impact as part of the review's alternative quantification).

\subsection{Initiating Events}

\subsection{Gemeral}

The scope of the initiating events review documentation included herein encompasses the following: a brief description of the selection and quantification of initiating events and the results of the BNL review of the initiating event analysis of the DCPRA, i.e., the completeness of the set of initiators considered and the frequency estimate assigned to each.

\subsection{Inithting Event Selection in the DCPRA}

In order to make the list of initiating events as complete as possible, PG\&E used three methods for initiating events identification. These were:

1. Master Logic Diagram (MLD)

2. Heat Balance Fault Tree (HBFT)

3. Failure Mode and Effects Analysis (FMEA)

The MLD is similar to a fault tree with "potential release of radioactive material" as the top undesired event. The diagram reflects the logical hierarchy of the safety functions of the plant systems. Its basic input events are the initiating events. By using the MLD method the DCPRA team identified most initiating event categories that were finally selected for quantification.

The HBFT method was used to enhance completeness. It is a special fault tree, whose undesired top event is "heat imbalance event occurs." The event is a consequence of a departure from equilibrium of thermal energy transfer from the reactor core to the environment. The input events of the HBFT are any event that causes a plant transient condition which challenges the plant control/safety systems to act. The application of the HBFT method not only resulted in enhancing completeness, but also proved to be helpful in defining finer structures for initiating event categories.

The FMEA method was used to systematically identify support and control system failure modes that give rise to common cause initiating events. (Observe that the FMEA method is a "bottom up" approach, in contrast with the previous two "top down" approaches.) Common cause initiating events also included spatially dependent physical interactions such as fires and floods, turbine missiles, sprays and pipe whips as well as truly external events such as earthquakes, aircraft accidents, etc.

The initiating event categories obtained as a result of the above identifying/selecting methods were further compared and cross-checked with lists of initiators given or analyzed in several reports, such as, the Diablo 
Canyon FSAR' (for plant-specific factors), WASH-1400, ${ }^{2}$ EPRI NP-2230, ${ }^{3}$ the PSA Procedures Guide, ${ }^{4}$ Indian Point Probabilistic Safety Study, and Seabrook Station Probabilistic Safety Assessment.6

The candidate initiating event categories were further screened by the quantification process, i.e., initiating ovents with low occurrence frequency and with less relevance to challenge safety systems were omitted.

With this procedure the DCPRA arrived at a rather comprehensive set of initiators consisting of 27 "internal" and 23 "external" initiating events. This set of initiating events is unusually large compared with that of previous PRAs.

The lit of the seven LOCA, 14 transients, six support system (common cause) failures, 14 fires, three floodfiet/apray, and six levels of earthquake initiators and their code designators are given in Table 3.4.1.

All the non-selsmic initiators were analyzed and quantified in the context of the full DCPRA plant model and in this sonse they were all considered in the core damage frequency analysis as "internal events."

\subsection{Qunifiention of the Finterwal Initiating Evento" in the DCPRA}

The 27 truly internal initiating events of the DCPRA were divided into two groups for quantification. The first group is composed of those initiators for which it was deemed that generic operating experience was applicable. The second group included initiators which were deemed to require plant-specific analysis. The quantification process for the first group entailed sophisticated Bayesian updating techniques which combined generic operating experience with plant-specific experience ( 4.7 years of operation). For the quantification of the socond group, system unavallability calculations were used. Initiators, whose frequencies were calculated by system unavailability calculations are marked with asterisks in Table 3.4.1.

The quantification of the "external" initiating events involved a rather complex spacial interaction study and a detailed impact analysis on plant systems. For fire scenarios (grouped in fire categories designated as FS1 through FS8) individual initiating event frequencies were calculated and they were fed into the plant model for core damage quantification.

Fire scenarios occurring in the control room (designator: CR) and the cable spreading room (CS) leading to core damage were not incorporated in the model as initiating events, they were modelled separately as direct core damage frequencies.

Flood scenarios were grouped into three initiating event categories: FS9, FS10 and FS11. Their initiator frequencies were determined individually and were fed into the plant model for core damage quantification, similarly to the FS1-FS8 fire scenario categories.

Soven truly "external" event initiators were also considered (i.e., aircraft impact, ship impact, external floods, hurricanes and tornadoes, hazardous chemical, turbine missiles, and external fires). Except hazardous chemical, these events were screened out by the DCPRA team as rather insignificant contributors to the core damage frequency, thus they were not considered further in the DCPRA (See Table 2.4.8).

Those initiators which were deemed to be significant contributors to the core damage frequency (i.e., those which were considered to constitute the risk after truncating, merging and correcting for scenarios with small core damage frequency) were included in the reduced plant model. These initiators are listed in Table 3.4.2a. 


\section{Reviow of the Internal Events Analyils}

(The reduced plant model is described in detail in Appendix D: DCPRA Dominant Accident Sequence Modol-DSM.)

The table lists the initiating ovent dedignators and the corresponding initiating frequencies. It also indicates the mean values of the generic prior diatributions applied in the Bayesian updating. Notice that several initiatore were found to provide negligible contribution to the total core damage frequency and therefore were loft out of the reduced plant model. These are:

- Interfacing Systems LOCA, RHR Discharge Side

- Closure of All MSIVs

- Inadvertent Opening of Main Steam Relief Valve

- Core Power Excursion

- Loss of All Charging Pumps and MSIV Closure (by fire)

- Loss of Component Cooling (by fire)

- Lose of Component Ventilation (by fire)

- Loss of 4kV Buses HO and HH (by fire)

The frequencies of the above initiators are listed in Table 3.4.2b. The external initiating event "hazardous chemical release" was not a significant contributor, however, it was included in the reduced plant model (see Table 3.4.2a) based upon a completeness argument. The control room (CR) and the cable spreading room (CS) core damage frequency contributions were combined in the DSM and were given a common designator: CRFIRE. Table 3.9.12 provides a breakdown of this set of initiators.

\subsection{Review of the Initiating Event Amblyis}

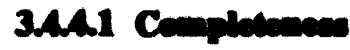

As demonstrated in Table 3.4.1 the DCPRA does not suffer from a lack of initiating events. The high degree of completeness is the consequence of applying mutually supplementing initiating event selection methods and a self- checking systematic comparison with other references. However, BNL noted that the screening process applied to the final list of initiators dismissed the following:

a. The traditional initiator "Loss of two (or more) $120 \mathrm{~V} \mathrm{AC} \mathrm{Instrument} \mathrm{Buses"} \mathrm{was} \mathrm{screened} \mathrm{out} \mathrm{from} \mathrm{the}$ original list of initiators as an extreme low frequency event. A recent BNL study (see also Appendix B2), however, found that the recent generic rate of reactor trips due to multiple inverter failure alone is .185 trips/year. From these trips a non negligible fraction might be selected to be applicable for Diablo Canyon Plant conditions. Each of the applicable events has the potential for impacting safety because of the additional equipment response and operator actions generally needed to bring the unit to a safe and controlled condition. In the DCPRA, the reactor trip initiator (RT) and the associated ovent tree do not account for events of the above type, since the RT event tree is not conditioned for simultaneous guaranteed failure of more than one instrument ac channel. Its contribution to the core damage frequency might be accounted for in the present framework of the DCPRA by being grouped together with the initiators "Loss of $480 \mathrm{~V}$ AC Switchgear Ventilation, LOSWV" (or with "Loss of One DC Bus, L1DC"). The Review of the Millstone 3 PRA $^{8}$ lists a mean value of 6.15E-02 year ${ }^{-1}$ for the frequency of the initiator: "Loss of a Pair of Vital 120V AC Buses." 
The review interest in th: analysis of the "loss of two (or more) vital $120 \mathrm{~V}$ ac instrument buses, stemmodirom the insights it might provide into plant dependencles, interactions and, therefore, safety."

b. Similarly, another traditional initiating event "Loss of Instrument Air" was not quantified and analyzed as a separate initiator. Instead, the DCPRA assumed that the instrument air system simply fails after each type of initiatingevent. By this assumption, the DCPRA introduced an unnecessary "conservatism" which ultimately led to a modelling artifact: instrument air appears to be the most important plant safety system. In addition, this assumption prevented the realistic assessment of the instrument air system's importance for safety. Thus, the only useful information about the effect of failures of the instrument air system at Diablo Canyon plant comes from the original initiator FMEA. (Results of the FMEA indicated that at Diablo Canyon the effect of loss of instrument air is somewhat similar to the effect of loss of main feedwater, TLMFW). BNL believes a realistic analysis of the loss of instrument air initiator would be beneficial for any safety insights for the DCPP.

c. BNL simply notes for completeness that it appears that none of the DCPRA initiator categories includes events classified in category [37] of EPRI-NP-2230: "Loss of Power to Necessary Plant Systems." The median frequency of these events was estimated by BNL using the data of NUREG/CR-3862 ${ }^{\circ}$ to be 2.87E-02 year ${ }^{-1}$. BNL identified several events of this kind during its search for events representing "Total Loss of Service Water Systems" (see Appendix A3) but did not take them into account in the calculation of that initiator.

\section{Inthating Bvent Proquencios}

BNL's review of the initiating event frequencies did not include a recalculation of each initiator frequency. However, to determine potential outliers, all initiating event frequencies listed in Tables 3.4.2a and 3.4.2b were compared with those of other recent PRAs or other data sources. From those initiating frequencies which were determined in the DCPRA by system analysis (fault trees), two specific initiators were chosen for detailed audit/requantification. The two initiators were "Loss of Auxiliary Saltwater (LOSW)" and "Loss of Component Cooling Water (LPCC)."

The objective of this section is to provide the observations gained from this portion of the review. The observations are made roughly in the order of the initiator's importance, i.e., the initiator's contribution to the core damage frequency (for ranking of initiator importance see Table 3.9.1a). 


\section{Loes of Onatte Power, LOOP}

Generic and aite-specific information was used in the DCPRA to evaluate the frequency of the loss of offsite power, LOOP, initiating event. This detailed information was not available for BNL. Neither was the information about the subdivision of this frequency according to its main sources. That is: 1) frequency of LOOP events affecting only Unit 1 or Unit 2,2 ) frequency of LOOP events affecting both units simultaneously (the DCPRA emphasized that DCPP never experienced a grid-related LOOP during the 4.7 years of operation included in the study), 3) LOOP events induced by non LOOP transients at Unit 1 and affecting that unit only (see, e.8., BNL's question on load rejection contribution to the LOOP frequency in the review of non-vital electric power system, Appendix B2), and 4) LOOP events at Unit 1 induced by non-LOOP transients at Unit 2 under any operational condition. (As this report was in its final editing stages, DCPP did experience a LOOP event, however, no attempt at that late stage was made to factor this event into the review analyais.)

A recent article from the EPRI Journal ${ }^{10}$ calls attention to the fact that solar magnetic storms represent a real threat to power grid integrity during the next 11 year cycle of increased sunspot-activity. The article goes on to discuss specific examples. This subject would not normally be a concern in a PRA, however, the article includes a map which shows most all of California within a "high potential" zone for grid failure and this could affect the LOOP component 2) mentioned above.

Based upon 1) the electrical system model of Unit 1, which considers the advantages due to the interconnections between the two units, but is tacit about any disadvantages arising from component 4) above of the LOOP initiators and 2) the recent EPRI report, BNL suggested that it would be very useful for PG\&E to reevaluate the derivation of their LOOP initiator frequeacy and document their findings accordingly. PG\&E did reevaluate the original derivation and concluded no changes were required. ${ }^{11}$ As a sensitivity calculation, BNL applied the assumption that one such solar event would happen on the PG\&E grid over the next 30 years with a 0.5 probability of causing a loss of offsite power at Diablo Canyon. When this contribution was added to the PG\&E LOOP value and substituted in the dominant sequence model, the nonseismic CDF increased by just over 4 percent. Subsequent to BNL's surfacing this issue within the DCPPRA review, the U.S. NRC issued Information Notice No. 90-42 (June 19, 1990) advising all licensees of this potential circumstance.

\section{Reactor Trip, RT}

The mean generic prior frequency for this initiator, $1.53 \mathrm{yr}^{-1}$ seems to be small compared with other generic RT data. This initiator is cross-referenced (see Table C1-6 and C1-5 of the DCPRA) with the following initiator categories of the EPRI-NP-2230 report: [38], [39], [40]. The review of the Millstone 3 PRA lists for these EPRI categories a combined median and a mean value of $2.32 \mathrm{yr}^{-1}$ and $3.03 \mathrm{yr}^{-1}$, respectively (see Table 3.1-3 in NUREG/CR-4142). Based on the data in NUREG/CR-3682 and the classification of EPRI-NP-801, ${ }^{12}$ (equivalent with the classification of EPRI-NP-2230) BNL obtained a median value of $2.18 \mathrm{yr}^{-1}$, near to the Millstone median. A subset of the data, the "spurious frequency of reactor trips" (due to testing of analog channels in the Reactor Protection System) alone provides a value of $0.5 \mathrm{yr}^{-1}$. This is the value used by the Westinghouse Owner's Group to request Technical Specification Relaxation for the Reactor Protection Instrumentation Systems from the NRC (see the Westinghouse reports WCAP-10271 ${ }^{13}$ and WCAP-10272-A ${ }^{14}$. The latter report also contains the NRC's approval of the request). 
In the Seabrook PRA; the mean frequency of the reactor trip initiator is: $3.13 \mathrm{yr}^{-1}$. The Midland PRA ${ }^{15}$ used for the same initiator: $6.11 \mathrm{yr}^{-1}$. Both values are "prior dominated" plant-specific values with zero plant evidence (since the plants had no operating experience).

The analysts preparing the DCPRA used special selection and screening criteria to arrive at their results on the prior frequency, RT. These are described in Section 3.4.5. The DCPRA mean value for RT was 1.14 events/year. It appears that the screening criteria employed and the blanket fashion in which it was applied in order to obtain the DCPRA value represents a lower bound mean value. BNL did not perform a Bayesian updating of the other data sources to obtain an alternative plant-specific mean frequency. However, the Millstone and BNL median values were close and therefore, one can take the Millstone mean value (3.03/yr) as indicative of long term previous industry experience and then take the specific industry performance for the years 1988 and 1989 (from the April 23, 1990 issue of "Inside NRC" - Volume 12, No. 9, as 2.26/yr and $1.85 / y r$, respectively) as indicative of the trend to fewer trips per year as the industry matures. The following sensitivity study (based upon the dominant sequence model) using these values gives an indication of the effect of the reactor trip initiator on the non-seismic CDF.

\begin{tabular}{|l|c|c|c|c|}
\hline \multicolumn{1}{|c|}{ Case } & $\begin{array}{c}\text { RT } \\
\text { Events/Year }\end{array}$ & $\begin{array}{c}\text { Unnormalized } \\
\text { Fussel-Vesely } \\
\text { Importance }\end{array}$ & $\begin{array}{c}\text { Resulting } \\
\text { Non-Seismic } \\
\text { CDF }\end{array}$ & $\% \Delta$ CDF \\
\hline DCPRA & 1.14 & $1.615-5$ & $1.7728-4$ & - \\
\hline Industry - 1989 & 1.85 & $2.621-5$ & $1.8733-4$ & 5.7 \\
\hline Industry - 1988 & 2.26 & $3.202-5$ & $1.9314-4$ & 9 \\
\hline Long Term Industry Experience & 3.03 & $4.293-5$ & $2.0405-4$ & 15 \\
\hline
\end{tabular}

\section{Turbine Trip, TT}

The mean generic prior frequency of the TT initiator, $1.08 \mathrm{yr}^{-1}$ is also smaller compared with other data. The DCPRA cross references this initiator with categories [33] and [34] of EPRI-NP-2230. The review of the Millstone 3 PRA provides for the sum of the EPRI categories a median value of $1.76 \mathrm{yr}^{-1}$ (see Table 3.1-2a of Reference 8). Based on the data in NUREG-3682, BNL obtained a median value of: $1.66 \mathrm{yr}^{-1}$. The "prior dominated" plant-specific mean TT initiator frequencies in the Seabrook and Midland PRAs are the same for both plants: $1.95 \mathrm{yr}^{-1}$. The Diablo Canyon plant-specific mean TT initiator value was $1.05 \mathrm{yr}^{-1}$. The following chart demonstrates the sensitivity of the non-seismic CDF to the TT initiator frequency using the dominant sequence model.

\begin{tabular}{|l|c|c|c|c|}
\hline \multicolumn{1}{|c|}{ Case } & $\begin{array}{c}\text { RT } \\
\text { Events/Year }\end{array}$ & $\begin{array}{c}\text { Unnormalized } \\
\text { Fussel-Vesely } \\
\text { Importance }\end{array}$ & $\begin{array}{c}\text { Resulting } \\
\text { Non-Seismic } \\
\text { CDF }\end{array}$ & $\% \Delta$ CDF \\
\hline DCPRA & 1.05 & $1.477-5$ & $1.7728-4$ & - \\
\hline Alternative & 1.95 & $2.743-5$ & $1.8994-4$ & 7.1 \\
\hline
\end{tabular}




\section{All Tranaient Categories}

A similar trend that the prior and consequently the posterior mean frequencies used for a given transient are smaller than those of other PRAs can be observed for practically each of the categories in the transient group of Table 3.4.1. The trend can' be demonstrated by comparing the sums of the mean prior and posterior frequencies of the initiators of that initiator group with similar values of other PRAs. The sum of the mean prior frequencies of the group "Transient" in Table 3.4.1 (i.e., RT+TT+LCV+... LOPF) is: $4.53 \mathrm{yr}^{-1}$. The sum of the mean posteriors (Diablo Canyon specific values) is: $3.79 \mathrm{yr}^{-1}\left(3.71 \mathrm{yr}^{-1}\right.$ in the dominant sequence model). Seabrook's sum of posterior means is: $10.80 \mathrm{yr}^{-1}$ (the categories are the same as those of the DCPRA). Midland's sum of posterior means is: $8.98 \mathrm{yr}^{-1}$ (Midland categories are: RT, TT, EF, FW). The Millstone Unit 3 sum of the medians is: $7.71 \mathrm{yr}^{-1}$ and the sum of the means is: $10.57 \mathrm{yr}^{-1}$ (the Millstone values correspond to the categories of EPRI-NP-2230, cross referenced by using Tables C.1-6 of the DCPRA). Additional comparison with other sums of transient values obtained from PRAs on Westinghouse plants, show the same discrepancy. The Westinghouse plants analyzed in NUREG-1150 ${ }^{16}$ have the following (plant-specific) values:

1. Surry Unit 1 transient categories: $T_{2}+T_{3}=8.16 \mathrm{yr}^{-1}$.

2. Zion, as reviewed in Reference 17 see p. $2-8$ of that reference) transient categories: $5+6+7+8+9+10+11 \mathrm{a}+12+13 \mathrm{a}=13.97 \mathrm{yr}^{-1}$.

3. Sequoyah transient categories: $T_{2}+T_{3}=7.80 \mathrm{yr}^{-1}$.

BNL offers the following sensitivity study to place the entire class of transient initiators into perspective for the Diablo Canyon plant. The previous two subsections addressed RT and TT individually and therefore they have been removed from the list for this sensitivity study. With the removal of RT and TT:

the DCPRA value becomes (3.79 $-1.14-1.05=) 1.60 \mathrm{yr}^{-1}$

the DSM value becomes $(3.71-1.14-1.05=) 1.52 \mathrm{yr}^{-1}$

the Seabrook value becomes $(10.8-3.13-1.95=) 5.72 \mathrm{yr}^{-1}$

Taking the ratio of Seabrook to Diablo Canyon $(5.72 / 1.60=3.575)$ and applying it to the dominant sequence model yields the following:

\begin{tabular}{|l|c|c|c|c|}
\hline \multicolumn{1}{|c|}{ Case } & $\begin{array}{c}\text { Transient } \\
\text { Events/Year }\end{array}$ & $\begin{array}{c}\text { Unnormalized } \\
\text { Fussel-Vesely } \\
\text { Importance }\end{array}$ & $\begin{array}{c}\text { Resulting } \\
\text { Non-Seismic } \\
\text { CDF }\end{array}$ & $\% \Delta$ CDF \\
\hline DCPRA & 1.52 & $2.348-5$ & $1.7728-4$ & - \\
\hline (DCPRA) (3.575) & 5.43 & $8.395-5$ & $2.3774-4$ & 34 \\
\hline
\end{tabular}

\section{Total Loss of Auxiliary Saltwater, LOSW}

The mean frequency of the LOSW initiator was derived in the DCPRA by an unavailability analysis (fault tree evaluation) of the AS system and not by reflecting appropriately screened industry experience. The analysis took into consideration the intertie to the parallel AS system of Unit 2. BNL performed a detailed review of the system analysis (see Appendix B3) and in addition conducted a data search and analysis to check 
whether the failure rates used in the quantification reflect the harsher environmental conditions that might be expected with a saltwater working fluid. The data search was directed also to obtain an industry-experience based frequency for this initiator. The review showed that the assumptions and failure values used in the DCPRA unavailability model to determine the frequency of LOSW were somewhat optimistic.

A sensitivity calculation performed by BNL (see Appendix B3) based on modified modelling assumptions but with the original failure rates resulted in a new point estimate mean initiator frequency BNL2: LOSW Cak $=$ 4.56E-04 $\mathrm{yr}^{-1}$.

A rough experience-basedBayesian estimate (San Onofre 2 and 3 event) provides the following value for the mean frequency of this initiator: $L O S W_{\mathrm{Bpp}}=2.23 \mathrm{E}-03 \mathrm{yr}^{-1}$. From the above two estimates, one may anticipate that the real value of the LOSW initiator frequency lies in the interval: $4.56 \mathrm{E}-04 \mathrm{yr}^{-1} \leq \mathrm{LOSW}<$ 2.23E-03 $\mathrm{yr}^{-1}$.

Based upon BNL's initial findings, PG\&E reevaluated their initiator frequency and offered an updated value 44 percent higher than the original. This represented a less than one percent increase in overall non-seismic CDF. In turn, in order to factor in an element of expert opinion and to provide an additional data point, BNL took the geometric mean of the above interval related to LOSW initiator frequency and this provided a 7.64 percent increase in total non-seismic CDF. This is the value used by BNL in section 3.10 (cases 5 and 6) concerning alternative quantification.

\section{Total Loss of Component Cooling Water System, LPCC}

BNL did not requantify the unavailability model of the CCWS which serves the basis for the determination of this initiator in the DCPRA. Instead a data search and analysis effort was carried out to provide an industry-experience-based frequency value (see Appendix B4). BNL's calculation resulted in a mean value of $\mathrm{LPCC}_{\mathrm{BNL}}=3.14 \mathrm{E}-3 \mathrm{yr}^{-1}$. BNL considered that a fraction of the initiating events represented "linked" initiators; e.g., when one of the CCW heat exchangers was in outage and the other heat exchanger was lost because of a failure of its associated saltwater system train. Depending on the perception of which LER events should be considered as conceivable priors, and as a result of the BNL review, PG\&E estimated a new mean initiating frequency to be: $L_{P C C}$ PGe $=2.88 E-04 \mathrm{yr}^{-1}$. This was 47 percent higher than the original DCPRA value listed in Table 3.4.2a.

If one takes the updated PG\&E value as a lower bound and the BNL value as an upper bound, the geometric mean derived therefrom would yield a 6.9 percent increase in non-seismic CDF when substituted into the dominant sequence model. This is the value used by BNL in Section 3.10 (cases 5 and 6) concerning alternative quantification.

\section{Other Common Cause Initiating Event Frequencies}

BNL did not perform a rigorous, detailed review of the remaining initiators belonging to the common cause group. These are: L1DC, LOSWV and LOCV. The frequencies of these initiators were obtained via plantspecific systems analysis which seemed to be reasonable. 


\section{Review of the Internal Events Analysis}

\section{LOCA Initiatore}

The categorization of LOCA events in the DCPRA is based on the analyses of generic Westinghouse plants. The LOCAs are:

- Excessive LOCA, ELOCA: Any LOCA more severe than the design basis LOCA (beyond the capability of the ECCS).

- Large LOCA, LLOCA: Dia > 6 inches (within the capability of the ECCS).

- Medium LOCA, MLOCA: $2<$ Dia $\leq 6$ inches.

- Small LOCA; Isolable, SLOCI: Dia $\leq 2$ inches; results from leaking PORV, Nonisolable, SLOCN: Dia $\leq 2$ inches; break or leak other than from PORV.

- Steam Generator Tube Rupture, SGTR.

- Interfacing Systems LOCA; VSI, VDI: LOCAs originating at an interface between RCS and low pressure systems with a release path bypassing containment. VDI is located on the discharge side of the RHR pump, and VST is located on the suction side.

BNL considers this selection of LOCA initiators to be consistent with those of other PRAs. The review found that the mean frequencies of the majority of the LOCA categories were comparable with those used in PRAs for other Westinghouse reactors.

Due to current regulatory interest and insights obtained from ongoing studies, BNL has the following comments on the frequencies of the "Interfacing LOCA" and "Nonisolable, Small LOCA" initiators:

a. The main frequencies of the interfacing LOCA initiators in the DCPRA were derived along the approach developed for the Seabrook Emergency Planning Study. ${ }^{18}$ The approach involved the application of: i) "gross reverse leakage" and "Sailure to reseat on demand" failure modes for the series of check valves in the RHR injection lines. The frequency of the gross reverse leakage failure mode was considered to be leak-rate dependent; ii) "Gross reverse leakage," "MOV fails to operate on demand", and "MOV disk failing open when indicating closed" failure modes for the series of MOVs in the RHR suction line. The frequencies of MOV leakage and failure to operate upon demand failure modes were assumed to be identical with those of the check valves; iii) Averaging of time-dependent failure rates over the time period between leak testing of the valves. Valve leak tests are performed at each refueling $(T=1.5$ year $)$.

b. The check valve leak failure frequencies play a crucial role in the initiator frequency calculation. These were determined by the authors of the Seabrook study by selecting LER events essentially at the RCSECCS interface (the majority of the events were leakage into the accumulators). To estimate the total number of checkvalve hours, the analysts considered the total population of check valves in the ECCS instead of the particular subset of check valves at the interfaces. This resulted in a substantial overestimate of check valve hours, i.e., underestimation of check valve leak failure rates and thus underestimation of the frequencies of the interfacing LOCA initiators.

c. The initiator frequency calculation apparently did not consider the number of cold shutdowns between refueling periods, and thus neglected the contribution of "disk fails to reseat after demand" failure mode to the initiator frequency. Similarly, the human factor contribution through testing and maintenance was completely neglected. 
d. The check valve failure analysis did not recognize that the frequent occurrence of leakage events into the accumulator was due to the unusual corrosive environment of boric acid affecting the outlet check valves of the accumulators. Consequently, it was not recognized that leakage events to the accumulators represent "small LOCA precursors" in that preferred direction.

e. The "Nonisolable, Small LOCA" initiating frequency seems to be underestimated from the point of view that industry-experiencedRCP seal LOCA events caused by mechanicalor maintenance-inducedfailures were neglected from the prior event sample. NUREG/CR- $4400^{19}$ identified 71 seal leakage events at Westinghouse RCP pumps up to 1985, with four events in the $25 \mathrm{gpm}-500 \mathrm{gpm}$ leak rate range. Based on this information, an annual exceedance frequency vs. leak rate curve was constructed for Westinghouse plants with four RCPs (sec Figure 3.4.1). From this curve the initiator frequency of seal LOCAs exceeding the makeup capability of a plant can be estimated. Taking the "best estimate" curve, the RCP seal LOCA initiating frequency, i.e., the frequency of seal leakage events exceeding $150 \mathrm{gpm}$ can be determined. The value is about $8.0 \mathrm{E}-03 \mathrm{yr}^{-1}$. This value, which relates essentially to a subset of unisolable small LOCA initiating events is still higher than the "updated" DCPRA value, which is 5.83E-03 $\mathrm{yr}^{-1}$.

Upon the request of BNL, PG\&E provided information about the fractional contribution of RCP seal LOCA events to the unisolable small LOCA frequency. PG\&E considered it to be zero based on a cursory review of the data in the period 1980-1985. BNL indicated that PG\&E may relatively easily correct the above problematic issues b) through e) by using Reference 18 and a recent BNL study on Interfacing Systems LOCA at PWRs. ${ }^{20}$ In terms of the overall impact on plant CDF, these issues would not be expected to have a significant influence. They may, however, be important for the level 2 and 3 portions of the DCPRA when that effort is undertaken.

\subsection{Selection of Generic Initiating Event Frequencies for the DCPRA}

In order to understand the difference in initiating event frequency with respect to other PRAs, upon BNL's request, PG\&E submitted the following clarification of their approach in selecting generic initiating events for the DCPRA. This is reiterated here verbatim.

"The two main sources for the generic plant data used in the development of transient initiating event frequencies were:

- an Idaho National Engineering Laboratory (INEL) study of transients at U.S. nuclear power plants (Reference 9). Events selected from this study were those causing forced shutdowns at PWR units from 1980 to 1983.

- a compilation of Licensee Event Reports (LERs) by Tennessee Valley Authority. PWR transients from 1984 through July 1987 were considered from this source.

The transient events collected from the above sources covering a period of 7.6 years (from 1980 through July 1987) were further screened to include only those events that are appropriate for use in a PRA of a nuclear plant in power operation. The criteria used in the screening process were:

1) Those trip events occurring at or below $25 \%$ power were excluded if they occurred during power ascent or during shutdown. Trip events at or below $25 \%$ power were included if they occurred 


\section{Review of the Internal Events Analysis}

during power descent or during prolonged operation at a low power level. It was assumed that the docay heat at low power levels during power ascent would not be significant.

2) Trip events occurring between $25 \%$ power and $50 \%$ power were excluded if they occurred during ascent and were also due to feedwater instability. Feedwater problems are extremely common during power ascent, and it was judged that these events would not be applicable to normal plant operating conditions.

Many trip events not appropriate for a PRA of a nuclear plant in power operation were excluded from the database when the above screening criteria were applied. Moreover, by considering PWR transients from the period 1980 through July 1987 only, trip events occurring during the first year of operation for many PWR units were also excluded from the database. These are PWR units whose first year of commercial operation is prior to 1980. It is evident from Reference 1 that, in general, nuclear reactors experienced many more trip events in their first year of operation than in subsequent years.

It is expected that the transient initiating event frequencies computed from the above sources would be lese than those values calculated from a PWR transient events database which includes events from all years of operation for all the FWR units (i.c., includes first year of operation). Furthermore, the screening criteria described above will also tend to yield lower values for initiating event frequencies."

BNL considers the above generic initiating event selection and screening procedures to be somewhat biased in an optimistic fashion as noted throughout Section 3.4.

\section{4 .6 References}

1. Units 1 and 2 Diablo Canyon Power Plant, "Final Safety Analysis Report Update," Pacific Gas and Electric Company, December 1988.

2. "Reactor Safety Study-An Assessment of Accident Risks in U.S. Commercial Nuclear Power Plant," WASH-1400 (NUREG-75/014), USNRC, October 1975.

3. McClymont, A. S. and B. W. Poelilonan, “ATWS: A Reappraisal-Part 3: Frequency of Anticipated Transients," EPRI NP-2230, January 1982.

4. "Probabilistic Safety Analysis Procedures Guide," NUREG/CR-2815, January 1984.

5. "Indian Point Probabilistic Safety Study," Power Authority of the State of New York, Consolidated Edison Company of New York, Inc., 1982.

6. "Seabrook Station Probabilistic Safety Assessment," prepared by Pickard, Lowe and Garrick, Inc., for Public Service Company of New Hampshire and Yankee Atomic Electric Company, PLG-0300, December 1983.

7. Gunther, W. E., R. Lewis, and M. Subudhi, "Detecting and Mitigating Battery Charger and Inverter Aging" NUREG/CR-5051, August 1988. 
3 Review of the Internal Events Analysis

8. Garcia, A. A., et al., "A Review of the Millstone 3 Probabilistic Safety Study," NUREG/CR-4142, April 1986.

9. Idaho National Engineering Laboratory, "Development of Transient Initiating Frequencies for Use in Probabilistic Risk Assessment," NUREG/CR-3862, May 1985.

10. "A Storm From the Sun, EPRI Journal, pp.14-21, July/August 1989.

11. PG\&E Letter No. DCL-90-046 from J. D. Shiffer to the U.S. NRC, February 16, 1990.

12. "ATWS: A Reappraisal Part III-Frequency of Anticipated Transients," EPRI-NP-801', July 1978.

13. "Evaluation of Surveillance Frequencies and Out of Service Times for the Reactor Protection Instrumentation System," WCAP-10271.

14. Jansen, R.L., et al., "Evaluation of Surveillance Frequencies and Out of Service Times for the Reactor Protection System," WCAP-10272-A, May 1986.

15. "Midland Nuclear Plant-Probabilistic Risk Assessment," Consumers Powers Company and Pickard, Lowe and Garrick, Inc., May 1984.

16. "Reactor Risk Reference Document," NUREG-1150, App. A-1, Volume 2, February 1987.

17. Berry, D.L., et al., "Review and Evaluation of the Zion Probabilistic Safety Study," NUREG/CR-3300, May 1984.

18. "Seabrook Station Risk Management and Emergency Planning Sensitivity Study," Prepared by Pickard, Lowe and Garrick, Inc., for Public Service Commission of New Hampshire and Yankee Atomic Electric Co., PLG-0432, December 1985.

19. Azarm, M. A., J. L. Boccio, S. Mitra, "The Impact of Mechanical and Maintenance Induced Failures of Main Reactor Coolant Pump Seals on Plant Safety," NUREG/CR-4400, December 1985.

20. Bozoki, G., P. Kohut, R. Fitzpatrick, "Interfacing Systems LOCA: Pressurized Water Reactors," NUREG/CR-5102, February 1989. 


\section{Review of the Internal Events Analysis}

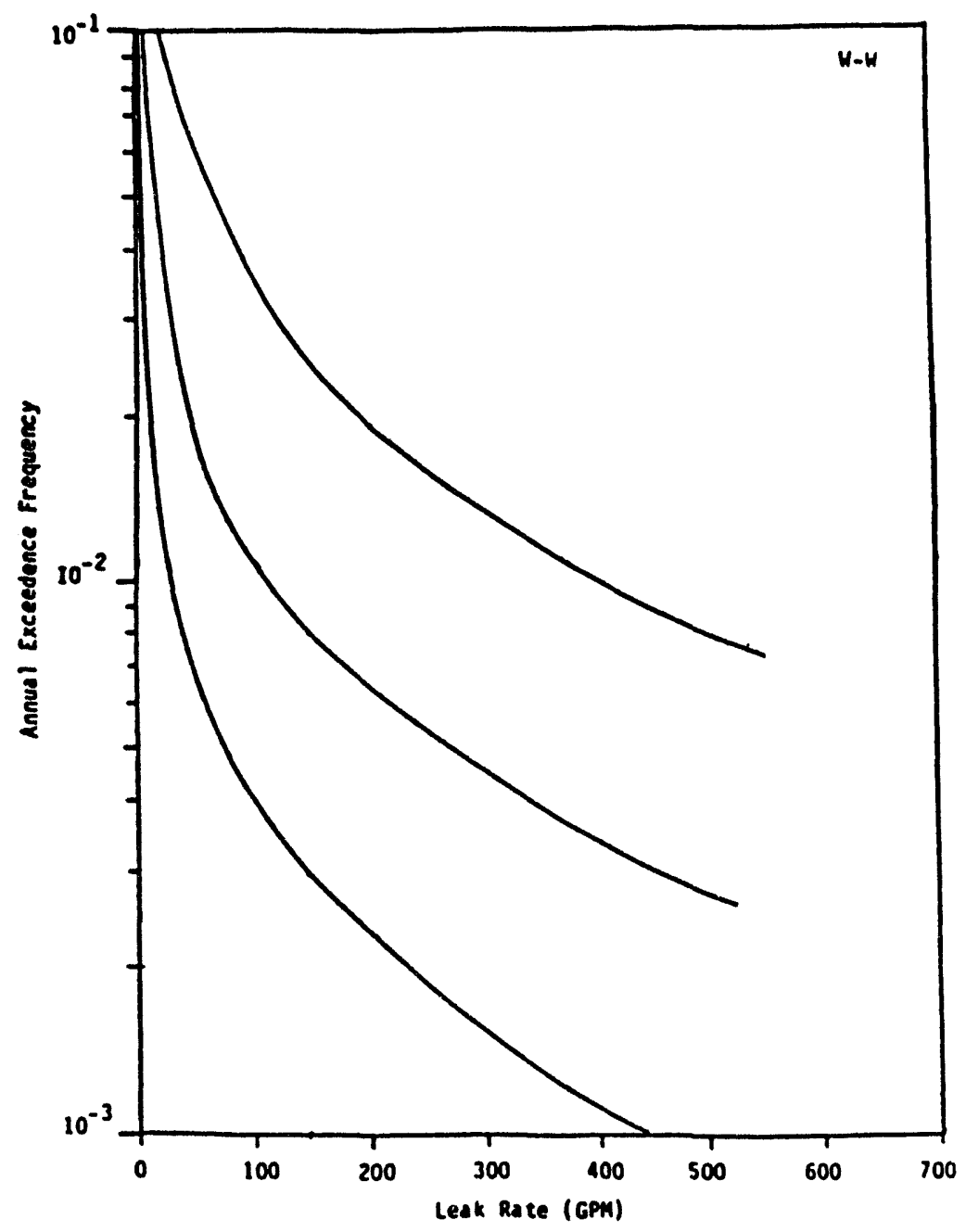

Figure 3.4.1 Annual Exceedance Frequency vs. Leak Rate for Westinghouse Plants with Four RCPs (lower bound, best estimate, upper bound)

(Figure Taken from Reference 20). 
3 Review of the Internal Events Analysis

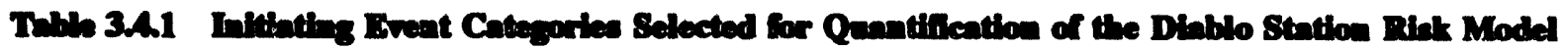

(Table 6.26 of DCPRA)

\begin{tabular}{|c|c|c|c|}
\hline Group & & $\begin{array}{l}\text { Initiating Event Categories Selected } \\
\text { for Separate Quantification }\end{array}$ & $\begin{array}{l}\text { Code } \\
\text { Desigrțator }\end{array}$ \\
\hline $\begin{array}{l}\text { Loss of Coolant } \\
\text { Inventory }\end{array}$ & $\begin{array}{l}1 . \\
2 . \\
3 . \\
4 . \\
5 . \\
6 . \\
6 \mathrm{a} . \\
6 \mathrm{~b} . \\
7 .\end{array}$ & $\begin{array}{l}\text { Excessive LOCA } \\
\text { Large LOCA } \\
\text { Medium LOCA } \\
\text { Small LOCA, nonisolable } \\
\text { Small LOCA, isolable } \\
\text { Interfacing systems LOCA } \\
\text { At RHR pump suction } \\
\text { At RHR pump discharge } \\
\text { Steam generator tube rupture }\end{array}$ & $\begin{array}{l}\text { ELOCA } \\
\text { LLOCA } \\
\text { MLOCA } \\
\text { SLOCN } \\
\text { SLOCI } \\
\text { VS } \\
\text { VD } \\
\text { SGTR. }\end{array}$ \\
\hline Transients & $\begin{array}{l}8 . \\
9 . \\
10 . \\
11 . \\
12 . \\
13 . \\
14 . \\
15 . \\
16 . \\
17 . \\
18 . \\
19 . \\
20 . \\
21 .\end{array}$ & $\begin{array}{l}\text { Reactor trip } \\
\text { Turbine trip } \\
\text { Loss of condenser vacuum } \\
\text { Closure of all MSIVs } \\
\text { Steam line break inside containment } \\
\text { Steam line break outside containment } \\
\text { Inadvertent safety injection } \\
\text { Main steam relief valve opening } \\
\text { Total main feedwater loss } \\
\text { (includes feedwater line break) } \\
\text { Partial main feedwater loss } \\
\text { Excessive feedwater } \\
\text { Closure of one main steam } \\
\text { isolation valve (MSIV) } \\
\text { Core power excursion } \\
\text { Loss of primary flow }\end{array}$ & $\begin{array}{l}\text { RT } \\
\text { TT } \\
\text { LCV } \\
\text { AMSIV } \\
\text { SLBI } \\
\text { SLBO } \\
\text { ISI } \\
\text { MSRV } \\
\text { TLMFW } \\
\text { PLMFW } \\
\text { EXFW } \\
\text { IMSIV } \\
\text { CPEXC } \\
\text { LOPF }\end{array}$ \\
\hline \multicolumn{4}{|l|}{$\begin{array}{l}\text { Common Cause } \\
\text { Initiating Events }\end{array}$} \\
\hline $\begin{array}{l}\text { Support System } \\
\text { Faults }\end{array}$ & $\begin{array}{l}22 . \\
23 . \\
24 . \\
25 . \\
26 . \\
27 .\end{array}$ & $\begin{array}{l}\text { Loss of offsite power } \\
\text { Loss of one DC bus } \\
\text { Total loss of auxiliary saltwater } \\
\text { Total loss of component cooling water } \\
\text { Loss of } 480-V \text { switchgear ventilation } \\
\text { Loss of control room ventilation }\end{array}$ & $\begin{array}{l}\text { LOOP } \\
\text { L1DC } \\
\text { LOSW } \\
\text { LPCC } \\
\text { LOSWV } \\
\text { LOCV }\end{array}$ \\
\hline Seismic Events & $\begin{array}{l}28 . \\
29 . \\
30 . \\
31 . \\
32 . \\
33 .\end{array}$ & $\begin{array}{l}0.2 \mathrm{~g} \text { to } 1.25 \mathrm{~g} \\
1.25 \mathrm{~g} \text { to } 1.75^{\mathrm{g}} \\
1.75 \mathrm{~g} \text { to } 2.0 \mathrm{~g} \\
2.0 \mathrm{~g} \text { to } 2.5 \mathrm{~g} \\
2.5 \mathrm{~g} \text { to } 3.0 \mathrm{~g} \\
3.0 \mathrm{~g} \text { to } 4.0 \mathrm{~g}\end{array}$ & $\begin{array}{l}\text { SEIS1 } \\
\text { SEIS2 } \\
\text { SEIS3 } \\
\text { SEIS4 } \\
\text { SEISS } \\
\text { SEIS6 }\end{array}$ \\
\hline
\end{tabular}


3 Review of the Internal Events Analysis

Toble 3.4.1 (conthend)

Group

Fire and Smoke

Flood, Jets, and Sprays (pipe breaks)
34. Loss of both motor-driven AFW pumps

35. Loss of all charging pumps and MSIV closure

36. Loss of component cooling

37. Loss of control ventilation

38. Loss of auxilliary saltwater

39. Loss of 4-kV buses HF and HO

40. Loss of 4-kV buses HO and HH

11. Loss of 4-kV buses HF, HG, and HH

42. Control room fire at vertical board VB-1

43. Control room fire at verical board VB-2

44. Control room fire at the interface of vertical boards VB-2 and VB-3

45. Centrol room fire at vertical board VB-4

46. Cable spreading room fire one

47. Cable spreading room fire two

48. Loss of all audiliary feedwater

49. Loss of both motor-driven AFW pumps

50. Loss of auxiliary saltwater
Code

Designator
FS1

FS2

FS3

FS4

FS5

FS6

FS7

FS8

CR1

CR2

CR3

CR4

CS1

CS2

FS9

FS10

FS11 
3 Review of the Internal Events Analysis

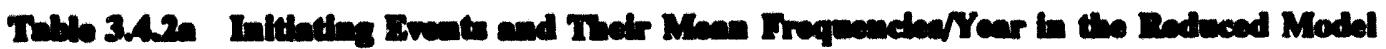

\begin{tabular}{|c|c|c|}
\hline $\begin{array}{l}\text { Initiating Event } \\
\text { Deaignator }\end{array}$ & Menn Frequency & $\begin{array}{c}\text { Generic Mean } \\
\text { Frequency }\end{array}$ \\
\hline LOSWV & $6.29 \mathrm{E}-05$ & - \\
\hline L1DC & $2.56 \mathrm{E}-02$ & $3.35-02$ \\
\hline LOOP & $9.10 \mathrm{E}-02$ & $1.40-01$ \\
\hline SLOCN & $5.26 \mathrm{E}-03$ & $5.83-03$ \\
\hline FS1 & 2.94E-04 & - \\
\hline SLBO & $5.53 E-03$ & $6.04-03$ \\
\hline $\mathbf{R T}$ & $1.14 E+\infty 0$ & $1.53+\infty 0$ \\
\hline $\mathbf{T T}$ & $1.05 \mathrm{E}+00$ & $1.08+00$ \\
\hline FS8 & 6.18E-06 & - \\
\hline PLMFW & 7.49E-01 & $1.13+00$ \\
\hline FS11 & 3.81E-04 & - \\
\hline LPCC & $1.96 \mathrm{E}-04$ & - \\
\hline MLOCA & 4.63E-04 & $4.65-04$ \\
\hline FS9 & 1.35E-05 & - \\
\hline EXFW & 2.79E-01 & $1.71-01$ \\
\hline LOSW & 9.74E-05 & - \\
\hline LLOCA & 2.02E-04 & $2.03-04$ \\
\hline SGTR & $1.71 \mathrm{E}-02$ & $2.88-02$ \\
\hline LOPF & $1.21 \mathrm{E}-01$ & $1.66-01$ \\
\hline SLBI & 4.63E-04 & $4.65-04$ \\
\hline IMSIV & 1.07E-01 & $8.43-02$ \\
\hline TLMFW & 9.98E-02 & $1.64-01$ \\
\hline VSI & 1.01E-06 & - \\
\hline SLOCI & 1.61E-02 & $2.30-02$ \\
\hline LCV & 8.73E-02 & $1.16-01$ \\
\hline LOCV & $7.99 \mathrm{E}-02$ & - \\
\hline ISI & 7.39E-02 & $3.03-02$ \\
\hline FS6 & 2.41E-05 & - \\
\hline ELOCA & $2.66 \mathrm{E}-07$ & $2.66-07$ \\
\hline FS5 & $5.26 \mathrm{E}-05$ & - \\
\hline FS10 & $1.40 \mathrm{E}-05$ & - \\
\hline HAZCHM & $4.39 \mathrm{E}-04$ & - \\
\hline CRFIRE & 3.17E-05 & - \\
\hline
\end{tabular}


3 Review of the Internal Events Analysis

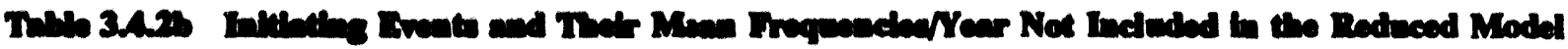

\begin{tabular}{|l|c|c|}
\hline $\begin{array}{c}\text { Initiating Evout } \\
\text { Deatgnator }\end{array}$ & Mean Frequency & $\begin{array}{c}\text { Generic Mean } \\
\text { Frequency }\end{array}$ \\
\hline VDI & $3.86-06$ & \\
\hline AMSIV & $1.74-02$ & $1.95-02$ \\
\hline MSRV & $3.59-03$ & $4.23-03$ \\
\hline CPEXC & $2.19-02$ & $2.72-02$ \\
\hline FS2 & $2.89-03$ & - \\
\hline FS3 & $3.05-06$ & - \\
\hline FS4 & $2.00-03$ & - \\
\hline FS7 & $1.43-05$ & - \\
\hline
\end{tabular}




\subsection{Event Troes}

The DCPRA event trees were not given a rigorously detailed review by BNL as part of the overall DCPRA review process. The basis for this was that there was an extremely detailed and comprehensive methodology applied to the event tree development and, therefore, BNL tried to concentrate resources on other areas of the PRA.

The applied methodology utilized event sequence diagrams (ESDs) and stressed the involvement of both PRA analysts and plant operations personnel. BNL did check for any obvious errors in the event tree logic structure but none were apparent. The following is one of the latest papers presented on this subject: F.R. Hubbard III, M.A. Waller, D.J. Wakefield, "The Use of Event-Sequence Diagrams in Probabilistic Risk Assessment," Vol. 60, p. $407-408$ in Transactions of the American Nuclear Society, 1989 Winter Meeting, San Francisco, California, TANSAO 60 1-792, 1989.

\subsection{Fault Trees}

The fault tree review was conducted as part of the systems analyses documented in Appendices $A$ and $B$. The system documentation associated with the DCPRA provided reliability block diagrams (as opposed to actual fault trees) containing supercomponents covering large portions of the system. BNL converted these diagrams into fault trees and used the SETS computer code to solve them. This allowed BNL to display the leading cut sets for those top events so modelled. Such cut sets are not provided within the DCPRA.

The quantification of the supercomponents was supplied in equation form by PG\&E. That is, in order for BNL to supply the value block for input to the SETS code, the equation for each of the supercomponents had to be solved as well as broken down to identify/verify its constituent parts. Each equation represented an expression that combined all the failure modes of each of the elements of the supercomponents. BNL also checked the equation against the plant drawings to verify that all major components/failure modes were included.

In order to then verify the various split fractions associated with each fault tree, BNL had to set various items to one or zero to define each boundary condition and then solve that version of the fault tree four times to account for the different postulated sets of system alignment as discussed in Section 3.2.

The BNL quantification/verification of the conditional split fractions in most cases provided good agreement with the PG\&E results. The difference in the majority of the cases coming from the use of Monte Carlo techniques by PG\&E and point estimates by BNL. There were a few notable exceptions as discussed in Appendices A and B, however, these were not due to the fault tree/reliabilityblock diagram structure or level of detail. BNL concluded that for the fault trees/reliability block diagrams that received detailed review, they adequately represented the Diablo Canyon Plant.

\subsection{Failure Data}

The DCPRA data base was developed by updating the proprietary PL\&G data base. The PL\&G data base was first screened to exclude failure events that were believed clearly not applicable to the Diablo Canyon plant. This screened data base then became the source of the "priors" for a Bayesian updating process. Diablo Canyon-specific data was used where available as the "experience" for updating and the resulting posterior distributions became the Diablo Canyon data base used in the DCPRA. Appendix H (specifically Section H.2) of the DCPRA provides a comprehensive treatise on the complete data analysis approach that was used and is therefore not repeated here. 
Appendix $C$ of this report presents the complete data base as provided to BNL by PG\&E and reflects its status as of July 8, 1988. Quantification of the conditional split fractions discussed in Section 3.9 and Appendix D was based upon this data base.

The BNL review scope in this area was limited to a review of the overall methodology and then was specifically guided by the results of the systems analysis reviews documented in Appendices A and B. BNL fully endorses the Bayesian updating approach used for the DCPRA. Specific comments on individual failure probabilities are addressed as part of the systems analysis reviews within Appendices A and B. Specific comments on initiator quantification are found in Section 3.4 .

As part of the systems analysis review of the Auxiliary Feedwater (AFW) system, BNL substituted generic failure probabilities into the AFW model to get an idea of how sensitive the results were to the Diablo Canyon-epecific data used in the DCPRA. This analysis is documented in Appendix A3 and showed that the results were not that sensitive to an alternate data base.

\subsection{Human Reliability Analysis (HRA) Methodology}

\subsubsection{Scope of the HRA Methodology Review}

The review of the Diablo Canyon Probabilistic Risk Assessment (DCPRA) HRA methodology centered on Section 3.5 and Appendix $G$ of the main report. Also reviewed were documents elaborating on the HRA prepared by PG\&E and provided to the NRC in response to questions and solicitations arising from this review. These latter documents were included in correspondence from PG\&E to the Nuclear Regulatory Commission (NRC) dated January 16, 1989, and December 8, 1989, respectively. The following paragraphs present questions which guided the HRA review (part of 3.8.1), an overview of the HRA performed as part of the DCPRA (3.8.2), an overview of the HRA review findings (3.8.3), a detailed discussion of each finding vis-a-vis questions guiding the review (3.8.4), and a summary of the HRA review (3.8.5). In addition, suggested revisionsfimprovements for Section 3.5 and Appendix $G$ to the DCPRA, mainly for the benefit of future users, are discussed in Section 6 of this report.

No attempt was made to replicate any of the analyses performed by the HRA team. Rather, reasonableness of results was judged based on the credibility of modeling, source data, and human factors assessment instruments employed. Therein, the review focused on data, methods, and comprehensiveness of analyses performed to aseese involvement of humans as precursors, initiator, and mediators, including recovery, in each DCPRA accident sequence. More specifically, the following nine questions guided this review.

1. To what degree were human factors professionals used in the DCPRA HRA?

2. What type of human systems analysis was performed to support plant model development, and to identify pertinent human task actions for inclusion in the event and fault trees?

3. What types of task actions (both cognitive and execution) were analyzed as part of each accident sequence? And, how were they chosen?

4. What person-centered (e.g., experience, fatigue, stress), task-centered (e.g., training, procedures), and environment-centered (e.g., supervision, team support, organizational support) performance shasping factors (PSFs) were scaled for human task actions included as precursors, initiators, or mediatoni in the event and fault trees? How were they chosen? And, what methods (including rationale for their choice) were used to scale each PSF?

5. What quantification methods (e.g., THERP, HCR, SLIM-MAUD) were used to estimates human errors on task actions selected for analysis? Why were these quantification methods chosen?

6. What were the sources of generic error data from which bounding values were derived for estimating human errors? Why were these sources selected?

7. What characterization or behavioral model of plant personnel was used to identify multiples and dividends of the base or point estimates of human error for the sensitivity analysis? 
8. What inferences (insights) are drawn (in line with information developed as part of 2 through 7 above) regarding the contribution of human error on overall plant risk?

9. To what extent were results documented to allow for auditing and/or replicating, or to allow for combining with data from other PRAs?

BNL conducted a full set of importance analyses for the various human actions/errors that are included in the DSM. These can be found in Section 3.9 and Appendix D. In addition, the quantification of selected actions was reviewed as part of the system analyses documented in Appendices A and B.

\subsection{Overview of the HRA}

The DCPRA HRA was performed by staff of PG\&E and PLG, closely supported by Diablo Canyon (DC) plant personnel. Based on discussions with PG\&E representatives, it was concluded that no human factors specialists were included on the PG\&E/PLG HRA team. The HRA segment of the DCPRA followed a sometimes iterative six step process:

- First, event sequence diagrams (ESDs) were used as task analysis tools to identify pertinent human actions that would be analyzed as part of the event and fault trees.

- Second, human task actions identified as pertinent were categorized as skill, rule, or knowledge based (Rasmusson's topology) using the Electric Power Research Institute's Human Cognitive Reliability (HCR) criteria.

- Third, performance shaping factors (PSFs) were identified by PG\&E and PLG HRA team members by means of a nominal group technique, for each human task action selected for analysis.

- Fourth, some 21 equipment and person centered PSFs, identified as part of the previous step, were ovaluated by DC operators using a survey instrument developed and pre-tested by the HRA team.

- Fifth, error probability, recovery, and uncertainty estimates were calculated employing the Nuclear Regulatory Commission's Success Likelihood Index Method (SLIM) as the quantification tool, and using Three Mile Island PRA human error data as bounding values.

- Sixth, HRA results documentation was guided by precedents established in earlier PRAs especially Three Mile Island.

\subsection{Sunmary of Findings}

\subsubsection{Poulive Finding}

The HRA performed by PG\&E/PLG is innovative in form if not completely in substance. Efforts were made to systematically select, analyze and document the effects of $\mathbf{7 5}$ different human task action types, primarily involving potential for errors of omission, as part of some $\mathbf{4 0 0}$ sequence analyses encompassing 12 frontline and 14 support systems. Calculated human error point estimates ranging from close to 1.0 to $1.6 \mathrm{E}-3$ appear reasonable based on the scaled PSF values, bounding values, and quantification methods employed. Uncertainty estimates generally falling between 1-2 magnitudes above and below the point estimates also appear reasonable. In summary, while the human factors qualifications of the PG\&E/PLG personnel involved in the HRA may be at issue, it appears that personnel involved made appropriate use of source data and quantification methods cited in the DCPRA main report (Section 3.5, Appendix G). Two other innovative features of the HRA should be noted. First, task analyses were constructed as ESDs relying on interviews, documentation reviews, and walk-throughs/talk-throughs, rather than relying primarily on normal operating and emergency operating procedures. This had the effect of producing more accurate and complete descriptions of what plant personnel actually do during off-normal conditions than can be achieved relying solely on written procedures. Second, human factors rating scales and plant personnel were used in 


\section{Review of the Internal Events Analysis}

evaluating the 21 PSFs considered in the HRA. This is a significant departure from more commonly used engineering judgement approaches to PSF scaling. This approach also allowed for better auditing of the quantitativeanalyses of human error and for gaining insights into strengths and weaknesses of person-centered, equipment-centered, and environment-centered factors which tend to enhance or exacerbate human performance.

\subsection{Negative Fadinge}

The following findings were derived using the nine questions enumerated under 3.8.1. They should be viewed as distractions rather than as refutations from the findings summarized under 3.8.3.1.

1. No certified human factors specialists participated as members of the HRA team.

2. The HRA focused primarily on potential errors of omission rather than commission; likewise it focused primarily on execution errors rather than on cognitive errors.

3. PSF evaluations, based on ratings by plant personnel, were often done based on single item rating scales.

4. It is not always clear which of the 21 PSFs scaled for the HRA were associated with each task action, by type and by analysis.

5. Generic data used for bounding point estimates and uncertainties were derived from the Three Mile Island PRA rather than from training simulator data collected by the HRA team at Diablo Canyon, or from an amalgamation of data from the two dozen PRAs that were available at the time.

6. The main report, Section 3.5 and Appendix G, leave the impression that the HCR method was used to analyze cognitive errors. Later correspondence from PG\&E to the NRC indicate that HCR was used only to categorize task actions (i.e., skill, rule, knowledge).

7. Environmental PSFs (e.g., habitability, supervision, working or organizational climate) were not included in the analysis.

8. The main report (Section 3.5 and Appendix G) leave the impression that a SLIM-MAUD "type" quantification method was used to analyze human error. Later correspondence indicates that the SLIM methodology, a limited version of SLIM-MAUD, was actually used.

\subsubsection{Discussion of each Finding Vis-A-Vis Review Guidelines}

\subsection{HRA Tean Members}

The HRA team was comprised of PG\&E and PLG scientists none of whith were certified human factors specialists. While this omission has been the rule in past PRAs, the credibility of the DCPRA could have been further enhanced if this type of expertise had been available, especially since a number of innovations were introduced into the analysis. In this regard, free standing task analyses were done to identify pertinent task actions and associated PSFs (the latter heavily reliant on behavioral and social science considerations). Second, rating scales were developed and implemented with plant personnel to evaluate PSFs (a process heavily reliant on psychometric scaling expertise). Third, the SLIM method, employing a nominal group approach, was used to derive human error point estimates and to do sensitivity analyses (the latter assuming a conceptual model of human performance under a wide range of task conditions mediated by the effects of person, equipment and environmental PSFs). In each of these instances, inclusion of one or more human factors specialists, bringing behavioral and social science, and psychometric perspectives to the team, would have contributed to a more complete analysis, would have enhanced the team's ability to gain insights from the analytic experience, and would have enhanced the team's ability to derived lessons learned for future analyses such as those to be performed for the Individual Plant Examination (IPE) Program. Greater involvement of certified human factors specialists would have more fully responded to Negative Finding 1 (3.8.3.2). 


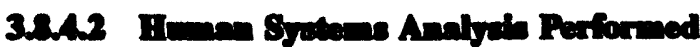

The HRA team did stand-alone task analyses of anticipated accident sequences, employing event sequence diagrams (ESDs), to identify pertinent task actions and associated PSFs. This innovation (not relying solely on normal and emergency operating procedures) reflects a conscious attempt by the team to elevate HRA beyond a peripheral consideration in the DCPRA main report. The ESDs emerging from this process along with narrative information necessary for the human actions review were not fully documented in the main PRA report or Appendix G. This omission somewhat limited the reviewers' ability to assess the degree to which inclusion of certified human factors practitioners on the team could have further exploited the procedure. As a minimum, such involvement would have sensitized the whole team to a broader mix of omission, commission, execution, and cognitive errors, therein, responding more coinpletely to Negative Finding 2 (3.8.3.2).

\section{Types of IImann Tark Actions Analyzed}

A total of 75 human task types, involving approximately 400 analyses, were included in the HRA. The scope of the analysis, therefore, compares favorably with other precedent setting HRAs such as those performed for the Oconee III and Arkansas Nuclear 1 PRAs. Limited attention of the HRA to errors of commission and cognition (e.g., decision making) is unfortunate because the HRA team did had available the HCR method for analyzing errors of commission; likewise it had available the THERP method described in NUREG/CR1278 for analyzing cognitive errors. Greater attention to these type errors would have responded more fully to Negative Finding 2 (3.8.3.2).

\subsection{A Performance Shaping Factors Evaluated}

Some 21 person and equipment centered PSFs were selected by the HRA team using the ESDs discussed above, and were scaled using a rating scheme with plant personnel. These human task actions and PSFs were subsequently used to analyze the likelihood of error for each of the 75 task actions types under the approximate $\mathbf{4 0 0}$ analysis conditions. Regarding PSF selection; the absence of environment centered PSFs from this constellation, and the potential influences that these environmental factors are believed to have on performance, may have had a deleterious effect on the accuracy of the HRA results. Closer attention to environment centered PSFs would have more fully responded to Negative Finding 2 (3.8.3.2). Regarding PSF scaling; the reviewers consider this to be an innovative way of developing such data. In some instances, however, PSFs were scaled using single items, therefore, potentially reducing intra- and inter-judge reliability. Inclusion of a human factors practitioner experienced in scale development and interpretation would most likely have precluded this type of problem, and would have responded more fully to Negative Findings 3,4 , and 7 (3.8.3.2).

\subsection{Qunntification Methods Used to Estimate Human Error Rates}

Two state of the knowledge quantification methods were used for the analysis (i.e., HCR, SLIM). According to the main report (Section 3.5 and Appendix G) HCR was used to analyze cognitive errors (diagnosis). Later correspondence to the NRC (January 16, 1989, and December 8, 1989, however, indicate that HCR was only used to categorize human task actions using the Rasmusson topologies (skill, rule, knowledge). According to the main report (Section 3.5, Appendix G) a SLIM-MAUD type method was used to do the main analyses. It was determined through subsequent interactions between the reviewers and PG\&E staff, that SLIM rather than SLIM-MAUD was used for quantifying human error likelihoods. This is an important distinction because SLIM is implemented differently than SLIM-MAUD, and some of its algorithms differ from SLIM-MAUD. This distinction is even more important for the DCPRA since SLIM was employed in a unique way. Instead of using SLIM as a basis for forming consensus judgements among subject matter experts, its rating and 


\section{Review of the Internal Events Analysis}

weighing inputs were based, in part, on actual field data. In summary, SLIM was used to quantify the effects of PSFs human error rates using real plant data rather than solely on judgements of subject matter experts. A more clear description of the method employed in the analyses, and the manner in which it was employed (real data rather than solely expert opinion), would have more fully responded to Negative Findings 4, 6, and 8 (3.8.3.2).

\section{Cenceric Buman Brror Datu Sources}

Source data used by the HRA team as bounding values for human error rate point estimation and for sensitivity testing were taken primarily from the Three Mile Island PRA. Relying solely on those data ostensibly introduced an unnecessary element of conservatism into the analysis. The team had available to it data from some two dozen other PRAs, as well as data derived from the plant training simulator. More stable bounding value data could have been derived from combining these data rather than relying solely on Three Mile Island data. Consideration of other PRA data as well as training simulator data for this purpose would have more fully responded to Negative Finding 5 (3.8.3.2).

\section{Sonctiving Modelting Apronach}

Sensitivity analyses performed using HRA data seem reasonable within the context of the findings presented above, although neither the main report nor the supplements, provided to the NRC in January and December 1989, made clear what kind of behavioral model was used to guide the analyses. Explication of the model used would have made the review more straightforward and probably more supportive of the reported results.

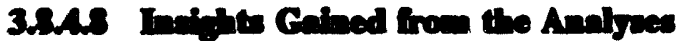

Insights gained from the HRA were stated in very general terms in Section 3.5 and Appendix $G$ of the main report. Human error was a "significant contributor" to bottom line risk. However, more specific delineations of the particular human task actions, associated PSFs, and qualitative information on these PSFs, would have enhanced the reader's appreciation of work done, and would have provided guidance to future HRA analysts for gaining insights into causal factors of human error, and potential remedial actions for overcoming these errors. Information developed in the HRA (stand-alone task analyses, PSFs scaling, innovative uses of HCR and SLIM) could have supported a much better delineations of insights gained from the HRA.

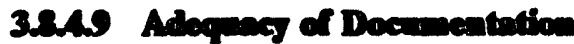

Again, this review of the Diablo Canyon Probabilistic Risk Assessment (DCPRA) HRA centered on Section 3.5 and Appendix $G$ of the main report. Also reviewed were documents elaborating on the HRA prepared by Pacific Gas and Electric (PL\&G) in response to questions and solicitations from the reviewers. These were included in correspondence from PG\&E to the Nuclear Regulatory Commission (NRC) dated January 16, 1989, and December 8, 1989, respectively. The latter two documents were necessitated because information initially provided in the main report was incomplete. It was concluded as part of this review that the main report is an incomplete, and sometimes inaccurate (e.g., applications of HCR and SLIM) representation of what was accomplished in the HRA. Just as important, the main report fails to detail the innovations that were introduced in the HRA, and the effects of these innovations on the overall results. Finally, as suggested by the discussions under 3.8.3.4.1 through 3.8.3.4.8, incomplete documentation was probably one contributor to the negative findings. It is suspected that further interactions between the reviewers and the HRA team would disclose that some of the negative findings discussed above were influenced by incomplete documentation on the tasks for which these analyses were carried out. 


\subsection{Summary of the HRA Methodology Review}

This review was conducted using the main report and supplements described in 3.8.4.9 above. Based on these documents it is concluded that the HRA team conducted a state of the knowleage analysis, and introduced several innovations ostensibly of interest to the larger HRA/PRA community. These included stand-alone task analyses using ESDs, systematic scaling of PSFs using plant personnel, and applying real PSF data in quantification of human error using an otherwise expert judgement SLIM approach. The quantitative results (point estimates and uncertainty bounds) emerging from this were reasonable when compared with data from other PRAs. In some instances the main report fails to document completely and accurately the analyses performed, the techniques employed to gather input data, and the manner in which quantification methods were used. Negative findings described under 3.8.3 and 3.8.4, while detracting from the HRA, do not compromise its overall acceptability.

\subsection{Accident Sequence Quantification}

\subsubsection{The Event Sequence Model of the DCPRA}

The event sequence model of the DCPRA consists of four parts:

1. The electrical support system event tree.

2. The actuation and mechanical support system event tree.

3. The early response frontline system event tree.

4. The long-term response frontline system event tree.

Figure 3.9.1 shows the schematic of the event sequence model. This model served as a basis to evaluate the plant response to each one of the 50 initiating categories. Depending on various combinations of plant equipment and operator response success or failure states, an event sequence either was terminated with no core damage (success) or lead to core damage. Since the plant response to many of the initiating event categories is quite similar, common event tree modules were developed. They were quantified, however, with different boundary conditions and different system top event values specific to each initiator. Figure 3.9.1 indicates the essential modules of the event sequence model.

The electrical/actuation-mechanical modules are common to all initiating events. These modules represent different support system states, i.e.,combinations of support system top event successes and failures that affect the frontline systems in similar ways. The number of different support system states after a reduction/combination process became 178 distinct states.

Next are the seven early response frontline system event tree modules. These are:

General Transient: For most initiating event categories that require active nuclear shutdown, heat removal or coolant injection.

Lange LOCA: For LOCA's having breaks in the RCS boundary with dia. $>6$ inches up to a full guillotine break of the cold leg piping requiring high capacity makeup.

Steam Generator Tube Rupture: Similar to the general transient, with special requirements for operator actions to eliminate the flow of reactor coolant though the ruptured steam generator and thus bypassing containment. 


\section{Review of the Internal Events Analysis}

Interfacing Systems LOCA: For high pressure/low pressure boundary failures when reactor coolant can bypass containment and equipment needed for control of the LOCA can also be damaged.

Seismic Events: Similar to the general transient module; additional details added to account for earthquake-induced dependent failures and specially required human actions.

Any Transient Without Trip (ATWT): Covers all the initiating events that require trip if the trip was identified as failed in the support system model; considers potential ensuing overpressurizations and special actions to provide long-term shutdown of the nuclear reaction.

Medium LOCA: For LOCAs having breaks in the RCS boundary with diameters in the range from 2 inches to 6 inches. Similar to the general transient module with modified top event success criteria to reflect conditions imposed by the break flow.

If the event sequence has not reached a successful conclusion, sequences continue to one of the long-term event tree modules. Sequences requiring long-term cooling continue into LT1. Essentially, the model for Diablo Canyon ends here, as the DCPRA is a Level 1 PRA. Figure 3.9.1 shows the generic model as it relates to Level 2 of PRA. In that case sequences that have already reached core damage would branch to LT2, LT3 or LT4 to be classified into plant damage states depending on the status of the containment safeguards.

In developing the ATWT event tree module, four initiating events were selected as representative of a range of challenges to the RCS pressure boundary and the plant safety systems. These were:

1. Loss of Auxiliary Saltwater or Loss of Component Cooling Water

2. Turbine Trip

3. Loss of Offsite Power

4. Loss of Main Feedwater

The loss of main feedwater was taken as representative for all initiators not mentioned above that are accompanied by failure of reactor trip, except for small LOCA. The small LOCA was not treated in the ATWT, because the safety requirements without reactor trip are claimed to be essentially the same as in the case when the reactor is successfully tripped.

It has to be emphasized that the event trees associated with the modules appearing in Figure 3.9.1 are essentially abstracted, simplified versions of the underlying event sequence diagram (ESD) models. They were quantified by using the appropriately selected top event split fractions discussed previously. For the DCPRA quantification, the RISKMAN family of computer codes was used.

\subsubsection{Some Technical Aspects of the Internal Event Sequence Quantification}

This section provides information about some technical aspects of the internal event sequence quantification process. Its content was developed mainly as a result of the review effort as the originally submitted DCPRA documentation did not present significant information in this area. It outlines the calculational approach followed by PG\&E to obtain core damage frequency values for the internal event sequences. 


\section{A. Quantification of the Support State Modules}

According to the DCPRA, the plant event sequence model was quantified for each initiating event and each support state. Since many of the internal (and seismic) event/support state combinations were extremely unlikely or physically not realizable, the DCPRA grouped them together. The review requested clarification about this procedure. The concern was the potential error in the plant response analysis if certain support states were neglected entirely. The response from PG\&E is reproduced here to offer some detailed insight about the early phases of the event sequence quantification:

"In regards to frequency binning of support states, it should be noted that the DCPRA does not discard any support states; all support states are retained either explicitly or in a bin. Support states are binned, if low enough in frequency, to a support state with the worst possible impacts (on the frontline systems). The frequency of this support state is added directly to the core damage frequency (it is not unused). The binning occurs in the following two steps:

The first step evaluates the combination frequency of the electric power event tree end states and the mechanical/actuation event tree end states without regard to impacts on frontline systems. If the combinational frequency of an electric power state (i.e., first stage of the support model) and a mechanical/actuation state (i.e., second stage of the support model) for all initiators is low enough (5.0E-10) then this support state is mapped to the bin support state. This cutoff is primarily used to filter out the zero frequency states (i.e., those combinations which are logically impossible) although it is possible that some very low frequency support states are binned in this process.

The second step of binning occurs after the support states have been defined in terms of their impacts on the frontline systems. This binning also is done on the electric power/mechanical and actuation event tree end state combination frequency. Two different cutoffs were used; for the sum of all non-seismic events, the cutoff was 5.0E-09 and for the sum of all seismic events the cutoff was 1.0E-08. Again, all support states with frequencies below these cutoffs were mapped to a "bin" support state with the worst possible impacts."

\section{B. Quantification of the Frontline System Modules}

The support states of the support modules were linked with the sequences of the frontline modules by using a computer code, SQLINK of the RISKMAN family of codes. From the vast amount of sequences which lead to core damage, SQLINK selected and retained the leading 1999 sequences. In other words, the DCPRA truncated all sequences whose frequency was below an effective cutoff value of approximately $8.0 \mathrm{E}-09 \mathrm{yr}^{-1}$. This de minimus value is somewhat higher than the traditional de minimus value used in the Reactor Safety Study (WASH-1400); 1.0E-09 $\mathrm{yr}^{-1}$. For practical purposes, PG\&E developed a reduced, Dominant Sequence Model (DSM) by selecting the leading 420 sequences (representing 88.1 percent of the total non-seismic core damage frequency). The corresponding effective cutoff value was: 1.1E-07 $\mathrm{yr}^{-1}$. (A description of the Dominant Sequence Model can be found in Appendix D.) PG\&E claims that after correcting the leading sequences (manually) for selected successful top events, requantifying them by using point estimated initiators and top event split fractions and renormalizing the total frequency of the $\mathbf{4 2 0}$ sequences to the total frequency from the SQLINK output, the DSM can be taken as an unbiased representative for the full (1999 sequences) model. 


\section{Review of the Internal Events Analysis}

The final values of the total non-seismic core damage frequencies were determined by multiplying a number of selected sequences by appropriate sequence recovery factors (for their definition see also Appendix D).

\section{Quantification of the Long Term Frontline System Module}

The quantification of the Long Term Frontline System Module LT1 was also performed by the code SQLINK. The reviewers noticed, however, that some of the sequences in the reduced model included top event split fractions associated with certain containment functions seemingly irrelevant to mitigate core damage (e.g., containment isolation). Their effect, at first, seemed simply to somewhat reduce the core damage frequency contribution of those sequences in which they appeared. Upon further review and discussions with PG\&E, it was determined that the corollary sequences (i.e., those with the failure state) were also in the overall model but were of too low a value to, in most cases, make the cutoff for the dominant sequence model. The basis for the inclusion of these top events was to facilitate the Level 2 PRA effort expected to follow.

\section{Special Methods}

Owing to their small core damage frequency, sequences belonging to eight of the initiating event categories are not represented in the DSM (see their list in Section 3.4.3 and in Table 3.4.2b). Upon BNL's request for an explanation, PG\&E supplied the following additional information. For three of the internal initiating events, 1) Closure of all MSIVs, 2) Main Steam Relief Valve Opening, and 3) Core Power Excursion, PG\&E calculated the core damage frequencies by the following special method:

"The conditional core damage frequency for these three initiators was assumed to be equal to the conditional core damage frequency for reactor trip initiating event after recovery; this assumption was based on the fact that the quantification of the event tree models for these initiating events are similar. The conditional core damage frequency for the reactor trip event was calculated by dividing the core damage frequency for reactor trip initiating event, by the reactor trip initiating event frequency. This conditional core damage frequency was then multiplied by the initiating event frequencies for "Closure of All MSIVs," "Main Steam Relief Valve Opening," and "Core Power Excursion" to calculate the corresponding core damage frequencies."

The core damage frequency due to interfacing systems LOCA (RHR pump discharge side) was estimated using a special interfacing LOCA event tree.

\section{E. Uncertainty Analyeis of the Total Internal Core Damage Frequency}

The uncertainties of the core damage frequencies due to (strictly) internal events and due to the non-seismic external events (fires, floods, chemical hazards) included in the DSM were determined by Monte Carlo techniques in the DCPRA. The associated probability distributions are shown in Figure 3.9.2. The figure also shows the probability distributions for seismic events and for all the initiators combined. The probability distributions were obtained by using the DSM model (400+ sequences). Although the DCPRA claims that the uncertainty calculations account for all identified sources of uncertainty in the model and the data, it seems that one aspect of the uncertainty is missing. There was no clear attempt to quantify uncertainties contributed by initiating events that were excluded from further analysis by the selection process and the screening calculations (see Section 3.4.4). A more complete treatment of uncertainties would have included additional work on the contribution to the core damage frequency of the excluded events. (See Section 4.7 .3 for a description and comments on the seismic uncertainty analysis). 


\subsubsection{Internal Bvent Sequence Characterietics}

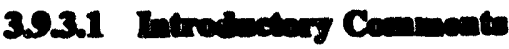

Initial documentation of the DCPRA and its results was limited to Chapter 6 of the LTSP Final Report. As such, a significant amount of information required for this review as well as insights that might be derived from the PRA were missing. This review has subsequently surfaced considerably more information and has cought to independently offer insights where feasible. To this end, BNL performed detailed 1) initiator, 2) system and 3) top event importance analyses for the whole model, both globally and also for each individual initiator/conditional split fraction based on the non-seismic Dominant Sequence Model (DSM). The results of these review efforts are presented in the following sections. As one would expect, several of the results will reiterate those of the original PRA, however, others provide some useful additional information.

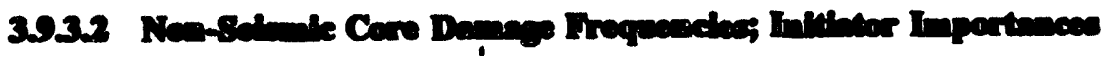

The core damage frequency contributions for the non-seismic initiating event categories listed in Table 3.4.2a were calculated by using the DSM (Appendix D). The core damage frequency contribution for each initiator was calculated as the difference between the total non-seismic core damage frequency, $\mathrm{CDF}_{\mathrm{T}}$ and the total non-seismic core damage frequency calculated with the frequency of the initiator of interest, $I_{i}$ set equal to zero, $\mathrm{CDF}_{\mathrm{T}}\left(\mathbf{L}_{1}=0\right)$. Expressed in a formula:

$$
\operatorname{CDF}\left(I_{i}\right)=C D F_{T}-C D F_{T}\left(I_{i}=0\right)
$$

It is easy to see that Equation 1 is the numerator in the expression describing the Fussel-Vesely importance of the $i$-th initiator to core damage:

$$
J_{F-V}\left(I_{i}\right)=\frac{C D F\left(I_{i}\right)}{C D F_{T}}=\frac{C D F_{T}-C D F_{T}\left(I_{i}=0\right)}{C D F_{T}}
$$

i.e., the unnormalized Fussel-Vesely initiator importance.

Table 3.9.1a lists the ranked unnormalized and normalized Fussel-Vesely importances of the initiating events included in the dominant sequence model.

In order to gain insights about the plant non-mitigation probability given the occurrence of an initiating event, another quantity: the conditional core damage probability was also calculated for each initiating event. This quantity was calculated as the difference between the total non-seismic core damage frequencies when the frequency of initiator of interest $\left(I_{i}\right)$, set to one, $C D F_{T}\left(I_{i}=1\right)$ and when it is set to zero, $C D F_{T}\left(I_{i}=0\right)$. Expressed in a formula:

$$
C C D P_{i}=C D F_{T}\left(I_{i}=1\right)-C D F_{T}\left(I_{i}=0\right)
$$

It is easy to recognize that Eq. (3) represents the partial derivative of the total non-seismic core damage frequency according to the $i$-th initiator frequency. This is also called "Birnbaum importance," 


$$
J_{D}^{\prime}=\frac{\partial\left(C D F_{T}\right)}{\partial I_{i}}-C D F_{T}\left(I_{i}-1\right)-C D F_{T}\left(I_{i}=0\right)=C C D P_{i}
$$

From Equations 1 and 3' one can see that the relationship between the unnormalized Fussel-Vesely and the Birnbaum importances has the following form:

$$
C D F\left(I_{i}\right)=I_{i} * C C D P_{i}=I_{i} * J_{B}^{l}
$$

The Birnbaum importance has the advantage that it is independent of the initiator frequency itself (which may change significantly) but actually measures the plant performance under the condition of the occurrence of that initiating event. Besides the Fussel-Vesely importance, Table 3.9.1a also shows the Birnbaum importances for each initiator. In order to see the relationship between the importances expressed by Equation 4, the initiator frequencies are also indicated.

It may also be of specific interest as to how many leading sequences contribute to the core damage frequency for a given initiator. This information is given in the last column of Table 3.9.1a.

Table 3.9.1b shows the core damage frequency contributions to the internal event core damage frequency due to initiating events not included in the DSM. Their total estimated contributions seem to be indeed negligible.

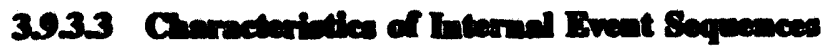

Based on the data given in Table 3.9.1a one can make the following observations about the characteristics of the internal event sequences:

- The majority (73\%) of the non-seismic core damage frequency is due to internal events.

- The LOOP initiator is by far the greatest contributor to the non-seismic core damage (24\%), in magnitude and in number of leading sequences. About 3/4 of the LOOP induced core damage (i.e., 17.5\%) is associated with extended unit blackout (for additional details see the Special Issues Importance Summary, Table 3.9.4).

- Initiators representing (common cause) support system failures other than LOOP (i.e., L1DC, LOSW, LPCC, LOSWV, LOCV) contribute about 9.6 percent to the non-seismic core damage frequency. Including LOOP, this initiator group causes $1 / 3$ of core damage, more than all the "externals" (fire, flood, etc.) combined. The plant mitigating capability against these initiators is varied (see the conditional core damage probabilities, i.e., Birnbaum importances); the worst is for the initiating event, Loss of $480 \mathrm{~V}$ Switchgear Ventilation, LOSWV. This is followed by the Loss of Component Cooling Water, LPCC, and the Loss of Auxiliary Saltwater, LOSW. Then LOOP, L1DC, and LOCV. (One should keep in mind that the ranking of the plant mitigating capability might be somewhat biased because many of the LOOP sequences were subjected to "sequence recovery" considerations, while others were not.) The average conditional core damage probability for this group of initiators is: 2.9804.

- Transients contribute to the non-seismic core damage frequency almost in the same proportion as the support system failures: 31 percent. Reactor trips, turbine trips, feedwater losses etc. are rather frequent initiating events. Their large occurrence frequc.acy represents a challenge to the plant's safety systems but their nature seems to be mild. The average conditional core damage probability for this group is one order of magnitude smaller than that of the group associated with support system failures: 
2.99-05. Among the transiente, the initiating event with the highest conditional probability of core damage is the steam line break accident, particularly if the break is inside the containment. The plant's conditional probability of failure to mitigate (Birnbaum importances) against the initiating events belonging to this group rank as follows: SLBI, SLBO, PLMFW, RT, TT, EXFW, LOPF, IMSIV, TLMFW, LCV, and ISI.

- The total contribution of initiators belonging to the Lass of Coolant Inventory group to the non-seismic core damage frequency is small: about 9 percent. However, the nature of these accidents is quite significant: the plant safety systems have the most trouble mitigating the excesaive LOCA, and interfacing syatems LOCA ovents. The conditional core damage probability (Birnbaum importance) ranking of the LOCAs is as follows: ELOCA, VSI (SS), LLOCA, MLOCA, SGTR, SLOCN, SLOCI.

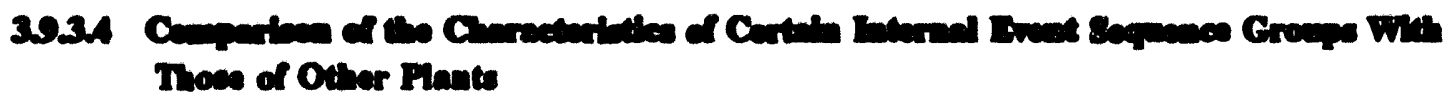

In order to compare the Diablo Canyon plant responses for various types of internal event sequences with those of other PWRe, nine PRAs were selected, the majority of them representing Westinghouse plants. These were: Seabrook, Zion, Sequoyah, Surry, Millstone 3, Indian Point 2 and 3, Oconee, and Yankee Rowe PRAs. It must be noted that all of these PRAs were done on different bases for different utilities by different analyats. Therefore, the following insights are provided to yield an enhanced perepective of the Diablo Canyon plant and not to provide a comprehenstve and rigorous basis for direct numerical comparisons.

To make the task of comparison tractable and meaningfuh, the average characteriatics of certain initiating event groups were compared. The grouping followed the traditional way of grouping, to allow the use of somewhat older data, as well. Thus, the LOCA initiators were categorized as large, intermediate, small, and interfacing LOCAs. (The large LOCAs include the excessive LOCA and reactor vessel rupture initiating events, as well.) The transient group included the common cause initiators associated with support system failures and the SGTR initiator.

Table 3.9.2a compares the core damage frequency contributions for these groups to the total internal core damage frequency. Table 3.9.2b compares the corresponding conditional core damage probabilities (except for interfacing systems LOCA, for which the conditional core damage probability is one or near one).

From Table 3.9.2a it can be seen that at six plants out of nine, transients contribute more to the internal core damage frequency, than the LOCAs. At three plants (Diablo Canyon 1, Seabrook, and Zion) the dominance of the transients is overwhelming. The dominance of the transients were pointed out only by recent PRAs, where common cause initiators due to support system failures such as loss of component cooling water, loss of service water etc. were analyzed more seriously. The other observation is that Yankee Rowe and Diablo Canyon have the smallest frequency for transient initiators. The PRAs for both plants utilized very similar screening criteria for generic data selection (see the discussion of the selection criteria for the DCPRA in Section 3.4).

Because of the broad variability in the data of Table 3.9.2a, the less fluctuating data of Table 3.9.2b are discussed further. By using this data, the conditional core damage probabilities; i.e., the noa-mitigating probabilities of the plants are compared. From this comparison one can infer, that given a large LOCA, among all the plants, the Diablo Canyon plant can mitigate this initiating event the most ineffectively. Apparently, there is a similar situation with the intermediate LOCA. As concerns small LOCA, the resilience 


\section{Roview of the Internal Events Anulyais}

of Diablo Canyon seems to be the beat. For transients, the Diablo Canyon again plant seems to provide the least mitigating capability compared to the other plants.

BNL bolieves that these differences are drtven predominantly by some of the event tree modelling assumptions of the DCPRA rather than inherent deficiencies in the Diablo Canyon design. A typical example of this is the use of the split fraction HS1. The reader should refer to the following sequences in Table 3.9.3: 3, 4, 6, 13, 64, 74, 78, 93, and 111. All of these sequences involve a transient and then the only legitimate other fallure is HS1 (operator fails to maintain the plant in a safe and stable hot standby condition). If these sequences were to be removed from the model, Diablo Canyon would not appear to handle transients less efriciently than the other plants in the comparison.

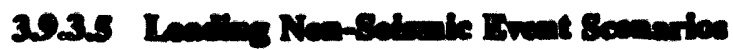

Individual scenarios having core damage frequency $\geq 1.0 \mathrm{E}-06 \mathrm{yr}^{-1}$ selected from the Dominant Sequence Model are listed in Part A of Table 3.9.3. The listing follows the ranking of the individual scenarios according to the contribution to the non-seismic core damage frequency. Besides the individual ranking the listing also provides information about the sequence ranking of appearance of the leading sequence of each given initiator.

In order to make the latter information more complete, in Part B of Table 3.9.3 the ranking of the leading sequences associated with initiators not listed in Part A are also presented.

In contrast with Table 6-60 of the DCPRA, the scenarios listed in Table 3.9.3 are shown in their entirety, i.e., each constituent top event split fraction is indicated. For convenience, the definitions of all the top event split fractions included in the table are also given in the note section of the same table. The definitions of the initiating events are provided in Table 3.9.1a.

By examining the table one can make several observations:

- Except for the block of control room/cable spreading room fire sequences, CRFIRE, there is no scenario which contributes more than 3 percent to the non-seismic core damage frequency.

- In spite of the fact that the initiating event LOOP is the main contributor to the non-seismic core damage frequency, only two LOOP sequences appear among the leading 23 scenarios and the rank of the first one is 17th.

- External scenarios tend to have higher rank than internal scenarios.

- Transients (mainly RT, TT, PLMFW initiators) appear predominately among the leading internal sequences as opposed to LOCAs.

- Among the initiators associated with support system failures, a Loss of Component Cooling Water (LPCC) sequence has the highest rank.

- Among the operational failures of the plant, loss of feed and bleed cooling (top event OB), loss of auxiliary feedwater system (top event AW), and occurrence of RCP seal LOCA (top events SE) are the most frequent.

The first observation implies that the present ranking of the leading sequences is not robust, i.e., very small changes in the sequence variables (i.e., initiator frequency, top event split fractions, recovery actions) may change the ranking significantly. Change in the ranking is fairly conceivable because recovery actions were not applied to a great many of the internal event sequences and the DCPRA considers the potential for additional recovery actions for numerous scenarios where recovery actions already were applied. 
The first observation has also a technical impact to the review process. The significance of changes or errors in the initiating event frequencies, top event split fractions, etc. cannot be estimated by simply changing several variables in a small group of leading sequences with high frequency as was the case in the majority of older PRAs. In the case of the DCPRA, where a relatively large number of sequences contribute almost uniformly to the core damage frequency, sensitivity calculations have to be carried out by performing more sophisticated importance analyses such as those done by BNL in this review.

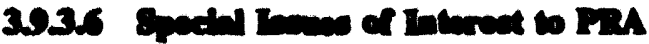

This section presents the results obtained by PG\&E concerning several issues of special interest to PRAs that were identified in the DCPRA, but somehow were not presented in the original version of the final report. They were recently submitted to BNL as a consequence of the review effort. The nature of the issues involves both the non-seismic and seismic initiating events, therefore the scope of the following presentation goes beyond the non-seismic scenario analysis. The presentation roughly follows the text of the PG\&E submittal.

There are six issues of special interest to PRAs identified in the DCPRA:

1. Station Blackout

2. Failure to Trip the Reactor (ATWT)

3. Reactor Coolant Pump Seal LOCA

4. Primary Relief Valves Open and Fail to Reclose

5. Feed and Bleed Cooling

6. PWR Pressurized Thermal Shock (PTS)

These issues are of interest because they are unresolved safety issues or have been shown to be significant contributors to risk in other PRAs.

The core damage frequency and percent contribution to core damage frequency of each of these categories (i.e., unnormalized and normalized Fussel-Vesely importances) are presented in Table 3.9.4. These quantities are provided for both non-seismic and seismic initiators.

The contributions to core damage frequency associated with non-seismic initiators were computed from the DSM by PG\&E. The scenarios in the DSM were sorted into special scenario groups indicated in the table and the frequencies of each group summed to determine contribution to each of the categories.

The scenarios associated with ATWT events in the DSM were supplemented with additional ATWT event sequences identified from a larger list of key sequences i.e., including sequences which were too low in frequency to be included in the DSM.

To normalize the unnormalized non-seismic Fussel-Vesely importances, the total non-seismic core damage frequency (Table 3.9.1a) was used.

For seismic initiators, the contributions to core damage frequency were obtained by PG\&E from the DCPRA seismic key event sequence list, not the seismic uncertainty model. The key sequence list (the top 791 core damage sequences) accounts for approximately 82 percent of the total seismic core damage frequency. It was assumed that the remaining 18 percent conttibution to the seismic core damage is distributed proportionally among each of the scenario groups of interest. The contributions from each of the scenario groups in the key 


\section{Roview of the Internal Events Analysis}

event sequence liat were therefore scaled up. The point estimate seismic core damage frequency from the key sequences is 3.43E-5 $\mathrm{yr}^{-1}$. This is the normalization value for the associated importances. The contributions from the scenario groups of interest shown in Table 3.9.4 are not mutually exclusive. Therefore, care must be taken when combining different scenario groups. For example, it would be incorrect to add the seismicinitiated station blackout "ATWT" contribution to the "total" seismic-initiated station blackout contribution since the "total" already contains the "ATWT" contribution. Notes to the table indicate the type of sequences that each of the groups contain.

The total core damage frequency (point estimate) according to PG\&E is: 2.12E-04 $\mathrm{yr}^{-1}$. This is the normalization value for the "total" importances calculated by BNL.

From Table 3.9.4 one can obtain perhaps one of most important insights of the whole DCPRA: the vulnerability ranking of the Diablo Canyon plant for these special failure categories (ranking is according to the "total importance):

\begin{tabular}{|c|c|c|c|c|}
\hline \multirow{2}{*}{\multicolumn{2}{|c|}{ 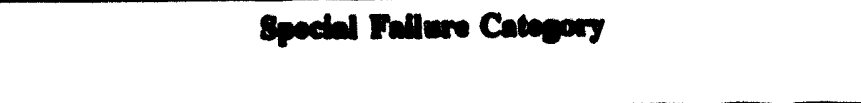 }} & \multicolumn{3}{|c|}{ Fescel-Vecely Importances (\%) } \\
\hline & & Non-Solvemic & Solemile & Total \\
\hline 1. & RCP San LOCA (With and Without Station Blackout) & 31 & 38 & 35 \\
\hline 2. & Sintion Blactiont & 18 & 79 & 28 \\
\hline 3. & Primary Reliof Valve Opea and Fail to Recloce & 20 & 3 & 17 \\
\hline 4. & Bloed and Feed Cooling & 12 & 0 & 10 \\
\hline s. & Preasuribed Thermal Shoct & 4 & 0 & 3 \\
\hline 6. & Fallure of Reactor Trip (ATWT) & .8 & 11 & 3 \\
\hline
\end{tabular}

It is noted here for completeness that the very low ATWT contribution reflects the fact that Diablo Canyon has made modifications to the protection system in accordance with the NRC's ATWT rule.

\subsubsection{Sycher LovilOparater Action Inportineses}

In order to gain insights into the vulnerability of the Diablo Canyon plant with respect to system level failures, a system level importance analysis was performed at BNL by using the Dominant Sequence Model. The analysis was separately carried out for support systems and frontline systems as well as importance calculations for operator and recovery action failures explicitly appearing in the event sequences as top event split fractions. The analysis was global, in that sense, that it did not distinguish between the various initiating events. In the analyses each system/operator action importance was determined by calculating the importance of its associated top event or an aggregate of top events appearing in the DSM.

Table 3.9.5 presents the unnormalized Fussel-Vesely importances for both the overall systems safety functions as well as the constituent top evenis. Part A lists the support system, Part B the frontline system and Part C the operator/recovery action importances. The system/operator action importances are given in ranked order.

The most important support systems are: 1) the diesel generator systems and 2) the Unit $1125 \mathrm{~V} \mathrm{dc}$ power system. The most important frontline systems are: 1) the auxiliary feedwater, and 2) the primary pressure relief systems. The most important operator action is: to maintain hot-standby given a transient. 


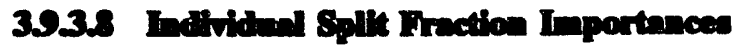

In order to gain insights into the importances of the individual top event split fractions, BNL performed a dedicated top event split fraction importance analysis. The results of that analysis are presented in Table 3.9.6. Following the structure of 'Table 3.9.5, parts A and B of Table 3.9.6 list the ranked top event split fraction importances for support systems and frontline systems respectively. Part $\mathrm{C}$ shows the ranked importances of the operator/recovery action top event split fractions. The ranking was done according to the unnormalized Fussel-Vesely importance values. For additional information, the table also indicates the number of occurrences of each top event split fraction within the DSM (i.e., the number of sequences in which it occurs).

To facilitate sensitivity analysis on the individual top event split fractions, the Birnbaum importance is also shown. A complete list of top event split fractions ranked according to their Birnbaum importance is given in Table D2.10 of Appendix D2.

The overall ranking of the leading top event split fraction (CSF) importances is as follows:

\begin{tabular}{|l|c|c|}
\hline & CSF & $\begin{array}{c}\text { Normalised } \\
\text { Fusel-Vecely }{ }^{+}(\%)\end{array}$ \\
\hline 1. Operator inability to maintain hot standby (everything available). & HS1 & 11.5 \\
\hline $\begin{array}{l}\text { 2. Loss of primary pressure relief (loss of PORV operability for feed } \\
\text { and bloed. No instrument air.) }\end{array}$ & OB1 & 9.9 \\
\hline $\begin{array}{l}\text { 3. Loss of primary pressure relief (for LOOP/SGTR, failure of 1/2 } \\
\text { PORVs or 1/3 SRVs). }\end{array}$ & PRD & 9.1 \\
\hline 4. Loss of DG13 (after loss of 4.15 kV bus HF). & GF1 & 8.6 \\
\hline 5. Failure to trip RCP, after lose of CCWS to prevent seal LOCA. & RP2 & 6.8 \\
\hline 6. Loes of DG12 (DG13 is mocesuful). & GG1 & 6.3 \\
\hline
\end{tabular}

${ }^{+}$For normalization, the total non-seismic core damage frequency was used.

\subsubsection{Syctem Inportances in Accident Sequences Indeced by Variows Initintors}

It is rather reassuring to be able to give quantitative answers to questions posed frequently in connection with nuclear plant safety: Given an initiating event of a certain type, which are those safety systems/operator actions whose unavailabilities dominate the failure to mitigate the variety of event scenarios that may follow that initiator? Or, to answer assertively to question formulated as follows: Given a safety system or operator action with its characteristic unavailability, which are those initiating events where this unavailability contributes most to the core damage frequency?

In order to gain such knowledge about the Diablo Canyon plant, BNL extended its system's importance analysis to individual initiating events. An analysis was performed for each of the initiating events of the Dominant Sequence Model by subsequently setting to zero all the initiating events in the DSM but the one of interest and setting that one to a value of 1.0 . 


\section{Review of the Internal Events Analysis}

Tables 3.9.7 and 3.9.8 present the results of these analyses for the internal and external (fire/flood scenarios) initiators, respectively. For each initiator, the unnormalized Fussel-Vesely importances of system/operator actions and associated top events were calculated.

Table 3.9.7 is rather large; it spreads over four sheets. When one scans the data column below a given initiator (put sheets 1,2, or 3,4 together vertically) one can read off the answer to a question of the first type above. When one scans the data row belonging to a system/operator action (put sheets 1,3 , or 2,4 together horizontally) one can get an answer to a question of the second type.

Thus, given the initiating event, RT; the ranking of system/operator action importances is:

1. Auxiliary Feedwater System

2. Maintain Control for Hot Standby

3. Primary RCS Pressure Relief (feed and bleed)

4. Instrument AC Power, etc.

Or, given the Auxiliary Saltwater System, the ranking of the initiating event importances is:

\section{Loss of One 125 V DC Bus, L1DC}

2. Loss of Offsite Power, LOOP

\subsubsection{Importances of System/Top Event Split Fraction Pairs}

Individual system/top event split fraction pair importances provide information that can be used to identify system/human action unavailabilities, whose simultaneous occurrence are critical with regard to the core damage frequency. The identification of these pairs is more relevant for plant safety from an operational point of view; it guides the personnel, e.g., to assess the advisability of permitting simultaneous activities (maintenances, tests) on two systems that may not be prohibited by the Technical Specifications.

Mathematically the definition of the pair importances is analogous to the single event importances, e.g., the definition of the Birnbaum pair importance of two top event split fractions, $T_{i}$ and $T_{j}$ with respect to the nonseismic core damage frequency, $C D F_{T}$ is given by the formula:

$$
D_{i j}^{B}=\frac{\partial(C D F)_{T}}{\partial\left(T_{i} T_{j}\right)}=C D F_{T}\left(T_{i}=T_{j}=1\right)-C D F_{T}\left(T_{i}=T_{j}=0\right),
$$

The corresponding unnormalized pair-wise Fussel-Vesely importance is:

$$
D_{I J}^{F-V}=\left(t_{i} t_{j}\right) * d_{I J}^{B}
$$

This importance characterizes the contribution of the "intersection" of the pair $\left(T_{i} T_{j}\right)$ to the total core damage frequency.

To obtain normalized pair-wise Fussel-Vesely importances, the above expression should be divided by the normalization constant; in this case the total non-seismic core damage frequency. 


\section{Review of the Internal Events Analysis}

The pair-wise system importances represent a generalization of the above concepts. They are determined by calculating the importances of the intersection between two aggregates of top event split fractions, where each aggregate contains the top event split fractions associated with a given system. The unnormalized FusselVesely importances of support system pairs, as well as those of frontline system-support system pairs are tabulated in matrix form in Tables 3.9 .9 and 3.9.10, respectively. Similar values in the tables express strongly correlated top events in the support system event tree module.

From Table 3.9.9 the overall ranking of the top five support system-support system pair importances is as follows:

1. Component Cooling Water - Diesel Generator Systems

2. Component Cooling Water - Vital 125 V DC Systems

3. Diesel Generator Systems - Vital 125 V DC Systems

4. Control Room Ventilation - Diesel Generator Systems

5. $480 \mathrm{~V}$ Switchgear Ventilation - Diesel Generator Systems

From Table 3.9.10 the overall ranking of the top five frontline system-support system pair importances is as follows:

1. Primary RCS Pressure Relief - Diesel Generator Systems

2. Auxiliary Feedwater System - Diesel Generator Systems

3. Primary RCS Pressure Relief - Instrument AC Power

4. Auxiliary Feedwater System - Instrument AC Power

5. Auxiliary Feedwater System - Vital 125 V DC Systems

The pair importances presented in this section reflect aggregated split fractions and in some cases aggregated top events to represent the system/function level. Unnormalized Fussel-Vesely importances as well as the associated Birnbaum importances were also calculated for a variety of combinations of all top event individual split fractions of the DSM. These are listed in ranked form (according to the unnormalized Fussel-Vesely importance) in nine tables (Tables D2.1 through D2.9) of Appendix D2. Each of the tables provides some additional insight into the plant safety. Among them, maybe the most enlightening are those pair importances which describe support system-operator/recovery actions (Table D2.8), frontline system-operator/recovery actions (Table D2.6) and operator/recovery-operator/recovery actions (Table D2.9) top event split fraction pairs. 
3 Review of the Internal Events Analysis

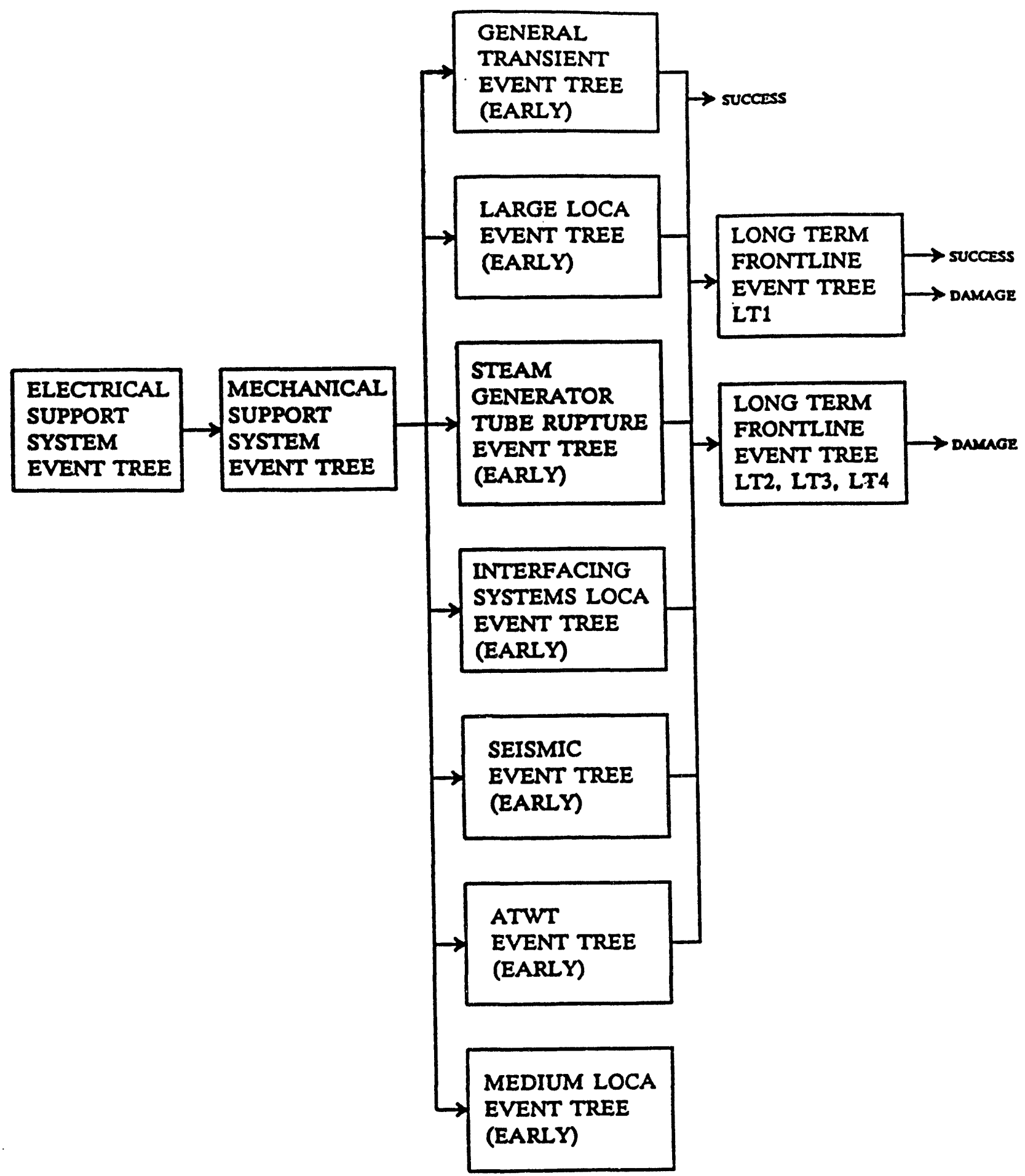

Foure 3.9.1 The Fourpart Event Sequeace moded 


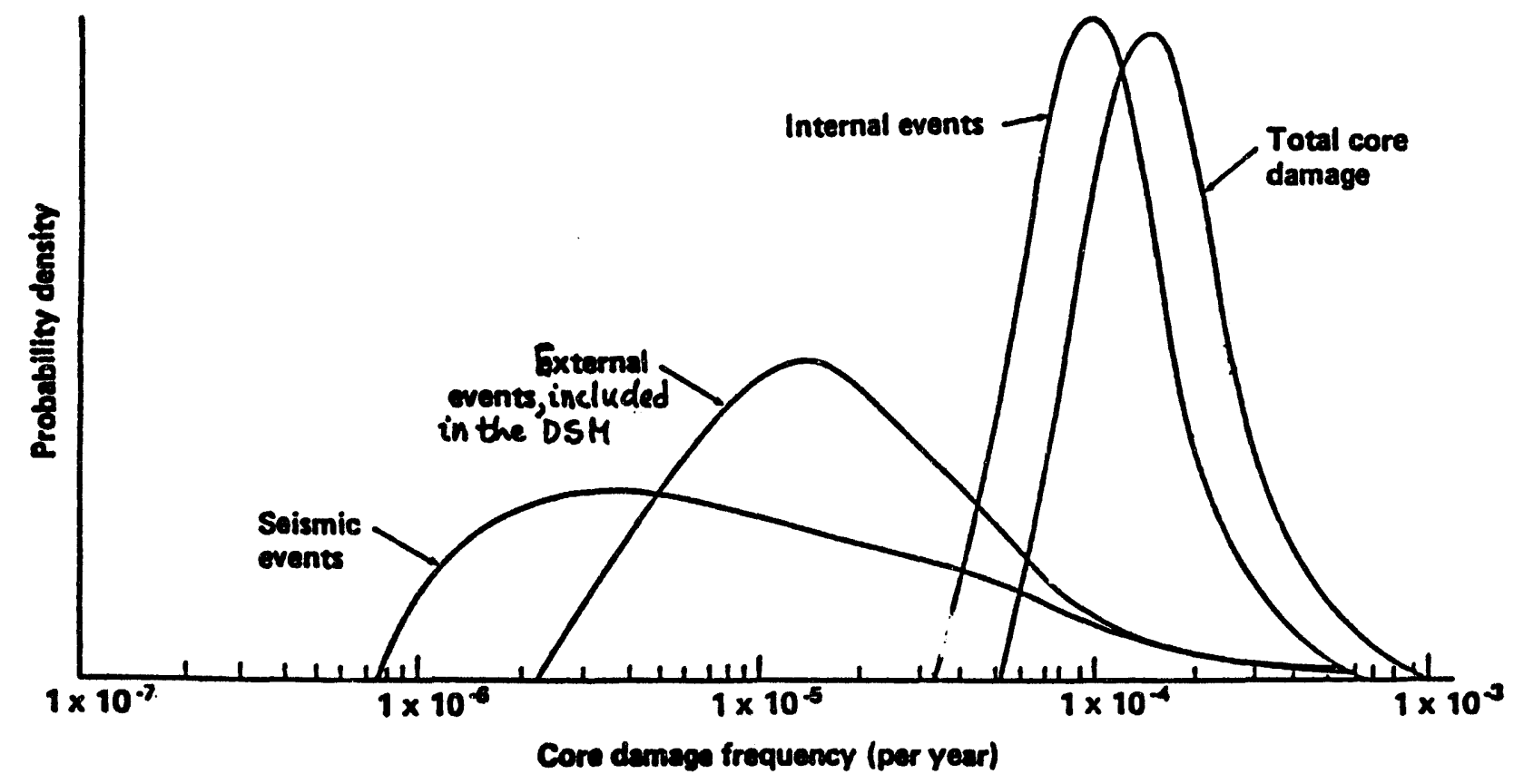

Fugure 3.9.2 Probability Dietributions for Total Core Damage Frequencies Dwe to Variows Groups of Inthiating Evente. The Leading Sequences of Internal and External Events Constitute the Dominant Sequence Model (DSM). 


\begin{tabular}{|c|c|c|c|c|c|c|c|}
\hline \multicolumn{3}{|r|}{ Invinding Bune } & \multicolumn{5}{|c|}{ 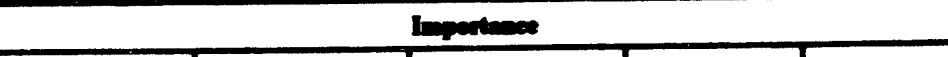 } \\
\hline (i) & Dealumerer & Conogers & $\begin{array}{c}\text { Propenoy, II } \\
\text { (Per Yant) }\end{array}$ & 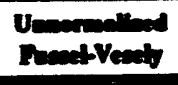 & $\begin{array}{c}\text { ProptVenty } \\
\text { (S) }\end{array}$ & 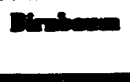 & Nen ef Co \\
\hline 1 & LOOP & Loss of Otwite Power & $9.10-02$ & $4.18-05$ & 23.57 & $459-04$ & 183 \\
\hline 2 & CRFIRE • & Control Room and Cable Spreading Room Fires & & $3.17-05$ & 17.87 & & 1 \\
\hline 3 & $\mathbf{R T}$ & Rescror Trip & $1.14+\infty$ & $1.62-05$ & 9.13 & $1.42-05$ & 34 \\
\hline 4 & $\mathbf{T T}$ & Turbine Trip & $1.05+\infty$ & $1.48-05$ & 8.34 & $1.41-05$ & 33 \\
\hline 5 & PLMFW & Partial Loses of Main Feedwater & $7.49-01$ & $1.00-05$ & 6.09 & $1.45-05$ & 26 \\
\hline 6 & LIDC & Loes of One DC Bus & 2.5602 & 9.5006 & 5.36 & $3.71-04$ & 34 \\
\hline 7 & Fs8 & Fire Scenario: Lowe of 4.16kV Buses HF, HO and HH & $6.18-06$ & $6.48-06$ & 3.65 & $100+\infty$ & 2 \\
\hline 8 & FS11 & Flood Scenario: Loes of Auxiliary Saltwater & $3.81-04$ & $6.20-06$ & 3.50 & $1.63-02$ & 4 \\
\hline 9 & MLOCA & Medium LOCA & $4.63-04$ & $5.97-06$ & 3.37 & $129-02$ & 7 \\
\hline 10 & SGTR & Steam Geserntor Tube Rupture & $1.71-02$ & $3.58-06$ & 2.22 & $2.10-04$ & 12 \\
\hline 11 & LPCC & Total Loes of Component Cooling Water & $1.96-04$ & $3.19-06$ & 180 & $1.63-02$ & 4 \\
\hline 12 & EXFW & Excesive Feedwater Flow & $2.79-01$ & $3.12-06$ & 1.76 & $1.12-05$ & 9 \\
\hline 13 & SLBO & Steam Line Break Outuide Containment & $5.53-03$ & 28006 & 158 & $5.06-04$ & 24 \\
\hline 14 & LOCA & Large LOCA & 2.02-04 & $258-06$ & 1.45 & $1.22-02$ & 4 \\
\hline 15 & SLBI & Steam Line Break Imide Containment & 4.63-04 & 23806 & 134 & $5.15-00$ & 8 \\
\hline 16 & SLOCI & Small LOCA; Isolable & $1.61-02$ & $1.81-06$ & 1.02 & $1.12-04$ & 6 \\
\hline 17 & LoswV & Loes of $480 \mathrm{~V}$ Switchgear Ventilation & $6.29-05$ & $1.61-06$ & .91 & $256-02$ & 6 \\
\hline 18 & FS1 & Fire Scenario: Lose of Both Motor-Driven AFW Pumpe & $2.94-04$ & $1.47-06$ & 83 & $5.00-03$ & 9 \\
\hline 19 & Losw & Total Loas of Auritiary Saltwater & $9.74-05$ & $1.45-06$ & 82 & $1.49-02$ & 2 \\
\hline 20 & LOCV & Loes of Control Room Ventilation & $7.99-02$ & 1.2406 & .70 & $1.55-05$ & 6 \\
\hline 21 & FS6 & Fire Scenario: Loses of 4.16kV Buses HF and HG & $2.42-05$ & $1.10-06$ & .62 & 45402 & 2 \\
\hline 22 & LOPF & Loses of Primary Flow & $1.21-04$ & $1.08-06$ & .61 & $889-06$ & 5 \\
\hline 23 & IMSTV & Choure of One MSIV & $1.07-01$ & $951-07$ & 53 & $889-06$ & 5 \\
\hline 24 & TLMFW & Total Lose of Main Feedwater & $9.98-02$ & 8.87-0 & so & $8.89-06$ & 5 \\
\hline 25 & SLOCN & Small LOCA; Non-lsolable & $5.26-03$ & $8.17-07$ & .46 & $155-04$ & 4 \\
\hline 26 & LCV & Loss of Condenser Vecuum & $8.73-02$ & $7.76-07$ & .44 & $8.89-06$ & 5 \\
\hline
\end{tabular}


Table 3.9.1n (comolanead)

\begin{tabular}{|c|c|c|c|c|c|c|c|}
\hline \multicolumn{3}{|r|}{ Inthelion Even } & \multicolumn{5}{|c|}{ 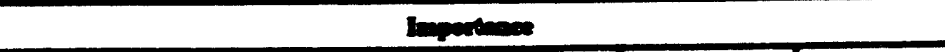 } \\
\hline $\mathrm{Na}$ & Dodengerer & Coners & 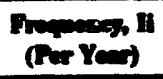 & 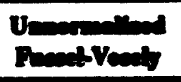 & $\begin{array}{l}\text { Puseot Venoly } \\
\text { (x) }\end{array}$ & Ensm & Ma etCo \\
\hline 27 & $\mathbf{F P S}$ & Food Soenariox Los of All AFW & $1.35-05$ & $6.87-07$ & 39 & $500-02$ & 2 \\
\hline 28 & IN & Indverteat Safety Injection Sigen & $7.39-12$ & $657-07$ & 37 & $\cos 05$ & $\mathbf{s}$ \\
\hline 29 & F5S & Fine Soenariox Lose of Amerifiary Salmwater & $5.26-05$ & s.71-0 & 32 & 1.0202 & 1 \\
\hline 30 & vsi(ss) & Inverficing LOCA (RHR Suction Side) & $1.01-06$ & 5.0007 & 28 & $4.95-01$ & 1 \\
\hline 31 & HAZCHM & Cremical Hazord (e.g. chlorimethmmonia relesues) & 4.38-04 & $351-07$ & 20 & 7.9904 & 1 \\
\hline 32 & ELOCA & Eracuive LOCA & $2.66-07$ & $266-07$ & .15 & $1.00+\infty 0$ & 1 \\
\hline 33 & FS10 & Flood Soenariox Loes of Both Molor-Driven AFW Pumpe & $1.00-08$ & $2.93-08$ & $\mathbf{e n}$ & 2.1003 & 1 \\
\hline & & Total Internal & & $129-04$ & 7272 & & \\
\hline & & Total "External" & & 48405 & 27.28 & & \\
\hline & & Total CDF (Dowinant Sequeace Model) & & $1.77-04$ & & & 452 \\
\hline
\end{tabular}

"Sum of six control room and cable spreading room fire sequences which break down as follows: CR-VB-1 CR Vertical Board-1: Loss of ASW, CCW controb

$1.08-04 \quad 1.25-06$

$8.00-05 \quad 1.16-06$

CR-VB-2 CR Vertical Board-2: LOss of PORV and Charging Pump controks

$9.36-05 \quad 3.15-06$

CR-VB-2/3 CR Vertical Boards 2 and 3, Interface: Loss of PORV and AFW controls

$9.74-05$

CR-VB-4

CR Vertical Board 4: Loss of 4.16kV Buses HF,HG \& HH

CSR-1

Cable Spreading Room: Loss of ASW, CCW controls

$5.49-04$

$6.01-06$

$9.25-04$

$7.90-06$

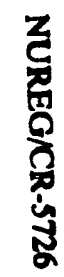

CSR-2

Cable Spreading Room: Loss of PORV and Fressurizer Instrumentation

$1.23-05$

$\begin{array}{rr}.70 & 1.16-02 \\ .65 & 1.45-02 \\ 1.76 & 3.37-02 \\ 3.38 & 6.17-02 \\ 4.45 & 1.43-02 \\ 6.93 & 1.33-02\end{array}$




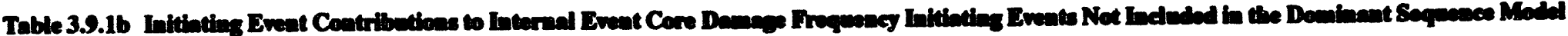

\begin{tabular}{|c|c|c|c|c|c|c|c|}
\hline \multicolumn{3}{|r|}{ 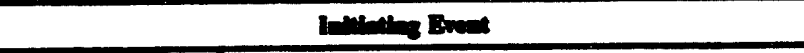 } & \multicolumn{5}{|c|}{ 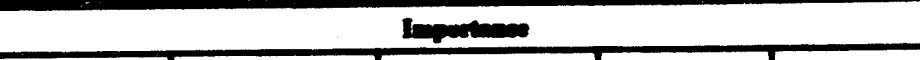 } \\
\hline (I) & 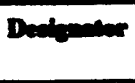 & Compers & 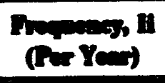 & 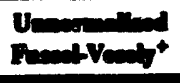 & 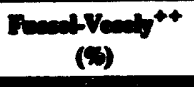 & Dint & 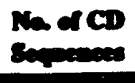 \\
\hline 34 & CPEXC & Core Pomer Bramaion & $2.19 \cdot 02$ & $3.11-07$ & .18 & $1.12-00^{\circ}$ & 34 \\
\hline 35 & AMSIV & Coame of All MSIVE & 1.7408 & $247-07$ & .14 & 1.0000 & 34 \\
\hline 36 & VDI & 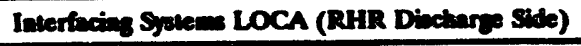 & $3.86-06$ & 52000000 & 103 & 15002 & 1 \\
\hline 37 & MSRIV & Inedverteant Opering of Main Stean Relibe Value & $359-03$ & 5.1000 & $\boldsymbol{\infty}$ & $1.4200^{\circ}$ & 34 \\
\hline & & 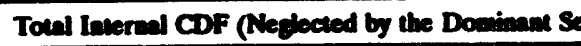 & coe Model) & & $6.67-07$ & & \\
\hline
\end{tabular}

+Estimated by special methods (see Section 3.9.2.C).

++For normalization, the total CDF (Dominant Sequence Model) was used.

Taken from Table 3.9.1a for RT initiator.

- Taken from Table 6-59 of the DCPRA. 


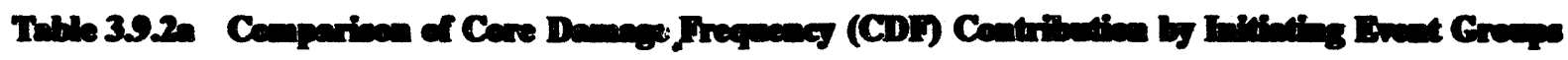

\begin{tabular}{|c|c|c|c|c|c|c|c|c|c|c|}
\hline \multirow[b]{2}{*}{$\begin{array}{l}\text { Initiating Event } \\
\text { Group }\end{array}$} & \multicolumn{2}{|c|}{ Diablo Canyon 1} & \multicolumn{2}{|c|}{ Seabrook } & \multicolumn{2}{|c|}{ Zion (Revined) } & \multicolumn{2}{|c|}{ Sequogah } & \multicolumn{2}{|c|}{ Surry } \\
\hline & \begin{tabular}{|l|} 
Initiator \\
Frequency
\end{tabular} & $\begin{array}{l}\text { CDF } \\
\text { Contribution }\end{array}$ & $\begin{array}{l}\text { Initiator } \\
\text { Frequency }\end{array}$ & $\begin{array}{l}\text { CDF } \\
\text { Contribution }\end{array}$ & $\begin{array}{l}\text { Initiator } \\
\text { Frequency }\end{array}$ & $\begin{array}{l}\text { CDF } \\
\text { Contribution }\end{array}$ & \begin{tabular}{|l|} 
Initintor \\
Frequency
\end{tabular} & $\begin{array}{l}\text { CDF } \\
\text { Contribution }\end{array}$ & \begin{tabular}{|l|} 
Initintor \\
Frequency
\end{tabular} & $\begin{array}{l}\text { CDF } \\
\text { Comentibution }\end{array}$ \\
\hline LOCA & & & & & & & & $\begin{array}{l}\text { Median } \\
\text { Values }\end{array}$ & & \\
\hline $\begin{array}{l}\text { Large (incl; } \\
\text { excessive, RV } \\
\text { rupture) }\end{array}$ & $2.02-04$ & $285-06$ & $2.03-04$ & $1.70-06$ & $9.40-04$ & $1.46-06$ & $5.00-04$ & $1.13-06$ & $5.00-04$ & $8.41-07$ \\
\hline Intermediate & $4.63-04$ & $5.97-06$ & $4.65-04$ & 9.91-07 & 9.40 .04 & $4.14-06$ & $1.00-03$ & $3.15-06$ & $1.00-03$ & 16006 \\
\hline Small & $2.14-02$ & $2.63-06$ & $1.73-02$ & $1.99-05$ & $3.50-02$ & 8.2406 & $200-02$ & $467-05$ & $2.10-02$ & $4.28-06$ \\
\hline Interface & $1.01-06$ & $5.00-07$ & 1.8406 & 1.8406 & $1.10-07$ & $1.10-07$ & 3.3007 & $3.30-07$ & $9.00-07$ & $9.00-07$ \\
\hline Total LOCA & & $1.20-05$ & & $2.44-05$ & & $1.40-05$ & & $5.13-05$ & & $7.62-06$ \\
\hline $\begin{array}{l}\text { Transients } \\
\text { (incl; SGTR, } \\
\text { common cause) }\end{array}$ & 3.80 & $1.17-04$ & 11.0 & $1.31-04$ & 14.1 & 26404 & 8.4 & $3.49-05$ & 8.4 & $1.72-06$ \\
\hline Total CDF & & $1.29-04$ & & $1.55-04$ & & $2.78-04$ & & $8.62-05$ & & $248-05$ \\
\hline & & & & & & & & Mean: $1.00-04$ & & \\
\hline $\operatorname{LOCA}(\%)$ & & 9.3 & & 15.7 & & 5.0 & & 59.5 & & 30.7 \\
\hline Transients (\%) & & 90.7 & & 84.3 & & 95.0 & & 40.5 & & 693 \\
\hline
\end{tabular}




\begin{tabular}{|c|c|c|c|c|c|c|c|c|c|}
\hline \multicolumn{8}{|c|}{ Table 3.9.2e (continend) } & \multirow{2}{*}{\multicolumn{2}{|c|}{ Yantee Rome }} \\
\hline \multicolumn{2}{|c|}{ Millstone 3} & \multicolumn{2}{|c|}{ Indian Point 2} & \multicolumn{2}{|c|}{ Indian Point 3} & \multicolumn{2}{|c|}{ Oconee } & & \\
\hline \begin{tabular}{|l} 
Initiator \\
Frequency
\end{tabular} & $\begin{array}{l}\text { CDF } \\
\text { Contribution }\end{array}$ & \begin{tabular}{|l|} 
Initiator \\
Frequency
\end{tabular} & $\begin{array}{l}\text { CDF } \\
\text { Contribution }\end{array}$ & $\begin{array}{l}\text { Initintor } \\
\text { Frequency }\end{array}$ & $\begin{array}{l}\text { CDF } \\
\text { Contribution }\end{array}$ & $\begin{array}{l}\text { Initiator } \\
\text { Frequency }\end{array}$ & $\begin{array}{l}\text { CDF } \\
\text { Comentibution }\end{array}$ & \begin{tabular}{|l|} 
Initintor \\
Froquency
\end{tabular} & $\begin{array}{l}\text { CDF } \\
\text { Cosetrituation }\end{array}$ \\
\hline & & & & & & & $\begin{array}{l}\text { Medina } \\
\text { Values }\end{array}$ & & \\
\hline $3.88-04$ & $2.67-06$ & $1.95-03$ & $1.61-05$ & $2.16-03$ & $181-05$ & 9.31-04 & $1.51-05$ & $263-04$ & $287-06$ \\
\hline $6.11-04$ & $5.49-06$ & $1.95-03$ & $130-05$ & Note 1 & Note 1 & $7.50-04$ & $4.62-06$ & $1.00-03$ & 16006 \\
\hline 9.07-03 & 1.58 .06 & $1.85-02$ & $1.65-05$ & $2.01-02$ & $8.50-056$ & $3.00-03$ & $0.10-06$ & 3.4403 & $5,12-06$ Nole 2 \\
\hline $1.90-06$ & $1.90-06$ & $4.60-07$ & $4.60-07$ & $4.64-07$ & $464-07$ & $1.40-07$ & $1.40-07$ & $200-07$ & $200-07$ \\
\hline & $1.15-05$ & & $4.61-05$ & & $1.16-04$ & & 16405 & & $132-05$ \\
\hline 10.9 & $3.42-05$ & 22.0 & 3.29-05 & 10.0 & $1.40-05$ & 7.0 & 3.76-05 & 3.54 & $3.80-06$ \\
\hline & 4.57-05 & & $7.90-05$ & & $1.30-04$ & & $5.40-04$ & & $1.70-05$ \\
\hline & 25.4 & & 58.2 & & 89.6 & & 30.3 & & 77.1 \\
\hline & 74.6 & & 41.8 & & 10.4 & & 69.7 & & 22.9 \\
\hline
\end{tabular}

- Core damage frequencies are based on internal initiating events only.

Note 1: Intermediate LOCA was not defined for this plant.

Note 2. Very small and small LOCA categories were added. 
Table 3.9.2b

Comparison of Conditional Core Damage Probabilities by

Initiating Event Groups

\begin{tabular}{|c|c|c|c|c|c|c|c|c|c|c|}
\hline $\begin{array}{l}\text { Initiating } \\
\text { Bvent Group }\end{array}$ & $\begin{array}{c}\text { Diablo } \\
\text { Canyon } 1\end{array}$ & Seabrook & $\begin{array}{c}\text { Zion } \\
\text { (revised) }\end{array}$ & Sequoyalf & Surry & Millstone 3 & $\begin{array}{l}\text { Indian } \\
\text { Point } 2\end{array}$ & $\begin{array}{l}\text { Indian } \\
\text { Point } 3\end{array}$ & Oconee & $\begin{array}{c}\text { Yankee } \\
\text { Rowe }\end{array}$ \\
\hline \multirow{3}{*}{$\begin{array}{l}\text { LOCAs } \\
\text { Lare } \\
\text { Intermediate }\end{array}$} & & & & & & & & & & \\
\hline & 1.41 .02 & $8.37-03$ & $1.55-03$ & $2.26-03$ & $1.68-03$ & $6.11-03$ & 8.21 .03 & $4.23-03$ & $9.68-03$ & $9.89-03$ \\
\hline & 1.29 .02 & $2.13-03$ & $4.40-03$ & $3.15-03$ & $1.60-03$ & $8.99-03$ & $6.67-03$ & $6.34-03$ & . & $6.16-03$ \\
\hline Small & $1.23-04$ & 1.15 .06 & $2.35-04$ & $2.34-03$ & 2.04-03 & $1.74-04$ & $8.92-04$ & $4.05-03$ & $2.03-03$ & $1.58-03$ \\
\hline Tranuients & $3.08-05$ & $1.19-05$ & 1.87 .05 & $4.15-06$ & $2.05-06$ & $3.14-06$ & $1.46-06$ & $1.40-06$ & $5.37-06$ & $1.05-06$ \\
\hline
\end{tabular}

Median Values 
Table 3.9.3

Event Scenarios Ranked According to Non-Selsmic CDF Contribution Dominant Sequence Model

\begin{tabular}{|c|c|c|c|}
\hline $\begin{array}{c}\text { CDF } \\
\text { RANK }\end{array}$ & CDF Contribution & \multicolumn{2}{|l|}{ Sequence } \\
\hline Part A: & \multicolumn{3}{|c|}{ Leadine Bvent Scenarios } \\
\hline 1 & $3.170-05$ & \multicolumn{2}{|l|}{ CRFIRE } \\
\hline 2 & $6.180-06$ & FS8 & $\cdot$ IAF $\cdot A F F \cdot A G F \cdot A H F \cdot C C F$ \\
\hline 3 & 5.377 .06 & RT & $\cdot \mathrm{IAF} \cdot \mathrm{HS} 1 \cdot / \mathrm{RF} 4 \cdot / \mathrm{Cl} 1$ \\
\hline 4 & 4.953-06 & TT & $\cdot \mathrm{IAF} \cdot \mathrm{HSI} \cdot / \mathrm{RF} 4 \cdot / \mathrm{Cl}$ \\
\hline 5 & $4.136-06$ & FS11 & $\cdot$ IAF $\bullet A S F \cdot R P 2 \cdot S E 1$ \\
\hline 6 & 3.533-06 & PLMFW & $\cdot \mathrm{IAF} \cdot \mathrm{HSI} \cdot / \mathrm{RF} 4 \cdot / \mathrm{CI} 1$ \\
\hline 7 & $2.283-06$ & MLOCA & $\cdot$ IAF ・ RF3 \\
\hline 8 & 2.128-06 & LPCC & - IAF * ASF * RP2 * SE1 \\
\hline 9 & $1.714-06$ & LIDC & $\cdot \mathrm{DGF} \cdot 12 \mathrm{~F} * 13 \mathrm{~F} * \mathrm{DH} 2 \cdot 14 \mathrm{~F} * \mathrm{AWA}$ \\
\hline 10 & $1.705-06$ & MLOCA & $\cdot$ IAF * LB2 * LA3 \\
\hline 11 & $1.699-06$ & LIDC & $\cdot \mathrm{DGF} \cdot 12 \mathrm{~F} \cdot 14 \mathrm{~F} \cdot \mathrm{AWA} \cdot \mathrm{AH} 4$ \\
\hline 12 & $1.524-06$ & FS11 & $\cdot$ IAF * ASF * /RP2 \\
\hline 13 & $1.398-06$ & EXFW & - IAF * HSI \\
\hline 14 & $1.267-06$ & LLOCA & - IAF・ACI \\
\hline 15 & $1.250-06$ & RT & OB1 $111 \cdot$ AW5 \\
\hline 16 & $1.280-06$ & $\mathbf{R T}$ & $\cdot \mathrm{OB} 1 \cdot 131 \cdot \mathrm{AWS}$ \\
\hline 17 & $1.241-06$ & LOOP & 'IAF* OGF* SW1 *AW3*VI2 \\
\hline 18 & 1.157 .06 & $\mathbf{R T}$ & - IAF* OB1 * AW1*/RF4*/CI1 \\
\hline 19 & $1.152-06$ & TT & - OB1 $\cdot 111 \cdot$ AWS \\
\hline 20 & $1.152-06$ & $\mathbf{T T}$ & - OB1 $131 \cdot$ AW5 \\
\hline 21 & $1.134-06$ & LOOP & - IAF * OGF * LA1 * PRD * REACO6* GH1 \\
\hline 22 & 1.132 .06 & $\mathbf{T T}$ & - IAF * OB1 * AW1 \\
\hline 23 & $1.057-06$ & Losw & $\cdot \mathrm{IAF} \cdot \mathrm{ASF} \cdot \mathrm{RP2} \cdot \mathrm{SE} 1$ \\
\hline \multicolumn{4}{|c|}{ Part B: Appearance of the First Event Scenario Associaled with a Given Initialor Not Listed Above } \\
\hline 32 & 8.269-07 & SGTR & - IAF $\cdot$ SLI * MUI \\
\hline 49 & $6.889-07$ & FS6 & - IAF * CC5 * AFF * AGF* RP2 \\
\hline 58 & 6.334-07 & SLBO & - IAF* LA1 * LB2* MS2*AWB \\
\hline 64 & $6.062-07$ & LOPF & : IAF* HS1 \\
\hline 66 & $5.861-07$ & SLOCI & - IAF * LA1 * LB2 * PRN \\
\hline 67 & $5.823-07$ & FS1 & - IAF $\cdot$ AW4 ${ }^{\circ}$ OB1 $\cdot / R F 4$ \\
\hline 68 & $5.710-07$ & FS5 & - IAF * ASF * RP2 * SE1 \\
\hline 74 & $5.360-07$ & IMSIV & - IAF* HSI \\
\hline 75 & $5.257-07$ & LOVC & * CVF * OSF * RT1 \\
\hline 78 & $5.000-07$ & TLMFW & - IAF * HS1 \\
\hline 79 & 4.999 .07 & VSI & - IAF*IT1 * ME1 \\
\hline
\end{tabular}


Table 3.9.3 (contlnued)

\begin{tabular}{|c|c|c|c|}
\hline $\begin{array}{c}\text { CDF } \\
\text { RANK }\end{array}$ & CDF Contribution & Sequerice & \\
\hline 81 & $4.816-07$ & SLBI & - MS2 $\cdot \mathrm{SA} \cdot \mathrm{SBE} \cdot \mathrm{OSF}$ \\
\hline 84 & 4.767-07 & LoswV & - SVF * SAI \\
\hline 93 & 4.373-07 & LCV & - IAF • HS1 \\
\hline 105 & 3.901-07 & FS9 & - IAF * OBI \\
\hline 111 & 3.702-07 & ISI & - IAF • HS1 \\
\hline 119 & 3.507-07 & HAZCHM & - ZHEHSS • POINTI \\
\hline 144 & 2.913-07 & SLOCN & $\cdot \mathrm{IAF} \cdot \mathrm{LA} 1 \cdot \mathrm{LB} 2 \cdot \mathrm{MU} 2 \cdot / \mathrm{Cl} 1$ \\
\hline 158 & 2.660-07 & ELOCA & - IAF \\
\hline 356 & 2.933-08 & FS10 & - IAF * AW4 * OB1 \\
\hline
\end{tabular}


Tablo 3.9 .3 (conthued)

\section{Dercriotion of Toe Ryents:}

\section{HAZCHM}

LAF

\author{
AFF,AGF,AHF \\ AH4 \\ DFF,DGF,DHF \\ DH2
}

OGF

GH1

sw1

I1F, 12F, BBF,IAF

I11,121,131,141

RT1

SA1

SAS

SBE

CVF
Desigator for all chemical relense sequences (in particular, chlorine and ammonia releases).

Obbal failure of inatrument air system.

Guaranteed fallure of vital 4.16kV ac buses: F, G, and $H$.

Fallure of vital 4.16kV ac bus H, given failure of de power train $G$ (DO-F).

Global failure of vital $125 \mathrm{~V}$ de power train F, G or $\mathrm{H}$.

Fallure of vital $125 \mathrm{~V}$ de power train $\mathrm{H}$, given availablo $480 \mathrm{~V}$ ac bus $1 \mathrm{H}$ and top eveat DF failure and top event DG success (DF-F, DG-S).

Global failure of son-vital ac power from 230kV switchyard.

Failure of diesel generator 11, all support systems available.

Swing diesel is aligned to Unit 2.

Global failure of inatrument ac channel I, II, II or IV.

Failure of inatrument ac channel I, II, III or IV, with all support syatem or previous trains available.

Failure of resctor protection syatem; $1 / 2$ trains, both SSPS signals generated.

Failure of aigaal generation (General Transient) by train $A$ of the SSPS; all support available.

Failure of signal generation (SLBIC) by train A of the SSPS; all four instrument channebs available.

Failure of signal generation (SLBIC) by train B, given failed train A; all four instrument channels available.

Global failure of control room ventilation system.

-The deacriptions for the majority of initiator designators are given in Table 3.9.1.

NUREG/CR-5726 

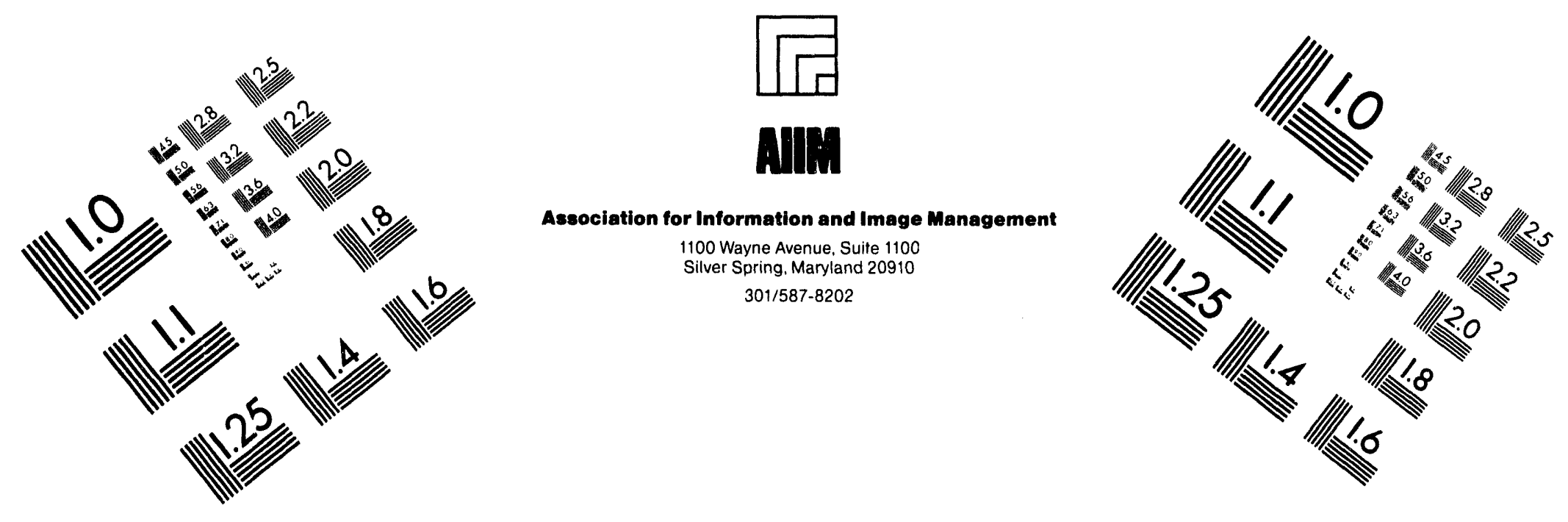

\section{Centimeter}

$\begin{array}{llllllllllllllll}1 & 2 & 3 & 4 & 5 & 6 & 7 & 8 & 9 & 10 & 11 & 12 & 13 & 14 & 15 & \mathrm{~mm}\end{array}$ IIII)

Inches
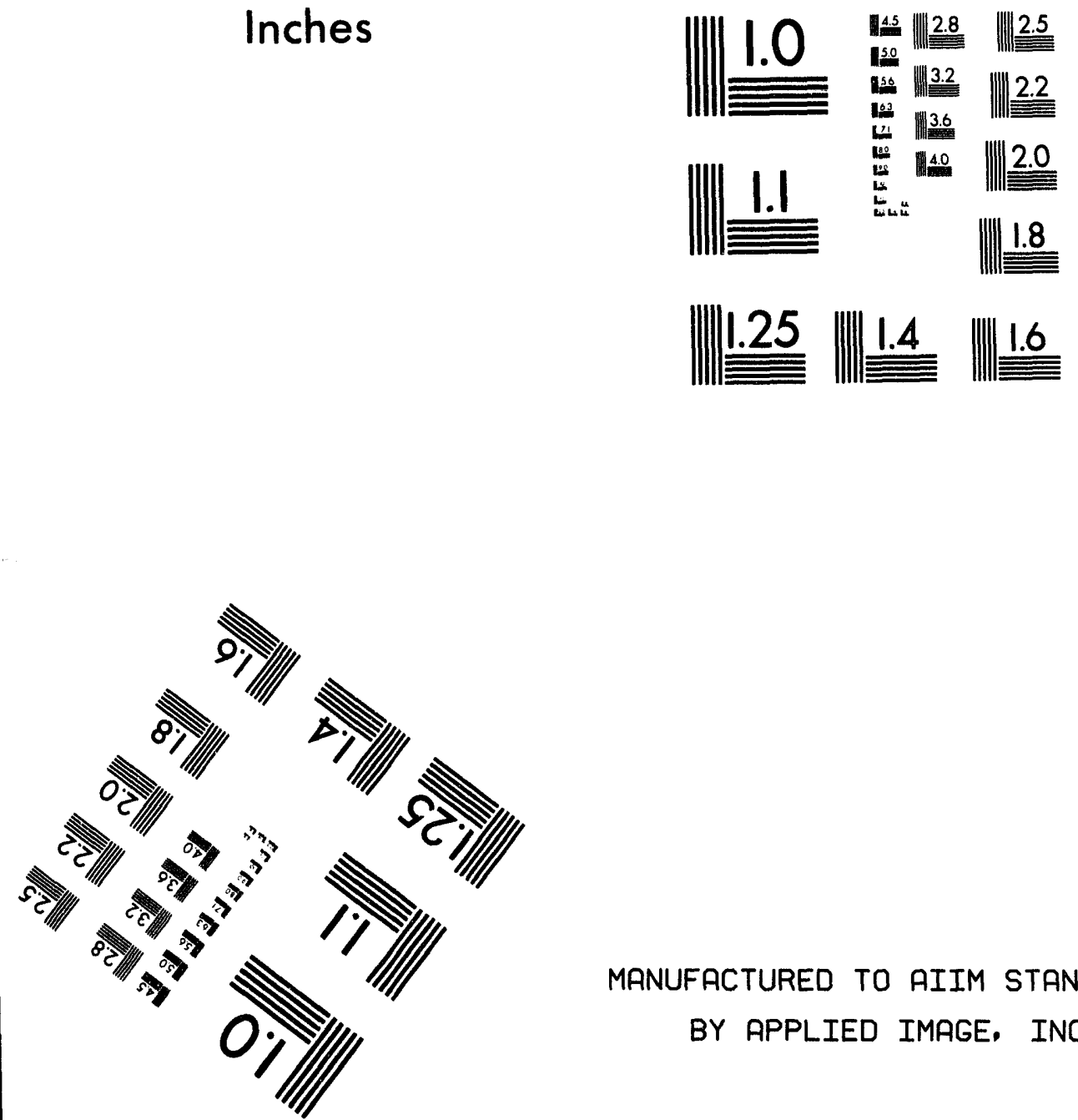

MANUFACTURED TO AIIM STANDARDS

BY APPLIED IMAGE, INC.

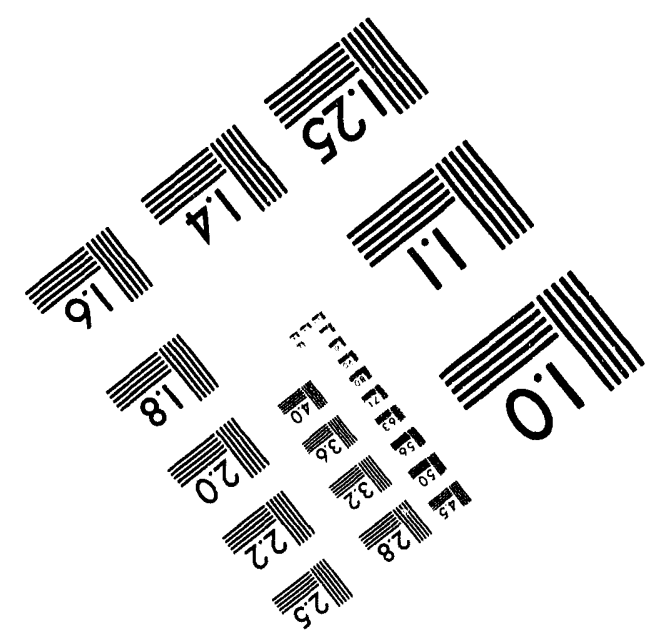



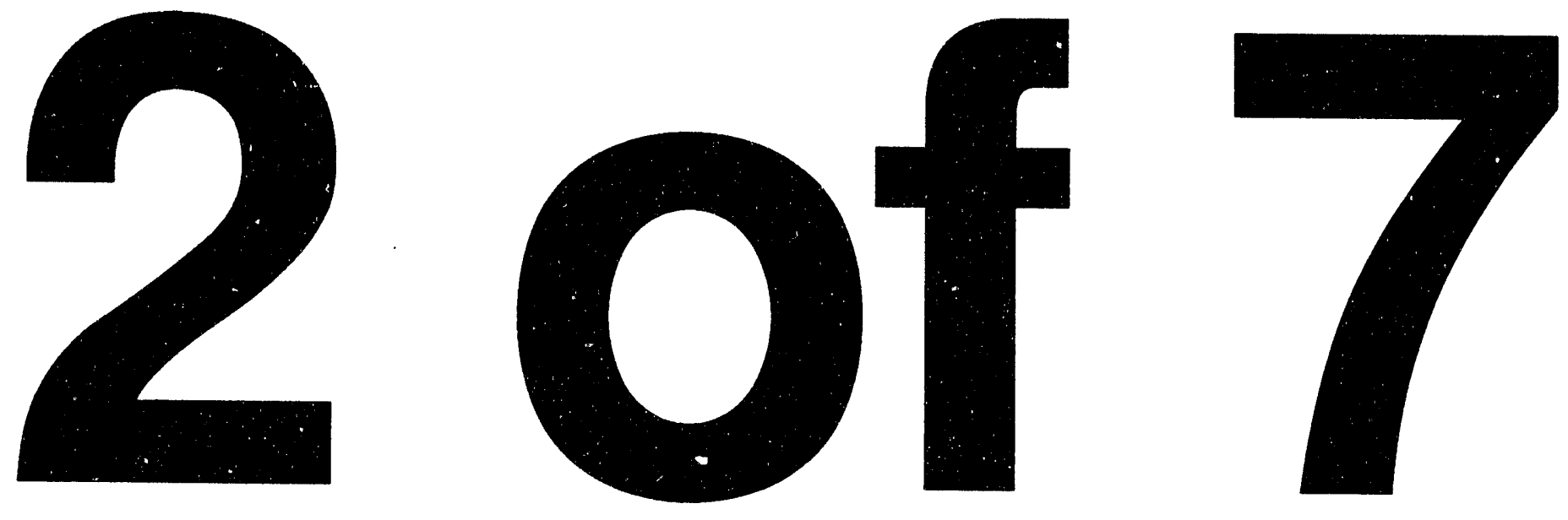
Table 3.9.3 (contlaned)

SVF

ASF

CCF

$\mathrm{CCA}$

AW1

AW3

AW4

AWs

AWA

AWB

AC1

LA1

LA3

LB2

MU1

MU2

PRD

PRN

OB1

MS2

Global failure of $480 \mathrm{~V}$ switchgear ventilation system.

Global failure of auxiliary saltwater system.

Global failure of component cooling water system.

Failure of component cooling water system, given loss of $4.16 \mathrm{kV}$ buses $F$ and $G$.

\section{Eailures of Auriliary Feedwater Syotem. Low Power}

All support system available.

Support for one MDP unavailable.

Support for two MDPs unavailable.

Support for all 10\% steam dump unavailable.

Support for all 10\% steam dump, one MDP and TDP unavailable.

One SG depressurizes, all support systems available.

\section{Failures of ECCS. Low Presure}

Failures of accumulators and cold leg path.

Failure of RHR train A, all support available (SLOCA).

Failure of RHR train A, all support available (MLOCA).

Failure of RHR train-B; given failed top event LA (SLOCA).

Failure of RHR suction from the hot leg or RWST makeup; power at ac buser $\mathbf{G}$ and $\mathbf{H}$ available.

Failure or makeup to RWST (via RFW pump); power available at ac buses $\mathbf{G}$ and $\mathrm{H}$.

\section{Fuilures of Primary RCS Presaure Relief}

1/2 PORVs or (1/3 SRVs) nicks open and is not isolated given LOSP/SGTR. No block valve available.

1/1 block valve clones, all support available.

Failure of feed and bleed (PORV opening and reclosure) given loss of instrument air.

Failure of main steamline isolation; given failed TT, all support svailable. 
Table 3.9.3 (continued)

MII

SE1

SL1

IT1

ME1

V12

Point1

HS1

REACO6

OSF

RP2

IRP2

RF3

/RF4

ZHEHSS
Success of containment penetration isolation (inboard or outboard isolation valves for penetrations $50,51,52$, and 45 are successful).

Failure to align backup cooling to charging pumps for RCP seal cooling; CCWS fails.

Failure of isolation of ruptured steam generator, all support available.

Failure of RHR piping integrity; VO successful (RHR pressure relief valves open).

RHR hole size $>4$ inches.

Failure of vessel integrity (due to pressurized thermal shock); loss of secondary heat sink.

Conditional probability of core damage in chemical sequences given that the CST was not initially replenished.

Failure of operator action to maintain hot standby (ioss of control after an accident); all support systems are available.

Failure to recover vital $4.16 \mathrm{kV}$ ac power before recirculation required.

Operator failure to actuate faiped SSPS automatic actuation.

Operator failure to trip RCPs to prevent LOCA; given loss of CCW. Operator success to trip RCPs to prevent LOCA, given loss of CCW.

Failure of manual switchover to recirculation after LLOCA or MLOCA.

Successful switchover to recirculation after core melt.

Operator failure to replenish the condensate storage tank. 
Table 3.9.4 Special Isoued Importance Summary

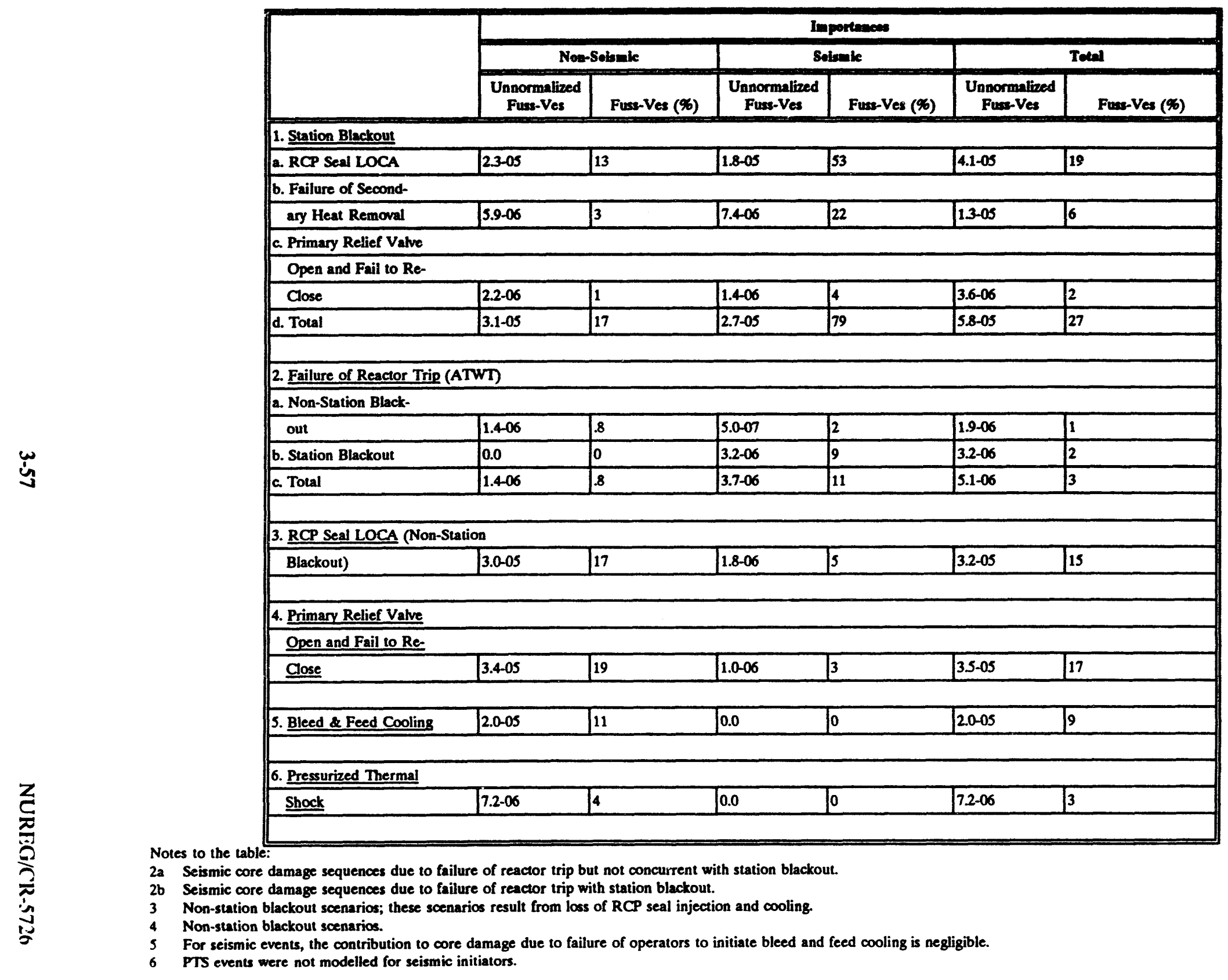


Table 3.9.5

System/Operator Action Importances for Non-Selsmic Core Damage Frequency Ranking According to System/Operator Action Importances

\section{A. Dominant Sequence Model, Support Systems}

\begin{tabular}{|c|c|c|c|c|}
\hline \multicolumn{2}{|c|}{ Support Systems } & \multicolumn{3}{|c|}{ Fussel-Vesely Importances } \\
\hline & \multirow[b]{2}{*}{$\begin{array}{l}\text { Assoclated Top } \\
\text { Event(s) }\end{array}$} & \multicolumn{2}{|c|}{ Unnormalized } & \multirow[b]{2}{*}{$\begin{array}{c}\text { System } \\
\text { Importance } \\
(\%)\end{array}$} \\
\hline & & $\begin{array}{l}\text { Top Event } \\
\text { Importance }\end{array}$ & System Importance & \\
\hline Diesel Generator Systems & & & $4.255-05$ & 24.0 \\
\hline \multirow[t]{3}{*}{ a. Unit 1 DGs } & GF & $1.517-05$ & & \\
\hline & GG & $1.983-05$ & & \\
\hline & GH & $2.139-05$ & & \\
\hline \multirow[t]{2}{*}{ b. Unit 2 DGs } & TG & 7.387-06 & & \\
\hline & TH & $7.099-06$ & & \\
\hline c. Swing Diesel Alignment & sw & $9.262-06$ & & \\
\hline $\begin{array}{l}\text { d. Diesel Fuel Oil } \\
\text { Transfer }\end{array}$ & FO & $7.004-06$ & & \\
\hline \multirow[t]{4}{*}{$\begin{array}{l}\text { Vital 125V DC Power, } \\
\text { Unit } 1\end{array}$} & & & $1.681-05$ & 9.5 \\
\hline & DF & $2.281-06$ & & \\
\hline & DG & 3.926-06 & & \\
\hline & DH & $1.006-05$ & & \\
\hline \multirow[t]{5}{*}{ Instrument AC Power } & & & $1.138-05$ & 6.4 \\
\hline & II & 3.675-06 & & \\
\hline & 12 & 1.771 .06 & & \\
\hline & I3 & $4.159-06$ & & \\
\hline & I4 & $1.771-06$ & & \\
\hline Component Cooling Water & CC & $1.065-05$ & $1.065-05$ & 6.0 \\
\hline \multirow[t]{5}{*}{ Vital AC Power, Unit 1} & & & $8.605-06$ & 4.9 \\
\hline & AF & 2.428 .06 & & \\
\hline & AG & 6.722-07 & & \\
\hline & AH & $5.500-06$ & & \\
\hline & SF,SG,SH & - & & \\
\hline \multirow{3}{*}{$\begin{array}{l}\text { Solid State Protection } \\
\text { System }\end{array}$} & & & $5.153-06$ & 2.9 \\
\hline & $\mathbf{S A}$ & $4.000-06$ & & \\
\hline & SB & $4.376-06$ & & \\
\hline $\begin{array}{l}480 \mathrm{~V} \text { Switchgear } \\
\text { Ventilation }\end{array}$ & SV & 4.411 .06 & $4.411-06$ & 2.5 \\
\hline
\end{tabular}


Table 3.9.5 (continued)

\begin{tabular}{|c|c|c|c|c|}
\hline \multicolumn{2}{|c|}{ Support Systoms } & \multicolumn{3}{|c|}{ Fussel-Vesely Importances } \\
\hline & \multirow[b]{2}{*}{$\begin{array}{l}\text { Assoclated Top } \\
\text { Event(s) }\end{array}$} & \multicolumn{2}{|c|}{ Unnormalized } & \multirow[b]{2}{*}{\begin{tabular}{|c} 
System \\
Importance \\
$(\%)$
\end{tabular}} \\
\hline & & $\begin{array}{l}\text { Top Event } \\
\text { Importance }\end{array}$ & System Importance & \\
\hline Auxiliany Saltwater & AS & $2.588-06$ & $2.588-06$ & 1.5 \\
\hline Control Room Ventilation & $\mathrm{CV}$ & 2.583-06 & $2.583-06$ & 1.5 \\
\hline Reactor Protection System & RT & $1.558-06$ & $1.558-06$ & 0.9 \\
\hline \multirow[t]{3}{*}{ Non-Vital Electric Power } & & & $1.309-06$ & 0.7 \\
\hline & OG & $1.309-06$ & & \\
\hline & NV & - & & \\
\hline \multirow[t]{4}{*}{$\begin{array}{l}\text { Vital AC and DC Power, } \\
\text { Unit } 2\end{array}$} & & & $7.511-07$ & 0.4 \\
\hline & BF & - & & \\
\hline & BG & $5.402-07$ & & \\
\hline & BH & 2.109-07 & & \\
\hline
\end{tabular}

B. Dominant Sequence Model Frontline Systems

\begin{tabular}{|c|c|c|c|c|}
\hline \multicolumn{2}{|c|}{ Frontline Systems } & \multicolumn{3}{|c|}{ Fussel-Vesely Importances } \\
\hline & \multirow[b]{2}{*}{$\begin{array}{l}\text { Aseociated Top } \\
\text { Event(a) }\end{array}$} & \multicolumn{2}{|c|}{ Unnormalized } & \multirow[b]{2}{*}{$\begin{array}{c}\text { System } \\
\text { Importance } \\
\text { (\%) }\end{array}$} \\
\hline & & $\begin{array}{l}\text { Top Event } \\
\text { Importance }\end{array}$ & System Importance & \\
\hline \multirow[t]{3}{*}{$\begin{array}{l}\text { Auxiliary Feedwater } \\
\text { System }\end{array}$} & & & $4.586-05$ & 25.9 \\
\hline & AW & $4.586-05$ & & \\
\hline & TD & - & & \\
\hline \multirow[t]{4}{*}{$\begin{array}{l}\text { Primary RCS Pressure } \\
\text { Relief }\end{array}$} & & & $3.717-05$ & 21.0 \\
\hline & PR & $1.689-05$ & & \\
\hline & PO & - & & \\
\hline & OB & $2.028-05$ & & \\
\hline \multirow[t]{7}{*}{ ECCS, Low Presoure } & & & $1.390-05$ & 7.8 \\
\hline & LA & $7.519-06$ & & \\
\hline & LB & $7.149-06$ & & \\
\hline & LV & $2.125-07$ & & \\
\hline & $\mathbf{R W}$ & 2.072-07 & & \\
\hline & VA & $2.292-07$ & & \\
\hline & VB & $7.663-07$ & & \\
\hline
\end{tabular}


Table 3.9.5 (continued)

\begin{tabular}{|c|c|c|c|c|}
\hline \multicolumn{2}{|c|}{ Frontline Systems } & \multicolumn{3}{|c|}{ Fussel-Vesely Importances } \\
\hline & \multirow[b]{2}{*}{$\begin{array}{l}\text { Associated Top } \\
\text { Event(s) }\end{array}$} & \multicolumn{2}{|c|}{ Unnormallzed } & \multirow[b]{2}{*}{$\begin{array}{c}\text { System } \\
\text { Importance } \\
(\%) \\
\end{array}$} \\
\hline & & $\begin{array}{l}\text { Top Event } \\
\text { Importance }\end{array}$ & System Importance & \\
\hline & $A C$ & $1.267-06$ & & \\
\hline & LI & - & & \\
\hline & MU & $1.918-06$ & & \\
\hline \multirow[t]{2}{*}{ ECCS, High Pressure } & & & $7.456-06$ & 4.2 \\
\hline & $\mathrm{CH}$ & 8.943-07 & & \\
\hline \multirow[t]{4}{*}{ ECCS, High Pressure } & SI & $7.268-07$ & & \\
\hline & HR & $1.085-06$ & & \\
\hline & RC & - & & \\
\hline & RF(-RF4) & 4.794-06 & & \\
\hline $\begin{array}{l}\text { Reactor Vessel Integrity } \\
\text { After Pressurized Thermal } \\
\text { Shock (PTS) }\end{array}$ & $\mathrm{VI}$ & $7.175-06$ & $7.175-06$ & 4.0 \\
\hline \multirow[t]{3}{*}{$\begin{array}{l}\text { Turbine Trip and Main } \\
\text { Steam Isolation }\end{array}$} & & & $5.984-06$ & 3.4 \\
\hline & TT & - & & \\
\hline & MS & $5.984-06$ & & \\
\hline Isolation of Ruptured SG & SL & $1.940-06$ & $1.940-06$ & 1.1 \\
\hline Interfacing LOCA Tree & & & $5.0-07$ & .3 \\
\hline \multirow[t]{4}{*}{ Top Events } & VO,VC,VR,SM & - & & \\
\hline & IT & $5.0-07$ & & \\
\hline & LW & - & & \\
\hline & $\mathrm{ME}$ & $5.0-07$ & & \\
\hline \multirow[t]{3}{*}{ Containment Isolation } & & & $3.524-07$ & .2 \\
\hline & CI & $3.524-07$ & & \\
\hline & $\mathrm{CP}, \mathrm{WL}$ & E & & \\
\hline \multirow[t]{3}{*}{ Containment Spray } & & & 2.982 .08 & .02 \\
\hline & CS & 3.364-10 & & \\
\hline & SR & $2.949-08$ & & \\
\hline
\end{tabular}


Table 3.9.5 (continued)

C. Dominant Sequence Model, Operator Recovery Actions

\begin{tabular}{|c|c|c|c|c|}
\hline \multicolumn{2}{|l|}{ Action } & \multicolumn{3}{|c|}{ Fussel-Vesely Importances } \\
\hline & \multirow[b]{2}{*}{$\begin{array}{l}\text { Associated Top } \\
\text { Event(s) }\end{array}$} & \multicolumn{2}{|c|}{ Unnormalized } & \multirow[b]{2}{*}{$\begin{array}{l}\text { Operator } \\
\text { Action } \\
\text { Imp. (\%) }\end{array}$} \\
\hline & & $\begin{array}{l}\text { Top Event } \\
\text { Importance }\end{array}$ & $\begin{array}{l}\text { Op. Action } \\
\text { Importance }\end{array}$ & \\
\hline $\begin{array}{l}\text { Maintain Control for Hot- } \\
\text { Standby After a Reactor } \\
\text { Trip }\end{array}$ & HS & $1.960-05$ & $1.960-05$ & 11.0 \\
\hline $\begin{array}{l}\text { Operator Trips RCPs After } \\
\text { Loss of CCW to Prevent } \\
\text { Seal LOCA }\end{array}$ & $\mathbf{R P}$ & $1.215-05$ & $1.215-05$ & 6.8 \\
\hline $\begin{array}{l}\text { Actions Needed to } \\
\text { Maintain RCP Seal Cooling }\end{array}$ & $\mathrm{SE}$ & 8.999-06 & $8.999-06$ & 5.1 \\
\hline \multirow[t]{6}{*}{$\begin{array}{l}\text { Electric Power Recovery } \\
\text { Factors }\end{array}$} & & & $5.958-06$ & 3.4 \\
\hline & REAC06 & \multicolumn{3}{|l|}{ 2.733-06 } \\
\hline & RESLC1 & \multicolumn{3}{|l|}{1.645 .06} \\
\hline & RESLC2 & \multicolumn{3}{|l|}{$1.484-06$} \\
\hline & RESLC3 & \multicolumn{3}{|l|}{$9.360-08$} \\
\hline & REAC12 & 2.925-09 & & \\
\hline $\begin{array}{l}\text { Secure SI Per Operating } \\
\text { Procedures Following } \\
\text { SGTR }\end{array}$ & OP & $1.643-06$ & $1.643-06$ & 0.9 \\
\hline $\begin{array}{l}\text { Operator Actuation of } \\
\text { SSPS Signal }\end{array}$ & OS & $1.069-06$ & $1.069-06$ & 0.6 \\
\hline \multirow[t]{10}{*}{$\begin{array}{l}\text { Various Human Failures in } \\
\text { Accident Recovery }\end{array}$} & & & $1.136-06$ & 0.6 \\
\hline & ZHEHSS & \multicolumn{3}{|l|}{$3.508-07$} \\
\hline & ZHESV3 & \multicolumn{3}{|l|}{ 2.874-07 } \\
\hline & ZHERP2 & \multicolumn{3}{|l|}{ 1.709-07 } \\
\hline & ZHEFO6 & \multicolumn{3}{|l|}{ 1.153-07 } \\
\hline & ZHEAW4 & \multicolumn{3}{|l|}{$8.748-08$} \\
\hline & ZHEOB2 & \multicolumn{3}{|l|}{$5.587-08$} \\
\hline & ZHEAW3 & \multicolumn{3}{|l|}{$2.584-08$} \\
\hline & ZHESW1 & \multicolumn{3}{|l|}{$2.236-08$} \\
\hline & ZHERE2 & \multicolumn{3}{|l|}{$2.018-08$} \\
\hline
\end{tabular}


Table 3.9.6

Top Event Importances - Conditional Split Fractions

(Ranking According to Fussel-Vesely Importance)

\begin{tabular}{|c|c|c|c|}
\hline \multicolumn{4}{|c|}{ A. Dominant Sequence Model, Support Systems } \\
\hline \multirow[b]{2}{*}{ Top Event } & \multicolumn{2}{|c|}{ Importances } & \multirow{2}{*}{$\begin{array}{c}\text { Occurrence } \\
\text { in the DSM } \\
\text { Scenarios }\end{array}$} \\
\hline & $\begin{array}{l}\text { Unnormalized } \\
\text { Fussel-Vesely }\end{array}$ & Birnbaum & \\
\hline GF1 & 1.517E-05 & 3.338E-04 & 69 \\
\hline GG1 & $1.125 \mathrm{E}-05$ & $2.504 \mathrm{E}-04$ & 43 \\
\hline GH1 & 9.579E-06 & 2.157E-04 & 47 \\
\hline GG2 & $8.576 E-06$ & $1.538 \mathrm{E}-04$ & 30 \\
\hline GH2 & 8.193E-06 & $1.504 \mathrm{E}-04$ & 44 \\
\hline sw1 & 7.248E-06 & $1.449 \mathrm{E}-05$ & 40 \\
\hline DH1 & 7.227E-06 & $1.031 \mathrm{E}-02$ & 34 \\
\hline CCS & 5.105E-06 & $1.779 \mathrm{E}-04$ & 17 \\
\hline DG1 & 3.926E-06 & $5.568 \mathrm{E}-03$ & 31 \\
\hline FO1 & 3.853E-06 & $1.784 E-02$ & 7 \\
\hline 131 & 3.799E-06 & 3.303E-03 & 7 \\
\hline I11 & 3.675E-06 & 3.196E-03 & 6 \\
\hline TH3 & $3.618 \mathrm{E}-06$ & 5.761E-05 & 18 \\
\hline TG2 & $3.478 \mathrm{E}-06$ & $6.339 \mathrm{E}-05$ & 26 \\
\hline GH3 & 2.887E-06 & 3.420E-05 & 7 \\
\hline DH2 & $2.833 \mathrm{E}-06$ & $4.059 \mathrm{E}-03$ & 3 \\
\hline DF1 & 2.821E-06 & 4.002E-03 & 14 \\
\hline AH4 & $2.809 \mathrm{E}-06$ & $4.059 \mathrm{E}-03$ & 3 \\
\hline TH2 & $2.722 \mathrm{E}-06$ & $4.969 \mathrm{E}-05$ & 16 \\
\hline SA1 & $2.692 \mathrm{E}-06$ & 3.549E-04 & 8 \\
\hline AH1 & 2.691E-06 & $3.889 \mathrm{E}-03$ & 22 \\
\hline AF1 & $2.428 \mathrm{E}-06$ & 3.509E-03 & 10 \\
\hline TG3 & 2.297E-06 & 3.610E-05 & 18 \\
\hline SB2 & $2.101 E-06$ & 8.755E-05 & 6 \\
\hline $\mathrm{CC3}$ & 1.817E-06 & 3.107E-03 & 8 \\
\hline 141 & 1.771E-06 & $3.074 \mathrm{E}-03$ & 5 \\
\hline 121 & 1.771E-06 & $3.074 \mathrm{E}-03$ & 5 \\
\hline SV1 & $1.672 \mathrm{E}-06$ & $9.780 \mathrm{E}-01$ & 12 \\
\hline sw2 & $1.601 E-06$ & 6.305E-04 & 3 \\
\hline CV3 & $1.596 \mathrm{E}-06$ & 2.811E-05 & 5 \\
\hline SV2 & $1.460 \mathrm{E}-06$ & $8.112 \mathrm{E}-03$ & 14 \\
\hline FOS & 1.385E-06 & $2.727 \mathrm{E}-05$ & 2 \\
\hline ASB & 1.371E-06 & $5.077 \mathrm{E}-05$ & 6 \\
\hline OG1 & $1.309 \mathrm{E}-06$ & $1.715 \mathrm{E}-03$ & 9 \\
\hline CC2 & $1.284 \mathrm{E}-06$ & $2.256 \mathrm{E}-03$ & 4 \\
\hline
\end{tabular}


Table 3.9.6 (continued)

\begin{tabular}{|c|c|c|c|}
\hline \multicolumn{4}{|c|}{ A. Dominant Sequence Model, Support Systems } \\
\hline \multirow[b]{2}{*}{ Top Event } & \multicolumn{2}{|c|}{ Importance } & \multirow{2}{*}{$\begin{array}{c}\text { Occurrence } \\
\text { in the DSM } \\
\text { Scenarios }\end{array}$} \\
\hline & $\begin{array}{l}\text { Unnormallzed } \\
\text { Fussel-Vesely }\end{array}$ & Birnbaum & \\
\hline RT7 & $1.032 \mathrm{E}-06$ & 5.347E-04 & 3 \\
\hline AS4 & $1.010 \mathrm{E}-06$ & 5.977E-05 & 3 \\
\hline TG1 & $1.008 \mathrm{E}-06$ & $2.283 \mathrm{E}-05$ & 3 \\
\hline SB1 & $9.748 \mathrm{E}-07$ & $1.301 \mathrm{E}-04$ & 6 \\
\hline $\mathrm{CC4}$ & 9.501E-07 & $3.558 \mathrm{E}-05$ & 2 \\
\hline FO4 & $9.300 \mathrm{E}-07$ & 4.115E-05 & 4 \\
\hline $\mathrm{CC1}$ & 9.025E-07 & $4.801 \mathrm{E}-02$ & 4 \\
\hline svs & 7.639E-07 & $1.042 \mathrm{E}-04$ & 9 \\
\hline AG1 & 6.772E-07 & $9.786 \mathrm{E}-04$ & 4 \\
\hline SAS & $6.682 \mathrm{E}-07$ & 4.773E-05 & 2 \\
\hline SA2 & $6.398 \mathrm{E}-07$ & $5.613 \mathrm{E}-05$ & 2 \\
\hline SB6 & 6.398E-07 & 7.581E-06 & 2 \\
\hline FO2 & $6.214 \mathrm{E}-07$ & 8.827E-05 & 8 \\
\hline $\mathrm{CC7}$ & 5.914E-07 & 8.921E-04 & 3 \\
\hline BG1 & 5.402E-07 & 3.7.52E-04 & 2 \\
\hline RT1 & 5.257E-07 & $7.990 \mathrm{E}-02$ & 1 \\
\hline SBE & $4.816 \mathrm{E}-07$ & $6.482 \mathrm{E}-06$ & 1 \\
\hline GH4 & 4.133E-07 & 9.225E-06 & 2 \\
\hline SW3 & $4.130 \mathrm{E}-07$ & 4.155E-07 & 9 \\
\hline SV3 & 4.057E-07 & 3.051E-03 & 4 \\
\hline 132 & 3.606E-07 & 2.072E-04 & 2 \\
\hline CV1 & 3.519E-07 & $4.630 \mathrm{E}-04$ & 1 \\
\hline $\mathrm{CV}_{2}$ & 3.323E-07 & $1.613 \mathrm{E}-05$ & 1 \\
\hline GHS & 3.195E-07 & $5.746 \mathrm{E}-06$ & 2 \\
\hline TG6 & 3.109E-07 & $5.746 \mathrm{E}-06$ & 2 \\
\hline CV6 & 3.023E-07 & 7.792E-06 & 1 \\
\hline TG5 & $3.010 \mathrm{E}-07$ & $6.780 \mathrm{E}-06$ & 2 \\
\hline TH6 & 2.983E-07 & $6.780 \mathrm{E}-06$ & 2 \\
\hline TH1 & 2.477E-07 & $5.598 \mathrm{E}-06$ & 4 \\
\hline FO3 & 2.145E-07 & $6.110 \mathrm{E}-04$ & 4 \\
\hline TH4 & 2.132E-07 & 2.579E-06 & 4 \\
\hline $\mathrm{BH} 1$ & 2.109E-07 & $1.465 \mathrm{E}-04$ & 1 \\
\hline SBC & $1.786 \mathrm{E}-07$ & 1.333E-05 & 1 \\
\hline AS5 & $1.776 \mathrm{E}-07$ & $4.960 \mathrm{E}-04$ & 1 \\
\hline sv4 & $1.089 \mathrm{E}-07$ & $4.236 \mathrm{E}-03$ & 2 \\
\hline AS3 & 2.949E-08 & 2.418E-04 & 1 \\
\hline
\end{tabular}


Table 3.9.6 (continued)

B. Dominant Sequence Model, Frontline Systems

\begin{tabular}{|c|c|c|c|}
\hline & \multicolumn{2}{|c|}{ Importances } & \multirow{2}{*}{$\begin{array}{l}\text { Occurrence } \\
\text { in the } \\
\text { DSM }\end{array}$} \\
\hline & $\begin{array}{l}\text { Unnormalized } \\
\text { Fussel-Vesely }\end{array}$ & Birnbaum & \\
\hline OB1 & $1.760 \mathrm{E}-05$ & $6.089 \mathrm{E}-04$ & 59 \\
\hline PRD & $1.618 \mathrm{E}-05$ & $3.316 \mathrm{E}-04$ & 43 \\
\hline AW4 & $9.760 \mathrm{E}-06$ & $1.346 \mathrm{E}-04$ & 60 \\
\hline AWS & $9.676 \mathrm{E}-06$ & $2.932 \mathrm{E}-04$ & 12 \\
\hline AW1 & 7.497E-06 & $2.010 \mathrm{E}-01$ & 21 \\
\hline AWA & $6.532 \mathrm{E}-06$ & $6.811 \mathrm{E}-05$ & 8 \\
\hline MS2 & $5.984 \mathrm{E}-06$ & $5.984 \mathrm{E}-06$ & 32 \\
\hline LA1 & 5.695E-06 & 2.792E-04 & 24 \\
\hline V12 & $5.569 \mathrm{E}-06$ & $2.531 \mathrm{E}-04$ & 16 \\
\hline AW3 & $5.029 \mathrm{E}-06$ & $4.056 \mathrm{E}-03$ & 33 \\
\hline LB2 & $4.958 \mathrm{E}-06$ & 2.128E-05 & 11 \\
\hline AW8 & $3.412 E-06$ & $2.774 \mathrm{E}-03$ & 18 \\
\hline RF3 & 3.278E-06 & $6.650 \mathrm{E}-04$ & 2 \\
\hline OB3 & 2.684E-06 & 7.158E-06 & 14 \\
\hline LB3 & $2.061 \mathrm{E}-06$ & $1.010 \mathrm{E}-04$ & 7 \\
\hline LA3 & $1.824 \mathrm{E}-06$ & 1.155E-04 & 2 \\
\hline AW9 & $1.688 \mathrm{E}-06$ & $1.197 \mathrm{E}-05$ & 8 \\
\hline AWB & $1.611 \mathrm{E}-06$ & $6.686 \mathrm{E}-0.5$ & 9 \\
\hline RF1 & $1.515 \mathrm{E}-06$ & $4.795 \mathrm{E}-04$ & 11 \\
\hline MU1 & $1.430 \mathrm{E}-06$ & $1.792 \mathrm{E}-04$ & 2 \\
\hline SL1 & $1.319 \mathrm{E}-06$ & 2.177E-04 & 2 \\
\hline AC1 & $1.267 \mathrm{E}-06$ & $2.020 \mathrm{E}-04$ & 1 \\
\hline V13 & $9.260 \mathrm{E}-07$ & $4.630 \mathrm{E}-04$ & 1 \\
\hline $\mathrm{CH} 2$ & 8.917E-07 & $6.324 \mathrm{E}-05$ & 13 \\
\hline HRB & $8.310 \mathrm{E}-07$ & 2.072E-04 & 2 \\
\hline VB1 & $7.544 \mathrm{E}-07$ & 2.072E-04 & 2 \\
\hline VI5 & $6.802 \mathrm{E}-07$ & $7.558 \mathrm{E}-0.5$ & 1 \\
\hline AW7 & $6.616 \mathrm{E}-07$ & $2.042 \mathrm{E}-03$ & 32 \\
\hline SL2 & $6.202 E-07$ & $9.513 \mathrm{E}-05$ & 7 \\
\hline PRN & $5.862 E-07$ & $7.653 \mathrm{E}-0.5$ & 1 \\
\hline IT1 & $4.999 \mathrm{E}-07$ & $5.050 \mathrm{E}-07$ & 1 \\
\hline ME1 & $4.999 \mathrm{E}-07$ & 9.999E-07 & 1 \\
\hline MU2 & $4.878 E-07$ & 4.169E-05 & 3 \\
\hline SI2 & 4.818E-07 & $3.011 \mathrm{E}-0.5$ & 3 \\
\hline HRD & 2.535E-07 & $5.560 \mathrm{E}-0.5$ & 1 \\
\hline SII & $2.450 \mathrm{E}-07$ & $7.513 \mathrm{E}-0.5$ & 3 \\
\hline
\end{tabular}




\begin{tabular}{|c|c|c|c|}
\hline \multicolumn{4}{|c|}{ B. Dominant Sequence Model, Frontline Systems } \\
\hline & \multicolumn{2}{|c|}{ Importances } & \multirow{2}{*}{$\begin{array}{l}\text { Occurrence } \\
\text { In the } \\
\text { DSM }\end{array}$} \\
\hline & $\begin{array}{l}\text { Unnormalized } \\
\text { Fussel-Vesely }\end{array}$ & Blrnbaum & \\
\hline CI1 & 2.414E-07 & $4.116 \mathrm{E}-05$ & 1 \\
\hline VA1 & 2.292E-07 & $5.970 \mathrm{E}-05$ & 2 \\
\hline LV1 & 2.125E-07 & $4.630 \mathrm{E}-04$ & 1 \\
\hline RW1 & $2.072 \mathrm{E}-07$ & $5.260 \mathrm{E}-03$ & 1 \\
\hline PRA & 1.233E-07 & $1.498 \mathrm{E}-05$ & 1 \\
\hline LB8 & 1.197E-07 & $3.192 \mathrm{E}-06$ & 1 \\
\hline CI2 & $1.110 \mathrm{E}-07$ & $1.924 \mathrm{E}-05$ & 2 \\
\hline SR2 & 2.949E-08 & $3.110 \mathrm{E}-06$ & 1 \\
\hline VB3 & 1.194E-08 & $3.110 \mathrm{E}-06$ & 1 \\
\hline LB1 & 9.977E.09 & $6.395 \mathrm{E}-07$ & 2 \\
\hline CH1 & $2.555 \mathrm{E}-09$ & $4.094 \mathrm{E}-06$ & 1 \\
\hline CS2 & $3.364 \mathrm{E}-10$ & $2.352 \mathrm{E}-08$ & 1 \\
\hline \multicolumn{4}{|c|}{ C. Dominant Sequence Model, Operator Recovery Actions } \\
\hline & \multicolumn{2}{|c|}{ Importances } & \multirow{2}{*}{$\begin{array}{l}\text { Occurrence } \\
\text { in the } \\
\text { DSM }\end{array}$} \\
\hline & $\begin{array}{l}\text { Unnormalized } \\
\text { Fussel-Vesely }\end{array}$ & Birnbaum & \\
\hline HS1 & $1.960 \mathrm{E}-05$ & $1.000 \mathrm{E}+00$ & 15 \\
\hline $\mathbf{R P 2}$ & $1.215 \mathrm{E}-05$ & $-6.622 \mathrm{E}-04$ & 23 \\
\hline SE1 & 8.999E-06 & $8.256 \mathrm{E}-04$ & 9 \\
\hline REAC06 & 2.733E-06 & $9.690 \mathrm{E}-06$ & 6 \\
\hline RESLC1 & $1.645 E-06$ & $3.545 \mathrm{E}-06$ & 22 \\
\hline OP1 & $1.643 \mathrm{E}-06$ & $3.716 \mathrm{E}-04$ & 3 \\
\hline RESLC2 & $1.484 \mathrm{E}-06$ & $3.885 \mathrm{E}-05$ & 6 \\
\hline OS1 & $1.069 \mathrm{E}-06$ & $5.347 \mathrm{E}-04$ & 3 \\
\hline ZHEHS5 & 3.508E-07 & $4.390 \mathrm{E}-05$ & 1 \\
\hline ZHESV3 & $2.874 \mathrm{E}-07$ & $7.706 \mathrm{E}-05$ & 14 \\
\hline ZHERP2 & $1.709 \mathrm{E}-07$ & 1.711E-05 & 2 \\
\hline ZHEFO6 & 1.153E-07 & $2.882 E-06$ & 7 \\
\hline RESLC3 & $9.360 \mathrm{E}-08$ & $1.085 \mathrm{E}-06$ & 9 \\
\hline ZHEAW4 & $8.748 E-08$ & $1.750 \mathrm{E}-05$ & 5 \\
\hline ZHEOB2 & $5.587 \mathrm{E}-08$ & $6.983 \mathrm{E}-07$ & 4 \\
\hline ZHEAW3 & $2.584 \mathrm{E}-08$ & $3.230 \mathrm{E}-06$ & 5 \\
\hline ZHESW1 & $2.236 \mathrm{E}-08$ & $6.315 \mathrm{E}-06$ & 3 \\
\hline ZHERE2 & $2.018 \mathrm{E}-08$ & $3.800 \mathrm{E}-06$ & 4 \\
\hline REAC12 & 2.925E-09 & $1.373 \mathrm{E}-08$ & 5 \\
\hline
\end{tabular}

Note: A ne ive Bimbaum insportance indicates that the complement of the even tominant in the overall CDF expression. 


\begin{tabular}{|c|c|c|c|c|c|c|c|c|c|c|}
\hline \multirow[b]{2}{*}{ Syateom/operator Aetion } & \multirow{2}{*}{ 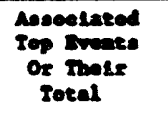 } & \multirow{2}{*}{$\operatorname{lom}_{3 p=0.10-02}^{\infty}$} & \multirow[b]{2}{*}{$\begin{array}{l}m x, 28-1.20 \\
\pi, 2 \pi=1.03\end{array}$} & \multicolumn{4}{|c|}{ 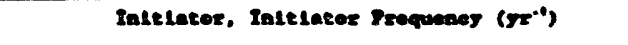 } & \multirow{2}{*}{ 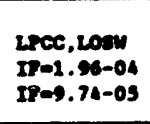 } & \multirow[b]{2}{*}{$\operatorname{mow}_{\mathrm{TP}-2.79-02}$} & \multirow{2}{*}{$\begin{array}{c}200 \\
\text { In-5.53-03 }\end{array}$} \\
\hline & & & & $x_{n=1.49-02}$ & 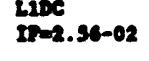 & 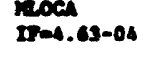 & $\ln _{-1.21-02}^{\cos }$ & & & \\
\hline 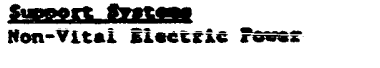 & 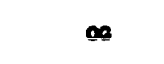 & Inleleator & $3.782-07$ & 2.927-97 & $\cdots$ & $\cdots$ & -.- & $\cdots$ & $\cdots$ & $\cdots$ \\
\hline 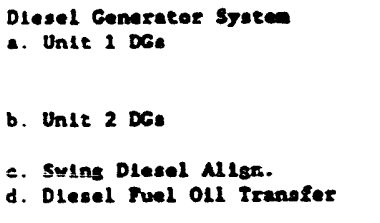 & 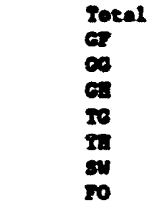 & 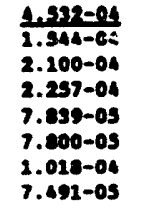 & 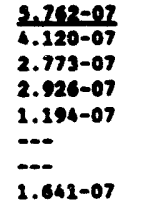 & 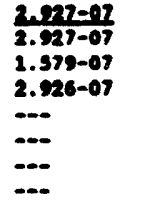 & $\begin{array}{l}\cdots \\
\cdots \\
\cdots \\
\cdots \\
\cdots\end{array}$ & $\begin{array}{l}\cdots \\
\cdots \\
\cdots \\
\cdots \\
\cdots\end{array}$ & $\begin{array}{l}\cdots \\
\cdots \\
\cdots \\
\cdots \\
\cdots\end{array}$ & $\begin{array}{l}\cdots \\
\cdots \\
\cdots \\
\cdots \\
\cdots\end{array}$ & $\begin{array}{l}\cdots \\
\cdots \\
\cdots \\
\cdots \\
\cdots\end{array}$ & $\begin{array}{l}\cdots \\
\cdots \\
\cdots \\
\cdots \\
\cdots\end{array}$ \\
\hline Instroment AC Powar & $\begin{array}{l}\text { Yoreal } \\
11 \\
12 \\
13 \\
14\end{array}$ & $\begin{array}{l}2.004-06 \\
\because-: \\
3.004-06 \\
\cdots-\end{array}$ & $\begin{array}{l}2.282-06 \\
1.097-06 \\
3.943-07 \\
1.097-06 \\
3.493-07\end{array}$ & $\begin{array}{l}2.292-06 \\
1.097-08 \\
2.015-07 \\
1.097-06 \\
3.493-07\end{array}$ & 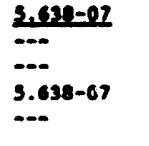 & $\begin{array}{l}\cdots \\
\cdots \\
\cdots \\
\cdots\end{array}$ & $\begin{array}{l}\frac{2.2512-03}{7.498-06} \\
33.756-06 \\
7.498-06 \\
3.756-06\end{array}$ & $\begin{array}{l}\cdots \\
\cdots \\
\cdots \\
\cdots\end{array}$ & $\begin{array}{l}\cdots \\
\cdots \\
\cdots\end{array}$ & $\begin{array}{l}\frac{1.509-04}{3.854-0.5} \\
1.656-0.05 \\
3.854-05 \\
1.665-05\end{array}$ \\
\hline Aurillery Seltwater & as & $2.702=05$ & $\cdots$ & $\cdots$ & 2.476-05 & $\cdots$ & $\cdots$ & Init1ator & $\cdots$ & $\cdots$ \\
\hline Vieal 125V dC Pomor, Unit 1 & $\begin{array}{l}\text { Jors1 } \\
\text { of } \\
\text { De } \\
\text { DE }\end{array}$ & $\begin{array}{l}2.069-09 \\
7.12506 \\
9.009-06 \\
1.290-05\end{array}$ & $\begin{array}{l}\frac{2.245-06}{1.320}-07 \\
4.422-07 \\
1.351-06\end{array}$ & $\begin{array}{l}1.292-08 \\
1.520-07 \\
1.422-07 \\
1.258-06\end{array}$ & \begin{tabular}{l}
$\frac{2.682-04}{3.750-05}$ \\
\hdashline$-107-00$
\end{tabular} & $\begin{array}{l}\cdots \\
\cdots \\
\cdots\end{array}$ & $\begin{array}{l}\frac{1.376-05}{4.597-06} \\
. .397-06 \\
. .571-06\end{array}$ & 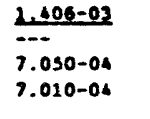 & $\begin{array}{l}\frac{1.692-06}{1.09} \\
4.307-07 \\
1.261-06\end{array}$ & $\begin{array}{l}\frac{1.049-04}{2.037-05} \\
-.230-05 \\
4.246-05\end{array}$ \\
\hline Component coollng Water & $\propto$ & $\$ .274-03$ & $2.023-06$ & $2.015=-06$ & 2.635-05 & $\cdots$ & $\cdots$ & $\cdots$ & 2.2133-03 & $\cdots$ \\
\hline Viteal AC Pover, UnAt 1 & $\begin{array}{l}\text { Totel } \\
\text { NF } \\
\text { NG } \\
\text { AN }\end{array}$ & $\begin{array}{l}\frac{2.035-08}{3.366-06} \\
4.944-06 \\
7.737-06\end{array}$ & \begin{tabular}{l}
$\frac{2.737-07}{1.246-07}$ \\
\hdashline $1.491-07$
\end{tabular} & $\begin{array}{l}\frac{2.827-07}{1.246-07} \\
\because-.581-07\end{array}$ & \begin{tabular}{l}
$\frac{2.667-04}{5.684-05}$ \\
\hdashline $1.097-04$
\end{tabular} & $\begin{array}{l}\cdots \\
\cdots \\
\cdots\end{array}$ & $\begin{array}{l}\cdots \\
\cdots \\
\cdots\end{array}$ & $\begin{array}{l}\cdots \\
\cdots \\
\cdots\end{array}$ & \begin{tabular}{l}
$\frac{0.581-07}{--}$ \\
\hdashline $.581-07$
\end{tabular} & \begin{tabular}{l}
$\frac{6.192-05}{2.000-05}$ \\
\hdashline $.192-05$
\end{tabular} \\
\hline Control Room Ventslaction & cr & $2.092-03$ & $\cdots$ & $\cdots$ & $\cdots$ & $2.699-04$ & $\cdots$ & $\cdots$ & $\cdots$ & $\cdots$ \\
\hline Solld State Protection Syateon & $\begin{array}{l}\text { Toesal } \\
\text { SA } \\
\text { se }\end{array}$ & $\frac{1.056-08}{--0}$ & $\begin{array}{l}\frac{7.269-07}{7.149-07} \\
7.149-07\end{array}$ & $\begin{array}{l}\frac{2.269-07}{7.149-07} \\
7.169-07\end{array}$ & $\begin{array}{l}\frac{2.719-06}{4.434-06} \\
3.285-06\end{array}$ & $\begin{array}{l}\frac{2.620-04}{9.620 .04} \\
9.620-04\end{array}$ & $\begin{array}{l}\cdots \\
\cdots\end{array}$ & $\cdots$ & $\cdots$ & $\cdots$ \\
\hline 480V Sultchgear Ventilat 10n & sv & $2.252-05$ & $3.363-07$ & $2.375=07$ & $\cdots$ & $\cdots$ & $\cdots$ & $\cdots$ & $1.7100-06$ & $\cdots$ \\
\hline Reactor Protection Syatta & RT & $\cdots$ & $3.3212=07$ & $3.521-07$ & $\cdots$ & $\cdots$ & $\cdots$ & $\cdots$ & $\cdots$ & $\cdots$ \\
\hline Vital $A C$ and $D C$ Pover, Unit 2 & $\begin{array}{l}\text { Joenal } \\
\text { or } \\
x \\
\text { in }\end{array}$ & $\begin{array}{l}\frac{2.254-06}{3-2-06} \\
3.037-06 \\
2.318-06\end{array}$ & $\begin{array}{l}\cdots \\
\cdots \\
\cdots\end{array}$ & $\begin{array}{l}\cdots \\
\cdots \\
\cdots\end{array}$ & $\begin{array}{l}\cdots \\
\cdots \\
\cdots\end{array}$ & $\begin{array}{c}\cdots \\
\cdots \\
\cdots\end{array}$ & $\begin{array}{l}\cdots \\
\cdots \\
\cdots\end{array}$ & $\begin{array}{l}\cdots \\
\cdots \\
\cdots\end{array}$ & $\cdots$ & $\begin{array}{l}\cdots \\
\cdots \\
\cdots\end{array}$ \\
\hline 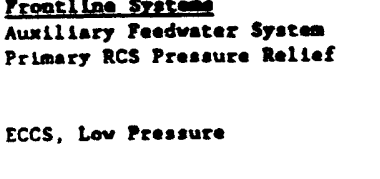 & $\begin{array}{l}\text { Nu } \\
\text { Toten } \\
\text { mo } \\
\text { on } \\
\text { Toteal } \\
\text { un }\end{array}$ & 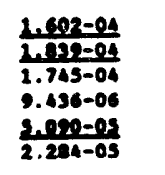 & 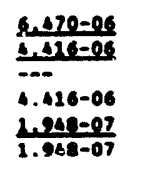 & \begin{tabular}{l}
$\frac{2.394-06}{1.486-06}$ \\
\hdashline $.494-06$ \\
$\frac{1.479-07}{2.773-07}$
\end{tabular} & 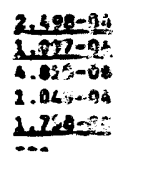 & $\begin{array}{l}\cdots \\
\cdots \\
\cdots \\
\frac{4.240-02}{3.621-03}\end{array}$ & $\begin{array}{l}\cdots \\
\cdots \\
\cdots \\
\cdots .3 \\
\frac{2.34-04}{4.981-05}\end{array}$ & $\begin{array}{l}\cdots \\
\cdots \\
\cdots \\
\cdots\end{array}$ & \begin{tabular}{l}
$\frac{2.637-06}{2.078-06}$ \\
\hdashline $1.078-06$ \\
$\cdots .0$ \\
$\cdots$
\end{tabular} & 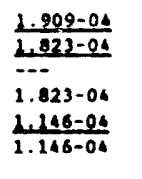 \\
\hline
\end{tabular}




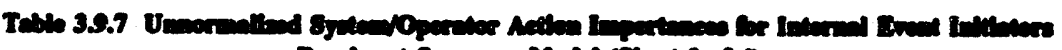

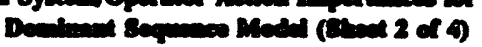

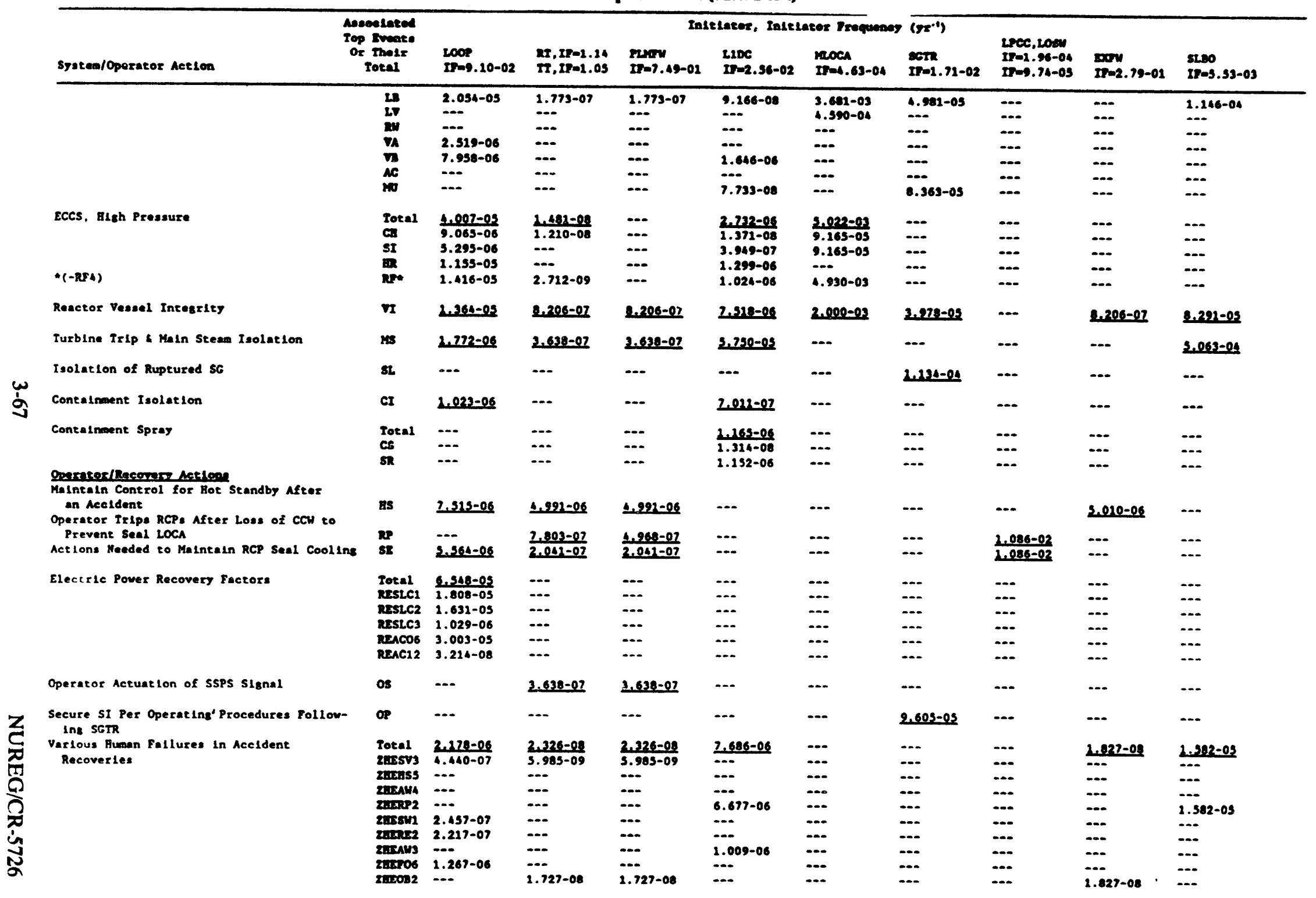




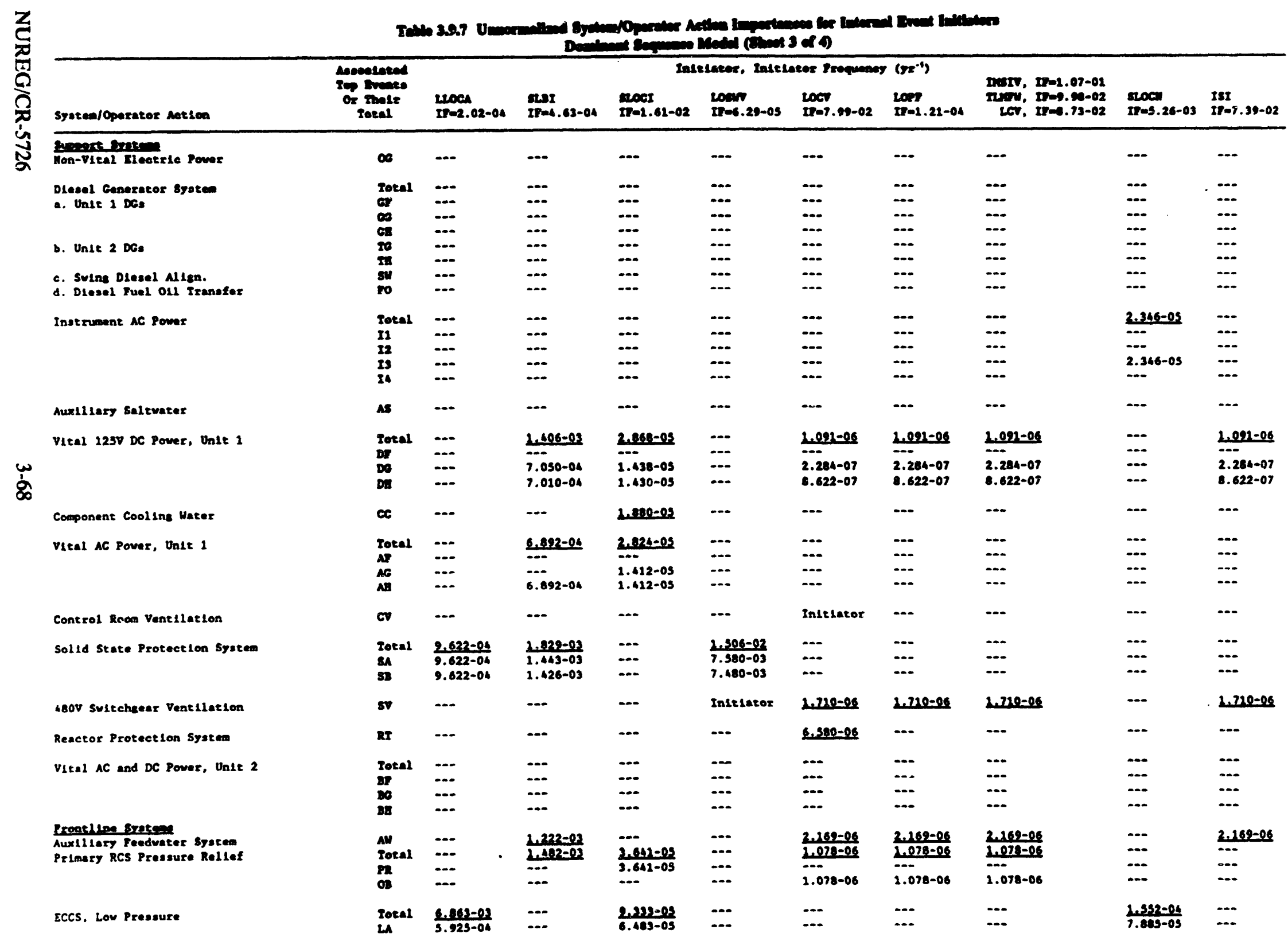




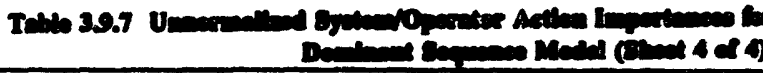

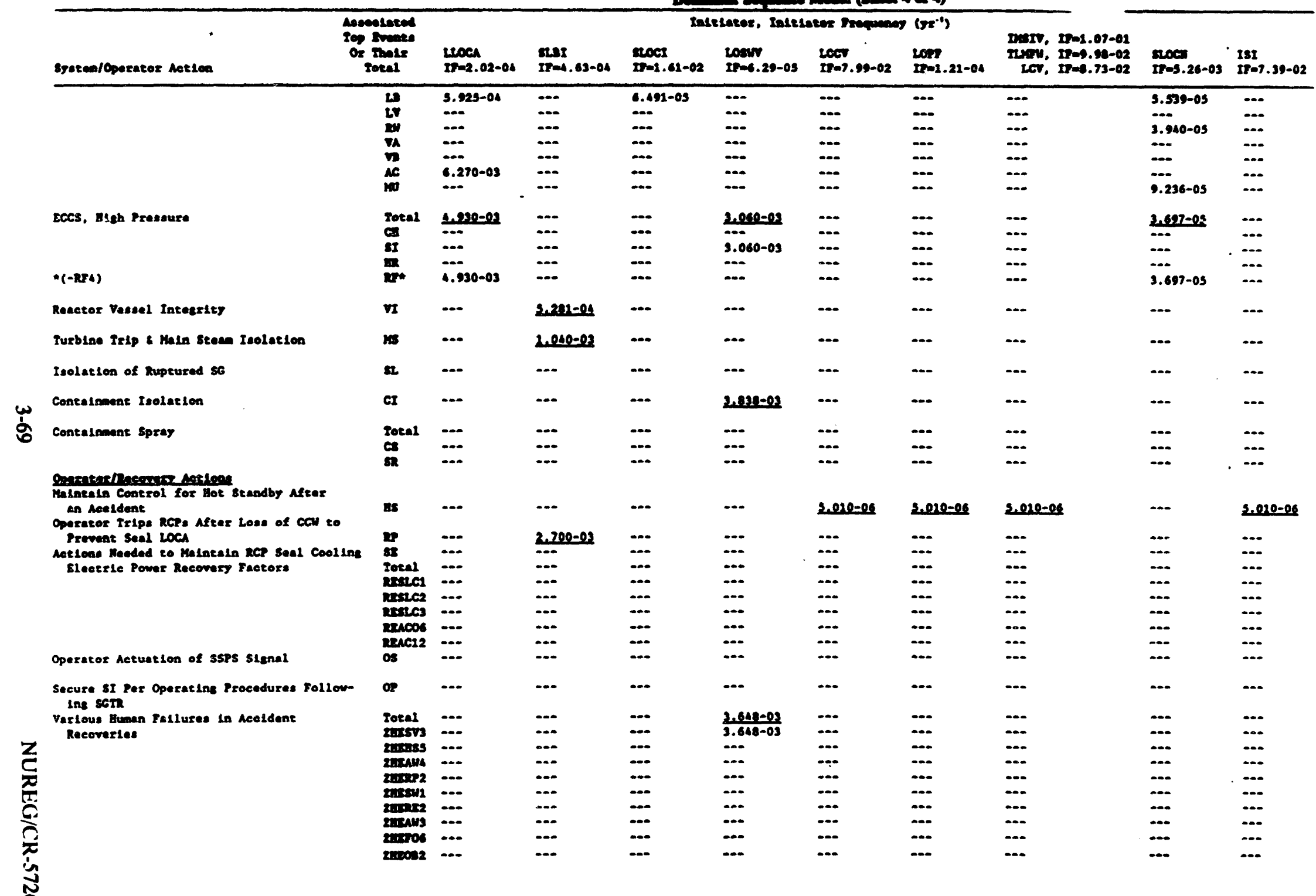




\begin{tabular}{|c|c|c|c|c|c|c|c|c|}
\hline \multirow[b]{3}{*}{ Syatem/Operator Aetion } & \multicolumn{7}{|c|}{ 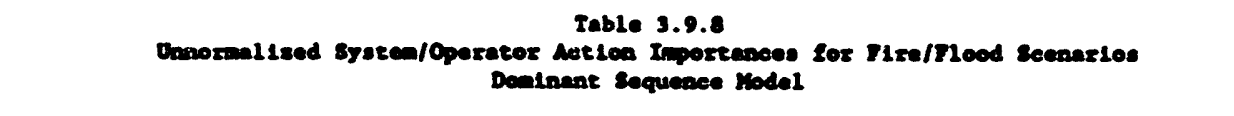 } & \\
\hline & \multirow{2}{*}{ 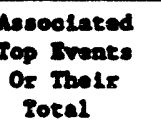 } & \multirow[b]{2}{*}{$\begin{array}{l}\text { TS12 } \\
\text { IF-3.18-04 }\end{array}$} & \multicolumn{5}{|c|}{ Intelatox, Inltiator Prequenes (yz-) } & \\
\hline & & & $\begin{array}{l}\text { Pso } \\
\text { IF=6.18-06 }\end{array}$ & $\begin{array}{l}\text { Fs1 } \\
\text { Ir-2.24-04 }\end{array}$ & $\begin{array}{l}786 \\
27-2.42-05\end{array}$ & $\underbrace{}_{I P-1.35-05}$ & $\begin{array}{l}\text { TrS } \\
\text { IT-5.26-05 }\end{array}$ & $\begin{array}{l}\text { PS10 } \\
\text { IY }=1.40-05\end{array}$ \\
\hline Suppors Syateme & & - & & & & & & \\
\hline Auxillary Seltvater & As & $\cdots$ & $\cdots$ & $\cdots$ & $2.6293-02$ & $\cdots$ & $\cdots$ & $\cdots$ \\
\hline VItal 225V DC Pover, Unit 1 & Iotal & 1.406-03 & $\cdots$ & 2.028-04 & $\cdots$ & -- & $\cdots$ & -.. \\
\hline & DE & $7.050-04$ & $\cdots$ & $7.050-04$ & $\cdots$ & $\ldots$ & $\cdots$ & $\ldots$ \\
\hline & DE & $7.010-04$ & $\cdots$ & $9.884-05$ & $\cdots$ & $\cdots$ & $\cdots$ & -.. \\
\hline Component cooling Water & cc & $\cdots$ & -- & $\cdots$ & $\underline{2.859-02}$ & $\cdots$ & $\cdots$ & $\cdots$ \\
\hline \multicolumn{9}{|l|}{ Erentline Systems } \\
\hline Auxhllary Feeduater Syatem & Aw & $\cdots$ & $\cdots$ & $\underline{1.296-03}$ & $\cdots$ & Inteleter: & --. & $2.095-03$ \\
\hline Primaiy RCS Pressure Rellef & Total & $\cdots$ & $\frac{6,880-02}{4.880-02}$ & $2.271-03$ & $\ldots$ & $2.890-02$ & $\cdots$ & $\underline{2.095-03}$ \\
\hline & 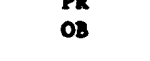 & $\cdots$ & & $2.171-03$ & $\cdots$ & $2.890-02$ & $\cdots$ & $2.095-03$ \\
\hline ECCS, Low Pressure & Tota1 & $\ldots$ & 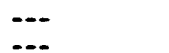 & $\frac{4.200-04}{3.874-04}$ & $\cdots$ & -- & 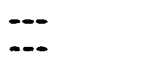 & 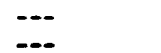 \\
\hline & LB & $\cdots$ & $\cdots$ & $3.773-06$ & $\cdots$ & $\cdots$ & $\cdots$ & $\cdots$ \\
\hline Reactor Vessel Integrity & vI & $\cdots$ & $\cdots$ & $1.682-03$ & $\cdots$ & 2.890-02 & --- & --- \\
\hline \multicolumn{9}{|l|}{ Operator Recovesy Aestions } \\
\hline $\begin{array}{l}\text { Operator Irlps RCPs After Loss of CCW to } \\
\text { Prevent Seal Loce }\end{array}$ & $\mathbf{R P}$ & $2.086-02$ & -.- & $\cdots$ & $4.562-02$ & $\cdots$ & $1.086-02$ & $\cdots$ \\
\hline Action Meeded to Malntaln RCP Seal Cooling & SE & 1.086-02 & $\cdots$ & $\cdots$ & $\cdots$ & $\cdots$ & $1.086-02$ & $\cdots$ \\
\hline
\end{tabular}


Iable 3.9.9

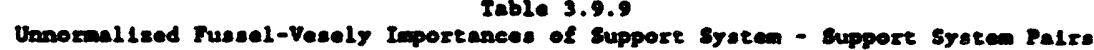

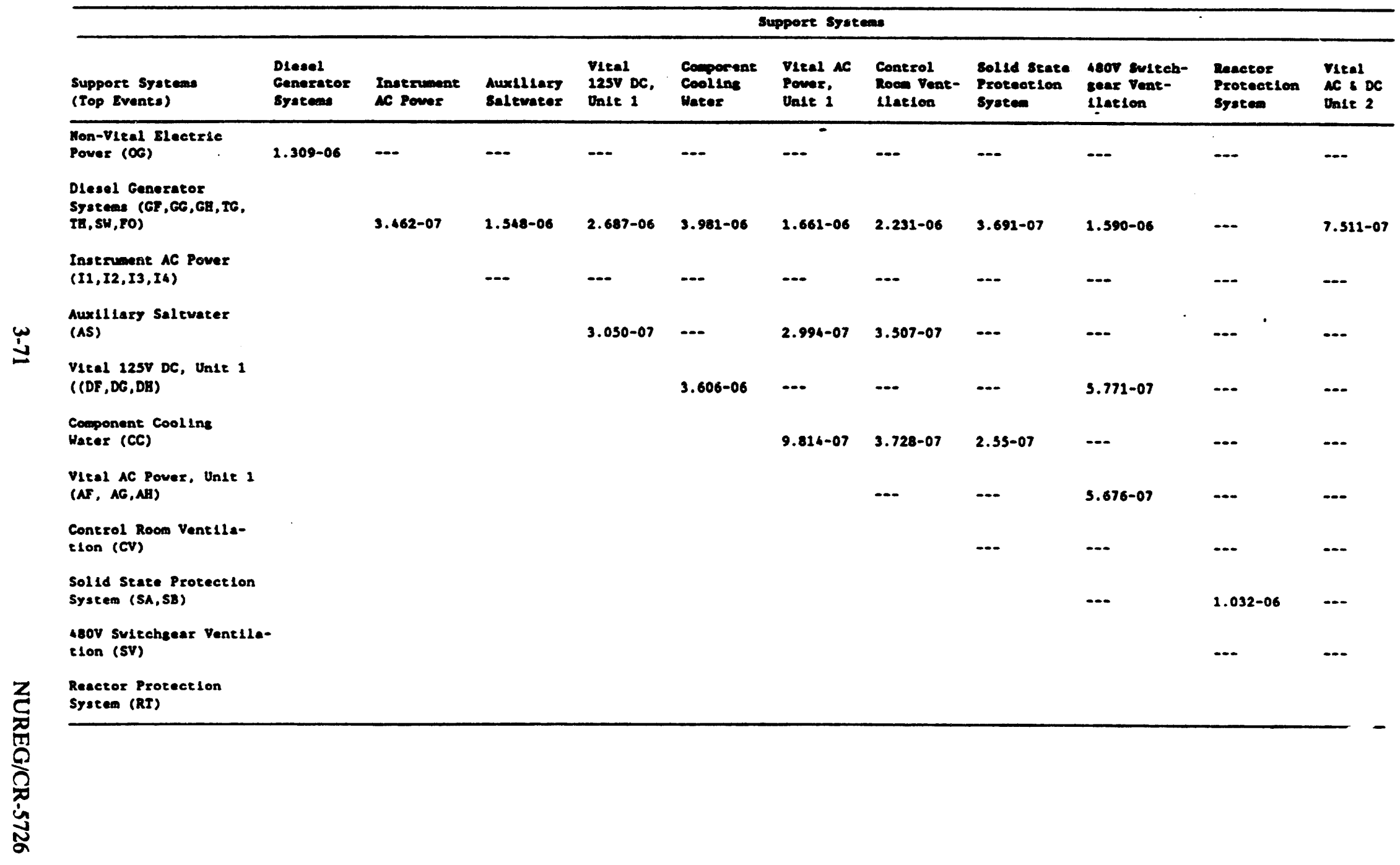




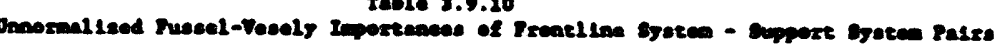

enporte system (Iop Dreate)

\begin{tabular}{|c|c|c|c|c|c|c|c|c|c|c|c|c|}
\hline $\begin{array}{l}\text { Frontl lae syatems } \\
\text { (Top Events) }\end{array}$ & 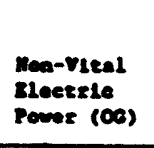 & 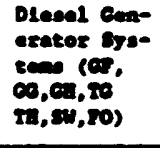 & $\begin{array}{l}\text { Inetru- } \\
\text { Pent AC } \\
\text { Power (II, } \\
\text { I2,I3,IA) }\end{array}$ & $\begin{array}{l}\text { Muselliars } \\
\text { Seltwates } \\
\text { (AS) }\end{array}$ & $\begin{array}{l}\text { Venel } 2280 \\
\text { DC, Dale } 1 \\
\text { (De, De, Dan) }\end{array}$ & $\begin{array}{l}\text { Componest } \\
\text { Cooilses } \\
\text { Water (C) }\end{array}$ & 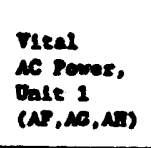 & $\begin{array}{l}\text { Control } \\
\text { Roen } \\
\text { Ventlln- } \\
\text { elea (CV) }\end{array}$ & 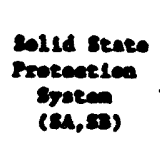 & $\begin{array}{l}\text { seop } \\
\text { sencehenar } \\
\text { vemelie- } \\
\text { tien (sv) }\end{array}$ & $\begin{array}{l}\text { Vital } \\
\text { Rowetor } \\
\text { Proceetion } \\
\text { Spated (RT) }\end{array}$ & $\begin{array}{l}\mathcal{A C}: D C \\
\text { Dast } 2 \\
(\boldsymbol{N}, 26,2 \mathrm{n})\end{array}$ \\
\hline $\begin{array}{l}\text { Auxillexy Feodvater } \\
\text { Systes (AW) }\end{array}$ & $3.960-07$ & 1.487-03 & $9.690-06$ & $\cdots$ & $0.450-06$ & $9.120-07$ & $4.396-06$ & $6.367-07$ & $1.439-07$ & $4.330-07$ & $\cdots$ & $4.218-07$ \\
\hline $\begin{array}{l}\text { Primary Res Pressure } \\
\text { Rellef (PR, 03) }\end{array}$ & $\cdots$ & $1.674-05$ & $1.057-05$ & $4.453-07$ & $1.617-06$ & $1.782-06$ & $1.714-06$ & $8.419-07$ & $3.204-07$ & $\cdots$ & $\cdots$ & $\cdots$ \\
\hline 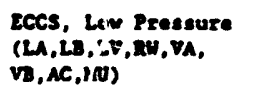 & $\cdots$ & $4.632-06$ & $1.234-07$ & $\cdots$ & $4.618-07$ & $\cdots$ & $4.929-07$ & $2.305-07$ & $\cdots$ & $\cdots$ & $\because=$ & $\cdots$ \\
\hline $\begin{array}{l}\text { ECCs, High Presesure } \\
\text { (CH, S1, RR, RF") }\end{array}$ & $\cdots$ & $3.647-06$ & $\cdots$ & $\cdots$ & $\cdots$ & $\cdots$ & $3.263-08$ & $\cdots$ & $\cdots$ & 2.465-07 & $\cdots$ & $\cdots$ \\
\hline $\begin{array}{l}\text { Reactor Vessel } \\
\text { Inceertey (VI) }\end{array}$ & $\cdots$ & $1.241-06$ & $2.798-07$ & $\cdots$ & $\cdots$ & $\cdots$ & $\cdots$ & $\cdots$ & $\cdots$ & $\cdots$ & $\cdots$ & $\cdots$ \\
\hline $\begin{array}{l}\text { Iurbine Irlp C Maln } \\
\text { Stean Iaolation (HS) }\end{array}$ & $\cdots$ & 2.612-07 & 8.315-07 & $3.030-07$ & $2.214-06$ & $3.180-07$ & $3.424-07$ & $\cdots$ & $1.351-06$ & $\cdots$ & $\cdots$ & $\cdots$ \\
\hline $\begin{array}{l}\text { Isolnetion of Ruptured } \\
\text { SG (SL) }\end{array}$ & $\cdots$ & $\cdots$ & $3.849-07$ & $\cdots$ & $2.354-07$ & $\cdots$ & $\cdots$ & $\cdots$ & $\cdots$ & $\cdots$ & $\cdots$ & $\cdots$ \\
\hline $\begin{array}{l}\text { Coneatinent toolation } \\
\text { (CI) }\end{array}$ & $\ldots$ & 9.309-08 & $\cdots$ & $\cdots$ & $\cdots$ & $\cdots$ & $\cdots$ & $\cdots$ & $\cdots$ & $\cdots$ & $\cdots$ & $\cdots$ \\
\hline $\begin{array}{l}\text { Conealnawent Spray } \\
\text { (CS, SR) }\end{array}$ & $\cdots$ & $\cdots$ & $\cdots$ & $\cdots$ & $\cdots$ & $\cdots$ & $\cdots$ & $\cdots$ & $\cdots$ & $\cdots$ & $\cdots$ & $\cdots$ \\
\hline $\begin{array}{l}\text { Interfac Lng LOCA } \\
\text { Event Tree Top Events } \\
\text { (vo, VC, VR, SH, IT, LH, } \\
\text { ME) }\end{array}$ & $\cdots$ & $\cdots$ & $\cdots$ & $\cdots$ & $\cdots$ & $\cdots$ & $\cdots$ & & & & & \\
\hline
\end{tabular}




\subsection{Summary and Analysis of Review Results}

Alternative quanatification has been offered throughont this report on a multitude of individual itemafiscues as a reant of the internal ovents review. This section collocts these individual itoms and incorporates them into a set of integrated CDF calculations.

Table 3.10.1 lins each of the items within the DCPRA internal events review for which alterantive quantification bas been offered and any significant differesce has been noted with the originalhupdated DCPRA. Table 3.10.1 aloo Hats the original values provided by PGEE (as used in BNL's calculations in Section 3.9) as well as the BNL alternative values used in senditivity extimates throughout the report.

Table 3.10.2 provides the results of a set of overall intermal eveat CDF calculations in an attempt to place the internal ovents review reents into an integratsd and proper perapective. Case 1 in the table is simply the solution to the orifinal DSM as described in Siction 3.9 (minus the external events). Case 2 represents Case 1 phes the throe updated initiators provided by PGEE as a reente of this reviow and it is our belief that this escentially represents PO\&E's updated DCPRA internal ovent recults. Case 3 represents Case 2 plus the mubetitution of the BNL eplit frection quantification from Table 3.10.1. Cace 4 represents Case 3 plus the mubetitution of the BNL value for LOOP. Case 5 represents Case 4 plus the BNL values for LOSW, LPCC and SLOCN. Came 6 reprecents the subatitution of all alternativa BNL values from Table 3.10.1 into the DSM.

BNL belioves that Case 4 represents the mont reascasable set of alternative quantification of those offered in Table 3.10.2. Case 2 is simply the updated POEE results and Case 3 does not include the solar magnetic norm contribution ideatified by BNL for the LOOP initiator as does Case 4. (Note: Following the completion of this atudy bet prior to the ismance of this report, Diablo Canyon experienced its fira LOOP ovent. Although not cansed by a solar magnetic storm, it further subatantiates the use of a higher LOOP initiating froquency.) Cases 5 and 6 are dominated by initiator frequenciea that are beavily dependent on bagterm indurary-wide experiesce. Baced upon Diablo Canyon's domonstrated ability to avoid plant transients as well as the industry trend to lower numbers of trasieat events per year, these cases are felt to be overty peasimiatic for formulating a beat eatimate type of value.

It should aleo be noted that the BNL CSF's include the removal of HS1 (HS1 $=0.0$ ) from the model as it is believed to be 100 concervative in the DCPRA. HS1 represents the complete failure of the operating atafi to maintain the plant in a safe bot shutdown condition following a trandient (predominantly RT and TT) with no other failures present.

The breakdown of initiator importance for case $4 \mathrm{can}$ be found in Table 3.10.3. This is an equivalent table to that of Table 3.9.1a (which is besed upon the original DSM/input data) minus the non-seismic external cvents. Case 2 is abo included in Table 3.10.3 a this represents a truer comparison between the updated DCPRA and BNL's alternative eet of quantification. (The updated input-j.e., three new initiator frequencies from PG\&E came after the importance calculations on the DSM were completed and therefore those calculations were not repeated. The changes, however, do not represent a significantly different view of the Diablo Canyon riak profile).

Table 3.10.4 covers Case 4 (the analog of Table 3.9.5 for Case 1) and provides the associated ranking of the syatem/operator action importances scoording to the semumptions of this case. It is interesting to note that for the sppport sydems, the largen increases in importance ranking came from the Diesel Generator Synteme, the Auriliary Saltwater Syatem and the Solid State Protection Syptem. For the frontline syatems, the bigest change was that the Auxiliary Feedwater Syatem and the Primary RCS Prescure Relief System (ranked 1 and 
2 in the DCPRA) wechanged pheces and are now ranked 2 and 1 respectively. And, mont intereatiag of all is the inportasce rauling within the operator/hecovery setions. For the case 4 analynis, the beading importance contributor (operator activation of SSPS) was significantly los important in Case 1 and the least important operator sction in Case 4 (fillure to meintain hot standby) was the leading contributor in case 1. This cementialiy represents a full reverial of these roles.

The above shorative results are offered as an additional set of seasitivity atudies that may provide further instigh to the future userefreviewer.

In enmmary, the internal ovents level 1 portion of the DCPRA represents a truly comprehensive and detailed effort. It has withetood dotalled scrutiay and is coneldored to represent a firm and reasonable basis for input into PGaE's Long Term Selsmic Program. 
Twhes 3.10 .1

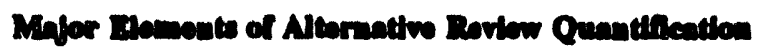

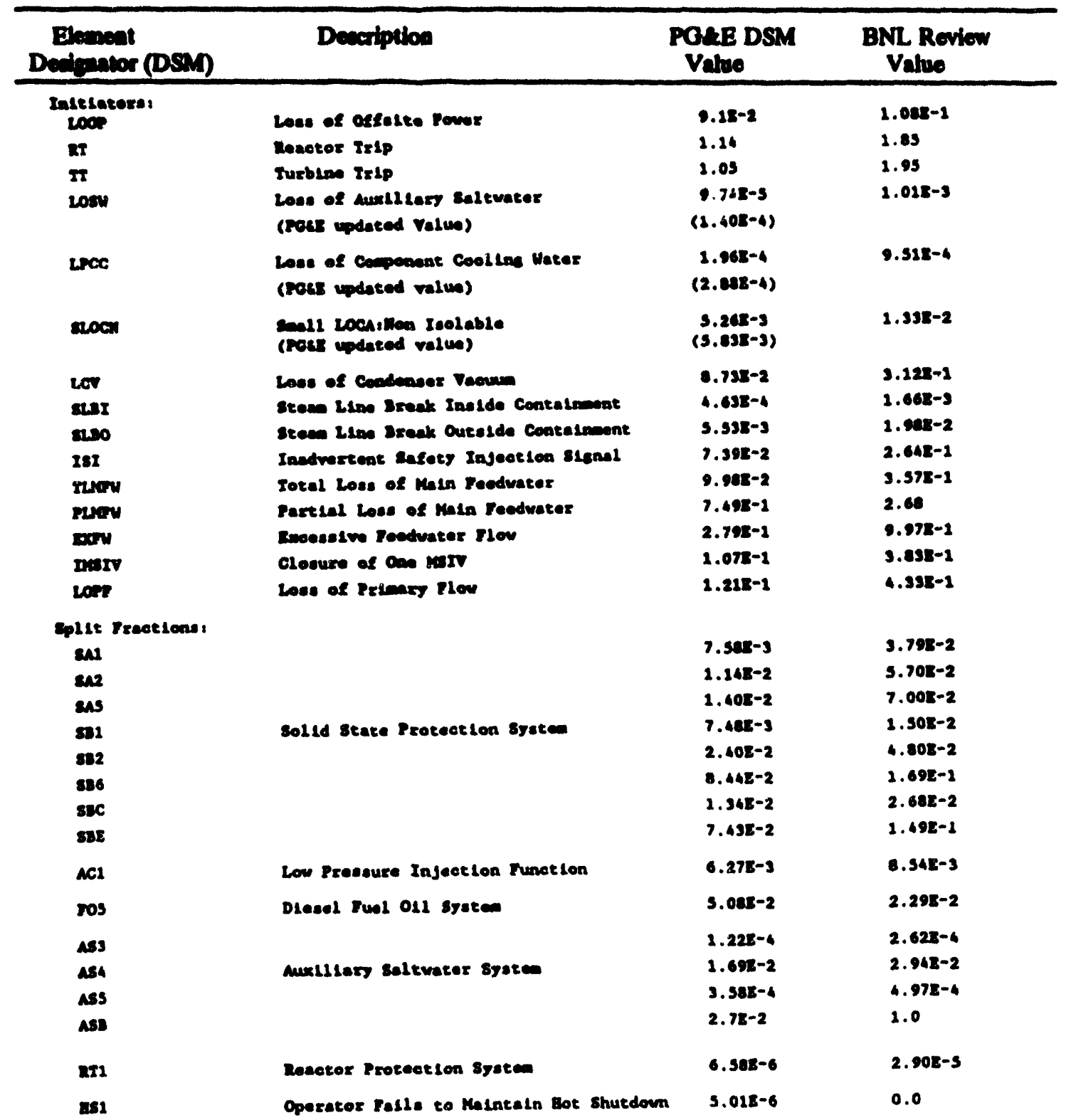


Twable 3.102

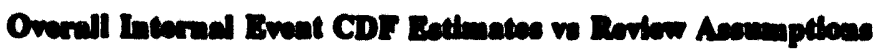

\begin{tabular}{|c|c|c|}
\hline Cane & Description & Point Entimate CDF $\left(\mathrm{rr}^{-1}\right)$ \\
\hline 1. & Orifinal POQE input for the DSM & $1.3 \mathrm{E}-4$ \\
\hline 2. & $\begin{array}{l}\text { Cave } 1 \text { + PGEE updated initiator } \\
\text { saquesciee (Tablo 3.10.1) }\end{array}$ & $1.3 \mathrm{E}-4$ \\
\hline 3. & Cane 2 + BNL CSPa & $2.0 \mathrm{E}-4$ \\
\hline 4. & Case 3 + BNLLLOP & $2.1 E-4$ \\
\hline s. & $\begin{array}{l}\text { Caw 4 + BNL values for } \\
\text { LOSW, LPCC and SLOCN }\end{array}$ & 2.4E-4 \\
\hline 6. & All BNL valuce from Table 3.10.1 & $3.3 E-4$ \\
\hline
\end{tabular}

Noto: Polat catimate reanlas presented in the drat verion of this report formed the bacti for the stafrs SER finding (NURBO-0675, Supplement No. 34) and were based on a alightly

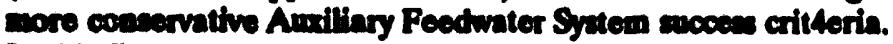
If this final verioan, this conservatien has beea removed. Overall conctudions are eacentially uschanged.) 
3 Roview of the Intereal Eveats Asalyais

Twabo 3.10 .3

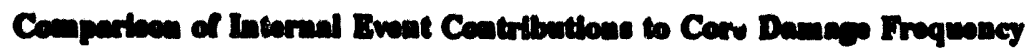

(Raaking of Top Tea Internal Event Contributors)

\begin{tabular}{|c|c|c|c|}
\hline \multicolumn{2}{|c|}{ Updated DSM - Cace 2 (POEE) } & \multicolumn{2}{|c|}{$\begin{array}{l}\text { Cano } 4 \\
\text { (DNL) }\end{array}$} \\
\hline Initiator & $\begin{array}{l}\text { Funol-Vecely } \\
\text { Importance } \\
\text { (\%) }\end{array}$ & Initiator & $\begin{array}{c}\text { Fuseel- } \\
\text { Vesely } \\
\text { Importance } \\
(\%) \\
\end{array}$ \\
\hline LOOP & 32 & LOOP & so \\
\hline $\mathbf{R T}$ & 12 & $\mathbf{R T}$ & 8 \\
\hline $\mathbf{T T}$ & 11 & $\mathbf{T T}$ & 8 \\
\hline PLMFW & 8 & PLMFW & 6 \\
\hline LIDC & 7 & LIDC & 5 \\
\hline MLOCA & 4 & MLOCA & 5 \\
\hline LPOC & 3 & SLBI & 4 \\
\hline $\operatorname{soTR}$ & 2 & LLCA & 2 \\
\hline EXPW & 2 & LPOC & 2 \\
\hline SIBO & 2 & LoswV & 2 \\
\hline
\end{tabular}


Table 3.10.4A

RANKING OF SUPPORT SYSTEMS IMPORTANCE

INTERNAL EVENTS - CASE 4

\begin{tabular}{|l|c|}
\hline \multicolumn{1}{|c|}{ Support System } & $\begin{array}{c}\text { System Importance } \\
(\%)\end{array}$ \\
\hline 1. Diesel Generator Systems & 51 \\
\hline 2. Auxiliary Saltwater & 29 \\
\hline 3. Solid State Protection System & 18 \\
\hline 4. Control Room Ventilation & 3 \\
\hline 5. Vital 125V DC Power, Unit 1 & 8 \\
\hline 6. Reactor Protection System & 6 \\
\hline 7. Instrument AC Power & 5 \\
\hline 8. Component Cooling Water & 5 \\
\hline 9. Vital AC Power, Unit 1 & 4 \\
\hline 10. 480V Switchgear Ventilation & 2 \\
\hline 11. Non-Vital Electric Power & 1 \\
\hline 12. Vital AC\&DC Power, Unit 2 & 0.4 \\
\hline
\end{tabular}


Table 3.10.4B

RANKING OF FRONT-LINE SYSTEMS IMPORTANCE

INTERNAL EVENTS - CASE 4

\begin{tabular}{|l||l||}
\hline \multicolumn{1}{|c||}{ Frontline System } & \multicolumn{1}{|c|}{$\begin{array}{c}\text { System } \\
\text { Importance } \\
\%\end{array}$} \\
\hline i. Primary RCS Pressure Relief & 28 \\
\hline 2. Auxiliary Feedwacer & 22 \\
\hline $\begin{array}{l}\text { 3. Turbine Trip and Main Steam } \\
\text { Isolation }\end{array}$ & 9 \\
\hline 4. ECCS, Low Pressure & 7 \\
\hline 5. ECCS, High Pressure & 4 \\
\hline 6. Reactor Vessel Integrity After PTS & 3 \\
\hline 7. Isolation of Ruptured Steam \\
\hline Generator
\end{tabular}


Table 3.10.4C

RANKING OI OPERATOR/RECOVERY ACTIONS IMPORTANCE

INTERNAL EVENTS - CASE 4

\begin{tabular}{|l|c|}
\hline \multicolumn{1}{|c|}{ Operator/Recovery Actions } & \multicolumn{1}{|c|}{$\begin{array}{c}\text { Action } \\
\text { Importance } \\
(\%)\end{array}$} \\
\hline Operator Fails to Actuate SSPS Signal & 5 \\
\hline $\begin{array}{l}\text { Failure to Recover 1 of } 3 \text { DGs with AFW } \\
\text { Available }\end{array}$ & 4.6 \\
\hline $\begin{array}{l}\text { Operator Fails to Trip RCPs After Loss of CCW } \\
\text { to Prevent Seal LOCA }\end{array}$ & 4.1 \\
\hline $\begin{array}{l}\text { Failure to Take Actions Needed to Maintain RCP } \\
\text { Seal Cooling }\end{array}$ & 2.8 \\
\hline Failure to Recover Normal Power in 6 Hours & 1.5 \\
\hline $\begin{array}{l}\text { Failure to Recover 1 of 3 DGs with AFW } \\
\text { Unavailable }\end{array}$ & 0.9 \\
\hline $\begin{array}{l}\text { Failure to Secure SI Per Operating Procedures } \\
\text { Following SGTR }\end{array}$ & 0.8 \\
\hline $\begin{array}{l}\bullet \\
\bullet\end{array}$ & \\
\hline $\begin{array}{l}\text { Failure to Maintain Control for Hot Standby After } \\
\text { a Reactor Trip }\end{array}$ & $($ neg.) \\
\hline
\end{tabular}




\section{REVIEW OF SEISMIC PRA}

\subsection{Introduction}

This section presents the results of a detailed review of the Seismic Probabilistic Risk Assessment portion of the DCPRA, as contained in Section 6 of the LTSP document. The necessity for and importance of a detailed review of the soismic PRA is discussed in Section 1 of this report. In the following, the major steps in the seismic PRA, namely:

a. Hazard Curve Development

b. Fragility Development

c. Systems Analysis (Event and Fault Trees)

d. Risk Integration and Uncertainty Analysis

are discussed separately in Sections 4.4 through 4.7. One unusual feature of the PRA is the use of average spectral acceleration rather than peak ground acceleration as the independent parameter for the hazard curve, fragilities and responses. This raises a number of interface questions which are discussed explicitly in Section 4.5.

\subsection{Detailed Description of DCPRA Approach Used in the Seismic Analysis}

In general, the PL\&G approach for seismic PRA is to develop a so called "frontline seismic event tree" which has top events which model both building failures and failures of essential safety systems. The single initiating event is the earthquake. Definitions of the top events in terms of Boolean expressions for component failures are developed in the report. These frontline seismic accident sequences either (a) lead directly to core damage with seismic failures only (b) require the addition of random failures for certain systems (as obtained from the internal events analysis) to create the conditions for core damage or (c) are "ok" sequences. In addition, each accident sequence on the frontline seismic is mapped to an end state which is used to determine the configuration of support systems. For example,

sequences are usually noted which lead to one of the following: (a) no seismic impact on support states (b) extended loss of offsite power (c) loss of all AC power, etc.

In order to quantify the accident sequences, both random and seismic failure probabilities were determined. In general, the seismic failure probabilities were developed by using the so-called "factor of safety" or "separation of variables" approach in which the median level of failure is characterized by the design capacity and the margin of safety associated with each aspect of the design calculation. Uncertainties (both random and systematic) are derived from data or estimated for each safety margin factor and combined assuming a log-normal model of conditional failure probability.

In addition to structural seismic failures of components and buildings, functional failure of electro-mechanical equipment due to relay chatter or circuit breaker trip (caused by the seismic shaking) were also considered. An extensive effort to identify all relays or breakers which were susceptible to ground motion (and which are also important to safety) was made as part of the DCPRA.

As part of developing the fragility functions for both components and structures, the response of each important structure (in terms of floor response spectra for each floor slab and shear and moment loads in load-bearing wall) must be determined. In the DCPRA, a detailed building response analysis was performed for the auxiliary building using dynamic structural models and suites of recorded earthquake (and artificial 


\section{Review of Soismic PRA}

earthquakes) time histories. This analysis was compared with the design analysis results, and the results of the comparison were used to scale the design responses of other structures important to the PRA, and provide appropriate measures of uncertainty for all required responses.

In addition to the seismically-induced failures, both random and human error failure probabilities were included in the seismic sequences. Random equipment failure rates were taken directly from the internal events portion of the PRA. Judgement was used to specify human error rates as a function of earthquake level.

In general, PLAG uses a two step approach to quantifying risk, a point estimate calculation of the mean core damage frequency followed by an uncertainty analysis. In the first step, a mean point estimate is computed using a mean hazard curve and mean values of the conditional component failures. In general, a family of hazard curves are developed and each hazard curve is associated with a judgmental weight. The numerical values of the family of hazard curves and their associated weights are presented in tabular format. From this table one can compute the ordinates of the mean hazard curve, taking into account the assigned weighing. $A$ number of increments are then defined, in this case six increments starting at $0.20 \mathrm{~g}$ and extending up to 4.0g. These increments or levels are used to report intermediate results. Typically, they use small intervals for low values of acceleration and fairly large intervals for higher values of acceleration.

A table of conditional mean failure fractions is presented for each component having a seismic failure mode. This table presents the mean conditional failure fractions for each interval of average spectral acceleration, such that this mean fraction - when multiplied by the incremental hazard curve frequency of that interval gives the correct total failure frequency for that component over the interval. It is computed by use of a code in which each acceleration interval is further subdivided into increments (either $.01 \mathrm{~g}$ or $0.5 \mathrm{~g}$ ) and then the failure fractions are computed at each sub-point in the interval and weighted by the hazard curve frequency. They are finally normalized to the numerical value of the hazard frequency in the increment. The net result is that the mean failure fraction, when multiplied by the reported increment frequency does yield the correct total failure frequency for that increment. This is done so that in the second stage analysis (the uncertainty analysis using Discrete Probability Distribution (DPD) arithmetic techniques as discussed later in this section) these incremental values can be used.

The accident sequences are evaluated by multiplying each Boolean expression for the top events in the accident sequence together, using the mean component failure fractions to evaluate the Boolean expressions. Correlation (other than $\mathbf{0 . 0}$ or $\mathbf{1 . 0}$ ) is not considered in this process. Finally, each accident sequence is multiplied by the incremental hazard curve frequency for that increment. A table is provided which gives the conditional mean values for the top events as computed from the Boolean definition and including the hazard curve values. This table is useful in that it usually shows the relative contributions of the components within each Boolean expression to the total and it is usually found that (at most) one or two components essentially dominate each Boolean expression. This is useful in interpreting the final results, in performing sensitivity studies, and in identifying critical components whose fragilities should be given special scrutiny.

Comple ment events are added to the accident sequences both in the reduced model for uncertainty analysis and in the point estimate accident sequences. (If neglected, this leads to unnecessary conservatism in the results.) In addition, random failures are added to the dominant accident sequences as required.

As the second step in their seismic PRA process, an uncertainty analysis is performed. The PL\&G approach makes use of a discrete probability distribution (DPD) arithmetic scheme which involves developing pairs of acceleration and probability frequency points and then combining them using DPD arithmetic. This approach 
is relatively laborious and requires considerable intermediate aggregation of groups of components at each stage of the process. This aggregation may lead to some uncertainty in the final result. In general, because their accident sequence and support state process develops many accident sequences (on the order of 2000-4000), PLQG does not use the full set of accident sequences to evaluate the uncertainty. Instead, using engineering judgement, they develop a subset of accident sequences. This is a fairly simple Boolean model involving perhaps only 20 to 30 components which models the dominant accident sequences for the entire plant. It is this model on which the uncertainty analysis is performed, rather than the more complete set of accident sequences derived from the frontline, mechanical support, and electrical support trees used in the point estimate calculation.

\subsection{Detalled Description of Review Approach}

The detailed review of the seismic PRA was performed by several different teams which focussed on the natural divisions of the PRA, namely,

a. Hazard Curve Development

b. Component and Structures Fragility Development

c. Systems Analysis (Event and Fault Trees)

d. Riak Integration and Uncertainty Analysis

The scope and objectives of the reviews of each of these areas are described below.

\section{(1) Review of the Hazard Curve}

The review of the development of the hazard curve was performed by the NRC staff. The family of hazard curves provided by PG\&E was examined for the basic methodology used to develop the curves, the bgical decision trees used to incorporate various assumptions as to types of faults, etc. and for appropriateness of basic ground motion data utilized in this process.

In addition, the hazard curves being developed are being keyed to spectral acceleration, rather than peak ground acceleration as has been done in the past. Implications of this new approach were examined.

(2) Review of Fragilities Development

Two separate aspects of the fragility development were considered - equipment fragility and building structural fragility. To perform this review, an initial two-day plant visit was required. In addition, copies of all supporting documentation (in particular, the engineering calculation sheets - usually handwritten) for all fragilities were made available. After preliminary review of the documentation, a meeting between the reviewers and the fragility development personnel was scheduled to allow discussion of the details of the fragility calculations.

A vital aspect of developing both component and building fragilities is the defendable determination of all floor slab special accelerations and all load-bearing wall shear and moment forces. This important aspect was reviewed by a) duplicating the DCPRA analysis of the auxiliary building using the same input and models as in the DCPRA (but different structural dynamic computer codes) and b) performing the analysis using alternate input assumptions. 


\section{Review of Seismic PRA}

The component fragility review was performed by Dr. M.K. Ravindra (EQE, Inc.). He reviewed the basis for the component fragilities, and compared the final values against the existing equipment seismic performance data base (observed performance during actual earthquakes) being developed and maintained by EQE, Inc. The structural fragility calculations were reviewed by Dr. J.J. Johnson (EQE, Inc.), who performed the independent response benchmarking studies.

\section{(3) Review of Event Trees and Fault Trees}

The frontline event tree plus the support trees were reviewed for completeness and appropriateness. In particular, proper inclusion of random failures and test/maintenance unavailabilities in the seismic sequences (which can be important at low earthquake levels) was examined. Assumptions underlying any credit taken for recovery were examined for appropriateness. Finally, any implicit assumptions as to correlation between seismically-induced failures were identified and examined. Review of the systems models was performed jointly by the Brookhaven National Laboratory team (who reviewed the fault trees as part of their review of the internal events portion of the DCPRA) and by Dr. M.P. Bohn of Sandia National Laboratories who reviewed the seismic systems models.

\section{(4) Review of Seismic Risk Integration}

The assembly process resulting in the final seismic core melt frequencies was reviewed. An independent calculation of key results was made. Key assumptions driving the final result were identified and limited sensitivity studies performed to highlight the potential impact of alternative assumptions. Areas examined included the following:

a. appropriateness of final sequences and method of screening used.

b. Proper inclusion of seismically-induced correlation.

c. Completeness of evaluation of initiating events.

d. Appropriateness of inclusion of seismically-induced secondary failures (e.g., failure of interior block walls damaging nearby equipment, etc.).

e. Assumptions as to correlation between modeling (as opposed to random) sources of uncertainties.

f. Method of inclusion of relay chatter/breaker trip.

g. Proper combination fragility failure modes.

h. Subjective weighting factors for the hazard curves and their impact on the final core melt frequencies.

i. Development of plant-level fragility functions.

The review of the seismic risk integration, including independent evaluation of the final risk results, was performed by Sandia National Laboratories personnel.

The review of the Diablo Canyon Seismic PRA took place interactively with PG\&E personnel over the course of $21 / 2$ years. During the "interactive phase" of the review, the review teams met with PG\&E and their contractor support personnel to review individual pieces of input as it was developed. A number of meetings were held based on preliminary aspects of the PRA. One of the earliest major inputs completed was that of development of the seismic fragilities. When this material was completed and documented, a detailed review was begun. Finally, an audit meeting was held to review detailed aspects of various parts of the fragility derivations. 


\section{Selsmic Hazand Analysis}

\subsubsection{Introduction}

The detailed review of the seismic hazard was conducted directly by NRC staff members, therefore, the detailed findings of that review are not reported here. However, for completeness, a brief description of the hazard methodology and hazard curves used in the DCPRA analysis is given in this section.

The objective of the seismic hazard analysis was to provide a probabilistic representation of the earthquake ground motion at the Diablo Canyon site for the DCPRA. The seismic hazard analysis considers all the seismic sources that can affect ground motion estimates at the site and compares the contribution of various earthquake source hypotheses. The seismic hazard estimate is then convolved with the plant fragility to arrive at the core damage frequency assessment for the plant.

The seismic hazards analysis considered characteristics of seismic sources, maximum magnitude distribution, rate of earthquake occurrence, and attenuation of ground motion to develop a probabilistic representation of earthquake ground motions expected at the site. The results (probabilities of specified levels of ground motion being exceeded) were used as seismic input in the DCPRA.

\subsubsection{Seismic Hazard Analysis Methodology}

Uncertainties in defining risk are accounted for by a logic tree format and are represented by a family of hazard curves. Logic trees are composed of nodes and branches. Each element in the logic tree has a set of nodes representing an uncertain state of nature and each branch represents discrete possible values for that state. Probabilities are assigned to each branch using subjective assessments and end branch probabilities are calculated as the product of all the intermediate branch probabilities. A seismic hazard analysis was performed for each end branch resulting in a single hazard curve. The hazard curves for all the branches in the logic tree are used to estimate seismic hazard at the site.

Logic trees present the following source characteristics of faults: sense of slip, dip angle, depth of seismogenic zone, length of fault, maximum magnitude, seismicity model, and rate of activity. Weights are assigned to each possible hypothesis for each characteristic. These weights were assigned by a panel of experts based on currently available data. Logic trees were developed for the Hosgri, Los Osos, San Luis Bay, Santa Lucia Banks, West Huasna, Lompoc, Rinconada, Nacimiento and San Andreas faults.

Earthquake recurrence relationships were selected for each seismic source. Due to the limited historical seismicity record, the rate of seismic activity was based on (1) seismic moment release inferred from estimated fault slip rates or (2) geologically estimated recurrence intervals for surface rupturing events (LTSP Final Report, Figure 3-13). PG\&E found the estimated recurrence rates to be conservative because the predicted recurrence rates for magnitude 5 and 6 earthquakes are higher than the historical seismicity observations to the region.

The logic trees used to calculate seismic hazard include the geological data used to calculate maximum magnitude distributions and the mean annual rate of potentially damaging earthquakes (greater than or equal to magnitude 5) for each fault. Three median ground-motion attenuation relationships were used (strike-slip, oblique and thrust faulting). Both uncertainty in ground-motion amplitude and site conditions were used in estimating hazard. The results were summed over all faults which affect the site to calculate the seismic 


\section{Review of Selsmic PRA}

hamard curves (plots of the probability of exceedance at different values of ground motion). This procedure takes into sccount randomioss in the following variables: fault geometry, location of rupture surface, magnitude rupture size, closest distance of this site to the rupture, and ground motion attenuation.

In the Diablo Canyon soismic hazard analysis, 20,700 branches were calculated from the logic tree. These hazard curves were reduced to summary curves (fractile hazard curves) which show the distribution of hazard at each ground motion amplitude (spectral acceleration in the frequency range 3 to $8.5 \mathrm{~Hz}$ ). To represent uncertainty in the hazard, the large number of hazard curves were aggregated into a limited number of agregate hazard curves (eight curves) that maintain the character of the original curves while providing results appropriate for the DCPRA. The aggregate hazard curves represent 90 percent of the total variance of the original set for all ground motion amplitudes. These aggregate curves were used as input for the DCPRA.

The Hoegri fuult zone was found to dominate the seiamic hazard at the site. The Los Osos and San Luis Bay faults anch wontribute only a few percent to the total hazard. Relative contributions to the total hazard from the other faults are insignificant. Sensitivity studies showed that important parameters are slip rate, maximum magnitude and ground motion attenuation.

Hazard analyses were performed in terms of response spectral accelerations to be consistent with plant fragility extimates. Estimates were made for 5 percent damped accelerations at 2, 4, 8, 14, 25, and $33 \mathrm{~Hz}$ and for average spectral sccelerations in the ranges of 3 to $8.5 \mathrm{hz}$ and 5 to $14 \mathrm{~Hz}$. Hazard curves for different styles of faulting representing the frequency range of 3 to $8.5 \mathrm{~Hz}$ were selected for use in the DCPRA because this parameter provided the least variability between different earthquake magnitudes. Fig. 4.4.1 shows the hazard curves used in the seismic portion of the DCPRA. 


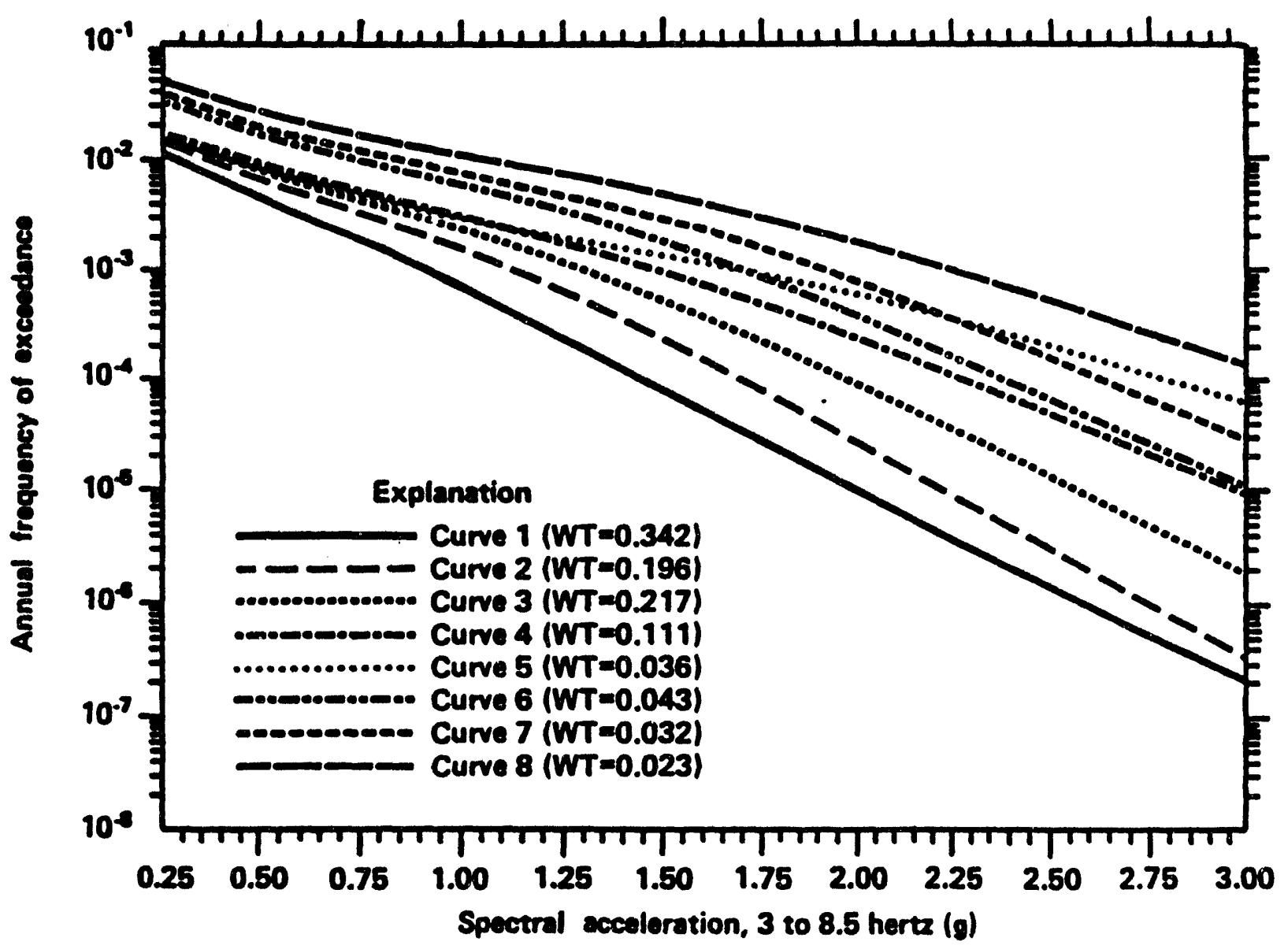

Fezure 4.4 .1

Total Acgrogate Basand Curves

(Azare 6-7 of the PRA) 


\subsection{Hazard/Fragillity Interface}

In most previous PRAs, the peak ground acceleration (pga) was used as the key parameter. The variability in the spectral shape was estimated in the previous PRAs by taking the ratio of spectral acceleration at a specific frequency to the pga. Since the logarithmic standard deviation of this ratio was found to vary over the frequency range of interest, it was conservatively assumed that this logarithmic standard deviation is frequency independent and equal to 0.25 or greater.

In the Diablo Canyon seismic PRA, the ratio of the spectral acceleration at any specific frequency to the average spectral acceleration (over the frequency range of $4.8 \mathrm{~Hz}$ to $14.7 \mathrm{~Hz}$ ) was found to have a constant logarithmic standard deviation of 0.18 for $5 \%$ damping. In addition, the randomness variability for earthquake directional effects alone was estimated to have an average value of about 0.12 . Thus the use of average spectral acceleration (over an appropriate frequency range) was felt to provide lower uncertainty than the use of peak ground acceleration (and hence reduce the uncertainty in the overall seismic risk) as well as preclude the possibility of "double counting" of uncertainty in the determination of fragilities and response analyses.

The independent parameter chosen for the DCPRA was the average of the ground motion response spectra (5\% damped) over the range of 3 to $8.5 \mathrm{~Hz}$. The hazard curves and the fragility median accelerations at failure were both expressed in terms of this independent parameter. This parameter can, of course, be related to the peak ground acceleration of the spectra from which it was developed. For comparison to the results of other seismic PRAs, the relation is

$$
(\mathrm{Sa})_{3-8.5}=2.34 * \mathrm{PGA}
$$

Where $(\mathrm{Sa})_{3-8.5}$ is the average of the ground motion response spectrum over the frequency interval of 3.0 to 8.5 Hertz.

Although the use of average spectral acceleration as the fragility parameter avoids the "so-called" double counting of variability introduced by attenuation and spectral shape factor, the reduction in the overall variability and the resulting reduction in the mean seismic CDF estimates are indeed small. It was concluded that the increase in the seismic margin (measured by the HCLPF capacity) may not be larger than $8 \%$ over that calculated using the pga and the previous seismic PRA methods; the decrease in the seismic CDF estimates is even smaller. These conclusions are very important for future seismic PRAs since the available seismic hazard results for different sites in the US east of the Rocky Mountains from the EPRI and NRC are in terms of peak ground acceleration and uniform hazard spectra whose uncertainty is not necessarily (or usually) constant over the 3.0 to $33 \mathrm{Hertz}$ frequency range of interest. However, the DCPRA results show that the frequency dependence of the uncertainty is not a major effect and can be neglected in future PRAs. Thus there is no need to revisit the seismic hazard studies and develop seismic hazard curves with the average spectral acceleration as the parameter as long as the spectral shape variability is consistently handled.

\subsection{Fragility and Building Response Analysis Review}

\subsubsection{Fragility and Building Response Analysis Methodology}

The fragility analysis method used in the Diablo Canyon seismic PRA has evolved over the last ten years and has been applied in seismic PRAs of over 25 nuclear power plants. So, in one sense, the methodology is mature. However, because of the high seismicity at the Diablo Canyon site, several aspects of the seismic 
PRA have been modified or performed in more detail than in previous applications. For example, the detailed probabiliatic response calculations performed on the auxiliary building, the detailed nonlinear analyses of the turbine building, and the large number of equipment items for which specific fragility calculations were performed represent non-typical applications of the methodology. The reference fragility parameter in the DCPRA is the 5 percent damped average spectral acceleration of the horizontal ground-motion averaged over the frequency range of $3.0 \mathrm{~Hz}$ to $8.5 \mathrm{~Hz}$. Table 4.6.1 presents the fragility functions used in the DCPRA, with the median values expressed in terms of average spectral acceleration. (To interpret these in terms of peak ground acceleration, it is only necessary to divide the median value by 2.34 . The random uncertainty $\beta_{R}$ and the modelling uncertainty $\beta_{U}$ are dimensionless, and apply to either representation.) The last column of Table 4.6.1 lists the average spectral acceleration aseociated with a high confidence of low probability of failure (the so-called HCLPF acceleration). These may also be scaled to peak ground acceleration in the same way.

The use of median-centered reference horizontal floor spectra in the development of equipment fragilities has afforded a realistic representation of the seismic capacity and reduced the overall variability. This approach of generating probabilistic responses has been used in selected past PRA's and may be used in future seismic margin/PRA studies to make a realistic assessment of response margins and variabilities.

This review is both global and specific. From a global viewpoint, an overall assessment of the implications of the response and fragility assessments on the seismic PRA was performed. From a specific standpoint, the review was focused on items that are risk significant or have generic implications.

\section{Praglity Amalyais Review Procedure}

The review focused on methodology and its application in the response and fragility areas. The review consisted of the following activities:

- Attending technical information meetings between the NRC and PG\&E held from 1987 through 1989.

- Performing a walkdown of the Diablo Canyon Nuclear Power Plant, focusing on the seismic risk important components and structures, in March 1988.

- Review of calculations and reports submitted by PG\&E.

- Independent calculations to verify various key aspects of the seismic PRA.

- Audit of calculations of selected components in June 1989.

In the response area, the auxiliary building and the turbine building were reviewed in detail. Independent response analyses and sensitivity studies were performed on the auxiliary building. The nonlinear analyses of the turbine building were reviewed in detail.

In the equipment fragility review, the review focused on identification of failure modes, information sources for fragility evaluation, i.e., loads, test response spectra, frequency calculations, etc., methods used in the derivation of median capacity and variability estimates $\left(\beta_{R}\right.$ and $\left.\beta_{U}\right)$, assumptions made on anchorage adequacy, and reasonableness of the final fragility estimates. The scope of the equipment fragility review did not permit independent fragility calculations by the reviewer. However, such independent calculations would not have provided much different conclusions or insights on the seismic capacities of components. It is recognized that the fragility evaluation requires the use of judgment on the part of the analyst in identifying the failure modes and estimating the seismic capacities of the component for these failure modes. There could be differences 


\section{Roviow of Solemic PRA}

in these judgments between difforent analyats. Therefore, the review concentrated on identifying such alforeaces and awowing the impact on the final fracility eatimates.

There was a cloes interaction between PO\&E and the reviewers throughout the program. The revilewers were presented with the mothodoby and results at different phases of the study. The questions raised and charifications sought by the reviewere were addresed in the final summary report, responses to reviow queations and apporting documents.

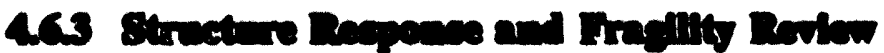

In the PLSC methodoloy, all fragilities are based on ground acceleration. Thus development of fragilities involves both extimates of responses at points where equipment is beated as well as eatimating the capacity of the components themelves. In the DCPRA all atructural responses wore scalod from dedgen calculations with the scaling dotermined based on a dotailed structural response evaluation of ono structure - the Auxiliary Building. Hence, as part of the Fragility Analyais reviow, it was considered easential to verify the DCPRA probabilitic responce study performed for the Auxiliary Building.

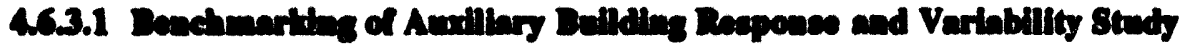

This rection focuenes on the response and variability atudy performed by POLE on the Diablo Canyon aurilingy building. The objective of the reviow was to reproduce and verify the simulation performed by PGEE and to perform senaitivity studies to identify parameters important to the seirmic PRA.

PG\&E utilibed the atudy: to benchmark a determiniatically calculated median response with one generated by probabilinic response analyais techniques; and to calculate estimates of variability in floor response epectral sccolerations as a function of frequency and elevation in the structure due to variability in ground motion, collhock properties and atructure dyanmic characteriatics. This study was abo used to quantify the portion of the "Atructure response fector" attributed to ground-motion spectral shape, structural damping structural frequency, atructural mode combination, earthquake directional combination, and soil structure interaction.

\section{Benchmertinc of Probebilintic Reanese Anntris}

Probabiliatic reiponce analyes of the Diablo Camyon auxiliary building were performed by PG\&E and verified herein. The probabilictic responce analydis methodology used to verify PG\&E's analyses parallols exactly that need by PGQE except that different atructural dynamic computer codes were used. The methodoloy used both by PGEE and in the roview is basically that doveloped for the US NRC Seismic Safety Margins Research Program (SSMRP) as implemented in the computer program SMACS (Ref. 6-2). The pertinent features of SMACS have been implemented in a veraion of CLASSI which was used in this benchmark evaluation. The mothod of analydis condists of conatructing best extimate models of rockffoundation/structure, asaigning best extimate parameters to their phydical characteriatica, and asdigning variability to a limited number of parameters of the rock/floundation/atructure system to represent variability in the system. A data set of ground motions is amembled or conatructed to represent variability in the free-field ground motion. The probabilistic reponse analyses then proceed by performing repeated determiniatic anabyes, each analyais simulating an earthquake cocurrence. By performing many such analywes and varying the free-field ground motion and values of the parameters, in-structure responses and their statistics can be calculated. 
Inout Motion. The bonchmark study used the identical ensemble of scceleration time histories as PG\&E's probablititic response analyail. Fifty-two horizontal ground motion time histories were used in the PG\&E audy. Twelve pair of orthogonal horizoutal time histories dertved from recordinga from oight earthquakes comprise twenty-four of the fifty-two. An additional fourteen pain of orthogonal horizontal time histories were aumerically generated. The twelve pairs of modified recorded motions are random with respect to north/houth or eachweat directions of the Diablo Canyon site, hence, the two components of each of the cots were interchanged to produce twenty-four sets of input motions for simulation purposes. The fourteen pain of numerically generated time histories were epecifically generated for the Diablo Canyon site and correspond to plant north/couth and eantweat, hence, they were applied in the appropriate direction. Therefore, for dmulation purposes, an ensemble of thirty-eight earthquakes comprise the data set. The fifty-two time histories are listed in Tables 4.6.2 (Table 6-5, DCPRA) and 4.6.3 (Table 6-6, DCPRA) for the modified recorded and numerically simulated ground motions, respectively. The time history scaling factor was dertived weh that the $3 \%$ damped spectral scceleration over the frequency range of 4.8 to 14.7 herte is $2.0 \mathrm{~g}$ for the average of the two componente.

Sinctmol Model. A simplified awdiliary building model was weed in the benchmark analyais and in anberequent sensitivity studies. Dynamic characteriatics of the auxiliary building are described by the modols fixed-base oifensyatem and modal damping factore. Figure 4.6.1 shows the 3-D lumped mase beam element model used in both PGdE's and present probabilitic response analysis. The fixed-base

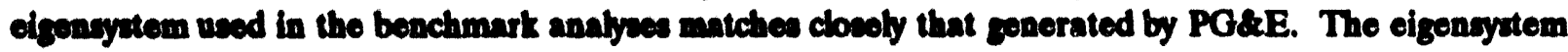
for the benchmart analycis wes recalculated fiven nodal maes values, their coordinate location, and beam element propertice. Comparison of the olgenvalues and man participation between the benchmark and POeE implified auriliary building models is gtwen in Table 4.6.4. The differences in the oigengystems are anall and do sot alpificantly contribute to difierences between in-atructure response.

Fonndntion Imoad ances. Impedance functions for the benchmark analyais initially assumed the foundation to be surface founded and based on the anene geometry and rock profile properties used by PG\&E (Table 4.6.5 and Fig. 4.6-2). Modification of the impedance functions to account for imbedment in the benchmark analycis was accomplished using the identical factors developed by PG\&E (Table 4.6.6). The benchmark analyais retained the frequency dependent nature of the impedance function while PG\&E's probabilistic analycis selocted the impedance values at $8 \mathrm{~Hz}$ and applied thum as a constant over the frequency range of the analydis. Table 4.6 .7 show a comparison of the impedance values at $8 \mathrm{~Hz}$ between those used in the benchmark and PGEE's analyses, including the imbedment factors.

Yarible Model Panmeter. Several discrete parameters were asoumed to be random variables and represented by probability distributions - rock modulus (stifiness of soil), structure frequency (stiffness of the Aructure) and atructure damping. Log normal diatributions were asaumed with median values defined by beat eatimates (rock modulus as given in Table 4.6.5, fixed-base structure frequencies times 0.90 to account for concrete cracking, and 7\% of critical atructural damping). Variabilities in the PGRE analyais were defined by log normal standard dovlations as given in Table 4.6.8. Thirty-eight earthquake simulations were performed to eatablish median and variability of in-atructure responses. Parameter values were celected by mb-dividing the probability diatributions into equally probable intervale and sampling from each of these intervaln; hence, each parameter value is equally probable. The thirty-eight combinations of parameter values were constructed by a Latin hypercube experimentaldesign. Two points of emphasis here -- two experimental deaigas were generated for the PG\&E analyses; one for the recorded time histories (total sample size of 24 earthquake simulations) and one for the numerically simulated time histories (total sample size of 14 earthquake simulations). Secondly, the parameter distributions were sub-divided into 


\section{Roviow of Solanic PRA}

$N+2$ equally probable intervals where $N$ equaled 24 or 14 respectively. The extreme high and low ranges were then diacarded to assure phyalcally meaningful samples. Tables 4.6 .9 and 4.6 .10 contain the sample values.

(i) Reeults of Benchmarking of PG\&E Probabiliatic Response Calculations

Table 4.6.11 compares the various appects of PG\&E's analysis with the benchmark analysis performed here. The only difference in the two is in the SSI area where the frequency-dependency of the impedance functions is retained. The beschmark analyses were performed and floor response spectra were calculated at several node points throughout the structure. The thirty-eight individual floor response spectra at a given location and direction were combined to obtain a median and $84 \%$ non-exceedance probability (NEP) spectrum. Figures 4.6.3 and 4.6.5 compare median responses calculated by PG\&E and the benchmark for the N-S and E-W directions; Figures 4.6.4 and 4.6.6 compare similarly the 84\% NEP spectra. They compare well as they should. Figures 4.6.7 thru 4.6.12 contain median and $84 \%$ NEP spectra at other elevations as calculated by the benchmark analysis.

\section{(ii) Results of Indepeadent Evaluation of Responses}

To supplement the benchmark analysis and investigate the effect of a different selection of parameter values on the response and its statistics, an independent verification of the probabilistic response analysis aspect of the PG\&E and benchmark analysis was performed. Table 4.6.12 itemizes each aspect of the analysis procedure with comparison to the PG\&E case. The only difference lies in the treatment of the variable parameters. One parameter was added, i.e., rock material damping consistent with Ref. 6-2. Sampling and the experimental design were based on a sample size of 38 earthquake simulations rather than two samples of sizes 24 and 14.

Figures 4.6.13 thru 4.6.16 compare floor response spectra (median and

84 percent NEP, N.S and E-W, for elev. $140 \mathrm{ft}$.) generated by PG\&E and generated by this independent analyais. The responses compare reasonably well with slight deviations in both the N-S and E-W directions.

(iii) Verification of Variability of Responses

Tables 4.6.13 and 4.6.14 compare log normal standard deviations of spectral accelerations at 5 percent damping averaged over various frequency ranges. These values are reasonably consistent and validate the PG\&E recommended ranges presented on p. 6-46 of the LTSP final report.

(iv) Results of Assessment of Apportioning of Variability

Two additional cases were analyzed to investigate the sources of variability in in-structure response spectra, i.e. attempt to separate composite variability into the portions due to ground motion and uncertainty in system properties. The first case held soil/structure properties at their nominal values and varied ground motion only. Thirty-eight simulations were performed and variability in floor response spectra was due to variability in the ensemble of ground motions only. The second case analyzed the auxiliary building for two artificial time histories generated to match the median ground response spectra of the thirty-eight motions in the N-S and E-W directions. Variability in system properties was identical to the benchmark case discussed previously. 
Tables 4.6.15 and 4.6.16 compare variability in response spectral accelerations due to the three cases:

- Bonchmark analysis.

- Ground motion variability only, soil/structure properties fixed at their nominal values.

- Single ground motion, soil/structure properties varied according to the Benchmark experimental design.

Assuming variability in in-structure response spectra due to ground motion variability only as random variability, variability in in-structure response spectra due to SSI/structure variability only as modeling uncertainty, and variability in in-structure response spectra due to both sources of variability as combined (or total) uncertainty, one can evaluate the square-root-of-the-sum-of-the-squares(SRSS) combination of random and modeling uncertainty to yield total uncertainty. Doing so in the average sense of Tables 4.6.15 and 4.6.16 demonstrated that SRSS of $\beta_{\mathrm{R}}$ and $\beta_{U}$ to obtain $\beta_{\mathrm{C}}$ is typically conservative and can be very conservative. That is, if the uncertainties (at different frequency ranges as shown on Tables 4.6.15 and 4.6.16) for the cases where only ground motion uncertainty or only soil-structure interaction uncertainties were included in the response calculation are combined in an SRSS sense, the results is often substantially greater than in the benchmark case where in both sources of uncertainty are simultaneously considered. For example, in Table 4.6.15 at elevation 100 feet and frequency range $11-30 \mathrm{Hertz}$, combining the uncertainty of the GMTH only case $(0.22)$ with the uncertainty in the SSITH only case $(0.08)$ in an SSRS sense gives a total uncertainty of 0.234 , which is substantially greater than that obtained in the benchmark case $(0.17)$ in which both sources of uncertainty are included together.

The lack of generic applicability of the SRSS rule for this case is due to the nonlinear nature of the equations of motion with respect to the frequency characteristics of the excitation and soil stiffness and damping and structure frequency and damping. Note, these observations apply to response uncertainty only and not to the combination of response and capacity uncertainties for which this investigation does not apply.

\subsubsection{Structural Fragility Review}

A number of structures were considered in the DCPRA, but only two played any significant role: the turbine building and the auxiliary building. As will be seen later, failure of the turbine building played a significant part in the final seismic risk results, while failure of the auxiliary building was a minor but non-negligible contributor.

The development of fragilities for all structures (except for turbine building) was based on the separation of variables approach. A description of the methodology, factors considered and sources of uncertainty was presented in reference 2 . The methodology and assumptions, failure correlations and ductility models described in this report were reviewed and found to be acceptable.

Due to the importance of the turbine building, an alternate calculation of structural failure level was made as described in reference 3. This report described the capacity analysis using the DRAIN-2D computer code and a non-linear hysteretic reinforced concrete shear wall constitutive model. Non-linear gap elements were used to model impact of the massive turbine pedestal with the remainder of the turbine building. Multiple time history analyses were made, and computed story drift values were compared with a story drift failure criteria (thought to be conservative) of 0.7 percent of the story height. Based on these analyses, a probabilistic failure function was developed. 


\section{Review of Seismic PRA}

Review of the turbine building seismic fragility assessment focused on the nonlinear dynamic analysis performed in a probabilistic manner to determine the fragility function. A review of the LTSP final report and reforence 3 along with meetings and discussions culminated in a series of questions ${ }^{4}$ to be addressed by PG\&E. These questions were discussed in a fragility meeting of June 16-17, 1989 and were addressed by formal responses.

These questions focused on modeling assumptions and details of the analysis. All questions were addressed adequately during the meeting of June 16-17, 1989 with the exception of the need to evaluate the assumption of impact occurring only at the center of the turbine pedestal whereas impact near the ends of the turbine pedestal would appear possible and have poteratially more severe consequences on the capacities of walls 19 and 31. The June 16-17, 1989 meeting defined additional studies for wall 31, examining in a limiting case the effect of the turbine pedestal impacting the turbine deck at close proximity to the wall connection. Reference 6 documents PG\&E's evaluation which shows this phenomenon to have minimal effect on the fragility function for the turbine building; thus, resolving this issue. The methodology used for the non-linear fragility an analyais as found to be acceptable. The non-linear analyses provided two important results. First, it provided an independent calculation of fragility from that provided by the method of separation of variables and secondly, it provided a substantiation of the underlying assumptions as to ductility and combinations of uncertainties which are implicit in the separation of variables approach. Overall, the non-linear turbine building analysis was felt to substantially contribute to the confidence level in the final seismic PRA results.

\section{Equipment Fragillity Review}

This section focuses on the equipment fragility review for components and equipment in the DCPRA. In the Diablo Canyon Seismic PRA, the median capacities of equipment were estimated using site-specific and plant-specific information. Median-centered horizontal floor spectra were estimated for selected floors of the important civil structures using ground motions matching the median ground response spectra, median-centered soil-structure interaction methods and parameters, and building structural parameters. For the west core of the auxiliary building, probabilistically based median-centered horizontal floor spectra were generated. This approach contrasts with many previous seismic PRAs wherein the design basis floor spectra were used to establish equipment capacity through the use of structural response factors of safety estimated to account for conservatism or unconservatism in the generation of the design flonr response spectra. The approsch followed in the LTSP provides a more realistic estimate of the median capacity with reduced uncertainty.

The randomness and uncertainty variabilities $\beta_{R}$ and $\beta_{U}$ are typically lower than those reported in previous seismic PRAs. The reasons are the use of median centered floor spectra and availability of qualification analysis and test reports specific to Diablo Canyon equipment. The analysts have systematically included the variabilities in different factors of safety by the "separation of variables" approach in arriving at the overall variabilities in the capacity for each equipment. The responses to formal review questions (Fragility Meeting. June 16-17, 1989) describe the bases for most of the variability assignments which rely rather heavily on profeasional judjment. However, the results are not very sensitive to these judgments because of the following. The overall variability (either $\beta_{R}$ or $\beta_{u}$ ) is obtained by taking the SRSS of the individual variabilities of different safety factors. Because of this process, only a few variabilities dominate. Therefore, it is not important to make a precise estimation of the variabilities of different safety factors. This is true even when the fragility parameters are used to calculate the seismic margin, the so called HCLPF capacity. The need for precise estimation of fragility parameters (specifically $\beta_{U}$ and $\beta_{R}$ ) become less when the final output are risk estimates (i.e., core damage frequency for example). 


\subsubsection{Fallure Modes and Issues Reviewed}

For each item of equipment, a review of the failure modes was made to assure that all reasonable failure modes were included. These included structural failure modes (e.g., buckling of tank walls and component supports, anchor bolt failures, and pressure boundary failure of piping) and functional failures (e.g., chatter and trip in electrical components, excessive blade deflection in fans, and shaft seizure in pumps).

Support failure modes. During the review and plant walkdown, the focus was on identifying potential failure modes. An example of the review is the identification of failure modes for the RHR heat exchanger. In Phase II, the heat exchanger support fragilities were calculated by extrapolating the Hosgri earthquake evaluation loads. For the Hosgri evaluation, the heat exchanger was modeled as fixed at the top and bottom and supported in the middle. It was pointed out in the review that the intermediate support would fail at higher accelerations and the anchor bolts would yield. Therefore, the horizontal frequency near failure level was evaluated by modeling the heat exchanger as a beam with the upper and lower support treated as pinned connections, and the intermediate support not included. This reduced the horizontal frequency from $29.6 \mathrm{~Hz}$ for the Hosgri evaluation to $11.9 \mathrm{~Hz}$ for the fragility evaluation.

In the PG\&E calculations submitted in response to Question 32c, two failure modes were identified. These were failure of anchor bolts at the pedestal and the upper N-S strut. For the upper support, the capacity was determined to be the sum of tension in the embedded studs and buckling load of the longer strut. From the sketch supplied by PG\&E, it appeared that a failure mode comprised of concrete pull out of the upper support could occur before yielding of the studs could take place. The imbedment length of these studs (7/8 in. diameter) was shown to be $4 \mathrm{in}$. If this failure mode was in fact the governing one, the median strength factor would have been less than $50 \%$ of what was estimated. In the audit review of calculations, this failure mode was examinedcarefully. PG\&E produced Drawing No. 439520 which showed that the actual imbedment length of these studs is $\mathbf{8 . 7 5}$ in. Therefore, concrete pullout of the studs would not happen before their tensile failure.

Failure modes based on qualification tests. Structural fragility of tested componeris was estimated by using a factor of 1.8 over the test response spectra (TRS) for those cases where there was no distress noted during or subsequent to the seismic qualification test. Herein, the median factor of 1.2 represents the ratio of test response to onset of distress, and the median of 1.5 represents the ratio of response at the onset of distress to onset of failure. The composite variability in the first factor was estimated to be $\beta_{C}=0.11$ whereas the uncertainty in the latter factor was estimated to be $\beta_{U}=0.04$. Although these median and variability values were estimated using judgment, they appear to be reasonable and the overall effect on the component fragility may have been conservatively estimated. In a few cases, excess conservatism in the total median factor of 1.8 has been demonstrated and removed. For the $4160 \mathrm{~V}$ Switchgear, the median capacity was calculated first by assuming it to be 1.8 times the ratio of TRS/RRS (Required Response Spectra). This approach led to an excessively conservative low capacity estimate for the component. To refine this evaluation, the potential failure mode for the component was examined. The failure mode was identified as carriage guide rod bending. For this failure mode, the median capacity was calculated as 7.44 $\mathrm{g}$ and the HCLPF capacity was obtained as $2.9 \mathrm{~g}$. Whereas, application of the 1.8 factor would have yielded a median capacity of $4.5 \mathrm{~g}$ and the HCLPF capacity of $1.8 \mathrm{~g}$.

Relay Chatter. PG\&E identified relays (see also Section 3.2) that affect components necessary for safe shutdown. Functional failure fragilities of relays were evaluated only for those relays which were considered to be chatter sensitive. The median strength factor for the chatter mode was estimated using 


\section{Review of Seismic PRA}

the Generic Equipment Ruggedness Spectrum (GERS), the cabinet amplification factor and the floor spectral acceleration. The relay chatter failure mode fragilities were derived for the diesel generator control panel, 4-KV switchgear, main control boards and hot shutdown panel. Except for the $4 \mathrm{kV}$ switchgear, the chatter failure mode capacities, were found to be sufficiently high so as not to contribute significantly to plant seismic risk. The $4-\mathrm{kV}$ switchgear, contains a large number of overcurrent relays, which are primarily sensitive to vertical excitations. The fragility parameters for relay chatter of the switchgear were estimated to be $3.53 \mathrm{~g}$ (median) and $1.31 \mathrm{~g}$ (HCLPF). It was demonstrated that the switchgear chatter failure mode is recoverable from the control room. Hence, the relay chatter failure mode is addressed in LTSP in detail and does not contribute significantly to the plant seismic core damage frequency.

Anchorage failure modes. The anchorage failure modes investigated included some of the recent generic issues such as edge distance, close spacing of bolts and cracked concrete. For example, the RHR heat exchanger is mounted on a pedestal with anchor bolts; the edge distance for anchor bolts appeared to be low. However, a closer examination revealed that the anchor bolts were $J$ bolts and are installed well inside the outer layer of reinforcement. There are a few items of equipment mounted on pedestals which may pose possible concerns with small edge distance; however, the seismic fragilities for these components were developed using specific information; the review and walkdown generally focused on this issue. Therefore, it is concluded that the anchor bolt failure modes are properly represented in the LTSP.

Development of vertical floor acceleration input for equipment frapility evaluation. A question often raised in seismic PRAs is how the vertical ground motion and uncertainties are combined with the horizontal ground motion. Appendix D of reference 2 describes the procedure used in developing the median centered vertical floor response spectra. Through selected examples, it is shown that the fraction of the total critical response attributable to vertical excitation is generally small and is unimportant in terms of contribution to plant seismic risk. The reviewer agrees that the incorporation of the vertical ground input by the so-called "Hosgri factoring method" described in this appendix is adequate for this purpose.

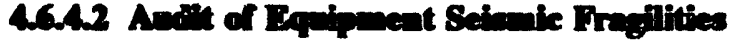

The purpose of the audit was to obtain additional information on equipment fragility calculations, and understand the sources of information and assumptions made in the identification of failure modes and their fragility estimation. The calculations were reviewed with the aid of equipment support drawings, qualification reports and photographs of the equipment items.

The major focus was on equipment items for which PG\&E had previously sent fragility calculations and had specifically addressed in response to NRC Question 32. The audit covered the following aspects:

- Identification of failure modes

- Information sources for fragility evaluation

- Method for deriving median, $\beta_{R}$ and $\beta_{U}$

- Anchorage

- Reasonableness of fragility estimates

- Detailed information available for review 
Specific findings for the components whose fragility derivations were examined during the audit are described below.

\section{$4160 \mathrm{~V}$ Switchear}

Two failure modes were identified by PG\&E: functional failure (chatter) and structural failure. The fragility for relay chatter was calculated using the GERS and modian cabinet amplification factors. The relay is sensitive to vertical seismic response and the vertical frequency of relays was estimated to be from $19-21 \mathrm{~Hz}$. The median spectral acceleration capacity in the chatter mode was estimated to be $3.53 \mathrm{~g}$ and the HCLPF capacity was obtained as $1.31 \mathrm{~g}$. It was stated in Ref. $6-1$ that relay chatter in this component is recoverable from the control room.

For structural failure, it is customary to use qualification test data; if there was no distress in the component in the qualification test, the median capacity is taken to be 1.8 times the ratio of TRS/RRS. As discussed previousty, this approach was determined to be excessively conservative for this component. Instead, the structural failure mode was identified as carriage guide rod bending. For this failure mode, the median capacity was calculated as $7.44 \mathrm{~g}$ and the HCLPF capacity was obtained as $2.96 \mathrm{~g}$. This a good example of evaluating the failure mode by analytical means.

The median factors in the fragility calculations were selected conservatively; therefore, the $\beta$ values are lower than the ones reported in previous seismic PRAs. Also note that median floor response spectra were calculated instead of being extrapolated as in previous seismic PRAs.

The intermediate values of $\boldsymbol{\beta}$ do not have a significant influence on the overall variability because of the way $\beta$ are combined.

\section{RHR Heat Exchange;}

In the PG\&E calculations submitted in response to Question 32c, two failure modes were identified. These were failure of anchor bolts at the pedestal and the upper N-S strut. For the upper support, the capacity was determined as the sum of tension in the embedded studs and the buckling load of the longer strut. The potential for the embedded studs pulling out of concrete before yielding could take place was examined. The imbedment length of the studs was found to be sufficient to prevent this failure mode as discussed previously ("Failure Modes").

The edge distance of anchor bolts in the pedestal was reviewed; it was determined that the anchor bolts are $\mathrm{J}$ bolts and are well inside the outer layer of reinforcement.

For the RHR heat exchanger, the two failure modes were estimated to have similar seismic capacities. The analysis correctly calculated the fragility of the component as the conditional probability that any one of the failure modes could occur.

Initial review of the calculations did not include frequency calculations for the heat exchanger used in the fragility estimation. Follow-up reviews demonstrated this frequency based on the pinned-pinned condition to be $11.9 \mathrm{~Hz}$ taking into account the hypothesized failure of the center support and moment resistance at the bottom. A frequency of $29.6 \mathrm{~Hz}$ was estimated in design for the condition of three active supports. 


\section{Reviow of Soirnic PRA}

The nozde bads on the heat exchanger were scaled from those obtained using Hosgri floor spectra to the median floor spectra at the piping frequency of $12 \mathrm{~Hz}$. Subsequent to the audit, PG\&E provided a justification for the piping frequency of $12 \mathrm{~Hz}$.

\section{CCW Heat Exchancer}

The controlling failure mode was identified as the longitudinal support concrete anchors. The review confirmed that the odge distance for the expansion anchor does not matter since the anchors go into the concrete alab pasaing through a thin grout pad.

\section{Soray Additive Tenk}

The anchorage was checked and found to have substantially higher capacity; the bolts are 1-1/8 in. diameter and $2 \mathrm{ft} .10 \mathrm{in}$. long. In the Hosgri evaluation, the fixed support was modified by adding another bolt. Therefore, the sliding support governs.

\section{D.G. Control Panel}

Of the two failure modes examined for this component, the structural failure modes were found to have lower seismic capacity. For most electrical components, functional failure capacity is usually smaller than structural failure capacity; however, for the D.G. control panel, the relay chatter failure mode had a higher capacity because of the following reasons: the relays are vertical response sensitive and the cabinet amplification factor of 1.5 is considered to be a median value. The Westinghouse ARD relays are sensitive to front-back response for which the cabinet amplification is asumed to be 3. These amplification factors are conservative because the cabinet is stiff (it is supported at the top) and the relays are mounted low in the cabinet.

The overtest factor for the side-to-side direction was derived from the qualification test data. For the vertical direction, the overtest factor was assumed to be the same as for the horizontal direction and no failure in the panel and anchorage was assessed.

The anchorage of the panel to the isolator and of the internals to the panel was reviewed and found to be not critical.

\section{Preagurizer Safoty and Relief Volves}

Safety valves are qualified to $6 \mathrm{~g}$ and the PORVs are qualified to $3 \mathrm{~g}$. The judgment made on the median ratio of allowable to the actual stress at the operability limit as 1.4 was reviewed and found to be acceptable. The overall fragility parameters for valves appear to be reasonable.

\section{Reactor Coolant Pump}

The fragility calculations for this component have been described in detail in the fragility methodology report. Excessive plastic bending of the lower motor stand is the critical failure mode. The major contributing factor to the median capacity is the inelastic energy absorption factor taking the median strain to failure as 4 percent. The resulting fragility estimates appear to be reasonable. 


\section{Control Rod Drive Mechaniem}

The failure mode is the yielding of the lead adepter at the RPV juncture. The streas reported by the NSSS vendor for the Double Design Earthquake was wed in deterwining the margin to yield. The strength factor and ductility factor contribute most to the overall fragility in terms of modian and $b$ values.

\section{Mrin Stenm PORV8}

This is qualified to 6g. The median spectral acceleration demand was estimated as about 3g. The fragility paramoters appear to be reasonable.

\section{Contrinment Fan Cooler}

The fallure mode identified is the cooler box foot platefimbedment weld. The analysis done for qualification was used in determining the safety margin for the weld. Being a shear failure of the weld, no account for ductility was taken. The fragility parameters appear to be reasonable.

\section{4kV Potential Transformer}

This component is supported on a stand instead of being on the top of the 4-kV switchgear as in most plants. The critical failure mode was identified as the shear failure of the frame leg/imbedment weld. The strength factor is high making the overall median capacity to be in the range of $10 \mathrm{~g}$ spectral acceleration.

\section{Safequard Relay Panel}

The relay chatter failure mode was considered not important by the system analysts. The cabinet is stout and the structural failure of the anchor plug welds has a substantial margin.

\section{DC Batteries}

Since the batteries are snugly held and adequate spacers are provided, the median failure/test factor was assumed to be 1.8. The TRS to RRS was also estimated as about 1.5. The fragility parameters for batteries appear to be reasonable.

\section{V DC Battery Racks}

The failure mode is the bending of the battery support rail at the 5 in. overhang of batteries. The fragility parameters were estimated using the Hosgri stress analysis results.

\section{V DC Battery Chargers}

In the qualification test there was no distress; therefore, a median factor for test/failure of 1.8 was used in the calculations. The TRS to RRS ratio was also found to be 2.45 . 


\section{Roviow of Solmic PRA}

\section{V Vinen}

Since the cabinet wes on the veree of failure at the end of qualification test, the median failure acceleration was taltea to be 15 percent above the teat level. There is sofficient margin in the TRS compared to the RRS. Therefore, the fractity parameters appear to be rescomable.

\section{V Brenter Penel}

Based on the bow atres at the median vertical response for the worst case

(19 in. panel), the median capacity is estimated to be about 184. Hence, this component was not studied further.

\section{Main Control Bonents and Control Console}

The main control board enclosure was structurally modified during the Hosgri reevaluation. The stress in the structural angle was assumed to be 85 percent of the AISC allowable and a redistribution factor of 1.6 was used in calculating the strength factor. The fragility parameters appear to be reasonable.

\section{Reactor Trip Switchear}

The fragility eatimation is based on the qualification test; since there was no distress observed during the test, a median factor of 1.8 was used for the test to failure ratio.

\section{Balance of Plant Piping}

Several failure modes were analyzed and the piping support fillet weld failure was identified as the critical mode. A factor between the total stress and the allowable was established by studying a number of large bore piping systems. A system ductility factor was used to allow for response in the ductile piping systems. The fragility parameters appear to be reasonable.

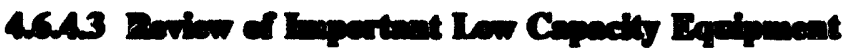

The review specifically focused on the low capacity equipment items that contribute significantly to the seismic risk of Diablo Canyon. The groups of components whose failure contribute significantly to core damage frequency are:

\section{- 4.16-kV vital AC switchgear}

- Loss of offsite power

- Excessive LOCAs

- All vital 125 DC

The fragility parameters for these equipment items were found to be reasonable. The documentation of fragility calculations and the supporting analysis and test data were examined in the audit and found to be acceptable. The sensitivity studies reported in Table 6-58 of the LTSP report indicate risk improvement if some of the components were further seismically upgraded. The conclusion is that there are no overwhelmingly weak links in the plant. 
Excessive LOCAs were based on the probability of piping failure. The treatment of piping, fragility in the DCPRA is aovel. The bacis for the fragility of BOP piping is generic failure of piping supports coupled with the probability of pipe break given support failure. The fragility function is estimated for a piping segment containing approximately 20 supports. A piping system may contain a number of piping segments. The study calculated the piping system fragility by assuming fallure to occur if one or more segments fail. The number of piping segments in each system was estimated for use in the risk quantification.

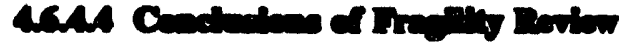

The level of effort in developing the DCPRA seismic fragilities for equipment is well beyond that expended on previous seismic PRAs. One major reason was that the expected high seismic levels limited screening of components on the basis of earthquake experience data and generic qualification test data and accentuated the need for apecific evaluation for most components. This level of effort is typically not needed for the execution of a seismic PRA for plants in the Eastern United States.

Using the results of the seismic PRA (the component and plant seismic margins reported in the LTSP Final Report); the margin against the 84 percent site specific ground motion was calculated. It is seen that all components whose failure will lead to seismic risk have at least $\mathbf{4 0}$ percent margin over the site specific ground motion. The modian capacities were shown to be much higher.

\section{Deferences}

1. Johnoon, J. J., Goudreau, G. L., Bumpus, S. E., and Maslenikov, O. R., "SSMRP Phase I Final Report: SMACS (Seismic Methodoby Analysis Chain with Statistics) (Project VII)," UCRL-53021, Vol. 9, NUREG/CR-2015, Vol. 9, Lawrence Livermore National Laboratory, Livermore, CA, 1981.

2. Kipp, T.R., D.A. Wesley, W.H. Tong, "Seismic Fragilities of Civil Structures and Equipment Components at the Diablo Canyon Power Plant," No. 1643.02. NTS Engineering, December 1988.

3. Kennedy, R. P., Wesley, D. A., and Tong, W. H., "Probabilistic Evaluation of the Diablo Canyon Turbine Building Seismic Capacity Using Nonlinear Time-History Analyses," No. 1643-01, NTS Engineering, December 1988.

4. Letter, J. J. Johnson to N. Chokshi, "Diablo Canyon Turbine Building Fragility Assessment," June 8, 1989.

5. Letter, J. D. Shiffer to U.S. Nuclear Regulatory Commission, Re: Docket No. 50-275, OL-DPR-80, Docket No. 50-323, OL-DPR-82, Diablo Canyon Units 1 and 2, Long Term Seismic Program Meetings, July 25, 1989, Enclosure 3 Fragility Analysis Meetingo June 16-17, 1989.

6. Letter, J. D. Shiffer to U.S. Nuclear Regulatory Commission, Re: Docket No. 50-275, OL-DPR-80, Docket No. 50-323, OL-DPR-82, Diablo Canyon Units 1 and 2, Response to Action Items trom the Long Term Seismic Program, Fragility Meeting, June 16-17, 1989, October 26, 1989. 


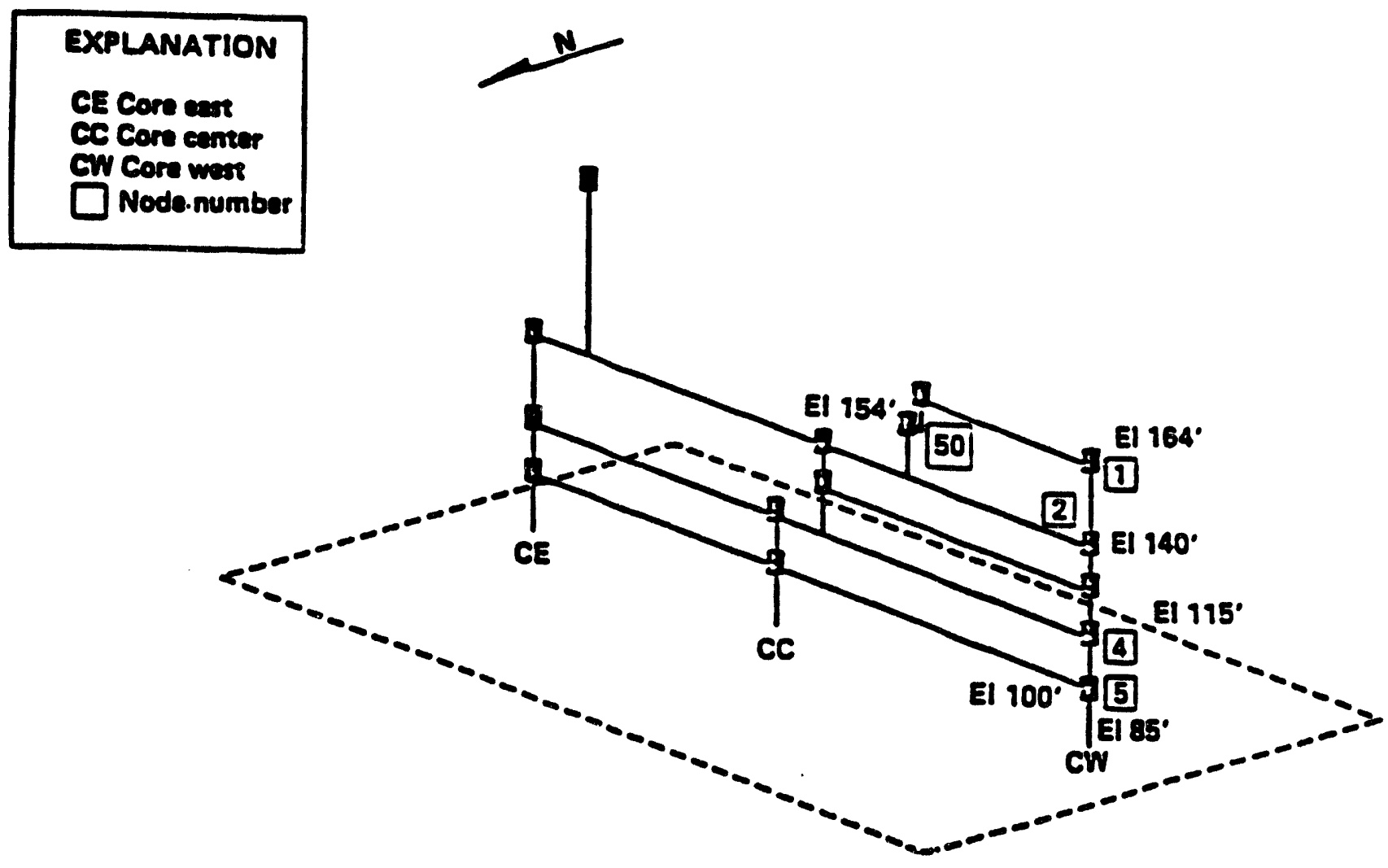

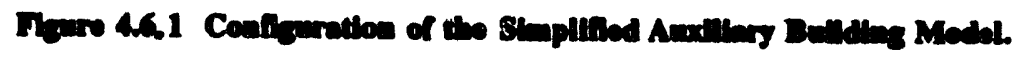



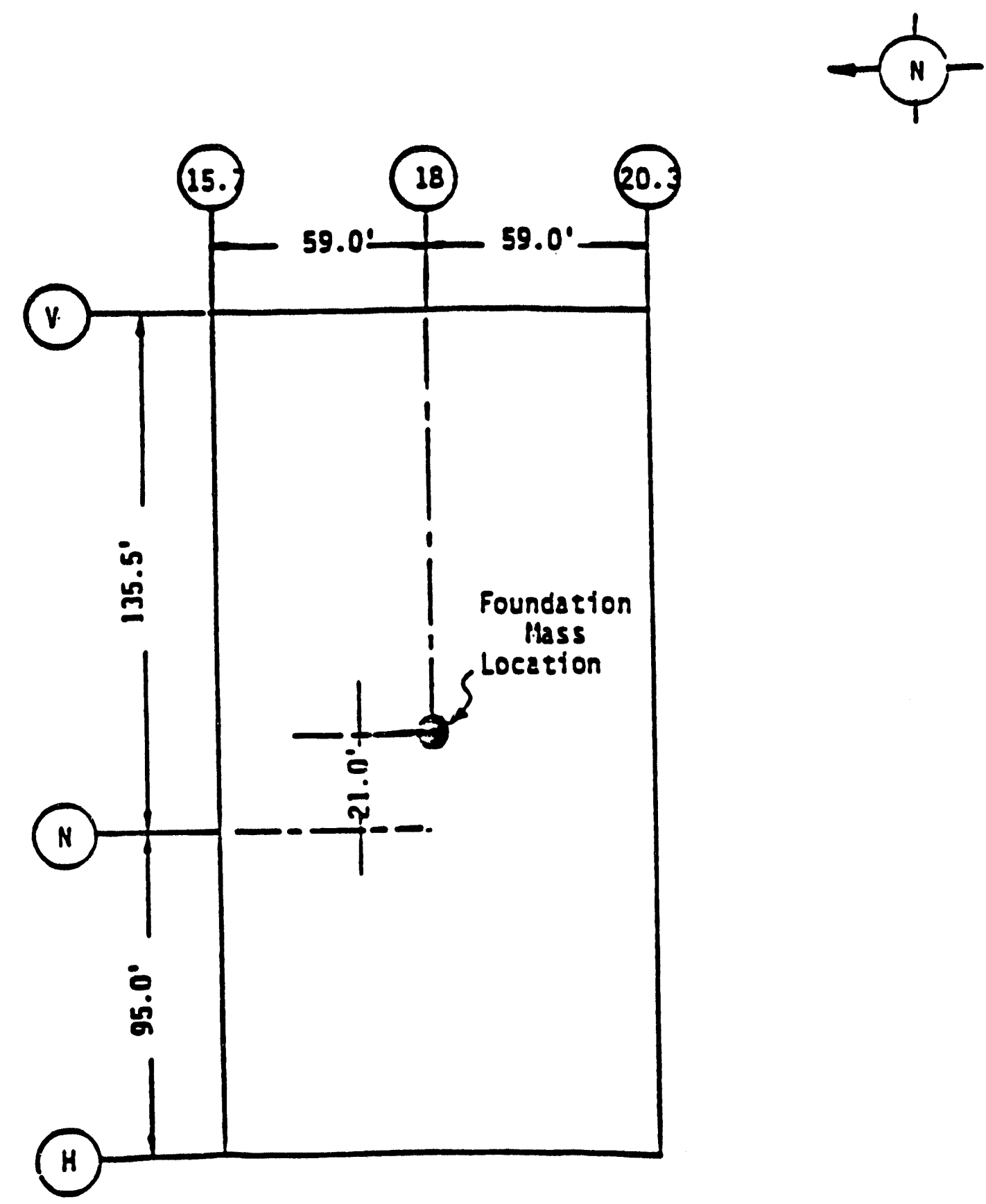

Dyare 4.6.2 Rigld rectangular foundation geomotry for the Simplinied Auxillary Bullding Model. 


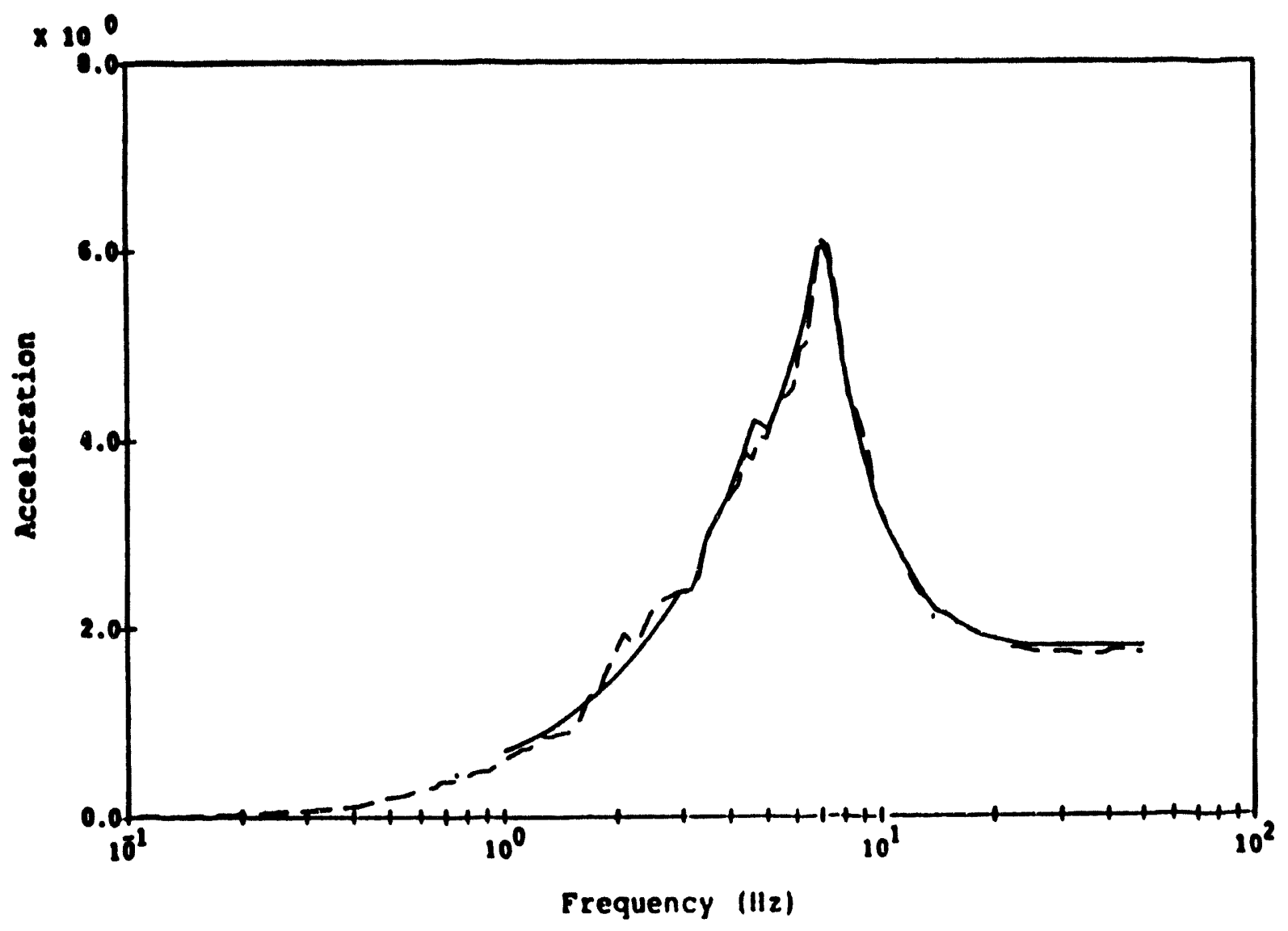

Legend:

Notes:

PGSE

Spectra calculated at 58 damping

Benchmark

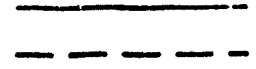
Accelerations in units of $g$

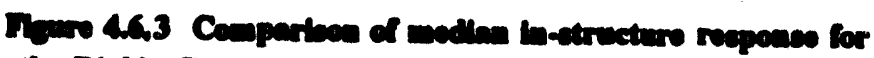

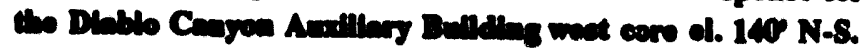




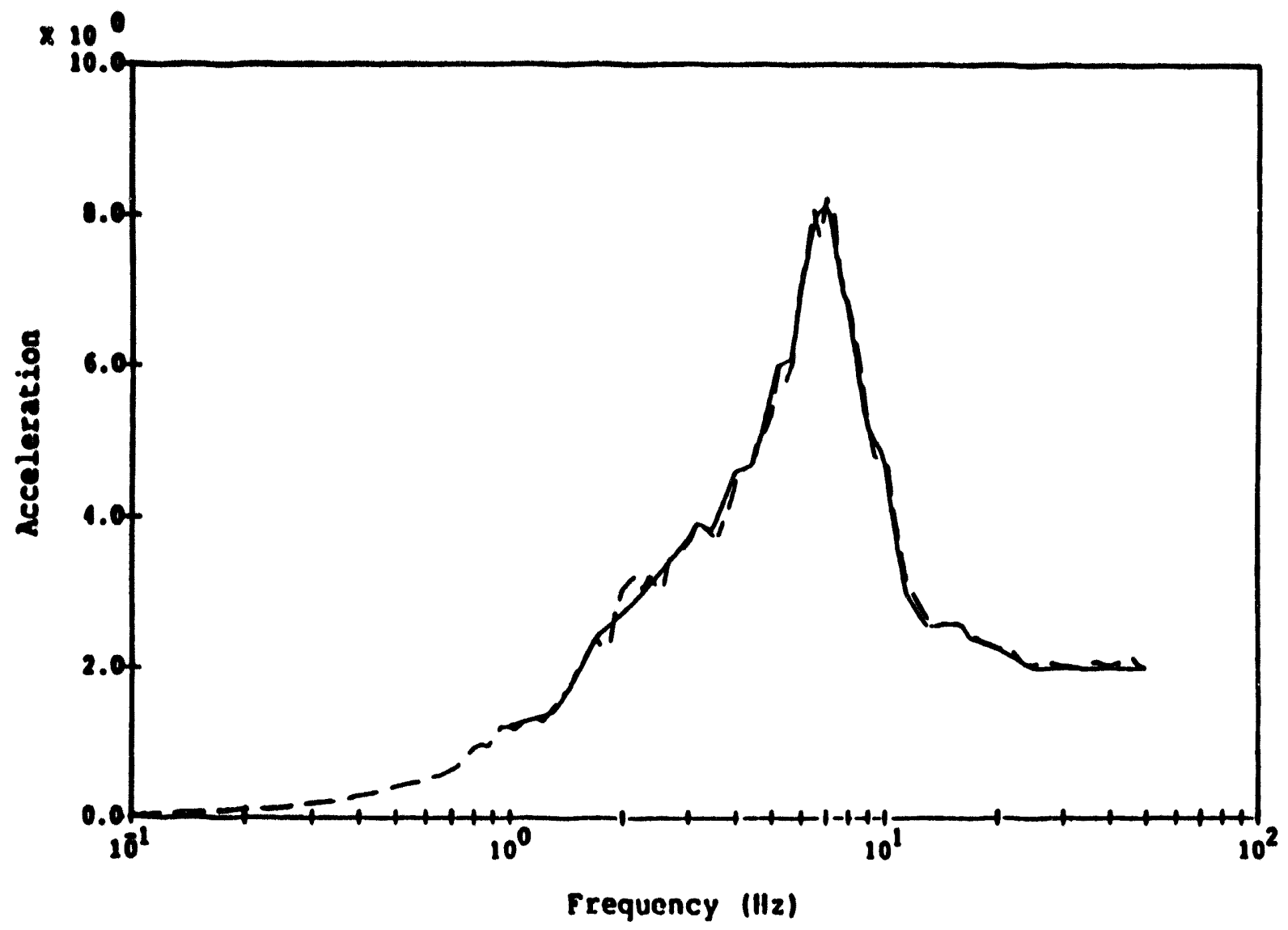

Legend:

PGSE

Benchmark
Notes:

Spectra calculated at 58 damping Accelerations in units of $\mathrm{g}$

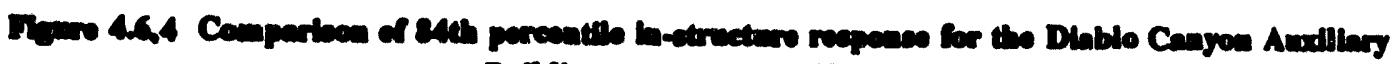
Bunding nocer cors ol. 140 N-S. 


\section{Reviow of Sulem PaA}

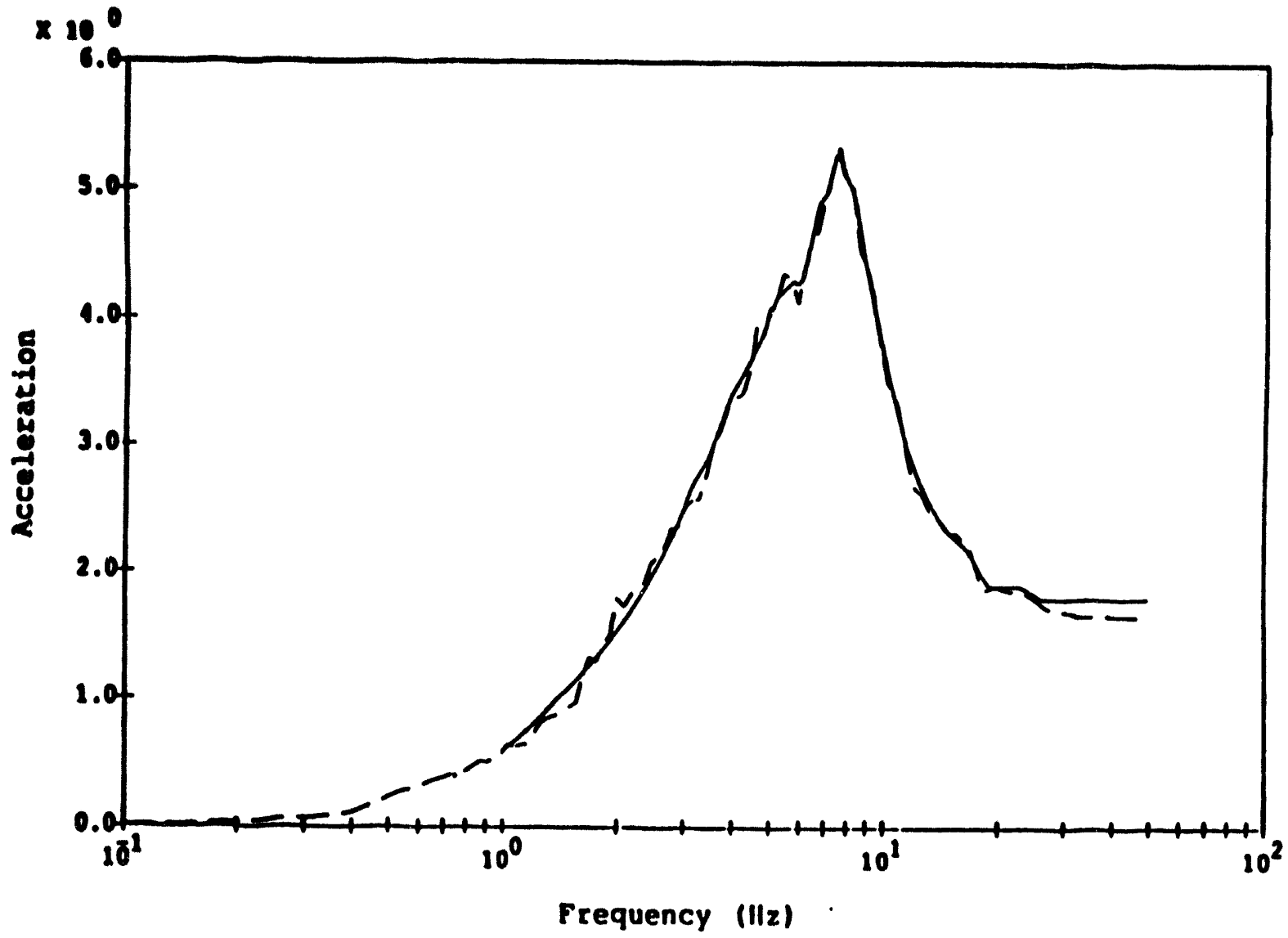

Legend:

PGSE

Note3:

Benchmark

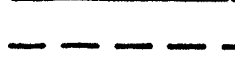

Spectra calculated at 58 damping Accelerations in units of $g$

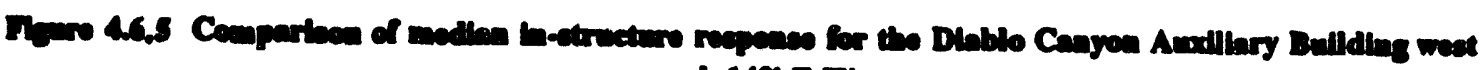
$\cos \operatorname{ch} 100 \mathrm{BW}$. 


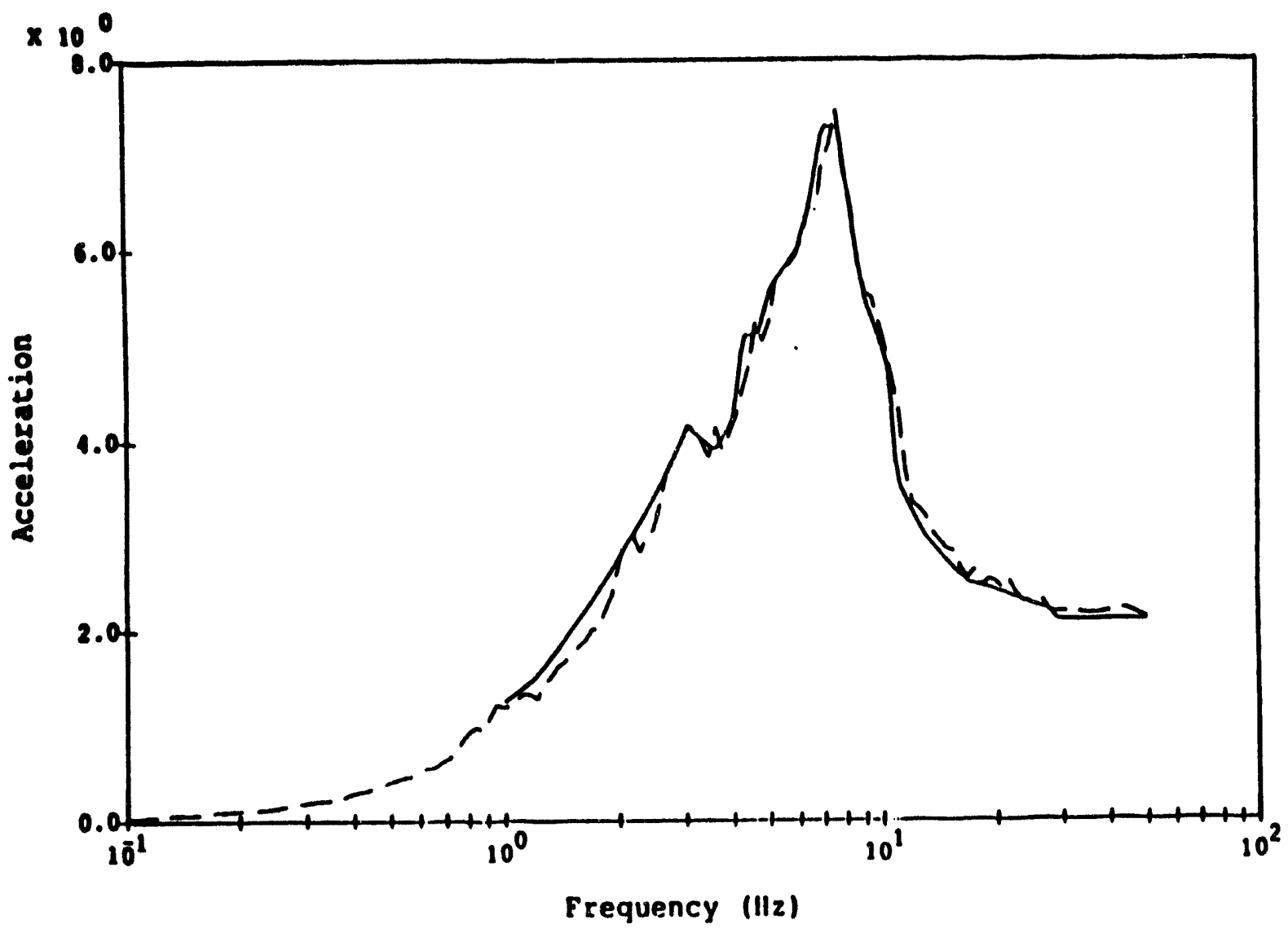

Legend:

PGEE

Benchmark
Notes:

Spectra calculated at 58 damping Accelerations in units of $\mathrm{g}$

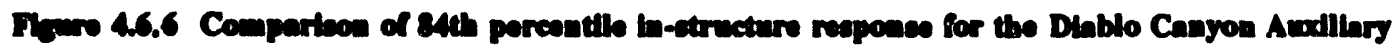
Buillare mat cose ol. $140 \mathrm{EW}$. 
4 Review of Seismic PRA

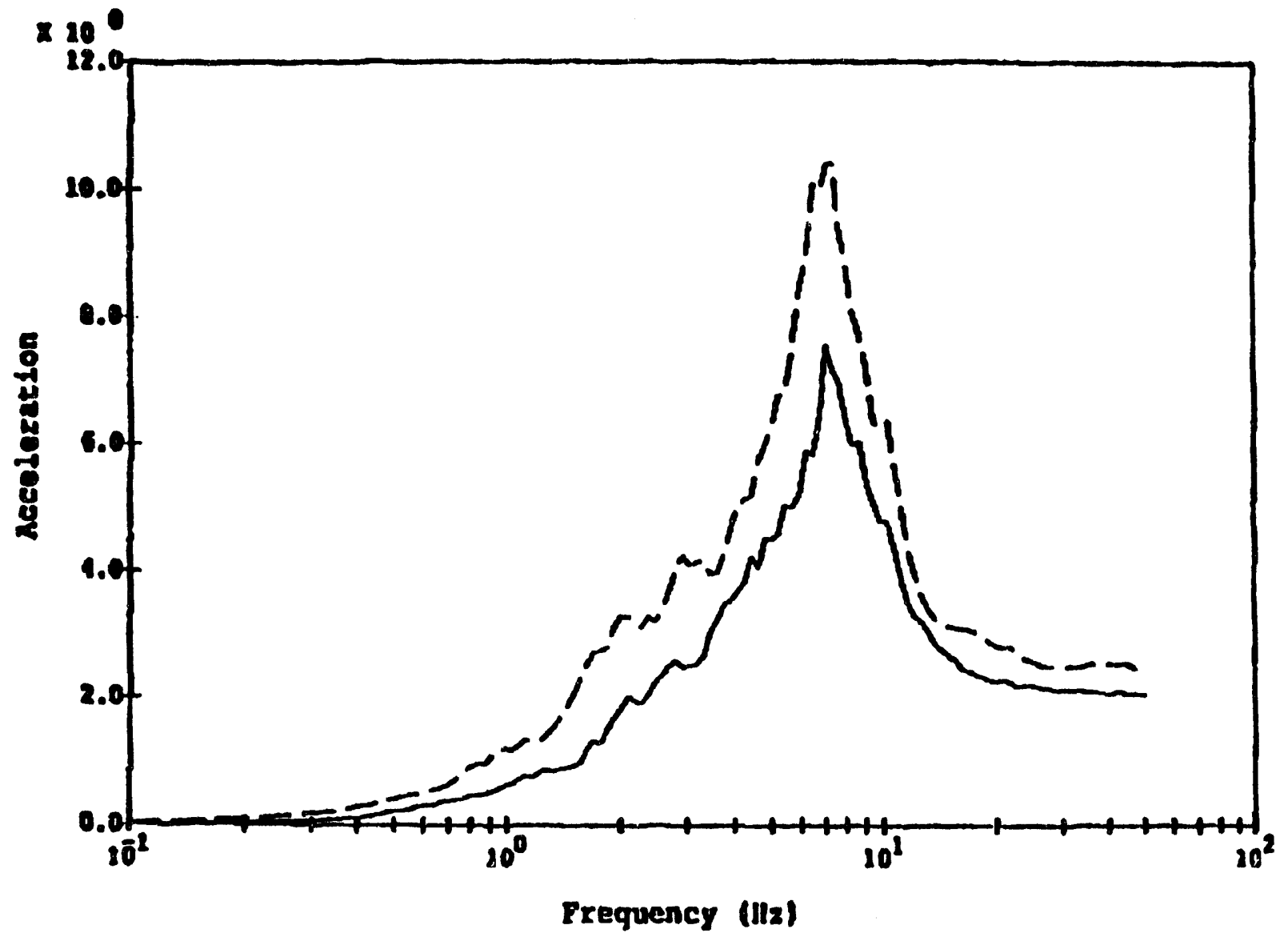

Iegend:

Notes:

Median

Spectra calculated at 51 damping

84th Percent1le

Acceleration in units of $\mathbf{g}$

Figure 4.6.7 Diablo Canyon Auxdliary Bullding benchmark analysis In-structure response core west el. $164^{\circ}$ N-S. 
4 Review of Seimic PRA

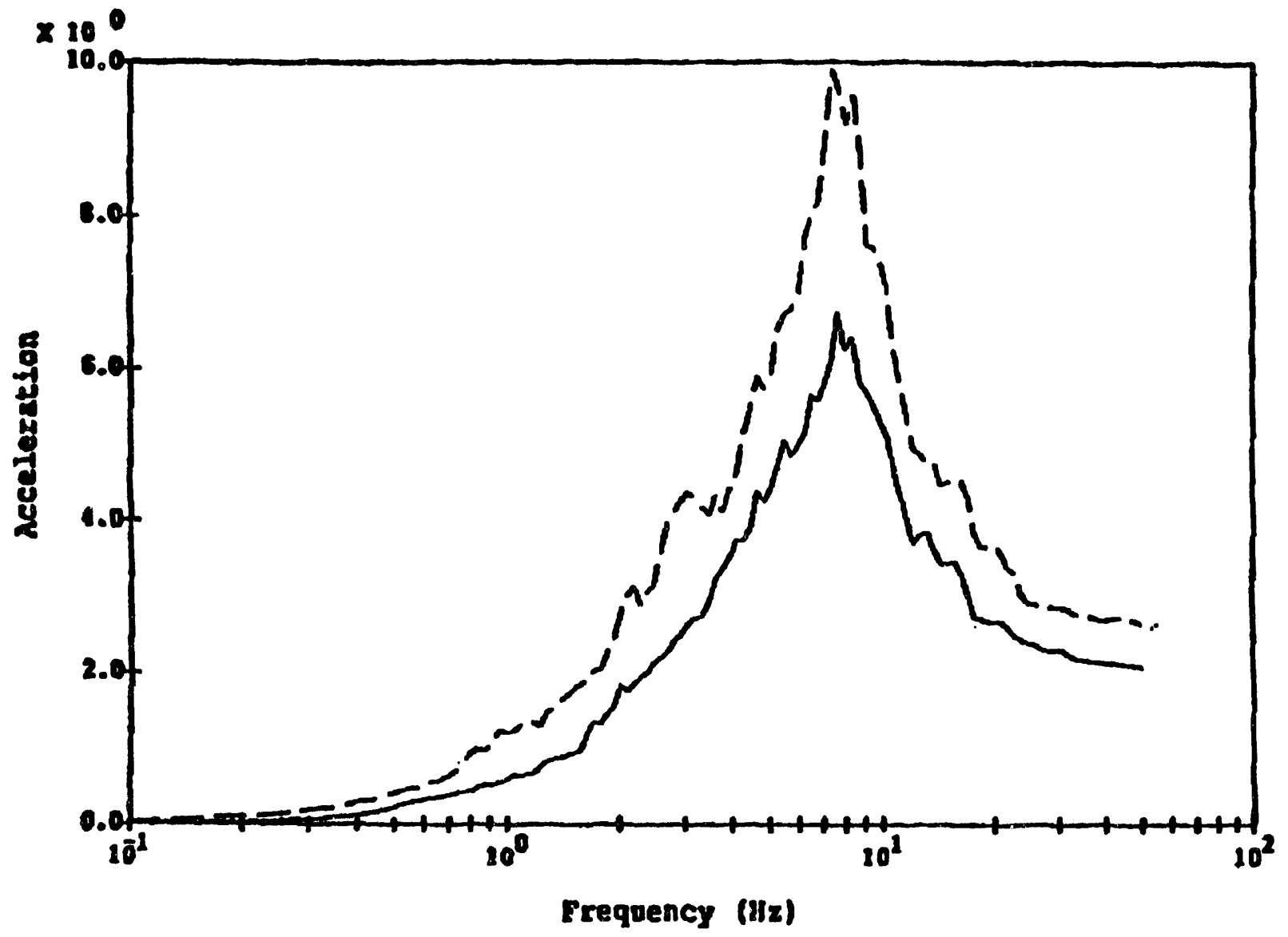

Legend:

Median

84th Percentile
Notes:

Spectra calculated at 58 damping Acceleration in units of $\mathbf{g}$

Figure 4.6.8 Diablo Canyon Auxillary Bullding benchmark analysis in-structure response core west el. $164 \mathrm{E}-\mathrm{W}$. 


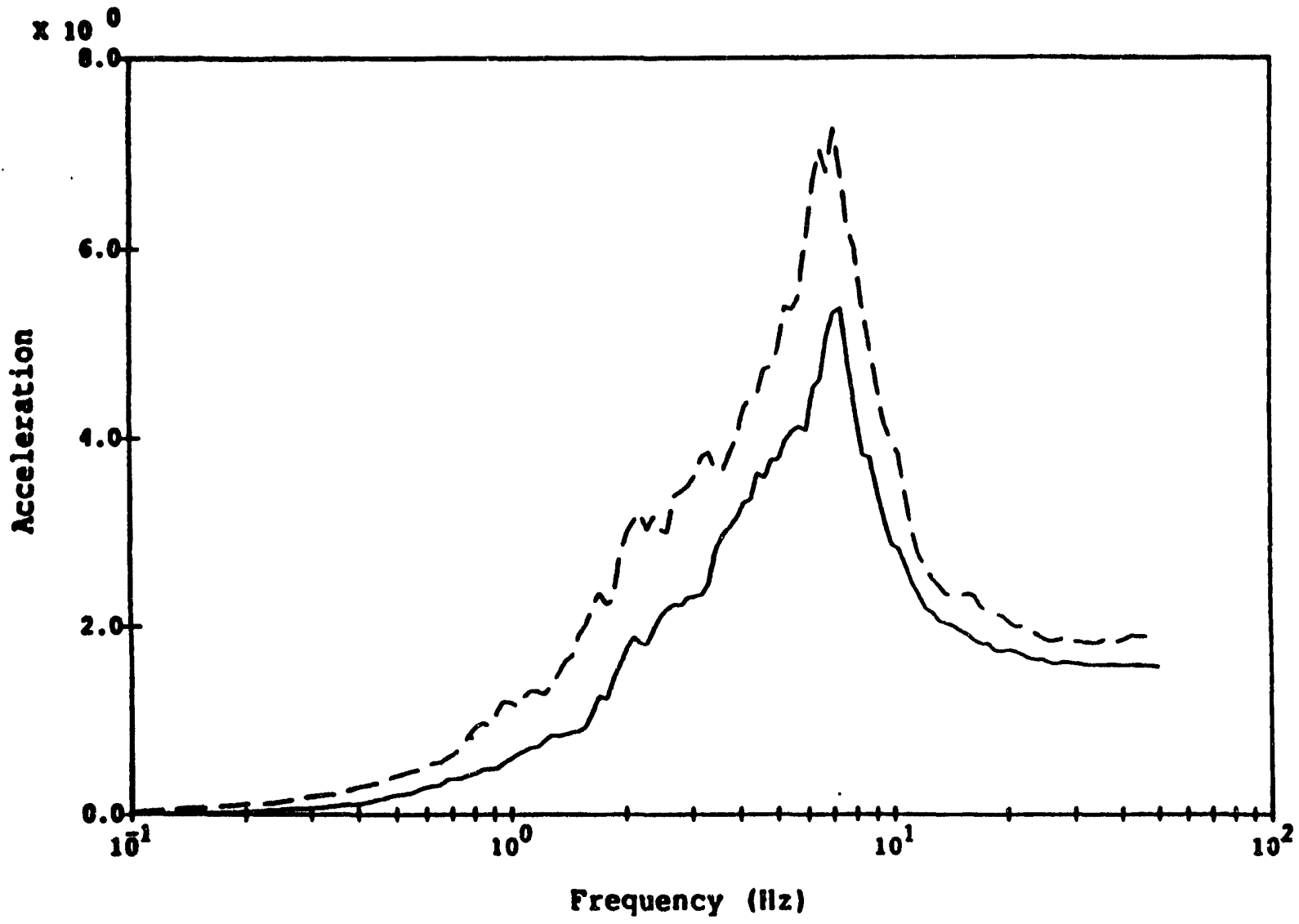

Legend:

Notes :

Median

Spectra calculated at 58 damping

84th Percentile $\quad-\ldots-\ldots$ Acceleration in units of $\mathbf{g}$

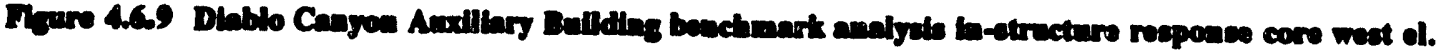
1250 N-S. 


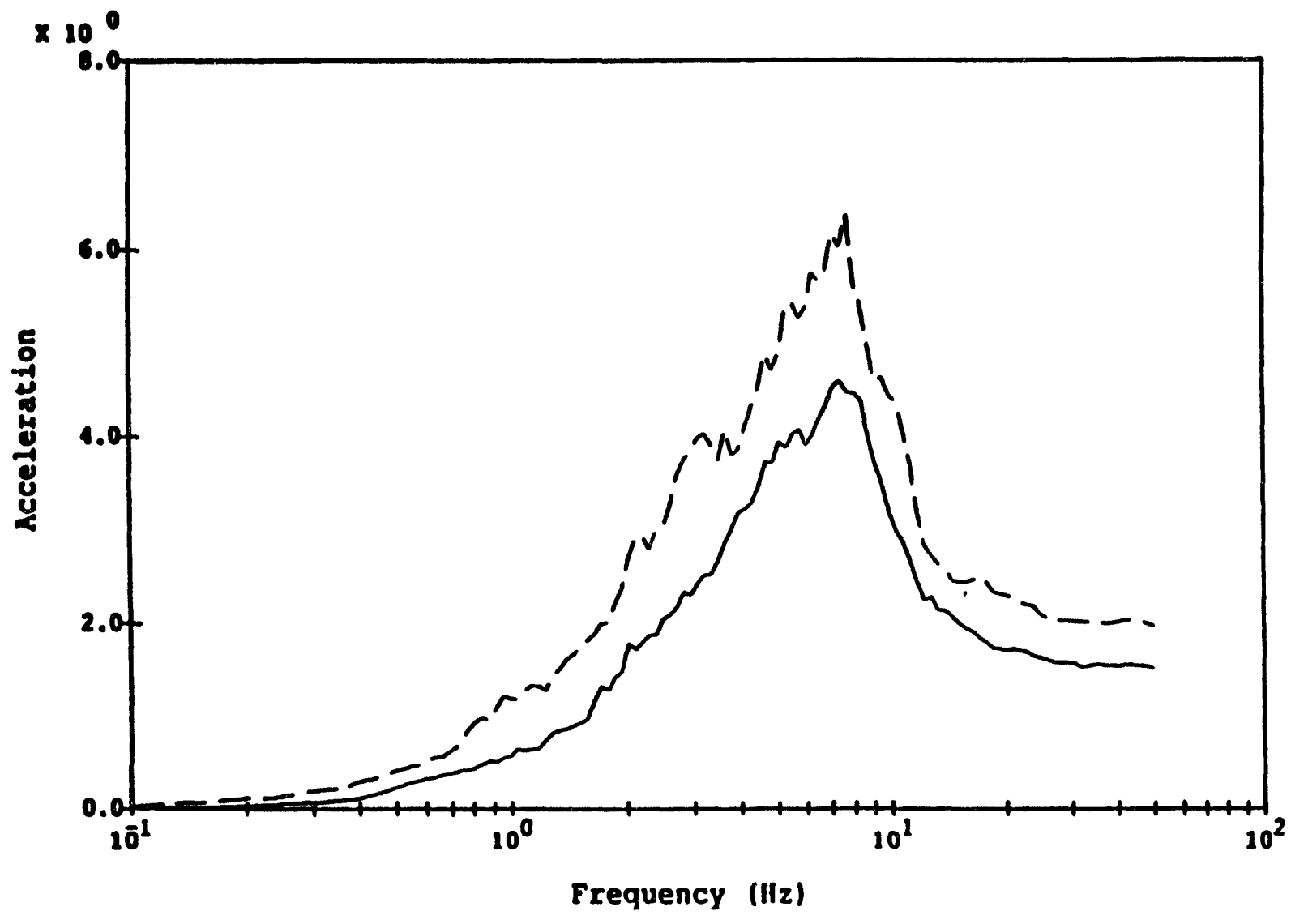

Legend:

Notes:

Median

Spectra calculated at 58 damping

84th Percentile Acceleration in units of $\mathrm{g}$

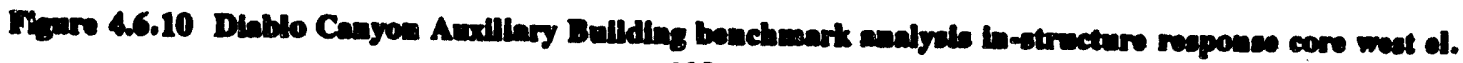
$128^{\circ} \mathrm{E}-\mathrm{W}$. 
4 Reviow of Selanic PRA

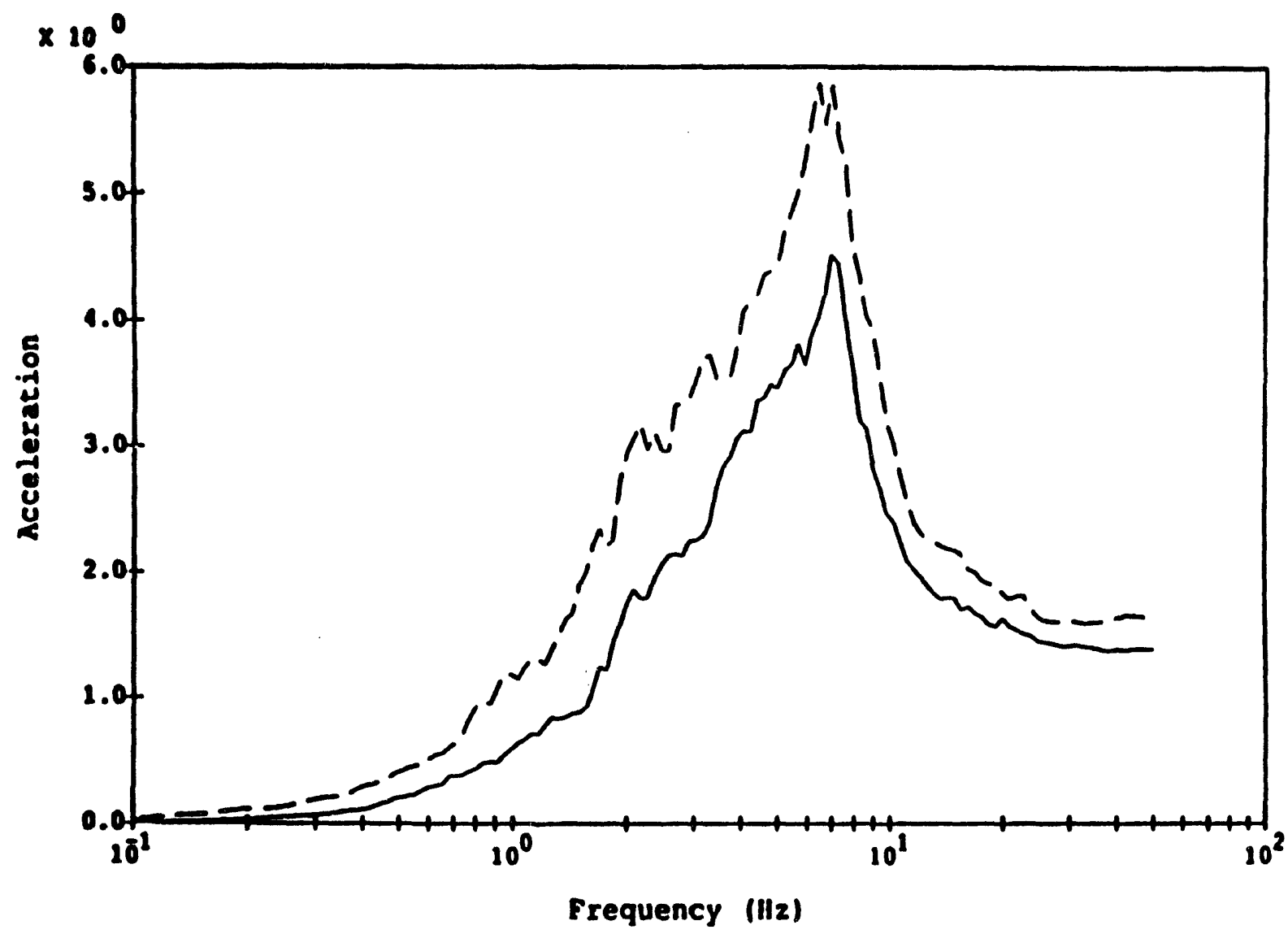

Legend:

Notes:

Median

Spectra calculated at 58 damping

84th Percent1le

$-\cdots-$

Acceleration in units of $\mathbf{g}$

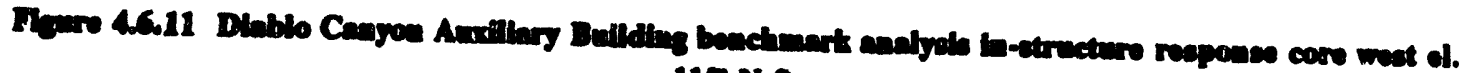
115 N-S.

NUREG/CR-5726 


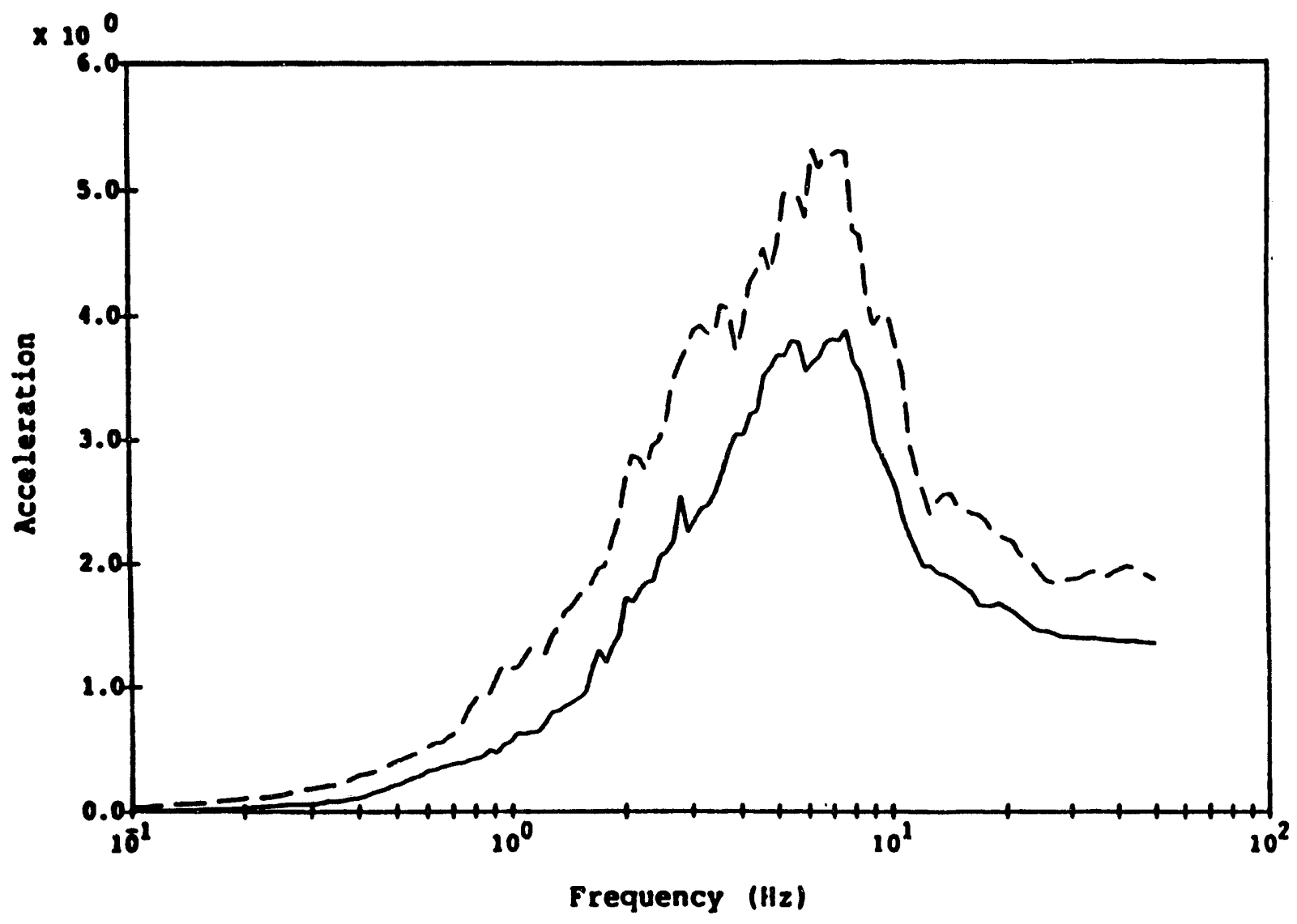

Iegend:

Notes:

Median

Spectra calculated at 58 damping

84th Percentile -

Acceleration in units of $g$

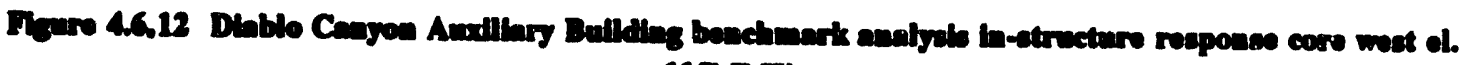
115 E.W. 
4 Reviow of Solemic PRA

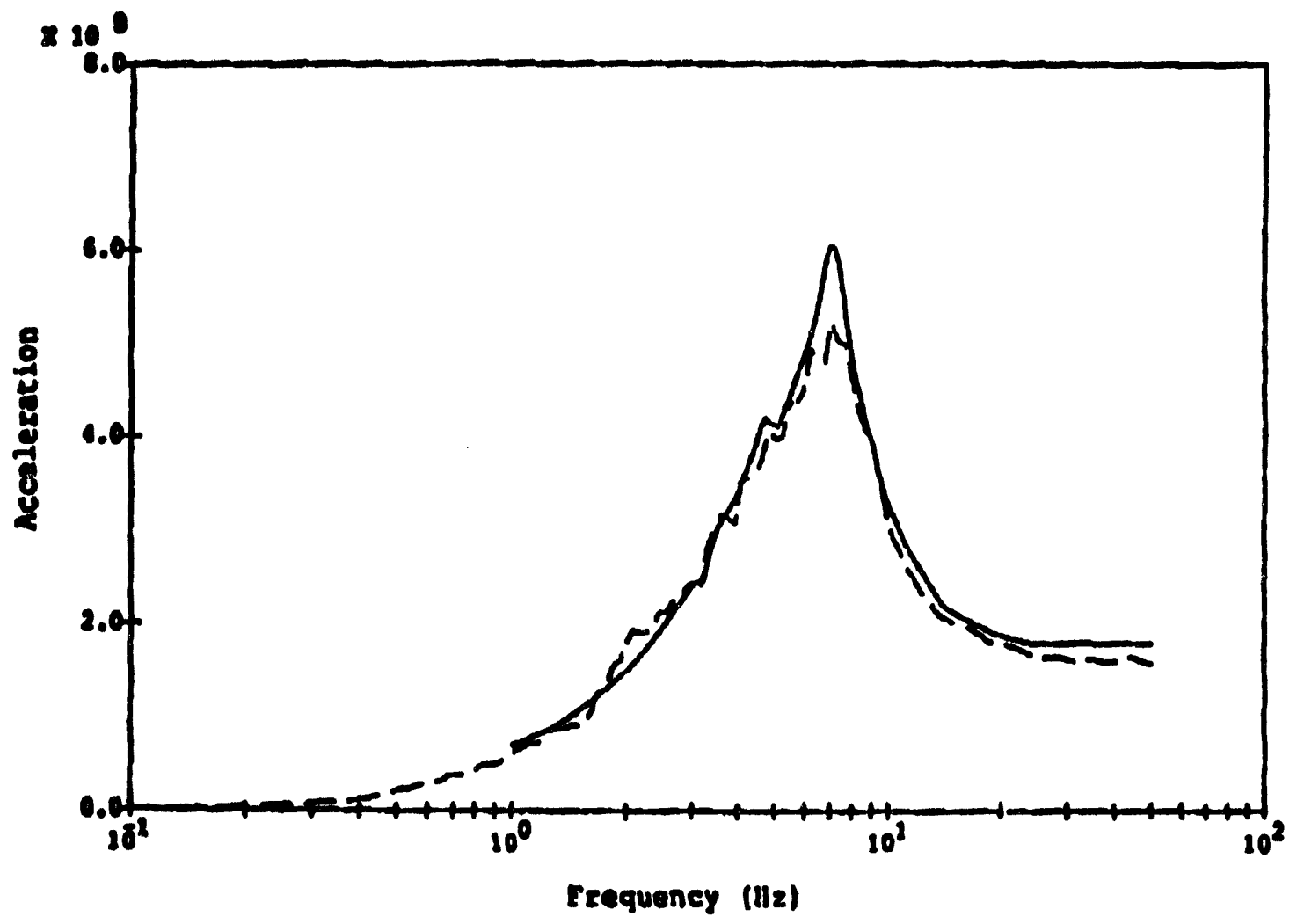

Legend:

PGE

Notes:

New Exp. Design

Spectra calculated at $5 t$ daapling Accelcrat dons in units of $g$

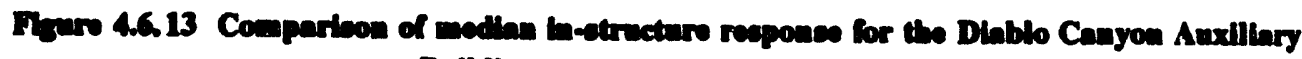
Bullding weat core d. 140 N.S Dir. 


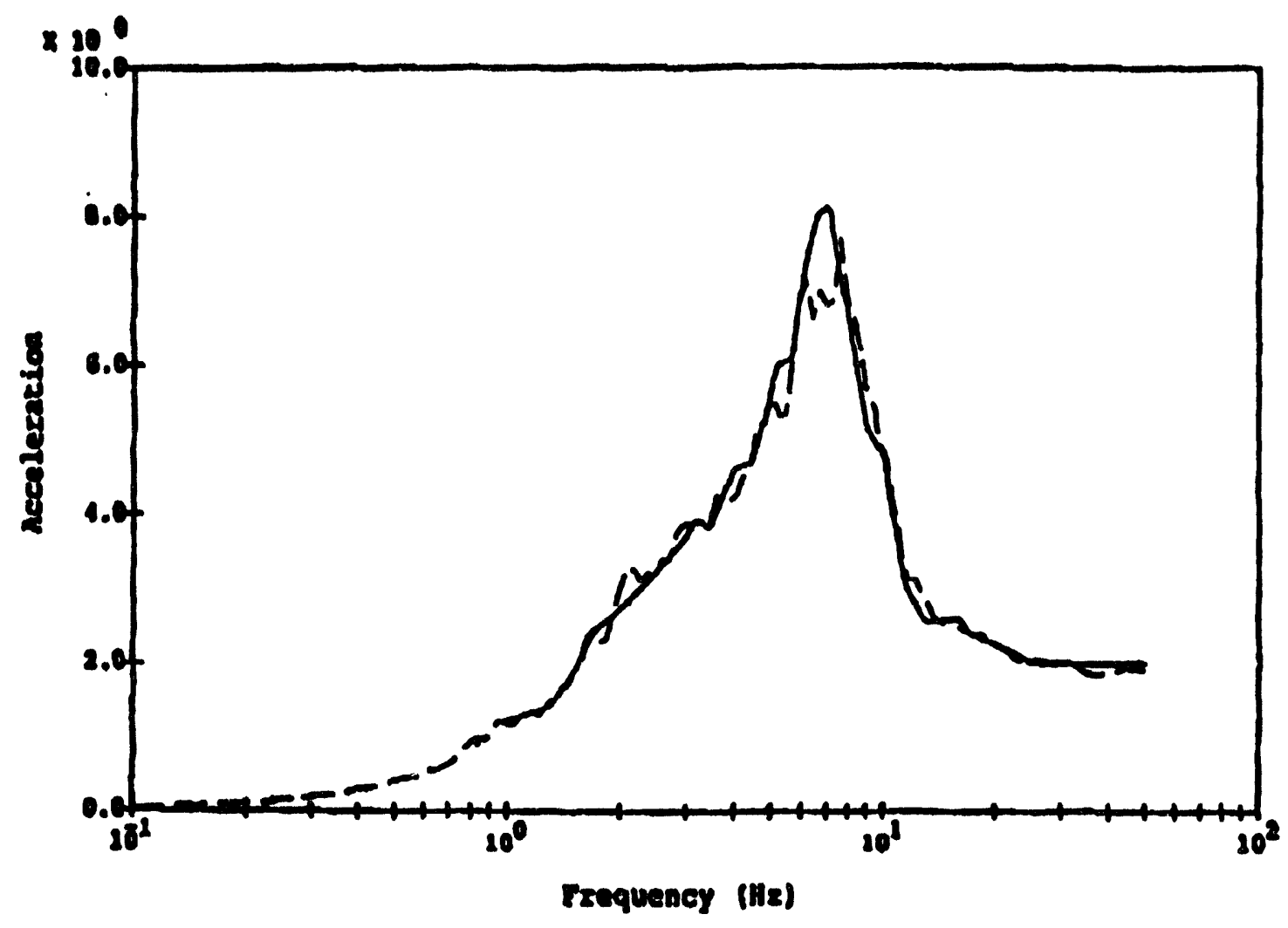

leogends

PGES

Now Exp. Deadgn

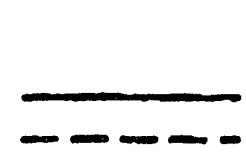

Motes:

Spectre caleulated at 35 damplng nccelerations in undte of $q$

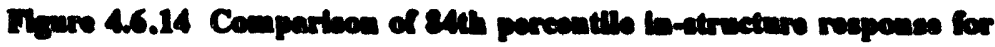

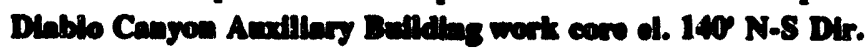




\section{Review of Selemic PRA}

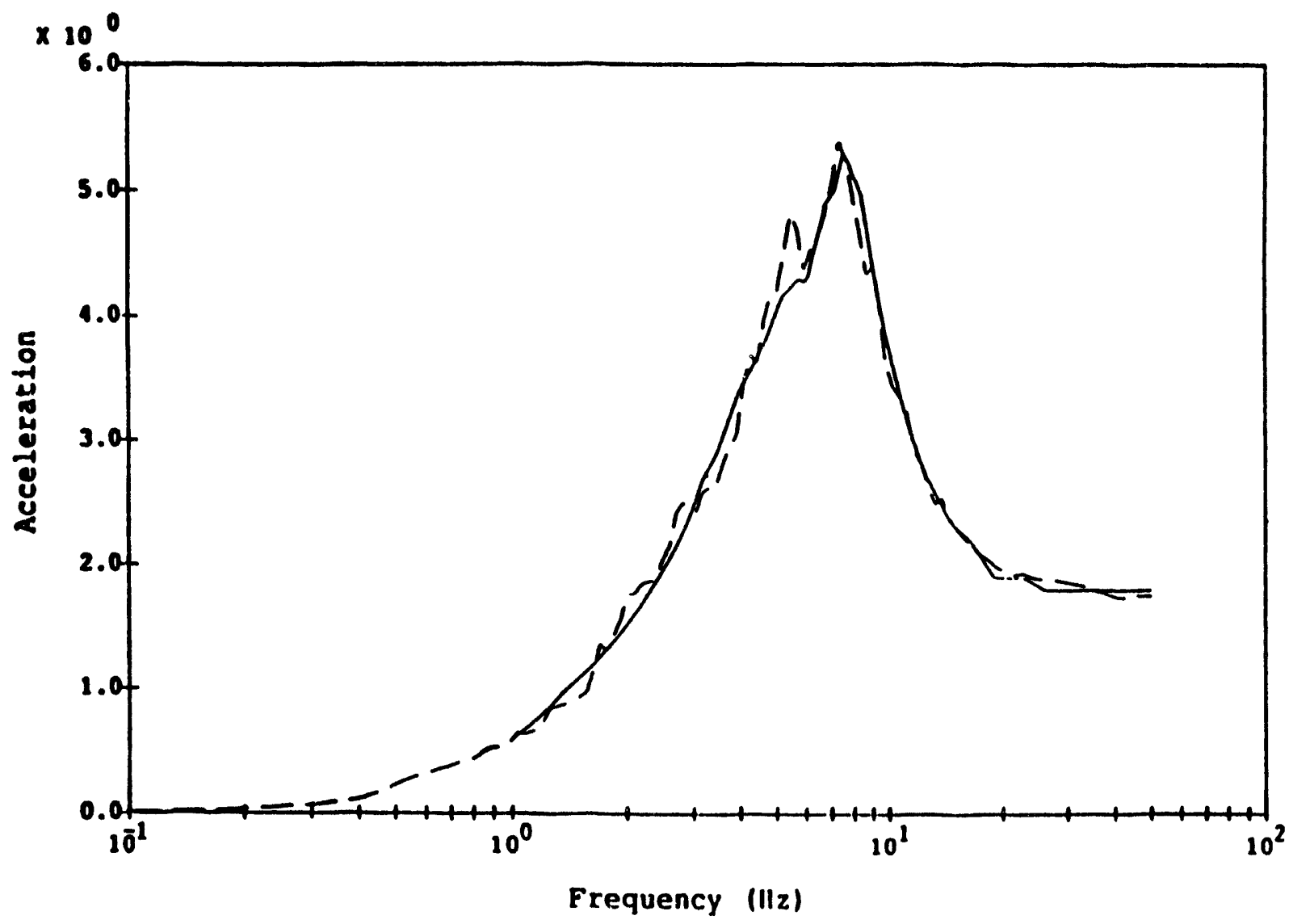

Legend:

\section{PGE E}

New Exp. Design
Notes:

Spectra calculated at 58 damping Accelerations in units of $g$

Fie 4.6.15 Comparison of Median in-etructure reapones for the Diablo Canyon Awxiliary Bullding weet core elev. $140^{\circ} \mathrm{E}=\mathrm{W}$ Dir. 


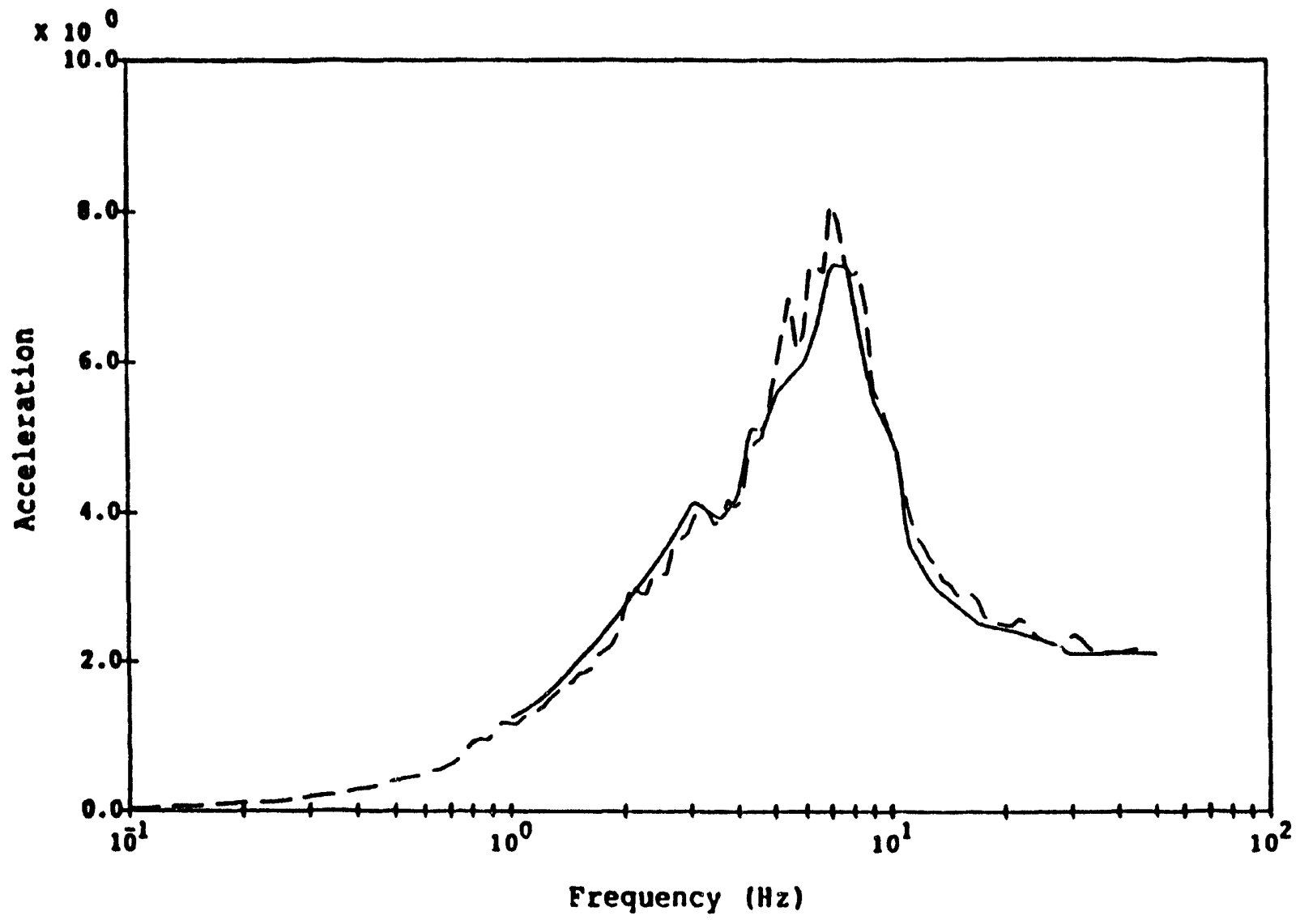

Legend:

Notes:

PGCE

New Exp. Design $\quad-\ldots-\ldots$

Spectra calculated at 58 damping Accelerations in units of $g$

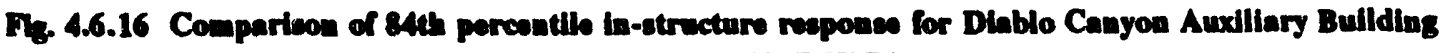
weat cors elov. 140 RW DHr. 
4 Reviow of Salonic PRA

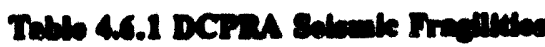

comoonne/strongturen.

Median daceleration $R$

Beta HCLPF U Value

1. Containment building

8.42

0.260

0.300

3.342

2. Concrote Internal blostructure

6.91

0.200

0.310

2.979

3. Intake etructure

8.55

0.280

0.310

3.230

4. Auxiliary bullaling

5.79

0.210

0.260

2.666

5. Turbine bullding ohear wall

4.87

0.260

0.330

1.840

6. Refueling water storage

9.92

0.290

0.360

3.394

7. Auxiliary saltwater piping

9.23

0.180

0.210

4.850

8. Reactor pressure veseel

8.71

0.250

0.330

3.345

9. Reactor internals

20.54

0.400

0.260

3.547

10. Stean generators

6.96

0.310

0.290

2.586

11. Power-operated reller valves

7.62

0.300

0.420

2.323

12. Reactor coolant pumpo

8.82

0.370

0.320

2.825

13. RHR pumps

8.31

0.330

0.220

3.353

14. RHR heat exchangers

8.090 .240

0.270

3.487

15. Safety injection accumulators

10.01

0.290

0.190

4.534

16. Boron injection tank

8.460 .270

$0.190 \quad 3.960$

17. CCN pumps

8.53

0.290

0.210

3.738

28. CCW heat exchangers

6.31

0.270

0.280

2.546

19. CCH surge tanis

7.22

0.330

0.220

2.913

20. Containment spray pumps

8.65

0.290

0.200

3.854

21. Spray additive tank

6.78

0.300

0.180

3.071

22. AFW pumps

7.71

0.290

0.210 .

3.379

23. Diesel generator fuel oll pumps/filter

8.33

0.270

0.230

3.650

24. Diesel generators

7.79

0.260

0.200

3.647

25. Diesel generator radiator/ water pump

8.78

0.290

0.240

3.662 
4 Roviow of Solmic PRA

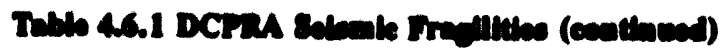
Gomponent/struatiuren

Modian Acenleration Beta

Beta HCLPF I valme

26. Diesel generator excitation cublele

27. Dlesel generator control panel

$\begin{array}{llll}7.40 & 0.290 & 0.350 & 2.574\end{array}$

28. Contalmant lan cooler

$$
4.55
$$

0.300

0.130

2.238

29. supply lans

8.10

0.310

0.330

2.818

30. supply/return sans

9.79

0.330

0.240

3.822

31. A-kv switchgear

21.16

0.330

0.300

3.947

32. Dus $G$ and $H$ potential

7.44

0.320

0.250

2.953 traneformer

10.83

0.320

0.380

3.469

33. 8efeguard relay panel

10.76

0.340

0.360

3.390

34. Batteries

6.04

0.300

0.180

2.736

35. Battery chargers

9.93

0.340

0.400

2.929

36. switchgear/breaker panel

6.67

0.350

0.280

2.359

37. Inverters

6.82

0.310

0.240

2.752

38. 4,260V/480V transformers

5.34

0.280

0.200

2.119

39. Auxiliary relay panel

$7: 25$

0.280

0.150

3.566

40. Main control boards

7.77

0.310

0.270

2.984

41. Hot shutdown panel

7.60

0.270

0.250

3.222

42. Process control and protection

10.78

0.399

0.280

3.569

43. Reactor trip switchgear

7.90

0.300

0.260

3.236

44. Pressure and $\Delta P$ transmitter

8.93

0.270

0.200

4.112

45. Impulse lines

7.09

0.280

0.320

2.634

46. Oefsite power, $230 \mathrm{kV}$

1.69

0.240

0.200

0.818

47. Offaite power, $500 \mathrm{kV}$

0.81

0.240

0.200

0.392

48. BOp piping and supports

11.22

0.390

0.400

3.047

49. Penetrations/penetration boxes

7.38

0.310

0.270

2.834 


\section{Reviow of Selemic PRA}

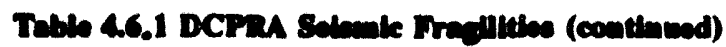

\section{Component/Structures.}

so. HVAC ducting and supports

51. Switchgear/strut

52. Chatter, main control board

53. Chatter, DG control panel

54. Chatter, 4-kV switchgear

55. Chatter, safoguards relay panel

56. Strut for turbine building

57. Bus $F$ potential transformer

58. Safeguard relay panel

59. Centrifugal charging pump
Median Beta Beta HCLPF Acceleration $R \quad U$ Value

$\begin{array}{rlll}9.78 & 0.350 & 0.480 & 2.486 \\ 7.07 & 0.310 & 0.250 & 2.806 \\ 10.00 & 0.010 & 0.010 & 9.675 \\ 7.77 & 0.250 & 0.140 & 4.083 \\ 3.53 & 0.350 & 0.250 & 1.312 \\ 10.00 & 0.010 & 0.010 & 9.675\end{array}$

6.71

0.250

0.320 .

2.620

5.85

0.310

0.380

1.874

5.81

0.340

0.360

1.830

10.16

0.310

0.190

4.452 


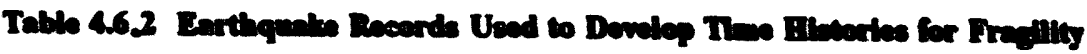

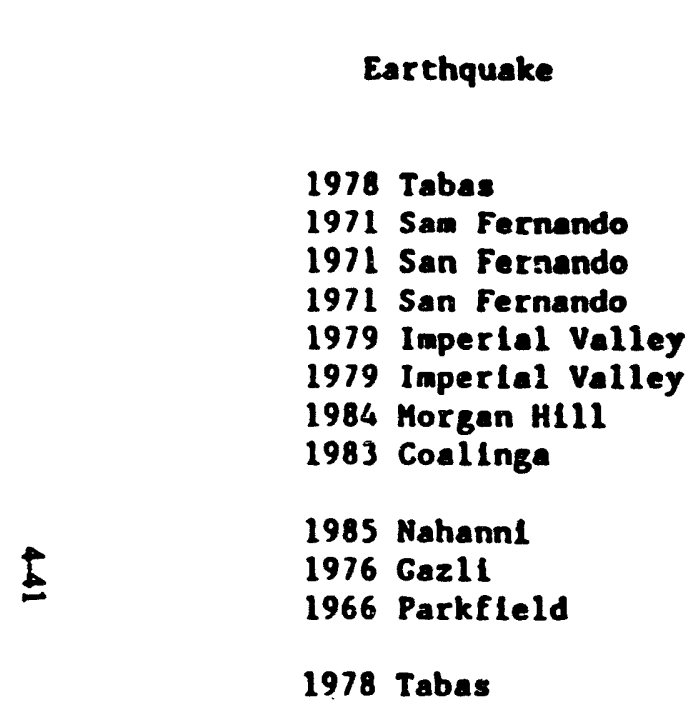

Recording

Station

Tabas

Pacolna Dam

Lake Huges No. 12

Castalc

Differential Array

El Centro No. 4

Coyote Lake Dam

Pleasant Valley Pump

Station (Switchyard)

site 1

Karakyr Point

Temblor

Dayhook
Magn. Dist. Style of

Used (km) Faulting Adjustment

Scalling

Factor

\begin{tabular}{|c|c|c|c|c|}
\hline $\begin{array}{l}7.4 \\
6.6 \\
6.6 \\
6.6 \\
6.5 \\
6.5 \\
6.2 \\
6.5\end{array}$ & $\begin{array}{r}3 \\
3 \\
20 \\
25 \\
5 \\
4 \\
0.1 \\
10\end{array}$ & $\begin{array}{l}\text { Thrust } \\
\text { Thrust } \\
\text { Thrust } \\
\text { Thrust } \\
\text { Strike-slip } \\
\text { Strike-sllp } \\
\text { Strike-sllp } \\
\text { Reverse }\end{array}$ & $\begin{array}{l}\text { None } \\
\text { None } \\
\text { Distance } \\
\text { Distance } \\
\text { Slte response } \\
\text { Slte response } \\
\text { Magnitude } \\
\text { Distance }\end{array}$ & $\begin{array}{l}0.98 \\
1.12 \\
1.01 \\
1.25 \\
1.46 \\
1.80 \\
1.21 \\
1.31\end{array}$ \\
\hline $\begin{array}{l}6.8 \\
6.8 \\
6.1\end{array}$ & $\begin{array}{r}6 \\
3 \\
10\end{array}$ & $\begin{array}{l}\text { Thrust } \\
\text { Reverse } \\
\text { Strike-slip }\end{array}$ & $\begin{array}{l}\text { None } \\
\text { None } \\
\text { Distance and } \\
\text { magnitude }\end{array}$ & $\begin{array}{l}0.84 \\
1.24 \\
2.13\end{array}$ \\
\hline 7.4 & 17 & Thrust & Distance & 1.45 \\
\hline
\end{tabular}


Table 46,3

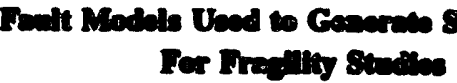

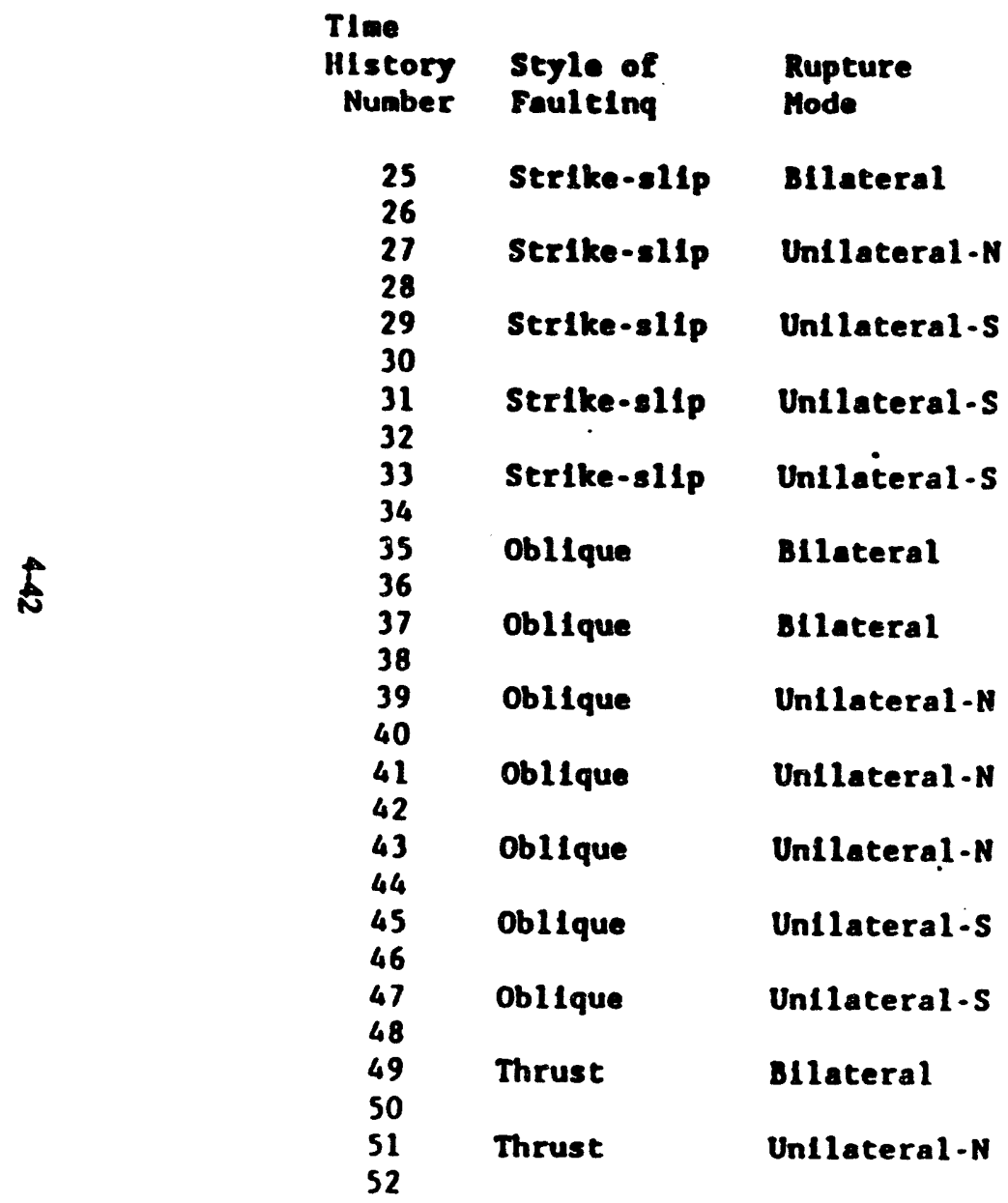

Source Functions

Coalinga aftershock

Imperial Valley aftershock

Imperlal Valley aftershock

Coalinga aftershock

Imperial Valley aftershock

Coalinga aftershock

Coallnga aftershock

Coalinga aftershock

Coalinga aftershock

Imperlal Valley aftershock

Coalinga aftershock

Imperlal Valley aftershock

Coalinga aftershock

Coalinga aftershock
Scalling

Factor

1.38

2.06

2.53

1.68

2.33

1.09

1.33

1.39

2.25

2.25

1.12

1.96

1.23

1.05 


\section{Frequency (Hz)}

Benchmark PG\&E

\section{Mass Particioation}

Benchanark

PGSE

\begin{tabular}{cccr} 
NS & \multicolumn{1}{c}{ EH } & \multicolumn{1}{c}{ NS } & \multicolumn{1}{c}{ EW } \\
0.0 & 2.2 & 0.0 & 2.2 \\
0.0 & 0.0 & 0.0 & 0.0 \\
2.3 & 0.0 & 2.3 & 0.0 \\
0.0 & 65.9 & 0.0 & 66.0 \\
60.2 & 0.0 & 60.2 & 0.0 \\
0.0 & 2.9 & 0.0 & 3.0 \\
0.0 & 0.4 & 0.0 & 0.4 \\
0.0 & 2.2 & 0.0 & 2.1 \\
5.8 & 0.0 & 5.8 & 0.0 \\
0.0 & 0.0 & 0.0 & 0.0 \\
0.0 & 0.1 & 0.0 & 0.0 \\
0.0 & 0.0 & 0.0 & 0.0 \\
0.0 & 0.0 & 0.0 & 0.0 \\
0.0 & 0.4 & 0.0 & 0.4 \\
0.0 & 0.3 & 0.0 & 0.2 \\
0.0 & 8.9 & 0.0 & 8.6 \\
0.0 & 0.0 & 0.1 & 0.0 \\
0.0 & 5.1 & 0.0 & 5.4 \\
0.0 & 1.0 & 0.0 & 0.9 \\
8.2 & 0.0 & 7.8 & 0.0 \\
\hline 76.5 & 89.45 & 76.2 & 89.0
\end{tabular}


4 Review of Seismic PRA

Tabbo 465 Fonndation Rock Profllo and Proportios

\begin{tabular}{ccccccc}
\hline & $\begin{array}{c}\text { Top of } \\
\text { Layer } \\
\text { Elev. } \\
(\mathrm{ft})\end{array}$ & $\begin{array}{c}\text { Thickness } \\
(\mathrm{f})\end{array}$ & $\begin{array}{c}\text { Shear Wave } \\
\text { Velocity } \\
(\mathrm{ft} / \mathrm{sec})\end{array}$ & $\begin{array}{c}\text { Poisson's } \\
\text { Ratio }\end{array}$ & $\begin{array}{c}\text { Mass } \\
\text { Dens1ty } \\
(\mathrm{k}-\mathrm{sec} 2 / \mathrm{ft})\end{array}$ & $\begin{array}{c}\text { Damping } \\
(\mathrm{8})\end{array}$ \\
\hline & & & & & & \\
1 & $8^{\circ}-0^{\prime \prime}$ & 10 & 2600 & 0.45 & .00435 & 2.0 \\
2 & $75^{\circ}-0^{\prime \prime}$ & 20 & 3300 & 0.43 & .00435 & 2.0 \\
3 & $55^{\circ}-0^{n}$ & 125 & 4000 & 0.37 & .00444 & 2.0 \\
4 & $-70^{\circ}-0^{\prime \prime}$ & INF & 4800 & 0.36 & .00463 & 2.0 \\
\hline
\end{tabular}


4 Review of Seimic PRA

Table 4.6,6 Bullding Inpadance Function Rebedmont Fectors

\begin{tabular}{lcc}
\hline Component & \multicolumn{2}{c}{ Embedment Factor } \\
& $K($ Real) & (Imaginary) \\
\hline$X(N-S)$ & 1.4 & 2.4 \\
$Y(E-W)$ & 1.0 & 1.8 \\
$Z$ (Vert.) & N/A & N/A \\
X-X & 1.0 & 1.8 \\
$Y-Y$ & 1.3 & 2.4 \\
$Z-Z$ & 2.0 & 3.0 \\
\hline
\end{tabular}




\section{Reviow of Solemic PRA}

Tablo 4.6 .7 Inpodance Traction Valuo Comparbee at 8 he

\begin{tabular}{|c|c|c|c|}
\hline $\begin{array}{l}\text { Component } \\
\text { (K-FT-SEC) }\end{array}$ & Benchwark & PGSE* & Ratio \\
\hline $\mathrm{K} 11$ & $3.26 \mathrm{E7}$ & $3.37 E 7$ & .97 \\
\hline $\mathbf{K} 22$ & $2.08 E 7$ & $2.15 E 7$ & .97 \\
\hline $\mathbf{k} 33$ & $3.68 \mathrm{E7}$ & $3.37 E 7$ & 1.09 \\
\hline$K 44$ & 2.77E11 & $2.98 E 11$ & .93 \\
\hline $\mathbf{k 5 5}$ & $1.405 \mathrm{E} 11$ & $1.51 E 11$ & .92 \\
\hline K66 & $3.60 E 11$ & $3.92 \mathrm{E} 11$ & .92 \\
\hline D11 & $7.171 \mathrm{ES}$ & $7.58 \mathrm{E} 5$ & .95 \\
\hline D22 & $4.47 \mathrm{ES}$ & 4.97E5 & .90 \\
\hline D33 & 6.9985 & $6.63 E 5$ & 1.05 \\
\hline$D 44$ & $5.50 \mathrm{Eg}$ & $2.99 E 9$ & 1.84 \\
\hline D55 & $2.77 \mathrm{E9}$ & $7.87 \mathrm{E} 8$ & 3.52 \\
\hline D66 & $2.33 \mathrm{Eg}$ & $2.65 \mathrm{Eg}$ & .88 \\
\hline
\end{tabular}


4 Review of Seimic PRA

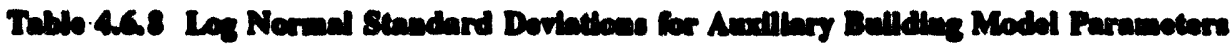

\begin{tabular}{ll}
\hline Parameter & $\beta$ \\
\hline Structure Frequency Rat10 & .25 \\
Structure Dampling & .35 \\
Rock Modulus Ratio & .45 \\
\hline
\end{tabular}


4 Review of Selemic PRA

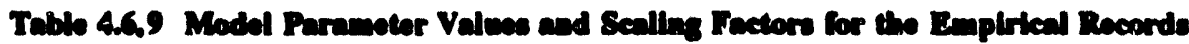

\begin{tabular}{|c|c|c|c|c|c|}
\hline \multirow{2}{*}{$\begin{array}{l}\text { Analysis } \\
\text { Number }\end{array}$} & $\begin{array}{l}\text { Input } \\
\text { Hist } \\
\text { Numb }\end{array}$ & $\begin{array}{l}\text { Time } \\
\text { ory } \\
\text { er }\end{array}$ & \multirow{2}{*}{$\begin{array}{l}\text { Structure } \\
\text { Damping } \\
\text { (8) }\end{array}$} & \multirow{2}{*}{$\begin{array}{c}\text { Structure } \\
\text { Frequency } \\
\text { Ratio }\end{array}$} & \multirow{2}{*}{$\begin{array}{l}\text { Rock } \\
\text { Modulus } \\
\text { Ratio }\end{array}$} \\
\hline & NS & EW & & & \\
\hline 1 & 1 & 2 & 6.80 & $0.950 \times 0.9-0.855$ & 1.335 \\
\hline 2 & 2 & 1 & 4.71 & $0.915 \times 0.9-0.824$ & 1.124 \\
\hline 3 & 3 & 4 & 9.46 & $0.983 \times 0.9-0.885$ & 0.771 \\
\hline 4 & 4 & 3 & 12.45 & $0.803 \times 0.9=0.721$ & 1.737 \\
\hline 5 & 5 & 6 & 4.34 & $0.903 \times 0.9=0.813$ & 1.081 \\
\hline 6 & 6 & 5 & 5.10 & $1.174 \times 0.9-1.057$ & 1.238 \\
\hline 7 & 7 & 8 & 5.82 & $0.814 \times 0.9-0.733$ & 1.486 \\
\hline 8 & 8 & 7 & 6.33 & $1.009 \times 0.9-0.908$ & 0.986 \\
\hline 9 & 9 & 10 & 10.09 & $1.217 \times 0.9-1.095$ & 2.187 \\
\hline 10 & 10 & 9 & 10.71 & $1.509 \times 0.9-1.358$ & 0.986 \\
\hline 11 & 11 & 12 & 4.05 & $0.644 \times 0.9-0.580$ & 1.434 \\
\hline 12 & 12 & 11 & 8.07 & $0.871 \times 0.9=0.784$ & 0.900 \\
\hline 13 & 13 & 14 & 6.28 & $0.855 \times 0.9-0.770$ & 0.540 \\
\hline 14 & 14 & 13 & 9.97 & $1.344 \times 0.9=1.285$ & 1.033 \\
\hline 15 & 15 & 16 & 7.29 & $1.068 \times 0.9-0.961$ & 1.651 \\
\hline 16 & 16 & 15 & 7.68 & $0.750 \times 0.9-0.675$ & 0.853 \\
\hline 17 & 17 & 18 & 5.49 & $1.428 \times 0.9-1.285$ & 0.934 \\
\hline 18 & 18 & 17 & 8.02 & $1.134 \times 0.9-1.012$ & 0.672 \\
\hline 19 & 19 & 20 & 5.33 & $0.957 \times 0.9-0.861$ & 1.167 \\
\hline 20 & 20 & 19 & 7.01 & $1.121 \times 0.9-1.009$ & 0.512 \\
\hline 21 & 21 & 22 & 6.08 & $1.047 \times 0.9=0.942$ & 0.697 \\
\hline 22 & 22 & 21 & 8.57 & $0.734 \times 0.9-0.661$ & 0.738 \\
\hline 23 & 23 & 24 & 8.73 & $1.264 \times 0.9-1.138$ & 1.311 \\
\hline 24 & 24 & 23 & 6.72 & $1.097 \times 0.9-0.987$ & 0.830 \\
\hline
\end{tabular}




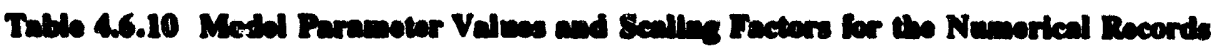

\begin{tabular}{|c|c|c|c|c|c|}
\hline \multirow{2}{*}{$\begin{array}{l}\text { Analysis } \\
\text { Number }\end{array}$} & $\begin{array}{l}\text { Input } \\
\text { Hist } \\
\text { Numb }\end{array}$ & $\begin{array}{l}\text { TIme } \\
\text { ory } \\
\text { ar }\end{array}$ & \multirow{2}{*}{$\begin{array}{c}\text { seructure } \\
\text { Damping } \\
\text { (\$) }\end{array}$} & \multirow{2}{*}{$\begin{array}{c}\text { Structure } \\
\text { Frequency } \\
\text { Ratic }\end{array}$} & \multirow{2}{*}{$\begin{array}{l}\text { Rock } \\
\text { Modulus } \\
\text { Ratio }\end{array}$} \\
\hline & NS & EW & & & \\
\hline 25 & 26 & 25 & 9.28 & $0.892 \times 0.9-0.803$ & 0.954 \\
\hline 26 & 27 & 28 & 5.42 & $0.865 \times 0.9=0.779^{\circ}$ & 0.566 \\
\hline 27 & 29 & 30 & 8.77 & $1.061 \times 0.9-0.955$ & 0.669 \\
\hline 28 & 31 & 32 & 7.90 & $1.218 \times 0.9-1.096$ & 1.510 \\
\hline 29 & 33 & 34 & 5.08 & $1.265 \times 0.9=1.139$ & 1.693 \\
\hline 30 & 35 & 36 & 10.57 & $0.801 \times 0.9=0.721$ & 0.924 \\
\hline 31 & 37 & 38 & 5.56 & $0.928 \times 0.9=0.835$ & 1.016 \\
\hline 32 & 39 & 40 & 7.08 & $0.811 \times 0.9=0.730$ & 1.190 \\
\hline 33 & 41 & 42 & 9.77 & $1.025 \times 0.9-0.923$ & 1.190 \\
\hline 34 & 43 & 44 & 6.05 & $1.180 \times 0.9-1.062$ & 0.747 \\
\hline 35 & 45 & 46 & 7.56 & $0.712 \times 0.9-0.641$ & 1.299 \\
\hline 36 & 47 & 48 & 6.58 & $1.430 \times 0.9-1.287$ & 1.098 \\
\hline 37 & 49 & 50 & 6.75 & $0.986 \times 0.9=0.887$ & 0.701 \\
\hline 38 & 51 & 52 & 4.35 & $1.129 \times 0.9-1.016$ & 0.864 \\
\hline
\end{tabular}




\section{Reviow of Solmenic PRA}

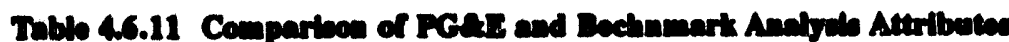

\begin{tabular}{|c|c|c|}
\hline & PGGE & $\begin{array}{l}\text { Benchmark } \\
\text { Analysis }\end{array}$ \\
\hline $\begin{array}{l}\text { Earthquake } \\
\text { Ground } \\
\text { Motions }\end{array}$ & $\begin{array}{l}\text { Ensemble of } 38 \\
\text { earthquake simulations } \\
\text { comprised of } 12 \text { palrs } \\
\text { recorded notions with } \\
N-S \text { and E-W components } \\
\text { 1nterchanged to produce } \\
24 \text { simulatlons and } 14 \\
\text { numerically simulated } \\
\text { earthquakes. Each ground } \\
\text { motion palr was scaled } \\
\text { to an average } 58 \text { damped } \\
\text { spectral acceleration of } \\
2.0 \mathrm{~g} \text { over the frequency } \\
\text { range of } 4.8 \text { to } 14.7 \mathrm{~Hz} \text {. }\end{array}$ & Same \\
\hline SSI Model & $\begin{array}{l}\text { Foundation Impedance } \\
\text { calculated for an assumed } \\
\text { flat surface foundation } \\
\text { using CLASSI. Frequency } \\
\text { - dependent impedances } \\
\text { approximated by values } \\
\text { at } 8 \text { Hz. Values corrected } \\
\text { for embedment based on } \\
\text { SASSI results. }\end{array}$ & $\begin{array}{l}\text { Same, except } \\
\text { both frequency. } \\
\text { dependent and } \\
\text { frequency. } \\
\text { independent } \\
\text { Impedances were } \\
\text { considered. }\end{array}$ \\
\hline Structure Model & $\begin{array}{l}\text { Three-stick simplified } \\
\text { auxiliary building model. }\end{array}$ & Same \\
\hline $\begin{array}{l}\text { Parameter Variation } \\
\text { and experimental } \\
\text { design }\end{array}$ & $\begin{array}{l}\text { Three parameters assumed } \\
\text { to be variable .. rock } \\
\text { shear modulus. fixed-base } \\
\text { structure frequencles, and } \\
\text { structure modsl damping; } \\
\text { (median, log-normal } \\
\text { standard deviation) were } \\
\text { (Table } 4.6-4,0.45) \text {, } \\
(0.9 \times \text { best est. fixed. } \\
\text { base freqs.. } 0.25) \text {, and } \\
(78,0.35) \text { respectively. }\end{array}$ & Same \\
\hline
\end{tabular}




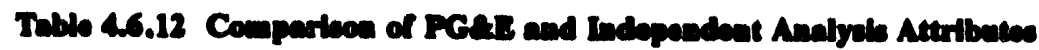

PG\&E

Table $4.6 \cdot 10$

Earthquake

Ground

Motions

SSI modeI

Seructure

model

Paraweter Varlation and experimental

design

Table 4.6-10

Table $4.6 \cdot 10$

Table $4.6 \cdot 10$
Independent

Analysis

Table $4.6-10$

Table $4.6 \cdot 10$

Table 4.6-10.

\author{
Four parameters \\ assumed to be \\ variable .. rock \\ shear modulus, rock. \\ material damping. \\ fixed-base structure \\ frequencies, and \\ structure modal \\ damping: (median, \\ log normal standard \\ deviation) were \\ (Table 4.6.4, 0.45) \\ (Table $4.6-4,0.5$ ). \\ $(0.9 \times$ best est. \\ fixed-base freqs. . \\ $0.25)$, and (78. \\ 0.35 ) respectively. \\ One experimental \\ design of $\mathrm{N}-38$ was \\ constructed.
}




\section{Reviow of Soismic PRA}

Table 4.6.13 North-South Roopones

Combined Vartabillty (Bc)

\begin{tabular}{lccc}
\multicolumn{3}{c}{ (a) Elevation $100 \mathrm{ft}$} \\
\\
& \multicolumn{3}{c}{ Frequency Range $(\mathrm{Hz})$} \\
& 3.5 to 5 & 5 to 11 & 11 to 30 \\
PGSE & & & \\
Benchmark & 0.24 & 0.27 & 0.18 \\
Independent & 0.24 & 0.26 & 0.17 \\
& 0.21 & 0.28 & 0.22
\end{tabular}

(b) Elevation $215 \mathrm{ft}$

3.5 to $5 \stackrel{\left.\begin{array}{c}\text { Frequency } \\ 5 \text { to }\end{array} \text { Range ( } 7 \text { to }\right)}{71} 11$ to 30

$\begin{array}{lllll}\text { PG\&E } & 0.24 & 0.32 & 0.27 & 0.18 \\ \text { Benchmark } & 0.24 & 0.31 & 0.26 & 0.26 \\ \text { Independenc } & 0.25 & 0.23 & 0.32 & 0.22\end{array}$

(c) Elevation $140 \mathrm{ft}$

0.37

0.36

0.29

0.18

0.25

0.33

0.31

0.40

0.18

0.27

(d) Elevation $164 \mathrm{ft}$.
Frequency Range ( $\mathrm{Hz}$ )

3.5 to $5 \quad 5$ to 7 to $11 \quad 11$ to 30

PG\&E

0.26

0.41

0.31

0.18

0.27

0.41

0.29

0.19

Independent

0.29

0.37

0.44

0.22 
Tablo 4.6.14 Eant-Woet Roupones

Combland Vartabulity (Be)

PG\&E

Benchmark

Independent

PG\&E

Benchmark

Independent (a) Elevacion $100 \mathrm{ft}$

Frequency Range ( $\mathrm{Hz}$ )

$\begin{array}{ccc}3.5 \text { to } 6 & 6 \text { to } 11 & 11 \text { to } 30 \\ 0.24 & 0.30 & 0.25 \\ 0.26 & 0.30 & 0.30 \\ 0.25 & 0.25 & 0.30\end{array}$

(b) Elevacion $115 \mathrm{ft}$

Frequency Range ( $\mathrm{Hz}$ )

3.5 to $6 \quad 6$ to $11 \quad 11$ to 30
0.24
0.30
0.25
0.27
0.32
0.29
0.27
0.32
0.30

(c) Elevation $140 \mathrm{ft}$

Frequency Range ( $\mathrm{Hz}$ )

3 to $11 \quad 11$ to 30

PG\&E

Benchmark

Independent

$\begin{array}{ll}0.31 & 0.25 \\ 0.30 & 0.25 \\ 0.34 & 0.23\end{array}$

(d) Elevation $164 \mathrm{ft}$ :

Frequency Range ( $\mathrm{Hz}$ )
3 to 11
11 to 30

PG\&E

0.35

0.26

0.34

0.26

0.35

0.28 
4 Roviow of Solonic PRA

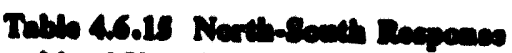

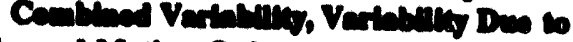

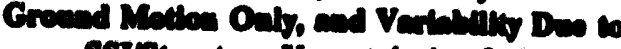

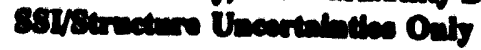

Benchmark GH TH only SSI/Str only

0.24

0.07

0.26

0.21

0.21

0.22

0.08

(b) Elevation $215 \mathrm{ft}$

$\begin{array}{cccc}3.5 \text { to } 5 & \begin{array}{c}\text { Erequency Range } \\ \text { 5 to } 7\end{array} & \text { 7 to } 11 & 11 \text { to } 30 \\ 0.24 & 0.31 & 0.26 & 0.16 \\ 0.23 & 0.22 & 0.21 & 0.18 \\ 0.11 & 0.21 & 0.30 & 0.12\end{array}$

(c) Elevation $140 \mathrm{ft}$

Benchmark

GM IH only

SSI/Str only

0.11

0.21

0.12

(a) Elevation 200 fts

0.5 to 5 Frequancy Range $(\mathrm{Hz}) \quad 11$ to 30 
4 Review of Seismic PRA

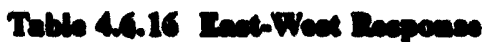

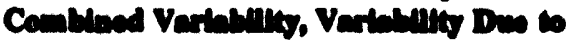

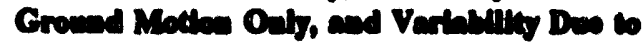

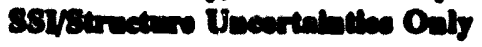

Benchmark GM TH only SSI/SEr only

Benchmark GM TH only SSI/Str only (a) Elevention $100 \mathrm{ft}$

Frequency Range ( $\mathrm{Hz}$ )

3.5 to 6

6 to 11

0.26

0.25

0.30

0.25

0.08

0.16

(b) Elevation $115 \mathrm{ft}$

Frequency Range ( $\mathrm{Hz}$ )

3.5 to $6 \quad 6$ to $11 \quad 11$ to 30

0.27

0.32

0.29

0.24

0.23

0.23

0.24

0.14

0.12 (c) Elevation $140 \mathrm{ft}$

Frequency Range ( $\mathrm{Hz}$ )
3 to 11
11 to 30

Benchmark

GM TH only

SSI/Str only
0.30
0.25
0.24
0.23
0.24
0.14

(d) Elevation $164 \mathrm{ft}$.

Frequency Range (Hz)
3 to 11
11 to 30

Benchmark

GI TH only

SSI/Str only

$\begin{array}{ll}0.34 & 0.26 \\ 0.25 & 0.27 \\ 0.28 & 0.14\end{array}$




\subsection{Systems Analysis and Risk Quantification}

\subsubsection{Systems Analysis}

The crux of the systems analysis for seismic events is contained in $t$ he Seismic Early Frontline event tree (Figure 6-47 of DCPRA), the Electric Power Support event tree (Figure 6-44 of DCPRA), the Actuation and Mechanical Support event tree (Figure 6-45 of DCPRA) and the Seismic Failure Impacts Table (Table 6-43 of DCPRA). In addition, "support system to support system" and "support system to frontline system" dependencies are given on Tables 2.1.1 and 2.1.2 respectively within Section 2 of this report.

The frontline event tree (Figure 4.7.1) defines the accident scenarios which follow the occurrence of an earthquake in terms of successes or failures of the top events identified in the tree. In keeping with the PL\&G methodology, the top events include support state frequencies, LOCA events, component failures (e.g., RWST, PORVs, etc.), frontline systems (e.g., charging system, auxiliary feedwater system, etc.) and finally, human errors. In general, failures of these top events may be due to either seismic or random causes.

As shown on Figure 4.7.1, there are 13 top events whose success or failure determines the scenario associated with an earthquake event. A description of each of these top events is presented in Table 4.7.1 (taken from the DCPRA). Note that both transients and LOCAs are included on this single tree. Small LOCAs are implied by failure of top event PR (pressure relief function) and failure of event SE (seal cooling). All larger LOCAs are contained in top event EL (excessive LOCA). The LOCAs associated with event EL are conservatively assumed to be beyond the capacity of the ECCS mitigating systems. This, of course, is a significant conservatism. If neither failure of events PR or EL occur, the remaining scenarios are associated with transients. The various types of transients (LOSP, station blackout, or general transients with a PCS initially available) are determined by the availability of the various electrical and mechanical support systems as determined by the electrical and mechanical support trees. Relay chatter is

explicitly included in this tree in event CT. This is the union of all events whose chatter could result in loss of all $\mathrm{AC}$ power to the site. Operator recovery of the various busses and switchgear failed by relay chatter is incorporated in event OC. Failure of event $O C$ results in continued loss of all vital ac power to the site. Instrumentation and signals to the control room operators are included in event ID. It is assumed that loss of all indications to the operator will lead directly to core damage, even if all other safety and mitigating systems are not failed mechanically. If relay chatter has occurred, and (among other things), failed the motor-driven AFW pumps, the event TD is asked which represents the status of the turbine driven auxiliary feedwater pump. This is asked primarily because it shortens the time available to allow successful recovery from the relay chatter event and thus affects the probabilities of non-recovery of relay chatter. Failure of the RWST (RW), the charging system (CH), and the safety injection system (SI) are the usual PWR safety mitigating systems used to respond to LOCAs or transients with AFWS unavailable. Similarly the auxiliary feedwater system (AW) is used to respond either to small LOCAs or normal transients. Event $O B$ is the operator action of initiating and performing feed and bleed given that the auxiliary feedwater system has failed. The event is modeled here consists entirely of human actions and not seismic mechanical failures of the equipment (injection pumps, PORVs) needed to perform feed and bleed. Finally, event HS is a general human error to fail to maintain the system in hot standby condition after this has successfully been reached.

Based on a review of seismic event trees associated with Westinghouse PWR reactors and other PRAs (NUREG 1150, TAP A-45, Zion and Indian Point) the top events included in the Diablo Canyon seismic early frontline tree are found to be a complete and logical set of systems and events which can be used to model 
accident sequences typical of commercial PWR reactors. In addition to the normal mitigating safety systems, instrumentation and relay chatter are explicitly included in a logical (if conservative) manner. Only failures of safety systems early in an accident scenario are included in this early frontline tree, and failures during later portions of the scenario (recirculation) are not explicitly included. However, this is consistent with past experience in all PRAs for PWRs which shows that sequences involving only late failures of the safety systems are negligible. Failure of the reactor protection system is not explicitly shown on this seismic early frontline tree, however, it is explicitly considered (on a train level) in the mechanical support tree which is used to describe initial conditions for the sequences shown on the early frontline tree. Further, sequences normally associated with loss of offsite power (LOSP) and with station blackout (SBO) are again not explicitly shown on this tree as they are explicitly contained in the various sequences in the electrical support tree, again which determines initial conditions for the early frontline tree. Thus, it is concluded that the sequence of scenarios and events which can be portrayed by the early frontline tree represents a reasonable and complete approach to modeling the Diablo Canyon seismic scenarios.

The early frontline tree shown on Figure 4.7.1 represents a very large number of accident sequences. Even though $\mathbf{4 2}$ specific end states are shown on this figure, the dotted lines indicate locations where portions of the tree are to be transferred and the tree expanded. A definition of the subtrees (transfers) is shown at the bottom of the figure. Altogether, this tree represents over 200 separate end states (i.e., accident sequences) which must be evaluated. Further, since these accident sequences may be evaluated with a variety of different initial conditions (as determined by the electrical and mechanical support event trees) a very large number of accident sequences must be evaluated. For these trees, approximately 3000 accident sequences were numerically evaluated, but only the dominant sequences were retained for further study.

The electric power support event tree is shown in Figure 4.7.2. In addition to the earthquake initiating event (IE) a total of 21 top events are modeled on this electric power support event tree. Definitions of these basic events are shown on Table 4.7.2. The top events on this tree are essentially the different trains of the various electrical support systems. Loss of offsite power is the first event shown (OG). The mechanical and actuation support event tree is shown on Figure 4.7.3. In this case, the various trains of actuation and mechanical support are shown as top events on this tree. Definitions of the top event on this tree are shown on Table 4.7.3. Together, the electric power and mechanical support event trees generate a large number of support system states (SS) to be used in evaluating the accident sequences as defined by the seismic early frontline tree. As described below, different end states from the mechanical event and electrical event trees are used to define different numerical failure probabilities for each of the top events on the seismic early frontline tree. Thus, the same top event will have a variety of numerical values depending on the support state conditions being evaluated. Thus, as is typical in the PL\&G methodology, a very large number of accident sequences must be evaluated and numerically screened before further evaluation is performed.

The impact of seismic component failures on the top events of the early frontline event tree and the electrical and mechanical support trees are given on the Seismic Failure Impact table (Table 6-43 of DCPRA). A modified version of this table showing the component failure expressions for each top event is shown in Table 4.7.4. In the DCPRA it is assumed that the various contributors are independent, and that their contributions can be combined by the "OR" logical operator. Thus, in effect, this table gives Boolean expressions for each top event in terms of component failures and serve as fault trees for the systems under consideration. In certain cases, the impacts of multiple redundant components were combined so as to conservatively estimate the failure probability of the top event, but this is not inappropriate provided undue conservatism does not skew the overall risk picture. The Boolean expressions implied by this table were reviewed and found to be appropriate. 


\section{Review of Seismic PRA}

A notable feature of these Boolean expressions for the top event failures is the explicit inclusion of piping failures. Piping associated with various systems were lumped into segments and a number of segments were aseociated with each top event system. A generic pipe support fragility was associated with each pipe segment and then the various segments associated with each system were "OR-ed" together to yield the piping failure contribution to the system failure probability. The effect is that the probability of failure increases (approximately linearty) with the number of piping segments included. This approach is conservative in that the inclusion of any degree of correlation between the piping failures would tend to reduce the contribution of the segments to the overall failure probability.

Another conservative assumption is made when a single system is modeled by component failures rather than having the various trains of the system modeled by component failures. For example, the component cooling water system (CC) is modeled by failure of the RHR heat exchangers or CCW pumps or CCW heat exchangers, etc. Since multiple heat exchangers or pumps are involved, it would be possible to break this out further and hence reduce conservatism in the modeling of this system. However, the approach taken is indeed conservative.

Note that when individual trains of this system are involved (for example, vital AC power trains AF, AG, and AH) the component failures contributing to these train failures involve both failures of components which affect only a single train (e.g., 4kV switchgear) and failures of buildings which may fail all trains of the system (e.g. turbine building shear wall). This mix of single component failures and more global failures is satisfactory provided that in numerically evaluating the split fractions, different split fractions are used depending on whether 1, 2, or 3 trains have failed. In determining these split fractions, account must be taken of the fact that the global failures affect more than one train at a single time. This has evidently been done as described in the point estimate calculations.

\section{Mean Point Detinnte Evaluation}

As described earlier, the initial quantification consisted of a mean point estimate evaluation of the all accident scenarios $s 0$ as to identify the main contributors to core damage and to delete negligible scenarios from future consideration. The actual sequences themselves, in terms of basic events, are given in Appendix $J$ to the DCPRA. The split fractions for the basic events (conditional on the earthquake level) are given on Table 6-46 of the DCPRA, at six different ground acceleration intervals. A sample of this table is given in Figure 4.7.4. It should be noted that this table is somewhat mislabeled and these should actually be labeled as mean conditional top event split fractions for the six acceleration ranges. Further, a point of confusion was that the values shown for the range $\mathbf{0 . 0 - 0 . 2}$ were not, in fact, used in the final quantification. These rather, represent random failures for the various top events in question. In addition, the numerical values presented are in fact a weighted average of the failure probabilities of the Boolean expressions representing the top events divided by the probability of the mean hazard curve for the interval in question. Thus, these values, when multiplied by the probability of the hazard curve interval, yield the correct unconditional probability of seismic failure for the top event. In addition, note that the top events have numbers appended to them. For example, top event DG occurs as either DG1 or DG2. The different numbers represent different support states and, in effect, allow for the fact that the Boolean expressions defining the top event DG include some single failures as well as some global failures. For example, if a accident sequence involved only the failure of DG then the split fraction DG1 would be used in evaluating the accident sequence. However, if the accident sequence involved failures of both busses DF and DG then the numerical values coinciding with DF1 and DG2 would be used in evaluating the accident sequence. 
A number of these mean split fractions were checked. It was found that, for those split fractions checked, reasonably close agreement was obtained. A difficulty in reviewing the split fractions used for the point estimate calculation is that there are some small numerical inconsistencies between DCPRA Table 6-44, which presents conditional mean failure fractions for key structures or components and Table 6-46 which gives the split fractions for the top events. For those top events which involve only a single component failure (e.g., offsite power, reactor trip, etc.) the numerical values of the split fractions should be identical. However, small discrepancies exist. The magnitudes of the discrepancies are not considered significant, and are undoubtedly due to minor changes in the mean values of the hazard curves utilized in evaluating these split fractions.

A related and important point is that nowhere in the DCPRA are the actual hazard curve increment frequencies presented. This is, of course, essential for checking the numerical values of the unconditional failure fractions for the top events or for the key structures and components, since these are weighted averaged values over the increment. For the purposes of the review, these hazard increment frequencies were provided on diskette to the reviewers.

In the numerical evaluation process, each sequence was evaluated for each of the six earthquake levels, and reported individually. This allowed an identification of which sequences dominated as well as which earthquake levels contributed for each accident sequence. A total of 791 non-negligible individual sequences were identified. As part of the review, the Boolean expressions for the 791 dominant sequences were obtained from PG\&E on a PC disk, and were reformatted and independently re-quantified using the split fractions of Table 6-46 of the DCPRA and the hazard curve increment frequencies provided by PG\&E.

After certain nomenclature issues were resolved, and after it was established that the split fractions for the 0.0-0.2g spectral acceleration level given on Table 6-46 of the DCPRA were not used in the mean point estimate calculation, a frequency of $2.87 \mathrm{E}-5$ per year for the 791 accident sequences was independently computed. This is nearly the same as the value of $2.80 \mathrm{E}-5$ per year reported in the DCPRA, which is, of course, as it should be given that the same Boolean expressions and input data were used.

In addition to the frequency of the 791 sequences provided to the reviewer on diskette, an evaluation of the frequency of the "remaining" accident sequences was performed and given as 6.30E-6. Thus, the total core damage frequency mean point estimate is $3.43 \mathrm{E}-6$ per year. As will be seen later, this is quite close to the mean values obtained from the uncertainty analysis calculation, and reported as 3.7E-6 per year as the final DCPRA seismic mean core damage frequency.

This independent evaluation, however, allowed us to rank the sequences in terms of their contribution to the overail core damage frequency, and to identify the subset of accident sequences which dominated the risk taking all earthquake levels into consideration. Where this was done, the somewhat surprising result was obtained that the risk was not dominated by a small set of sequences, but rather was spread out over at least 150 logical sequences to account for $90 \%$ of the total core damage frequency.

This is partially accounted for by the fact that each of the 791 sequences corresponds to one specific earthquake level. That is, if a logical sequence were important at all six earthquake levels, there would be six separate accident sequences occurring in the 791 dominant sequences reported. A second cause for the large number of sequences is described below. 
The top ten sequences only accounted for $57 \%$ of the total as shown on Table 4.7.5. These top 10 dominant accident sequences (based on contributions from all earthquake levels) were derived from the mean point estimate accident sequences provided (at different earthquake levels) on the PC diskette by PG\&E. These are shown in Table 4.7.5 along with their total frequency and percent contribution to the total (mean) core damage frequency.

To understand these sequences requires a certain amount of work, since (at first glance) these sequences do not make logical sense. The basis for these seeming errors is the approach of using two support trees (electrical and mechanical) which are not rigorously (in a Boolean logic sense) connected to the main seismic frontline tree. This approach - if implemented correctly - will yield the correct numerical answer, but (as will be seen) the approach yields individual accident sequences which are difficult to understand and review.

To see this, consider dominant sequence \#1 on Table 4.7.5. The failure events are loss of offsite power (OG1) and failure of all three trains of on-site emergency AC power (events AF1, AG2, AH3). But by definition of AF1, AG2, AH3, the simultaneous failure of all three trains fails all AC power regardless of whether LOSP occurs or not. (For example, AF1 includes failure of the 4KV switchgear in that train, and offsite power is routed through this switchgear, as is emergency power from the diesels. Hence, whether or not LOSP occurs is irrelevant.) Thus, the LOSP failure event OG1 is not required, and in a true Boolean logic sense, dominant accident sequence \#1 is non-minimal in comparison to dominant sequence \#3, which involves failure of only the events AF1*AG2*AH3.

Similarly, dominant sequence \#5 involves both direct failure of all $\mathrm{AC}$ power (events $\mathrm{AF1}$ *AG2*AH3) as well as failure of all $\mathrm{AC}$ power due to relay chatter (event CT2). So this accident sequence is also non-minimal. In the same fashion, dominant sequences $\# 2, \# 7, \# 8, \# 9$ and $\# 10$ all involve failure events not required for core damage and are - at first glance - logically incorrect. These apparent discrepancies can be resolved by looking at dominant sequences $\# 1, \# 3, \# 5$ and $\# 7$ as a group - as shown in Table 4.7.6. All sequences involve the terms $A F 1 * A G 2 * A H 3$, but failures of the auxiliary feedwater system (AFW4) and failures due to relay chatter (CT2) and their complements are also present. Consider now a simple event tree involving only OG1, CT2 and AW4 as shown in Figure 4.7.5. (This is a subset of the Electric Power Support tree). A total of 8 outcomes are possible given success or failures of OG1, CT2 and AW4 (Labeled A, B, C,......H). By examining the successes or failures in the dominant sequences $\# 1, \# 3$, \#5 and \#7, one can identify to which branch on the simple event tree of Figure 4.7.5 each corresponds. Thus, dominant sequence \#1 involving OG1*(1-CT2)*(1-AW4) corresponds to branch E. Sequence \#3 involving (1-OG1)*(1-CT2)*(1-AW4) corresponds to branch A. Sequence \#7 involving OG1*AW4*(1-CT2) corresponds to branch $F$. And finally, sequence \#5 must correspond to branch $G$. No specific sequences were found corresponding to the other branches, but clearly they must be significantly smaller than the four sequences reported and hence were undoubtedly not reported. Now if the numerical values of all four sequences are added, one obtains just the probability of the term $A F 1^{*} \mathrm{AG2} 2^{*} \mathrm{AH} 3$ and, in fact, the actual numerical values of OG1, CT2 and AW4 are irrelevant.

Thus, when all four sequences (\#1,\#3,\#5,\#7) are combined one gets the logically and numerically correct single dominant accident sequence involving only the simultaneous failures of the three $\mathrm{AC}$ power trains AF1*AG2*AH3. 
In similar fashion, one must consider the dominant sequences $\# 4$, $\# 8$ and $\# 9$ together as shown in Table 4.7.6. Again, in this case the terms OG1, CT2 and RT7 are superfluous, and the actual failure cut set is only DF1*DG2 which is the simultaneous failure of all DC power for control and instrumentation. (This was assumed to lead to core damage regardless of LOSP or other failures).

Finally, sequences $\# 2, \# 6$ and $\# 10$ must also be considered as group. Together, these represent the logically correct accident sequences involving OG1* GF1*GG2*GH3 which is LOSP in conjunction with simultaneous loss of all three emergency diesel generators.

Thus, the top 10 accident sequences derived from the 791 sequences provided on diskette actually represent only three independent (and logically correct) dominant accident sequences:

\section{Sequence}

AF2*AG2*AH3*

DF1"DG2*

OG1*GF1"GG2*GH3* complement events

complement events

complement events
Percent

Contribution

$31 \%$

$10 \%$

$16 \%$

Total $\quad 57 \%$

and each of these is a station blackout scenario involving only electrical power trains. It is only when the 791 mean point estimate sequences are grouped appropriately (as described above) that one can relate them to the simpler block model and accident sequences used in the uncertainty analysis (discussed below).

\subsubsection{Uncertainty Amalysis}

As described earlier, a separàte uncertainty analysis quantification was performed following the mean point estimate calculation. As a first step, the dominant accident sequences identified in the mean point estimate calculation were used as the basis for constructing a simple block diagram model of core damage for the DC plant. This simplified block diagram in effect provides Boolean expressions which encompass the dominant failure scenarios, and which are simple enough so that the Discrete Probability Distribution approach to uncertainty analysis (developed by PL\&G) can be conveniently applied.

The logical block diagram was not reported in the DCPRA although the numerical results for total core damage frequency are listed on Table 6-54 of the DCPRA. At NRC request, this block diagram was made available, and an uncertainty analysis was performed independently using an alternate approach, namely a full Monte Carlo analysis of the accident scenarios implied by the logical block diagram. As in the DCPRA evaluation, the random/human error basic events were fixed at their mean point estimate values, while lognormal fragility functions (as characterized by their random uncertainty $\beta_{r}$ and modelling uncertainty $\beta_{u}$ ) as provided in Table 6-40 of the DCPRA were used.

The seismic logical block diagram is shown in Figure 4.7.6. Circled numbers are seismic component numbers which correspond to the fragility descriptions on Table 4.6.1. The " + " denotes the logical union "OR"

operator, while the " $x$ " denotes the logical intersection "AND" operator. Twelve "boxed" blocks are shown, each corresponding to a functional accident scenario. Boxes containing a single (engineering notation) 


\section{Review of Seismic PRA}

numerical value represent dominant groups of random failures of components. A description of each scenario block - as provided by PG\&E - is presented in Table 4.7.7.

The hazard curves used in the independent Monte Carlo uncertainty analysis were the identical set of eight discrete hazard curves whose non-exceedance probability ordinates (and associated weights) were given on Table 6-38 of the DCPRA. (The values given in the initial draft were incorrect. After this was pointed out, corrected values were provided to the reviewers and corrected in an addendum.)

Table 4.7.8 compares the seismic core damage frequency percentiles (as given on Table 6-54 of the DCPRA) with those independently computed using a Monte Carlo process. This shows that the mean values of total core damage frequency were computed to be nearly the same by both DPD and Monte Carlo. The lower 5 percent values were also quite close, while the 95 percent value computed by Monte Carlo was 55 percent greater than that computed by the DPD method. This gives rise to a smaller error factor (17 vs 26) being computed by the DPD method than by the Monte Carlo method. Overall, however, the results agree quite well between the two methods. (This is, to our knowledge, the first independent evaluation of the DPD uncertainty analysis approach available in the public literature).

Table 4.7.9 summarizes the ranking of the twelve blocks (accident sequences) as computed in the Monte Carlo independent uncertainty analysis. The dominant sequences are (a) loss of all onsite ac power (station blackout), (b) loss of offsite power, and (c) loss of all dc power. This is consistent with both the point estimate evaluation (Section 4.7.2) and the "issues" studies summarized in Section 4.2.

Table 4.7.10 shows the (unconditional) contributions of each of the twelve accident scenarios (blocks) on the simplified logic block diagram for different intervals on the hazard curve. Figure 4.7.7 compares the contributions at various earthquake intervals as computed by the DPD approach (as presented on Figure 6-49 of the DCPRA) versus the Monte Carlo approach. It can be seen that, because of the rather coarse interval on spectral acceleration used in the DCPRA, the contributions at the upper and lower intervals have been overestimated. However, both approaches show that most of the risk occurs due to earthquakes with average spectral accelerations in the $1.7 \mathrm{~g}$ to $3.0 \mathrm{~g}$ range. This can also be seen from the mean plant level fragility curve presented in Figure 4.7.8. This curve shows that the mean conditional probability of core damage is very small below $2.0 \mathrm{~g}$ average spectral acceleration.

Finally, an evaluation of the risk reduction potential (RRP) for each of the components was made using the simplified logic block model. The risk reduction potential is defined as the percentage decrease in seismic core damage frequency which results if the probability of failure of that component is set equal to zero, and the seismic core damage frequency is re-evaluated. Table 6-58 of the DCPRA presented such values (denoted as "Impact If Very Strong that is, If Impervious to Earthquake"). Table 4.7.11 lists the RRP for all components in the block model, and compares them to those reported in Table 6-58 of the DCPRA. In general, for those components whose RRP values were listed in the DCPRA, the agreement is reasonably good. The RRP of the various diesel generator failure modes was found to be 16 percent while the DCPRA listed only 10 percent. The RRP of switchgear/breaker panels was found to be 6.6 percent, but was not listed in the DCPRA, although components with smaller values of RRP were listed. Finally, the RRP for BOP piping and supports was found to be 5.8 percent as contracted to the DCPRA value of 2.2 percent. Overall, however, the agreement is quite good.

In conclusion, the comparison studies between the DPD method and the Monte Carlo method of uncertainty analysis show that similar results are obtained (by the two quite different approaches) and that the core damage frequencies and percentiles are - from a computational standpoint - verified. 


\subsection{Seandtutty Stadles}

Using the uncertainty anabysis block model, a number of sensitivity studies were performed to estimate the effect of certain assumptions in modeling techniques used in the DC seismic PRA. In each case, a mean point estimate calculation based on the block model was performed and changes in mean seismic core damage frequency were computed. Eech study is reported separately and, in general, the changes in the seismic core damage frequency from the different sensitivity studies are not independent and are certainly not additive.

a) Impact of pipe conditional failure fraction. In the modeling of pipe failures, different pipe segments were identified for the various safety systems and the probability of failure of each segment was estimated as the probability of the pipe support failure (generic fragility) multiplied by conditional fraction that failure of the pipe support would result in double ended pipe fracture. In the uncertainty analysis block model, this conditional failure fraction varied between 0.05 to 0.25 , depending on the system and type of piping being analyzed. The basis for the choice of these different fractions was not described. Hence, in this study, the sonditional pipe break failure fraction was varied (the same for all systems) from a lower value of 0.0 to an upper value of 1.0 (that is, support failure equals pipe failure). The results of this study are summarized below:

\begin{tabular}{|l|l|l|}
\hline $\begin{array}{c}\text { Conditional Prob } \\
\text { (Plpe Break) }\end{array}$ & Prob (Core Damage) & \multicolumn{1}{|c|}{$\%$ Diff } \\
\hline 0.0 & & $5 \%$ lower \\
\hline 0.05 & & $4 \%$ lower \\
\hline 0.10 & $4.01-5$ & $3 \%$ lower \\
\hline 0.20 & $4.12-5$ & $0.2 \%$ lower \\
\hline 0.25 & $4.17-5$ & $1 \%$ higher \\
\hline 0.40 & $4.33-5$ & $5 \%$ higher \\
\hline 0.50 & $4.44-5$ & $7 \%$ higher \\
\hline 1.00 & $4.96-5$ & $20 \%$ higher \\
\hline
\end{tabular}

As can be seen from the values above, the effect of varying the conditional pipe break failure fraction was relatively small. In fact, if the conditional failure fraction is taken as 1.0 (certainty), the result is only a 20 percent increase in the total core damage frequency. Thus, overall, the seismic total core damage frequency results are not particularly sensitive to the assumed failure fraction. This is not surprising since the overall generic fragility for the piping supports has a relatively high median value and, thus, is not expected to play a major role in the final core damage frequency results.

b) Impact of correlation on uncertainty analysis. As described earlier, correlation was incorporated somewhat differently in the point estimate model (in which the various support trains were considered explicitly) and in the final block model used for the uncertainty analysis (in which the failure of one train was assumed to imply the failure of all trains). Further, it was seen that the uncertainty analysis 


\section{Review of Seismic PRA}

gave slightly higher mean core damage frequencies due to this more conservative assumption with respect to correlation. A sensitivity study was performed to estimate the impact of correlation on the block model uncertainty analysis. This was done by assuming that similar trains of the same support systems were identical (e.g., had the same random and seismic fragilities) and that the responses seen by these similar support trains were identical. It was assumed that the respon ses were fully correlated, but that there was no correlation between the fragility failure modes of the support systems. The uncertainty analysis block model was modified to incorporate these assumptions and the frequency of core damage was reevaluated. When this was done, the mean point estimate core damage frequency was reduced to $2.8 \mathrm{E}-5$ per year, a reduction of 33 percent. Thus, it can be seen that a reduction in core damage frequency could be achieved if a more complete description of correlation were included in the systems models.

c) Impact of range of integration. As described in the DCPRA, the seismic integration was performed up to an upper limit average spectral acceleration of $4.0 \mathrm{~g}$, and a total of 6 (unequal) intervals were used in the integration scheme. To verify that this range of integration was adequate, a mean point estimate calculation using the block model was performed in which the range of integration was increased to $5 \mathrm{~g}$ and then $6 \mathrm{~g}$ average spectral acceleration. These two calculations showed increases in mean core damage frequency from the base case value (4.15E-5) to $4.51 \mathrm{E}-5$ and $4.60 \mathrm{E}-5$, respectively. Thus it can be seen that the range of integration considered in the DCPRA was adequate, and if a greater range of integration were used, an increase of about 12 percent would be expected.

d) Impact of ceramic insulator fragility. A key feature in the results of the DCPRA results from the fact that the fragility taken for the $230 \mathrm{kv}$ ceramic insulators in the switchyard was taken to have a median average spectral acceleration of $1.66 \mathrm{~g}$. Scaling to peak ground acceleration, this corresponds to a median peak ground acceleration value of $0.72 \mathrm{~g}$. This is at least twice as high as any ceramic insulator fragility median used in past PRAs. The basis for this median value came from segregating the recorded earthquake performance data on ceramic insulators into several groups. It was found that the so called "dead tank" type of ceramic insulator performed significantly better than other ceramic insulators typically used in commercial power plants. Further, it was verified that the ceramic insulators in the $230 \mathrm{kv}$ switchyard were of the dead tank variety and hence, it was appropriate to use a higher median value for their seismic fragility. This played a substantial role in the results of the DCPRA in that it greatly lessened the probability of loss of offsite power and reduced the importance of the LOSP corresponding sequences.

To esamine the effect of the ceramic insulator fragility mean on the core damage frequency predicted for Diablo Canyon, a sensitivity study was performed in which the ceramic insulator fragility median was varied from $0.3 \mathrm{~g} \mathrm{pga}$ to $0.72 \mathrm{~g} \mathrm{pga}$ and the core damage frequency was recomputed. The results of this calculation are shown as a continuous curve on Figure 4.7.9. It can be seen that the mean core damage frequency increased from 4.15E-5 to a value of 6.25E-5 at the weaker limit. Thus, the effect of going from a median ceramic fragility value of $0.72 \mathrm{~g}$ (pga) down to a more usual value of $0.3 \mathrm{~g}(\mathrm{pga})$ is an increase in the mean core damage frequency of $50 \%$.

e) Sensitivity Study on Human Actions in DCPRA Seismic Analysis

A number of human recovery actions were identified for the seismic analysis in Table 6-51 of the DCPRA. Many of these were associated with post-earthquake actions taken to recover offsite or onsite power lost due to seismic component failures, and which involved replacement battery chargers or 
portable power generators being put in place to replace damaged components. Although considerable thought was given to these post-earthquake options, for the final results of the seismic DCPRA it was decided to take no credit for their use. Thus events ZHEAC2, ZHEHS2, and ZHERE6 denoted as earthquake related recovery actions on Table 6-51 of the DCPRA were not used in the final quantification.

However, three important human actions were considered in the final quantification, and these were incorporated as a function of earthquake level - which is an advancement over past seismic PRAs. These human error events are:

ZHECT1 Failure to reset of control power circuits (from control room) that were tripped as a result of relay chatter during an earthquake.

ZHELA2 Failure to turn off RHR pumps following an SI signal with the RCS at high pressure. After 4 hours, this error is assumed to fail the pumps, which resultsin loss of ability to establish sump recirculation.

ZHESE1 Failure to align fire water system to provide RCP seal cooling, given loss of normal ASW or CCW This results in a seal LOCA.

These three human error events were explicitly included in the block model used for the uncertainty analysis as shown on Figure 4.7.6 and explained on Table 4.7.7.

These events were modeled as being (stepwise) dependent on earthquake level as shown below:

\begin{tabular}{|l|c|c|c|c|}
\hline & & \multicolumn{3}{|c|}{ Average Spectral Acceleration } \\
\hline Event & $\begin{array}{c}\text { Random } \\
\text { Value }\end{array}$ & $<1.75 \mathrm{~g}$ & 1.75 to $<2.5 \mathrm{~g}$ & 2.5 to $4.0 \mathrm{~g}$ \\
\hline ZHECT1 & 0.002 & 0.002 & 0.01 & 0.06 \\
\hline ZHELA2 & 0.0047 & 0.0047 & 0.02 & 0.20 \\
\hline ZHESE1 & 0.01 & 0.01 & 0.07 & 0.40 \\
\hline
\end{tabular}

The random values for these events were taken from Table 6-52 of the DCPRA, and is the mean value used in the internal events analysis for the corresponding non-seismic event. The earthquake ievel-dependent values were provided directly by PG\&E in response to reviews questions, and are not in the DCPRA.

In one sense, the human action failure rates can be viewed as being somewhat optimistic, as they assume the random (non-stressed) failure rate for all earthquake levels up to 1.75 of average spectral acceleration, which corresponds to $0.75 \mathrm{~g}$ of peak ground acceleration. However, these three actions are all performed in the control room (rather than requiring an operator to leave the control room to perform the action) which tends to increase the likelihood of success. Engineering judgement was used to establish the numerical values of these human errors as a function of the three earthquake levels, but they seem quite reasonable to the revieviers. (Nominally, the failure rate increases by a factor of 5 for each earthquake level). 


\section{Roviow of Seimic PRA}

To evaluate the sensitivity of the final results to the assumed earthquake levels at which these human error rates were increased, the mean core damage frequency was reevaluated with the threshold levels decreased from $(1.75 \mathrm{~g}, 2.5 \mathrm{~g})$ down to $(0.8 \mathrm{~g}, 1.2 \mathrm{~g})$. The result was an increase in mean core damage frequency from 4.13E-s to 4.61E-5, an increase of 11 percent. Thus the mean point eatimate results are not very sensitive to the stepwise threshold levels at which the human error probabilities are increased to reflect the stress due to larger earthquakes. The lack of sensitivity is due to the fact that the human error events are "OR-ED" in conjunction with seismically-induced component failures which soon dominate the final results as higher earthquake levels are considered.

\subsection{Summary and Conclusions}

In general, the seismic portion of the DCPRA was found to be a defendable and detailed state-of-the-art seismic risk assessment. Due to the nature of the numerous conservative assumptions made in modeling systems and including correlation on component failures, it is felt that the mean core damage frequency computed is somewhat conservative.

The DCPRA involved a number of relatively novel considerations. First of all the examination and inclusion of relay chatter effects has advanced the state-of-the-art. The detailed level to which circuits were examined for the potential impact of relay chatter and its documentation goes well beyond that performed in any previous seismic PRA. The detailed comparison of the nonlinear response of the Turbine Building and comparison against drift limits against the engineering factor of safety approach provided a meaningful validation of the later more simplified method, which has been used in all the commercial seismic PRAs to date. This study provides considerable confidence in the use of the factor of safety approach for predicting failure levels of both structures and components. The use of average spectral acceleration as the independent seismic parameter (both for the seismic hazard curves and for the component fragilities) provided a consistent means of eliminating double counting in assigning uncertainties to both the hazard curve and the fragility. Overall, however, the use of average spectral acceleration versus peak ground acceleration was found to play little role in determining the overall magnitude of the risk of core damage frequency at Diablo Canyon. This in itself is of interest because of the fact it substantiates the use of peak ground acceleration in all past seismic PRAs.

The DCPRA fragilitieswere found to have been derived by state-of-the-art and relatively mature methodology which has been applied to more than 25 power plants in the past. A review of the identified and analyzed modes found that the appropriate failure modes had been identified. A review of the anchor bolt failure modes showed that important recent generic issues (such as edge distance, bolt spacing, and concrete cracking effects) were properly included and taken into account. The methods and the basis for assigning the random and modeling uncertainties to the fragilities were found to be reasonable. No significant unresolved remain for the fragilities in the DCPRA.

The procedure used for quantifying the risk of core damage was found to be acceptable and the quantification itself was found to be accurate. An independent evaluation of the block model used to perform the uncertainty analysis by a completely independent approach (Monte Carlo) was found to produce final distributions on core damage frequency that were reasonably close to those reported for the DCPRA. The main discrepancy was some difference in the $95 \%$ percentile values. The same dominant contributors to core damage frequency were found and the percentage contributions for these dominant contributors were found to be nearly the same. 
In general, a major deficiency of the DCPRA was that the final report was not adequate to allow a meaningful review without extensive and continuing interactions with both PG\&E and contractor personnel. If the goal is to produce a report that can be reviewed (if not reproduced) by the data contained in the report and its appendices, then a somewhat different structure and additional data may be required.

A detailed review of the DCPRA was considerably complicated by the PL\&G methodology on which it was based. This methodology, as applied in the Diablo Canyon PRA, involves significant "hands on" intermediate steps which were both difficult to document and to review. In particular, the methodology utilizing a separate simple systems model for the final uncertainty analysis as contrasted to a very detailed model for the point estimate calculations provides a large source of difficulty in reviewing the final results (as was described earlier). In addition, the block uncertainty analysis model seems to require considerable amount of analysts judgement to develop, which in itself is difficult to document or review. Considering the large number of mean point estimate accident sequences involved in the first step of the analysis, it is likely that future versions of the report would have to provide this information on PC diskette in order for any meaningful review to be performed. It should be noted that the PG\&E personnel performing the PRA were more than helpful in resolving these issues as they arose. Given the level of effort that went into the development of the hazard curves, the very detailed fragility calculations for all components, and the level of effort into developing accident sequences and correctly incorporating complement events, it is felt that the DCPRA represents a state-of-the-art and defendable seismic PRA and that no unresolved issues remain concerning the final results. 
4 Review of Seismic PRA

\subsubsection{References}

1. Letter, D. A. Brand to U.S. Nuclear Regulatory Commission, Re: Docket No. 50-275, OL-DPR-80, Docket No. 50-323, OL-DPR-82, Diablo Canyon Units 1 and 2, Long Term Seismic Program Completion, July 31, 1988, Enclosure, "Long Term Seismic Program Final Report."

2. Johnson, J. J., Goudreau, G. L., Bumpus, S. E., and Maslenikov, O. R., "SSMRP Phase I Final Report: SMACS (Seismic Methodology Analysis Chain with Statistics) (Project VIII)," UCRL-53021, Vol. 9, NUREG/CR-2015, Vol. 9, Lawrence Livermore National Laboratory, Livermore, CA, 1981.

3. Kennedy, R. P., Wesley, D. A., and Tong. W. H., "Probabilistic Evaluation of the Diablo Canyon Turbine Building Seismic Capacity Using Nonlinear Time-History Analyses," No. 1643-01, NTS Engineering, December 1988.

4. Letter, J. J. Johnson to N. Chokshi, "Diablo Canyon Turbine Building Fragility Assessment," June 8, 1989.

5. Letter, J. D. Shiffer to U.S. Nuclear Regulatory Commission, Re: Docket No. 50-275, OL-DPR-80, Docket No. 50-323, OL-DPR-82, Diablo Canyon Units 1 and 2, Long Term Seismic Program Meetings, July 25, 1989, Enclosure 3 Fragility Analysis Meeting, June 16-17, 1989.

6. Letter, J. D. Shiffer to U.S. Nuclear Regulatory Commission, Re: Docket No. 50-275, OL-DPR-80, Docket No. 50-323, OL-DPR-82, Diablo Canyon Units 1 and 2, Response to Action Items from the Long Term Seismic Program, Fragility Meeting, June 16-17, 1989, October 26, 1989.

7. Kipp, T.R., D.A. Wesley, and W.H. Tong, "Seismic Fragilities of Civil Structures and Equipment Components at the Diablo Canyon Power Plant No. 1643.02, NTS Engineering, December 1988. 


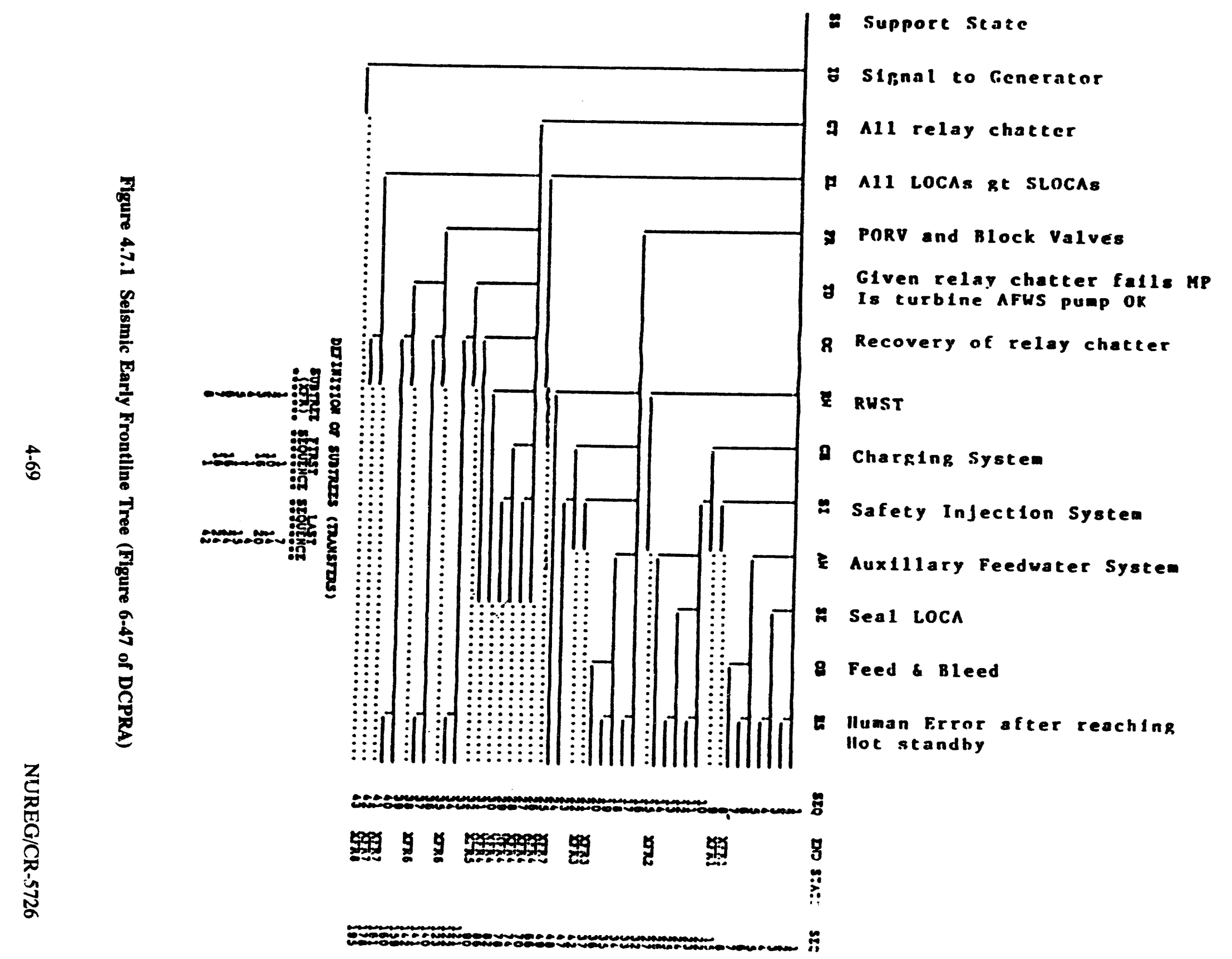



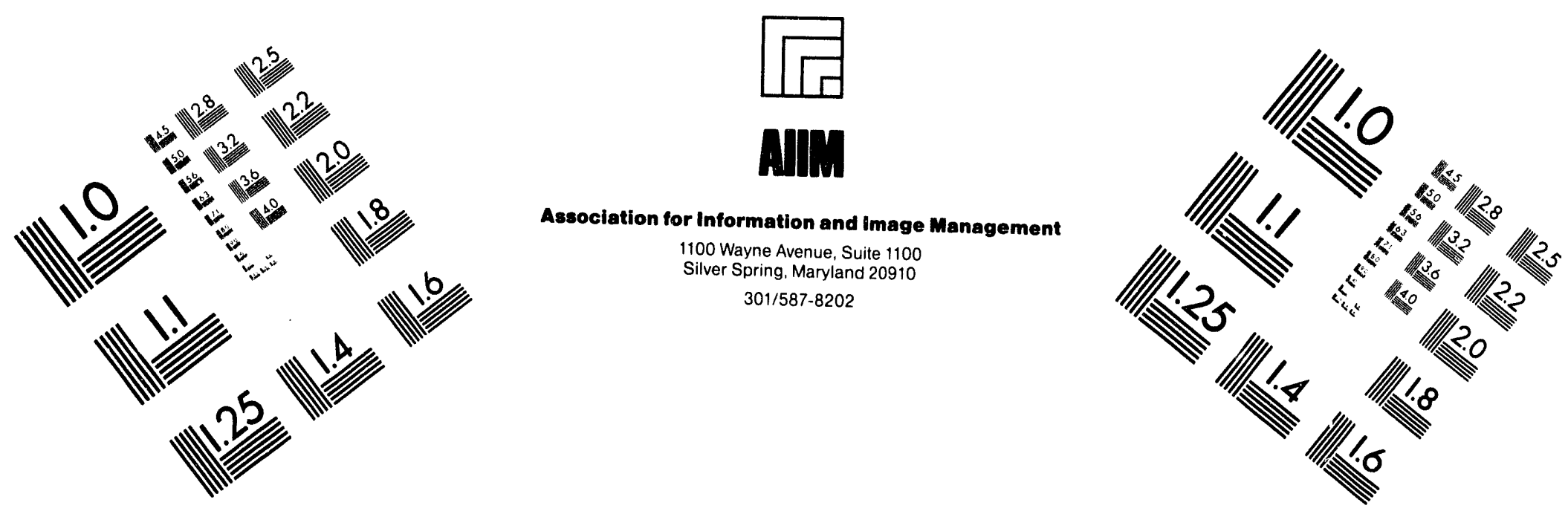

Centimeter

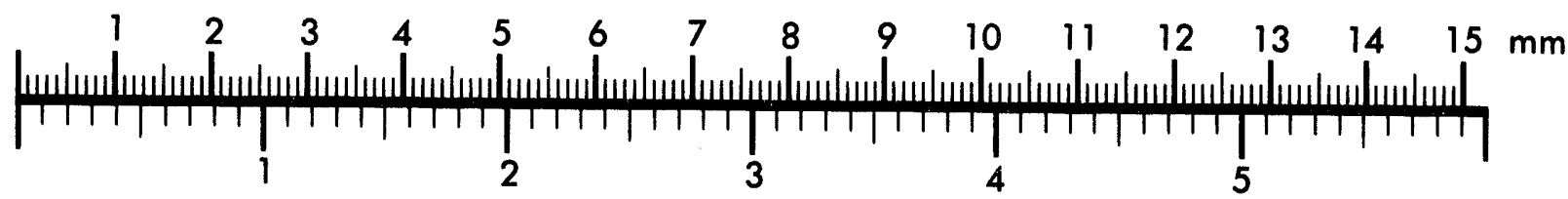
Inches
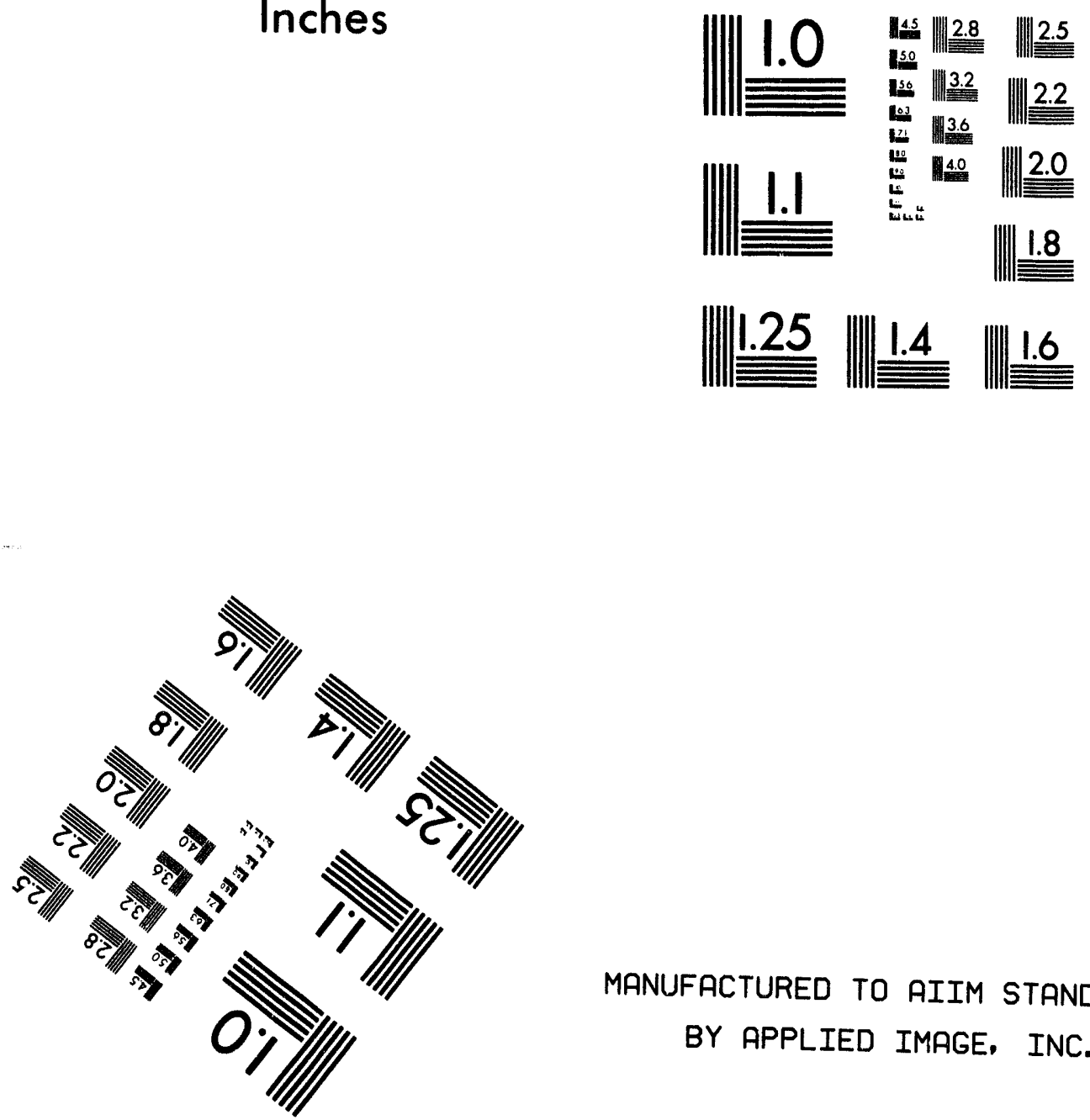

MANUFACTURED TO AIIM STANDARDS BY APPLIED IMAGE, INC.

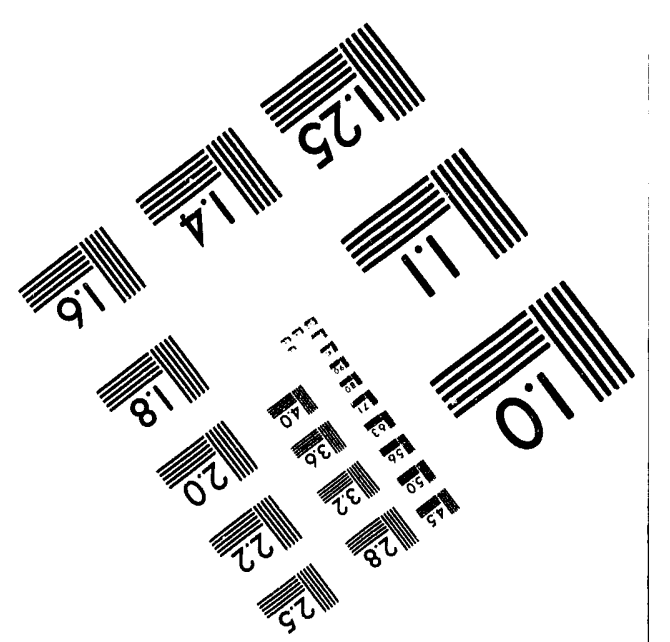



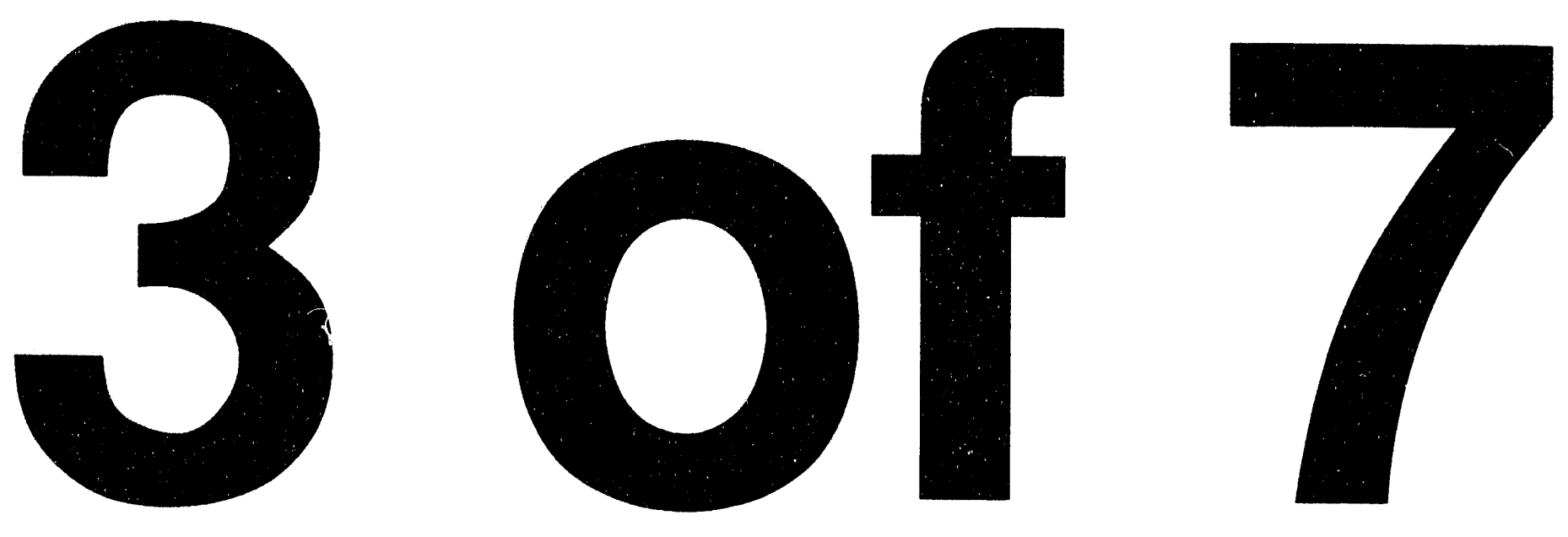

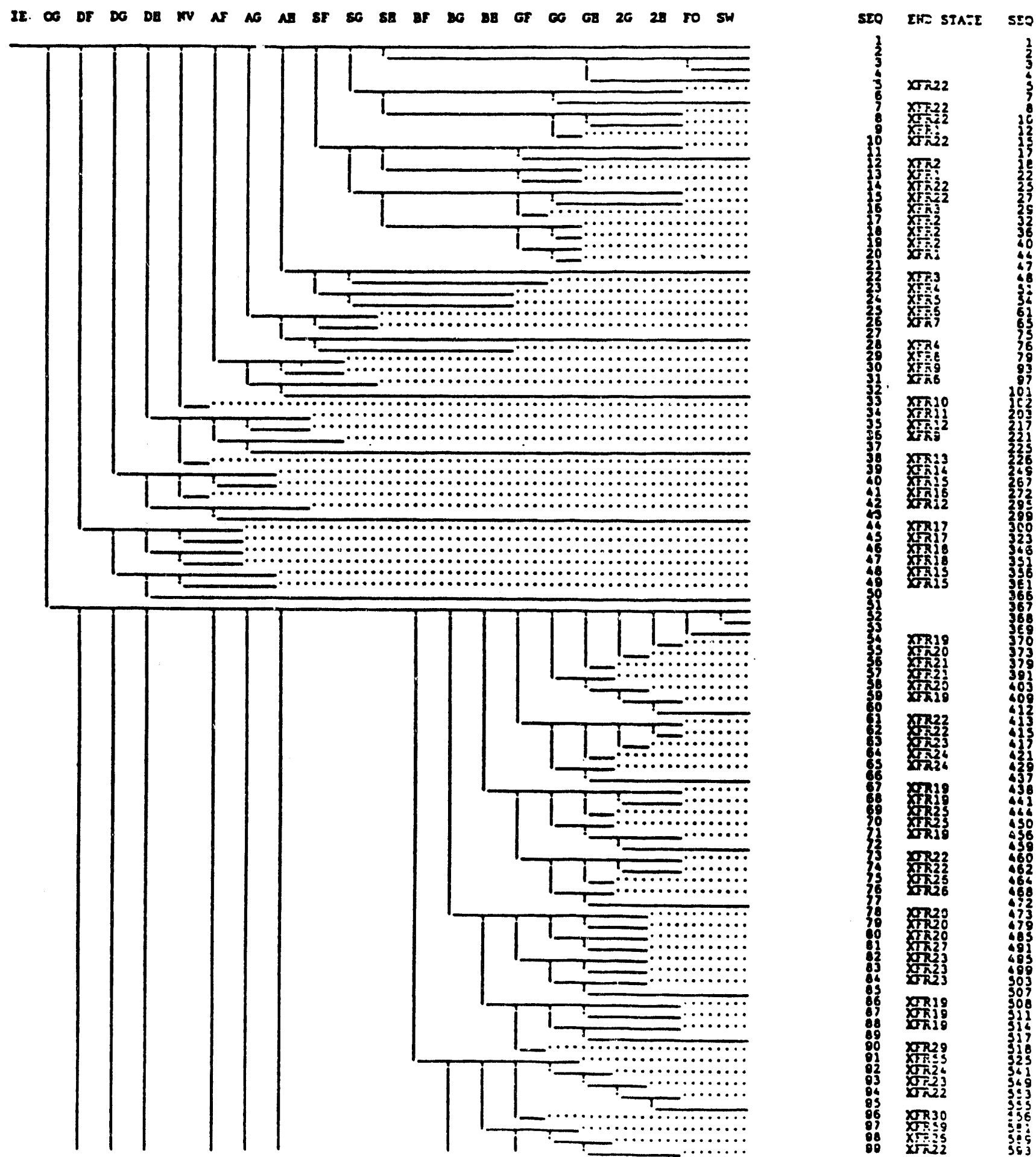

Tygure 4.7.2 DCPRA Elocturical Support Tree (Fione 6-4A of DCT:-A) 
4 Review of Seismic PRA


Nown 4.7.2 (contlaned) DCPRA Eloctrical Support Tree (Thare 6-44 of DCPRA) 

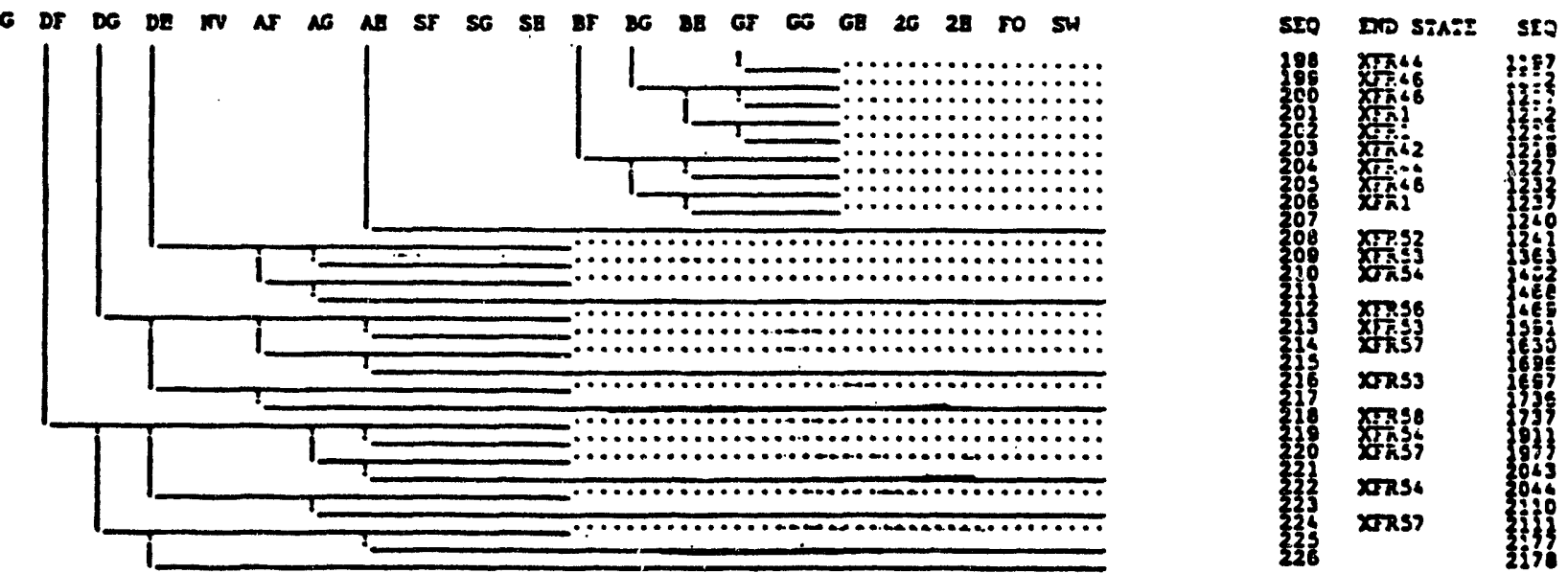

DEInITION of suatrers (IRUistras)

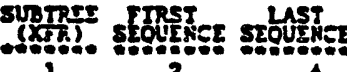

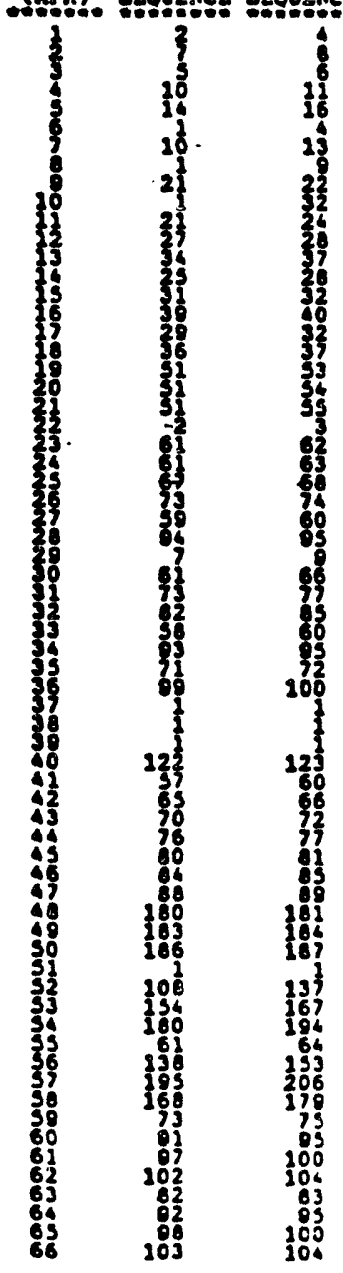

Tezare 4.7 .2 (continend)

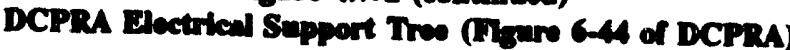


4 Revicw of Seismic PRA
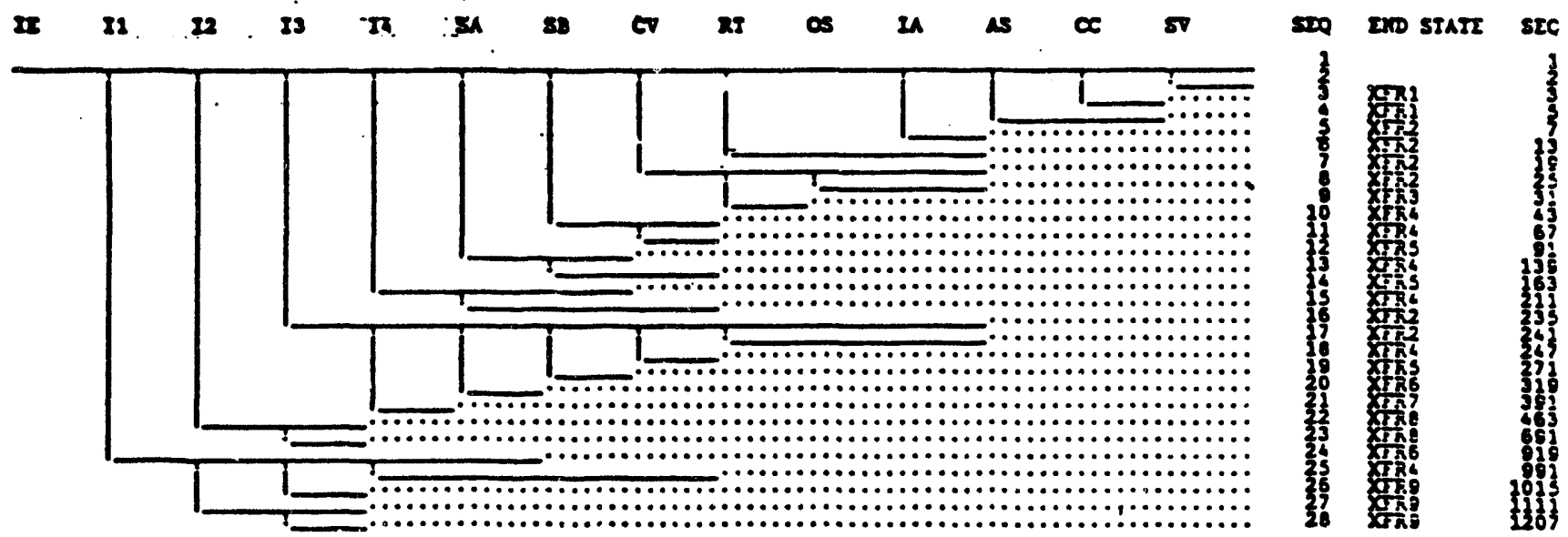

Deringtzan of suthers (munsturs)

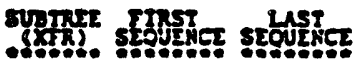

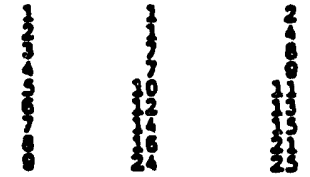

Pyare 4.7.3 DCPRA Actuation and Mechanleal Support Tree Figure 6-45 of DCPRA) 
4 Review of Seismic PRA

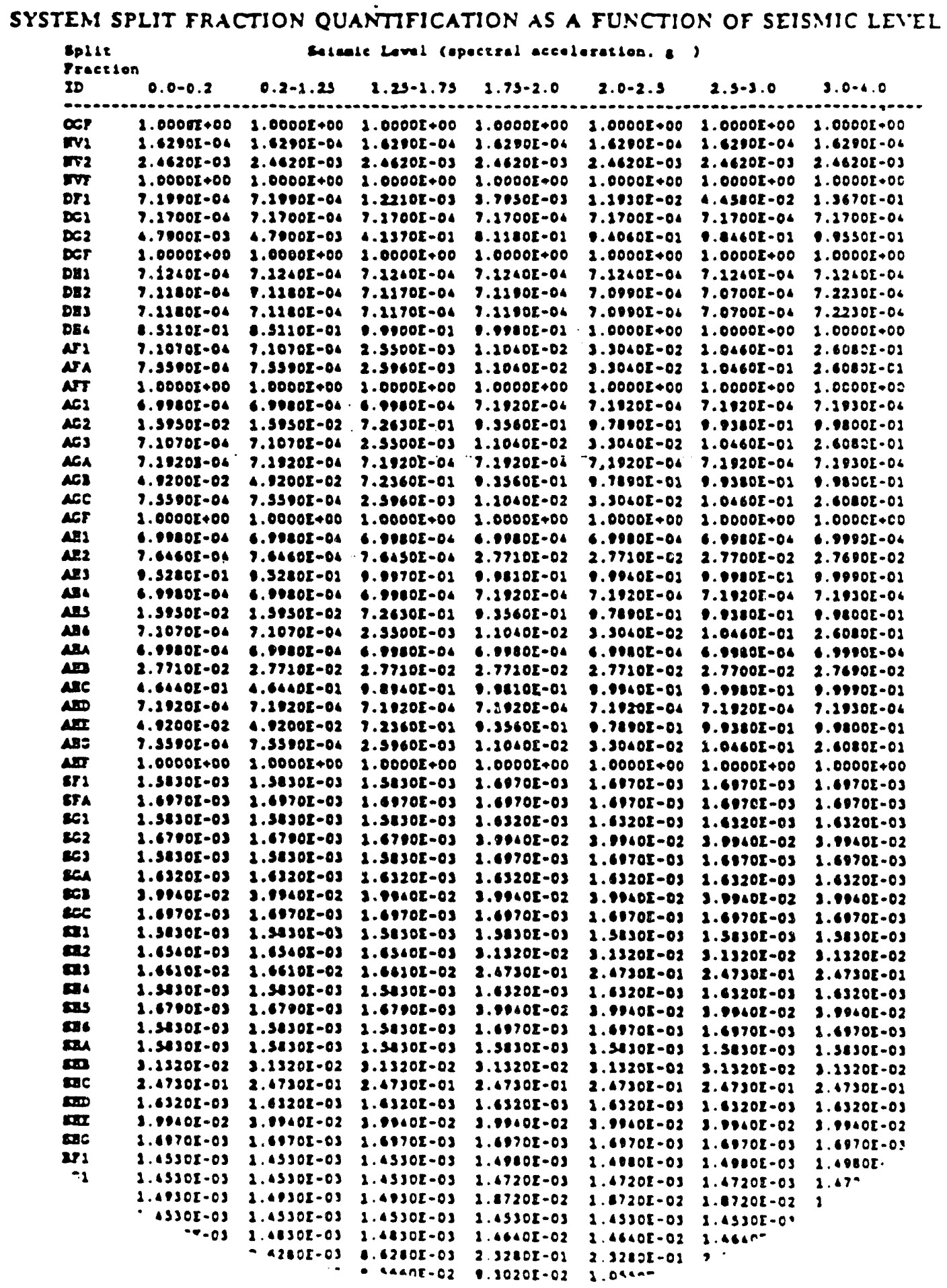

Mgare 4.7.4 Sample Sheet of DCPRA Table 6-46 


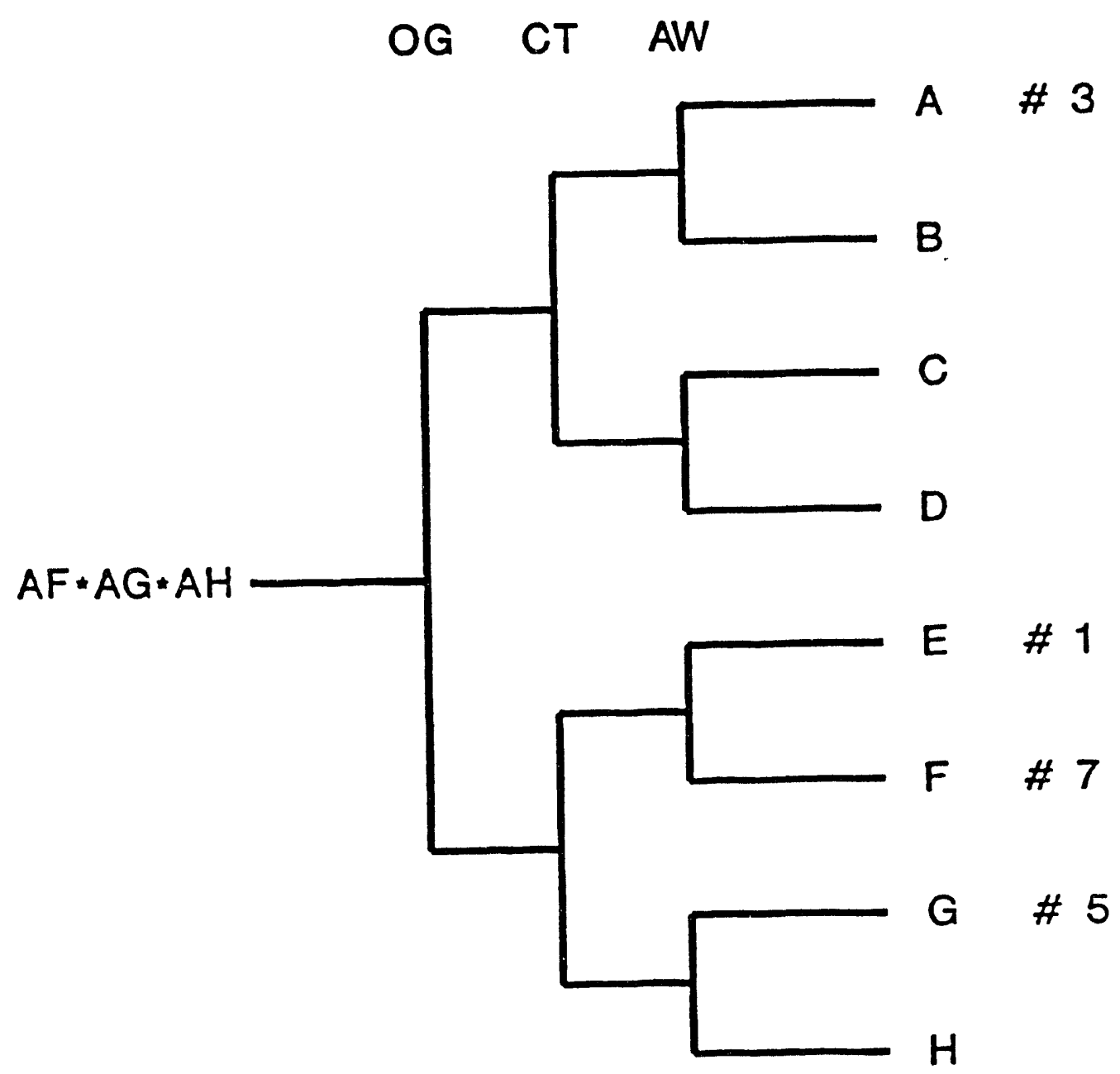

Figure 4.7.5 Partial Event Tree for Electric Power 


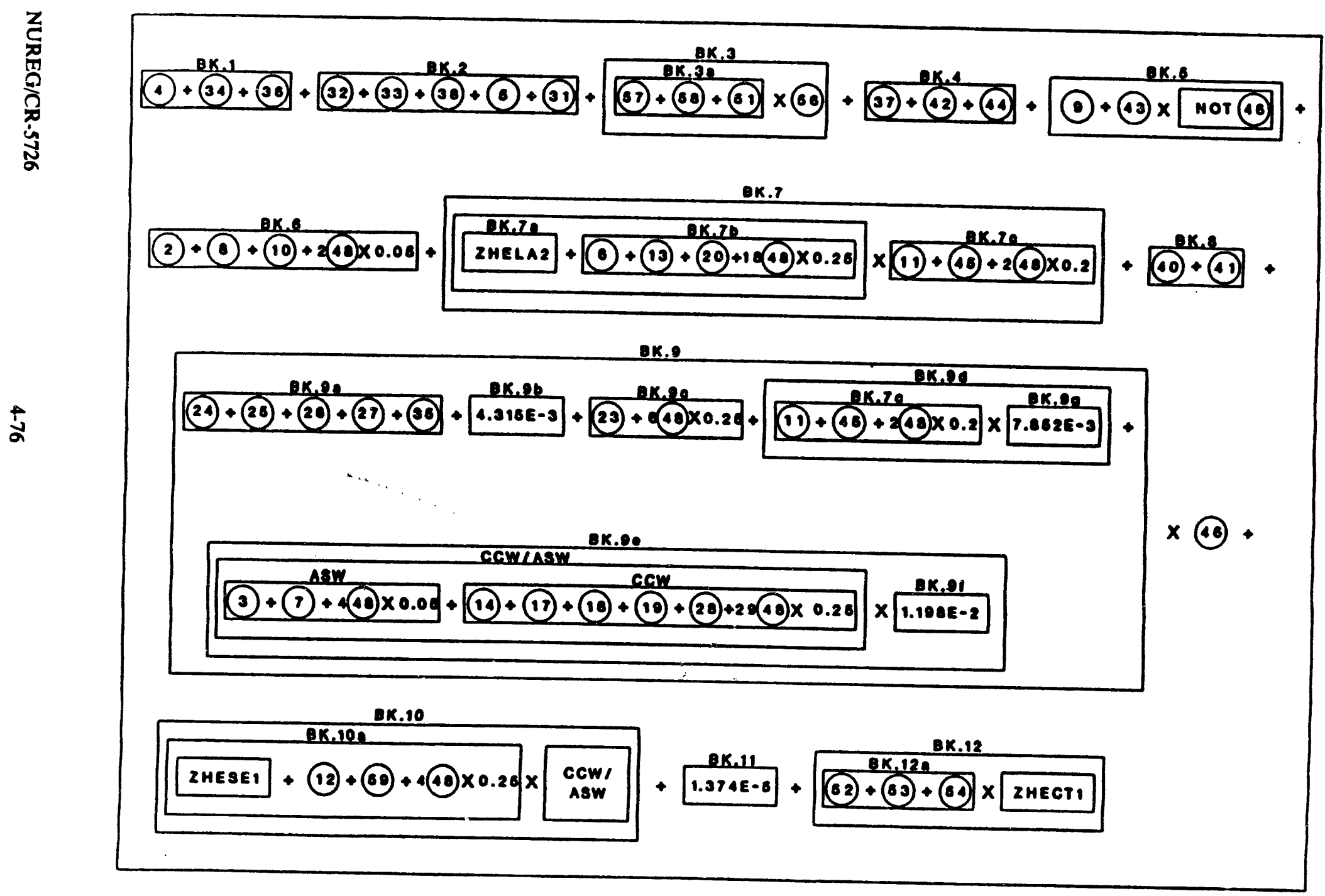


4 Review of Seismic PRA

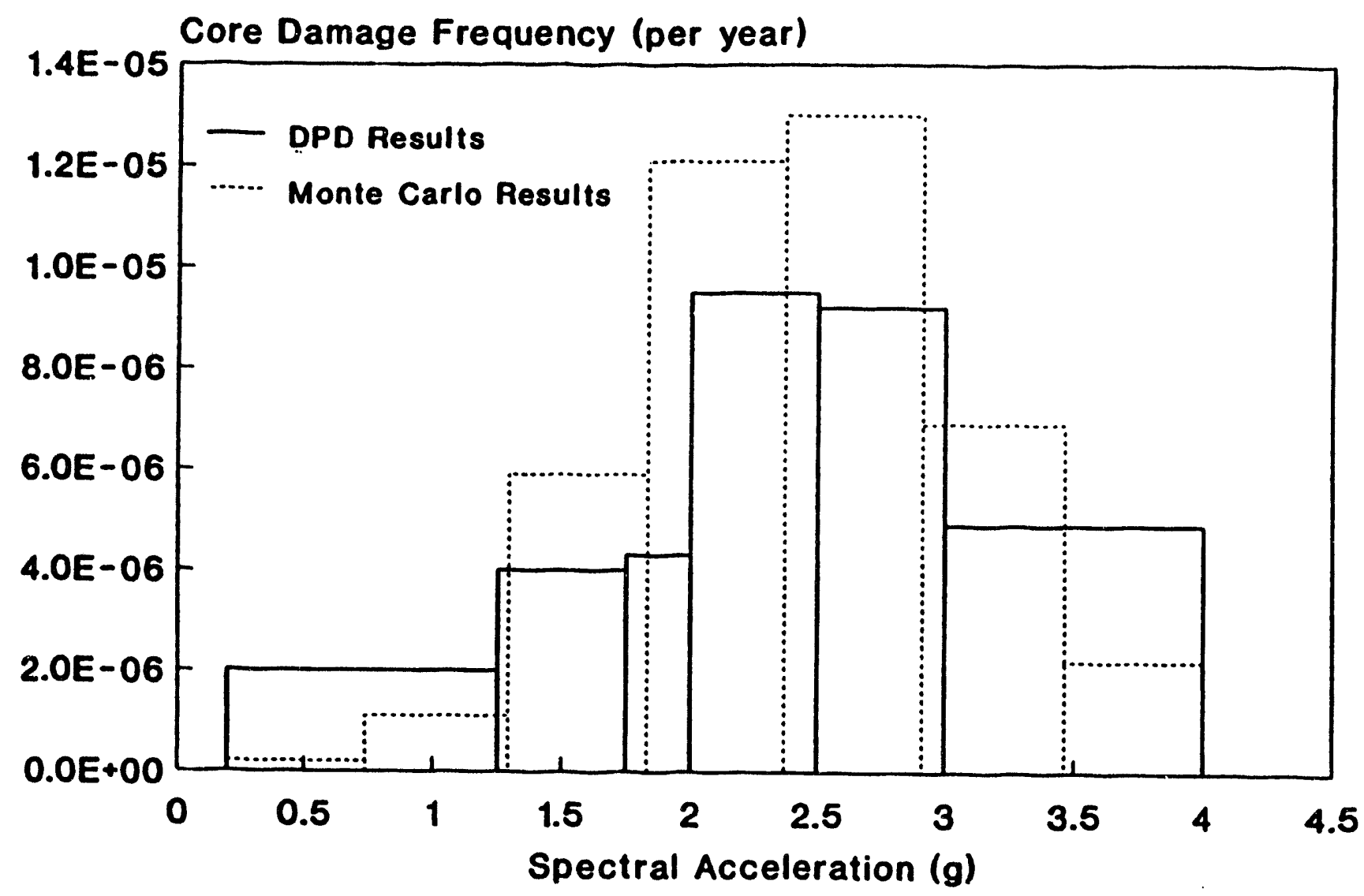

Figure 4.7.7 Contribution of Each Earthquake Range to the Seismic Core Damage Froquency (DPD Versus Monte Carto) 
4 Review of Seismic PRA

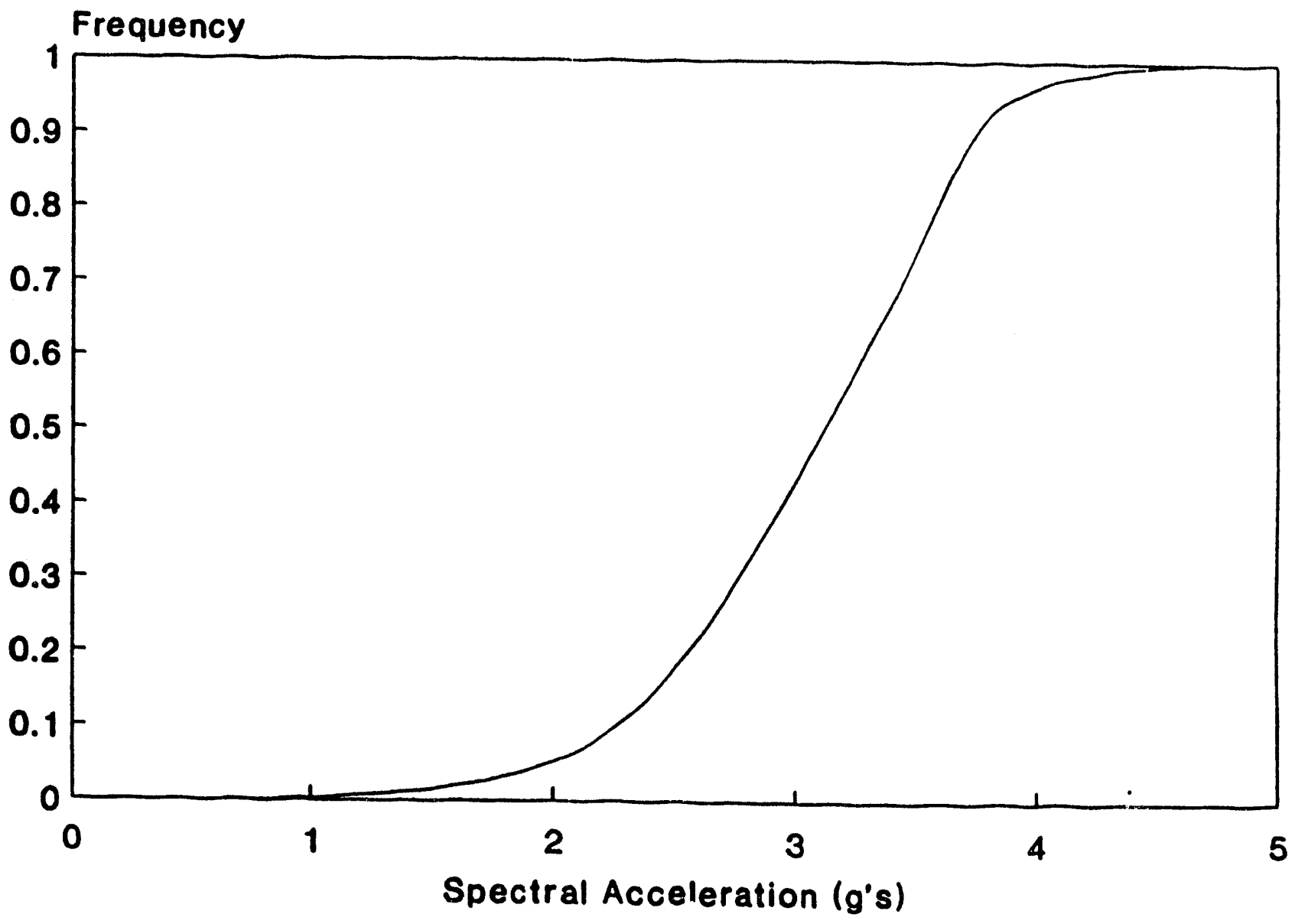

Figure 4.7.8 Plant Total Mean Fragility Curve 
4 Revicw of Seismic PRA

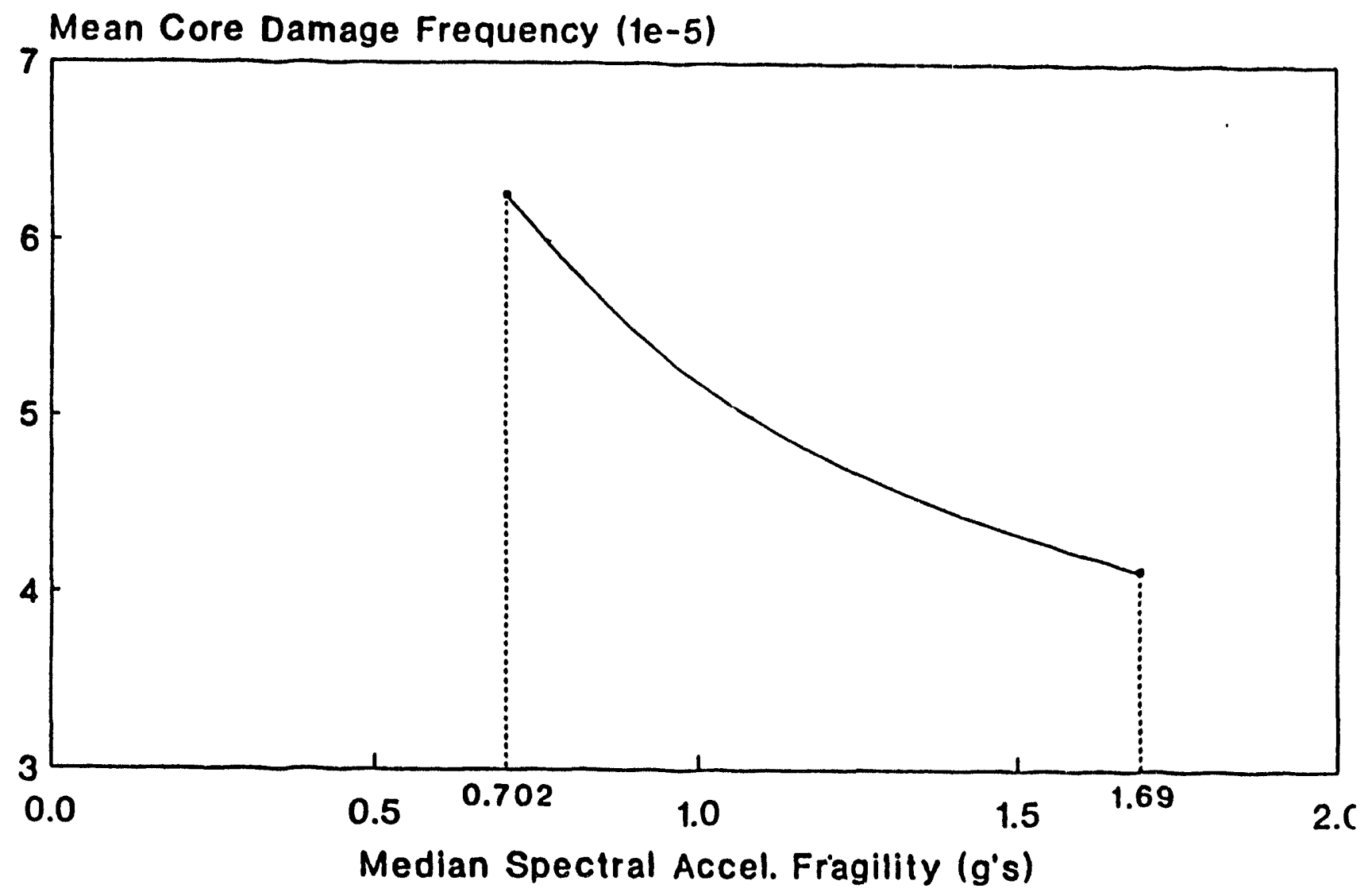

$0.702 \mathrm{~g} \mathrm{Sa} \cdot 0.3 \mathrm{~g} P G A$

Pigure 4.7.9 Sensitivity Stedy on 230Kr Fradility Median 
Table 4.7.1 Descriptions of Top Events on Selomic Early Frontline Tree (from Chapter 6 of ACPRA)

Top Event ID. Represents a loss of indications to the operators, and this is assumed to lead directly to core damage.

Top Event CT. Represents the summation of all relay chatter fragilities resulting in a loss of all $\mathrm{AC}$ power.

Top Event EL. This is the summation of all LOCAs greater than a small LOCA. Such LOCAs are all conservatively assumed to be excessive, that is, modeled as if the ECCS systems are not effective at mitigating them.

Top Event PR. This top event models the RCS pressure relief function. At least one of three pressurizer PORV's must open to relieve RCS pressure, if required, and all three PORVs must reseat. Failure of Top Event PR implies that a PORV train is open and a small LOCA has developed. The operator action to isolate a stuck-open PORV by closing the PORV block valve is also considered in this top event. The likelihood that a PORV block valve is closed initially is also considered. If all three PORVs fail to open when challenged, the pressurizer safety valves are then assumed challenged and all three must reseat.

For loss of offsite power events, the pressurizer PORV's are assumed.to be challenged.

For all transients, the pressurizer PORV's would not be challenged, if automatic reactor trip is successful. Successful reactor trip is also assumed if the operators manually trip the reactor in the first minute after a fallure of an automatic reactor trip. However, for these cases of successful reactor trip, the PORVs would likely be challenged.

Therefore, it is assumed that all three PORVs would be challenged if automatic reactor trip fails, even if the operator successfully trips the reactor as a backup.

Top Event ID. Represents the turbine-driven AFW pump in order to ask if, under the condition of relay chatter causing loss of the motor-driven AFW pumps, the turbine-driven AFW pump is operational. Fallure of all AFW shortens the time avallable for successful recovery from relay chater.

Top Event OC. Represents operator recovery from relay chatter. This event is only asked if Top Event CT fails. Different human error rates are used depending on the status of auxiliary feedwater and whether a LOCA has occurred. Failure of this event is assumed to result in a continued loss of all vital AC. 
If a Phase B safety injection signal occurs (for example, due to a steam Ine break Inside containment). CCW flow to header $C$ is isolated, which isolates cooling to the posirive displecement charging pump and RCP thermal barrier cooling. Then, Top Event SE can only be successful if the operators successfully stop the RCPs in time; that is. Top Event RP is successful. Then to protect the RCP seals, the operator must use one of the centrifugal charging pumps. The seal injection path from the charging pumps is then modeled in this top event. If CCW header $C$ is isolated. Top Event CH must have succeedad for this path to be viable.

For success without any CCW cooling, the operators must provide alternate cooling frow the fire main to a charging punp heat exchangers for RCP seal injection as described in plant procedures. The pump suction may be frow either the VCT or the RWST. A makeshift systen for temporary cooling of the positive displacement charging pump (that is, wet rags and portable fans) could also te established, although such actions are not currently covered by procedures.

This top event is not asked in the event if Top Event PR fails (that is, a PORV opened and falled to reseat) because a LOCA is then already known to have occurred. The consequential failure of the RCP seals is judged to be unimportant.

Top Event $O B$. This top event represents the operators inltiating bleed and feed cooling. provided all secondary cooling falls, by manually inftiating a safety injection signal, then resetting it, and by restoring instrument alr to the containment. This cooling path also requires that at least two PORVs are avallable and are held open for 6 hours that 1s, the time it takes to reduce RCS pressure to allow closed loop RHR cooling. Th? operator also trips the RCPs by procedure. Cooling through the pressurizer safety valves alone, without the PORVs, is assumed unsuccessful because of insufficient makeup flow at their pressure reliaf setpoint. The procedural actions to open the reactor vessel head vents and depressurize one stean generator to acmospheric pressure, If only two PORV trains are avallable, are assumed unnecessary. Only two PORVs ax connected to a nitrogen supply header supplied by backup nitrogen accumulators. Instrument air bust be restored to the containaent for operation of the third PORV. The PORVs are qualified for the environment after a stean IIne break inside the containment and therefore are expected to remain functional during bleed and feed sequences.

Top Event HS. This top event models whether hot standby conditions can be maintained successfully. If Top Events HS falls, It is assumed that long-term actions to control AFW, provide AFW makeup, or establish closed loop RHR cooling have failed and damage has occurred. There is plenty of time for a successful operator response if something does go wrong in the long-term after conditlons have stabilized. Plant operations may decide to cooldown to cold shutdown conditions or to return to power after the cause of the Plant trip is identified and resolved. This analysis assumes that they would only attempt to cool down to cold shutdown if a LOCA is in progress. If a LOCA is not in progress, the plant would return to power from hot standby. 
Top Event RW. This top event models the availabilicy of the refueling water storage tank. The RWST must maintain its structural integrity. contain the minimum inventory required by technical specifications (i.e.. 400,000 gallons), and be properly vented.

Top Event CH. One of two centrifugal charging pumps starts automatically on a safety injection signal, taking suction from the RWST. High pressure flow from the charging pimps is used for either RCP seal injection or for reactor vessel makeup. The analysis for Top Event $\mathrm{CH}$ considers the flow paths through the boron injection tank to the RCS cold legs. Cooling of the RCP seals is considered in Top Event SE.

No credit is given to the positive displacement charging pump for RCS Injection because it has limited capacity and is not normally operated. The positive displacement punp is considered in Top Event SE, however, for seal injection.

Top Events SI. This top event nodels the safery injection pumps starting automatically on a safety injection signal to provide injection to the RCS. At least one of two pumps starts and operates, taking suction from the RWST. This top event is asked, even if Top Event CH is successful, to determine whether two high pressure recirculation paths from the discharge of the RHR pumps are available in the long-term.

Top Event AW. This top event represents the availability of feedwater pumped fron the condensate storage tank to at least one intact stean generator by the auxiliary feedwater system. One of three steam generators is assumed to be required for steam line break events inside the containwent because one stean generator will have depressurized. The operators are instructed to isolate flow to the affected stean generator unless no other stean generators are intact. Therefore, for a stean line break outside the containment and the MSIVs falling to close, the operators would establish flow to one of the depressurized steam generators. In this case, the operator action to reestablish flow is nodeled, and any one of the four steam generacors is assumed required. Also, for steam line break events, one steam supply path to the turbine. driven AFW pumps is assuned to be unavallable 50 percent of the tine: that 1s. the stean line break is assuned to occur on either stean generator 1-2 or 1-3 50 percent of the tine.

At least $460 \mathrm{gpm}$ of flow is required. One of three AFW pumps is, therefore, needed for success. Event AW also represents the main steam valves needed for cooling. The 10 percent atnospheric steam dump valves and steav generacor rellef valves on each stean generator are assumed necessary for cooling the respective stean generutor.

Top Event SE. This top event models RCP seal integrity. If CCW flow to headers $A$ and $B$ is successful. RCP pump thermal barrier cooling is also assumed successful if flow through $\mathrm{CCU}$ header $\mathrm{C}$ continues. Therefore, if $C C W$ flow to headers $A$ and $B$ is successful. Top Event SE models the availability of cooling to header $C$ of the $\mathrm{CCW}$ system. 
Table 4.7.2 Definitions of Basic Events on Electric Power Support Tree

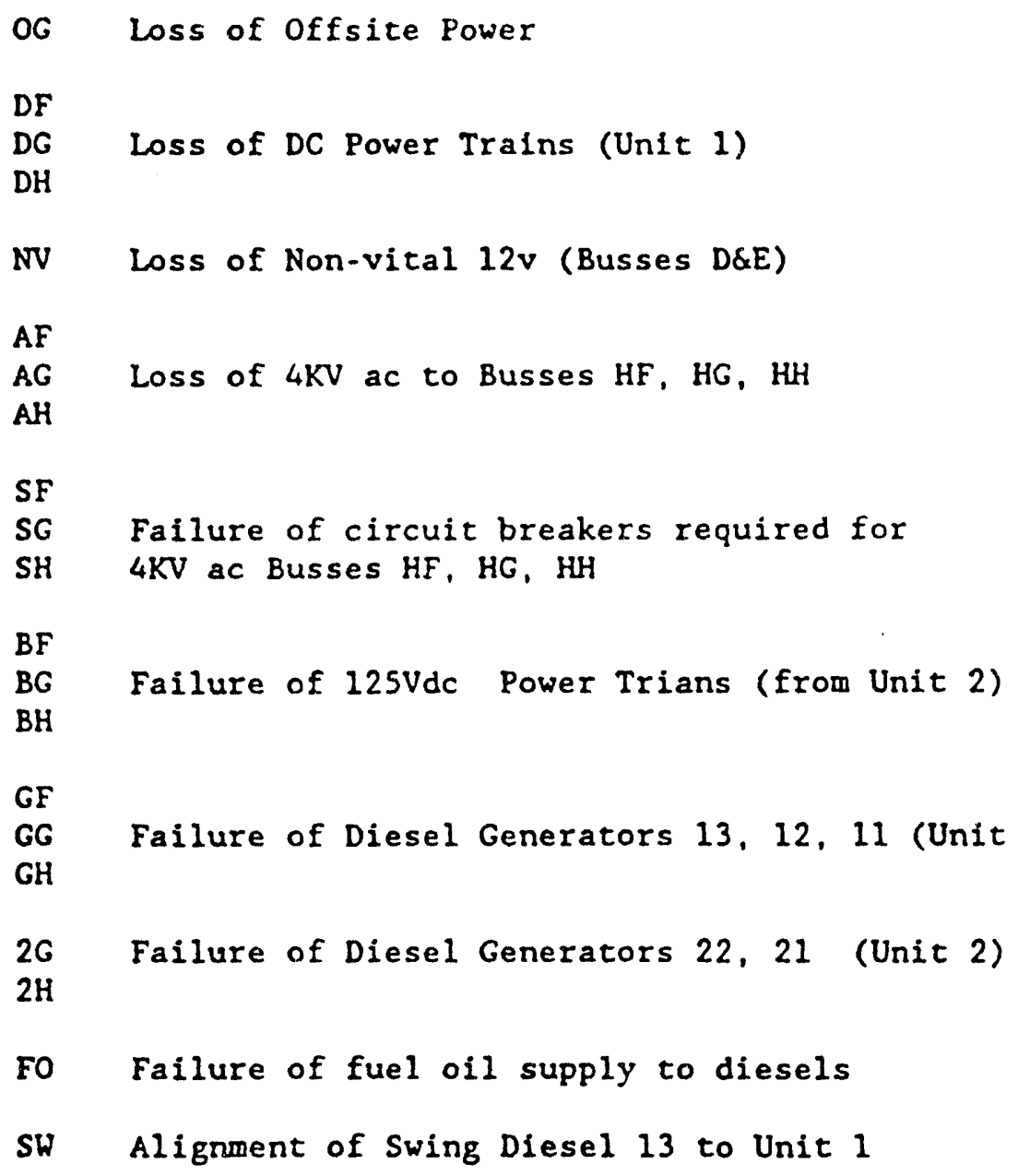




\title{
4 Review of Seismic PRA
}

Table 4.7.3 Definitions of Basic Events in Actuation and Mechanical Support Tree

\author{
I1 \\ I2 Failure of Vital AC instrument channels \\ I3 I, II, III, IV \\ I4 \\ SA Failure of Solid State Protection.System (SSPS) \\ SB Trains A, B \\ CV Failure of Control Room HVAC \\ RT Failure of both auto and manual (backup) reactor trip \\ OS Manual actuation of safety equipment normally activated \\ by SSPS (if SSPS has failed) \\ IA Instrument Air (always assumed failed) \\ AS Availability of Auxiliary Saltwater System \\ CC Availability of Component Cooling Water System \\ SV Availability of Ventilation for $480 \mathrm{~V}$ Switchgear
}


4 Review of Seismic PRA

Table 4.7.4 Doolean Expreadiona for Fallure Events (Dertived from Table 6-43 of DCPRA)

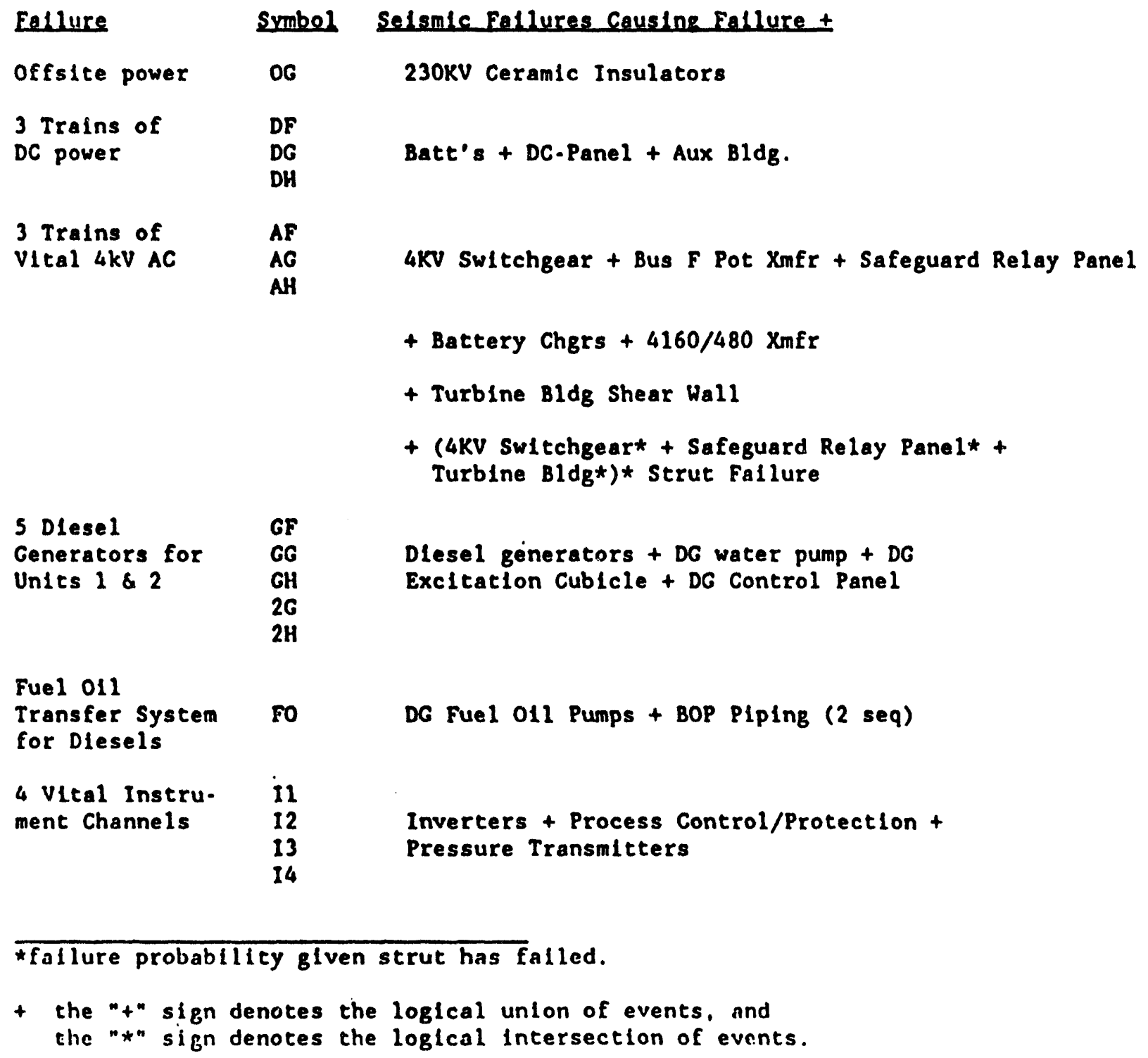


Table 4.7.4 (continued)

\begin{tabular}{|c|c|c|}
\hline $\begin{array}{l}\text { Reactor } \\
\text { Trip }\end{array}$ & $\mathbf{R T}$ & Reactor Intervals \\
\hline $\begin{array}{l}\text { Partial } \\
\text { Reactor } \\
\text { Trip }\end{array}$ & $\begin{array}{c}\mathrm{RT} \\
(\operatorname{part1a1})\end{array}$ & Reactor Trip Switchgear \\
\hline $\begin{array}{l}\text { Control Room } \\
\text { Ventilation }\end{array}$ & CS & Control Room Fans + HVAC Ducting \\
\hline $\begin{array}{l}\text { Component } \\
\text { Cooling Water } \\
\text { System }\end{array}$ & CC & $\begin{array}{l}\text { RHR Heat Xcter + CCW Pump + CCW Heat Xcher } \\
+ \text { CCW Surge Tank + BOP piping }(29 \text { seq })+ \\
\text { Containment Fan Coolers }\end{array}$ \\
\hline $\begin{array}{l}\text { Aux111ary } \\
\text { Salt water } \\
\text { System }\end{array}$ & AS & $\begin{array}{l}\text { Intake structure }+ \text { aux. saltwater plping }+ \\
\text { BOP piping. }(4 \text { seq) }\end{array}$ \\
\hline $\begin{array}{l}480 \text { switchgear } \\
\text { Ventilation } \\
\text { System }\end{array}$ & $\begin{array}{l}\text { sv } \\
(\operatorname{part1a1})\end{array}$ & HVAC Ducting \\
\hline $\begin{array}{l}\text { Relay } \\
\text { Panel } \\
\text { Chatter }\end{array}$ & $\mathrm{CT}$ & $\begin{array}{l}\text { Maln Control Board Chatter + DG Control } \\
\text { Chatter + Safeguard Relay Panel Chatter }\end{array}$ \\
\hline $\begin{array}{l}\text { Excess Ive } \\
\text { LOCA } \\
\text { Seq) }\end{array}$ & EL & $\begin{array}{l}\text { Concrete Internal walls + reactor pressure } \\
\text { vessel + steam generators + BOP piping ( } 1\end{array}$ \\
\hline $\begin{array}{l}\text { Containment } \\
\text { Isolation }\end{array}$ & CI & Steam generators \\
\hline $\begin{array}{l}\text { Control Room \& } \\
\text { RSP Indications }\end{array}$ & ID & Main control boards + Remote Shutdown panel \\
\hline
\end{tabular}


Table 4.7.4 (continued)

\begin{tabular}{|c|c|c|}
\hline $\begin{array}{l}\text { Refuelling water } \\
\text { Storage Tank }\end{array}$ & RW & $\begin{array}{l}\text { RWST Tank + RHR pumps + Safety Injection }+ \\
\text { Spray Addltive Tank + BOP piplng }(18 \text { seq })\end{array}$ \\
\hline $\begin{array}{l}\text { Pressurizer } \\
\text { Rellef Valves } \\
\text { (small LOCAs) }\end{array}$ & PR & PORVs + Impulse Lines + BOP plping ( 1 seq) \\
\hline $\begin{array}{l}\text { RCP Seal } \\
\text { Cooling } \\
\text { (Seal LOCA) }\end{array}$ & SE & Reactor Coolant Pumps + BOP piping (4 seq) \\
\hline $\begin{array}{l}\text { Charging } \\
\text { Pumps }\end{array}$ & $\mathrm{CH}$ & Boran Injection Tank + BOP plping ( 3 seq) \\
\hline $\begin{array}{l}\text { Aux1llary } \\
\text { Feedwater } \\
\text { System }\end{array}$ & AW & AFW pumps + BOP piping ( 6 seq) \\
\hline $\begin{array}{l}\text { Contalnment } \\
\text { Spray Pump } 1,2 \\
\text { (partial) }\end{array}$ & CS & Spray additive tank \\
\hline $\begin{array}{l}\text { Large hole in } \\
\text { Contalnment }\end{array}$ & $\mathrm{CP}$ & Contalnment Bldg \\
\hline
\end{tabular}


4 Review of Seismic PRA

Table 4.7.5 Top Ten Point Estimate Accident Sequences

Sequence

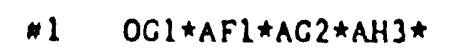

$(1 \cdot C T 2) \star(1-D F 1) \star(1-A W 4) \star(1-P R A) \star(1-R H 1) \star$ $(1 \cdot E L 1) *(1 \cdot O S 1) *(1-112) *(1-I D 1) *(1-R T 1) *$ $(1 \cdot C P 1) \star(1 \cdot S A 1) \star(1 \cdot S B 1)$

*2 $\mathrm{OCl} 1$ CF $1 \star \mathrm{CC} 2 \star \mathrm{CH} 3 \star$

$(1 \cdot C T 2) *(1-A F 1) *(1-D F 1) *(1-A W 4) *(1-P R A) \star$ $(1-R \omega 1) \star(1-E L 1) *(1-O S 1) \star(1-112) \star(1-1 D 1) \star$ $(1 \cdot R T 1) *(1-C P 1) *(1-S A 1) \star(1-S B 1) *(1-132) *$ $\left(1-B E^{-1}\right) *(1 \cdot B C 1)$

*3AF1*AC2*AH3*

$(1-.01) \star(1-A S 4) \star(1-C T 2) \star(1-A W 4) \star(1-D F 1) \star$ $(1-P R A) \star(1-R W 1) \star(1-E L 1) *(1-C V 1) *(1-112) \star$ $(1 \cdot S A 1) \star(1 \cdot S B 1) \star(1-I D 1) \star(1-R T 1)$

$\approx 4 \quad 0 C 1 * D F 1 * D C 2 *$

$(1-R T 7) *(1-C T 2) \star(1-P R A) *(1-R W 1) \star(1-E L 1)$ * $(1 \cdot I D 1) *(1 \cdot C P 3)$

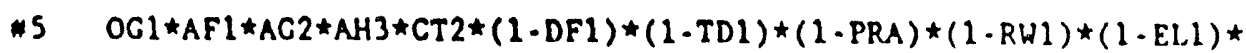
$(1 \cdot O C 1) \star(1-O S 1) \star(1-112) \star(1 \cdot 1 D 1) \star(1-R T 1) \star$ $(1 \cdot C P 1) \star(1-S A 1) \star(1-S B 1)$

46

$O C 1 \star C F 1 \star C C 2 \star C H 3 \star C T 2 \star(1-A F 1) \star(1-D F 1) \star(1-T D 1) \star(1-P R A) \star(1-R H 1) \star$ $(1-E L 1) *(1-O C 1) *(1-O S 1) *(1-112) *(1-I D 1) \star$ $(1-R T 1) \star(1-C P 1) *(1-S A 1) *(1-S B 1) *(1-132) \star$ $(1-B F 1) *(1-B G 1)$

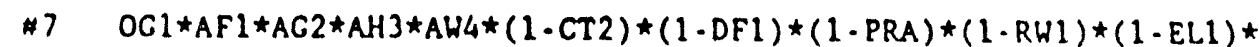
$(1-O S 1) *(1-I 12) *(1-I D 1) *(1-R T 1) *(1-C P 1) *$ $(1 \cdot S A 1) *(1 \cdot S B 1)$

$\star 8 \quad O C 1 \star D F 1 \star D C 2 \star R T 7 \star \quad(1 \cdot C T 2) \star(1-P R A) \star(1-C P 3) \star(1-R W 1)$

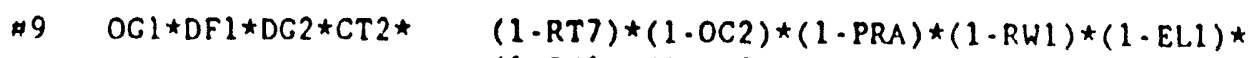
$(1 \cdot ; 01) \star(1 \cdot C P 3)$

10 OC $1 \star C F 1 \star C C 2 \star C H 13 \star A W 4 \star(1-C T 2) \star(1 \cdot A F 1) \star(1-D F 1) \star(1-P R A) \star(1-R W 1) \star$ $(1-E L 1) *(1 \cdot 0 S 1) \star(1-112) *(1-1 D 1) *(1-R T 1) \star$ $(1 \cdot C P 1) *(1 \cdot S A 1) *(1-S B 1) *(1-132) *(1 \cdot B F 1) *$

Top 10 Total
Erequency of Total

$5.3 E .6 \quad 181$

$3.3 E \cdot 6 \quad 111$

$2.1 E \cdot 6$

7 .

$1.7 E \cdot 6$

61

$1.2 E \cdot 6$

41

7. 3E-7

31

5.BE.7

28

5. $7 E \cdot 7$

21

$5.5 E \cdot 7$

21

3. $6 \mathrm{E} \cdot 7$

11

$\overline{1.64 \mathrm{E} \cdot 5}$

578

NUREG/CR-5726 
Table 4.7.6 Top Ten Point Estimate Sequences - Grouped

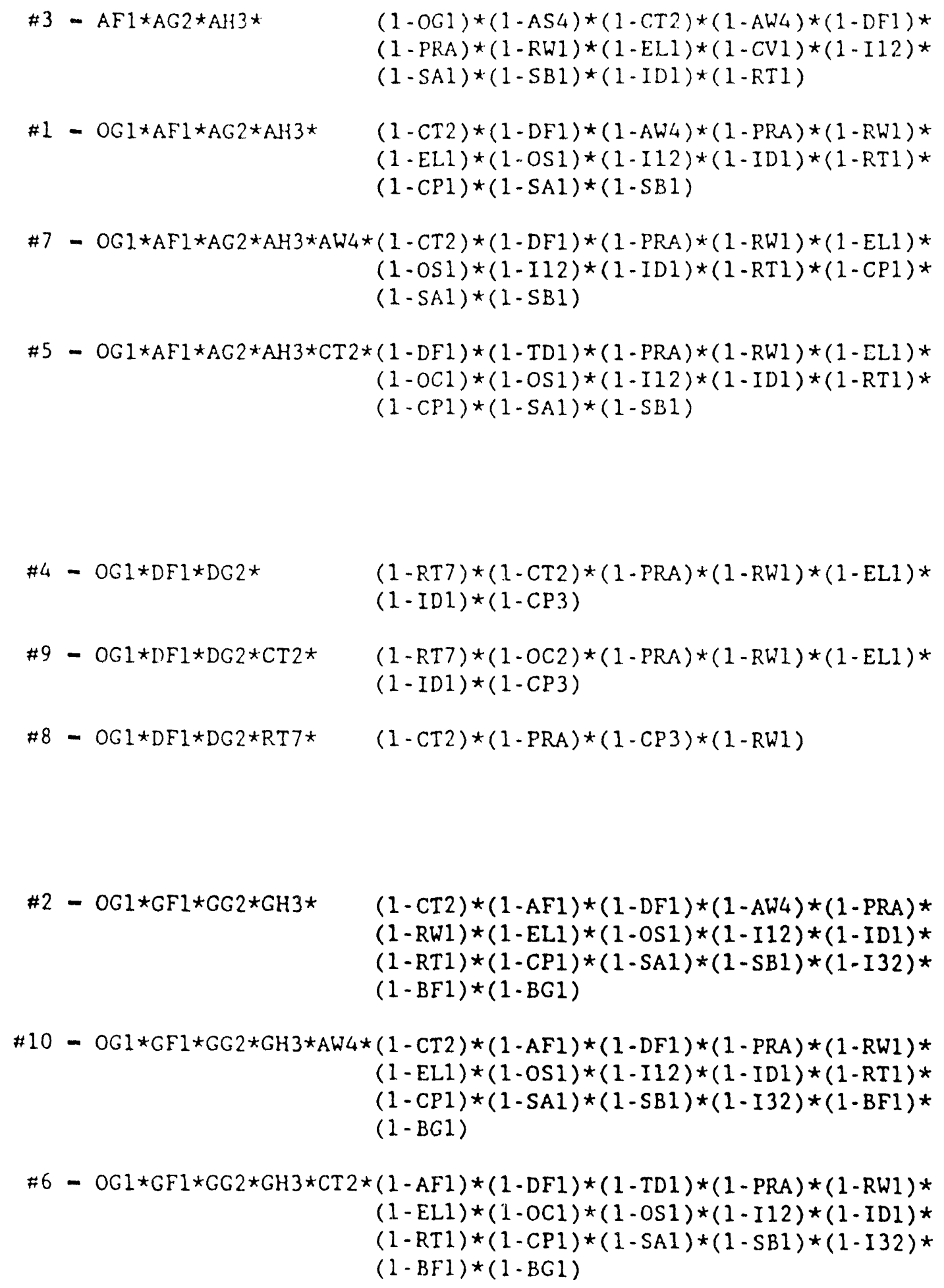


4 Review of Scismic PRA

Table 4.7.7 Description of Lopical Blocks As Provided by PG\&E

\section{BLOCK 1}

This Block includes the seismic fragilities assumed to result in a total loss of all $125 \mathrm{~V} \mathrm{DC}$. The auxiliary building fragility was included among this group. All equipment in the auxiliary building and the control room is assumed lost if this fragility event fails. The total loss of all DC is used as a surrogate for this impact. Loss of $125 \mathrm{~V} \mathrm{DC}$ also implies loss of the associated train of $\mathrm{AC}$ power.

\section{BLOCK 2}

This Block includes the seismic fragilities which result in loss of all emergency AC power. Curve 32 of Table 6-40 in reference 1 is labeled "Buses $G$ and $H$ potential transformers." It is actually the fragility for the Bus $F$ potential transformer. Although the corresponding potential transformers for Buses $G$ and $H$ are stronger than the one for Bus $F$, the seismic core damage model conservatively assumed that all three emergency buses fail if the weaker transformer fails.

\section{BLOCK 3}

Block 3 includes the fragilities that result in failure of all three trains of emergency AC power but which also involve failure of the strut for the turbine building. In the process of developing the fragilities, it was recognized that the turbine building strut failure would change the response of certain components in the turbine building. Separate fragility curves were therefore, developed for the Bus F potential transformer, the safeguard relay panel, and for the $4 \mathrm{kV}$ switchgear for the conditions where the turbine building strut failure occurs. Curve 56 (i.e., that for the strut) is, therefore. combined with the three fragilities using a logical "AND" operation.

\section{BLOCK 4}

Block 4 includes just the fragility curves that, if failed, are modeled as a loss of all vital instrumentation. No credit was assumed for shutting down without instrumentation; consequently, core damage was conservatively assumed to occur.

\section{BLOCK 5}

Block 5 models the components that, if failed, could result in a failure to trip the reactor. For the reactor trip switchgear, it was recognized that failure of this component would not prevent reactor trip if offsite power was also lost. Consequently, Fragility 43 is combined with a logical "AND" with the complement of the loss of offsite power fragility.

\section{BLOCK 6}

Block 6 includes the component failure modes which are considered to lead to an excessive LOCA. One piping segment is modeled for each of the charging and RHR system piping connections in their interface with the reactor coolant system boundary. The quantity 0.05 is the probability that a failure of the most stressed pipe support in these segments would lead to a complete severance of the pipe. In actuality, even if these pipes completely rupture, the resulting flow area is insufficient to result in an excessive LOCA; i.e., beyond 
the design basis of the ECCS. However, to simplify the model, this conservative assumption was made. Therefore, this failure mode of either of these two pipe segments is assumed to lead to core damage.

\section{BLOCK 7}

Block 7 models the seismic failures that result in a LOCA with failure of either RCS injection or recirculation from the containment support. The LOCA failure modes are included in Block 7C. Two pipe segments are modeled; i.e., charging and the RHR system connections. The factor 0.20 is the likelihood of a small break in the most stressed segment of these pipes given that the pipe support fails; this results in a LOCA from the RCS. Block 7B includes the failure modes that result in a failure of high head injection. Fragilities for the RHR and containment spray pumps are included here because the limiting seismic failure mode is a break in the pump boundary, which is assumed to drain the RWST, resulting in failure of RCS injection. A total of 18 pipe segments are also included. Again, failure of any one of these 18 segments is postulated to result in a draining of the RWST. The factor of 0.25 is the assumed probability of any size pipe break given failure of the most limiting pipe support in one of these segments. Block 7A models an operator error to align recirculation from the containment sump. Action ZHELA2 models the operator action to turn off the RHR pumps following an SI signal with the RCS at high pressure. Failure to turn off these pumps within about 4 hours is assumed to result in failure of the pumps due to overheating while operating on miniflow; this, subsequently, results in the failure of the ability to establish recirculation from the containment sump.

\section{BLOCK 8}

Block 8 considers seismic failure of the two sets of control panels; i.e., the main control boards and the hot shutdown panel. Rather than further considering the operator action to transfer control to the hot shutdown panel, given loss of the main control boards, the seismic model conservatively assumes that failure of either control station leads directly to core damage.

\section{BLOCK 9}

Block 9 considers the important system failure combinations (i.e., both seismic and non-seismic failures) that involve a loss of offsite power. Block 9A includes the different seismic failure modes of the diesel generators. The battery chargers (i.e., fragility curve 35) are also included in this assessment. It is assumed that their loss eventually leads to a loss of DC, which is needed for continued operation of the diesels. Block 9B is a constant which accounts for all the non-seismic failure combinations of the emergency diesel generators. Block $9 \mathrm{C}$ models the seismic failures of the diesel generator fuel oil transfer system. Even a small break in any one of the six segments modeled is conservatively assumed to fail both trains of fuel oil. Block 9D consists of two terms. Block 7C is as described previously; i.e., it accounts for the seismic failure modes, which lead to a small LOCA. The constant in Block 96 accounts for the non-seismic failure modes that, together with a small LOCA, lead to core damage. Block 9E models the scenarios which involve failure of either component cooling water or auxiliary saltwater together with loss of power to the charging pumps. The Block labeled CCW/ASW, models the CCW and ASW seismic failure modes. It is assumed that a complete guillotine rupture of the ASW piping is required to cause loss of the ASW system. For the CCW system, even a small pipe break is assumed sufficient. The constant modeled by Block $9 \mathrm{~F}$ accounts for the non-seismic failure combinations that, together with a loss of RCP seal cooling caused by a seismic failure of CCW or ASW, lead to core damage. These non-seismic failure combinations involve a loss of onsite emergency power to the charging pumps consequently resulting in a failure of seal injection and RCS makeup. Blocks 9F and 10A (to 


\section{Review of Seismic PRA}

be discussed in the next section), together, model all the failure combination that prevent the operators from aligning the firewater system to the charging pumps in the event CCW or ASW failures occurs.

\section{BLOCK 10}

Block 10 accounts for scenarios that involve a seismic failure of either CCW or ASW. Block CCW/ASW is as described above when discussing Block 9E. The failures in Block 10A model all the important failure combinations which preclude the operators from aligning firewater to the charging pumps for continued RCP seal injection. The operator action itself is modeled by ZHESE1. This human error rate is modeled as being dependent on the seismic level; i.e., the human error rate increases as the earthquake level increases.

\section{BLOCK 11}

Block 11 accounts for all the non-seismic failure combinations, except those involving a loss of offsite power, which result in core damage. Examples of these sequences are failure of all vital AC and failure of all vital DC.

\section{BLOCK 12}

This Block accounts for the scenarios that result in core damage because of relay chatter. Block 12A models the three separate fragilities modeled for relay chatter. Any one of these events is modeled as leading to an initial loss of all AC. The relay chatter recovery action is ZHECT1; as with ZHESE1, the human action is dependent upon the seismic level. Given the occurrence of relay chatter and the failure to recover from it, the model assumes that core damage results. 
Table 4.7.8 Comparison of DCPRA Seismic Core Damage

Frequency Percentiles With Monte Carto Results

\begin{tabular}{|l|c|c|c|c|c|}
\hline & \multicolumn{5}{|c|}{ Total Core Damage Frequency Distribution } \\
\hline & $5 \%$ & $50 \%$ & Mean & $95 \%$ & EF \\
\hline DCPRA & $9.3 \mathrm{E}-7$ & $6.2 \mathrm{E}-6$ & $3.7 \mathrm{E}-5$ & $1.1 \mathrm{E}-4$ & 17 \\
\hline Monte Carlo & $1.26 \mathrm{E}-6$ & $8.89 \mathrm{E}-6$ & $4.15 \mathrm{E}-5$ & $1.77 \mathrm{E}-4$ & 26 \\
\hline
\end{tabular}




\section{Review of Seismic PRA}

Table 4.7.9 Ranking of Block Model Accident Sequences from Monte Carlo Uncertainty Analysis

BLOCK ACC. Seq,

1 Loss of all $125 \mathrm{~V} \mathrm{DC}$

2 Loss of all AC power

3 Loss of 3 trains AC

4 Loss of vital instrumentation

5 RPS failure to trip

6 Excessive LOCA

7 LOCA w/O ECCS injection

8 Control panels fail

9 Loss of offsite power

$10 \mathrm{CCW}$ or ASW systems fails

11 Random failures

12 Relay chatter fails AC power

\section{Ranking}

No 3

No 1

No 12

No 6

No 10

No 4

No 9

No 8

No 2

No 5

No 11

No 7
Contribution

$12.4 \%$

38.7

0.4

2.9

0.7

5.8

1.5

2.0

26.6

5.3

0.6

2.9 
4 Review of Seismic PRA

Table 4.7.10 Accident Sequence Mean Frequency Inerements for Intervals on the Hazard Curve

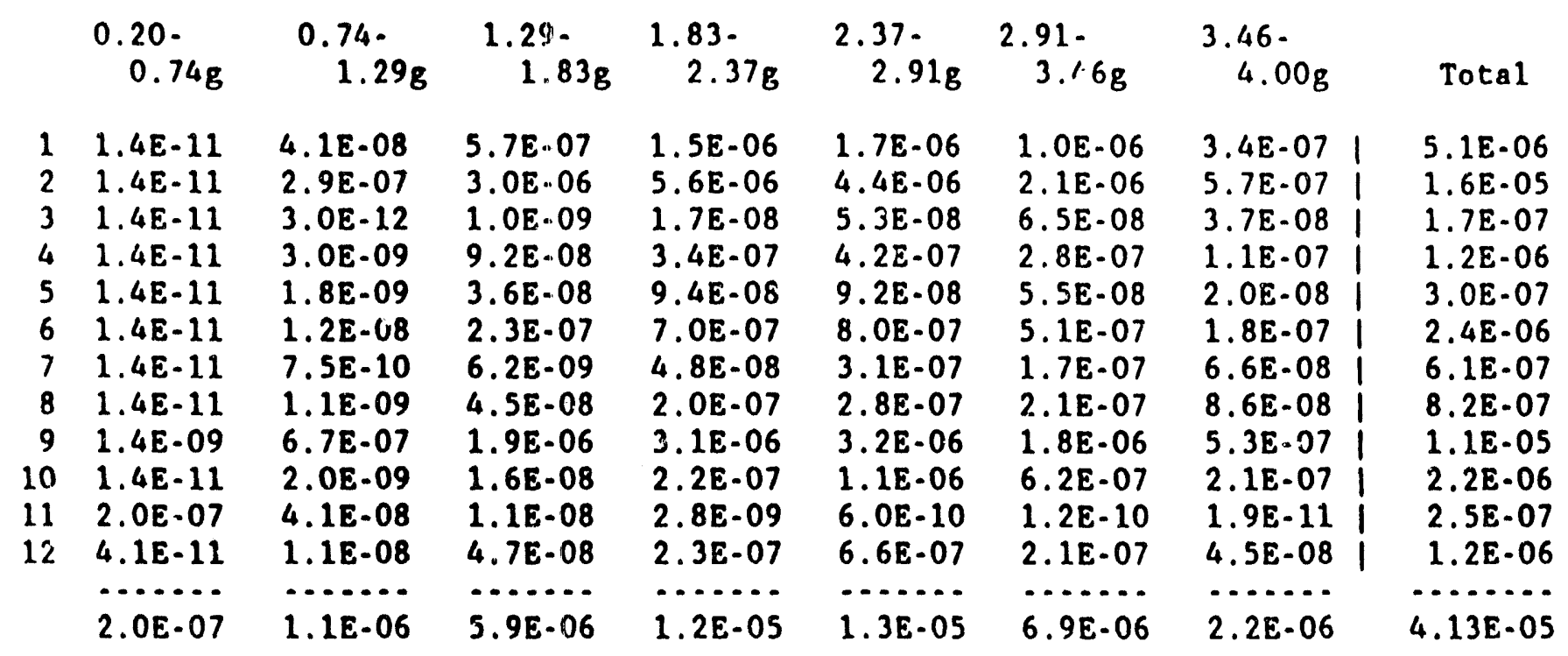


4 Review of Seismic PRA

Table 4.7.11 Component Risk Reduction Potentials as Given in the DCPRA and as Computed by Monte Carlo Approach

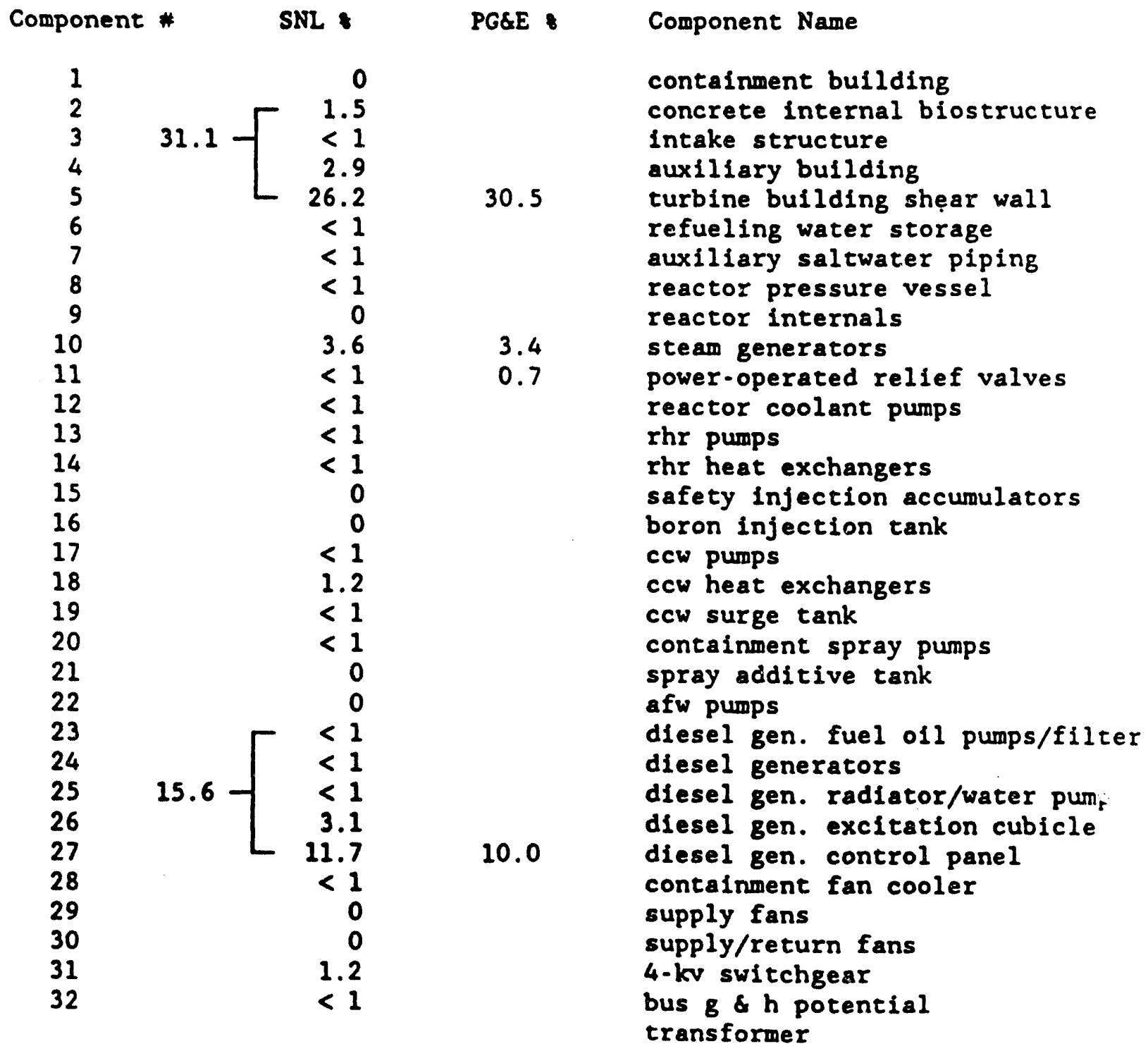


Table 4.7.11 (continued)

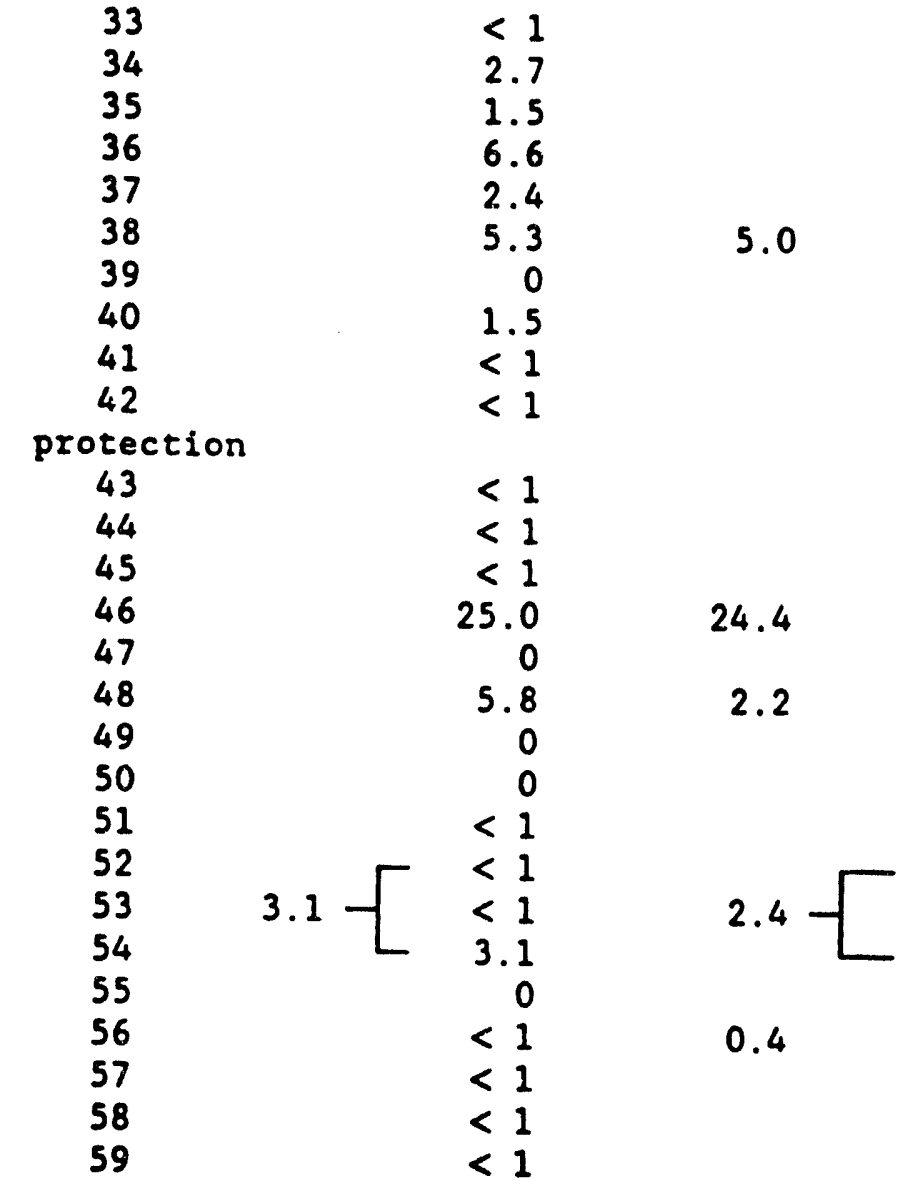

safeguard relay panel

batteries

battery chargers

switchgear/breaker panel

inverters

$4,160 v / 480 v$ transformers

auxiliary relay panel

main control boards

hot shutdown panel

process control and

reactor trip switchgear

pressure \& del p transmitter

impulse lines

offsite power, $230 \mathrm{kv}$

offsite power, $500 \mathrm{kv}$

bop piping and supports

penetration(s) boxes

hvac ducting and supports

switchgear/strut

chatter, main control board

chatter, dg control panel

chatter, 4-kv switchgear

chatter, safeguards relay panel

strut for turbine building

bus $f$ potential transformer

safeguard relay panel

centrifugal charging pump 


\section{OTHER EXTERNAL EVENTS}

\subsection{Fire}

\subsubsection{Introduction}

The scope of the fire analysis review was limited to a review of the methodology and the dominant fire core damage scenarios as reported in the DCPRA. Two of the control room fire scenarios were requantified, and a scenario initiated by a fire on the turbine operating deck was also requantified. Importance analysis of the fire scenarios included within the non-seismic Dominant Sequence Model (DSM) can be found in Table 3.9.1a and Table 3.9.8.

\subsubsection{Methodology}

The methodology used in the fire portion of the PRA appears to be fully acceptable, and was judged to be capable of finding any fire vulnerabilities in the plant, as well as ranking them in the order of importance. First, a screening analysis was performed, in which it was conservatively assumed that a fire in any given fire area failed all equipment in that area. Fire and smoke propagation through fire doors left open, and through other pathways, was included. For scenarios surviving the screening analysis, a more detailed analyses including operator recovery actions and uncertainties in the estimated core damage frequency were performed. For scenarios requiring more detailed analysis, estimates of geometric factors and severity factors were made. The geometric factor gives the fraction of the fire area in which the fire must start in order to fail the target equipment. The severity factor gives the fraction of the fire frequency for fires at least severe enough to fail the target equipment. If the net result of a fire scenario was an initiating event with failures of mitigating systems at a frequency which was small compared to the frequency of the corresponding sequence from internal events, then the scenario was excluded from further analysis. For example, sheet 9 of table F.3-3 of the PRA gives an analysis of a fire in a particular location in the containment building which can as a consequence cause a small LOCA (by inadvertent opening of a PORV). However the frequency estimated, SE-5/yr, is small compared to the frequency of small LOCAs from other causes, and hence the scenario was eliminated from further consideration.

The geometric factors and severity factors were obtained by engineeringjudgement by the analyst, and did not make use of calculations with fire propagation codes. In the NUREG-1150 studies in which fire was considered, the geometric factor for a fire of a given severity was obtained by the use of a fire propagation code. However, it appears to the reviewers that an experienced fire analyst can make judgements concerning geometric and severity factors with adequate accuracy.

\subsubsection{Turbine Building Fire Scenarios}

The dominant turbine building fire scenario in the PRA was sequence FS8 in Table 6-61. There is no discussion of this sequence in the text of the fire appendix, Appendix F of the DCPRA. However, the quantification is given on sheets $98 \mathrm{ff}$ of Table F.3-3 of the DCPRA. A fire starts on the operating deck of the turbine building, and smoke from this fire goes through vents to the safety related $4 \mathrm{kV}$ switchgear located one floor below, and fails all three safety-related trains of $4 \mathrm{kV}$ busses $(F, G$, and $H)$. A significant feature of the Diablo Canyon plant is the fact that the safety-related 4KV switchgear are located in the turbine building. This means that a large fire in the turbine building has the potential for causing core damage. 


\section{Other External Events}

We have requantified the fire sequence initiated by a fire on the turbine operating deck. In the fire data base developed by Wheelis (NUREG/CR-4586), there were nine turbine operating deck fires in about 700 turbinebuilding years, which gives an estimate of $.013 / y r$ as the initiating event frequency. The PRA used 2E-3/yr as the initiating event frequency. The PRA estimate was based on weighing the estimate of the frequency for all fires in turbine buildings by the area fraction for the turbine building operating deck. However, a disproportionate number of fires that occur in turbine buildings occur on the operating deck, and, since there is enough operating data to obtain an estimate from experience directly, we believe that this is more appropriate. The PRA estimated that the fraction 2.5E-3 of the fires would result in smoke going through vents to the switchgear rooms (one floor below) and failing the switchgear, leading to core damage. If we use the same conditional probability (conditional frequency, in probability-of-frequency terminology) of leading to core damage in our estimate, we obtain 3.3E-5/yr as our estimate of core damage from a turbine building operating deck fire.

A review of international experience for fires beginning on operating decks of turbine buildings can also be used to help quantify this sequence. No systematic review was made, but there have been at least three very serious turbine operating deck fires in international experience: a fire at the Muehleberg nuclear power plant in Switzerland on July 28, 1971, a fire in Taiwan (Maanshan-1 nuclear power plant, July 7, 1985) and one in Spain (Vandellos-1 nuclear power plant, October 19, 1989). In the Vandellos-1 fire (See Nucleonics Week, December 4, 1989), burning oil ran down into the lower levels of the turbine building, damaging some equipment there. In addition, water from firefighters' hoses and a broken condenser intake pipe failed major safety systems. This fire illustrates the fact that in a severe turbine operating deck fire, water may be used, and the water can itself cause damage. In addition, there is the possibility that the fire itself can spread, by burning oil, to lower floors, and fail safety-related equipment there, if fire barriers fail. There are about 5600 reactor-years world-wide at the present time. Hence the frequency of very severe fires on turbine operating decks is estimated at 5.4E-4/yr. If, for such a fire at Diablo Canyon, there is a

6 percent chance of the fire failing the $4 \mathrm{kV}$ switchgear, either by smoke, or water damage through some unspecified path, or by the spreading of the fire through failure of a fire barrier, one obtains 3.2E-5/yr for the core damage frequency from this sequence, about the same as estimated above. Of course, there are considerable uncertainties in this estimate.

PG\&E, in letter No. DCL-90-156 (June 18, 1990), submitted clarifying information on the DCPRA fire risk analysis. This analysis supports the PRA estimate of the conditional probability of core damage, given a fire on the turbine operating deck, but does not address the frequency of turbine operating deck fires. Our reestimate of the frequency of core damage from turbine operating deck fires comes from a re-estimate of the frequency of turbine operating deck fires.

\subsubsection{Requantification of Two Control Room Fire Scenarios}

Two control room fire scenarios were requantified. Each scenario involves loss of auxiliary saltwater (ASW) or component cooling water (CCW). On loss of component cooling water (either directly or indirectly through loss of ASW), the reactor coolant pumps (RCPs) must be tripped in a short time (about 10 minutes), or there will be bearing failure of the RCPs, shaft vibration, and seal failure, with a small LOCA resulting. These scenarios must be distinguished from another way of getting a RCP LOCA, when CCW is lost. This other way involves loss of seal injection and CCW to the thermal barriers of the RCP seals, and results in a seal LOCA even for a tripped RCP, but in a much longer time frame, of about 1.5 hours. If a LOCA occurs, and the control room is evacuated, it is unlikely that the LOCA can be mitigated from the hot shutdown panel, since there are limited ECCS controls. This assumption is consistent with the DCPRA: in one of the 
dominant scenarios in Table 6-61 of the DCPRA, a cable spreading room fire in which a PORV sticks open, no credit is given for establishment of recirculation from outside the control room.

In the NUREG-1150 fire study for Surry, it was estimated that in 10 percent of all control room fires heavy smoke will fill the control room within 5 to 10 minutes. For such fires, it is unlikely that the RCP pumps will be tripped before leaving the control room. If the pumps are not tripped from the control room, it is unlikely they will be tripped in time from outside the control room. First, the operators would need to recognize the need to trip the pumps, and, secondly, it is not possible to trip the pumps from the hot shutdown panel. It would be necessary to 80 to the non-safety-related $12 \mathrm{kV}$ switchgear room, located 5 floors below the control room. No credit is given for re-initiation of ASW and CCW from the hot shutdown panel before the RCP seal LOCA, given that the RCP pumps are not tripped.

At a meeting with representatives of PG\&E (April 3, 1990, in Rockville, Md.), it was learned that the main control board panels (vertical) each have their own smoke alarm. Because of this, it was judged that fire suppression before control room evacuation was more likely to be successful at Diablo Canyon than at Surry, where these smoke alarms are not present. It was, therefore, assumed that in only 6 percent of control room fires would heavy smoke fill the control room and force evacuation before the RCPs were tripped.

For those fires which did create heavy smoke within 5 to 10 minutes, it was considered that the fire was sufficiently severe that a fire anywhere on a given control board would fail all equipment on that control board.

The two control room scenarios which were requantified were scenarios CR1 and CR4 from the PRA, appendix F. Scenario CR1 involves a fire at board VB-1 which fails the ASW/CCW control circuits. Scenario CR4 involves a fire at board VB-4 which fails the $4 \mathrm{kV}$ safety-related busses $F, G$, and $H$, and hence fails ASW/CCW, but leaves the non-safety-related reactor coolant pumps operating.

Quantification of the CR1 scenario is as follows:

Freq. of control room fires:

Fraction of fires with heavy smoke:

Prob. fire is on board VB-1:

Core damage frequency from scenario:
$5 \mathrm{E}-3 / \mathrm{yr}$

.06

$94 / 2260$

$1.2 \mathrm{E}-5 / \mathrm{yr}$

The probability the fire is on board VB-1 was the median estimate obtained in the DCPRA, and was obtained in the DCPRA as the ratio of the base area of VB-1 to the base area of all control panels in the control room.

The quantification of the CR4 sequence is the same, except that the probability the fire is on board VB-4 is .052 , taken from the DCPRA. (The DCPRA assumed that the fire had to be on 50 percent of the board to fail the $4 \mathrm{kV}$ circuits, but we are assuming that, for those fires creating heavy smoke, the fire is sufficiently severe so that a fire anywhere on the board will fail these circuits.)

The result obtained is:

Core damage frequency from CR4 scenario $=1.6 \mathrm{E}-5 / \mathrm{yr}$. 


\section{Other External Events}

In response to a request for additional information, PG\&E revised their analysis of the control room fire sequences (See letter from J.D. Schiffer of PGi\&E to U.S. NRC, dated May 3, 1990, PG\&E letter no. DCL-90-118). The review analysis differs from PG\&E's revised analysis primarily in assuming that in all fires so severe as to cause evacuation from the control room, all circuits on a board would fail (given that a fire initiated on the hoard), while PG\&E assumed that the geometry/severity factor was independent of whether or not the control room would have to be evacuated. Additionally, for those sequences in which the control room had to be evacuated, the review analysis gave no credit for operator action to trip the reactor coolant pumps, while PG\&E gave some credit. Experiments at Sandia National Laboratory (see NUREG/CR-4547, Vol. 2) have shown that a fire in a control cabinet can lead to dense smoke resulting in total obscuration of the room within 6-15 minutes of fire ignition. (In fact, in the Sandia experiments, this occurred in all cases.) For such fires, it seems inappropriate to give credit for operator action in tripping the reactor coolant pumps before leaving the control room. In the revised analysis, PG\&E obtained a frequency of 6.5E-6 per year for scenario CR1, and a frequency of $2.4 \mathrm{E}-6$ per year for scenario CR4.

The instrumentation at the hot shutdown panel (HSDP) at Diablo Canyon is not independent of the instrumentation in the control room. As noted in the May 3, 1990 letter from PG\&E to the NRC, if an instrumentation circuit should fail in the control room, the instrumentation at the HSDP will fail offscale. However, there is a dedicated shutdown panel (DSP), located elsewhere, where some essential instrumentation, including reactor coolant system temperature and pressure, pressurizer level, and steam generator level, is provided. The fact that, for a fire in the control room which affects instrumentation there, coordination is required from two separate locations, will increase the human error probability. We can make a rough estimate of the contribution to the core damage frequency from these sequences as follows:

\begin{tabular}{|l|c|}
\hline Frequency of control room fires: & $5 \mathrm{E}-3 / \mathrm{yr}$ \\
\hline Probability fire affects instrumentation: & .1 \\
\hline Probability of control room evacuation: & .06 \\
\hline $\begin{array}{l}\text { Probability of human error leading to core damage, from the need to } \\
\text { coordinate shutdown actions from two separate locations, the HSDP } \\
\text { and the DSP, in cases where instrumentation is affected at the HSDP. }\end{array}$ & .1 \\
\hline Sequence frequency: & $3 \mathrm{E}-6 / \mathrm{yr}$ \\
\hline
\end{tabular}

The above estimate is rather rough, but indicates that the sequence is not of very great importance.

\subsubsection{Cable Spreading Room Fires}

Two of the dominant fire core damage scenarios were initiated by fires in the cable spreading room. A brief review of these sequences found no obvious errors; the review was not in sufficient depth to determine independent estimates of the geometric or severity factors. One notes that, at least explicitly, there is no consideration of the fact that fire suppression activities may control the fire before the targets are damaged. Especially for that fraction of fires where the fire was started because of activities in the cable spreading room, it may be appropriate to give credit for fire suppression activities. No changes were made in the frequencies 
of the cable spreading room fire scenarios. The core damage frequency from the two dominant cable spreading room fires was 2.0E-5/yr in the PRA.

\subsubsection{Summary of Analysis of Review Results}

Table 5.1.1 gives the results obtained in the requantification of the fire scenarios, and compares them to the results obtained in the DCPRA, as revised by PG\&E in their May 3, 1990 letter. The mean core damage frequency from all fire scenarios is estimated at 3.5E-5/yr in the DCPRA, and the review estimate is 8.0E-5/yr. The dominant fire scenarios initiated in the control room and the cable spreading room are more or less typical of scenarios obtained in other fire PRAs. Although fires affecting electrical switchgear have also been important in other fire PRAs, one thing that is different about Diablo Canyon is the location of the safetyrelated switchgear in the turbine building, where it can possibly be damaged by a fire on the turbine operating deck. Uncertainties include uncertainties in the fire initiator frequency, and the geometric/severity factors. In addition, the Sandia fire risk scoping study (NUREG/CR-5088) identified several previousty unaddressed issues, including the adequacy of fire barrier qualification methods. If fire barriers should fail much earlier than now expected, then there would be a higher probability of zone-to-zone fire propagation, which might increase severe core damage frequency from fires.

In Summary, a fairly rigorous fire analysis was performed by PG\&E and it appears to have captured the major fire scenarios associated with the plant. Alternative quantification offered by the review is approximately a factor of two higher than the DCPRA results. In both cases these are point estimates with large uncertainty bands. 
Table 5.1.1 Fire Scenario Core Damage Frequencles

\begin{tabular}{|l|l|c|c|}
\hline \multicolumn{1}{|c|}{ Scenario } & $\begin{array}{c}\text { DCPRA } \\
\text { Frequency }\end{array}$ & $\begin{array}{c}\text { Review } \\
\text { Frequency }\end{array}$ \\
\hline 1. & CS2 (Cable Spreading Room Fire) & $1.2 \mathrm{E}-5 / \mathrm{yr}$ & $1.2 \mathrm{E}-5 / \mathrm{yr}$ \\
\hline 2. & CS1 (Cable Spreading Room Fire) & $7.9 \mathrm{E}-6 / \mathrm{yr}$ & $7.9 \mathrm{E}-6 / \mathrm{yr}$ \\
\hline 3. & FS8 (Turbine Operating Deck Fire) & $6 \mathrm{E}-6 / \mathrm{yr}$ & $3.2 \mathrm{E}-5 / \mathrm{yr}$ \\
\hline 4. & CR1 (Control Room Fire on board VB-1) & $6.5 \mathrm{E}-6 / \mathrm{yr}$ & $1.2 \mathrm{E}-5 / \mathrm{yr}$ \\
\hline 5. & CR4 (Control Room Fire on board VB-4) & $2.4 \mathrm{E}-6 / \mathrm{yr}$ & $1.6 \mathrm{E}-5 / \mathrm{yr}$ \\
\hline & Sum, all fire scenarios & $3.5 \mathrm{E}-5 / \mathrm{yr}$ & $8.0 \mathrm{E}-5 / \mathrm{yr}$ \\
\hline
\end{tabular}




\subsection{Non-Seismic/Non-Fire External Events}

The internal flood analysis within the DCPRA followed the same methodology as the other spatiallydependent external events, i.e. a complex spatial interaction study coupled with a detailed impact analysis on plant systems. Flooding-type events ranked third in the external event category behind seismic and fire. Importance analyses of the flood scenarios (FS9, FS10, and FS11) included within the DSM can be found in Table 3.9.1a and Table 3.9.8. A detailed review beyond the importance analyses was not deemed necessary for the flooding sequences.

As discussed in Section 3.4, seven truly external events were also considered as potential initiators (aircraft impact, ship impact, external floods, hurricanes and tornadoes, hazardous chemical release, turbine missiles and external fires). The DCPRA showed all of these events provided low core damage risk. PG\&E included a hazardous chemical release sequence within the DSM based upon a completeness argument as it constituted only 0.2 percent to the overall DSM CDF (see Table 3.9.1a). 


\section{RESULTS AND CONCLUSIONS}

\subsection{Summary of Results}

The DCPRA was given a detailed and broad-scoped review. The basis for such a comprehensive review originates with a license condition that required a full reevaluation of the seismic risk of the plant. As part of the reevaluation, both probabilistic and determiniatic analyses were required. The seismic portion of the PRA, therefore, required special review attention. In order to provide a base from which to develop a seismic or other external event PRA model, the internal events PRA including all the systems analyses must first be formulated. Therefore, the internal event portion of the PRA was also given an in depth review. As firerelated initiating events contributed a measurable fraction of the overall CDF, these too were included in the review.

As the result of a fairly interactive reviow procese, PG\&E made a number of changes to their original submittal. There are, therefore, two sots of results discussed in this section as provided by PG\&E (original and updated).

In a number of areas, the review has ofiered alternative quantification to that presented in the DCPRA. Table 6.1.1 provides an overall picture of the DCPRA results (both original and updated) versus those of this review. (Mean point estimate vahes for the soirmic CDF are used rather as an estimate from the uncertainty analysis to be consistent with eatimates for other initiators).

The primary reason for the difieresce in internal event quantification presented in Table 6.1.1 between the PG\&E and BNL resolis is the allernate condilional split fractions quantifled for the auxiliary saltwater system and a higher initiating frequency for the LOOP oveat. Overall, requantification has not significantly changed indights regarding which initiating ovents are the major contributors to the core damage frequency.

As seen from Tabie 6.1.1, the reviow did sot propose athernative remitts for the seismic-induced CDF as the roviow found that there were wo aignificant deagreements in the hazard and fragility estimates used in the DCPRA. In pad PRA reviown, alterantive ectiantes of these two parameters have been generally the cause of different CDP eutimates. Partly, this is the result of the interactive mode in which this review was conducted. This node of reviow idontifled woveral lawes and offered a number of sensitivity studies early in the review such that the resolutions were bxopporated in the final analysis or issues were shown to be not important. However, the main reacon for the fower dlangreements is the very rigorous and detailed plant/sitespecific analyses performed for both the haserd and fragility extimates in the DCPRA by PG\&E. Sensitivity studies in the seianic analyais were performed as part of the reviow to verify the DCPRA analyses and draw additional iasights.

A number of the fire sequences were alos requantified. The reasons for the higher fire-induced CDF eatimated by the reviow ane two fold: 1) higher eatimate of frequency of the turbine deck fires; and 2) less credit to several operator actions weed in the DCPRA to mitigate the fire scenarios.

From an overall perrpective, (in the conteat of the license condition and the LTSP program) the seismic events are not dominant contributors to the CDP culimates for the Diablo Canyon plant. The overall seismic contribution in percentages varies from about 10 percent (review) to about 20 percent (DCPRA). Both the internal events and fire events contribute more to the CDF eatimates than the seismic events based upon the results of this review. 


\section{Results and Conclusions}

A comparative analydis of the overall results of the DCPRA with those of nine other PRAs was also conducted. The estimated CDFs for the Diablo Canyon plant were found to be similar to those compuied for other comparable PWRs. 
6 Results and Conclusions

Table 6.1.1 Overall DCRPA Mean CDF Estimates

\begin{tabular}{|l|l|l|l|}
\hline & \multicolumn{1}{|c|}{$\begin{array}{c}\text { Initial DCPRA } \\
\text { Submittal }\end{array}$} & $\begin{array}{c}\text { Updated DCPRA } \\
\text { (Based Upon Review } \\
\text { Feedback) }\end{array}$ & $\begin{array}{c}\text { Alternative Results } \\
\text { Offered by the Review }\end{array}$ \\
\hline Internal Events & $1.3 \mathrm{E}-4(65 \%)$ & $1.3 \mathrm{E}-4(62 \%)$ & $2.1 \mathrm{E}-4(63 \%)$ \\
\hline Seismic Events & $3.7 \mathrm{E}-5(19 \%)$ & $3.7 \mathrm{E}-5(18 \%)$ & $3.7 \mathrm{E}-5(11 \%)$ \\
\hline Fire Events & $2.8 \mathrm{E}-5(14 \%)$ & $3.5 \mathrm{E}-5(17 \%)$ & $8.0 \mathrm{E}-5(24 \%)$ \\
\hline $\begin{array}{l}\text { Other External Events } \\
(0.8 ., \text { Foods) }\end{array}$ & $6.9 \mathrm{E}-6(3 \%)$ & $6.9 \mathrm{E}-6(3 \%)$ & $6.9 \mathrm{E}-6(2 \%)$ \\
\hline Total & $2.0 \mathrm{E}-4$ & $2.1 \mathrm{E}-4$ & $3.3 \mathrm{E}-4$ \\
\hline
\end{tabular}


6 Results and Conclusions

\subsection{Conclusions}

\subsubsection{General}

The following conclusions/observations are applicable to the entire PRA:

- The PRA methodology used in the DCPRA represents "state-of-the-art" methodology, and in many cases advanced the state of the art.

- The involvement of the PG\&E personnel in conducting and maintaining the DCPRA was extensive. (This is an important point in that the license condition mandated a significant degree of "hands on" involvement.)

- Several plant modifications, based on the results of the early phases of the PRA study, have been accomplished within the plant and were incorporated into the DCPRA models.

- The review of the DCPRA was made difficult by the lack of adequate details in the original documentation coupled with a very complex plant model consisting of over 2000 sequences (encompassing 178 distinct support states) for the internal events alone.

Specific conclusions with respect to the internal and seismic events are described in the following subsections.

\subsubsection{Intermal Events Analysis}

- The internal events are the major contributors to the total core damage frequency (roughly 65\%).

- The Loss of Ofisite Power (LOOP) initiator is by far the greatest contributor to the internal event core damage frequency (roughly $1 / 3$ to $1 / 2$ ) and in number of leading sequences. About $3 / 4$ of the LOOPinduced core damage is ascociated with extended unit blackout.

- Initiators representing support system (common cause) failures other than LOOP (i.e., Loss of one de bus (L1DC), Total loss of auxiliary saltwater (LOSW), Total loss of component cooling water (LPCC), Loss of $480 \mathrm{~V}$ switchgear ventilation (LOSWV), Loss of control room ventilation (LOCV)) contribute in the range of 9-11 percent to the internal event core damage frequency.

- Transients (other than Loop) contribute between 25 and 40 percent to the internal event core damage frequency.

- The total contribution to the internal event core damage frequency of initiators belonging to the Loss of Coolant Inventory group is small, about 5 to 10 percent.

- By examining the leading sequences, the following observation is made regarding the leading individual sequences. No single internal event sequence contributes more than 3 percent of to the non-seismic CDF. 
- Among the operational failures of the plant, loss of feed and bleed cooling, loss of auxiliary feed water system, and occurrence of RCP seal LOCA are the most frequent.

- The following scenarios and their contribution to the non-seismic CDF are: Station Blackout (18\%); RCP Seal LOCA (with and without station blackout - 31\%); Primary Relief Valve Opens and Fails to Reclose (12\%); Bieed and Feed Cooling (12\%); and Pressurized Thermal Shock (4\%).

- The most important frontline systems were the auxiliary feedwater system and primary system pressure relief.

- The most important support systems were the diescl generator systems, the $125 \mathrm{~V}$ dc power systems and the auxiliary saltwater system.

- Based upon the review requantification, operator failure to actuate SSPS (upon failure to automatically actuate) went from one of the lesser important operator actions to the most important.

\subsubsection{Seismic Events Analysis}

- The seismic core damage frequency computed in the PRA has withstood a detailed review, and was found to be a reasonable estimate taking into consideration uncertainties inherent in such estimates.

- The overall seismic CDF profile is dominated by accident sequences leading to station blackout (79\%). These may be due to loss of offsite power (LOOP) in conjunction with failures in the emergency diesel generator system, or due to failures of buildings (i.e., Turbine Building) or components (e.g., $4 \mathrm{kV}$ switchgear or $4 \mathrm{kV} / 480 \mathrm{~V}$ transformers) which can lead to station blackout with or without loss of offsite power.

Of these blackout sequences, 53 percent are those not directly failing the auxiliary feedwater system, but leading to reactor coolant pump seal cooling failures, and hence, small LOCAs. Direct failures of the AFWS account for 22 percent of the station blackout sequence contributions.

- The single greatest contributor is the failure of the turbine building, followed by the loss of $230 \mathrm{kV}$ offsite power. There appear to be no overwhelmingly weak links in the plant.

- The seismic hazard analysis provided a reasonable probabilistic representation of the earthquake ground motions at the site considering the information developed in the LTSP program. The Hosgri fault zone was found to dominate the seismic hazard at the site. The Los Osos and San Luis Bay faults each contribute only a few percent to the total hazard. Relative contributions to the total hazard from the other faults is insignificant. Sensitivity studies showed the important parameters are slip rate, maximum magnitude, and ground motion attenuation. The spectral shape and uncertainties used in the fragility analysis are thought to be a reasonable estimate and consistent, in most part, with the detailed ground motion studies carried out in the LTSP. 


\section{Results and Conclusions}

- The fragility parameters for the structures and equipment were found to be reasonable. The level of effort in developing the seismic fragilities for equipment went well beyond those expended on previous seismic PRAs. A review of the identified and analyzed modes found that the appropriate failure modes have been considered.

- Based on the systems review, it appears that the sequence of scenarios and events which can be portrayed by the early front line tree represents a reasonable and complete approach to modeling the Diablo Canyon seismic scenarios.

- Using the results of the seismic PRA, the component and plant seismic margins were derived, and the margin against the 84 percent site-specific ground motion was calculated. It can be seen that all components whose failure will lead to seismic core damage have at least $\mathbf{4 0}$ percent margin over the sitespecific ground motion. The median capacities were shown to be much higher.

- The largest contribution to the seismic-induced CDF comes from earthquakes with average spectral accelerations in the $2 \mathrm{~g}$ to $3 \mathrm{~g}$ range. This provides an important insight that for the high seismic sites, the seismic CDF estimates appear to be governed by seismic levels at or near the HCLPF value.

\subsection{Suggested Enhancements for Future DCPRA Use}

Based upon the detailed review performed on the DCPRA and the fact that PG\&E has stated that this PRA will be continually updated and used in support of future activities, the following items are offered as suggested enhancements:

- Instrument Air System should be realistically modelled. (It currently is modelled as a guaranteed failure.)

- The HRA documentation should be augmented to describe:

a. how HCR and SLIM were used and for what purposes;

b. which PSF scale values were analyzed as part of each human task action under the various analyses;

c. what general and specific insights (causal factors for significant contributions to risk) were derived from the analysis along with recommended remedial actions taken/planned to overcome each deficiency;

d. general and specific insights gained from the HRA; and

e. any lessons learned from applying SLIM in a non-consensus expert judgement mode.

- In the main body of the report, the dominant seismic accident sequences from the po int estimate model should be presented (and collapsed as necessary) and discussed so that the reader has a clear picture of the risk profile. 
- Explain and present an example showing how the seismic point estimate mean component fragilities were calculated. This is especially important as, given the way they are currently calculated (by averaging over a hazard curve increment), the reader cannot reproduce the numbers presented.

- Explain and present an example showing how the mean point estimate seismic split fractions were calculated including showing how calculations representing conditional failure probabilities were calculated. Again, these are vital to reviewing the point estimate model and the reader should have an example to show how they can be reproduced from the tables of fragility, median values and uncertainties.

- In presenting the 791 seismic accident sequences including complement factors, it is very important that the complement factors be consistently applied (as they were inserted by hand) because, as explained in the body of this report, these sequences must be collapsed to be meaningful. Collapsing these sequences is considerably hampered by the fact that a number of complement factors (when near unity) were merely deleted from the accident sequences. This greatly increased the difficulty in identifying similar accident sequences in the collapsing process.

- The mean hazard curve frequencies associated with the various spectral increments should be included explicitiy in the report as it is these values that were multiplied by the failure fractions to obtain the unconditional accident sequence probabilities.

- The seismic block diagram uncertainty analysis model should be fully documented in the final report. Much insight can be gained from this model and it is the basis of the uncertainty analysis results. In addition, the random blocks should be explained as to their meaning and how they were computed.

\subsection{Final Remarks}

There were two primary goals associated with the review of the DCPRA. The first was to ensure that the DCPRA was sufficiently complete and accurate to provide a reasonable foundation upon which the necessary elements of the Diablo Canyon Long Term Seismic Program (LTSP) could be based. The second was to provide quality feedback, where appropriate, so that the DCPRA might become an even more useful tool in any future applications.

We believe that both goals were met in that the review was sufficiently rigorous and broad enough in scope to conclude with a high degree of confidence that the DCPRA does indeed provide a reasonable foundation to support the LTSP and sufficient feedback was provided such that some elements of the DCPRA were modified during the review land others have been identified by PG\&E for future revisions as a result of the review.

One of the key elements of the review process turned out to be its interactive nature. There were a number of meetings where preliminary results of specific review areas were presented and discussed. For example, all eight system analysis reviews documented in Appendices A and B were forwarded through NRC to PG\&E and meetings were held to discuss the preliminary findings. A number of meetings were also held covering the seismic aspects of the PRA. 


\section{Results and Conclusions}

As with any large and complex piece of work such as the DCPRA, it is almost impossible to document every detail, assumption, success criterion, etc. Therefore, when the meetings were held, much of the open item material was found to be because of insufficient documentation. Other open items were shown to have merit with some being dismissed as having very low impact and others accepted in whole or in part as feedback into the DCPRA.

Finally, we believe that the rather sophisticated importance analyses carried out by BNL provided a large number of insights with respect to the Diablo Canyon plant that were not otherwise available. 


\section{APPENDIX A \\ System Analyses for Selected Frontline Systems}

A1: High Pressure Injection System

A2: Low Pressure Injection System

A3: Auxiliary Feedwater System 


\section{APPENDIX A1: HIGH PRESSURE INJECTION SYSTEM}

\section{A1.1. Introduction}

\section{A1.1.1 Objective}

The objective of this appendix is to summarize the results of reviewing the unavailability analysis of the high pressure injection functions of the emergency core cooling system described in the DCPRA. ${ }^{1}$

\section{A1.1.2 Organization}

Section A1.2 provides a brief description of the configurations and the functions, the dependency on support equipment, the surveillance and maintenance conditions, the unavailability modelling in the DCPRA, and the original results obtained. The purpose of this approach is to present stand alone documentation to which the review's findings can be directly compared. Section A1.3 contains the results of the BNL review and presents the findings.

For completeness, the ranked cut sets of hardware unavailabilities (both independent and total) obtained by BNL are given for certain top events in Attachment A1.A.

\section{A1.2. Unavailability Modelling of High Pressure Functions of the ECCS in the DCPRA}

\section{A1.2.1 System Description, Configarations and Fanctions}

The emergency core cooling system (ECCS) of Diablo Canyon Unit I consists of four systems: the safety injection (SI) system, the residual heat removal (RHR) system, the accumulators, and a portion of the chemical and volume control system (CVCS). Different configurations of these systems serve to perform the high pressure injection (HPI) and high pressure recirculation (HPR) functions of the ECCS. For the HPI function, the emergency water source is the refueling water storage tank (RWST). For the HPR function, the containment recirculation sumps (CRS) are used as water sources. The changeover from HPI function to HPR function involves a system reconfiguration (valve realignment performed by the operator).

The HPI functions of the ECCS include:

a. providing makeup water for lost RCS water after a small or medium LOCA when the pressure of the reactor coolant system (RCS) is above the shutoff pressure of the accumulators and the RHR pumps (170 psi),

b. providing water "feed" for steam "bleed" after certain transients when this operation is required or providing "feed cooling" if steam-generator cooling is lost, and

c. providing boration given an anticipated transient without trip (ATWT) or cooldown transient.

The HPR function provides long-term heat removal via the RHR heat-exchangers under post-LOCA conditions. 
Appendix A1

\section{A1.2.2 Top Event Definitions, Success Criteria}

Associated with HPI and HPR functions the DCPRA defines five top events to be used in the frontline system event trees for various initiators. These top events essentially represent the unavailabilitiesof particular ECCS configurations as defined in the accident sequences. The unavailabilities of the top events are quantified for different boundary conditions, i.e., when all the support systems are available as well as when various portions are unavailable. The designators of these five top events are: $\mathrm{CH}, \mathrm{HI}, \mathrm{HR}, \mathrm{RC}$, and RF. Their definitions are given in Table A1.2.1. The associated system configurations are shown in Figures A1.2.1 through A1.2.3. In the figures, the areas with shaded contours represent the supercomponents of the configurations modelled in the DCPRA.

The success criteria for each of the top events are also described in Table A1.2.1. They relate to the specific boundary condition represented by each of the support systems being available after a reactor trip. (The success criteria for all the boundary conditions can be found in Chapter E. 4 of the DCPRA.) For comparison, in Table A1.2.1 the success criteria for HPI and HPR functions of the ECCS required by the DCFSAR ${ }^{2}$ are also indicated. One can see that the top event success criteria and the assumptions used in the logic models fairly well cover those of the FSAR.

\section{A1.2.3 Logic Models, Dependencies on Support Systems}

The logic models of the top events describe the system configurations shown in Figures A1.2.1 through A1.2.3. These indicate the logic relationships among the supercomponents and the dependencies on trains or supercomponents of relevant support systems whose unavailabilities determine the boundary conditions for the top events.

Figures A1.2.4 and A1.2.5 show the logic diagrams for top events $\mathrm{CH}$ and SI, respectively. Figure A1.2.6 presents the logic diagrams for top event $H R$, when

1) the RHR discharge is aligned to the CH or the SI pumps (see the diagram via broken line), and when

2) the RHR discharge is aligned to an operating SI pump.

Figure A1.2.7 shows the logic diagram for top event RC. The logic diagram for top event RF is presented in Figure A1.2.8.

\section{A1.2.4 Boundary Conditions of Top Events}

Top events $\mathrm{CH}$ and SI were evaluated for five and four boundary conditions, respectively. These boundary conditions were required for no LOOP and LOOP initiating events and for unavailabilitiesof certain support system trains or certain supercomponents. Top event HR was evaluated for 15 boundary conditions. These boundary conditions are defined by the unavailabilities of various support system supercomponents, charging pumps, safety injection pumps and RHR pumps. Top events RC and RF were evaluated for two and four boundary conditions resulting from the unavailabilities of RHR trains and various combinations of LOCA size, the available time for recirculation switchover and the unavailability of containment spray. The detailed list of the above boundary conditions is given in Table A1.2.2. 
Appendix AI

\section{A1.25 Quantincation of Top Erent Split Fractions}

The methodology of systems analysis applied in the DCPRA requires that the top event "split fractions" associated with each boundary condition reflect the notion that a top event occurs when a system (or its portion) is in one of the following mutually exclusive alignments: 1) normal alignment, 2) testing alignment, 3) maintenance alignment, and 4) misalignment. Thus, the contribution to the system unavailability from a specific alignment is determined by the conditional system unavailability, given that the system is in that alignment multiplied by the fraction of time that the system spends in that alignment. That is the way that the DCPRA considers the constraints due to Technical Specifications disallowing simultaneous maintenance or test activitieson redundant components and the human errors causing the system or its components (usually occurring after these activities) to be inoperable.

Table A1.2.3 lists the values of top event split fractions for each houndary condition quantified by PG\&E. The table presents the total unavailabilities (TTL), along with the main contributors to the total unavailabilities, such as hardware (HW), maintenance (MN), test (TS), and human error (HE). At a given boundary condition the hardware contribution relates to the normal alignment, when no test or maintenance activities are being performed. To provide complete information the table also indicates the two constituent parts of the hardware contribution to the unavailability: the independent (HWI) and the dependent (HWD common cause) failures of the supercomponents.

One notices that for the high pressure top events there is no contribution to the total unavailability due to testing. This fact arises because, according to the DCPRA:

a. Routine shift checks verify the standard valve alignment and do not change the state of any valves.

b. Monthly checks verify whether the injection flow paths are operable or not. According to the reviewer's understanding these "operability" checks represent stroke testing of SI discharge and suction valves, RHR suction and heat exchanger outlet valves). These tests are of short duration.

c. Quarterly tests are either of very short duration or performed during cold shutdowns.

(To be more specific, the quarterly tests represent:

1. Operability testing of centrifugal charging and safety injection pumps (centrifugal charging pumps are aligned normally, but SI pumps are aligned for recirculation to RWST). In addition, they include stroke testing of valves mentioned above at the monthly testing.

2. Operability flow testing of check valves and ECCS flow balancing tests are performed during cold shutdowns.)

d. Leak testing of check valves and pressure isolation MOVs are performed at refuelings (and after each maintenance).

The maintenance contribution was calculated by assuming that unscheduled maintenances are performed as required on both the pumps and valves. It was also assumed that a valve in maintenance will fail only the associated supercomponent. The DCPRA states that unscheduled maintenance of motor-operated valves of the ECCS is usually performed without isolating the valves. 
According to the models, significant human error may occur only when the operators must switch over from injection to recirculation and realign the flow paths. This is reflected in the HE contributions to the unavailability of top event RF. For the other top events, human errors do not contribute significantly to the total unavailability, because it is assumed that after test or maintenance activities the next shift would detect a misalignment.

\section{A1.3 Results of the BNL Review}

\section{A1.3.1 General}

A thorough review of the high pressure top event logic diagrams and the corresponding fault trees (the fault trees are not reproduced here, they can be found in Chapter E.4 of the DCPRA) was performed by BNL based on the drawings and information provided by $P G \& E^{3}$ The information included the Technical Specifications, operating and surveillance test procedures relevant to the high pressure top events, fluid flow and actuation logic diagrams.

\section{A1.3.2 Logic Diagrams, Fault Trees}

The review found that the logic diagrams and the fault trees adequately represent the failure conditions of the high pressure functions of the ECCS. The failure modes of each of the supercomponents for the various top events have been determined correctly. For audit calculation purposes, all the top events were requantified by BNL. The requantification was done using the SETS code ${ }^{4}$ and allowed identification of the most important minimal cut sets contributing to the hardware unavailabilities. These cut sets are inaccessible for direct review in the DCPRA. Attachment A1.A lists the ranked cut sets for various boundary conditions of top events $\mathrm{CH}, \mathrm{SI}$, and HR. The definitions of the basic events appearing in the cut sets are identical to those given in Chapter E-4 of the DCPRA.

\section{A1.3.3 Audit of Top Event Unavailability (Split Fraction") Calculations}

BNL performed audit calculations for each of the boundary conditions of all the top events by using the results obtained by the SETS-code and applying the same input data, maintenance frequency and duration, and human factors which were used in the DCPRA calculations. The obtained values are presented in Table A1.2.3, denoted by "BNL" to be compared with the values given in the DCPRA (see the entries denoted by "PG\&E").

BNL also carried out sensitivity calculations for top events $\mathrm{CH}$ and SI, in order to check the validity of the assumption made in the DCPRA, that in the case of the LOCAs the leak occurs in cold leg 1 (see the notes in Figures A1.2.4 and A1.2.5, respectively). This assumption was made by PG\&E to simplify the quantification of these split fractions.

\section{A1.3.4 Comments/Findings}

The BNL audit calculations resulted in the following comments. (The following comments relate directly to the comment numbers given in Table A1.2.3). 
Appendix A1

1. The hardware unavailability value given by PG\&E for the boundary condition HRA was in disagreement with the BNL value. According to the boundary condition definition given in the DCPRA for the HRA case (4kV Buses $F$ and $H$ failed), BNL believed that the value should be equal to 1.0. This boundary condition results in failure to isolate the RWST from the high pressure pumps, and therefore, according to the assumed success criterion No.1, the high pressure recirculation function would fail.

2. If one compares the PG\&E values for boundary conditions HRS (4kV Bus F failed) and HRA (4kV Buses $F$ and $H$ failed) one finds them to be very close to each other, in spite of the increased number of unavailable buses. Upon preliminary observation, this suggests a possible inconsistency.

3. The PG\&E calculation of HRC is in disagreement with the BNL value as well as from the comparison of the PG\&E values for boundary conditions HR8 and HRC. HR8 is defined as a state of greater system unavailability conditions than that of HRC. Despite this, the PG\&E split fractions are almost the same.

4. The unavailability calculations for $\mathrm{RCl}$ and $\mathrm{RC2}$ seem to be inconsistent with the failure rate values provided to BNL by PG\&E.

PG\&E responded with the following additional information. In terms of split fraction HRA, PG\&E pointed out that high pressure recirculation is not required to the safety injection pumps for this boundary condition. The comparison of HRA with HR5 should be numerically close as both cases have similar cut sets. HRS is dominated by valve $8974 \mathrm{~B}$ failing to close on demand or in maintenance $(90 \%)$ and HRA is dominated by valve $8804 \mathrm{~A}$ failing to open on demand or in maintenance (89\%). PG\&E identified three additional cut sets for HRC beyond those genesated by BNL during the review. When the missing cut sets were added to the BNL cut sets the resulting quantification was in excellent agreement with the PG\&E value. Again, PG\&E pointed out that HR8 and HRC had functionally very similar boundary conditions and therefore would be expected to be relatively close numerically. The difference between BNL and PG\&E values for RC1 and RC2 was attributed to Monte Carb versus point estimate quantification techniques. None of the above split fractions survived the cut-off for remaining in the reduced model.

The sensitivity calculations for top events $\mathrm{CH}$ and SI performed at BNL by assuming that a leak may occur in any of the four cold legs showed only insignificant increases in the values of these split fractions (since value changes occurred in third decimal place they are not indicated in Table A1.2.3). Thus, the simplifying assumption used by the DCPRA in the evaluation of top events CH and SI represents an acceptable approximation.

BNL pursued an in-depth review of the HPI function system analysis and after weighing the additional input from PG\&E found it to be a fully adequate characterization. 
Appendix A1

\section{A1.4 References}

1. Final report on the Diablo Canyon Long-Term Seismic Program, Pacific Gas and Electric Co., Diablo Canyon Power Plant, Docket Nos. 50-275 and 50-323, July 1988.

2. Units 1 and 2 Diablo Canyon Power Plant, "Final Safety Analysis Report Update," Pacific Gas and Electric Co., December 1988.

3. PG\&E letters to NRC signed by J.D. Shiffer, No. DCL-88-238, October 10, 1988 and No. DCL-88-260, October 28, 1988.

4. Worrel, R.B. and Stack, D.W., "A SETS User's Manual for the Fault tree Analyst," Sandia National Laboratories, NUREG/CR-0465, SAND77-2051, November 1978. 


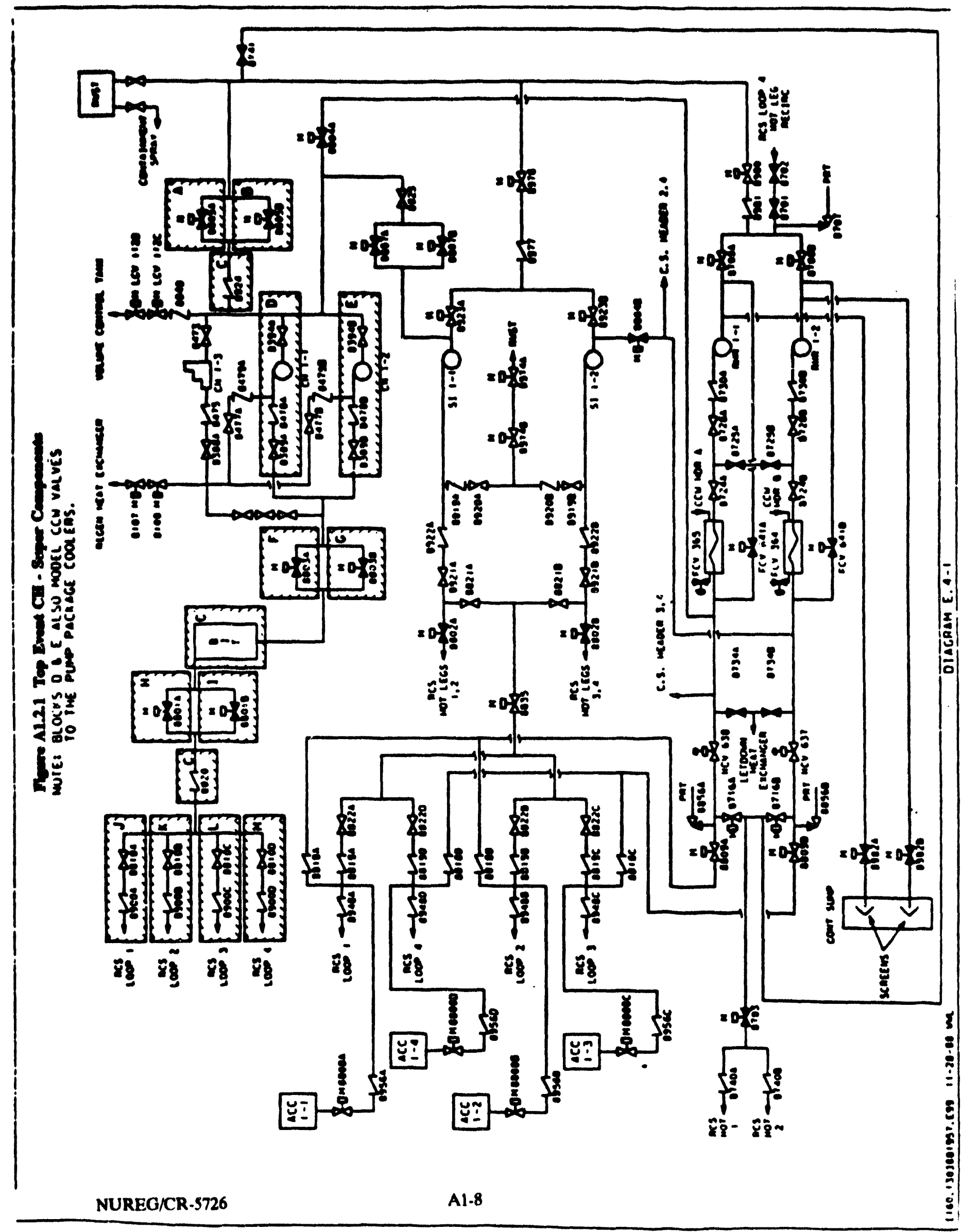




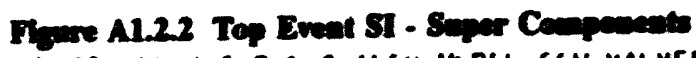

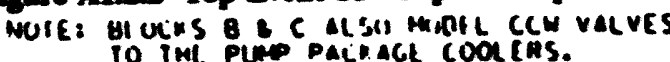

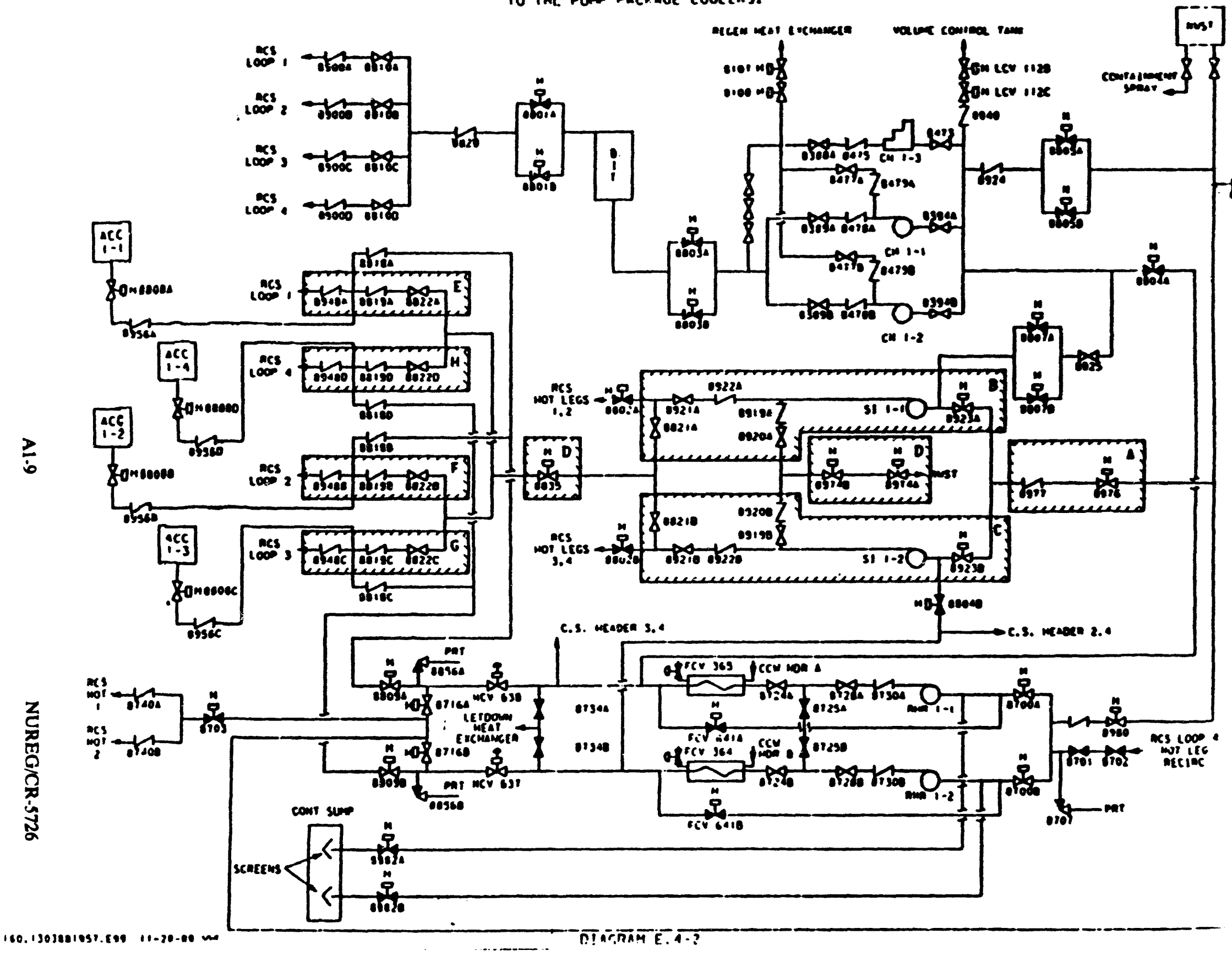




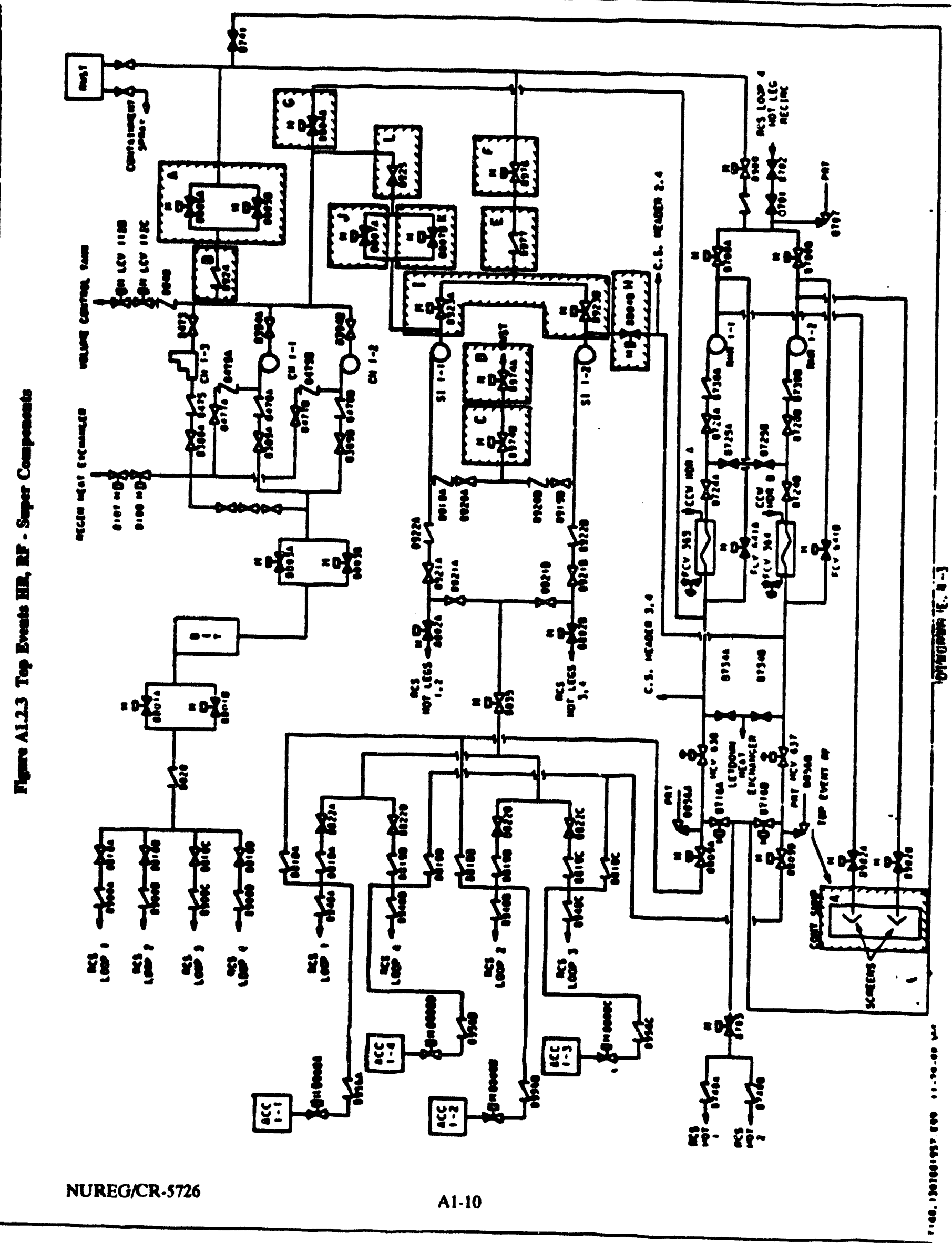




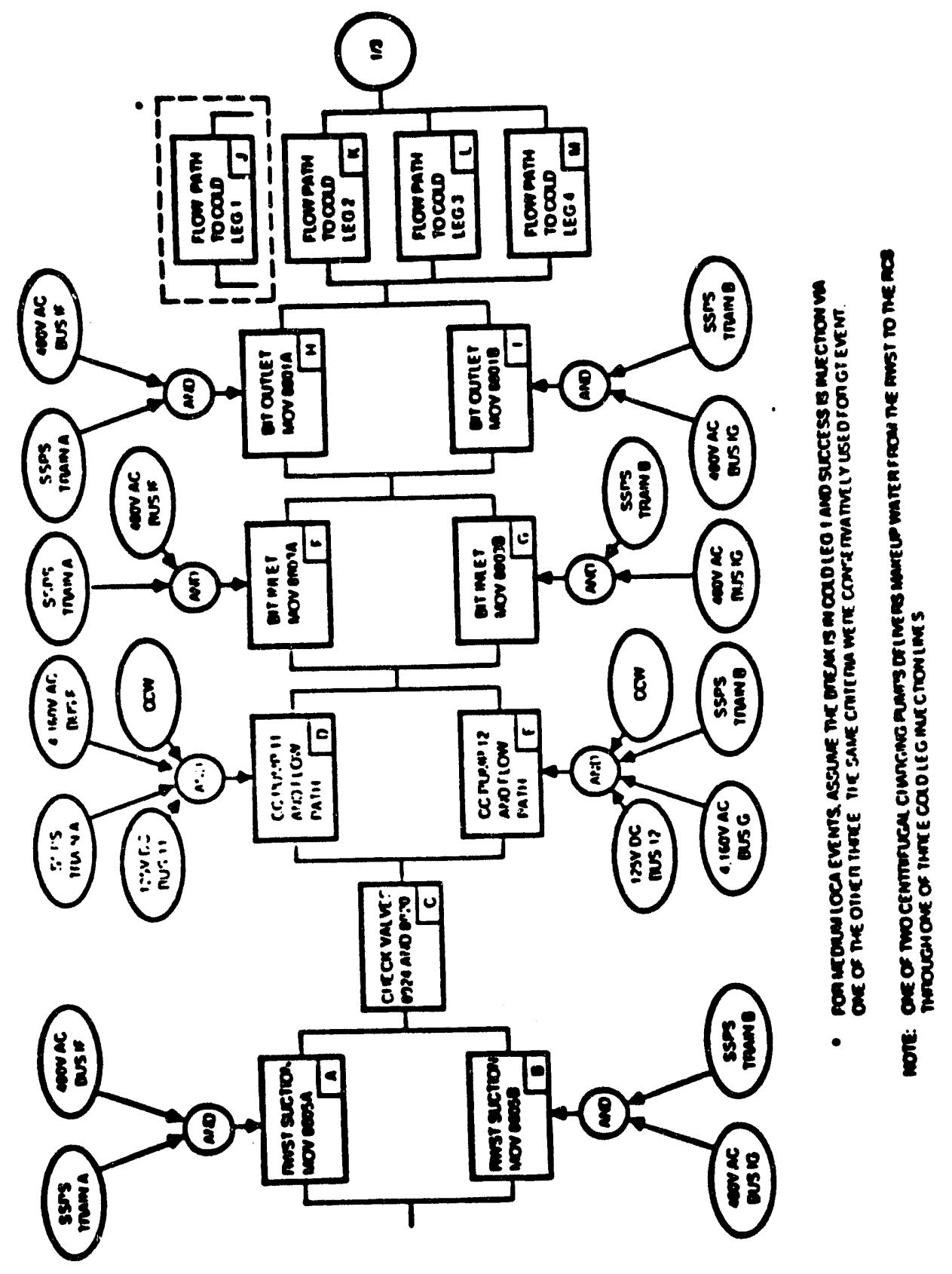

Figure A1.2.4 Logic diagram for top event $\mathrm{CH}$ (charging function) 


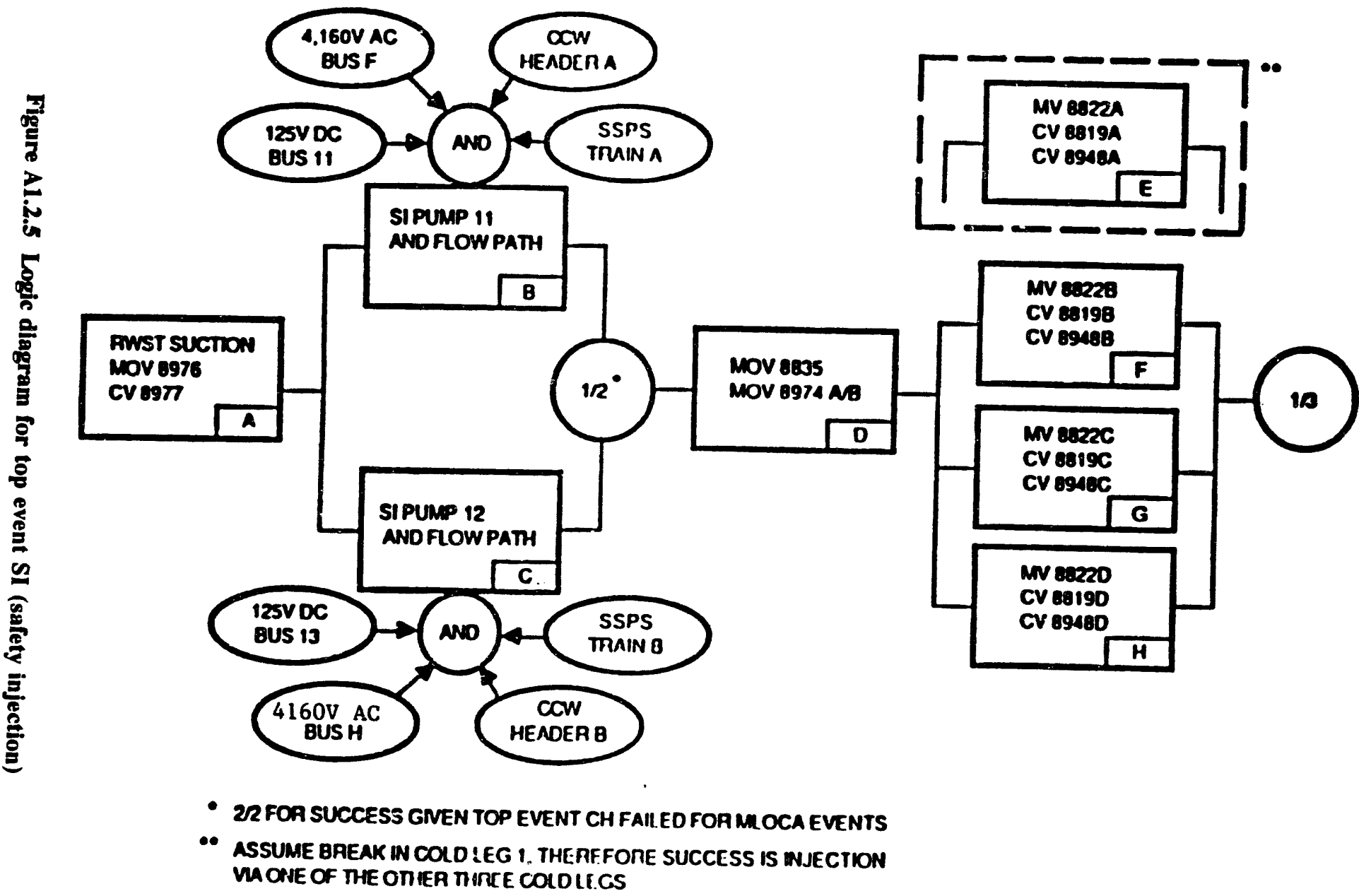




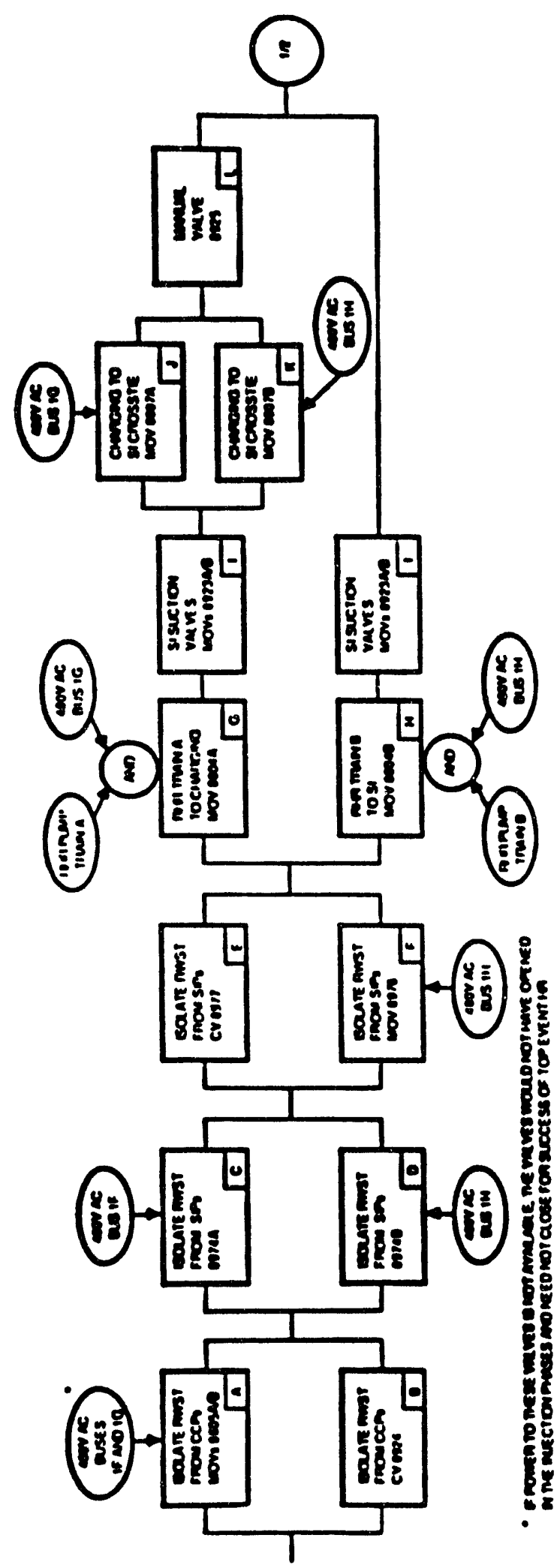

Appendix A1

a. RHR diachange aligaed to SI puap or chargiag purap (top events SI and CB seccesefulu). Dingram via broken "bypasaing" lime.

b. RHR discharge aliged to an operating high pressure pump (top event CH assumed failed).

Fyare A1.2.6 Loje diagran for top event BR: cold leg recirculation with RER pumps 
Appendix Al

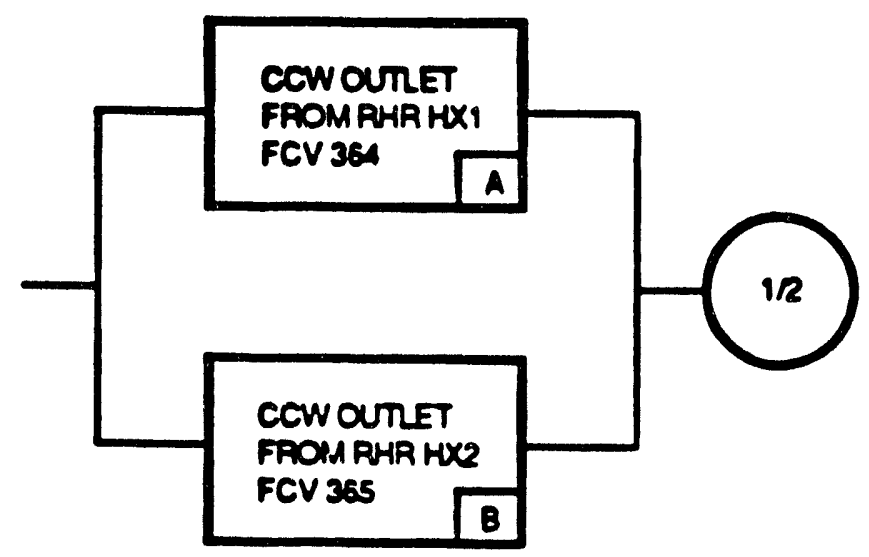

' I/I IF TOP EVENT LIOR LB FAILED

Figure A1.2.7 Logic diagram for top event RC Recirculation cooling unavailable

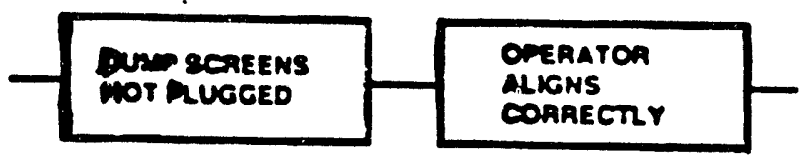

Figure A1.2.8 Logic diagram for top event RF

Operator aligns system for recirculation and sump screens remain unplugged 
Table A1.2.1

Top Event Definitions and Success Criteria*

High Pressure Functions of ECCS

Top Event

Designator

$\mathrm{CH}$

SI

Successful high pressure flow via safety injection pumps to the RCS.
HR ECCS aligns for recirculation.

RC Recirculation heat removal via the component cooling water system.

RF Containment sump available and operator switches to recirculation cooling.

\section{Top Event Success Criteria}

One of two centrifugal charging pumps must start and deliver water to the RCS through at least one cold leg injection line. It is assumed that a LOCA will prevent use of one of the four potential cold leg injection lines. Water is supplied from the RWST during injection and from the RHR pump discharge (i.e., from sumps) during recirculation.

For small LOCA and successful Top Event CH, Top Event SI is not required. For a medium LOCA or a small LOCA and failed $\mathrm{CH}$, then success criteria are the same as for $\mathrm{CH}$, but safety injection pumps supply the water. For a medium LOCA and failed $\mathrm{CH}$, then both safety injection pumps must start and inject water through at least one cold leg.

During recirculation only one safety injection or one charging pump is required to take suction from RHR train discharge and deliver water through one cold leg.

The discharge of at least one RHR train taking suction from the recirculation sumps is aligned to the suction of at least one charging or safety injection pump. The flow path from the RWST must also be isolated.

One of two RHR heat exchanger component cooling water outlet valves must open and stay open.

Sump screens stay unplugged for 24 hours and operator action successful. 
Appendix A1

Table A1.2.1 (contineed)

FSAR Success Criteria

1. The HPI success criterion for a transient or small LOCA during injection phase is one SI or centrifugal charging pump delivering water to at least two cold legs for six hours.

2. The HPI success criterion for a medium LOCA is two of the four SI and centrifugal charging pumps delivering water to at least two cold legs for two hours.

3. The HPR success criterion for a transient or small LOCA is one RHR pump and one centrifugal charging or SI pump delivering cooling water to at least two RCS cold legs for 18 hours. Prior to HPR, during the HPI mode, the RHR pumps were in the mini flow recirculation mode for six hours or tripped.

-The top event success criteria described in this table relate to the condition when all support systems are available. 
Table A1.2.2

Boundary Conditions for ECCS Top Eventa, High Pressure

1. Top Event $\mathrm{CH}$ :

(CH1) All support available.

(CH2) No LOSP, loss of $4160 \mathrm{~V}$ Bus F or LOSP, loss of $4160 \mathrm{~V}$ Bus F or G. (The standby pump train is available only.)

(CH3) No LOSP, loss of $4160 \mathrm{~V}$ Bus G or $125 \mathrm{~V}$ dc Bus 12 .

(CH4) LOSP, all support available.

2. Top Event SI:

(SI1) All support available (1/2).

(SI2) Loss of $4160 \mathrm{~V}$ Bus F or H or $125 \mathrm{~V}$ de Bus 11 or 13 (one SI train is available only, 1/1).

(SI3) Medium LOCA, all support available, top event CH failed (2/2).

3. Top Event HR:

(HR1) All support available.

(HR2) All support available, top event CH or SI failed.

(HR3) All support available, top even LA or LB failed.

(HR4) All support available, top event CH or SI failed and LA or LB failed.

(HRS) 4kV Bus F failed.

(HR6) 4kV Bus F failed, top event CH or SI failed.

(HR7) 4kV Bus F failed, top event LA or LB failed.

(HR8) $4 k V$ Bus F failed, top event CH or SI and top events LA or LB failed.

(HR9) $4 k V$ Bus $F$ and $4 k V$ Bus $G$ failed.

(HRA) 4kV Bus $F$ and $4 k V$ Bus $H$ failed.

(HRB) $4 k V$ Bus $G$ failed.

(HRC) $4 \mathrm{kV}$ Bus $\mathrm{G}$ failed, top event $\mathrm{CH}$ or SI failed.

(HRD) 4kV Bus $H$ failed.

(HRE) 4kV Bus $\mathrm{H}$ failed, top event CH or SI failed.

4. Top Event RC:

(RC1) Both RHR pump trains operable.

(RC2) One RHR pump train operable.

5. Top Event RF:

(RF1) Switchover failure to recirculation after SLOCA or bleed and feed conditions (B/F) with CS failed.

(RF2) Switchover failure to recirculation after SLOCA or bleed and feed conditions (B/F) with CS success.

(RF3) Switchover failure to recirculation after LLOCA or MLOCA initiating event, with CS success.

(RF4) Switchover failure to recirculation after core melt.

CHF, SIF, HRF denote guaranteed failures. 
Appendix Al

Table A1.2.3

Unavailability Values ("Split Fraction") for Emergency Core Cooling System High Pressure Function

\begin{tabular}{|c|c|c|c|c|c|c|c|c|c|c|}
\hline $\begin{array}{l}\text { Top } \\
\text { Event }\end{array}$ & Case & - Calc. & TTL & BW & HWI & EWD & $\mathbf{M N}$ & Ts & HE & $\begin{array}{c}\text { Comment } \\
\text { No. }\end{array}$ \\
\hline \multirow[t]{8}{*}{ CA } & cal & PGCE & $6.234-4$ & $6.066-4$ & $3.528-4$ & $2.339-4$ & $1.677-5$ & & & \\
\hline & & BAL & $6.232-4$ & $6.071-4$ & $3.523-4$ & $2.549-4$ & $1.607-5$ & & & \\
\hline & $\mathrm{CA} 2$ & PGSE & $1.412-2$ & $8.337-3$ & $8.078-3$ & $2.591-4$ & $5.786-3$ & & & \\
\hline & & BRL & $1.409-2$ & 8. $458-3$ & $8.058-3$ & $3.9913-4$ & $5.796-3$ & & & \\
\hline & CA3 & PGEE & $1.162-2$ & $5.829-3$ & $5.570-3$ & $2.591-4$ & $5.786-3$ & & & \\
\hline & & BAL & $1.143-2$ & $5.802-3$ & $5.547-3$ & $2.548-4$ & $5.796-3$ & & & \\
\hline & CaA & PGEE & $7.948-4$ & $7.597-4$ & $3.611-4$ & $3.985-4$ & $3.512-5$ & & & \\
\hline & & BNL & $7.927-4$ & $7.581-4$ & $3.583-4$ & $3.998-4$ & $3.461-5$ & & & \\
\hline \multirow[t]{6}{*}{$8 I$} & SII & PGEE & $3.252-3$ & $3.128-3$ & $2.921-3$ & $2.064-4$ & $1.240-4$ & & & \\
\hline & & BXL & $3.179-3$ & $3.056-3$ & $2.857-3$ & $1.994-4$ & $1.232-4$ & & & \\
\hline & SI2 & PGEE & $1.596-2$ & $9.328-3$ & $9.128-3$ & $2.007-4$ & $6.631-3$ & & & \\
\hline & & BRL & $1.593-2$ & $9.310-3$ & $9.108-3$ & $2.022-4$ & $6.621-3$ & & & \\
\hline & SI3 & POEE & $2.886-2$ & $1.560-2$ & $1.540-2$ & $2.012-4$ & $1.326-2$ & & & \\
\hline & & BRL & $2.884-2$ & $1.560-2$ & $1.540-2$ & $1.991-4$ & $1.324-2$ & & & \\
\hline \multirow[t]{28}{*}{$\mathbf{E R}$} & BR1 & PGCE & $2.111-4$ & $2.039-4$ & $7.468-6$ & $1.964-4$ & $7.262-6$ & & & \\
\hline & & BRL & $2.099-4$ & $2.029-4$ & $6.587-6$ & $1.963-4$ & $6.993-6$ & & & \\
\hline & HR2 & PGEE & $1.908-3$ & $8.092-4$ & $6.127-4$ & $1.965-4$ & $1.099-3$ & & & \\
\hline & & BML & $1.860-3$ & $7.977-4$ & $6.013-4$ & $1.964-4$ & $1.062-3$ & & & \\
\hline & AR3 & PGEE & $4.010-3$ & $2.366-3$ & $2.169-3$ & $1.964-4$ & $1.644-3$ & & & \\
\hline & & BRL & $3.941-3$ & $2.351-3$ & $2.155-3$ & $1.963-4$ & $1.584-3$ & & & \\
\hline & GR4 & PGEE & $4.325-3$ & $2.680-3$ & $2.399-3$ & $2.809-4$ & $1.649-3$ & & & \\
\hline & & BNL & $4.251-3$ & $2.657-3$ & $2.376-3$ & $2.806-4$ & $1.594-3$ & & & \\
\hline & HRS & PGLE & $2.291-3$ & $1.735-3$ & $1.539-3$ & $1.964-4$ & $5.555-4$ & & & 2 \\
\hline & & BLL & $2.280-3$ & $1.727-3$ & $1.530-3$ & $1.963-4$ & $5.531-4$ & & & \\
\hline & HR6 & PGEE & $3.988-3$ & $2.341-3$ & $2.144-3$ & $1.965-4$ & $1.647-3$ & & & \\
\hline & & BNL & $3.965-3$ & $2.322-3$ & $2.125-3$ & $1.964-4$ & $1.643-3$ & & & \\
\hline & HR7 & PGEE & $6.082-3$ & $3.897-3$ & $3.701-3$ & $1.964-4$ & $2.187-3$ & & & \\
\hline & & BNL & $6.057-3$ & $3.875-3$ & $3.679-3$ & $1.963-4$ & $2.182-3$ & & & \\
\hline & ER8 & PGCE & $6.402-3$ & $4.211-3$ & $3.931-3$ & $2.809-4$ & $2.193-3$ & & & 3 \\
\hline & & BRL & $6.369-3$ & $4.181-3$ & $3.900-3$ & $2.806-4$ & $2.188-3$ & & & \\
\hline & HR9 & PG\&E & $6.081-3$ & $3.897-3$ & $3.700-3$ & $1.964-4$ & $2.187-3$ & & & \\
\hline & & BNE. & $6.057-3$ & $3.875-3$ & $3.678-3$ & $1.963-4$ & $2.182-3$ & & & \\
\hline & BRA & PGEE & $2.361-3$ & $1.818-3$ & $1.733-3$ & 8. $440-5$ & $5 \cdot 429-4$ & & & 1,2 \\
\hline & & BAR & 1.000 & 1.000 & & & & & & \\
\hline & ERB & PGEE & $4.010-3$ & $2.365-3$ & $2.169-3$ & $1.964-4$ & $1.644-3$ & & & \\
\hline & & BRL. & $3.991-3$ & $2.351-3$ & $2.155-3$ & $1.963-4$ & $1.640-3$ & & & \\
\hline & enc & PGEE & $6.430-3$ & $4.236-3$ & $3.956-3$ & $2.809-4$ & $2.195-3$ & & & 3 \\
\hline & & BRL & $4.536-3$ & $2.351-3$ & $2.155-3$ & $1.963-4$ & $2.185-3$ & & & \\
\hline & ERD & PGEE & $4.559-3$ & $3.473-3$ & $3.275-3$ & $1.976-4$ & $1.087-3$ & & & \\
\hline & & BAL & $4.552-3$ & $3.463-3$ & $3.266-3$ & $1.963-4$ & $1.090-3$ & & & \\
\hline & ERE & PGEE & $8.664-3$ & $5.948-3$ & $5.666-3$ & $2.826-4$ & $2.717-3$ & & & \\
\hline & & Bat & $8.641-3$ & $5.917-3$ & $5.637-3$ & $2.806-4$ & $2.724-3$ & & & \\
\hline \multirow[t]{4}{*}{ RC } & RC1 & PGEE & $4.430-5$ & $4.366-5$ & $4.752-7$ & $4.318-5$ & $6.433-7$ & & & 4 \\
\hline & & BR2 & $4.389-5$ & $4.325-5$ & $3.463-7$ & $4.290-5$ & $6.413-7$ & & & \\
\hline & $\mathrm{RC2}$ & PGEE & $1.178-3$ & $6.348-4$ & $5.917 \times 4$ & $4.318-5$ & $5.429-4$ & & & 4 \\
\hline & & BNL & $1.176-3$ & $6.314-4$ & $5.885-4$ & $4.290-5$ & $5.449-4$ & & & \\
\hline \multirow[t]{8}{*}{$\mathbf{R P}$} & RFI & PGEE & $3.161-.3$ & $1.485-4$ & $1.485-4$ & & & & $3.013-3$ & \\
\hline & & BRL & $3.149-3$ & $1.493-4$ & $1.493-4$ & & & & $3.000-3$ & \\
\hline & RF2 & PGCE & $3.373-3$ & $1.485-4$ & $1.485-4$ & & & & $3.225-3$ & \\
\hline & & BARL & $3.349-3$ & $1.493-4$ & $1.493-4$ & & & & $3.200-3$ & \\
\hline & RF3 & PGEE & $4.930-3$ & $7.983-4$ & $7.983-4$ & & & & $4.136-3$ & \\
\hline & & BaL & $4.904-3$ & $8.070-4$ & $8.070-4$ & & & & $4.100-3$ & \\
\hline & RP4 & PGSE & $5.471-2$ & $4.790-2$ & $4.790-2$ & & & & $7.164-3$ & \\
\hline & & BNL & 5. 516-2 & $4.840-2$ & $4.840-2$ & & & & $7.100-3$ & \\
\hline
\end{tabular}

The comments are listed by number in Section A1.3.3 in the text. 


\section{Attachment A1.A}

Hardware Unavailability Cut Sets for the

High Pressure Functions of the

Emergency Core Cooling System

Top Events: $\mathrm{CH}, \mathrm{SI}, \mathrm{HR}$ 
Appendix Al

Top livenis: CII

Boundary condition designator: CIII

Hardware unavailability cut sets due to independent failures; HWI =

1. 3.4337E-04

BKC +

2. $2.4223 \mathrm{E}-06$

$B K F *$ BKG +

3. $2.4223 \mathrm{E}-06$

BKA * BKB +

4. $2.4223 \mathrm{E}-06$

$\mathrm{BKH} * \mathrm{BKI}+$

5. $1.6282 \mathrm{E}-06$

$\mathrm{BKD}$ * BKE +

6. $5.7588 \mathrm{E}-11$

$B K K * B K L$ * BKM

Leading hardware unavailability cut sets due to dependent and independent failures; HW =

1. 3.4337E-04

BKC +

2. $8.4300 \mathrm{E}-05$

$\mathrm{D} 2 \mathrm{VM} 002+$

3. $8.4300 \mathrm{E}-05$

$\mathrm{D} 2 \mathrm{VM} 003+$

4. $8.4300 \mathrm{E}-05$

D2VM001 +

5. 2.4223E-06

$\mathrm{BKF} * \mathrm{BKG}+$

6. 2.4223E-06

BKI * BKI +

$\mathrm{BKA} * \mathrm{BKB}+$

7. $2.4223 \mathrm{E}-06$

$\mathrm{BKD} * \mathrm{BKE}+$

8. $1.6282 \mathrm{E}-06$

D2VCOD1 +

10. 1.9200E-07

D2PCGR +

$+\ldots$

Boundary condition designator: $\mathrm{CH} 2$

Hardware unavailability cut sets due to independent failures; HWI =

1. 3.0455E-03

BKE +

2. $1.5564 \mathrm{E}-03$

BKG +

3. 1.5564E-03

BKB +

4. $1.5564 \mathrm{E}-03$

$\mathrm{BKI}+$

5. $3.4337 \mathrm{E}-04$

$\mathrm{BKC}+$

6. $5.7588 \mathrm{E}-11$

BKK * BKL * BKM

Leading hardware unavailability cut sets due to dependent and independent failures; HW =

1. 3.0455E-03

2. $1.5564 \mathrm{E}-03$

3. $1.5564 \mathrm{E}-03$

4. $1.5564 \mathrm{E}-03$

5. 3.4337E-04

6. $1.4500 \mathrm{E}-04$

7. $8.4300 \mathrm{E}-05$

8. $8.4300 \mathrm{E}-05$

9. $8.4300 \mathrm{E}-05$

10. 1.5600E-06

11. 1.9200E-07
BKE +

BKI +

BKG +

BKB +

$\mathrm{BKC}+$

D2PCGS +

D2VM002 +

D2VM003 +

D2VM001 +

D2VCOD1 +

D2PCGR +

$+\ldots$ 
Boundary condition designator: $\mathrm{CH} 3$

Hardware unavailability cut sets due to independent failures; HWI =

1. $1.5564 \mathrm{E}-03$

BKF +

2. 1.5564E-03

BKA +

3. $1.5564 \mathrm{E}-03$

BKH +

4. $5.3462 \mathrm{E}-04$

BKD +

5. $3.4337 \mathrm{E}-04$

$\mathrm{BKC}+$

6. $5.7588 \mathrm{E}-11$

BKK * BKL * BKM

Leading hardware unavailability cut sets due to dependent and independent failures; HW =

1. 1.3564E-03

BKH +

2. 1.5564E-03

BKF +

3. $1.5564 \mathrm{E}-03$

BKA +

4. $5.3462 \mathrm{E}-04$

BKD +

5. 3.4337E-04

$\mathrm{BKC}+$

6. $8.4300 \mathrm{E}-05$

$\mathrm{D} 2 \mathrm{VM} 001+$

7. $8.4300 \mathrm{E}-05$

$\mathrm{D} 2 \mathrm{VMO03}+$

8. 8.4300E-05

$\mathrm{D} 2 \mathrm{VMOO2}+$

9. $1.5600 \mathrm{E}-06$

D2VCOD1 +

10. 1.9200E-07

D2PCGR +

$+\ldots$

Boundary condition designator: $\mathrm{CH} 4$

Hardware unavailability cut sets due to independent failures; HWI =
1. 3.4337E-04
2. 7.6354E-06
3. $2.4223 E-06$
4. $2.4223 \mathrm{E}-06$
5. $2.4223 E-06$
6. $5.7588 \mathrm{E}-11$

$\mathrm{BKC}+$

BKDLP * BKELP +

BKF * BKG +

BKA * BKB +

BKH * BKI +

$B K K$ * BKL * BKM

Leading hardware unavailability cut sets due to dependent and independent failures; HW =

1. 3.4337E-04

2. $1.4500 \mathrm{E}-04$

BKC +

3. $8.4300 \mathrm{E}-05$

D2PCGS +

4. $8.4300 \mathrm{E}-0 \mathrm{~S}$

$\mathrm{D} 2 \mathrm{VMOO2}+$

$\mathrm{D} 2 \mathrm{VMO01}+$

$\mathrm{D} 2 \mathrm{VMOO3}+$

5. $8.4300 \mathrm{E}-05$

BKDLP * BKELP +

7. $2.4223 \mathrm{E}-06$

BKF * BKG +

8. $2.4223 \mathrm{E}-06$

BKA * BKB +

9. 2.4223E-06

10. 1.5600E-06

$\mathrm{BKH}$ * BKI +

11. 1.9200E-07

D2VCOD1 +

D2PCGR +

$+\ldots$ 
Appendix AI

\section{Top Events SI}

Boundary condition designator: SII

Hardware unavailability cut sets due to independent failures; IIWI =
1. 2.3493E-03
BKD +
2. 4.6790E-04
$\mathrm{BKA}+$
3. $3.9568 \mathrm{E}-05$
$\mathrm{BKB} * \mathrm{BKC}+$
4. $1.6950 \mathrm{E}-10$
BKG * BKH

Leading hardware unavailability cut sets due to dependent and independent failures; HW = 1. 2.3493E-03

2. $4.6790 \mathrm{E}-04$

BKD +

3. $1.9300 \mathrm{E}-04$

$\mathrm{BKA}+$

4. $3.9568 \mathrm{E}-05$

D2PSIS +

5. $4.5700 \mathrm{E}-07$

$\mathrm{BKB}$ * BKC +

D42VCOD12 +

6. $4.5700 \mathrm{E}-07$

D42VCOD14 +

7. $4.5700 \mathrm{E}-07$

D42VCOD23 +

8. $4.5700 \mathrm{E}-07$

D41VCOD24 +

9. 4.5700E-07

D41 VCOD14 +

10. 4.5700E-07

D41VCOD12 +

$+\ldots$

Boundary condition designator: S12

Hardware unavailability cut sets due to independent failures; HWI =
1. $6.2903 \mathrm{E}-03$
2. 2.3493E-03
BKC +
3. $4.6790 \mathrm{E}-04$
BKD +
$\mathrm{BKA}+$
4. $1.6950 \mathrm{E}-10$
BKF * BKG * BKH

Leading hardware unavailability cut sets due to dependent and independent failures; HW =

1. $6.2903 \mathrm{E}-03$

BKC +

2. 2.3493E-03

BKD +

3. $4.6790 \mathrm{E}-04$

$\mathrm{BKA}+$

4. $1.9300 \mathrm{E}-04$

D2PSOS +

5. $4.5700 \mathrm{E}-07$

D42VCOD12 +

6. $4.5700 \mathrm{E}-07$

D42VCOD14 +

7. 4.5700E-07

D42VCOD23 +

8. $4.5700 \mathrm{E}-07$

D41VCOD24 +

D41VCOD14 +

9. 4.5700E-07

10. 4.5700E-07

D41VCOD 12 +

$+\ldots$ 
Boundary condition designator: $\$$ \$I3

Hardware unavailability cut sets due to independent failures; IIWI =

1. $6.2903 \mathrm{E}-03$

BKB +

2. 6.2903E-03 BKC +

3. 2.3493E-03 BKD +

4. 4.6790E-04 BKA +

5. $1.6950 \mathrm{E}-11 \quad \mathrm{BKF}$ * BKG * BKH

Leading hardware unavailability cut sets due to dependent and independent failures; $\mathrm{HW}=$

1. $6.2903 E-03$

2. $6.2903 \mathrm{E}-03$

3. $2.3493 E-03$

4. $4.6790 \mathrm{E}-04$

5. $1.9300 \mathrm{E}-04$

6. $4.5700 \mathrm{E}-07$

7. $4.5700 \mathrm{E}-07$

8. $4.5700 \mathrm{E}-07$

9. 4.5700E-07

10. 4.5700E-07

BKB +

$\mathrm{BKC}+$

$\mathrm{BKD}+$

BKA +

D2PSIS +

D42VCOD12 +

D42VCOD14 +

D42VCOD23 +

D41VCOD24 +

D41VCOD14 +

$+\ldots$ 
Appendix Al

\section{Ton livents IIIR}

Boundary condition designator: HR1

Hardware unavailability cut sets due to independent failures; HWI =
1. 2.4223E-06
BKH * BKG +
2. $2.3298 \mathrm{E}-06$
$\mathrm{BKC} * \mathrm{BKD}+$
3. $9.2653 \mathrm{E}-07$
4. $6.0579 \mathrm{E}-07$
BKI * BKG +
5. $3.0289 \mathrm{E}-07$
BKA * BKB +
$\mathrm{BKF} * \mathrm{BKE}$

Leading hardware unavailability cut sets due to dependent and independent failures; $\mathrm{HW}=$
1. $1.2300 \mathrm{E}-04$
D2VMOC +
2. $8.4300 \mathrm{E}-05$
D2VMOD +
3. $2.4223 \mathrm{E}-06$
$B K{ }^{*} \mathrm{BKH}+$
4. $2.3298 \mathrm{E}-06$
$B K C *$ BKD +
5. $9.2653 \mathrm{E}-07$
$\mathrm{BKI}$ * BKG +
6. $6.0579 \mathrm{E}-07$
BKA * BKB +
7. $3.0289 \mathrm{E}-07$
$\mathrm{BKF} * \mathrm{BKE}+$
$+\ldots$

Boundary condition designator: HR2

Hardware unavailability cut sets due to independent failures; HWI =
1. 5.9531E-04
BKI +
2. $2.4223 \mathrm{E}-06$
BKH * BKG +
3. $2.3298 \mathrm{E}-06$
$\mathrm{BKC} * \mathrm{BKD}+$
4. $6.0579 \mathrm{E}-07$
BKA * BKB +
$\mathrm{BLK} * \mathrm{BKH}+$
$\mathrm{BKF} * \mathrm{BKE}+$
5. 3.4072E-07
$\mathrm{BKH} * \mathrm{BKJ} * \mathrm{BKK}$

Leading hardware unavailability cut sets due to dependent and independent failures; $H W=$

1. 5.9531E-04

2. 1.1200E-04

BKI +

3. 8.4300E-05

D2VMOC +

4. $2.4223 \mathrm{E}-06$

D2VM001 +

BKH * BKG +

5. $2.3298 \mathrm{E}-06$

6. $6.0579 \mathrm{E}-07$

7. $3.4072 \mathrm{E}-07$

8. $3.0289 \mathrm{E}-07$

BKC * BKD +

BKA * BKB +

BKL * BKH +

BKF * BKE +

9. 1.3120E-07

BKH * D2VM002 + $+\ldots$ 
Boundary condition designator: HR3

Hardware unavailability cut sets due to independent failures; HWI =
1. 1.5564E-03
2. $5.9531 \mathrm{E}-04$
$\mathrm{BKH}+$
3. $2.3298 \mathrm{E}-06$
BKI +
4. $6.0579 \mathrm{E}-07$
$\mathrm{BKC} * \mathrm{BKD}+$
BKA * BKB +
5. $3.0289 \mathrm{E}-07$
$B K F * B K E$

Leading hardware unavailability cut sets due to dependent and independent failures; HW =

1. $1.5564 \mathrm{E}-03$

2. $5.9531 \mathrm{E}-04$

$\mathrm{BKH}+$

3. $1.1200 \mathrm{E}-04$

$\mathrm{BKI}+$

$\mathrm{D} 2 \mathrm{VMOC}+$

4. $8.4300 \mathrm{E}-05$

$\mathrm{D} 2 \mathrm{VM} 001+$

5. $2.3298 \mathrm{E}-06$

6. $6.0579 \mathrm{E}-07$

$\mathrm{BKC} * \mathrm{BKD}+$

BKA * BKB +

7. $3.0289 \mathrm{E}-07$

$\mathrm{BKF} * \mathrm{BKE}+$

$+\ldots$

Boundary condition designator: HR4

Hardware unavailability cut sets due to independent failures; HWI =
1. 1.5564E-07
2. $5.9531 \mathrm{E}-04$
BKG +
BKI +
3. $2.1892 \mathrm{E}-04$
BKL +
4. 2.4223E-06
$\mathrm{BKJ} * \mathrm{BKK}+$
5. $2.3298 \mathrm{E}-06$
$\mathrm{BKC} * \mathrm{BKD}+$
6. $6.0579 \mathrm{E}-07$
$\mathrm{BKA} * \mathrm{BKB}+$
7. $3.0289 \mathrm{E}-07$
$B K F *$ BKE

Leading hardware unavailability cut sets due to dependent and independent failures; $\mathrm{HW}=$

1. $1.5564 \mathrm{E}-03$

2. $5.9531 \mathrm{E}-04$

3. $2.1892 \mathrm{E}-04$

4. $1.1200 \mathrm{E}-04$

5. $8.4300 \mathrm{E}-05$

6. $8.4300 \mathrm{E}-05$

7. $2.4223 \mathrm{E}-06$

8. $2.3298 \mathrm{E}-06$

9. $6.0579 \mathrm{E}-07$

10. 3.0289E-07
BKG +

BKI +

BKL +

D2VMOC +

D2VM002 +

D2VM001 +

BKJ * BKK +

BKC * BKD +

BKA * BKB +

BKF * BKE +

$+\ldots$ 
Appendix A1

Boundary condition designator: HR5

Hardware unavailability cut sets due to independent failures; HWI =

1. $1.5264 \mathrm{E}-03$

$\mathrm{BKD}+$

2. $2.4223 \mathrm{E}-06$

$\mathrm{BKH} * \mathrm{BKG}+$

3. $9.2653 \mathrm{E}-07$

4. $3.0406 \mathrm{E}-07$

$\mathrm{BKI} * \mathrm{BKG}+$

$\mathrm{BKAl} * \mathrm{BKB}+$

5. $3.0289 \mathrm{E}-07$

$\mathrm{BKF} * \mathrm{BKE}$

Leading hardware unavailability cut sets due to dependent and independent failures; HW =
1. $1.5264 \mathrm{E}-03$
2. $1.1200 \mathrm{E}-04$
BKD +
$\mathrm{D} 2 \mathrm{VMOC}+$
$\mathrm{D} 2 \mathrm{VM001}+$
3. $8.4300 \mathrm{E}-05$
BKH * BKG +
4. $2.4223 \mathrm{E}-06$
5. $9.2653 \mathrm{E}-07$
6. $3.0406 \mathrm{E}-07$
7. $3.0289 \mathrm{E}-07$
BKI * BKG +
BKA1 * BKB +
BKF * BKE +
$+\ldots$

Boundary condition designator: HR6

Hardware unavailability cut sets due to independent failures; HWI =

1. $1.5264 \mathrm{E}-03$

2. $5.9531 \mathrm{E}-04$

BKD +

3. $2.4223 \mathrm{E}-06$

4. $3.4072 \mathrm{E}-07$

5. $3.0406 \mathrm{E}-07$

6. $3.0289 \mathrm{E}-07$

BKH * BKG +

BKL * BKH +

BKA1 * BKB +

BKF * BKE +

$+\ldots$

Leading hardware unavailability cut sets due to dependent and independent failures; HW =

1. $1.5264 \mathrm{E}-03$

2. $5.9531 \mathrm{E}-04$

BKD +

BKI +

3. $1.1200 \mathrm{E}-04$

D2VMOC +

4. $8.4300 \mathrm{E}-05$

D2VM001 +

5. $2.4223 \mathrm{E}-06$

6. 3.4072E-07

7. $3.0406 \mathrm{E}-07$

8. $3.0289 \mathrm{E}-07$

9. $1.3120 \mathrm{E}-07$

10. 3.7700E-09

BKH * BKG +

BKL * BKH +

BKAl * BKB +

BKF * BKE +

BKH * D2VM002 +

$\mathrm{BKH} * \mathrm{BKJ} * \mathrm{BKK}+$

$+\ldots$ 
Boundary condition designator: HR7

Hardware unavailability cut sets due to independent failures; HWI =
1. 1.5564E-03
BKH +
2. 1.5264E-03
$\mathrm{BKD}+$
3. $5.9531 \mathrm{E}-04$
BKI +
4. $3.0406 \mathrm{E}-07$
$\mathrm{BKAl} * \mathrm{BKB}+-$
5. $3.0289 \mathrm{E}-07$
$\mathrm{BKF} * \mathrm{BKE}$

Leading hardware unavailability cut sets due to dependent and independent failures; HW =
1. 1.5564E-03
2. $1.5264 \mathrm{E}-03$
BKH +
3. $5.9531 \mathrm{E}-04$
$\mathrm{BKD}+$
4. $1.1200 \mathrm{E}-04$
BKI +
5. $8.4300 \mathrm{E}-05$
D2VMOC +
6. $3.0406 \mathrm{E}-07$
D2VM001 +
7. $3.0289 \mathrm{E}-07$
$\mathrm{BKA1} * \mathrm{BKB}+$
$\mathrm{BKF}$ * $\mathrm{BKE}+$
$+\ldots$

Boundary condition designator: HR8

Hardware unavailability cut sets due to independent failures; HWI =
1. $1.5564 \mathrm{E}-03$
2. $1.5264 \mathrm{E}-03$
BKG +
BKD +
3. $5.9531 \mathrm{E}-04$
BKI +
4. $2.1892 \mathrm{E}-04$
BKL +
5. $2.4223 \mathrm{E}-06$
$\mathrm{BKJ} * \mathrm{BKK}+$
6. $3.0406 \mathrm{E}-07$
BKA1 * BKB +
7. $3.0289 \mathrm{E}-07$
$B K F *$ BKE

Leading hardware unavailability cut sets due to dependent and independent failures; $H W=$ -
1. $1.5564 \mathrm{E}-03$
2. $1.5264 \mathrm{E}-03$
BKG +
BKD +
3. $5.9531 \mathrm{E}-04$
BKI +
4. $2.1892 \mathrm{E}-04$
$\mathrm{BKL}+$
5. $1.1200 \mathrm{E}-04$
$\mathrm{D} 2 \mathrm{VMOC}+$
6. $8.4300 \mathrm{E}-05$
D2VM002 +
D2VM001 +
BKJ * BKK +
BKA1 * BKB +
8. $2.4223 E-06$
9. $3.0406 \mathrm{E}-07$
$+\ldots$ 


\section{Appendix A1}

Boundary condition designator: HR9

Hardware unavailability cut sets due to independent failures; HWI =
1. $1.5564 \mathrm{E}-03$
$\mathrm{BKH}+$
2. 1.5264E-03
BKD +
3. $5.9531 \mathrm{E}-04$
$\mathrm{BKI}+$
4. $3.0289 \mathrm{E}-07$
$\mathrm{BKF} * \mathrm{BKE}+$
5. $2.3348 \mathrm{E}-09$
$B K A 2 * B K B$

Leading hardware unavailability cut sets due to dependent and independent failures; $\mathrm{HW}=$

1. $1.5564 \mathrm{E}-03$

2. $1.5264 \mathrm{E}-03$

BKH +

$\mathrm{BKD}+$

3. $5.931 \mathrm{E}-04$

$\mathrm{BKI}+$

4. $1.1200 \mathrm{E}-04$

D2VMOC +

5. $8.4300 \mathrm{E}-05$

D2VM001 +

$\mathrm{BKF} * \mathrm{BKE}+$

6. $3.0289 \mathrm{E}-07$

$\mathrm{BKA} 2 * \mathrm{BKB}+$

7. $2.3348 \mathrm{E}-09$

$+\ldots$

Boundary condition designator: HRB

Leading hardware unavailability cut sets due to independent failures; HWI =
1. $1.5564 \mathrm{E}-03$
2. $5.9531 \mathrm{E}-04$
3. $2.3298 \mathrm{E}-06$
4. $3.0406 \mathrm{E}-07$
5. $3.0289 \mathrm{E}-07$
BKH +
$\mathrm{BKI}+$
$B K C * B K D+$
$\mathrm{BKA1} * \mathrm{BKB}+$
$\mathrm{BKF} * \mathrm{BKE}$

Leading hardware unavailability cut sets due to dependent and independent failures; $\mathrm{HW}=$

1. $1.5564 \mathrm{E}-03$

2. 2.9531E-04

3. $1.1200 \mathrm{E}-04$

4. $8.4300 \mathrm{E}-05$

5. $2.3298 \mathrm{E}-06$

6. $3.0406 \mathrm{E}-07$

7. $3.0289 \mathrm{E}-07$
BKH +

BKI +

D2VMOC +

D2VMO01 +

BKC * BKD +

BKAl * BKB +

BKF * BKE + 
Boundary condition designator: HRC

Hardware unavailability cut sets due to independent failures; HWI =
1. $1.5564 \mathrm{E}-03$
2. $5.9531 \mathrm{E}-04$
BKH +
$\mathrm{BKI}+$
3. $2.3298 \mathrm{E}-06$
4. $3.0406 \mathrm{E}-07$
$\mathrm{BKC} * \mathrm{BKD}+$
$\mathrm{BKA1} * \mathrm{BKB}+$
5. $3.0289 \mathrm{E}-07$
$\mathrm{BKF} * \mathrm{BKE}$

Leading hardware unavailability cut sets due to dependent and independent failures; $\mathrm{HW}=$
1. 1.5564E-03
2. 5.9531E-04
BKH +
3. $1.1200 \mathrm{E}-04$
$\mathrm{BKI}+$
4. $8.4300 \mathrm{E}-05$
$\mathrm{D} 2 \mathrm{VMOC}+$
D2VM001 +
5. $2.3298 \mathrm{E}-06$
6. $3.0406 \mathrm{E}-07$
$\mathrm{BKC} * \mathrm{BKD}+$
$\mathrm{BKA1} * \mathrm{BKB}+$
$\mathrm{BKF} * \mathrm{BKE}+$
7. $3.0289 \mathrm{E}-07$
$+\ldots$

Boundary condition designator: HRD

Hardware unavailability cut sets due to independent failures; HWI =
1. $1.5564 \mathrm{E}-03$
BKG +
2. $1.5264 \mathrm{E}-03$
$\mathrm{BKC}+$
3. $1.8286 \mathrm{E}-04$
$\mathrm{BKE}+$
4. $6.0579 \mathrm{E}-07$
$\mathrm{BKA} * \mathrm{BKB}$

Leading hardware unavailability cut sets due to dependent and independent failures; $\mathrm{HW}=$
1. $1.5564 \mathrm{E}-03$
2. $1.5264 \mathrm{E}-03$
BKG +
$\mathrm{BKC}+$
$\mathrm{BKE}+$
3. $1.8286 \mathrm{E}-04$
D2VMOC +
5. 8.4300E-05
$\mathrm{D} 2 \mathrm{VM} 001+$
6. $6.0579 \mathrm{E}-07$
$\mathrm{BKA} * \mathrm{BKB}+$
$+\ldots$ 
Appendix Al

Boundary condition designator: HRF

Hardware unavailability cut sets due to independent failures; $H W I=$
1. $1.5564 \mathrm{E}-03$
2. $1.5564 \mathrm{E}-03$
BKG +
BKJ +
3. $1.5264 \mathrm{E}-03$
$\mathrm{BKC}+$
4. $5.9531 \mathrm{E}-04$
$\mathrm{BKI}+$
5. $2.1892 E-04$
$\mathrm{BKL}+$
6. $1.8286 \mathrm{E}-04$
$\mathrm{BKE}+$
BKA * BKB

Leading hardware unavailability cut sets due to dependent and independent failures; HW =

1. 1.5564E-03

2. $1.5564 \mathrm{E}-03$

BKJ +

BKG +

$\mathrm{BKC}+$

$\mathrm{BKI}+$

BKL +

$\mathrm{BKE}+$

D2VMOC +

D2VM001 +

D2VM002 +

BKA * BKB +

$+\ldots$ 


\section{APPENDIX A2: LOW PRESSURE INJECTION SYSTEM}

\section{A2.1 Introduction}

\section{A2.1.1 Objective}

The objective of this appendix is to summarize the results of reviewing the unavailability analysis of the low pressure injection functions of the emergency core cooling system described in the DCPRA. ${ }^{1}$

\section{A2.1.2 Organimation}

Section A2.2 provides a brief description of the configurations and the functions, the dependency on support equipment, the surveillance and maintenance conditions, the unavailability modelling in the DCPRA, and the original results obtained. The purpose of this approach is to present stand alone documentation to which the review's findings can be directly compared. Section A2.3 contains the results of the BNL review and presents the findings.

For completeness, the ranked cut sets of hardware unavailabilities (both independent and total) ohtained by BNL are given for certain top events in Attachment A2.A.

\section{A2.2 Unavailability Modelling of Low Pressure Functions of the ECCS in the DCPRA}

\section{A2.2.1 System Deseription, Configarations and Punctions}

The emergency core cooling system (ECCS) of Diablo Canyon Unit I consists of four systems: the safety injection (SI) system, the residual heat removal (RHR) system, the accumulators, and a portion of the chemical and volume control system (CVCS). Different configurations of these systems serve to perform the low pressure injection (LPI) and low pressure recirculation (LPR) functions of the ECCS. For the LPI function, the emergency water sources are the RWST and the accumulators. For the LPR function, either the containment recirculation sump (CRS) or the RCS hotleg (and if they are not available, make-up to the RWST) is used as a water source. The change from the LPI function to LPR function involves a system reconfiguration (valve realignment performed by the operator).

The LPI function of the ECCS is to provide makeup water for lost RCS water after a LOCA when the pressure of the reactor coolant system (RCS) falls below the shutoff pressure of the accumulators and the RHR pumps.

The LPR function of the ECCS is to provide long-term heat removal via the RHR heat-exchangers under post-LOCA conditions.

\section{A2.2.2 Top Event Definitions, Success Criteria}

Associated with LPI and LPR functions the DCPRA defines nine top events to be used in the frontline system event trees for various initiators. These top events essentially represent the unavailabilitiesof particular ECCS portions or components as defined in the accident sequences. The unavailabilities of the top events are quantified for different boundary conditions, i.e., when all the support systems are available as well as when 
Appendix A2

their various portions are unavailable. The designators of these nine top events are: $L A, L B, L V, R W, V A$, VB, AC, LI, and MU. Their definitions are given in Table A2.2.1. The associated system configurations are shown in Figures A2.2.1 through A2.2.6. In the figures, the areas with shaded contours represent the supercomponents of the configurations modelled in the DCPRA.

The success criteria for each of the top events are also described in Table A2.2.1. They relate to the specific boundary condition represented by each of the support systems being available after a reactor trip. (The success criteria for all the boundary conditions can be found in Chapter E.4 of the DCPRA.) For comparison, in Table A2.2.1 the success criteria for LPI and LPR functions of the ECCS required by the DCFSAR ${ }^{2}$ are also indicated. One can see that the top event success criteria and the assumptions used in the logic models relate well to those of the DCFSAR for transients as well as small and medium LOCAs. For large LOCAs, the success criteria applied in evaluating top events $A C$ and $L I$ is not as straight-forward a match to the success criteria defined in the DCFSAR. Simply stated, the DCFSAR requires two of four injection paths and the DCPRA requires one of the three intact injection paths (the fourth having the break). The reasons that the DCPRA applies this new set of success criteria for large LOCAs are given as follows (quotation from p.E.5-45 of the DCPRA):

"Based on the report "Safety Evaluation of the RHR Crosstie Line Isolation" prepared by Westinghouse (dated July 10, 1987) and received by PLG from PG\&E on July 27, 1987 (Chron F502246), a success criteria of 1 out of 4 injection paths can be justified for the large LOCA event for the first fuel cycle of Unit 2 if it is recognized that this analysis is not entirely applicable to Unit 1 or the subsequent fuel cycles of Unit 2 . However, the conditions are considered sufficiently close and the conservatisms in the Westinghouse evaluation are quite significant. The same success criteria is judged to be adequate for both Units 1 and 2 in the PRA model. Therefore, the ECCS success (for large LOCA) is defined in this analysis to require injection through one of the remaining three intact cold leg injection paths. For the analysis of the ECCS top events it is assumed that the rupture occurred in cold leg 1 . The 1 of 3 success criteria is conservatively used for all other initiating events also. This reduces the number of boundary conditions that need to be quantified and is just slightly conservative."

Based upon the above quotation and the fact that previous PRAs have used a $2 / 3$ success criterion, BNL conducted a sensitivity analysis to investigate the impact of a $2 / 3$ success criterion vs. the $1 / 3$ used in the DCPRA. The details of this analysis can be found in Section A2.3.2.

\section{A2.2.3 Logie Models, Dependencies on Support Systems}

The logic models of the top events describe the system configurations shown in Figures A2.2.1 through A2.2.6. These indicate the logic relationships among the supercomponents and the dependencies on trains or supercomponents of relevant support systems whose unavailabilities determine the boundary conditions for the top events.

Figures A2.2.7a and A2.2.7b show the logic diagrams for top events LA and LB, respectively. Figure A2.2.8 presents the logic diagram for top events LV and Figure A2.2.9 shows the logic diagram for top event RW. In Figure A2.2.10 the logic diagrams are shown for top event VA, when:

1. the RHR suction train $A$ is aligned to the containment sump (see Figure A2.2.10a), and when

2. the RHR suction train B is aligned to the containment sump (see Figure A2.2.10b). 
Figures A2.2.11 and A2.2.12 show the logic diagrams for top events AC and LI, respectively. The logic diagram for top event MU is given in Figure 2.13.

\section{A2.2.4 Boundary Conditions of Top Events}

Top events LA and LB were evaluated for four and ten boundary conditions, respectively. In both cases, these boundary conditions resulted from different initiating events, the need for bleed and feed, and from unavailabilities of certain support system trains or certain supercomponents. In the case of top event LB, additional boundary conditions were required to account for the success or failure of top event, LA. Top events LV and RW were evaluated only once for all conditions (no support required). Top event VA was evaluated for two boundary conditions; when all support was available and when the failure was guaranteed. Top event VB was evaluated for four boundary conditions by also taking into account the success or failure of top event VA. Top event AC was determined only once (no support required), for the large LOCA initiator. Top event $L$ I was quantified for two cases; a) for all conditions except large LOCA, and b) for large LOCA given failure of top event AC. Top event MU was calculated for three boundary conditions resulting from the unavailabilities of support system components and previous frontline system failures. One of its boundary conditions (MV2) was used to account the unavailability of makeup water to the RWST, involving the spent-fuel pit pump. The detailed list of the above boundary conditions is given in Table A2.2.2.

\section{A2.25 Qunntification of Top Event Split Fractions}

The methodology of systems analysis applied in the DCPRA requires that the top event "split fractions" associated with each boundary condition reflect the notion that a top event occurs when a system (or its portion) is in one of the following mutually exclusive alignments: 1) normal alignment, 2) testing alignment, 3) maintenance alignment, and 4) misalignment. Thus, the contribution to the system unavailability from a specific alignment is determined by the conditional system unavailability, given that the system is in that alignment multiplied by the fraction of time that the system spends in that alignment. That is the way that the DCPRA considers the constraints due to Technical Specifications disallowing simultaneous maintenance or test activitieson redundant components and the human errors causing the system or its components (usually occurring after these activities) to be inoperable.

Table A2.2.3 lists the values of top event split fractions for each boundary condition quantified by PG\&E. The table presents the total unavailabilities (TTL), along with the main contributors to the total unavailabilities, such as hardware (HW), maintenance (MN), test (TS), and human error (HE). At a given boundary condition the hardware contribution relates to the normal alignment, when no test or maintenance activities are being performed. To provide complete information the table also indicates the two constituent parts of the hardware contribution to the unavailability: the independent (HWI) and the dependent (HWD, i.e., common cause) failures of the supercomponents.

One notices that for the low pressure top events there is no contribution to the total unavailability due to testing. This fact arises because, according to the DCPRA:

a. Routine shift checks verify the standard valve alignment and do not change the positioning of any valves.

b. Monthly checks verify whether the injection flow paths are operable or not (these "operability" checks are essentially valve stroke testings performed for RHR pump section and heat exchanger outlet valves). These tests are of short duration.

c. Quarterly tests are either of very short duration or performed during cold shutdowns. 


\section{Appendix A2}

(To be more specific, the quarterly tests represent:

1. Operability testing of RHR pumps (the pumps are aligned for recirculation to RWST, their normal power alignment).

2. Operability flow testing of check valves is performed during cold shutdowns.)

d. Leak testing of check valves and pressure isolation MOVs are performed at refuelings (and after each maintenance). Functional tests of the ECCS are performed also during refuelings.

The maintenance contribution was calculated by assuming that unscheduled maintenances are performed as required on both the pumps and valves. It was also assumed that a valve in maintenance will fail only the associated supercomponent. The DCPRA states that unscheduled maintenance of motor-operated valves of the ECCS is usually performed without isolating the valves. Therefore, valve positions were assumed to be those required by Technical Specifications.

Operator failures were considered for the following activities:

While the RCS pressure is still high, the RHR pumps having been started by the SI signal must shut off before they overheat. Later, when the RCS pressure is appropriatcly reduced, the RHR pumps are manually started. The associated operator failures are treated in top events LA and LB. (The realignment from the RWST to the containment sump was considered in top event RF and described in the discussion of the HPR function in Appendix A1.) The realignment failure for hotleg recirculation by using hotleg suction path to the RHR pumps (following a transient, small LOCA or steam generator tube rupture) is treated in top event MU. This also includes the operator failure to initiate a makeup to the RWST if recirculation cooling is unavailable. Human errors due to misalignment do not contribute significantly to the total unavailability, because it is assumed that after test or maintenance activities the next shift would detect a misalignment.

\section{A2.3 Results of the BNL Review}

\section{A2.3.1 General}

A thorough review of the low pressure top event logic diagrams and the corresponding fault trees (the fault trees are not reproduced here, they can be found in Chapter E.4 of the DCPRA) was performed by BNL based on the drawings and information provided by PG\&E. ${ }^{3}$ The information included the Technical Specifications, operating and surveillance test procedures relevant to the low pressure top events, fluid flow and actuation logic diagrams and revised results for six of the low pressure split fractions. The revised split fractions from PG\&E were in response to the preliminary findings by BNL and have been incorporated in Table A2.2.3.

\section{A2.3.2 Logic Diagrams, Fault Trees}

The review found that the failure modes of each of the supercomponents involved in the logic diagrams for various top events were determined correctly. The logic diagrams and associated fault trees for the top events adequately represent the failure conditions of the low pressure functions of the ECC'S.

In the case of top events $\mathrm{LI}$ and $\mathrm{AC}$, however, the review pursued the following points which are associated with the interpretation of the large LOCA success criterion:

a. The large LOCA success criterion for the ECCS described in the DCFSAR requires two of the four LP injection pathways to be available. These pathways are interpreted by the DCPRA (as it was described above in Section A2.2.2) as allowing one of the pathways to be that which contains 
the break and one other from the remaining three intact pathways. A more conservative success criterion would have been to consider two available pathways from the intact three as was done, e.g., in the Indian Point PRA. The DCPRA large LOCA success criterion was defined essentially on the basis of a Westinghouse study (mentioned previously).

b. The present large LOCA success criterion requires one available injection path out of the three intact pathways. In addition, it assumes that the rupture occurred in cold leg 1 , instead of assuming that the rupture may occur in any of the four cold legs. This latter assumption, when used under appropriate conditions, can result in significant savings of time and effort for the PRA team. However, the review included the investigation as to whether accuracy was lost due to this assumption.

c. The logic diagram for top event AC does not consider common cause failures for the outlet check valves (valve transfer closed or fail to operate on demand failure modes) of the accumulators. The general directives of the DCPRA systems analysis do not allow the taking into account common cause failures of check valves found in four separate lines from four different water sources. However, in this case, the environmental effect due to deposition of boric acid common in the four accumulators represents "a common cause" for check valve failures.

In order to scrutinize the quantified values of split fractions associated with the low pressure functions of the ECCS, BNL performed audit calculations for each of these top events.

For top events $\mathrm{LI}$ and LC, BNL performed additional sensitivity calculations by assuming the same (1/3) success criterion for large LOCA as was assumed in the DCPRA but allowing the rupture to occur in any of the four cold legs.

In addition, BNL performed a cursory sensitivity calculation for top event LI by assuming that the large LOCA success criterion was 2/3, as was assumed in the Indian Point PRA, and by assuming that the rupture occurred in cold leg 1 . This allows a direct measure of the sensitivity to the DCPRA success criterion:

The common cause failure of the accumulator outlet check valves was not included in the BNL calculations because it would require reconfiguration/requantification of the associated fault trees as well as the fact that the plant-specific failure of these check valves when exposed to boric acid deposition and corrosion has not been observed. This item is therefore left as a potential refinement should PG\&E decide at some later date to upgrade the PRA.

The requantification at BNL was done either by PC calculations (for simple top events) or by using the SETS code. The use of the SETS code allowed the identification of the most important cut sets contributing to the hardware unavailabilities. These cut sets are inaccessible for direct review in the DCPRA. Attachment A2.A lists the ranked cut sets for various conditions of top events $\mathrm{LI}$ and $\mathrm{AC}$. The definition of the basic events appearing in the cut sets are identical to those given in Chapter E-4 of the DCPRA.

\section{A23.3 Audit of Top Event Unavailability ("Split Fraction") Calculations}

The audit calculations for each of the boundary conditions of all the top events were performed at BNL by using the results obtained by the SETS-code or by PC calculations. In the audit calculations the same input data, maintenance frequency and duration, and human factors were applied which were used in the DCPRA. The obtained values are presented in Table A2.2.3, denoted by "BNL" or "BNL1" to be compared with the values given in the DCPRA (the DCPRA values are denoted by "PG\&E"). The entries into Table A2.2.3 from 


\section{Appendix A2}

the sensitivity calculations allowing the rupture to occur in any of the four cold legs (success criterion is still $1 / 3$ ) are denoted by "BNL2." The entries from the sensitivity calculation, when one uses success criterion $2 / 3$ for top event LI and one assumes that the rupture occurs in cold leg 1 are denoted by "BNL3." In the sensitivity calculations the component failure rates were identical to those used in the audit calculations.

\section{A23.4 Commenter/Findinge}

The BNL audit calculations resulted in the following comments. (The following comments relate directly to the comment numbers given in Table A2.2.3.).

1. A comparison of the AC and LI split fraction values obtained by using the simplifying assumption that the rupture occurred in cold leg 1 (see the entries denoted by "BNLI") with those obtained when it is assumed that the rupture may occur in any of the four cold legs (see the entries denoted by "BNL2"), shows that the use of the latter assumption provides (on average) higher values.

The reason why the application of the simplifying assumption is not as accurate for top events $\mathrm{LI}$ and AC as it is for top events CH and SI (see Appendix AI - on the HPI functions of the ECCS) is connected with differences in the design features of the injection headers of the RHR system and of the charging and safety injection systems. In the case of the RHR each of two header systems feeds its own two branch lines. In the case of the charging and safety injection systems, one header feeds all four branch lines.

The impact of this finding on the overall CDF is negligible $(0.25 \%$ increase) as none of the LI split fractions survived truncation for the DSM and only AC1 survived from the AC split fractions.

2. The use of a more conservative large LOCA success criterion (i.e., two available pathways required from the intact three legs) would result in an increase of the LII split fraction by more than a factor of 570. However, PG\&E subsequently provided sufficient information to demonstrate the validity of the original success criterion.

3. Small discrepancies were identified between PG\&E and BNL in the quantification of certain HWIs (independent hardware unavailabilities). Another small discrepancy between the DCPRA and BNL was found in the calculation of LI2. BNL believes this to be the difference between the Monte Carlo and mean value approaches.

Overall, the BNL audit calculations are in good agreement with those obtained by PG\&E. 
Appendix $\mathbf{A 2}$

\section{A2.4 References}

1. Final report on the Diablo Canyon Long-Term Seismic Program, Pacific Gas and Electric Co., Diablo Canyon Power Plant, Docket Nos. 50-275 and 50-323, July 1988.

2. Units 1 and 2 Diablo Canyon Power Plant, "Final Safety Analysis Report Update," Pacific Gas and Electric Co., December 1988.

3. PG\&E letters to NRC signed by J.D. Shiffer, No. DCL-88-238, October 10, 1988 and No. DCL-88-260, October 28, 1988.

4. Indian Point Probabilistic Safety Study, Power Authority of the State of New York, Consolidated Edison Company of New York, Inc., Spring 1982.

5. Worrel, R.B. and Stack, D.W., "A SETS User's Manual for the Fault tree Analyst," Sandia National Laboratories, NUREG/CR-0465, SAND77-2051, November 1978. 


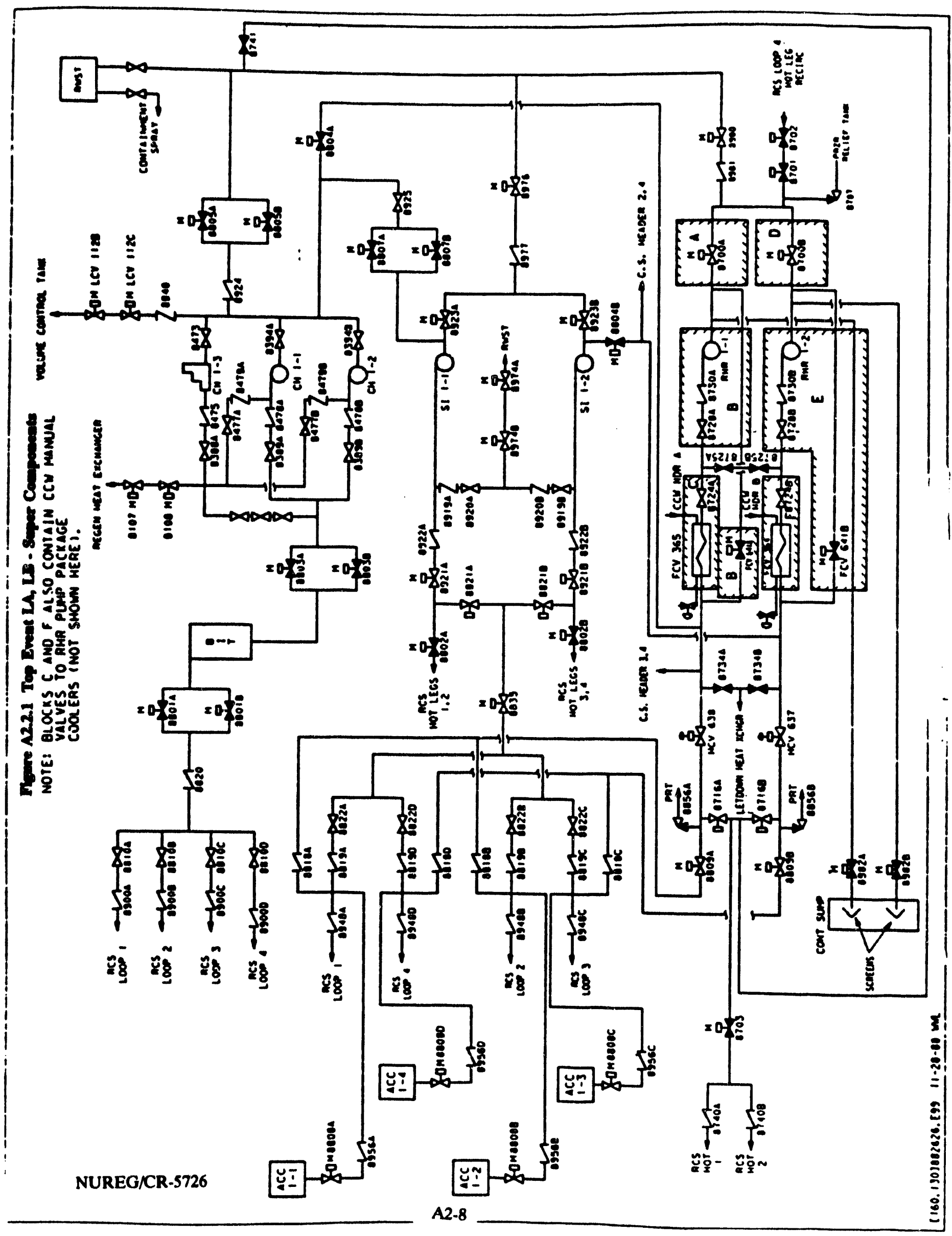




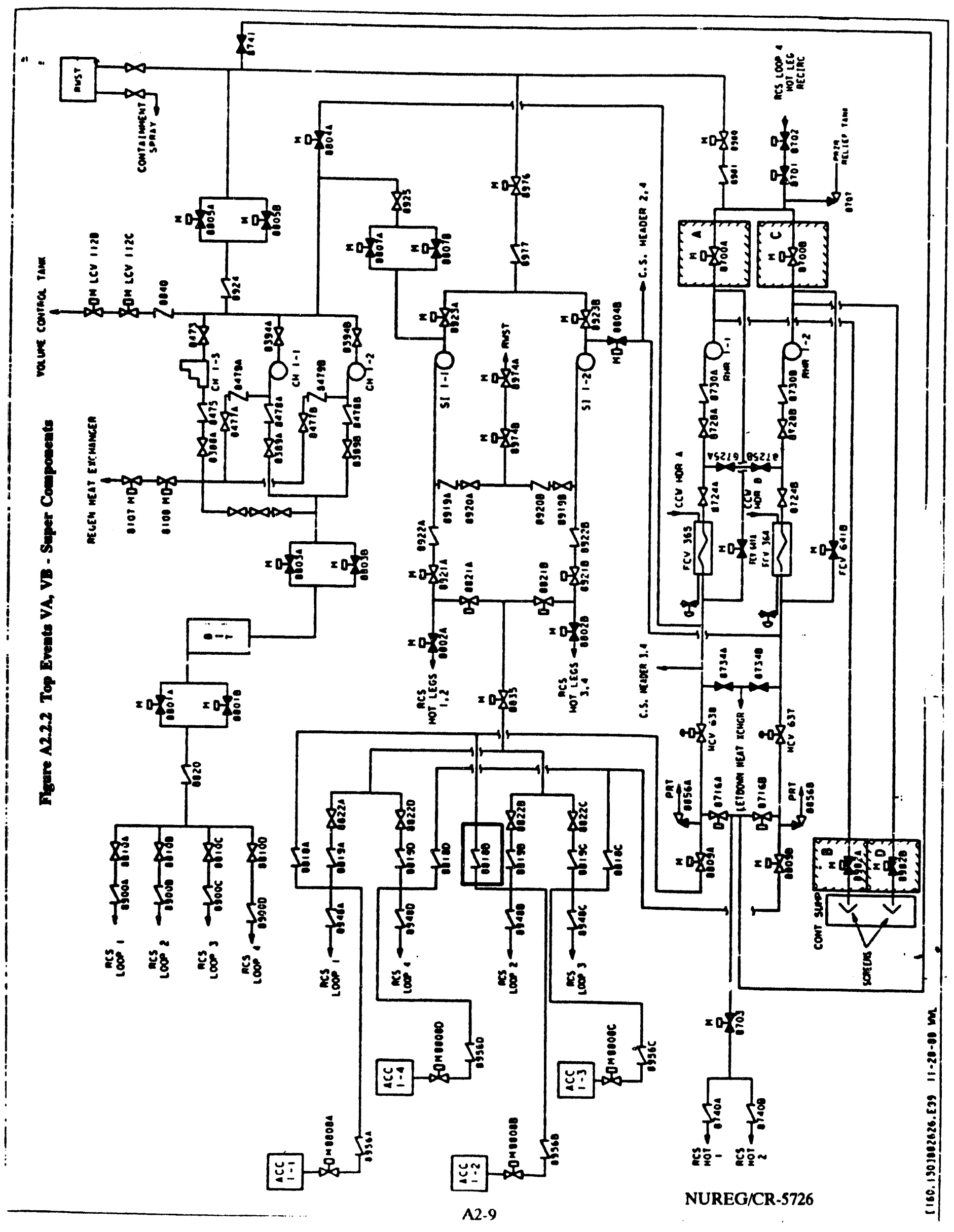




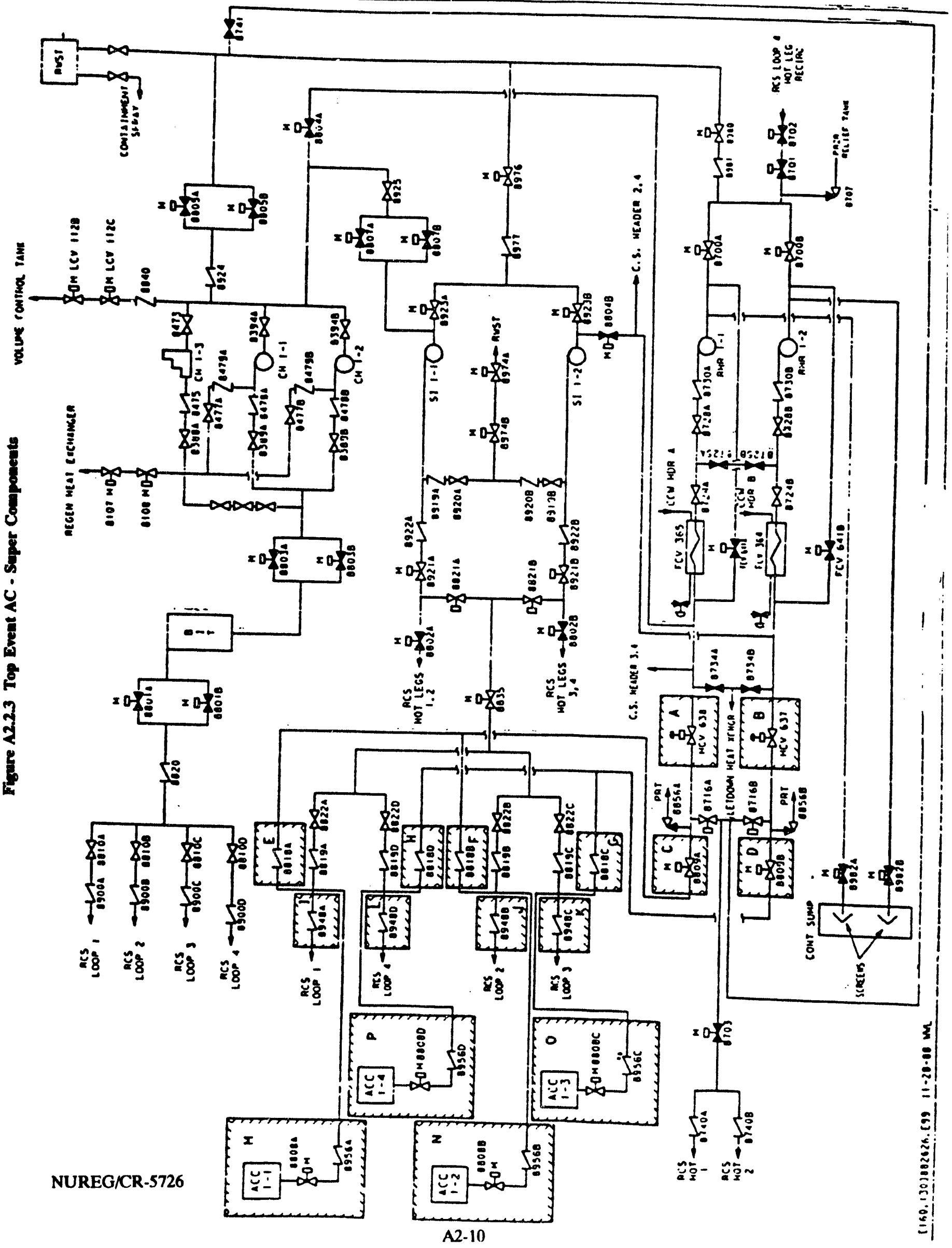




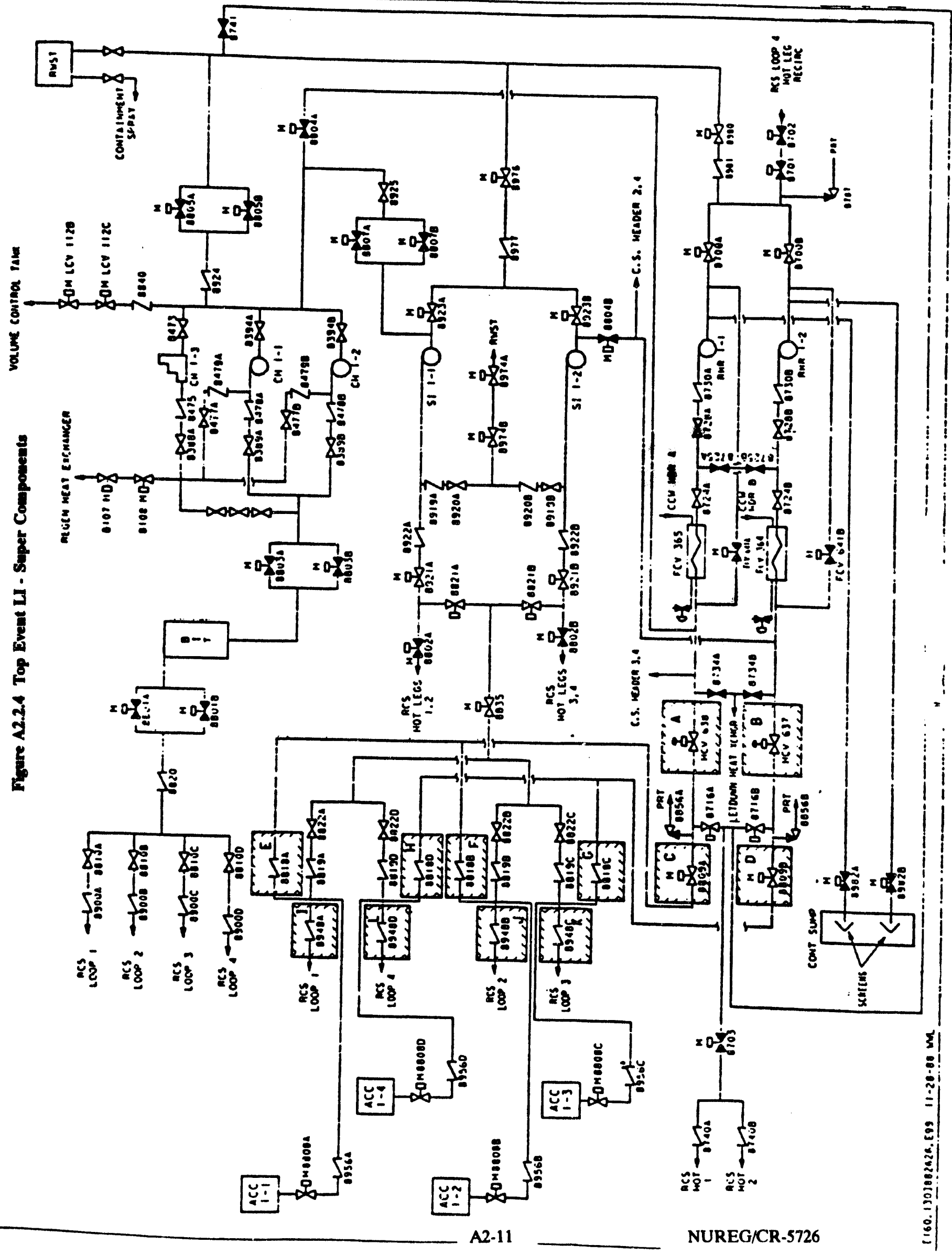




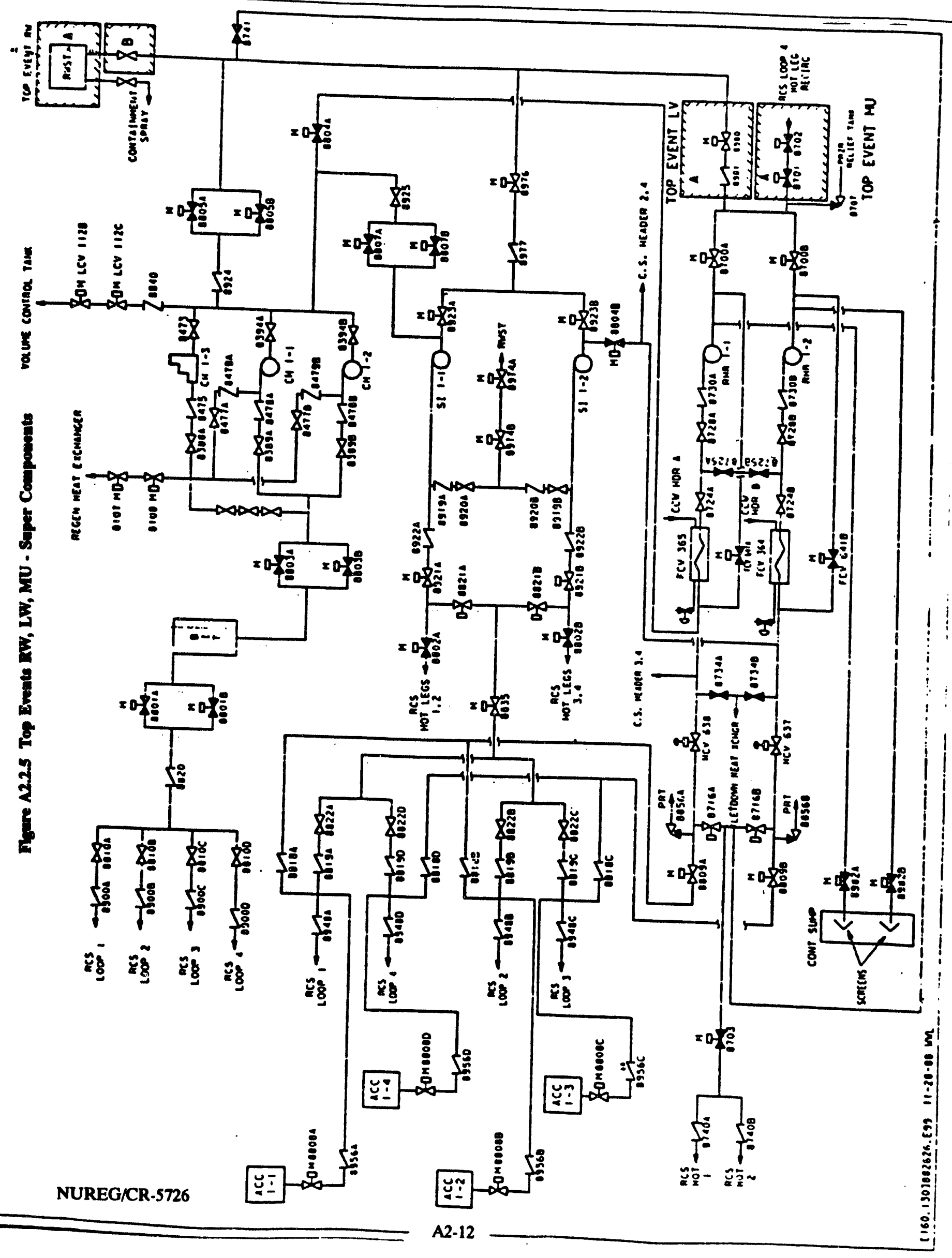




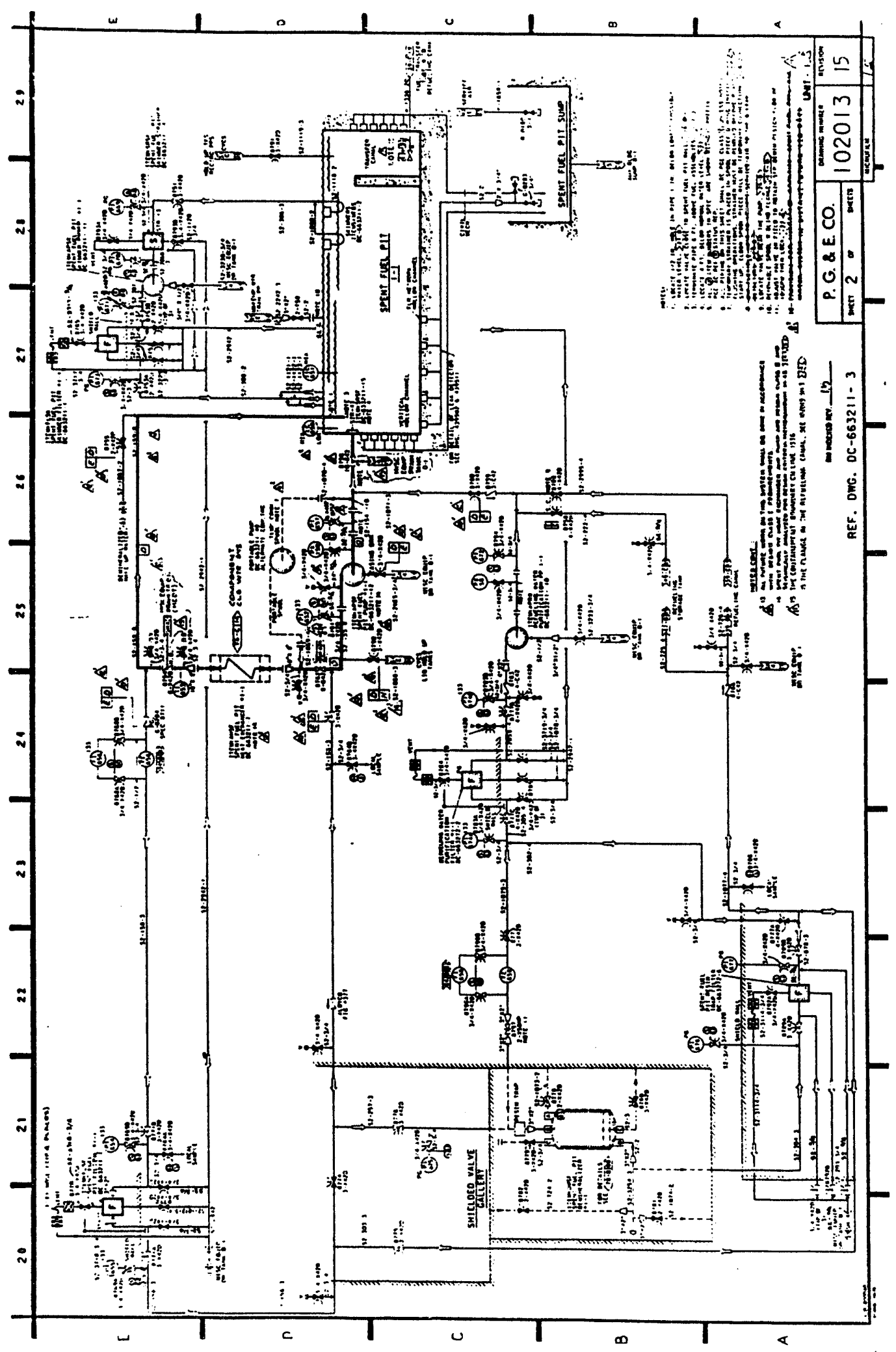

Figure A2.2.6 Top event RW. Supercomponent B. Makeup line to RWST from spent fuel pit via the spent fuel pit pump 1-1 
Appendix A2

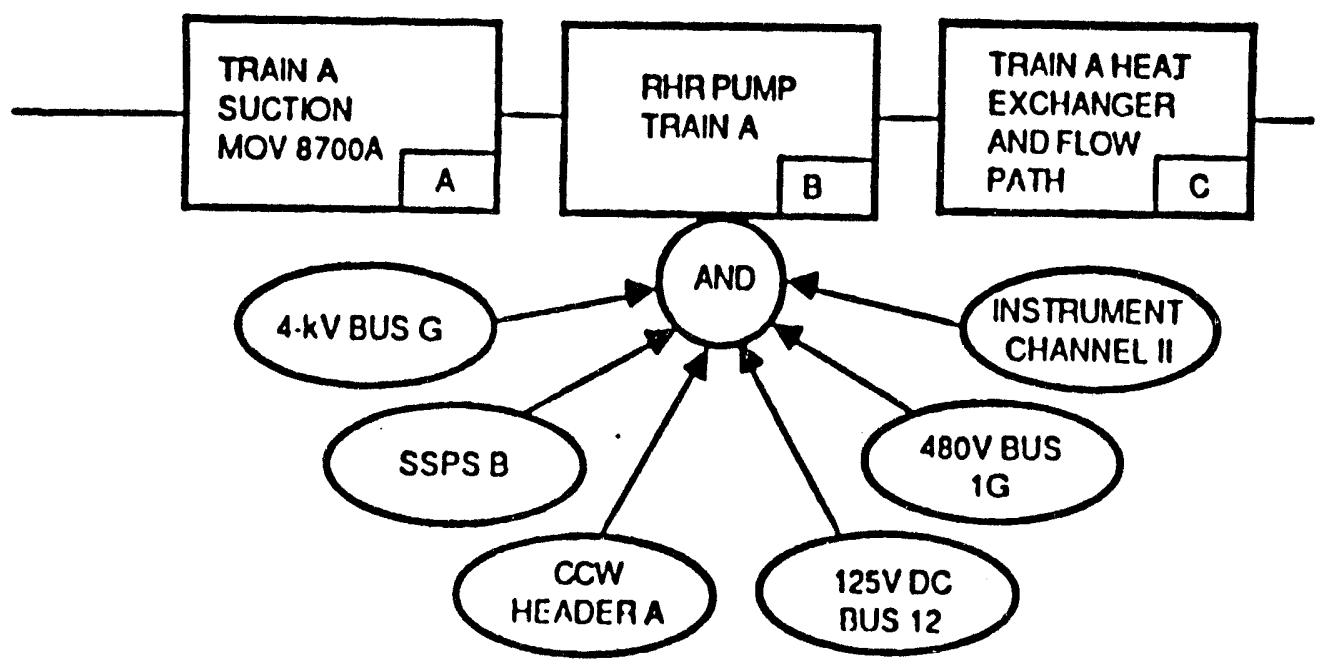

Figure A2.2.7a Logic diagram for top event LA

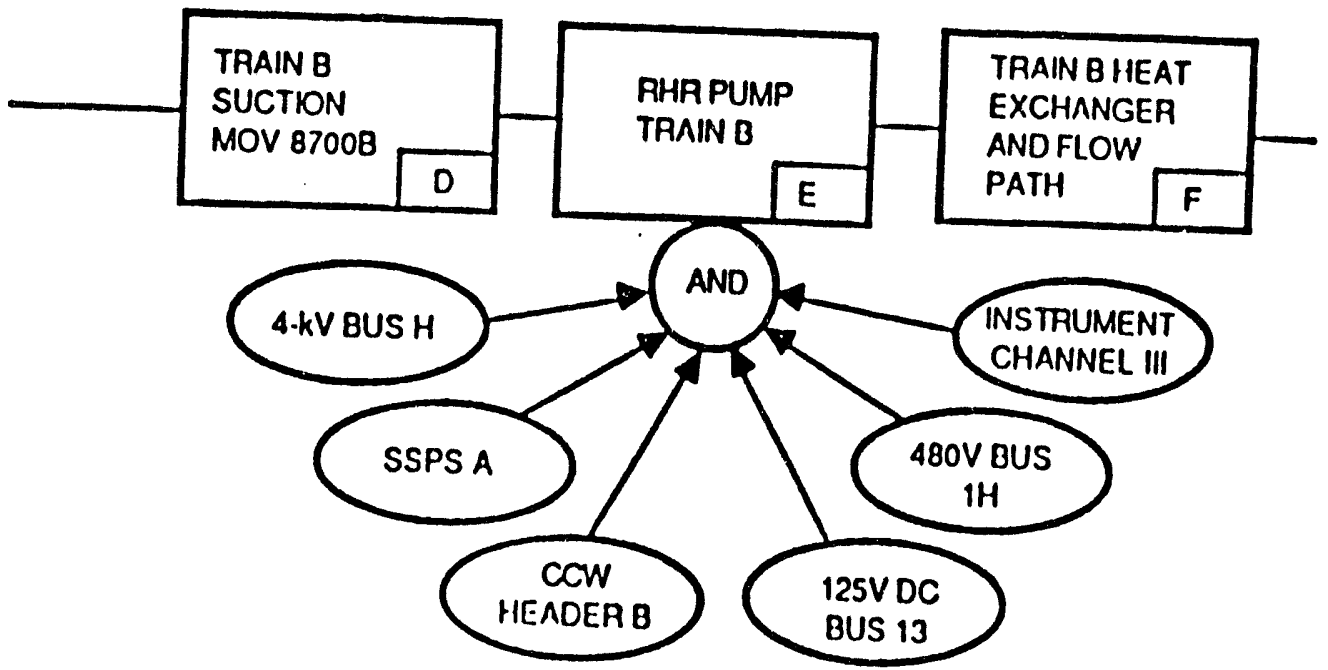

Figure A2.2.7b Logic diagram for top event LB 


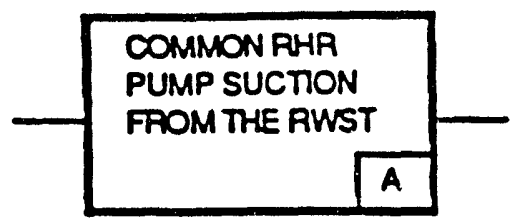

Figure A2.2.8 Logic diagram for top event LV

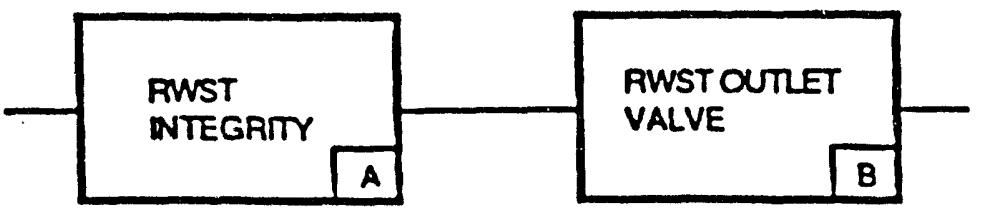

Figure A2.2.9 Logic diagram for top event RW (suction from RWST to the charging, RHR, and safety injection pumps) 
Appendix A2

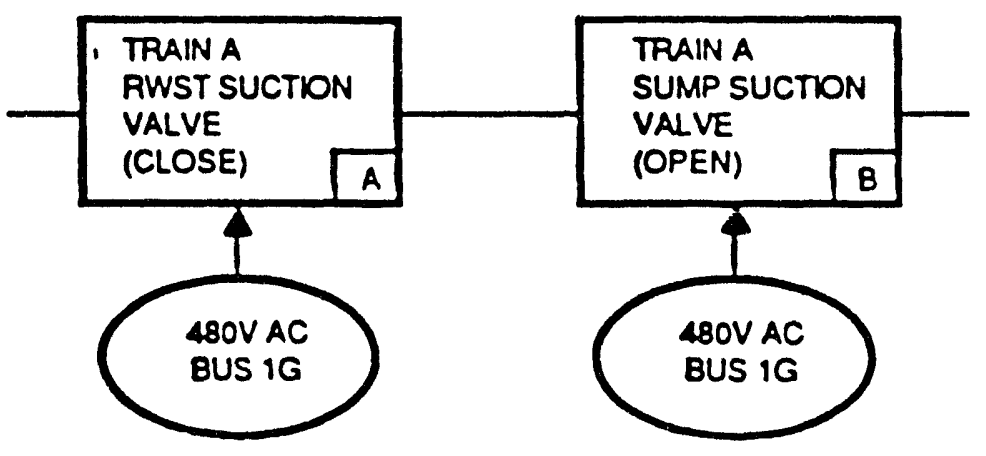

Figure A2.2.10a Logic diagrams for top event VA (suction to RHR system from containment sump train A)

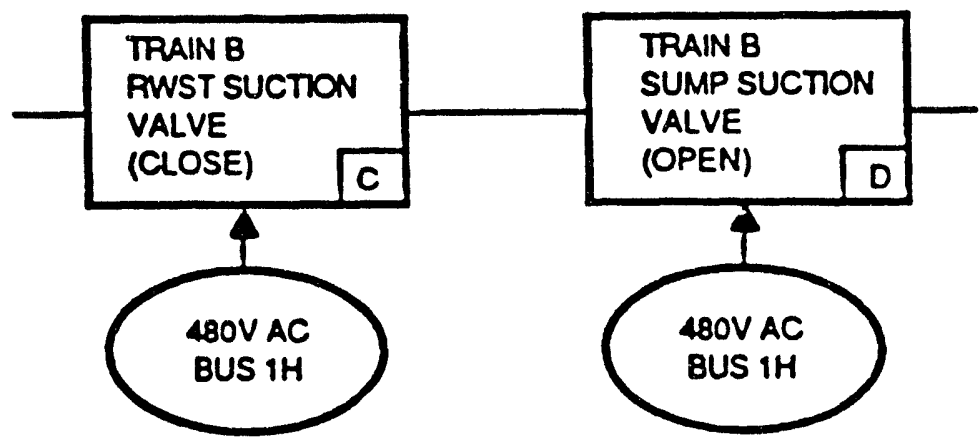

Figure A2.2.10b Logic diagrams for top event VB (suction to RHR system from containment sump train B) 
Appendix A2
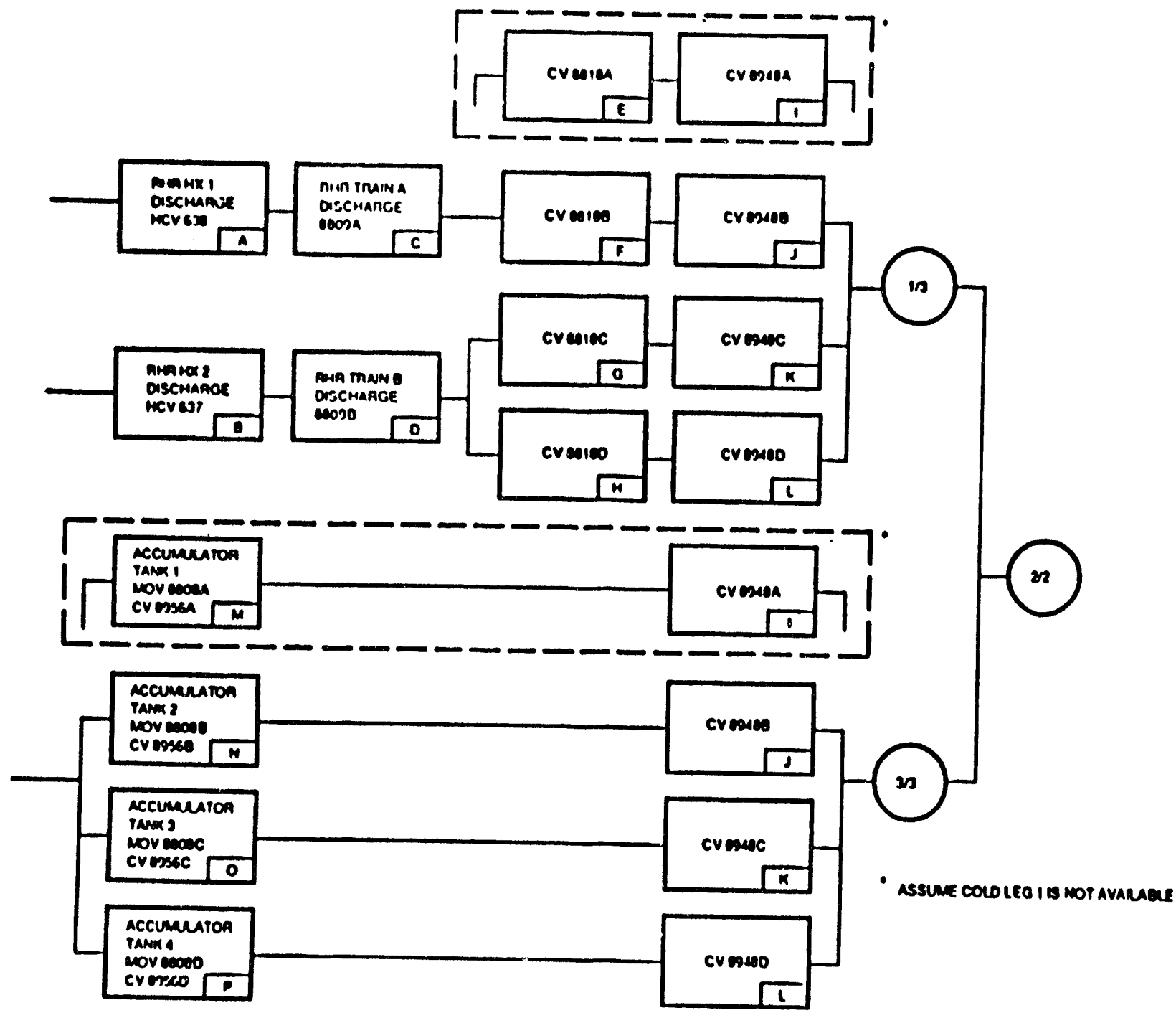

Figure A2.2.11 Logic diagram for top event AC 
Appendix A2
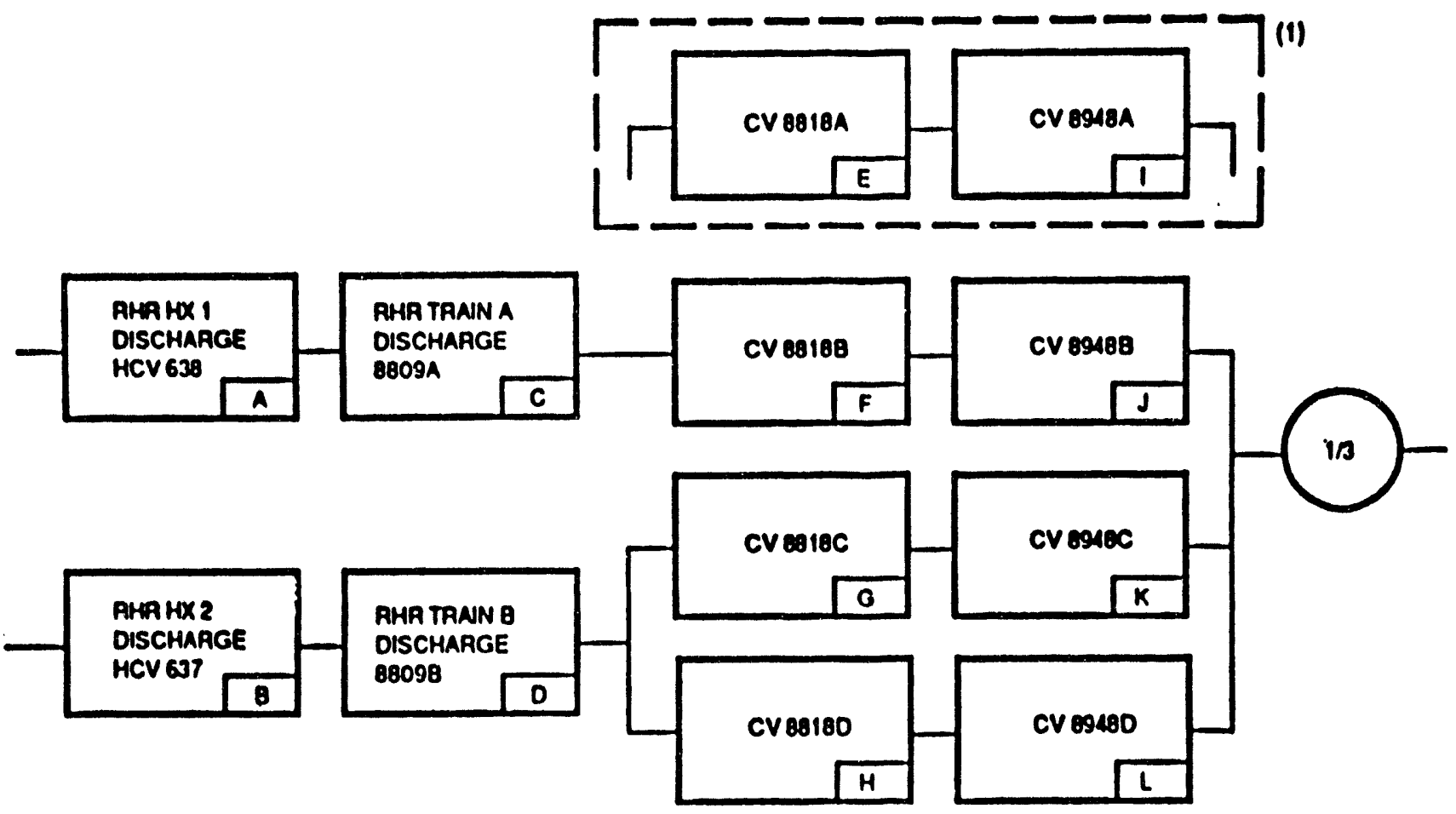

(i) ASSUME COL LEO I IS RUPTUAEO. THEREFORE ONE OF THE REMUININO THAEE COL O LECS REOUIRED FOR SUCCESS OF LI

Figure A2.2.12 Logic diagram for top event LI 

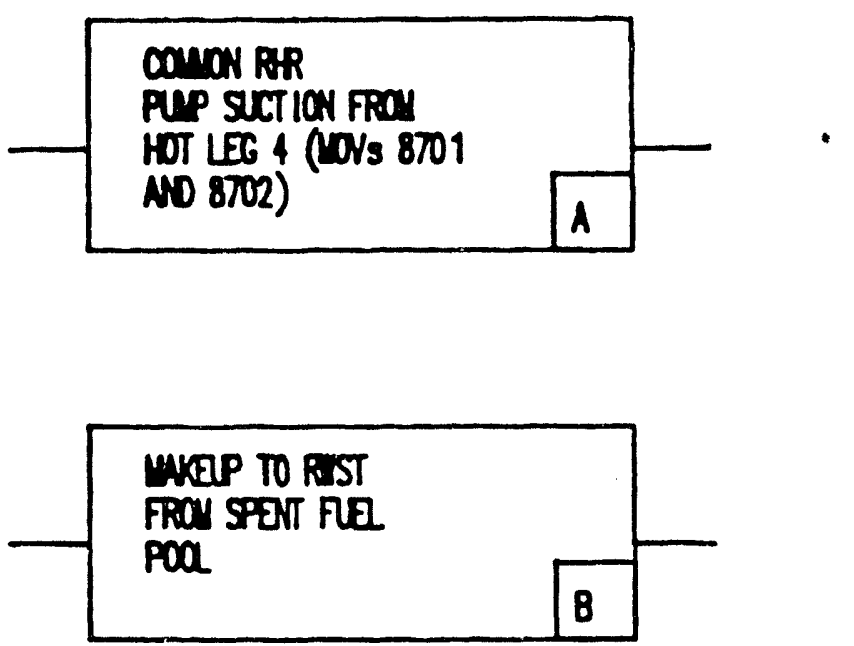

Figure A2.2.13 Logic diagram for top event MU 
Table A2.2.1

Top Event Definitions and Success Criteria*

Low Pressure Functions of ECCS

Top Event
Designator

LA

LB

LV RHR suction valves on suction header.

RW RWST available.

VA Sump valves open to RHR train A.

VB Sump valves open to RHR train B.

AC Injection path available and accumulators discharge.

LI Injection path available.

MU RHR suction from the hot leg and RWST makeup.

\section{Top Event Success Criteria}

RHR pump train $A$ must start and run, delivering water to the RCS. The RHR pump must be shut down for high pressure scenarios and restarted if pressure is sufficiently low. At least one cold leg path must be available.

RHR pump train $B$ must start and run, delivering water to the RCS if RHR train A fails. The RHR pump must be shut down for high pressure scenarios and restarted if pressure is sufficiently low. At least one cold leg path must be available.

RHR suction valves on suction header from RWST to RHR trains remain open.

RWST is available, and the manual valve on the RWST discharge line remains open.

RHR train A suction path from the RWST must close, and RHR train B suction path from the containment sump must open and remain open.

RHR train B suction path from the RWST must close, and RHR train B suction path from the containment sump must open and remain open.

At least one cold leg injection path is available (from three), and three accumulators discharge into the RCS. The fourth accumulator (with the associated injection path) is assumed to discharge out the break.

At least one cold leg injection path is available (from three).

Suction path from RCS hot leg 4 to an RHR train is available, and operator correctly aligns path. RWST refilled by operator when required. 
Table A2.2.1 (continued)

\section{FSAR Success Criteria}

1. The RHR shutdown cooling success criterion for a transient or small LOCA is at least one RHR pump delivering cooling water to at least one of the operating cold legs for 24 hours.

2. The LPI success criterion for a large LOCA is at least one RHR pump delivering makeup water to at least two cold legs for one hour. Three of the accumulators are required for one hour for accumulator system success. The other accumulator is assumed to discharge into the ruptured leg and is therefore unavailable.

3. The LPR success criterion for a large LOCA is at least one RHR pump delivering makeup water to at least two cold legs for 23 hours.

*The top event success criteria described in this table relate to the condition when all support systems are available. 
Appendix A2

Table A2.2.2

Boundary Conditions for ECCS Top Events, Low Pressure

1. Top Event LA:

1. (LA1) All support available (SLOCA Case).

2. (LA2) All support available (Bleed \& Feed Case).

3. (LA3) All support available (LLOCAMLOCA Case).

4. (LAF) Guaranteed failure.

2. Top Event L.B:

1. (LB1) All support available. Top event LA successful (SLOCA).

2. (LB2) All support available. Top event LA failed (SLOCA).

3. (LB3) Top event LA guaranteed failure (SLOCA).

4. (LB4) All support available. Top event LA successful (B \& F).

5. (LB5) All support available. Top event LA failed (B \& F).

6. (LB6) Top event LA guaranteed failure (B \& F).

7. (LB7) All support available. Top event LA successful (LLOCA).

8. (LB8) All support available. Top event LA failed (LLOCA).

9. (LB9) Top event LA guaranteed failure (LLOCA).

10. (LBF) Guaranteed failure.

3. Top Event RW:

1. (RW1) All conditions (no support requireu).

4. Top Event VA:

1. (VA1) All support available.

2. (VAF) Guaranteed failure.

5. Top Event VB:

1. (VB1) All support available. Top event VA successful.

2. (VB2) All support available. Top event VA failed.

3. (VB3) Top event VA guaranteed failure.

4. (VBF) Guaranteed failure.

6. Top Event AC:

1. (AC1) Large LOCA initiating event. All conditions (no support required).

7. Top Event LI:

1. (LI1) All conditions except large LOCA initiating event (no support required).

2. (LI2) LLOCA initiating event: given failure of top event AC.

8. Top Event MU:

1. (MU1) Power available at $\mathrm{AC}$ buses $\mathrm{G}$ and $\mathrm{H}$.

2. (MU2) Power available at AC bus G (makeup to RWST via SFP pump).

3. (MUF) Guaranteed failure. 

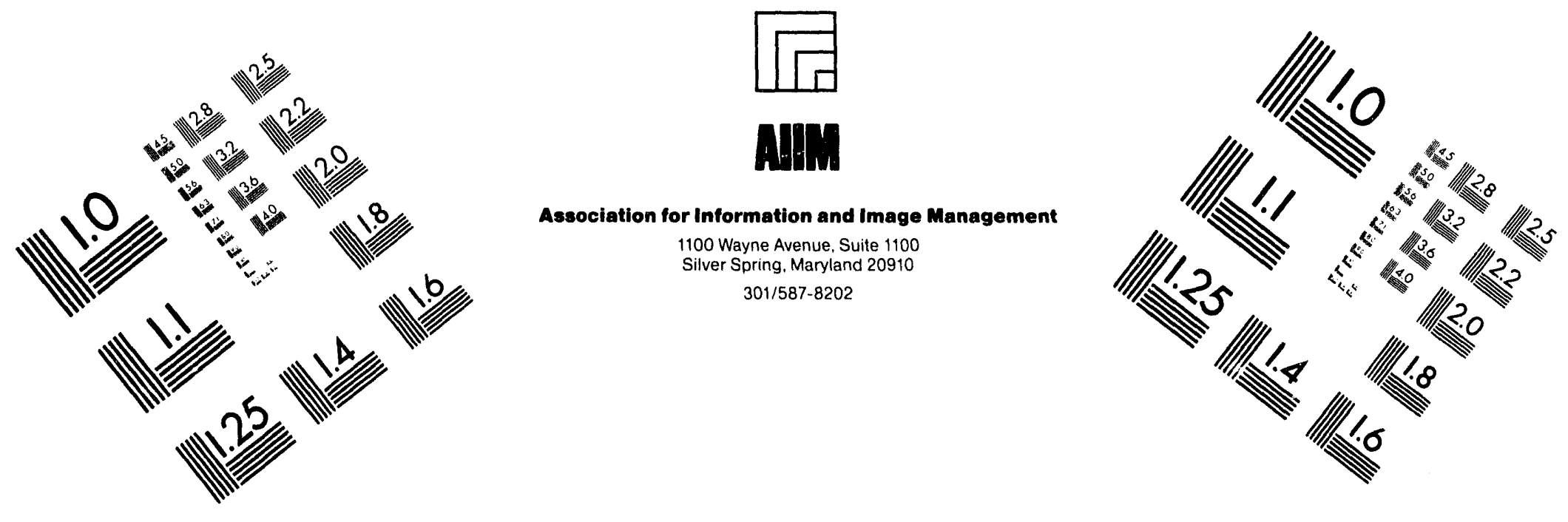

\section{Centimeter}

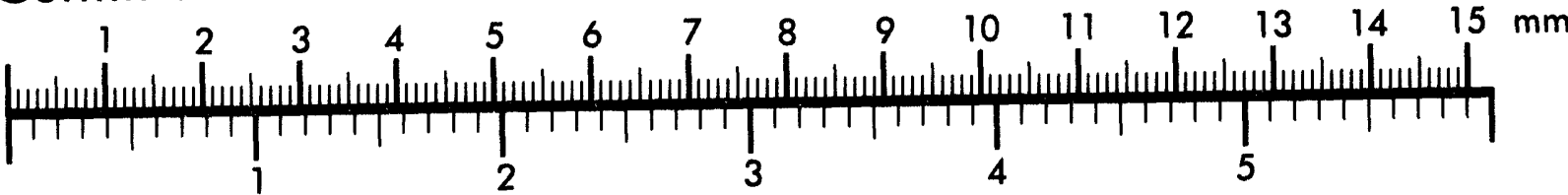
Inches
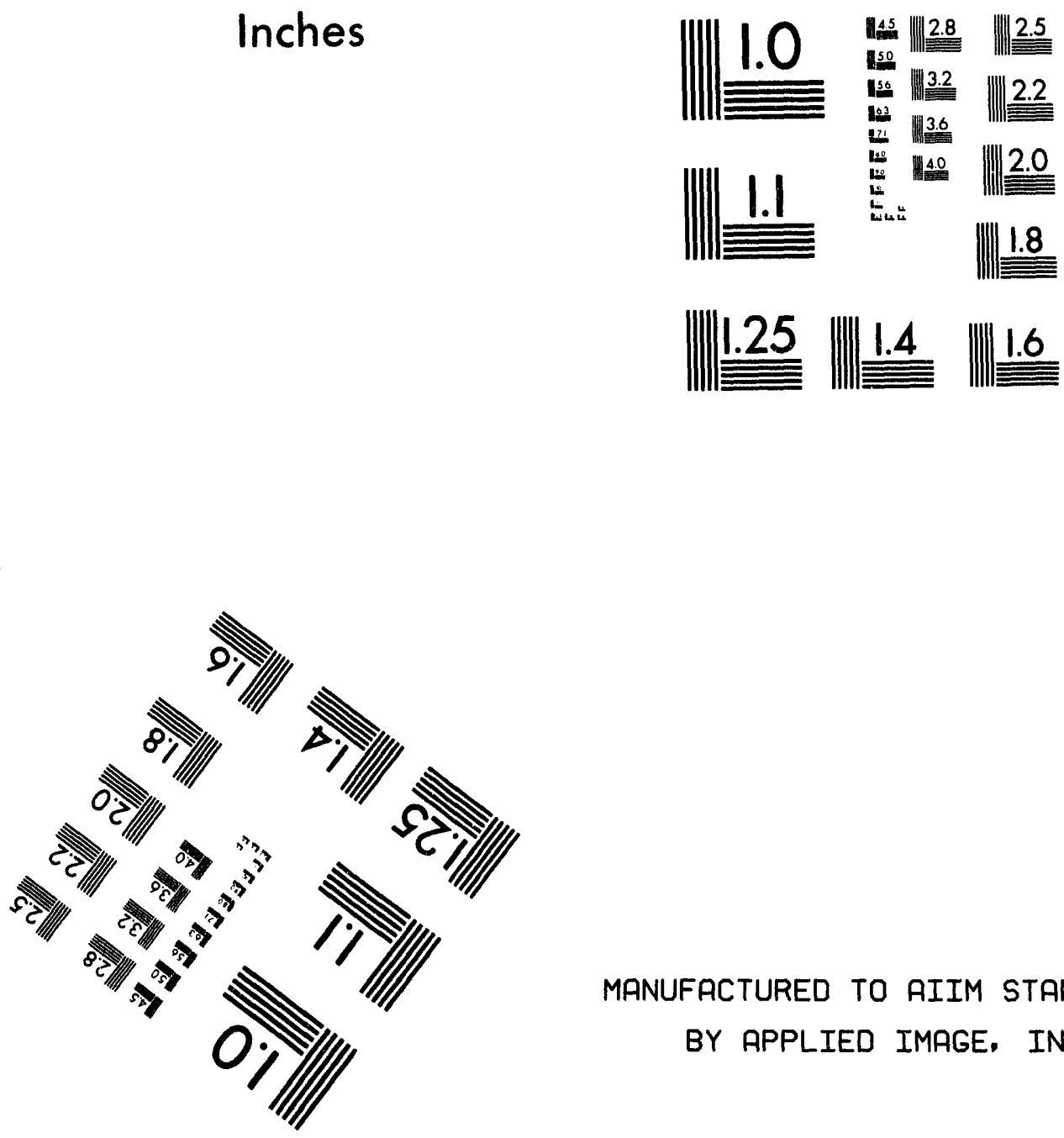

MANUFACTURED TO AIIM STANDARDS BY APPLIED IMAGE, INC.

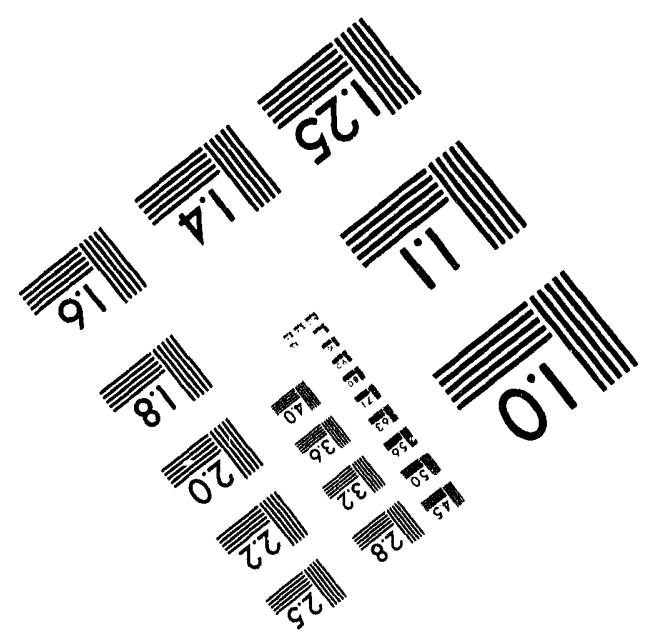



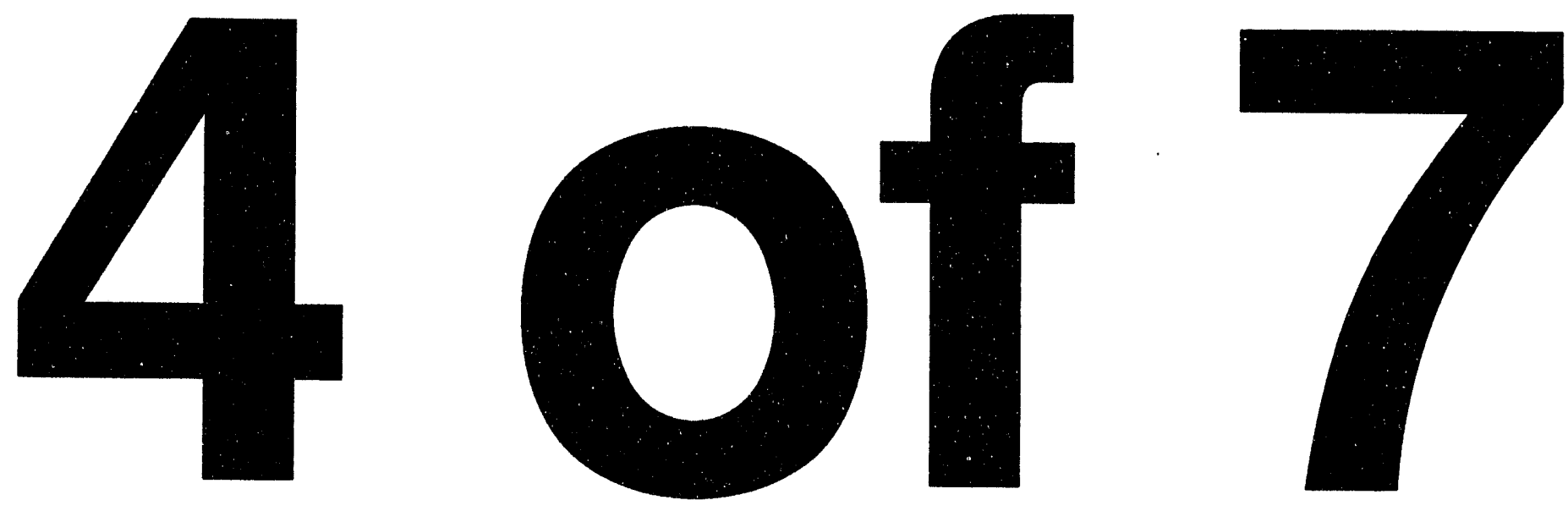
Tabie A2.2.3

Unavallability Values ("Split Fraction") for Dmergeacy Core Cooling System Low Presenure Function

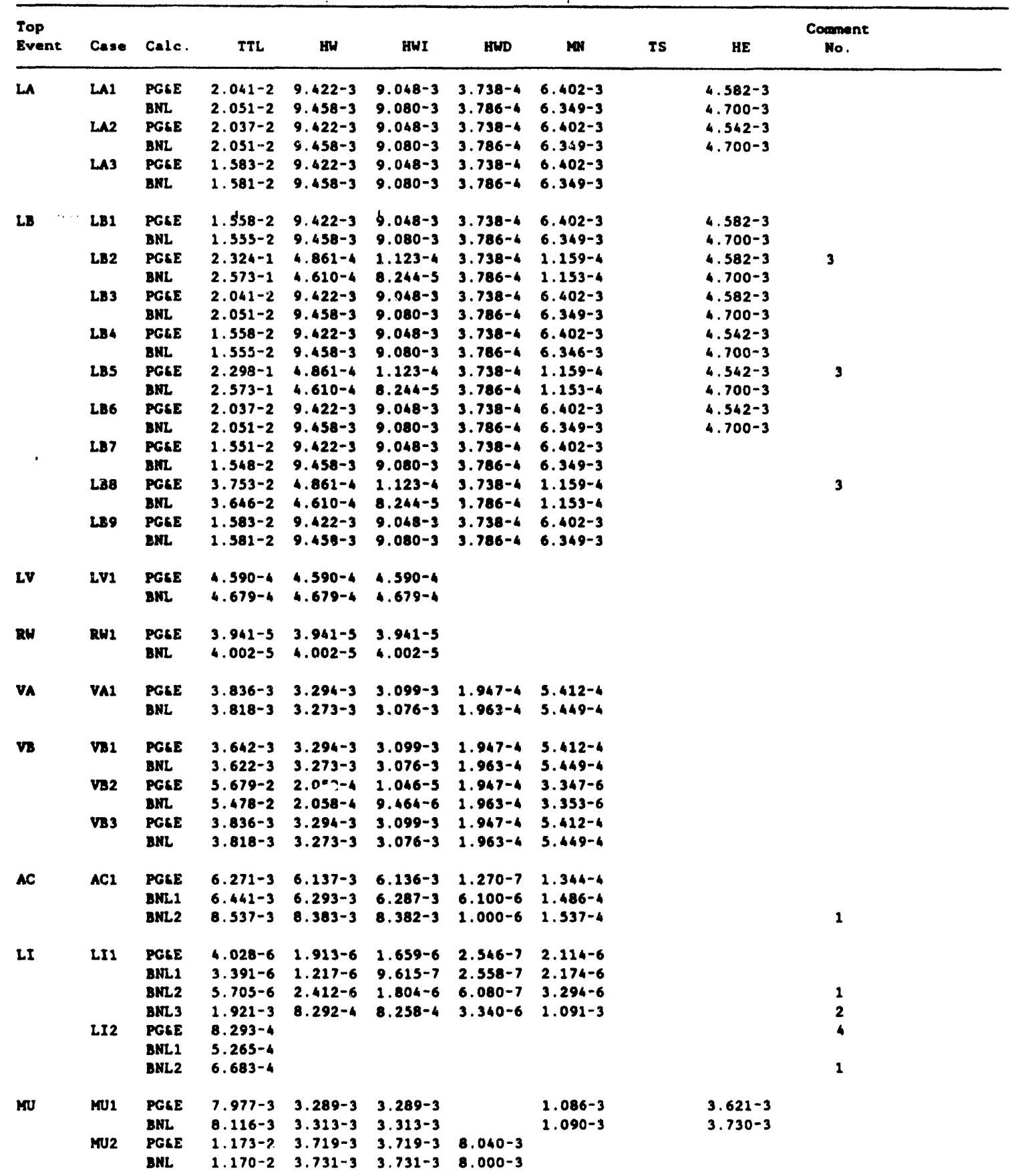

The comments are listed by number in Section A2.3.4 of the text. 
$0 \forall$ '17 :squarz do1

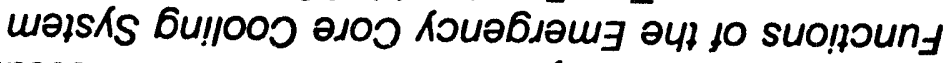

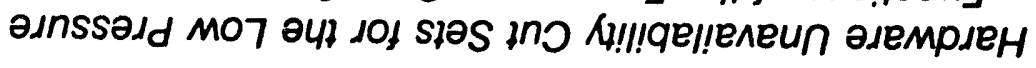

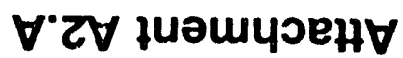




\section{Top Events: LI}

Boundary condition designator: LI1

Hardware unavailability cut sets due to independent failures; HWI =

Case A

Success Criterion: $1 / 3$

Rupture in Cold Leg 1
Case B

Success Criterion: 1/3

Rupture in any of the Four Cold Legs
1. 1.9651E-07

2. 1.6917E-07

3. 1.6917E-07

4. $1.4564 \mathrm{E}-07$

5. $7.5470 \mathrm{E}-08$

6. $7.5450 \mathrm{E}-08$

7. $\quad 7.5470 \mathrm{E}-08$

8. 7.5470E-08

9. 7.5470E-08

10. $7.5470 \mathrm{E}-08$

11. $7.5470 \mathrm{E}-08$

12. $7.5470 \mathrm{E}-08$

13. $6.4972 \mathrm{E}-08$

14. $6.4972 \mathrm{E}-08$

15. $6.4972 \mathrm{E}-08$

16. $6.4972 \mathrm{e}-08$

17. $6.4972 \mathrm{E}-08$

18. $6.4972 \mathrm{E}-08$

19. $6.4972 \mathrm{E}-08$

20. 6.4972E-08 


\section{Top Events: LI}

Boundary condition designator: I.I1

Hardware unavailability cut sets due to dependent and independent failures; HW =

Case A

Success Criterion: $1 / 3$

Rupture in Cold Leg 1
Case B

Success Criterion: $1 / 3$

Rupture in any of the Four Cold Legs
1. $\quad 1.9651 \mathrm{E}-07$

2. 1.6917E-07

3. 1.6917E-07

4. 1.4564E-07

5. $7.5470 \mathrm{E}-08$

6. $7.5470 \mathrm{E}-08$

7. $6.8100 \mathrm{E}-08$

8. $6.8100 \mathrm{E}-08$

9. $6.4972 \mathrm{E}-08$

10. $6.4972 \mathrm{E}-08$

11. $5.7600 \mathrm{E}-08$

12. $5.7800 \mathrm{E}-08$
LIBKC * LIBKD +
LIBKA * LIBKD +
LIBKC * LIBKB +
LIBKA * LIBKB +
LIBKD * LIBKJ +
LIBKD * LIBKE +
G41VCOD +
G42VCOD +
LIBKB * LIBKE +
LIBKB * LIBKJ +
T41VCOD234 +
T42VCOD234 +

1. 1.9651E-07

2. 1.6917E-07

3. $\quad 1.6917 \mathrm{E}-07$

4. $1.4564 \mathrm{E}-07$

5. $7.5470 \mathrm{E}-08$

6. $\quad 7.5470 \mathrm{E}-08$

7. $7.5470 \mathrm{E}-08$

8. $7.5470 \mathrm{E}-08$

9. 7.5470E-08

10. $7.5470 \mathrm{E}-08$

11. $7.5470 \mathrm{E}-08$

12. $7.5470 \mathrm{E}-08$

13. $6.8100 \mathrm{E}-08$

14. $6.8100 \mathrm{E}-08$

15. $6.4972 \mathrm{E}-08$

16. $6.4972 \mathrm{E}-08$

17. $6.4972 \mathrm{E}-08$

18. $\quad 6.4972 \mathrm{E}-08$

19. $6.4972 \mathrm{E}-08$

20. $6.4972 \mathrm{E}-08$

21. $6.4872 \mathrm{E}-08$

22. $6.4972 \mathrm{E}-08$

23. $5.7600 \mathrm{E}-08$

24. $5.7600 \mathrm{E}-08$

25. $5.7600 \mathrm{E}-08$

26. $\quad 5.7600 \mathrm{E}-08$

27. $5.7600 \mathrm{E}-08$

28. $5.7600 \mathrm{E}-08$

29. $5.7600 \mathrm{E}-08$

30. $5.7600 \mathrm{E}-08$

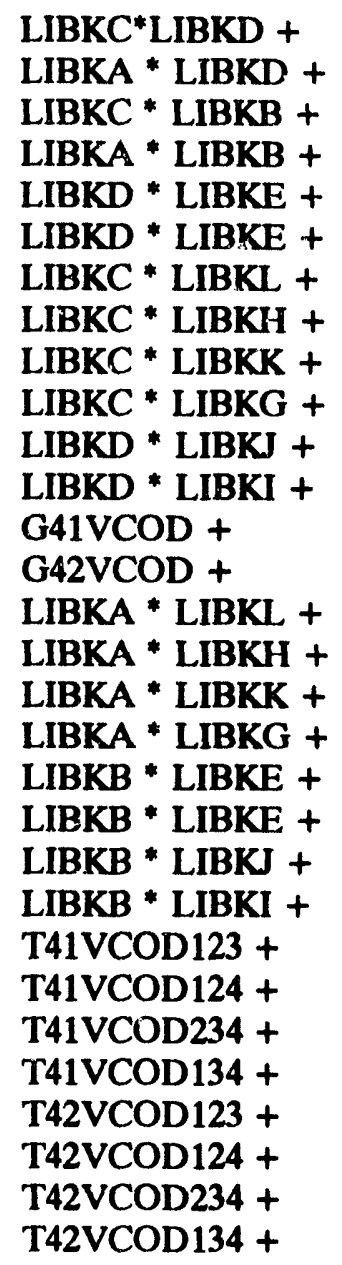


Top Events: AC

Boundary condition designator: $\mathrm{AC1}$

Hardware unavailability cut sets due to independent failures; HWI =

Case A

Success Criterion: $1 / 3$

Rupture in Cold Leg 1
Case B

Success Criterion: $1 / 3$

Rupture in any of the Four Cold Legs
1. 1.9251E-03

2. 1.9251E-03

3. 1.9251E-03

4. 1.7025E-04

5. 1.7025E-04

6. 1.7025E-04

7. $1.9651 \mathrm{E}-07$

8. 1.6917E-07

9. 1.6917E-07

10. $1.4564 \mathrm{E}-07$

11. $7.5470 \mathrm{E}-08$

12. $6.4972 \mathrm{E}-08$

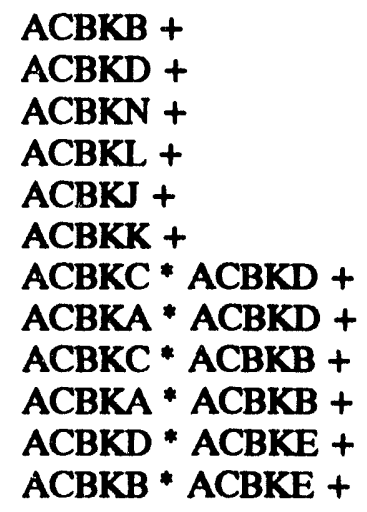

1. 1.9251E-03

2. $\quad 1.9251 \mathrm{E}-03$

3. 1.9251E-03

4. 1.9251E-03

5. 1.7025E-04

6. 1.7025E-04

7. $1.7025 \mathrm{E}-04$

8. 1.7025E-04

9. 1.9651E-07

10. 1.6917E-07

11. $1.6917 \mathrm{E}-07$

12. $1.4564 \mathrm{E}-07$

13. $7.5470 \mathrm{E}-08$

14. $7.5470 \mathrm{E}-08$

15. $7.5470 \mathrm{E}-08$

16. $7.5470 \mathrm{E}-08$

17. $6.4972 \mathrm{E}-08$

18. $6.4972 \mathrm{E}-08$

19. $6.4972 \mathrm{E}-08$

20. $6.4972 \mathrm{E}-08$

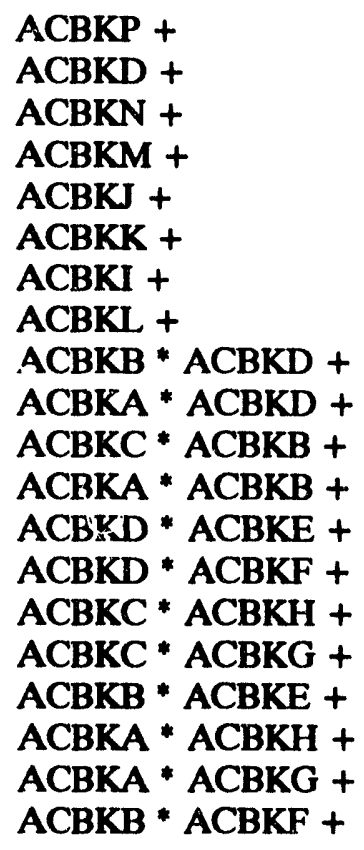




\section{Top Events: AC}

Boundary condition designator: $\mathrm{AC1}$

Hardware unavailability cut sets due to dependent and independent failures; HW =

Case A

Success Criterion: $1 / 3$

Rupture in Cold Leg 1
Case B

Success Criterion: $1 / 3$

Rupture in any of the Four Cold Legs

1. $1.9251 \mathrm{E}-03$

2. $1.9251 \mathrm{E}-03$

3. $1.9251 \mathrm{E}-03$

4. 1.9251E-03

5. 1.7025E-04

6. $1.7025 \mathrm{E}-04$

7. $1.7025 \mathrm{E}-04$

8. $1.7025 \mathrm{E}-04$

9. 1.9651E-07

10. 1.6917E-07

11. $1.6917 \mathrm{E}-07$

12. $1.4564 \mathrm{E}-07$

13. 7.5470 E- 08

14. $7.5450 \mathrm{E}-08$

15. $7.5470 \mathrm{E}-08$

16. $7.5470 \mathrm{E}-08$

17. $\quad 6.8100 \mathrm{E}-08$

18. $6.8100 \mathrm{E}-08$

19. $\quad 6.4972 \mathrm{E}-08$

20. $6.4972 \mathrm{E}-08$

21. $6.4972 \mathrm{E}-08$

22. $6.4972 \mathrm{E}-08$

23. $\quad 5.7600 \mathrm{E}-08$

24. $5.7600 \mathrm{E}-08$

25. $5.7600 \mathrm{E}-08$

26. $5.7600 \mathrm{E}-08$

27. $5.7600 \mathrm{E}-08$

28. $5.7600 \mathrm{E}-08$

29. $\quad 5.7600 \mathrm{E}-08$

30. $5.7600 \mathrm{E}-08$
ACBKP + ACBKO + ACBKN + ACBKM + ACBKL + ACBKK + ACBKJ + ACBKI + ACBKC * ACBKD + ACBKA * ACBKD + ACBKC * ACBKB + ACBKA * ACBKB + ACBKD * ACBKF + ACBKD * ACBKF + ACBKC * ACBKH + ACBKC * ACBKG + G41VCOD + G42VCOD + ACBKB * ACBKE + ACBKA * ACBKH + ACBKA * ACBKG + ACBKB * ACBKF + T41VCOD124 + T41VCOD234 + T41VCOD134 + T41VCOD123 + T42VCOD124 + T42VCOD234 + T42VCOD123 + T42VCOD134 + 


\section{APPENDIX A3: AUXILIARY FEEDWATER SYSTEM}

\section{A3.1 Introduction}

\section{A3.1.1 Objectives}

The main objective of this report is to summarize the results of reviewing the unavailability analysis of the Auxiliary Feedwater System (AFWS) in an updated version ${ }^{1}$ prepared for the DCPRA. ${ }^{2}$ The updated version was made by PG\&E after a BNL/NRC/PG\&E meeting held in Rockville, Maryland on September 12-15, 1988, where preliminary findings of this system's review ${ }^{3}$ were presented.

\section{A3.1.2 Organization}

Section A3.2 provides a brief description of the functions and system configuration, the dependency on support systems, the surveillance and maintenance conditions, the unavailability modelling of the AFW system in the DCPRA, and the updated results ${ }^{1}$ obtained. The purpose of this approach is to present to the reader stand alone documentation to which the review findings (presented in Section A3.3) can be directly compared. Section A3.3 contains the results of the BNL review and presents the findings.

For completeness, the ranked cut sets of hardware unavailabilities (both independent and total) obtained by BNL for certain top event split fractions are given in Attachment A3.A.

\section{A3.2 Unavailability Modelling of the Auxiliary Feedwater System}

\section{A3.2.1 Functions, System Description, Configuration}

The Auxiliary Feedwater System is an alternate to the Main Feedwater System (MFWS) when the MFWS is not available to provide heat removal capability on the secondary side of the Reactor Coolant System. It is designed to cool the reactor core safely after a reactor trip. It would also be used in the event of an ATWS. The AFWS is also operated for all startup, shutdown and hot standby conditions.

The Diablo Canyon Unit 1 AFWS consists of two motor-driven (MDP) and one turbine-driven (TDP) pumps, their associated trains which feed into four feedwater lines (downstream of the four feedwater isolation valves), and a preferred water supply from the Condensate Storage Tank. The flow goes to the four steam generators, where it is heated to steam. The RCS heat is removed by dumping the steam via the $40 \%$ dump valves to the condenser (if it is available) or vented to the atmosphere by the $10 \%$ dump vales and/or (if the initial pressure spike is high) by the safety valves. Each of the motor-driven pumps can feed two steam generators while the turbine-driven pump can feed all four steam generators.

The system configuration is shown in Figure A3.2.1. The figure shows the Raw Water Reservoir and the pathway to the Fire Water Tank as alternate water sources. In addition, it indicates the steam lines from steam generators 2 and 3 back to the turbine of the TDP.

The preferred mode of operation is to use the two MDPs for startup and shutdown but only the TDP can operate if ac powe: is lost. Upon demand, the TDP starts automatically together with the MDPs. If the MDPs start successfully, the operator shuts off the TDP in order to restart it again if later there were MDP failures. 
Appendix $\mathbf{A 3}$

\section{A3.2.2 Top Event Definition, Success Criteria}

Associated with the unavailability of the AFW functions, the DCPRA defines two top events to be used in the main event trees. The designators of these top events are: AW and TD. The top event AW includes the two MDP and the TDP trains, the water supply and steam rejection (via 10\% steam dump or steam generator safety valves) paths. Top event TD includes the TDP, the water supply and the steam rejection paths. It is used only in the quantification of seismic event trees, when the $4 \mathrm{kV}$ load breakers are locked out due to relay chatter and consequently the MDPs are unavailable. The definitions of the top events are given in Table A3.2.1. The AFW is assumed to be in standby mode prior to an initiating event.

The success criteria for the unavailability modelling of both top events, AW and TD are also described in Table A3.2.1. They relate to the specific boundary condition represented by each of the support systems being available after a reactor trip. Notice, if the reactor does not trip, the success criterion for top event AW is more strict (see "high power" requirements). The success criteria for all the boundary conditions can be found in Chapter E.2 of the DCPRA). For completeness, the success criteria, the LOCA and test requirements given in the DCFSAR and DC Technical Specifications are also listed in the Table. One can see the top event success criteria cover well those of the DCFSAR ${ }^{4}$ for all initiating events.

\section{A3.2.3 Logic Models, Dependencies on Support Systems}

The logic model of the top events AW and TD shown in Figure A3.2.2 describes the logic relationships among the supercomponents defined by areas designated with broken lines in Figure A3.2.1. Figure A3.2.2 indicates also the dependencies on trains or supercomponents of relevant support systems whose unavailabilitiesplay a role in the definitions of the top event boundary conditions.

\section{A3.2.4 Boundary Conditions of Top Events}

Top event AW was evaluated for 13 boundary conditions, depending on the initiator, the reactor power level, and the unavailabilitiesof certain support system trains or certain AFW supercomponents. Top event TD was evaluated for three boundary conditions. The quantified split fraction values are identical to those of top event $\mathrm{AW}$ under similar boundary conditions.

The detailed list of the boundary conditions is given in Table A3.2.2.

\section{A3.2.5 Quantincation of Top Event Split Fractions}

The methodology of systems analysis applied in the DCPRA requires that the top event "split fraction" (associated with a system under a given boundary condition) should reflect the notion that the system (or its portion) in question is in one of the following mutually exclusive alignments: 1) normal alignment, 2) testing alignment, 3) maintenance alignment, or 4) misalignment. Thus, the contribution of the system unavailability from a specific alignment is determined by the conditional system unavailability, given that the system is in that alignment multiplied by the fraction of time that the system spends in that alignment. That is the way that the DCPRA considers the constraints imposed by Technical Specifications which disallow simultaneous maintenance or test activities on redundant components and the human errors causing the system or its components (usually occurring after these activities) to be inoperable. 
Table A3.2.3 lists the values of the SW and TD split fractions associated with the various boundary conditions quantified by PG\&E. The table presents the total unavailabilities (TTL), along with the main contributors to the total unavailabilities, such as hardware (HW), mainten se (MN), test (TS), and human error (HE). At a given boundary condition the hardware contribution relisces to the normal alignment, when no test or maintenance activities are being performed. To provide complete information, the table also indicates the two constituent parts of the hardware contribution to the unavailability: the independent (HWI) and the dependent (HWD, i.e., common cause) failures of the supercomponents.

The DCPRA used two sets of fault trees to distinguish between the "low power" and "high power" success criteria. This explains the marked differences between the calculated "low" and "high power" split fraction values. The BNL review focused on the "low power" case.

The DCPRA unavailability model assumes that only the $10 \%$ steam-dump valves and backup safety relief valves are viable steam relief paths, i.e., the model considers that the condenser and hence the $40 \%$ dump valves are unavailable. (If the $40 \%$ steam valves were available, they would be the preferred steam dump paths to the condenser. However, they will not be available if the condenser is unavailable or Main Steam Line Isolation occurs.) It further assumes that the use of the Raw Water Reservoir as a water source requires operator action (open valves FCV-436 and FCV -437) upon the failure of the CST. The human error probability was taken to be unity thus having only CST failure as part of the hardware unavailability. (In the BNL audit calculations, the human error contribution was explicitly treated as a part of the total human error, HE.) The model considers that the system starts automatically and the flow is automatically controlled by level control valves at the discharge side of the MDP. Operators manually control the flow from the TDP and shut it down if the flow is sufficient froin the MDPs.

In the quantification, common cause failures were used:

a. between the MDPs (not between the TDP and MDPs),

b. between the check valves in parallel lines (e.g., inlet lines to the TDP),

c. between the four $10 \%$ steam dump valves, and

d. between the safety relief valves on a single steam generator. (Failure of the stean generator relief valves to reclose was evaluated if the $10 \%$ steam dump valve failed. 20 open/shut cycles were assumed to calculate the conditional probability of reclosure failure.)

Different block failure 13tes were applied for the "P-blocks" (i.e., blocks N, O,P and Q in Figure A3.2.1) in quantifying split fractions depending on the boundary conditions (i.e., whether they included the unavailability of support to the $10 \%$ steam dump valves or not).

Unscheduled maintenance was modelled for the TDP and MDPs. Maintenance of the $10 \%$ steam dump valves was included in the rate of "valve failure to open given a demand" failure mode.

The recirculation tests for the TDP and MDPs were included in the unavailability model, because they require isolation of certain AFWS components.

Human errors were considered in restoring these components to their operable conditions. Those were modelled as failure events disabling the AFWS between tests. Human errors in accessing the Raw Water Reservoir were discussed above. 


\section{A3.3 Results of the BNL Review}

\section{A3.3.1 General}

A thorough review of the top event logic diagrams and the corresponding fault trees related to the "low pressure" boundary conditions was performed by BNL (the fault trees are not reproduced here, they can be found in Chapter E.2 of the DCPRA). The fault trees associated with the "high pressure" boundary conditions were not reviewed. The reason for this is that the high pressure boundary conditions appear only in the ATWS event tree and the contributions of the ATWS sequences to the total core damage frequency are negligible. The review is based, as was mentioned in the introduction, on a revised version ${ }^{1}$ of the original submittal. ${ }^{2}$

\section{A3.3.2 Logic Dlagrams, Fault Trees}

The unavailability model of the AFWS represents one of the systems in the DCPRA which have been analyzed in the most detailed way. It serves as a "showcase" for demonstrating the application of a new approach of system analysis suggested by Fleming, Mosleh and Deremer, in Reference 5. These authors analyzed, as an example, a three train auxiliary feedwater system, similar to that at the Diablo Canyon plant and provided guidelines for application of the new approach. As part of the review process, BNL checked whether the model developed in the DCPRA was consistent with the guidelines described in that paper. The review found that the independent failure modes of each supercomponent involved in the logic diagrams were determined correctly and the associated (independent) fault trees adequately represented the failure conditions of the AFWS.

In the treatment of the common cause failures (dependent fault trees), however, the review identified some inconsistencies with the recommendations given in Reference 5. Reference 5 states:

"An important characteristic of this system (AFWS) is that, although diversity is employed in pump drivers, all three mechanical pumps are identical."

Consequently, in the example analysis common cause failures were applied for the pumps failing all the three AFWS trains, dominating the system unavailability.

In the DCPRA there is no common cause failure modelled between all the AFWS pumps. The DCPRA assumes common cause failures only for the two motor pumps. Similarly, it assumes common cause failures between check valves and MOVs belonging only to the trains containing similar motor drivers, i.e., to the motor trains or to the turbine train. This treatment results in complete independence between the motor trains and turbine train. As a result it provides, e.g., in the case of the boundary condition when the low power success criterion is used and all support systems are available an unavailability value of AW1(PG\&E) $=3.73-5 /$ demand. This result is somewhat surprising in that if is compared with the value given in the conclusion of Reference 5, and taking into account that essentially the same organization was responsible for both values:

"The three-train AFWS analyzed in this paper (Reference 5) is a rather typical configuration found in several existing U.S. power plants. The system was analyzed using U.S. industry-wide experience data that were screened for applicability to a 
specific plant's design. The results indicate that a realistic failure frequency with all support systems available is about $1 \times 10^{-3} /$ demand. The result is corroborated by three different parametric models: the Basic Parameter, Multiple Greek Letter, and Binomial Failure Rate (with lethal shocks) models."

The difference between the above mentioned unavailability values do not arise from the failure rates used in the calculations because the failure rates applied in both cases (DCPRA and Reference 5) were found to be rather close; the data source was, in both cases, the data base developed by the PG\&E consultant, PL\&G.

The omission of potential common cause failures between the motor trains and the turbine train appears to result in an underestimation of the split fractions for the "low pressure" cases. If this is truly the case, then it would result in even greater underestimation for boundary conditions when the "high pressure" success criterion is used.

\section{A3.3.3 Audit Calculations for Top Event Split Fractions ("Low Pressure")}

In order to scrutinize the quantified values of split fractions AW and TD (associated with the "low pressure" case) given in the DCPRA, audit calculations were performed at BNL for each of these split fractions. The requantification was done by using the SETS code ${ }^{6}$ and by PC software developed at BNL. The use of the SETS code allowed the identification of the most important cut sets contributing to the hardware unavailabilities. These cut sets are inaccessible for direct review in the DCPRA. Attachment A3.A lists the ranked cut sets for selected boundary conditions. The definitions of the basic events appearing in the cut sets are identical to those given in Chapter E.2 of the DCPRA. In the audit calculations practically the same input data, maintenance frequency and duration, and human factors were applied which were used in the DCPRA (see columns "PG\&E" and "BNL1" in Tables A3.2.4 and A3.2.5). The obtained split fraction values for various boundary conditions are presented in Table A3.2.3, denoted by "BNL1" to be compared with the values quoted from the DCPRA, and denoted by "PG\&E" in Table A3.2.3. Table A3.2.3 also contains the definitions of certain quantities as used in the audit calculations to make clear the meaning of these quantities under different buundary conditions, even if their notations sometimes are the same.

The BNL audit calculations resulted in the following comments:

1. There was an apparent non-coherent condition in the AFW model (see also Comment No.1 in Table A3.2.3). A comparison of split fractions AW5 and AW7 with AW8 (as calculated by PG\&E) shows that AW5 and AW7 have a higher unavailabilities than AW8, in spite of the fact that they involve less unavailable components than AW8. In the BNL calculations this inconsistency did not show up (see also Section A3.3.5).

2. In several cases there were some major or minor differences between the results of PG\&E and BNL1.

\section{A3.3.4 Sensittvity Calculations for Split Fractions by Using Difierent Set of Data}

The methodology of the DCPRA puts an emphasis on the application of data appropriately selected for the conditions of the Diablo Canyon plant and updated according to the Diablo Canyon experience. 


\section{Appendix A3}

It was therefore of interest to see the sensitivity of the split fraction values (and through this the methodology of the systems analysis in the DCPRA) when one simply uses the numerical parameters from other data bases.

For this purpose the data bases given in the Seabrook ${ }^{7}$ and Millstone $^{8}$ PRAs were used. From these data bases numerical paraneters, appropriately selected for the AFWS unavailability analysis were taken. (If the data bases did not contain a given parameser, the DCPRA value was applied.) Tables A3.2.4 and A3.2.5 list the failure modes and components involved in the AFWS unavailability model together with the numerical parameters selected from the above data bases (see Column "BNL2"). For comparison, those numerical parameters which were used in the original analysis and in the BNL audit calculations are also presented (see Columns "PL\&G" and "BNL1").

The split fraction values obtained by the sensitivity study are shown in Table A3.2.3 (denoted by "BNL2"). By comparing the "BINL2" values with those of "BNL1", one observes that except for the boundary condition AW1, there is a rough agreement between them. This result shows that the majority of the split fractions were not that sensitive to the use of a reasonable generic data source.

\section{A3.3.5 Findinga/Conclusions}

Sensitivity caiculations showed that the majority of split fractions would not be seriously influenced if instead of plant-specific data, generic type numerical parameters had been used in the fault tree quantification of the Diablo Canyon standby safety systems.

The BNL review identified the following items that required resolution for completion of the review:

1. Should common cause failures have been modelled between the turbine and two motor driven pump trains?

2. What was the reason for the lack of agreement between BNL (BNL1) and PG\&E calculations as listed in Table A3.2.3 given that BNL used the DCPRA model and data?

3. Why was there an apparent non-coherence between support states AW5, AW7 and AW8?

PG\&E's response to item 1 essentially stated that no such common cause coupling was found in the Diablo Canyon design. BNL believes that this item should have been in the model for completeness. The exact effect of adding this elemejat to the model is unknown as it was not explored further in the review. However, it would tend to push the quantification of the split fraction somewhat higher.

For item 2, the disparities between the BNL calculations (BNL1) and the DCPRA (PG\&E) were determined to be a result of the Monte Carlow approach used in the DCPRA and the mean values used by BNL.

The apparent non-coherence between support states AW5, AW7 and AW8 was explained by PG\&E as a truncation anomaly and upon recalculation, the PG\&E point estimate values were in gool agreement with 
those of BNL. However, substitution of these values into the dominant sequence model had a significant effect on the calculated non-seismic CDF. BNL's results were as follows:

\section{Case}

Original PG\&E values (AW5, AW7, AW8)

BNL AFW Review Values (AW5, AW7, AW8)

PG\&E Point Estimate Values (AW5, AW8)

Final PG\&E Values (AW7, AW8)
Total Non-Seismic CDF

$2.6684 \mathrm{E}-4(151 \%)$

$2.4542 \mathrm{E}-4(138 \%)$

2.2887E-4 (129\%)

$1.7728 \mathrm{E}-4(100 \%)$

In order to clear up the confusion associated with these various sets of numbers, BNL asked for clarification and a detailed response from PG\&E was supplied in their May 3, 1990 letter to the NRC (PG\&E Letter No. DCL-90-118). The question and answer are reproduced here for completeness.

\section{Item 9:}

Two split fractions for the Auxiliary Feedwater (AW7 and AW8) system were revised in the uncertainty analysis of the non-seismic dominant sequences. PG\&E should provide these values to BNL along with a discussion on how these values were determined. Also, PG\&E should consider NRC Information Notice No. 89-58 in re zard to whether the PRA model addresses this issue.

\section{Response to Item 9:}

The split fraction values which should be used in the dominant sequence model for AW7 and AW8 are 3.238E-4 and 1.225E-3 respectively. The following discussion describes how these values were determined.

In the original auxiliary feedwater model the following conservative modelling assumptions were made:

1. A support system condition involving failure of one instrument channel was modelled as if both instrument channels that supply the steam generator $10 \%$ atmospheric steam dumps are unavailable.

2. No credit was taken for aligning the backup regulated transformer to the failed instrument channel so as to power the $10 \%$ steam dumps.

3. Given the unavailability of the $10 \%$ steam dumps, the model assumed 20 safety valve challenges and that all five safety valves may lift for each challenge. Each safety valve is required to reclose after each challenge.

To reduce the number of minimum cut sets in the AFW system equation file, certain low

frequency cut sets were removed based on their relative importance; this was done using a point estimate quantification. These cut sets were related to split fractions AW7 and AW8. Mean values of the split fractions AW7 and AW8, obtained from a Monte Carlo calculation using the truncated equations, were used in the event tree quantification. These values are presented in Column 2 of the table below.

During BNL's review of the AFW system model, BNL identified inconsistencies in the values of the AFW system split fractions. PG\&E determined that these inconsistencies were due to the truncated cut sets. Some of the cut sets became significant contributors to the AFW system unavailability when the model was quantified using Monte Carlo quantification because of the products of correlated variables. To resolve the 


\section{Appendix A3}

inconsistencies, the cut sets were restored to the equation file and the equation model requantified. The results are shown in Column 3 of the table below. The AFW split fraction values used in the DCPRA event tree model quantification and hence, in the development of the DCPRA dominant sequence model were, however, adequate for determining the important core damage sequences associated with the unavailability of the AFW system. This is because the values of AW7 and AW8 used to develop the dominant sequence model are higher than the revised values of AW7 and AW8 which were used for the uncertainty analysis of the dominant sequences.

The results of event tree quantification revealed that split fractions AW7 and AW8 were in a number of relatively high frequency core damage sequences, and that the conservative assumptions in the original $A F W$ system model contributed to their importance. These sequences involved the failure of one instrument bus which was assumed to fail all four $10 \%$ steam dump valves; in actuality, it takes the failure of two instrument buses to render all four $10 \%$ steam dumps unavailable. A more realistic analysis of the AFW system model was then performed by assuming that all safety valve failures to reclose were negligible for split fractions AW7 and AW8. This assumption is reasonable because loss of one instrument channel bus does not disable all of the $10 \%$ steam dumps, and with the availability of the $10 \%$ steam dumps the steam generator safety valves would not be challenged.

The reanaiysis involved the revision of an equation in the AFW system model equation file (see Figure E.2-16 of the DCPRA report, Sheet 16) which contained the variable " $\mathrm{C}$ ". The variable " $\mathrm{C}$ " is the failure probability of one or more of the five safety valves on a single steam generator to reclose successfully in each of the 20 cycles. " $\mathrm{C}$ " was set equal to zero in the AFW system model equaticn file, implying that all safety valve failures to reclose were negligible. This resulted in the mean values for AW7 and AW8 as shown in Column 4 of the table below: note, the values in Column 5 were used for the uncertainty analysis of the dominant sequence model.

Split Fraction Values for AW7 and AW8 ${ }^{1}$

Used in Event
Tree Quantifica-
tion (EFT2 Terms
Truncated)

AW7 6.269-3

AW8 7.759-3

\begin{tabular}{c} 
EFT2 Terms Restored \\
to AFW Equation File \\
\hline Variable $\quad$ Variable \\
$\begin{array}{cc}\text { C not Zero } & \text { C Set to Zero }\end{array}$
\end{tabular}

3.499-2

5.318-2
Used in

Dominant

Sequence

Model $^{5}$

3.238-4

$1.225-3$

1 Monte Carlo mean values.

2 Computer file PGE.1123EVENT.TREES>INTERNALS>MFF.RM3. Also see Appendix J, Table J-8.

3 Computer file PGE.1123> IBM.SYSTEMS>ADDENDUMS>AFW1004M.CTS.

4 Computer file PGE.1123> IBM.SYSTEMS>ADDENDUMS>AFW1006M.CTS.

5 Computer file PGE.1123> EVENT.TREES $>$ INTERNALS $>$ PMODEL $>$ DBF.RM3.SAVE. Also see Appendix J, Table J-3.

The resulting distributions for AFW split fractions AW7 and AW8 from the reanalysis of the AFW system model were used in the uncertainty analysis of the non-seismic dominant sequences. The characteristics of these distributions are provided below (5). These are the values which should be used in the dominant sequence model.

\begin{tabular}{|c|c|c|c|c|}
\hline & Mean & 5th & 50th & 95th \\
\hline & $3.238-4$ & $8.715-5$ & $2.234-4$ & 6.907 \\
\hline$A$ & $1.225-3$ & $3.861-4$ & $9.393-4$ & $2.372-3$ \\
\hline
\end{tabular}


NRC Information Notice No. 89-58 (1) describes an event that occurred at Diablo Canyon during power operation involving isolation of one of the two steam supply paths to the turbine-driven AFW pump (for maintenance) coincident with removal of a notor-driven AFW pump from service. In the post TMI reanalysis of main feedwater line break for Diablo Canyon, it was assumed that AFW would be supplied to two steam generators in order to prevent the pressurizer from filling with liquid coolant. With one of the two steam pressurizer from filling with liquid coolant. With one of the two steam supply valves to the AFW pump turbine isolation, only one steam generator may be supplied with AFW if the break occurs on the line feeding the steam generator that provides the remaining steam supply to the turbine-driven AFW pump and if the inoperable motor-driven AFW pump is associated with two steam generators with unbroken feedwater lines. Because of this, the NRC recommended that the turbine-driven AFW pump should be considered inoperable at Diablo Canyon (with respect to the emergency requirements) when one of the two steam paths is isolated.

From a system reliability standpoint, however, the turbine-driven AFW pump is still available even with only one operable steam supply path, since its design function to provide cooling water to all four steam generators can still be accomplished. An additional failure in the remain.ng steam supply path is required before the turbine-driven AFW pump is rendered unavailable. With the consideration of the joint frequency of maintenance-relatedisolation of one steam supply path and an additional failure in the remaining path, it was judged in DCPRA that this combined frequency is insignificant compared to the unavailability of turbinedriven AFW pump due to other causes. Maintenance of the steam supply valve(s) was, therefore, not modelled explicitly in DCPRA.

Nonetheless, in the DCPRA any maintenance event contributing to the unavailability of an AFW pump (turbine-driven or motor-driven) train was grouped together with the maintenance events for the corresponding AFW pump. The unavailability of an AFW pump train due to maintenance activities was then calculated based on the frequency and duration of the maintenance events on its respective pump. As such, unavailability of a motor-driven AFW pump due to maintenance (including events related to maintenance on steam generator level control valves as discussed in Information Notice No. 89-58) was modelled explicitly in the AFW system analysis of DCPRA. The mean frequency and duration of maintenance on a motor-driven AFW pump were estimated in DCPRA to be 5.53-4 per hour and 21 hours respectively. The mean maintenance unavailability of a motor-driven AFW pump is thus approximately 1.16E-2.

The isolation of one AFW pump turbine steam supply valve described in Information Notice No. 89-58 is the only occurrence of AFW steam supply isolation at Diablo Canyon since commercial operation. During that event, the steam supply valve was isolated for about 49 hours. Assuming the average frequency and duration of maintenance on steam supply valve are once every 3 years and 49 hours respectively, the estimated mean unavailability of the AFW pump turbine steam supply valve due to maintenance would be approximately $1.9 \mathrm{E}$ 3. Even without considering the frequency of an additional failure in the remaining steam supply path, this is only about $2.5 \%$ of the total due to other causes, which is approximately 7.3E-2. The impact of the steam supply valve isolation event on the unavailability of the AFW turbine pump train is therefore not significant.

If the uravailability of a motor-driven AFW pump due to maintenance is also considered in conjunction with the isolation of an AFW pump turbine steam supply valve, the combined unavailability is about 2.2E-5. This is less than $2 \%$ of the unavailability of one turbine-driven and one motor-driven AFW pump due to all causes, which is $1.2 \mathrm{E}-3$. If the frequency of the additional failure in the remaining AFW turbine steam supply path is also accounted for, the combined frequency should be much less than $1 \%$ of the unavailabilityof two pumps. After the January 1989 event, PG\&E has revised the procedures to make it clear that the turbine-driven pump should be declared inoperable when one AFW pump turbine steam supplies is isolated. If a motor-driven AFW pump also becomes unavailable during the period when one steam supply is isolated, the unit will be shutdown after six hours. This will prevent the recurrence of the January 1986 event and further reduce its contribution to system unavailability. 


\section{Appendix A3}

\section{Reference}

1. NRC Information Notice No. 89-58: Disablement of Turbine-Driven Auxiliary Feedwater Pump Due to Closure of One of the Parallel Steam Supply Valves, August 3, 1989.

The final updated PG\&E values for AW7 and AW8 as described above were used by BNL in the calculations found in Section 3.9 and Appendix D.

\section{A3.4 References}

1. PG\&E Letter to NRC signed by J.D. Shiffer, No. DCL-89-010, January 16, 1989.

2. Final report on the Diablo Canyon Long-Term Seismic Program, Pacific Gas and Electric Co., Diablo Canyon Power Plant, Docket Nos. 50-275 and 50-323, July 1988.

3. G. Bozoki, R. Fitzpatrick, M. Sabek, "Review of the Diablo Canyon PRA: Some Preliminary Results," presented at the NRC/PG\&E meeting, Rockville, MD, September 12, 1988.

4. Units 1 and 2 Diablo Canyon Power Plant, "Final Safety Analysis Report Update," Pacific Gas and Electric Co., December 1988.

5. K. Fleming, A. Mosleh, R. Deremer, "A Systematic Procedure for the Incorporation of Common Cause Events into Risk and Reliability Models," Nuclear Engineering and Design, Vol. 93, 1986.

6. R. Worrel, D. Stack, "A SETS User's Manual for the Fault Tree Analyst," Sandia National Laboratories, NUREG/CR-0465, SAND77-2051, November 1978.

7. Seabrook Station Probabilistic Safety Assessment, PLG-300, December 1983.

8. Millstone Unit 3 Probabilistic Safety Study, Westinghouse Electric Corporation, August 1983. 


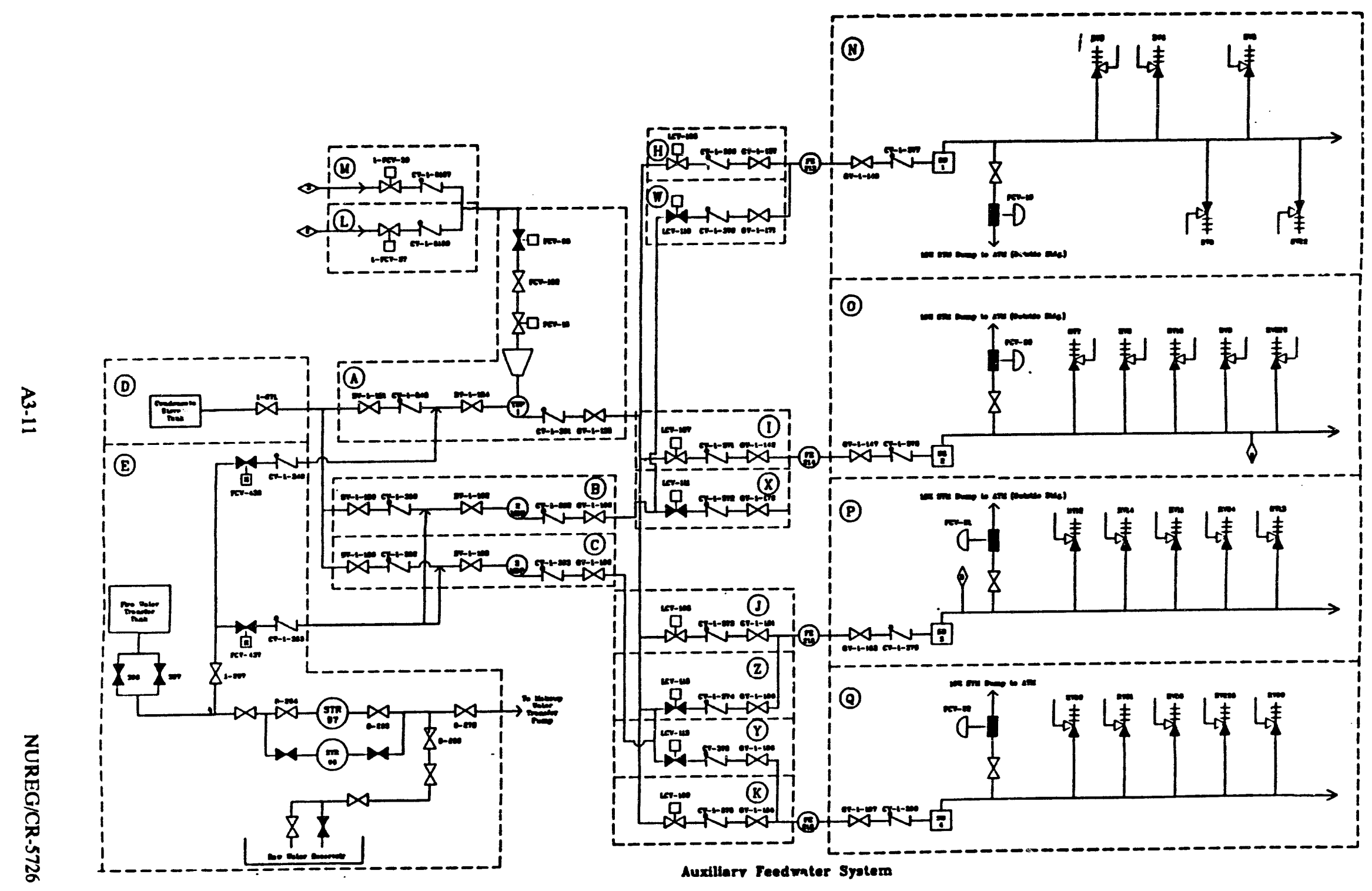

Figare A3.2.1 Top events AW and TS - Sapercomponents 
Appendix A3

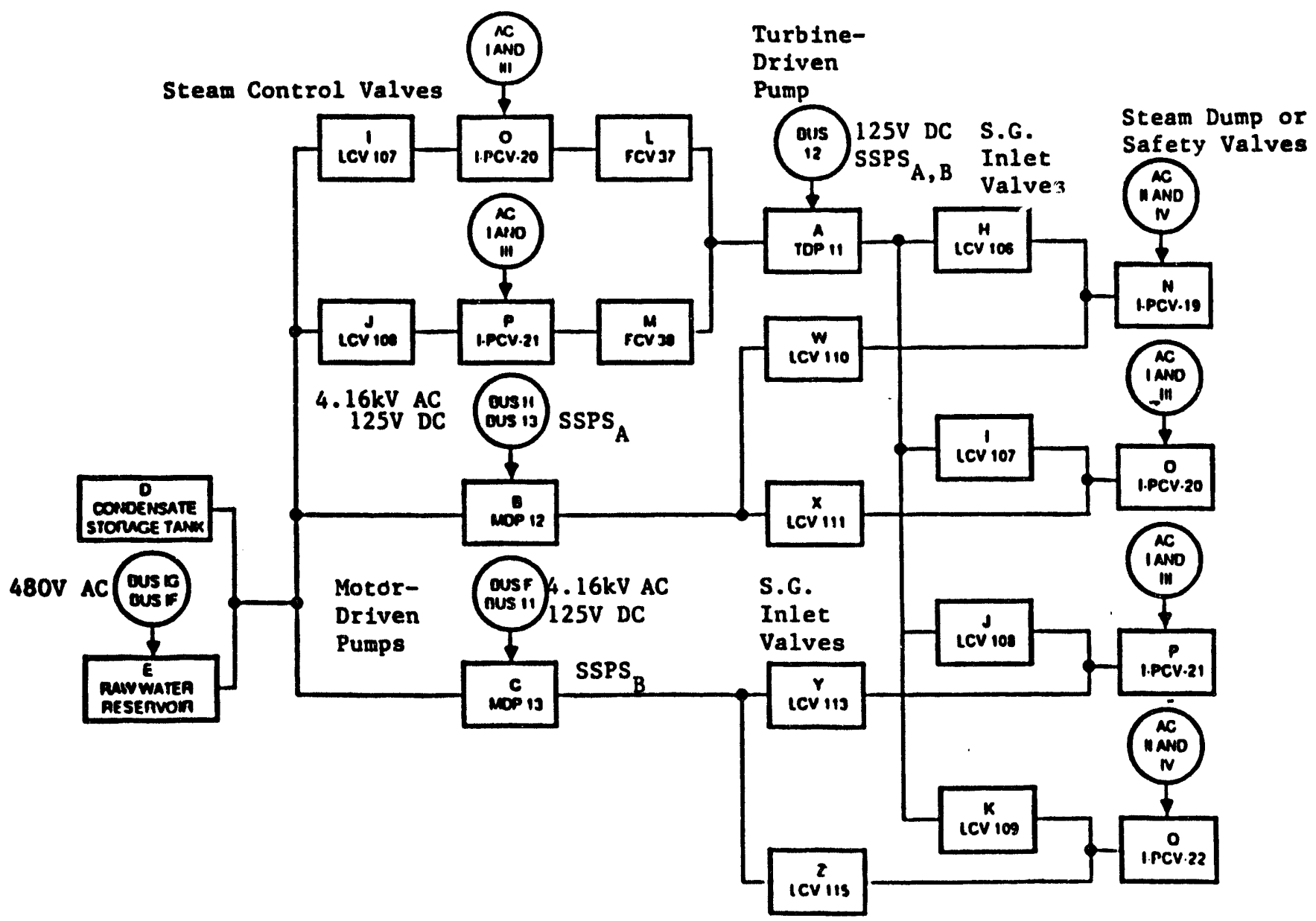

400V AC Dame 1F, 1G, 1A provide motive power to the turbine-ditven panp control valves and all SG inlet valves

Awelliary bullding ventilation provides roov cooling for MDPs 12 and 13

Figure A3.2.2. Lopic diagram for top events AW and TD - Auxiliary Fendwatar Syation: 
Table A3.2.1

Top Event Definitions and Success Criteria* Auxiliary Feedwater System

\begin{tabular}{cll}
\hline $\begin{array}{c}\text { Top } \\
\text { Event } \\
\text { Designator }\end{array}$ & \multicolumn{1}{c}{ Top Event Definition } & \multicolumn{1}{c}{ Top Event Success Criteria } \\
\hline AW & $\begin{array}{l}\text { Suciessful start and run** of } \\
\text { the auxiliary feedwater system and steam } \\
\text { rejection path through either the 10\% } \\
\text { dump valves or steam generator safety } \\
\text { relief valves. Successful reclosure of all } \\
\text { steam rejection valves is also included. }\end{array}$ & $\begin{array}{l}\text { If reactor trip succeeds, the flow } \\
\text { equivalent of at least one (or three) } \\
\text { auxiliary feedwater pumps must flow } \\
\text { through at least one steam generator and } \\
\text { be rejected through the 10\% steam dump } \\
\text { valves of the safety relief valves ("Low } \\
\text { Power" Requirements). }\end{array}$ \\
& $\begin{array}{l}\text { If the reactor does not trip, the flow } \\
\text { equivalent of all three auxiliary feedwater } \\
\text { pumps through at least two steam } \\
\text { generators is assumed to be required } \\
\text { ("High Power" Requirements). }\end{array}$ \\
& $\begin{array}{l}\text { Successful start and run of the turbine- } \\
\text { driven auxiliary feedwater pump, flow } \\
\text { path, and steam rejection path with } \\
\text { successful reclosure of steam rejection } \\
\text { valves. }\end{array}$ & $\begin{array}{l}\text { The flow equivalent of a motor-driven } \\
\text { auxiliary feedwater pump must flow } \\
\text { through at least one steam generator and } \\
\text { be rejected through the 10\% steam dump } \\
\text { or safety relief valves ("Low Power" } \\
\text { Requirements). }\end{array}$ \\
\hline TD & &
\end{tabular}

*The top event success criteria described in this table relate to the condition when all support systems are available.

**24 hours of continuous operation is used as the length of time for success.

\section{FSAR Success Criteria}

1. FSAR 6.5.1.1: The AFW system must function under the following conditions:

1a. Loss of main feedwater with offsite power available.

1b. Loss of main feedwater without offsite power available.

2a. Feedwater pipe rupture.

2b. Secondary steamline pipe rupture.

3. Loss of all ac power (station blackout)

4. Loss of coolant accident.

5. Cool down.

It is noted in FSAR 6.5.1.1.4 that LOCAs do not impose any AFW flow requirements in excess of those of other initiating event types. 
Table A3.2.1 (continued)

2. FSAR Table 6.5-1: For loss of main feedwater during station blackout, the criteria is that the reactor coolant system pressure not exceed the design pressure. A note on this table indicates that flow to a single SG is sufficient. For all other accident types the pressure requirement is the same, but there are also the requirements of 10CFR100 dose limits (secondary steamline rupture, feedline rupture, and LOCA) and 10CFR50 PCT limits.

LCOs: Tech. Spec. 3.7.1.2: At least three AFWS pumps shall be operable with the MDPs fed off separate vital buses and the one TDP powered by an operable steam supply system. If one AFWS pump inoperable: restore within 72 hours or be in at least hot standby within six hours and hot shutdown within the following six hours. If two AFWs pumps inoperable: be in at least hot standby within six hours and hot shutdown within the following six hours. If three AFWS pumps inoperable: immediate operator action to restore at least one AFWS pump to operable status as soon as possible.

\section{Tests:}

1. Each AFWS pump is tested at least once per 31 days.

2. Non-automatic non-secured values are checked for correct position once every 31 days.

3. AFWS pumps and valves are to be tested via an auxiliary feedwater activation signal at least once per 18 months. 


\section{Table A3.2.2}

Boundary Conditions for AFWS Top Events

\section{Top Event AW:}

1. AW1, all support system available, low power*.

2. AW2, all support system available, high power**.

3. AW3, support for one MDP unavailable, low power.

4. AW4, support for two MDPs unavailable, low power.

5. AW5, support for all (4) $10 \%$ steam dump (air operated) valves unavailable, low power.

6. AW6, support for all (4) $10 \%$ steam dump (air operated) valves unavailable, high power.

7. AW7, support for all (4) $10 \%$ steam dump (air operated) valves and to TDP unavailable, low power.

8. AW8, support for all (4) $10 \%$ steam dump (air operated) valves and to one MDP unavailable, low power.

9. AW9, support for all (4) $10 \%$ steam dump (air operated) valves and to two MDPs unavailable, low power.

10. AWA, support for all (4) $10 \%$ steam dump (air operated) valves and to (1 MDP + TDP) unavailable, low power.

11. AWB, one steam generator depressurizes. Loss of TDP and the MDP due to failure to isolate faulted SG. All other support system available, low power.

12. AWC, ATWS with turbine trip, TT, successful. All other support system available. TDP or both MDPs feeding two SGs required.

13. AWF, guaranteed failure.

2. Top Event TD (Seismic Events):

1. TD1, support for two MDPs unavailable, low power.

2. TD2, support for all (4) $10 \%$ steam dump (air operated) valves and to two MDPs unavailable.

3. TDF, guarantced failure.

*Low power; success criterion; 1 AFP to 1 SG.

"High power; success criterion; 3 AFPs to 2 SGs. 
Appendix $\mathbf{A 3}$

Table A3.2.3

Unovallabillty Valwes (8pllt Prections) for the Avoillary Foedweer Syntem Tunction

Top Eronte: AW, TD

\begin{tabular}{|c|c|c|c|c|c|c|c|c|c|c|}
\hline $\begin{array}{l}\text { Top } \\
\text { Event }\end{array}$ & Case & Calc. & TIL & HW & HWI & HWD & $\mathbf{M N}$ & $\mathbf{T S}$ & HE & Comment \\
\hline \multirow[t]{15}{*}{ AW } & AW1 & $\begin{array}{c}\text { PG\&E } \\
\text { BNL1 } \\
\text { BNL2 }\end{array}$ & $\begin{array}{l}3.727-5 \\
4.113-5 \\
1.402-4\end{array}$ & $\begin{array}{l}2.606-5 \\
1.578-5 \\
1.047-4\end{array}$ & $\begin{array}{l}1.420-5 \\
6.591-7 \\
1.610-6\end{array}$ & $\begin{array}{l}1.187-5 \\
1.512-5 \\
1.031-4\end{array}$ & $\begin{array}{l}1.543 .6 \\
1.679 .6 \\
4.917 .6\end{array}$ & $\begin{array}{l}7.016-6 \\
7.436-6 \\
5.521-6\end{array}$ & $\begin{array}{l}2.650-6 \\
1.623-5 \\
2.509-5\end{array}$ & \\
\hline & AW/2 & PGAE & $1.174-1$ & $5.732-2$ & $5.715-2$ & 1.7304 & 8.332 .3 & $3.780-2$ & $1.396-2$ & \\
\hline & & BNL1 & $\cdots$ & $\cdots$ & $\cdots$ & -- & - & - & - & \\
\hline & & BNL2 & $\cdots$ & $\cdots$ & $\cdots$ & -- & - & $\cdots$ & $\cdots$ & \\
\hline & AW3 & $\begin{array}{l}\text { PGCE } \\
\text { BNL1 } \\
\text { BNL2 }\end{array}$ & $\begin{array}{l}1.239-3 \\
1.492-3 \\
1.128-3\end{array}$ & $\begin{array}{l}1.8054 \\
2.107-4 \\
4.5804\end{array}$ & $\begin{array}{l}1.6774 \\
1.948-4 \\
3.010-4\end{array}$ & $\begin{array}{l}1.273-5 \\
1.594-5 \\
1.570-4\end{array}$ & $\begin{array}{l}1.509-4 \\
1.833-4 \\
1.916-4\end{array}$ & $\begin{array}{l}6.542-4 \\
7.740-4 \\
1.378-4\end{array}$ & $\begin{array}{l}2.531-4 \\
3.242-4 \\
3.410-4\end{array}$ & \\
\hline & $\frac{A W 4}{T D 1}$ & $\begin{array}{l}\text { POAE } \\
\text { BNL1 } \\
\text { BNL2 }\end{array}$ & $\begin{array}{l}7.250-2 \\
8.413-2 \\
7.521-2\end{array}$ & $\begin{array}{l}5.108-2 \\
6.273-2 \\
6.314-2\end{array}$ & $\begin{array}{l}5.107-2 \\
6.272-2 \\
6.280-2\end{array}$ & $\begin{array}{l}1.384-5 \\
1.500-5 \\
3.400-4\end{array}$ & $\begin{array}{l}2.777-3 \\
2.778-3 \\
2.778-3\end{array}$ & $\begin{array}{l}1.399-2 \\
1.390-2 \\
4.577-3\end{array}$ & $\begin{array}{l}4.655-3 \\
4.723-3 \\
4.717-3\end{array}$ & \\
\hline & Aws & $\begin{array}{l}\text { PO\&E } \\
\text { BNL1 } \\
\text { BNL2 }\end{array}$ & $\begin{array}{l}3.296-2 \\
4.934-3 \\
5.799-3\end{array}$ & $\begin{array}{l}3.249-2 \\
4.061-3 \\
5.270-3\end{array}$ & $\begin{array}{l}3.247-2 \\
4.040-3 \\
5.081-3\end{array}$ & $\begin{array}{l}2.195-5 \\
2.100-5 \\
1.890-4\end{array}$ & $\begin{array}{l}1.128-4 \\
1.237-4 \\
1.496-4\end{array}$ & $\begin{array}{l}5.562-4 \\
5.160-4 \\
1.126-4\end{array}$ & $\begin{array}{l}2.120 .4 \\
2.231-4 \\
2.699-4\end{array}$ & 1 \\
\hline & AW6 & PORE & $2.005-1$ & 1.519 .1 & $1.512-1$ & $1.747-4$ & $8.332-3$ & 3.592 .2 & $1.419-2$ & \\
\hline & & BNL1 & - & $\cdots$ & -- & - & - & - & - & \\
\hline & & BNL2 & - & $\cdots$ & $\cdots$ & - & - & - & $\cdots$ & \\
\hline & AW7 & $\begin{array}{l}\text { PG\&E } \\
\text { BNL1 } \\
\text { BNL2 }\end{array}$ & $\begin{array}{l}3.499-2 \\
7.011-3 \\
7.866-3\end{array}$ & $\begin{array}{r}3.282-5 \\
4.492-3 \\
6.460-3\end{array}$ & $\begin{array}{l}5.320-4 \\
4.323-3 \\
5.570-3\end{array}$ & $\begin{array}{l}3.229-2 \\
1.690-4 \\
8.900-4\end{array}$ & $\begin{array}{l}4.3574 \\
3.6624 \\
4.2064\end{array}$ & $\begin{array}{l}1.798-3 \\
1.531-3 \\
2.664-4\end{array}$ & $\begin{array}{l}7.8034 \\
6.344-4 \\
7.284-4\end{array}$ & 1 \\
\hline & AW8 & $\begin{array}{l}\text { POAE } \\
\text { BNL1 } \\
\text { BNL2 }\end{array}$ & $\begin{array}{l}7.9 \times-3 \\
2.384-2 \\
2.586-2\end{array}$ & $\begin{array}{l}3.900-3 \\
2.002-2 \\
2.370-2\end{array}$ & $\begin{array}{l}1.704-4 \\
2.000-2 \\
2.350-2\end{array}$ & $\begin{array}{l}3.730-5 \\
2.000-5 \\
2.000-4\end{array}$ & $\begin{array}{l}5.581-4 \\
5.317-4 \\
5.800-4\end{array}$ & $\begin{array}{l}2.552-4 \\
2.374-3 \\
5.807-4\end{array}$ & $\begin{array}{l}9.883-4 \\
9.150-4 \\
9.981-4\end{array}$ & 1 \\
\hline & $\begin{array}{l}\text { AW9 } \\
\text { TD2 }\end{array}$ & $\begin{array}{l}\text { POAE } \\
\text { BNL1 } \\
\text { BNL2 }\end{array}$ & $\begin{array}{l}1.410-1 \\
1.411-1 \\
1.372-1\end{array}$ & $\begin{array}{l}1.225-1 \\
1.255-1 \\
1.331-1\end{array}$ & $\begin{array}{l}5.095-2 \\
1.255-1 \\
1.328-1\end{array}$ & $\begin{array}{l}7.156-2 \\
3.810-6 \\
3.000-4\end{array}$ & $\begin{array}{l}2.777-3 \\
2.778-3 \\
2.778-3\end{array}$ & $\begin{array}{l}1.377-2 \\
1.390-2 \\
4.577-3\end{array}$ & $\begin{array}{l}4.731-3 \\
4.723-3 \\
4.716-3\end{array}$ & \\
\hline & AWA & $\begin{array}{l}\text { POAE } \\
\text { BNL1 } \\
\text { BNL2 }\end{array}$ & $\begin{array}{l}9.585-2 \\
8.803-2 \\
8.410-2\end{array}$ & $\begin{array}{l}7.813-2 \\
6.592-2 \\
7.570-2\end{array}$ & $\begin{array}{l}3.220-3 \\
6.575-2 \\
7.460-2\end{array}$ & $\begin{array}{l}7.522-2 \\
1.700-4 \\
1.100-3\end{array}$ & $\begin{array}{l}2.777-3 \\
2.778-3 \\
2.778-3\end{array}$ & $\begin{array}{l}1.152 .2 \\
1.161-2 \\
1.760-3\end{array}$ & $\begin{array}{l}4.731-3 \\
9.433-3 \\
9.417-3\end{array}$ & \\
\hline & AWB & $\begin{array}{l}\text { PG\&E } \\
\text { BNL1 } \\
\text { BNL2 }\end{array}$ & $\begin{array}{l}2.414-2 \\
2.240-2 \\
1.509-2\end{array}$ & $\begin{array}{l}4.808-3 \\
3.883-3 \\
5.840-3\end{array}$ & $\begin{array}{l}3.054-3 \\
3.104-3 \\
4.790-3\end{array}$ & $\begin{array}{l}1.755-3 \\
1.790-4 \\
1.050-3\end{array}$ & $\begin{array}{l}2.777-3 \\
2.778-3 \\
2.778-3\end{array}$ & $\begin{array}{l}1.190 .2 \\
1.161-2 \\
1.760-3\end{array}$ & $\begin{array}{l}4.655-3 \\
4.723-3 \\
4.717-3\end{array}$ & \\
\hline
\end{tabular}


Appendix A3

Trbble 13.2 .3 (continued)

Top

Event

$\begin{array}{ccc}\text { Case } & \text { Cak. } & \text { T } \\ & & \\ \text { AWC } & \text { PG\&E } & 2.4 \\ & \text { BNL1 } & - \\ & \text { BNL2 } & -\cdot \\ \text { AWF } & \text { PG\&E } & 1.0 \\ \text { TDF } & \text { BNL1 } & 1.0 \\ & \text { BNL2 } & 1.0\end{array}$

\section{Notntion}

TIL = Total unavailability.

HW = Unavailability due to hardware contribution which is the sum of independent failures and common cause failures.

HWI = Unavailability due to independent failures.

HWD = Unavaliability due to common cause failures.

TS = Unavailability due to tea.

MN = Uanvailability due to maintenance.

HE = Unavailability due to human error contribution.

\section{Definition of Verions Omantities Used in the Caleulntion}

The teat, maintenance, and human error contributions are calculated for each split fraction by using the following quantities (PGQE notations):

\section{Ror AW1}

EFT1 = Total hardware unavailability when the TDP is unavailable, all support is available.

EFT2 = Total hardware unavailability when one MDP is unavailable, all support is available.

\section{Bor AWB}

EFT3 = Total hardware unavailability when two MDPs are uravailable, all support is available.

EFT4 = Total hardware unavailability when one MDP and the TDP are unavailable, all support is available.

\section{Bor AWS}

EFT1 - Tntal hardware unavailability when the TDP is unavailable, and support to all $10 \%$ steam dump valves is unavailable.

EFT2 = Total hardware unavailability in case when one MDP is unavailable, and support to all 10\% steam dump valves is unavailable.

\section{Eor AW7}

EFT4 = Total hardware unavailability when one MDP and the TDP are unavailable, and support to all 10\% steam dump values is unavailable. 


\section{Appendix A3}

Table $\mathbf{A 3 . 2 3}$ (continmad)

\section{ForAW8}

EFT3 = Total hardware unavailabllity when two MDPs are unavailable, and support to all $10 \%$ steam dump valves is unavailable.

EFT4 = The same as at AW7.

\section{Comments}

1. There was an inconsistency between these split fractions in that the more degraded support state (AW8) had a bower unavailability than the lesser degraded support states (AW5 and AW7). This was subsequently corrected by PG\&E. 
Table A3.2.4

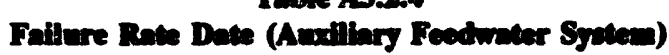

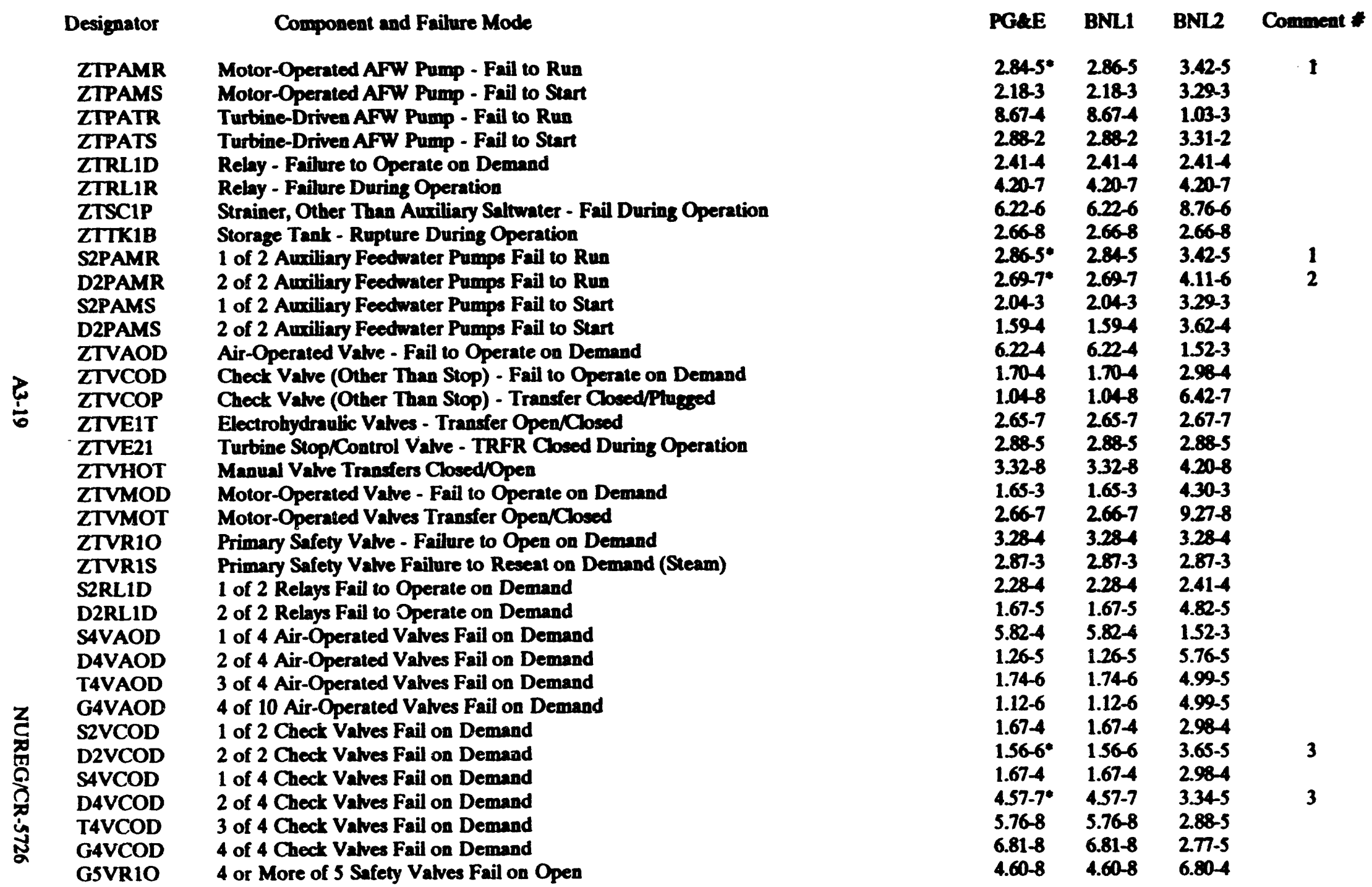


Appendix $\mathbf{A 3}$

\section{Comments to Table A3.2.4}

1. These values are presumably interchanged.

2. This value seems to be too small. The small value might be the result of inappropriate Bayesian updating. In Reference 5, Table 6, e.g., the beta factor for the failure mode "motor fail to run" if $\beta_{M R}=0.6$ yielding a double failure rate of $1.7 \times 10^{-5} / \mathrm{hr}$.

3. These values also seem to be too small. The cause might be also due to inappropriate Bayesian updating. 
'I:ablo A.1.2.5

Maintenance Durations, D (hr.) und Maintenance Frequency, F (per year) Auxiliary Feedwater System

\begin{tabular}{lllll}
\hline Designator & Component & PG\&E & BNL1 & BNL2 \\
\hline \multirow{2}{*}{ ZMPAMD } & Motor-Driven AFW Pump & 21 & 21 & 20.9 \\
ZMPATD & Turbine-Driven AFW Pump & 17 & 17 & 20.9 \\
ZMVMSD & Mainstenm Atmospheric Dump Valves & 55 & 55 & 132 \\
ZMPAMF & AFW Motor-Driven AFW Pump & $5.53-4$ & $5.53-4$ & $8.42-5$ \\
ZMPATF & AFW Turbine-Driven AFW Pump & $8.08-4$ & $8.08-4$ & $2.19-4$ \\
ZMVMSF & Mainsteam Atmospheric Dump Valves & $1.19-4$ & $1.19-4$ & $2.7-5$
\end{tabular}




\section{Attachment A3.A}

Hardware Unavailability Cut Sets for the

Auxiliary Feedwater System (Case: "Low Pressure," "BNL1")

Top Events: AW, TD 
Boundary Condition Designator: AW1

Leading hardware unavailability cut sets due to independent failures; HWI =

$\begin{array}{lll}1 & 6.0230 \mathrm{E}-07 & \mathrm{ZTA} * \mathrm{~S} 2 \mathrm{~B} 8 * \text { S2BC }+ \\ 2 & 5.4831 \mathrm{E}-08 & \mathrm{ZTD} * \mathrm{ZTE}+\end{array}$

Leading hardware unavailability cutsets due to independent and dependent failures; HW =

$\begin{array}{lll}1 & 1.0571 E-05 & \text { ZTA * D2BC + } \\ 2 & 4.5337 E-06 & \text { G4P * } \\ 3 & 6.0230 E-07 & \text { ZTA * S2B8 * S2BC + } \\ 4 & 5.4831 E-08 & \text { ZTD * ZTE + } \\ 5 & 4.2705 E-09 & \text { ZTA * G4CWXYZ + } \\ 6 & 1.5256 E-09 & \text { S2BB * T4POPQ + } \\ 7 & 1.5256 E-09 & \text { S2BC * T4PNOP + }\end{array}$




\section{Appendix A3}

Boundary Condition Designator: AWS

Leading hardware unavailability cutsets due to independent and dependent failures; HW =

\begin{tabular}{|c|c|c|}
\hline 1 & $3.9076 \mathrm{E}-03$ & S4PN * S4PO * S4PP * S4PQ + \\
\hline 2 & 4.8436E-05 & S2BC * S4PN * S4PO * S4PP + \\
\hline 3 & 4.8436E-05 & $\mathrm{S} 2 \mathrm{BB} * \mathrm{S4PO} * \mathrm{S4PP} * \mathrm{S4PQ}+$ \\
\hline 4 & $1.2149 \mathrm{E}-05$ & ZTA * S2BC * S4PN * S4PO + \\
\hline 5 & $1.2149 \mathrm{E}-05$ & ZTA * S2BB * S4PP * S4PQ + \\
\hline 6 & 1.0571E-05 & ZTA * D2BC + \\
\hline 7 & $1.0538 \mathrm{E}-05$ & D2BC * S4PO * S4PP + \\
\hline 8 & 4.4036E-06 & S4PN * S4PO * S4PP * S4YZ + \\
\hline 9 & 4.4036E-06 & S4PO * S4PP * S4PQ * S4YW + \\
\hline 10 & 6.0230E-07 & ZTA * S2BB * S2BC + \\
\hline 11 & 6.0040E-07 & S2BB * S2BC * S4PO * S4PP + \\
\hline 12 & 2.7614E-07 & ZTA * S4PN * S4PP * S4PQ * S4YZ + \\
\hline 13 & 2.7614E-07 & ZTA * S4PN * S4PO * S4PQ * S4YY + \\
\hline 14 & $6.8100 \mathrm{E}-08$ & G4P + \\
\hline 15 & 5.4831E-08 & $\mathrm{ZTD} * \mathrm{ZTE}+$ \\
\hline 16 & 5.4659E-08 & S2BB * S4PP * S4PQ * S4JI + \\
\hline 17 & 5.4659E-08 & S2BC * S4PW * S4PO* S4JJ + \\
\hline 18 & 5.4585E-08 & S2BC * S4PO * S4PP * S4YW + \\
\hline 19 & 5.4585E-08 & S2BB * S4PO * S4PP* S4YZ + \\
\hline
\end{tabular}


Boundary Condition Designator: AW5

Leading hardware unavailability cutsets due to independent failures; HWI =

$\begin{array}{lll}1 & 3.9076 E-03 & \text { S4PN * S4PO * S4PP * S4PQ + } \\ 2 & 4.8436 E-05 & \text { S2BB * S4PO * S4PP * S4PQ + } \\ 3 & 4.8436 E-05 & \text { S2BC * S4PN * S4PO * S4PP + } \\ 4 & 1.2149 E-05 & \text { ZTA * S2BB * S4PP * S4PQ + } \\ 5 & 1.2149 E-05 & \text { ZTA * S2BC * S4PN * S4PO + } \\ 6 & 4.4036 E-06 & \text { S4PN * S4PO * S4PP * S4YZ + } \\ 7 & 4.4036 E-06 & \text { S4PO * S4PP * S4PQ * S4YW + } \\ 8 & 6.0230 E-07 & \text { ZTA * S2BB * S2BC + } \\ 9 & 6.0040 E-07 & \text { S2BB * S2BC * S4PO * S4PP + } \\ 10 & 2.7614 E-07 & \text { ZTA * S4PN * S4PO * S4PQ * S4YX + } \\ 11 & 2.7614 E-07 & \text { ZTA * S4PN * S4PP * S4PQ * S4YX + } \\ 12 & 5.4831 E-08 & \text { ZTD * ZTE + } \\ 13 & 5.4659 E-08 & \text { S2BC * S4PN * S4PO * S4JJ + } \\ 14 & 5.4659 E-08 & \text { S2BB * S4PP * S4PQ * S4II + } \\ 15 & 5.4585 E-08 & \text { S2BB * S4PO * S4PP * S4YZ + } \\ 16 & 5.4585 E-08 & \text { S2BC * S4PO * S4PP * S4YW + } \\ 17 & 5.2190 E-08 & \text { S2BC * S2MM * S4PN * S4PO + } \\ 18 & 5.2190 E-08 & \text { S2BB * S2ML * S4PP * S4PQ + } \\ 19 & 1.3691 E-08 & \text { ZTA * S2BC * S4PO * S4YW + }\end{array}$




\section{Appendis A3}

Boundary Condition Designator: AW8

Leading hardware unavailability cutsets due to independent and dependent failures; $H W=$

\begin{tabular}{|c|c|c|}
\hline 1 & $1.5629 \mathrm{E}-02$ & SAPO * S4PP * S4PQ + \\
\hline 2 & $3.9200 \mathrm{E}-03$ & ZTA * SAPP * SAPQ + \\
\hline 3 & $1.9434 \mathrm{E}-04$ & ZTA $*$ S2BC + \\
\hline 4 & 1.9373E-04 & S2BC * SAPO * SAPP + \\
\hline 5 & 1.7637E-05 & SAPP * SAPG * SAII + \\
\hline 6 & 1.7613E-0S & S4PO * S4PP * S4YZ + \\
\hline 7 & $1.6840 \mathrm{E}-05$ & S2ML * S4PP * S4PQ + \\
\hline 8 & 1.0571E-05 & ZTA * D2BC + \\
\hline 9 & $1.0536 \mathrm{E}-05$ & D2BC * S4PO * S4PP + \\
\hline 10 & 4.4175E-C6 & ZTA $*$ SAPP * S4YZ + \\
\hline 11 & 4.4175E.06 & ZTA * SAPQ * SAYY + \\
\hline 12 & 2.1862E-07 & S2BC SAPP * SAJI + \\
\hline 13 & 2.1862E-07 & S2BC * S4PO * S4JJ + \\
\hline 14 & 2.0874E-07 & S2BC * S2MM * S4PO + \\
\hline 15 & 2.0874E-07 & S2BC * S2ML * S4PP + \\
\hline 16 & 1.1426E-07 & D4POP : S4PQ + \\
\hline 17 & $1.1426 \mathrm{E}-07$ & D4POQ * S4PP + \\
\hline 18 & $1.1426 \mathrm{E}-07$ & S4PO * D4PPQ + \\
\hline 19 & 9.7516E-08 & D3ML * S4PP * S4PQ + \\
\hline 20 & 6.8100E-08 & G4P + \\
\hline 21 & $5.7300 \mathrm{E}-08$ & T4POPQ + \\
\hline 22 & 5.4831E-08 & ZTD $*$ ZTE \\
\hline 23 & 2.8358E-08 & ZTA $*$ D4CYZ + \\
\hline 24 & 2.8358E-08 & ZTA * D4PPQ + \\
\hline
\end{tabular}


Boundary Condition Designator: AW8

Leading hardware unavailability cutsets due to independent failure; HWI =

\begin{tabular}{|c|c|c|}
\hline 1 & $1.5629 \mathrm{E}-02$ & SAPO * SAPP * SAPQ + \\
\hline 2 & $3.9200 \mathrm{E}-03$ & ZTA * SAPP * SAPQ + \\
\hline 3 & $1.9434 \mathrm{E}-04$ & ZTA * SBBC + \\
\hline 4 & $1.9373 \mathrm{E}-04$ & S2BC * SAPO * SAPP + \\
\hline 5 & 1.7637E-05 & SAPP * SAPQ * SAII + \\
\hline 6 & 1.7613E-0S & SAPO * SAPP * SAYZ + \\
\hline 7 & $1.6840 \mathrm{E}-05$ & S2ML * S4PP * S4PQ + \\
\hline 8 & 4.4175E-06 & ZTA * SAPQ * SAYY + \\
\hline 9 & 4.4175E-06 & ZTA * S4PP * S4YZ + \\
\hline 10 & 2.1862E-07 & S2BC * SAPO * SAJJ + \\
\hline 11 & 2.1862E-07 & S2BC * SAPP * SAII + \\
\hline 12 & 2.0874E-07 & S2BC * S2MM * S4PO + \\
\hline 13 & 2.0874E-07 & S2BC * S2ML * S4PP + \\
\hline 14 & 5.4831E-08 & $\mathbf{Z T D} * \mathbf{Z T E}+$ \\
\hline 15 & $1.9876 \mathrm{E}-08$ & SAPP * S4JI * S4YZ + \\
\hline 16 & $1.8978 \mathrm{E}-08$ & S2ML * S4PP * S4YZ + \\
\hline 17 & 4.9783E-09 & ZTA * S4YY* S4YZ + \\
\hline 18 & 4.9693E-09 & * SAJJ * S4YY + \\
\hline 19 & 4.7448E-09 & S2MM * S4PO * S4PQ * S4YY + \\
\hline
\end{tabular}




\section{APPENDIX B}

\section{Syctom Anahyses for Solected Support Systems}

81: Diced Conorator \& Dicsel Fual Transfor Systoms

B2: Electrical Power Syatoms

Bs: Aundllary Saltwater Syatem

B4: Component Coollng Water Systom

B5: Solld state Protection/Reactor Protection Systoms 


\section{APPENDIX B1: DIESEL GENERATOR \& DIESEL FUEL TRANSFER SYSTEMS}

\section{B1.1 Introduction}

\section{B1.1.1 Objoctives}

The main objective of this appendix is to summarize the results of reviewing the unavailability analysis of the Diesel Generator and Diesel Fuel Transfer Systems described in the DCPRA. ${ }^{1}$ The review was carried out with epecial attention to the details of the unavailability modelling of the maintenance activities on the DGs. (This particular emphasis was prompted by a concurrent request of the Pacific Gas and Electric Co. to change the Allowed Outage Time (AOT) of the DGs from the present outage of three days to seven days, and the fact that the study aupporting this request derived data on expected core damage frequency changes based mainly on the DCPRA.)

\section{B1.12 Orvelintion of the Repert}

Section B1.2 provides condensed descriptions of the configurations and functions of the Diesel Generator and the Diesel Fuel Oil Transfer Systems. It also describes the dependency of these systems on support equipment, the surveillance and maintenance conditions, the unavailability modelling in the DCPRA, and the original PRA results. The purpose of this approach is to present stand alone documentation to which the review's findings can be directly compared. Section B1.3 contains the results and findings of the BNL review.

For completeness, the ranked cut sets of hardware unavailabilities (both independent and total) obtained by BNL for various diesel configurations are given in Attachment B1.A.

\section{B1.2 Unavallability Modelling of the Diesel Generator and Diesel Fuel Oil Transfor Systems}

\section{B1.21 Diesel Gemerntor Sydtem Deweription, Configurntions and Punctions}

The Diesel Generator System at the Diablo Canyon plant consists of five diesel generators: two dedicated to Unit 1, two dedicated to Unit 2, and one (a "swing diesel") shared between the two units. According to, the DCFSAR, ${ }^{3}$ the individual diesel generator units are isolated from each other and from other equipmen!. The swing diesel is physically located in Unit 1. Each diesel generator supplies power to its associated 4.16kV vital bus (H, G, and F - Units 1 and 2). In the event of a loss of electrical power from the main generator (due to a unit trip, a safeguard signal or a loss of voltage on a vital bus) the vital $4.16 \mathrm{kV}$ buses are automatically disconnected from the main generator and transferred to the offsite standby source. (The Unit 1 main generator provides power through auxiliary transformer 12. The standby power is provided through startup transformers 11 and 12.) If this transfer is unsuccessful or the standby power is unavailable, the diesel generators must start and provide power to the affected buses. The diesel generators start on undervoltage sigmals from their respective buses, load onto those buses (the output breakers are normally open), initiate reloading of the vital loads and continue delivering power at normal frequency to the buses. A safety injection actuation signal (SIS) from either Train A or B of the SSP System will also start the diesels (Train A will start 11 and 13, Train B will start 11 and 12). 
The swing diesel (13) may supply power to either Unit 1 or Unit 2 vital Bus F. It will start with an undervoltape or an SI signal from either unit (SSPS Train A). Because the output is not shared simultaneoushy by the units, only one of its two circuit breakers is closed at a time. The breakers have individual sets of control and protection circuits. If one of the units recelves an SI sigal (earlier than the other), it is given priority of using the swing diesel.

The DGs are $2750 \mathrm{~kW}, 18$ cylinder, vee conffguration, ALCO units. Each unit consiats of a self-contained diesel engine directly connected to an alternating current generator. Each diesel has dual train electrical starting circuits and air system with turbocharger, ventilation, fuel oil system, self-contained radiator cooled jacket cooling water system, lube oll aystem, and speed control governor system.

- Each independent starting circuit has its own de power source (DG11; de panels 13, 12. DG12; de panels 12, 11. DG13; de panels 11, 13. DG21; de panels 22, 21. DG22; dc panels 23, 22). The operating control circuit is common. Without control power a unit keeps running. A mechanical trip handle, located in the diesel compartment serves to shut the unit down.

- The air start system consists of two trains. Each train includes a compreseor, a dryer, an air receiver and two air-drtven motors. Air from receivers is fed through regulator valves and up to the starting air system solenoid valves. Only one motor is needed to start a diesel. Power supply to the compreseor trains are provided by 480V ac buses: [DG11; Trains A and B; 1H, 1G. DG12; Trains A and B; 1G, 1F. DG13; Trains A and B; 1F (backup 2F), 1H (backup 2F). DG21; Trains A and B; 2G, 2F. DG22; Trains A and $B ; 2 H, 26$.$] One solenoid control valve of an air driven motor in each compressor train gets its "open"$ signal from the normal control, the other solenoid valve receives signal from the backup control. Upon initiation of a start, the solenoid valves open supplying air to the motors. After initiation, pressure switches located on the discharge of the jacket water pump shuts off the air supply. The air start system supplies air to the Level Control Valves (LCVs) of the diesel fuel oil day tanks. There is one air supply line per LCV.

- The air start system also includes an air operated turbocharger for quick starting and load pickup. The associated air subsystem consists of one turbo air compressor, one starting air receiver tank, and an air dryer. Two solenoid operated shutoff valves, one on each of the two supply lines, control the air supply to the turbocharger. A solid state speed-loss sensor controls the turbo-assist air supply to prevent a critical loss of speed when a sudden large load increase occurs.

- Each diesel has also another air system: the combustion air and exhaust system (ventilation), containing the intake and exhaust silencers and the two motor-driven crankcase exhauster fans.

- The engine fuel oil system involves the fuel oil day tank. Fuel oil is supplied by the Diesel Fuel Oil Transfer System (see its description in Section B1.2.2). The fuel oil level in the day tank is controlled by two redundant level control valves (LCVs). Each LCV has two $480 \mathrm{~V}$ ac control power sources; a normal supply and a backup supply. The power sources for LCVs associated with the primary fuel oil transfer pump (Train 02) are: 480V $a$ buses $1 G$ and 2G. Power sources for LCVs associated with the secondary fuel oil transfer pumps (Train 01) are: $480 \mathrm{~V}$ ac buses, $2 \mathrm{H}$ and $1 \mathrm{H}$. The valves may be actuated also manually. 


\section{Appondix B1}

- The cooling of a diecel unit is provided by a closed loop jacket cooling water system. The jacket water pump takes water from the lube oil cooler and the turbocharger aftercooler. There is a 50-gallon expanaion tank conanected to the suction addo of the pump. The pump diccharges water through the engine block and turbochareer to a common return line. Encine water temperature is maintained at $170^{\circ} \mathrm{F}$ by a thermoutatically controlled three-way valve eot. Overheated water is cent to a water radiator, where it is cooled by forced air (ongine driven fan) taken from outside the building.

- The lubricating oil syatem condiats of an oll reservoir, an engine driven pump and a heat exchanger. The heat exchanger is cooled by the ongine jacket cooling water aytem. Lubricating oil temperature is thermoutatically controlled. The oil is kept in the range of $90^{\circ} .110^{\circ} \mathrm{F}$ circulated by a small pre-circulation pump evea if the cenerator is idle, to reduce wear during the engine start period. The diesel automatically stope if the oll presoure drops below 40 paig.

- To control the fuel delivery and therefore the engine's apeed and generator output frequency to a predotermined value, an engine governor apeed control unit is used. The governor has electrical and mechanical controls; both of which sct through a hydraulic actuator to control the fuel supply.

The diesels cannot respond to a start aignal under the following conditions:

1. Shutdown relay tripped.

2. Manual teat condition.

3. Low fuel lovel in the day tank.

4. Low pressure in both starting air receivers.

5. Loss of de control power.

6. Voltage regulator on manual.

The eventual problems of the diecels are annunciated by various alarms (14 groups of signals) in the control room.

The loads of the diesels are listed in Table B1.2.1. Each diesel has enough capacity to handle some extra startup load. The bading of the diesels during the recirculation phase of a LOCA is under the control of the operator.

Each generator compartment is provided with an automatic flooding $\mathrm{CO}_{2}$ gas system for fire protection.

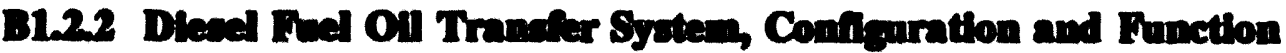

The diesel fuel oil transfer system maintains a supply of fuel oil to each DG day tank from two large underground storage tanks (capacity: 40,000 gallons per tank). It contains two trains (01 and 02 ), each having a rotary screw type positive displacement pump. These pumps are self priming. A single pump has enough capacity (55 gpm at 50 psig) to supply all the five diesels. (The fuel consumption rate is about $3.2 \mathrm{gpm}$ per DG). Each pump train has a fuel oil distribution header supplying all five of the DGs. Manual crosstie valving between headers allow either transfer pump to deliver to either header. Also, it is possible to pump from either of the storage tanks. 
Local controls for the aystem are bocated at each DG. There are two sets of controls; one for pump train 01 and another for pump train 02 . These are the LCV switches: a total of 10 (5x2). Each switch starts the tranefer pumps and opens the LCV of its respective train. The pump start levels are different: 252 gallons for train 01 and 271 gallons for train 02 . Once a pump is started it will remain running until shut down by the operator. If all the LCVs are closed (the day tanks are full) the fuel oil will recirculate back to the main atorage tank.

The motors that drive the pumps are powered by $480 \mathrm{~V}$ vital ac buses (pump train 01 by either bus $1 \mathrm{H}$ or $2 \mathrm{H}$, from Units 1 and 2 respectively, and pump train 02 by either bus $1 G$ or 2G). A manual transfer switch determines the alignment, the only criterion for alignment is that the pumps should be powered by different unita.

The operation of the oll fuel transfer syatem is made on a demand basis: when one of the day tanks reaches a low lovel set point, the fuel transfor pumps start and remain running until all diesels have been shut down. For the six hour mission time (24 hours for seismic events) of the diesels, the fuel transfer system must remain functioning to replenish the fuel supply to each running diesel. The minimum total storage in the storage tanks is sufficient for seven days of power generation.

The importance of the operability of the fuel oil transfer system for the plant safety is obvious: if the fuel transfer system is unavailable, it results in failure of all the DGs of both units, Unit 1 and Unit 2. For events when both ac powered fuel transfer pumps might become unavailable, a dedicated portable fuel oil driven pump is kept at hand. This pump takes suction directly from the main storage tank and connects to one of the fuel delivery headers. Flexible hoses are used to make the appropriate connections.

\section{B1.23 Top Bvent Definitions, Success Criteria}

Associated with the unavailability of the diesel generators, the DCPRA defines six top events in the electric part of the support system event tree. The designators of these top events and their relationships with the diesels are:

- Top Event GF - Diesel Generator 13 ("swing diesel")

- Top Event GG - Diesel Generator 12

- Top Event GH - Diesel Generator 11

- Top Event 2G - Diesel Generator 21

- Top Event 2H - Diesel Generator 22

- Top Event SW - Unit alignment of the swing diesel, 13

If the offaite grid is available (top event $O G$ in the support system event tree is successful) only the " $G$ " events (GF, GG, GH) re questioned in the support systems event tree. If the offsite grid fails, all the five top events are questioned. The boundary conditions of these top events depend on the status of the preceding diesel generators in the event tree. Thus, top event GF has only one boundary condition (GF1) corresponding to the case when all support is available. GG has three boundary conditions (GG1, when GF succeeded; GG2, when GF failed; and GG3, when GF was bypassed, i.e., not demanded). Similarly GH has 6, 2G has 10, and 2H has 15 boundary conditions. Top event SW has four boundary conditions: one for LOCAs; one for LOOPs, when an equal number of diesels are operating at Unit 1 and Unit 2; and two for LOOPs, when an unequal number of diesels are operating at the two units. 
Appendix B1

Only one top event is defined in the DCPRA for the support system event tree associated with the diesel fuel oll transfer system. The designator of this top event is: LO. It is evaluated for six boundary conditions, depending on the availability of $480 \mathrm{~V}$ ac buses at both of the units (i.e., 1G, 2H, 2G, and 1H).

The success criteria of the above top events are described in Table B1.2.1. The Technical Specification requirements with respect to the operability of the associated systems are also indicated.

\section{B12. Lexte Model of the Dlecels and Dieael Prel Oll Trander System Dependency on Other Support Sydeme}

The generic reliability block diagram for the diesel generators is shown in Figure B1.2.1. The diagram is constructed from blocks (supercomponents) of the DG system. The boundaries of the supercomponents (for instance: GH-1, GH-2A, GH-2B) are indicated in Figures B1.2.2 through B1.2.9. Notice, that the equipment boundaries for each of the diesels start with the diesel generator and include the output breaker, the fuel oil day tank, the day tank level control valves, and the undervoltage and transfer control relays. The diesel starting air system was not modelled separately because it was included as part of the diesel start failure data.

The reliability block diagram shows the dependencies on the supercomponents of the plant (ac and dc) electrical systems.

The reliability block diagram for the diesel fuel oil transfer system (Top Event, FO) is presented in Figure B1.2.10. The boundaries of the pump train blocks are indicated in Figure B1.2.11. The reliability block diagram shows also the system dependencies on other supercomponents of the plant (ac and dc) electrical systems.

\section{B1.25 Qmantification of Top Event Split Practions}

The definitions of the boundary conditions and the associated split fractions for top events associated with the DG system are listed in Table B1.2.3. Table B1.2.4 presents a similar list for the diesel fuel oil transfer system (Top Event, FO).

Table B1.2.5 presents the values of diesel generator related top event split fraction values quantified by PG\&E. Notice, that to provide better train-wise dependency tracking in the event tree model, the split fractions are expressed in terms of unavailabilities of various diesel state combinations (conditional split fractions, CSF). The arithmetic is explained in the DCPRA, Chapter D.2.1.5. The table presents also the total unavailability value (TTL) used in the calculation of each CSF, along with the main contributors to the total unavailabilities, such as hardware (HW), maintenance (MN), test (TS), and human error (HE). At a given boundary condition the hardware contribution relates to the normal alignment, when no test or maintenance activities are being performed. To provide complete information, the table also indicates the two constituent parts of the hardware contribution to the unavailability: the independent (HWI) and the dependent (HWD) (i.e., common cause) failures of the supercomponents of the diesels.

The maintenance contribution is a significant contributor to the total unavailability. The DCPRA assumes that, due to Technical Specification limitations, only one diesel or level control valve may be in maintenance at a time. The following relevant quantities are used in the maintenance unavailability quantification: 
Diesel maintenance frequency, ZMDGSF: $7.74-4 / \mathrm{hr}$ (Mean Value). Variance $=2.33-8,5$ th Percentile $=5.25$ 4, Median $=7.52-4$, 95th Percentile $=9.66-4$.

Diesel maintenance duration, ZMGSD: $1.01+1 \mathrm{hr}$ (Mean Value). Variance $=3.99$, 5th Percentile $=6.65$, Median $=9.74$, .5 th Percentile $=13.3$.

Level control valve maintenance frequency, ZMGNDF: $2.03-5 / \mathrm{hr}$ (Mean Value). Variance $=3.52-11$, 5 th Percentile $=1.14-5$, Median $=1.91-5,95$ th Percentile $=2.97-5$.

Level control valve maintenance duration, ZMGN3D: $1.89+1 \mathrm{hr}$ (Mean Value). Variance $=597.0$, 5th Percentile $=1.54$, Median $=10.1,95$ th Percentile $=51.3$.

Notice that the total maintenance unavailability of a diesel unit is determined by the diesel (as defined in DCPRA) maintenance unavailability plus the LCV maintenance unavailability. When a diesel is unavailable (not for reason of preventive maintenance) the other diesels must be surveillance tested once within 24 hours to verify operability. The DCPRA includes the unavailability contribution due to this type of test in the maintenance unavailability (MN).

The test contribution to the total unavailability is modelled in the DCPRA as to be due to the scheduled monthly surveillance tests, which include the manual test of the fuel transfer system to the diesels and the quarterly stroke test of the LCVs.

There is no explicit human error contribution to the total unavailability, because human errors occurring after maintenances and tests due to leaving diesel components in misalignment are included in the maintenance and test contributions.

Table B1.2.6 lists the split fraction values for the various boundary conditions of the FO top event. The table, as the previous one, details the hardware (independent and dependent components), maintenance test and human error contributions to the total unavailability values. Notice there are no explicit test or human error contributions. All the tests on fuel oil transfer system can be performed without making the system inoperable, human errors occurring leaving a fuel oil transfer train in misalignment after maintenance are included in unavailability values due to maintenance.

\section{B1.2.6 Quamtification of Seismic Split Fractions for DG Top Events}

The basis for detailing the seismic split fraction quantification for the DG top events is to provide insight into how the maintenance unavailability (and through it, the AOT) affects the seismic top events and consequently the seismic contribution to the core damage frequency. (This particular investigation was done as part of the parallel BNL. DG AOT review as discussed in Section B1.1.) 
Appendix B1

All diesel generator components susceptible to failures by seismic events contribute to the diesel unavailability. The components considered to be the most vulnerable to seismic effects are the following:

$\begin{array}{ll}\text { Component } & \text { Frepility Designator } \\ \text { DG Control Panel } & \text { ZDGCPN } \\ \text { DG Excitation Panel } & \text { ZDGEXC } \\ \text { DG Radiator/Water Pump } & \text { ZDGRWP } \\ \text { Dieeel Generator Itsolf } & \text { ZDGSLGN }\end{array}$

By using the conditional seismic failure probabilities ("fragilities"), the DCPRA combines them into a "seismic term" denoted by SEIST. SEIST has seven values corresponding to the seven seismic levels (i.e., spectral acceleration ranges) defined in the DCPRA. The seven SEIST values were determined by the mean fragilities of the diecel components listed in Table 6-44 on p.6-175 of Reference 1.

In order to calculate seismic split fractions, the DCPRA combines the SEIST values with the total unavailability values (TTL) coming from the conventional handware, maintenance, test and human failures. In the case of seismic events, however, the DCPRA (correctly and innovatively) treats many human failures as seismic-level-dependent; that is, the humen factor probabilities are also dependent upon the seismic level.

To be more specific, the human failure which affects the TTL unavailabilities is the "failure to reestablish fuel oil transfer to day tanks by aligning a portable fuel oil transfer pump (see also Figure B1.2.10) and by controlling the day tank LCVs manually;" its designator is ZHEF06. For numerical values as a function of seismic level, Appendix G of the DCPRA Table G.1-2.4

By using the resultent unavailabilities (SEIST + seismic level dependent TTL) the conditional seismic split fractions were determined for each diesel top event according to the rules of the sequential diesel failure model. These split fractions are listed as a function of the seismic level in Table B1.2.7. Each value of the table has a slight AOT dependence through the maintenance contribution to the TTL component of the unavailability. 


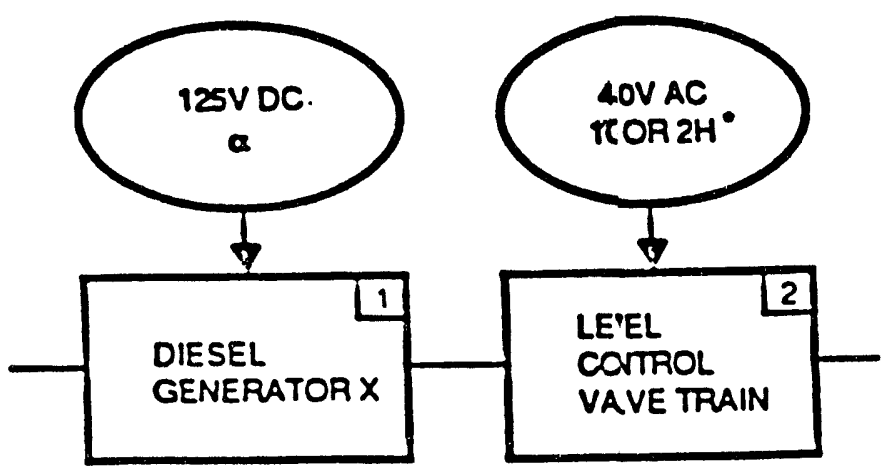

\begin{tabular}{|l|l|}
\hline$x$ & $a$ \\
\hline 13 & 11 \\
12 & 12 \\
11 & 13 \\
21 & 22 \\
22 & 23 \\
\hline
\end{tabular}

DIESEL GENERATOR CONTROL POVIER

- THESE ARE THE NORMAL FOWER SUPPLIES FOR THE TWO LCV TRAINS: THE BACKUPS ARE $2 G$ ANE IH RESPECTIVELY

Figure B1.2.1 Reliability block diagram for the diesel generators 


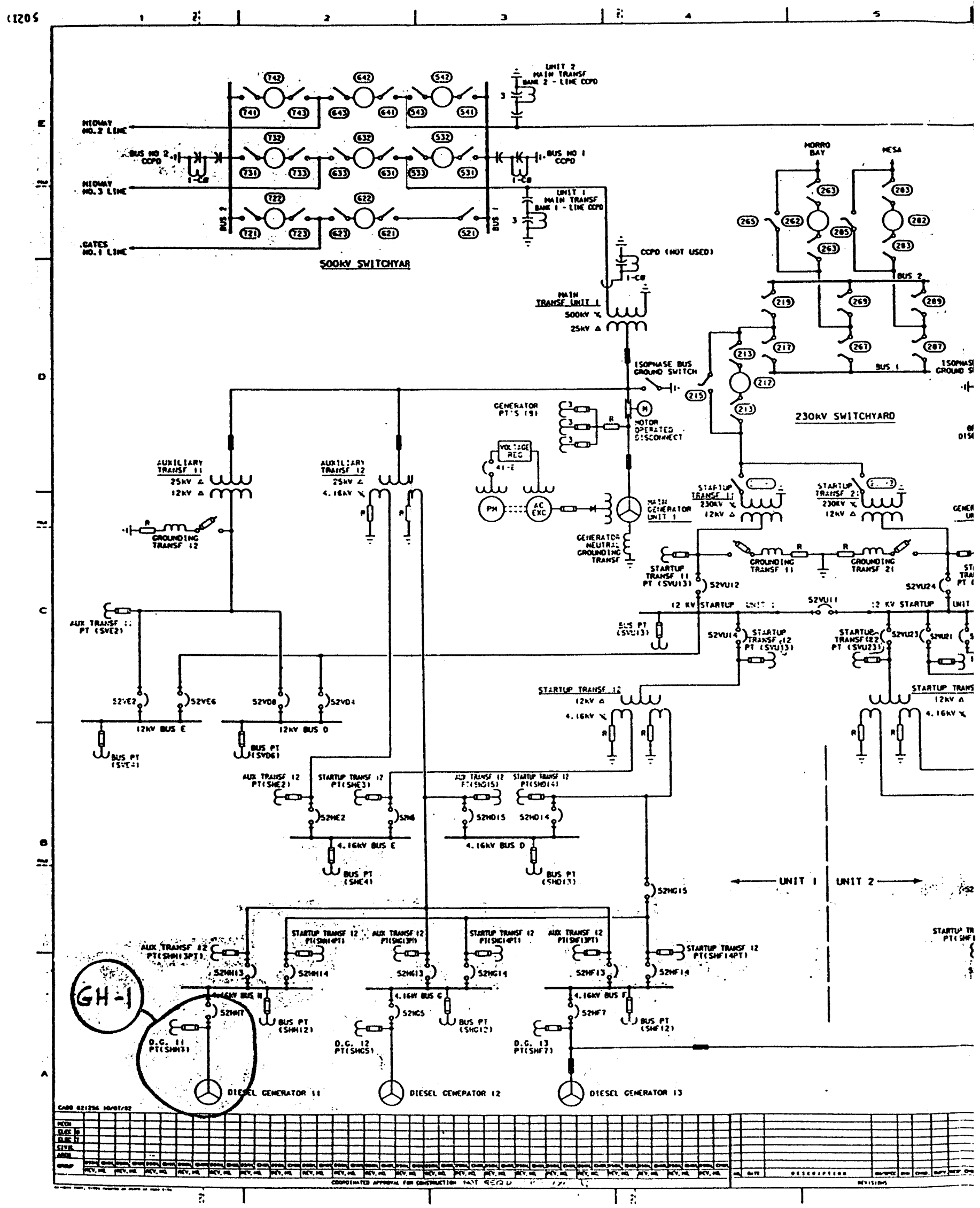




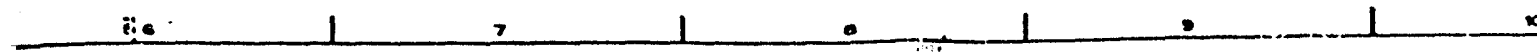

索

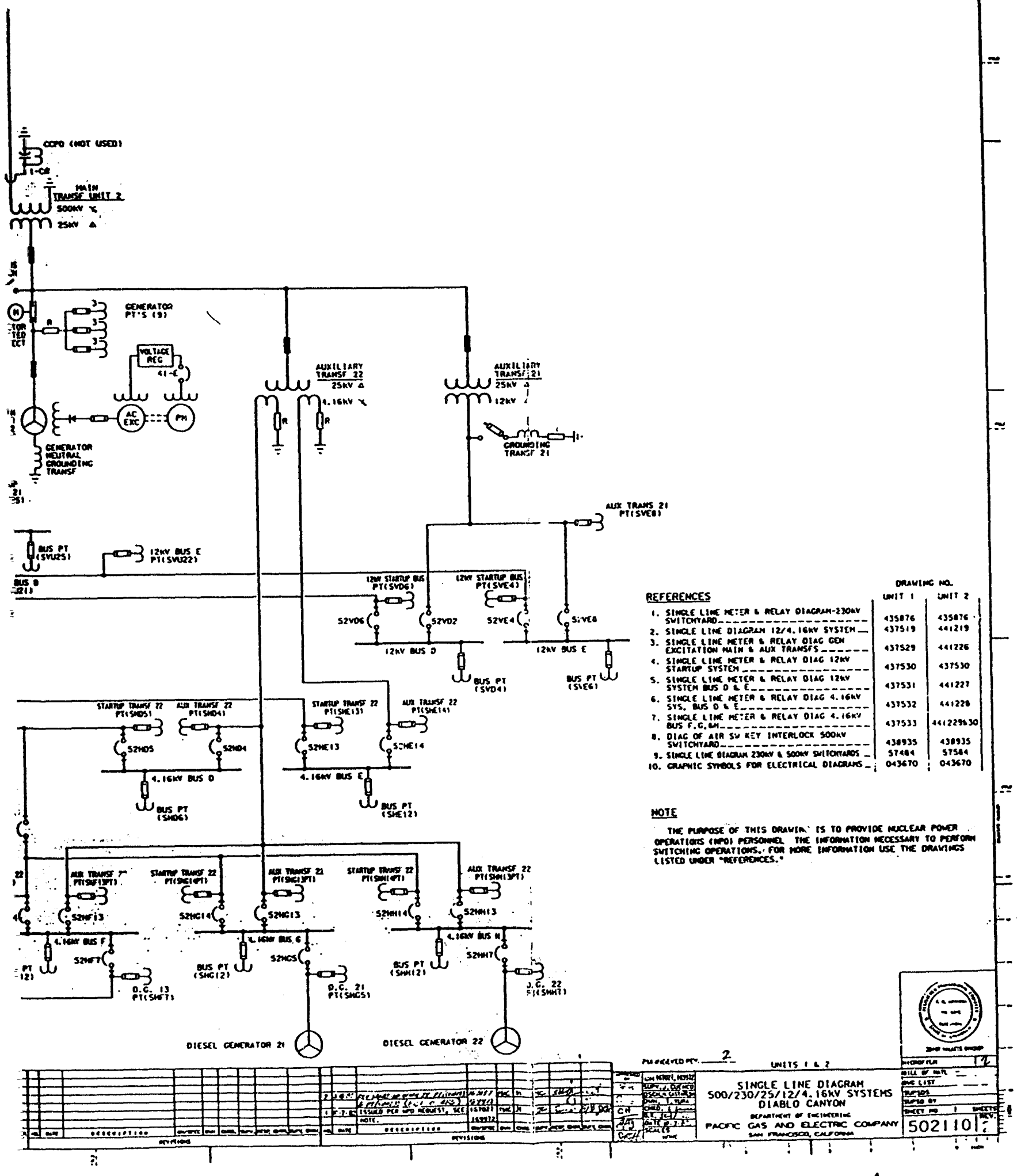

DIAGRAM D.2.1.5 - I 


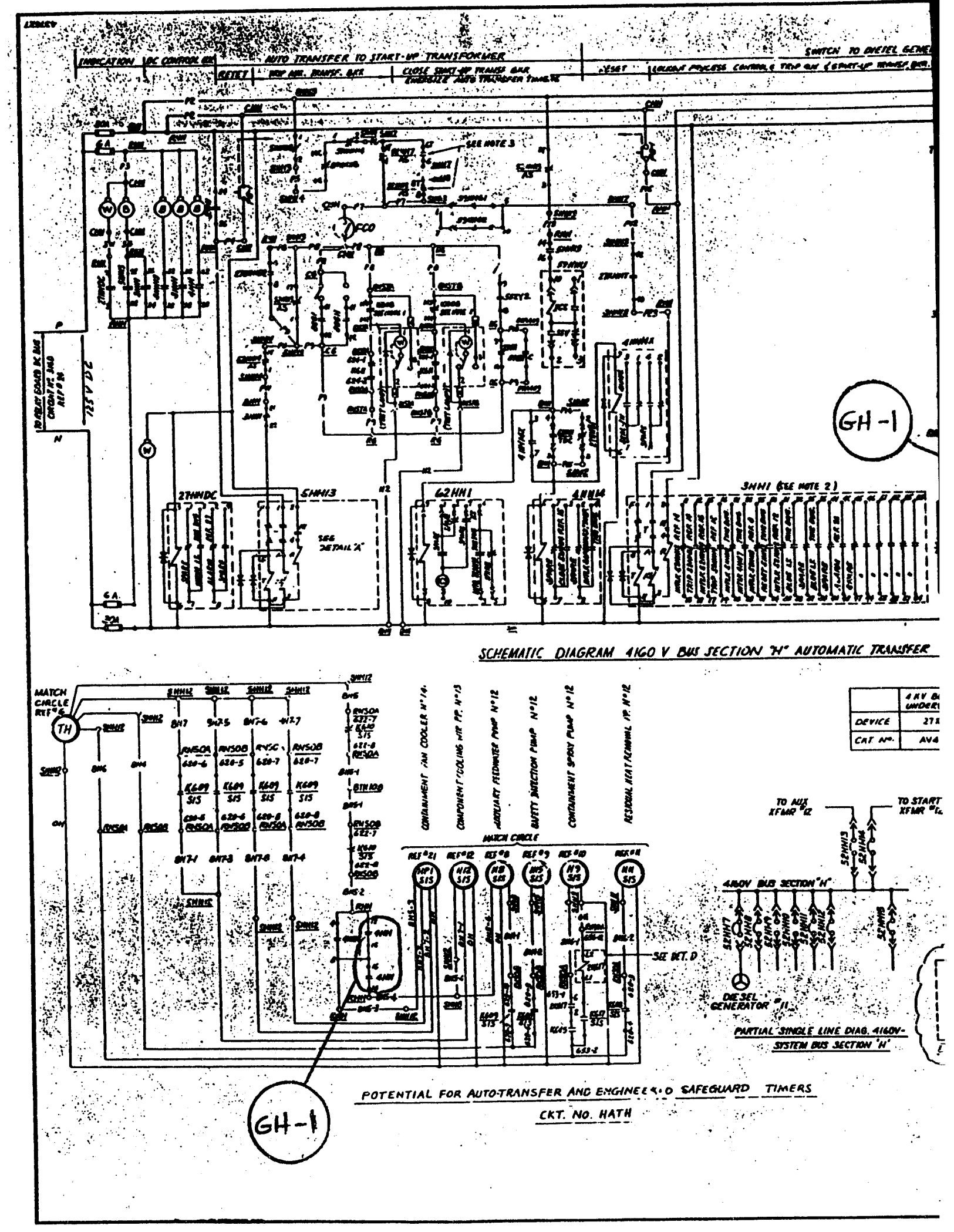

Dowre B1.2.3 Diesel Cewerntorn; Seperoomponents 


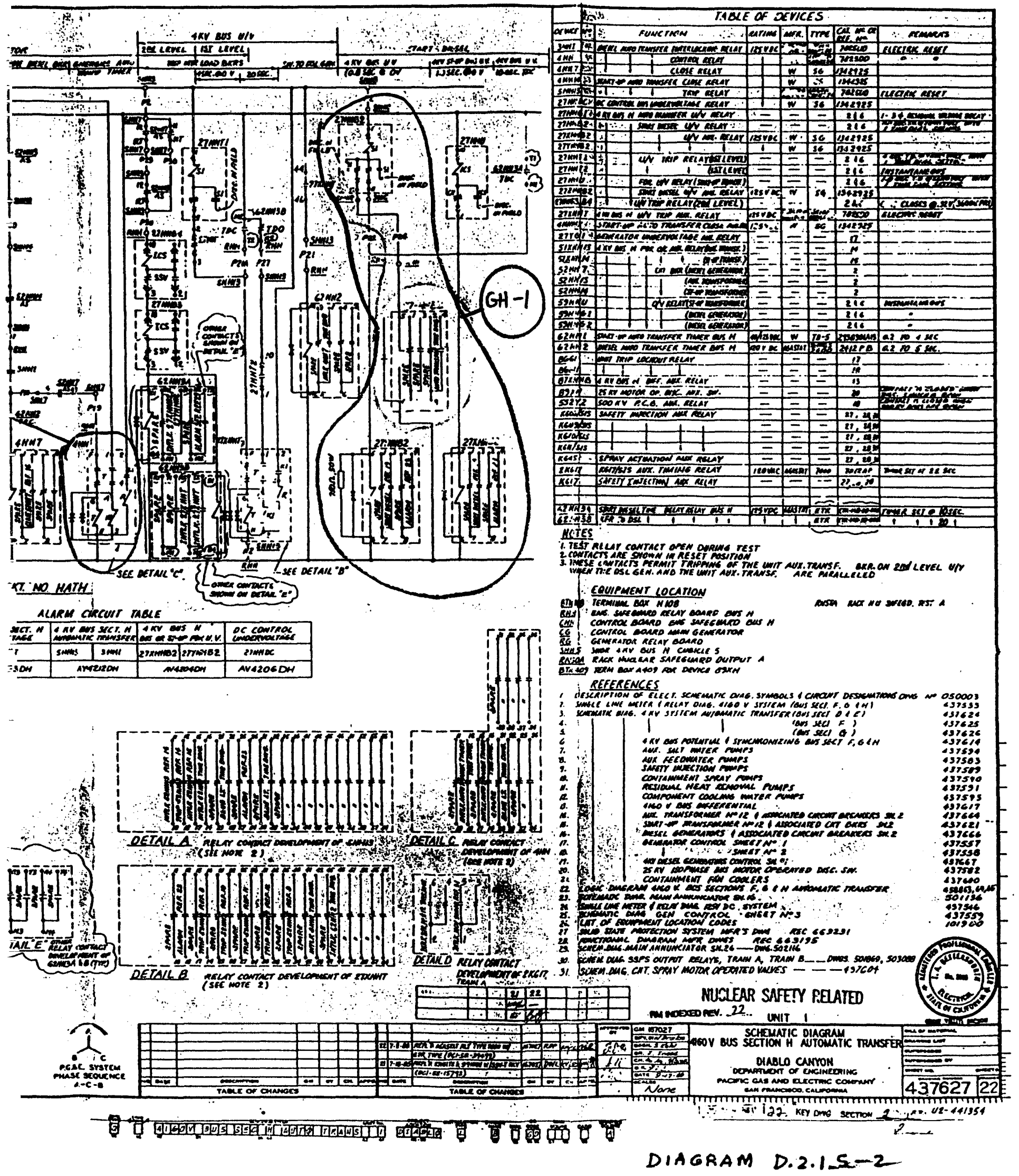




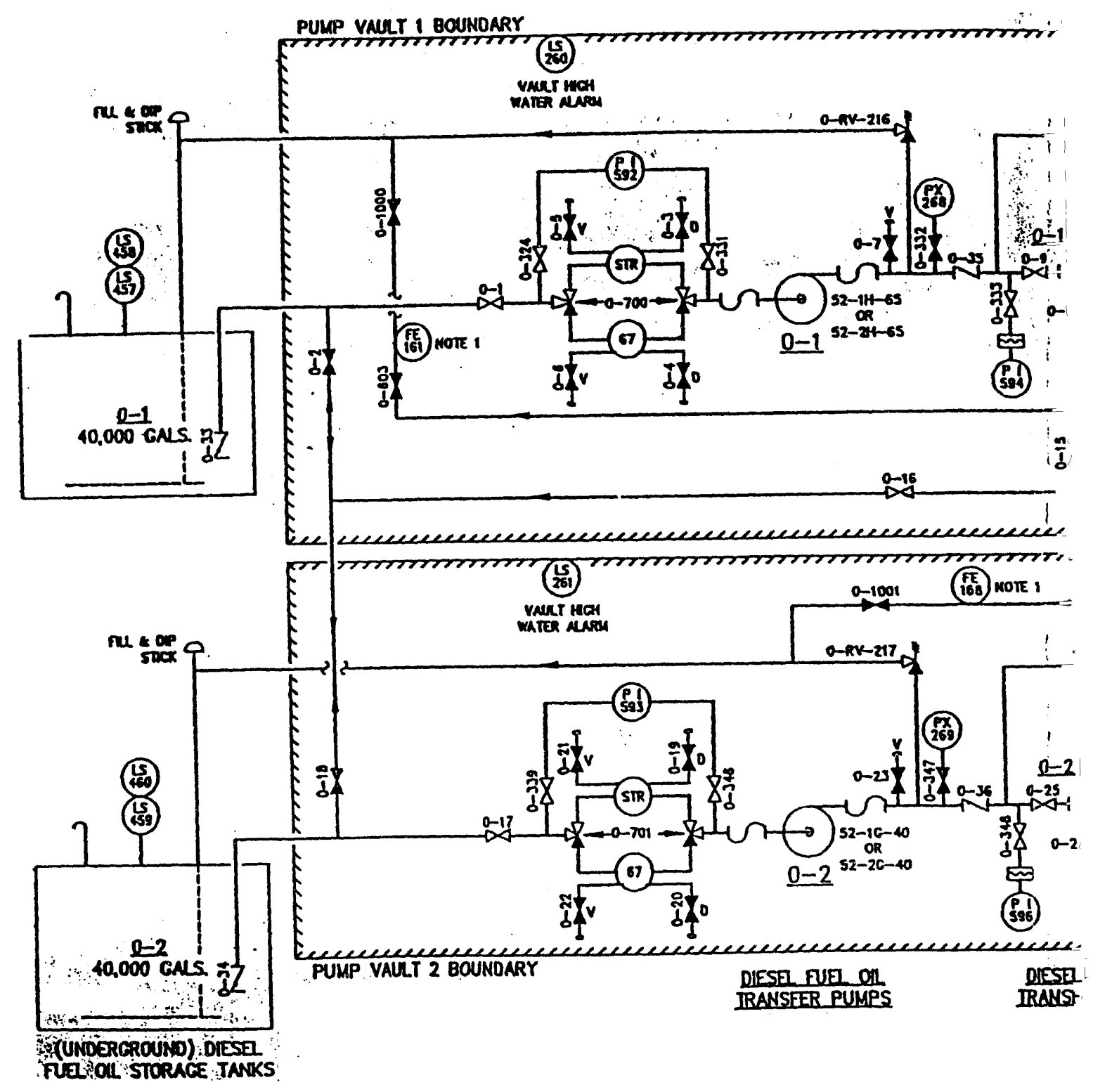

DIESEL FUEL OIL SYSI 


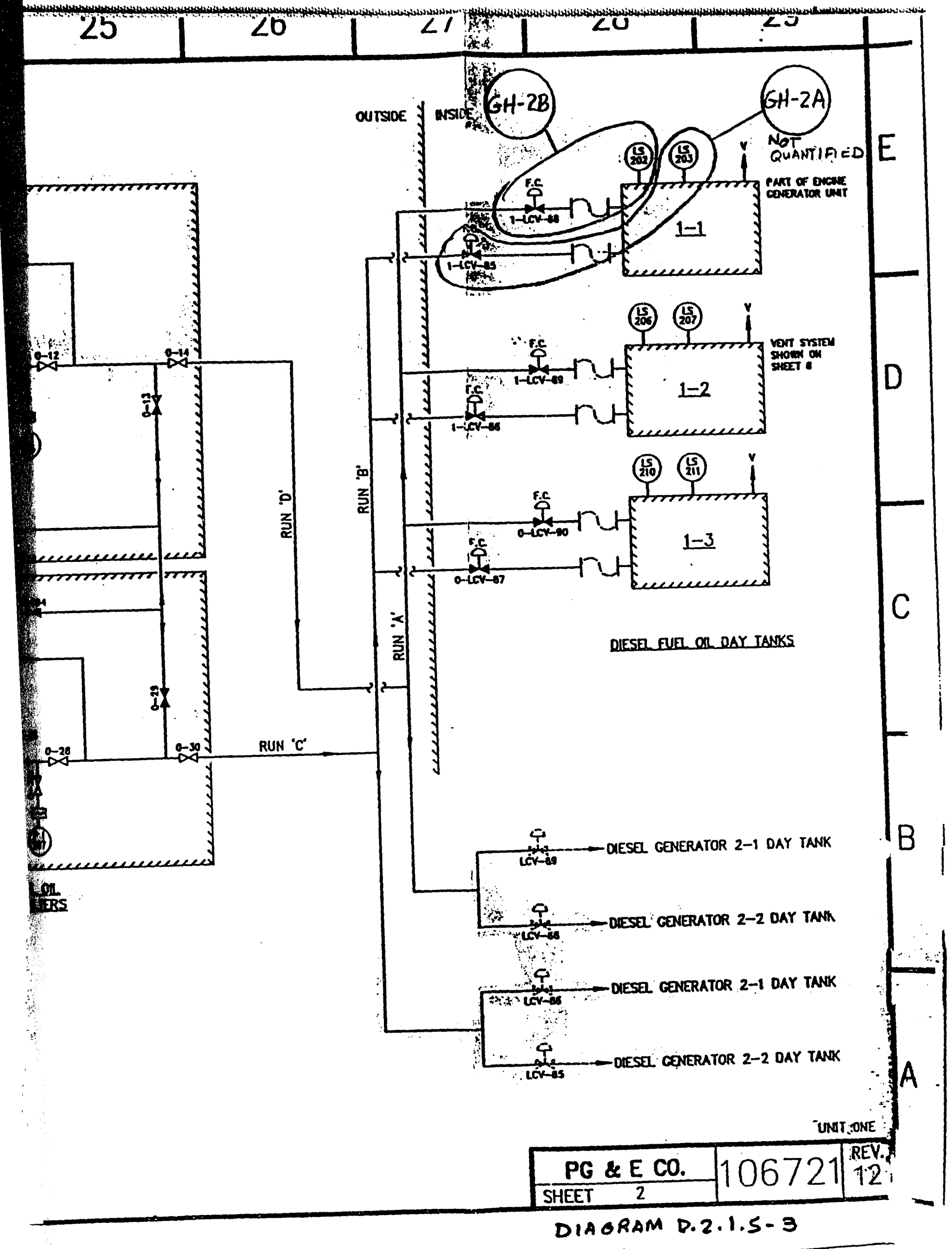




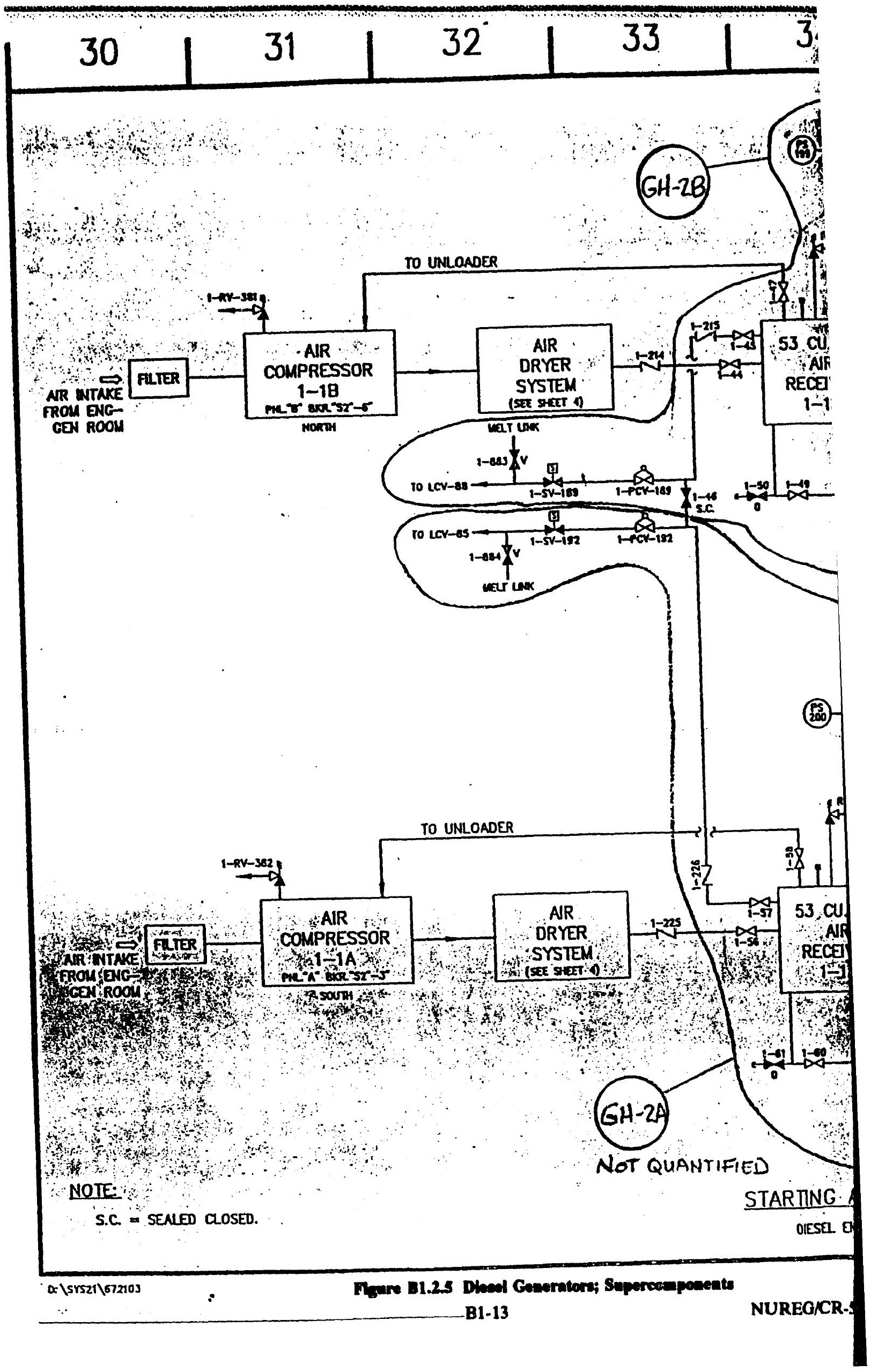




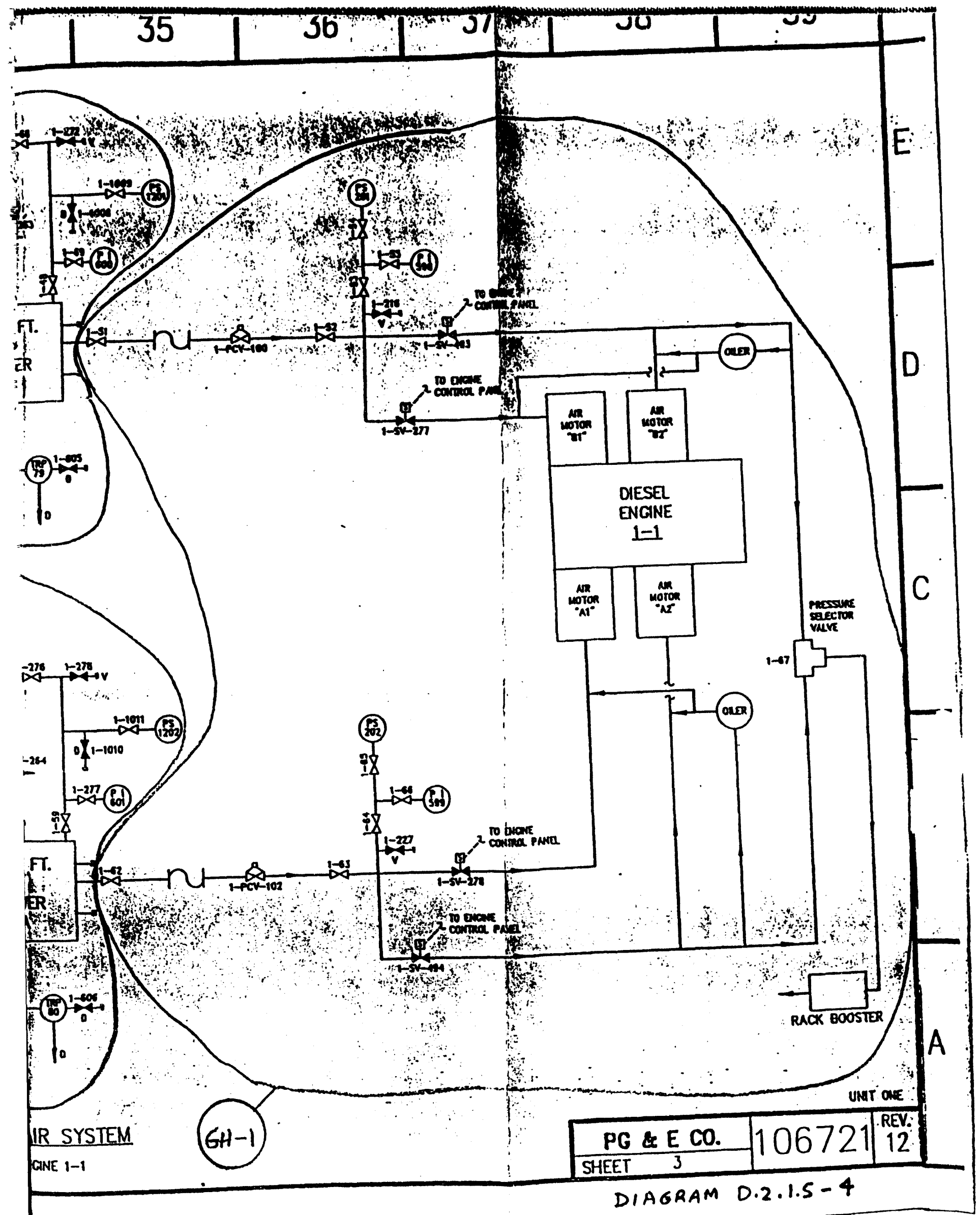




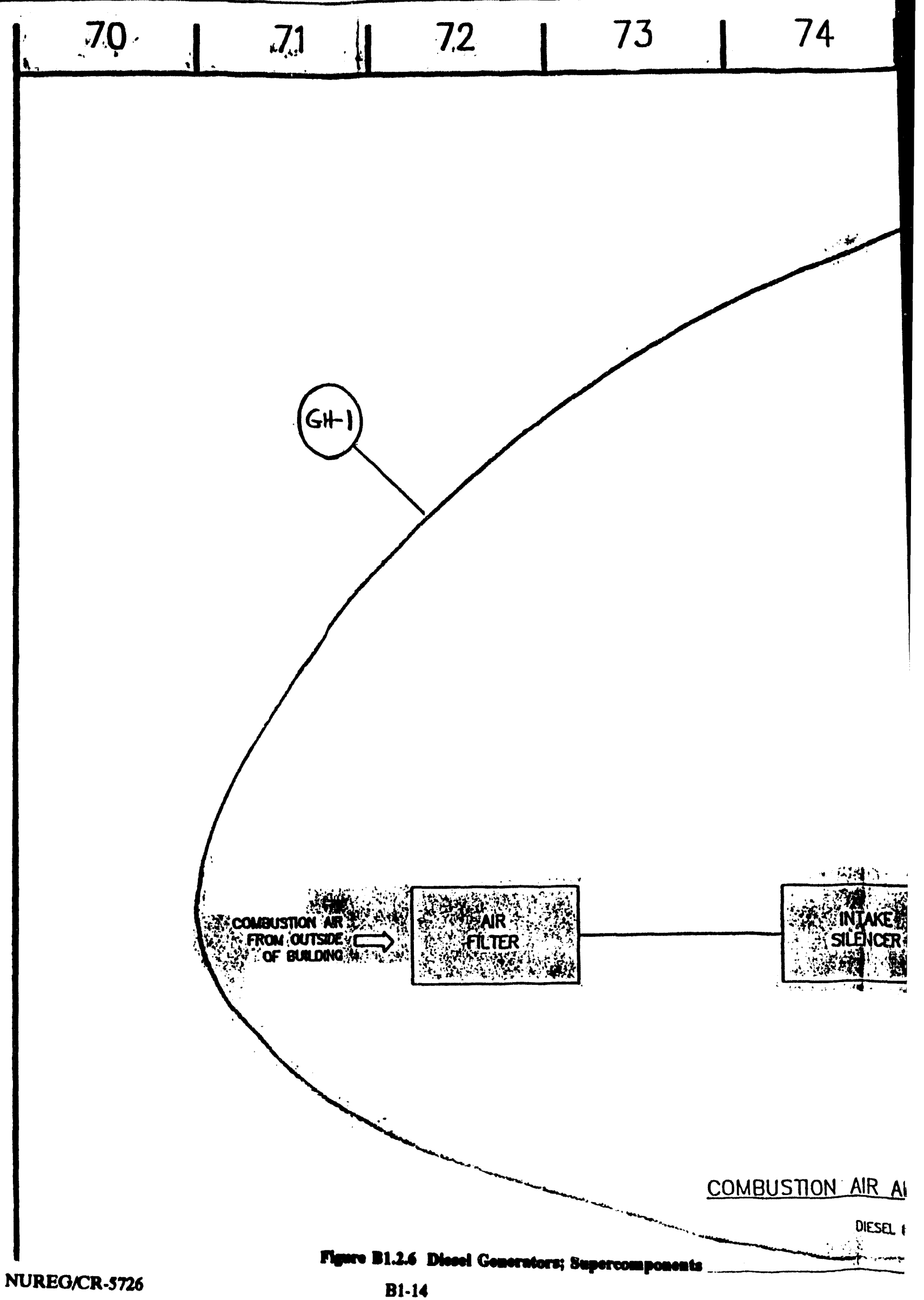




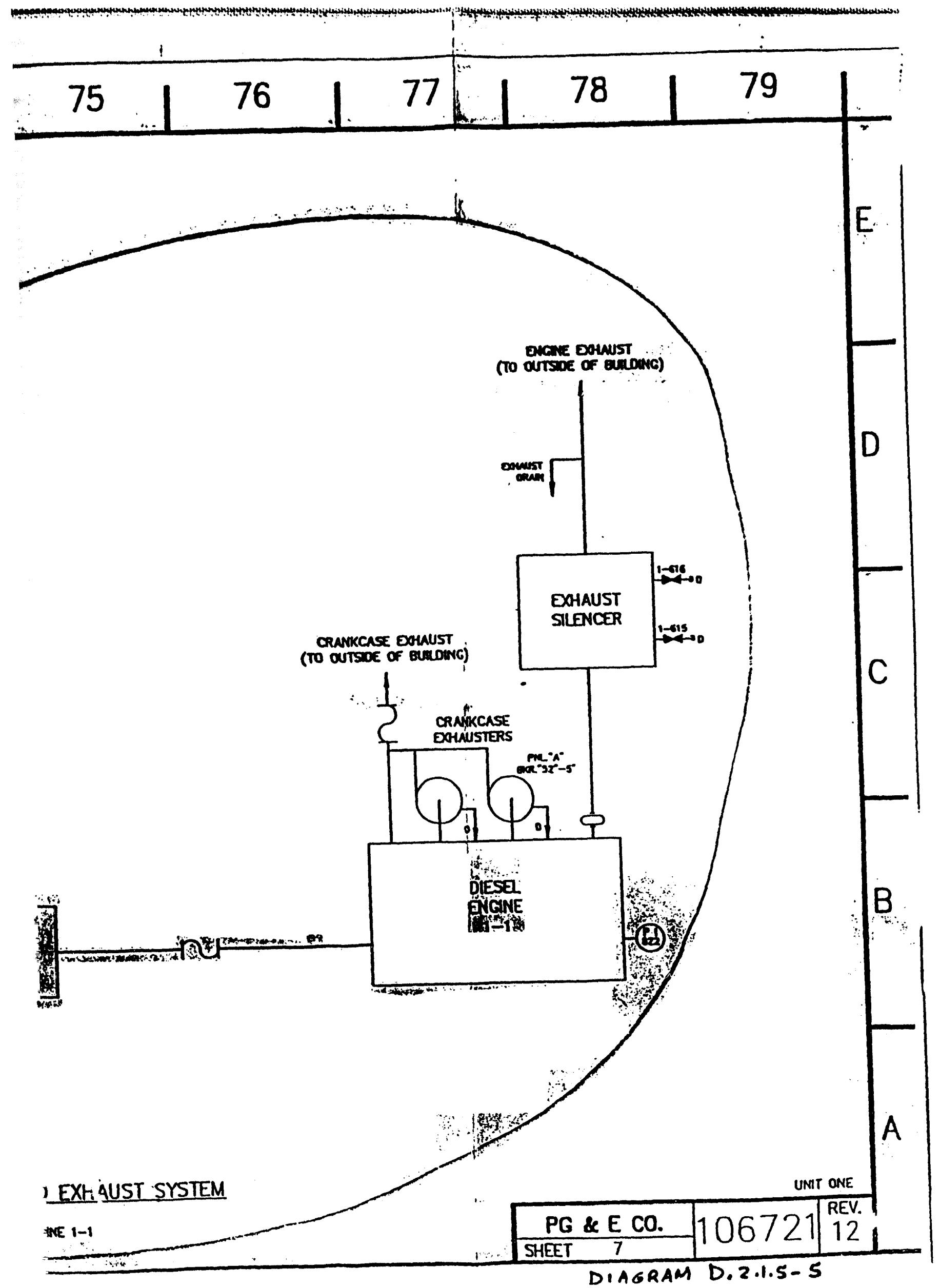




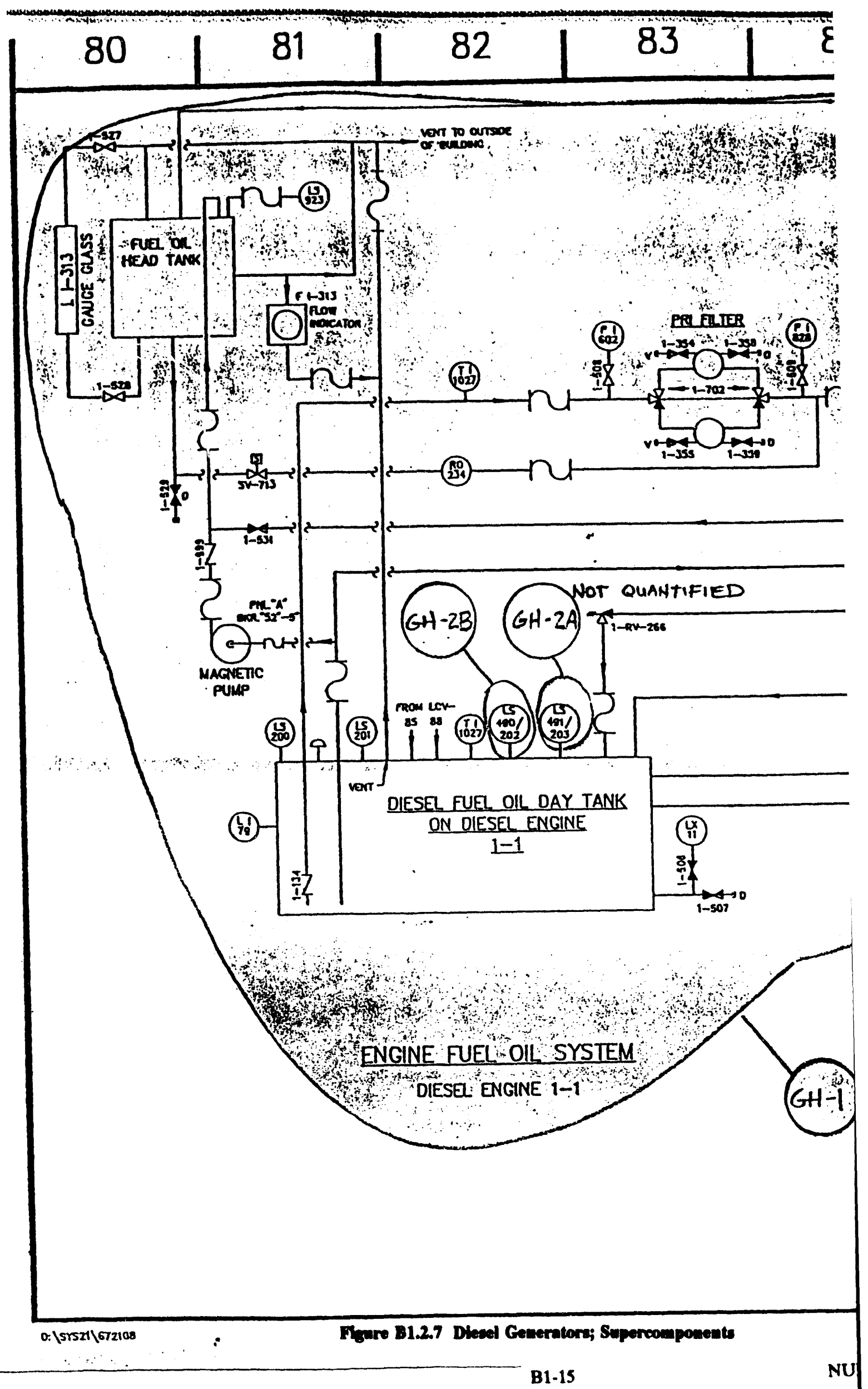




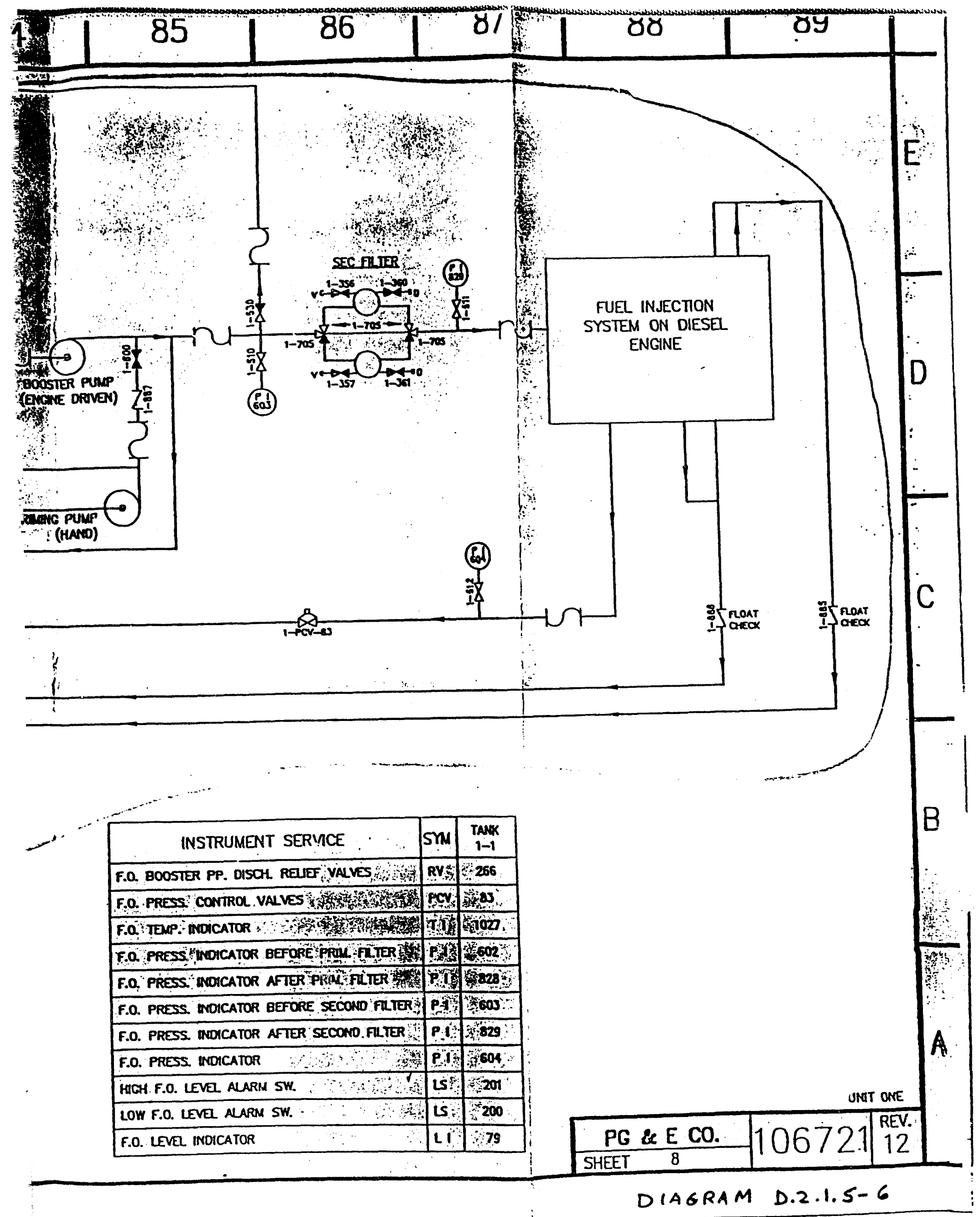




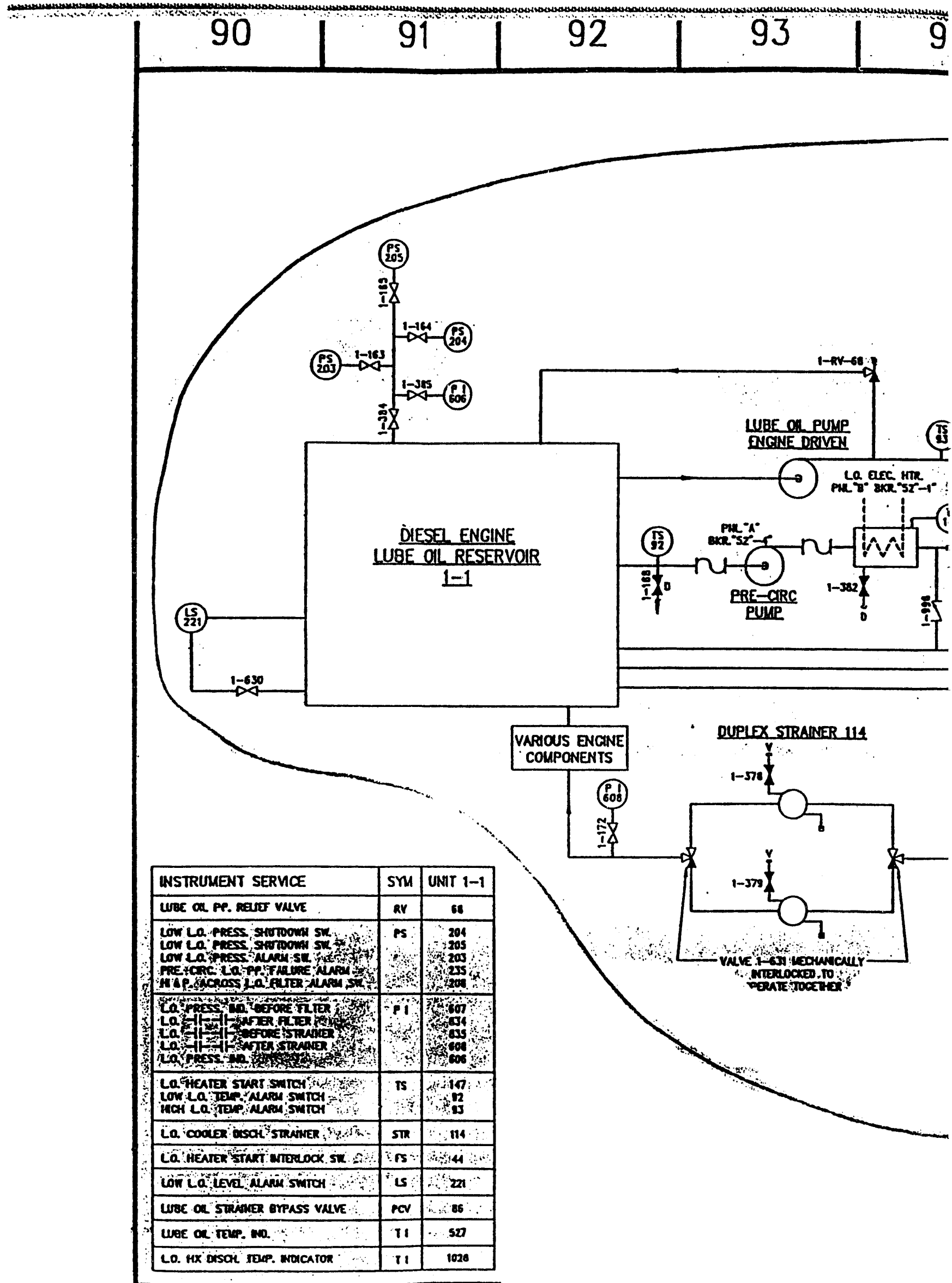




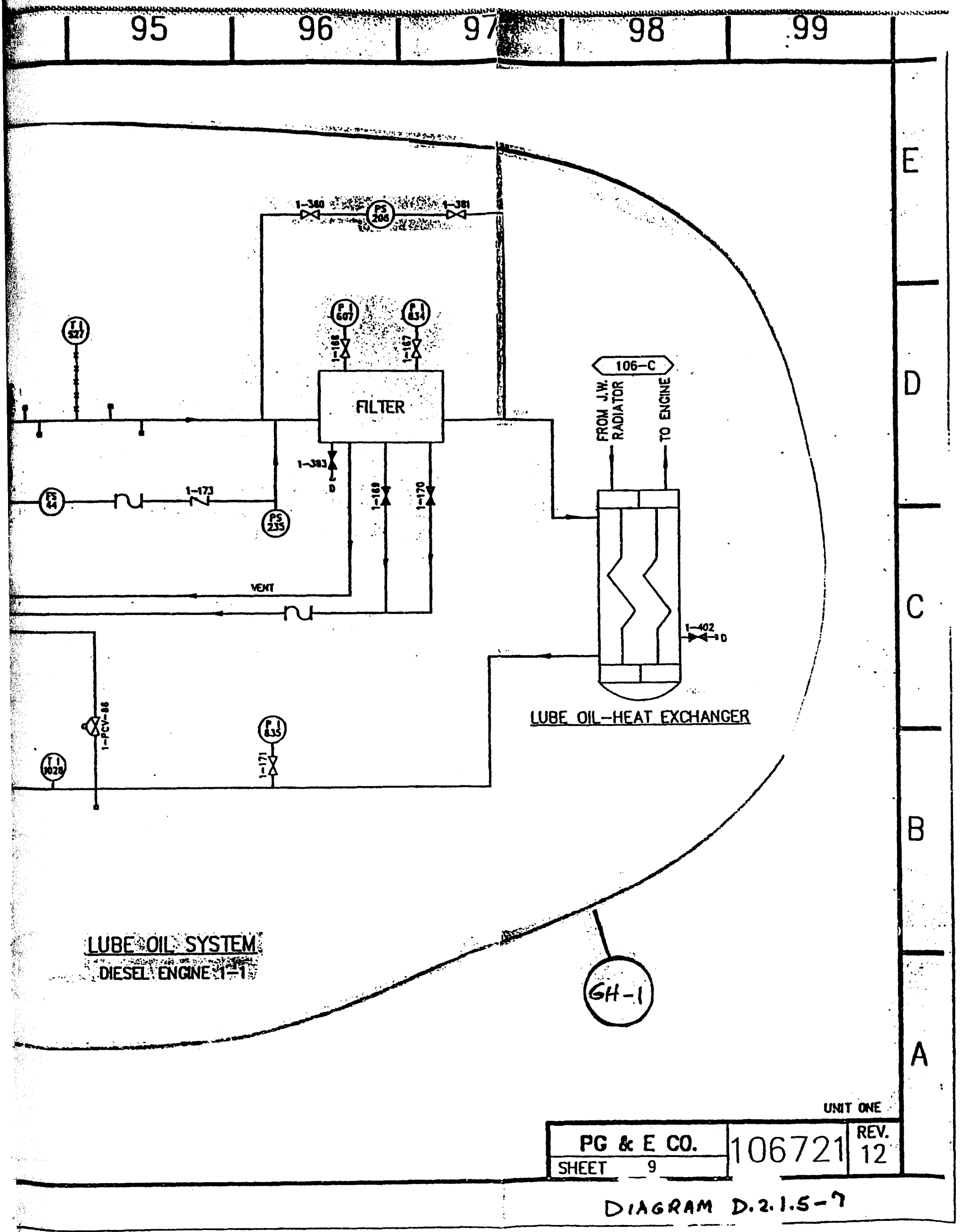




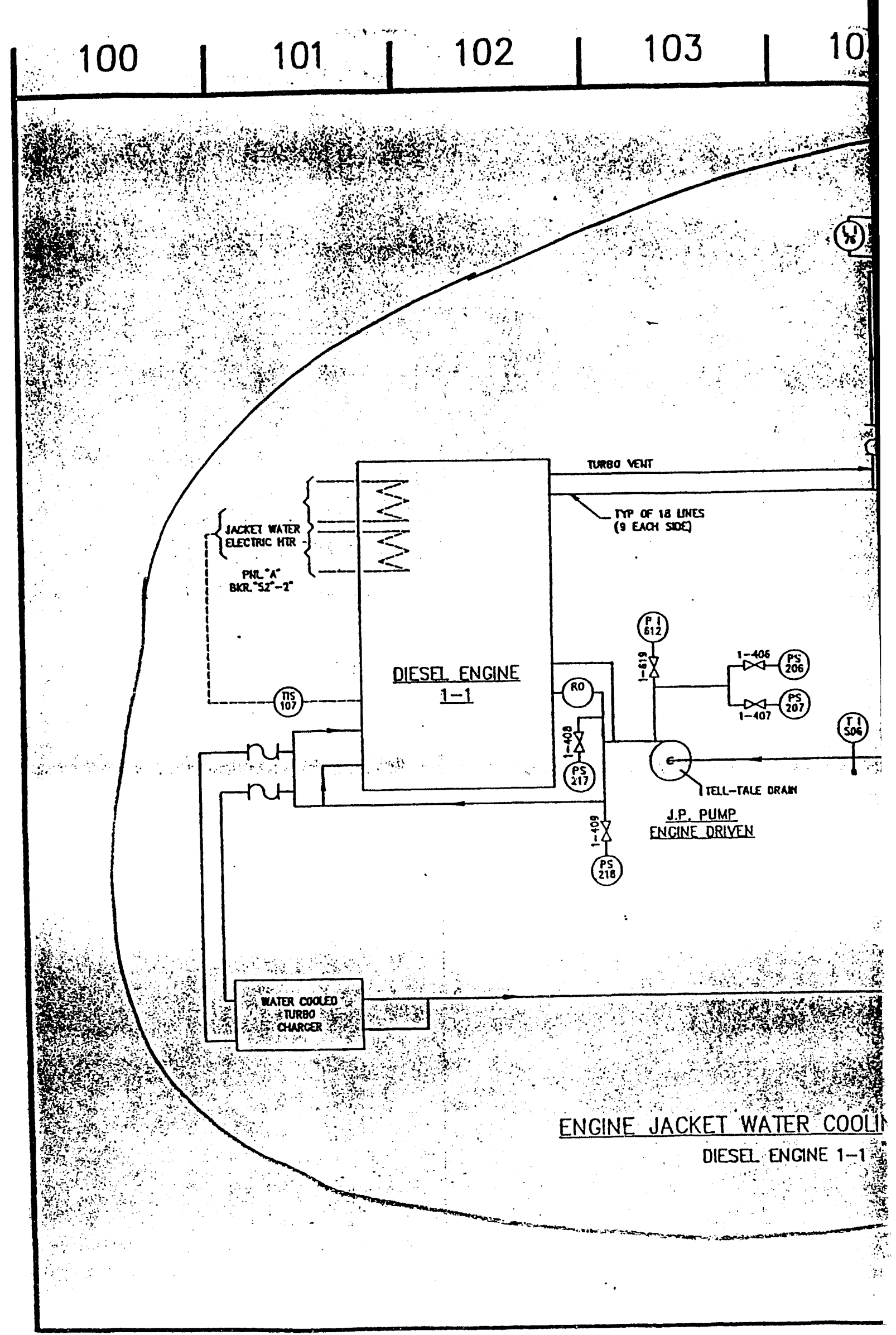

$0: \backslash 5 r \$ 21 \backslash 672110$

Fzure B1.2.9 Dievel Generntors; Supercomponents 


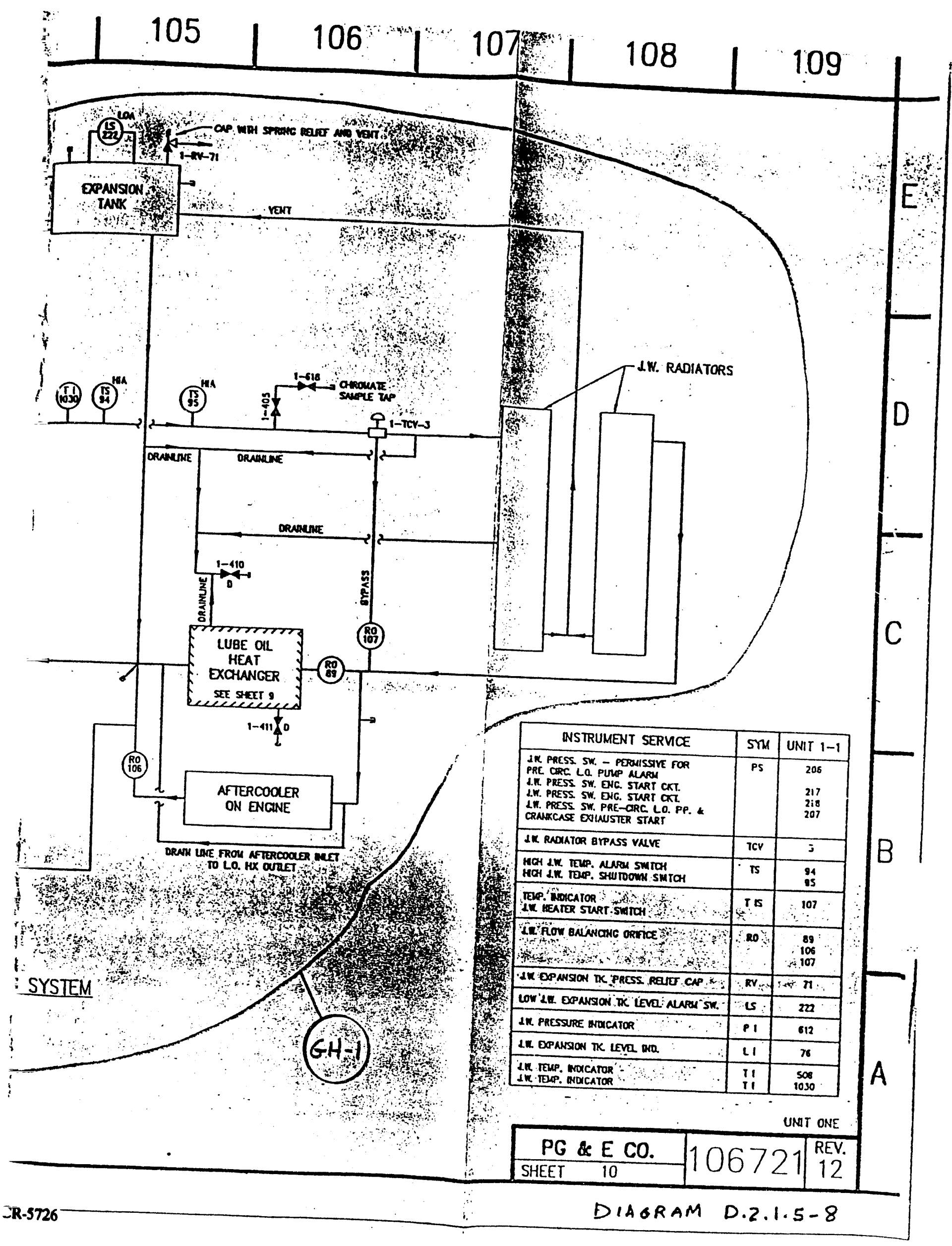




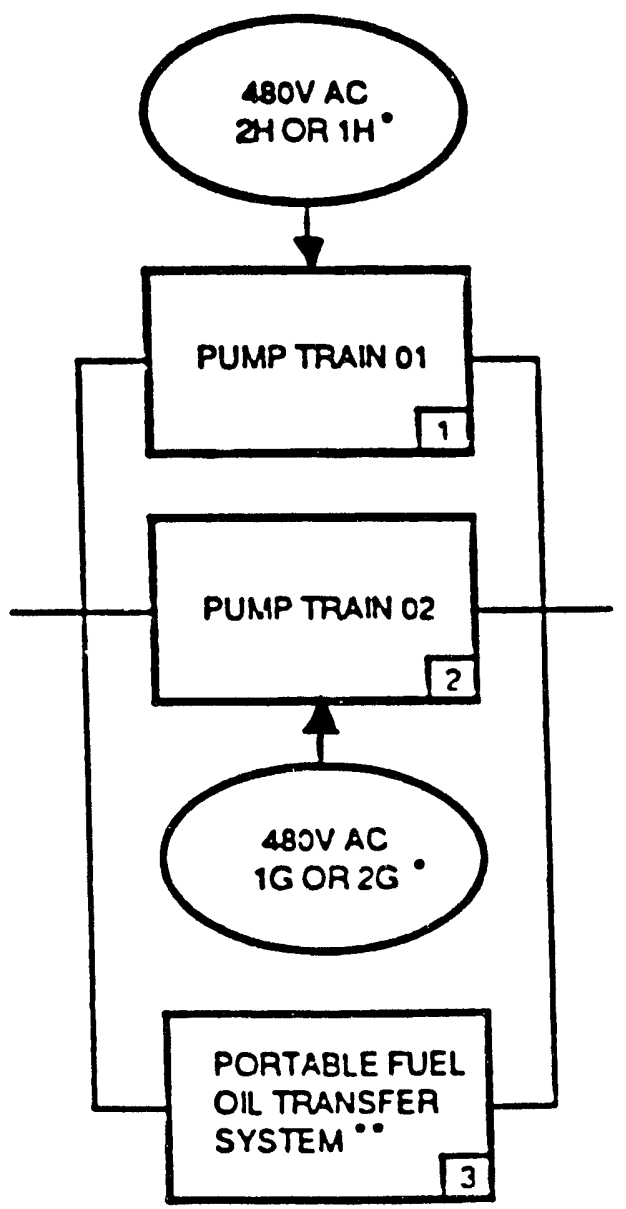

- backup power source

- the portable fuel oll transfer system is eVAluated IN THE ELECTRIC POWER RECOVERYMODEL

Figure B1.2.10 Reliability block diagram for the diesel fuel oil transfer system (Top Event, FO) 


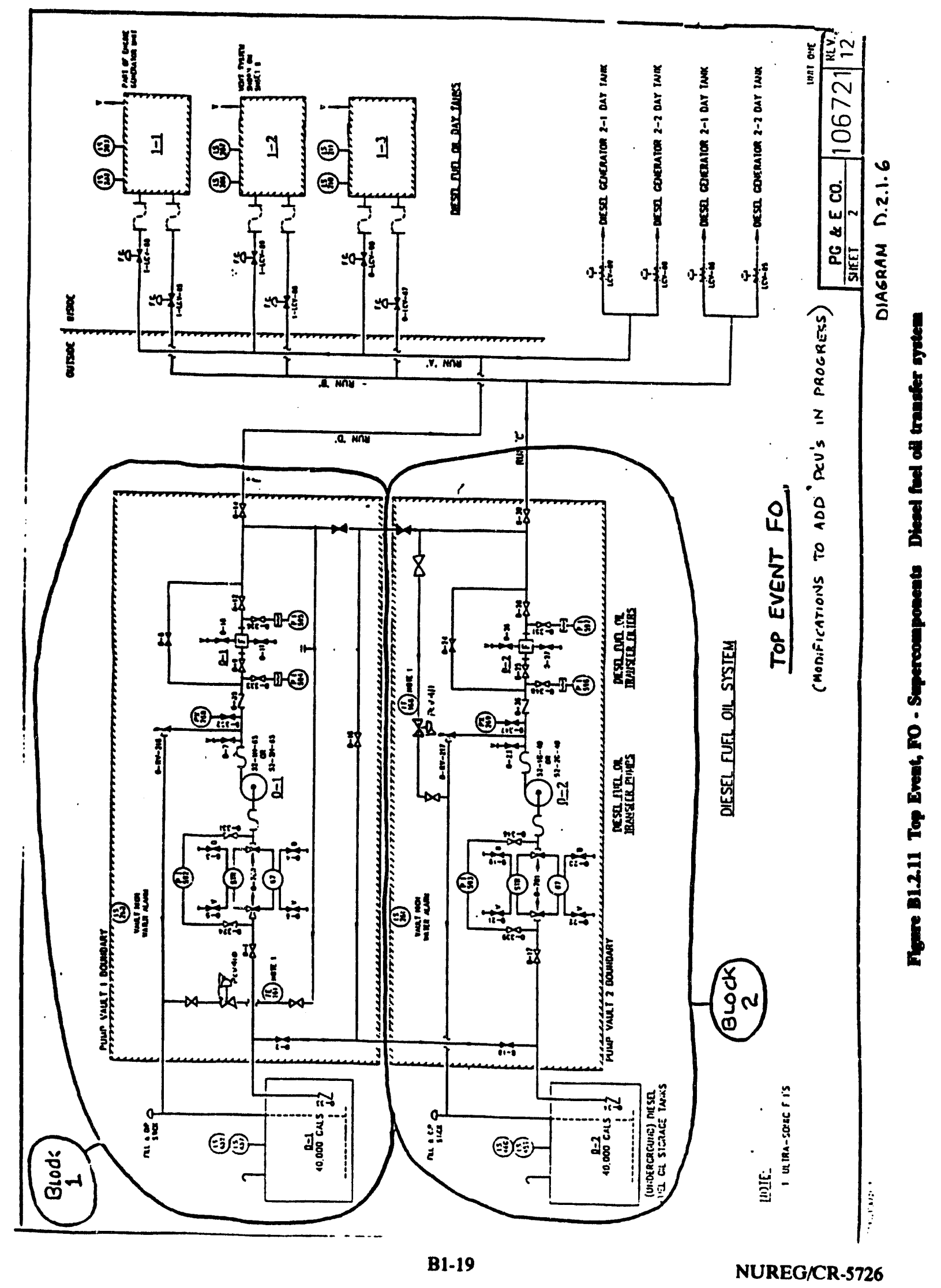


Table B1.2.1

\begin{tabular}{|c|c|c|}
\hline Bus & DG & Vital Safety-Related Loads \\
\hline $\mathbf{F}$ & 1-3 (Swing) & $\begin{array}{l}\text { Centrifugal Charging Pump No.1 } \\
\text { Safety Injection Pump No.1 } \\
\text { Containment Fan Cooler Unit No.2 } \\
\text { Containment Fan Cooler Unit No.1 } \\
\text { Component Cooling Water Pump No.1 } \\
\text { Auxiliary Saltwater Pump No.1 } \\
\text { Auxiliary Feedwater Pump No.3 }\end{array}$ \\
\hline $\mathbf{G}$ & $1-2(2-1)$ & $\begin{array}{l}\text { Centrifugal Charging Pump No.2 } \\
\text { Residual Heat Removal Pump No.1 } \\
\text { Containment Fan Cooler Unit No.3 } \\
\text { Containment Fan Cooler Unit No.5 } \\
\text { Component Cooling Water Pump No.2 } \\
\text { Auxiliary Saltwater Pump No.2 } \\
\text { Containment Spray Pump No.1 }\end{array}$ \\
\hline $\mathbf{H}$ & $1-1(2-2)$ & $\begin{array}{l}\text { Safety Injection Pump No.2 } \\
\text { Residual Heat Removal Pump No.2 } \\
\text { Containment Fan Cooler Unit No.4 } \\
\text { Component Cooling Water Pump No.3 } \\
\text { Auxiliary Feedwater Pump No.2 } \\
\text { Containment Spray Pump No.2 }\end{array}$ \\
\hline
\end{tabular}


Table B1.2.2

Top Event Definition and Success Criteria

Diesel Generator and Diesel Fuel Transfer Systems

Top Event
Designator

GF

GG

GH

2G

2H

SW

Swing diesel alignment.

DG13 is normally aligned to Unit 1.

FO

Diesel fuel oil transfer system provides fuel oil for each of the DGs for six hours (24 hours for seismic events).

\section{Top Event Success Criteria}

$F_{1,2}$ Each top event is successful if the $G_{1} \quad$ corresponding DG starts on undervoltage $\mathrm{H}_{1} \quad$ signal from its bus, takes bus loads and $G_{2}$ continues powering loads for the $\mathrm{H}_{2}$ appropriate mission times (6 hours or 24 hours).

The value of SW determines whether DG13 goes to Unit 2. A value of 0 indicates it does not, a value between 0 and 1 represents the probability that it does.

One of two pumps starts on low day tank level and refills each day tank for the period that each diesel operates.

\section{FSAR Success Criteria:}

Any two of three DGs and their associated buses are adequate to serve the vital loads necessary for safe shutdown of a single unit (although one DG may supply power to two vital buses at the same time, no credit is currently given this mode of operation).

The diesel fuel oil transfer system must remain operable and deliver fuel to each of the DGs for the time the DGs are required to operate. There must be enough fuel in storage tanks for seven days of power generation.

\section{Technical Specifications:}

With a single DG inoperable, demonstrate the operability of the remaining ac sources within 24 hours. Restore the diesel within 72 hours.

With two DGs inoperable, demonstrate the operability of the two offsite ac circuits (one 230kV and one $500 \mathrm{kV}$ line) within one hour and at least once every eight hours. Restore at least two of the inoperable diesels within two hours. 
Appendix B1

Table B1.2.3

Boundary Condition and Split Fraction Identifications

for Top Events GF, GG, GH, 2G, 2H, and SW

Top

Diesel Conditions

$\begin{array}{lllllll}\text { Event } & \text { Case } & 13 & 12 & 11 & 21 & 22\end{array}$

Comments

GF = Unavailability of DG13 under the following conditions:

GF1

All support available.

GG = Unavailability of DG12 under the following conditions:

$\begin{array}{ll}\text { GG1 } & 0 \\ \text { GG2 } & 1 \\ \text { GG3 } & -\end{array}$

Offsite grid succeeded, GF succeeded.

Offsite grid succeeded, GF failed.

Offsite grid succeeded, GF bypassed (not demanded)

GH = Unavailability of DG11 under the following conditions:

$\begin{array}{lll}\text { GH1 } & 0 & 0 \\ \text { GH2 } & 0 & 1 \\ \text { GH3 } & 1 & 1 \\ \text { GH4 } & 0 & - \\ & & \\ \text { GH5 } & 1 & - \\ \text { GH6 } & - & -\end{array}$

Offsite grid succeeded, both GF, GG succeeded. Offsite grid succeeded, GF-S/F, GG-F/S (two possible combinations).

Offsite grid succeeded, both GF, GG failed.

Offsite grid succeeded, GF-S/B, GG-B/S (two possible combinations).

Offsite grid succeeded, GF-F/B, GG-B/F (two possible combinations).

Offsite grid succeeded, both GF, GG bypassed.

2G = Unavailability of DG21 under the following conditions:

2G1 0000

$2 G 2 \quad 0 \quad 0 \quad 1$

$2 G 3 \quad 0 \quad 1 \quad 1$

$2 \mathrm{G} 4 \quad 1 \quad 1 \quad 1$

26500.

Offsite grid failed, all GF, GG, and GH succeeded.

Offsite grid failed, two of GF, GG, and GH succeeded, the third failed (three possible combinations).

Offsite grid failed, two of GF, GG, GH failed, the third succeeded (three possible combinations).

Offsite grid failed, all GF, GG, GH failed.

Offsite grid failed, two of GF, GG, GH succeeded, the third bypassed (three possible combinations). 
Appendix B1

Table B1.2.3 (continued)

Top

Diesel Conditions

Event

$\begin{array}{llllll}\text { Case } & 13 & 12 & 11 & 21 & 22 \\ 2 G 6 & 0 & 1 & - & & \\ 2 G 7 & 1 & 1 & - & \\ 2 G 8 & 0 & - & - \\ 2 G 9 & 1 & - & -\end{array}$

\section{Comments}

Offsite grid failed, one of GF, GG, GH succeoded, one failed, the third bypassed (six possible combinations).

Offsite grid failed, two of GF, GG, GH failed, the third bypassed (three possible combinations). Offaite grid failed, one of GF, GO, OH succeeded, the other two bypassed (three possible combinations).

Offsite grid failed, one of GF, GG, GH failed, the other two bypassed (three possible combinations). 2GA-.-Offsite grid failed, all of GF, GG, GH bypassed.

$2 H=$ Unavailability of DG22 under the following conditions:

$\begin{array}{lllll}2 \mathrm{H} 1 & 0 & 0 & 0 & 0 \\ 2 \mathrm{H} 2 & 1 & 0 & 0 & 0 \\ & & & & \\ 2 \mathrm{H} 3 & 1 & 1 & 0 & 0 \\ & & & & \\ 2 \mathrm{H} 4 & 1 & 1 & 1 & 0 \\ & & & & \\ 2 \mathrm{H} 5 & 1 & 1 & 1 & 1 \\ 2 \mathrm{H} 6 & 0 & 0 & 0 & - \\ & & & & \\ 2 \mathrm{H} 7 & 0 & 0 & 1 & - \\ & & & & \end{array}$

Offsite grid failed, all of GF, GG, GH, $2 G$ succeodod.

Offsite grid failed, one of GF, GG, GH, $2 G$ failed, the other three succeeded (four possible combinations).

Offsite grid failed, two of GF, GG, GH, $2 G$ failed, the other two succeeded (six possible combinations).

Offsite grid failed, three of GF, GG, GH, $2 G$ failed, the fourth succeeded (four possible combinations).

Offsite grid failed, all of GF, GG, GH, $2 G$ failed. Offsite grid failed, three of GF, GG, GH, 2G succeeded, the fourth bypassed (four possible combinations).

Offsite grid failed, two of GF, GG, GH, 20 succeeded, one failed, the fourth bypassed (12 possible combinations).

Offsite grid failed, two of GF, GG, GH, $2 G$ failed, one succeeded, the fourth bypassed (12 possible combinations). 
Appendix B1

Table B1.2.3 (continued)

Top

Event

Diesed Conditions

$\begin{array}{llllll}\text { Cnec } & 13 & 12 & 11 & 21 & 22 \\ 2 H 9 & 1 & 1 & 1 & - \\ 2 H A & 0 & 0 & - & - \\ 2 H B & - & - & 1 & 0 \\ 2 H C & - & - & 1 & 1 \\ 2 H D & - & - & - & 0 \\ 2 H E & - & - & - & 1\end{array}$

$2 \mathrm{HO}$

sw . swo

SW1

SW2

SW3

Offsite grid failed, three of GF, GG, GH, 20

failed, the fourth bypassed (four possible combinations).

Offsite grid failed, two of GF, GG, GH, 20 succeeded, the other two bypassed (six possible combinations).

Offsite grid failed, two of GF, GG, GH, 20 bypessed, one failed, the fourth succeoded (12 possible combinations).

Offsite grid failed, two of GF, GG, GH, 2G bypassed, the other two failed (six possible combinations).

Offsite grid failed, three of GF, GG, GH, 2G bypassed, the fourth succeeded (four possible combinations).

Offsite grid failed, three of GF, GG, GH, 20 bypassed, the fourth failed (four possible combinations).

Offsite grid failed, all of GF, GG, GH, $2 G$ bypessed.

LOCA, the swing diesel locked to the Unit 1. LOSP, with equal chance for swing diesel to operate on each unit.

LOSP, with more DGs aligned to Unit 2 than Unit 1.

LOSP, with more DGs aligned to Unit 1 than Unit 2.

Notes: $0=$ Succeoded

1 = Failed

- = Bypassed 
Table B1.2.4

Diesel Fuel Oil Transfer System Boundary

Conditions for Top Event, LO

\section{Split Fraction ID}

\begin{tabular}{|c|c|}
\hline FO1 & All support available. \\
\hline FO2 & Support available to one train only. \\
\hline FO3 & $\begin{array}{l}1 / 2 \text { normal support available; recover support to the other train by } \\
\text { realignment to beckup support. }\end{array}$ \\
\hline FO4 & $\begin{array}{l}2 / 2 \text { normal support unavailable; recover supports by realignment to } \\
\text { backups. }\end{array}$ \\
\hline FO5 & $\begin{array}{l}2 / 2 \text { normal supports unavailable; recover only } 1 / 2 \text { backup support by } \\
\text { realignment. }\end{array}$ \\
\hline 06 & All support unavailable (guaranteed failure). \\
\hline
\end{tabular}


Appendix B1

Table 21.2.5

Unavallability Valwes (Conditional Split Fractions) for the

Dised Gesernter System

\begin{tabular}{|c|c|c|c|c|c|c|c|c|c|c|}
\hline $\begin{array}{l}\text { Top } \\
\text { Event }\end{array}$ & Case & Calc. & CSF & TTL & KW & HWI & HWD & TS & $\mathbf{M N}$ & $\mathrm{HE} \stackrel{\text { Coment }}{*}$ \\
\hline $\mathbf{G F}$ & GF1 & $\begin{array}{l}\text { PGGE } \\
\text { BNL }\end{array}$ & $\begin{array}{l}4.523-2 \\
4.571-2\end{array}$ & $\begin{array}{l}4.554-2 \\
4.603-2\end{array}$ & $\begin{array}{l}3.703-2 \\
3.754-2\end{array}$ & $\begin{array}{l}3.689-2 \\
3.695-2\end{array}$ & $\begin{array}{l}1.393-4 \\
5.860-4\end{array}$ & $\begin{array}{l}2.950 .4 \\
2.934 .4\end{array}$ & $\begin{array}{l}8.217-3 \\
8.198-3\end{array}$ & .. \\
\hline \multirow[t]{3}{*}{ GG } & GG1 & $\begin{array}{l}\text { PGSE } \\
\text { BNL }\end{array}$ & $\begin{array}{l}4.477-2 \\
4.527-2\end{array}$ & $\begin{array}{l}4.554-2 \\
4.603-2\end{array}$ & as GFI & & & & & \\
\hline & GG2 & $\begin{array}{l}\text { PGSE } \\
\text { BNL }\end{array}$ & $\begin{array}{l}5.561-2 \\
5.474-2\end{array}$ & $\begin{array}{l}2.702-3 \\
2.540-3\end{array}$ & $\begin{array}{l}1.749 .3 \\
1.581 .3\end{array}$ & $\begin{array}{l}1.536-3 \\
1.366-3\end{array}$ & $\begin{array}{l}2.129-4 \\
2.149-4\end{array}$ & $\begin{array}{l}4.989 .5 \\
4.980-5\end{array}$ & $\begin{array}{l}9.025-4 \\
9.089-4\end{array}$ & $\begin{array}{l}- \\
\cdots\end{array}$ \\
\hline & G63 & $\begin{array}{l}\text { PGSE } \\
\text { BNL }\end{array}$ & $\begin{array}{l}4.523-2 \\
4.571-2\end{array}$ & $\begin{array}{l}4.554-2 \\
4.603-2\end{array}$ & as GF1 & & & & & \\
\hline \multirow[t]{6}{*}{ GH } & GH1 & $\begin{array}{l}\text { PGSE } \\
\text { BNL }\end{array}$ & $\begin{array}{l}4.436-2 \\
4.490-2\end{array}$ & $\begin{array}{l}4.554-2 \\
4.603-2\end{array}$ & as GF1 & & & & & \\
\hline & GH2 & $\begin{array}{l}\text { PGQE } \\
\text { BNL }\end{array}$ & $\begin{array}{l}5.408-2 \\
5.322-2\end{array}$ & $\begin{array}{l}2.702-3 \\
2.540-3\end{array}$ & as GG2 & & & & & \\
\hline & GH3 & $\begin{array}{l}\text { PGEE } \\
\text { BNL }\end{array}$ & $\begin{array}{l}8.265-2 \\
8.097-2\end{array}$ & $\begin{array}{l}2.339-4 \\
2.066-4\end{array}$ & $\begin{array}{l}1.264-4 \\
1.034-4\end{array}$ & $\begin{array}{l}7.438-5 \\
5.057-5\end{array}$ & $\begin{array}{l}5.204-5 \\
5.284-5\end{array}$ & $\begin{array}{l}3.173-5 \\
3.128-5\end{array}$ & $\begin{array}{l}7.566-5 \\
7.194-5\end{array}$ & $\begin{array}{l}\cdots \\
\cdots\end{array}$ \\
\hline & GH4 & $\begin{array}{l}\text { PGGE } \\
\text { BNL }\end{array}$ & $\begin{array}{l}4.477-2 \\
4.527-2\end{array}$ & $\begin{array}{l}4.554-2 \\
4.603-2\end{array}$ & as GF1 & & & & & \\
\hline & GH5 & $\begin{array}{l}\text { PGGE } \\
\text { BNL }\end{array}$ & $\begin{array}{l}5.561-2 \\
5.474-2\end{array}$ & $\begin{array}{l}2.702-3 \\
2.540-3\end{array}$ & as GG2 & & & & & \\
\hline & GH6 & $\begin{array}{l}\text { PGGE } \\
\text { BNL }\end{array}$ & $\begin{array}{l}4.523-2 \\
4.571-2\end{array}$ & $\begin{array}{l}4.554-2 \\
4.603-2\end{array}$ & as GFI & & & & & \\
\hline \multirow[t]{4}{*}{26} & $2 G 1$ & $\begin{array}{l}\text { PGEE } \\
\text { BNL }\end{array}$ & $\begin{array}{l}4.396-2 \\
4.453-2\end{array}$ & $\begin{array}{l}4.554-2 \\
4.603-2\end{array}$ & as GF1 & & & & & \\
\hline & $2 G 2$ & $\begin{array}{l}\text { PGQE } \\
\text { BNL }\end{array}$ & $\begin{array}{l}5.364-2 \\
5.271-2\end{array}$ & $\begin{array}{l}2.702-3 \\
2.540-3\end{array}$ & as GG2 & & & & & \\
\hline & $2 G 3$ & $\begin{array}{l}\text { PG\&E } \\
\text { BNL }\end{array}$ & $\begin{array}{l}6.250-2 \\
6.246-2\end{array}$ & $\begin{array}{l}2.339-4 \\
2.066-4\end{array}$ & as $\mathrm{GH}_{3}$ & & & & & \\
\hline & $2 G 4$ & $\begin{array}{l}\text { PGQE } \\
\text { BNL }\end{array}$ & $\begin{array}{l}2.898-1 \\
2.910-1\end{array}$ & $\begin{array}{l}6.369 \cdot 5 \\
5.995-5\end{array}$ & $\begin{array}{l}2.597 .5 \\
2.363 .5\end{array}$ & $\begin{array}{l}4.314-6 \\
1.874-6\end{array}$ & $\begin{array}{l}2.166-5 \\
2.176-5\end{array}$ & $\begin{array}{l}3.049 .5 \\
3.017 .5\end{array}$ & $\begin{array}{l}7.221-6 \\
6.176-6\end{array}$ & $\begin{array}{l}-. \\
-.\end{array}$ \\
\hline
\end{tabular}


Table B1.25 (continued)

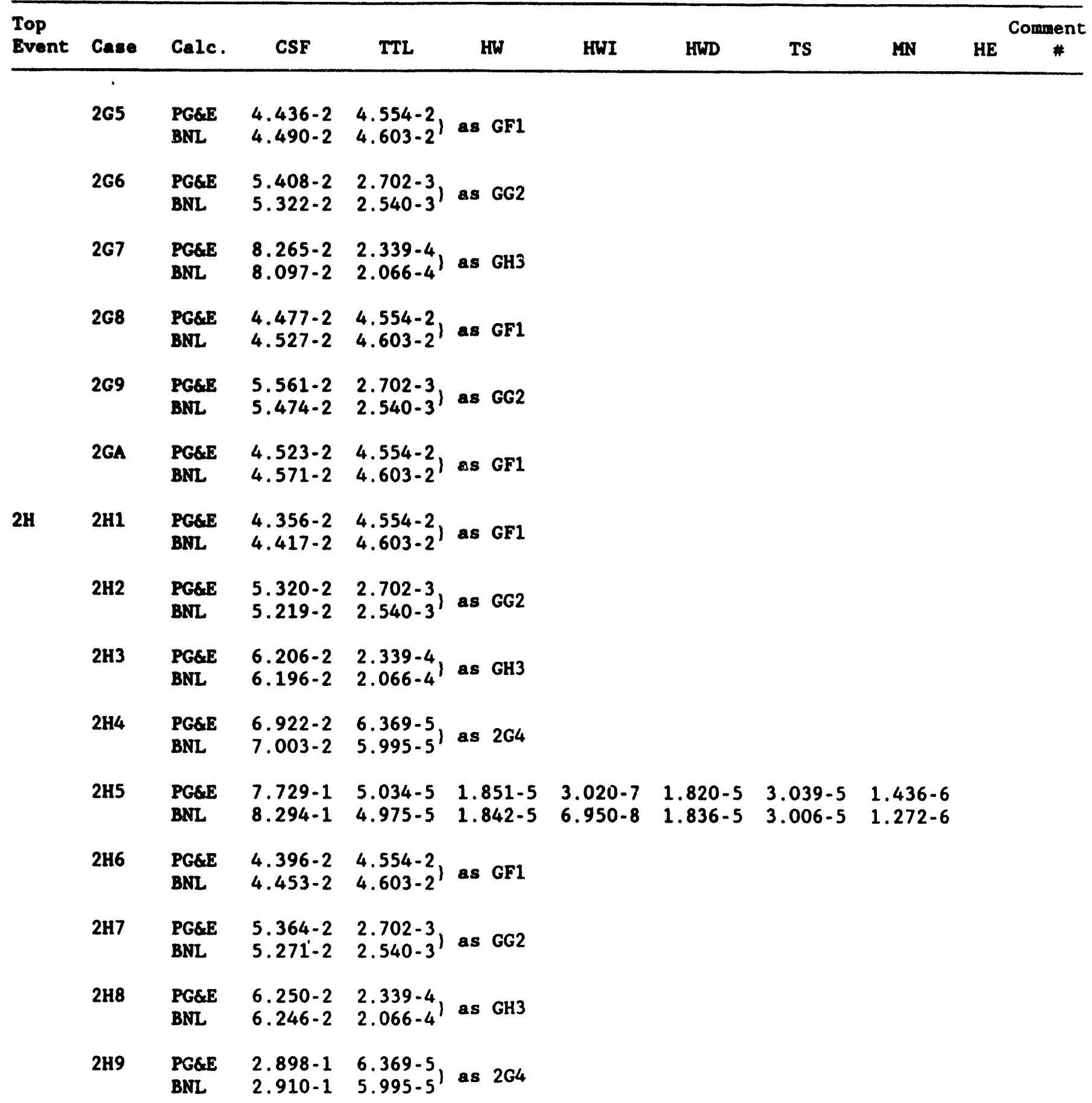


Appendix B1

Table B1.2.5 (continend)

\begin{tabular}{|c|c|c|c|c|c|c|c|c|c|c|c|}
\hline $\begin{array}{l}\text { Top } \\
\text { Event }\end{array}$ & Case & Calc. & CSF & TTL & HW & HWI & HWD & TS & $\mathbf{M N}$ & $\mathrm{HE}$ & $\begin{array}{c}\text { Comment } \\
*\end{array}$ \\
\hline & 2HA & $\begin{array}{l}\text { PG\&E } \\
\text { BNL }\end{array}$ & $\begin{array}{l}4.436-2 \\
4.490-2\end{array}$ & $\begin{array}{l}4.554-2 \\
4.603-2\end{array}$ & as GF1 & & & & & & \\
\hline & $2 \mathrm{HB}$ & $\begin{array}{l}\text { PG\&E } \\
\text { BNL }\end{array}$ & $\begin{array}{l}5.408-2 \\
5.322-2\end{array}$ & $\begin{array}{l}2.702-3 \\
2.540-3\end{array}$ & as $G G 2$ & & & & & & \\
\hline & $2 \mathrm{HC}$ & $\begin{array}{l}\text { PG\&E } \\
\text { BNL }\end{array}$ & $\begin{array}{l}8.265-2 \\
8.098-2\end{array}$ & $\begin{array}{l}2.339-4 \\
2.066-4\end{array}$ & as $\mathrm{GH} 3$ & & & & & & \\
\hline & 2HD & $\begin{array}{l}\text { PG\&E } \\
\text { BNL }\end{array}$ & $\begin{array}{l}4.477-2 \\
4.527-2\end{array}$ & $\begin{array}{l}4.554-2 \\
4.603-2\end{array}$ & as GF1 & & & & & & \\
\hline & $2 \mathrm{HE}$ & $\begin{array}{l}\text { PG\&E } \\
\text { BNL }\end{array}$ & $\begin{array}{l}5.561-2 \\
5.474-2\end{array}$ & $\begin{array}{l}2.702-3 \\
2.540-3^{\prime}\end{array}$ & as GG2 & & & & & & \\
\hline & $2 \mathrm{HG}$ & $\begin{array}{l}\text { PG\&E } \\
\text { BNL }\end{array}$ & $\begin{array}{l}4.523-2 \\
4.571-2\end{array}$ & $\begin{array}{l}4.554-2 \\
4.603-2\end{array}$ & as GF1 & & & & & & \\
\hline \multirow[t]{4}{*}{ Sw } & swo & $\begin{array}{l}\text { PG\&E } \\
\text { BNL }\end{array}$ & & $\begin{array}{l}0.000 \\
0.000\end{array}$ & & & & & & & \\
\hline & SW1 & $\begin{array}{l}\text { PG\&E } \\
\text { BNL }\end{array}$ & & $\begin{array}{l}5.000-1 \\
5.000-1\end{array}$ & & & & & & & \\
\hline & SW2 & $\begin{array}{l}\text { PG\&E } \\
\text { BNL }\end{array}$ & & $\begin{array}{l}1.767-3 \\
1.770-3\end{array}$ & & & & & & & \\
\hline & sw3 & $\begin{array}{l}\text { PG\&E } \\
\text { BNL }\end{array}$ & & $\begin{array}{l}9.981-1 \\
9.982-1\end{array}$ & & & & & & & \\
\hline
\end{tabular}

Note: "I as " means that the remaining values in those lines are the same as the referenced spilt fraction occurring earlier in the table. 
Table B1.2.6

Unavallability Values (Split Fractiona) for the

Diesel Fuel Tranofer System

\begin{tabular}{clllllllll}
\hline Top Event & Case & Calc. & TTL & HW & HWI & HWD & TS & MN & HE \\
\hline FO & FO1 & PG\&E & $2.164-4$ & $1.919-4$ & $1.176-5$ & $1.802-4$ & 0.0 & $2.445-5$ & 0.0 \\
& & BNL & $2.092-4$ & $1.848-4$ & $8.533-6$ & $1.763-4$ & 0.0 & $2.447-5$ & 0.0 \\
& \multirow{2}{*}{ FO2 } & PG\&E & $7.040-3$ & $3.113-3$ & $2.933-3$ & $1.802-4$ & 0.0 & $3.930-3$ & 0.0 \\
& & BNL & $7.048-3$ & $3.097-3$ & $2.921-3$ & $1.763-4$ & 0.0 & $3.951-3$ & 0.0 \\
& \multirow{2}{*}{ FO3 } & PG\&E & $3.509-4$ & $1.919-4$ & $1.176-5$ & $1.802-4$ & 0.0 & $2.445-5$ & 0.0 \\
& & BNL & $3.460-4$ & $1.848-4$ & $8.533-6$ & $1.763-4$ & 0.0 & $2.447-5$ & 0.0 \\
& FO4 & PG\&E & $2.263-2$ & $1.919-4$ & $1.176-5$ & $1.802-4$ & 0.0 & $2.445-5$ & 0.0224 \\
& & BNL & $2.250-2$ & $1.848-4$ & $8.533-6$ & $1.763-4$ & 0.0 & $2.447-5$ & 0.0223 \\
& \multirow{2}{*}{ FO5 } & PG\&E & $5.079-2$ & $3.113-3$ & $2.933-3$ & $1.802-4$ & 0.0 & $3.930-3$ & 0.0224 \\
& & BNL & $2.292-2$ & $3.097-3$ & $2.921-3$ & $1.763-4$ & 0.0 & $3.951-3$ & 0.0223 \\
& FOF & PG\&E & 1.0 & & & & & & \\
& BNL & 1.0 & & & & & & \\
\hline
\end{tabular}


Appendix B1

Table B1.2.7a

Conditional Split Trections for DG Top Eveats as a Function of Seinmic Level

PG\&E

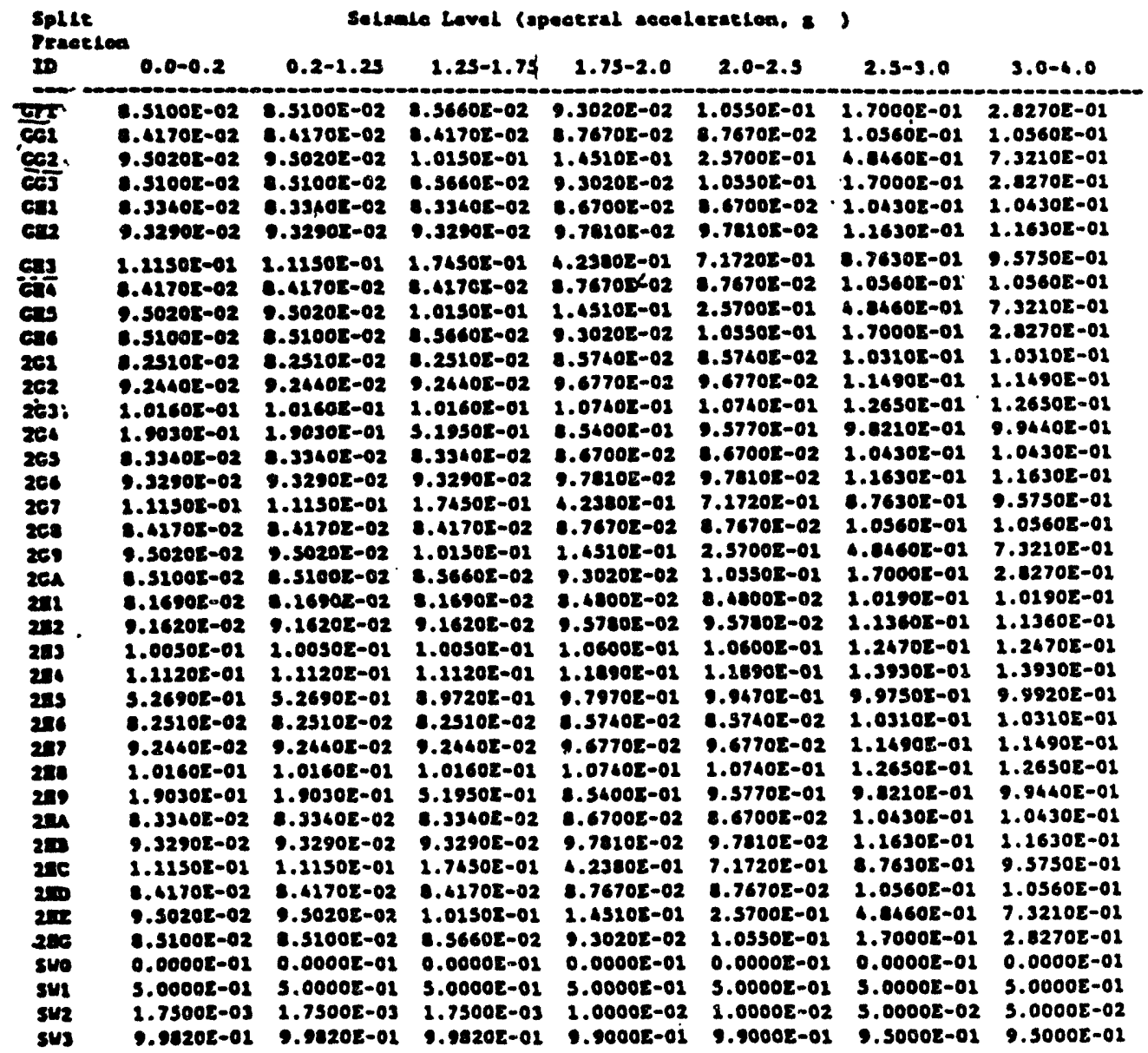


Appendix B1

Table B1.2.7b

Conditional Split Fractions for DG Top Events as a Function Selamic Level

BNL

\begin{tabular}{|c|c|c|c|c|c|c|c|}
\hline \multirow{2}{*}{$\begin{array}{l}\text { Splise } \\
\text { Pereesson } \\
\text { ID }\end{array}$} & \multicolumn{7}{|c|}{ teale Level (apeoeral secolezael } \\
\hline & $0.0-0.2$ & $0.2-2.25$ & .75 & $1.75-2.0$ & $2.0-2.3$ & $2.5-3.0$ & $3.0-6.0$ \\
\hline 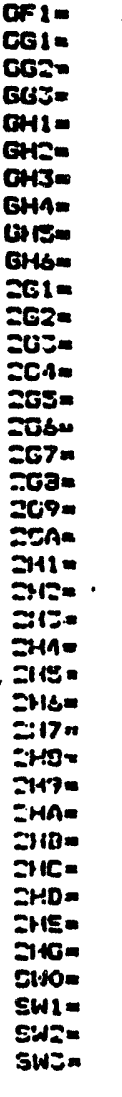 & 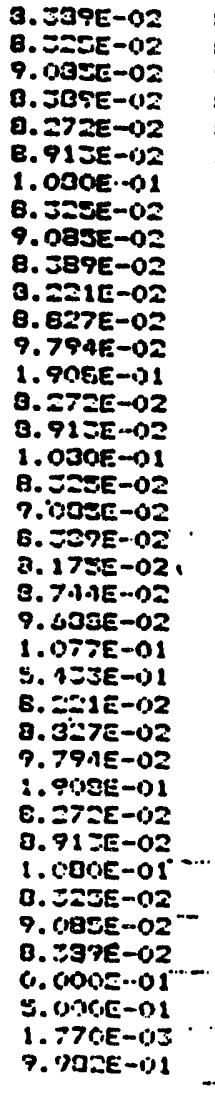 & 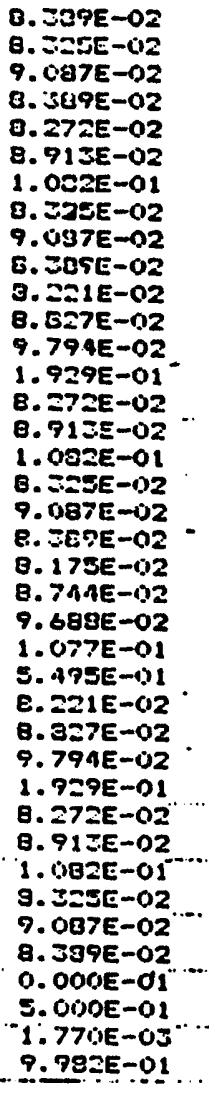 & 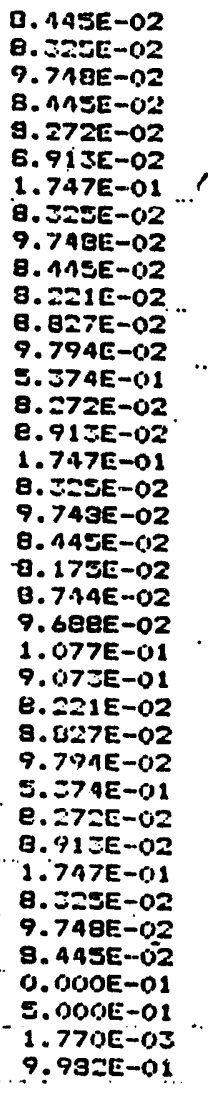 & 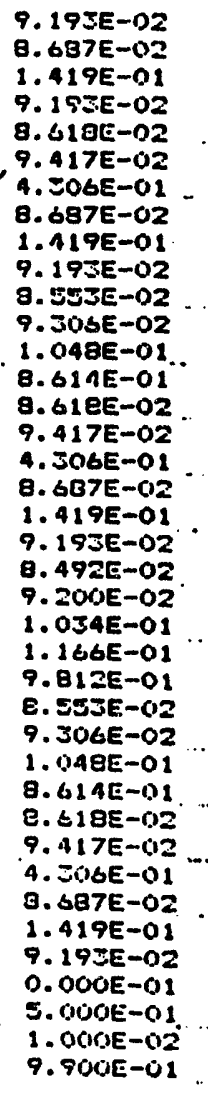 & 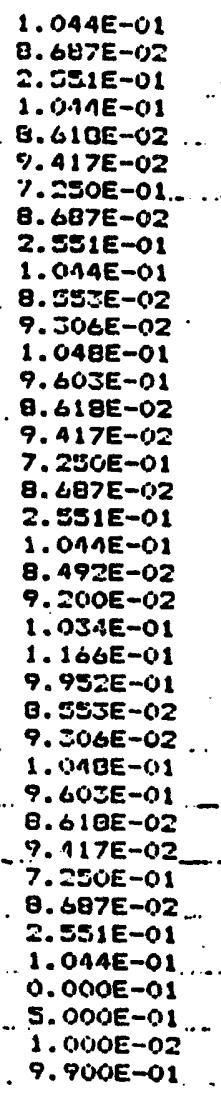 & 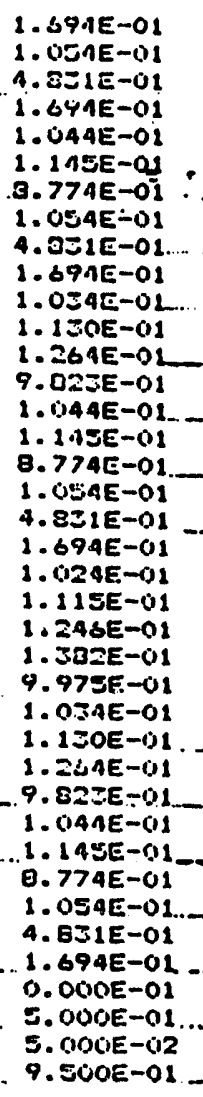 & 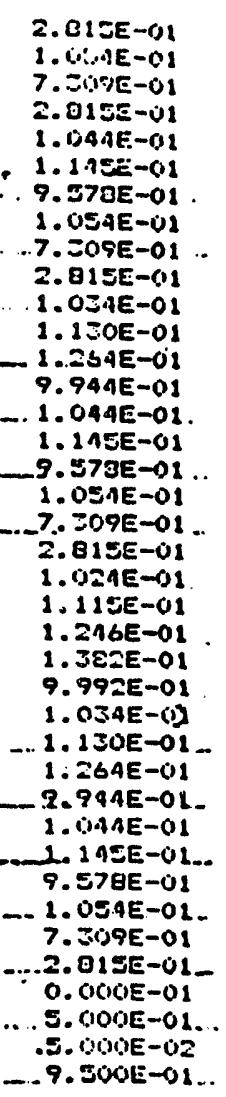 \\
\hline
\end{tabular}




\section{B13 Results of the BNL Review}

\section{B1.3.1 General}

The unavailability modelling of the Diesel Generators and the Diesel Fuel Transfer System in the DCPRA were reviewed by BNL with special emphasis because of the following:

a. The DGs are the most important support systems; impacting the safety of the majority of plant operations, including cold shutdown.

b. As discussed in Section B1.1, a request for changing the Allowed Outage Time (AOT) of the Diesel Generators was submitted to the NRC by PG\&E and the study ${ }^{2}$ supporting the request was based mainly on the DCPRA. BNL reviewed this study in a parallel effort to this review.

Therefore, to check the adequacy of the DCPRA modelling for "system- specific" effects which may also influence granting permission for AOT changes, BNL used the following approach: BNL compared the vendor-specific (ALCO) diesel failure events with those obtained from generic diesel data. This was done to see how well the DCPRA model reflects the vendor-specific "experience" and to estimate the expected downtime distribution of the diesels. The evaluation was carried out by reviewing the failure modes and maintenance unavailabilities involved in the diesel model. In order to check for calculational inconsistencies, all of the split fractions were recalculated (seismic inclusive).

\section{B1.3.2 Comparison of ALCO Type DG Failures With All Types of DG Failures}

In order to see whether the ALCO-type DGs used at the Diablo Canyon power plant have some dominant subsystem- or component-specific failure modes (and thus, some subsystem or component specific expected downtimes) BNL compared the leading failure contributions of subsystems and components of ALCO diesels with those of all other types of DGs. The data were taken from a recent study performed at Battelle on aging of diesel components. ${ }^{3}$ Table B1.3.1 presents the results. One can see that the Instruments and Control System's (and within it the governor's) failures are the main contributors to the generic failures of ALCO diesels. Also with ALCO diesels, the Cooling System and to a lesser extent the Lubrication System seem to be more prone to failures than in the total generic DG population. A positive feature of the ALCO diesels is that the starting system appears to be less vulnerable to failure than the generic DG population. Finally, the ALCO fuel system does not seem to be any more prone to failures than the generic one.

\section{B1.3.3 Remarks on the Unavailability Modelling of the Diesels and Fuel Oil Transfer System in the DCPRA}

a. The system modelling of the DGs in the DCPRA represents an elaborate sequential unavailability analysis of a "five train" system, where one train (the swing diesel) plays a special role. There is no question that the approach used is mathematically appealing because it uses the symmetry aspects of the diesel configuration and renders the results of the analysis very suitable for integration into the DCPRA.

b. In contrast with the systems modelling, the unavailability modelling of the individual diesels (the fault tree modelling) was kept simplistic by using the standard "diesel fails to start and run" failure modes. The diesel starting air system (i.e., air compressors, receivers, etc.) was not modelled separately because it was considered to be included as part of the diesel start failure data. An attempt was made to display some components of the diesel subsystems in the model. This effort, however, tended to be inconsistent in that 
only some support failures were modelled and inconsequential in that the modelled failures were of such

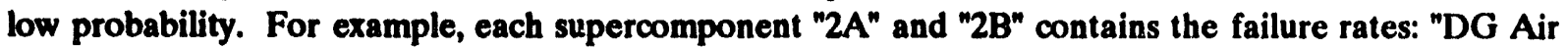
Receiver - Rupture During Operation: ZTTK1B = 2.66-8/hr," "Air Check Valve - Transfer Closed During Operation: ZTVCOP $=1.04 .8 / \mathrm{hr}$," etc. The failure contribution of the turbocharger, however, with an estimated ${ }^{2}$ failure probability of $2.73-4 / d$ was neglected.

Another example: while the diesel supercomponent boundaries indicate several subsystems as part of the supercomponents (see Figures B1.2.6, B1.2.8, and B1.2.9), one cannot find any representative component failure rate contributing to the combined unavailability of those supercomponents. Such subsystems are: the cooling, the lubrication and the combustion air systems. Table B1.3.1 shows that the cooling system is the second largest contributor to the failure of the ALCO type diesels.

c. The DCPRA models the maintenance frequency and duration of the LCVs as separate quantities from those of the diesels. Given that the day tank and other fuel system components were included in the maintenance data of the diesel, it was interesting to note that the LCVs were treated separately. Given that they were treated separately, the mean and 95th percentile of the "effective" downtime distribution of the diesel system would be determined by the combination of the diesel and the LCV maintenance duration distributions (the 95th percentile value of LCV maintenance duration is 51.3 hours).

d. The DCPRA considers only unscheduled maintenances performed on Unit 2 diesels as contributing to the unavailabilitiesof the associated top events, " $2 \mathrm{G}^{\text {" and }}$ " $2 \mathrm{H}$." Unavailabilitiesdue to large overhauls lasting over a protracted period of time performed when Unit 1 is operating and Unit 2 is in refueling (or cold shutdown) (say two times 10 to 16 days each) were not included in the model.

e. In Table B1.2.6 the PG\&E total split fraction value, FO5 was in variance with that obtained by BNL. The probable cause of the discrepancy is that the human error contribution was double counted in the DCPRA. The PG\&E value is seemingly also in contradiction with the PG\&E seismic values given at the lowest three seismic levels in Table 6-46 (p.6-182) of Reference 1.

f. The detailed analysis of the Fuel Oil Transfer System (see DCPRA Figure D.2.1-3 Sheet 3 of 4) contains the following item (Item No.12): "In an emergency where it is necessary to get into the fuel oil pump vault to manipulate valves, it may take several hours to get security to open the vault." This item renders questionable the estimates of the human factors (among others the value of ZHEF06 used in the diesel analysis) considered for recovery of the Fuel Oil Transfer System and through it, the recovery of electrical power.

g. Among the DG failure-related LERs filed by the Diablo Canyon power plant, ${ }^{6}$ there was one failure in the Fuel Oil Transfer System which would affect all the DGs. This common cause failure involved the degradation of the diesel oil in the underground reserve tanks caused by fungi. According to PG\&E, the problem does not exist any more. However because of its peculiarity and importance it is quoted here:

LER 88-14. This report is being voluntarily submitted for information purposes only as described in Item 19 of Supplement No.1 to NUREG-1022. On May 4, 1988, during performance of surveillance test procedure (SRP) M-96, "diesel generator 24 hour load test," the diesel generator (DG) 1-1 load decreased below the value specified in the SRP acceptance criteria. An investigation showed that a high differential pressure existed across the primary fuel oil filter. After switching to the standby primary fuel oil filter, the load returned to the required value. An investigation determined that the DG day tank contained a fungus and that the first primary filter was clogged by fungus. The other DG day tanks also contained a fungus and fungus spores were found in the main storage tanks. The fuel oil in the day tanks was diocided and filtered until the fuel oil met the criteria of STP M-108, "diesel fuel oil analysis," for particulate contamination, flash point, API gravity and viscosity. The day tanks were drained, inspected and cleaned. The bottom of main storage tanks $0-1$ and $0-2$ were suctioned out and 


\section{Appendix B1}

a biocide was added. A biocide program will be developed and implemented to inhibit the growth of fungus in the DG fuel oil storage system. Also, a sampling and inspection program for the DG day tanks will be developed. Both will be incorporated into plant procedures.

\section{B1.3.4 Audit Calculations}

In order to scrutinize the quantified split fractions themselves, BNL performed audit calculations for each of the split fractions associated with each of the boundary conditions. The calculations were extended for both non-seismic (mission time: 6 hours) and seismic (mission time: 24 hours) cases. Seismic calculations were not performed for the Fuel Oil Transfer System. In these audit calculations the same assumptions, input data, maintenance and test frequency and duration, as well as mean fragility and human factor values were used as in the DCPRA. The SETS code ${ }^{7}$ and locally generated PC software were used for the computations. The use of the SETS code allowed the identification of the most important cut sets contributing to the hardware unavailabilities. These cut sets are not readily accessible for direct review in the DCPRA. Attachment B1.A lists the ranked cut sets for single, double, triple, quadruple and quintuple diesel failures. The definition of the basic events appearing in the cut sets are identical to those given in Chapter D.2.1.5 of the DCPRA.

The results obtained by the audit calculations are presented in Tables B1.2.6 and B1.2.7.b for the DGs and for the Fuel Oil Transfer System, respectively. They are denoted by "BNL" to be compared with the values given in the DCPRA (denoted by "PG\&E").

By comparing the PG\&E and BNL results one can see that there is an overall agreement between the data. The agreement is even better, if one takes into account that BNL used point estimates, while PG\&E mainly used a Monte-Carlo approach in the split fraction quantification.

\section{B1.3.5 Conclusions}

The BNL review identified several inconsistencies and neglection of failures of diesel subsystems in the unavailability modelling of diesel generators in the DCPRA and the omission of the unavailability contribution from Unit 2 (and swing) diesel overhauls. The combined effect of these neglections may result in underestimation of the associated top event split fractions and through them the expected core damage frequency value of Unit 1.

As an overall sensitivity study on the Diesel Generator and Diesel Fuel Transfer Systems, the BNL values for the conditional split fractions found in Tables B1.2.5 and B1.2.6 were substituted into the dominant sequence model. The overall unnormalized Fussel-Vesely importance of this class of events was 4.255-05 (PG\&E values) and 4.115-05 (BNL values) respectively. This demonstrates excellent agreement between BNL and PG\&E. 


\section{B1.4 References}

1. Final report on the Diablo Canyon Long-Term Seismic Program, Pacific Gas and Electric Co., Diablo Canyon Power Plant, Docket Nos. 50-275 and 50-323, July 1988.

2. Diesel Generator Allowed Outage Time Study, Pacific Gas \& Electric Company, May 1989.

3. Units 1 and 2 Diablo Canyon Power Plant, "Final Safety Analysis Report Update," Pacific Gas and Electric Co., December 1988.

4. PG\&E letters to NRC signed by J.D. Shiffer, No. DCL-88-238, October 10, 1988, No. DCL-88-260, October 28, 1988, No. DCL-88-285, November 29, 1988, No. DCL-88-297, December 9, 1988, No. DCL89-010, January 16, 1989, and No. DCL-89-152, June 2, 1989.

5. Hoopingarner, K.R., Vause, J.W., Dingee, D.A., Nesbitt, J.F., "Aging of Nuclear Station Diesel Generators: Evaluation of Operating and Expert Experience," NUREG/CR-4590, August 1987.

6. DOE/RECON, Nuclear Safety Information Center (NSIC), 1963 to present.

7. Worrel, R.B. and Stack, D.W., "A SETS User's Manual for the Fault Tree Analyst," Sandia National Laboratories, NUREG/CR-0475, SAND77-2051, November 1978. 
Appendix B1

Table B1.3.1

Systems and Components Contributing Most to Failures at All Types of DGs and at ALCOType DGs

Systems and Components

Instrument and Controls System

Governor

Sensors

Relays

Startup Components

Fuel System

Piping on Engine

Injector Pumps

Fuel Oil Pumps

Starting System

Controls

Starting Air Valve

Starting Motors

Air Compressor

Switchgear System

Breakers

Relays

Instrument and Controls

Cooling System

Pumps

Heat Exchangers

Piping

Lubrication System

Heat Exchangers

Pumps

Lube Oil
Percent of

All Failures

25

26

10

3

2

2

11

3

2

10

10

3

5

1

9

2

2

2

7

2

2

1

28

26
10 3

2

1

10

Percent of Failures at ALCO DGs

15

3

1

1

1

1

5

6

4

4

1

14

1

1

6

8

3

3

Other Systems

Date Base: 1984 failure event recorded between 1974 and 1984 in Reference 5 .

Nuclear plants where ALCO Diesel Generators have been used in 1984:

Indian Point 1 and 2, Power Authority of the State of NY

Salem 1 and 2, Public Service Electric and Gas Company

Palisades, Consumers' Power Company

Pilgrim 1, Boston Edison

Ginna, Rochester Gas and Electric 
Appendix B1

\section{ATTACHMENT B1.A}

HARDWARE UNAVAILABILITY CUT SETS FOR THE

DIESEL GENERATORS 
Appendix B1

Hardware Unavailability Cut Sets in Case of One DG Failure

Total Hardware, HW =

$\begin{array}{lll}1 & 3.6736 E-02 & \text { FID1F + } \\ 2 & 2.1339 E-04 & \text { HEV * FIV1F } \\ 3 & 1.1073 E-04 & \text { DDC + } \\ 4 & 1.1073 E-04 & \text { DDA + } \\ 5 & 1.1073 E-04 & \text { DDD + } \\ 6 & 1.1073 E-04 & \text { DDB + } \\ 7 & 1.7703 E-05 & \text { GDG + } \\ 8 & 1.6417 E-05 & \text { TDA + } \\ 9 & 1.6417 E-05 & \text { TDC + } \\ 10 & 1.6417 E-05 & \text { TDB + } \\ 11 & 1.6417 E-05 & \text { TBD + } \\ 12 & 1.6417 E-05 & \text { TDF + } \\ 13 & 1.6417 E-05 & \text { TDD + } \\ 14 & 5.8176 E-06 & \text { HEV * DVC + } \\ 15 & 5.8176 E-06 & \text { HEV * DVA + } \\ 16 & 5.8176 E-06 & \text { HEV * DVD + } \\ 17 & 5.8176 E-06 & \text { HEV * DVS + } \\ 18 & 4.1268 E-07 & \text { HEV * TVA + } \\ 19 & 4.1268 E-07 & \text { HEV * TVC + } \\ 20 & 4.1268 E-07 & \text { HEV * TVE + }\end{array}$

Independent Hardware, HWI =

$1 \quad 3.6736 \mathrm{E}-02 \quad$ FID1F +

2 2.1339E-04 HEV * FIV1F 
Hardware Unavailability Cut Sets in Case of Two DG Failures

Total Hardware, HW =

$\begin{array}{lll}1 & 1.3495 E-03 & \text { FIDIF * FID1G + } \\ 2 & 1.1073 E \cdot 04 & \text { DDA + } \\ 3 & 1.7703 E-05 & \text { GDG + } \\ 4 & 1.6417 E-05 & \text { TDB + } \\ 5 & 1.6417 E-05 & \text { TDA + } \\ 6 & 1.6417 E-05 & \text { TDC + } \\ 7 & 7.8392 E-06 & \text { FID1F * HEV * FIV1G + } \\ 8 & 7.8392 E-06 & \text { HEV * FIV1F * FID1G + } \\ 9 & 5.8176 E-06 & \text { HEV * DVA + } \\ 10 & 4.0678 E-06 & \text { FID1F * DDF + } \\ 11 & 4.0678 E-06 & \text { FIDIG * DDC + } \\ 12 & 4.0678 E-06 & \text { FID1F * DDG + } \\ 13 & 4.0678 E-06 & \text { FID1F * DDE + } \\ 14 & 4.0678 E-06 & \text { FID1G * DDD + } \\ 15 & 4.0678 E-06 & \text { FID1G * DDB + } \\ 16 & 1.1384 E-06 & \text { HEV * FIV1F * FIV1G + } \\ 17 & 6.0309 E-07 & \text { FID1F + IDI + }\end{array}$

Independent Hardware, HWI =
1 1.3495E-03
FID1F* FID1G +
2 7.8392E-06
FID1F * HFV * FIV1G +
3. 7.8392E-06
HEV * FIV1F * FID1G +
4 1.1384E-06
HEV * FIV1F * FIVIG 


\section{Appendix B1}

Hardware Unavailability Cut Sets in Case of Three DG Failures

Total Hardware, HW =

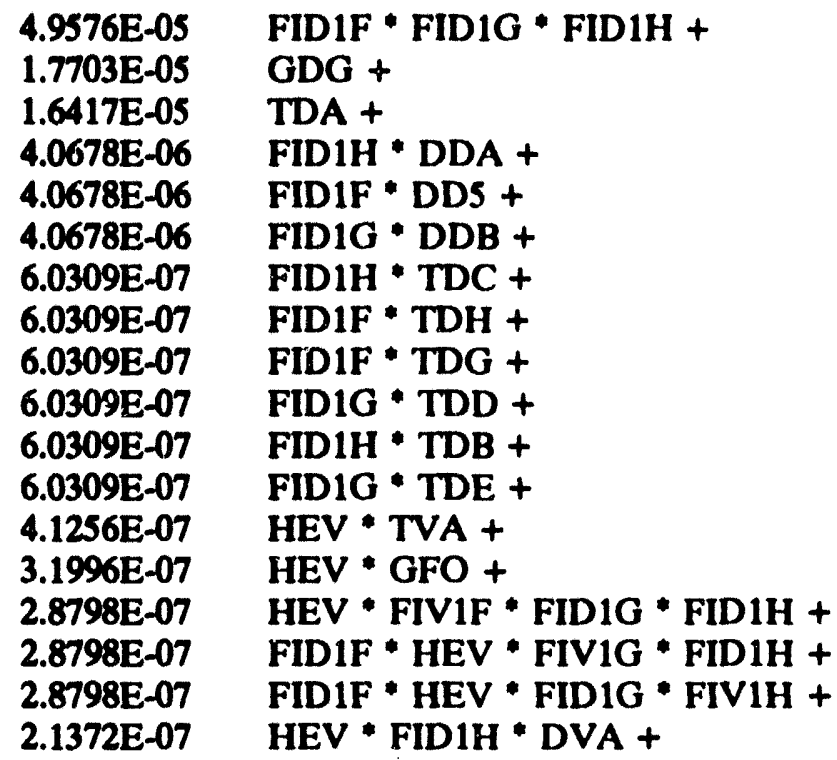

Independent Hardware, HWI =

$\begin{array}{ll}1 & 4.9576 \mathrm{E}-05 \\ 2 & 2.8798 \mathrm{E}-07 \\ 3 & 2.8798 \mathrm{E}-07 \\ 4 & 2.8798 \mathrm{E}-07 \\ 5 & 4.1821 \mathrm{E}-08 \\ 6 & 4.1821 \mathrm{E}-08 \\ 7 & 4.1821 \mathrm{E}-08 \\ 8 & 6.0733 \mathrm{E}-08\end{array}$

FID1F * FID1G * FIDIH +
FID1F * HEV * FIV1G * FID1H +
HEV * FIV1F * FID1G * FID1H +
FID1F * HEV * FID1G * FIV1H +
FID1F * HEV * FIV1G * FIV1H +
HEV * FIV1F * FIV1G * FID1H +
HEV * FIV1F * FID1G * FIV1H +
HEV * FIV1F * FIV1G * FIV1H +


Hardware Unavailability Cut Sets in Case of Four DG Failures

Total Hardware, HW =

$\begin{array}{ll}1 & 1.7703 \mathrm{E}-05 \\ 2 & 1.3212 \mathrm{E}-06 \\ 3 & 6.0309 \mathrm{E}-07 \\ 4 & 6.0309 \mathrm{E}-07 \\ 5 & 6.0309 \mathrm{E}-07 \\ 6 & 6.0309 \mathrm{E}-07 \\ 7 & 3.1996 \mathrm{E}-07 \\ 8 & 1.4943 \mathrm{E}-07 \\ 9 & 1.4943 \mathrm{E}-07 \\ 10 & 1.4943 \mathrm{E}-07 \\ 11 & 1.4943 \mathrm{E}-07 \\ 12 & 1.4943 \mathrm{E}-07 \\ 13 & 1.4943 \mathrm{E}-07 \\ 14 & 2.2155 \mathrm{E}-08 \\ 15 & 2.2155 \mathrm{E}-08 \\ 16 & 2.2155 \mathrm{E}-08 \\ 17 & 2.2155 \mathrm{E}-08 \\ 18 & 2.2155 \mathrm{E}-08 \\ 19 & 2.2155 \mathrm{E}-08 \\ 20 & 1.5160 \mathrm{E}-08\end{array}$

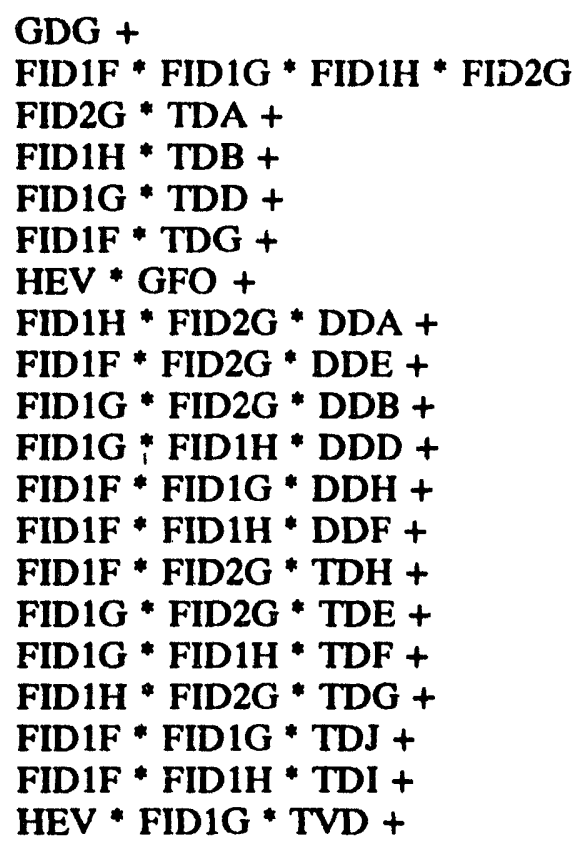

Independent Hardware, HWI =

$\begin{array}{lll}1 & 1.8212 E-06 & \text { FID1F * FID1G * FID1H * FID2G + } \\ 2 & 1.0579 E-08 & \text { FID1F * HEV * FIV1G * FID1H * FID2G + } \\ 3 & 1.0579 E-08 & \text { HEV * FIV1F * FID1G * FID1H * FID2G + } \\ 4 & 1.0579 E-08 & \text { FID1F * HEV * FID1G * FID1H * FIV2G + } \\ 5 & 1.0579 E-08 & \text { FID1F * HEV * FID1G * FIV1H * FID2G + } \\ 6 & 1.5363 E-09 & \text { FID1F * HEV * FIV1G * FID1H * FIV2G + } \\ 7 & 1.5363 E-09 & \text { FID1F * HEV * FIV1G * FIV1H * FID2G + } \\ 8 & 1.5363 E-09 & \text { HEV * FIV1F * FID1G * FID1G * FIV2G + } \\ 9 & 1.5363 E-09 & \text { HEV * FIV1F * FID1G * FIV1H * FID2G + } \\ 10 & 1.5363 E-09 & \text { HEV * FIV1F * FIV1G * FID1h * FID2G + } \\ 11 & 1.5363 E-09 & \text { FID1F * HEV *FID1G * FIV1H * FIV2G + } \\ 12 & 2.2311 E-10 & \text { HEV * FIV1F * FIV1G * FIV1H * FID2G + } \\ 13 & 2.2311 E-10 & \text { FID1F * HEV * FIV1G *FIV1H * FIV2G + } \\ 14 & 2.2311 E-10 & \text { HEV * FIV1F * FID1G * FIV1H * FIV2G + } \\ 15 & 2.2311 E-10 & \text { HEV * FIV1F * FIV1G * FID1H * FIV2G + } \\ 16 & 3.2400 E-11 & \text { HEV * FIV1F * FIV1G * FIV1H * FIV2G }\end{array}$


Appendix B1

Hardware Unavailability Cut Sets in Case of Five DG Failures

Total Hardware, HW =

$\begin{array}{lll}1 & 1.7703 E-05 & \text { GDG }+ \\ 2 & 3.1996-07 & \text { HEV * GFO + } \\ 3 & 6.6905 E-08 & \text { FID1F * FID1G * FID1H * FID2H + } \\ 4 & 2.2155 E-08 & \text { FID1F * FID2G * TDH + } \\ 5 & 2.2155 E-08 & \text { FID1G * FID2G * TDE + } \\ 6 & 2.2155 E-08 & \text { FID1G * FID2H * TDD + } \\ 7 & 2.2155 E-08 & \text { FID2G * FID2H * TDA + } \\ 8 & 2.2155 E-08 & \text { FID1H * FID2H * TDB + } \\ 9 & 2.2155 E-08 & \text { FID1F * FID2H * TDG + } \\ 10 & 2.2155 E-08 & \text { FID1H * FID2G * TDC + } \\ 11 & 2.2155 E-08 & \text { FID1G * FID1H * TDF + } \\ 12 & 2.2155 E-08 & \text { FID1F * FID1H * TDI + } \\ 13 & 2.2155 E-08 & \text { FID1F * FID1G * TDJ + } \\ 14 & 5.4896 E-09 & \text { FID1G * FID2G * FID2H * DDB + } \\ 15 & 5.4896 E-09 & \text { FID1G * FID1H * FID2H * DDD + }\end{array}$

Independent Hardware, HWI =

$\begin{array}{lll}1 & 6.6905 E-08 & \text { FID1F * FID1G * FID1H * FID2G * FID2H + } \\ 2 & 3.8864 E-10 & \text { FID1F * HFV * FIV1G * FID1H * FID2G * FTD2H + } \\ 3 & 3.8864 \text { E-10 } & \text { HEV * FIV1F * FID1G * FID1H * FID2G * FID2H + } \\ 4 & 3.8864 E-10 & \text { FID1F * HEV * FID1G * FID1H * FID2G * FIV2H + } \\ 5 & 3.8864 E-10 & \text { FID1F * HEV * FID1G * FID1H * FIV2G * FID2H + } \\ 6 & 3.8864 E-10 & \text { FID1F * HEV * FID1G * FIV1H * FID2G * FID2H + } \\ 7 & 5.6439 E-11 & \text { FID1F * HEV * FIV1G * FID1H * FID2G * FIV2H + } \\ 8 & 5.6439 E-11 & \text { FID1F * HEV * FIV1G * FID1H * FIV2G * FID2H + }\end{array}$




\section{APPENDIX B2: ELECTRICAL POWER SYSTEMS}

\section{B2.1 Introduction}

\section{B2.1.1 Objective}

The objective of this appendix is to summarize the results of reviewing the unavailability analyses of the following electric power systems in the DCPRA: ${ }^{1}$

- Nonvital Electric Power System

- Vital 125V DC System

- Vital AC System

- Unit 2 Vital AC and DC Systems

- Instrument AC System

This Appendix reflects BNL's understanding of the subject systems. ${ }^{2,3}$

\section{B2.1.2 Organization}

Section B2.2 provides condensed descriptions about the configurations and functions of the above electric power systems. It also describes the dependency of these systems on support equipment, the surveillance and maintenance conditions, the unavailability modelling in the DCPRA, and the original DCPRA results. The purpose of this approach is to present stand alone documentation to which the review's findings can be directly compared. Section B2.3 contains the results and findings of the BNL review.

\section{B2.2 Unavailability Modelling of Electric Power Systems}

\section{B2.2.1 General}

The electric power systems are analyzed in the DCPRA as a series of top events in the support system event trees.

The electric systems and the associated top events are as follows:

Electric System

\author{
Nonvital Electric Power System \\ Vital 125V DC System \\ Vital AC Power System, Unit 1 \\ Vital AC and DC Power, Unit 2 \\ Instrument AC Power System
}

Top Event Designator

$$
\begin{aligned}
& \text { OG, NV } \\
& \text { DF, DG, DH } \\
& \text { AF, AG, AH, SF, SG, SH } \\
& \text { BF, BG, BH } \\
& \text { I1, I2, 13, I4 }
\end{aligned}
$$

The subsequent sections provide the condensed descriptions of the electrical system analyses. Figure B2.2.1 shows an overall schematic of the electrical distribution for Unit 1. The definitions and success criteria of the associated top events are detailed in Tables B2.2.1a through B2.2.1f. 
Appendix B2

\section{B2.2.2 Nonvital Electric Power System}

\section{B2.22.1 Puction, Configration, Operation}

The functions of this "nonvital" electric power system are:

- To provide power to the plant's vital $(4.16 \mathrm{kV})$ and nonvital $(12 \mathrm{kV}$ and $4.16 \mathrm{kV})$ buses during normal operation.

- To provide power to the plant's $4.16 \mathrm{kV}$ and $12 \mathrm{kV}$ buses during startup, plant trips, or situations when the plant is not able to shed loads down to house levels.

- To transfer power generated at Diablo Canyon to PG\&E's distribution system.

The first function is provided by the plant main generator. Under normal operating conditions the main generator supplies power to the $4.16 \mathrm{kV}$ vital buses and to the nonvital $12 \mathrm{kV}$ and $4.16 \mathrm{kV}$ buses (see Figure B2.2.1 and in more detail Figure B2.2.2). Power is generated at $25 \mathrm{kV}$ and auxiliary transformer 11 drops this voltage down to the $12 \mathrm{kV}$ level to power nonvital $12 \mathrm{kV}$ buses $\mathrm{D}$ and E. Auxiliary transformer 12 drops the $25 \mathrm{kV}$ down to the $4.16 \mathrm{kV}$ level to power the $4.16 \mathrm{kV}$ vital $\mathrm{F}, \mathrm{G}, \mathrm{H}$, and nonvital $\mathrm{E}, \mathrm{D}$ buses.

The second function is satisfied by the $230 \mathrm{kV}$ system (switchyard). Under plant conditions when the main generator cannot supply house loads (see above), the $230 \mathrm{kV}$ switchyard provides power to the $12 \mathrm{kV}$ and 4.16kV buses. Startup transformer 11 (SU11) delivers power to the $12 \mathrm{kV}$ SU (startup) bus. This bus supplies power to the nonvital 12kV E, D buses and to startup transformer 12 (SU12). Startup transformer 12 has two secondary sides: one side supplies $4.16 \mathrm{kV}$ to the vital F, G, H, and the nonvital D buses and the other side supplies also $4.16 \mathrm{kV}$ to the nonvital $\mathrm{E}$ bus. The breaker OCB 212 is normally closed (one can identify it in Figure B2.2.2). It connects the $230 \mathrm{kV}$ switchyard to the plant via the startup transformers. Thus, the secondary side of SU12 is energized at all times down to the $4 \mathrm{kV}$ level. Breakers 52HF14, 52HG14 and 52HH14 keep the vital F, G and H buses (Figure B2.2.2) separated from the standby power source. Given a loss of offsite power event or a large drop in the load, the plant is designed to run back to house loads (about $50 \mathrm{MW}$ ) and not trip.

The third function is satisfied by the $500 \mathrm{kV}$ system. This system (switchyard) may also be used as a backup for the second function. To align the system for backup, however, requires long duration (several hours) operator action, therefore it was not quantified in the event tree analysis.

\section{B2.2.2.2 Unovallability Modelling (Nonvital Electric Power System)}

For the unavailability analysis the nonvital electric power system was considered to be composed of two subsystems: the standby offsite power to the $4 \mathrm{kV}$ vital buses $(F, G$ and $H)$ and the nonvital $12 \mathrm{kV}$ buses (D and E). The associated top events are: $O G$ and NV. Top event $O G$ questions the availability of power from the offsite grid ( $230 \mathrm{kV}$ switchyard) to the $4.16 \mathrm{kV}$ vital buses after an initiating event. Top event NV questions the availability of the nonvital $12 \mathrm{kV}$ buses after a plant initiating event. The success criteria of these top events are described in Table B2.2.1a. Technical Specification and FSAR ${ }^{2}$ requirements with respect to the operability of the subsystems are also indicated. The reliability block diagrams of top events OG and NV are shown in Figure B2.2.3. The diagram is constructed from blocks (supercomponents) whose boundaries are indicated in Figure B2.2.2. 
The reliability block diagrams (Figure B2.2.3) show the dependencies on other electrical systems: $230 \mathrm{kV}$ offsite power and dc power (dc train 12 supplies power to the controls of $12 \mathrm{kV}$ bus $\mathrm{D}$ and dc train 13 supplies the controls of bus E). Each of the plant switchyards $(500 \mathrm{kV}$ and $230 \mathrm{kV})$ has its own battery (and battery chargers) providing motive power for its respective circuit breakers. It is claimed that given a loss of offsite power, the charge on these batteries will last longer than the station batteries due to the small loads. The $4.16 \mathrm{kV}$ buses $\mathrm{E}$ and $\mathrm{D}$ supply power to the $500 \mathrm{kV}$ and $230 \mathrm{kV}$ switchyard battery chargers and air compressors, respectively. The auxiliary power for the $\mathrm{SU}$ transformers is taken from the $4.16 \mathrm{kV}$ bus $\mathrm{E}$. Both sets of auxiliary and startup transformers require $480 \mathrm{~V}$ power to operate cooling fans and cooling oil pumps. Buses $11 \mathrm{D}$ and $11 \mathrm{E}$ provide this power.

Upon loss of auxiliary power the $12 \mathrm{kV}$ buses automatically transfer to the startup bus and all five $4.16 \mathrm{kV}$ buses automatically transfer from auxiliary transformer 12 to startup transformer 12 . Upon an SI signal the vital buses transfer immediately, the nonvital ones after the main unit trip. Simultaneously a signal opens the auxiliary feeder breakers and closes the startup feeder breakers.

\section{B2.2.2.3 Quantification of Top Event Split Fractions (Nonvital Electric Power System)}

The definitions of the boundary condition split fractions associated with top events OG and NV are listed in Table B2.2.2a. The quantification of $O G$ top events involved the following assumptions:

- When the plant trips and the $500 \mathrm{kV}$ circuit breakers 532 and 632 do not open, the nonvital $12 \mathrm{kV}$ and 4.16kV buses will not transfer over to the startup power; i.e., feeders from the auxiliary transformers 11 and 12 need not open to keep the $500 \mathrm{kV}$ and the $230 \mathrm{kV}$ systems separated.

- Loss of offsite power initiator includes failure events to accomplish load rejection to house loads.

- No credit for backfeed from $500 \mathrm{kV}$.

- Circuit breaker 212 may be bypassed for maintenance (by using air switches 213 and 215).

The quantification of top event NV involved the assumption that maintenance of auxiliary feeder breakers does not contribute to the unavailability since given an initiating event, the buses would be realigned to the backup startup power supply.

For both top events it was assumed that breaker maintenance is performed in less than one hour, because usually they replace the existing breaker with an operable spare. Breaker overhauls are performed at every five or six years. Transformer maintenance is performed about once per year for about eight hours. This is done while the plant is at power. Other preventative maintenance activities are done during plant shutdowns.

Table B2.2.3a presents the quantified values of the split fractions (entries denoted by PG\&E). To provide complete information, besides the total value of a split fraction (TTL) the table also indicates the main contributors, such as unavailabilities due to hardware failures (HW), maintenance (MN), test (TS), and human error (HE). The table also provides the constituent parts of the hardware unavailability: the independent (HWI) and the dependent (HWD, i.e., common cause) failures.

Notice, there is no contribution due to test and human error. Technical Specifications require only system operability checks once every seven days by verifying correct breaker alignment and power availability. 


\section{Appendix B2}

The seismic analysis included only the $230 \mathrm{kV}$ offsite grid fragility. All the other components were assumed to have higher capacities and therefore the offsite grid was considered to be the limiting case. It is mentioned that the switchyard/plant isolation breakers 542 and 632, which are Westinghouse sulfur-hexafluoride breakers will be replaced by seismically much better qualified GE/Hitachi dead-tank SF6 breakers by the end of 1987 .

\section{B2.2.3 Vital $125 \mathrm{~V}$ DC System}

\section{B2.23.1 Fection, Configaration, Operation}

The vital $125 \mathrm{~V}$ dc system provides power to controls, protection circuitry (equipment) and instrumentation and annunciators throughout the plant. The system is configured from three $125 \mathrm{~V}$ dc subsystems: $125 \mathrm{~V}$ dc train 11, 125V dc train 12, and 125V dc train 13 (see Figure B2.2.1). Each subsystem consists of a 60 cell $125 \mathrm{~V}$ battery, a $125 \mathrm{~V}$ distribution switchgear assembly, and a battery charger. The battery chargers are located in the dc switchgear room (see also Figures B2.2.4.1, B2.2.4.2, and B2.2.4.3 for details). The switchgear assemblies each include a completely enclosed $125 \mathrm{~V}$ dc bus, circuit breakers, fuses, metering equipment, and two distribution panels. One of the panels supplies the vital loads and the other supplies the nonvital loads; they are physically separated on the left and right sides of the switchgear. Breakers on the panels may be used to disconnect all non-Class $1 \mathrm{E}$ loads from the batteries.

A total of five battery chargers are supplied; three chargers serve two of the $125 \mathrm{~V}$ dc buses (11 and 12$)$ and two chargers serve dc bus 13 . Under normal operation, each bus is powered by one battery charger and the battery charger provides the dc power for the plant. Buses 11 and 12 share a single backup battery charger in case either primary charger should become unavailable. A second battery charger is a backup charger for bus 13 . Technical specifications require that chargers 11,12 , and 13 be the normally aligned chargers. $480 \mathrm{~V}$ vital buses power the battery charges (charger 11 from bus $1 \mathrm{~F}$, charger 12 from 1G, charger 121 from bus $1 \mathrm{H}$, charger 131 from bus $1 F$, charger 132 from bus $1 \mathrm{H}$ ). Each of the chargers is connected to a dc bus through a thermal-magnetic breaker located in the dc switchgear. Manual transfer is required to align the backup battery charger.

Distribution panels 11 and 12 are connected to their respective buses by drawout (manually operated air) breakers. Panel 13 is hardwired to its bus. The batteries are sized to provide sufficient power to operate the dc loads for the time necessary to safe shutdown should a $480 \mathrm{~V}$ ac source to one or more battery chargers be unavailable. Batteries have a minimum two hour capacity.

Should a failure occur on any $125 \mathrm{~V}$ dc circuit on panel 11 or 12 , the breaker to this circuit would trip to isolate the failure. If the circuit breaker failed the drawout breaker would trip to isolate the whole panel from the battery (i.e., would cause the loss of all the loads). For panel 13 there is no drawout breaker. The isolation of this panel would have to occur through a 3000A fuse. Natural ventilation is sufficient for the battery rooms to maintain safe levels of hydrogen gas generated during charging.

The most important loads on the $125 \mathrm{~V}$ dc system are: the inverters, the $4 \mathrm{kV}$ feeder breaker controls, the diesel generator controls, control room main annunciator system and the $480 \mathrm{~V}$ motor control center relay boards. Detailed lists of loads are given in Figures B2.2.4.1 through B2.2.4.3. 
Appendix B2

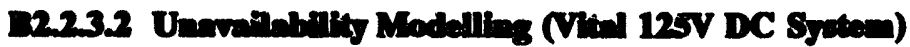

For unavailability modelling, the vital $125 \mathrm{~V}$ dc system was considered as a three train system: after an initiating event the unavailability of one, two and three trains were determined. Three top events are associated with the model: DF, DG and DH, where the first letter identifies the dc power and the second the vital ac bus to which it supplies control power. These top events question the availability of vital $125 \mathrm{~V}$ dc power on dc buses (and distribution panels) 11, 12 and 13, respectively. The success criteria for these top events are described in Table B2.2.1b. Technical Specification and FSAR requirements with respect to the operability of the system are also indicated. A representative reliability block diagram for the top events DF, DG and DH is shown in Figure B2.2.5. The diagram is constructed from blocks (supercomponents) whose boundaries are indicated in Figures B2.2.4.1, B2.2.4.2, and B2.2.4.3, respectively. Notice, top events DG and DH contain similar components to the blocks for top event DF; however, for top event DH, there are differences in blocks 1 and 3. Block DH-1 does not contain the circuit breaker that blocks DF-1 and DG-1 have, and block DH-3 has one more fuse than blocks DF-3 and DG-3. The reliability block diagram shows that the model is conservative because the failure of a battery charger does not immediately fail the system. (In some PRAs the battery charger and battery are treated as parallel components, but it is not always true that a battery charger's capability is sufficient to function adequately without its associated battery.)

The reliability block diagram (Figure B2.2.5) also shows the dependency on other electrical systems: the vital $480 \mathrm{~V}$ buses. There is no common cause failure mode modelled between the three dc trains (except seismic and ventilation; ventilation is needed to supply cooling to the $125 \mathrm{~V}$ dc switchgear rooms which also house the battery charger, but ventilation is powered by the $480 \mathrm{~V}$ buses).

\section{B2.233 Quantisication of Top Event Split Practions (Vitw 125V DC Syatem)}

Technical Specifications restrictions, that one train may be unavailable due to maintenance at any one time, introduce certain dependencies between the trains and this renders the split fractions conditional on the success or failure of the preceding dc train in the event tree. A single train unavailability will be the sum of the hardware unavailability (HW) and the maintenance (MN) unavailability. Since there are no scheduled tests performed resulting in an unavailable dc train, there is no test contribution to the unavailability.

The definitions of the boundary conditions and the associated conditional top event split fractions are listed in Table B2.2.2b. The quantification of the top event split fractions involved the following assumptions:

- Redundant battery chargers and associated breakers are given credit only during charger/breaker performance test and charger/ breaker maintenances.

- Panel feeder breaker (dc trains 11 and 12) maintenance is included in the maintenance of the panel itself.

- Vital $480 \mathrm{~V}$ ac buses $1 F, 1 \mathrm{G}, 1 \mathrm{H}$ are available.

- During switching of ac power to the battery chargers, the batteries supply power to the loads.

- All scheduled maintenance and testing is performed in a way that does not disable the system during power operation.

Table B2.2.3b presents the.quantified values of the conditional split fractions; CSF. To provide complete information the table also indicates the total value of a split fraction (TTL) and other contributors. The dominant contributors to the hardware failure are battery failure on demand and battery charger failure during operation. 


\section{Appendix B2}

In terms of seismic failures, the batteries, buses and breaker panels are modelled. (Seismic failures of the battery charger are modelled with the vital ac system.) The auxiliary building is modelled with the dc system, because failure of the dc system impacts all the important support and frontline systems.

\section{B2.2.4 Vital AC System - Unit 1}

\section{B2.24.1 Punction, Configaration, Opernation}

The function of the vital ac system is to provide power to safety related equipment under all foreseeable conditions.

The DCPRA considered this system as composed of six subsystems: Three of the subsystems are the 4.16kV $(F, G, H)$ and $480 \mathrm{~V}(1 \mathrm{~F}, 1 \mathrm{G}, 1 \mathrm{H})$ vital ac buses and the associated hardware at Unit 1, the other three are the standby startup feeder breakers to these buses (see Figures B2.2.1 and B2.2.7.1).

During normal operation the $4.16 \mathrm{kV}$ system is powered through auxiliary transformer 12 . The standby power source is the $230 \mathrm{kV}$ system via the startup transformers SU11 and SU12 and the $12 \mathrm{kV}$ startup (SU) bus. The $480 \mathrm{~V}$ vital buses $1 \mathrm{~F}, 1 \mathrm{G}$, and $1 \mathrm{H}$ are fed from the $4.16 \mathrm{kV}$ vital buses $F, G, H$, respectively, through $4160 / 480 \mathrm{~V}$ transformers. The control power to the $4.16 \mathrm{kV}$ breakers is provided by the $125 \mathrm{~V}$ dc system (manual transfer is also possible):

DC panel 11 to $4.16 \mathrm{kV}$ bus $\mathrm{F}$

DC panel 12 to $4.16 \mathrm{kV}$ bus $\mathrm{G}$

DC panel 13 to $4.16 \mathrm{kV}$ bus $\mathrm{H}$

In the case of an auxiliary feeder breaker trip the $4.16 \mathrm{kV}$ buses automatically transfer to the standby sources. Transfer is also initiated by various plant conditions listed in Table B2.2.4. This table shows the transfers and the diesel states as a function of plant conditions. The transfer usually occurs after a short delay $(.8 \mathrm{sec})$ to allow for voltage decay. Usually only low voltage loads operated by magnetic controllers (such as containment fan coolers) are tripped and restarted. In the case when there is an SI signal or in other conditions the transfer is immediate, the "loads do not strip" (see these notes also in the table) and the diesels start. If there is a LOSP, direct transfer to the diesel occurs. If in $10 \mathrm{sec}$ after a transfer to the startup transformer undervoltage was detected on the vital buses, a $2 / 2$ relay logic (relays 27 HFB3 and 24HFB4) would start the diesel and load stripping would occur in 20 seconds.

In order that a transfer could occur, certain permissives have to be satisfied.

For transfers without and with an SI signal:

1. Presence of the initiating signal.

2. No undervoltage on the startup transformer.

3. No electrical fault on the bus.

4. Startup and diesel feeder breaker tripped.

5. Transfer to diesel not in progress.

6. Auto transfer switch cut in main control room. 
For transfer to the diesel:

1. Vital bus undervoltage.

2. No electrical fault on the bus.

3. Diesel is up to rated voltage.

Table B2.2.4 also lists the most important loads on the 4.16kV system. The loads on the vital $480 \mathrm{~V}$ buses are: primary makeup water pumps, lighting, batter' chargers and inverters, diesel generator auxiliary loads, ventilation fans and containment fan coolers.

The 4.16kV switchgear is located indoors and requires ventilation; the breakers are in individual cubicles cooled by fans powered from the associated $480 \mathrm{~V}$ bus. The $480 \mathrm{~V}$ ac and $125 \mathrm{~V}$ dc vital switchgear compartments (see Figure B2.2.6b) are supplied cooling air by two trains of supply and exhaust fans. The power supplies to these trains are $480 \mathrm{~V}$ buses $1 \mathrm{~F}$ and $1 \mathrm{H}$. Each room has an inlet and outlet duct with a fuseable link fire damper. The inadvertent closing of the fire dampers was included in the unavailability model of the vital ac system.

The failures of the vital ac system represent potential for event initiation: loss of a 4.16kV train could result in loss of component cooling water or auxiliary saltwater. Loss of $480 \mathrm{~V}$ could cause a loss of switchgear ventilation (later a loss of inverters, instrument buses) and trigger a plant trip.

\section{B2.242 Unovailability Modelling (Vital AC Syatem - Unit 1)}

The Unit 1 vital ac electric system unavailability model is represented by six top events corresponding to the six electric subsystems. These are: AF, AG, AH, and SF, SG, SH. In the electrical support system event tree top events $A F, A G$, and $A H$ question the availability of vital ac power on $4.16 \mathrm{kV}$ buses $F, G, H$ and $480 \mathrm{~V}$ buses 1F, 1G, 1H, respectively. Top events SF, SG, SH question the closure of circuit breakers 52HF14, 52HG14, 52HH14 supplying 4.16kV buses F, G, H, respectively, from the startup transformer following a plant trip. The success criteria for these top events are described in Table B2.2.1c. Technical Specification and FSAR requirements with respect to the operability of the system are also indicated. The reliability block diagrams of both types of top events, AF and SF are shown in Figure B2.2.6. The diagrams are constructed from blocks (supercomponents) whose boundaries are indicated in Figures B2.2.7.1 and B2.2.7.2. The reliability diagrams also show the dependency on dc power. If any dc train is unavailable, the corresponding ac train is also assumed to be unavailable.

\section{B2.2.4.3 Quantification of Top Event Split Fractions (Vital AC System - Unit 1)}

The quantification of the top events associated with the vital ac system involved the following assumptions:

- The 4.16kV switchgear room does not require ventilation.

- The two trains of fańs for the $480 \mathrm{~V}$ switchgear rooms would not be out for maintenance at the same time.

- Detection of power loss on the $4.16 \mathrm{kV} / 480 \mathrm{~V}$ buses would be immediate (not by the weekly surveillance check).

- Maintenance of $4.16 \mathrm{kV}$ breakers would only take one hour (replacement of a spare breaker).

- Breakers, buses, transformers for ac trains require infrequent maintenance. 


\section{Appendix B2}

Common cause failure was assumed between breakers to open (auxiliary feeder breakers) and to close (startup feeder breakers) on demand. These common cause failures were assumed to be recoverable (even for three trains) for all accidents except for large and medium LOCAs, because of the insufficient time available. Recovery would consist of operator action to manually operate the feeder breakers in the $4.16 \mathrm{kV}$ switchgear rooms.

Due to the common cause failure (and recovery) as well as Technical Specification dependencies, the split fractions evaluated are conditional on the success or failure of the preceding top events in the electrical support system event tree. The dependency on the dc system has also complicated the quantification of the vital ac systems split fractions. Table B2.2.2c lists the definitions of the boundary conditions and the associated conditional top event split fractions. The quantified values of the conditional split fractions are listed in Table B2.2.3c (entries denoted by PG\&E).

Notice that the maintenance contribution to the unavailability is small because the components modelled require infrequent maintenance. There are no test or human error contributions because relevant tests are done during shutdown and human errors committed would be detected before resuming operation.

Seismic unavailability is modelled for the following plant components:

- Turbine Building Shear Wall

- 125V DC Battery Chargers

- 4.16kV/480V Transformers

- Turbine Building Strut

- 4.16kV Switchgear (in the case of when the strut failed and when not failed)

- 4.16kV Safeguard Relay Panel (for bus transfer in the case when the strut failed and when not failed)

- 4.16kV Unit 2 Bus F Potential Transformer (in the case of when strut failed and when not failed).

\section{B2.25 Vithl AC/DC Systems; Unit 2}

\subsubsection{Cewerd}

This system is modelled in the DCPRA primarily to determine the unavailability of the Unit 2 Auxiliary Saltwater System, where a crosstie to Unit 1 is beneficial. For seismic initiators the crosstie is not modelled.

\section{B2.25.2 Panctions, Configaration, Operation}

The vital ac/dc system of Unit 2 is modelled in the DCPRA for the case when offsite power is unavailable. The systems function, during such an event, is to provide motive and control power to the engineered safety loads (Auxiliary Saltwater System) of Unit 2. The system was analyzed to determine the unavailability of the combined $4.16 \mathrm{kV}$ ac, $480 \mathrm{~V}$ ac, and $125 \mathrm{~V}$ dc power to these loads.

The system, similarly to the vital ac and dc systems of Unit 1, was considered to be composed of three subsystems representing the three vital ac and dc trains of Unit 2 . Correspondingly, there are three top events associated with the subsystems: BF, BG and BH. These top events describe the unavailabilities of:

Unit $2,4.16 \mathrm{kV}$ bus $\mathrm{F}, 480 \mathrm{~V}$ bus $2 \mathrm{~F}$, and $125 \mathrm{~V}$ dc train 21 ; Top Event BF. 
Appendix B2

Unit 2, 4.16kV bus G, $480 \mathrm{~V}$ bus $2 \mathrm{G}$, and $125 \mathrm{~V}$ dc train 22; Top Event BG.

Unit 2, 4.16kV bus H, 480V bus $2 \mathrm{H}$, and $125 \mathrm{~V}$ dc train 23; Top Event BH.

The configurations and operations of the buses and trains are similar to those of Unit 1 described in Sections B2.2.3 and B2.2.4.

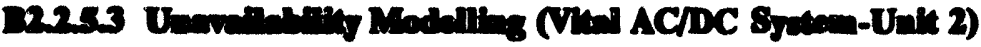

Top events BF, BG, and BH are equivalent to the combined top events AF and DF, AG and DG, AH and DH previously discussed. The reliability block diagram for top event BF is shown in Figure B2.2.8. Block diagrams for top events BG and BH are similar. The diagrams are constructed from blocks (supercomponents) whose boundaries are indicated in Figures B2.2.9.1 and B2.2.9.2. Block 4 (backup charger 2-21) is treated as being unavailable; thus the block diagram reduces to five blocks in series. The success criteria of the top events are described in Table B2.2.1d. Technical Specifications and FSAR requirements with respect to the operability of the system are the same as those given in Table B2.2.1b and B2.2.1c for Unit 1 and are therefore not repeated.

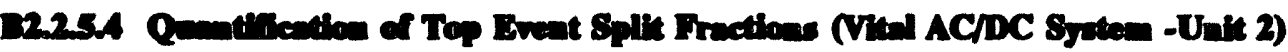

Unit $2 \mathrm{ac} / \mathrm{dc}$ train $\mathrm{H}$ is slightly different from trains $\mathrm{F}$ and $\mathrm{G}$. Train $\mathrm{H}$ has one less breaker but one additional fuse. This difference is not modelled. It does not affect the results since the two failure frequencies are of the same order.

The assumptions concerning the failure rates of the components, test and maintenance conditions, common cause failures and recoveries, operational conditions, human errors, etc. are the same described in Sections B2.2.3 and B2.2.4 for the Unit 1 vital dc and ac systems.

Due to Technical Specification dependencies and common cause failures, the quantified split fractions are conditional on the success or failure of the preceding top events in the electrical support system event tree. The definitions of the boundary conditions and the associated conditional top event split fractions are shown in Table B2.2.2d. The quantified values of the conditional split fractions are given in Table B2.2.3d (entries denoted by PG\&E).

One can see from the data that there are only small maintenance and no test or human error contributions to the unavailability of top events BF, BG, and BH. This is a consequence of the assumptions made; the contribution to the maintenance unavailability is small because the components modelled require infrequent unscheduled maintenance (during operation) and tests. Scheduled maintenance and tests that would make the system unavailable are done during shutdown and it is unlikely that any human error committed would not be detected before resuming operation.

Seismic unavailability was not explicitly modelled. It was assumed that seismic failures for similar components are correlated and do not allow recovery by crosstying buses. Thus, it was decided that seismic unavailability does not impact the model. 


\section{B2.2.6 Instrument AC System}

\section{B2.2.6.1 Dunction, Comfiguration, Operation}

The function of the instrumeat ac system is to maintain a supply of $120 \mathrm{~V}$ ac to the vital instrument loads of the plant unit. The system consists of four channels: I, II, III, and IV. Channels I, III, and Channels II, IV aro somowhat difierent: for Chennels I and III, each involves two inverters and two distribution panels; while for Channols II and IV, each represents only one inverter and one distribution panel. There are three power sources for the inverters:

- $480 \mathrm{~V}$ vital bus,

- $480 \mathrm{~V}$ vital bus via a battery charger, and

- 125V de battery.

The inverters feed the $120 \mathrm{~V}$ ac panels. In addition, there is a standby transformer/regulator set (transformer) that may subetitute for one of the inverters (when the inverter is in unscheduled maintenance). For clarification, more details are given in the following system description table (see also Figure B2.2.1):

\section{Instrument AC Systom Description}

\begin{tabular}{|c|c|c|c|c|c|}
\hline \multirow[t]{3}{*}{ Channel } & \multirow[t]{3}{*}{ Panel } & \multirow{3}{*}{$\begin{array}{l}\text { Panel Power } \\
\text { Sources }\end{array}$} & \multicolumn{3}{|c|}{ Support Systems } \\
\hline & & & \multicolumn{2}{|c|}{ For Inverters } & \multirow{2}{*}{$\begin{array}{c}\text { For X-r } \\
480 \text { V AC }\end{array}$} \\
\hline & & & $480 \mathrm{~V} A C$ & 125 V DC & \\
\hline \multirow[t]{2}{*}{ I } & PY-11 & Inv.11, X-r & 1F & 11 & 10 \\
\hline & PY-11a & Inv.11 $u^{*}$, X-r & 1F & 11 & $1 G$ \\
\hline II & PY-12 & Inv.12, X-r & 10 & 12 & $1 G$ \\
\hline \multirow[t]{2}{*}{ III } & PY-13 & Inv.13, X-r & 1H & 13 & $1 G$ \\
\hline & PY-13a & Inv.13a*, X-r & $1 \mathrm{H}$ & 13 & $1 G$ \\
\hline IV & PY-14 & Inv.14, X-r & 1H & 12 & $1 G$ \\
\hline
\end{tabular}

*Inverters 11a and 13a are not safety related (only supplemental) equipment; they are required for modelled systems in the plant model, e.g., the 10\% steam dump valves.

Adequate cooling to the inverters is maintained by the $480 \mathrm{~V}$ switchgear ventilation (the non-reviewed Top Event SV queations its availability). One has to keep in mind that the dc trains supply support power to the ac trains. Therefore, after a plant trip if a dc train is unavailable, the associated ac train will also be unavailable. It is clear from the above table that for Channels I, II, and III there is such dependency. Channel IV is different because its dc and ac support systems are independent.

Among the instrument ac system loads the most important are the Nuclear Steam Supply System (NSSS) instrumentation and the Solid State Protection System.

For operating the inverters there is no preferred source of power; the inverter has an input diode, which gates the highest instantaneous voltage source. The $480 \mathrm{~V}$ ac and $125 \mathrm{~V}$ dc feeder breakers are closed. Plant statistics show that $90 \%$ of the. load is supplied by the dc source and $10 \%$ is supplied by the ac source. If both sources fail the standby transformer may be used to supply any one of the power panels. The transformer is 
fed from vital bus 10 by the breaker 52-1G-43. If an inverter failed it would take 15 minutes to realign a penel to the standby transformer (close the feeder breakers).

Given low of 20 power, the inverter switches to the battery without dolay. After ac is restored (diesel generator) there is a 40 second dolay to switch beck to $480 \mathrm{~V}$ ac to allow voltage to stabilize. Loss of ac power to the invertere is alarmed in the control room.

No scheduled maintenance is performed on the inverters while at power. Testing is done during refueling (18 month intervals). The availability of the instrument ac system is very important for plant safety. Loss of two inetrument ac channols could lead to a plant trip; if this happens whilo the plant is above $10 \%$ power, the plant will trip becauce the instruments indicate that a RCP has failed. In addition, even a loss of one channel could result in a plant trip, because loss of power to steam flow control instruments (on the Hagan racks) may cause wrong steam flow control, which could then cause a plant trip.

\section{B2.2.6.2 Unavailability Modelling (Instrument iC System)}

Four top events represent the channels of the instrument ac system. They are: 11, 12, 13, and 14. These top ovents are in the mechonical and actuation systems support ovent tree (not in the electrical support ovent treo). They question the availability of $120 \mathrm{~V}$ instrument ac distribution Channels I, II, III, and IV, respectively. The success criteria of these top events are deecribed in Table B2.2.1e. Technical Specification and FSAR requirements with respect to the operability of the system are also indicated. The reliability block diagrams of the top events are shown in Figure B2.2.10. The diagrams are constructed from blocks (supercomponents) whose boundaries are marked in Figures B2.2.11.1 through B2.2.11.4. The reliability diagrams also show the dependency on the supporting power supplies (notice the difference for top event I4).

\section{B2.2.6.3 Qunatification of Top Event Split Fractions (Instrument AC System)}

The quantification of the top events associated with the instrument ac system involved the following main essumptions:

- Although the inverters have both an ac and a dc power source, the breakers for these sources are modelled as being both required; this was done to reduce the number of split fractions and boundary conditions. The procedure is conservative.

- There is no common cause failure mode between instrument ac system channels.

- Any or all of the instrument channels may be in maintenance and aligned to the backup transformer. This assumption is considered to be conservative since the plant is required to shutdown when more then one panel is not powered from its inverter source.

- To realign a distribution panel to the backup power supply ( $\sim 15$ minutes) the channel is considered to be unavailable. No human error is assumed in aligning the beckup.

The top event boundary conditions were determined on the availabilities of the support systems to the inverter and the backup transformer. They are listed in Table B2.2.2d. Each top event has essentially three boundary conditions. For Top Event I2, two additional split fractions are required for seismic events; these are 123 and 124. These conditional split fructions calculate the conditional probability of failing instrument Channel II, given failure of instrument Channel I for boundary condition 1 and boundary condition 2, respectively. 


\section{Appendix B2}

Table B2.2.3d presents the quantified values of the (non-conditional and conditional) split fractions (see entries denoted by PG\&E). Notice, that there is no contribution due to common cause failures, test and human errors. (Technical Specifications require only the verification of the alignment of the buses at least once every roven days.) The maintenance contribution appears because of the time period required to align the backup transformer for unscheduled maintenance of an inverter.

For seismic initiators, only the inverters, process control and protection racks, and pressure delta-p transmitters were modelled. The remaining components have median fragilities greater than $10 \mathrm{~g}$ and therefore were not modelled. 



\section{Centimeter}

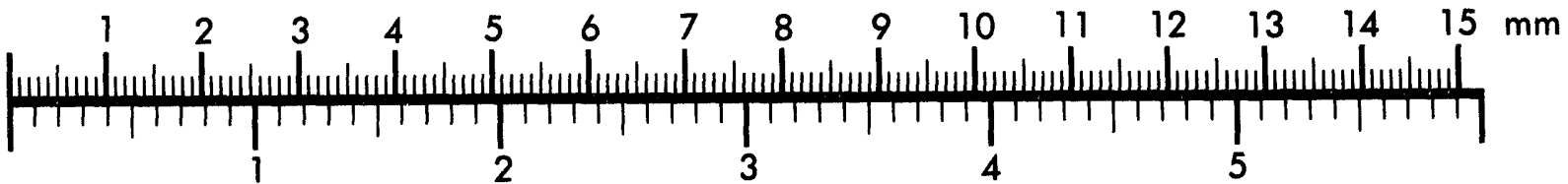
Inches
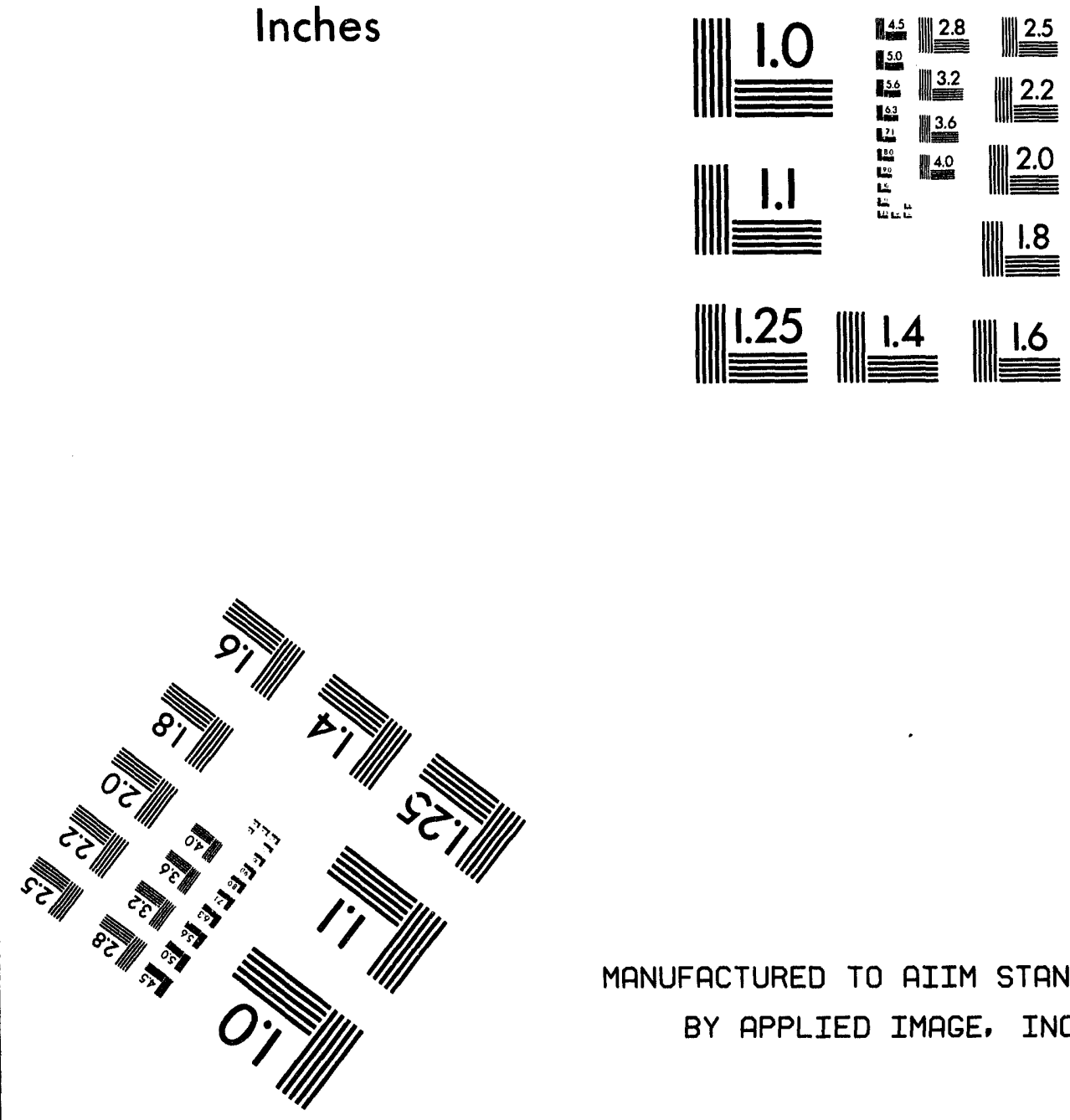

MANUFACTURED TO AIIM STANDARDS BY APPLIED IMAGE, INC.

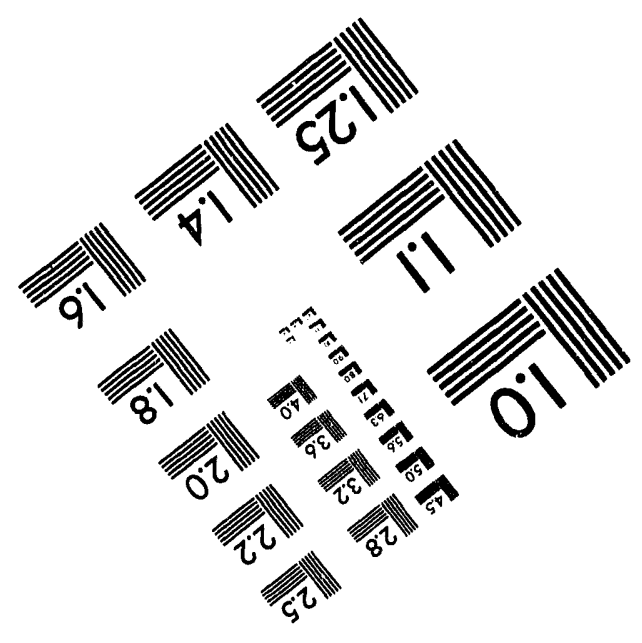



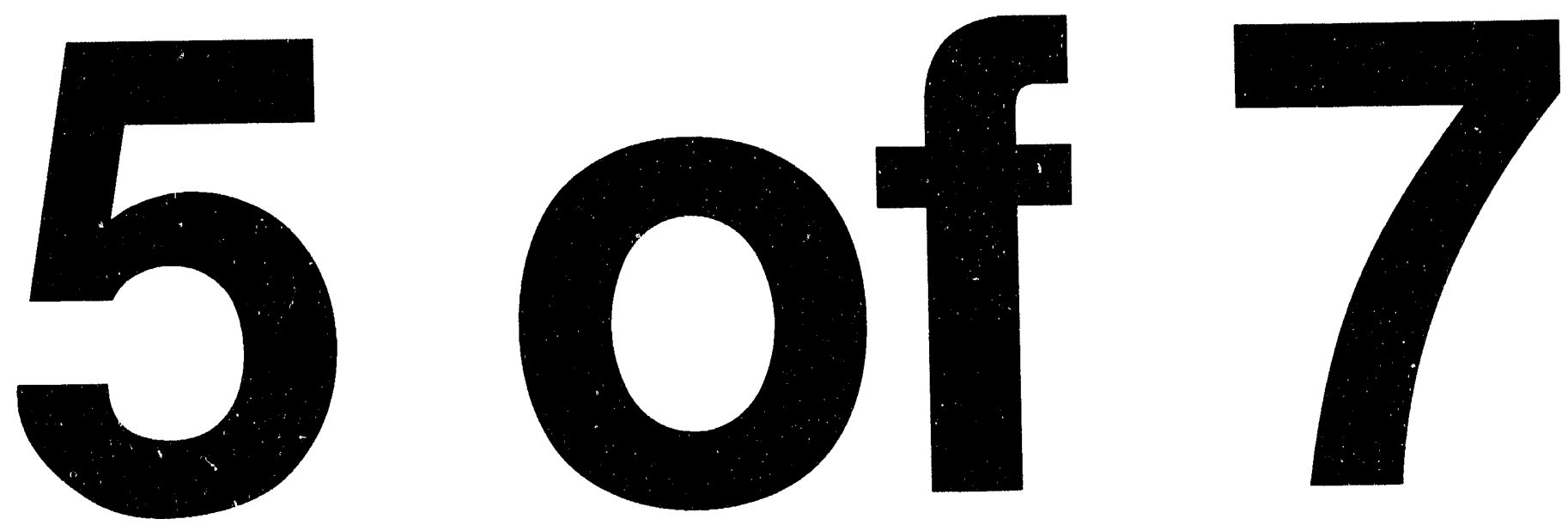


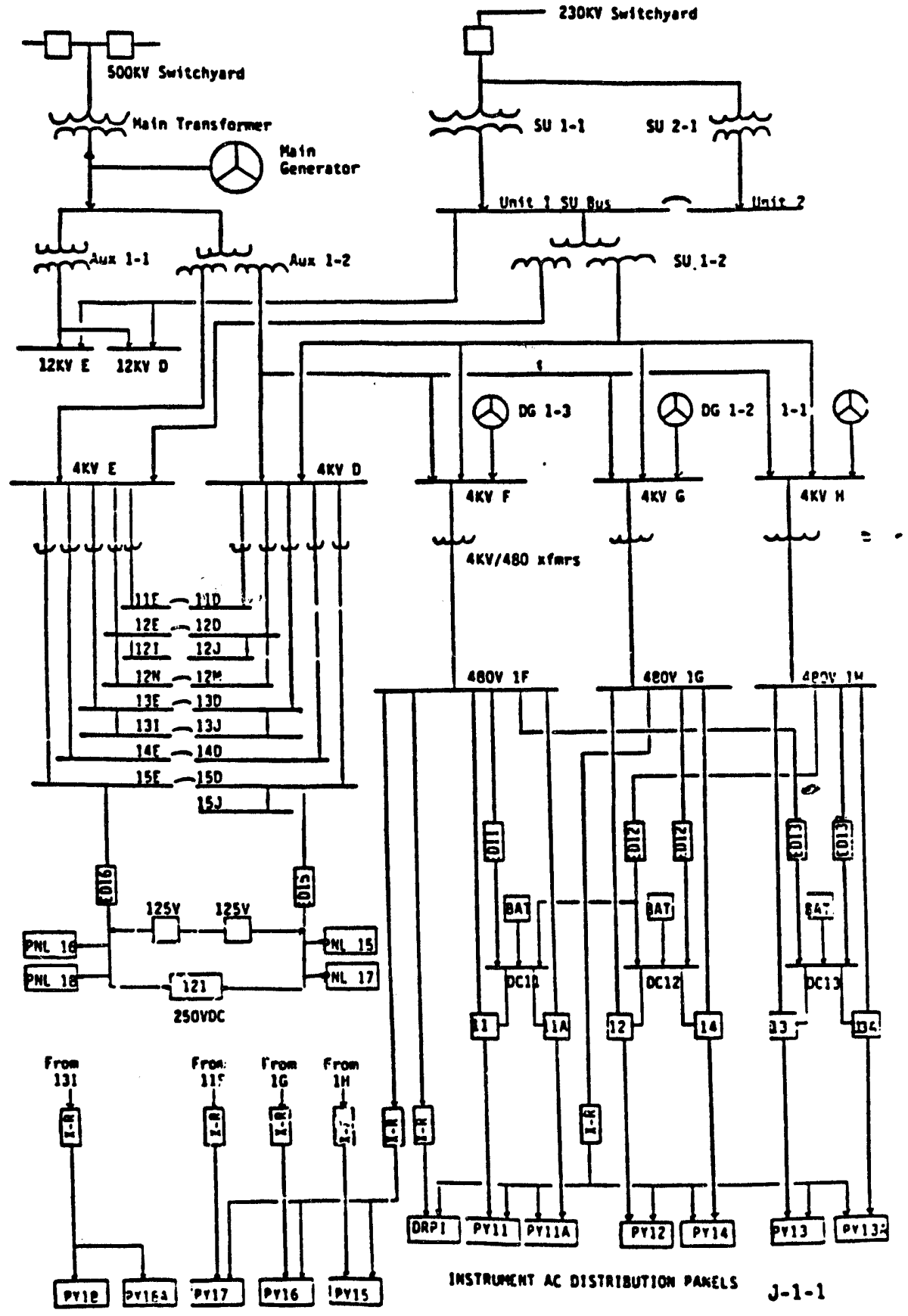

Figure B2.2.1 Electrical distribution overview for Unit 1 of the Diablo Canyon Power Plant

B2-13

NUREG/CR-5726 


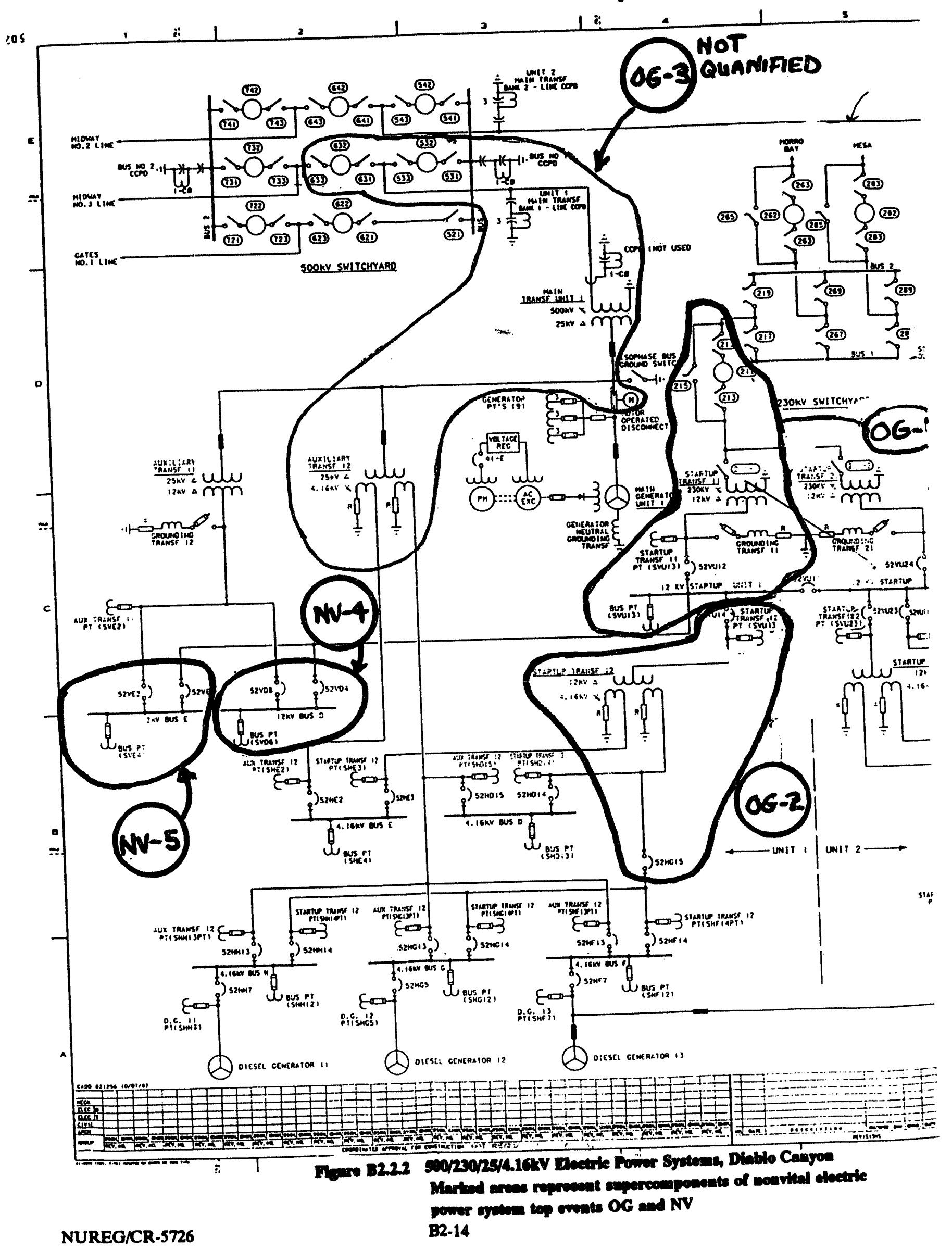




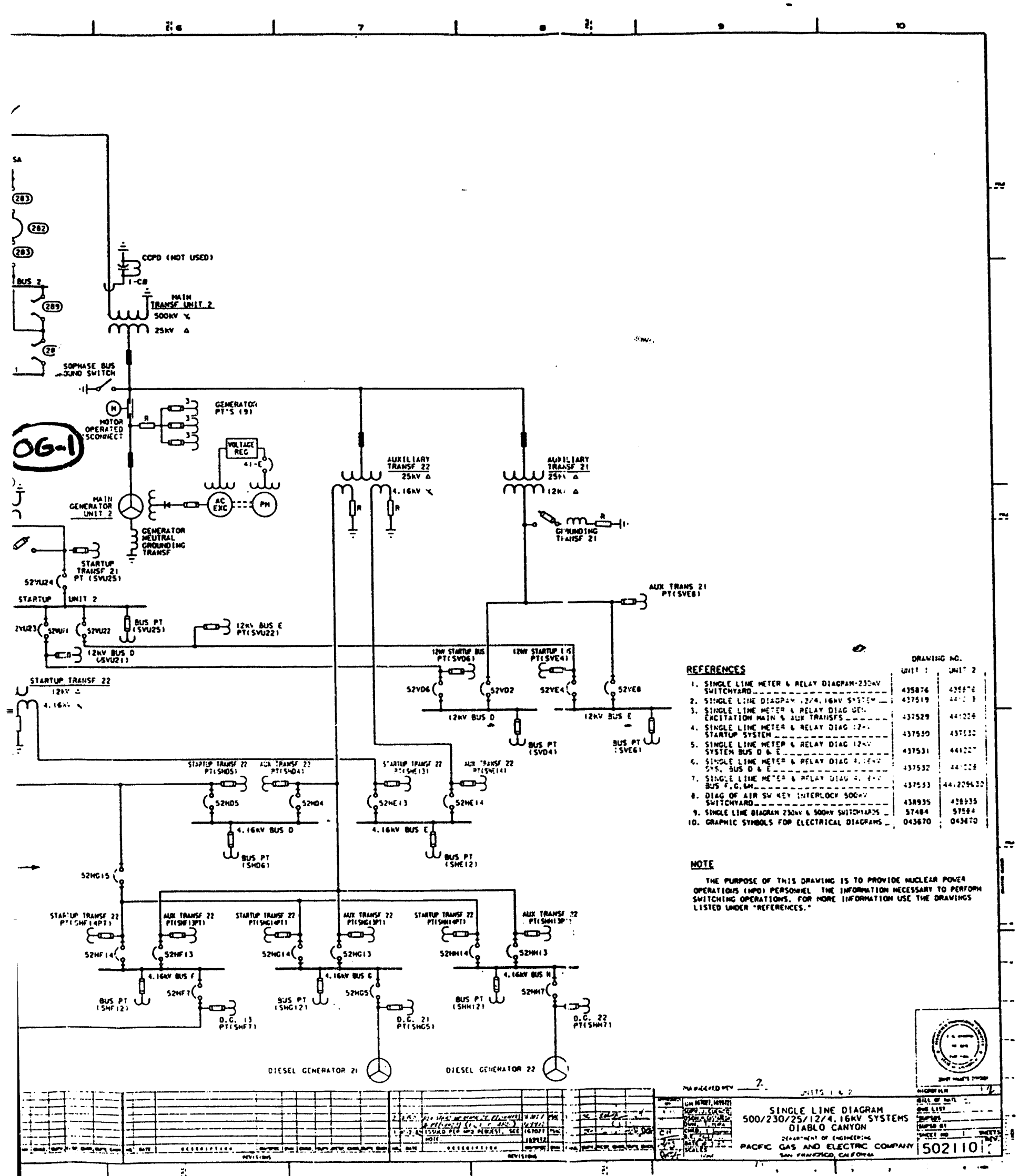

DIAGRAM D.2.1.1-1 


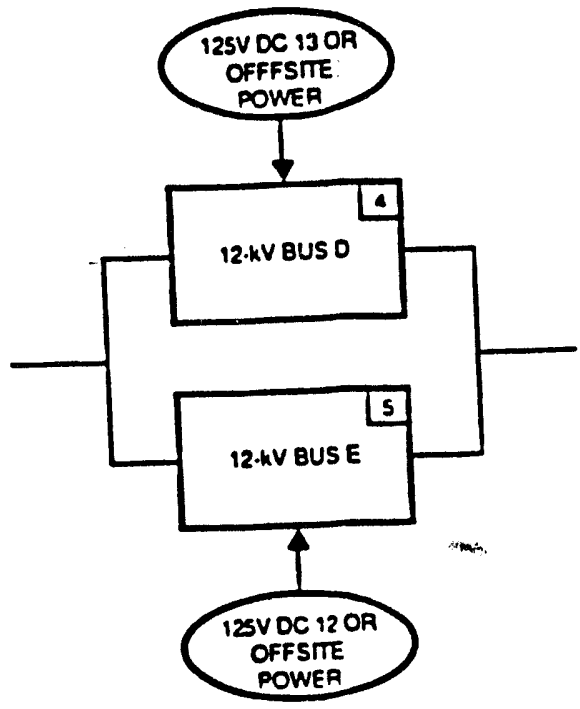

Top Event OG

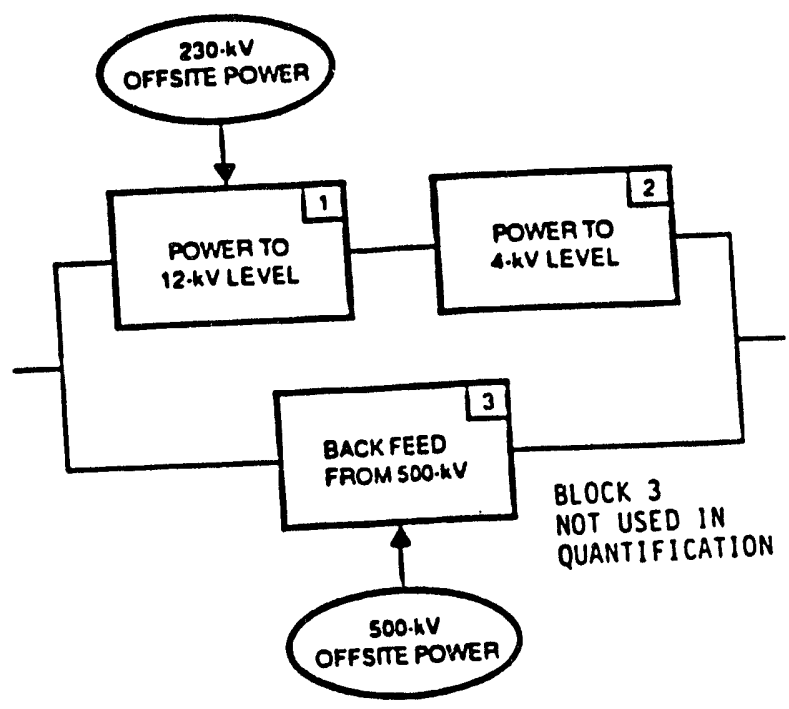

Top Event NG

Figure B2.2.3 Reliability block diagrams for nonvital electric power system top events OG and NG 


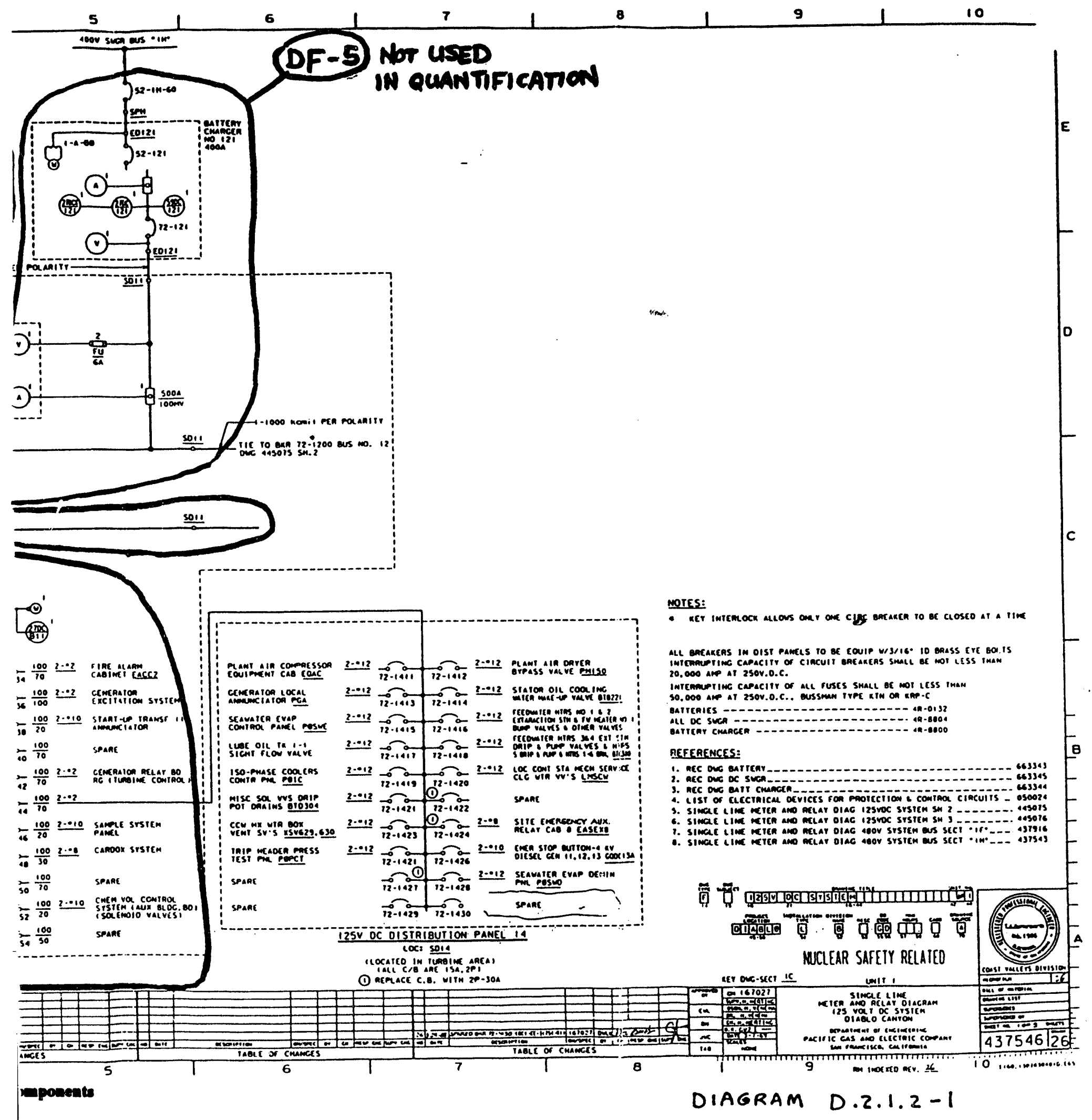




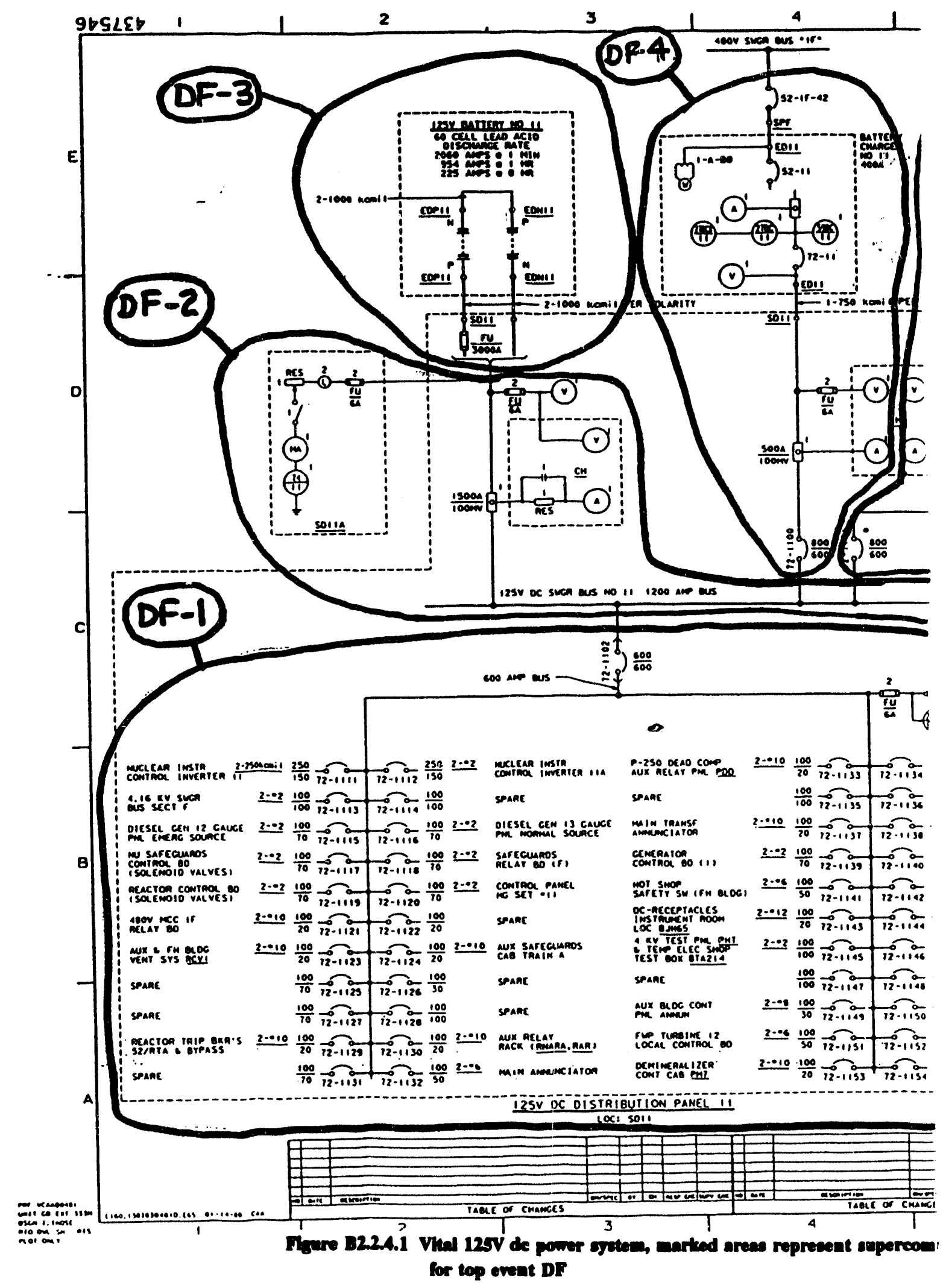

NUREG/CR-5726

B2-16 

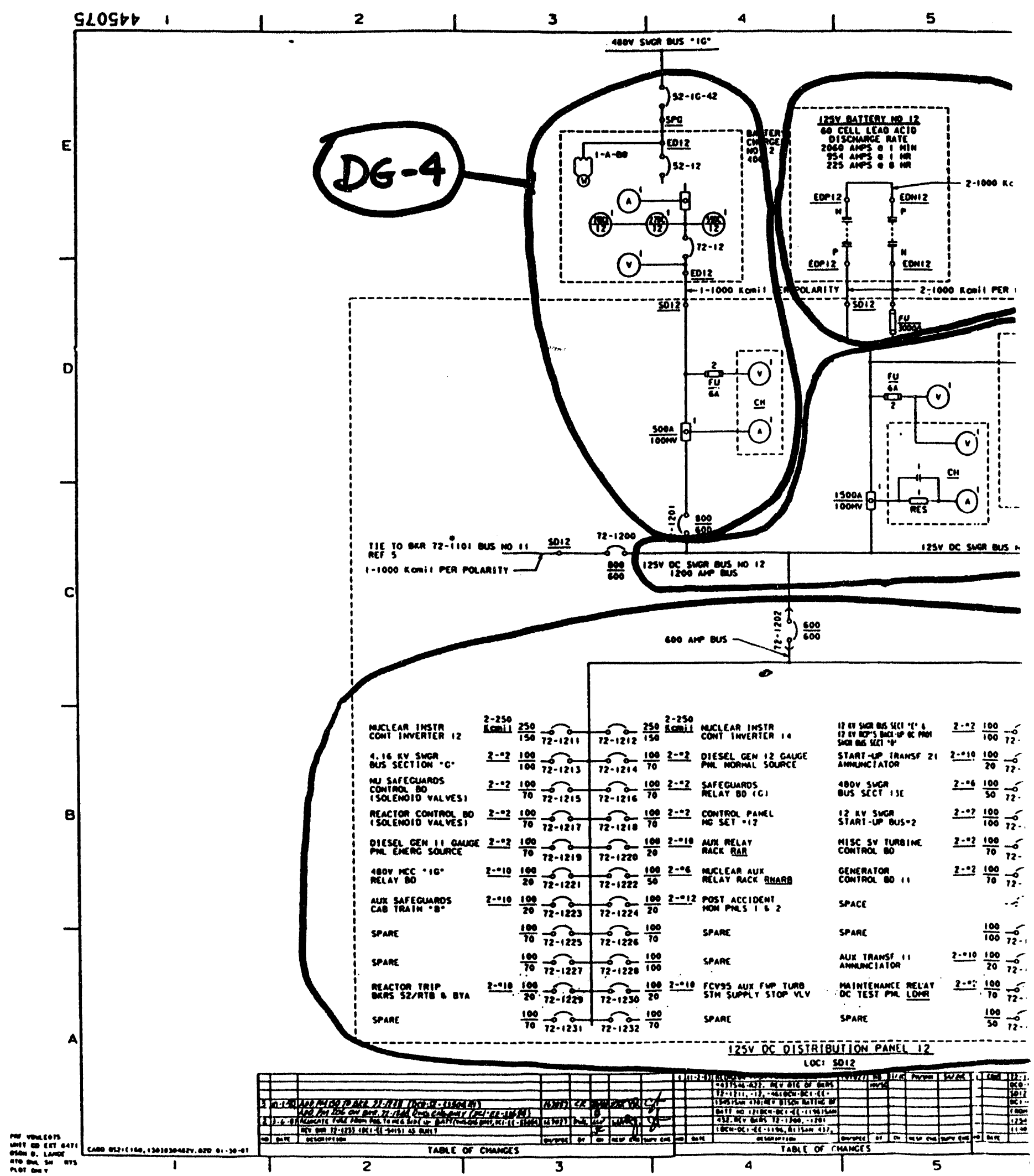

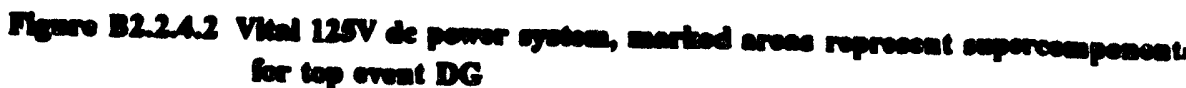




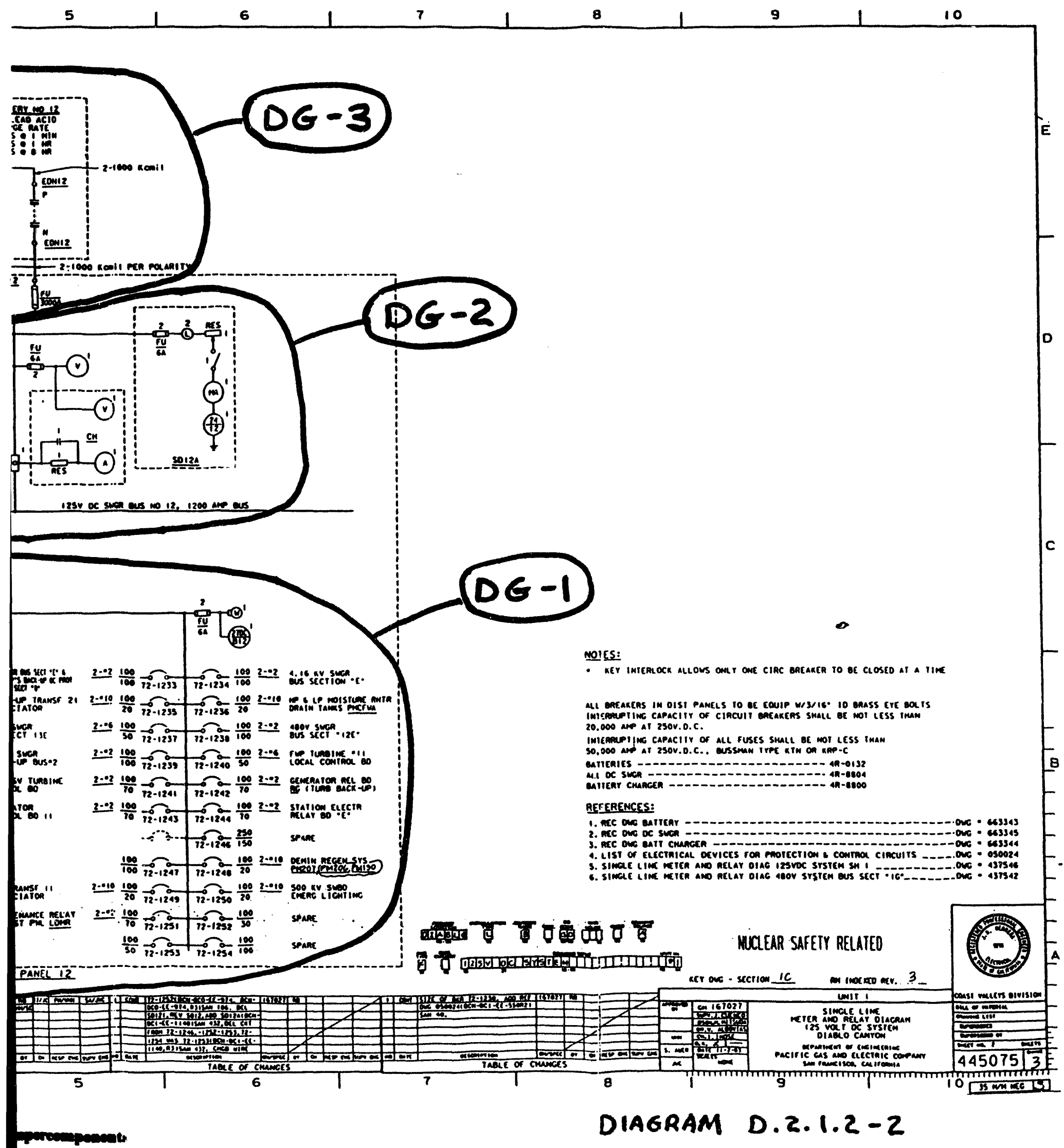

NUREG/CR-5726 


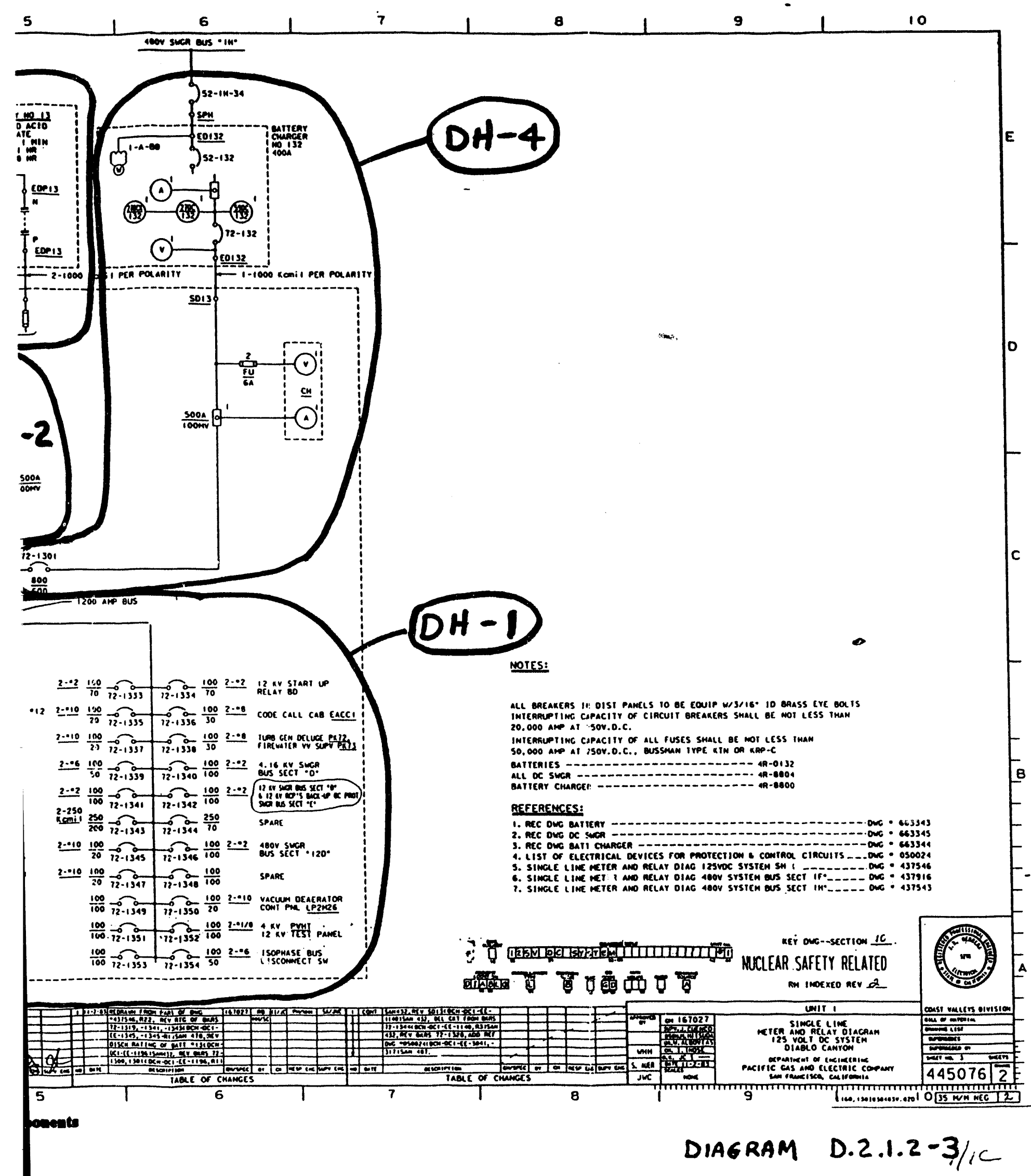




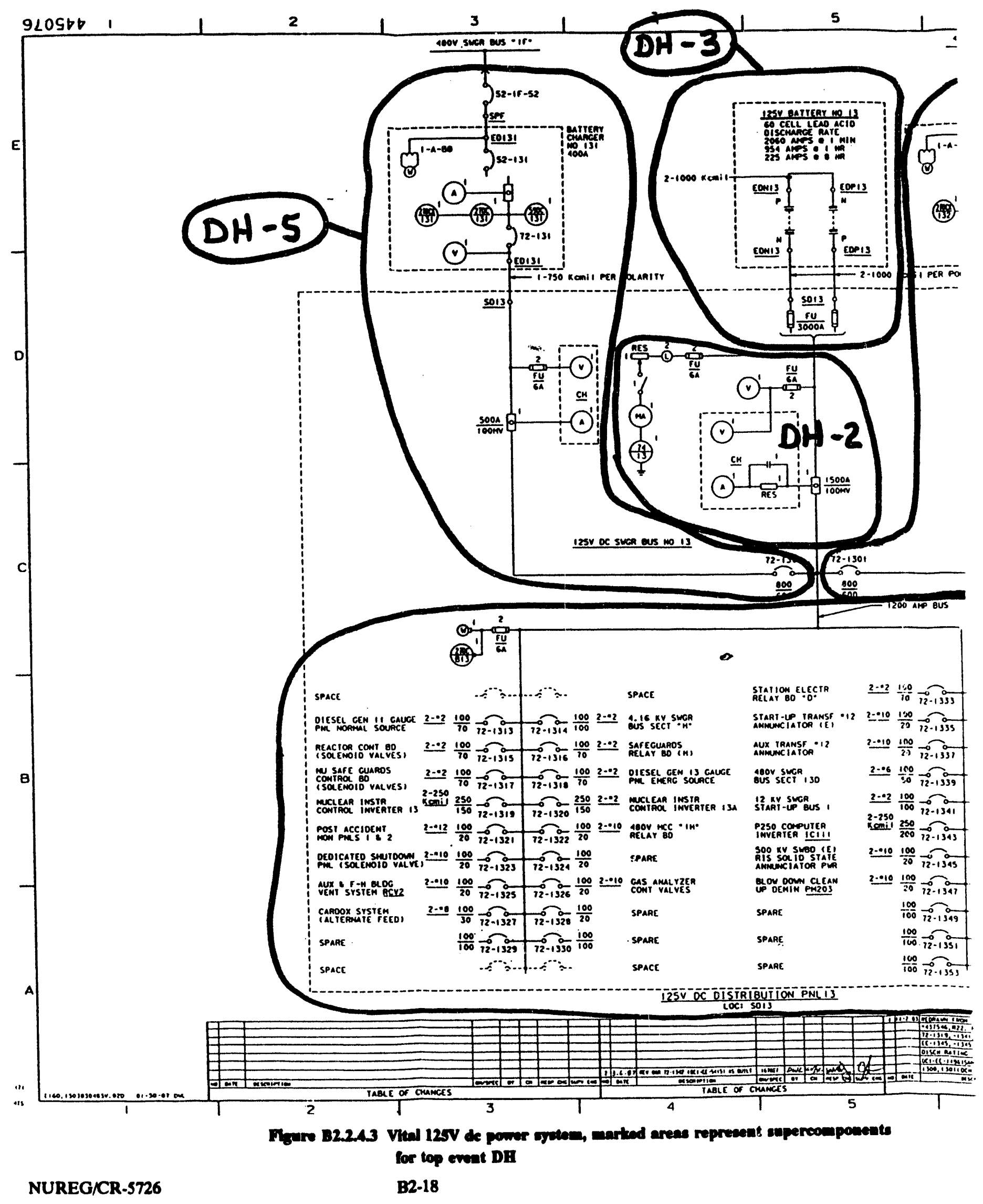


Appendix B2

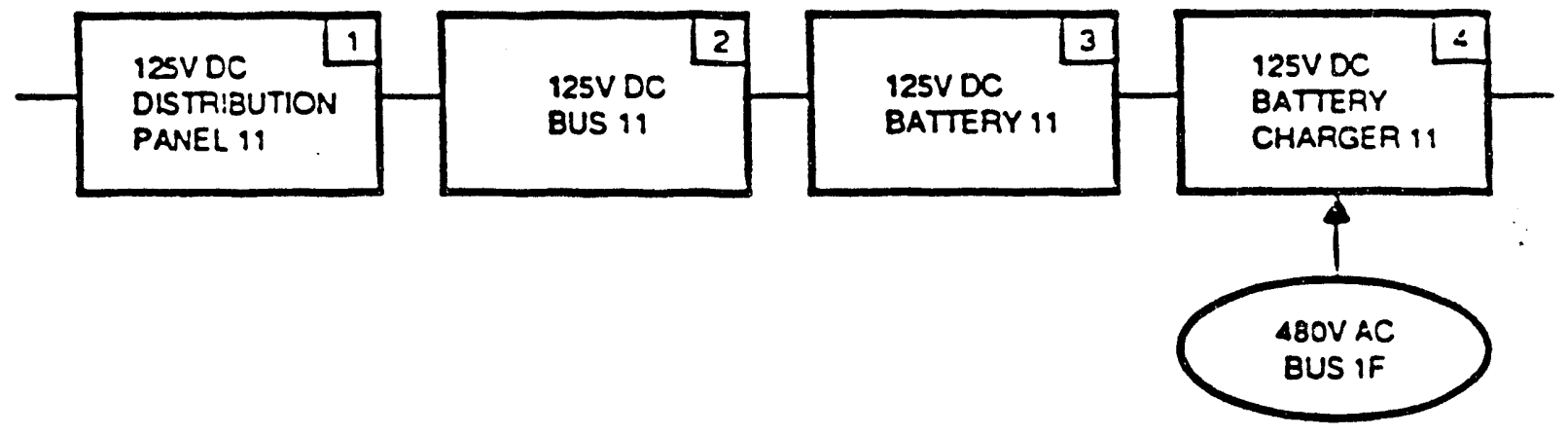

Figure B2.2.5 Reliability block diagram for vital $125 \mathrm{~V}$ de power system top event DF (block diagrams for top events DG and DH are similar) 


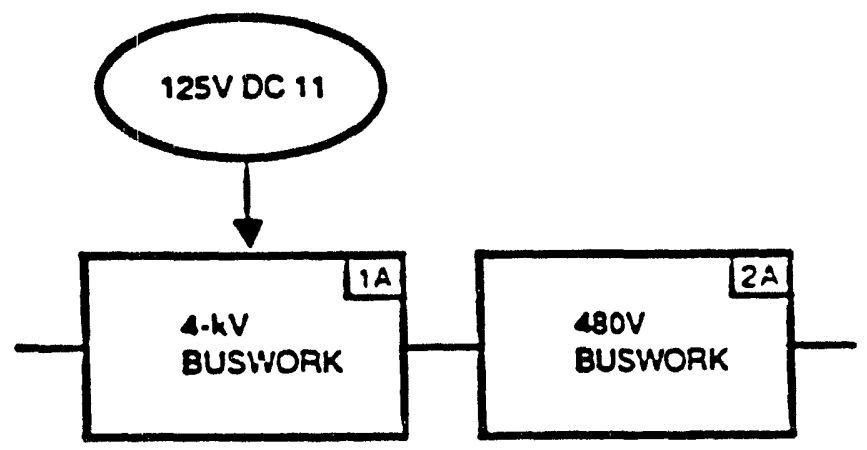

Top Event AF

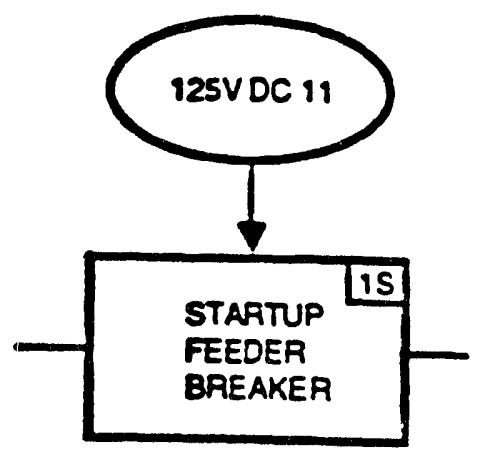

Top Event SF

Figure B2.2.6 Reliability block diagrams for vital ac electric power system top events AF and SF 


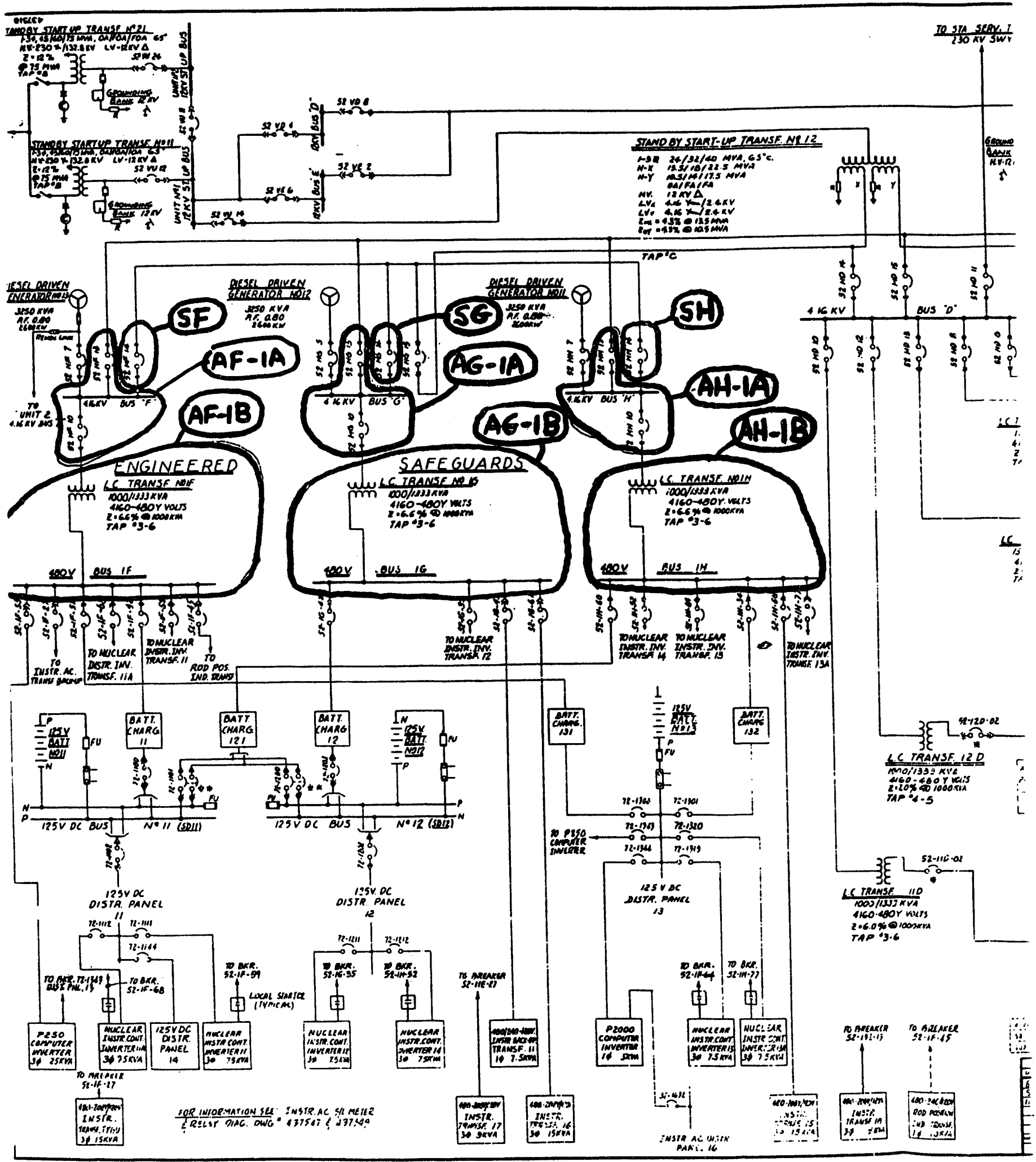

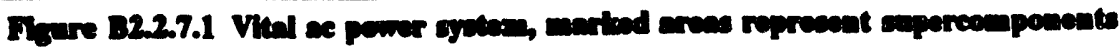

(blodte) fer top ownte AF, AG, AR, and 8F, 8G, 8I

B2-21

NUREG/CRST26 


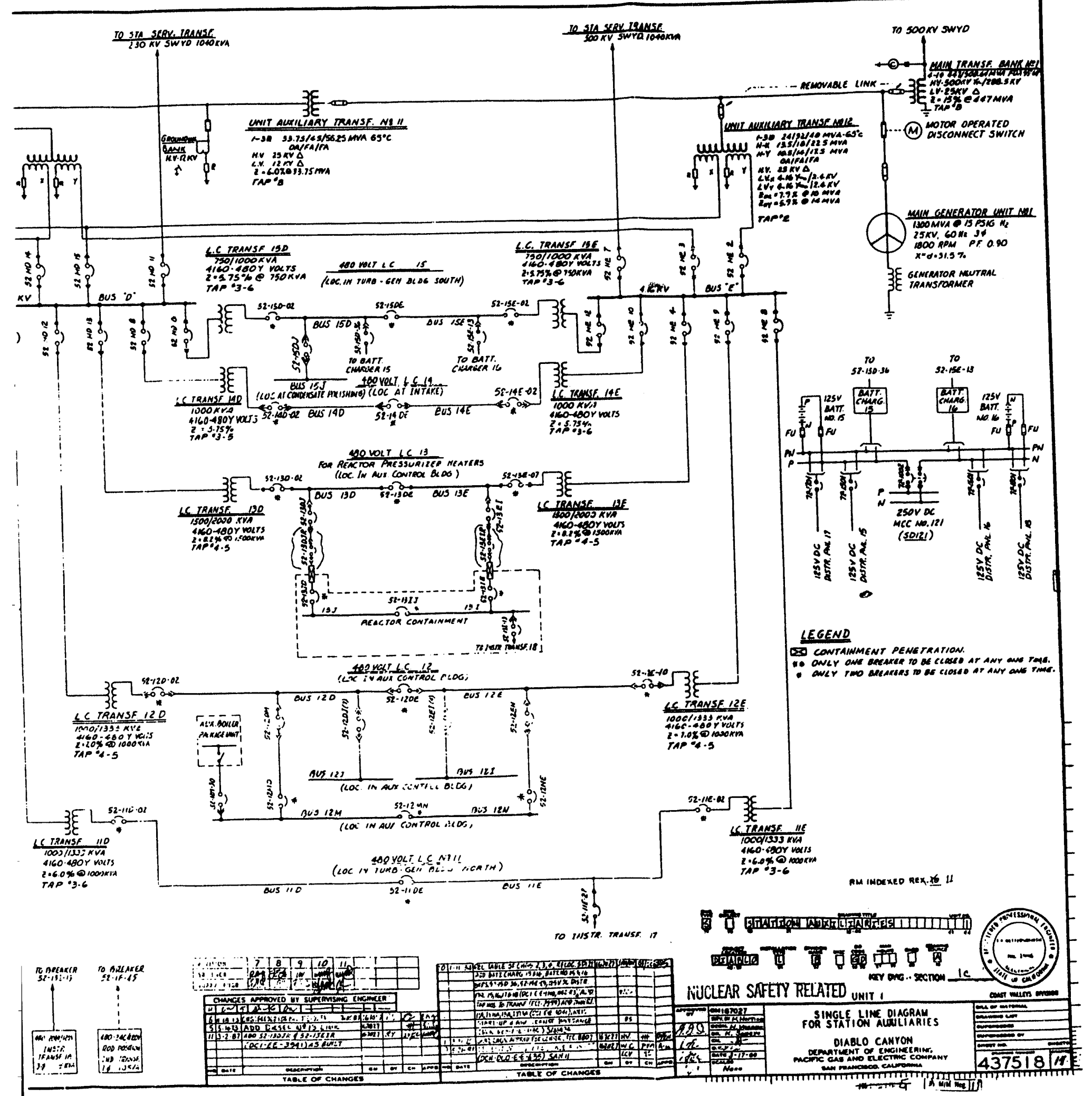

DIAGRAM D.2.1.3-1

JREG/CRS726

$1 / \mathrm{c}$ 


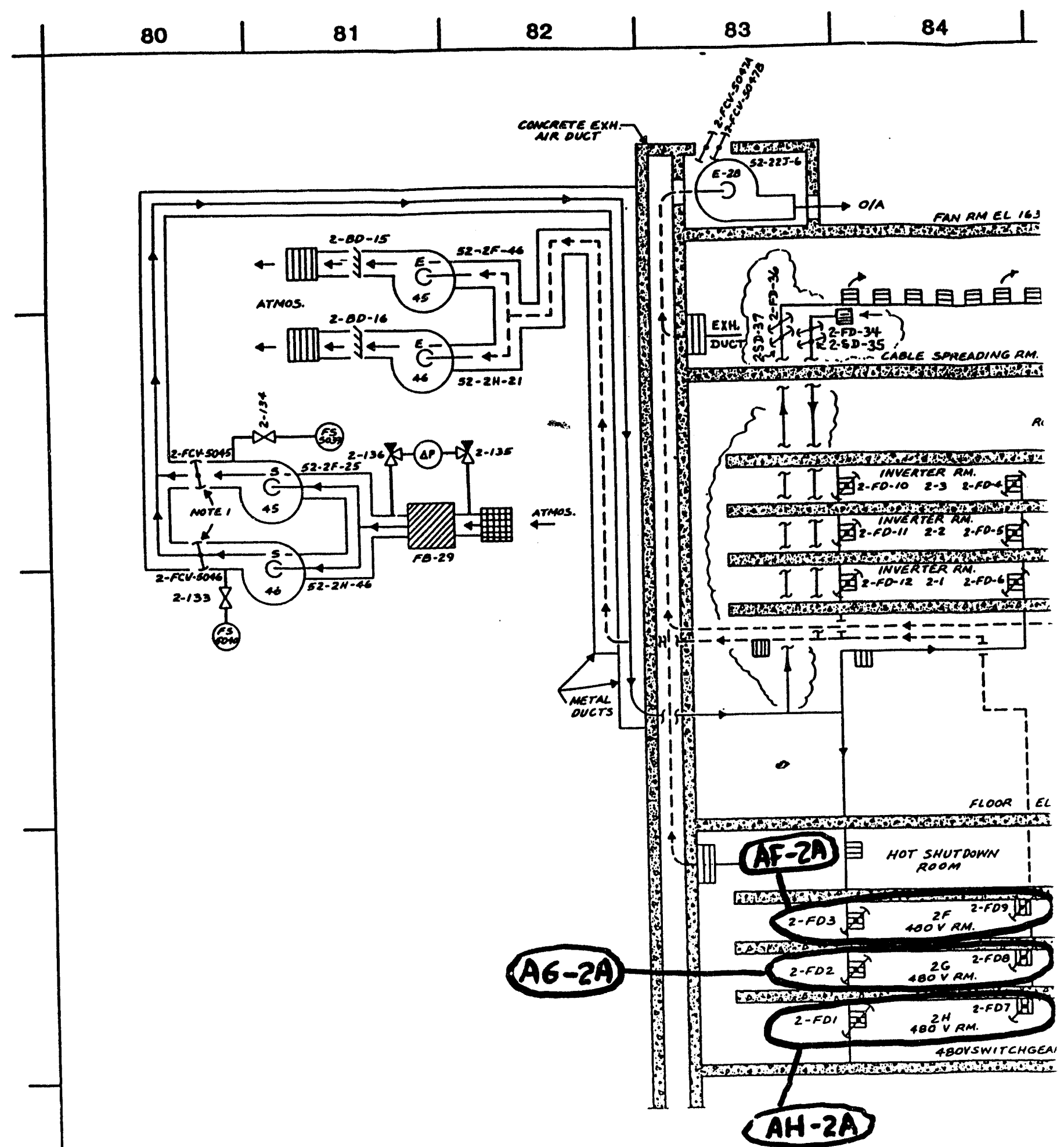

AIIX/LIARY BLDG - ARI

NOTE: 1.) DAMPERS ARE SPECIALLY ELECTRO-

HYDRANLICALLV OPERATED. LOCATED

ON BLDG. ROOF' AT $164^{\prime} E L$.

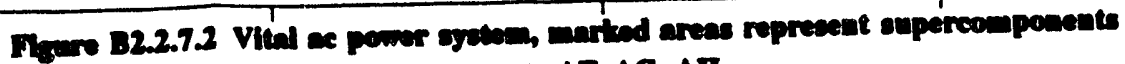
(blocke) for top events AF, AG, AB 
14 FAN RM EL 169:-

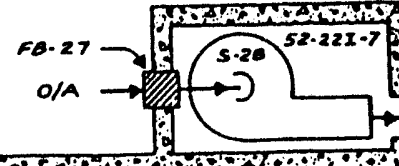
JTAR FAN

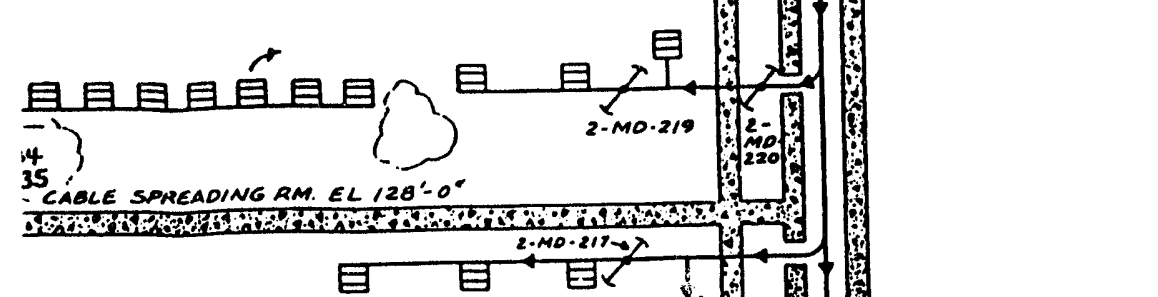
ROO ORIVE.MG AREA 2-MO-2IS)

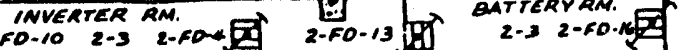

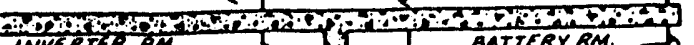

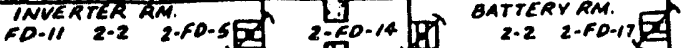

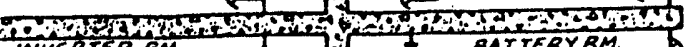

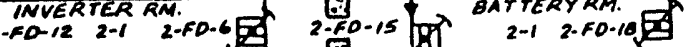
(1)

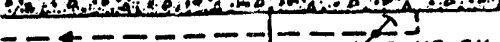
ニニニニニニース

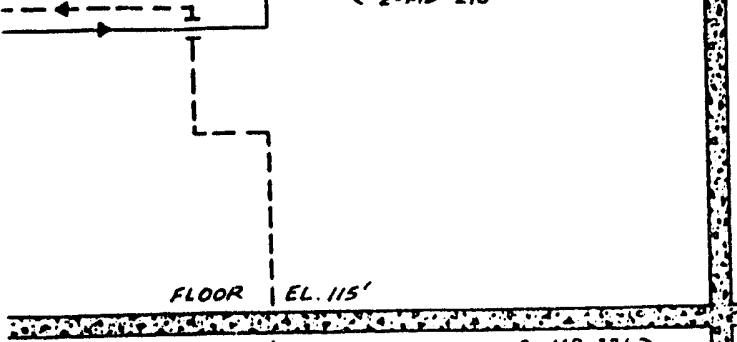
$\frac{480 \text { V RM. }}{480 V \text { WWITCHGEAR RM. EL 100' }}$ CONCAETE SUPPLY
AIR SHAFT

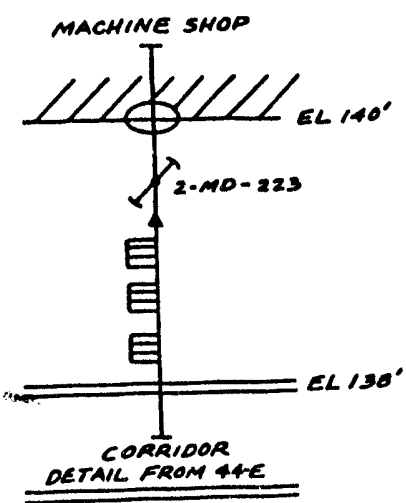




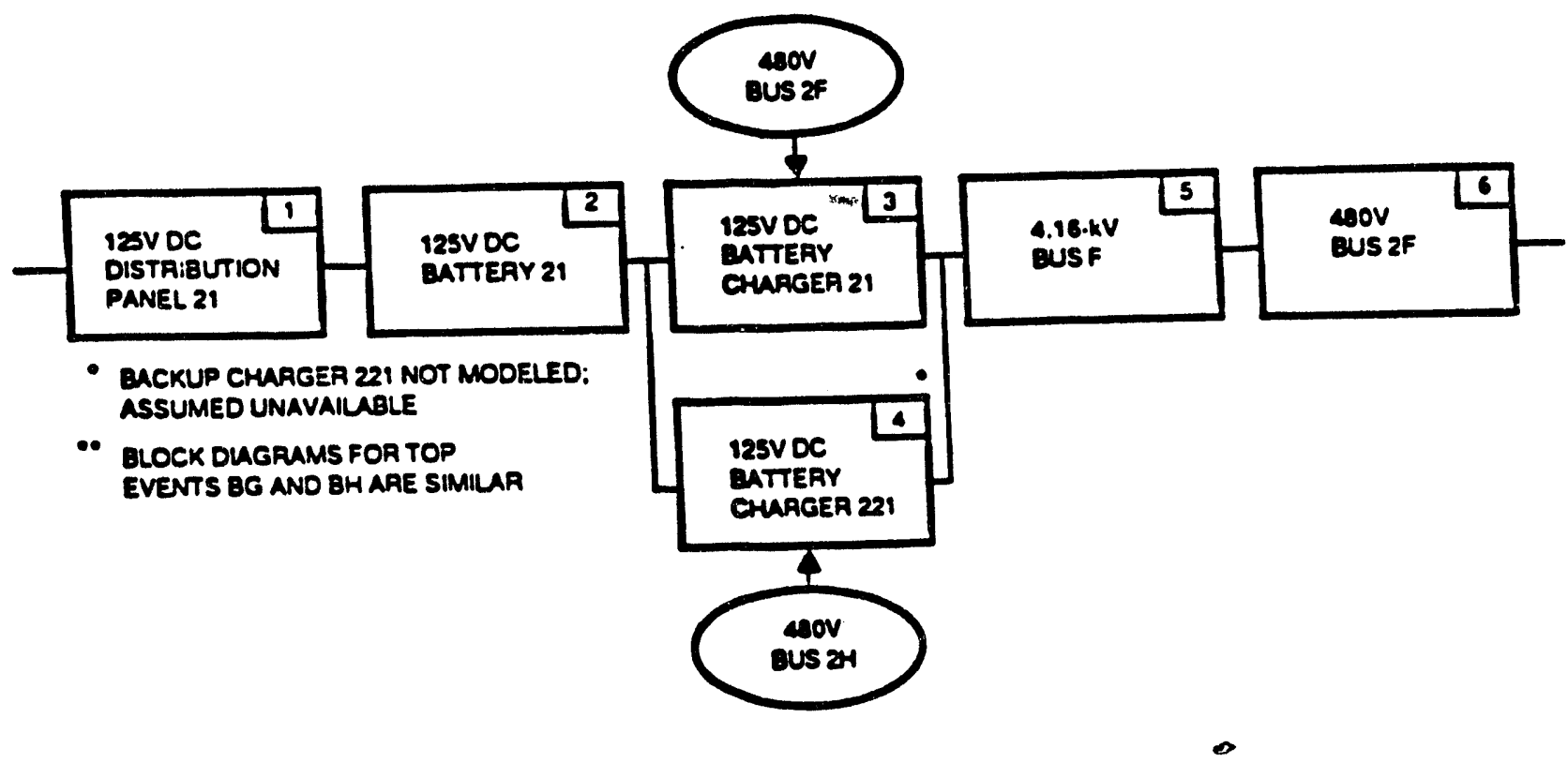

Degure 82.2.8 Rellability block dingran for Unit 2 vital ae/de ayatom* top event BPeo 


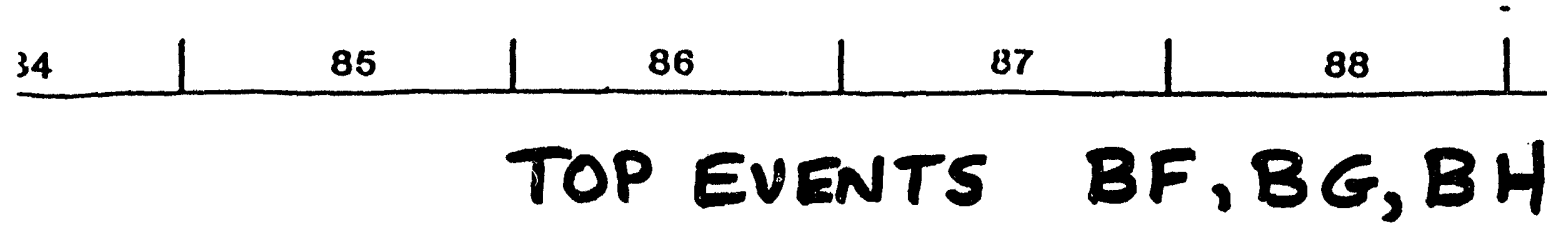

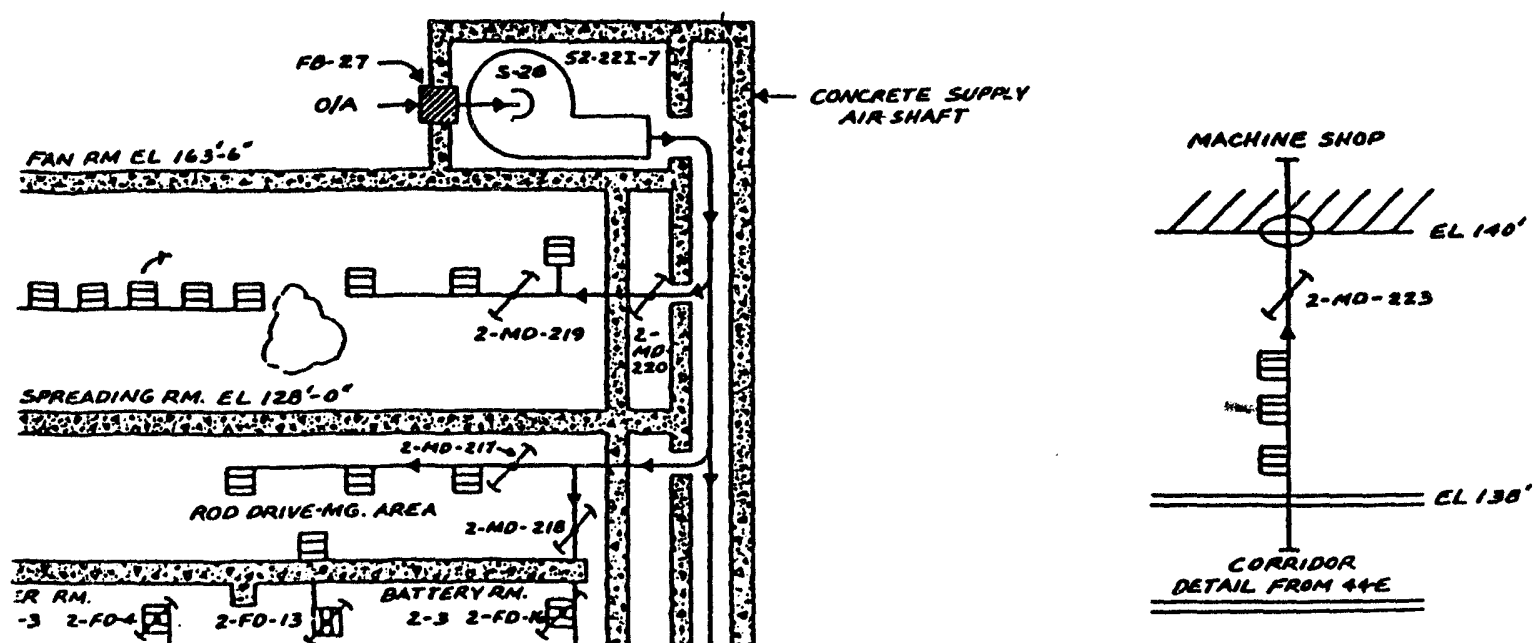

730.

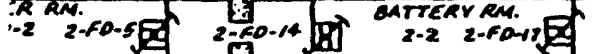

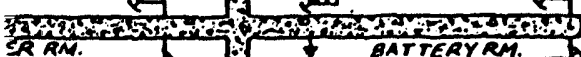

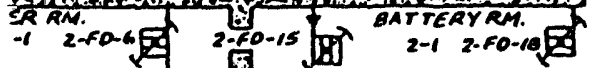

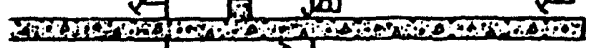

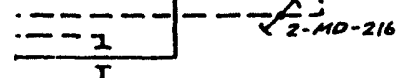

1

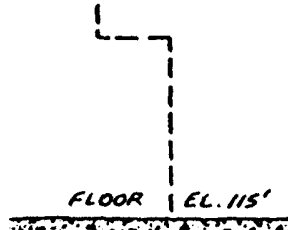

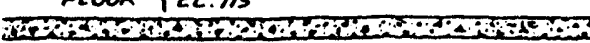

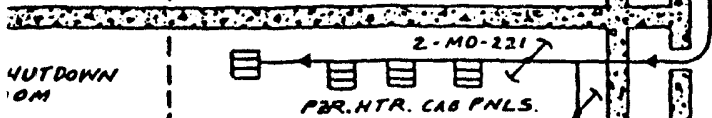

$\frac{2-609}{2-104}$

if $\mathrm{AM}$.

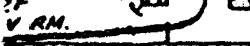

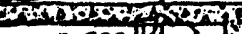

$\checkmark$ RM.

$\checkmark R M$ Ja)

amparestes.

2-FOT

$\checkmark$ ant

CBOH SWITCKGEAR RM. EL 100

R. CAOPNLS.
$2 \cdot M 0-222$

LrG.orst.

$-m$

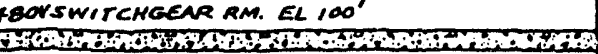

UNTT 2 


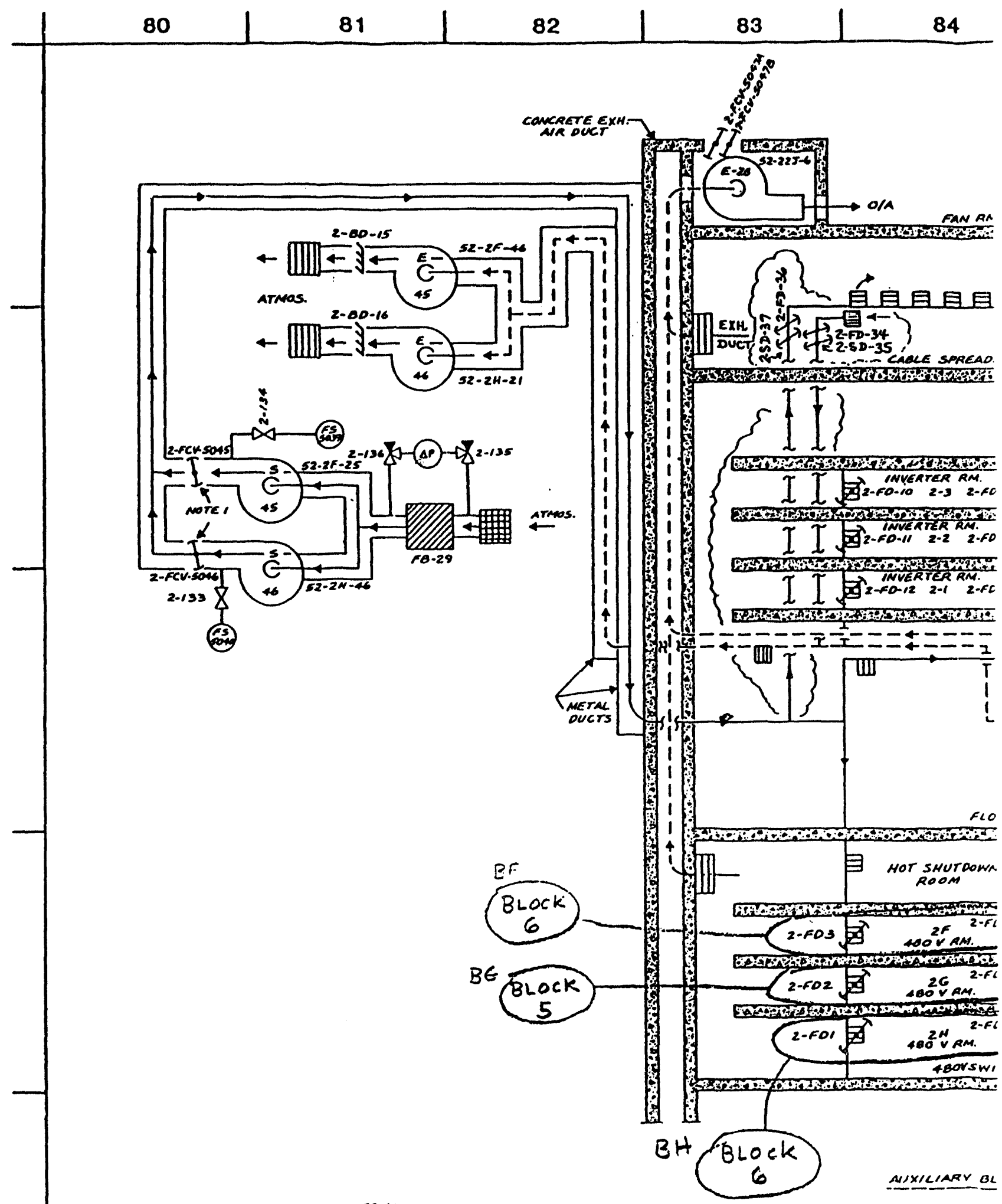

NOTE: 1.) DAMPERS ARE SPCCIALLY ELECTRO-

2) FAvs S4t sto ces द E4t

FANS 545, SAH, CAS Y E46 LOCATED
ON BLDG. ROOF'AT 164'EL. 


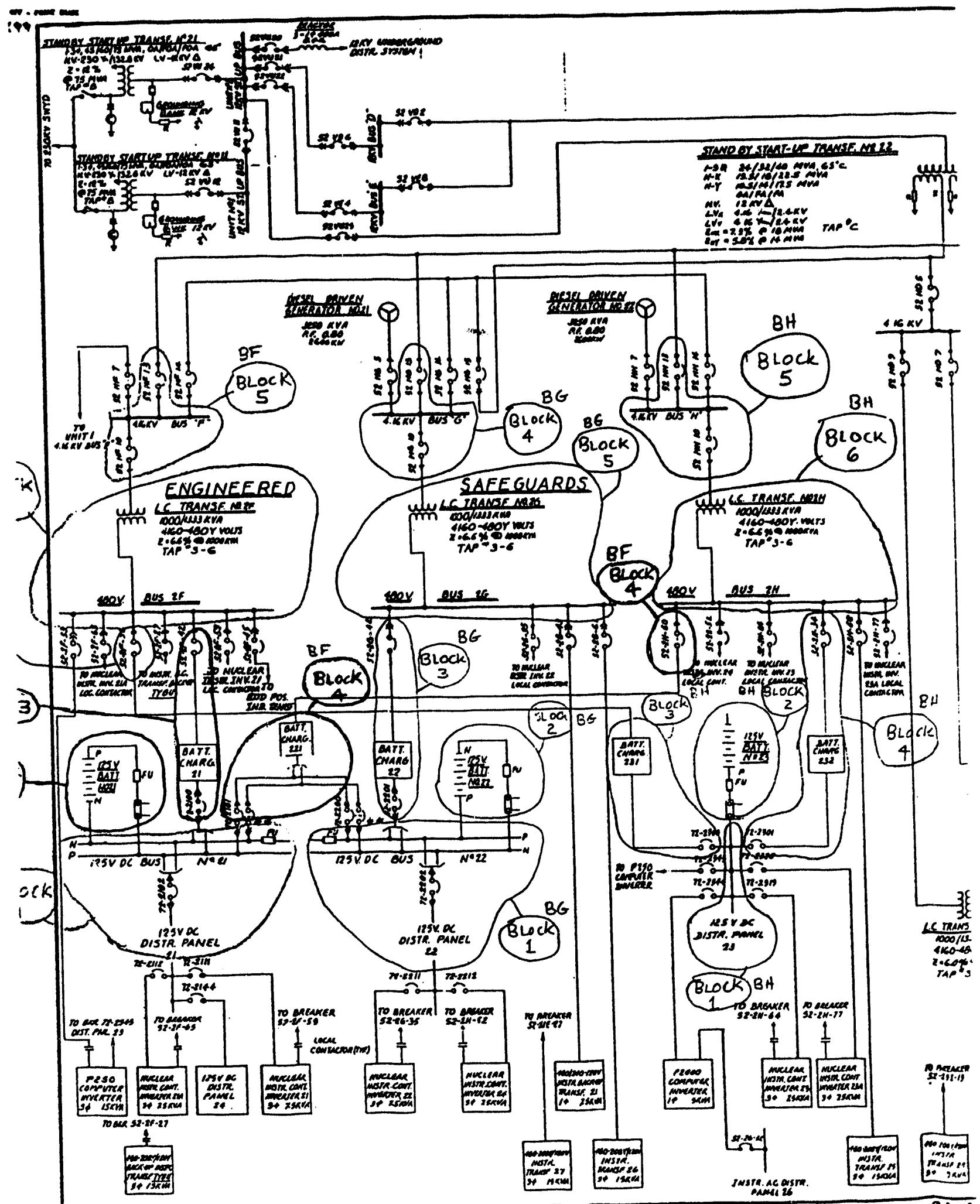

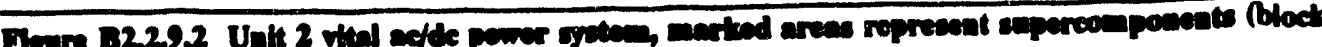
top evente BF, DG, and DE 


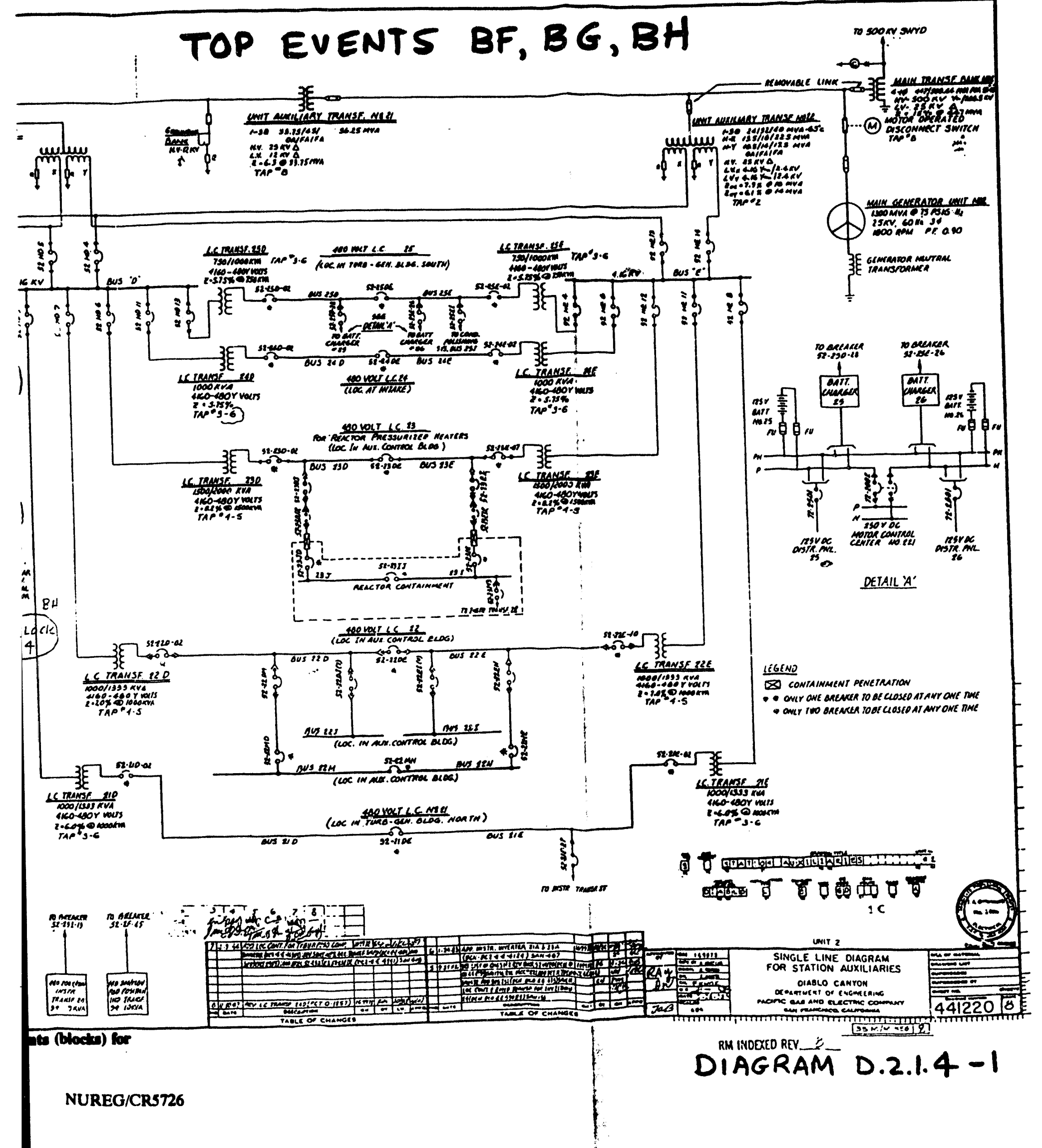



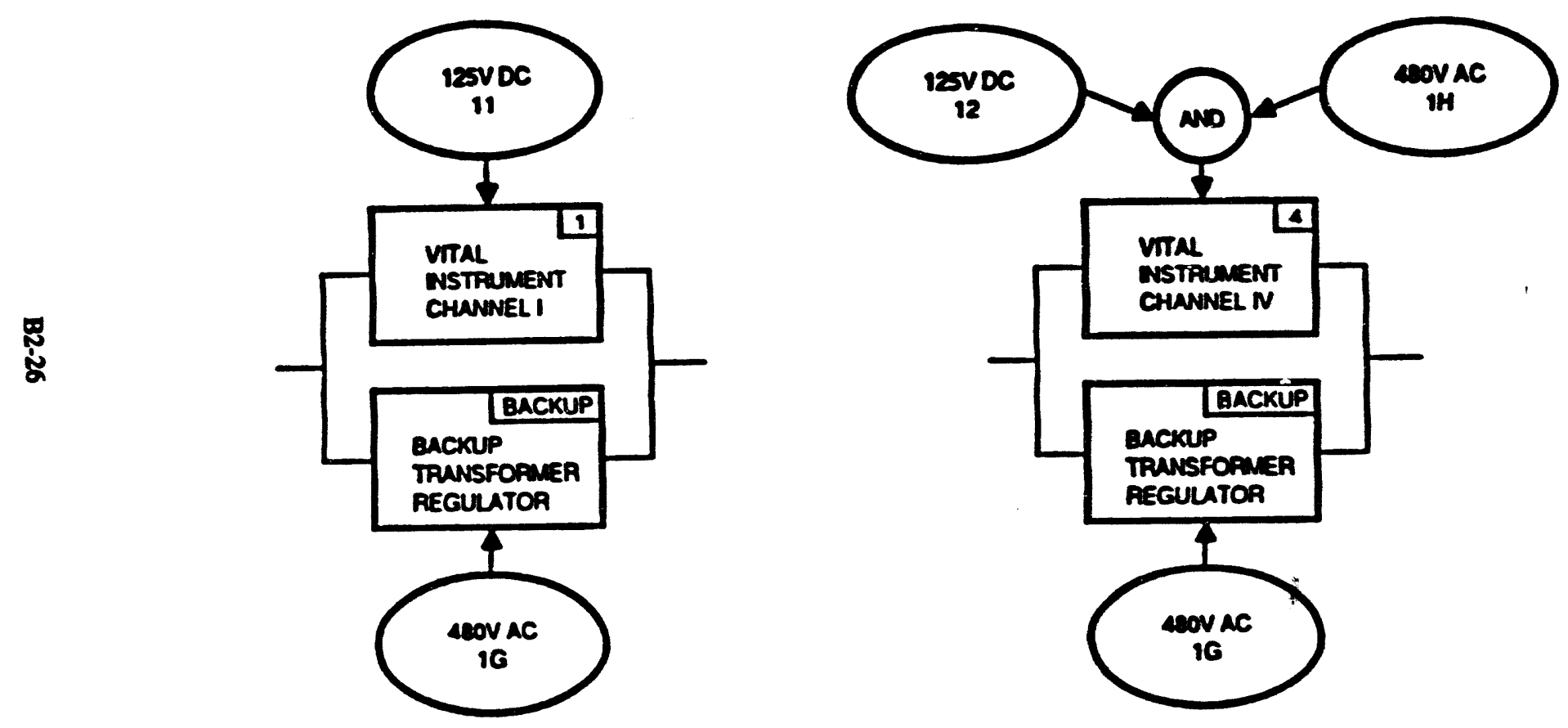

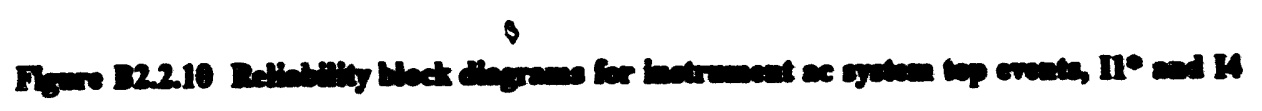




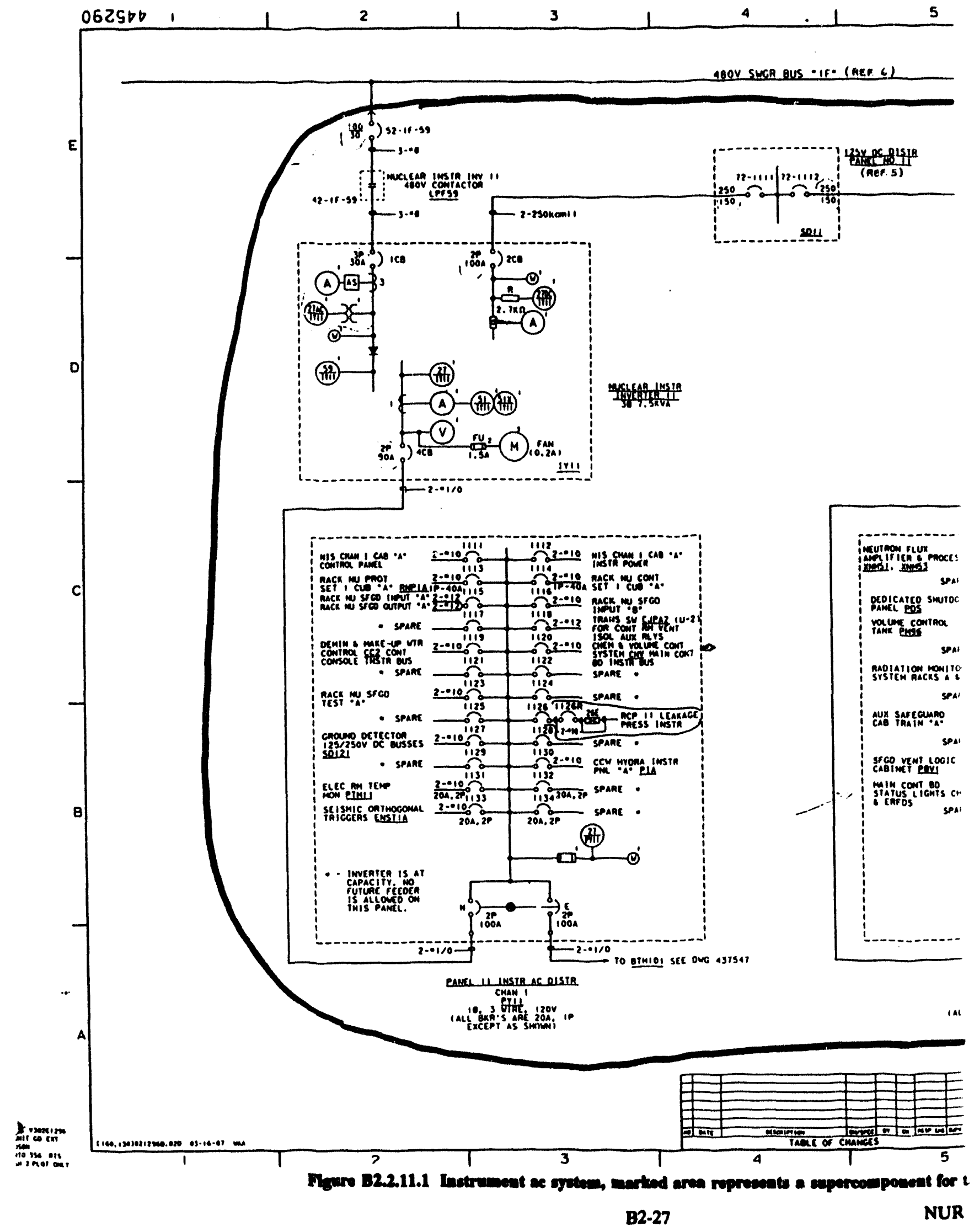




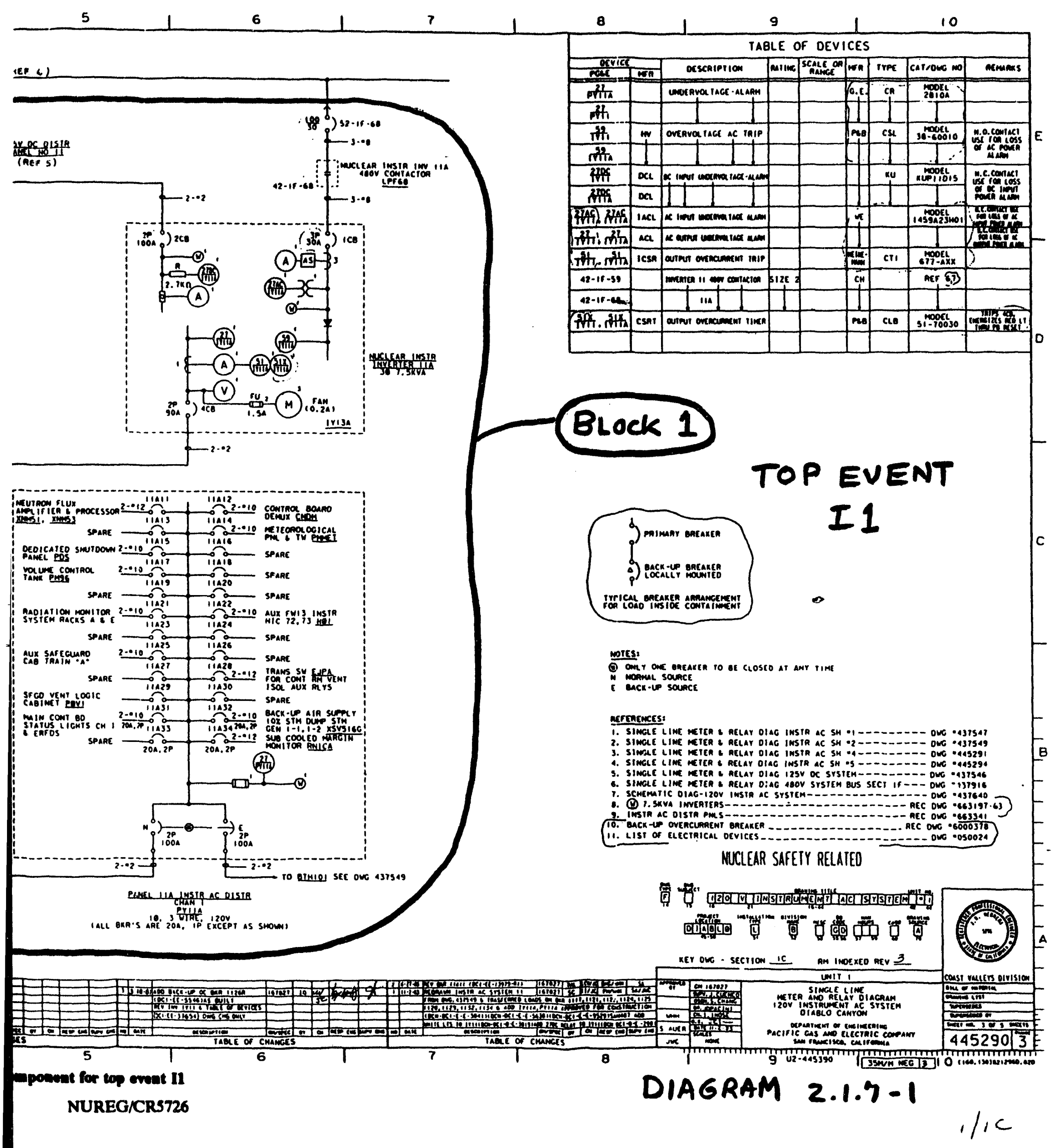




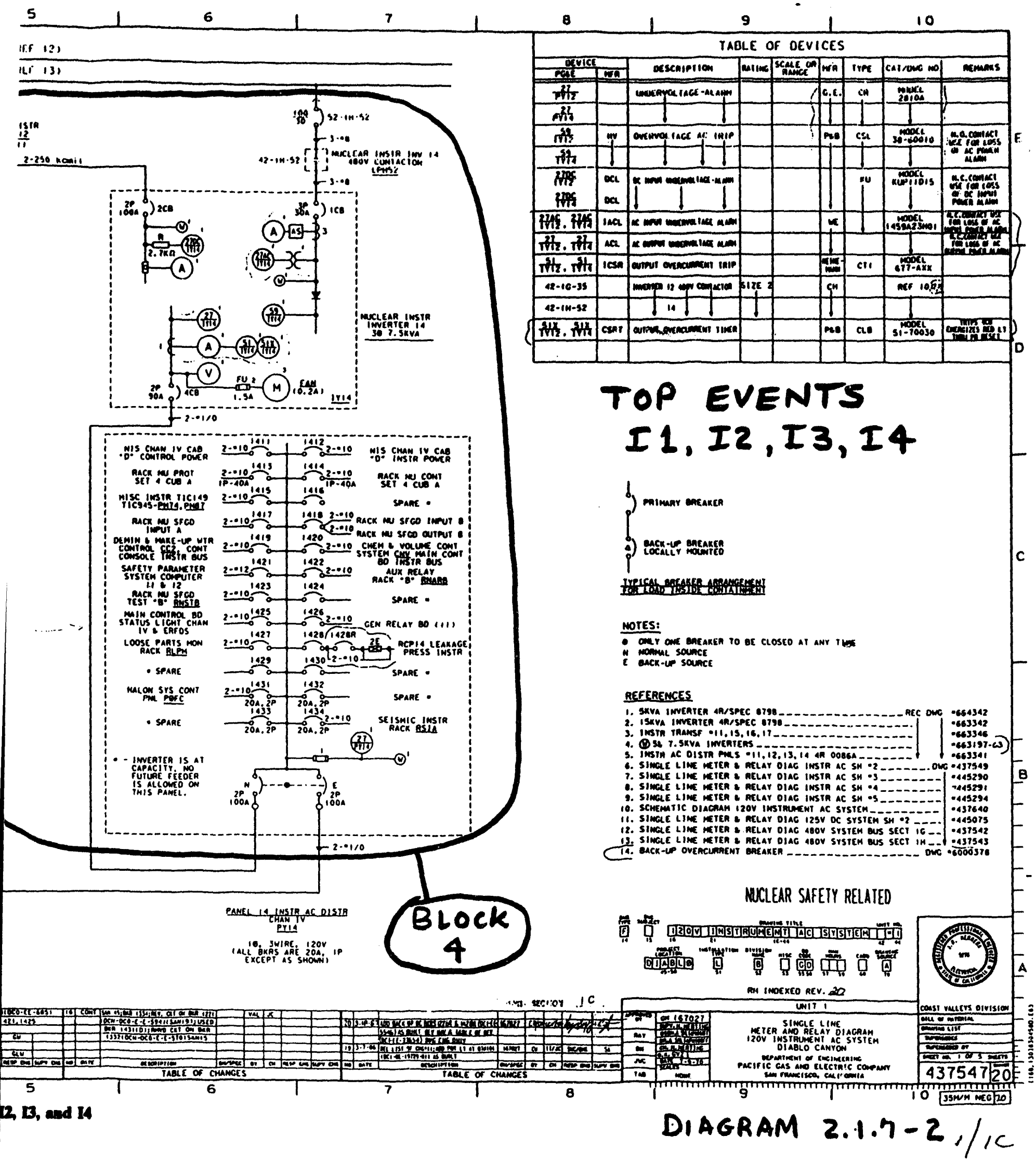




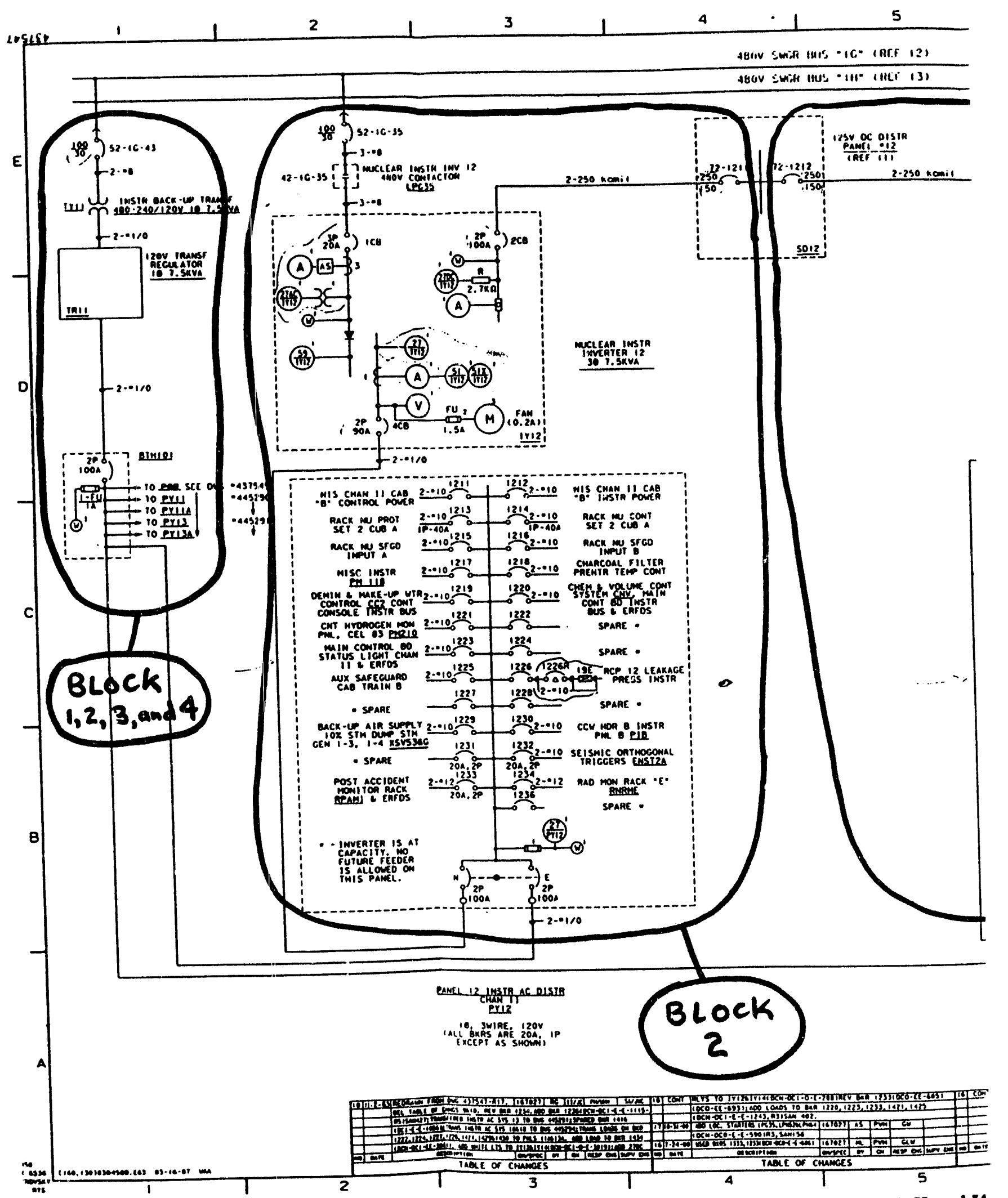

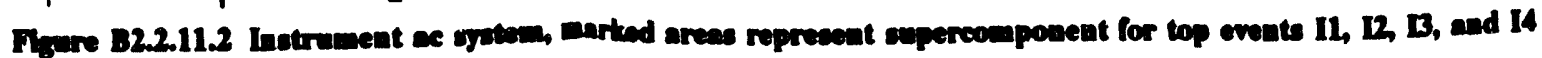




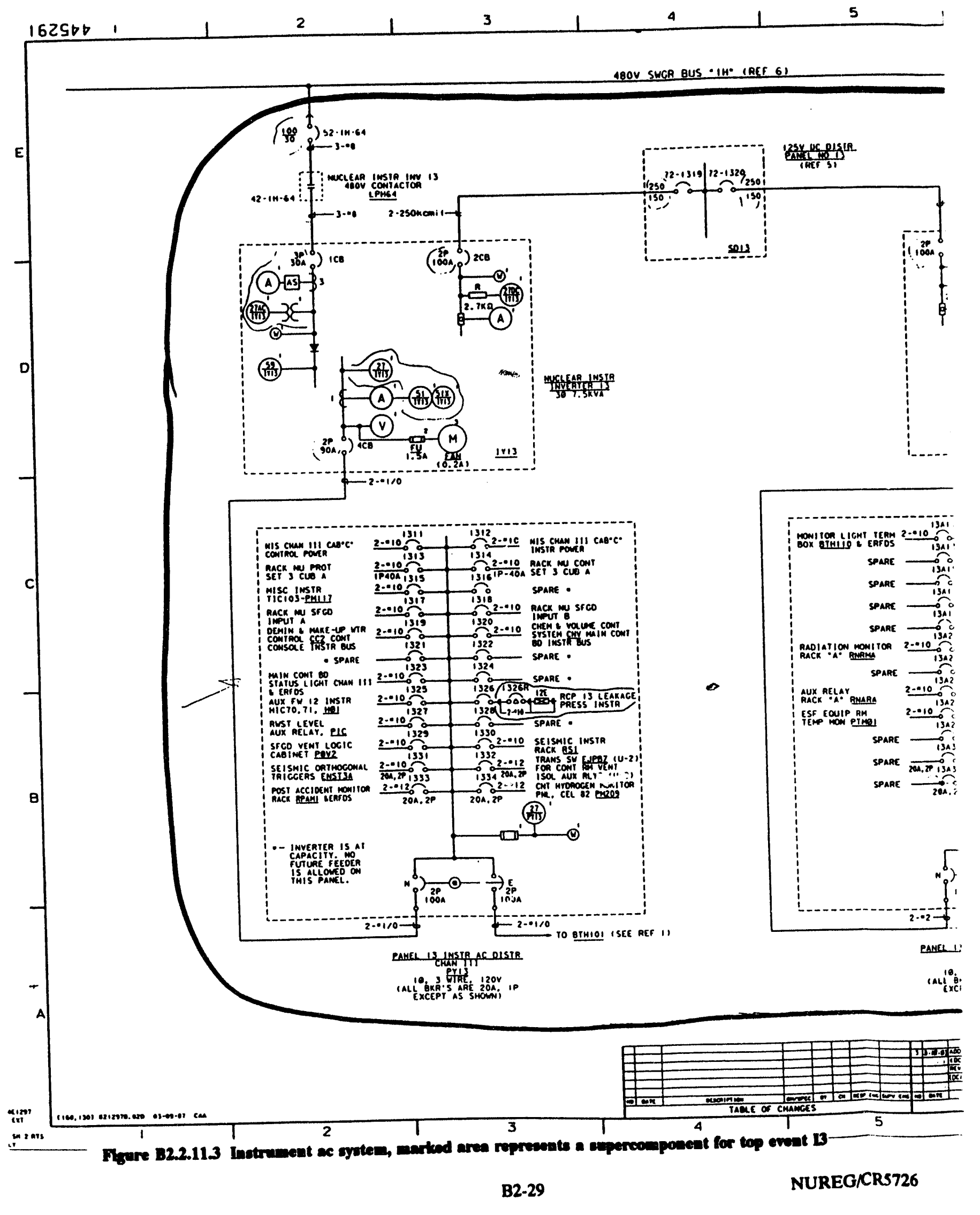




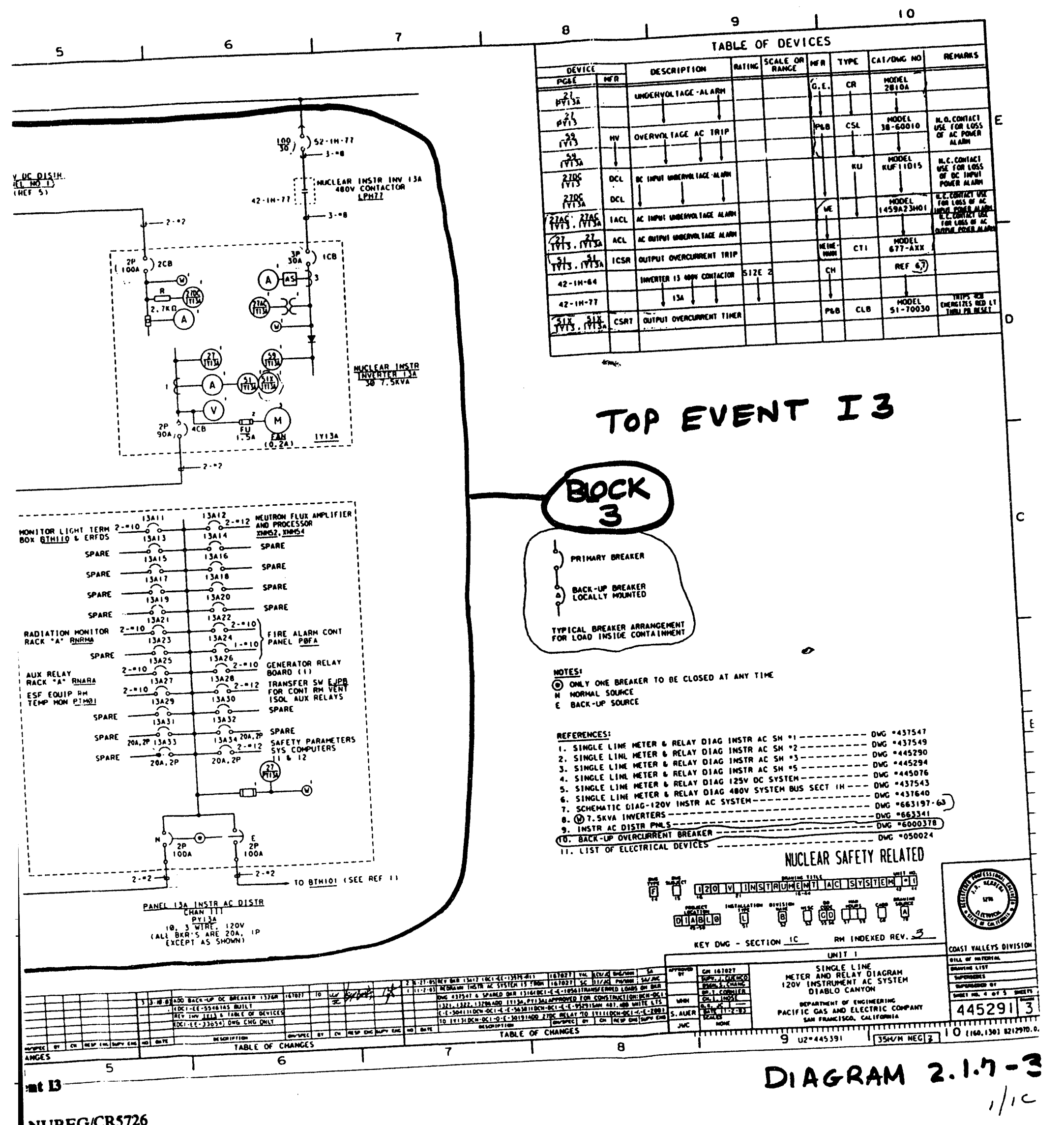

NUREG/CR5726 
$\wedge$

FN AM CL $163^{\circ}: 6^{\circ}$

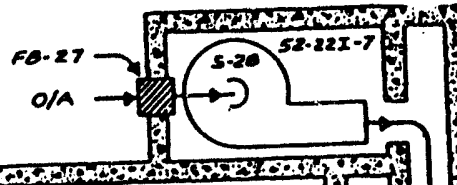

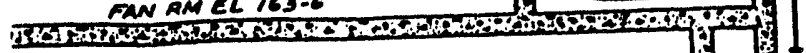
크를 ;<smiles>C#CC#CC(C#C)(C#CC#C)CC</smiles>
CÁCE SPREADING RM. EL $120^{\prime}-0^{\circ}$ (8) BLock 3 and 4

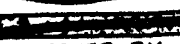
INUEATER AMM
- cancaete supply AIR SMANT

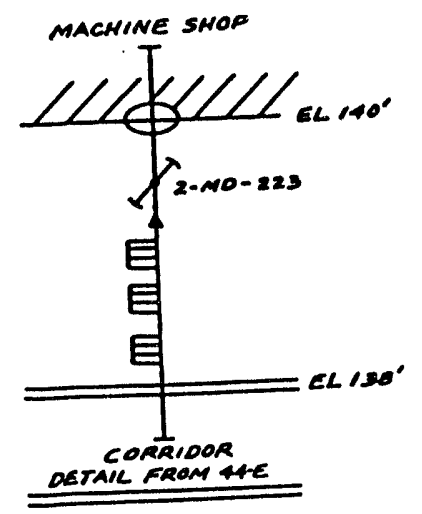

TOP EVENTS

$$
\text { IL, I2, I3, I4 }
$$




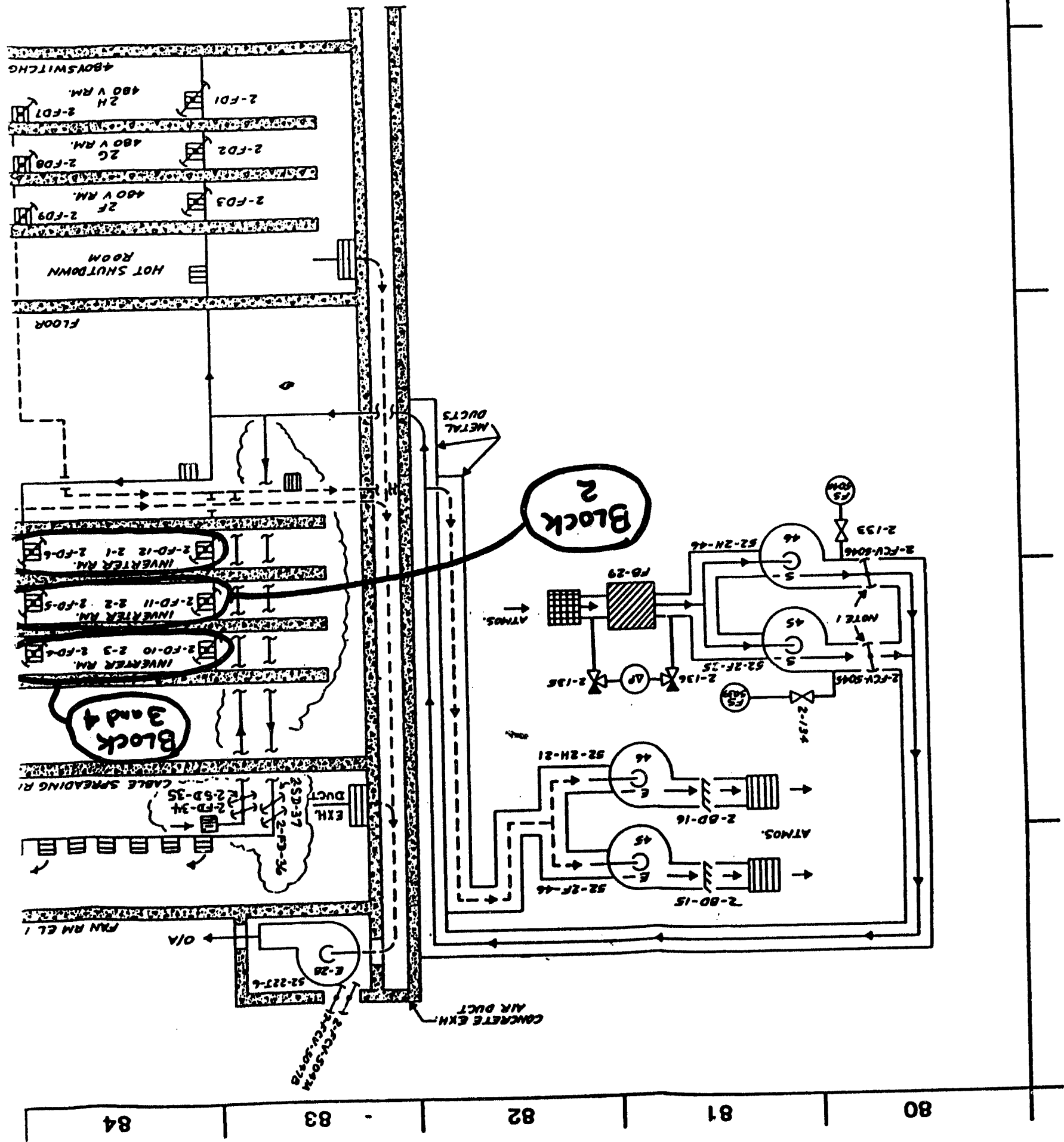


Table B2.2.1a

Top Event Definitions and Suscese Criberia

Electrical Power Sydems

Nonvital Dectric Syotem

Top Event

Deaifuntor

$\mathbf{O G}$

NV
Top Event Definition

Nonvital ac power from 230kV swilchyand.

Nonvital ac power from $12 k v$ buses.

\section{Top Erent Succes Criteria}

Power is mintained from the $230 \% \mathrm{~V}$ switchyand down to the $4.16 \mathrm{kV}$ vital bueses $\mathrm{F}$, G, H for 24 bours following an initiating ovent.

One of two $12 k \mathrm{~V}$ buses $(\mathrm{D}, \mathrm{E})$ transfers to standby ofisite power source and remain carcized for 24 hours following an initieting event.

\section{PSAR Succese Criterin:}

Explicitly are not epecified, however one can infer the following:

1. If the unit trips, the unit must switch to the $230 \mathrm{KV}$ system via the startup transformers to maintain power to the plant loads.

2. If the $230 \mathrm{KV}$ system is unavailable, the vital 4.16kV buses must be isolated from both the auxiliary and startup power systems so that the diesel generators may supply the loed.

\section{Technical Soecification (LCOs):}

1. For continuous operation, two independent offite circuits must be maintained (one $500 \mathrm{kV}$ and one $230 \mathrm{KV}$ line).

2. If one officte circuit is lost it must be restored within $\mathbf{7 2}$ hours or else the unit must shuddown.

3. If one officite circuit and one diesel generator is out of serrice they must be restored within 12 hours. 
Appeadix B2

Table B2.2.1b

Top Event Definitions and Success Criteria

Electrical Power Systems

Vital 125V DC System

Top Event

Dodiznator

DF
Top Event Definition

125V DC Power

\section{Top Event Success Criteria}

Each de power train is successful if it remains do-energized for 24 hours following an initiating event. If ac power is lost oach battery is required to power each train for two hours, and provide power to its respective diesel generator.

\section{BSAR Succese Criterin}

Explicitly are not specified, however, one can infer that in the event that any or all $480 \mathrm{~V}$ vital buses are lost, the $125 \mathrm{~V}$ batteries will provide de control power necessary to shut down safely the plant.

\section{Technical Specifications (LCOs):}

If one battery or charger or both are inoperable, restore within two hours or be in hot standby within the next six hours or in cold shutdown within the following 30 hours. 
Table B2.2.1c

\section{Top Event Definitions and Success Criteria \\ Electrical Power Systems \\ Vital AC System - Unit 1}

\section{Top Event \\ Deciznator}

AF, AG, AH

Top Event Definition

Vital AC Buses

SF; SG, SH Startup Feeder Breakers

\section{Top Event Success Criteria}

Eech $4.16 \mathrm{kV}$ bus is successful if it isolates from the auxiliary power source and each $4.16 \mathrm{kV}$ and $480 \mathrm{~V}$ vital train remains energized for 24 hours. (Success of this ovent implies that given failure of top event $O G$ the corresponding diesel generator must start and load to power the equipment supplied from the vital train. Failure of this event means that vital ac is not available even if the startup transformer and the diesel could supply ac power.)

Each standby startup foeder breaker to each $4.16 \mathrm{kV}$ bus is successful if it energizes the bus and remains energized for 24 hours. (These top events are asked if the corresponding top events AF, AG, AH are successful. Success of these top events and of $O G$ implies that vital ac power is available on the corresponding buses. If $O G$ fails and these top events are not asked the corresponding diesels must start and load to power the equipment from these buses.)

\section{ESAR Success Criteris:}

During a plant trip or loss of offsite power, the system must maintain power to at least two of three vital $4.16 \mathrm{kV}$ buses. Any two of the three vital buses are adequate to serve at least the minimum required ESF loads of a unit.

\section{Technical Specifications (LCOs):}

- With one $4.16 \mathrm{kV}$ and/or associated $480 \mathrm{~V}$ vital buses not energized, must re-energize within eight hours or be in hot standby within the next six hours and cold shutdown within the following 30 hours.

- Loss of diesel generator 13 which supplies vital bus F of both units would require both units to shutdown if not restored within 72 hours.

- If one diesel or one offsite circuit is unavailable the other two power trains must be verified within one hour and the unavailable source restored within 72 hours. 
Appendix $\mathbf{B 2}$

Table B2.2.1d

Top Event Definition and Succees Criteria

Eloctrical Powrer Systems

Vital AC/DC Syetem - Unit 2

\section{Top Erent}

Dexiguator

BF, BG, BH
Top Erent Definition

Vital AC/DC Electric Power

Subsystems of Unit 2
Top Event Success Criteria

$4.16 \mathrm{kV}$ bus $(\mathrm{F}, \mathrm{G}, \mathrm{H})$ at Unit $2,480 \mathrm{~V}$ bus $(2 \mathrm{~F}, 2 \mathrm{G}, 2 \mathrm{~F})$ and $125 \mathrm{~V}$ de panel $(21,22,23)$ reepectively, must remain available for 24 hours. (The success of top event BF implies that if offsite power is not available, swing diesel 13 aligns to Unit 2, power is available at the sssociated buses.) 
Table B2.2.1e

Top Ereat Definition and Succesen Criteria

Electrical Power Syctemens

Instrument AC Syetem

Top Ereat

Dechemator

I1

12

13

14
Top Event Definition

Instrument AC Channols

\section{Top Erent Succeses Criteria}

Eech chennel is succeseful if $120 \mathrm{~V}$ ac power to the loads on the instrument ac distribution pands (panels PY-11, PY-11a for Channel I, panel PY-12 for Channel II, panols PY-13, PY-13a for Channel III, and panel PY-14 for Chennel IV) is maintained for 24 hours.

ESAR Succese Criterin: Similar to that appliod in the modelling.

Technical Specifications (2COn): With one vital instrumeat ac bus not energized by its aseociated inverter or with one inverter not connected to its aneociated de bus, re-energize the vital instrument bus from another source within two hours or bo in hot standby within six hours and cold shutdown within 30 hours; re-energize the vital instrumeat $a$ bus from its aseociated dc bus within 24 bours or be in hot standby within the next six hours and in cold shutdown within the following 30 hours. 
Appendix $\mathbf{B 2}$

Table B2.2.2a

Boundary Condition and Split Fraction Identifications for

Top Events 06 and NV

Nonvital Electric Power Syatem

Top Erent

OO

NV

NV1

NV2

NV3

\section{Definition}

Given offiside grid arceess.

Given offeide grid failure (ouaranteed failure).

Given all support available.

OO succeeded, dc 12 or de 13

failed.

OO failed, de 12 and de 13

failed (guaranteed failuro).

Table B2.2.2b

Boundary Condition and Split Fraction Identifications

for Top Events DF, DG, and DH

Vital 125V DC System

Top Erent

DF

DG

DH
Split Fraction ID

DF1

DG1

DG2

DGF

DH1

$\mathrm{DH} 2$

DH3

DH4

\section{Definition}

Vital $480 \mathrm{~V}$ bus $1 \mathrm{~F}$ available.

Vital 480V bus $1 G$ available, DF succeeded.

Vital $480 \mathrm{~V}$ bus 16 available, DF failed.

Guarantood failure.

Vital $480 \mathrm{~V}$ bus $1 \mathrm{H}$ available, DF succeeded, DG succeeded.

Vital $480 \mathrm{~V}$ bus $1 \mathrm{H}$ available, DF succeeded, DG failed.

Vital $480 \mathrm{~V}$ bus $1 \mathrm{H}$ available, DF failed, DG succeeded.

Vital $480 \mathrm{~V}$ bus $1 \mathrm{H}$ available, DF failed, DO failed. 
Table 82.2.2c

Boundary Coadition and Conditional Split Praction Identification

for Top Events AF, AG, AH, and SF, SG, SH

Vital AC Syatem - Unit 1

\section{Top}

Event

$\mathbf{A F}$

AO

AH

SF

\section{Splile Draction}

Identification

AF1

APA

AFF

AO1

AO2

AO3

AOA

AGB

$A G C$

AOP

AH1

AH2

AH3

AH4

AH5

AH6

AHA

AHB

AHC

AHD

AHE

AHG

AHF

SF1

SFA

\section{Definition}

All oupport available, with recovery from common cauve breaker failure. All aupport aveilable with no recovery from common cause breaker failure. Ouaranteed failuro.

In the following the notations: DF-S, DF-F, and DO-S, DG-P, represent the axceses (S) and failure (F) of de trains 11 and 12, respectively. The notation AF-S, AF-F, AG-S, and AO-F represent the amo for ec trains F and $G$, respectively.

DF-S, AF-S, with recovery.

DP-S, AP-F, with recovery.

DP-F, with recovery.

DF-S, AF-S, no recovery.

DF-S, AF-F, no recovery.

DF-F, no recovery.

Guarnateod failure.

DF-S, DG-S, AF-S, AG-S, with recovery.

DF-S, DG-S, AF-S, AG-F, or DF-S, DG-S, AF-F, AG-S, with recovery. DF-S, DG-S, AF-F, AG-F, with recovery.

DF-S, DG-F, AF-S or DF-F, DG-S, AG-S, with recovery.

DF-S, DG-F, AF-F or DF-F, DG-S, AG-F, with recovery.

DF-F, DG-F, with recovery.

DF-S, DG-S, AF-S, AG-S, no recovery.

DF-S, DG-S, AF-S, AG-F or DF-S, DG-S, AF-F, AG-S, no recovery.

DF-S, DG-S, AF-F, AG-F, no recovery.

DF-S, DG-F, AF-S or DF-F, AG-S, AG-S, no recovery.

DF-S, DG-F, AF-F or DF-F, DG-S, AG-F, no recovery.

DF-F, DG-F, no recovery.

Guaranteod failure.

All support available with recovery.

All support available, no recovery. 
Appoadix $\mathbf{B 2}$

Table B2.2.2c(continued)

\begin{tabular}{|c|c|c|}
\hline \multirow[t]{2}{*}{$\begin{array}{l}\text { Top } \\
\text { Event }\end{array}$} & $\begin{array}{l}\text { Split Frnetion } \\
\text { Identifiention }\end{array}$ & Deifution \\
\hline & & In the following the notation B atunds for "bypeased" state. \\
\hline SO & $\begin{array}{l}S O 1 \\
S G 2 \\
S O 3 \\
S O A \\
S G B \\
S G C\end{array}$ & $\begin{array}{l}\text { SP-S, with recovery. } \\
\text { SF-P, with recovery. } \\
\text { SF-B, with recovery. } \\
\text { SF-S, no recovery. } \\
\text { SF-F, no recovery. } \\
\text { SF-B, no recovery. }\end{array}$ \\
\hline SH & $\begin{array}{l}\text { SH1 } \\
\text { SH2 } \\
\text { SH3 } \\
\text { SH4 } \\
\text { SH5 } \\
\text { SH6 } \\
\text { SHA } \\
\text { SHB } \\
\text { SHC } \\
\text { SHD } \\
\text { SHE } \\
\text { SHO }\end{array}$ & $\begin{array}{l}\text { SF-S, SG-S, with recovery. } \\
\text { SF-S, SG-F or SF-F, SG-S, with recovery. } \\
\text { SF-F, SG-F, with recovery. } \\
\text { SF-S, SG-D or SF-D, SG-S, with recovery. } \\
\text { SF-F, SG-D or SF-D, SG-F, with recovery. } \\
\text { SF-D, SG-D, with recovery. } \\
\text { SF-S, SG-S, no recovery. } \\
\text { SF-S, SG-F or SF-F, SG-S, no recovery. } \\
\text { SF-F, SG-F, no recovery. } \\
\text { SF-S, SG-D or SF-D, SG-S, no recovery. } \\
\text { SF-F, SG-B or SF-B, SG-F, no recovery. } \\
\text { SF-D, SG-B, no recovery. }\end{array}$ \\
\hline
\end{tabular}


Tabie B2.2.2d

Boundary Conditions and Conditional Spllt Fraction Identification

for Top Events BF, BG, BA

Vital AC/DC System - Unit 2

$\begin{array}{lll}\begin{array}{l}\text { Top } \\ \text { Event }\end{array} & \begin{array}{c}\text { Split Praction } \\ \text { Identinication }\end{array} & \text { Definition } \\ \text { BF } & \text { BF1 } & \text { Offrite grid failed, OG-F. } \\ \text { BG } & \text { BG1 } & \text { OG-F, ac/dc train F Unit } 2 \text { successful, BF-S. } \\ & \text { BG } & \text { OG-F, ac/dc train F Unit } 2 \text { failod, BF-F. } \\ \text { BH } & \text { BH1 } & \text { OG-F, BF-S, BG-S. } \\ & \text { BH2 } & \text { OG-F, BF-S, BG-F, or OG-F, BF-F, BG-S. } \\ & \text { BH3 } & \text { OG-F, BF-F, BG-F. }\end{array}$

Table B2.2.2e

Boundary Conditions and Split Fraction Identification

for Top Events I1, 12, 13, and I4

Instrument AC System

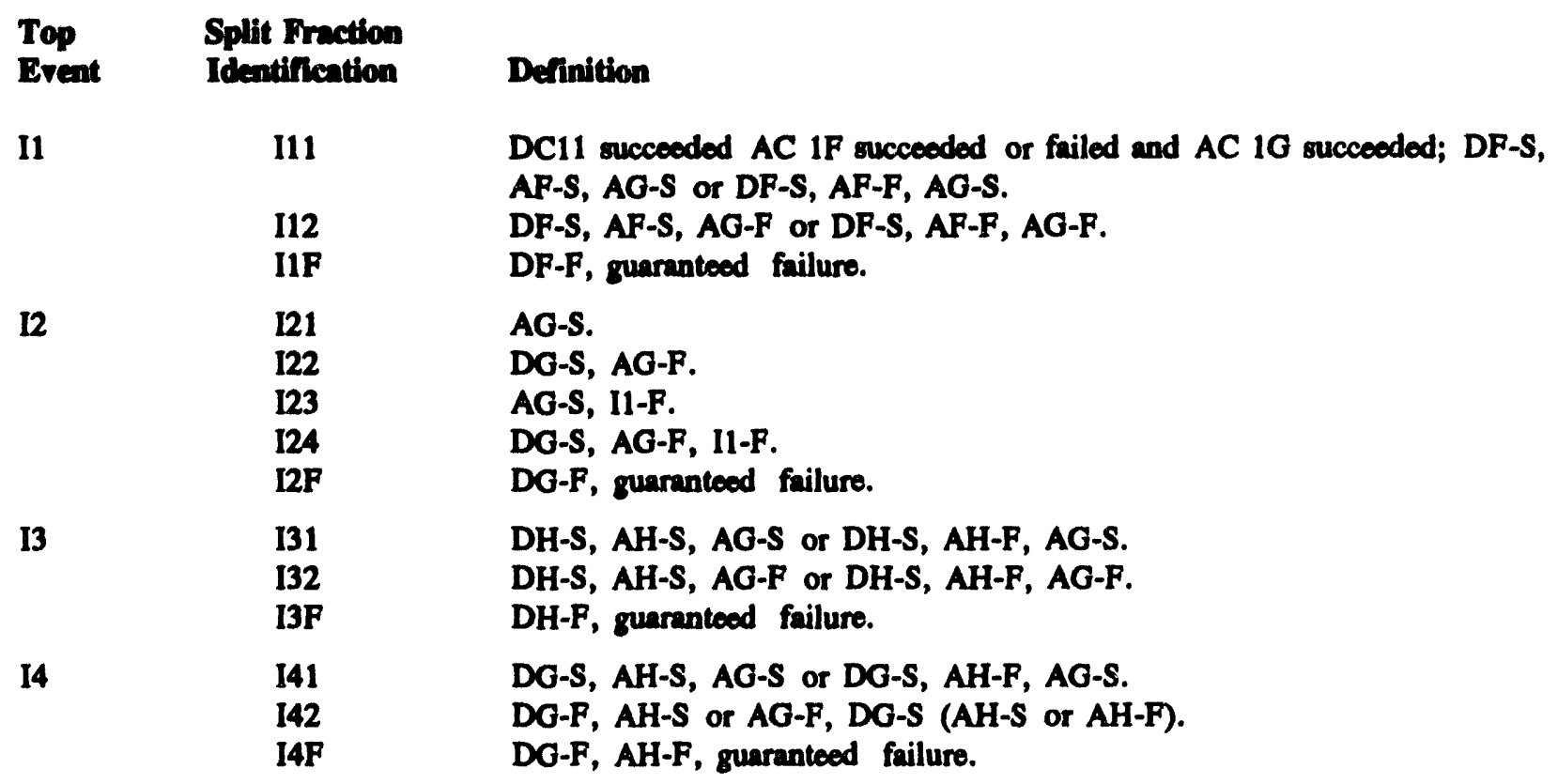


Appendis B2

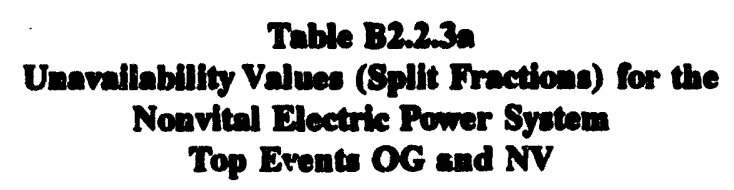

\begin{tabular}{|c|c|c|c|c|c|c|c|c|c|c|}
\hline $\begin{array}{l}\text { Top } \\
\text { Drent }\end{array}$ & Cace & Calc. & TTL & $\mathbf{H W}$ & nWW & EWD & $T S$ & $\mathbf{M N}$ & $\mathbf{H E}$ & $\begin{array}{c}\text { Comment } \\
\text { No. }\end{array}$ \\
\hline \multirow[t]{4}{*}{ OG } & OG1 & PG\&E & $7.629-4$ & $4.813-4$ & $4.813-4$ & 0.0 & & $2.816-4$ & & \\
\hline & & BNL & $7.592-4$ & $4.841-4$ & $4.841-4$ & 0.0 & & $2.751-4$ & & \\
\hline & OGF & PG\&E & 1.0 & & & & & & & \\
\hline & & Bisl & 1.0 & & & & & & & \\
\hline \multirow[t]{6}{*}{ NV } & NV1 & PG\&E & $1.629-4$ & $1.621-4$ & $8.471-6$ & $1.537-4$ & & $7.645-7$ & & \\
\hline & & BNL & $1.623-4$ & $1.616-4$ & $4.561-6$ & $1.570-4$ & & $7.688-7$ & & \\
\hline & NV2 & PG\&E & $2.455-3$ & $2.285-3$ & 2.131-3 & $1.537-4$ & & $1.705-4$ & & \\
\hline & & BNL & $2.460-3$ & 2.293-3 & $2.136-3$ & $1.570-4$ & & $1.677-4$ & & \\
\hline & NV3 & PG\&E & 1.0 & & & & & & & \\
\hline & & BNL & 1.0 & & & & & & & \\
\hline
\end{tabular}


Appendir B2

Table B2.2.3b

Unavallability Values (Conditional Split Dractions)

for the Vital 125V DC Syatem

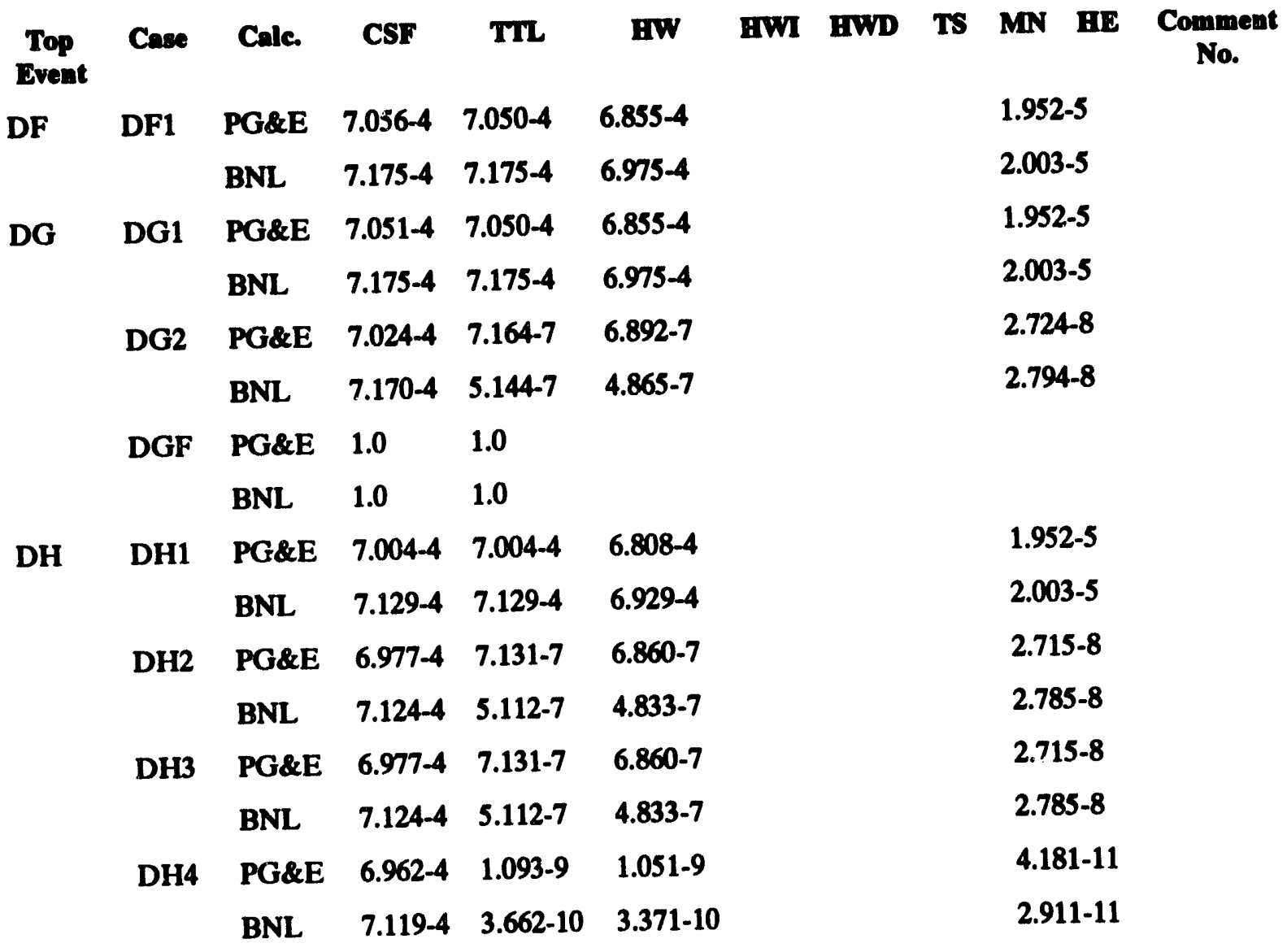


Appendix B2

Table B2.2.3c

Unavailability Values (Conditional Split Fractions) for the Vital AC System

\begin{tabular}{|c|c|c|c|c|c|c|c|c|c|c|c|}
\hline $\begin{array}{l}\text { Top } \\
\text { Event }\end{array}$ & Cave & Cale. & $\operatorname{css}$ & TTL & HW & HW & HWD & TS & $\mathbf{M N}$ & $\mathrm{HE}$ & $\begin{array}{c}\text { Comment } \\
\mathrm{No}_{\mathrm{O}} .\end{array}$ \\
\hline $\mathbf{A P}$ & AP1 & Paes & $6.922-4$ & $6.922-4$ & $6.569-4$ & $6.568-4$ & $1.003-7$ & & 3.526-5 & & \\
\hline & & BNL & $7.002-4$ & $7.002-4$ & $6.645-4$ & $6.644-4$ & $1.036-7$ & & 3.568-5 & & \\
\hline & APA & PGeE & $7.392-4$ & $7.392-4$ & $7.039-4$ & $6.568-4$ & 4.713-5 & & $3.526-5$ & & \\
\hline & & BNL & $7.453-4$ & $7.453-4$ & $7.096-4$ & $6.644-4$ & $4.524-5$ & & $3.568-5$ & & \\
\hline$\Delta G$ & $A G 1$ & PGeE & $6.921-4$ & $6.922-4$ & \multirow[b]{2}{*}{ fes AP1 } & & & & & & \\
\hline & & BNL & $7.001-4$ & $7.002-4$ & & & & & & & \\
\hline & $A G 2$ & PGeE & $8.371-4$ & $1.077-6$ & $1.029-6$ & $9.720-7$ & 5.699-8 & & $4.805-8$ & & $*$ \\
\hline & & BNL & $7.827-4$ & $5.480-7$ & $5.006-7$ & $4.414-7$ & $5.923-8$ & & $4.742-8$ & & \\
\hline & $A G 3$ & PGeE & $6.922-4$ & $6.922-4$ & \multirow{2}{*}{ Ias AP1 } & & & & & & \\
\hline & & BNL & $7.002-4$ & $7.002-4$ & & & & & & & \\
\hline & AGA & PGeE & $7.126-4$ & $7.392-4$ & \multirow{2}{*}{ Aas } & & & & & & \\
\hline & & BNL & $7.195-4$ & $7.453-4$ & & & & & & & \\
\hline & $A G B$ & Paer & $5.179-2$ & $2.770-5$ & $2.765-5$ & $9.720-7$ & 2.668-5 & & $5.128-8$ & & $*$ \\
\hline & & BNL & $3.537-2$ & $2.636-5$ & $2.631-5$ & 4.4147 & 2.587-5 & & 5.064-8 & & \\
\hline & $A G C$ & PGQE & $7.392-4$ & $7.392-4$ & \multirow{2}{*}{$\begin{array}{l}\text { las } \\
\text { AFA } \\
\end{array}$} & & & & & & \\
\hline & & BNL & $7.453-4$ & $7.453-4$ & & & & & & & \\
\hline $\mathbf{A H}$ & AH1 & PGeE & $6.921-4$ & $6.922-4$ & \multirow[b]{2}{*}{ Ias AF1 } & & & & & & \\
\hline & & BNL & $7.001-4$ & $7.002-4$ & & & & & & & \\
\hline & AH2 & PGRE & $8.005-4$ & $1.077-6$ & \multirow{2}{*}{ Jas AG2 } & & & & & & $*$ \\
\hline & & BNL & $7.617-4$ & $5.480-7$ & & & & & & & \\
\hline & AH3 & PGQE & $4.724-2$ & $1.656-8$ & 1.6448 & $2.876-9$ & $1.357-8$ & & 1.153-10 & & $*$ \\
\hline & & BNL & $2.771-2$ & 1.519-8 & $1.513-8$ & 2.933-10 & $1.484-8$ & & 5.359-11 & & \\
\hline & AH4 & PGRE & $6.921-4$ & $6.922-4$ & \multirow[b]{2}{*}{ \}a AFI } & & & & & & \\
\hline & & BNL & $7.001-4$ & $7.002-4$ & & & & & & & \\
\hline & AHS & PGeE & $8.371-4$ & $1.077-6$ & \multirow{2}{*}{ Jas AG2 } & & & & & & $*$ \\
\hline & & BNL & $7.827-4$ & $5.480-7$ & & & & & & & \\
\hline & AH6 & PGeE & $6.922-4$ & $6.922-4$ & \multirow[b]{2}{*}{ Jes AF1 } & & & & & & \\
\hline & & BNL & $7.002-4$ & $7.002-4$ & & & & & & & \\
\hline & AHA & PGeE & $6.921-4$ & $7.392-4$ & \multirow{2}{*}{ AFA } & & & & & & \\
\hline & & BNL & $7.001-4$ & $7.453-4$ & & & & & & & \\
\hline
\end{tabular}


Table B2.2.3e (Continued)

\begin{tabular}{|c|c|c|c|c|c|c|c|c|c|c|c|}
\hline Top & Case & Cale. & $\operatorname{CSP}$ & TTL & HW & HWI & HWD & TS & $\mathbf{M N}$ & $\mathbf{H E}$ & $\begin{array}{c}\text { Comment } \\
\text { No. }\end{array}$ \\
\hline & AHB & PGeE & 4.419-2 & $2.770-5$ & \multirow{2}{*}{ AGB } & & & & & & $*$ \\
\hline & & BNL & $2.765-2$ & $2.636-5$ & & & & & & & \\
\hline & AHC & PGeE & $3.028-1$ & $6.232-6$ & $6.229-6$ & $2.876-9$ & $6.226-6$ & & $2.882-9$ & & $*$ \\
\hline & & BNL & $2.459-1$ & $6.483-6$ & $6.480-6$ & 2.933-10 & $6.480-6$ & & $2.816-9$ & & \\
\hline & AHD & PGRE & $7.126-4$ & $7.392-4$ & \multirow{2}{*}{ AFA } & & & & & & \\
\hline & & BNL & $7.195-4$ & $7.453-4$ & & & & & & & \\
\hline & AHE & PGeE & $5.179-2$ & $2.770-5$ & \multirow{2}{*}{ AGB } & & & & & & $*$ \\
\hline & & BNL & $3.537-2$ & $2.636-5$ & & & & & & & \\
\hline & AHG & PGeE & $7.392-4$ & $7.392-4$ & \multirow{2}{*}{ APA } & & & & & & \\
\hline & & BNL & $7.453-4$ & $7.453-4$ & & & & & & & \\
\hline \multirow[t]{4}{*}{ SF } & SP1 & PGQE & $1.598-3$ & 1.598-3 & $1.533-3$ & $1.533-3$ & 2.448-7 & & $6.500-5$ & & \\
\hline & & BNL & $1.583-3$ & 1.583-3 & $1.520-3$ & $1.520-3$ & $2.604-7$ & & $6.288-5$ & & \\
\hline & SFA & PG\&E & $1.708-3$ & $1.708-3$ & 1.643-3 & 1.533-3 & $1.100-4$ & & $6.500-5$ & & \\
\hline & & BNL & $1.696-3$ & $1.696-3$ & $1.634-3$ & $1.520-3$ & $1.137-4$ & & $6.288-5$ & & \\
\hline \multirow[t]{12}{*}{ SG } & SG1 & PGRE & 1.598-3 & 1.598-3 & \multirow{2}{*}{ Jes SP1 } & & & & & & \\
\hline & & BNL & $1.583-3$ & $1.583-3$ & & & & & & & \\
\hline & SG2 & PG\&E & $1.740-3$ & $6.446-6$ & $6.257-6$ & $6.115-6$ & $1.420-7$ & & $1.891-7$ & & $*$ \\
\hline & & BNL & $1.674-3$ & 2.651-6 & $2.459-6$ & $2.310-6$ & 1.494-7 & & $1.912-7$ & & \\
\hline & sG3 & PGEE & $1.598-3$ & 1.598-3 & \multirow{2}{*}{ Jas SP1 } & & & & & & \\
\hline & & BNL & $1.583-3$ & $1.583-3$ & & & & & & & \\
\hline & SGA & PG\&E & $1.645-3$ & $1.708-3$ & \multirow{2}{*}{ Jea SFA } & & & & & & \\
\hline & & BNL & $1.631-3$ & $1.696-3$ & & & & & & & \\
\hline & SGB & PG\&E & $5.312-2$ & $6.947-5$ & $6.926-5$ & $6.115-6$ & $6.315-5$ & & $2.032-7$ & & $*$ \\
\hline & & BNL & $3.995-2$ & $6.777-5$ & $6.756-5$ & $2.310-6$ & $6.525-5$ & & $2.054-7$ & & \\
\hline & sGc & PG\&E & 1.708-3 & 1.708-3 & \multirow{2}{*}{ Jas SFA } & & & & & & \\
\hline & & BNL & $1.696-3$ & $1.696-3$ & & & & & & & \\
\hline SH & SH1 & PG\&E & 1.598-3 & 1.598-3 & \multirow{2}{*}{ Jas SF1 } & & & & & & \\
\hline & & BNL & $1.583-3$ & $1.583-3$ & & & & & & & \\
\hline & SH2 & PG\&E & 1.699-3 & 6.446-6 & \multirow{2}{*}{ Jas SG2 } & & & & & & $*$ \\
\hline & & BNL & $1.650-3$ & $2.651-6$ & & & & & & & \\
\hline & $\mathrm{SH} 3$ & PG\&E & $3.033-2$ & $1.051-7$ & $1.041-7$ & $6.496-8$ & $3.912-8$ & & $1.053-9$ & & $*$ \\
\hline & & BNL & $1.595-2$ & 4.228-8 & 4.182-8 & $3.511-9$ & $3.831-8$ & & 4.639-10 & & \\
\hline & SH4 & PG\&E & 1.598-3 & 1.598-3 & \multirow[b]{2}{*}{ fas SF1 } & & & & & & \\
\hline & & BNL & 1.583-3 & $1.583-3$ & & & & & & 1 & \\
\hline
\end{tabular}


Table B2.2.3e (Continued)

\begin{tabular}{|c|c|c|c|c|c|c|c|c|c|c|c|}
\hline $\begin{array}{c}\text { Top } \\
\text { Event }\end{array}$ & Cave & Cale. & CSF & TTL & HW & HWI & HWD & $\mathbf{T S}$ & $\mathbf{M N}$ & $\mathbf{H E}$ & $\begin{array}{c}\text { Comment } \\
\mathbf{N} \text {. }\end{array}$ \\
\hline & SH5 & PGeE & $1.740-3$ & $6.446-6$ & \multirow{2}{*}{ fas SG2 } & & & & & & $*$ \\
\hline & & BNL & 1.6743 & $2.651-6$ & & & & & & & \\
\hline & SH6 & PGRE & 1.598-3 & $1.598-3$ & \multirow{2}{*}{ Jas SF1 } & & & & & & \\
\hline & & BNL & 1.583-3 & $1.583-3$ & & & & & & & \\
\hline & SHA & PG\&E & $1.598-3$ & $1.708-3$ & \multirow{2}{*}{ Jas SFA } & & & & & & \\
\hline & & BNL & $1.583-3$ & $1.696-3$ & & & & & & & \\
\hline & SHB & PGQE & $4.421-2$ & $6.947-5$ & \multirow{2}{*}{ \}as SGB } & & & & & & $*$ \\
\hline & & BNL & 3.133-2 & $6.777-5$ & & & & & & & \\
\hline & SHC & PG\&E & 2.901-1 & $1.634-5$ & $1.633-5$ & $6.496-8$ & $1.626-5$ & & $1.315-8$ & & * \\
\hline & & BNL & 2.471-1 & $1.674-5$ & $1.673-5$ & $3.511-9$ & $1.673-5$ & & $1.274-8$ & & \\
\hline & SHD & PG\&E & $1.645-3$ & $1.708-3$ & \multirow{2}{*}{ \}as SFA } & & & & & & \\
\hline & & BNL & $1.631-3$ & $1.696-3$ & & & & & & & \\
\hline & SHE & PG\&E & $5.312-2$ & $6.947-5$ & \multirow{2}{*}{ Jas SGB } & & & & & & * \\
\hline & & BNL & $3.995-2$ & $6.756-5$ & & & & & & & \\
\hline & SHG & PG\&E & $1.708-3$ & $1.708-3$ & \multirow{2}{*}{ Jas SFA } & & & & & & \\
\hline & & BNL & $1.696-3$ & $1.696-3$ & & & & & & & \\
\hline
\end{tabular}

* All of these conditional split fractions involve powers $(\geq 2)$ of unavailabilities whose quantifications require convolutions of unavailability distributions. Since for audit calculations BNL used point value approximation the BNL results should be considered as lower limits of the correct (PG\&E) values.

Note: "\} as _ " means that the remaining values in those lines are the same as the referenced split fraction occurring earlier in the table. 


\section{B2.2.3d}

Unavailability Values (Conditional Split Fractions) for the Vital AC/DC System - Unit 2

\begin{tabular}{|c|c|c|c|c|c|c|c|c|c|c|c|}
\hline $\begin{array}{c}\text { Top } \\
\text { Erent }\end{array}$ & Case & Cak. & CSF & TTL & HW & HWI & HWD & TS & MN & HE & $\begin{array}{c}\text { Comment } \\
\text { No. }\end{array}$ \\
\hline BF & BF1 & PG\&E & $1.440-3$ & $1.440-3$ & $1.386-3$ & $1.386-3$ & $1.074-7$ & & $5.410-5$ & & \\
\hline & & BNL & $1.431-3$ & $1.431-3$ & $1.376-3$ & $1.376-3$ & $1.036-7$ & & $5.571-5$ & & \\
\hline BG & BG1 & PG\&E & $1.440-3$ & $1.440-3$ & & & & & & & \\
\hline & & BNL & $1.431-3$ & $1.431-3$ & Jas BF1 & & & & & & \\
\hline & BG2 & PG\&E & $1.476-3$ & $2.930-6$ & $2.775-6$ & $2.713-6$ & $6.182-8$ & & $1.546-7$ & & $*$ \\
\hline & & BNL & $1.471-3$ & $2.105-6$ & $1.952-6$ & $1.892-6$ & $5.930-8$ & & $1.533-7$ & & \\
\hline BH & BH1 & PG\&E & $1.440-3$ & $1.440-3$ & & & & & & & \\
\hline & & BNL & $1.431-3$ & $1.431-3$ & jas BF1 & & & & & & $*$ \\
\hline & BH2 & PG\&E & $1.476-3$ & $2.930-6$ & & & & & & & \\
\hline & & BNL & $1.461-3$ & $2.105-6$ & jas BG2 & & & & & & \\
\hline & BH3 & PG\&E & $1.187-2$ & $2.439-8$ & $2.390-8$ & $7.794-9$ & $1.610-8$ & & $4.884-10$ & & $*$ \\
\hline & & BNL & $8.486-3$ & $1.786-8$ & $1.754-8$ & $2.603-9$ & $1.493-8$ & & $3.262-10$ & & $*$ \\
\hline
\end{tabular}

*These conditional split fractions involve powers $(\geq 2)$ of unavailabilities whose quantifications require convolutions of unavailability distributions. Since for audit calculations BNL used point value approximation the BNL results should be considered as lower limits of the correct (PG\&E) values.

Note: "\} as _ "means that the remaining values in those lines are the same as the referenced split fraction occurring earlier in the table. 
Appeadix B2

Table B2.2.3e

Unavallability Values (Split Fractions and Conditional Split

Fractions) for the Instrument AC System

Top Erents: 11, 12, 13, 14

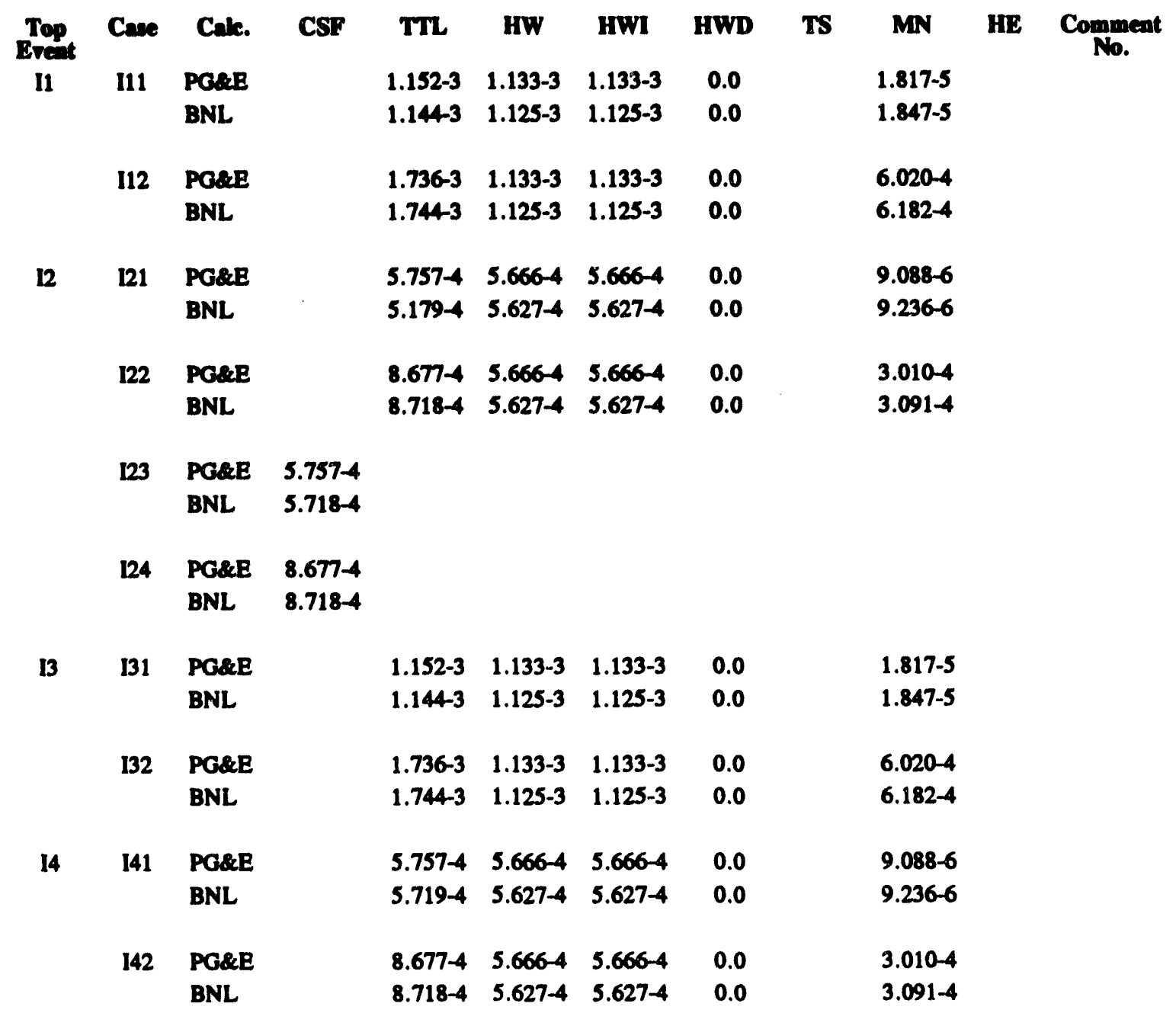


Table B2.2.4

Vital 4.16:V Bus Loading

\section{Fant Condition 4.16 kV Bus Operation}

Lose $500 k V+$ Stay on aux. transformer.

shed load

Loes 500kV +

230kV shed

loed

Loee 500kV +

unit trip

Two 500kV

breakers open

+ unit trip

Unit trip + SI

SI (alone)

Unit trip + Transfer to diesel.

LOSP (all)

Unit trip + Transfer to diesel.

LOSP + SI

Londs:

System/Component

- 480 MCC*

- Auxiliary Saltwater

- Centrifugal Charging

- Reciprocating Charging

- Component Cooling Water

Safety Injection

Residual Heat Removal

Contrinment Spray **

Auxiliary Feedwater

Containment Fan Coolers* (MCC load)

- System used during normal operation.

* These never trip (except containment fan coolers).

* Loed only if "P" (Phase B) signal is present.

\section{Diesd Operation \\ Start Load}

$\begin{array}{ll}\text { No } & \text { No } \\ \text { Yos } & \text { No }\end{array}$

No No

No No

Yes No

Yes No

Yes Yes

Yes Yes

$\begin{array}{lcc}\text { Bus E } & \frac{\text { Bus G }}{\text { F }} & \frac{\text { Bus H }}{\mathbf{H}} \\ 1 & 2 & \\ 1 & 2 & \\ & 3 & \\ 1 & 2 & 3 \\ 1 & & 2 \\ & 1 & 2 \\ 3 & 1 & 2 \\ 2 & 3 & 2 \\ 1 & 5 & \end{array}$




\section{B2.3 Results of the BNL Review}

\section{B2.3.1 General}

The unavailability analyais of the Electrical Power System in the DCPRA was reviewed by BNL with an emphasis on complotences and adequacy in modelling the electrical systems. In addition, to check for calculational inconsistencies,

all of the split fractions (w/o soismic) were recalculated (audited).

\section{B2.3.2 Observations on the Umavallability Modelling of Eloctuic Power System}

The review of the Electric Power Systems resulted in the following obeervations and questions.

\section{B2.3.2.1 Nonvital Electric Power System}

1. The startup transformers (SU-11 and SU-12) are depicted in the nonvital electric power system description (Figure D.2.1-1,Sheet 4) as somewhat complex systems; e.8.,transformer SU-11 has two cooling oil pumps and 25 cooling fans (powered via breaker 52-11D-23 from bus 11D 480V) as well as radiators. It was stated that the transformers can carry only up to $60-70 \%$ of the load without cooling. Upon further discussions with PG\&E it was established that the transformers can handle 100\% of the load in the short term but for long term aging considerations the supplemented cooling is desirable.

2. It was not clear if the switch yard/plant breakers (542 and 632) had already been replaced by "seismic resistant" Hitachi breakers or not. PG\&E documented that the breakers were indeed changed out.

3. Assumption 2 for quantifying Top Event OG states that failures to accomplish load rejection down to house loeds are included in the loss of power initiator. Upon further questioning, PG\&E stated that no credit was taken for Diablo Canyon's load rejection capability in the model and that the generic data used for the LOOP initiator most likely did contain a contribution from load rejection failure but it was not easily identifiable.

4. Block 3 shown in Figures B2.2.2 and B2.2.3 was not developed at the equation level. It was said that it might be modelled as a recovery action if it were needed. Upon further investigation by BNL, PG\&E stated that the recovery action would have been backfeeding power from the $500 \mathrm{kV}$ grid which was never made part of the model.

\subsubsection{Vital 125V DC Syter}

1. Figure B2.2.1 as well as Figures B2.2.4.1 and B2.2.4.2indicate a bus tie between dc buses 11 and 12 (which might be in use during maintenance). The system analysis does not consider common cause failures between dc trains. Upon further investigation, PG\&E explained that the two bus-tie breakers are interlocked to prevent simultaneous closure. The purpose of this tie is to allow connection of the space charger. 
2. In modelling of the olectrical recovery actions, the DCPRA states (p.3-5-18): "besed on the actual plant operation data, PG\&B electrical dovign personnel cetimated an exteaded battery availability of more then 12 hours with no reduction in de loads during a station blackout." In the unavailability analysis of the dieal generator it was sesumed that the batteries are not recoverable after depletion. Depletion timo was taken to be 12 hours. PG\&B was asked to clarify the consistency of the assumptions used for battery depletion time in the DCPRA (2 hours or 12 hours) and its impact on accident sequences where battery depletion is important (operation of turbine driven AFW pump, etc.). PG\&E responded with the following:

For scenarios in which de power is successful but ac power is lost, credit is only given for electric power recovery within the 12-hour battery depletion time. This treatment of the de power unavailability is conservative in the estimate of core damage frequency, especially when battery depletion is important.

\subsubsection{Vital AC Sydem, Unit 1}

1. The system analysis stated that the $4.16 \mathrm{kV}$ switchgear room needs cooling (heavy equipment being used during normal operation) via cooling fans. The unavailability modelling of the system assumes that the 4.16kV switchgear room does not require ventilation. PG\&E was asked to provide clarification of this situation. PG\&E stated that although cooling fans are part of the plant design they are not required in order to keep the room below critical temperatures.

2. The $480 \mathrm{~V}$ switchgear room ventilation was considered so important that a top event was dedicated to it (Top Event SV; not reviewed in detail). BNL questioned the fact the "failure of fire damper" in the 480V switchgear room (a fairly infrequent event) was included in the top event analysis of the vital ac system and not in that of the switchgear room. The PG\&E response clarified the situation in that first, this is not applicable to the $4.16 \mathrm{kV}$ switchgear room and second, that each of the $480 \mathrm{~V}$ switchgear rooms has its own damper that could block ventilation to only its own room as is reflected in the model.

3. It appeared that the failures of the hardware (relays, electronics) associated with the permissives (allowing/disallowing power source transfer) were not modelled. PG\&E responded that these elements were included in the boundary conditions and not as specific elements in the top event analysis.

\subsubsection{Vitul ACSCSyden - Unit 2}

The Unit 2 ac/dc system unavailability is modelled in the DCPRA as a combination of the vital ac and dc systems as was done for Unit 1 with the only difference that Unit 2 components are substituted for the Unit 1 components. This approach compelled BNL:

1. to reiterate all the observations made in the previous two sections (Sections B2.3.2.2and B2.3.2.3)also with respect to the Unit 2 vital ac/dc system, and

2. to disagree cn the assumptions made in the analysis about maintenance, tests and human failure contributions to the total unavailability. This latter item is detailed below.

The DCPRA apparently overlocked the fact, that throughout a time period while Unit 1 is in operation, Unit 2 will have one refueling (and/or several cold shutdowns). During a refueling (or cold shutdowns) the 


\section{Appeadix B2}

components of the Unit 2 vitul so/do aystem could be mbjected to longer hating echeduled maintenance and tede (required by Techaical Specificadion, etc.) which could render the various trains of the vital sec/de ayetem unavailable for protrected periods of tims. Therefore, the contributions dus to these sebeduled

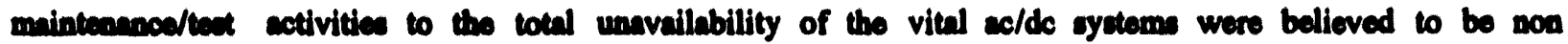
segligible. POEE' respones to this isave indicated that aince Unit 2 olectrical power is basically modelled in aupport of the Unit 2 Auxiliary Saltwater Syetem, the following addreaced this concern: 1) like trin mintenance for the so/de power and auxiliary altwater would be scheduled simultaneoualy and 2) thewe duration were reflected in the POEB update of the auxiliary caltwater syatems analysis.

Given the PO\&B remponse, BNL atill feols that there may be some new requences (due to a coupling of failures between Unit 1 and Unit 2) that would appear and contribute to the total core damage frequeacy. This expectation is baned on the similar conditions that arise when 0.8., the swing dienel is in overtaul or the trin aseociated with BF is unavailable. These types of new sequences were neatly calculated in PO\&B's Dienel Generator Allowed Outage Time Study." A similar calculation bere would aleo have been benoficial.

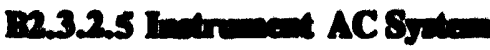

1. The unavailability analyais of the Instrument AC Systom assumes no common cause failures between the instrument sc syetem's channols. On the other hand, the analysis calls attention to the condition that two or more simultaneous instrument $a$ channel failures reault in a reactor trip, i.o., inetrument ac channel failures reprecent a potential eveat initiator. Indeed, the DCPRA ideatified by the Master Logic Diagram (MLD) method the "Loss of Instrumeat AC Power" as an initiator catogory; MLD-20. However, the DCPRA did not analyzo this event catogory and stated (ece Table C.1-3 of DCPRA): "a failure mode and offect analysis shows that failure of more then one instrument chunnel is a low frequency ovent and is not included as a separate initiating oveat." Table C.1-4 roiterates: "The plant will be tripped by lose of RCP if more then one instrument ac channel failure occurs. Random failure of more than one paseive system is an oxtremoly low frequency eveat and therefore is not included as a separate initiating ovent. However, multiple failure of instrument channels due to external causes (0.8., carthquake and $480 \mathrm{~V}$ switchgear ventilation) are addreseed."

In order to check whether the DCPRA's claim about the negligible occurrence frequency of multiple instrument channel failures was valid or not, BNL referred to a recent study ${ }^{7}$ conducted on inverter aging. According to this study, in the nine years from 1976 to 1984 (i.e., during 720 reactor years of operation) there were 42 reactor trips that reoulted from (multiple) inverter failures, i.e., .058 trips/reactor year. From 1984 to 1986 (i.e., during 308 reactor years) 57 reactor trips occurred due to (multiple) inverter failures, i.e.,.185 tripe/reactor year (in both cases the majority of the trips occurred during high power operation).

Each reactor trip has the potential for impacting safety because of the additional equipment response and operator actions generally needed to bring the plant to a safo and controlled condition. In the DCPRA the reactor trip initiator, RT, and the aseociated ovent tree does not account for events of the above type, since the RT ovent tree is not conditioned for simultaneous guaranteed failure of more than one instrument ac channol.

The closest ovent tree involving simultaneous guaranteed failures of instrument ac channels is the one aseociated with the initiator "Loss of One DC Bus" with an initiator frequency; LIDC $=2.56-2 /$ year. 
In order to menke a rough eximato of the impect of the seglection of the low of inatrumeat we power initivitor from the DCPRA model to the core damage frequency, the simpleat way appeared to bo to ineremes the value of the LIDC initiator, 0.8., by the weighted avernge of the above reactor trip frequescies, 1.0., 0.096 tripa/renctor year. Then, the now value of the initiator would be: LIDC $=0.096$ $+.0256=0.122 /$ year. BNL then performed a censitivity atudy uaing this now frequency to provide an upper bound on the poesiblo impect. The upper bound calculation yielded a $20 \%$ increave in total nonseiemic CDP uaing the dominent sequeace model.

2. Pigure B2.3.1 shows the distribution of cauces of invertor failures for LER oveats in the 1984-1986 period (figure is aleo taken from Roference 7). One observes that $18 \%$ of the inverter failures were caused by percoanel error (mado during unscheduled maintenance, teat, elc.). Therefore, it would aleo seem that humen error contributions should be explicitly included in the eplit fractions of the instrument ac system.

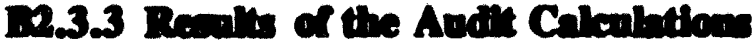

In order to scrutinize the quantified values of the eplit fractions themeelves, BNL performed audit calculations for ench of the eplit fractions aseociated with each of the boundary conditions. Soismic aplit fractions were not chocked. The reacon for the detriled audit was that these eloctrical syatem associated eplit fractiona determine the aupport eystem ovent tres: somo of the fault trees because of their simplicity, were originally "hend calculated" in the DCPRA. In the BNL calculations the SETS code ${ }^{6}$ and locally generated PC coftware wero ueed. In theee audit calculations tho amo aseumptions, input data, humen orror probabilities as well as mainteanance and teet frequency and duration values were ued as in the DCPRA.

The results obtained by the eudit calculations are presented in Tablen B2.2.3a through B2.2.3d. They are denoted by "BNL" to be compared with the values given in the DCPRA (denoted by "PO\&E"). By comparing the PO\&B and BNL renulte, coe can wee that there is an overall agreement between the data. The agreement is oven better if one takes into sccount that BNL used point extimates while PG\&E used a Monto-Carlo approsech in the split fraction quantification.

\section{D2.3.4 Comments on the LOOP Initintor}

In a very recent article in the BRRL Joum ${ }^{7}$ there is a discussion concerning solar magnetic storms. This aubject would not normally be a concern in a PRA, however, the article includes a map which shows all of Californin within a "high-potential" zone and includes a discussion with specific examples that demonstrates a real threat to power grid integrity. Banod upon this article, BNL requested that PG\&B evaluate whether or not this phenomeson would have an impact on the derivation of thoir LOOP initiator frequency. PG\&E looked into this pheoomenon and stated that it would not change their thinking/derivation concerning the DCPRA LOOP initiator frequescy. As a sensitivity study, BNL mado the following assumptions: during the next 30 years, one disnuptive ovent would occur on the PG\&E grid with a 0.5 probability of causing \& LOOP ovent at Diablo Canyon. When this contribution was addod to the PG\&E LOOP value and substituted into the dominant sequence model, the non-seismic CDF increased by just over $4 \%$. 
Appeandix $\mathbf{B 2}$

\subsubsection{Cenchering}

The BNL roviow ideatified everen inconaistencies and potential omineions in the unavailability modelling of

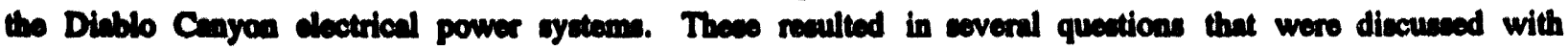
POAB a highlighted berin. The combined results of the ideatified omissions may reault in a slight underentiontion of the expected core damage frequency of Unit 1. BNL's audit calculations were in close agresenent with those within the DCPRA concerning the electrical power aystem.

\section{B2.4 Reforences}

1. Final report on the Diablo Canyon Loag-Term Soismic Program, Pacific Gas and Blectric Co., Diablo Canyon Power Plant, Docket Nos. 50-275 and 50-323, July 1988.

2. Units 1 and 2 Diablo Canyoa Power Plant, "Final Safety Andyeis Report Update," Pacific Gas and Blectric Co., December 1988.

3. PGeB lettere to NRC signed by J.D. Shiffer, No. DCL-88-238, October 10, 1988, No. DCL-88-260, October 28, 1988, No. DCL-88-285, November 29, 1988, No. DCL-88-297, December 9, 1988, No. DCL89-010, Jenuary 16, 1989.

4. Diead Generator Allowed Outage Timo Study, Pacific Gas \& Electric Company, May 1989.

5. W.B. Gunther, R. Lowis and M. Subudhi, "Detecting and Mitigating Battery Charger and Inverter Aging," NUREG/CR-5051, BNL-NUREG-52108, August 1988.

6. R.B. Worrel and D.W. Stack, "A SETS User's Manual for the Fault Tree Analyst," Sandia National Laboratorien, NURBC/CR-4075, SAND77-2051, November 1978.

7. "A Storm From the Sun," EPRL Jonme, July/August 1989, pp. 14-21. 


\section{Percent of Failures}

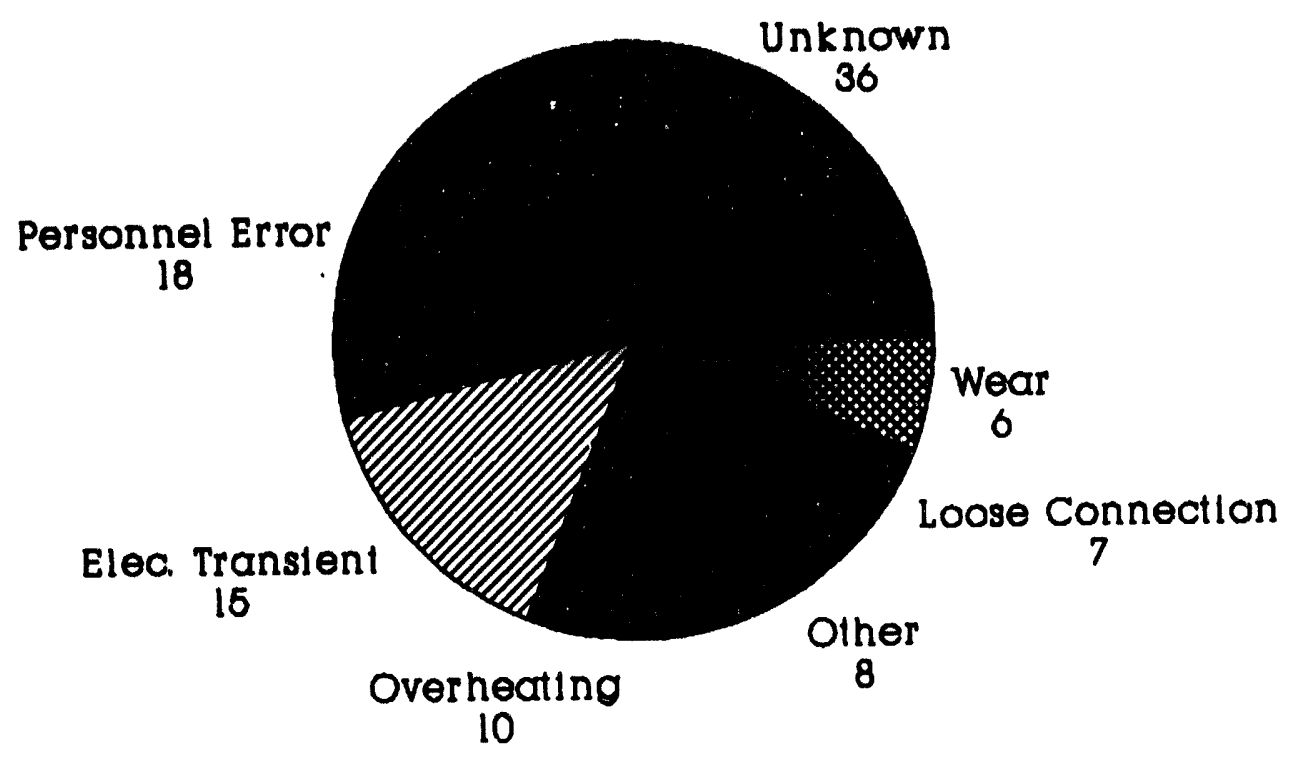

Figure B2.3.1 Inverter failure causes (1984-1986 LERs). 


\section{APPENDIX B3: AUXILIARY SALTWATER SYSTEM}

\section{B3.1 Introduction}

\section{B3.1.1 Objectives}

The main objective of this appendix is to summarize the results of reviewing the unavailability analysis of the auxiliary saltwater (ASW) system described in the DCPRA. ${ }^{1}$ An additional objective is to determine a new value for the initiator "Total Loss of Auxiliary Saltwater (LOSW)" based on generic plant experience appropriately updated for Diablo Canyon with Bayesian techniques. This was done to compare with the DCPRA value obtained by, calculating the total yearly failure frequency of the ASW system.

\section{B3.1.2 Organimation}

Section B3.2 provides a brief description of the configurations and the functions, the dependency on support equipment, the surveillance and maintenance conditions, the unavailability modelling in the DCPRA, and the original DCPRA results. The purpose of this approach is to present stand alone documentation to which the review's findings can be directly compared. Section B3.3 contains the preliminary results and findings of the BNL review as well as a new value for the LOSW initictor.

As this particular systems analysis review was conducted and documented in a letter report to NRC midway through the overall DCPRA review, PG\&E was able to respond to the preliminary findings and in this particular case made changes to their model. Section B3.4 briefly describes the changes made and PG\&E's new results as well as BNL's updated findings/conclusions.

For completeness, the documentation of the data used by BNL for determination of the initiator frequency (LOSW) is presented in Attachment B3.A. In addition, the ranked cut sets of hardware unavailabilities (both independent and total) obtained by BNL for a representative condition (AS1) and the calculated initiator values are given in Attachment B3.B.

\section{B3.2 Unavailability Modelling of the Auxiliary Saltwater System in the DCPRA}

\section{B3.2.1 System Deseription, Configurations and Functions}

The function of the Diablo Canyon auxiliary saltwater (ASW) system is to provide cooling water to transfer heat from the component coling water (CCW) system to the Pacific Ocean. The ASW system of Unit 1 consists of two trains. Each train includes the motor-operated ASW bay gate, and ASW pump, the discharge check and manual isolation valves, the secondary side of the Component Cooling Water (CCW) System heat exchanger, and the exhaust fan that supplies ASW pump room ventilation, when the pump is running.

Two normally open train-to-train crosstie valves insure that each pump can serve both CCW heat exchangers. If Unit 1 ASW pumps fail, Unit 2 pumps are able to provide flow to Unit 1 equipment through opening of a normally closed unit crosstie valve. Equipment that can be considered common to both ASW pump trains of Unit 1 are the traveling screen and the train-to-train crosstie valves. 


\section{Appendix B3}

The ASW system is normally operating with one pump running and one CCW heat exchanger in service. The non-operating ASW pump is in a standby mode. It starts automatically
a. on low header pressure,
b. bus transfer to startup power,
c. diesel generator start, or on
d. safety injection signal.

If the ocean temperature exceeds $64^{\circ} \mathrm{F}$ it is manually started. The ASW pump bay gates are normally open so the standby pump has an available suction source. ASW ventilation fans start automatically when the ASW pumps start and stop when the puinps stop.

\section{B3.2.2 Top Event Definition, Success Criteria}

Associated with the unavailability of the ASW function, the DCPRA defines only one top event to used in the support system event tree ("mechanical" part). The designator of this top event is: AS. It is evaluated for 14 boundary conditions depending on the initiator and/or the unavailability of ASW trains of Unit 1 and Unit 2. One of the boundary conditions, "Loss of ASW Supply tc Unit 1 (designator: ASI)" was taken as an initiator among one of the initiator groups of the DCPRA called: "common cause initiating events." The name of the boundary condition as initiator is: "Total Loss of Auxiliary Saltwater (LOSW)" as was mentioned in Section B3.1.1. Its value is computed as: $\mathrm{ASI}=\mathrm{LOSW}$.

The AS model assumes that initially pumps 1-1 (Unit 1) and 2-1 (Unit 2) are the normally running pumps. The other two pumps are in standby.

The success criteria of the top event AS is described in Table B3.2.1 for post accident injection and recirculation phases, as well as for normal plant cooldown. For comparison, the success criteria for ASW required by the DCFSAR ${ }^{2}$ are also indicated.

\section{B3.2.3 Logic Model, Dependency on Other Support Systems}

The logic model of the top event AS describes the system configuration shown in Figures B3.2.1 and B3.2.2. The logic model is constructed from blocks (supercomponents) of AS components. The boundaries of the blocks are given in Figures B3.2.1 and B3.2.2.

The logic model itself is shown by the diagram in Figure B3.2.3. The diagram indicates the logic relationship among the blocks and the dependencies on trains or supercomponents of the plant (ac and dc) electrical systems. The start signal to the standby ASW pump is provided either by auto start circuitry or by the Solid State Protection System (SSPS) given an event that generates such an actuation signal.

\section{B3.2.4 Boundary Conditions of Top Event AS}

Top event AS was evaluated for 14 boundary conditions (including the initiating condition, ASI). The boundary conditions (except ASI) resulted from initiating events with an without offsite power available and the combination of various states of the electrical systems of both Units 1 and 2. To be more specific, when offsite power was assumed to be available, only the auto start of the standby ASW pump on low discharge 
pressure was modelled and all Unit 2 support systems were taken to be available. In the case of loss of offsite power, the ASW pumps were required to restart automatically and function after the vital busses had been re-energized. In this case it was not assumed that all Unit 2 support systems were available.

Different operator failures, were applied for modelling the opening of the inter-unit crosstie valve depending upon whether this operation required remote or local actions. The detailed list of the boundary condition definitions and the designators of the associated top event split fractions are given in Table B3.2.2.

\section{B3.25 Quantification of Top Event Split Praction, AS}

The methodology of systems analysis applied in the DCPRA requires that the top event "split fraction" (associated with a system under a given boundary condition) should reflect the notion that the system (or its portion) in question is in one of the following mutually exclusive alignments: 1) normal alignment, 2) testing alignment, 3) maintenance alignment, or 4) misalignment. Thus, the contribution to the system unavailability from a specific alignment is determined by the conditional system unavailability, given that the system is in that alignment multiplied by the fraction of time that the system spends in that alignment. That is the way that the DCPRA considers the constraints imposed by Technical Specifications which disallow simultaneous maintenance or test activities on redundant components and the human errors causing the system or its components (usually occurring after these activities) to be inoperable.

Table B3.2.3 lists the values of AS split fractions associated with the various boundary conditions quantified by PG\&E. The table presents the total unavailabilities(TTL), along with the main contributors to the total unavailabilities, such as hardware (HW), maintenance (MN), test (TS) and human error (HE). At a given boundary condition the hardware contribution relates to the normal alignment, when no test or maintenance activities are being performed. To provide complete information, the table also indicates the two constituent parts of the hardware contribution to the unavailability: the independent (HWI) and the dependent (HWD, i.e., common cause) failures of the supercomponents.

The maintenance alignment is a significant contributor to the unavailabilitybecause it includes the demusseling and chlorination of the trains. Demusseling occurs every 60 days and takes four to five hours per train. During normal maintenance, only the pump of the train in maintenance is unavailable; the two heat exchangers still get cooling water from the running pump or through the crosstie to Unit 2. During demusseling, however, since the intertrain crosstie is closed, the train in maintenance would appear to become complete isolated and unavailable. In this case, the DCPRA changed the success criterion of the ASW system from $2 / 2$ to $1 / 1$. Unit 2 train demusseling and maintenance are modelled identically.

The test alignment is a small contributor to the unavailability because of the relatively short duration involved. During pump start testing the standby pump does not get a start signal if a low pressure condition develops on the discharge header (e.g., due to failure of the running pump) because the pressure sensor is isolated. The status of the ASW system in that case is equivalent to the case when a pump is in maintenance. The ASW pump operability test does not alter the normal configuration except once per year, when the vacuum breakers are tested. Vacuum breakers are used on this system to prevent the occurrence of water hammer (see more about this later in this appendix). During this test the intertrain crosstie is closed, and a situation is similar to demusseling occurs.

Unavailability contributions due to operator failures to realign the system after test or maintenance were assumed to be negligible. This is because the crosstie valves and the motor-operated bay gates have position 


\section{Appendix B3}

indicators in the control room and maintenance procedures require that the open status of the discharge isolation valves and the service readiness of the discharge pressure switch should be verified before an ASW pump would be returned to service.

\section{B3.26 Quantification of the Initiator: "LOSW"}

The DCPRA models and quantifies the initiator LOSW as loss of all ASW supply to both of the Unit 1 CCW heat exchangers. The plugging of the ASW traveling screens is not included in the quantification, because given plugging the DCPRA assumes completely successful and timely mitigating actions.

Two fault trees were constructed by PG\&E to determine the initiator frequency: one, describing the yearly failures of the ASW system during normal operation, and one describing the yearly failures of the system which occur when the running and the standby trains are rotated ( 26 times per year). The fault trees involve independent and dependent component failures of Unit 1 and Unit 2 trains as well as failures occurring during maintenance. The numerical results of the quantification obtained by PG\&E are indicated at boundary condition ASI and denoted by "LOSW" in Table B3.2.3.

\section{B3.3 Results of the BNL Review}

\section{B3.3.1 General}

Special attention was directed by BNL to the review of the unavailability modelling and quantification of the auxiliary saltwater system based upon the following:

1. the DCPRA uses a "non-plant-specific experience-based"value for the LOSW initiator derived from a fault tree,

2. the system is exposed to rather harsh environmental effects (biological fouling, salt water, etc.), and

3. it is an important support system impacting the safety of the majority of plant operations, including cold shutdown.

For the review, therefore, the following approach was used to check the adequacy of the DCPRA modelling for "system-specific" effects. BNL performed a survey of failure events involving the Service Water (SW) Systems at U.S. PWRs by using the RECON ${ }^{3}$ data base and the NPE ${ }^{4}$ operating events listing. After having determined the nature and characteristics of these failures, an evaluation was made as to how well the DCPRA model reflects this 'experience.' The evaluation was carried out by a thorough review of the failure modes involved in the AS top event logic diagram and by comparing the failure rates occurring in the associated fault trees (including those describing the initiator - LOSW) with failure rates used by the DCPRA in the fault trees for other standby systems. In order to check for calculational consistencies, all of the fault trees ivere requantified. (The fault trees are not reproduced here, they can be found in Chapter D.2.6 of the DCPRA.) Furthermore, sensitivity calculations were carried out to determine the impact of changes in the assumptions concerning the availability of the Unit 2 ASW trains. Finally, an attempt was made to independently determine a Diablo Canyon-specific LOSW initiator frequency based upon experience data. 
Appendix B3

\section{B3.3.2 Results of the Survey on SW Systems Fallures}

The results of the BNL survey on failures of SW systems at U.S. PWRs are presented in Attachment B3.A. The failure events are classified into three groups:

a. operating events involving the total loss of the SW system due to component failures or due to environmental effects (Table B3.A.3),

b. operating events involving the total loss of the SW system due to system interactions (e.g., electrical failures) or other initiators (e.g., flooding) (Table B3.A.4), and

c. operating events involving the partial degradation of the SW system due to any cause (Table B3.A.5).

The results also revealed that partial degradation of the SW system is rather frequent and there are some dominant failure modes of the $\mathrm{SW}$ system as a result of proneness to failure of certain components. These dominant failure modes are:

a. Biological fouling and/or sediment deposition. This is an indication that the quality of the cooling water is not very well controlled since SW systems are typically of an open-cycle design. (Systems of open cycle design take and discharge cooling water from and to an ultimate heat sink such as: ocean, lake, river, pond, etc.) The affected components are generally strainers and heat exchangers which become clogged and restrict the flow of the cooling water.

b. Unusually high rate of corrosion of pipe walls, tubes, valves, and consequent leakage. Additionally, mechanical and electrical problems with the operation of the SW pumps.

Failures which lead to the complete loss of the SW function typically involve:

c. The unavailability of the intake structure due to

1. problems associated with the traveling screens (clogging, corrosion, loss of motive power), and

2. cold weather (icing) or flooding.

d. Loss of motive or control power to the operating train (systems interaction) associated with loss of redundancy owing to maintenance or procedural failures.

e. Mechanical or design failures of the SW pumps.

A significant failure mode of SW systems can occur with piping of steep slope. The steep slope creates a situation which may be conducive to water hammer, such as the event that happened at Diablo Canyon in 1982. The following is a quote from Diablo Canyon LER-275/82-10-07 (see Appendix A for further details).

"the (Auxiliary Saltwater) system is susceptible to water hammer effects during anticipated operational transients. These transients include pump trip and restart sequences such as would occur following a loss of offsite power. The peak pressure observed during testing exceeded the 100 psig system design pressure specified in the FSAR. The cause of the system water hammer is believed to be water column separation and subsequent column recombination at a point of significant piping slope change."

The recovery times of the observed SW failure events (as estimated by examining the time evolution of the various events) indicate a distribution extending from a representative time period of $\mathbf{1 - 2}$ hours to more unpredictable time periods of a few hours or of even one or more days (weather, flooding). 


\section{Appendix B3}

It is noted that biological fouling and/or sedimeatation do not tend to cause total loss of the SW system even though these are the dominant causes of partial degradation. This may be explained by the relatively long time available for preventive actions after the failure/degradation is recognized for a given train or component.

\section{B3.3.3 Modelling of the ASW; the Logic Diagrams, Fault Trees}

The review of the unavailability modelling of the ASW system performed by BNL was based upon the latest version of the DCPRA information provided by PG\&E $E^{S}$ and the data obtained from the BNL survey of SW failures described above. The information included the fluid flow diagrams with indication of the supercomponents, Technical Specifications, FSAR, as well as operating and surveillance test procedures relevant to the ASW system.

The review found that the DCPRA unavailability model of the ASW system only weekly reflects the industrywide proneness of an SW train to be randomly blocked or to be prevented from functioning properly (e.g., by leaking) because of the environmental effects prevalent in such systems. Specific observations include:

1. Consider, e.g., the failure mode "Failure of the traveling screens, (ZTSC3P, plugging)." This is a common cause failure in the ASW unavailability model which one can take to be representative of some environmental effects. It was assumed in the DCPRA that this failure mode fails both trains at Unit 1 or both trains at Unit 2 but was not considered as a common mode failure for both units taken together. In other words, the cut set for common mode failure of the traveling screens for both units would be the Unit 2 common mode failure ANDED with the Unit 2 common mode failure. Even so, the DCPRA analysis identified it to be a leading contributor to the ASW systems' unavailability.

In the initiator model, however, this failure mode was not included for Unit 1, on the basis that complete recovery was assumed. As was mentioned in Section B3.3.2, experience indeed gives some indication that these types of failures can be recovered (similar to loss of offsite power initiators). To neglect them completely, however, is judged to be somewhat optimistic.

2. Demusseling (and chlorination) is performed on average once per 60 days for about 4.5 hours per train and has been assumed to be so effective, that the DCPRA did not consider the blockage of the ASW trains as a conceivable failure mode. Neither the ASW unavailability model, nor the model of the component cooling water system takes into account the "plugging of the shell side of the CCW heat exchangers."

3. The ASW unavailability model does not contain failure events which would reflect the frequent leakage/rupture failure events caused by corrosion and observed at numerous other plants.

4. In the ASW model, the same values were taken for the rate of the failure mode "pump fails to start" and for the pump maintenance frequency and duration as those used for pumps of standby safety systems or systems operating in closed cycles and using treated water.

5. As a consequence of neglecting the higher failure rate data observed throl ghout the industry for SW train components, the unscheduled switchover frequency between running and standby trains and the unscheduled maintenance contribution to the system's overall unavailability may be somewhat underestimated.

As concerns other aspects of the modelling, the review identified the following items:

6. The DCPRA changed the success criteria from $2 / 2$ to $1 / 1$ during demusseling activities and during testing of the vacuum breakers in order to avoid the otherwise guaranteed failure condition during these periods per the model's assumptions. 
7. The DCPRA assumes that when an ASW train at Unit 2 is unavailable (failure state or in maintenance/demusseling/test) the other train of Unit 2 can still provide enough cooling flow for the CCW heat exchangers of both units. BNL requested that this assumption be supported by engineering calculations.

8. The DCPRA considers only unscheduled maintenance for Unit 2 trains. Large train overhauls lasting over a protracted period of time performed when Unit 2 is in cold shutdown were not included in the model. During this time the full flow from the running ASW pump of Unit 2 is needed for Unit 2. These periods of complete unavailability of Unit 2 (in terms of Unit 1) should have been represented in the ASW unavailability model, particularly in the fault trees for the LOSW initiator. Similarly, periods when Unit 2 goes to cold shutdown or during warm ocean water conditions (when two ASW trains per unit are required) were omitted from the determination of the initiator frequency.

9. The ASW unavailability model is tacit about the possible occurrence of waterhammer given loss of offsite power. According to $P G \& E$, the plant eliminated this problem by applying vacuum breakers (see LER No.82-009-01T-1, quoted also in Attachment B3.A).

\section{B3.3.4 Audit and Sensitivity Calculations}

In order to scrutinize the quantified split fractions themselves, BNL performed audit calculations for each of the split fractions associated with each of the boundary conditions. In these calculations the same assumptions, input data, maintenance frequency and duration values were used as in the DCPRA. The SETS$\operatorname{code}^{6}$ and locally generated PC software were used for the computations. The use of the SETS code allowed the identification of the most important cut sets contributing to the hardware unavailabilities. These cut sets are not readily accessible for direct review in the DCPRA. Attachment B3.B lists the ranked cut sets for AS1 and for the initiator, LOSW, as example calculations. The definition of the basic events appearing in the cut sets are identical to those given in Appendix D.2.6 of the DCPRA, except HW1, HW2, HW3, HW11, HW22, and HW33. The definitions of the latter events are indicated in Attachment B3.B.

The results obtained by the audit calculations are presented in Table B3.2.3, denoted by "BNL1," to be compared with the values given in the DCPRA (PG\&E). BNL also performed a sensitivity calculation for all the boundary conditions to see the impact on the split fractions of abandoning the DCPRA's assumption that a Unit 2 ASW train would still be available for Unit 1 even if the other Unit 2 train had failed or was in maintenance/demusseling/test. The results of this sensitivity calculation are denoted by "BNL2" in Table B3.2.3. One can observe that this latter assumption results in a considerable increase in the split fractions associated with some boundary conditions.

\section{B3.3.5 Comments/Findings}

The BNL review and calculations resulted in the following comments:

1. In the expanded block level fault tree (offsite power available) given in Figure D.2.6-5 there were some inconsistencies:

a. In the sheet 1 of 5 (page D.2.6-38) for the events "loss of flow to header 11 only" and "loss of flow to header 12 only" one should use "AND" gates, instead of "OR" gates, given in the figure.

b. In the sheets 4 of 5 and 5 of 5 (pages D.2.6-41, 42) the failure modes of the running and standby pumps were reversed.

2. For the failure modes "pump fails to start," "pump fails to run," as well as for similar failure modes of the fans, the single failure rates used in the fault tree quantification were higher than the total failure rates. 


\section{Appendix B3}

3. The DCPRA fault trees deacribing the initiator LOSW did not include unavailability contributors due to
a. failure of the Unit 1 train-to-train crosstie,
b. the maintenance of the unit-to-unit crosstie,
c. the demusseling of standby trains at both units,
d. failure of the demusseling process, and
e. failure of the traveling screen at Unit 1 (but was considered in block E for Unit 2).

4. While it was assumed for the LOSW fault trees that failures of the traveling screen at Unit 1 can be successfully mitigated, there was no maintenance duration defined with these actions.

The audit calculations denoted by BNL1 incorporate items 1 and 2 above. BNL2 calculations include these corrections and involve (as discussed before) the assumption that a Unit 2 ASW train cannot provide water to Unit $1 \mathrm{CCW}$ heat exchangers if the other Unit 2 train is down due to failure/maintenance/ demusseling/test. In addition, the BNL2 calculation for LOSW incorporates items 3 and 4 above as well as a provision addressing train rotations and some scheduled outages of Unit 2 trains resulting in an unavailability value (for both trains) of .07 .

\section{B3.3.5 Determination of Initiator Frequency, LOSW Based on Industry Experience}

In order to compare the LOSW initiator frequencies obtained in the DCPRA by using ASW unavailability models with values based on industry experience, BNL msde an attempt to determine such Diablo Canyonspecific values by applying a Bayesian tochnique. ${ }^{7}$

Two approaches were used in the calculations:

a. The first approach was based on the observed frequency of appropriately selected LOSW events whose potential occurrence was deemed possible at the Diablo Canyon plant. According to this approach, the mean initiator frequency of LOSW events (non-recoverable within some time $t$ ) can be calculated if the Unit 1 ASW trains were independent of the Unit 2 trains, by the expression:

A

$\operatorname{LosW}_{\mathrm{E}}(1)=.85 * \operatorname{Los} W(1) * P(T \geq t)$

and, if the Unit 1 trains are dependent on Unit 2 trains (actual case) by the formula:

A

$\operatorname{LOSW}_{E}(1,2)=.85 * \operatorname{LOSW}(1) * P(T \geq t) * R_{C}(2 * 1)$

$$
=\operatorname{LosW}_{\mathrm{E}}^{\mathrm{A}}(1) * \mathrm{R}_{\mathrm{C}}(2 * 1)
$$

where LOSW(1) is the "posterior" mean frequency of the selected LOSW events. The selected events are counted independently; i.e.,events in which two units were involved counted twice. $P(T \geq t)$ is the probability that the time to recover a LOSW event will last longer than some given time, $t$. $R_{C}(2 * 1)$ is the conditional probability that given loss of both ASW trains at Unit 1, the Unit 2 trains also become unavailable. This quantity can be calculated by an ASW unavailability model. And .85 is the assumed capacity factor of a Diablo Canyon plant unit. 
b. The second approach derives the mean initiator frequency directly from the experienced frequency of selected LOSW events when SW trains of two units were lost. The mean frequency of these ovents non-recoverable within some given time $t$ can be obtained by the formula: B

$\operatorname{LosW}_{\mathrm{E}}(1,2)=.85 * \operatorname{LOSW}(1,2) * P(T \geq t)$

where LOSW $(1,2)$ is the "posterior" mean frequency of LOSW events experienced at twin units. The two other quantities in Eq.2 are the same as those defined above.

The advantage of Approach A (compared to Approach B) is that its "statistical basis" is better than that of Approach B. However, its disadvantage is that Eq.1b is a hybrid expression; it still needs the calculated quantity $\mathbf{R}_{\mathbf{C}}(2 * 1)$.

The posterior frequency distributions of the quantities $\operatorname{LOSW}(1)$ and $\operatorname{LOSW}(1,2))$ occurring in the above equations were determined by BNL using two-stage Bayesian updating calculations.

The first seven events of Table B3.A.3 and the reactor-years listed in Table B3.A.1 (except those of Diablo Canyon Units 1 and 2) were taken as "experience," and zero number of LOSW events during the operation times of both Diablo Canyon units was taken as "evidence for the LOSW(1) frequency updating calculation. By assuming lognormal prior and posterior frequency distributions and by using "best estimate" parameters for the prior of the second stage updating, the obtained Diablo Canyon specific posterior mean, median, standard deviation, 5th and 95th percentile values are presented in Table B3.2.4 (see "experience based values").

The calculation of the frequency distribution $\operatorname{LOSW}(1,2)$ is based on the San Onofre events, when Unit 2 and Unit $3 \mathrm{SW}$ trains were lost, and on the (overlapping) reactor-years associated with multi-plants listed in Table B3.A.2. The Diablo Canyon-specific posterior values are also given in Table B3.2.4(see also "experience based values").

In order to determine the recovery probability of LOSW events, all the events listed in Table B3.A.3 were used. Event No.8 was also included in the sample to represent some fraction of LOSW events which are nonrecoverable within (say) 12 hours. An exponential distribution was assumed for the recovery probability density function:

$$
f(t)=\lambda e^{-\lambda t}, t \geq 0, \lambda \geq 0 .
$$

Thus, the distribution function

$$
F(t)=P(T \leq t)=\int_{0}^{t} f(x) d x,
$$

gives the probability that a LOSW event will be recovered within $t$ hours, and

$$
P(T \geq t)=1-F(t)
$$

provides the probability that the time to recover a LOSW event will be longer than some given time, $t$.

The cumulative distribution of the LOSW events as a function of the time to recover, the fitting curve, (Eq.5), as well as the ninety percent uncertainty bounds are shown in semi-logarithmic representation in Figure B3.2.4. The maximum likelihood estimate of the parameter $\lambda$ is given by the expression:

$$
\lambda=N / \Sigma t_{i}=.271 / \text { hour }
$$




\section{Appendix B3}

where $t_{1}, t_{2}, \ldots, t_{N}$ represent the sample data, and $1 \hat{\lambda}=\bar{t}$ is the mean time to recovery.

Based on a rough estimate of the heat capacity of the water available in the CCW system given a LOSW ( $t_{1}$ $1 / 2$ hour) and the time necessary to develop a seal LOCA with appreciable leak rate given unavailable cooling ( $t_{2} \sim 1.5$ hours), the critical time for non-recovery of LOSW events was taken to the $t_{1}+t_{2} \sim 2$ hours. At this point in time the probability of non-recovery of an LOSW event was estimated from the best fitting curve in Figure B3.2.4 to be $P(T \geq 2)=.57$.

The conditional probability $R_{C}(2 \mid 1)$, that Unit 2 ASW trains become unavailable for Unit $1 \mathrm{CCW}$ heat exchangers given loss of both ASW trains at Unit 1 was determined by the ratio:

$$
R_{C}(2 \mid 1)=\frac{\operatorname{Losw}_{C}(1,2)}{\operatorname{LOSW}_{c}(1)}
$$

where LOSW $(1,2)$ denotes the calculated frequency of total loss of ASW trains at both Diablo Canyon Units (Units 1 and 2), and LOSW ${ }_{C}(1)$ denotes the calculated frequency of total loss of ASW trains belonging only to Unit 1.

The value of $\operatorname{LOSW}_{C}(1,2)$ is identical to the values of LOSW listed in Table B3.2.3 for the cases "BNL1" and BNL2." For the sake of completeness, however, they are again presented in Table B3.2.4 (see "model based values"). Table B3.2.4 also shows the corresponding values for $\operatorname{LOSW}_{C}(1)$ and $R_{C}(2 \mid 1)$.

For comparison, a ratio based on the experienced data and defined as

$$
R_{E}=\frac{\operatorname{LOSW}(1,2)}{\operatorname{LOSW}(1)}
$$

is also calculated and presented in Table B3.2.4. This value is indicative of the large dependence between the failures of the ASW trains at twin units.

After all the necessary quantities were determined, Eqs.1a, $1 \mathrm{~b}$, and 2 were evaluated. The obtained mean values for the initiator frequencies,

$\operatorname{LOSW}_{\mathrm{B}}^{\mathrm{A}}(1), \operatorname{LOSW}_{\mathrm{E}}^{\mathrm{A}}(1,2)$ and $\operatorname{LOSW}_{\mathrm{E}}^{\mathrm{B}}(1,2)$ are given in Table B3.2.4 (see"experience" and "experience and model" base values).

A comparison of the purely model-based initiator values with those obtained by experience or by "experience and model," shows that there is a satisfactory agreement between the following values:

a. If Unit 1 ASW trains were independent of Unit 2 trains:

$$
\operatorname{LOSW}_{\mathrm{C}}(1)=5 \cdot 16-3 / \mathrm{ry}(" \mathrm{BNL} 2 ") \text { and } \operatorname{LOS} W_{E}^{A}(1)=4.14-3 / r y \text {. }
$$


b. Actual situation of interdependency between units:

$$
\operatorname{LOSW}_{C}(1,2)=5.11-4 / \mathrm{ry}(" B N L 2 ") \text { and } \operatorname{LOSW}_{\mathrm{B}}^{A}(1,2)=4.56-4 / \mathrm{ry} \text {. }
$$

Since these values are higher than the initiator values $\left(\operatorname{LOSW}_{C}(1,2)\right)$ calculated by using the original PG\&E assumptions (PG\&E, BNL1) it appeared that the DCPRA could have undereatimated the real value of the LOSW initiator by at least a factor of 5 . The underestimation would be further exacerbated

if one compares the experience-besed value, $\operatorname{LOSW}_{\mathrm{E}}^{\mathrm{B}}=2.23-3 / \mathrm{ry}$ with the frequencies given above at $b$, because even those values may undereetimate the real value. According to BNL's calculations, the "real" value of the LOSW initiator, $\operatorname{LOSW}_{R}(1,2)$ should lie in the interval:

$$
\operatorname{Los}_{\mathrm{E}}^{\mathrm{B}}(1,2)=2.23-3 / \mathrm{ry}>\operatorname{Los}_{\mathrm{R}}(1,2) \geq \operatorname{Los}_{\mathrm{E}}(1,2)=4.56-4 / \mathrm{ry} \text {. }
$$

Fine-tuning this interval would require much more realistic modelling of both the inter-unit dependencies of the ASW trains as well as the use of more accurate information about the unavailabilities of the Unit 2 trains. Therefore, there is obviously a large amount of uncertainty associated with this interval. In order to factor in an element of expert opinion and to provide an additional data point for the sensitivity analysis (see Section B3.4), BNL took the geometric mean of the above interval (denoted: BNL "Midway" $=1.01-3 / r y$ ).

\section{B3.4 Overall Findings/Conclusions}

As a result of the BNL system analysis review of the Auxiliary Saltwater System, PG\&E performed a number of additional calculations. One of the calculations demonstrated that only under certain circumstances (not all) could a single Unit 2 ASW pump supply both Unit 1 (via the crosstie) and Unit 2 with sufficient ASW flow. PG\&E then carried out a sensitivity study to determine the impact of this finding on their overall results. PG\&E determined that the change in non-seismic CDF was not appreciable and stated that none of the conditional split fractions in Table A3.2.3 required requantification as a result.

In terms of the initiator frequency, LOSW, PG\&E did accept some (not all) of the BNL work and developed a new value for this initiator. This value was not presented in time for BNL to incorporate it into the dominant sequence model for BNL's quantification purposes. However, the following chart shows the relationship between the original and updated PG\&E values and the BNL work.

\begin{tabular}{|l|c|c|c|c|}
\hline \multicolumn{1}{|c|}{ Case } & $\begin{array}{c}\text { LOSW } \\
\text { (Events/Year) }\end{array}$ & $\begin{array}{c}\text { Unnormalized } \\
\text { Fusset-Vesely } \\
\text { Importance }\end{array}$ & $\begin{array}{c}\text { Resulting } \\
\text { Non-Seismic } \\
\text { CDF }\end{array}$ & $\% \Delta$ CDF \\
\hline PG\&E Original & $9.74-5$ & $1.447-6$ & $1.7728-4$ & - \\
\hline PG\&E Updated & $1.40-4$ & $2.080-6$ & $1.7791-4$ & 0.36 \\
\hline BNL1 & $1.62-4$ & $2.407-6$ & $1.7824-4$ & 0.54 \\
\hline BNL2 & $4.56-4$ & $6.775-6$ & $1.8260-4$ & 3.00 \\
\hline BNL "Midway" & $1.01-3$ & $1.500-5$ & $1.9083-4$ & 7.64 \\
\hline BNL-Upper Bound & $2.23-3$ & $3.313-5$ & $2.0896-4$ & 17.87 \\
\hline
\end{tabular}




\section{Appendix B3}

Baeed upon the above chart, it is clear that the LOSW initiator (eccording to the dominent sequence model) is not an important contributor to the Diablo Canyon core damage frequency. In addition, the chart demonatrates that BNL's quantification offort using the original PG\&B value, rather than the updated value, does not provide a distorted picture of the overall Diablo Canyon core damage profile.

\section{B3.5 References}

1. Final report on the Diablo Canyon Long-Torm Seismic Program, Pacific Gas and Electric Co., Diablo Canyon Power Plant, Docket Nos. 50-275 and 50-323, July 1988.

2. Units 1 and 2 Diablo Canyon Power Plent, "Final Sefoty Analysis Report Update," Pacific Gas and Electric Co., Docember 1988.

3. DOE/RECON, Nuclear Safoty Information Center (NSIC), 1963 to present.

4. Nuclear Power Experience, NPE, Published by the S.M. Stoller Corp.

5. PG\&E letters to NRC signed by J.D. Shiffer, No. DCL-88-238, October 10, 1988, No. DCL-88-260, October 28, 1988, No. DCL-88-285, November 29, 1988, No. DCL-88-297, December 9, 1988, and No. DCL-89-010, January 16, 1989.

6. R. Worrel, D. Stack, "A SETS User's Manual for the Fault Treo Analyst," Sandia National Laboratories, NUREG/CR-0465, SAND77-2051, November 1978.

7. C. Park, "Bayes: A Two-Stage Bayesian Update Procedure for Data Specialization for the Plant-Specific Risk and Reliability Analysis," BNL Internal Memorandum, March 19, 1987. 


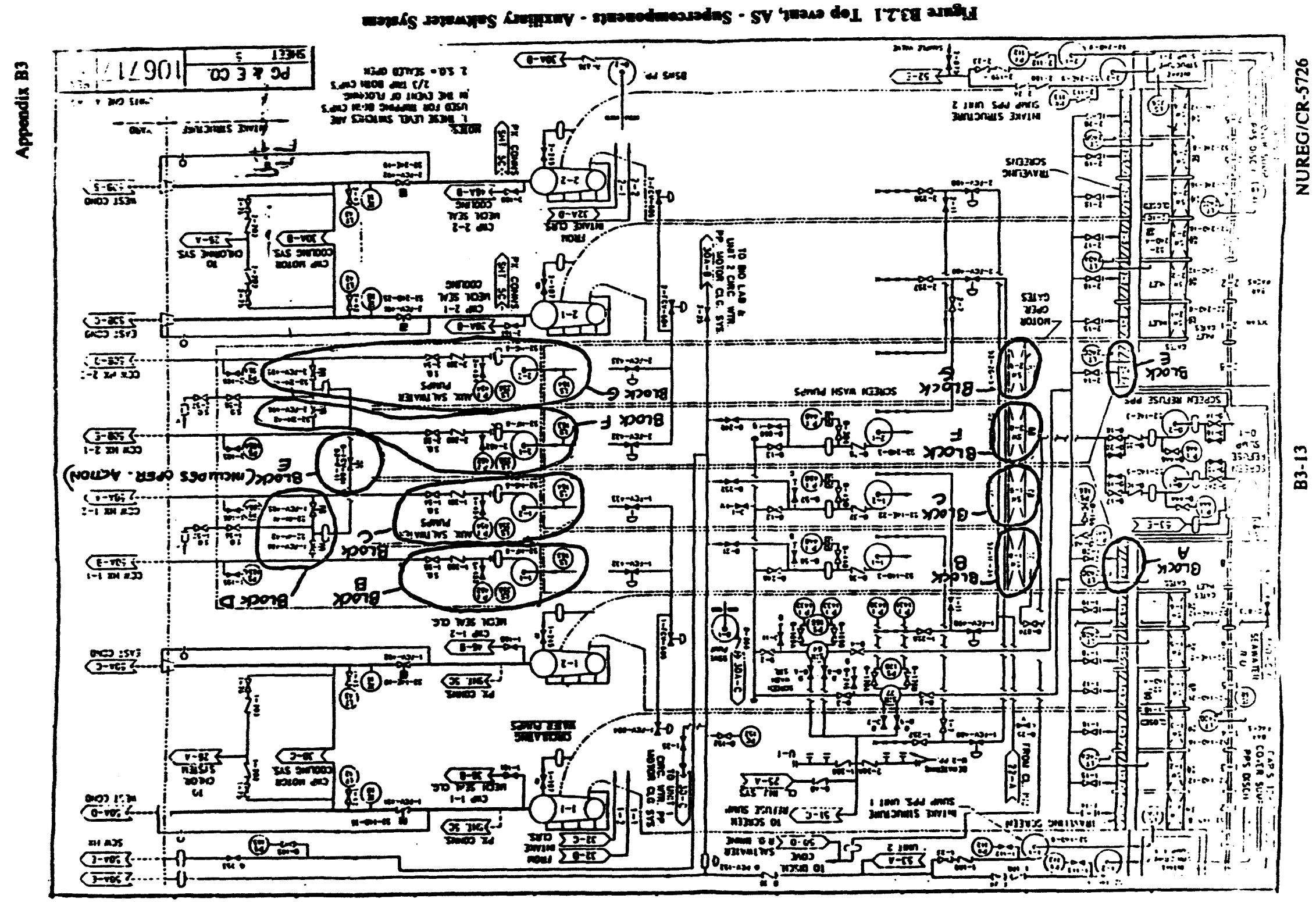




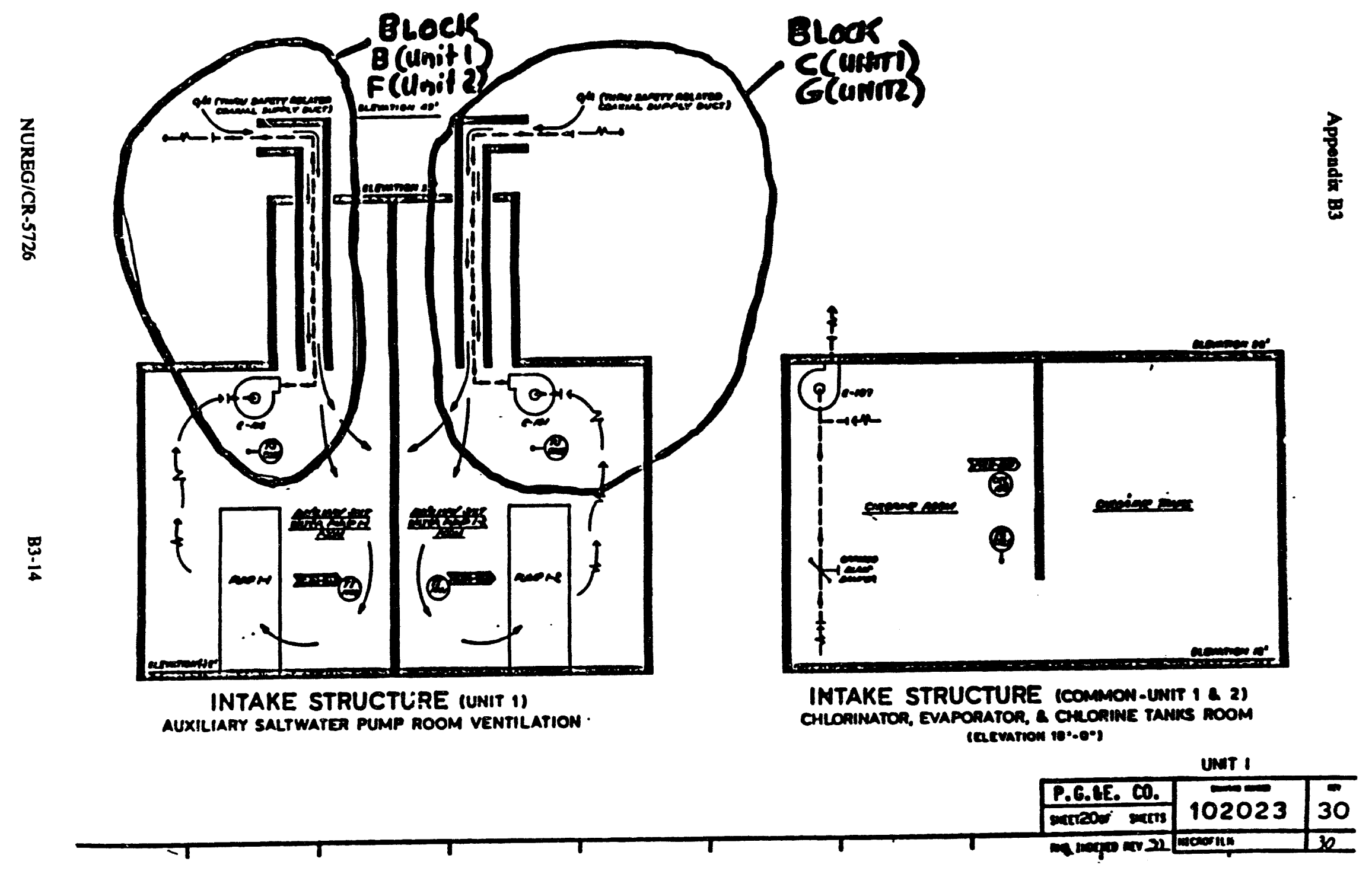

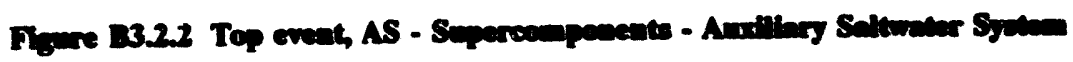


Appendix B3

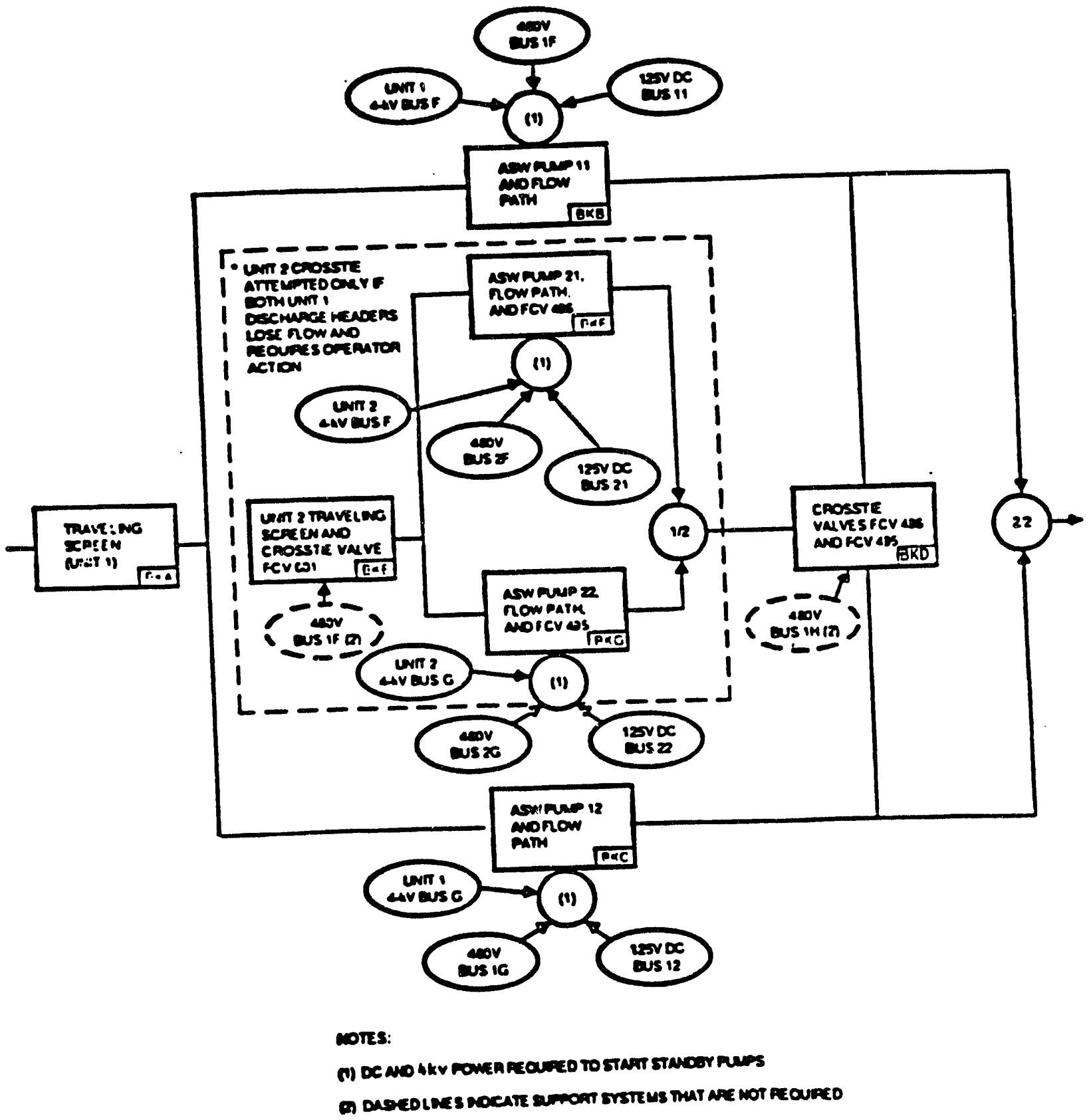

Foure 13.2.3 Logic diagram for top eveat, AS and initiator, LOSW 


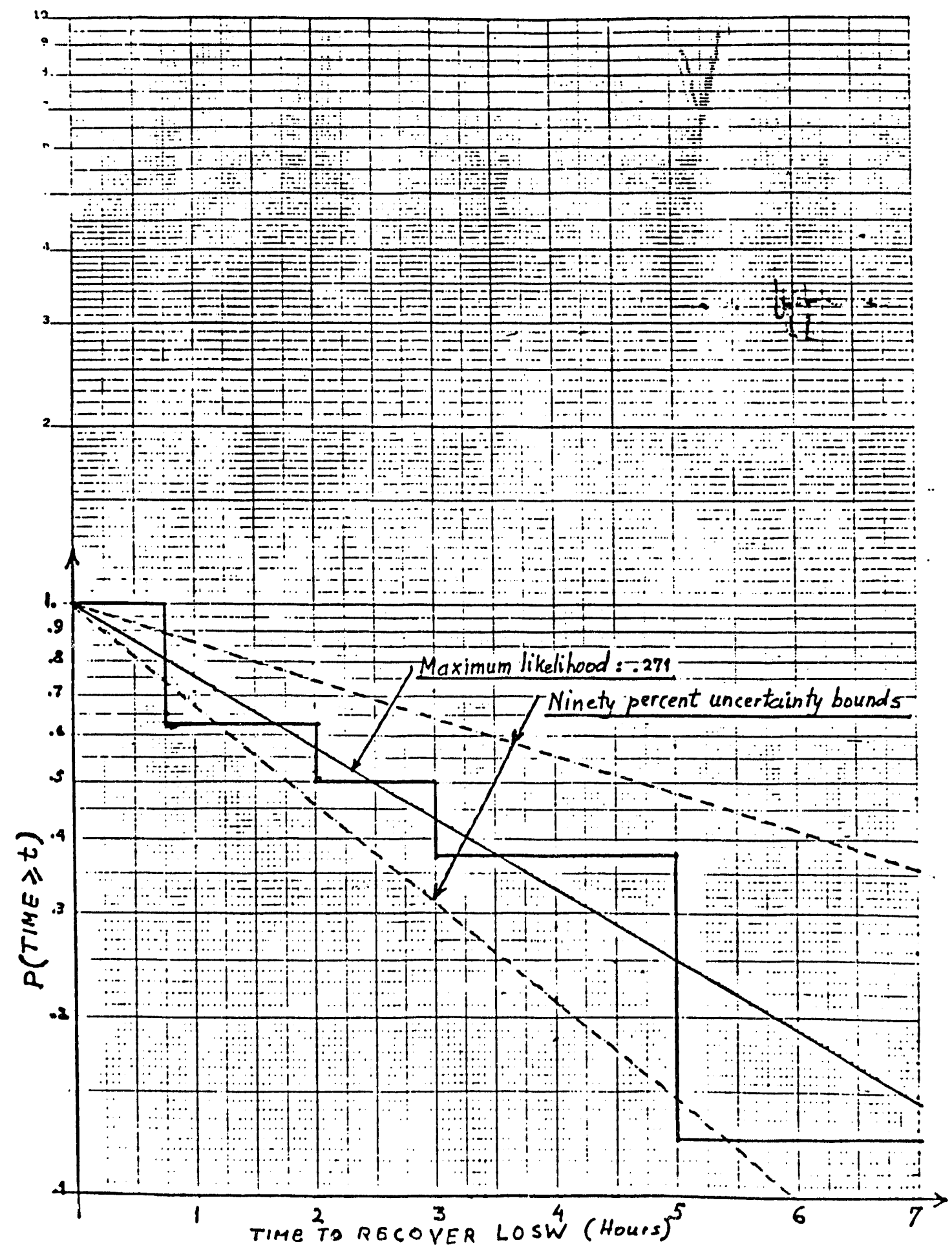

Figure B3.2.4 Exponential model for non-recovery of LOSW events 
Table B3.2.1

Top Event Definition and Success Criteria

Auxiliary Saltwater Function

\section{Top Event Top Event \\ Designator Definition}

AS ASW provides cooling water to Unit 1

CCW heat exchangers during 24 hours following an initiating event.

\section{Top Event Success Criteria}

1. Post accidental injection and recirculation phases. Cooling water is required to be available to both $\mathrm{CCW}$ heat exchangers of Unit 1 for all initiating event (of course, except LOSW). Event if both Unit 1 ASW pump trains fail, top event AS still succeeds if the operator aligns a Unit 2 ASW pump train to supply the Unit $1 \mathrm{CCW}$ heat exchangers by opening a crosstie valve.

2. Under normal plant cooldown conditions. Two operable ASW pump trains to two $\mathrm{CCW}$ heat exchangers are required for success (trains are operated separately). The unavailability of $\mathrm{CCW}$ heat exchangers is modelled in the CCW analysis.

FSAR Success Criteria:

Applicability: Modes: Power operation, 1; Startup, 2; Hot standby, 3; Hot shutdown, 4. At least two auxiliary saltwater trains shall be operable.

Action: With only one ASW train operable, restore at least two trains to operable status within 72 hours or be in at least hot standby within the next six hours, in cold shutdown (Mode 5) within the following 30 hours. 


\section{Table B3.2.2 \\ Boundary Conditions for Top Event, AS}

Split Fraction ID

AS1

AS2

AS3

AS4

AS5

AS6

AS7

AS8

AS9

ASA

ASB

ASC

ASI

ASF

\section{Boundary Condition}

No LOSP. All pump trains available (2 running, 2 standby). Power at Unit 1 available for 4.16kV Busses $F$ and $G$ and $125 \mathrm{~V}$ DC Bus 12. Open crosstie valve, remotely: OP1.

No LOSP. Three pump trains available (Train 11 fails). $4.16 \mathrm{kV}$ Bus $F$ failed at Unit 1. Open crosstie valve, manually: OP2.

No LOSP. Three pump trains available (Train 12 fails). $4.16 \mathrm{kV}$ Bus $\mathrm{G}$ or $125 \mathrm{~V}$ DC Bus 12 failed at Unit 1. OP1.

No LOSP. Two pump trains available (Trains 11 and 12 fail). $4.16 \mathrm{kV}$ Bus $\mathrm{F}$ and 4.16kV Bus $G$ or $125 \mathrm{~V}$ DC Bus 12 failed at Unit 1 . OP2.

LOSP. Three pump trains available (Train 11 fails). $4.16 \mathrm{kV}$ Bus at Unit 1 fails. OP2.

LOSP. Three pump trains available (Train 21, Unit 2, fails). 4.16kV Bus at Unit 2 fails. OP1.

LOSP. Two pump trains available (Trains 11 and 12 fail). 4.16kV Busses $F$ and G failed. OP2.

LOSP. Two pump trains available (Trains 11 and 21 or 22 fail). $4.16 \mathrm{kV}$ Bus $\mathrm{F}$ at Unit 1 and $4.16 \mathrm{kV}$ Busses $F$ or $G$ at Unit 2 failed. OP2.

LOSP. Two pump trains available (Trains 12 and 21 failed). $4.16 \mathrm{kV}$ Bus $G$ at Unit 1 and $4.16 \mathrm{kV}$ Bus $F$ at Unit 2 failed. OP1.

LOSP. Two pump trains available (Trains 21 and 22 failed). $4.16 \mathrm{kV}$ Busses $F$ and $G$ at Unit 2 failed. Useless operator action to open crosstie valve, because both Unit 2 trains are unavailable, OPF.

LOSP. One pump train available (Trains 11,12 , and 21 or 22 failed). $4.16 \mathrm{kV}$ Busses $F$ and $G$ failed at Unit 1 and $4.16 \mathrm{kV}$ Busses $F$ or $G$ at Unit 2 failed. OP2. LOSP. One pump train available (Trains 11 or 12 and 21 and 22 failed). $4.16 \mathrm{kV}$ Busses $F$ or $G$ at Unit 1 and $4.16 \mathrm{kV}$ Busses $F$ and $G$ at Unit 2 failed. OPF. Initiator. Total loss of ASW for Unit 1.

Guaranteed failure. 
Appendix $\mathbf{B 3}$

Table B3.2.3

Unarailability Velues (Split Fractions) for the Auxiliary

Saltwater System Dunction

\begin{tabular}{|c|c|c|c|c|c|c|c|c|c|c|}
\hline $\begin{array}{l}\text { Top } \\
\text { Event }\end{array}$ & Case & Calc. & $\mathbf{T L}$ & HW & HW & IWD & $\mathbf{M N}$ & TS & HE & $\begin{array}{c}\text { Comment } \\
\text { No. }\end{array}$ \\
\hline \multirow[t]{11}{*}{ AS } & AS1 & $\begin{array}{l}\text { PGQE } \\
\text { BNL1 } \\
\text { BNL2 }\end{array}$ & $\begin{array}{l}1.849-6 \\
1.809-6 \\
3.256-6\end{array}$ & $\begin{array}{l}1.598-6 \\
1.555-6 \\
1.555-6\end{array}$ & $\begin{array}{l}1.574-6 \\
1.531-6 \\
1.531-6\end{array}$ & $\begin{array}{l}2.406-8 \\
2.340-8 \\
2.340-8\end{array}$ & $\begin{array}{l}2.459-7 \\
2.493-7 \\
1.666-6\end{array}$ & $\begin{array}{r}4.811-9 \\
4.774-9 \\
3.505-8\end{array}$ & - & \\
\hline & AS2 & $\begin{array}{l}\text { PGQR } \\
\text { BNL1 } \\
\text { BNL2 }\end{array}$ & $\begin{array}{l}3.550-4 \\
3.614-4 \\
4.532-4\end{array}$ & $\begin{array}{l}2.458-4 \\
2.480-4 \\
2.480-4\end{array}$ & $\begin{array}{l}2.452-4 \\
2.474-4 \\
2.474-4\end{array}$ & $\begin{array}{l}6.438-7 \\
6.340-7 \\
6.340-7\end{array}$ & $\begin{array}{l}1.068-4 \\
1.108-4 \\
2.014-4\end{array}$ & $\begin{array}{l}2.531-6 \\
2.579-6 \\
3.779-6\end{array}$ & - & \\
\hline & AS3 & $\begin{array}{l}\text { PGaE } \\
\text { BNL1 } \\
\text { BNL2 }\end{array}$ & $\begin{array}{l}1.224-4 \\
1.631-4 \\
2.6174\end{array}$ & $\begin{array}{l}2.349-5 \\
2.361-5 \\
2.361-5\end{array}$ & $\begin{array}{l}2.344-5 \\
2.356-5 \\
2.356-6\end{array}$ & $\begin{array}{l}5.028-8 \\
5.000-8 \\
5.000-8\end{array}$ & $\begin{array}{l}9.778-5 \\
8.722-5 \\
1.796-4\end{array}$ & $\begin{array}{l}1.151-6 \\
5.226-5 \\
5.847-5\end{array}$ & - & \\
\hline & ASA & $\begin{array}{l}\text { PGRE } \\
\text { BNL1 } \\
\text { BNL2 }\end{array}$ & $\begin{array}{l}1.686-2 \\
1.666-2 \\
2.944-2\end{array}$ & $\begin{array}{l}1.664-2 \\
1.644-2 \\
1.644-2\end{array}$ & $\begin{array}{l}1.664-2 \\
1.644-2 \\
1.644-2\end{array}$ & $\begin{array}{l}5.223-7 \\
5.200-7 \\
5.200-7\end{array}$ & $\begin{array}{l}2.189-4 \\
2.215-4 \\
1.291-2\end{array}$ & $\begin{array}{l}5.233-6 \\
5.222-6 \\
3.044-4\end{array}$ & - & \\
\hline & AS5 & $\begin{array}{l}\text { PGeR } \\
\text { BNL1 } \\
\text { BNL2 }\end{array}$ & $\begin{array}{l}3.582-4 \\
3.637-4 \\
4.968-4\end{array}$ & $\begin{array}{l}2.481-4 \\
2.502-4 \\
2.502-4\end{array}$ & $\begin{array}{l}2.453-4 \\
2.474-4 \\
2.474-4\end{array}$ & $\begin{array}{l}2.804-6 \\
2.800-6 \\
2.800-6\end{array}$ & $\begin{array}{l}1.083-4 \\
1.115-4 \\
2.438-4\end{array}$ & $\begin{array}{l}1.909-6 \\
1.943-6 \\
2.837-6\end{array}$ & - & \\
\hline & AS6 & $\begin{array}{l}\text { PG\&E } \\
\text { BNL1 } \\
\text { BNL2 }\end{array}$ & $\begin{array}{l}7.857-6 \\
7.068-6 \\
4.559-5\end{array}$ & $\begin{array}{l}5.321-6 \\
4.573-6 \\
4.573-6\end{array}$ & $\begin{array}{l}2.912-6 \\
2.191-6 \\
2.191-6\end{array}$ & $\begin{array}{l}2.408-6 \\
2.382-6 \\
2.382-6\end{array}$ & $\begin{array}{l}2.499-6 \\
2.460-6 \\
4.008-5\end{array}$ & $\begin{array}{l}3.518-8 \\
3.500-8 \\
9.316-7\end{array}$ & - & \\
\hline & AS7 & $\begin{array}{l}\text { PG\&E } \\
\text { BNL1 } \\
\text { BNL2 }\end{array}$ & $\begin{array}{l}1.693-2 \\
1.674-2 \\
3.166-2\end{array}$ & $\begin{array}{l}1.667-2 \\
1.647-2 \\
1.647-2\end{array}$ & $\begin{array}{l}1.665-2 \\
1.646-2 \\
1.646-2\end{array}$ & $\begin{array}{l}1.424-5 \\
1.410-5 \\
1.410-5\end{array}$ & $\begin{array}{l}2.595-4 \\
2.629-4 \\
1.291-2\end{array}$ & $\begin{array}{l}4.651-6 \\
4.648-6 \\
2.283-3\end{array}$ & - & \\
\hline & AS8 & $\begin{array}{l}\text { PG\&E } \\
\text { BNL1 } \\
\text { BNL2 }\end{array}$ & $\begin{array}{c}4.709-4 \\
4.685-4 \\
1.082-2\end{array}$ & $\begin{array}{l}2.806-4 \\
2.780-4 \\
4.193-3\end{array}$ & $\begin{array}{l}2.660-4 \\
2.635-4 \\
4.157-3\end{array}$ & $\begin{array}{l}1.461-5 \\
1.450-5 \\
3.647-5\end{array}$ & $\begin{array}{l}1.881-4 \\
1.881-4 \\
6.511-3\end{array}$ & $\begin{array}{l}2.324-6 \\
2.343-6 \\
1.142-4\end{array}$ & - & \\
\hline & AS9 & $\begin{array}{l}\text { PG\&E } \\
\text { BNL1 } \\
\text { BNL2 }\end{array}$ & $\begin{array}{l}2.741-4 \\
2.635-4 \\
1.065-2\end{array}$ & $\begin{array}{l}1.016-4 \\
9.549-4 \\
4.023-3\end{array}$ & $\begin{array}{l}8.702-5 \\
8.124-5 \\
3.987-3\end{array}$ & $\begin{array}{l}1.455-5 \\
1.425-5 \\
3.650-5\end{array}$ & $\begin{array}{l}1.705-4 \\
1.661-4 \\
6.510-3\end{array}$ & $\begin{array}{l}2.007-6 \\
1.963-6 \\
1.142-4\end{array}$ & - & \\
\hline & ASA & $\begin{array}{l}\text { PG\&E } \\
\text { BNL1 } \\
\text { BNL2 }\end{array}$ & $\begin{array}{l}1.834-4 \\
1.799-4 \\
1.799-4\end{array}$ & $\begin{array}{l}1.315-4 \\
1.271-4 \\
1.271-4\end{array}$ & $\begin{array}{l}1.172-4 \\
1.130-4 \\
1.130-4\end{array}$ & $\begin{array}{l}1.424-5 \\
1.409-5 \\
1.409-5\end{array}$ & $\begin{array}{l}5.104-5 \\
5.190-5 \\
5.190-5\end{array}$ & $\begin{array}{l}9.113-7 \\
9.171-7 \\
9.171-7\end{array}$ & - & \\
\hline & ASB & $\begin{array}{l}\text { PG\&E } \\
\text { BNL1 } \\
\text { BNL2 }\end{array}$ & $\begin{array}{l}2.699-2 \\
2.697-2 \\
1.000\end{array}$ & $\begin{array}{l}2.063-2 \\
2.053-2 \\
1.000\end{array}$ & $\begin{array}{l}2.060-2 \\
2.049-2\end{array}$ & $\begin{array}{l}3.674-5 \\
3.647-5\end{array}$ & $\begin{array}{l}6.369-3 \\
6.457-2\end{array}$ & $\begin{array}{l}1.141-4 \\
1.142-4\end{array}$ & - & \\
\hline
\end{tabular}


Appendix B3

Talie B3.2.3 (comatinued)

Top

\begin{tabular}{|c|c|c|c|c|c|c|c|c|c|}
\hline \multirow[t]{2}{*}{ Event } & Cane & Calc. & TIL & $\mathbf{H W}$ & IW & ITW & $\mathbf{M N}$ & TS & H: \\
\hline & ASC & $\begin{array}{l}\text { PGeE } \\
\text { BNLL1 } \\
\text { BNL2 }\end{array}$ & $\begin{array}{l}1.065-2 \\
1.179-2 \\
1.179-2\end{array}$ & $\begin{array}{r}4.171-3 \\
4.193-3 \\
4.193-3\end{array}$ & $\begin{array}{r}4.134-3 \\
4.157-3 \\
4.157-3\end{array}$ & $\begin{array}{l}3.674-5 \\
3.647-5 \\
3.647-5\end{array}$ & $\begin{array}{l}6.369-3 \\
6.457-3 \\
6.457-3\end{array}$ & $\begin{array}{l}1.141-4 \\
1.141-4 \\
1.1414\end{array}$ & - \\
\hline LOSW & ASI & $\begin{array}{l}\text { PGQE } \\
\text { BNL1 } \\
\text { BNL2 }\end{array}$ & $\begin{array}{l}9.734-5 \\
9.588-5 \\
5.108-4\end{array}$ & $\begin{array}{l}9.734-5 \\
9.588-5 \\
5.108-4\end{array}$ & $\begin{array}{l}9.004-5 \\
8.894-5 \\
4.581-4\end{array}$ & $\begin{array}{l}6.975-6 \\
6.943-6 \\
5.270-5\end{array}$ & - & - & - \\
\hline & ASF & PG\&E & $\begin{array}{l}1.0 \\
1.0\end{array}$ & & & & & & \\
\hline
\end{tabular}


Table B3.2.4

Unavailability Model and Experience Based Frequencies of the LOSW Initiator

Unit capacity factor: 85

Probability of Non-Recovery of LOSW, P(T $\geq 2)=.57$

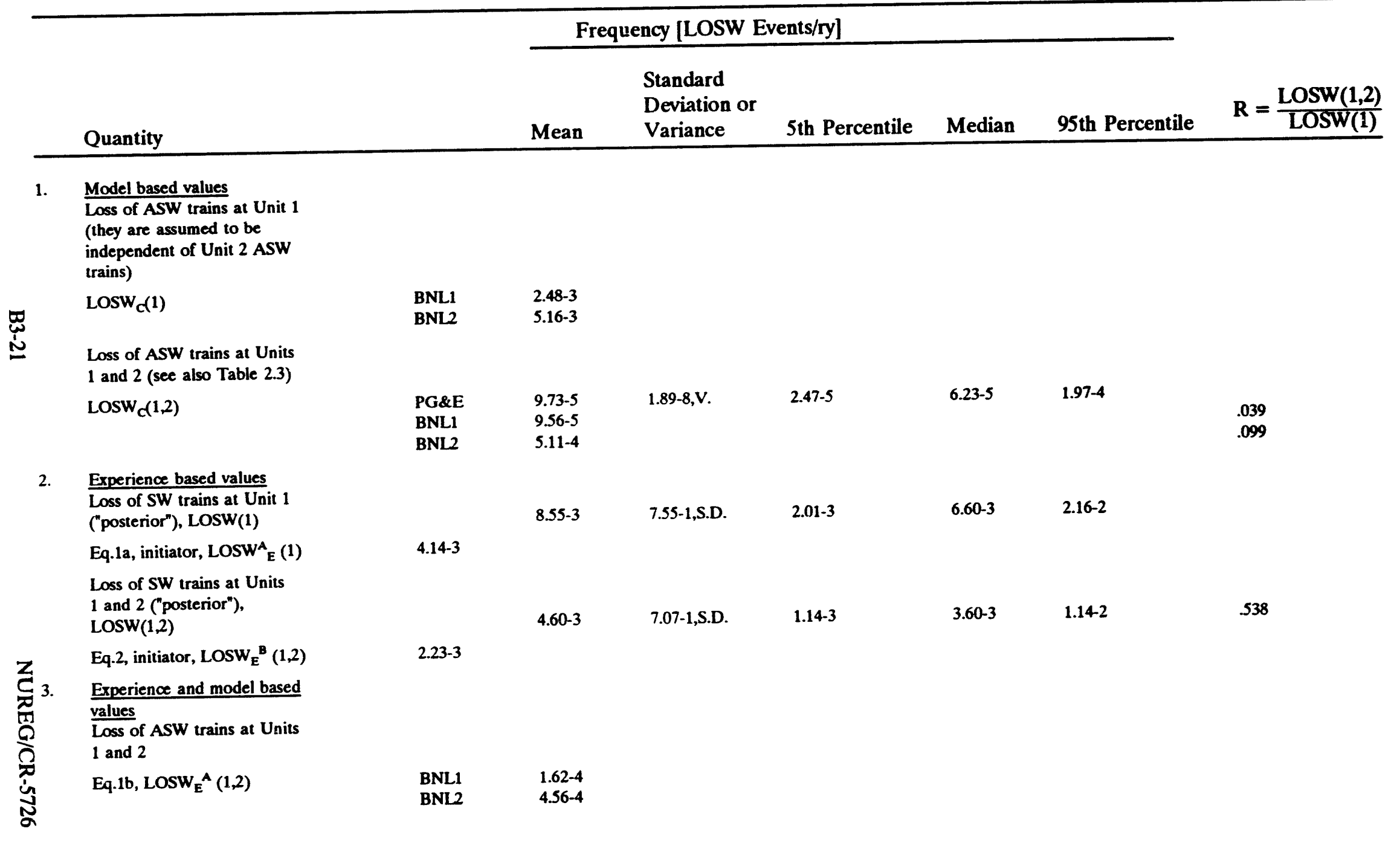




\section{ATTACHMENT B3.A}

\section{INFORMATION ON FAILURES OF SERVICE WATER SYSTEMS}

\section{W}


This attachment provides the documentation of information used to analyze the nature of failures occurring at the Service Water Systems of US PWRs and to determine the frequency of the initiator "Total Loss of Auxiliary Saltwater, LOSW" for Diablo Canyon based on generic plant experience.

The information includes:

a. the total time exposure of service water systems (SWS) at US PWRs (Table B3.A.1),

b. $\quad$ the classification of SWSs for multi unit PWR sites (Table B3.A.2),

c. a list of failure events obtained by a survey of the RECON data base ${ }^{3}$ and the NPE operating event listings ${ }^{4}$ when the SWS is completely lost for one or more units due to failures of the system itself or due to certain activities at the unit which is down (Table B3.A.3),

d. a list of failure events when the SWS is completely lost or susceptible to fail due to systems interaction (Table B3.A.4),

e. a detailed description of the events listed in Tables B3.A.3 and B3.A.4, and

f. a list of failure events when the SWS becomes partially degraded (Table B3.A.5). 
Table B3.A.1

Total Time Exposure of Service Water Systems at U.S. PWRs

\begin{tabular}{|c|c|c|}
\hline Plant Name & $\begin{array}{l}\text { Start of } \\
\text { Commereial } \\
\text { Operation }\end{array}$ & $\begin{array}{l}\text { Number of } \\
\text { Years to } \\
\text { End of } 1988\end{array}$ \\
\hline Calvert Cliffs 1 & $5 / 75$ & 14.27 \\
\hline Calvert Cliffs 2 & $4 / 77$ & 12.5 \\
\hline Haddam Neck & $1 / 68$ & 21.5 \\
\hline Indian Point 2 & $7 / 74$ & 15.0 \\
\hline Beaver Valley 1 & $4 \pi 7$ & 12.3 \\
\hline Beaver Valley 2 & $11 / 87$ & 1.7 \\
\hline Three Mile Island 1 & $9 / 74$ & 14.8 \\
\hline Three Mile Island 2 & $12 / 78$ & 5.8 \\
\hline Main Yankee & $12 / 72$ & 16.0 \\
\hline Indian Point 3 & $8 / 76$ & 12.9 \\
\hline Millstone 2 & $12 / 75$ & 13.0 \\
\hline Millstone 3 & $4 / 86$ & 3.3 \\
\hline Salem 1 & $6 / 77$ & 12.1 \\
\hline Salem 2 & $10 / 81$ & 7.8 \\
\hline Robert E. Ginna & $3 / 70$ & 19.3 \\
\hline Yankee & $6 / 61$ & 28.1 \\
\hline Zion 1 & $12 / 73$ & 15.6 \\
\hline Zion 2 & $9 / 74$ & 14.8 \\
\hline Byron 1 & $9 / 85$ & 3.8 \\
\hline Byron 2 & $8 / 87$ & 1.9 \\
\hline Braidwood 1 & $3 / 88$ & 3 \\
\hline Braidwood 2 & $9 / 88$ & .1 \\
\hline Palisades & $12 / 71$ & 17.6 \\
\hline Donald C. Cook 1 & $8 / 75$ & 13.9 \\
\hline Donald C. Cook 2 & $7 / 78$ & 11.0 \\
\hline Prairie Island 1 & $12 / 73$ & 15.6 \\
\hline Prairie Island 2 & $12 / 74$ & 14.6 \\
\hline Fort Calhoun 1 & $9 / 73$ & 15.8 \\
\hline Davis-Besse 1 & $11 / 77$ & 11.7 \\
\hline Callaway 1 & $4 / 85$ & 4.3 \\
\hline Point Beach 1 & $12 / 70$ & 18.6 \\
\hline Point Beach 2 & $10 / 72$ & 16.8 \\
\hline Kewaunee & $6 / 74$ & 15.1 \\
\hline Wolf Creek & 9/85 & 3.8 \\
\hline Joseph M. Farley 1 & $12 / 77$ & 11.6 \\
\hline Joseph M. Farley 2 & $7 / 81$ & 8.0 \\
\hline Arkansas Nuclear One 1 & $12 / 74$ & 14.6 \\
\hline Arkansas Nuclear One 2 & $3 / 80$ & 9.3 \\
\hline Robinson 2 & $3 / 71$ & 18.3 \\
\hline Shearon Harris & $5 / 87$ & 2.2 \\
\hline Oconee 1 & $7 / 73$ & 16.0 \\
\hline Oconee 2 & $9 \pi 74$ & 14.8 \\
\hline Oconee 3 & $12 / 74$ & 14.6 \\
\hline McGuire 1 & $12 / 81$ & 7.6 \\
\hline
\end{tabular}


Table B3.A.1 (continued)

$\begin{array}{llc} & \begin{array}{l}\text { Start of } \\ \text { Commercial } \\ \text { Oporation }\end{array} & \begin{array}{c}\text { Number of } \\ \text { Yearn to } \\ \text { End of 1988 }\end{array} \\ \text { Plant Name } & & \\ & 3 / 84 & 5.3 \\ \text { McGuire 2 } & 6 / 85 & 4.1 \\ \text { Catawba 1 } & 8 / 86 & 2.9 \\ \text { Catawba 2 } & 12 / 72 & 16.6 \\ \text { Turkey Point 3 } & 9 / 73 & 15.8 \\ \text { Turkey Point 4 } & 12 / 76 & 12.6 \\ \text { St. Lucie 1 } & 8 / 83 & 5.9 \\ \text { St. Lucie 2 } & 3 / 77 & 11.7 \\ \text { Crystal River 3 } & 5 / 87 & 2.2 \\ \text { Vogtle 1 } & 9 / 85 & 3.8 \\ \text { Waterford 3 } & 1 / 84 & 5.5 \\ \text { Virgil C. Summer 1 } & 7 / 81 & 8.0 \\ \text { Sequoyah 1 } & 6 / 82 & 7.1 \\ \text { Sequoyah 2 } & 12 / 72 & 16.6 \\ \text { Surry 1 } & 5 / 73 & 16.2 \\ \text { Surry 2 } & 6 / 78 & 11.1 \\ \text { North Anna 1 } & 12 / 80 & 8.6 \\ \text { North Anna 2 } & 1 / 86 & 3.5 \\ \text { Palo Verde 1 } & 9 / 86 & 2.8 \\ \text { Palo Verde 2 } & 2 / 88 & 0.4 \\ \text { Palo Verde 3 } & 3 / 88 & 0.3 \\ \text { South Texas Project 1 } & 5 / 85 & 4.2 \\ \text { Diablo Canyon 1 } & 3 / 86 & 3.3 \\ \text { Diablo Canyon 2 } & 5 / 76 & 13.2 \\ \text { Trojan } & 4 / 75 & 14.3 \\ \text { Rancho Seco } & 1 / 68 & 21.5 \\ \text { San Onofre 1 } & 8 / 83 & 5.9 \\ \text { San Onofre 2 } & 4 / 84 & 5.3 \\ \text { San Onofre 3 } & & \end{array}$

Total: PWR (72) $=751.4$ Reactor Years

Note: Use of the commercial operation date precludes an indeterminate amount of system operation time prior to that point. An attempt has been made to correlate the reported failures to this same time frame. 
Table B3.A.2

Clacelification of Service Water Syutems for Multi-Unit PWR SItes

\section{Muld-Unit Plants}

ANO 1 \& 2

Beaver Valley 1 \& 2

Braidwood 1 \& 2

Byron 1 \& 2

Calvert Cliffs 1 \& 2

Catawba 1 \& 2

Cook 1 \& 2

Diablo Canyon 1 \& 2

Farloy 1 \& $2^{1}$

Indian Point ${ }^{2}$

McGuire 1 \& 2

North Anna 1 \& $2^{3}$

Oconee 1 \& 2

Oconee 3

Palo Verde 1, 2 \& $3^{4}$

Point Beach 1 \& 2

Prairie Island 1 \& 2

\section{Service Water Pumpe/1 Unit}

$2+1$ Swing
$2+1$ Swing
2
2
3
2
2
2
2
9

Salem $1 \& 2$

San Onofre 1, $2 \& 3^{5}$

Sequoyah 1 \& 2

South Texas 1 \& 2

St. Lucie 1 \& 2

Surry $1 \& 2^{6}$

Turkey Point 3 \& 4

Vogtle 1 \& 2

Watts Bar 1 \& 2

Zion 1 \& 2

\section{Success Criterion \\ for Mighest Load}

1 of 2

1 of 2

1 of 2

1 of 2

2 of 3

1 of 2

1 of 2

1 of 2

1 of 2

3 of 9

1 of 2

1 of 2

1 of 1

1 of 2

1 of 2

3 of 6 (2 units)

1 of 2
Separate Water Source (Or Intake)

Yes

Yes

Yes - two separate intakes from the same source.

Yes

Yes

Yes - two separate intakes from the same source.

$$
\begin{array}{ll}
6 & 4 \text { of } 6 \\
4 & 1 \text { of } 4 \\
4 & 2 \text { of } 4 \\
3 & 2 \text { of } 3 \\
2+1 \text { Swing } & 1 \text { of } 2 \\
3 / 2 \text { units } & 2 \text { of } 3 / 2 \\
3 & 2 \text { of } 3 \\
6 \text { (3 trains) } & 2 \text { of } 3 / / \\
4 & 2 \text { of } 4
\end{array}
$$$$
3 / 2 \text { units } \quad 2 \text { of } 3 / 2 \text { units }
$$$$
6 \text { (3 trains) } \quad 2 \text { of } 3 / \text { trains }
$$$$
31 \text { of } 3
$$

${ }^{1} \mathrm{SW}$ pumps take suction from the SW wet pit, which is directly supplied by 5 pumps/1 unit from the ultimate heat sink (river) and the success criteria for these pumps are 2-out-of-5.

${ }^{2}$ Both essential and non-essential loads are included.

${ }^{3}$ Two auxiliary SW pumps are available. However, the power supply for these pumps are non-safety related.

The ESW system is on standby during normal operation. There is no crossties between the units.

${ }^{5}$ Each SW train has two pumps, but only one is powered during normal operation.

${ }^{6}$ The SW pumps ( $3 / 2$ units) are only for emergency purposes. The normal supply is by gravity flow from the circulating water system. 
Table R3.43

Total Loss of the Service Water System

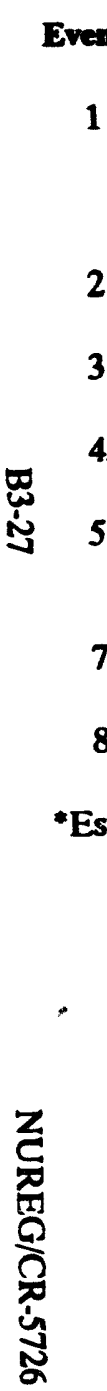

Reference

LER-206/80-06

LER-320/81-11

LER-311/83-32

LER-361/83-72

LER-413/85-68

LER-302/86-02

LER-269/86-11
Recovery

-45 Min.

$\geq 2$ Hours*

$\sim 1$ Hour

$>5$ Hours*

$\sim 45$ Min.

$\geq 3$ Hours*

$-$

\section{Description}

One ESW pump shaft sheared due to excessive vibration, the discharge valve of the standily pump didn't open and the auriliary pump lost suction.

One ESW pump lost due to vibration other pump unavailable.

Flooding ESW bay due to a gasket failure.

Traveling screens were damaged, CCW heat exchangers clogeod.

Both ESW trains declared inoperable due to torque switch problems on the discharge valves.

All ESW pumps are shut down, two divers drowned.

Loss of LPSW suction due to inadequate design.

Estimated. 


\section{Appendix B3}

Description of Operatine Events Involvine the Total Los of the SW System Function (Table B3.A.3).

\section{San Onofre 1 - LER-206/1980-006}

During normal operation, the south salt water cooling pump (SCP) discharge pressure dropped sharply. The north salt water cooling pump (NCP) automatically started on low pressure. However, its discharge POV failed to open. The auxiliary salt water cooling (ACP) was then started but flow could not be established. As a result of (1) excessive vibration, the shaft of the (SCP) sheared, (2) mechanical failure, the (NCP) POV did not open, and (3) apparent inadequate prime, the (ACP) lost suction. The POV on the (NCP) was manually opened and the (ACP) regained suction.

\section{TMI 2 - LER-320/1981-001}

April 23, 1981, the "A" nuclear service river water pump was started for operation. The pump exhibited high vibrations and high current readings. An evaluation showed that the pump should be declared inoperable to prevent further damage. The inoperable status resulted in a violation of Tech Spec since the "B" pump had been declared inoperable in October 1979. The cause of this event was most likely due to excessive clearance at the bottom of the pump which caused excessive vibration leading to damage. Procedures were rewritten to ensure that backup pumps are powered to provide cooling water to operating diesels.

\section{Salem 2 - LER-311/1983-032}

On June 23, 1983, during routine shutdown operation, an equipment operator performing routine surveillance discovered a large leak in the No. 2 service water bay. Due to the accumulation of approximately six feet of waidr in the bay, and an apparently continuing rise in the water level following an initial attempt to isolate the leak, all service water pumps were stopped, resulting in the loss of flow to the boron injection, residual heat removal and diesel generator system. Investigation revealed that the leakage was due to a failed gasket in the joint downstream of check valve 225; the gasket failure was attributed to an isolated problem in installation related to poor access to the joint. The connection had recently been opened then remade during cleaning of the No. 21 nuclear header. Related problems with the bay sump pumps and alarms will be corrected by a design change.

\section{4,5. San Onofre 2 \& 3 - LER-361/1983-072. LER-362/1983-041}

On July 6, 1983 at 0030 while Unit 2 was in mode 5 and Unit 3 was in mode 4 operator observed that the Unit 3 circulating water system traveling screen water level differential pressure was off scale indicating clogging of the screens. The screen wash system was actuated to clear the screens of marine debris. The screen wash system failed to clear the screen. The inability to clear the screens resulted in high $\mathrm{CCW}$ heat exchangers (Unit 2 train A and Unit 3 trains A and B) differential pressure being alarmed in the control room at 0210 on July 6, 1983, and at 0227 SCW flow was reduced to the point that the heat exchangers were declared inoperable. This resulted in exceeding limiting condition for operation (LCO) 3.7.4 for Unit 3, only, since the LCO is applicable to modes 1 through 4 and Unit 2 was in mode 5. Exceeding LCO 3.7.4, for Unit 3, resulted in invocation of LCO 3.0.3. Visual inspection of the traveling screens after the incident revealed that several screen panels were dislodged from their housings either before or during this event resulting in marine debris to be carried into the circulating water pump forebay. To preclude concurrent fouling of both trains of CCW heat exchangers during excessive marine debris buildup in a single intake structure, $\mathrm{C}$ system operating procedure is being revised.

\section{Catawba 1 - LER-413/1985-068}

On November 25, 1985, the in service test on the nuclear service water (RN) header 1B supply isolation valve was performed. While stroking the valve, it stopped in the intermediate position. Train B of RN was declared inoperable and train $A$ of $R N$ was placed in service. Upon starting $R N$ pump $1 A$, the discharge isolation valve also stopped in the intermediate position. Train $A$ of $R N$ was declared inoperable and Technical Specification 3.0.3 was entered due to the simultaneous inoperability of hoth trains of RN. Both trains of RN were inoperable for $\mathbf{4 3}$ minutes until the RN header 1B supply isolation valve was opened and train $B$ of $R N$ was declared operable. Investigation revealed that the torque switches for the valves were set at the low end of 
the allowable tolerance. These settings did not allow the valves to open completely. Therefore, this incident is classified as a design deficiency. Unit 1 was at $45 \%$ power.

\section{Crystal River - LER-302/1986-002}

On January 10, 1986, Crystal River Unit 3 was in mode 5 during an outage. The intake structure was being cleaned and inspected by two contract divers. At 1615 , one diver failed to reappear following his dive. The second diver attempted to locate and rescue the missing diver but was himself drowned. When the second driver was reported to be in trouble, all seawater pumps taking suction at the intake structure were secured, thus disabling both trains of the decay heat removal system. The body of the second diver was recovered shortly thereafter. The first diver was found to have been drawn into the $48^{\text {" }}$ suction line of the 'A' emergency nuclear services and decay heat seawater system pumps (both pumps were running at the start of the event). The body of the first diver was recovered in the auxiliary building. All seawater pumps were voluntarily secured and/or disabled in an attempt to prevent loss of life.

\section{Oconee 1 - LER-269/1986-011}

On October 1, 1986, with Units 1 and 3 at 100\% full power, and Unit 2 shutdown for refueling, a load shed test on Unit 2 was performed. Suction to the low pressure service water (LP) pump was lost about one hour into the test. The loss prime in the condenser circulating water (CCW) siphon flow (or emergency $\mathrm{CCW}$ ) system was the cause for the loss of the LP pumps. The emergency condenser circulating cooling water (ECCW) system is required to provide water through the main condenser for decay heat removal during loss of all ac power event (station blackout). The immediate corrective action was to analyze the failures that occurred during the load shed test, and shut down Oconee Units 1 and 3. Subsequent corrective actions included redesign of the CCW pump flanges and determination of the design basis of the ECCW system. The root cause of this event is the inadequate design and testing of the ECCW system. This led to a failure of the ECCW system to perform the intended function as described in the final safety analysis report (FSAR).

Events not included in the statistics.

\section{San Onofre 3 LER-362/1986-011}

Power level $-100 \%$ at 1550 on August 4, 1986, saltwater cooling (SWC) flow through train a component cooling water heat exchanger (CCWHX) decreased, due to fouling with marine growth, to below the postulated design basis flow rate required for removal of CCW heat loads (critical CCW loop), and was therefore declared inoperable. At this time Train B CCWHX was operating with reverse SWC flow to remove similar fouling which had previously take place. At 1605 , operators commenced realignment of Train B CCWHX SWC flow to the normal direction in order to return one train of CCW to its design configuration and thereby increase heat removal capability of that train. During the realignment, both trains of the SWC system were considered to be inoperable contrary to technical specification limiting condition for operation (LCO) 3.7.4, and LCO 3.0.3 was'entered. Train B SWC system was returned to operable status within thirty minutes, and at 1635, LCO 3.0.3 was exited. As corrective action, operating procedures will be revised to minimize the effect of marine fouling on the operability of the SWC system. 
Table B3.A.4

Total Loss of Service Water Due to Other Initiators

\begin{tabular}{|c|c|c|c|}
\hline Event & Plant & Reference & Recovery \\
\hline 1 & Salem 1 & NPE/PWR-2 & Few days* \\
\hline 2 & Farley 1 & NPE/PWR-2 & ～ 3 Days \\
\hline 3 & Salem 1 & LER-272/82-15 & $\sim 1$ Hour \\
\hline 巳ّ 4 & Calvert Cliffs & LER-318/82-54 & $\sim 30$ Min. \\
\hline 5 & Palisades & LER-255/84-01 & $\sim 1$ Hour \\
\hline 6 & Salem 1 & LER-272/84-14 & 1 Hour \\
\hline
\end{tabular}

\section{Description}

Winter storm shuts down the ESW system. Traveling VIII-110, 1976screens blocked by ice.

Flooding of the intake structure. VIII-155, 1978

Vital bus 1A tripped, operating ESW train is lost, other train n maintenance.

Power was lost on a $4 \mathrm{kV}$ bus resulting in the loss of ESW pump on the operating loop. Other train in maintenance.

Offsite power removed, no operable service water pump supplied by the operating diesel.

Vital bus 1A failed, bus 1B in maintenance, bus 1C didn't energize, loss of ESW system. 
Description of Operating Events Involving the Total Loss of the SW System Due to Systems Interaction (Table B3.A.4).

\section{Salem 1 - NPE/PWR2, VIII-110, 1976}

Numerous problems were experienced with the plant service water system. The first serious problem was noted in January 1976 when a winter storm shut down the system. Icing due to wind whipped spray and screen wash spray created four inches of ice on the operating deck of the structure making it hazardous to operators and caused the traveling screens in operation to ice-over thereby restricting flow to the pumps. Screens which were out of service froze in their tracks causing shear pins to fail when the screen was started. The eventual buildup of ice and debris resulted in the shutting down of the remaining pumps due to low flow. Some modifications were made to the system, however, the major improvement, a heated protective housing, had not yet been installed.

\section{Farley 1 - NPE/PWR-2, VIII-155, 1978}

At -2100 hours on January 25 th, $600 \mathrm{~V}$ load centers $1 \mathrm{H}$ and $1 \mathrm{~J}$, which were located in the river water structure, were de-energized when flooding of this structure occurred. The flooding was the result of high Chattahoochee River levels following heavy rains. The water level in the train A side of the river water structure was ${ }^{-1} \mathrm{ft}$. The river level at this time was -110 feet mean sea level (MSL). The river water pumps were still operable. They set up temporary sump pumps to supplement the permanently installed pumps. The Tech Specs required that load centers $1 \mathrm{H}$ and $1 \mathrm{~J}$ be operable, energized, and aligned to an operable DG.

At 2300 hours a $50 \%$ reduction in turbine load was initiated. Power to river water pumps $8 \mathrm{~A}, 9 \mathrm{~A}, 10 \mathrm{~A}$ was racked out at 2330 hours. At 0007 hours on January 26th, the unit was at $40 \%$ reactor power and 430 MWe. At 0040 hours a further load reduction was initiated at $5 \mathrm{MW} / \mathrm{min}$ to place the unit in hot standby as required by the Tech Specs. At 0045 hours power to river water pumps $4 B$ and $5 B$ was racked out, and the rate of load reduction was increased to $10 \mathrm{MW} / \mathrm{min}$ to have the unit in hot standby within the required one hour. At 0055 hours emergency service water recirculation flow to the pond (ultimate heat sink) was initiated. At 0135 hours the unit was taken off line and at 0136 hours the reactor was manually tripped. The water level in the river water structure train A section reached -5 feet; train B section reached 2 feet. The river reached a maximum level of -115 feet MSL at the river water structure.

Water had entered the structure through a hole in each river water pump baseplate and through the gland seal leakoff line on each pump. Additional leakage occurred through compression type cable penetrations of structure.

\section{Salem 1 - LER-272/1982-015}

Number 1A vital bus tripped resulting in a loss of component cooling water (CCW) and service water (ESW) flows; the redundant $\mathrm{CCW}$ and pumps were tagged out for maintenance. All charging pumps, boron injection flow paths, residual heat removal (RHR) loops and diesel generators were declared inoperable due to no CCW or flow. A wire to the TD5 undervoltage relay had shorted to the feeder cubicle door, causing the 1A vital bus infeed breaker to trip without automatic transfer. CCW and flows were restored.

\section{Calvert Cliffs 2 - LER-318/1982-054}

At 0547 , during normal shutdown operation in mode 6 , power was lost to $244 \mathrm{kV}$ bus resulting in the loss of 22 saltwater pumps and 22 LPSI pumps, thereby disabling the only operable shutdown cooling loop. Power was restored to $244 \mathrm{kV}$ bus and shutdown cooling flow restored at 0605 . The redundant shutdown cooling loop was out of service for maintenance. Vendor failure report indicated the cause of the power supply failure to be cracked printed circuit board.

\section{Palisades - LER-255/1984-001}

On January 8, 1984, the Palisades Nuclear Plant experiences a complete loss of all normal communications links between the plant, the NRC and state/local authorities. The event was precipitated by the need to isolate 


\section{Appendix B3}

a faulty switchyard breaker. To accomplish the isolation, it was necessary to interrupt the offsite power supply to the plant. At the time of the event, Palisades was in a refueling outage with all fuel removed from the reactor and one diesel generator inoperable. While operating procedures require two operable diesel generators prior to removing offsite power, the shift supervisor proceeded with the evolution after determining the safety of the fuel would not be jeopardized. In preparing for the evolution, the operators failed to realize that there would be no operable service water pumps supplied by the operating diesel. Consequently, after 50 minutes the diesel overheated due to lack of cooling water and was manually tripped. The resulting loss of onsite ac power caused a loss of all plant telephones and radios for 45 minutes. Onsite power was subsequently re-energized from the switchyard, resulting in the restoration of normal communications.

\section{Salem 1 - LER-272/1984-014}

On June 5, 1984, during a refueling outage, 1A vital bus was de-energized when the $1 \mathrm{~A}$ vital bus infeed breaker failed to close during breaker testing. Since 1B vital bus was de-energized for inspection at the time, a blackout loading signal started $1 \mathrm{~A}$ and $1 \mathrm{C}$ diesels and opened the $1 \mathrm{C}$ vital bus infeed breaker, de-energizing $1 \mathrm{C}$ vital bus. 1A diesel loaded, but because the $1 \mathrm{C} 125 \mathrm{~V}$ dc bus was de-energized for maintenance, the 1C safeguards equipment cabinet (SEC) was completely de-energized. This prevented $1 \mathrm{C}$ diesel from loading. $1 \mathrm{C}$ vital bus remained de-energized, resulting in a loss of service water cooling. Numerous control room indicators failed to mid-scale, leading the shift to believe that the $1 \mathrm{C}$ vital bus was still energized. As a result, the diesels ran for an extended period of time without cooling water; although, no diesel damage occurred. The root cause of this event was the lack of adequate procedural and/or administrative controls to ensure sufficient electrical systems remained in an operable status during a period when the plant was in a configuration which was not covered by the Tech Specs (i.e., defueled).

\section{Diablo Canyon - LER-275/82-10-18}

Testing performed on the auxiliary saltwater (ASW) system has revealed that the system is susceptible to water hammer effects during anticipated operational transients. These transients include pump trip and restart sequences such as would occur following a loss of offsite power. The peak pressure observed during this testing exceeded the 100 psig system design pressure specified in the FSAR. The cause of the system waterhammer is believed to be water column separation and subsequent column recombination at a point of significant piping slope change. Further evaluation of the event and ASW system design is being conducted. Results of the evaluation will be reported in a revision to this LER.

\section{Diablo Canyon - LER-275/84-03-02}

Prior to fuel load, testing on the Auxiliary Saltwater (ASW) System has shown that the system is susceptible to water hammer effects during anticipated operational transients. These transients include pump trip and restart sequences which would occur following a loss of offsite power. Peak pressures observed exceeded the 100 psig system design pressure specified in the FSAR and some valve damage did occur.

The cause of ASW System water hammer is water column separation and subsequent column recombination at a point of significant piping slope change. Corrective actions included the replacement of damaged valves, additional engineering analysis, the installation of vacuum breakers and further testing to ensure system operability.

Pacific Gas and Electric Company, Diablo Canyon Unit 1, Docket No. 50-275, LER

82-009-01T-1 - Supplemental Information.

Engineering analysis has shown the root cause of the Auxiliary Saltwater (ASW) System water hammer to be water column separation (resulting in vacuum formation) and subsequent column recombination at the point of significant piping slope change. A detailed inspection of the ASW was conducted after the pressure transients to inspect for damage. This inspection also included the ASW pump discharge check valves. There 
was no evidence of deformation in system piping due to the water hammer (pressure transient). Two butterfly valves, the ASW Pump 1-2 discharge isolation valve and the Component Cooling Water heat exchanger 1-1 inlet isolation valve, suffered damaged valve discs. These valves were replaced. In 1983, vacuum breakers were installed in the ASW system to reduce the pressure transients of subsequent column recombination. Further testing conducted after vacuum breaker installation verified that, for all operating conditions of the ASW system, pressure transients greater than maximum allowable system pressure will not occur. 
Table B3Ass

Operating Events Involving the Degradation of the Service Water System

Plant

San Onofre 1

Haddam Neck

Ginna

Indian Point 2

LER-247/80-16

/81-09

/81-10

/81-11

/81-21

/82-13

/82-26

/82-31

/82-33

/82-37

/83-07

/83-10

/84-11

/84-21

/85-13

/87.11

Turkey Point 3

LER-250/86-08

/86-18

$186-24$

Turkey Point $4 \quad$ LER-251/84-18

/87-16

$187-28$

\section{Description}

Pipe support installation error in SW system.

Pipe support corroded on one SW pump.

Discharge valve on pump failed to open automatically.

HX partially blocked, marine growth.

Pressure switch failed, pump discharge valve closed.

Intake structure flooded to dangerous levels, inadequate maintenance procedures.

One pump bearing degraded, other pump out for maintenance, auxiliary SW pump put in service.

Discharge valve opens, reverse flow through pump resulting in damage.

Corrosion of the intake structure.

SW leak in fan cooler due to corrosion.

SW filter plugged.

SW flood protectors are ineffective.

SW valve failed to open to AFW pump.

SW leak in fan cooler coils.

SW pipe wall thinning.

Valve seat problem, reduces pump capacity.

Pipe wall thinning, corrosion.

SW pipe leak.

SW pump vibration excessive.

Impeller wear of three SW pumps.

SW leak in containment.

SW leak in fan coolers.

SW leak in fan coolers.

Strainer plugged.

Pump inoperable, rope tied the impeller.

Leak into the CCW pump.

SW pump discharge valves leak.

SW leak in fan coolers.

SW pumps fail performance tests, vortexing.

SW system design deficiency.

SW system design deficiency.

SW pump inoperable.

Strainer removed for longer period as allowed.

SW pump tripped, electrical problems.

Two of three SW pumps are inoperable. 
Table B3.A.S (continued)

Plant

Palisades

Reference

LER-255/82-24

$186-24$

$186-36$

H. B. Robinson 2 LER-261/81-19

$182-13$

$183-03$

/83-05

183-06

183-14

$183-22$

183-27

Oconee 1

LER-269/80-02

$180-04$

$180-24$

$180-30$

/81-14

186-02

/87.04

Oconee 2

LER-270/80-10

/81-01

Salem 1

LER-272/80-22

$180-23$

$180-24$

$180-39$

$180-49$

$/ 80-60$

$/ 81-03$

/81-10

$181-11$

/81-12

/81-31

$181-39$

181.46

/81.64

/81-67

181-69

$181-71$

$181-76$

/81-77

$181-80$

/81-83

$181-90$

/81-94

\section{Description}

SW design problem.

Loss of coolers, SW valve problems.

SW pumps performs below requirements.

SW booster pump tripped, bearing and breaker problems.

SW pump failed to restart, blown fuse.

Leak in the CF cooler.

Two of four SW booster pumps lost.

SW pump and its replacement fails, longer in AOT than allowed.

SW leak at CF cooler.

SW leak at CF cooler.

SW leak at CF cooler.

HPSW inoperable, motor insulation broke down.

HPSW inoperable, motor cooler leakage.

Automatic initiation of HPSW was affected by construction.

Valve failed to close in SW system.

HPSW pumps A and B had no control powers, breakers were open, jockey pump used in place.

Seismic design deficiency in LPSW system.

SW heat exchanger capacity reduced, biological fouling.

SW valves fail in closed position.

Improper alignment of SW valves.

SW solenoid valve failure isolates CFCU coil.

SW flow reduced to CFCU, inoperable flow transmitter.

Solenoid on SW line failed, no flow to CFCU.

Solenoid on SW line failed, no flow to CFCU.

SW piping leak at charging pump.

SW valve mispositioned, all DG inoperable.

SW pipe leak, CCW HX removed from service.

SW pipe leak, CF coil inoperable.

SW pipe leak, CF coil inoperable.

SW hose leak.

SW pipe leak, CFCU inoperable.

SW pipe leak, CFCU inoperable.

SW valve failure blocks flow to CFCU.

SW pipe leak at CFCU.

SW valve failure reduces flow to CFCU.

SW valve failure reduces flow to $\mathrm{CCW} \mathrm{HX}$.

SW flow XMTR line plugged.

SW pipe leak at CFCU.

SW pipe leak at CFCU.

SW pipe leak at CFCU.

SW pipe leak, charging pump operation affected.

SW pipe leak, $C C W \mathrm{HX}$.

SW pipe leak, CFCU. 
Table B3.A.5 (continued)

Plant

Plant

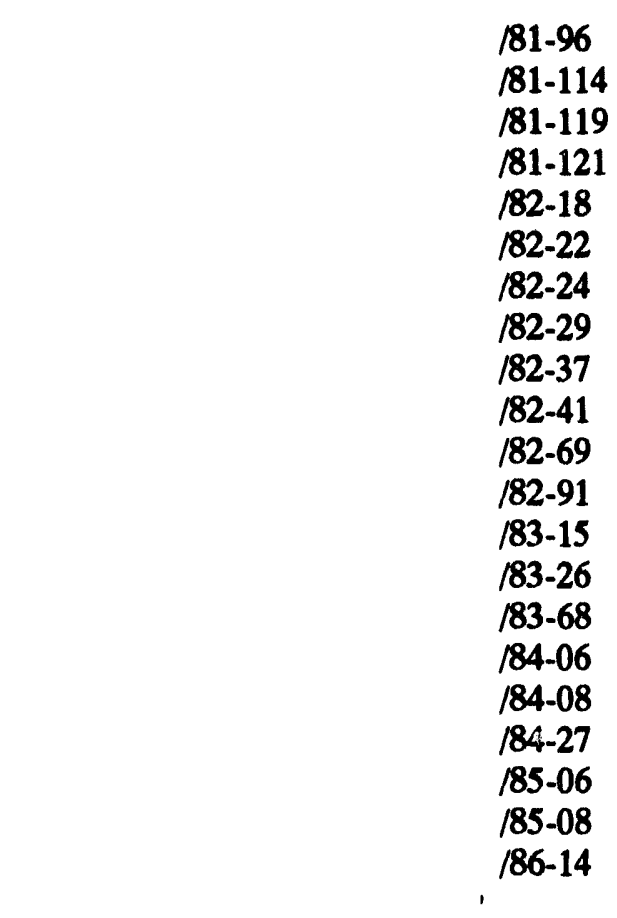

Surry 1

LER-280/80-54

/80-65

/82-100

/82-124

/83-42

/86-24

/86-30

/86-31

/86-34

187-02

/87-03

/87-05

187-06

/87-07

/87-08

/87-18

/87-21

/88-07

Surry 2

LER-281/80-28

/80-37

/81-21

/81-34

/81-47

/81-51

/81-73

\section{Description}

SW pipe leak, CFCU.

SW pipe leak, CFCU.

SW pipe leak, charging pump.

SW pipe leak, CFCU.

SW value leaks in containment.

SW flow control valve fails, reduces flow to CFCU.

SW flow control valve fails, reduces flow to CFCU.

SW flow control valve fails, reduces flow to CFCU.

SW flow control valve fails, reduces flow to CFCU.

SW pipe leak, charging pump affected.

SW pipe leak, charging pump affected.

SW leak, CCW HX.

SW valve malfunction, DG inoperable.

SW valve plugged, CFCU inoperable.

$S W$ line freezes - fire $O G$ inoperable.

SW line leak, CFCU.

SW pipe corrosion near CCW HX.

SW pipe leak at CFCU.

SW pipe leak at CFCU.

SW pipe leak at CFCU.

SW valve to turbine lube oil fails, reactor trip.

SW MOV failed to cycle.

SW MOV failed due to marine growth.

Loss of one SW pump due to personnel error.

SW inlet valve to RS HX was inadvertently closed, loss of one train.

SW strainer clogged.

SW lines in chillers are clogged.

SW lines clogged, marine growth.

SW strainer malfunction, personnel error.

SW strainer clogged.

SW valve malfunction, chiller affected.

SW valve malfunction, chiller affected.

SW strainer malfunction, chiller affected.

SW low flow to chiller, electrical trouble.

SW leak at chiller.

SW valve malfunction affecting chiller.

SW strainer clogged.

SW strainer clogged.

SW flow problems (manual control).

Check disk missing in SW subsystem.

SW strainer clogged, charging pump affected.

SW strainer clogged, charging pump affected.

SW strainer clogged, charging pump affected.

SW MOV breaker open at CCW HX.

SW MOV failed to close.

SW MOV malfunction. 
Table D3.A.5 (continued)

Plant

Deference

181.76

$182-02$

182-09

182-39

$182-45$

$182-49$

$182-50$

182-52

182.54

183-25

183-26

$183-50$

185-02

$186-06$

Prairie Island 1

LER-282/83-18

B3-21

185-03

185-16

187-07

187-08

Fort Calhoun 1

LER-285/81-05

187-01

Indian Point 3

LER-286/81-04

183-06

187-07

Oconee 3

LER-287/81-10

183-08

TML-1

LER-289/80-15

Zion 1

LER-295/80-18

$180-24$

181-07

183-32

184-04

185-39

186-01

Crystal River 3

LER-302/84-11

\section{Description}

SW MOV malfunction.

SW check valve failed on booste: pump discharge.

SW valve failure, flow obstructed.

SW MOV flooded.

SW MOV breaker open - CCW HX.

SW atrainer leaking.

SW arainer clogged, booster pump lost.

SW flow indicator fails, reduces flow.

SW MOV Ilooded.

SW MOV malfunction.

SW MOV breaker open.

SW strainer clogged.

Improper aligament of SW flow to $\mathrm{HX}$.

SW leak in containment spray HX.

Intake device fails, some SW pumps tripped.

SW isolation MOV failed.

SW value inadvertently closed.

SW leak at CFCU.

SW booster pump fails due to deposition.

SW booster pump air bound, procedural error.

Relay problems in the starting circuit.

Three SW pumps are unavailable for two hours.

SW supply to non-escential HDR lost, both supply pumps are in maintenance.

Seismic restraining plates removed, possible failure during a DBA.

Pipe snubbers failed.

SW value air line break.

SW valve failed, CFCU affected.

SW RTD failed.

SW valve failed, AFW pump affected.

SW pump failed to start, electrical switch problem.

SW pipe section made to non-safety specifications.

SW MOV failure.

SW leak at CFCU.

SW crosstie valve between two units cycled, loss of SW, standby pump started.

SW valves inadvertently closed, isolates AFW for three weeks.

185-24

185-35

$187-20$
SW pump discharge check valve stuck open.

Cracked pipe support pedestal at CCW HX.

Design deficiency, fire may affect various pumps.

Design discrepancy in SW system temperatures. 
Table B3.A.s (continued)

Piant

Zion 2

Kewaunee

Prairie Island

Maine Yankee

Salem 2
LER-309/81-07

/83-15

/83-17

/83-33

Reforence

LER-304/80-17

180-30

/81-14

/81-17

$181-36$

182-09

183-29

$183-40$

183.45

184-13

/85-04

LER-305/80-35

/81-01

/81-07

/82-05

/82-33

/83-05

/83-21

/83-24

/83-25

/83-27

/83-37

/84-18

/86-15

LER-306/80-32

LER-311/81-04

/81-10

/81-38

$181-64$

$181-90$

$181-94$

/81-99

/81-114

/81-115

/81-117

/81-118

/82-06

\section{Description}

SW pump disabled due to electrical fault at dc bus. SW MOV failure.

SW MOV failure.

SW valve inoperable, loss of initiating signal.

SW valve inoperable, loss of initiating signal.

SW valves fail, silt deposition.

SW valves fail, electrical problems.

SW valves fail, electrical problems.

SW leak at CFCU.

SW leak, tube degradation.

Control valve malfunction.

SW pump fail to start.

SW valve failure, $C F C U$ inoperable.

SW pump failed to start.

SW pump failed to start.

SW MOV failed to open.

SW MOV malfunction.

SW pump unavailable, strainer tested.

SW pump failed.

Flow indicator failed, SW pump unavailable.

Pipe leak due to corrosion at CCW HX.

SW strainer leaked, SW pump unavailable.

Silt deposition in CFCU coils reduces flow.

SW valve failed in closed position.

Intake area isolated for one unit, causing a loss of SW pump on the other unit.

SW cooling to SCC interrupted due to overload.

SW pump tripped, redundancy reduced.

SW MOV failed to operate.

SW MOV failed to operate.

SW pipe leak at CFCU.

SW pump failed, another in maintenance.

SW leak at CFCU.

SW leak at CFCU.

SW leak at CFCU.

SW leak at CFCU.

Instrument line clogged with silt, valve inoperable.

SW leak at CFCU.

SW leak at CFCU.

Line clogged with silt, limit SW failure on MOV.

SW leak.

Valve stuck closed at CFCU. 
Table B3.A.5 (continued)

Plant

Reference

182-17

182-28

$182-35$

182-39

$182-40$

$182-41$

$182-46$

$182-49$

182-50

182-58

$182-63$

182-65

$182-70$

$182-73$

$182-74$

$182-75$

/82-77

$182-78$

/82-80

$182-83$

182-84

$182-86$

$182-88$

/82-89

/82-91

/82-92

/82-93

/82-96

182-98

/82-99

$/ 82-100$

/82-101

/82-105

/82-109

/82-112

$/ 82-113$

/82-115

/82-117

/82-119

/82-120

/82-122

/82-123

/82-128

/82-130

/82-135

/82-136

\section{Description}

Valve stuck closed, line clogged with silt.

SW leak at CFCU.

SW valve inoperable.

SW leak at CFCU.

SW leak at CFCU.

Marine growth reduces flow to CFCU.

Oysters reduce flow to CFCU.

Oysters reduce flow to CFCU.

Oysters reduce flow to CFCU.

Oysters reduce flow to CFCU.

One vital bus lost, diesel didn't load.

Valve failure stopped flow to CFCU.

SW leak at CFCU.

SW leak at CFCU.

SW leak at CFCU.

SW leak at CFCU.

SW leak at CFCU.

SW leak at CFCU.

SW leak at CFCU.

Oysters reduce flow to CFCU.

SW leak at CFCU.

One train of SW lost, one pump failed, other maintenance.

Low flow to CFCU due to valve problems.

SW leak at CFCU.

SW leak at CFCU.

SW leak at CFCU.

SW leak at CFCU.

Silt buildup in line reduces flow to CFCU.

Silt buildup in line reduces flow to CFCU.

Silt buildup in line reduces flow to CFCU.

Silt buildup in line reduces flow to CFCU.

Silt buildup in line reduces flow to CFCU.

Silt buildup in line reduces flow to CFCU.

Silt buildup in line reduces flow to CFCU.

SW leak at CFCU.

SW leak at CFCU.

SW leak at CFCU.

Oysters block SW valve.

SW leak due to corrosion from silt.

SW leak due to corrosion from silt.

SW leak due to corrosion from silt.

Flow controller setpoint is incorrect, low flow.

SW leak due to silt buildup.

Reduced flow to CFCU, silt buildup.

SW leak at CFCU.

SW leak at CFCU.

B3-39

NUREG/CR-5726 
Appendix B3

Table B3.A.5 (continued)

Plant

Rancho Seco

ANO-1

Cook 1

LER-315/80-29

/81-04

/81-15

/82-06

/82-09

$182-43$

$182-48$

$182-94$

$/ 82-95$

/83-14

Cook 2

LER-316/81-03

/82-02

/82-11

/82-80

/83-97

Calvert Cliffs 1

LER-317/80-27

$180-32$

$180-41$

\section{Deseription}

SW strainer plugged, one train lost.

Valve problem reduces flow to CFCU.

SW air-operated valve failure to DG cooling.

Valve failed to open, both CCW HX unavailable.

SW leak at CFCU.

Design deficiency, cable separation didn't satisfy Appendix $\mathbf{R}$ requirements.

SW pump leaked, a number of pumps unavailable.

SW pump breaker didn't close.

Lube oil cooler malfunction.

SW pump tripped, no apparent cause.

SW pump failed to start.

Snubbers failed in the SW system.

Incorrect personnel actions in tests.

SW pump breaker not properly documented.

Snubbers and pipe system in operation in spite of incorrect acceptance criteria.

Pipe to spray pump bearing plugged.

Incorrect level switches could prevent the starting of the SW pumps.

Deficiency in SW pipe system design.

Deficiency in SW pipe system design.

MOVs are tested not in accordance with requirements.

MOV failed.

Relays lead to malfunction of SW pump circuitry.

MOV failed to close at CCW HX.

SW leak at $\mathrm{CCW} \mathrm{HX}$.

SW pump discharge valve failed, loop unavailable.

SW MOV failed at CCW HX.

MOV leaked.

SW valve left in closed position, containment spray HX unavailable.

Silt buildup in strainer.

Strainer shut down, enabling SW pump.

Sw MOV tested less frequently as required.

SW pipe leak at CCW HX.

$S W$ valve leak at $C C W H X$.

SW MOV electrical ground problem.

Air bound due to instrument air cooler tube leak

Valve failed, corrosion causes seizure.

Cooler failed. 
Appendix B3

Taible B3.A.S (continued)

Plant

Calvert Cliffs 2

TMI-2

Sequoyah 1
Roforence

180-52

181-04

/81-10

/81-29

/81.63

181-77

182-32

183-67

183-74

LER-318/80-17

181-09

181-53

/82-34

/82-35

/82-51

/83-17

LER-320/80-08

181-02

/81-37

$/ 82-03$

LER-327/80-75

181-72

/81-95

/81-97

/81-101

/82-17

/82-27

/82-35

$183-10$

/83-26

/83-182

184-69

$185-05$

186-28

186-29

/86-41

$187-08$

$187-11$

$187-27$

$187-37$

$187-45$

/87-51

\section{Description}

SW leak, HX tube failed. SW MOV failed open at CFCU discharge. SW valve malfunction.

Pipe support design deficiency.

SW valve operator failed at CCW HX.

Solenoid valves in SW are underrated.

SW MOV electrical trouble.

Valve failed at ECCS pump room air cooler.

Operator disconnected, SW pump lost, one train inoperable.

SW HX leaked.

SW MOV didn't operate, electrical trouble.

Reduced SW flow due to valve problem.

SW valve failed, $\mathrm{HX}$ inoperable.

Valve broken, leak at SW HX.

SW loop degraded due to valve failure.

Power on valves lost, one train unavailable.

SW pump locked out, not on standby.

SW unavailable to DG, improper operator action.

SW pump lost, mechanical trouble.

SW pump failed to start, loose connections.

SW pump failed, personnel error.

SW pipe hanger removed, not reinstalled.

Strainer failed.

Protective device not installed to prevent SW system damage from a steam line break.

Inadequate flow to safety equipments.

Inoperable snubbers.

Clams block flow to CS HX.

Solenoid valve failed closed to air rompr. $H X$.

SW valve failed to DG.

SW valve failed to $D G$.

Relay failure in SW electrical system.

SW valve overload improperly set.

SW pipe inadequately supported for seismic event.

Design deficiency in SW pump instruments.

SW pump failed to meet test acceptance criteria.

Misaligned valve, improper flow to DG.

SW valves are not tested as required by TS.

SW valves are not tested as required by TS.

SW valves are not tested as required by TS.

Inadequate calculations, design deficiency on coolers.

Inadequate design for traveling screen speed SW.

SW valves are not tested as per TS. 
Appendix B3

Table B3.A.5 (continued)

Plant

Reference

$187-64$

187.65

/87-71

Sequoyah 2

LER-328/82-47

$185-06$

Beaver Valley 1 LER-334/80-27

$180-42$

$180-65$

180-68

/82-19

$/ 82-60$

St. Lucie 1

Millstone 2

North Anna 1

LER-338/80-22

/80-10

/80-16

/81-24

$/ 81-46$

$/ 81-71$

$181-83$

$/ 82-06$

$/ 82-81$

/83-04

$183-48$

/85-04

\section{Description}

SW spool pieces out of tolerances.

Screen was pumps are not tested regularly.

Electrical interlock on strainers disabled.

Reduced flow to CS HX, valve position adjusted.

$S W$ valve in closed position, indicator plate mislabelled.

SW check valve eroded to DG.

Pipe line improperly restrained for seismic event.

SW pipe over-stressed, design deficiency.

Check valves installed backwards.

SW leak at CS HX.

Starter on MOV failed.

SW pump not tested as required.

SW pump A seized, $C$ unavailable.

Strainer leaked.

Strainer drive motor had loose mounting bolts.

SW strainer leaked.

Solenoid failed, DG inoperable.

SW pipe leaked.

SW pump leaked.

Misalignment of $\mathrm{HX}$ components.

SW pump/strainer leaked.

Pipe hangers undersized.

Strainer plugged, loss of one header.

Pipe hanger failed.

SW pump failed to start on loss of offsite power.

SW pump failed to start.

Improperly positioned control valve.

SW pump manually started on loss of offsite power sequencer failed.

Increased stresses on pipe/valve supports.

Increased stresses on pipe/valve supports.

Increased stresses on pipe/valve supports.

SW pipe leaked.

SW pipe leaked.

SW pipe leaked.

SW pipe leaked.

SW pipe leaked.

SW pipe leaked.

SW MOV failed, trouble with operator.

SW pipe leaked.

SW supply line not adequately covered against tornados. 

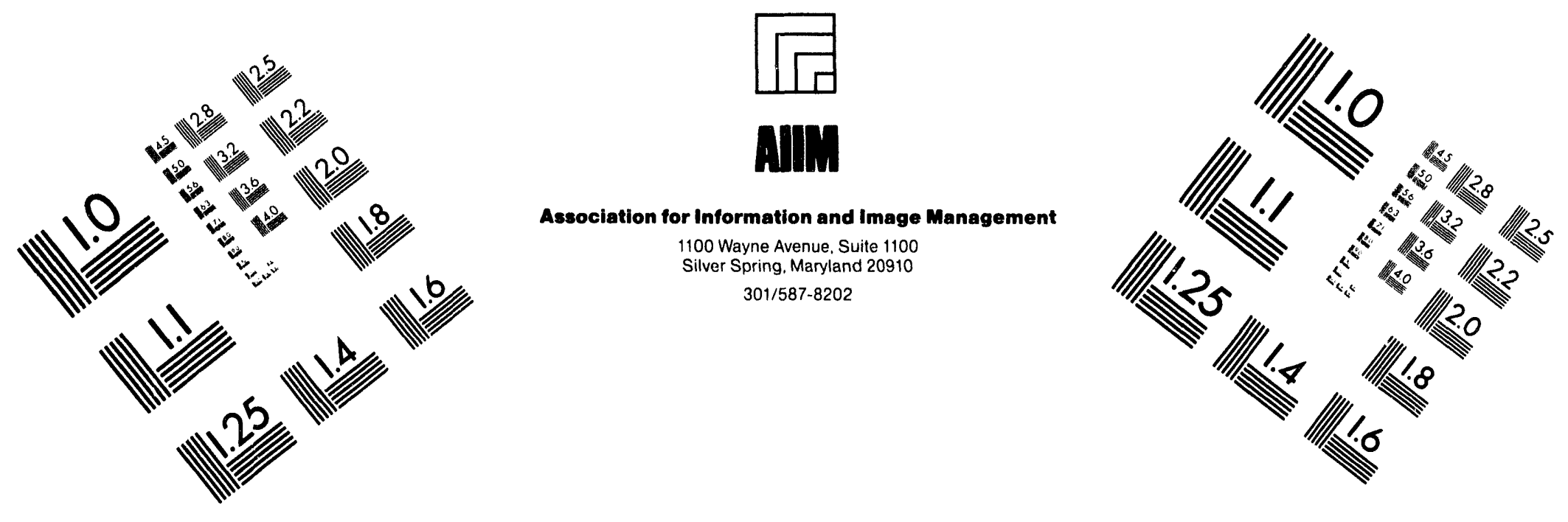

\section{Centimeter}

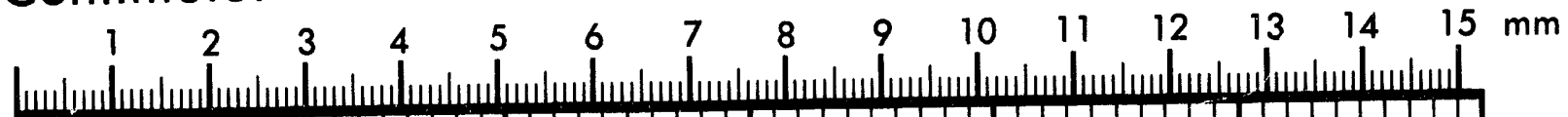

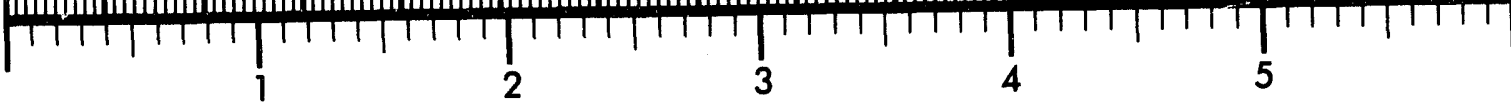
Inches
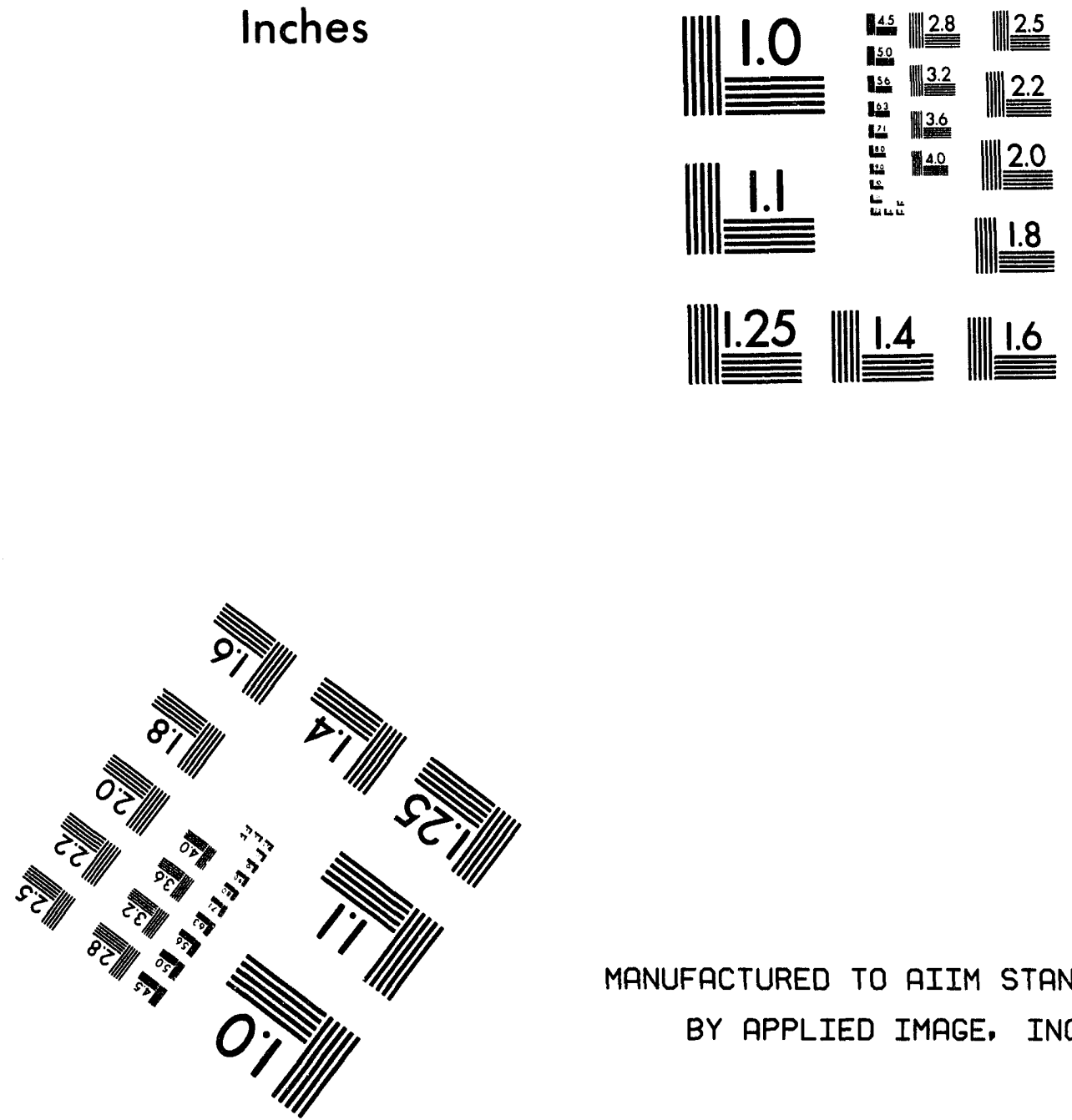

MANUFACTURED TO AIIM STANDARDS

BY APPLIED IMAGE, INC.

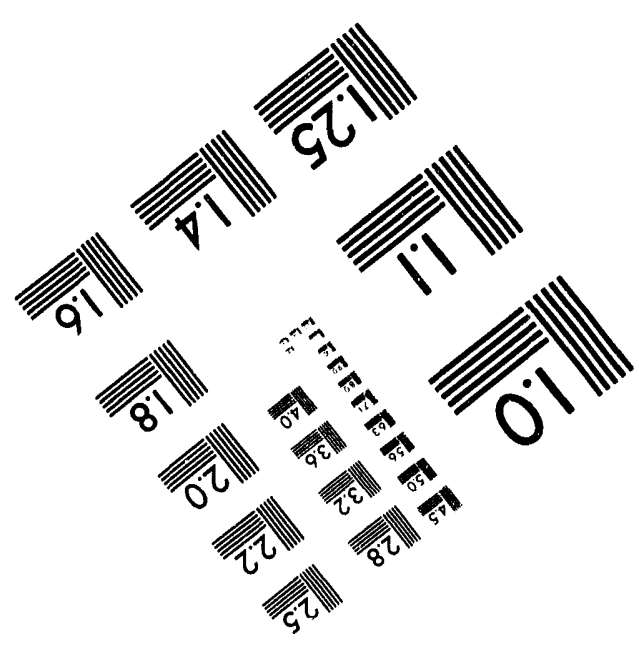



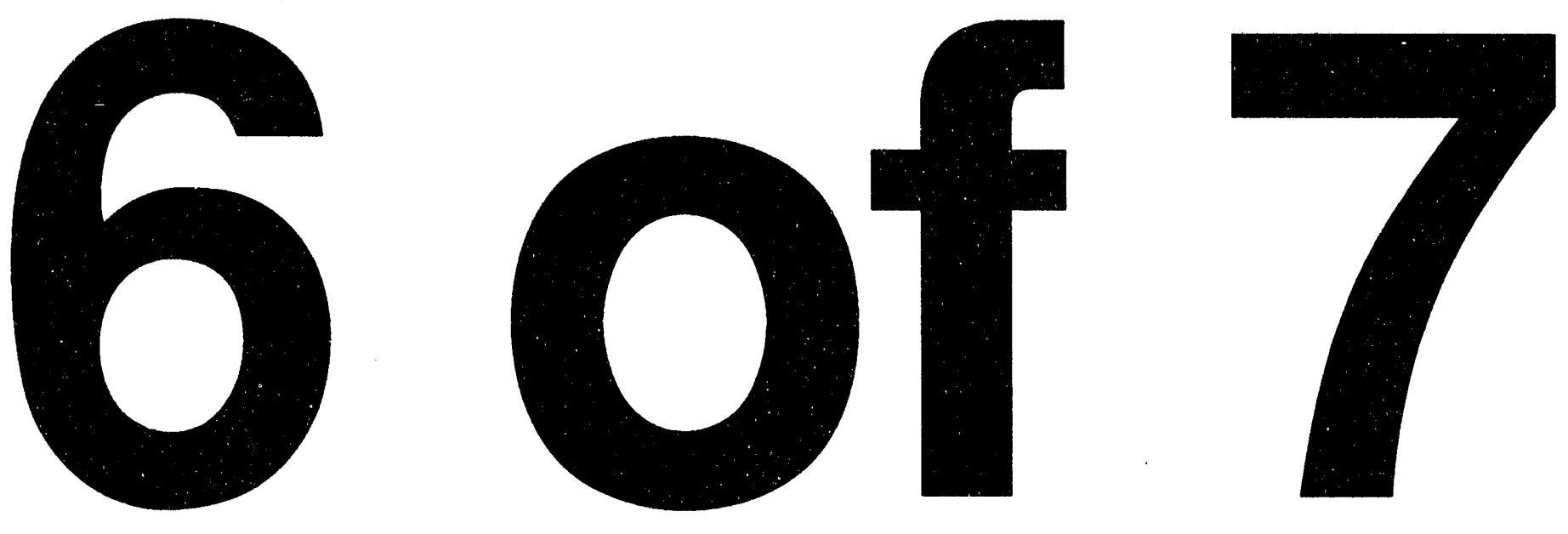
Appendix B3

Table B3.A.5 (continued)

Plant

Reference

/82-03

/83-06

/83-27

McGuire 1

LER-369/81-138

/83-21

183-84

185-30

186-06

186-19

187-09

/87-18

188-03

McGuire 2

LER-370/85-01

/87-14

/87.17

St. Lucie 2

LER-389/83-54

186-01

Summer 1

LER-395/82-30

/83-33

183-49

/85-14

186-12

887-10

Shearon Harris

LER-400/87-59

188-06

188-08

Catawba 1

LER-413/85-04
185-26

/85-32

186-24

$186-27$

$186-53$

186-57

$187-08$

/87-35

$187-36$

LER-423/86-56

/87-01

\section{Description}

Reduced flow through seal water cooler of SI pump.

Improper switch and valve settings.

Over-tight SW valve failed to operate.

SW system leak.

Valve actuator replaced incorrectly.

SW pump inoperable, one train unavailable.

Valve locked in incorrect position.

SW system no fully tested.

SW valve not tested as per requirements.

Improperly positioned valve.

Inadequate test performance of SW pumps.

Mispositioned control valve at $\mathrm{CCW}$ HX.

Valve not locked open as per requirements.

One SW pump tripped, other in maintenance.

Both SW trains are in maintenance.

SW pipe leak.

SW pipe leak.

Check valve stuck closed.

Speed switch failed, one train inoperable.

Check valve failed to close on reverse flow.

SW pump lost during DG test, RHR transient.

SW pump failed to start, faulty relay.

Screen pump failed to start, loose connections.

Travelling screen didn't start at loss of offsite power.

SW valves failed, debris.

Emergency SW pump unavailable due to test.

Loss of SW to RCP motor, improper airline design.

Loss of suction to SW pumps, incorrect valve operation.

SW intake aligned to standby source, personnel error.

Misalignment of SW intake.

Misalignment of SW intake.

Misalignment of SW intake.

SW MOV torque switches improperly set.

Tornado missile cover missing on SW pipe manways.

Incorrect procedures could prevent SW train operation.

Incorrect crossover supply alignment.

No flow to SI HX, valve closed.

SW low pressure causes turbine/reactor trip. 
Table B3.A.5 (continued)

Plant

Vogtle 1

Reference

Seabrook

LER-424/87-03

LER-443/87-25

Byron 1

LER-454/86-31

Byron 2

LER-455/87-03

Braidwood 1

LER-456/87-16

Wolf Creek

LER-482/85-12

/85-69

186-44

Callaway 1

LER-483/87-24

South Texas 1

LER-498/87-03

/87-18

$188-20$

/88-23

Palo Verde 1

LER-528/86-14

/86-37

Crystal River 3

LER-302/87-20

\section{Description}

Incorrect sealant used in penetrations.

Incorrect test monitoring for Sw pump vibrations.

Both SW strainers improperly tested.

SW makeup pumps out of service.

Incomplete test of SW systems.

SW MOV didn't close properly.

Travelling screens collapse due to plant growth.

SW valve failed to operate.

SW valve not tested as required.

SW pump tripped, discharge check valve stuck closed.

SW pipe leak, one train inoperable.

Screen wash booster pump inoperable.

Test on screen wash booster pump performed not as frequently as required.

SW pump failed to start, faulty relays.

SW pump failed to start.

On September 3, 1987, Crystal River Unit 3 (CR3) was operating at approximately $63 \%$ rated thermal power. An NRC audit of plant cooling water systems revealed that the ultimate heat sink (UHS) temperature exceeded the maximum value assumed in the plant design basis. Also, the plant Tech. Spec. limit for UHS temperature was higher than the design basis. This event was the result of an inadequate plant design specification. The maximum seawater temperature specified for plant design was $85^{\circ} \mathrm{F}$, while actual temperatures exceed this value during the summer months. The Tech.Spec crror appears to have been caused by inadvertently selecting a temperature limit from a closed cycle cooling loop rather than the UHS design specification. Analyses indicate that the nuclear services closed cycle cooling system $105^{\circ} \mathrm{F}$. Temperature limit can be met with seawater temperatures as high as $92.4^{\circ} \mathrm{F}$. FPC continues to evaluate the past operability of the decay heat closed cycle cooling system when the seawater temperature exceeded $85^{\circ} \mathrm{F}$. The results of the evaluation are due to be submitted to the NRC in a separate report by July $29,1988$. 


\section{ATTACHMENT B3.B}

HARDWARE UNAVAILABILITY CUT SETS FOR

THE AUXILIARY SALTWATER SYSTEM

Top Event: AS

Initiator: LOSW 
Appendix B3

Top Event AS

Boundary condition designator: AS1

Hardware unavailability cut sets due to dependent and independent failure; HW =

$\begin{array}{ll}1 & 1.2962 E-06 \\ 2 & 1.3552 E-07 \\ 3 & 4.9457 E-08 \\ & \\ 4 & 3.6275 E-08 \\ 5 & 1.6176 E-08 \\ 6 & 8.9693 E-09\end{array}$

$7 \quad 5.8375 \mathrm{E}-09$

$8 \quad 3.4400 \mathrm{E}-09$

$9 \quad 1.2472 \mathrm{E}-09$

$10 \quad 2.6989 \mathrm{E}-10$

$11 \quad 1.7070 \mathrm{E}-10$

$12 \quad 1.7070 \mathrm{E}-10$

$13 \quad 1.3023 \mathrm{E}-10$

$14 \quad 1.3023 \mathrm{E}-10$

$15 \quad 1.3023 \mathrm{E}-10$

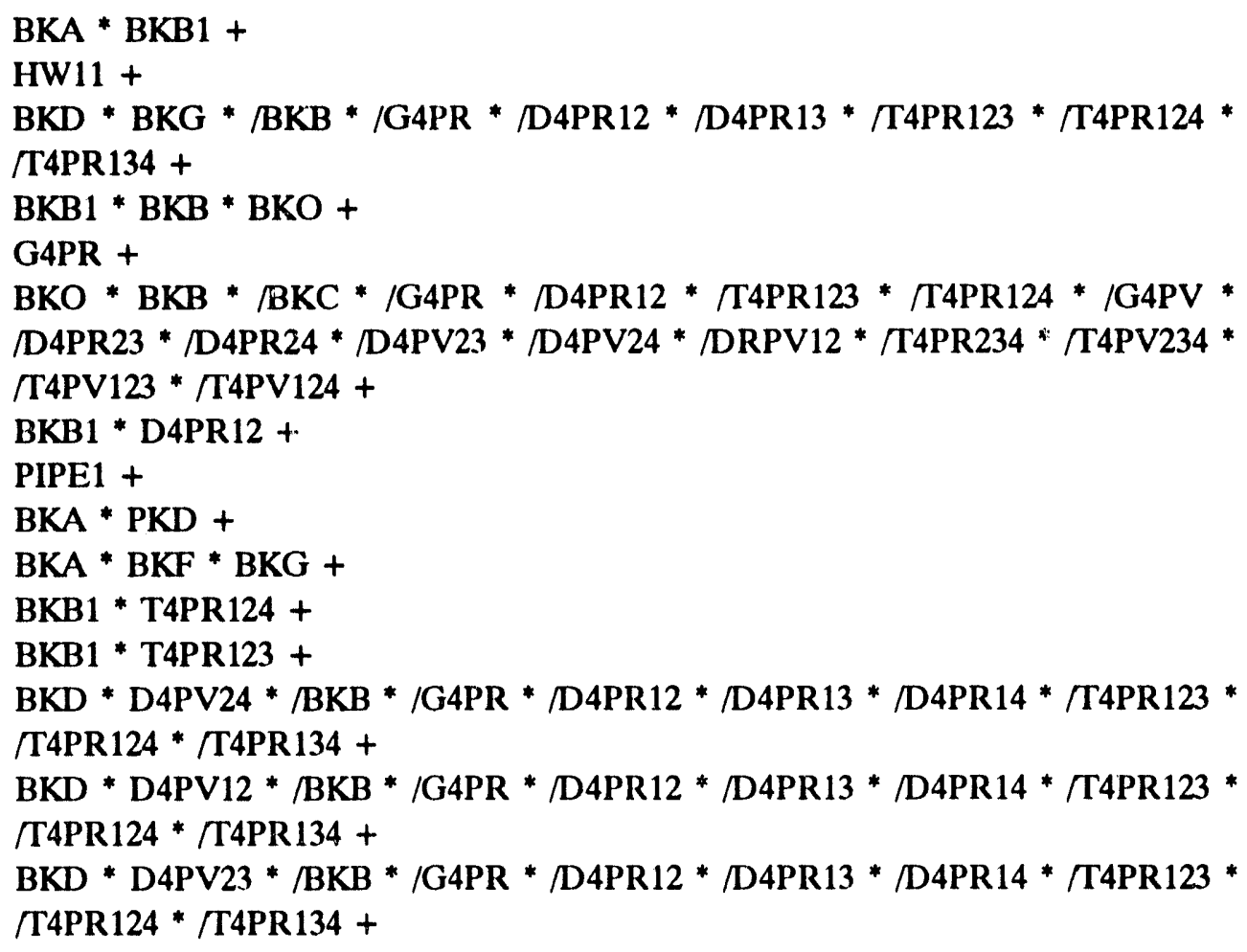

Hardware unavailability cut sets due to independent failures; HWI =

$\begin{array}{ll}1 & 1.2962 \mathrm{E}-06 \\ 2 & 1.3552 \mathrm{E}-07 \\ 3 & 4.9457 \mathrm{E}-08 \\ 4 & 3.6275 \mathrm{E}-08 \\ 5 & 8.9697 \mathrm{E}-09 \\ 6 & 3.4400 \mathrm{E}-09 \\ 7 & 1.2472 \mathrm{E}-09 \\ 8 & 2.6989 \mathrm{E}-10\end{array}$

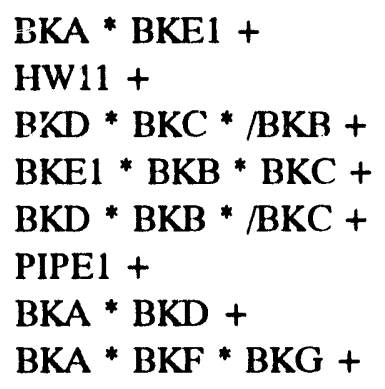

HW11 $=$ BKBT $^{*}$ ZTVCOO 
Appendix B3

$$
\begin{aligned}
& \text { Initiator: LOSW } \\
& 1 \text { - During Normal Operation (BNL1 Calculation) }
\end{aligned}
$$

Hardware unavailability cut sets due to dependent and independent failure; HW =

$\begin{array}{ll}1 & 3.6893 \mathrm{E}-05 \\ 2 & 2.5614 \mathrm{E}-05 \\ 3 & 1.7057 \mathrm{E}-05 \\ 4 & 9.0116 \mathrm{E}-06 \\ 5 & 5.0186 \mathrm{E}-06 \\ 6 & 1.5967 \mathrm{E}-06 \\ 7 & 4.6689 \mathrm{E}-08 \\ 8 & 4.6689 \mathrm{E}-08 \\ 9 & 2.6596 \mathrm{E}-08 \\ 10 & 2.6596 \mathrm{E}-08 \\ 11 & 2.6126 \mathrm{E}-08 \\ 12 & 2.6126 \mathrm{E}-08 \\ 13 & 2.6126 \mathrm{E}-08 \\ 14 & 1.4064 \mathrm{E}-08 \\ 15 & 1.4051 \mathrm{E}-08 \\ 16 & 1.2795 \mathrm{E}-08 \\ 17 & 1.0640 \mathrm{E}-08 \\ 18 & 1.0640 \mathrm{E}-08 \\ 19 & 6.0611 \mathrm{E}-09 \\ 20 & 5.6213 \mathrm{E}-09\end{array}$

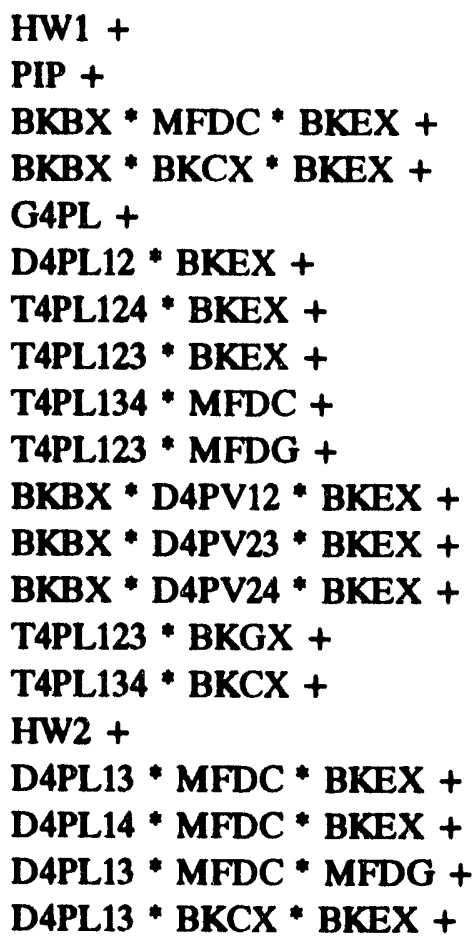

Hardware unavailability cut sets due to independent failure; HWI =

$\begin{array}{ll}1 & 3.6893 \mathrm{E}-05 \\ 2 & 2.5614 \mathrm{E}-05 \\ 3 & 1.7057 \mathrm{E}-05 \\ 4 & 9.0116 \mathrm{E}-06 \\ 5 & 1.2795 \mathrm{E}-08 \\ 6 & 3.3978 \mathrm{E}-09 \\ 7 & 1.7967 \mathrm{E}-09 \\ 8 & 1.7951 \mathrm{E}-09 \\ 9 & 9.4923 \mathrm{E}-10\end{array}$

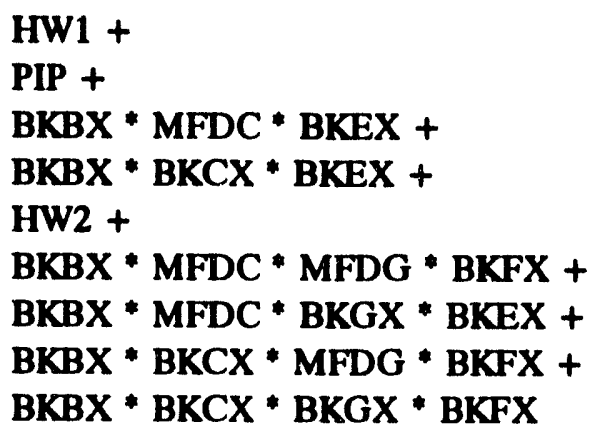

HW1 $=$ BKBXT*ZTVCOD

HW2 $=$ BKBXT $^{*}\left(B_{K C T}^{*} Z T V C O D\right)$ 
Initiator: LOSW

\section{1 - During Normal Operation (BNL2 Calculation)}

Hardware unavailability cut sets due to dependent and independent failure; HW =

$\begin{array}{lll}1 & 1.1913 E-04 & \text { BKBX * MFDC * COLD + } \\ 2 & 9.2449 E-05 & \text { HW11 + } \\ 3 & 5.2221 E-05 & \text { BKBX * BKCX * COLD + } \\ 4 & 3.7404 E-05 & \text { BKBX * MFDC * BKEX + } \\ 5 & 3.6637 E-05 & \text { BKBX * MFDC * MFDG + } \\ 6 & 2.5614 E-05 & \text { PIP + } \\ 7 & 1.0177 E-05 & \text { BKSX * BKCX * BKEX + } \\ 8 & 9.9771 E-06 & \text { BKBX * MFDC * BKGX + } \\ 9 & 9.9681 E-06 & \text { BKBX * BKCX * MFDG + } \\ 10 & 9.2523 E-06 & \text { D4PL12 * COLD + } \\ 11 & 5.0186 E-06 & \text { G4PL + } \\ 12 & 3.9911 E-06 & \text { T4PL123 + } \\ 13 & 3.9911 E-06 & \text { T4PL124 + } \\ 14 & 3.2833 E-06 & \text { BKA * COLD + } \\ 15 & 2.7145 E-06 & \text { BKBX * BKCX * BKGX + } \\ 16 & 2.2333 E-06 & \text { BKBX * DRPV24 + } \\ 17 & 1.8031 E-06 & \text { D4PL12 * BKBX + } \\ 18 & 1.7661 E-06 & \text { D4PL12 * MFDG + } \\ 19 & 1.7661 E-06 & \text { D4PL14 * MFDC + } \\ 20 & 1.7661 E-06 & \text { D4PL13 * MFDC + } \\ 21 & 9.9806 E-07 & \text { BKBX * MFDC * BKFX + } \\ 22 & 6.3984 E-07 & \text { BKA * BKEX + } \\ 23 & 6.2672 E-07 & \text { BKA * MFDG + } \\ 24 & 4.8095 E-07 & \text { D4PL12 * BKCX + }\end{array}$

HW11 $=$ BKBT $^{*}$ ZTVCOO 
Appendix B3

$$
\begin{aligned}
& \text { Initiator: LOSW } \\
& \text { 1. During Normal Operation (BNL2 Calculation) }
\end{aligned}
$$

Hardware unavailability cut sets due to independent failure; HWI =

$\begin{array}{lll}1 & 1.9193 E-04 & \text { BKBX * MFDC * COLD + } \\ 2 & 9.2449 E-05 & \text { HW11 + } \\ 3 & 5.2221 E-05 & \text { BKBX * BKCX * COLD + } \\ 4 & 3.7404 E-05 & \text { BKBX * MFDC * BKEX + } \\ 5 & 3.6637 E-05 & \text { BKBX * MFDC * MFDG + } \\ 6 & 2.5614 E-05 & \text { PIP + } \\ 7 & 1.0177 E-05 & \text { BKBX * BKCX * BKEX + } \\ 8 & 9.9771 E-06 & \text { BKBX * MFDC * BKGX + } \\ 9 & 9.9681 E-06 & \text { BKBX * BKCX * MFDG + } \\ 10 & 3.2833 E-06 & \text { BKA * COLD + } \\ 11 & 2.7145 E-06 & \text { BKBX * BKCX * BKGX + } \\ 12 & 9.9006 E-07 & \text { BKBX * MFDC * BKEX + } \\ 13 & 6.3984 E-07 & \text { BKA * BKEX + } \\ 14 & 6.2672 E-07 & \text { BKA * MEDG + } \\ 15 & 2.6937 E-07 & \text { BKBX * BKCX * BKFX + } \\ 16 & 1.7067 E-07 & \text { BKA * BKGX + } \\ 17 & 3.2064 E-08 & \text { HW22 + } \\ 18 & 1.6936 E-08 & \text { BKA * BKEX + } \\ 19 & 1.7924 E-09 & \text { BKBX * MFDC * BKDC + } \\ 20 & 4.8768 E-10 & \text { BKBX * BKCX * BKDC + } \\ 21 & 3.0662 E-11 & \text { BKA * BKDC }\end{array}$

HW11 $=$ BKBXT * ZTVCOO

HW22 $=$ BKBXT * $($ BKCT * ZTVCOO) 


\section{Initiator: LOSW \\ 2. During Pump Rotation (BNL1 Calculation)}

Hardware unavailability cut sets due to dependent and independent failure; HW =

$\begin{array}{lll}1 & 1.2972 \mathrm{E}-08 & \text { BKCY * BKBY * BKEX + } \\ 2 & 1.8744 \mathrm{E}-10 & \text { HW3 + } \\ 3 & 4.1754 \mathrm{E}-11 & \text { D4PV12 * BKBY * BKEX + }\end{array}$

Hardware unavailability cut sets due to independent failure; HWI =

$\begin{array}{lll}1 & 1.2972 E-08 & \text { BKCY * BKBY * BKEX + } \\ 2 & 1.8744 \mathrm{E}-10 & \text { HW3 + }\end{array}$

HW3 $=$ BKCYT*BKBYT*ZTVCOD 
Appendix B3

\author{
Initintor: LOSW \\ 2 - During Pump Rotation (BNL2 Calculation)
}

Hardware unavailability cut sets due to dependent and independent failure; HW =

$\begin{array}{lll}1 & 7.5169 E-08 & \text { BKCY * BKBY * COLD + } \\ 2 & 1.4649 E-08 & \text { BKCY * BKBY * BKEX + } \\ 3 & 1.4348 E-08 & \text { BKCY * BKBY * MFDG + } \\ 4 & 3.9874 E-09 & \text { BKCY * BKBY * BKGY + } \\ 5 & 3.5692 E-09 & \text { D4PV24 * BKBY + } \\ 6 & 6.9168 E-10 & \text { BKCY * D4PR14 + } \\ 7 & 6.9168 E-10 & \text { BKCY * D4PR13 + } \\ 8 & 4.6969 E-10 & \text { HW33 + } \\ 9 & 4.2403 E-10 & \text { T4PV124 * BKBY + } \\ 10 & 4.2403 E-10 & \text { T4PV234 * BKBY + } \\ 11 & 3.8775 E-10 & \text { BKCY * BKBY * BKEY + } \\ 12 & 2.9237 E-10 & \text { G4PV * BKBY + } \\ 13 & 2.4196 E-10 & \text { D4PV23 * BKBY * COLD + } \\ 14 & 2.4196 E-10 & \text { D4PV12 * BKBY * COLD + } \\ 15 & 4.7153 E-11 & \text { D4PV23 * BKBY * BKEX + } \\ 16 & 4.7153 E-11 & \text { D4PV12 * BKBY * BKEX + } \\ 17 & 4.6889 E-11 & \text { BKCX * D4PR12 * COLD + } \\ 18 & 4.6186 E-11 & \text { D4PV12 * BKBY * MFDG + } \\ 19 & 4.6186 E-11 & \text { D4PV23 * BKBY * MFDG + } \\ 20 & 2.8745 E-11 & \text { T4PV123 * BKBY * COLD + } \\ 21 & 2.5433 E-11 & \text { BKCY * G4PR + }\end{array}$

Hardware unavailability cut sets due to independent failure; HWI =

$\begin{array}{lll}1 & 7.5169 E-08 & \text { BKCY * BKBY * COLD + } \\ 2 & 1.4649 E-08 & \text { BKCY * BKBY * BKEX + } \\ 3 & 1.4348 E-08 & \text { BKCY * BKBY * MFDG + } \\ 4 & 3.9074 E-19 & \text { BKCY * BKBY * BKGY + } \\ 5 & 4.6969 E-10 & \text { HW33 + } \\ 6 & 3.8775 E-10 & \text { BKCY * BKBY * BKFY + } \\ 7 & 2.5433 E-11 & \text { BKCY * G4PR + } \\ 8 & 7.0199 E-13 & \text { BKCY * BKBY * BKDC }\end{array}$

HW33 $=$ BKCYT * BKBYT * ZTVCOO 


\section{APPENDIX B4: COMPONENT COOLING WATER SYSTEM}

\section{B4.1. Introduction}

\section{BA1.1 Objectives}

The main objective of this appendix is to summarize the results of reviewing the unavailability modelling of the Component Cooling Water System (CCWS) and the Reactor Coolant Pump (RCP) Seal Cooling equipment described in the DCPRA. ${ }^{1}$ An additional objective is to determine a BNL value for the initiator "Total Loss of Component Cooling Water (LOCCW)" based on genericplant experience appropriately updated for Diablo Canyon using Bayesian techniques. This was done to compare with the PG\&E value obtained by calculating the total yearly failure frequency of the $\mathrm{CCW}$ system via fault tree analysis.

\section{B41.2 Organization}

Section B4.2 provides a brief description of functions and the configurations, the dependency on support equipment, the surveillance and maintenance conditions, the unavailability modelling of the CCWS as given in the DCPRA, and the original PRA results. Similarly, Section B4.4 describes the approach used by PG\&E to analyze the unavailability of the equipment necessary to maintain RCP seal cooling and the corresponding PRA results. The purpose of this approach is to present stand alone documentation to which this review's findings can be directly related or compared. Section B4.3 contains the results of the BNL review of the CCWS and presents a new value determined for the LOCCW initiator. Section B4.5 presents some additional information requested by $\mathrm{BNL}$ in reviewing the unavailability analysis of $\mathrm{RCP}$ seal cooling. Section B4.6 provides a summary of the review results/findings.

For completeness, the documentation of the information used by BNL for determination of the initiator frequency (LOCCW) is presented in Attachment B4.A.

\section{B4.2 Unavailability Modelling of the Component Cooling Water System in the DCPRA}

\section{B4.2.1 System's Functions, Description and Operation}

The functions of Component Cooling Water System (CCWS) at Diablo Canyon are:

a. to supply cooling water to vital and non-vital loads after an accident,

b. to provide cooling water to various plant components during normal operation, and

c. to provide cooling water to the RHR system during plant cooldown.

The CCWS also represents a monitored intermediate barrier between radioactive fluids and the Auxiliary Saltwater System to which it rejects its heat.

The CCWS consists of three CCW pumps, two CCW heat exchangers, an internally baffled surge tank, and two chemical addition tanks. Its piping consists of three parallel loops. Two are separable redundant vital service loops, " $A$ " and " $B$ ", serving only the unit's emergency safety feature equipment and post-LOCA sample cooler. A miscellaneous service loop, " $\mathrm{C}$ ", serves non-vital equipment. The loads on the three loops are listed in Table B4.2.1. The system's drawing is shown in Figure B4.2.1. The Reactor Coolant Pump (RCP) seal 


\section{Appendix B4}

water heat exchanger, as well as the RCP thermal barrier and motor oil coolers do not represent "vital" loads as they are located on Loup C.

The CCWS is normally operating with all loops in service. Usually, two CCW pumps and one CCW heat exchanger are in operation. The third pump and the second heat exchanger are in standby. The standby pump starts on low pressure in loops A or B. (The pump breaker will not close until lube oil pressure is 6 psig; the lube oil pressure is provided by a lube oil pump.) With ocean water temperature in excess of $64^{\circ} \mathrm{F}$, two CCW heat exchangers are in service.

The flow through the heat exchangers is controlled from the control room by switching the CCW heat exchanger control valves.

During cooldown, all loops are operated with two or three pumps and two heat exchangers. If one of the pumps or one of the heat exchangers is inoperative, orderly shutdown is not affected, but the time for cooldown is extended.

Following an initiating event an "S" signal starts all non-operating $\mathrm{CCW}$ pumps. (The S signal bypasses the lube oil pressure interlock.) A transfer to emergency power trips all three $\mathrm{CCW}$ pumps on under voltage, then restarts the three pumps when bus voltage is restored. Loop $C$ is automatically isolated on high-high containment pressure (Phase $B$ isolation, "Pn signal) or it can be isolated manually. The operator can also reduce flow to the containment fan coolers.

\section{B4.2.2 Top Event Definition, Success Criteria}

Associated with the unavailability of the CCW function, the DCPRA defines only one top event to be used in the support system event tree ("mechanical" part). The designator of this top event is: CC. It is evaluated for nine boundary conditions depending on the initiator and/or the unavailability of certain trains of support systems.

One of the boundary conditions (designator: $\mathrm{CCI}$ ) was taken as an initiator among one of the initiator groups of the DCPRA called "common cause initiating events." The initiator name "total loss of LPCC" indicates the initiating event frequency when all the $\mathrm{CCW}$ pumps fail. Its value is computed as: $\mathrm{LPCC}=\mathrm{CCI}$.

The success criteria of the top event CC are described in Table B4.2.2 for post accident injection and recirculation phases as well as for normal plant operation and cooldown. The table also indicates the applicable Technical Specifications. A comparison of the top event success criteria with the success requirements for this system described in the DCFSAR ${ }^{2}$ showed that the top event success criteria cover those given in the DCFSAR.

\section{B4.2.3 Logic Model, Dependency on Other Support Systems}

The logic model of the top event CC describing the CCW system configuration is shown in Figure B4.2.2. The CCWS is modelled with one heat exchanger, because the second heat exchanger is isolated during normal operation and there is no operating procedure for placing the standby heat exchanger in service following failures of the operating one. The isolation valve for Loop C (FCD-355) is not modelled because flow to Loop $C$ is not required for system success. (Loop $C$ is located within the reactor primary shield wall. It is the most vulnerable of the CCW loops to a failure concurrent with a major LOCA.) The DCPRA assumes 
that excessive leakage from the CCWS would be discovered and corrected prior to any initiating event; therefore, failure of makeup to the surge tank will not fail the system function during the 24 hour mission time. It assumes also that pumps 1-1 and 1-2 are the running pumps and pump 1-3 is in standby, and a check valve failure at the discharge side of the standby pump pioduces sufficient bypass flow to fail the system function.

\section{B4.2.4 Boundary Conditions of Top Event CC}

Boundary conditions include loss of offsite power and degraded states of support systems such as: vital 4.16kV ac buses, vital $480 \mathrm{~V}$ ac and $125 \mathrm{~V}$ dc buses, the SSPS trains A and B. The nine split fraction boundary conditions cover all combinations of support system effects on the CCWS.

The failure of the operater action to throttle CCW flow to the containment fan coolers is included in the unavailability mojel. The detailed list of the boundary condition definitions and the designators of the associated top event split fractions are given in Table B4.2.3.

\section{B4.2.5 Quantification of Top Event Split Fractions, CC}

Table B4.2.4 lists the values of CC split fractions associated with the various boundary conditions quanaifi:d by PG\&E. The table presents the total unavailabilities(TTL) along with the main contributors to the totg: unavailabilities, such as hardware (HW), maintenance (MN), test (TS), and human error (HE). At a given boundary condition the hardware contribution relates to the normal alignment of the CCWS, when no test or maintenance activities are being performed. The table also indicates the two constituent parts of the hardware contribution to the unavailability, the independent (HWI) and the dependent (HWD, i.e., common cause) failures of supercomponen $s$ of the CCWS. The definition and the failure rates of the supercomponents of the CCWS are given in Chapter D.2.7 of the DCPRA and are therefore not repeated here.

The CCW pumps are tested for auto start on low header pressure at a nominal three year frequency. The CCW pumps and their respective discharge check valves are tested for operability on a quarterly basis. When the CCW heat exchanger outlet valves are tested and the RCPs are running both heat exchangers must be in service (the valve would be closed for less than one minute). Since these tests were not considered as making the system unavailable, there was no contribution due to test included in the system unavailability model. Similarly, following a test, misalignment errors were assumed to be insignificant on the basis that several independent errors would have to be made to make the system unavailable.

Maintenance on the heat exchangers was not included because only one heat exchanger is modelled (no unavailability is incurred if the standby heat exchanger happens to be in maintenance).

The impact of seismic failure is modelled by assuming structural failures in the vital loads (see Loops $A$ and $B$ in Table B4.2.1). 


\section{Appendix B4}

\section{B4.26 Quantincation of the Initiator: LPCC}

The DCPRA modelled and quantified the initiator LPCC as loss of all CCW pumps or loss of two CCW pumps in the event if the third pump would be in maintenance. In the calculation it was assumed that there would be a weekly changeover between the operating and standby pumps. The numerical result of the quantification is indicated at boundary condition $\mathrm{CCI}$ and denoted by "LPCC" in Table B4.2.4. BNL review comments on LPCC are found in Section B4.3.5. 

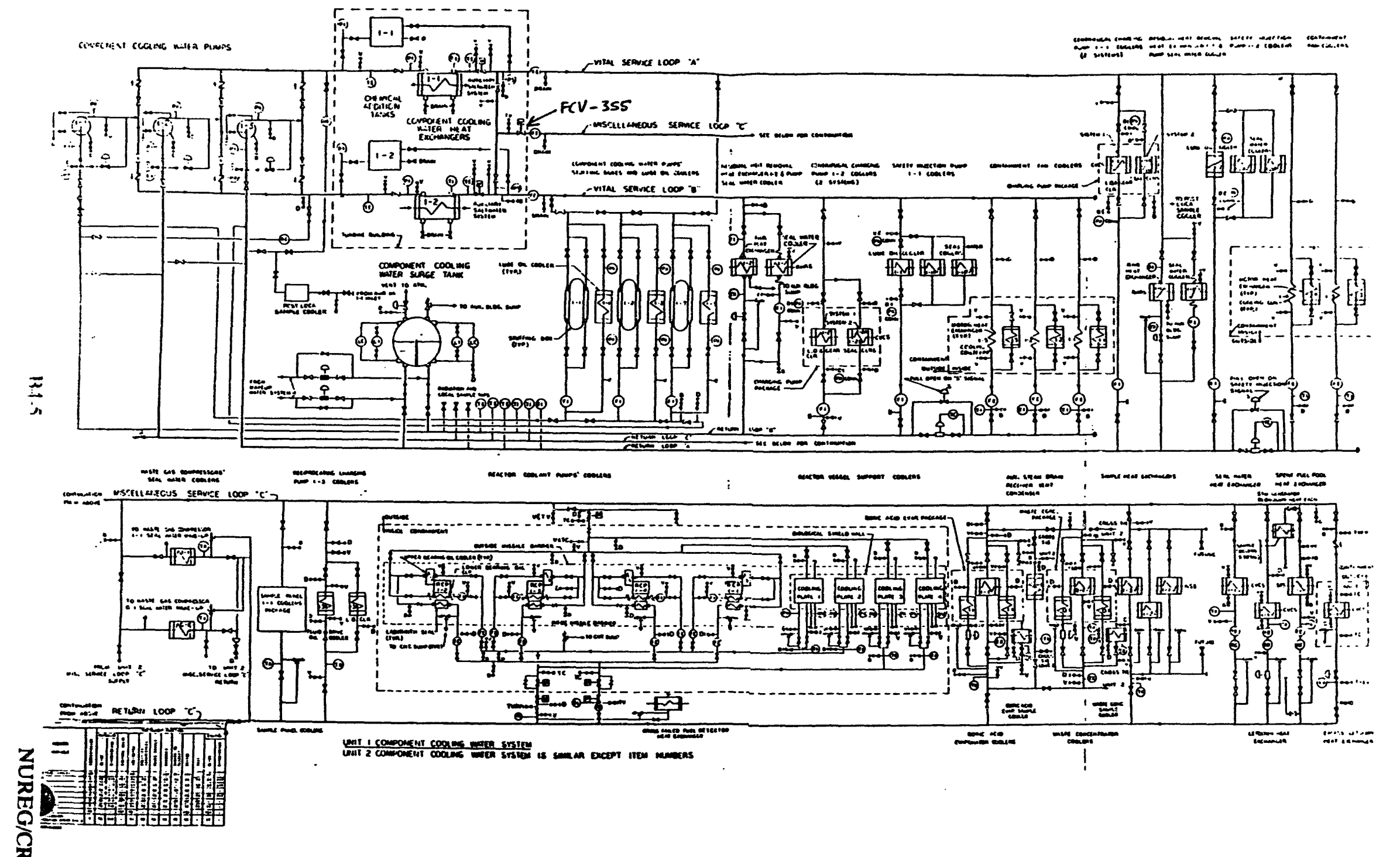

Figure B4.2.1 Component Cooling Water System 
Appendix B4

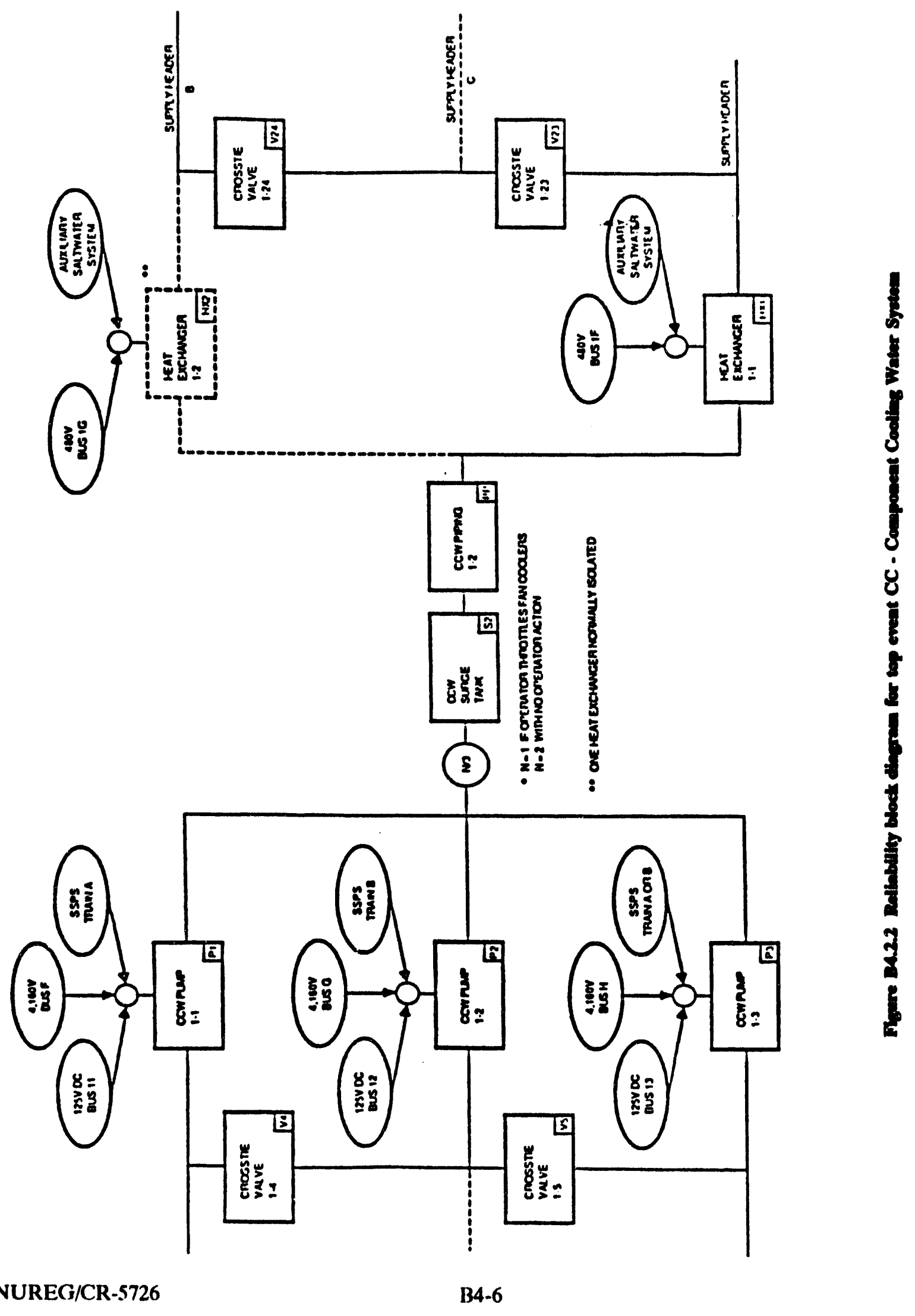


Appendix B4

Table B4.2.1

Component Cooling Water System Loads

Loop A Loop B Loop C

Containment fan coolers

23

Residual heat removal heat exchangers

11

Residual heat removal pump seal water coolers

11

Centrifugal charging pump oil and seal water coolers

11

Safety injection pump oil and seal water coolers

1

Component cooling water pump oil coolers and stuffing boxes

Post-LOCA sampling cooler

Spent fuel pool heat exchanger

Reactor coolant pump

Seal water heat exchanger

Letdown heat exchanger

Excess letdown heat exchanger

NSSS sample heat exchangers

Failed fuel detector heat exchanger

Steam generator blowdown sample heat exchangers

Reactor coolant pump thermal barriers and motor oil coolers

Reciprocating charging pump coolers

Boric acid evaporator condenser, distillate cooler, vent condenser, and sample cooler

Waste concentrator condenser, distillate cooler, vent condenser, and sample cooler

Auxiliary steam drain receiver vent condenser

Waste gas compressors

Reactor vessel support coolers 
Table B4.2.2

Top Event Definitions and Success Criteria

Component Cooling Water System Function

Top Event

Degignator

$\mathrm{CC}$

\section{Top Event Definition}

CCWS provides cooling water to vital components during 24 hours following an initiating event.

\section{Technical Specifications}

\section{LCOs:}

1. May operate 72 hours with one vital loop of $\mathrm{CCW}$ inoperable; if two vital loops are inoperable, must be in at least hot standby within the next six hours and in cold shutdown within the following 30 hours.

2. When ocean water temperature exceeds $64^{\circ} \mathrm{F}$ two $\mathrm{CCW}$ heat exchangers must be in operation within eight hours. 
Table B4.2.3

Boundary Conditions for Top Event, CC

Split Fraction ID

$\mathrm{CC1}$

CC2

$\mathrm{CC3}$

$\mathrm{CC4}$

CC5

CC6

$\mathrm{CC7}$

CCI

CCF
Boundary Condition

All support available (N/3 pumps starts and/or runs).

Loss of $4 \mathrm{kV}$ Bus $\mathrm{H}$ (N/2 pumps runs).

Loss of $4 \mathrm{kV}$ Bus $\mathrm{G}$ (N/2 pumps starts and/or runs).

Loss of $4 k V$ Buses $F$ and $G$ (1/1 pump runs).

Loss of $4 \mathrm{kV}$ Buses $\mathrm{F}$ and $\mathrm{G}$ (1/1 pump starts and runs).

LOSP - All support available (N/ 3 pumps starts and runs).

LOSP - Loss of one $4 \mathrm{kV}$ Buses $\mathrm{F}, \mathrm{G}$, or $\mathrm{H}$ (N/2 pumps starts and runs).

Initiating event frequency (all pumps fail).

Guaranteed failure.

Note: $N=1$ if operator throttles fan coolers.

$\mathrm{N}=2$ if operator fails to throttle fan coolers. 
Table B4.2.4

Unavailability Values (Split Fractions) for the

Component Cooling Water System Function

\begin{tabular}{|l|c|c|c|c|c|c|c|c|c|c|}
\hline $\begin{array}{c}\text { Top } \\
\text { Event }\end{array}$ & Case & Calc. & TTL & HW & HWI & HWD & MN & TS & HE & $\begin{array}{c}\text { Comment } \\
\text { No. }\end{array}$ \\
\hline CC & CC1 & PG\&E & $1.878-5$ & $1.840-5$ & $1.835-5$ & $5.555-8$ & $3.808-7$ & $\ldots .$. & $\ldots$ & \\
\hline & CC2 & PG\&E & $5.689-4$ & $3.981-4$ & $3.978-4$ & $3.547-7$ & $1.708-4$ & $\ldots .$. & $\ldots .$. & \\
\hline & CC3 & PG\&E & $5.849-4$ & $4.141-4$ & $4.137-4$ & $3.547-7$ & $1.708-4$ & $\ldots$ & $\ldots$ & \\
\hline & CC4 & PG\&E & $2.674-2$ & $1.450-3$ & $1.449-3$ & $6.622-7$ & $1.019-2$ & $\ldots .$. & $1.510-2$ & \\
\hline & CC5 & PG\&E & $2.865-2$ & $3.373-3$ & $3.373-3$ & $6.622-7$ & $1.019-2$ & $\ldots$ & $1.510-2$ & \\
\hline & CC6 & PG\&E & $2.431-5$ & $2.255-5$ & $1.955-5$ & $3.003-6$ & $1.763-6$ & $\ldots$ & $\ldots$ & \\
\hline & CC7 & PG\&E & $6.625-4$ & $4.437-4$ & $4.355-4$ & $8.139-6$ & $2.189-4$ & $\ldots$ & $\ldots .-$ & \\
\hline LPCC & CCI & PG\&E & $1.965-4$ & $2.231-4$ & $2.019-4$ & $2.114-5$ & $\ldots$ & $\ldots . .-$ & $8.037-6$ & 1 \\
\hline & CCF & PG\&E & 1.0 & & & & & & & \\
\hline
\end{tabular}

1. The $\mathrm{CCI}$ value is indicated here. $\mathrm{CCl}=.85^{*} \mathrm{TTL}$, where .85 is the capacity factor of Diablo Canyon Unit 1 . 
Appendix B4

\section{B4.3 Results of the BNL Review}

\section{B4.3.1 General}

In spite of the fact that the CC split fractions were not subjects for full quantitative audit calculations, BNL performed a quite thorough qualitative review of the unavailability modelling of this top event. The thorough qualitative review was done because the CCWS is an important support system impacting the safety of the majority of plant operations, including cold shutdown.

Special attention was directed to the determination of the initiator frequency, when the CCWS is completely lost, because the DCPRA uses a "non-plant-specific experience-based"value for this initiator based upon fault tree analysis.

An approach, similar to that applied in reviewing the Auxiliary Saltwater System was used: check the adequacy of the DCPRA modelling for "system-specific" effects derived from applicable experience. For that purpose BNL obtained information from a recent study ${ }^{3}$ investigating the operating experience of the CCWSs at U.S. PWRs. From this study (which is based mainly on analyzing NPRDS events) one can extract information about the nature and the main characteristics of generic CCWS failures. In addition, BNL performed a survey of CCWS failure events at PWRs by using the RECON ${ }^{4}$ data base to obtain information about the characteristics of those events in which the $\mathrm{CCW}$ function was completely lost or had the potential for such a complete loss. After having ascertained the nature and characteristics of these failures, an evaluation was made as to how well the DCPRA model reflected this experience. Finally, an attempt was made to determine a Diablo Canyon-specific "Loss of Component Cooling Water," LOCCW initiator frequency based upon industry experience as a prior and updated accordingly.

\section{B4.3.2 Results of the Survey on the CCW Systems Failures}

The CCWS is a continuously operating system, like the Auxiliary Saltwater System (ASW), but in contrast with the ASW, it is a closed system circulating treated water. Its predominant failure mode was found (based on 1179 NPRDS records by Reference 3) to be: leakage (37\%), associated mainly with both pumps and valve failures. The second failure node (12\%) was found to be: loss of function and failure to meet specification. Valve does not close (9\%), does not open (5\%), incorrect signals (9\%), plugging (4\%), noise/vibration (3\%), short circuit (2\%), and other failures (together $11 \%$ ) were found to represent the other characteristic failure modes of the CCWS. The majority of the CCWS failures resulted in degraded operation of the system or in a loss of redundancy. Valves ( $\sim 53 \%)$, pumps $(\sim 21 \%)$ and load heat exchangers $(\sim 12 \%)$ were found to be the components having most of the failures (roughly reflecting their occurrence frequency in the CCWS design). Pump failures were dominated by seal and bearing failures (resulting in leakage), while valve failures were dominated by valve operator failures and wear of the valve seats.

For the present study the distribution of pump and valve failure modes is very important. These were found to be:

Pump failures: $\quad$ leakage (49\%), fail to run (23\%), vibration (11\%), fail to start (5\%), low output $(4 \%)$, other $(8 \%)$.

Valve failures: $\quad$ leakage (30\%), spurious operation (27\%), fail to open (25\%), fail to close (2\%), other /unknown (16\%). 


\section{Appendix B4}

The description of events (found by the BNL survey) which resulted in or had the potential to result in a complete loss of CCWS is given in Attachment B4.A. Failures which lead to the complete loss of the CCW function typically involved:

a. Loss of an operating CCW heat exchanger while the other was in maintenance. The loss was caused either by a closed outlet valve or loss of shell side (service water) cooling flow (clogging, leak).

The events of the latter type are essentially failure events "linked" with the Service Water System ("linked initiators").

b. Loss of the CCW pumps (in the reported case: due to flooding by human error).

The recovery times of the $\mathrm{CCW}$ failure events (as estimated by the time evolution of the various events) indicate a distribution similar to that of the service water, extending from a representative 1-2 hours to more predictable time periods of a few hours or of even one or more days (fixing corroded CCW heat exchangers).

\section{B43.4 Comments on the CC Top Event Modelling}

The review of the unavailability modelling of the CCWS was based upon an updated version of the DCPRA information ${ }^{5}$ and the information obtained from the CCWS failures described above.

a. The review found that the DCPRA model of the CCW used the same or even lower failure rate values for the continuousty running $\mathrm{CCW}$ pumps as was used for standby pumps (this is against the industry wide experience with the CCWS mentioned in the previous section). Compare, e.g., the pump fails to run failure mode:

S3PCCR, 1 of 3 CCW pumps fail to run: 2.91-5/hour.

While for standby systems:

S2PAMR, 1 of 2 Auxiliary Feedwater pumps fail to run; 2.86-5/hour

S2PCSR, 1 of 2 Spray pumps fail to run; 3.48-5/hour

S2PRHR, 1 of 2 RHR pumps fail to run; 3.11-5/hour

S2PS1R, 1 of 2 SI pumps fail to run; 3.48-5/hour

Similarly:

S3PCCS, 1 of $3 \mathrm{CCW}$ pumps fail to start; 1.76-3/d. This value should be compared with:

S2PAMS, 1 of 2 Auxiliary Feedwater pumps fail to start; 2.04-3/d

S2PCCS, 1 of 2 Spray pumps fail to start; $2.54-3 / \mathrm{d}$

S2PRHR, 1 of 2 RHR pumps fail to start; 2.22-3/d

S2PSIS, 1 of 2 SI pumps fail to start; $2.59-3 / \mathrm{d}$

The CCW pump "fails to start" failure rate itself seems to be rather low, considering that the normal start of the $\mathrm{CCW}$ pumps goes through interlocks which prevent the start of these pumps if the lube oil pressure is below 6 psig. This latter requirement, however, involves the operation of lube oil motors and heat exchangers which themselves are subject to failures.

b. The DCPRA is tacit about the other failure modes of the pumps, such as leakage, vibration, and low (insufficient) output, which are the generic characteristic failure modes of the CCW pumps, mentioned previously. 
c. The DCPRA is also tacit about one of the most important failure modes of the CCW heat exchanger, the clogging of the shell side. This failure has caused most of the industry outages of CCW heat exchangers. The DCPRA simply modelled a CCW system having only a single, maintenance-free CCW heat exchanger with a rarely occurring "tube or shell rupture" failure mode.

\section{D43.5 Determisation of the Initiator Frequency, LOCCW, Based on Induetry Inperience}

In order to avoid the pitfalls of determining the initiator frequency of "Total Loss of Component Cooling Water-LOCCW' events, based on unavailability modelling of the CCWS (i.e., fault trees), an attempt was made by BNL to determine this frequency based on industry experience.

The approach used to obtain a Diablo Canyon-specific value is similar to that applied to obtain an initiator frequency for the Loss of Saltwater System (Appendix B3). Using a double Bayesian updating technique, ${ }^{6}$ a plant- specific posteriur mean frequency was calculated by using the Diablo Canyon experience and a prior distribution consisting of appropriately selected observed LOCCW events, whose potential occurrence was deemed possible at the Diablo Canyon plant.

a. For the updating calculation, events without the non-applicable (N/A) signs in Table B4.A.2 and the reactor years listed in Table B3.A.1 (except those of Diablo Canyon Units 1 and 2) were taken as "experience". For "evidence", zero number of LOCCW events during the operation times of both Diablo Canyon units was taken. By assuming lognormal prior and posterior frequency distributions and by using "best estimate" parameters for the prior in the second stage updating. the obtained Diablo Canyon specific posterior mean, median, standard deviation, 5th and 95th percentile values are given below:

\section{Total Loss of Component Cooline Water, LOCCW*. Events/ny}

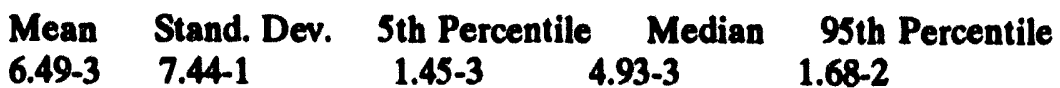

"LOCCW is applied as designator for these events instead of LPCC used in DCPRA. PG\&E considers only the tótal loss of CCW pumps, as the sole originator of these events.

b. To estimate the recovery probability of LOCCW events, all the events listed in Table B4.A.2 were used. Those events which were omitted from the frequency calculations were also included in the sample to represent some fraction of LOCCW events which are non-recoverable within (say) 16 hours.

Figure B4.3.1 shows in semi-logarithmic representation the cumulative distribution of LOCCW events as a function of the time to recover, the best fitting curve, $P(T \geq t)$ (providing the probability that the time to recover a LOCCW event, "T", will be longer than some given time " $t "$ ), as well as the $90 \%$ uncertainty bounds.

The maximum likelihood estimate of the parameter of an exponential recovery probability density function is

$\lambda=\mathrm{N} / \mathbf{2} \mathbf{t}_{\mathbf{i}}=.16 /$ hour, 


\section{Appendix B4}

where $t_{1}, t_{2}, \ldots t_{N}$ represent the individual recovery times in the sample and $1 / \lambda=\mathfrak{t}$ is the mean time to recovery.

Based on an optimistic estimate of the heat capacity of the water available in the CCW system given a LOCCW event ( $t_{1}-1 / 2$ hour) and the time necessary to develop an RCP seal LOCA with appreciable leak rate leading to core uncovery given unavailable cooling $\left(t_{2}-1.5\right.$ hours $)$, the critical time for non-recovery of an LOCCW event was taken to be $t_{1}+t_{2} \sim 2$ hours. At this point in time the probability of non-recovery of an LOCCW event was estimated from the best fitting curve in Figure $B 4.2 .3$ to be $P(T \geq 2)=0.74$. (To apply this non-recovery factor for all of the initiating events is optimistic, because, it is applicable only - strictly speaking - for that fraction of the initiating events when the circulation of the $\mathrm{CCW}$ does not stop. For the other fraction of initiating events, when the CCW circulation stops, a sizeable contribution from RCP seal failure and core uncovery may occur earlier than two hours.)

c. A fraction of the initiating events (see Table B4.A.2) represent "linked" initiators, i.e., events when one of the CCW heat exchangers was in outage and the other heat exchanger was lost because of a failure of its associated service water system train. In the case of Diablo Canyon, this "linked" $(L=1 / 2)$ fraction of the total events has to be multiplied by the conditional probability that given loss of both ASW trains at Unit 1 , the Unit 2 trains also become unavailable: $R_{E}(2 \mid 1)$. The "experience" value for this latter quantity was taken from Table B3.2.4: $R_{E}(2 \mid 1)=.538$.

Thus, the mean initiator frequency of LOCCW events (non-recoverable within some tin. $t$ ) can be calculated by the expression:

$$
\operatorname{LOCCW}(T \geq t)=.85 * P(T \geq t) * \operatorname{LOCCW}_{\text {Menn }} *\left[(1-L)+L * R_{E}(2 \mid 1)\right]
$$

where the quantity .85 is the capacity factor of Diablo Canyon Unit 1 and the other quantities were defined above.

By substituting their numerical values, the mean frequency of LOCCW initiator is:

$$
\operatorname{LOCCW}(T \geq 2)=.85 * .74 * 6.49-3 *\left[1 \frac{1}{2}+1 / 2 * .538\right]=3.14-3 \text { events } / r y
$$

This frequency is one order of magnitude higher than that originally obtained in the DCPRA by fault tree calculation. 


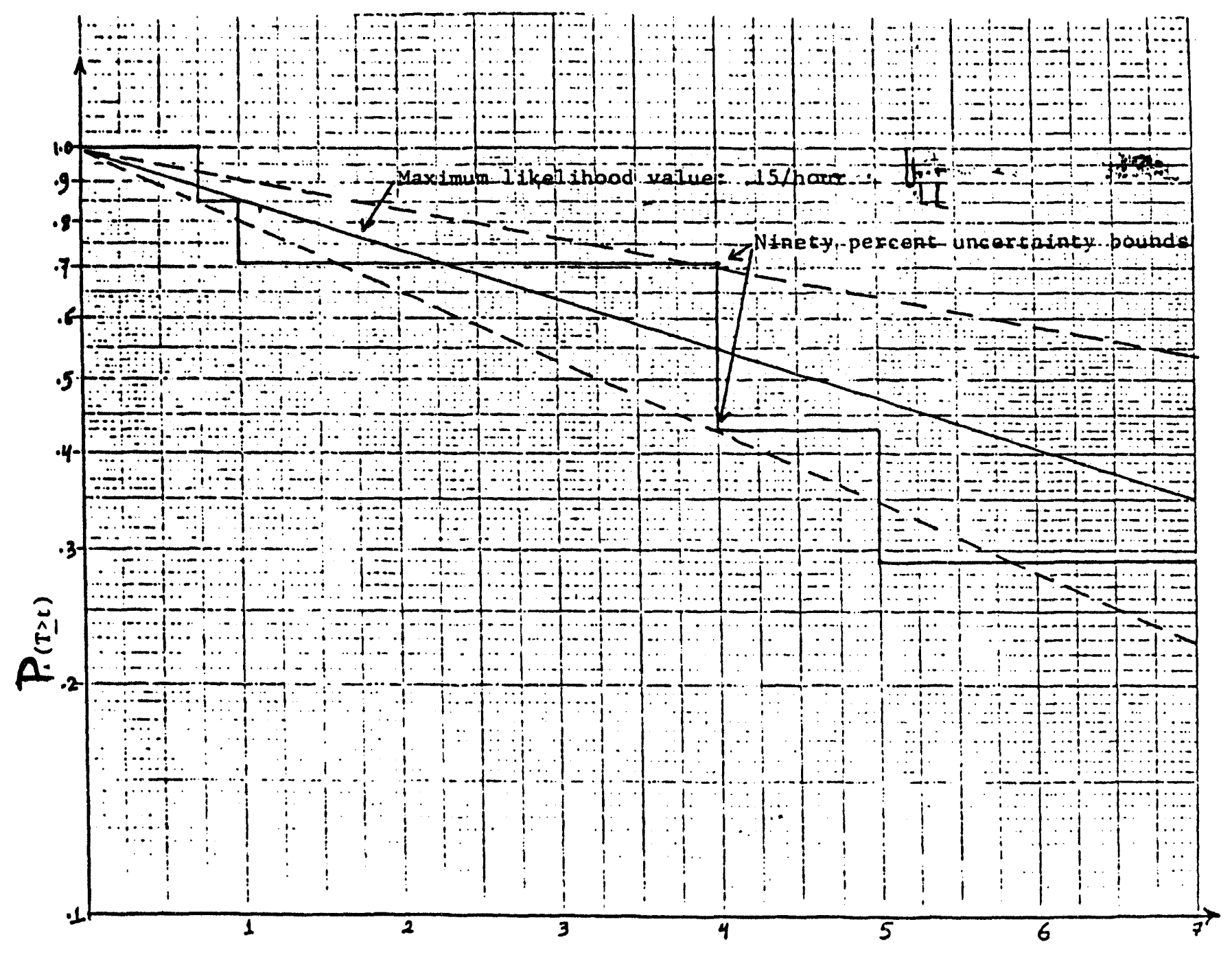

Time to Recover LOCCW (Hours)

Figure B4.3.1 Exponeatial model for nom-recovery of LOCCW events 
Appendix B4

\section{B4.4 Unavailability Analysis of the Equipment Necessary to Maintain Reactor Coolart Pump Seal Cooling, Top Event: SE}

\section{B4.4.1 General}

The unavailability analysis of the equipment necessary to maintain RCP seal cooling is strongly correlated with the analysis of the unavailability of CCWS. Therefore, it was deemed to be appropriate to include it with the review of the CCWS.

\section{B442 Equipment Description, Definition and Boundary Conditions of Top Event, SE}

Top event SE represents the unavailability of the equipment providing cooling to the RCP seals to keep intact their integrity. The equipment consists of seal cooling injection pathways associated with a seal cooling injection source and backup pathways with the RCP thermal barrier cooling acting as a backup cooling source. The seal injection water can be supplied by any one of the three charging pumps. The cooling for the thermal barrier heat exchanger is provided by circulation of $\mathrm{CCW}$. The split fractions for SE are evaluated for both non-seismic and seismic initiating events.

Four different boundary conditions were modelled. They are as follows:

a. For event sequences in which there is a guaranteed chance for success; the split fraction designator is SEO. Its value is:

SEO $=0.0$. (Guaranteed success)

This is assumed to be true if CCW flow to Loops $A, B$, and $C$ is available.

b. If the $\mathrm{CCW}$ is unavailable, the seal cooling can only be recovered by manual operator action (ZHESE1); such as restoring seal injection by providing cooling to the charging pumps (i.e., to the charging pump heat exchangers) from the fire water sprinkler system as described in the plant procedures. The charging pump, otherwise should be operable. The pump suction is from the RWST or from the Volume Control Tank. This means that the success of boundary condition 2 requires the success of top event $\mathrm{CH}$. In addition, it requires the successful trip of the RCPs within five minutes after the loss of CCWS (i.e., it requires the success of top event RP). If the centrifugal charging pumps are not available(quotation from p.6-142 of Ref.1) "a makeshift system for temporary cooling of the positive displacement charging pump (that is, wet rags and portable fans) could also be established, although such actions are not currently covered by procedures." The designator of this split fraction is: SE1. Since the unavailability of seal injection paths and the fire water system is much less than the failure frequency of the operator recovery action only the operator action, was included for non-seismic quantification of this split fraction:

$$
\text { SE1 }=\text { ZHESE } 1=9.907-3 \text {. }
$$

c. When either the CCWS is unavailable for thermal barrier cooling (all support systems are available) or when the CCWS Loop C is isolated (e.g., P-signal occurred due to steam line break inside the containment or power is lost to two vital buses, prompting the operator to isolate Loop 
C) so that thermal barrier cooling, as well as, cooling to the positive displacement charging pump is lost, injection flow still can be maintained by a charging pump (cooling to the charging pumps via CCW Loops A or B is available). The success of this boundary condition requires the success of top event $\mathrm{CH}$ (i.e., the cooling flow from the RWST by an operating charging pump should be available). The designator of the corresponding split fraction is SE2. By the same reason as discussed for boundary condition 2 above, for non-seismic quantification of this split fraction DCPRA used a value of:

$$
\text { SE2 }=0.0 \text {. }
$$

d. Split fraction SEF is used in conditions where seal cooling is guaranteed to be unavailable; e.g., if CCWS Loop C is isolated and the charging pumps fail, or CCWS is unavailable and RCPs are allowed to run. The value of this split fraction is:

$$
\mathrm{SEF}=1.0 \text {. (Guaranteed failure) }
$$

\section{B45 Additional Infornation Requested as Part of the Review}

In order to validate the DCPRA unavailability analysis for the seal injection and the fire water system paths, BNL requested the following:

1. A schematic flow diagram of the present status of the RCP seal cooling design at the Diablo Canyon plant. The flow diagram should indicate the injection and seal leakoff systems, all components (valves, heat exchangers, joints, rubber line sections, etc.) in the pathways which served the basis of the seismic analysis, as well as of the hardware unavailability estimate, that led to the statement used in calculating the split fractions of SE1 and SE2 (that the unavailability of injection path plus charging punap cooling paths and the RCP seals are negligible compared with the human failure probability).

2. Some details of the hardware unavailability calculation of RCP seal equipment: supercomponents and associated failure rates.

3. Frequency of RCP seal LOCA initiator, i.e., its fractional contribution to the small LOCA (isolable and unisolable) initialior frequency.

\section{B46 Comments/Findings}

BNL did not conduct as in depth an audit of this system as those of the previous support systems documented in this appendix. This system wasl originally not on the list of systems to be audited in the review. However, its importance to the overall Diablo Canyon plant led BNL to at least qualitatively examine the system analysis. Investigation of the initiator frequency, LPCC, was included in the original review plan and that helped to tie these aspects of the review together. Section B4.3.4 contains BNL's comments on the systems analysis and it is not felt that they would have a major impact on the core damage frequency. 


\section{Appendix B4}

With respect to the initiator frequency, LPCC, in the DCPRA model, its very definition leaves it lacking. LPCC was limited to loss of the three CCW pumps. BNL belicved a more global loss of CCW initiator was called for and proceeded to develop one. The experiential evidence included in Attachment B4.A was not fully accepted by PG\&E on the basis that certain events did not directly apply to Diablo Canyon. However, PG\&E did update their model/calculations and derived an updated value of LPCC as $2.88-4 / \mathrm{yr}$ which is a $47 \%$ increase over the original value. The following chart is included as an attempt to put this situation into perspective.

$\begin{array}{lllll}\text { Case } & \begin{array}{l}\text { Loss of CCW } \\ \text { (Events/Year) }\end{array} & \begin{array}{l}\text { Unnormalized } \\ \text { Fussel-Vesely } \\ \text { Importance }\end{array} & \begin{array}{l}\text { Non-Seismic } \\ \text { CDF }\end{array} & \% \Delta \text { CDF } \\ \begin{array}{l}\text { PG\&E Original } \\ \text { Value }\end{array} & 1.96-4 & 3.187-6 & 1.7728-4 & \cdots \\ \text { PG\&E Update } & 2.88-4 & 4.684-6 & 1.7877-4 & 0.8 \\ \text { BNL Value } & 3.14-3 & 5.106-5 & 2.2515-4 & 27 \\ \text { "Midway" } & 9.51-4 & 1.547-5 & 1.8955-4 & 6.9\end{array}$

In summary, BNL believes that the PG\&E value is still too low (as discussed previously) and PG\&E believes that BNL's value includes events not applicable to the Diablo Canyon plant. Given the high uncertainty associated with these values, we have attempted to apply an element of expert opinion to derive an additional data point. If one takes the PG\&E updated value as a lower bound and the BNL value as an upper bound and then derives the geometric mean thereof (9.51-4, denoted as "Midway" above) the resulting increase in non-seismic CDF becomes $6.9 \%$.

\section{B4.7 References}

1. Final report on the Diablo Canyon Long-Term Seismic Program, Pacific Gas and Electric Co., Diablo Canyon Power Plant, Docket Nos. 50-275 and 50-323, July 1988.

2. Units 1 and 2 Diablo Canyon Power Plant, "Final Safety Analysis Report Update," Pacific Gas and Electric Co., December 1988.

3. J. Higgins, R. Lofaro, M. Subudhi, R. Fullwood, J. Taylor, "Operating Experience and Aging Assessment of Component Cooling Water Systems in Pressurized Water Reactors," NUREG/CR-5052, July 1988.

4. DOE/RECON, Nuclear Safety Information Center (NSIC), 1963 to present.

5. PG\&E letters to NRC signed by J.D. Shiffer, No. DCL-88-297, December 9, 1988, and No. DCL-89-010, January 16, 1989.

6. C. Park, "Bayes: A Two-Stage Bayesian Update Procedure for Data Specialization for the Plant-Specific Risk and Reliability Analysis," BNL Internal Memorandum, March 19, 1987. 


\section{ATTACHMENT B4.A}

\section{INFORMATION ON FAILURES OF COMPONENT}

\section{COOLING WATER SYSTEMS}


Appendix B4

\section{Attachment B4.A}

\section{Information on Failures of the Component Cooling Water System}

This attachment provides the documentation of information used to determine the frequency of the initiator "Total Loss of Component Cooling Water, LOCCW" for Diablo Canyon Unit 1, based on generic plant experience.

The information includes:

a. Characterization of Component Cooling Water Systems (from Reference 3) for plants which have experienced "Total Loss of CCW" events (Table B4.A.1).

b. A list of failure events obtained by a survey of the RECON ${ }^{4}$ data base, when the CCWS is completely lost due to failures of the system itself or due to linked failures with the Service Water System (Table B4.A.2).

c. A detailed description of the events listed in Table B4.A.2, and of the events that occurred at Surry Units 1 and 2, when the Charging Pump Cooling Water System was lost. The Surry events were omitted from Table B4.A.2, because the Surry units have CCWS of limited design purpose; this system cools only the charging pumps. 
Table BaA.1

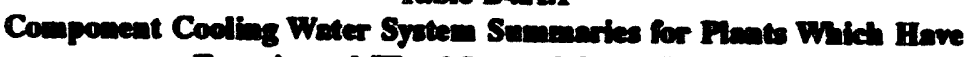

Experieaced Total Lose of CCWs" Events

Pinat,
System Vendor

Salem 2, Westinghouse

Turkey Point 3, Westinghouse

$\stackrel{+}{\stackrel{+}{+}}$

Indian Point 2, Westinghouse

Cakert Cliffs 1 and 2

Combustion Engineering

San Onofre 2 and 3

Combustion Engineering

Surry 1 and 2 Chg.P CW

Westinghouse

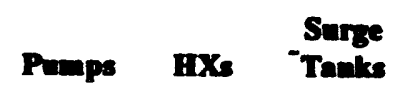

3

2

1

1

RHR HX, RCPm and t.b, LDHX, SWHX, XIDHX, RHR-P, SIP, Chg.-P

3

1

RHRHX, RCPm and t.b, Non-Reg. HX, XLDHX, SWHX, Misc., RHR-P, SI-P, Chg.-P, SFPHX, Cont. CRD ctr

RHX, SFPHX, SWHX, XLDHX, Non-Reg. HX, RHR-P, SI-P, Recirc.-P, Chg.-P, Misc., RCPm and t.b

Comments

No cross connection between Units 1 and 2.

Some cross connection at loads between Unit 3 and 4.

2

SDHX, LPSI-P, HPSI-P, LDHX, Misc., RCPm and SC, CRDM clg (cooling)

SDHX, LDHX, SFPHX, HPSI-P, LPSI-P, CS-P, CCW-P, RCPm and SC, Misc., Cont. Air Circ., CR- Chiller, CEDM cir.

Chg.-P 
Appendix B4

Table B4.A.1 (continued)

Abbreviations

CEDM

Chg.-P

CR

CS

Cont.

CCW

CRDM clg.

CRD chg.

CEDM clr.

HPSI

LPSI

LD

HX

Misc.

Non-Reg.

$\mathbf{P}$

RCPm and t.b

Recirc

RHR

RHX

SC

SDHX

SI

SFP

SWHX

XLD
Control Element Drive Mechanism

Charging Pump

Control Room

Containment Spray Fan Coolers

Containment

Component Cooling Water

Control Rod Drive Mechanism Cooling

Control Rod Drive Cooling

Control Element Drive Mechanism Cooler

High Pressure Safety Injection (CE)

Low Pressure Safety Injection

Letdown

Heat Exchanger

Miscellaneous Loads

Non-Regenerative

Pump

Reactor Coolant Pump Motor and Thermal Barrier

Recirculation

Residual Heat Removal

Residual Heat Exchanger

Seal Cooler

Shutdown Heat Exchanger

Safety Injection

Spend Fuel Pool

Service Water Heat Exchanger

Excess Letdown 
Table B4.A.2

"Total Loss of the Component Cooling Water System" Eveats

\begin{tabular}{|c|c|c|c|c|c|}
\hline Event & Plant & Reference & Recovery & Description & Comments \\
\hline 1,2 & Calvert Ciffs 1,2 & LER-317/84-5 & 16 hrs. & $\begin{array}{l}\text { At Unit } 1, \text { CCHXs } 11 \text { and } 12 \text { outlet channel heads } \\
\text { had three areas with apparent through wall weepage } \\
\text { due to corrosion. Similar failures at Unit } 2 \text { CCHXs } \\
21 \text { and } 22 \text { (CCHXs were made from cast iron). }\end{array}$ & N/A \\
\hline 3 & Salem 2 & LER-311/85-18 & $\geq 4$ hrs. & $\begin{array}{l}\mathrm{CCHX}(\mathrm{No} .22) \text { service water outlet valve failed to } \\
\text { the closed position (vibration caused the valve } \\
\text { actuator to separate from the valve stem). The } \\
\text { redundant CCHX (No.21) was in maintenance. }\end{array}$ & \\
\hline 4 & San Onofre 2 & LER-361/84-46 & $1 \mathrm{hr}$. & $\begin{array}{l}\text { Train "A" CCWHX was out of service for cleaning. } \\
\text { Train "B" CCWHX indicated fault condition (high } \\
\text { differential pressure). }\end{array}$ & \\
\hline 5 & Indian Point 2 & LER-247/84-011 & See notes & $\begin{array}{l}\text { While at cold shutdown for a refueling outage, all } \\
\text { component cooling pumps were disabled due to } \\
\text { looding of the pump compartment. Water entered } \\
\text { the compartment through an opening in the service } \\
\text { water piping after a valve had been removed for } \\
\text { maintenance. }\end{array}$ & $\bullet$ \\
\hline 6 & Turkey Point 3 & LER-250/86-18 & 5 hrs.* & $\begin{array}{l}\text { CCWHX "B" was taken out of service for cleaning. } \\
\text { Subsequently intake cooling water inlet temperature } \\
\text { increased such that the three CCWHXs were } \\
\text { required to be in service. }\end{array}$ & N/A \\
\hline
\end{tabular}


Table BAM22 (conotinand)

\begin{tabular}{|c|c|c|c|c|c|}
\hline Eveat & Plant & Reference & Recovery & Deecription & Conments. \\
\hline 7 & San Onofre 3 & LER-362/86-11 & $3 / 4 \mathrm{hr}$. & $\begin{array}{l}\text { CCWHX "B" was taken out of service for cleaning. } \\
\text { The salt water flow through CCWHX "A" was } \\
\text { blocked due to fouling with marine growth. }\end{array}$ & Linked initiator \\
\hline \multicolumn{6}{|l|}{ Notes: } \\
\hline \multicolumn{6}{|c|}{ - 2nd motor recovery: $4 \frac{1}{4}$ hours. } \\
\hline \multicolumn{6}{|c|}{ - 3rd motor recovery: $6 \% / 4$ hours. } \\
\hline \multicolumn{6}{|c|}{ Comments: } \\
\hline $\begin{aligned} \mathrm{N} / \mathrm{A} & = \\
& =\end{aligned}$ & $\begin{array}{l}\text { ot applicable at } \\
\text { timated. }\end{array}$ & & & & \\
\hline
\end{tabular}




\section{Calvert Cliffs 1 LER-317/1984-005}

Power level $-100 \%$ on $5 / 2 / 84$, during Unit 2 refueling outage a through wall hole occurred during removal of the graphite layer on one \#22 component cooling heat exchanger ( $\mathrm{CCHX}$ ) channel head. The graphite layer was being removed in preparation for coal tar epoxy application. On 5/3/84, a second hole was created during graphite removal, prompting notification to the Nuclenr Regulatory Commission. A visual examination was subsequently conducted on the operating \#11, \#12, and \#21 (CCHX) and service water heat exchanger (SRW HX) channel heads. The \#11 and \#12 C.CHX outlet channel heads had three areas with apparent through wall weepage. On 5/6/84, Unit 1 shutdown and all Unit 1 and Unit 2 CCHX and SRW HX were opened as conditions permitted. Due to the size, location, and number of helow minimum wall areas found on the channel heads, several repairs were pursued. Encapsulations were installed on \#12 and \#22 CCHX channel heads, while new channel heads were installed on \#11 and \#21 CCHX. Bolted plate patches were installed on \#12 and \#22 SRW HX to correct the deficiencies. Numbers 11 and 21 SRW HX did not need any repairs. However, all CCHX and SRW HX channel heads were coated with coal tar epoxy to prevent future corrosion. New channel heads for all CCHX and SRW HX will be installed during the next outage of sufficient duration. An expanded surveillance program for cast iron components in the salt water system is being developed.

\section{Salem 2 LER-311/1985-018}

Power level - $100 \%$ on $8 / 27 / 85$, No.22 component cooling water heat exchanger (CCHX) service water outlet valve (22SW356) failed to the closed position. Attempts to jack the valve open failed to adequately restore service water flow to the heat exchanger. Because the redundant CCHX (No.21) was out of service for maintenance at the time, Tech. Spec. 3.0.3 was entered, and a controlled shutdown was initiated. The malfunction of $22 S W 356$ was attributed to a vibration induced failure which caused the valve actuator to separate from the valve stem. The vibration resulted from the prior removal of the cavitrol tube bundle from No.22 CCHX service water control valve (22SW 127), due to plugging and deterioration. Investigation revealed that operation with this tube bundle removed has caused turbulence downstream of the control valve. Due to the close proximity of this valve to the heat exchanger outlet valve (22SW356). The turbulence caused $22 S W 356$ to vibrate which resulted in actuator damage. The valve actuator was replaced, tested and No.22 $\mathrm{CCHX}$ restored to an operable status. A new cavitrol tube hundle for $22 \mathrm{SW} 127$ is presently scheduled for delivery by 11/85. Replacement of this component should alleviate the vibration problem associated with $22 S W 356$.

\section{San Onofre 2 LER-361/1984-046}

Power level - 100\%. On 8/15/84, at 1130, with both Units 2 and 3 in Mode 1 the local readout for salt water cooling flow to the train "B" component cooling water (CCW) heat exchanger indicated a fault condition. The train 'A' CCW heat exchanger indicated a fault condition. The train 'A' C.W heat exchanger was out of service for cleaning. Because a high differential pressure existed across the train ' $B$ ' CCW heat exchanger, it was conservatively assumed that train ' $B$ ' saltwater cooling flow was less than the flow required for system operability. Train 'B' CCW was declared inoperable, and LCO 3.0.3 was invoked on Unit 2. Emergency chiller E-335 was declared inoperable since train 'B' CCW was supplying its cooling water. Loss of E-335 renders two vital inverters inoperable in each unit, and LCO 3.0.3 was also invoked for Unit 3. Shutdown of both units was initiated. A train 'B' salt water cooling pump in the Unit 2 intake was started, and the differential pressure across the heat exchanger decreased. At 12.30 the salt water cooling flow indication was restored. Salt water cooling flow was determined to be above the minimum required flow, and LCO 3.0 .3 was 


\section{Appendix B4}

exited. The salt water cooling flow indication was restored by switching readout channels. It is suspected that the initial fault indication was due to reading an inoperable channel.

\section{Indian Point 2 LER-247/84-011}

Date of event - 081384. Power level - 100\%. On August 13,1984, at 1050, while at cold shutdown for a refueling maintenance outage, two operating component cooling water pumps and subsequently the standhy pump automatically tripped on receipt of an over current protection signal. The over current condition was caused by wetting of the component cooling water pump motors with service water. Leakage through a service water valve permitted service water flow into the component cooling water pump compartment through an opening in the service water piping being prepared for a test. The central control room was promptly notified of water conditions in the compartment and the CCR operators immediately secured the operating service water pumps which stopped the flow. The water was pumped and drained from the compartment. No.21 component cooling pump was flushed with fresh water, dried and returned to service at 1344 .

\section{Turkey Point 3 LER-250/1986-018}

Power level - $100 \%$. While Unit 3 was at $100 \%$ power, a unit shutdown was commenced due to exceeding administrative guidelines for intake cooling water (ICW) system operation. These guidelines were established by engineering based on a postulated failure of temperature control valve, TCV-2201 during a design basis event, which could degrade the ability to provide the required ICW system flow through the CCW heat exchangers during a design basis event. These guidelines establish limits on IC W inlet temperature, component cooling water (CCW) heat exchanger cleanliness and lineup, and ICW flow raltes for unit operation. On April 16, 1986, the 3B CCW heat exchanger was taken out of service for cleaning. Plant conditions were evaluated at that time and it was determined that the administrative guidelines were met. Subsequently, ICW inlet temperatures increased such that three CCW heal exchangers were required to be in service for Unit 3 operation. At that time, the $3 \mathrm{~B}$ C.CW heat exchanger was still out of service which placed Unit 3 outside of the administrative guidelines requiring a Unit 3 shuldown. A Unit 3 shutdown was commenced and was stopped when the $3 B$ CCW heat exchanger was placed back in service. Cause of event: while the 3B CCW heat exchanger was being cleaned, ICW inlet temperatures increased such that three CC $\mathrm{W}$ heat exchangers were required by the special administrative guidelines, to be in service for Unit 3 operation.

\section{San Onofre 3 LER-362/1986-011}

Power level $-100 \%$ at 1550 on August 4, 1986, saltwater cooling (SWC) flow through train a component cooling water heat exchanger (CCWHX) decreased, due to fouling with marine growth, to below the postulated design basis flow rate required for removal of CCW heat loads (critical C.CW loop), and was therefore declared inoperable. At this time Train B CCWHX was operating with reverse SWC flow to remove similar fouling which had previously taken place. At 1605 , operators commenced realignment of Train B CCWHX SWC flow to the normal direction in order to return one train of CCW to its design configuration and thereby increa: : heat removal capability of that train. During the realignment, both trains of the SWC system were considered to he inoperable contrary to technical specification limiting condition for operation (LCO) 3.7.4, and LCO 3.0.3 was entered. Train B SWC system was returned to operable status within thirty minutes, and at 1635, LCO 3.0 .3 was exited. As corrective action, operating procedures will he revised to minimize the effect of marine fouling on the operability of the SWC systent. 


\section{SUIrY L LER-28:0/84-011}

Power level - 100\%. On 5/18/84 operations personnel performing a system walkdown following maintenance discovered the intended heat sink for the charging pump component cooling water system was isolated. The charging pump component cooling water isolated to intermediate seal cooler 1-SW-E-1B and service water isolated to intermediate seal cooler 1-SW-E-1A. A review of plant logs and operator interviews has confirmed that both intermedinte seal coolers were isolated during two separate events. The first event started $5 / 16$ at 2045 hours, when 'B' cooler was improperly placed in service and ' $A$ ' cooler was removed from service until 2125. The second event started 2140 hours on the same day when ' $A$ ' cooler was again removed from service with 'B' cooler remaining improperly valved in service. Both coolers were isolated for a total of 40 minutes during the first event and 32 hours for the second event. Due to the complexity of the maintenance involved, the existing procedures were not adequate to provide the necessary valve alignments. Immediate corrective actions involved placing ' $B$ ' intermediate senl cooler in service to provide a necessary heat sink and making ' $A$ ' cooler available as a backup. To prevent recurrence, maintenance operating procedures were written to ensure control of removal and return to service of the intermediate seal coolers.

\section{Surry 2 LER-281/86-010}

Power level $100 \%$. On 7/11/86 with Unit 1 in refueling shutdown and Unit 2 at 100\% power, operators were attempting to return the ' $A$ ' charging pump component cooling water pump to service following emergency maintenance. At 15.18 hours, the redundant ' $B$ ' pump, which had heen supplying cooling water to the charging pump seal coolers, lost discharge pressure. This resulted in hoth pumps heing inoperable. It is assumed that air introduced into the system during maintenance on the ' $A$ ' pump caused the ' $B$ ' pump to become vapor bound. The ' $A$ ' pump was vented, water was added to the system, and the pump was returned to service at $\mathbf{1 8 . 2 5}$ hours. Subsequently, operability of the 'B' pump was demonstrated, and it was also returned to service.

\section{Surry 2 LER-281/188-009}

Power level - 100\%. On April 20, 1988 at 12.27 hours, with Unit 1 in a refueling outage and Unit 2 at 100\% reactor power, the "A" and "B" component cooling water (EIIS-CC) heat exchangers (EIIS-HX) (CCHX) were declared inoperable. The " $C$ " and "D" CCHXS had previously heen removed from service for maintenance. Unit 2 entered a six hour clock to hot shutdown in accordance with technical specifications 3.0.1. An engineering review of the potential dependence of CCHXS on the station vacuum priming (VP) system had determined that five VP valves (EIIS-ISV) which isolate the CCHXS service water (SW) piping from the VP lines, were not seismically mounted. In a seismic event, the potential would have existed for rupture of the VP piping and air ingress into the CCHX, breaking the siphon effect on the SW, and causing a loss of SW to the heat exchangers. The seismic supports were installed and the " $A$ " and "B" CCHXS were returned to service at 17.10 hours on April 20,1988 and T.S. 3.0.1 was exited. Additional engineering evaluations will be performed to assess the necessary design changes to permit future $\mathrm{CCHX}$ operation with vacuum priming in service. The VP valves will remain normally closed pending the results of the engineering evaluations. 


\section{APPENDIX BS: SOLID STATE PROTECTION/REACTOR PROTECTION SYSTEMS}

\section{B5.1 Introduction}

\section{B5.1.1 Objecthes and Background}

One of the main tasks to be performed within the framework of reviewing the DCPRA outlined in BNL's approsech to the DCPRA review (see Section 3.2), was to scrutinize the unavailability annlyses of several selected support and frontline systems. The ohjective of this appendix is to provide the results of reviewing the unavailability analyses of the Solid State Protection System (SSPS) and the Reactor Protection System (RPS). According to the DCPRA, the SSPS and RPS represent the only non-plant-specific (i.e., generic) gystems ausalyzod in the PRA. The systems were provided by Westinghouse and helong to designs of fairly recent vintage.

The generic review methodology described in the PRA Review Manual' suggests that a comparison be made between the results obtained for the unavailabilities of systems of these types in a PRA under review and the results obtained in unavailability studies (if they exist) dedicated to "generic" systems. Recently, the Westinghouse Electric Corporation conducted very comprehensive unavailability studies on the Solid State Protection ${ }^{2}$ (from now on to be referred to as "WOGI") and Reactor Protection Systems ${ }^{3}$ (from now on to be referred to as "WOG2") on behalf of the Westinghouse Owners Group (WOG). The studies served as bases for requesting certain changes in the surveillance requirements of the Technical Specifications for these systems from the NRC. Both studies were reviewed hy BNL. BNL conducted a thorough audit calculation for the SSPS" (to be referred to as "BNLl") and a time-dependent Markovian analysis for the RPS" (to be referred to as "BNL2").

Therefore, for a comparative unavailability analysis of the DCPRA models and results, the WOG/BNL models were selected as bases. In order to render these models comparable to the conditions and assumptions used in the DCPRA, both models (WOG and BNL) were modified accordingly prior to the comparative analysis.

\section{B5.12 Organization}

This appendix documents the results of the comparative analyses noted above and is organized as follows: Section BS.2 describes the SSPS and its testing provisions/methods. Section B5.3 presents the comparison of the approaches used in the DCPRA and in the WOG/BNL calculations to model the SSPS and the results obtained. Section B5.4 discusses the RPS, its testing methods and the results of those comparative analyses.

\section{B5.2 Solid State Protection System}

\section{B5.2.1 Syatem Description}

The Solid State Protection System provides actuation signals to emergency safeguard equipment and to the reactor protection system when process and nuclear parameters exceed certain preset limits ensuring that safe operating conditions exist at all times. 


\section{Appendix BS}

The main components of the SSPS arc:

1. the analog channels,

2. the combinational logic units, and

3. the actuation relays.

\section{B5.2.1.1 Analog Channels}

An analog channel involves: an analog sensing device (sensor/ transmilter), a loop power supply, a signal conditioning circuit, and a signal comparator. The sensing device monitors a given process or nuclear parameter, such as pressure, level, flow, temperature or flux, etc. The parameter signals are converted to proportional voltage signals hy the power supply of the loop (Figure B5.2.1). The sensed signal is "shaped" by the signal conditioning circuit (signal modifiers). The shaped signal is compared with a preset parameter value by the comparator (bistable). The comparator controls two output relays; one of them provides input signals to the combinational logic train A and the other to combinational logic train $B$.

\subsection{Combinational Logic Unit and Master Relays}

The combinational logic unit is a dual train electronic system. Trains $A$ and $B$ contain several $2 / 4,2 / 3$, and $1 / 2$ logic circuits built on universal logic (UL) cards. The analog channel output relays operate grounding contacts at the inputs of the combinational trains. A trip signal is generated in each of the trains if an appropriate number of card inputs are grounded. Outputs of various logic circuits in each of the trains can be further interconnected by using additional logic circuits to achieve desired reactor trip and safeguard initiator signal combinations. The safeguard initiator signals drive the master relays by creating a current flow which energizes them. The block diagram of a typical SSPS is shown in Figure B5.2.2.

\subsubsection{Slave Relaye}

Given an initiator signal, the energized master relays close contacts in the slave relay circuits and energize master relays close contacts in the slave relay circuits and energize the associated slave relays. The slave relays activate the safety systems by energizing contacts in motor starters, solenoid circuits, etc. Usually each slave relay activates several safety system components. The number of master and slave relays energized is dependent upon the complexity of a given protective function required by a specific initiating event. The SSPS trains are train oriented: ESFAS train A energizes train $A$ of a safety system, etc.

Figure B5.2.3 shows the schematics of slave relay arrangements. Figure B5.2.4 presents the parameter signals and the master and slave relay arrangements modelled in the DCPRA which generate actuation signals for various safety functions.

\section{B5.2.2 Testing of the ESFAS}

\section{B522.1 Teating of the Analog Channels}

The functional testing of the analog channels is performed at power. Its purpose is to verify the entire operation of the channel excluding the sensor. Calibration and verification of proper operation of the sensors (the associated electronics included) is usually performed at shutdown. The functional testing scheme of the 
analog channels for the SSPS is also shown in Figure B5.2.1. The sensor is disconnected during testing. By using test jacks, test signals are sent through the circuit. A proving lamp is connected to the output of the bistable; usually the bistable is adjusted to ensure that the whole channel performs as required. The input relays of the logic trains are energized from outside circuits if the channel is tested in bypass. The input relays are de-energized if the channel is tested in trip.

During normal operation, a failure of a sensor or a loop power supply would cause abnormal indication and/or alarms. The status lights are checked by operators every shift, therefore, an analog channel failure is detectable within eight hours.

\section{E52.22 Teatiog of the Combinational Logic Units}

While a plant is at power, each of the combinational logic trains (located in separate cabinets) is allowed to be tested or maintained separately in "bypass" condition. Time sequenced pulses are applied to the logic circuits through switches located on a logic test panel dedicated to each train (semi-automatic tester). The pulses check the logic, but are of such a short duration that slave relay (or trip breaker) actuation is not possible. The semi-automatic tester allows quick and efficient testing of all the possible logic combinations of actuate or non-actuate conditions as well as the effects of the permissives. If one train is in test or in maintenance, the other is charged with providing all the safety function signals. It is not possible to lock out both logic trains without tripping the reactor. The tests of the combinational logic trains are performed according to a staggered testing schedule.

\section{BS.2.23 Teding of the Actuation Relays}

The master relays are "continuity" tested as part of the logic test to demonstrate total circuit operation. The master relays are actuated during master relay testing and proper contact operation is checked. Figure B5.2.3 also shows the test conditions for the actuation relays. Proper contact operation is verified by "continuity" checking of the associated slave relay. This test is performed by applying a voltage to the master relay contact which demonstrates the continuity but which is insufficieni to activate the slave relay.

The "actuation" test of a slave relay is performed individually by energizing the relay and demonstrating proper contact operation. Proper contact operation can be demonstrated with or without operating the associated equipment. The slave relay test sometimes requires the reconfiguration of the equipment to be tested in such a way that the test would not cause adverse effects on the plant operation. After the test, the equipment has to be returned to its normal operating configuration. Therefore, associated with each slave relay test there is also a potential for human error in that the personnel conducting the test could fail to return the equipment to its proper operating configuration. At Diablo Canyon the test of the slave relays is performed at shutdown. (This condition, therefore, was considered in the modified WOG/BNL modelling.) 
Appendix BS

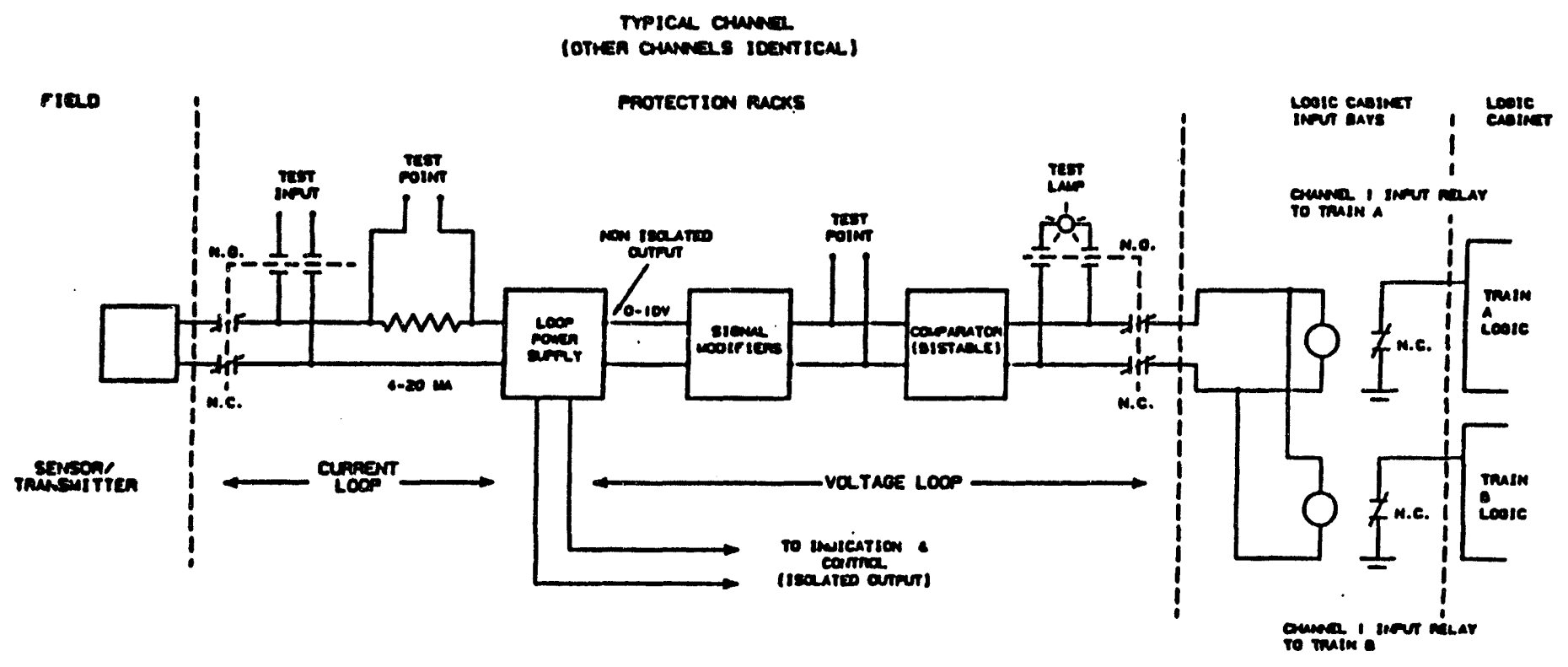

Figure B5.2.1 Analog channel block diagram 


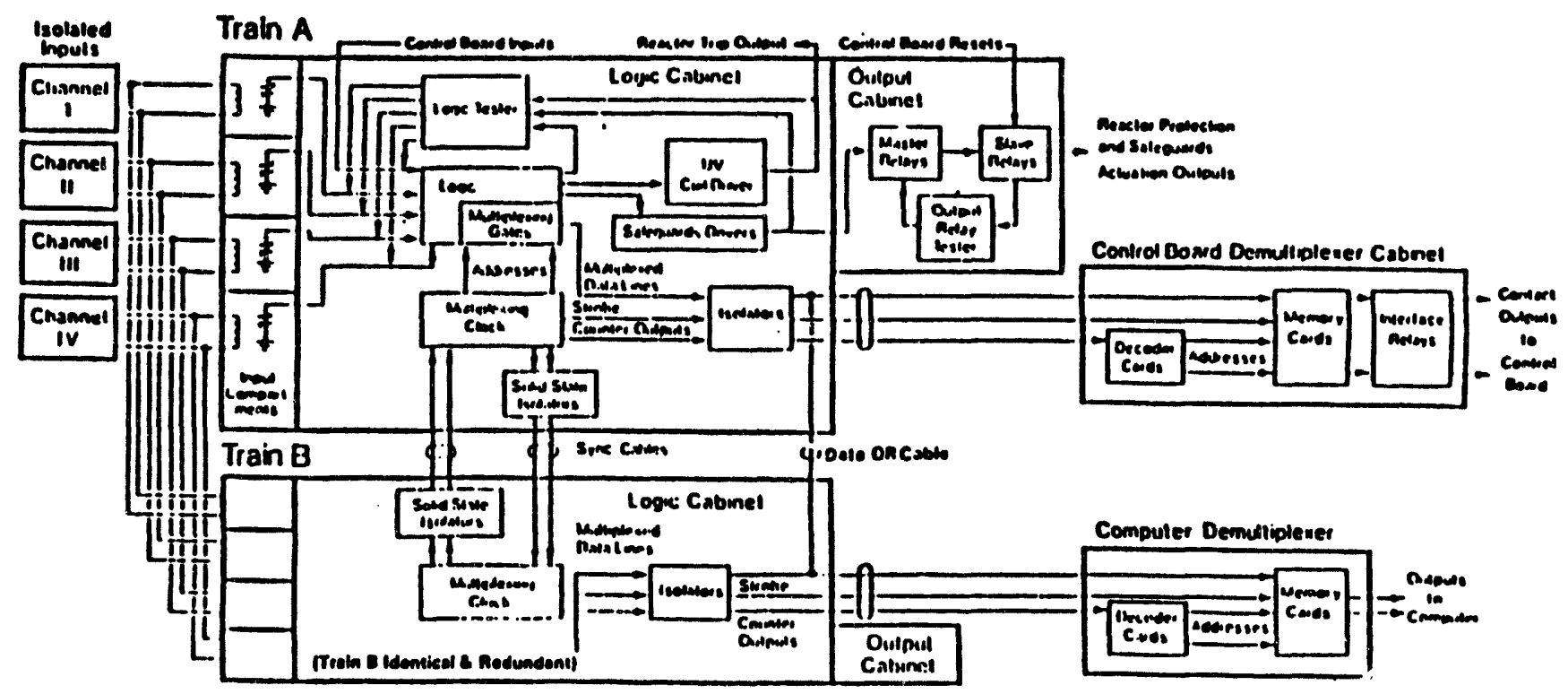

Figure BS.2.2 Block diagram of a typical Solid State Protection System 
Appendix B5

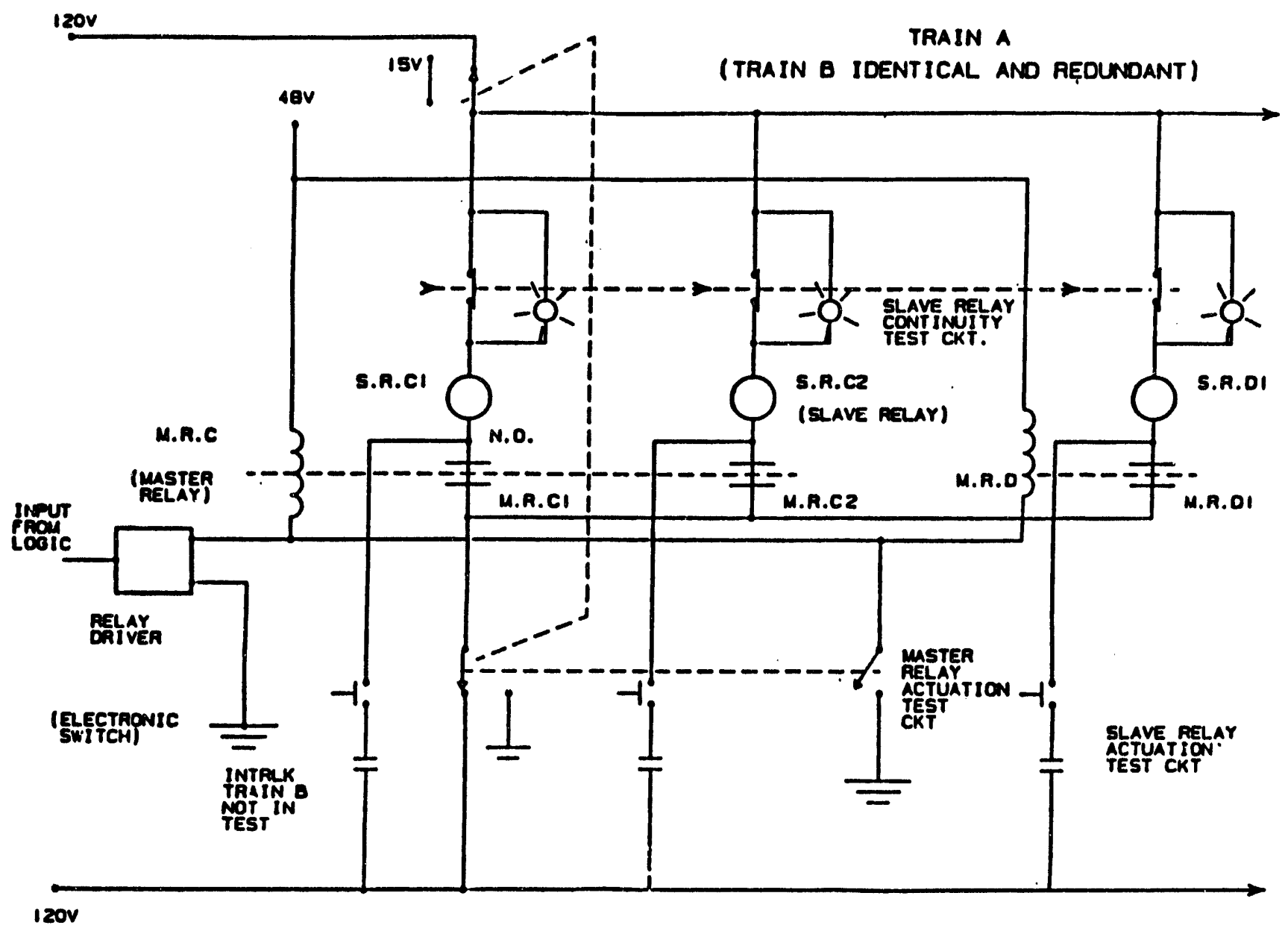

Figure B5.2.3 Schematic of actuation relays 


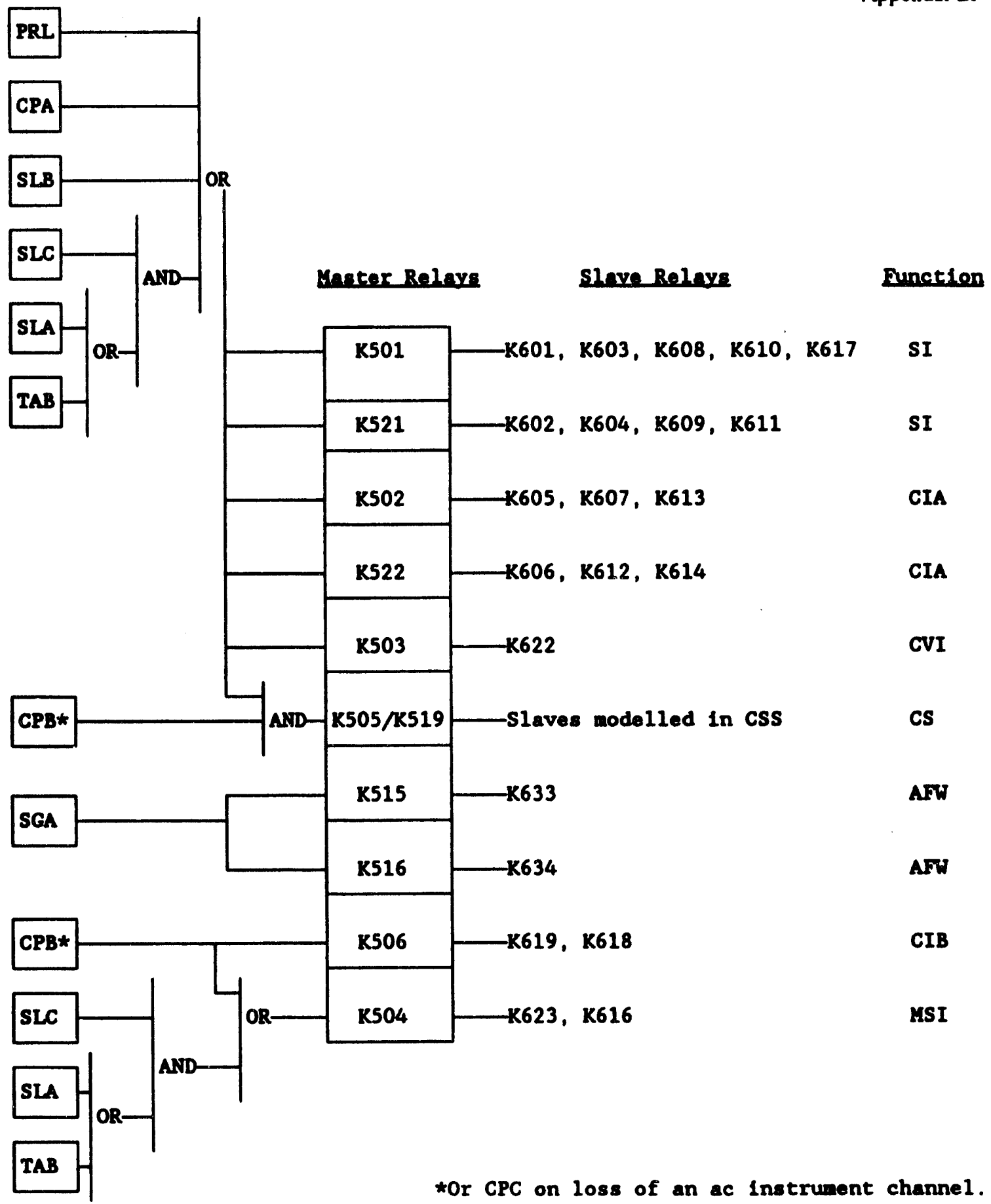

Figure B5.2.4 SSPS block logic. 


\section{Logic}

\section{CSS Containment Spray System}

CPA Containment Pressure High

$2 / 3$

CPB Containment Pressure High-High

$2 / 4$

SGA Steam Generator Level Low-Low

$2 / 3$ per $S G$ (for $1 / 4 \mathrm{SG}$ )

PRL Pressurizer Pressure Low

$2 / 4$

SLA Streamline Pressure Low

$1 / 1$ per loop for $2 / 4$ loops

SLB Streamline Differential Pressure High

$2 / 3$ per loop for $1 / 4$ loops

SLC Streamline Flow High

$1 / 2$ per loop for $2 / 4$ loops

TAB Low-Low Tang

AFW Auxiliary Feedwater Startup $1 / 1$ per loop for $2 / 4$ loops

SI Safety Injection and Associated Actions

CIA Containment Isolation, Phase A

CIB Containment Isolation, Phase B

CVI Containment Vent Isolation

CS Containment Spray

MSI Main Streamline Isolation 
Appendix B5

\section{B5.3 Comparison of SSP Systems Analysis of DCPRA with that of WOG/BNL}

In this section the SSP systems analysis is compared with that of WOG/BNL. Only those aspects of both approaches are discussed which are deemed to be relevant for clear understanding of the differences.

\section{B5.3.1 Unavailability Modelling of the SSPS Signals in DCPRA}

In the DCPRA six classes of initiating events were selected for which the unavailabilities of the SSPS were modelled. This selection was based on a unique set of safety functions required to be actuated by the SSPS given any type of initiating events. Table B5.3.1 lists the modelled initiating event classes with the required safety functions to be actuated. The table also lists the (minimum) number of master and slave relays per SSPS train which are involved in generating the appropriate safety system responses. The success criterion of the SSPS is: at least one of the two trains must produce an actuation signal for all necessary safety functions; that is; each slave relay (appropriately identified in the DCPRA) must produce actuation signals in at least one SSPS train.

Notice, this DCPRA success criterion is conservative, because it lumps together the success of the diverse safety functions. If any one required safety function fails, all the diverse functions are also assumed to be lost. (This conservatism also exists at the train level: if one safety function on train $A$ is lost, all train $A$ actuation signals are assumed lost.)

Figure B5.3.1 shows, as an example, the master fault tree for a class of initiating events: steam generator tube rupture. Given this initiator, the model assumes a diversity of parameter signal failures which may contribute to the failure of an SSPS train (see the SSPS block diagram on Figure B5.2.4). These are: Pressurizer Low Pressure (2/4), Steam Generator Low-Low Level (2/3 on 2/4 SG), High Steam Flow (2/4), or the combined T Average Low-Low (2/4), and Low Streamline Pressure (2/4) signals. In addition, the whole train is assumed to fail if either the power supply or the logic or any of the master or slave relays (in this case 8 master relays and 20 slave relays) fail.

On the component level, the model includes the failures of the bistables, the input relays, the signal transmitters, the failures of the input, master and slave relays, as well as the power supplies converting instrument ac to 48 and $15 \mathrm{~V}$ dc. The failure of the logic card is not modelled in terms of the components, it is characterized by a single overall failure rate.

The model assumes common cause failures between bistables and input relays for a particular function (there is no assumption for overall failure of the sensor signals). Common cause failures for master and slave relays are modelled for all two-member cutsets based on two or more failures out of the total number of relays (11 master and 22 slave relays). Common cause failure is also considered between the logic cards.

The DCPRA includes the unavailability contribution due to surveillance performed during power operations. This unavailability contribution is considered only for the analog channels and logic cabinets. Table B5.3.2 presents the relevant data (and their designators). The only maintenance event modelled in the DCPRA is the repair of randomly failing power supplies. The relevant information is also given in Table B5.3.2. Human error is modelled only for miscalibration of analog channels. 


\section{Appendix B5}

The effects of the unavailability of the ac instrument channels are included in the various boundary conditions, for which the various fault trees (corresponding to the initiating events) were evaluated. Table B5.3.3 reproduces the basic SSPS signal unavailabilities (split fractions) of a single train and of the whole system for various boundary conditions. The table separately shows the total unavailability, as well as the unavailability contributions due to independent and dependent hardware failures, test, maintenance, and human errors.

According to this model the leading contributor to a single train unavailability is independent hardware failures $(\sim 80 \%)$. The leading contributors to total systems failure are: the human errors of miscalibration and dependent hardware failures.

\section{B5.3.2 Unavailability Modelling of SSPS Signals by the WOG/BNL Approach}

In the WOG/BNL modelling of the SSPS (WOGI ${ }^{2}$ and $B N L 1^{4}$ ) the unavailabilities of the various safety function actuation signals are not lumped together, but rather are individually calculated. Table B5.3.4 lists the various safety function actuation signals considered in the analyses. The number of master and slave relays per train involved in each of these safety function actuation signals are also given in the table. The success criterion of the system is similar to that used in the DCPRA: each slave relay must produce actuation signals in at least one SSPS train.

The fault tree model of each safety function actuation signal was evaluated for various process parameter signals and logic. Table B5.3.5 presents a subset of those safety function actuation signals which were selected according to their relevance to Diablo Canyon.

The basic structure of the fault tree models for the various safety function actuation signals is somewhat similar to that of the DCPRA. However, in contrast with the DCPRA models, the diversity of process parameter signals was reduced (in most cases only one type of process parameter signal was assumed). On the other hand, the modelling of the permissives, which was neglected in the DCPRA, was consider ed.

The detailed fault trees are rather intricate and complex. The level of detail is shown to minute electronic parts, therefore, they are not shown here. They can be found in Appendix C of WOG1.2 The fault trees usually consist of three parts: a top fault tree, one or more middle fault trees, and the analog channel fault trees. The top fault tree describes the master and slave relays. The middle fault trees describe the master relay drivers and the logic cards including the permissive circuits. The analog channel fault trees describe the sensors, the power supply, the signal conditioning and signal comparator circuits. The rates of various failure modes of the components were taken from the Westinghouse data base, Military Handhook 217C, and IEEE 500.

Common cause failures were modelled for the analog channels, the logic cabinets and the master and slave relays. For the analog channels the Atwood/Binomial failure rate method was used. For the logic cabinets as well as the master and slave relays the beta factor method was applied. Human errors such as miscalibration or misposition of sensors, amplifiers, etc., were considered only in the analog channel fault trees, hy using the guidelines of Swain's Human Reliability Handbook.

The modelled surveillance conditions are given in Table B5.3.2. This table also shows the maintenance conditions considered in the WOG1/BNL1 calculations. A comparison with the conditions used in the DCPRA shows two minor differences: 
a. The WOGI/BNLI falculations consider the unavailability contribution of the master relays due to test.

b. In addition, they assume once/year maintenance for the analog channels, logic and master relays. This assumption was deemed to be more conservative than the randomly occurring maintenances modelled in the DCPRA.

Notice that the unavailability contributions due to test and maintenance of the slave relays were not taken into account in either approach.

Table B5.3.5 gives the system unavailabilities for the various safety function actuation signals for two cases. In the first case the effects of common cause failures are not considered and in the second case when the common cause failures are included in the results.

A breakdown of the results is given in Table B5.3.6 for two safety function signals: the safety injection signal and the auxiliary feedwater pump signal (the results of the calculations are presented in similar format as those given in the DCPRA).

The analysis provided the following findings: in general, common cause failures (logic trains, master and slave relays) are the main contributors to overall SSPS unavailability. The main contributors to train unavailabilities are: independent hardware failures (mainly master and slave relay failures due to mechanical binding and short circuits) and unavailability due to test. Analog channel contribution to signal unavailability proved to be negligible. Sensitivity calculations assuming more diversity in the parameter signals gave similar results. ${ }^{2}$

\section{B5.33 Comparison of the DCPRA and WOG1/BNL1 Results}

A comparison of the data given in Table B5.3.3 with the results shown in Tables B5.3.5 and B5.3.6 shows that the DCPRA appears to systematically underestimate the SSPS signal unavailabilities. In the worst case the underestimation contains a factor of about 4.6, however the situation is exacerbated by the fact that the DCPRA gives the unavailability for a group of safety function signals (i.e., an OR gate) while the WOG1/BNL1 results relate to the unavailability of a single safety function signal alone. In other words, a direct comparison would yield a larger discrepancy.

BNL believes the root cause of the discrepancy is that an oversimplified fault tree model was used for the SSPS in the DCPRA. In order to place this finding in perspective, BNL performed an importance analysis and a sensitivity study on the DCPRA reduced model provided by PG\&E. The importance analysis showed that the contribution to core damage frequency (CDF) for system failure (both trains failing) was about $2 \%$ of the total non-seismic CDF and the sensitivity study showed that multiplying each of the SA split fractions by a factor of five and the SB split fractions by a factor of two increased the overall non-seismic CDF by $18 \%$. BNL therefore believes this discrepancy does not have a significant impact on the DCPRA results, especially considering the modelling conservatisms discussed previously (e.g., a single signal failure fails a channel, etc.). 
Appendix BS

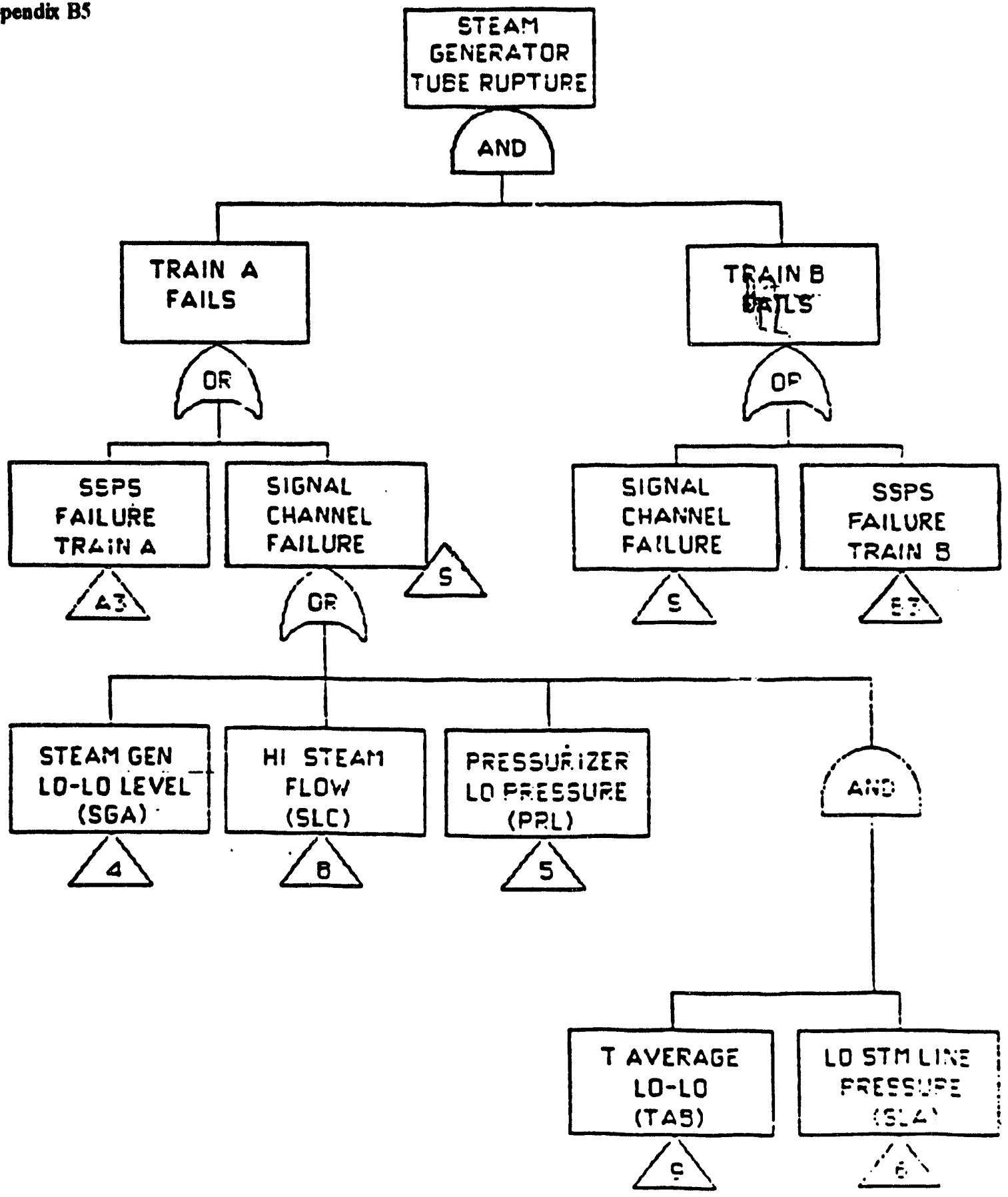

Figure B5.3.1.a SSPS master fault tree for steam generator tube rupture initiator in DCPRA 


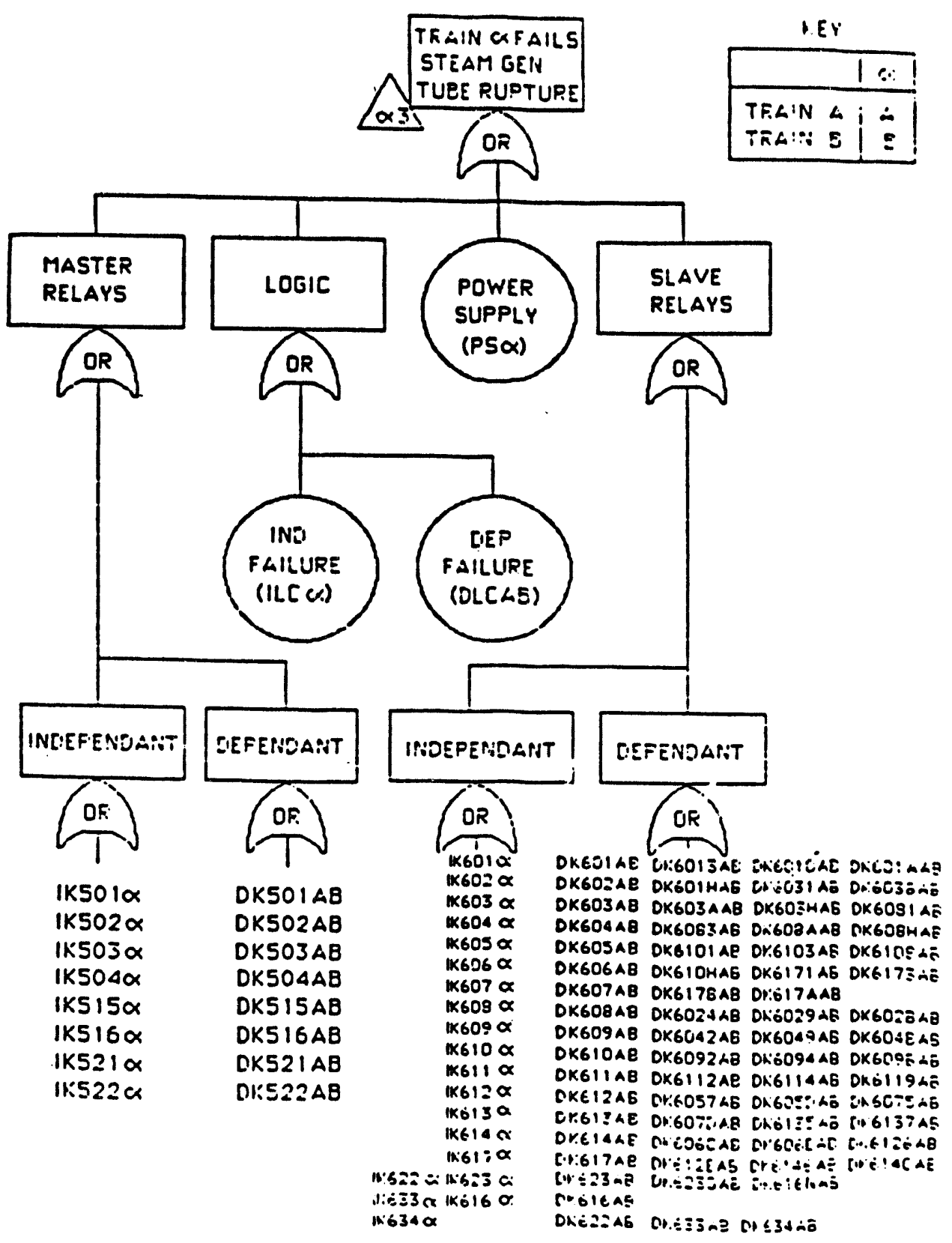

Figure B5.3.1.b SSPS master fault tree 
Appendix BS

Table B5.3.1

SSPS Safety Functions Modelled in

Diablo Canyon PRA

\begin{tabular}{|c|c|c|c|}
\hline Initiating Event & Safety Punction & $\begin{array}{l}\text { Required } \\
\text { Master Relays } \\
\text { Per Train }\end{array}$ & $\begin{array}{c}\text { Required } \\
\text { Slave Relays } \\
\text { Per Train }\end{array}$ \\
\hline \multirow[t]{5}{*}{ General Transient* } & Aux. Feed. Trains & 2 & 2 \\
\hline & Main Steamline Isol. (M) & 1 & 2 \\
\hline & Cont. Isol. Phase ، $(\mathrm{T})$ & 2 & 6 \\
\hline & Cont. Vent Isol. & 1 & 1 \\
\hline & Total & 6 & 11 \\
\hline \multirow[t]{6}{*}{ Large LOCA (LLOCA) } & Safety Injection (S) & 2 & 9 \\
\hline & Cont. Isol. Phase A (T) & 2 & 6 \\
\hline & Cont. Isol. Phase B (P) & 1 & 2 \\
\hline & Cont. Vent. Isol. & 1 & 1 \\
\hline & Cont. Spray (P) & 2 & 2 \\
\hline & Total & 8 & 18 \\
\hline Steam Gen. Tube & Safety Injection (S) & 2 & 9 \\
\hline \multirow[t]{5}{*}{ Rupture (SGTR) } & Cont. Isol. Phase A (T) & 2 & 6 \\
\hline & Cont. Vent Isol. & 1 & 1 \\
\hline & Aux. Feed. Train & 2 & 2 \\
\hline & Main Stenmline Isol. (M) & 1 & 2 \\
\hline & Total & 8 & 20 \\
\hline Steamline Break & Safety Injection (S) & 2 & 9 \\
\hline \multirow[t]{7}{*}{ Ins. Cont. (SLBIC) } & Aux. Feed. Trains & 2 & 2 \\
\hline & Cont. Isol. Phase A (T) & 2 & 6 \\
\hline & Cont. Isol. Phase B (P) & 1 & 2 \\
\hline & Cont. Vent Isol. & 1 & 1 \\
\hline & Main Steamline Isol. (M) & 1 & 2 \\
\hline & Cont. Spray (P) & 2 & 2 \\
\hline & Total & 11 & 22 \\
\hline Steamline Break & Safety Injection (S) & 2 & 9 \\
\hline Outside Cont. (SLBOC) & Aux. Feed. Trains & 2 & 2 \\
\hline
\end{tabular}


Tuble B5.3.1 (continued)

\begin{tabular}{llcc}
\hline Initiating Event & \multicolumn{1}{c}{ Safoty Function } & $\begin{array}{c}\text { Required } \\
\text { Master Relays } \\
\text { Per Truin }\end{array}$ & $\begin{array}{c}\text { Required } \\
\text { Slave Relays } \\
\text { Per Truin }\end{array}$ \\
\hline & Cont. Isol. Phnse A (T) & 2 & 6 \\
Cont. Vent Isol. & 1 & 1 \\
Main Steamline Isol. (M) & 1 & 2 \\
& Total & 8 & 20 \\
& & & \\
Small LOCA (SLOCA) & Safety Injection (S) & 2 & 9 \\
& Cont. Isol. Phase A (T) & 2 & 6 \\
& Cont. Vent Isol. & 1 & 2 \\
& Aux. Feed. Trains & 2 & 2 \\
& Main Stenmline Isol. (M) & 1 & 20
\end{tabular}

- Reactor trip, turbine trip signal unavailabilities are modelled presumably with this initiating event. 
Table B5.3.2

SSPS Surveillance Modelling

\begin{tabular}{|c|c|c|}
\hline & $\begin{array}{l}\text { Modelled in } \\
\text { DCPRA } \\
\text { (Designator) }\end{array}$ & $\begin{array}{l}\text { Modelled in } \\
\text { WOGI/BNLI }\end{array}$ \\
\hline \multicolumn{3}{|l|}{ Logic Cabinets } \\
\hline Test interval (month) & 2 (TS2F) & 2 \\
\hline Test time (hour) & 2 (ZHDSS2) & 1.5 \\
\hline Maintenance interval (month) & Unscheduled* & 12 \\
\hline Maintenance time (hour) & Plant-specific** & 2 \\
\hline \multicolumn{3}{|l|}{ Master Relay } \\
\hline Test interval (month) & $?$ & 2 \\
\hline Test time (hour) & ? & 1.5 \\
\hline Maintenance interval (month) & $?$ & 12 \\
\hline Maintenance time (hour) & $?$ & 2 \\
\hline \multicolumn{3}{|l|}{ Slave Relay } \\
\hline Test interval (month) & $-\cdots$ & $\cdots$ \\
\hline Test time (hour) & $\cdots$ & $\cdots$ \\
\hline Maintenance interval (month) & $\cdots$ & $\cdots$ \\
\hline Maintenance time (hour) & $\cdots$ & $\cdots$ \\
\hline \multicolumn{3}{|l|}{ Analog Channel } \\
\hline Test interval (month & 1 (TSIF) & 1 \\
\hline Test time (hour) & 2 (ZHDSS2) & 2 \\
\hline Maintenance interval (month) & $\cdots$ & 12 \\
\hline Maintenance time (hour) & $\ldots$ & 1 \\
\hline
\end{tabular}

"ZTPSIR (Power Supply Failure Rate) $=1.71-5 / \mathrm{hr}$.

* ZMGNBF (Time to Repair Failed Power Supply).

?The test and maintenance of master relays are apparently not accounted for in the DCPRA model. 
Table B5.3.3

SSPS Signal Unavallabilities

(Split Fractions in Diablo Canyon PRA)

\begin{tabular}{|c|c|c|c|c|c|c|c|c|}
\hline Case & TTL & $\mathbf{H W}$ & HWI & HWD & TS & $\mathbf{M N}$ & $\mathrm{HE}$ & Comment \# \\
\hline \multicolumn{9}{|c|}{ A: Single Train (Train A) Failure } \\
\hline SA1, SB3 & $6.47-3$ & $5.68-3$ & $5.68-3$ & $1.48-10$ & $7.89-4$ & $4.23-10$ & $4.18-6$ & \\
\hline SA2 & $9.69-3$ & $8.50-3$ & $8.50-3$ & $1.39-6$ & $7.89-4$ & $4.23-10$ & $3.98-6$ & \\
\hline SA4, SBB & $9.95-3$ & $9.16-3$ & $9.15-3$ & $1.39-6$ & $7.89-4$ & $4.23-10$ & $6.28-6$ & \\
\hline SA5 & $1.18-2$ & $1.06-2$ & $1.06-2$ & 1.39 .6 & $7.89-4$ & $4.23-10$ & $4.00-4$ & \\
\hline SA7 & $9.95-3$ & $9.15-3$ & $9.15-3$ & $3.20-11$ & $7.89-4$ & $4.23-10$ & $4.18-6$ & \\
\hline SAB, SBN & $9.95-3$ & $9.15-3$ & $9.15-3$ & $1.48-10$ & $7.89-4$ & $4.23-10$ & $4.18-6$ & \\
\hline
\end{tabular}

B: System (Trains $A$ and $B$ ) Failure

$\begin{array}{llllllll}\text { SB2 } & 9.86-5 & 8.54-5 & 1.14-5 & 7.40-5 & 9.02-6 & 5.74-12 & 4.18-6 \\ \text { SB6' } & 6.07-4 & 1.96-4 & 2.19-5 & 1.74-4 & 1.36-5 & 8.12-12 & 3.98-4 \\ \text { SBA' } & 2.17-4 & 1.96-4 & 2.26-5 & 1.74-4 & 1.46-5 & 8.68-12 & 6.28-6 \\ \text { SBE' } & 6.37-4 & 2.20-4 & 2.56-5 & 1.94-4 & 1.69-5 & 9.09-12 & 3.40-4 \\ \text { SBJ' } & 2.13-4 & 1.94-4 & 2.18-5 & 1.72-4 & 1.45-5 & 8.68-12 & 4.18-6 \\ \text { SBM' } & 2.13-4 & 1.94-4 & 2.18-5 & 1.72-4 & 1.45-5 & 8.68-12 & 4.18-6\end{array}$

Split Fraction Identification:

SA1, SB3 General transient, all needed electrical power is avallable, or ac Instr. Chnl. I is down.

SA2 Large LOCA, all needed electrical power Is available.

SA4, SBB Steam Generator Tube Rupture (SGTR), all needed electrical power is available, or ac Inst. Chnl. I is down.

SA5 Steamline Break Inside Containment (SLBIC), all needed electrical power is available.

SA7 Steamline Break Outside Containment (SLBOC), all needed electrical power is available.

SA8 Small LOCA, all needed electrical power is available.

SB2 - General transient, both trains, $A$ and $B$, fail.

SB6. Large LOCA, both trains, $A$ and $B$, fail.

SBA' Steam Generator Tube Rupture (SGTR), both trains, A and B, fail.

SBE' Steamline Break Inside Containment, both trains, $A$ and $B, f a i l$.

SBJ' Steamline Break Outside Containment, both trains, $A$ and $B$, fail.

SBM' Small LOCA, both trains, A and B, fail. 
Appendix BS

Table B5.3.3 (Continued)

\section{Notations}

IIL - Total unavallability.

HW - Unavailability due to hardware contribution which is the sum of Independent failures and comnon cause fallures.

hWI - Unavailability due to independent failures.

HWD - Unavallability due to common cause fallures.

TS - Unavailability due to test.

INW - Unavallability due to maintenance.

HE - Unavallability due to human error contribution. 
Table B5.3.4

Master/Slave Relay Arrangements for Various

Safety Function Actuation Signals

\begin{tabular}{llll}
\hline Safety Function Actuation Signal & $\begin{array}{l}\text { Master } \\
\text { Relays* }\end{array}$ & \multicolumn{1}{c}{ Slave Relays* } \\
\hline 1. Safety Injection & $\begin{array}{l}\text { A } \\
\text { B }\end{array}$ & $\begin{array}{l}\text { A1, A2, A3 } \\
\text { B1, B2, B3 }\end{array}$ \\
2. Steam Line Isolation & A & A1, A2 \\
3. Main Feedwater Isolation & A & A1, A2 \\
4. Auxiliary Feedwater Pump Start & A & A1, A2 \\
5. Containment Spray & A & A1, A2 \\
6. Containment Isolation & A & A1, A2 \\
\hline
\end{tabular}

* Relays per SSPS train as applied in the unavailability analysis. 
Appendix B5

Table B5.3.5

SSPS Signal Unavallabilities (WOG1/BNL1)

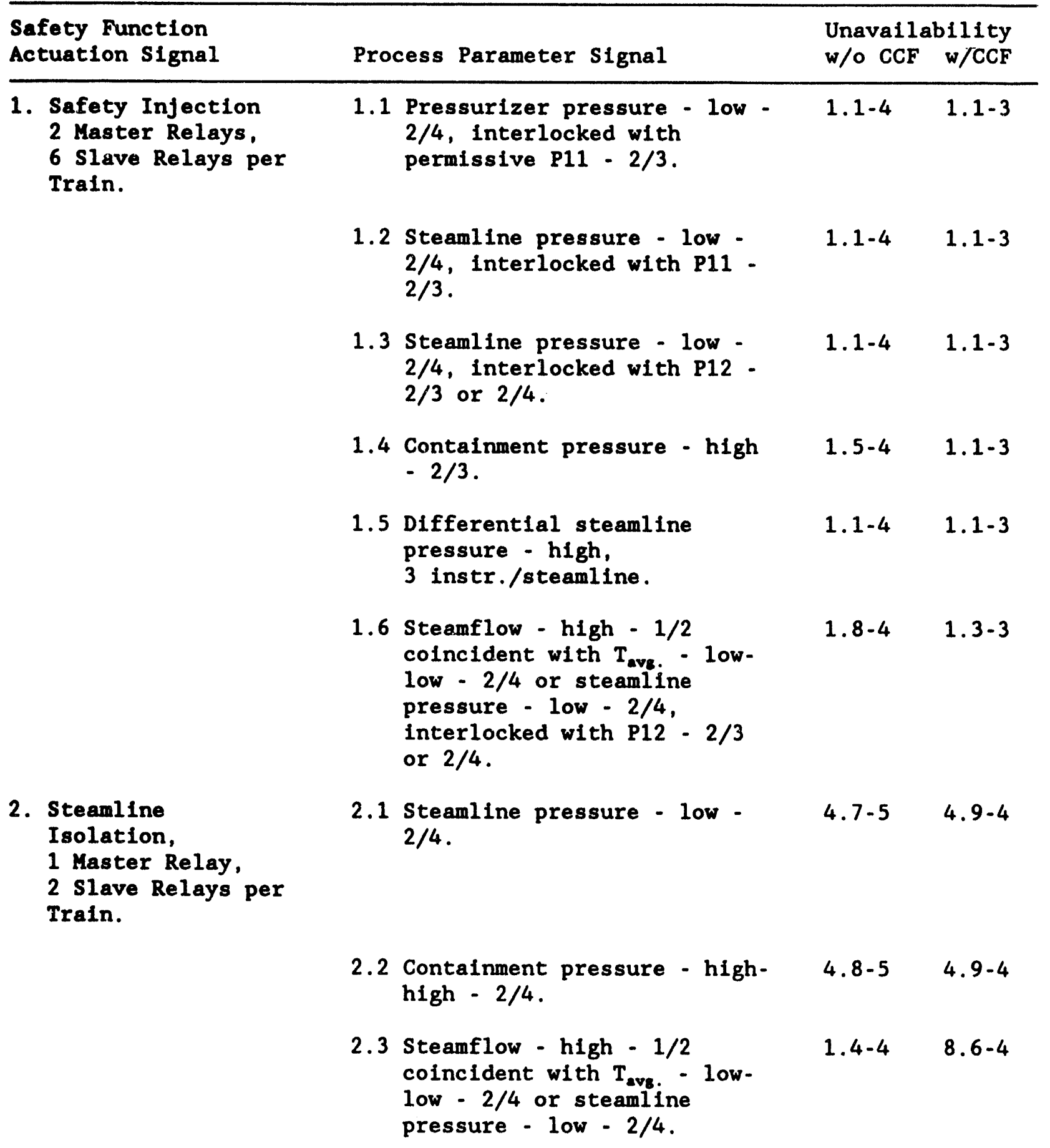


Table B5.3.5 (Continued)

\begin{tabular}{|c|c|c|c|c|}
\hline $\begin{array}{l}\text { Safety Function } \\
\text { Actuation Signal }\end{array}$ & Proce & ess Parameter Signal & $\begin{array}{l}\text { Unavaila } \\
\text { w/o CCF }\end{array}$ & $\begin{array}{l}\text { oility } \\
\text { w/CCF }\end{array}$ \\
\hline & $\begin{array}{r}2.4 \mathrm{~s} \\
2 \\
1 \\
1 \\
k\end{array}$ & $\begin{array}{l}\text { Steamline pressure - low - } \\
2 / 4 \text { and steamflow - high - } \\
1 / 2 \text { coincident with } T_{\text {ave }}- \\
\text { low-low }-2 / 4 \text { interlocked, } \\
\text { with } \mathrm{P} 12-2 / 3 \text { or } 2 / 4 \text {. }\end{array}$ & $4.7-5$ & $5.0-4$ \\
\hline & $2.5 \mathrm{~s}$ & $\begin{array}{l}\text { Steamflow - high }-1 / 2 \\
\text { interlocked with P12 }-2 / 4 \\
\text { coincident with SI. }\end{array}$ & $1.4-4$ & $8.2-4$ \\
\hline $\begin{array}{l}\text { 3. Containment } \\
\text { Isolation Phase B, } \\
\text { Containment Spray } \\
1 \text { Master Relay, } \\
2 \text { Slave Relays per } \\
\text { Train. }\end{array}$ & $3.1 \mathrm{c}$ & $\begin{array}{l}\text { Containment pressure - high- } \\
\text { high }-2 / 4 \text {. }\end{array}$ & $1.1-4$ & $9.6-4$ \\
\hline \multirow{4}{*}{$\begin{array}{l}\text { 4. Auxiliary Feedwater } \\
\text { Pump Start Signal, } \\
1 \text { Master Relay, } \\
2 \text { Slave Relays per } \\
\text { Train. }\end{array}$} & 4.1 & $\begin{array}{l}\text { Steam generator water level } \\
\text { low-low }-2 / 4 \text { in one loop. }\end{array}$ & $6.1-5$ & $5.7-4$ \\
\hline & 4.2 & $\begin{array}{l}\text { Steam generator water level } \\
- \text { low-low }-2 / 3 \text { in one loop. }\end{array}$ & $1.3-4$ & 6.4-4 \\
\hline & $4.3 \mathrm{I}$ & RCP bus undervoltage $-2 / 3$. & $1.8-4$ & $7.6-4$ \\
\hline & 4.41 & $\begin{array}{l}\text { RCP bus undervoltage }-1 / 2 \\
\text { twice. }\end{array}$ & $1.5-4$ & $7.2-4$ \\
\hline $\begin{array}{l}\text { 5. Main Feedwater } \\
\text { Isolation, } \\
1 \text { Master Relay, } \\
2 \text { Slave Relays per } \\
\text { Train. }\end{array}$ & 5.1 & $\begin{array}{l}\text { Steam generator water level } \\
\text { - high-high }-2 / 3 \text { in one } \\
\text { loop. }\end{array}$ & $1.3-4$ & $6.4-4$ \\
\hline
\end{tabular}

CCF - Common cause failures. 
Appendix BS

Table B5.3.6

SSPS Signal Unavallabilities Calculated by WOG1/BNL1

\begin{tabular}{lllllllll}
\hline Case & TTL & HW & HWI & HWD & TS & MN & HE & Comment \#
\end{tabular}

A: Single Train Failure

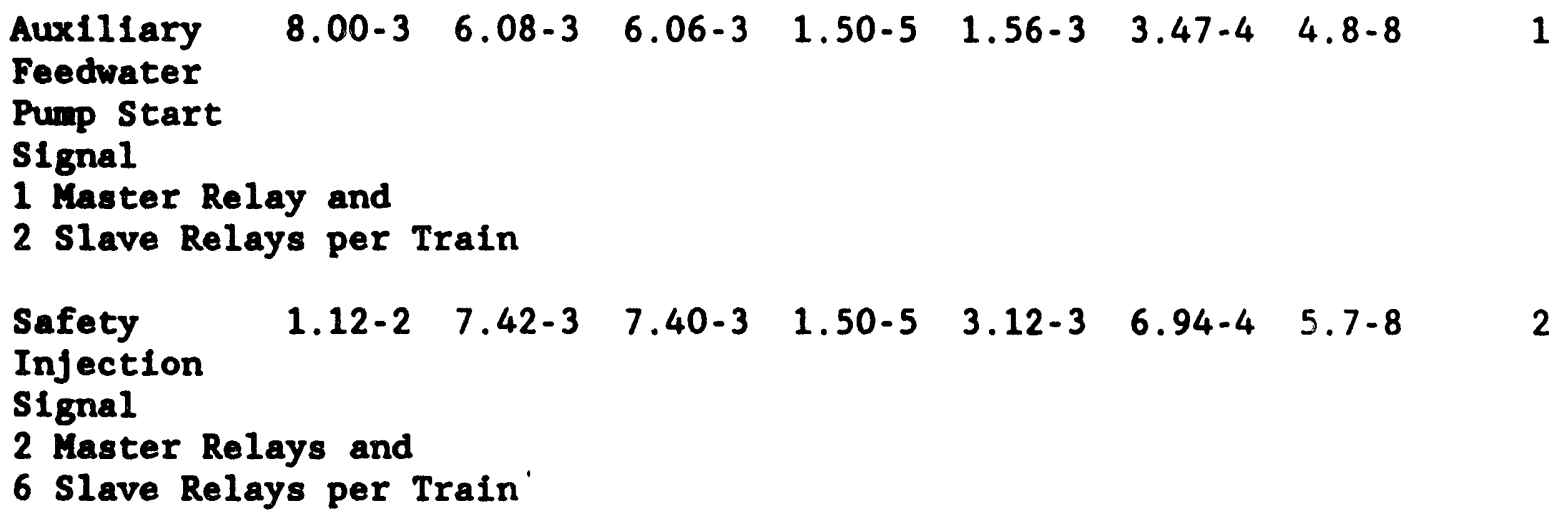

B: System Fallure

\begin{tabular}{|c|c|c|c|c|c|c|}
\hline $\begin{array}{l}\text { Auxiliary } 5.66-4 \quad 5.43-4 \\
\text { Feedwater } \\
\text { Pump Start } \\
\text { Signal } \\
1 \text { Master Relay and } \\
2 \text { Slave Relays per Train }\end{array}$ & $3.67-5$ & $5.06-4$ & $1.90-5$ & $4.22-6$ & $4.8-8$ & $\begin{array}{l}1 \text {, See } \\
\text { also } \\
\text { Table } 5 .\end{array}$ \\
\hline $\begin{array}{l}\text { Safety } \quad 1.08-3 \quad 1.02-3 \\
\text { Injection } \\
\text { Signal } \\
2 \text { Master Relays and } \\
6 \text { Slave Relays per Train }\end{array}$ & $5.48-5$ & $9.64-4$ & $4.62-5$ & $1.03-5$ & $5.7-8$ & $\begin{array}{l}2 \text {, See } \\
\text { also } \\
\text { Table } 5 .\end{array}$ \\
\hline
\end{tabular}

1. The process parameter signal is: steam generator level low-low $(2 / 4$ in 1 loop). The Auxiliary Feedwater Pump Start Signal is also indicative of Steamline Isolation and Main Feedwater Isolation.

2. The process parameter signal is: low pressurizer pressure $(2 / 4$ interlocked with P-11 2/3). The Safety Injection Signal is also indicative of Containment Spray Actuation and Phase B Isolation.

\section{Notations}

TTL - Total unavailability.

HW - Unavailability due to hardware contribution which is the sum of independent fallures and common cause failures.

HWI - Unavailability due to independent failures.

HWD - Unavailability due to common cause failures.

TS - Unavailability due to test.

MN - Unavailability due to maintenance.

$\mathrm{HE}$ - Unavailability due to human error contribution. 
Appendix B5

\section{B5.4 Comparison of RPS Unavailabilities Obtained in DCPRA and in WOG/BNL Calculations}

\section{B5.4.1 System Description}

The Reactor Protection System (RPS) could be considered a continuation of the SSPS in the sense that it trips the reactor on a "trip" signal from the SSPS. The RPS also trips the reactor if loss of power occurs, or the plant operator manually actuates the system. The RPS consists of two trains each containing two undervoltage coils in an energized condition and associated breakers. One undervoltage coil for the reactor trip breaker and one for the bypass breaker. When an SSPS train generates a trip signal the UV coils are de-energized. This will open the reactor trip and bypass (if closed) breakers removing power from the control rods, allowing the rods to fall into the core. A schematic of the RPS at Diablo Canyon is shown in Figure B5.4.1. The success of the RPS is defined as at least 52 of the 53 control rods successfully inserted into the core on demand.

\section{B5.4.2 Testing of the RPS}

Testing of the analog channels and logic is essentially identical with that described in Sections B5.2.2.1 and B5.2.2.2. When the breaker actuation test is performed, the associated bypass trip breaker is closed to prevent an unwanted reactor trip. The manual trip test can be performed by using four push buttons.

\section{B5.43 Unavailability Modelling of the RPS in the DCPRA and in the WOG/BNL Calculations}

\section{BSA3.1 The RPS Fault Tree Model in the DCPRA}

The RPS fault tree model of the DCPRA is shown in Figure B5.4.2. It is a block level fault tree with identified common cause events ( $\alpha$-factor model). The block events involve the control rod insertion failure, circuit breaker failures, undervoltage rely and trip coil failures, bypass undervoltage relay, bypass circuit breaker, and shunt trip coil failures.

The surveillance and maintenance conditions of the RPS modelled in the DCPRA are shown in Table B5.4.1. The model correctly describes the staggered testing of the trip breakers. The effect of loss of $\mathrm{dc}$ power, instrument ac and loss of SSPS signals are considered in the houndary conditions. The quantification indicated that the common cause failures of the circuit breakers and trip coils dominate the system unavailability.

\section{R5.4.32 The RPS Unavailability Models in the WOG/BNL Calculations}

In the WOG2 calculation ${ }^{3}$ a set of fault trees was used to quantify the unavailabilities of the RPS for various trip signals. The fault trees are rather complex as they were developed and updated over many years. The NRC and BNL have scrutinized them and they are therefore not reproduced here. Representative fault trees can be found in Reference 4.

The trip signal unavailabilities obtained in these calculations relate to the whole system including the analog channels and the logic. The fault tree model in the DCPRA does not show these components because that approach considers them by the houndary conditions. 


\section{Appendix B5}

The WOG2 calculations contain the complete set of unavailability contributors; random, common cause, and human errors as well as unavailabilities due to test and maintenance. The common cause and human errors for the analog channels and logic portions of the RPS are identical to those described earlier for the SSPS modelling by WOG1/BNLI (Section B5.3). Additional common cause failures have been quantified for the reactor trip and bypass breakers. The surveillance and maintenance conditions considered are given in Table B5.4.1 for comparison with those used in the DCPRA.

As a representative result, we give here the trip signal unavailability prompted by pressurizer low pressure (2/4) parameter signal - RPS trip failure: 2.9-5/d (w/o CCF) and 1.2-4/d (w/CCF). These values were obtained without considering diversity of parameter signals. With diversity, RPS trip failure became $1.44-5 / \mathrm{d}$.

BNL provided an independent analysis of the unavailability of the RPS in References 5 and 6 (BNL2 results). BNL utilized a time-dependent (Markovian) model. The model thoroughly analyzed the dynamic behavior of the RPS. It also considered the common mode failures of all the main components of the full system (including the analog channels and logic units). The surveillance and maintenance data are identical to those listed in Table B5.4.1 for the WOG2/BNL2 calculations - RPS trip failure (BNL2-Markov) = 2.9-5/d.

\section{B5.4.3.3 Comparison of the RPS Unavailabilities}

In order to compare the DCPRA results with those obtained in the WOG/BNL calculations, a representative DCPRA system unavailability value (RTI) is reproduced here. This was obtained under the boundary condition when two SSPS signals are received and all power is available. The total failure of the RPS to initiate reactor trip was originally calculated in the draft DCPRA to be: RTI $=9.32-6 /$ demand. And, except for the operator-initiated trip given SSPS failure, the other houndary conditions resulted in only slightly increased values.

The BNL review of the RPS was essentially completed during the "interaction" phase of the DCPRA review and as such was based upon draft material. When the final version of the DCPRA was submitted, BNL discovered that RT1 was given a new, lower value (6.58-6/demand). The following sensitivity study is offered to help place this situation in context with the overall PRA results.

\begin{tabular}{lcccc}
\hline Case & $\begin{array}{c}\text { RT1 } \\
\text { Failures/Demand }\end{array}$ & $\begin{array}{c}\text { Unormalized } \\
\text { Fussel-Vesely } \\
\text { Importance }\end{array}$ & $\begin{array}{c}\text { Resulting } \\
\text { Non-Seismic } \\
\text { CDF }\end{array}$ & \% $\Delta$ CDF \\
\hline PG\&E Final & $6.58-6$ & $5.257-7$ & $1.7728-4$ & $\ldots$ \\
PG\&E Draft & $9.32-6$ & $7.447-7$ & $1.7749-4$ & 0.12 \\
BNL & $2.90-5$ & $2.317-6$ & $1.7907-4$ & 1.01 \\
\hline
\end{tabular}

The results obtained in the DCPRA seem to be somewhat lower than those obtained in the WOG2/BNL2 calculations. However, since in the DCPRA calculations the analog channel/ogic unavailabilities are not explicitly included (only through the houndary conditions) the obtained conditional unavailability values can be taken as reasonable. In terms of the importance of RTl, it is clear that the model is not very sensitive to changes in this split fraction. This result is at least partially due to the fact that the model accounts for the fact that Diablo Canyon has undergone modifications with respect to the RPS in accordance with the ATWS Rule. 


\section{B5.5 References}

1. El-Bassioni et al., PRA Review Manual, NUREG/CR-3485, September 1985.

2. WOG1; Andre, G., Howard, R., Jensen, R., and Leonelli, K., "Evaluation of Surveillance Frequencies and Out-of-Service Times for the Engineered Safety Features Actuation System," WCAP-10271 Supplement 2, February 1986 and WCAP-10271 Supplement 1, Revision 1, March 1987.

3. WOG2; Jansen, R., Lijewski, L., Masarik, R., "Evaluation of Surveillance Frequencies and Out-of-Service Times for the Reactor Protection Instrumentation System," WCAP-10271-P-A, May 1986.

4. BNL1; Bozoki, G., Aliefendioglu, K., Fitzpatrick, R., Yoon, W., "A Review of the Westinghouse Owner's Group Technical Specification Relaxation Analysis for the Engineered Features Actuation System, Draft Report for NRC, April 1988.

5. BNL2; Papazoglou, 1. and Cho, N., "Evaluation of Surveillance Frequencies and Out-of-Service Times for the Reactor Protection System (WCAP-10271)," Letter Report to NRC, July 13, 1983.

6. BNL2; Papazoglou, I. and Cho, N., "Probabilistic Evaluation of Surveillance and Out-of-Service Times for the Reactor Protection Instrumentation System, Draft Report, April 1984. 
Appendix BS
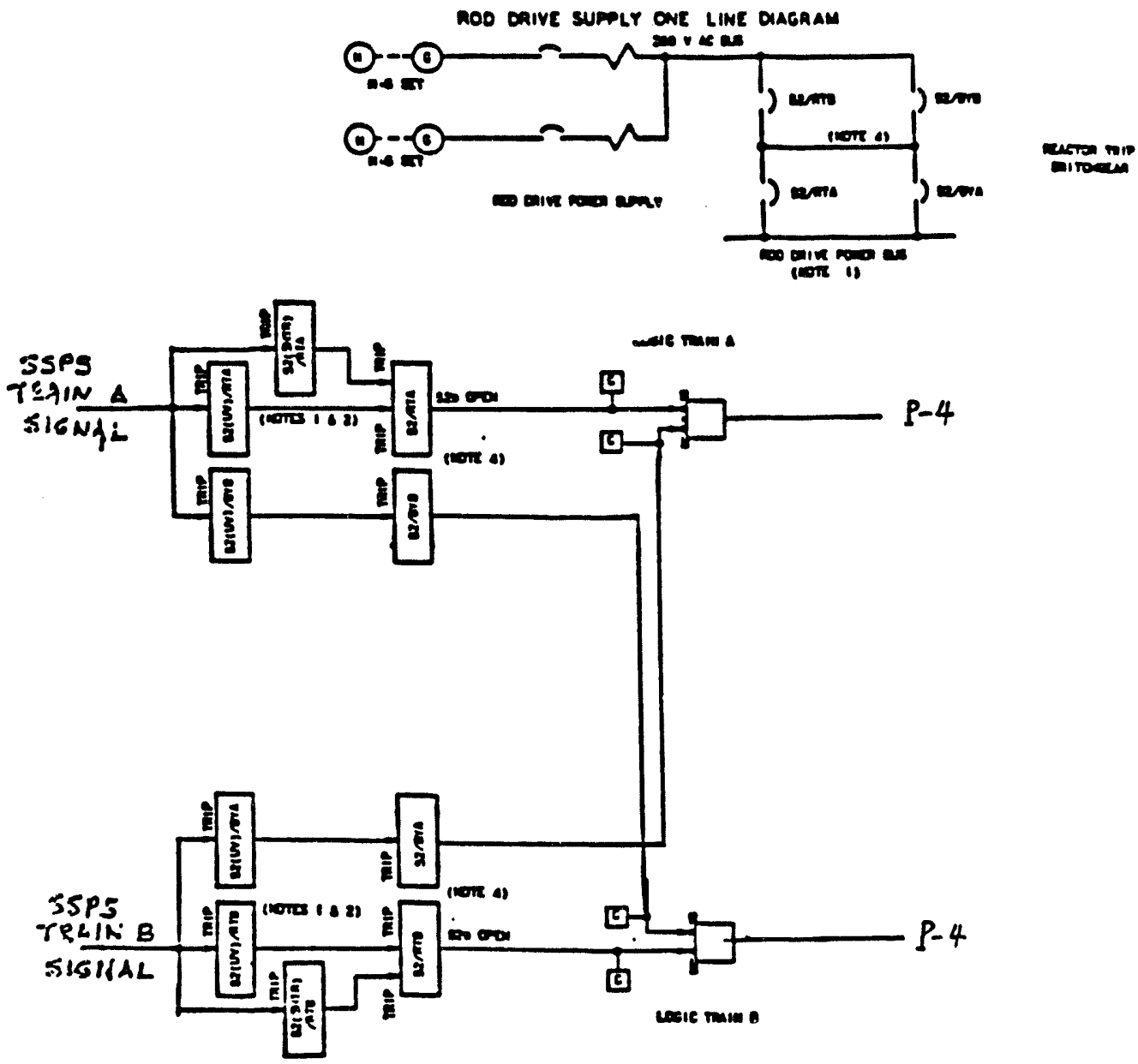

(n)

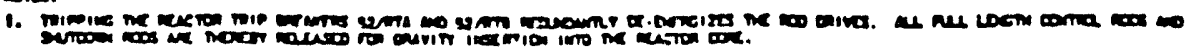

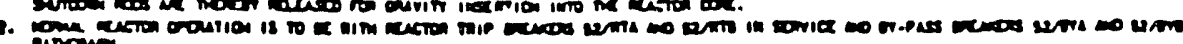

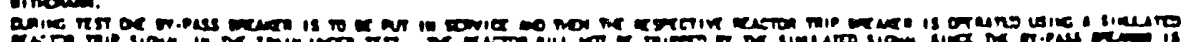

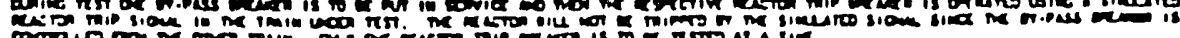

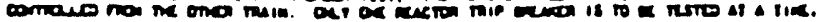

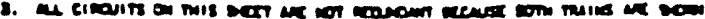

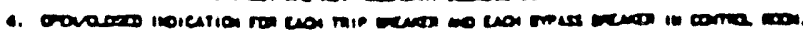

Figure B5.4.1 Schematic of Reactor Protection System in DCPRA 
Appendix BS

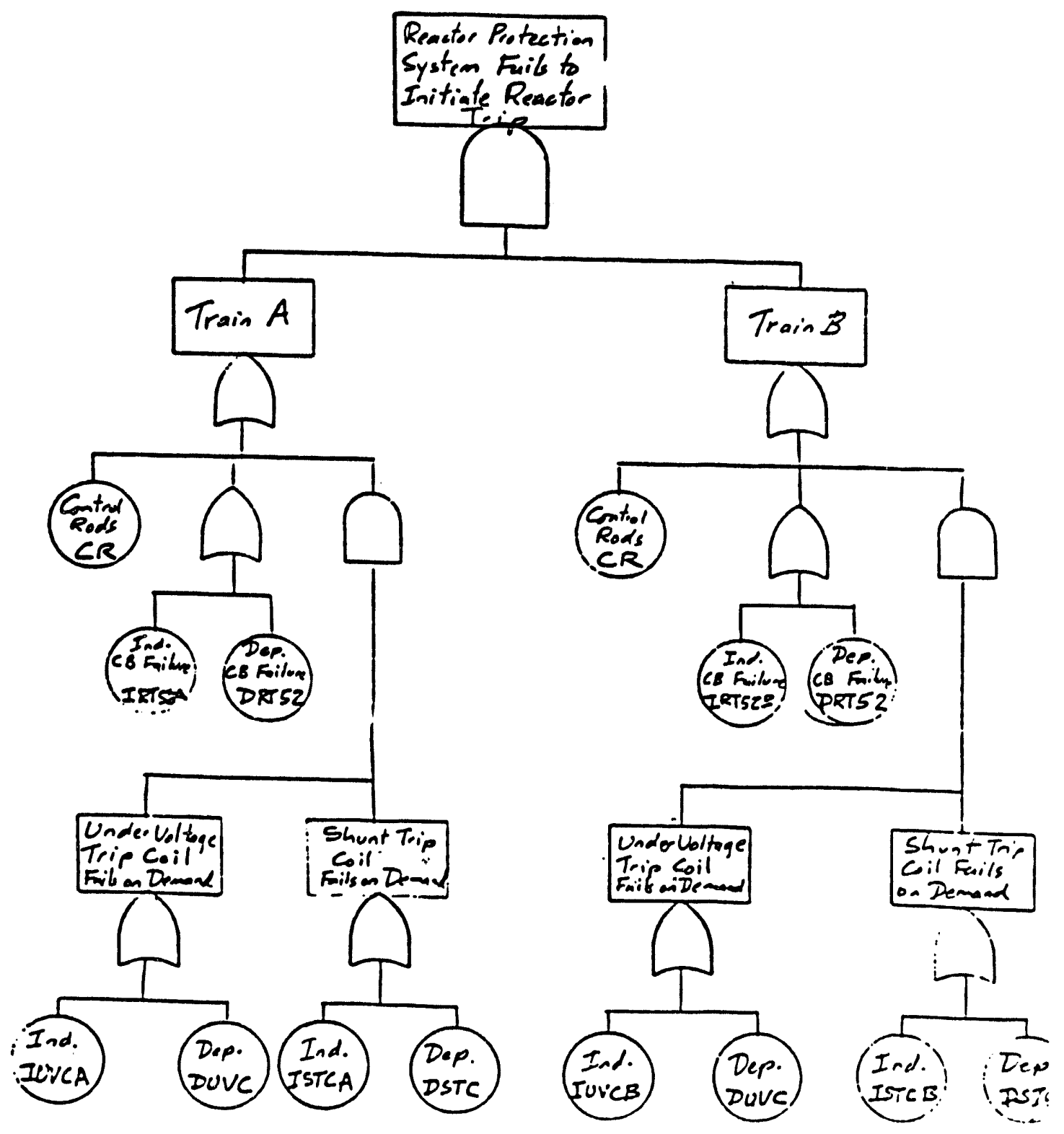

Figure B5.4.2 RPS fault tree with common cause identified in DCPRA 
Table B5.4.1

RPS Surveillance Modelling

\begin{tabular}{|c|c|c|}
\hline Component & $\begin{array}{l}\text { Modelled in } \\
\text { DCPRA } \\
\text { (Designator) }\end{array}$ & $\begin{array}{l}\text { Modelled by } \\
\text { WOG2/BNL2 }\end{array}$ \\
\hline \multicolumn{3}{|l|}{ Logic for Breaker Actuation: } \\
\hline Test interval (month) & 2 (TS2F) & 2 \\
\hline Test time (hour) & 2 (ZHDSS2) & 2 \\
\hline Maintenance interval (month) & Unscheduled* & 12 \\
\hline Maintenance time (hours) & Plant-specific ** & 6 \\
\hline \multicolumn{3}{|l|}{ Breakers: } \\
\hline Test interval (month) & 1 & 2 \\
\hline Test time (hour) & 2 & 2 \\
\hline Maintenance interval (month) & 6 & 12 \\
\hline Maintenance time (hour) & 1 & 6 \\
\hline \multicolumn{3}{|l|}{ Analog (Sensor) Channels: } \\
\hline Test interval (month) & $* * *$ & 1 \\
\hline Test time (hour) & $\ldots 2$ & \\
\hline Maintenance interval (month) & $\cdots$ & 12 \\
\hline Maintenance time (hour) & $\ldots 1$ & \\
\hline
\end{tabular}


APPENDIX C

DCPRA BASIC EVENT DATA DISTRIBUTIONS 
STR IILE

$\operatorname{matax}$

997a 8IL

1. $\operatorname{sen} 16$ cen

2.

3. $8 \operatorname{sen} 20$

4. Bseresc oca

5. rrousc och

- iscoric cel

7. osenzc con

- rscarc cen

- escarc ca

10. 830010 CCA

11. Dxas 10 ocs

12. sscasio ces

13. D3C110 ce

14. $\mathrm{rac0} 10 \mathrm{cos}$

15. $820030 \mathrm{CCA}$

16. D20030 cen

17. exerm ces

10. oxerm cen

10. sacrirs ces

20. Dexerm ces

21. sameco cCA

23. D20m00 cCA

23. arnos ces

24. Dempos cea

25. Inve00 ocs

26. cronco oca

27. $220000 \mathrm{CCA}$

20. D200000 CCA

20. ssposs ceA

30. Dsposs cen

31. Is0Gss CCA

32. espoess ceA

33. esposi cen

34. Desoesi cen

35. $13 \operatorname{sus} 1 \mathrm{CA}$

36. essess ceA

37. $8500 \mathrm{sec}$ CA

36. DsDoser CCA

39. Issocse cen

40. ospose cca

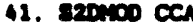

42. D201000 CCA

43. searbo cal

44. Dedroo CCA

45. reorsoo col

46. ceovico ced

47. ssmulr CCA

46. DSTIIR $\propto \mathrm{CA}$

49. TsтmL col

5o. ostrulR oca

31. ssrmis cCA

32. Dsmits CCA

53. ISPm1s CCA

54. osrisis ceA

55. s4rmat CC:

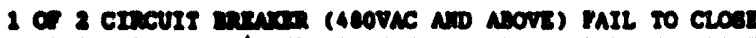

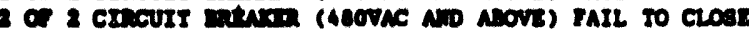

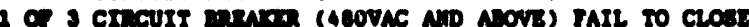

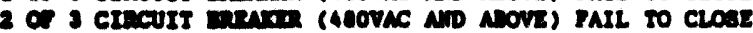

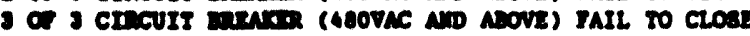

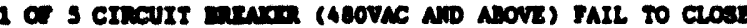

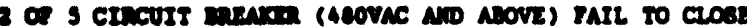

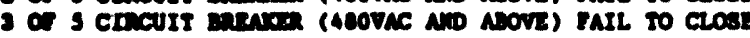

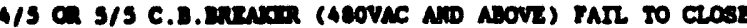

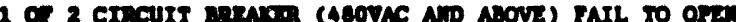

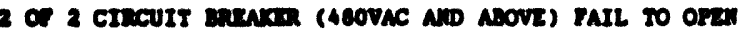

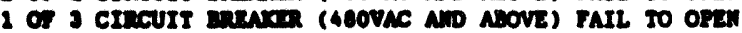

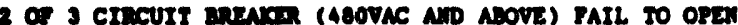

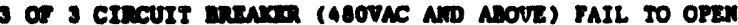

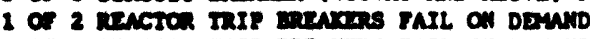

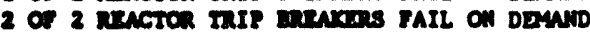

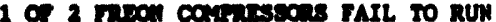

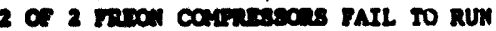

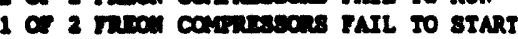

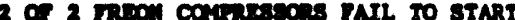

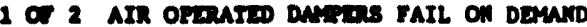

2 Or 2 AIR ORERATE DANTERS FAIL ON DEMND

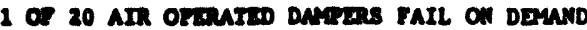

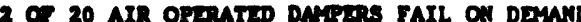

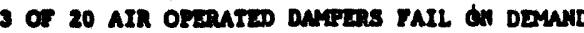

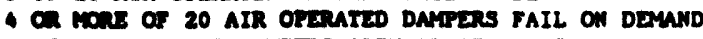

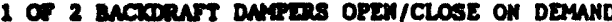

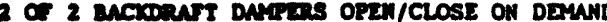

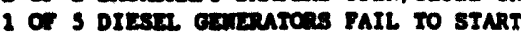

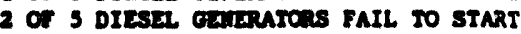

3 of 5 DIEsE Gxirnatons FAIL TO START

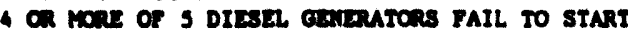

1 OF I DIEser cearmatons FAIL to RUM DURING 18T kR.

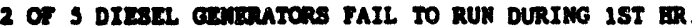

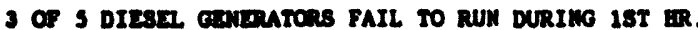

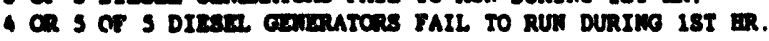

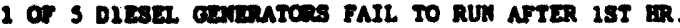

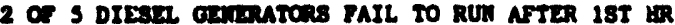

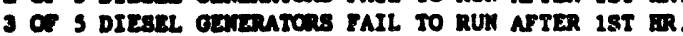

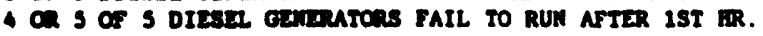

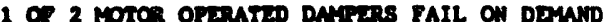

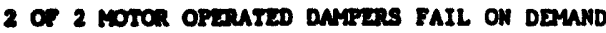

1 of a roton OPEATIED DNAPERs FAIL ON DEMAND

2 OF - MOTCR OPERATED DAyPens FAIL ON DDWHD

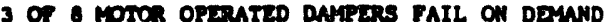

- OR MOEE OF - MOTOR OPERATED DAMPERS FAIL ON DEMAND

1 of 5 Cowralinent FAM COOLERS FAIL TO RUN

2 OF 5 CONTAIMENT TAM COOLRRS FAIL. TO RUN

3 of I COMTAIMERT FAN COOLERS FAIL TO RUH

- ar 5 or 5 contaImeart FAN COOLERS FAIL TO RUN

1 OF 5 CONTAIMEET FN COOLERS FAIL TO START

2 OF I CONTAIMILNT PAM COOLERS PAIL TO START

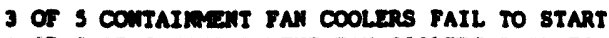

- OR 5 OF 9 COWTAIment FN COOLERS FAIL TO START

1 OF - ROOM VENT. FAMS FAIL TO RUN
$1.502-03$

1. $122-04$

1. 505-03

4. $468-05$

1. 65E-05

1.502-03

$3.108-05$

$4.335-06$

2. 868-06

. 055-04

$4.505-0$

6. 058-0

1.94z-05

6. 44E-06

1. 53E-03

1.14E-04

3.34E-05

3. $398-07$

2. 35E-03

2. 268-06

7. 285-06

5. 468-05

7. 272-04

1. 53E-05

2. $155-08$

1. $402-06$

2.52E-04

1.058-0

1. 50E-02

8.27z-06

5. 235-07

6. $175-07$

6. 63E-03

3.485-05

4. 855-06

6.152-06

2.075-03

5. 085 -06

1.272-06

1. $402-06$

2. 575-03

2. $005-06$

2.678-03

3.70E-05

$7.778-06$

3.06E-06

1.262-05

$7.068-08$

2. $465-10$

6. 55E-10

1.60E-03

7. 192-00

6.24E-07

4. 18E-07

6. 65E-06 3.208-08 3. 502-00 6.685-09 1.078-09 3. 535-08 3. 018-09 $3.002-11$ 4. 468-11 3. 725-07 5. 308-08 5. $682-07$ 1.105-08

$1.615-10$

$1.005-06$

1. 15E-08

2. $325-08$

4.318-13

3.058-06

1. $418-11$

325 -07

2. $468-09$

1.52E-07

2.205-10

6. $435-12$

$4.39 z-12$

8.63E-08

7. $908-10$

3. 012-05

$4.64 E-11$

5. 165-13

1. $015-12$

1.34E-05

5. $822-10$

2.675-11

6.162-11

$4.055-00$

5. $44 E-11$

$4.11 \mathrm{E}-12$

6.47E-12

$1.528-06$

3. $00 \mathrm{E}-08$

1.51E-00

2. 73E-08

0.162-11

3. $805-11$

7.04E-11

$6.32 z-17$

1. $015-18$

$7.00 E-10$

1.41E-06

3. $17 z-11$

7.03E-13

6. $045-13$

2. $80 \mathrm{E}-11$

2.

$0.438-06$

2.605-04

3. 385-07

2.25E-07

2. 802-04

$3.305-07$

4.808-08

2.008-00

. $538-05$

2.015-06

3. 508-05

1.84E-07

3. 62E-08

3. 805-04

1. 51E-05

2. $805-06$

1. 045-08

2. 50E-04

$4.978-00$

.375-04

6. 5ez-00

2.35E-04

1. 685-07

2. $488-08$

1. $468 \mathrm{E}-08$

S. 16E-05

1.73E-06

7. 485-03

0.465-07

-. 71E-00

7. 578-09

3. 662-03

7.782-08

3.758-07

3. 65E-07

2.11E-04

$4.385-07$

$4.635-08$

$4.798-08$

$0.935-04$

2. $565-05$

9.82E-04

1.78E-06

7.675-0

5. 90 E-08

4. 18E-06

7. 33E-10

1.07E-12

3. 03E-11

3. 23E-04

7. 63E-07

3.04E-08 
8.10.

58. Darman ces 57. Torman so. asrma cos 30. surmas col co. Darrias ca 61. Intria $\mathrm{CO}$ 43. esrrias cas 63. ت11010 ca 4. Dncid ca bs. gapar cas 6. derara cen 67. sapara ces co. Dapara cC co. sarata ca 70. Darata ca

71. TARAM ces

72. Garns ce 73. sarass $C \mathrm{C}$ 74. Darnse ces 75. rapass col 78. enzare col 71. Esrocan cos 74. osrecen col 79. rarces con \%. Eurces co 42. Dirces cas 42. rancos cos e3. envoe cos 4. Darocen cen 15. Baroos cas e. Daroes cas 07. sarean ces 4. Darcos cos 19. $8250 \mathrm{cos}$ 6. Daros $\mathrm{CO}$ 01. s2510 CCI 22. Darite Ca 93. sarvos $\mathrm{CC}$ 4. D2trus Cos 05. E250 $\mathrm{CO}$ 6. D25 COI 97. $2350 \mathrm{CC}$ ต. D2ros Cel ต. E2ron $c$ 100. D2For CC: 101. sarsis $\mathrm{CC}$. 102. D25ars COS. 103. $\operatorname{sen} 11000$ 104. DE2L10 COC 105. 8321000 106. Dinis 1000 107. T32u1d 000 108. SARL10 COC 100. DARL10 COC 120. TARL10 Coc 111. $\operatorname{callil} 1000$ 112. $\sin 210 \operatorname{coc}$ 114. Tsin.10 $\operatorname{coc}$ 115. $\operatorname{csin} 2000$ 116. $\operatorname{senh} 1000$ 117. Dersid CCC 110. Tarrid COC 110. $\cosh 10 \mathrm{CCC}$ 120. $\sin 10 \mathrm{CCC}$ 121. Danid CCC 122. TE2:10 CCC 123. $\cosh 10 \mathrm{COC}$ 126. SADL10 CCC
Me or Distnusurion

2004 goca verr. The garl to gum

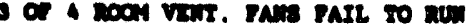

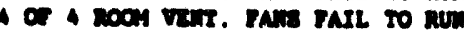

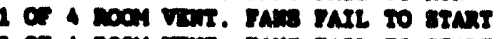

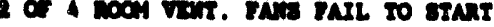

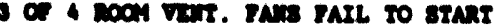

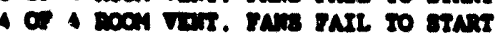

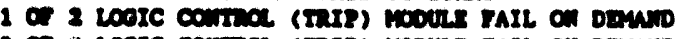

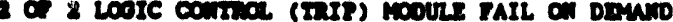

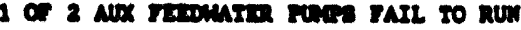

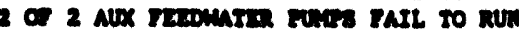

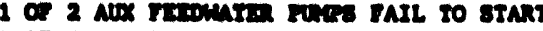

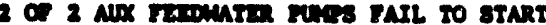

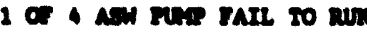

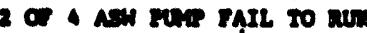

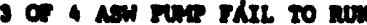

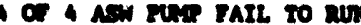

1 O 4 AN THR TAIL 20 etart

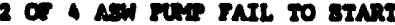

3 o. an pur rath to etant

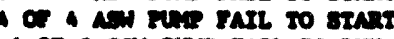

1 or a con rase rafr to and

2013 con proe rart to sur

3 o 3 con mas rarl 50 mm

1 o 3 con rust parl 50 stans

2 OI 3 con rap TAIL 20 stall

30 con mai rarh 50 stans

1 of 2 cunotmo purts raIl to gun

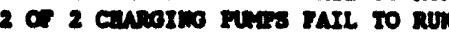

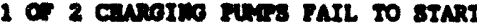

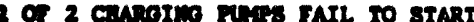

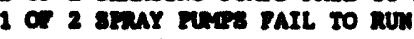

2 C 2 simaY raws rath to RUM

1 of 2 smaY Thips TaIL to start

2 of 2 srax furts rafl to stakt

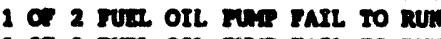

2 of 2 rve Orl rate rall to ram

1 of 2 rur orl war Yarl to stari

2 Of 2 rusL OIL TWR PAIL 20 START

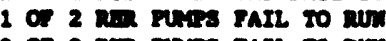

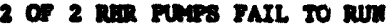

1 O 2 nuh gurps FaIL to stakt

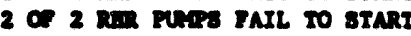

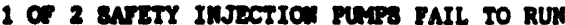

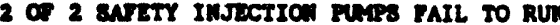

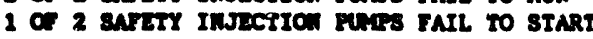

2 O 2 SATLTY IMJECTION FUTS TAIL TO START

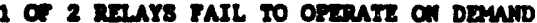

2 of 2 menars paIL to orevate on Demnd

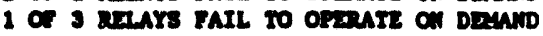

2 O 3 mars Parl to OFenate On DDMND

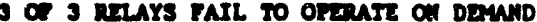

1 o 4 retars rall on dawnd

2 or a matays raIl on Dewro

3 or a maYs pall on Dewnod

- Co a relars raIl on dawdo

1 or 5 rmaYs paIl on dewid

2 of 5 retars raIL on daund

3 o s morars rarl on dammo

- Co 5 or 5 Renars rall on demud

1 o - Relars faIl on domid

2 Of Rerars gaIl ON Downd

3 or a relays paIl on demind

- Or - melars FaIl on Demind

1 or 11 melars rall on Demind

2 Of 11 meLaYs gaIl of DDMND

3 of 11 ReLAYs raIL On DEMND

- OR mose of 11 retays faIl ON DEMnid

1 OF 22 RELAYS FAIL OI DEMNo

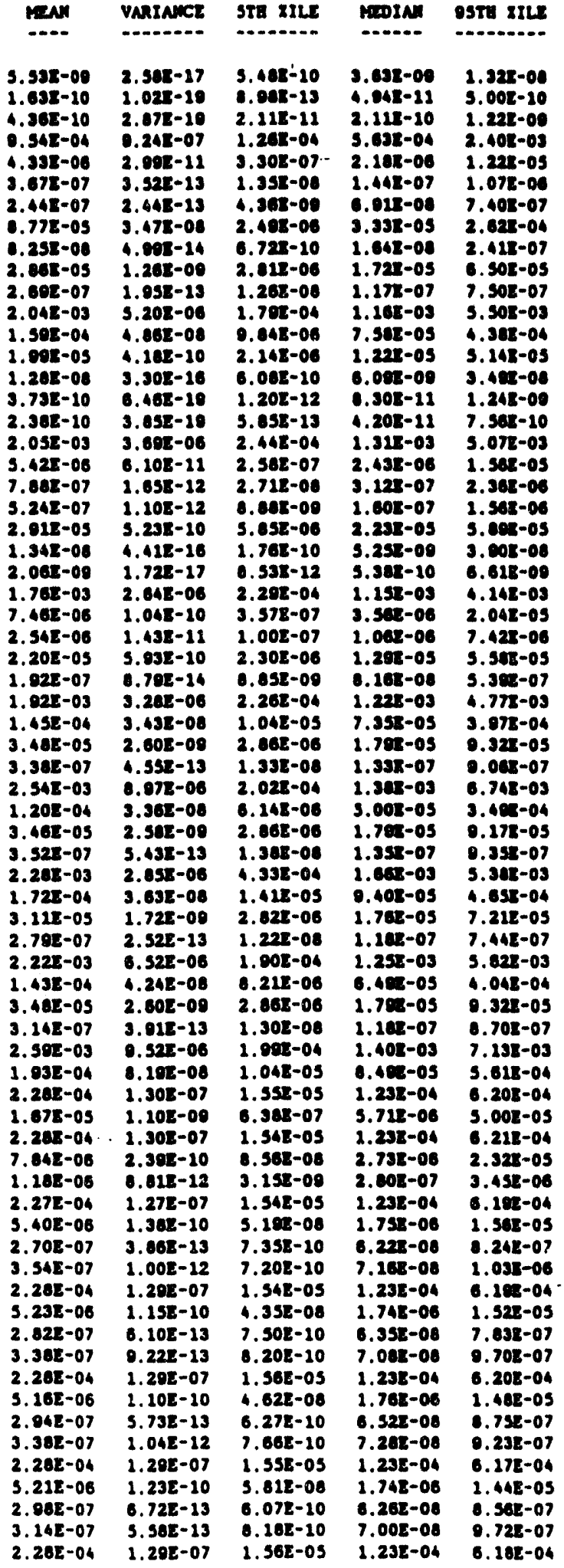


Aprenoux C

DCPRA oata gase

s.10.

123. Dinl20 coc

228. MILL $C 0$

127. Gisio coc

121. 82sted coc

120. D2stod coc

130. $22 \sin 000$

131. D2xind Coc

132. B3:50 coc

133. D3sino $\infty$

134. I3suno coc

135. S43ind CCC

136. DAsind CCe

137. T4sindo cec

130. Gasind coc

130. 82:120 coc

140. D2sind coc

141. 800ind coc

142. Doesind coc

143. mont coc

144. $\operatorname{cosin} 10 \mathrm{ccc}$

145. salno cos

146. Denive Cec

147. Tasinto coces

140. enenis coe

14. Exuted coc

150. Devies cos

151. evver c0e

152. Devace cec

153. 83vado coe

154. DJvaco CCe

155. T3Faco coc

153. sAvaco coc

157. DAvaco Coc

158. T4VMco cor

159. CAvLCo coc

19. 100100 co

101. Dovnco cos 182. Tovace coc 103. Govero cer 184. Exoos con 185. Drvoce cot 1C5. ssv000 000 197. pervos 0 1CA. Trvoo cos 160. s4v000 cen 170. DH weos ex 171. 24v00 con 172. asteos cos 173. suveo cor 176. Duteos con 175. T0w000 000 176. esteos cos 177. Eveos con 17. vincos cas 179. I0v000 Co: 109. Concos ces 181. Esticos con 182. Dewcos con 103. strves CCD 184. D4Vead CCD 185. T4Vea con 14. ब4V20 cos 107. s2moc CCD 1es. Davioc CCD 189. $824 \mathrm{~m} 00 \mathrm{CCD}$ 100. DrWino CCD 191. $224 \mathrm{rmo} \mathrm{CCD}$ 192. D2VTro CCD 193. $\$ 3 \mathrm{Vm} 10 \mathrm{CCO}$
MNE of DIstRrsurton

2 or 22 mars raIL on Dand

3 or 22 mats raIl on bernid

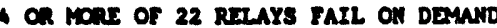

OF 2 RT stant IRIP CoILs FAIL ON DEMAND

2 or 2 RT SEUT IRIP COILS FAIL ON DEWND

2 or 2 arstantss raIt cn Derato

2 or 2 arsquats FAIL $\mathrm{cm}$ Danid

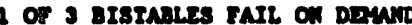

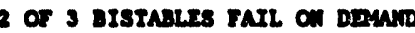

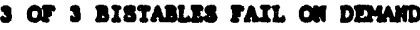

2 or a Dstants Fath on Dawn

2 or 4 Distants rafl a Dawn

3 or a bistabs garl an Dawk

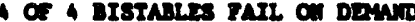

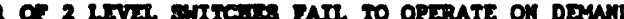

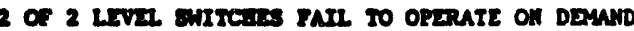

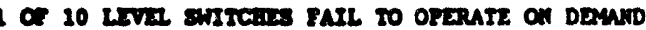

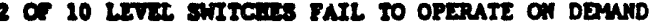

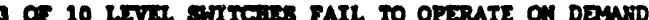

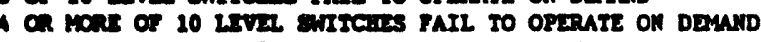

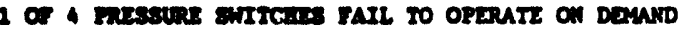

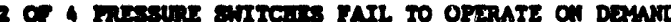

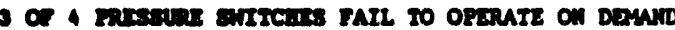

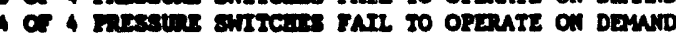

1 c. 2 R.I. iv cons FArL on Dawro

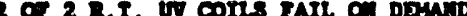

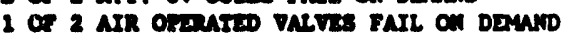

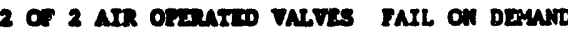

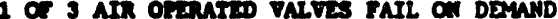

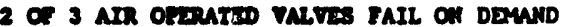

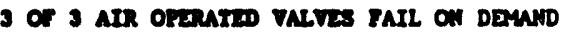

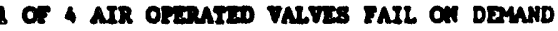

2 or 4 arr orante varves raIl on Danid

3 or 4 aIn ortated varves faIl on demand

- Or 10 arr Orgatro valves faIl on deMnd

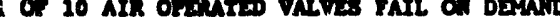

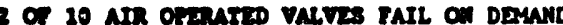

3 or 10 AIR orderte vatues FaIl on dewno

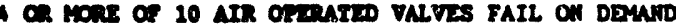

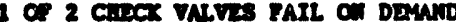

2 or 2 ciser valves raIl of Dewnd

1 or 3 Gaser varvis raIl on Dewn

2 co 3 cunces valves Farl on Dawis

3 or 3 cirser valves Farl on Daynid

1 or 4 Casex valves FAIl on DEshiD

2 of a cueck valves rarl of Dawio

3 o. Guder valves rath or Drand

- or a cidor valves raIl on danno

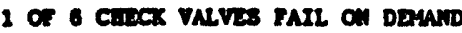

2 cr 8 Cules valves rall on Dawno

3 o. 6 crinct vanves rath on Dound

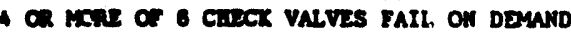

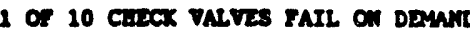

2 OF 10 Creec valves FaIl on Drand

3 or 10 cleex valves Fatl on Danno

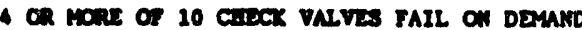

1 Or 2 ANA BTRM SUPTLX LIME VALVES FAIL ON DDMAND

2 OF 2 ANA STEAN SUPFLY LIML VALVES FAIL OH DXMAP

1 OF 4 TURATM CONTEOL/8TOR VALVES FAIL ON DEMND

2 Of IURAIR COMTBOL/stoP VALVES FAIL ON DEWND

3 OR 4 TURBTHE COISBOL/STOP VALVES FAIL ON DBMAND

4 OF 4 TUREIRE CONITOL/STOP VALVES FAIL ON DEMAHD

1 OR 2 moton OTRRATLD VALVES FAIL TO CLOSE OA DEMAND

2 OF 2 MOTOR OFERATED VALVES FAIL TO CLOSE OA DEMMND

1 OF 2 MOTOR OFtRATED VALVES FAIL TO OPEN ON DEMAN

2 OF 2 MOTOR OPLRATED VALVES FAIL TO OPEN ON DEMND

1 OF 2 PRESSURE RJCULATIKG VALVES FAIL TO OPEN ON DEMND

2 OF 2 FRESSURE RJeULATIME VALVES FAIL TO OREH ON DXMAND

1 of 3 RELIEF valves raIL to OPEA

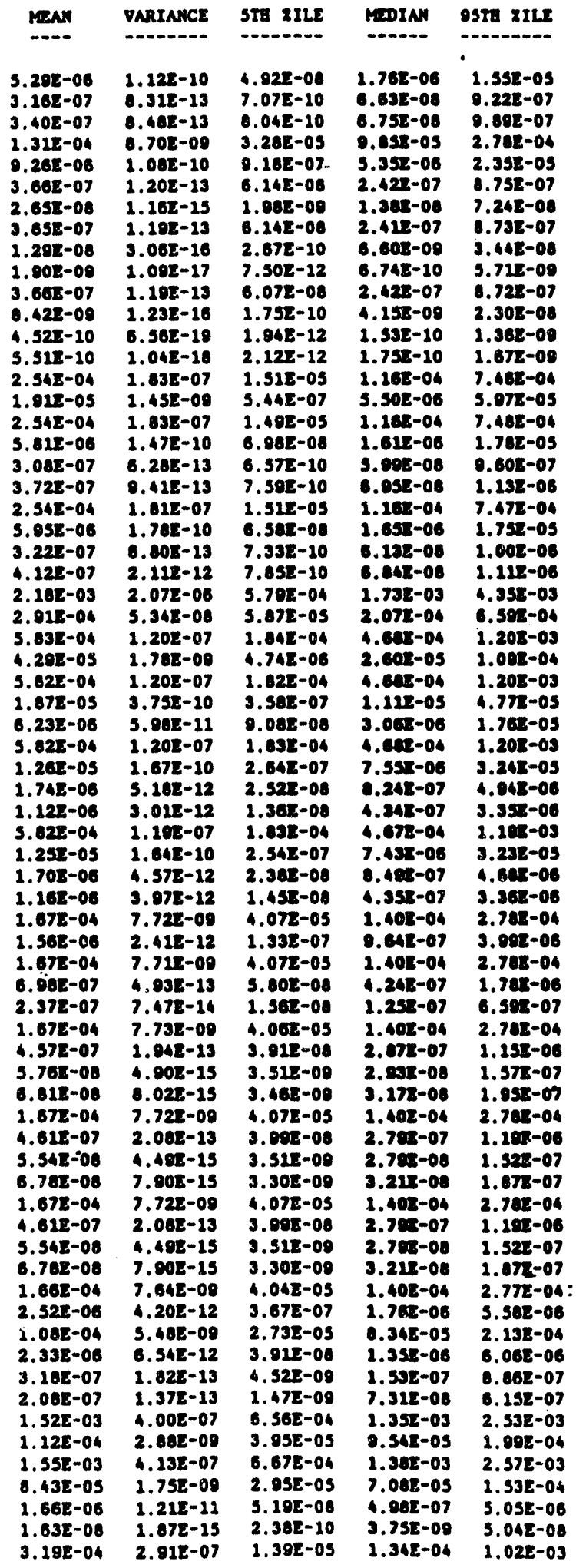


APPENDIXC

DCPRA DATA base

s.mo.

104. DJVR10 CC

195. I3VR10 cCD

106. SSVR10 CCD

197. DSVR10 CCD

108. ISVR10 CCD

100. GSVR1O CCD

200. S3VRIS CCD

201. DJVRIS CCD

202. I3VR1S CCD

203. SSVRIH CCD

204. DJVR2H CCD

205. TSVRIW CCD

206. 83VR3O CCD

207. DJViso CCD

208. T3VR30 COD

800. suviss CCD

210. DJva3s CCD

211. T3Vmas $\mathrm{CCD}$

212. 83VR3W CCD

213. DJVR3H CCD

214. T3VR3W cCD

215. S2VSOD CCD

218. Davsos CCD

217. $53 v 500 \mathrm{cCO}$

218. DJVECD CCD

210. r3vsOD CCD

220. ZTBATD 125V DC BATTERY - FAILURE OF OUTPUT OA DEMAND

222. ZTECHR BATMRY CEAPTIR - FAIL DURTHG OPARTIO

223. EIES1R DUSSES - FAIL DURIMO OPERATIOI

224. 2TCB1C CIRCUIT BREAKER (480 VAC AND ABOVE) - FAIL TO CLOSE ON DEMAND

225. ETC:20 CIRCUIT BRENCRR (480 VAC AND ADOVT) - FAILURE TO OPEN ON DEMAND

226. 2TCO1t CIRCUIT BREATXR (480VAC NTD ABOVE)-TRNUSFER OPEN DURING OPER.

227. 2TCe2C CIRCUIT BKR(AC OR DC.LT.480V) - FAILURE TO CLOSE ON DEMND

228. zTC220 CTnCUIT IKR(AC OR DC. IT 480V) - EATLIRE TO OPEN ON DEMAND

220. 2TCB2T CIRCUIT DREAKER ( $4480 V$ DC) IRAMSFER OPEN/CLOSED DUR. OPER.

230. 2ICB3D REACTOR IRIP BRENCXR MECBNISA - FAIL TO OPERATE ON DEMND

231. 2TCETR COARESSOR FEOA - FAIL TO RUI

232. ETCETS COMPRESSOR - FRTOM - FAIL TO START

233. ZTCRA SINGLE COIEROL ROD - FAIL TO IASERT ON DEMAND

234. CR MO MORE TRAM ONE CONTROL ROD FAILS

235. 2TDO2L RADOCY FATLUR: OF DISK LEADIRG TO LEAK RATE OF 150 GPM

236. 2T0021 RATDOA FAILURE OF DISK LEADIME TO LEAK RATE OF $1700 \mathrm{GPM}$

237. ITDOJL RARDCA FAILURE OF DISK LEADING TO LEAK RATE OF BOO GPM

238. ZTDAOD AIR OPERATED DAMPXR - FAIL TO OPERATE ON DEMAND

239. ZTDAOF AIR OPERATLD DAMPER - FAILURE TO IRAHSFER TO FAILED POSITION

240. ZTDAOT AIR OPERATED DAYPERS - TRAISFER OPEN/CLOSED

241. ZTDADD ACTDRAFT DAYPER - FAILURE TO OPEN OA DEAND

242. 2TDUDT MCTORAFT DAYPER - TRAMSFER CLOSED

243. ZTDFRI FIRE DAMPER - IMADVERTANT ACTUATION

244. ZTDESI DIESX GENXRATOR - FAIL TO RUN DURINO FIRST HR. OF OPERATION

243. ZTDGS2 DIESEL GENERATOR - FAIL TO RUI AFTER FIRST HOUR OF OPERATION

246. ZTDESS DIESEL GENERATOR - FAIL TO START

247. ZTOHOT MUIUAL DAMPLR - IRNISFER ORH/SHUT DURING OPERATION

248. ZTDEDD WOTOR OPERATLD DAMPXR - FAIL TO OPMRATE ON DEMAND

249. ZTOKOT EOTCR OPERATED DNAPERS - IRAMSFER OPEN/CLOSED

250. 2TDRYP AIR DRYER - FAILURE DURING OPERATION

251. ZTDSTD DISK FAILURE OA DENAD

252. ZTrLIP VENTILATION FILIER - PLUCOED

253. ITTL2P VENTILATIOA LOUVRE PLUGGED

254. ZTTL3P FUEL OIL FILTER - PLUSGED

255. 2TTHLR COATAIMELT FAN COOLERS - FAIL TO RUH

256. ZTHIS CONTAIMENT FAN COOLERS - FAIL TO START

257. ZTFW2R VERTILATION FANS - FAIL TO RUN

258. 2TFW2S VENTILATION FANS - FAIL TO START

250. 2TTU1R FUSE - FAIL OPEN DURIMG OPERATION

260. ZTEXR GEAT EXCHANGDR - EXCESSTVE LEAK, PLUCOING

261. ZTIMVR INVERTER - FAILURE DURIHG OPERATION

262. ITLCID IRIP LOGIC MODUTE - FAILURE TO IRIP ON DEMNND

\begin{tabular}{|c|c|c|c|c|}
\hline TSAN & VARIAHCE & STH RILE & MODIAN & 9STE XIL \\
\hline 6 & $258=$ & $\begin{array}{l}36 z-08 \\
63 z-09\end{array}$ & $\begin{array}{l}07 \\
08\end{array}$ & 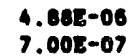 \\
\hline$E-04$ & $\begin{array}{l}01 E-07 \\
085-12\end{array}$ & $\begin{array}{l}39 z-05 \\
.805-08\end{array}$ & $\begin{array}{l}06 \\
07\end{array}$ & -0 \\
\hline$E-08$ & $03 E-14$ & $30 \mathrm{E}-10^{\circ}$ & 35 & $2.205-07$ \\
\hline $605-08$ & $90 E-14$ & $.308-10$ & 00 & $1.385-0$ \\
\hline $775-03$ & $.04 E-05$ & $.48 \tau-05$ & $1.118-03$ & 7.7 \\
\hline O4E-0S & $5.87 E-10$ & .525-07 & 2.87 & -0 \\
\hline $18 z-06$ & $.025-10$ & $.07 E-07$ & 1.6 & -0 s \\
\hline $03 z-02$ & $.20 E-02$ & $.095-03$ & $0.30 z-02$ & 2.3 \\
\hline 665-04 & $3.228-07$ & $.125-06$ & $1.512-04$ & -03 \\
\hline 57E-04 & $2.12 E-07$ & $3.34 E-06$ & 0.3 & 7.8 \\
\hline $.88 z-03$ & $7.695-06$ & $.528-04$ & $2.938-03$ & -03 \\
\hline $23 E-04$ & $1.918-08$ & $2.04 E-06$ & $6.868-05$ & 0 \\
\hline $092-05$ & $3.17 E-00$ & $5.87 E-07$ & 1.94E-05 & -04 \\
\hline $30 E-02$ & $.72 z-04$ & $5.66 z-03$ & $1.73 z-02$ & \\
\hline $38 E-04$ & 6. 84E-07 & $.90 \varepsilon-05$ & $4.262-04$ & -03 \\
\hline $46 E-04$ & $.005-07$ & $.40 \mathrm{E}-06$ & 1.1 & -04 \\
\hline $31 E-02$ & $.08 E-02$ & $.90 x-03$ & -02 & -01 \\
\hline $09 E-03$ & $2.31 E-05$ & $.74 E-05$ & 03 & $z-03$ \\
\hline $78 E-04$ & $2.03 E-06$ & $.42 E-06$ & 3.30 & $=-03$ \\
\hline $.21 E-03$ & $1.08 \mathrm{z}-05$ & $.725-05$ & 8.01 & E-03 \\
\hline 61E-04 & $.26 E-07$ & $.385-08$ & 4.60 & -04 \\
\hline $.20 E-$ & $1.03 \mathrm{E}-05$ & $7.70 z-05$ & 8.0 & -03 \\
\hline $.62 E-05$ & $3.64 E-08$ & $6.70 E-07$ & 2.1 & -04 \\
\hline $18 E-05$ & $1.278-08$ & $2.36 E-08$ & 2.0 & -05 \\
\hline $.84 E-04$ & $2.15 E-07$ & $7.51 z-05$ & 3.2 & $:-03$ \\
\hline $.09 E-07$ & $9.07 E-13$ & $.75 E-08$ & 3.7 & -06 \\
\hline $.50 E-06$ & $4.12 E-11$ & 5.7 & 3.9 & $=05$ \\
\hline $.48 E-07$ & $1.63 E-13$ & $7.398-08$ & $=08$ & -06 \\
\hline $.61 E-03$ & $3.94 E-06$ & $2.80 z-04$ & -03 & $:-03$ \\
\hline $.408-04$ & $6.545-07$ & $.055-05$ & 3.8 & -09 \\
\hline $28 E-07$ & $1.57 z-12$ & $.08 z-08$ & -07 & $3-06$ \\
\hline $27 E-04$ & $2.318-07$ & $3.48 z-06$ & $8.008-05$ & $E-04$ \\
\hline $398-04$ & $3.15 E-06$ & $2.39 \mathrm{E}-05$ & $3.285-04$ & $05-03$ \\
\hline 6 & -14 & -08 & $1.412-07$ & -07 \\
\hline 3 & $8-06$ & -04 & $5-03$ & $2-03$ \\
\hline 3 & 2. & 06 & $5-05$ & ? \\
\hline $35 z-03$ & $5.07 E-06$ & $.512-04$ & $3-03$ & Us \\
\hline $20 \mathrm{E}-05$ & $3.50 \mathrm{E}-08$ & $.00 E-06$ & $x=05$ & -0 \\
\hline $16 \Sigma-05$ & $1.425-09$ & & & \\
\hline $67 E$ & $1.01 E-08$ & $.64 E-05$ & $.705-04$ & -03 \\
\hline $15 z-05$ & $.23 E-08$ & $.498-06$ & $2.27 z-05$ & $E-0$ \\
\hline $38 E-C$ & $1.468-07$ & $.512-06$ & $3.672-05$ & E-04 \\
\hline$\Delta 12-04$ & -07 & $z-04$ & $=-04$ & -03 \\
\hline $66 \mathrm{E}$ & 07 & .06 & 14 & \\
\hline 55E & 7.0 & $8-06$ & $z-06$ & -06 \\
\hline & 1. & -05 & & $.2-06$ \\
\hline $04 E-08$ & $3.64 \Sigma-17$ & $2.43 E-08$ & $2-00$ & $2.105-08$ \\
\hline 208 & $0.025-1$ & $.60 z-09$ & -08 & $12-07$ \\
\hline $76 E-0$ & $E-0$ & $65 E-03$ & $=-03$ & -02 \\
\hline R & -06 & $.14 E-0$ & $1.435-03$ & -03 \\
\hline $59 \varepsilon-02$ & $3.85 E-05$ & 6.958-03 & $1.37 E-02$ & $2.725-02$ \\
\hline $20 E$ & 8.0 & $1.69 z-08$ & 1.4 & $1.32 x-07$ \\
\hline $67 \mathrm{E}$ & -06 & $1.03 E-03$ & -03 & 4.8 \\
\hline 7 & & & & $=08$ \\
\hline 008 & 4.1 & $3.04 E-08$ & $=-07$ & 2. \\
\hline & & & & -04 \\
\hline $.45 E-07$ & -12 & $3.04 E-08$ & 4.07 & $8-06$ \\
\hline 07 & s. & $2-08$ & 4.10 & $E=07$ \\
\hline 061 & 7 & $.04 E-0$ & $4.18 z-07$ & $3 z-08$ \\
\hline & & -06 & & $2.005-05$ \\
\hline 62 & 1. & $1.25 E-04$ & $1.225-03$ & $3.35 E-03$ \\
\hline & & -06 & 6.8 & $1.50 z-05$ \\
\hline 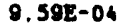 & -07 & $1.22 E-04$ & $5.528-04$ & $e-08$ \\
\hline & 0 & 2. $63 \mathrm{E}-08$ & $z-07$ & 2. $.83 E-00$ \\
\hline . & $5-12$ & $3.168-07$ & 1. & 3. \\
\hline . & 20 & 80 & & \\
\hline 52 & $8-08$ & $.43 E-06$ & $=-05$ & $2.44 z-0$ \\
\hline
\end{tabular}


263. ZTLCIR IRLP LOOTC MOOULE - TAILURE DURING OPERATION

264. ITPAN HOTOR OPLRATED NTW TURP - FAIL TO RUN

265. ITPANS MOTOR OPERATED APW PURP - BAIL TO START

2. 93E-06

$3.84 E-11$

$8.358-08$

$1.258-00$

$0.392-00$

2. $342-05$

$1.242-09$

2. $838-06$

$1.702-05$

$6.658-05$

$2.18 E-03 \quad 3.87 E-06 \quad 1.03 E-04 \quad 1.24 E-03 \quad 3.36 E-03$

266. ETPASR AUX SALTMATER TURP - FAIL TO RUM

267. ETPASS AUX SALTHATER PUEP - FAIL TO START

260. 2TPATR TURBIIL DRIVEX AWW PUAP - FAIL TO RUN

260. ITPATS TURAIRE DRIVUM AFW FUAR - FAIL TO START

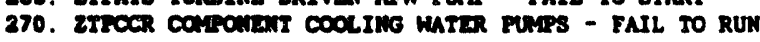

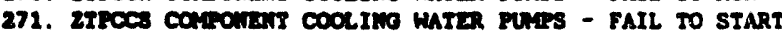

$1.978-05$

4. $18 \mathrm{E}-10$

$2.008-08$

$1.202-05$

$678-0$

$3.792-06$

2.395-04

$1.30 z-03$

$4.758-05$

$\begin{array}{lllll}.67 E-04 & 1.83 E-06 & 3.89 E-05 & 4.32 E-04 & 2.43 E-03\end{array}$

2.89E-05 $5.22 E-10 \quad 4.24 E-00 \quad 2.142-05$ S.44E-0S

$1.76 E-03 \quad 2.84 E-06 \quad 2.28 E-04 \quad 1.18 E-03 \quad 4.00 E-03$

2.20E-05 6.00E-10 2.17E-06 $1.30 \mathrm{E}-05 \quad 3.15 \mathrm{E}-05$

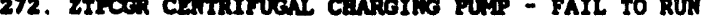

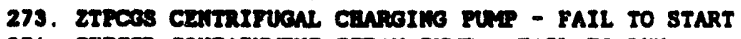

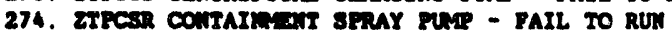

275. ZTPCSB COMTATMELent SPRAY PUIP - TAIL TO START

276. ETPTUR FUE OIL IRNASTER PUNP - TAIL TO RUH

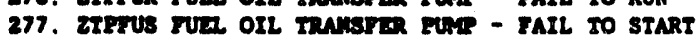

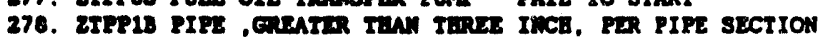

2.05E-03

$3.412-05$

$.78 E-06$

$2.392-04$

3. $192-03$

$2.838-03$

Q.72E-06

2.83z-08

$1.30 \mathrm{E}-03$

$3.395-05$

2. $41 E-00$

$2.002-04$

2. $44 E-03$

3. $38 \mathrm{E}-06$

2. $83 \mathrm{E}-08$

$8.60 E-10$

1. 65E-17

$3.605-04$

$0.60 \mathrm{E}-09$

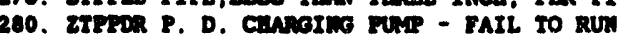

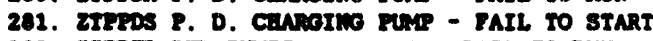

202. ITFRER RER PUATE 2-1, 1-2 - FAIL TO RUI

203. ETPEAs RER guap - TAIL TO START

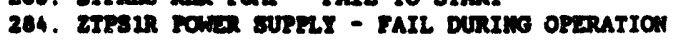

205. ZTTSIR SATETY IMJECTIOH PUSP - FAIL. TO RUI

286. 2TFBIS 8ATETY IMJECTIO PUAP - FAIL TO START

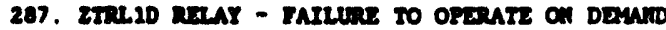

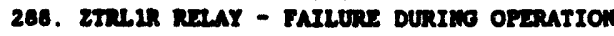

200. ZTSCIP ITMINER, OTIIAR TBAM AUX BALT WATER - FAILURE DURING OPERATIOM

$2.735-0.05$

$1.65 E-15$

$1.96 z-12$

$1.435-03$

6.10E-09

$1.802-05 \quad 8.238-05$

$1.732-03 \quad 3.082-03$

2.265-03

$1.278-09$

$1.98 E-11$

2.028-08

$3.06 E-05$

$4.68 \mathrm{E}-06$

2. $498-06$

$1.80=-09$

2.02E-08

2. 33E-03

1.71E-0s

.67E-09

2. $48 \mathrm{E}-04$

$1.495-05$

1. $40 \mathrm{E}-03$

7.04E-05

1.802-04

3. 41E-05

$0.04 E-10$

$1.1802-00$

2.76E-03

2. $47 E-08$

2. $835-06$

1.755-0s

6.10z-03

$7.25 \%-05$

2. 418-06

1.10z-05

2. 05E-06

$1.802-05$

$4.392-05$

4. 202-07

1. $47 \mathrm{E}-07$

1. $412 \mathrm{E}-0 \mathrm{~S}$

1.47E-03

-.19E-05

6. 22E-06

2. 67E-13

2. 83E-08

1.358-04

7.765-03

$4.075-08$

.47E-11

$0.082-07$

1. $002-07$

6. $40 \mathrm{E}-04$

8. 07E-06

$0.018-07$

6. 48E-07

3. $008-06$

1. $418-06$

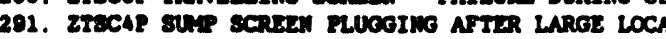

202. zTSCSP SUAP SCREEA RLVCOIM AFTER CORE MELT

293. ZTSEOC ESTAS/LOP SEOUEACER - DAMND

$4.04 E-02$

$3.462-03$

Q.518-05

2.015-06

$4.895-04$

5.71E-03 2.04E-02

$2.40 \mathrm{E}-06$

2. 37E-11

6. $035-08$

$1.765-14 \quad 2.70 z-00$

9.092-07

205. 2TSPRI TIRE SPRIMLER WEA IMADVERTAMT ACTUATION

296. 2TSTCD REACTOA TRIP BREAKER (SEUITI IRIP COIL)-FAIL TO OPERATE ON DEMAND

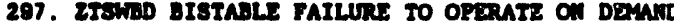

290. ZTEADI BISTABLE SPURIOUS OPERATIOA

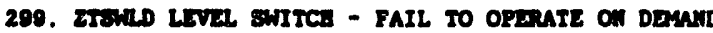

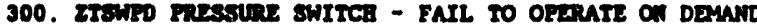

301. ITTK1B STOQhas IANK -- RUPTURE DURING OPARATIOY

302. ZTTRFR FLW TRUSEITTER - PAIL DURING OPERATION

303. ETTRLR LEVE TRAMSAITTER - FAILURE DURIMG OPERATION

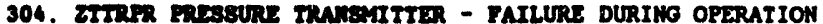

305. zTIRTR TEATEATURE TRANSMITTER - FAIL TO OPERATE ON DEMAND

306. zTUVED REACTOR IRIP ANEAKER UNDERVOLTAGE COIL - FAIL TO OPEN ON DEMAND

307. 2TVAOO AIR OPERATED VALVE - FAIL TO OPERATE ON DEMAND

308. 2TVMO AIR OTEUTED VALVE FAIL TO TRAMBELR TO FAILED POSITION OH DENAD

309. 2TVAOT ATR OPLRATED VALVES TRANBTER OPEW/CLOSED

310. 2TVCOO CHLCK VALVE (OTHIRR TANM STOP) - FAIL TO OPERATE ON DEMAND

311. ZTVCOL CEDCK VALVE (OTER TEAN STOR) - GROSS LEAKAGE DURING OPERATION

312. 2TVCOO MODNALLY OFA CEX VALVE (OTHIR TRAN STOP) - FAIL TO PESEAT

313. ETVCOP CBDOK VALVAS (OTHER TEN STOP) TRNISFER CLOSED/PLUGGED

314. ETVCSD STOP CuLCK VALVES - FAIL TO OPLRATE ON DEMAND

315. ETVCSL STOP CADCK VALVES - EXCESSIVE LEAKAGE

316. ETVCST STOP CHECK VALVES - TRANBFER OPEN/CLOSED

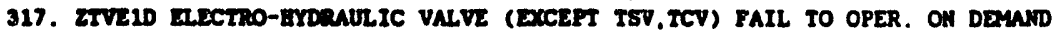

316. ZTVE1T ELECTBOHYORAULIC VALVES - TRAMBTER OPEN/CLOSED

319. ZTVE21 TUREIRE 3TOP/CONTROL VALVE - IRPR CLOSED DURING OPERATION

320. ZTVE22 TURBIME STOP/COWTRO VALVE IRANSFER OPEN DURING OPERATION

321. ZTVE2D TUREINE STOP/COMTROL VALVE EAILURE TO OPERATE ON DEMAND

322. ITVBOT MUNUAL VALVE TRAHSFERS CLOSED/OPEM

323. ZTVICX VALVE (HOTCR-OPZRATED OR CEDCK) - DISC RUPTURE

324. 2TVMOD MOTOR OPLRATED VALVE - FAIL TO OPERATE ON DEMAND

325. 2TMEL MOV PAILURE TO CLOSE ON DENAND WHILE SHOWIHG CLOSED

326. ZTVNOT MOTOR OPERATED VALVES IRAMSFER OPEN/CLOSED

327. 2TVECT PRESSURE CONTROL VALVE, SELF CONTAINED FAILURE DURING OPERATION

326. ZTVRRO FUEL OIL PRESSURE REGULATING VALVE, FAILURE TO OPEN ON DDMAND

329. ZTVPRT FUEL OIL BRESSURE REGULATIMG VALVE, TRANSFER CLOSED DURING OPERA

330. ZTVR1O PRIMURY SAFETY VALVE - FAILURE TO OPEN ON DEMAND

331. ZTVR1S PRIMARY SAFETY VALVE FAILURE TO RESEAT ON DEMAND (STEAM)

$1.398-04$

$1.475-12$

$2.705-09$
$1.10 E-07$

9.375-07

$118-00$

2. $435-03$

1.46x-01

3.025-00 $2.008-07$

$\begin{array}{llll}0.04 E-00 & 3.27 E-05 & 1.05 E-04 & 2.018-04 \\ 1.36 E-13 & 5.98 E-08 & 2.58 z-07 & 0.162-07\end{array}$

$1.518-10$

2.218-06

2.602-04

2. 56E-09

$.162-07$

2. $602-04$

2.662-08

6. 25E-06

1. $378-05$

1.418-05

$4.015-07$

$4.618-06$

2.09E-07 $\quad 1.41 E-05$

1.255-04

1.25E-0h

$7.602-04$

$3.17 E-15 \quad 7.50 E-10 \quad 1.04 E-08 \quad 7.63 E-08$

2.87E-11 6.03E-07

4. $1828-00$

$7.60 \mathrm{E}-06$

$1.205-10$

3.51E-06

1.125-05

1. $415-05$

$5.50 E-11 \quad 8.11 E-07$

1. 518-05

1. $20=-10$

3. 51E-06

$4.60 \mathrm{E}-06$

1.702-05

2. $462-03$

6. 22E-04

2.71E-08

6. $43 \mathrm{E}-04$

1.12E-05

3. 34E-05

1.41E-07

1. 58E-04

1.95E-03

$4.02 E-03$

2.668-04

2. 295-07

17:-07

7. 57E-08

5.008-04

1. 23E-03

1.53E-13 1.74E-08

$1.045-04$

7.625-04

5. 362-07

. 55E -00

$4.05 E-05$

1. 245-07

5. $912-07$

$4.26 E-04$

4. 152-13

0. 21E-08

1.412-04

2. 84E-04

1. n8E-06

1.108-05

3. 46. -07

1.34E-04

1.378-06

1.04E-08 5.60z-17

2. $43 E-09$

$7.00 \mathrm{E}-08$

1. 585-03

1.24E-00

$.07 E-05$

5.365-07

$1.042-00$

ค. 15E- 13

6. 21E-08

$4.025-04$

2. 105-08

5. 64E- -17

2. $43 E-00$

3.85-07

1. 378-0

. 12E-04

$26 \mathrm{E}-07$

.66E-04

7.80z-00

6.365-04

2. 102-08

.65E-07 
Napavoux $C$

DCPRA OATA BASE

S.Mo. MAN OF DIstrutBurion

332. ZTVLH FRIMRY SAFLXY VALVE - FAILUR TO RESEAT AFTER WATER RELIEF 333. ZTVLO RELIEF VALVE (EXCEPT PORV, BATLTY) FAILURE TO OPEN ON DEMAND 334. ITVET RLLIEF VALVE (EXCLFT FONV, sAFETY) - FRBATURE OPEN

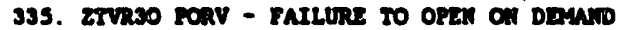

336. ZTVRs POAV - FAILURE TO RESEAT ATTAR STEAM RELIEF

337. ZTVR3 PORV - FAILUR TO RESEAT ATTER UATXR RELIEF

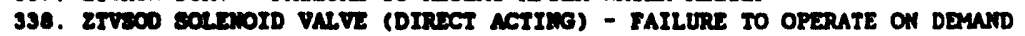

330. 2TVEOT SOLmoID VALVE (OIRECT ACIIMG) - IRAHSFER OPEM/CLOSED DURIMO OPER

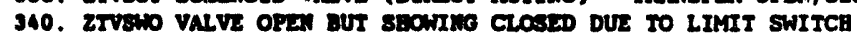

341. ZTVICD AIR ORER. ERESSURE CONTROL VALVE - FAIL TO OPERATE OA DEMND

342. ZTVTC AIR OPAR. FESSURE COMTROL VALVE - FAIL TO TRFR TO FAILED POS.

343. ZTVICT AIR OPER. FRESURE CONIROL VALVE - TRRR OPEN/SHUT DUR. OPERATIOA

344. ZTRIR IRANGPCiNA (MAIM, STARTUR, AUKILIARY) - FAILURE DURING OPERATION

345. ZTRER IRNIBICHigs (LOAD CENTER) - FAILURE DURING OPERATION

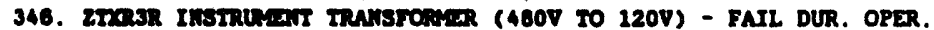

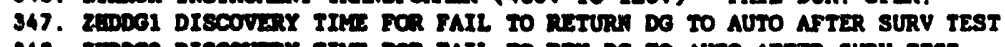

348. ZiDOE DISCOVARY TITE POR RAIL TO RTM DE TO AUTO AFTER SURV TEST

348. ziDDes DISCOV TIRE FOA FAIL TO RTI FTP ND LCV CIRLS TO AUTO ATTER TEST

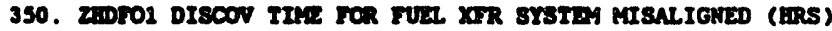

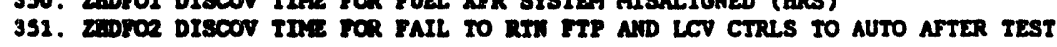

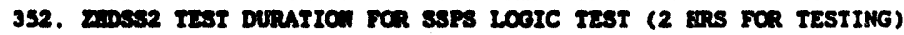

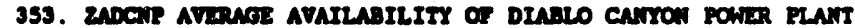

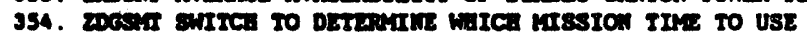

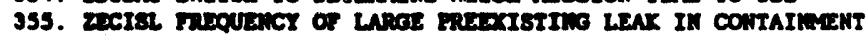

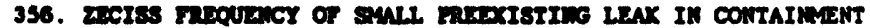

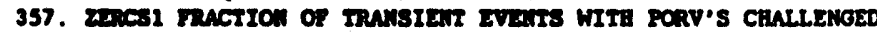

358. Easot whold or VALV LIFTS WLIL MT2R RELIEF - SGTR.SL

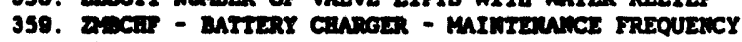

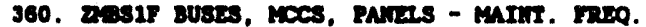

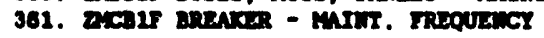

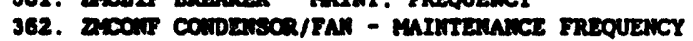

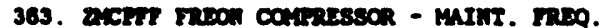

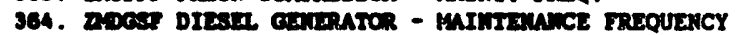

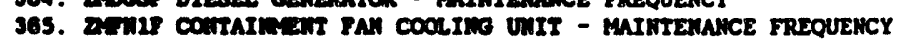

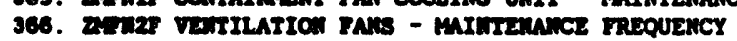

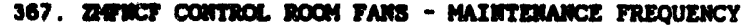

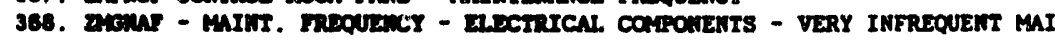

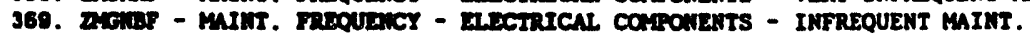

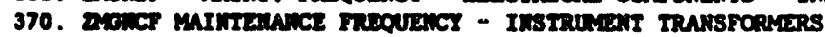

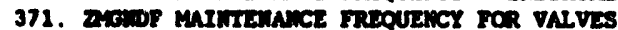

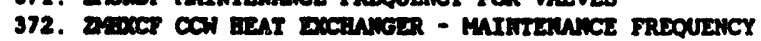

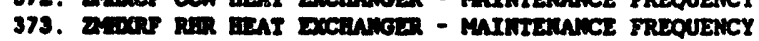

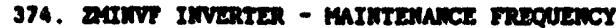

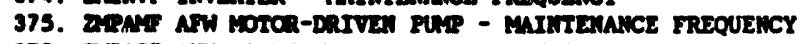

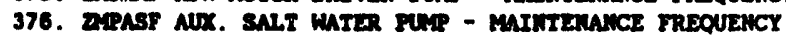

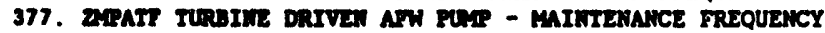

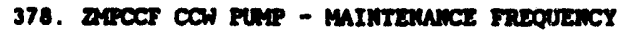

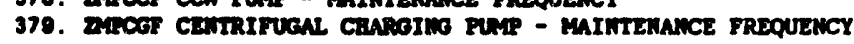

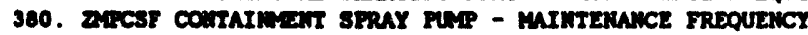

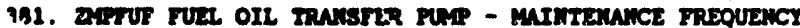

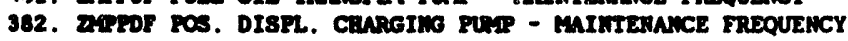

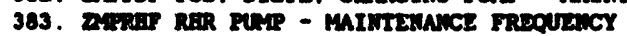

384. ZRSIF SAFETY InJECTIOA PLAP - MIMTEWANCE FREOUENCY

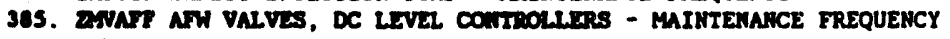

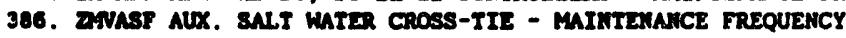

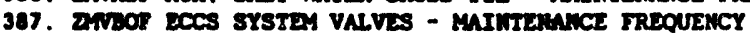

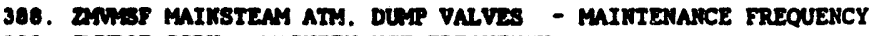

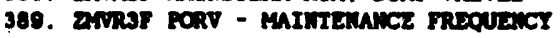

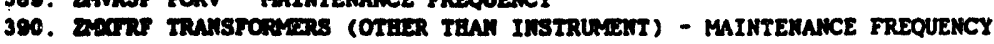

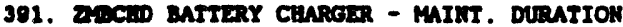

392. ancond Condenson/FAN - MInt. DURATIOA

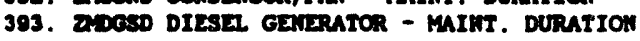

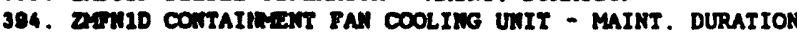

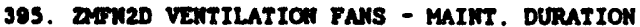

306. ZATICD COATROL ROCA FASS - MAIRT. DURATIOH

307. ZNEA2D MAIT. DURATION FOR VALVES WITH TECH SPEC LIMITS OF 24 HRS.

398. 2NEA30 MAINT. DURATIOA FOR VALVES WITH TECR SPEC LIMITS OF 72 HRS.

399. ZMENAD MAIT. DURA. FOR DOUIP. OTELA THAN VALVES, PUMPS, HXRS-72 HRS IS

400. 2XOASD MINT. DURA. FOR EQUIP. OTEER THAN VALVES, PUMPS, HXRS-SHORT
MEAN

\section{VARTAMCE}

$1.012-01$

$2.42 E-05$

6. 06E-08

$4.212-03$

2. $50 \mathrm{E}-02$

1. $01 \mathrm{E}-01$

$2.43 E-03$

1.27E-08

1.08E-04

$1.525-03$

2. 66E-04

2.675-07

1. $53 \mathrm{E}-06$

6.66E-07

$1.55 \mathrm{E}-08$

1. $725+00$

$1.672+00$

1. $38 x+01$

8. 51E+01

1. 38E+01

1. $17 E+00$

8. 502-01

$1.00 z+00$

1. 44E-03

3. $80 \mathrm{z}-03$

$1.00 E+00$

$4.80 \mathrm{E}+00$

2. 15E-05

1. $60 \mathrm{E}-06$

4. 80E-06

$0.005-05$

1. 56E-04

7. 74E-04

5. 0ot-05

1. 63E-04

3. 48E-04

2. 61E-06

2. 44E-05

4. 18E-00

2. 03E-05

$4.90 \mathrm{E}-04$

1. $025-05$

3. 608-05

5. 538-04

2. 80z-06

6. 08E-04

2. $90 \mathrm{z}-04$

2. $895-04$

2. 40E-04

2.30E-04

3. $485-04$

3.265-04

3.71E-04

5. 93E-05

๑.31E-05

4. $438-05$

1. 195-04

3.81E-05

2. 508-08

6. 14E+01

2. 11E+01

$1.01 E+01$

2. $08 E+01$

6. $395+01$

$3.38 E+01$

$4.05 E+00$

$1.88 E+01$

1.31E+01

$6.28 E+00$
1.25E-02

2. $44 E-08$

4. 24E-11

9.11E-06

$3.25 \mathrm{E}-04$

1. 25E-02

2. $44 z-05$

3. 32E-12

2. 20E-08

1. $895-06$

3. 17E-07

2. 535-13

2. $99 E-12$

$4.03 E-13$

7.08E-12

$1.66 z+00$

2. $13 E+00$

$4.60 \mathrm{E}+01$

$4.525+03$

$4.60 x+01$

1. $82 E-01$

$0.008-01$

$0.00 \mathrm{E}-01$

1.51E-05

5.37E-05

$0.00 E-01$

$3.468+01$

1. 10z- 10

$3.78 E-12$

1. $45 \mathrm{z}-11$

3. 62E-08

3.66E-08

2.335-08

$9.915-10$

4.01E-08

1.578-08

2. 365-11

4.84E-10

$6.32=-11$

3. 525-11

2. 27E-08

3. 58E- 10

5. 37z-10

2. 58t-08

1.172-08

7.585-08

8.56E-08

1.21E-08

1. $025=08$

$0.00 z-00$

2. 685-08

1. $47 \mathrm{E}-08$

1. 685-08

$1.128-00$

1.51E-00

0.33E-11

6. 98E-10

3. $492-10$

1.20E-11

1. $90 \mathrm{E}+02$

0.30z+01

3. $90 \mathrm{E}+00$

$6.05 E+01$

1.78E+03

1.34E+02

1. $13 E+01$

5. $97 \mathrm{E}+02$

$4.21 E+02$

4. $45 E+01$
5TH XIL

2. $80 E-03$

7. 55E-07

1.08E-06

. 95E-04

5. 85E-03

2. $88 \mathrm{E}-03$

7. 64E-05

.21E-08

1.75E-0S

2. 83E- 04

$7.57 \mathrm{E}-08$

1. 785-08

2. 83E-07

1.31E-07

7.445-08

6. 33E-02

6. 25E-02

2. $30 E+00$

3. $30 x+00$

$2.30 \mathrm{E}+00$

1.67E-01

1.25E-02

$1.00 E+00$

1.76E-0S

1.26E-04

5. 005-02

1.25E-01

$7.225-06$

$1.245-07$

5. 595-07

2. 25E-0s

1.298-05

5. 255-04

1. $49 \mathrm{E}-05$

- 22E-05

$1.62 \mathrm{E}-04$

1. A4E-07

2. 24E-06

1. 30z-07

1. 14E-05

2. 45E-04

1. 55E-06

1. 32E-05

3. 13E-04

1. 18E-04

3. 98E-04

1.37E-04

1. 10E-04

0.37E-05

6.12E-05

1.06E-04

1. 31E-04

1. 625-04

1. B5E-05

3. 58E-0S

2.75E-05

7. 84E-05

8. $90 \mathrm{E}-06$

1. 14E-07

3. $092+01$

$7.55 E+00$

$6.65 E+00$

0. $83 E+00$

1. $97 \mathrm{E}+02$

1. $48 E+01$

6. 83E-01

1. $54 E+00$

7. 84E-01

5. $46 E-01$

MIDIAY

100

$0.725-06$

3. $948-08$

. 285-03

1. 875-02 


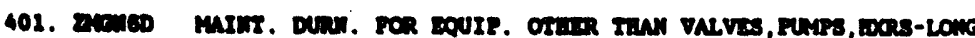

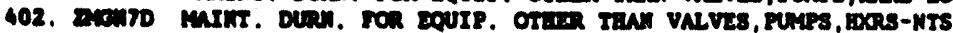

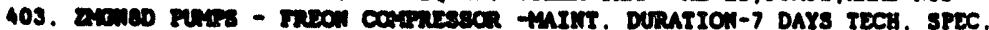

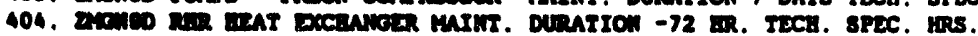

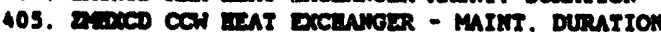

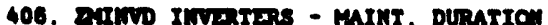

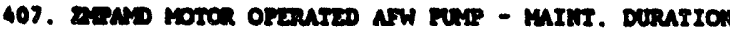

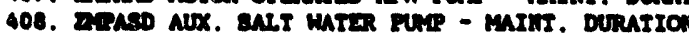

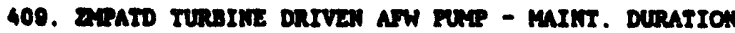

410. anCed CON FuRP - MINTEMANCE DURATION

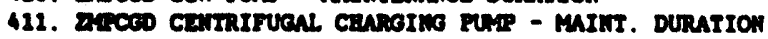

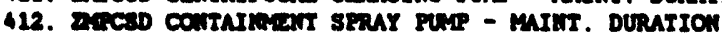

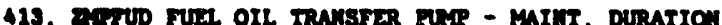

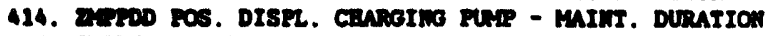

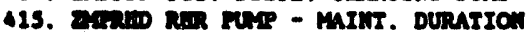

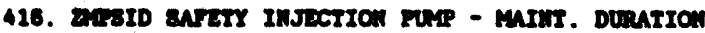

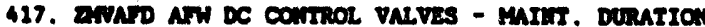

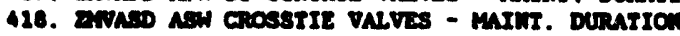

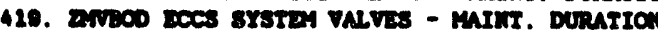

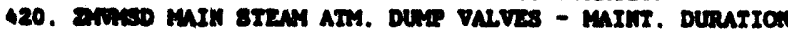

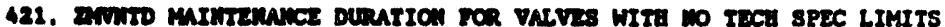

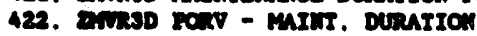

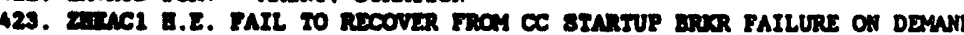

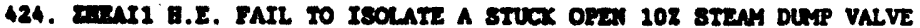

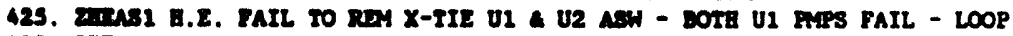

426. zanAs2 B.E. FAIL TO LOC X-TIE U1 \& U2 AEN CR YAIL TO OPEN X-TIE VALVE

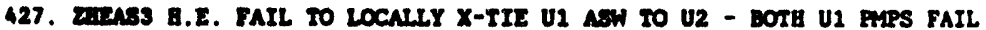

428. 2HDOC1 B.E. FAIL TO LOC ISOLATE GEU FOW WIEN ONRY 1 CCW PMP IS AVAIL

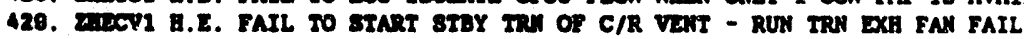

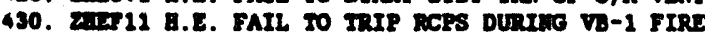

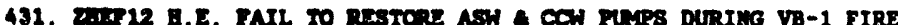

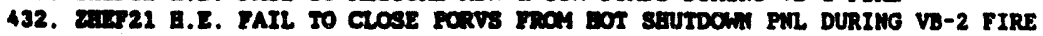

433. Lin 31 B.E. FAIL to CLOSE RORVS Fway EOT STDN PAL - VB-2/VB-3 FIRE

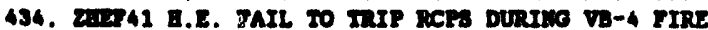

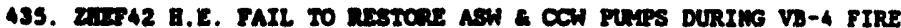

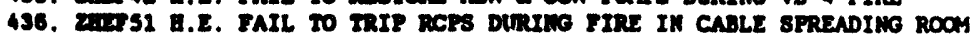

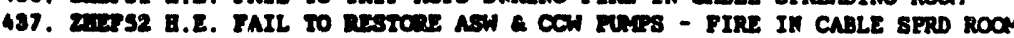

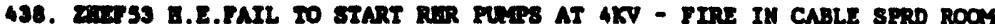

430. 2mP61 B.E.TAIL TO CLOSE PORVS FRON BOT STDH PML - FIRE IN CBL SPRD ROO

440. 20004 G.E. FAIL TO REALIGH FUEL XRR PP POWLR SOURCE TO OPPOSITE UNIT

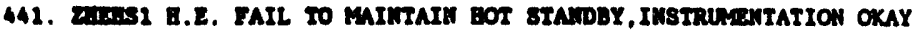

442. ZadAB2 FAIL TO InSTALL PORTABLE GLULRATCR TO PROVIDE VITAL AC

443. ZULAB3 B.E. BANE AS FOR ZELES1, BUT AFTER A LOCA

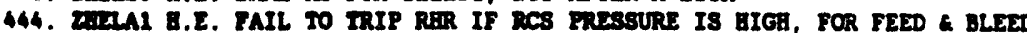

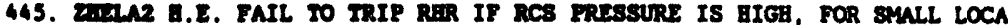

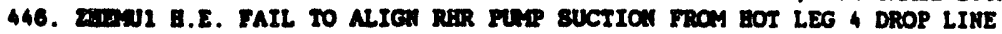

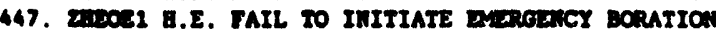

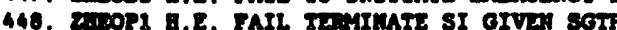

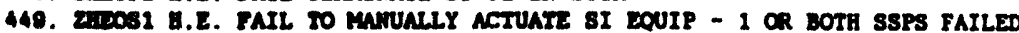

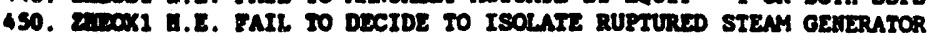

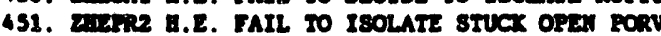

452. E.dHR3 g.E. FAIL TO ISOLATE AM ISOLABLE LOCA WITH PORV BLOCK VALVE

453. ZESTRA B.E. FAIL TO ISOLATE STUCK OPEA PORV W/O RX TRIP

454. LaAE2 घ.E. FAIL TO X-TIE 2 VITAL DUSES - STATIOW BLACKOUT \& RECOVERY

455. EUANEA M.E. TAIL TO $X$-TIE VITAL POWIR SOURCE TO FUEL OIL TRAYSFER PUMP

458. ZIDES M.E. FAIL TO DEPRESS S/OS DURAMO BTATION BLACKOUT (SEISMIC COND)

457. 2.1.NT B.E. TAIL TO SHITCE TO RECIRC FROM INJ MOOE (ECCS) SLOCA W/O CS

456. zearr2 घ.E. FAIL TO SHITCH TO RECIRC FROM IMJ HODE (ECCS) MLOCA/LLOCA

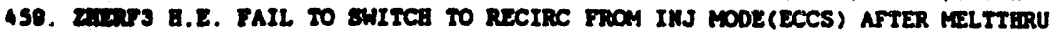

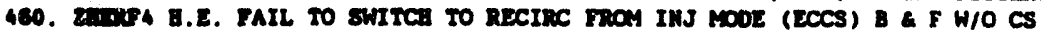

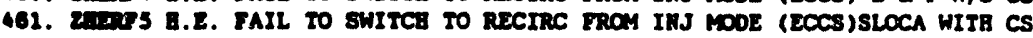

462. ZIIT1 a.2. FAILURE TO TRIP RCPS GIVEM CCW FAILS

463. Eusms1 B.E. FAIL TO SBUTDOWN REACTOR W ROOS WITHIM 10 MINS

464. ZMART H.E. FAIL TO RRESS MUTUAL PUSH BUTTOH TO IRIP REACTOR

465. ZUART2 B.E. FAIL TO DEEMLRTILE RPS BUS TO IRIP REACTOR

466. 2monol B.E. FAIL TO ISOLATE A STUCK OPEA SAFETY VALVE

467. DULaR1 H.E. FAIL TO ALIGH FOR CS RDCIRC - SUMP RECIRC SUCCESS

468. ZELSV2 H.E. FAIL TO OPEN DOORS TO INVERTER \& 4 BOV SWITCHGEAR ROOWS

460. LHESWl B.E. FAIL TO REALIOT SWTMG DG TO OPPOSITE UNIT
MENH

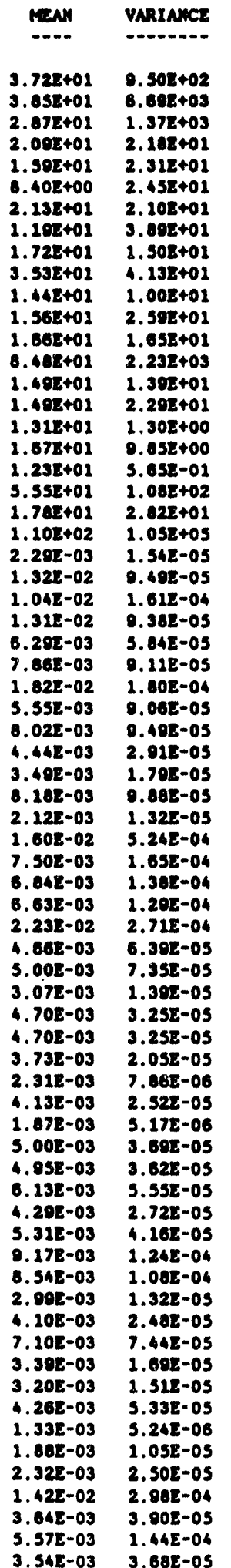

STB 2ILE

$0.208+00$

$1.378+00$

$2.305+00$

$2.21 \mathrm{E}+01$

$7.778+00$

$2.285+00$

$1.362+02$

$3.60 z+00$

$1.092+01$

2. $435+01$

Q.122 +00

$7.308+00$

9. $462+00$

$2.168+01$

$7.20 z+00$

6. $615+00$

$1.12 x+01$

$1.162+01$

$1.002+01$

3. $702+01$

$7.21 E+00$

4.542-01

1.352-04

3. $415-03$

1. 23E-03

3. $392-03$

7. 425-04

9.265-04

4.692-03

3.202-04

0.455-04

5.23E-04

4.112-04

$0.655-04$

1.255-04

$7.002-04$

4. 44E-04

4.05E-04

3.03E-04

5.75E-03

2.768-04

2. $985-04$

3.612-04

3. 54E-04

5. 54E-04

$4.302-04$

2.728-04

4.878-04

2.218-04

5. 90 E-04

5. 84E-04

7. 23E-04

5.068-04

6.268-04

1.085-03

1.018-03

3. 525-04

4. 835-04

8. 372-04

$4.002-04$

3.77E-04

2. 525-04

7.90E-05

1.12E-04

8. 125-05

1.68E-03

2. $168-04$

1. 03E-04

2. 09E-04
MEDIAN

9STH 2ILE

$2.752+01 \quad 7.41 E+01$

$1.372+01 \quad 1.17 E+02$

$1.57 E+02 \quad 7.27 E+01$

$1.048+02 \quad 2.77 z+01$

$1.405+012.362+01$

$.052+001.675+01$

$2.05 x+01 \quad 2.03 x+01$

$0.000+00 \quad 2.17 E+01$

$1.65 z+01 \quad 2.33 z+01$

$3.35 E+01 \quad 4.55 z+01$

$1.372+01 \quad 1.058+01$

$1.458+012.352+01$

$1.502+01 \quad 2.32 E+01$

$7.003+01 \quad 1.50 z+02$

$1.418+012.202+01$

$1.303+01 \quad 2.278+01$

$1.205+01 \quad 1.402+01$

$1.50 t+012.10 z+01$

$1.222+01 \quad 1.332+01$

$5.375+01 \quad 7.292+01$

$1.705+012.662+01$

$1.642+02 \quad 4.128+02$

$1.058-03 \quad 7.062-03$

$1.045-02 \quad 3.115-02$

6.335-03 3.15E-02

1.04E-02 3.00E-02

$3.825-03 \quad 1.005-02$

..77E-03 2.37E-02

1.44E-02 $4.205-02$

2.558-0.3 $1.018-02$

. $800-03 \quad 2.425-02$

2.60z-03 1.34E-02

2.11E-03 1.05E-02

$0.00303 \quad 2.478-02$

0.73E-04 $\quad 7.27 z-03$

6.135-03 4.508-02

3.458-03 2.582-02

$3.148-03 \quad 2.358-02$

3.05E-03 2.265-02

$1.762-02 \quad 5.258-02$

$2.14 \mathrm{E}-03 \quad 1.60 \mathrm{E}-02$

2.302-03 1.725-02

1.865-03 $9.255-03$

2.858-03 $1.428-02$

2. $258-03 \quad 1.428-02$

2.268-03 $1.12 E-02$

1.40E-03 6.98E-03

$2.505-03 \quad 1.258-02$

1.14E-03 5.65E-03

3.032-03 1.518-02

$3.008-03 \quad 1.408-02$

$3.725-03 \quad 1.058-02$

2.605-03 1.205-02

3.225-03 1.605-02

5. 505-03 2.77E-02

5.10E-03 2.305-02

1.818-03 $0.012-03$

$2.402-03 \quad 1.24 \mathrm{E}-02$

$4.318-03 \quad 2.14 \mathrm{E}-02$

2.05E-03 $1.02 E-02$

1.94E-03 $\quad 0.64 E-03$

$1.968-03 \quad 1.462-02$

6.13E-04 4.50E-03

$0.66 E-04 \quad 6.47 E-03$

0.44E-04. $0.428-03$

$0.628-03 \quad 4.208-02$

1.67E-03 1.25E-02

2.03E-03 2.02E-02

1.63E-03 $1.21 E-02$ 
NPFendux C

copan Data anse

s.no.

MASE OF DISTRINUT1OA

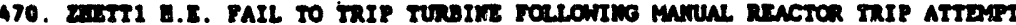

471. zETT2 घ.E. FAIL TO TRIP TUROIRE W/EO mWNAL REACTOR TRIP ATTDAPT

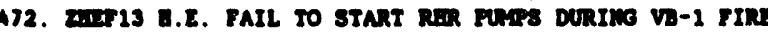

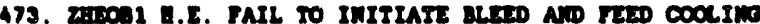

474. LEDOAl G.E. FAll TO COWrROR ANH FLON TO RUPTURED STENM GENERATOR

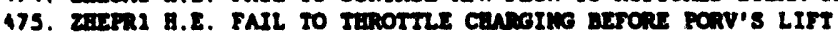

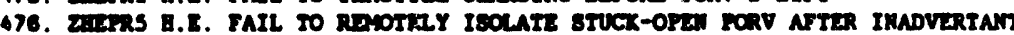

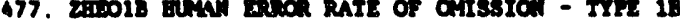

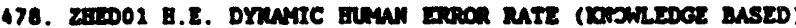

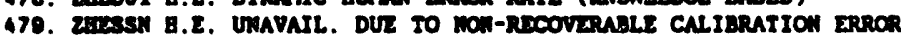

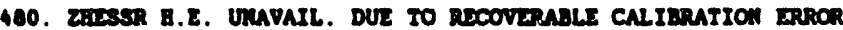

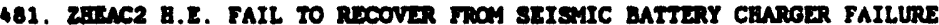

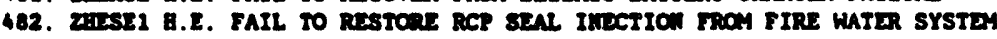

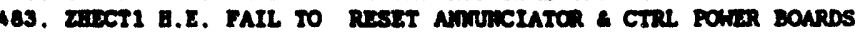

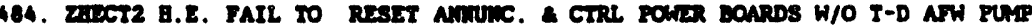

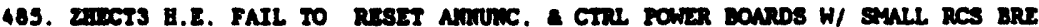

486. ZHECTA B.E. TAIL TO RESET ANNUINC. C CTRL POWLR BOARDS W/ BOTE OF TEE A

467. zaLPOS H.E. FAIL TO REALIOA PVEL OIL TTR PUMP GIVEW 1 LOSS OF POWER, 1

488. ZMT00 E.E. FAIL TO ALICY A DXDICATID, PCRTABLE FUR. OIL TRANSTER PUMP

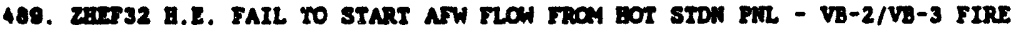

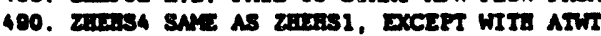

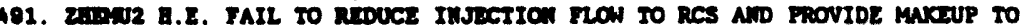

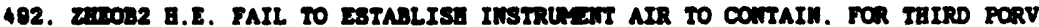

493. z.teck1 B.E. FAIL to COOL DOW AND DEFressurire RCS

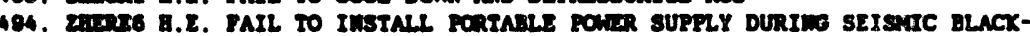

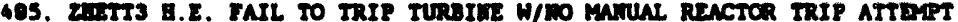

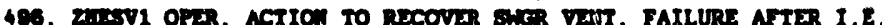

407. zCaLde comraiment BurLDIng

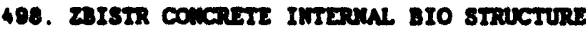

400. ZIIETR IITAKE STRUCTURE

500. ZNBLDG AUXILIARY BUILDING

501. ETBSER TUROIWE BUTLDTMG STEAR WNL

502. ZESTK REVUE.ING MATER STORAGE TAMK

503. ZASPIP ACX. SALTMATER PIPINO

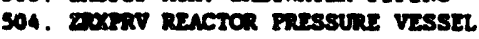

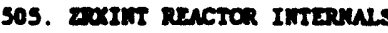

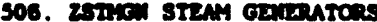

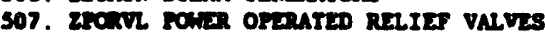

s0s. anctie rencton coorntr plaps

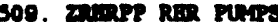

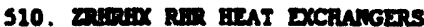

511. zsince 81 nccuivinatoss

512. 2001TK BOEOW IMJECTION IANR

513. roONPP CCH PUAPS

514. roontx COW HEAT ExCaniczrs

515. ZeCirTK CCW SUREE IANX

516. 203par CS plemps

517. ZSRATK SERAY ADOITIVE TAMK

538. ZSBDRR ANW PUAPS (STENM DRIVEN)

510. 2DCFPH DC FUER OIL PLAPE, IIITTE

520. zostem DIESEL GENARATORs

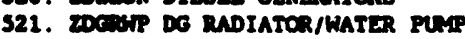

322. zDCExC DG Excitation CUDICAL

523. 2DECPR DE COMrROA PNIEI

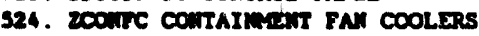

525. ZSUEFI SUPPLY FAN

326. ZENTT SUPPLY/RETURN FAYS

527. ZEMETR AKV SHITCBCEAR

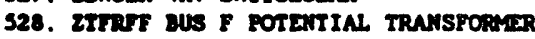

329. zosTER SAFECUNAD RELAY PANEL

530. ZaATRY BATTERIES

531. ZaTCE BATTERY CRARGERS

332. 2SUEDP SWITCBGEAR/BREAKCRR PANET

533. ZIWVTR INVERTERS

534. ZTRAHS 4180/480V TRNISFORELRS

535. ZNRTM AUXILIARY RELAY PANEL

536. ancirts MAIM CONTROL BOARDS

537. ZESPML HOT SEUTDON PANER

530. 2PCAPS PROCESS CONTROL AND PROTECTION CABIMETS

\section{MEN}

3. $182-03$

$0.835-03$

7.012-03

$1.362-02$

1. 325-02

1.28E-02

$4.505-03$

$4.708-03$

1.00E-01

$4.012-04$

$2.095-06$

$3.018-03$

$1.00 E-02$

$1.902-03$

$4.002-03$

$4.002-03$

$8.002-03$

2.00E-02

$4.00 \mathrm{E}-02$

6. $502-03$

6. 15E-03

0.00E-03

8.005-02

$7.60 \mathrm{E}-02$

$0.90 \mathrm{z}-03$

5. 32E-02

$6.835-04$

$0.00 z-01$

$1.00 z+00$

$0.002-02$

$0.008-01$

$0.00 \mathrm{E}-01$

$0.005-01$

$0.005-01$

$0.002-02$

0.00 E-01

$0.008-01$

$0.00 E-01$

$0.005-01$

$0.00 E-01$

$0.005-01$

$0.00 E-01$

$0.008-01$

$0.002-01$

$0.005-02$

$0.00 E-01$

$0.008-01$

$0.00 E-01$

$0.00 \mathrm{E}-01$

$0.005-01$

$0.00 E-01$

$0.00 E-01$

$0.002-01$

$0.002-01$

$0.002-01$

$0.002-01$

$0.005-01$

$0.00 E-01$

$0.00 E-01$

$0.00 \mathrm{E}-02$

$0.00 E-01$

$0.00 E-01$

$0.00 E-01$

$0.005-01$

$0.005-01$

$0.00 E-01$

$0.008-01$

$0.008-01$

0.00 E-01
VARIAICE

405-05

20:-04

1. $452-04$

3. $808-04$

. 58E-04

.35E-04

. 005-05

. 768-0s

1. 10E-02

. 68z-08

1. $18 \mathrm{E}-12$

4. 22E-05

5. 43E-05

1. 17E-05

4.71E-05

.72E-05

$1.00 z-04$

2. 17E-04

2. 365-03

1.24E-04

1. 23E-04

1.80E-04

$3.47 E-03$

$4.588-03$

2.94E-04

3. 83E-03

5. 965-08

$0.002-01$

$0.005-01$

$0.005-01$

$0.00 z-01$

$0.002-01$

$0.005-01$

$0.00 \mathrm{r}-01$

$0.00 \mathrm{E}-01$

$0.005-01$

$0.00 \mathrm{z}-01$

$0.005-01$

$0.002-01$

$0.005-01$

$0.005-01$

$0.002-01$

$0.002-01$

$0.005-02$

$0.002-01$

$0.002-01$

$0.008-01$

$0.002-01$

0.008-01

$0.005-01$

$0.005-01$

$0.008-01$

$0.002-01$

$0.005-01$

$0.005-01$

0.008-01

$0.005-01$

$0.005-01$

$0.00 \mathrm{E}-01$

$0.00 z-01$

$0.00 E-01$

$0.008-01$

$0.008-01$

$0,005-02$

$0.005-01$

$0.00 \mathrm{E}-01$

$0.008-01$

$0.00 \mathrm{E}-01$

$0.00 \mathrm{E}-01$
STH IILE

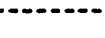

.735-04

235-04

4. 13E-04

$6.73 E-04$

. 53E-04

. 32E-04

5. 31E-04

5. 40z-04

.025-02

$3.60 \mathrm{E}-05$

1.00 E-07

.05E-04

2. 57E-03

1. 18E-04

2.378-04

.37E-04

4. 74E-04

5. 25E-03

$72=03$

3. 858-04

1. 80E-04

4.74 E-04

$.06 z-02$

2.34E-03

5. $925-04$

2. 14E-03

. 972-06

$0.002-01$

$1.00 E+00$

$005-01$

$0.002-01$

$0.005-01$

$0.005-01$

$0.005-01$

$0.005-02$

$0.005-02$

$0.005-01$

$0.005-01$

$0.002-01$

$0.00 z-01$

$0.005-01$

$0.002-01$

$0.002-01$

$0.005-01$

$0.00 \mathrm{E}-01$

$0.008-01$

$0.005-01$

$0.00 \mathrm{E}-01$

$0.005-01$

$0.005-01$

$0.005-01$

$0.002-01$

$0.005-01$

$0.00 \mathrm{z}-01$

0.002-01

0.008-01

$0.008-01$

$0.005-01$

0.00E-01

$0.002-01$

$0.005-01$

$0.005-01$

$0.00 \mathrm{E}-01$

$0.00 E-01$

$0.005-01$

0.00 E-01

$0.00 E-01$

$0.00 E-01$

$0.002-01$

MaxaM

95TE RILE

$1.022-03 \quad 0.542-03$

1.062-03 3.03E-02

3.22E-03 2.41E-02 
APEENOXX C

DCPra Data ease

S.10. $\quad$ mage or DIstrisurion

539. RTENE REACTCR IRIP SitrChOSAR

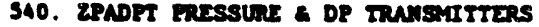

541. ZTMUS IHITLSE LIMTS

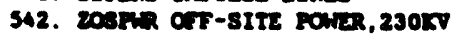

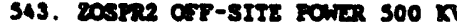

544. acopts DOP PIPING ND sUPFonTs

S4s. zerein Fenteratroms

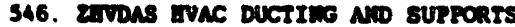

S47. Ginge2 SintCGOEAR /STRUT FAILTD

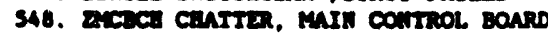

s49. zocrea cantres, of conmol pantl.

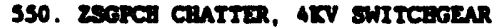

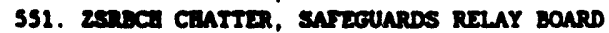

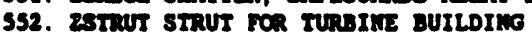

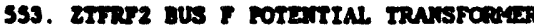

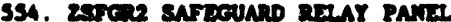

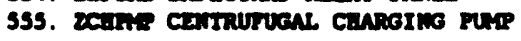

MEAH VARIANCE STE TILE MLDTA gSTE TILE

\begin{tabular}{|c|c|c|c|c|}
\hline $\begin{array}{l}.005-01 \\
.005-01\end{array}$ & $\begin{array}{l}0.002-01 \\
0.005-01\end{array}$ & $\begin{array}{l}0.00 z-01 \\
0.00 \Sigma-01\end{array}$ & $\begin{array}{l}0.00 z-01 \\
0.00 z-01\end{array}$ & $\begin{array}{l}0.00 z-01 \\
0.00 E-01\end{array}$ \\
\hline $\begin{array}{l}.00 z-02 \\
.00 z-01\end{array}$ & $\begin{array}{l}0.00 z-01 \\
0.00 z-01\end{array}$ & $\begin{array}{l}0.00 \mathrm{E}-01 \\
0.00 \mathrm{E}-01\end{array}$ & $\begin{array}{l}0.00 E-01 \\
0.00 E-01\end{array}$ & $\begin{array}{l}0.00 E-01 \\
0.00 E-01\end{array}$ \\
\hline $002-01$ & $0.002-01$ & $0.00 \mathrm{E}-01$ & $0.002-01$ & $0.00 E-01$ \\
\hline $002-01$ & $0.00 E-01$ & $0.008-01$ & $0.002-01$ & $0.008-01$ \\
\hline $002-01$ & $0.00 z-02$ & $0.00 E-01$ & $0.002-01$ & $0.005-01$ \\
\hline $.002-02$ & $2 z-01$ & $0.00 z-01$ & $0.002-01$ & $0.00 \varepsilon-01$ \\
\hline $.008-01$ & $0.008-01$ & $0.008-01$ & $0.008-01$ & $0.00 \mathrm{E}-01$ \\
\hline $002-01$ & $0.005-01$ & $0.00 t-01$ & $0.002-01$ & $0.002-01$ \\
\hline $.00 E-02$ & $0 z-01$ & $0.00 E-01$ & $0.002-02$ & $0.00 z-02$ \\
\hline $00 \mathrm{E}-01$ & $=-01$ & $0.00 \mathrm{E}-01$ & $.005-01$ & $0.00 z-01$ \\
\hline $.002-01$ & -01 & $0.00 z-01$ & $0.008-01$ & $0.002-01$ \\
\hline $.00 \mathrm{E}-01$ & 0.0 & $0.00 \mathrm{E}-01$ & $0.00 E-01$ & 0.00 \\
\hline $.002-01$ & $0.005-01$ & $0.00 \mathrm{E}-01$ & $0.008-01$ & $0.005-01$ \\
\hline $.00 \mathrm{E}-01$ & 01 & $0.00 \mathrm{E}-01$ & $0.002-02$ & $0.00 z-01$ \\
\hline $.008-01$ & $0.002-01$ & $0.00 \mathrm{E}-01$ & $0.00 z-01$ & $0.008-02$ \\
\hline
\end{tabular}




\section{APPENDIX D \\ DCPRA DOMINANT ACCIDENT SEQUENCE MODEL}

D1: Description of the Diablo Canyon Reduced Core Damage Frequency Model

D2: Pair Importance Calculations by Conditional Split Fractions 
Appendix D1: Description of the Diablo Canyon Reduced Core Damage Frequency Model

\section{D1.1 Introduction}

As discussed in Section 3.2, BNL proposed to develop a reduced core damage frequency model composed of the leading accident sequences in order to pursue insights into the DCPRA and/or the plant itself. As it turned out, when BNL requested that PG\&E supply a listing of the dominant accident sequences totaling a minimum $80-90 \%$ of the internal events core damage frequency, BNL was informed that PG\&E was developing its own reduced model and it was offered to BNL. The PG\&E reduced model included both the internal as well as the leading non-seismic external event dominant contributors and covered approximately $88 \%$ of the total non-seismic core damage frequency as determined by the DCPRA.

BNL subsequently requested a brief description of the PG\&E dominant sequence model and was provided with the following which we quote here without further comment:

"Non-Seismic Dominant Sequence Model Development - The dominant sequence model is a compilation of the highest frequency sequences which lead to core damage. These sequences were compiled by the computer code SQLINK. SQLINK was used to link the support model sequences with the frontline model sequences and generate a listing of core damage sequences. A cutoff of 1.0E-6 was used in SQLINK; this cutoff operates as follows: sequences with frequenc, greater than $1.0 \mathrm{E}-6$ times the total core damage frequency (prior to recovery) were retained by SQLINK for inclusion in the dominant sequence model. The highest frequency sequence excluded by SQLINK due to this cutoff would be approximately 6.0E-10.

Additionally, the maximum number of sequences which can be processed by SQLINK is 1999 . The DCPRA quantification reached this limit; due to reaching this limit the highest frequency sequence excluded from the SQLINK output was approximately 8.0E-8.

Neither the cutoff nor the storage limitation affected the composition of the dominant sequence model. The dominant sequence model contains the first 420 sequences contained in the SQLINK output. These 420 sequences total $88.1 \%$ of the total non-seismic core damage frequency. The largest sequence excluded from the dominant sequence model, but contained in the SQLINK output, had a frequency of 1.1E-7 (i.e., sequence 421).

The dominant sequence model was developed by writing the sequences in the form of equations; each sequence is written as the product of an initiating event and the failed split fractions. This process was automated by using the computer code RMODEL: however, split fractions for successful top events were not automatically included in these equations. The most important success terms were manually added to the dominant sequence model where the rare event approximation was not appropriate. The dominant sequence model was then requantified using point estimates for initiating events and split fractions. The resulting value, from the $\mathbf{4 2 0}$ sequences, did not equal the total core damage frequency before any truncation. The $\mathbf{4 2 0}$ sequence total was actually higher. The result from the 420 sequences differed because of the missing success terms (those not included in the sequences) and because of the limited number of sequences included in the model; this indicated that the absence of success terms in the dominant sequence model out weighed the total frequency of the sequences below the 420th. 
Therefore, to make the total frequency from the dominant sequence model match the total frequency from the SQLINK output, a ratio was applied to the dominant sequence model total.

Finally, selected sequences in the dominant sequence model were multiplied by recovery factors; the recovery factors were described previously in PG\&E letter number DCL-89-283 dated November 13, 1989.

In summary, the most important cutoff or limitation with respect to the development of the nonseismic dominant sequence model is the limit of $\mathbf{4 2 0}$ sequences. Sequences with frequency as high as $1.0 \mathrm{E}-7$ are not explicitly included in the dominant sequence model. In principle, however, the frequency of these sequence is included because the total core damage frequency from the dominant sequence model prior to recovery was adjusted to match the total core damage frequency without any truncation."

Upon receipt of the floppy disk, BNL solved the provided set of equations as a FORTRAN expression and obtained the same results as PG\&E. This was done simply as a QA check of the floppy disk not the model and data. In order to pursue insights into the model, BNL reconfigured the model into input compatible with the SETS code. The purpose for using the SETS code was that the faithfulness to a Boolean expression could be investigated, the individual sequences could be quantified and ranked, and then local software could be applied to the SETS output for post-processing purposes.

BNL first converted the four hundred plus equations in the PG\&E model into one large equation containing 420 sequences. The ratio discussed in the above quote was of no further interest (beyond the QA check) to BNL and was therefore discarded. Table D1.1 contains the final form of the model and associated quantification that all succeeding BNL calculations were performed on and the remainder of this appendix details its evolution. (Table D1.1a contains the Boolean model, and Tables D1.1b through D1.1f contain the quantification for the initiators, frontline system split fractions, support system split fractions, human actions and recovery events, and elements added by BNL to preserve the original quantification respectively.)

The 420 sequence version of the model contained six sequences that had combinations of elements OR'ed together within them. Table D1.2 shows these six sequences as modified by BNL and originally input to the SETS code. In final version (Table D1.1) the original six sequences were left with the AW split fractions and the ZHE terms were substituted in and became sequences 421 through 426 . The hazard chemical sequence became sequence 427 and the control room fire sequences (grouped together) became sequence 428 . The remainder of the equations define the recovery actions (see Table D1.3) and the complemented events that PG\&E placed into the model per their description above.

The first input to SETS was the 420 sequence version with the BNL-defined combination events (COMB_), no complement event definitions, and no substitution for the recovery events. This run revealed that sequence 43 had a ratio in it expressed as AW1/AW3 while SETS interpreted it as an erroneous cut set with a complemented event $(S)$ in it. This was fixed by substituting the new event AW1BAW3 for this ratio and adding the numerical value of the ratio to the Valueblock. Given the above mentioned split of sequence 43, AW1BAW3 wound up in the final model in both sequence 43 and 426 . Also, in sequence 63 there was a factor of two within the cut set. This was changed to the variable name FACTR2 and given a value of one in the Valueblock (as SETS believes it to be a probability), however, in the post processing computations on which all BNL results are based it was given its original value of two. With the above changes made the SETS code was successful in reading and quantifying the sequences. Again, reactor trip (RT) and turbine trip (TT) 
Appendix D

were set to one for the SETS calculation and to their original values $(\mathrm{RT}=1.14$ and $T \mathrm{TT}=1.05)$ for the later BNL quantification.

The next step was to solve the model with the COMB_elements removed and the six additional sequences added. This step flagged two problems as the quantification for this run was less than the original run. One problem was traced to sequence $\mathbf{4 3}$ which with the substitution became:

LOOP * OGF * SW1 * IAF * AW3 * OB1 * AW1BAW3 * AW3.

The SETS code did a Boolean reduction on this sequences as AW3 appeared twice and simply threw away the second one. In order to preserve the sequence quantification, the second AW3 was changed to DUMMXAW3 and given the same quantification.

The second problem was that a number of sequences were thrown away by the SETS code as it interpreted them as non-minimal cut sets. BNL investigated and found that the cause was the lack of an expressed success state in the nine sequences shown in Table D1.4. BNL, therefore, added the variable DUMMY to each of these sequences in order that no sequence would be thrown away and gave it the value of 1.0 to preserve the quantification.

When the equations for the recovery actions were substituted into the model the following problem was discovered. Sequence 10 became:

\section{LOOP * OGF * GH1 * TG2 * SW1 * IAF* AW4 * GF1S * FF1S * TH25* [(ZHESW1 + AW4) * RESLC1]}

which has two AW4's in it. BNL changed the definition of RSEQ10 to include DUMMYAW4 substituted for the second AW4 to preserve the quantification.

The final step was to add sequences 427 and 428 as discussed above. Sequence 427 has a 0.1 factor which was renamed POINT1. When the $\mathbf{4 2 8}$ sequences were expanded with the substitutions of the recovery actions within SETS the result became $\mathbf{4 5 2}$ "cut sets" defining the top event TOP. Using this model and the accompanying data yields: TOP $=1.7728 E-04$. The SETS solution (with RT, TT and FACTR2 reduced to 1.0) was: $T O P=1.7457 \mathrm{E}-04$.

PG\&E supplied two updates to the original model sent to BNL. The first was an update of six split fractions following the BNL review of the ECCS low pressure injection function. The current model reflects these changes. The second update was to correct an error discovered in Sequence 43 . The original sequence provided to BNL was the following:

$$
\text { LOOP * OGF * SW1 * IAF * AW3 * OB1 * (AW1/AW3 * (ZHESW1 + AW3)) }
$$

which was split into two sequences as discussed previously. The updated version was as follows:

$$
\text { LOOP * OGF * SW1 * IAF * AW3 * OB1 * (AW1/AW3 + ZHESW1 + AW3) }
$$

As can be seen from above, the correction (when expanded) would have added an additional cut set to the model and would have increased the two in the model by a factor of (AW1/AW3) ${ }^{-1}$. This would not have had an appreciable effect on the model as discussed in Table D1.3 or on the BNL quantification and therefore BNL did not make this change to the model and rerun the calculations. 
Appendix D

Table D1.1a

Eodecod Core Daneer Truqueney Modal

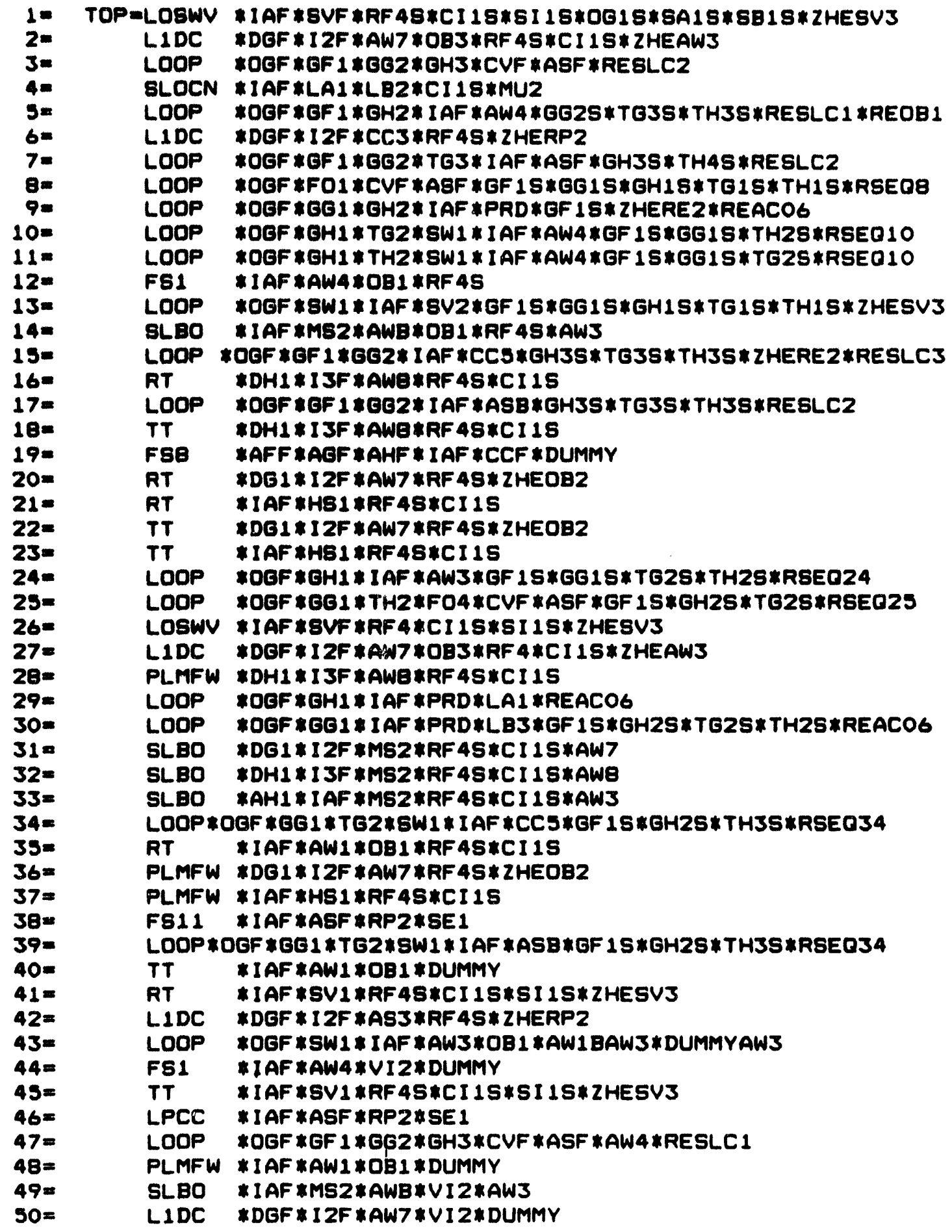




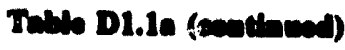

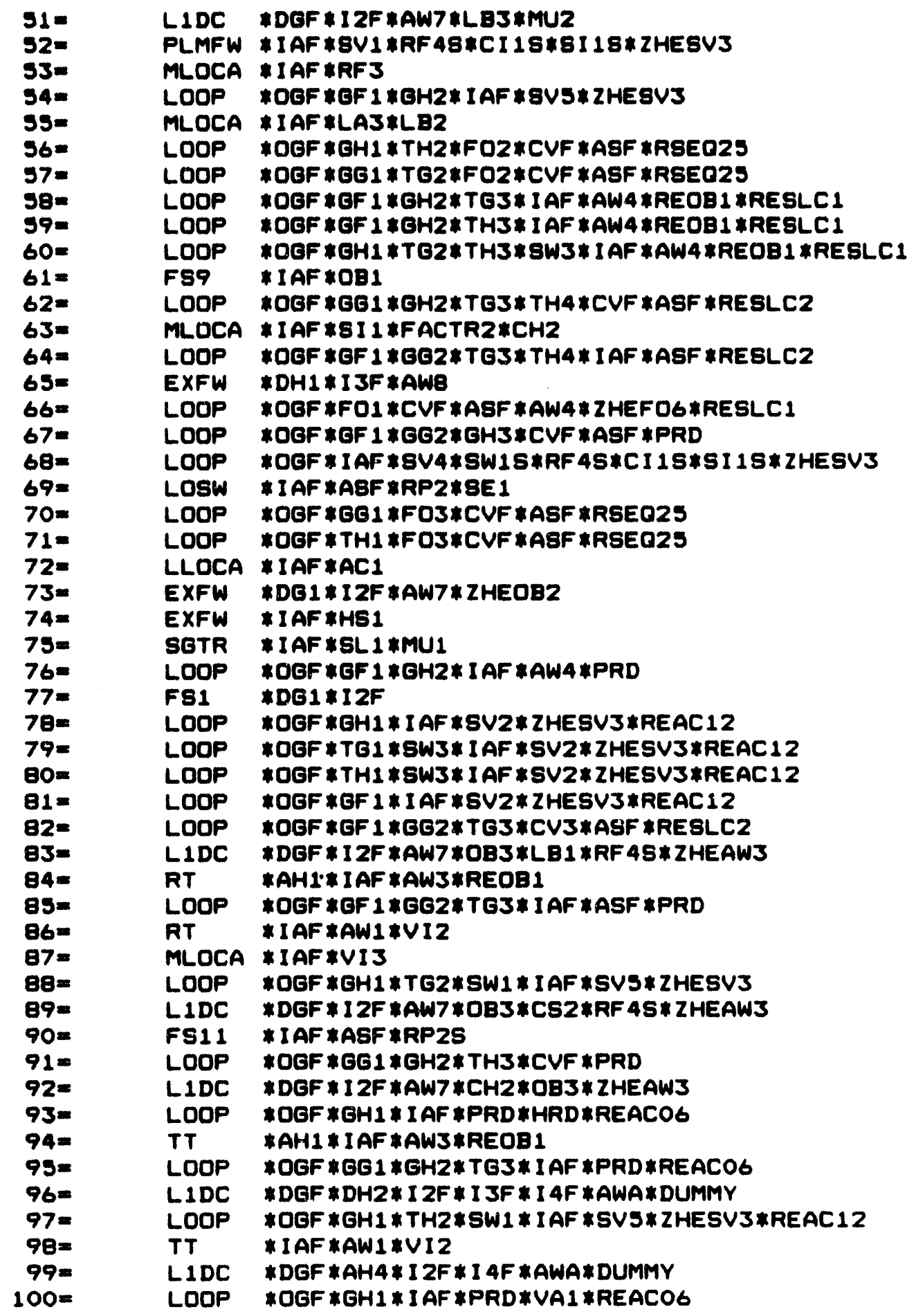




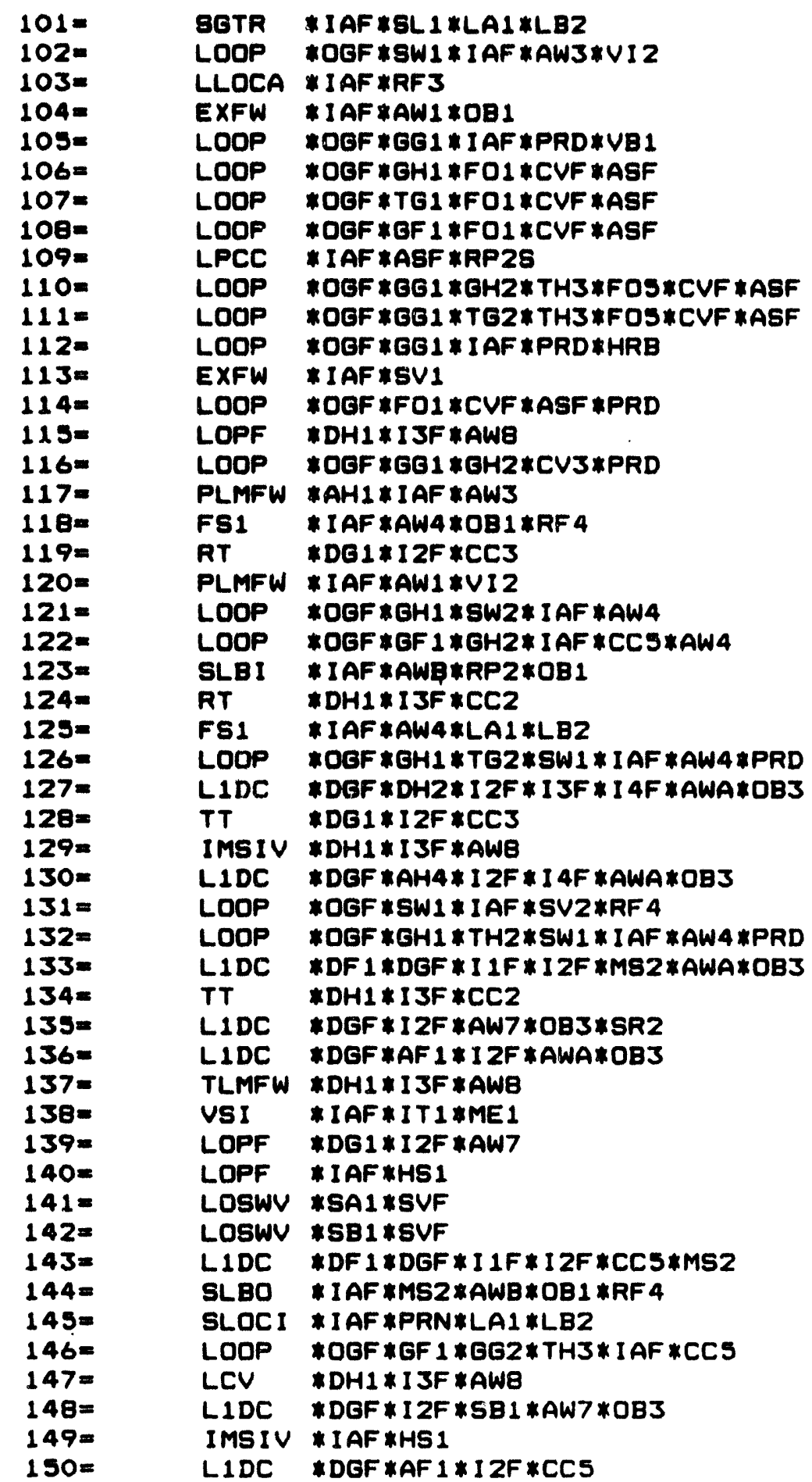


Appendix D

Table D1.1a (continued)

\begin{tabular}{|c|c|c|}
\hline $\begin{array}{l}151= \\
152=\end{array}$ & $\begin{array}{l}\text { IMSIV } \\
\text { FS9 }\end{array}$ & $\begin{array}{l}\text { *DG1*I2F*AW7 } \\
* I A F * V I 2\end{array}$ \\
\hline $153=$ & SLBI & *SAS*SBE *OSF *MSZ \\
\hline $154=$ & SLBO & *I31*MS2*OB 1 \\
\hline $155=$ & L $1 D C$ & *DGF*DH2*I 2F*I IF* I 4F*CC4 \\
\hline $156=$ & SLBO & * I 11 *MS2*OB 1 \\
\hline $157=$ & LOOP & *OGF*DH1*GF 1*I3F*AW9 \\
\hline $\begin{array}{l}158= \\
159=\end{array}$ & $\begin{array}{l}\text { SLBO } \\
\text { LOOP }\end{array}$ & $\begin{array}{l}\text { *IAF*MS2*AWB*LA1*LB2 } \\
* \text { OGF*GH1*TH2*SW1*CV6*AW4 }\end{array}$ \\
\hline $160=$ & L 1DC & *DGF*AH4* I 2F * I 4F*CC4 \\
\hline $161=$ & LOOP & *OGF*GG1*TH2*FO4*CVF*ASF*AW4 \\
\hline $162=$ & RT & *DF 1*I IF*AWB*OB 1 \\
\hline $163=$ & TLMFW & *DG1*I2F *AW7 \\
\hline $164=$ & LOOP & *OGF *GF $1 * G G 2 * T H 3 *$ I AF *ASB \\
\hline $165=$ & TLMFW & * IAF*HS1 \\
\hline $166=$ & LOQP & *OGF*DF $1 *$ GH1* I 1F*AW9 \\
\hline $167=$ & MLOCA & *SA2*5B6*05F \\
\hline $168=$ & LOOP & *OGF*GH1* IAF *PRD*RF 1 \\
\hline $169=$ & LOOP & *OGF*GG $1 * G H 2 * S W 2 *$ I AF*CCF \\
\hline $170=$ & SGTR & * IAF*OP $1 *$ I I 5 \\
\hline $171=$ & SLBI & *SBC*OSF *RP 2 *OB 1 \\
\hline $172=$ & SLBI & *SA5*OSF *RP 2 *OB 1 \\
\hline $173=$ & L1DC & *DGF*I2F*AW7*VB1 \\
\hline $174=$ & LOOP & *OGF*GG $1 *$ I AF *PRD*RF 1 \\
\hline $175=$ & PLMFW & *DG1*I 2F*CCJ \\
\hline $176=$ & TT & *DF 1 * I 1F*AWB*DB 1 \\
\hline $177=$ & LOCV & *DH1*I3F*CVF*AWB \\
\hline $178=$ & RT & *131*AW5*OB1 \\
\hline $179=$ & LOCV & *CVF*RT 1 *OSF \\
\hline $180=$ & RT & *DH1*I3F*AWB*RF 4 \\
\hline $181=$ & RT & * I 11 *AW5*OB 1 \\
\hline $182=$ & ISI & *DH1*IJF*AWB \\
\hline $183=$ & L1DC & *DGF* I 2F *AW7*HRB \\
\hline $184=$ & PLMFW & *DH1*IJF*CC2 \\
\hline $185=$ & LDOP & *OGF *BG $1 *$ GF $1 * G G 2 *$ IAF *ASF \\
\hline $186=$ & FS6 & *AFF*AGF* I AF *CC5*RPZ \\
\hline $187=$ & $\mathbf{R T}$ & *SA1*SB2*RT7*OSF \\
\hline $188=$ & LCV & *DG $1 *$ I 2F*AW7 \\
\hline $189=$ & LOSW & *IAF *ASF *RP2S \\
\hline $190=$ & LCV & * IAF *HS1 \\
\hline $191=$ & LOOP & *OGF *SW 1 * I AF *CC7*SE 1 \\
\hline $192=$ & TT & *I31*AW5*0B1 \\
\hline $193=$ & TT & * I 11 *AW5*OB 1 \\
\hline $194=$ & TT & *DH1*I JF *AWB*RF 4 \\
\hline $195=$ & LOOP & *OGF*DH1*I 3F*AWB \\
\hline $196=$ & L1DC & *DGF*I 2F *AW7*OBJ*CI 2 \\
\hline $197=$ & RT & *SA1*SB2*OS1*MS2 \\
\hline $198=$ & LOPF & * IAF *AW 1 *OB 1 \\
\hline $199=$ & TT & *SA1*5B2*RT7*OSF \\
\hline $200=$ & LDOP & *OGF*GF 1 *GH 2 *CV 2 *AW4 \\
\hline
\end{tabular}


Appendix D

Table D1.1a (continued)

\begin{tabular}{|c|c|c|}
\hline $\begin{array}{l}201= \\
202=\end{array}$ & $\begin{array}{l}\text { LOOP } \\
\text { SLOCI }\end{array}$ & $\begin{array}{l}\text { *OGF*GF } 1 * \text { GG } 2 * C V 3 * C C 5 \\
\text { *IAF*CC1 }\end{array}$ \\
\hline $203=$ & LOOP & *OGF*GG $1 *$ I 32*PRD \\
\hline $204=$ & LOOP & *OGF*GG $1 * G H 2 *$ I AF *CC5*PRD \\
\hline $205=$ & LOOP & *OGF*TH1*SW3*IAF*AW3*OB 1 \\
\hline $206=$ & LOOP & *OGF*TG1*SW3*IAF*AW3*OB 1 \\
\hline $207=$ & LQOP & *OGF*GF 1 *GG2*TH3*FO4*CVF*ASF \\
\hline $\begin{array}{l}208= \\
209=\end{array}$ & $\begin{array}{l}\text { LOOP } \\
\text { LOOP }\end{array}$ & $\begin{array}{l}\text { *OGF*GF } 1 * \text { *G } 2 * \text { I AF*SV5*AW9 } \\
\text { *OGF*GF } 1 \text { * I AF*AW3*OB } 1\end{array}$ \\
\hline $210=$ & RT & *IAF*HS1*RF 4 \\
\hline $211=$ & LOCV & *DG $1 * I 2 F * C V F * A W 7$ \\
\hline $212=$ & TT & *SA1*SB2*0S1*MSZ \\
\hline $213=$ & LOCV & *CVF*HS1 \\
\hline $214=$ & LOOP & *QGF*GF $1 *$ GG2* I AF *CC5*PRD \\
\hline $215=$ & RT & *DG $1 * 12 F * A W 7 * R F 4$ \\
\hline $216=$ & LOOP & *OGF*GF $1 * G H 2 *$ I AF *AW4*CH2*DUMMY \\
\hline $217=$ & ISI & * IAF*HSI \\
\hline $218=$ & IS I & *DG $1 * I 2 F * A W 7$ \\
\hline $219=$ & LOOP & *OGF*GF $1 *$ GG $2 * C V 3 * A S B$ \\
\hline $220=$ & SGTR & * IAF*OP $1 * M U 1$ \\
\hline $221=$ & LOOP & *OGF*GF 1 *GH2* I AF *CC5*PRD \\
\hline $222=$ & SLBI & *DG1*I2F \\
\hline $223=$ & LOPF & *IAF*SVI \\
\hline $224=$ & LOOP & *OGF*GH1*TG2*SW1*IAF*CC5*AW4 \\
\hline $\mathbf{2 2 5}=$ & SLBI & *DH1*I3F \\
\hline $226=$ & IMSIV & * IAF*AW $1 * 0 B 1$ \\
\hline $227=$ & SLBI & *AHI*IAF*RPZ \\
\hline $228=$ & RT & * IAF*CC $1 * R P 2 * S E 1$ \\
\hline $229=$ & TT & *IAF*HS1*RF 4 \\
\hline $230=$ & LOOP & *OGF*GF 1 *GG2* I AF*ASB*PRD \\
\hline $231=$ & LOOP & *OGF*GH1*TH2*SW1*IAF*CC5*AW4 \\
\hline $232=$ & TT & *DG $1 *$ I 2F*AW7*RF 4 \\
\hline $233=$ & ELOCA & * IAF \\
\hline $234=$ & LOOP & *OGF*GH1*IAF*AW3*RF 4 \\
\hline $235=$ & TLMFW & * IAF *AW $1 * O B 1$ \\
\hline $236=$ & MLOCA & *CVI*OSF \\
\hline $237=$ & PLMFW & *DF 1*I IF*AWE*OB1 \\
\hline $238=$ & LOQP & *OGF*DG 1*I2F *AW7 \\
\hline $239=$ & LIDC & *DGF* I 2F*AW7*OB3*VB3 \\
\hline $240=$ & $T T$ & *IAF*CC 1*RP $2 *$ SE 1 \\
\hline $241=$ & LOOP & *OGF*GG1*GH2*IAF*AW3 \\
\hline $242=$ & SGTR & *I31*SL2 \\
\hline $243=$ & SGTR & * I11*SL2 \\
\hline $244=$ & IMSIV & * IAF*SV1 \\
\hline $245=$ & LOOP & *OGF*BG1*GH1*SW1*IAF*AW4 \\
\hline $246=$ & SLBO & *DF 1*I 1F*MS2*OB1 \\
\hline $247=$ & FSB & *AFF*AGF *AHF* I AF *CCF *PRD \\
\hline $248=$ & PLMFW & *I31*AW5*OB1 \\
\hline $249=$ & SLBO & *AF $1 *$ I AF *MS2*OB 1 \\
\hline $250=$ & LOOP & *QGF*GF $1 *$ GG2* IAF*AW3 \\
\hline
\end{tabular}


Appendix D

Table D1.1a (continuod)

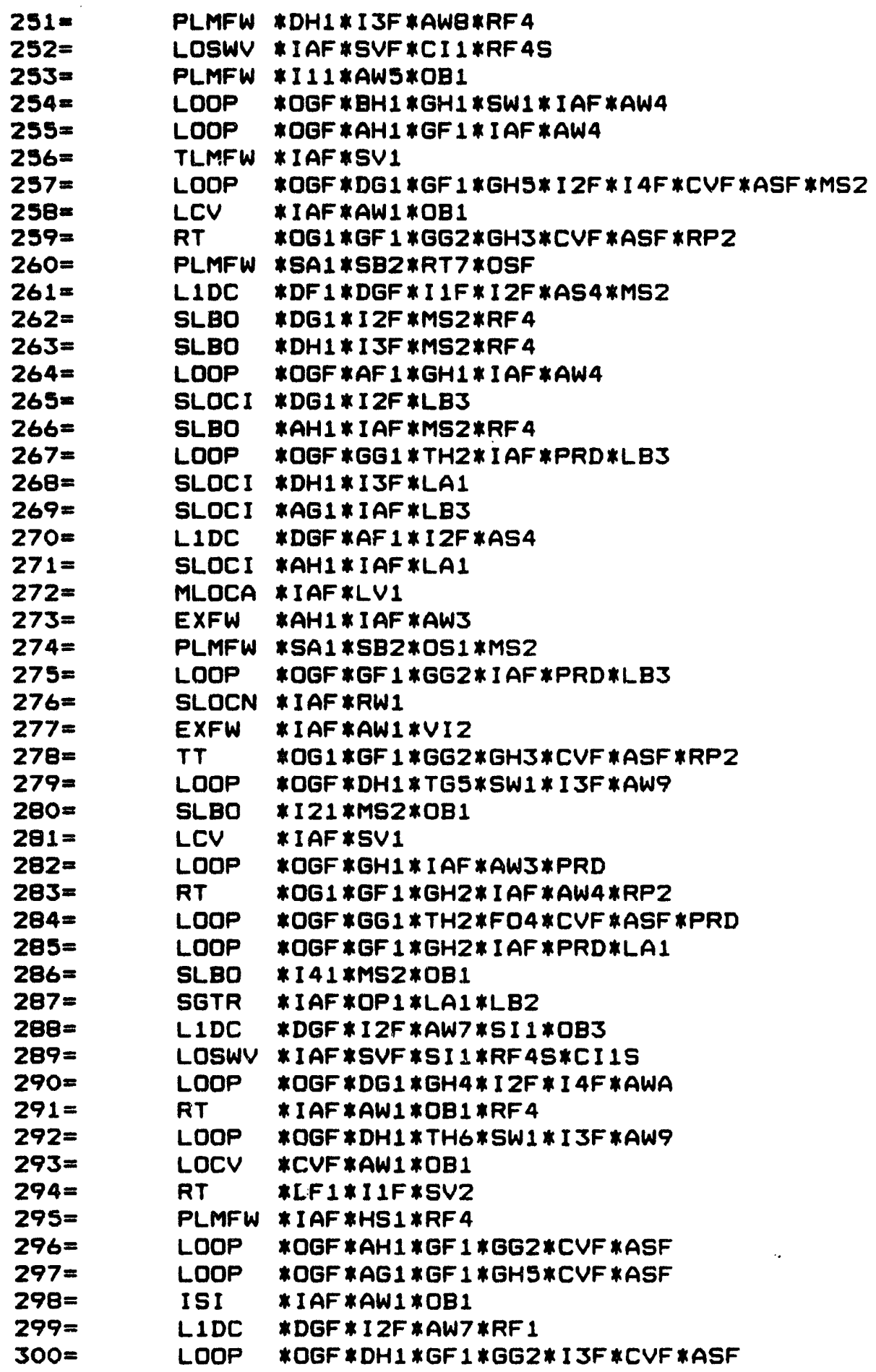


Appendix D

Table D1.1a (continued)

\begin{tabular}{|c|c|c|}
\hline $\begin{array}{l}301= \\
302=\end{array}$ & $\begin{array}{l}\text { PLMFW } \\
\text { RT }\end{array}$ & $\begin{array}{l}\text { *DG1* I } 2 F * A W 7 * R F 4 \\
\text { *AF } 1 * \text { I AF*SV2 }\end{array}$ \\
\hline $303=$ & TT & *OG1*GF 1 *GH2* IAF*AW4 *RP 2 \\
\hline $304=$ & RT & *IAF *AWI *LA1 *LB2 \\
\hline $305=$ & LOOP & *OGF*GG1*CV3*PRD*LB3 \\
\hline $306=$ & RT & *I21*AW5*OB1 \\
\hline $307=$ & TT & *IAF*AW $1 * O B 1 * R F 4$ \\
\hline $\begin{array}{l}308= \\
309=\end{array}$ & $\begin{array}{l}\text { PLMFW } \\
\text { TT }\end{array}$ & $\begin{array}{l}\text { *IAF*CC1 *RP } 2 * S E 1 \\
* D F 1 * I 1 F * S V 2\end{array}$ \\
\hline $310=$ & RT & *141*AW5*0B1 \\
\hline $311=$ & LOOP & *OGF*DG $1 *$ GF $1 *$ I 2F *AWA \\
\hline $312=$ & FS6 & *AFF *AGF * IAF *AS4*RP 2 \\
\hline $313=$ & LOOP & *OGF*AF $1 *$ GG 1 *GH2*CVF*ASF \\
\hline $314=$ & LQQP & *OGF *GF $1 * G G 2 * G H 3 * S B 1 * C V F * A S F$ \\
\hline $315=$ & LOOP & *OGF *GG 1 *GH2* I AF *PRD*SI 2 \\
\hline $316=$ & LOOP & *OGF *GF 1*GG2* I AF *PRD*S I 2 \\
\hline $317=$ & LOCV & *CVF*SV1 \\
\hline $318=$ & TT & *AF $1 *$ I AF *SV2 \\
\hline $319=$ & LOOP & *OGF *SW1* IAF*AW3*OB 1 *RF 4 \\
\hline $320=$ & IS I & *IAF*SVI \\
\hline $321=$ & TT & * I AF*AW1*LA1*LB2 \\
\hline $322=$ & LOOP & *OGF*DF $1 * G G 1 * G H 2 * 11 F * C V F * A S F$ \\
\hline $323=$ & LOOP & *OGF*AG1*GF $1 *$ TGG* IAF*ASF \\
\hline $324=$ & RT & *IAF*SV1*RF 4 \\
\hline $325=$ & TT & *I21*AWS*OB1 \\
\hline $326=$ & LOOP & *OGF*DG1*GF $1 *$ TG6* I 2F*ASF \\
\hline $327=$ & LLOCA & *SA2*SB6*OSF \\
\hline $328=$ & LOOP & *OGF*GG1*SW2*IAF*CC5 \\
\hline $329=$ & TT & * I 41*AW5*OB1 \\
\hline $330=$ & SLOCN & * I AF *RF 1 *MU2 \\
\hline $331=$ & LOOP & *OGF*SW1*IAF*AW3*LA1*LB2 \\
\hline $332=$ & FS1 & * IAF*AW4*VI $2 * R F 4$ \\
\hline $333=$ & LOOP & *OGF*DF 1*GG1*TG2*I 1F*ASF \\
\hline $334=$ & FSS & * IAF *ASF *RP $2 * S E 1$ \\
\hline $335=$ & RT & *0G1*GF $1 *$ GG2*TG3* I AF*ASF *RP2 \\
\hline $336=$ & L1DC & *DGF*12F*132*AW7 \\
\hline $337=$ & LOOP & *OGF*AF $1 *$ GG $1 *$ TG2* I AF *ASF \\
\hline $338=$ & FS11 & *DG1*I2F*ASF \\
\hline $339=$ & FS10 & *IAF*AW4*OBI \\
\hline $340=$ & FS11 & *DHI*I JF *ASF \\
\hline $341=$ & LOOP & *OGF*GG1*GH2*IAF*PRD*CH2 \\
\hline $342=$ & TT & *IAF*SVI*RF 4 \\
\hline $343=$ & SLBI & *IAF*AWB*RP $2 * V I 2$ \\
\hline $344=$ & LOOP & *OGF*GH1*TH2*FO2*CVF *ASF*AW4 \\
\hline $345=$ & LOOP & *OGF*GG1*TG2*F02*CVF *ASF*AW4 \\
\hline $346=$ & LOOP & *OGF*GG1*TG2*SW1*I AF*CC5*PRD \\
\hline $347=$ & LOOP & *OGF*GF $1 * G H 2 * S B 1 * A W 4$ \\
\hline $34 B=$ & LOOP & *OGF*GH1*TG2*SW1* I AF *5VS*AWG \\
\hline $349=$ & SGTR & *DF 1*I 1F*SL2 \\
\hline $350=$ & PLMF & *OG1*GF 1 *GG2* \\
\hline
\end{tabular}

D1-11

NUREG/CR-5726 
Appendix D

Table D1.1n (continued)

\begin{tabular}{|c|c|c|}
\hline $\begin{array}{l}351= \\
352=\end{array}$ & $\begin{array}{l}\text { LQOP } \\
\text { TT }\end{array}$ & $\begin{array}{l}* 0 G F * G H 1 * T G 2 * 5 W 1 * I A F * A W 4 * C H 2 \\
* O G 1 * G F 1 * G G 2 * T G 3 * I A F * A S F * R P 2\end{array}$ \\
\hline $353=$ & LIDC & *DGF * I $2 F$ *CC $3 * P R A$ \\
\hline $354=$ & SGTR & *DHI*I JF*5L2 \\
\hline $355=$ & RT & *DH1*I3F*SV3 \\
\hline $356=$ & SGTR & *DG1*I2F*SL2 \\
\hline $357=$ & LOOP & *OGF*GH1*TH2*SW1* IAF*SV5*AW9 \\
\hline $358=$ & LOOP & *OGF*GH1* I AF *CC7*PRD \\
\hline $359=$ & FS1 & *DH1*I3F*AW9 \\
\hline $360=$ & LOOP & *OGF*GH1*TG2*SW1*IAF*CC5*PRD \\
\hline $361=$ & $\mathbf{R T}$ & *AH1*IAF*SV3 \\
\hline $362=$ & LOOP & *OGF*GH1*TH2*SW1*IAF*AW4*CHZ \\
\hline $363=$ & LOOP & *OGF *GG 1 *GH2*TG3*TH4*CVF*ASF *AW4 \\
\hline $364=$ & LOOP & *OGF *GG1*TG2*SW1* IAF *ASB*PRD \\
\hline $365=$ & LOOP & *OGF*SW1* IAF*AS5*SE 1 \\
\hline $366=$ & LOOP & *OGF*SW1*IAF*SV2*SI2 \\
\hline $367=$ & LOOP & *OGF*GG 1 *IAF*CC7*PRD \\
\hline $368=$ & LOQP & *OGF*GH1*TH2*SW1* IAF *CC5*PRD \\
\hline $369=$ & EXFW & *DG1*I2F*CC3 \\
\hline $370=$ & FS1 & *IAF*AW4*OB $1 *$ LA 1 \\
\hline $371=$ & FSI & * IAF*AW4*OB1*LB1 \\
\hline $372=$ & T.T & *DH1*I3F*5V3 \\
\hline $373=$ & PLMFW & *OG $1 *$ GF $1 *$ GH2* I AF *AW4*RP2 \\
\hline $374=$ & LPCC & *DG1*I2F *ASF \\
\hline $375=$ & LODP & *DGF* I AF*HS1*DUMMY \\
\hline $376=$ & LPCC & *DH1*I3F*ASF \\
\hline $377=$ & TT & *AH1*IAF*5V3 \\
\hline $378=$ & EXFW & *DH1*I3F*CCZ \\
\hline $379=$ & LOOP & *OGF *SW1*IAF*HS1 \\
\hline $380=$ & L1DC & *DGF* I 2F*SA1*CC3 \\
\hline $381=$ & L1DC & *DGF*I2F*SB $1 * \mathrm{CC} 3$ \\
\hline $382=$ & LOOP & *OGF*GH1*TG2* I AF*AW3 \\
\hline $383=$ & PLMFW & * I AF *AW1 *OB1*RF 4 \\
\hline $384=$ & LOOP & *OGF*GG1*IAF*SV4 \\
\hline $385=$ & PLMFW & *DF 1*I1F*SV2 \\
\hline $386=$ & LOOP & *OGF*DH1*GG1*I JF*PRD \\
\hline $387=$ & LOOP & *OGF*GG1* I AF*AW1 \\
\hline $388=$ & LOOP & *OGF*GF 1*GH2*TG3*TH4*IAF*AW4 \\
\hline $389=$ & LDOP & *DGF*GH1*TH2* I AF *AW3 \\
\hline $390=$ & SGTR & * I $21 *$ SL 2 \\
\hline $391=$ & LOOP & *OGF*AH1*GG $1 *$ I AF *PRD \\
\hline $392=$ & LQDP & *OGF*GF 1 *GG2*TG3*SB1*ASF \\
\hline $393=$ & LOOP & *OGF*AG $1 * G H 4 *$ I AF *PRD \\
\hline $394=$ & PLMFW & *AF $1 *$ I AF* SVZ \\
\hline $395=$ & LQOP & *OGF*GG1*TG2*SW1*IAF*AW3 \\
\hline $396=$ & SGTR & * I $41 *$ SL 2 \\
\hline $397=$ & SLBO & * I AF *MS2*AWB*VI $2 * R F 4$ \\
\hline $398=$ & LDOP & *OGF*GF 1*GH2*TG3* IAF*SV5 \\
\hline $399=$ & PLMFW & * I AF *AW1*LA1*LB2 \\
\hline $400=$ & LIDC & *DGF* I 2F *AW7*VI 2*RF 4 \\
\hline
\end{tabular}


Appendix D

Table D1.1a (contineod)

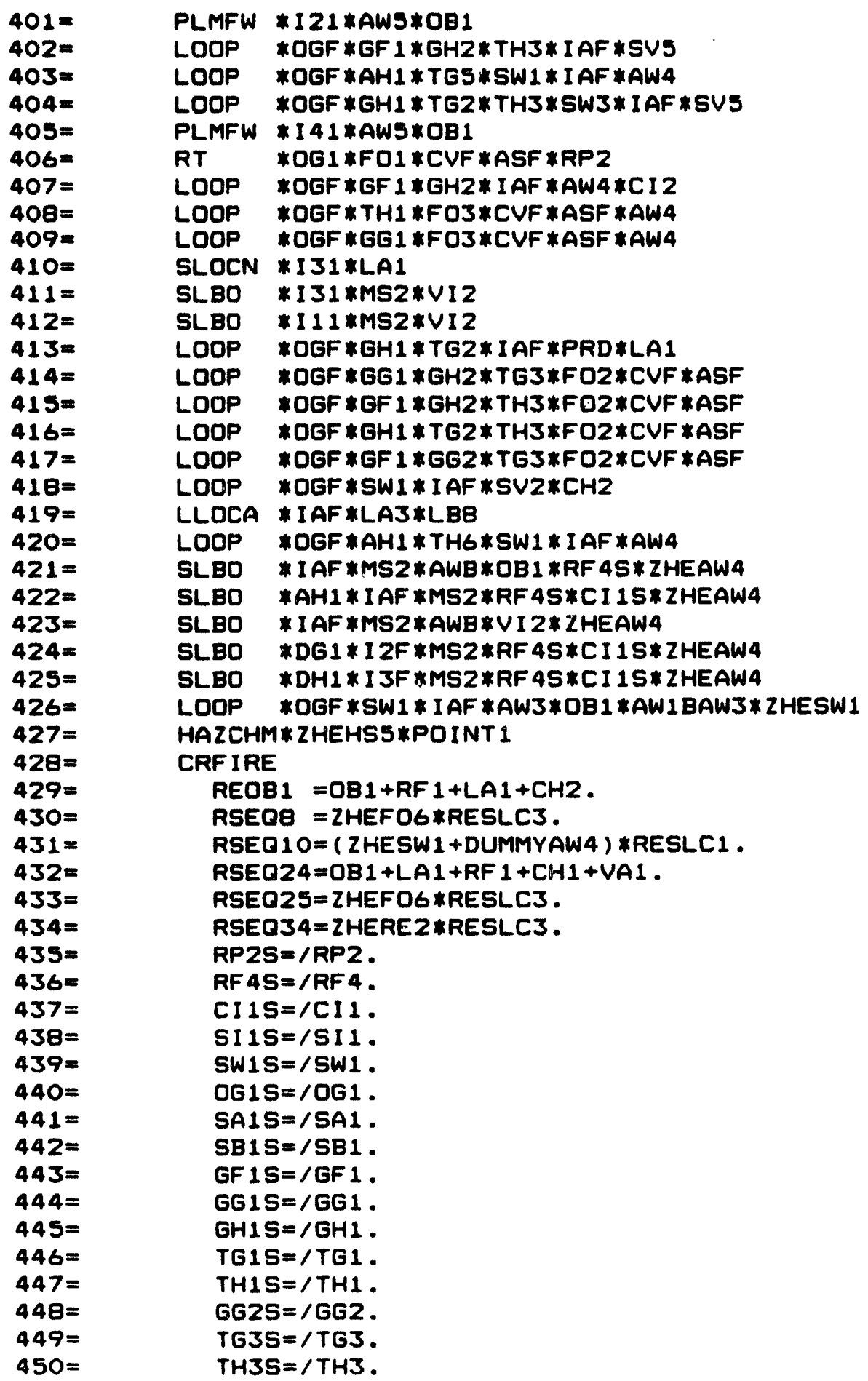


Appendix D

Table D1.1a (continued)

$451=$

$G H 3 S=/ G H 3$.

$452=$

$T H 45=/ T H 4$.

$453=$

TH2S $=/ T H 2$.

$454=$

TG2S $=/ T G 2$.

$\mathrm{GH} 25=/ \mathrm{GH} 2$.

PRDS $=/$ PRD .

$456=$

$457=$

OB3S $=/ 0 B 3$.

$458=* E O R$ 
Appendix D

Table D1.1b

Reduced Model Input Data - Initiators

\#

NAME

VALUE

$\begin{array}{ll}3 & \text { LOSWV } \\ 13 & \text { LIDC } \\ 19 & \text { LOOP } \\ 27 & \text { SLOCN } \\ 61 & \text { FS1 } \\ 64 & \text { SLBO } \\ 70 & \text { RT } \\ 75 & \text { TT } \\ 76 & \text { FSB } \\ 90 & \text { FLMFW } \\ 95 & \text { FS11 } \\ 103 & \text { LPCC } \\ 104 & \text { MLOCA } \\ 111 & \text { FS9 } \\ 116 & \text { EXFW } \\ 120 & \text { LOSW } \\ 123 & \text { LLOCA } \\ 125 & \text { SGTR } \\ 144 & \text { LOPF } \\ 146 & \text { SLBI } \\ 148 & \text { IMSIV } \\ 153 & \text { TLMFW } \\ 154 & \text { VSI } \\ 159 & \text { SLOCI } \\ 161 & \text { LCV } \\ 176 & \text { LOCV } \\ 179 & \text { ISI } \\ 181 & \text { FS6 } \\ 190 & \text { ELOCA } \\ 209 & \text { FS5 } \\ 210 & \text { FS1O } \\ 217 & \text { HAZCHM } \\ 220 & \text { CRFIRE }\end{array}$

$6.29000 E-05$

2.56000E-02

$9.10000 E-02$

$5.26000 E-03$

2.94000E-04

5.53000E-03

1. $14000 E+00$

1. $05000 E+00$

6.1800OE-O6

$7.49000 E-01$

$3.81000 E-04$

1.96OOOE-04

$4.63000 E-04$

1. 35000 E-05

$2.79000 E-01$

9.74000E-05

2.02000E-04

1.7100OE-02

$1.21000 E-01$

$4.63000 E-04$

1. $07000 E-01$

$9.98000 \mathrm{E}-02$

$1.01000 E-06$

$1.61000 \mathrm{E}-02$

8.73000E-02

$.7 .99000 E-02$

$7.39000 E-02$

2.41000E-05

2.66000E-07

$5.26000 E-05$

1. 40000E-05

4.39000E-04

3.17000E-OS 
Table D1.1e

Reduced Model Inpat Date - Prontlies Syctome

VALUE

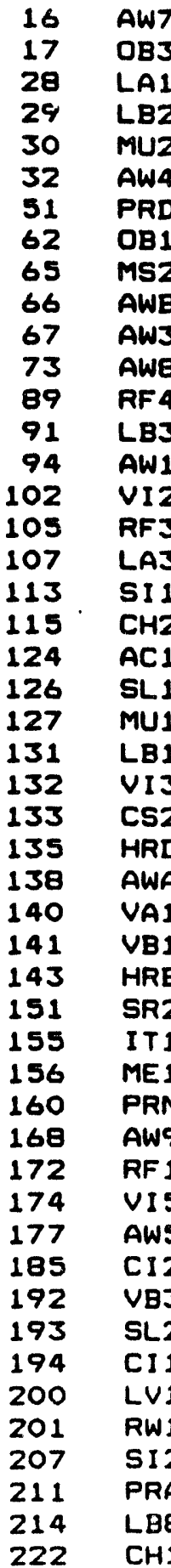

3.24000E-04

$3.75000 E-01$

2. O4OOOE-02

2. 33OOOE-01

1. $17000 E-02$

7. 2500OE-02

4.8BOOOE-02

$2.89000 E-02$

1. OOOOOE+OO

2. 41000E-02

1.24000E-03

$1.23000 E-03$

5. 4700OE-02

2. 04000E-02

3.7300OE-OS

2. 20000E-02

4.93000E-03

1. 58OOOE-02

3.25OOOE-OJ

1. $41000 E-02$

$6.27000 E-03$

$6.06000 E-03$

7.98OOOE-O3

1. 56OOOE-02

2. OOOOOE-03

1. 43OOOE-O2

4. 56OOOE-03

$9.59000 E-02$

3.8400OE-03

$3.64000 E-03$

4.01000E-03

9.4 BOOOE-O3

$9.90000 E-01$

5. OOOOOE-01

$7.66000 E-03$

$1.41000 E-01$

$3.16000 E-03$

$9.00000 E-03$

3. 30000E-02

5.770OOE-03

3.8400OE-03

$6.52000 E-03$

4. $06000 E-03$

4. 59000E-04

$3.94000 E-05$

1. $60000 E-02$

8. 23000E-03

3. $75000 E-02$

$6.24000 E-04$

D1.16 
Appendix D

Table D1.1d

Rodweed Modal Inpet Data - Support Syotem

VALUE

\begin{tabular}{|c|c|c|}
\hline 4 & IAF & 1. OOOOOE +00 \\
\hline 5 & SVF & $1.00000 E+00$ \\
\hline 14 & DGF & 1. OOOOOE +OO \\
\hline 15 & $I 2 F$ & $1.00000 E+00$ \\
\hline 20 & OGF & 1. OOOOOE+OO \\
\hline 21 & GF 1 & 4. 5200OE-02 \\
\hline 22 & GG2 & 5.56OOOE-02 \\
\hline 23 & GH3 & B.270OOE-02 \\
\hline 24 & CVF & 1. OOOOOE +OO \\
\hline 25 & ASF & 1. OOOOOE+OO \\
\hline 31 & GH2 & 5.4100OE-02 \\
\hline 38 & CC3 & $5.85000 E-04$ \\
\hline 40 & TG3 & 6.25000E-02 \\
\hline 43 & FO1 & 2. $16000 E-04$ \\
\hline 50 & GG 1 & 4. $48000 E-02$ \\
\hline 54 & GH1 & 4. $44000 E-02$ \\
\hline 55 & TGZ & 5.3600OE-02 \\
\hline 56 & SW1 & 5.00000E-01 \\
\hline 59 & THZ & 5.32000E-02 \\
\hline 63 & SV2 & 1.8000OE-04 \\
\hline 68 & CCs & 2.87000E-02 \\
\hline 71 & DH1 & $7.01000 E-04$ \\
\hline 72 & ISF & $1.00000 E+00$ \\
\hline 74 & ASB & $2.70000 E-02$ \\
\hline 77 & AFF & 1. OOOOOE+OO \\
\hline 78 & AGF & $1.00000 E+00$ \\
\hline 79 & AHF & $1.00000 E+00$ \\
\hline 80 & CCF & 1. OOOOOE+OO \\
\hline 82 & DG1 & 7.05000E-04 \\
\hline 86 & FO4 & $2.26000 E-02$ \\
\hline 92 & AHI & $0.92000 E-04$ \\
\hline 98 & SV 1 & $1.71000 E-O 6$ \\
\hline 99 & AS3 & $1.22000 E-04$ \\
\hline 106 & SV5 & $7.33000 E-03$ \\
\hline 108 & FOZ & $7.04000 E-03$ \\
\hline 109 & TH3 & $6.21000 E-02$ \\
\hline 110 & SW3 & $9.94000 E-01$ \\
\hline 112 & TH4 & $6.92000 E-0 Z$ \\
\hline $11 \theta$ & SV4 & $2.57000 E-O 5$ \\
\hline 121 & $\mathrm{FO3}$ & $3.51000 E-04$ \\
\hline 122 & TH1 & $4.36000 E-02$ \\
\hline 129 & TG1 & 4.40000E-OZ \\
\hline 130 & CV3 & $5.68000 E-02$ \\
\hline 136 & $\mathrm{DH} 2$ & $6.98000 E-0$ \\
\hline 137 & I 4F & $1.00000 E+O C$ \\
\hline 139 & $\mathrm{AH4}$ & $6.92000 E-0$ \\
\hline 142 & F05 & $5.08000 E-0$ \\
\hline 145 & SW2 & $2.54000 E-0$ \\
\hline 147 & $\mathrm{CC2}$ & $5.69000 E-0$ \\
\hline 149 & DF 1 & $7.05000 E-00$ \\
\hline
\end{tabular}

D1-17

NUREG/CR-5726 
Troble D1.1d (condineod)

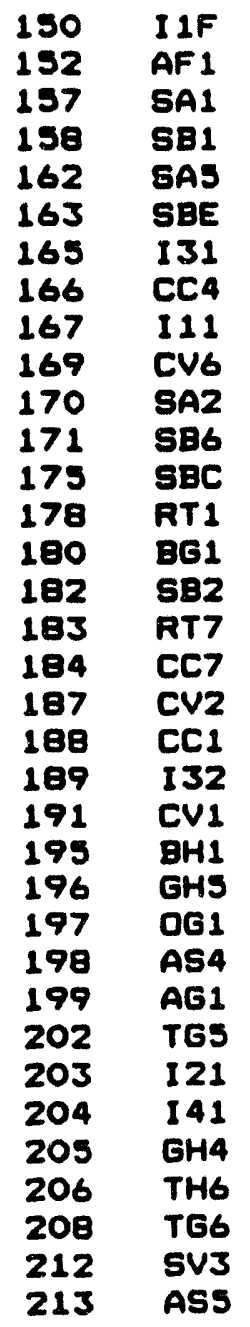

$1.00000 E+00$

$6.92000 E-04$

$7.58000 E-03$

$7.48000 E-03$

$1.40000 E-02$

$7.43000 E-02$

$1.15000 E-03$

$2.67000 E-02$

$1.15000 E-03$

3. 88000E-02

$1.14000 E-02$

8.4400OE-02

1. $34000 E-02$

$6.58000 E-06$

$1.44000 E-03$

$2.40000 E-02$

$1.93000 E-03$

$6.63000 E-04$

2.06000E-02

$1.88000 E-05$

$1.74000 E-03$

$7.60000 E-04$

$1.44000 E-03$

$5.56000 E-02$

$7.63000 E-04$

$1.69000 E-02$

$6.92000 E-04$

$4.44000 E-02$

$5.76000 E-04$

5. $76000 E-04$

4. 4800OE-O2

$4.40000 E-02$

5.41000 E-02

1. $33000 E-04$

3. 58OOOE-04 
Appeadth D

Table D1.10

Rodwead Model Inpet Data - Buman Actions + Rocovery

,

NAME

value

$\begin{array}{ll}12 & \text { ZHESU3 } \\ 18 & \text { ZHEAW3 } \\ 26 & \text { RESLC2 } \\ 36 & \text { RESLC1 } \\ 39 & \text { ZHERP2 } \\ 52 & \text { ZHERE2 } \\ 53 & \text { REACO6 } \\ 69 & \text { RESLC3 } \\ 83 & \text { ZHEOB2 } \\ 84 & \text { HS1 } \\ 96 & \text { RP2 } \\ 97 & \text { SE1 } \\ 117 & \text { ZHEFO6 } \\ 128 & \text { REAC12 } \\ 164 & \text { OSF } \\ 173 & \text { OP1 } \\ 186 & \text { OS1 } \\ 215 & \text { ZHEAW4 } \\ 216 & \text { ZHESW1 } \\ 218 & \text { ZHEHSS }\end{array}$

$3.73000 E-03$

8.00000E-03

3.82000E-02

4.64000E-01

$9.99000 E-03$

$5.31000 E-03$

2.82000E-01

8.63000E-02

8.00000E-02

$5.01000 E-06$

$9.96000 E-01$

$1.09000 E-02$

$4.00000 E-02$

$2.13000 E-01$

$1.00000 E+00$

4.42000E-03

$2.00000 E-03$

5. $00000 E-03$

3.54000E-03

$7.99000 E-03$

D1-19

NUREG/CR-5726 
Table D1.1f

Reduced Model Input Data - Elements Added by BNL

$\begin{array}{rlr}\# & \text { NAME } & \text { VALUE } \\ 81 & \text { DUMMY } & 1.00000 E+00 \\ 100 & \text { AW1BAW3 } & 3.01000 E-02 \\ 101 & \text { DUMMYAW3 } & 1.24000 E-03 \\ 114 & \text { FACTR2 } & 2.00000 E+00 \\ 219 & \text { POINT1 } & 1.00000 E-01 \\ 221 & \text { DUMMYAW4 } & 7.25000 E-02\end{array}$


Appendis D

Tablo D1.2

Radread Modal Soqueneces Whit "OR" Operalors

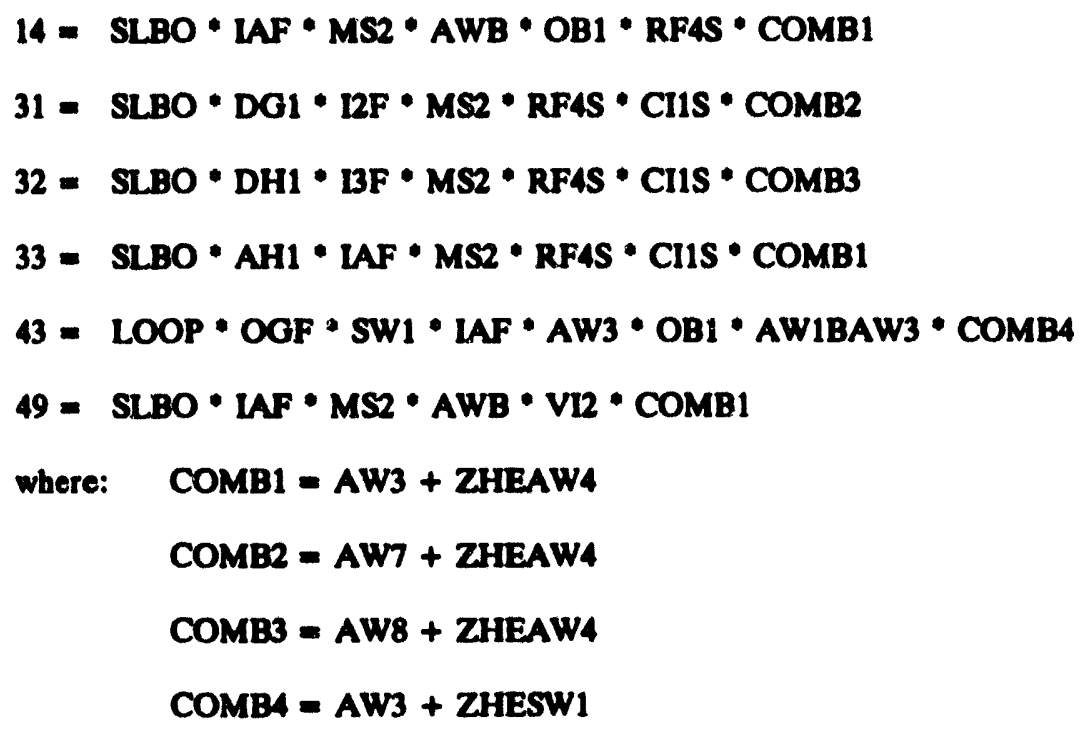



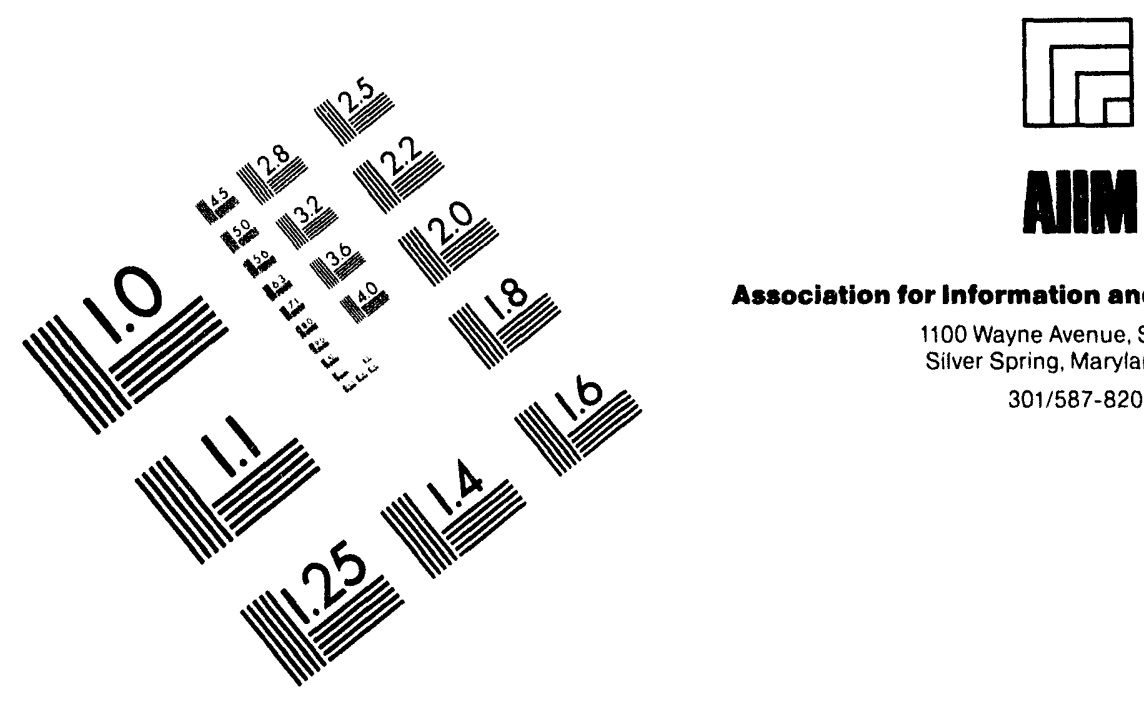

Association for Information and Image Management

1100 Wayne Avenue, Suite 1100

Silver Spring, Maryland 20910

$301 / 587-8202$

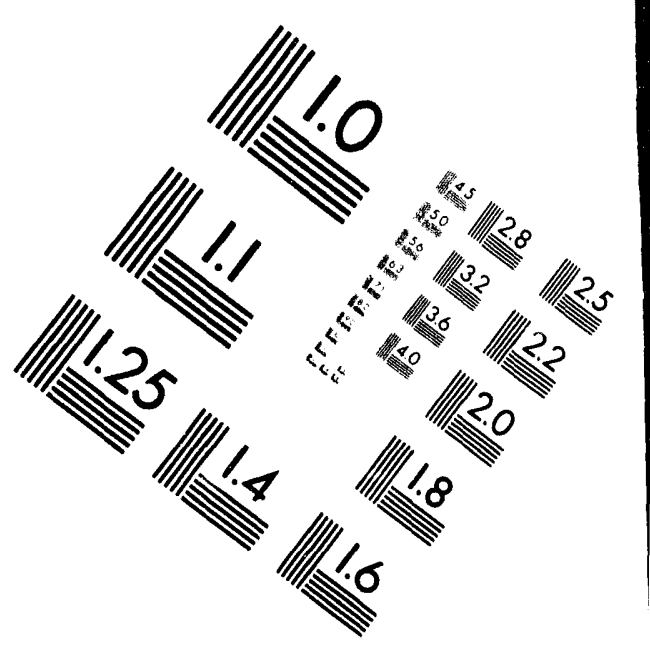

\section{Centimeter}

$\begin{array}{llllllllllllllll}1 & 2 & 3 & 4 & 5 & 6 & 7 & 8 & 9 & 10 & 11 & 12 & 13 & 14 & 15 & \mathrm{~mm}\end{array}$

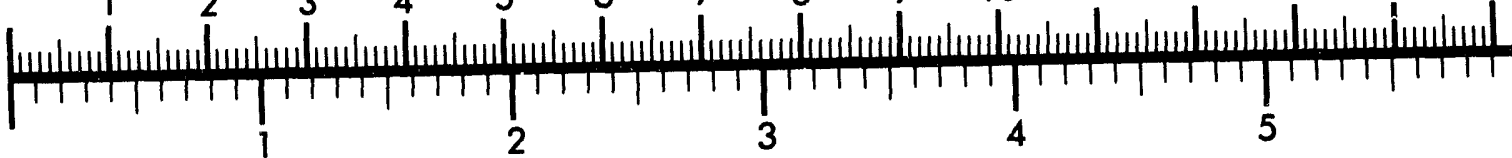
Inches
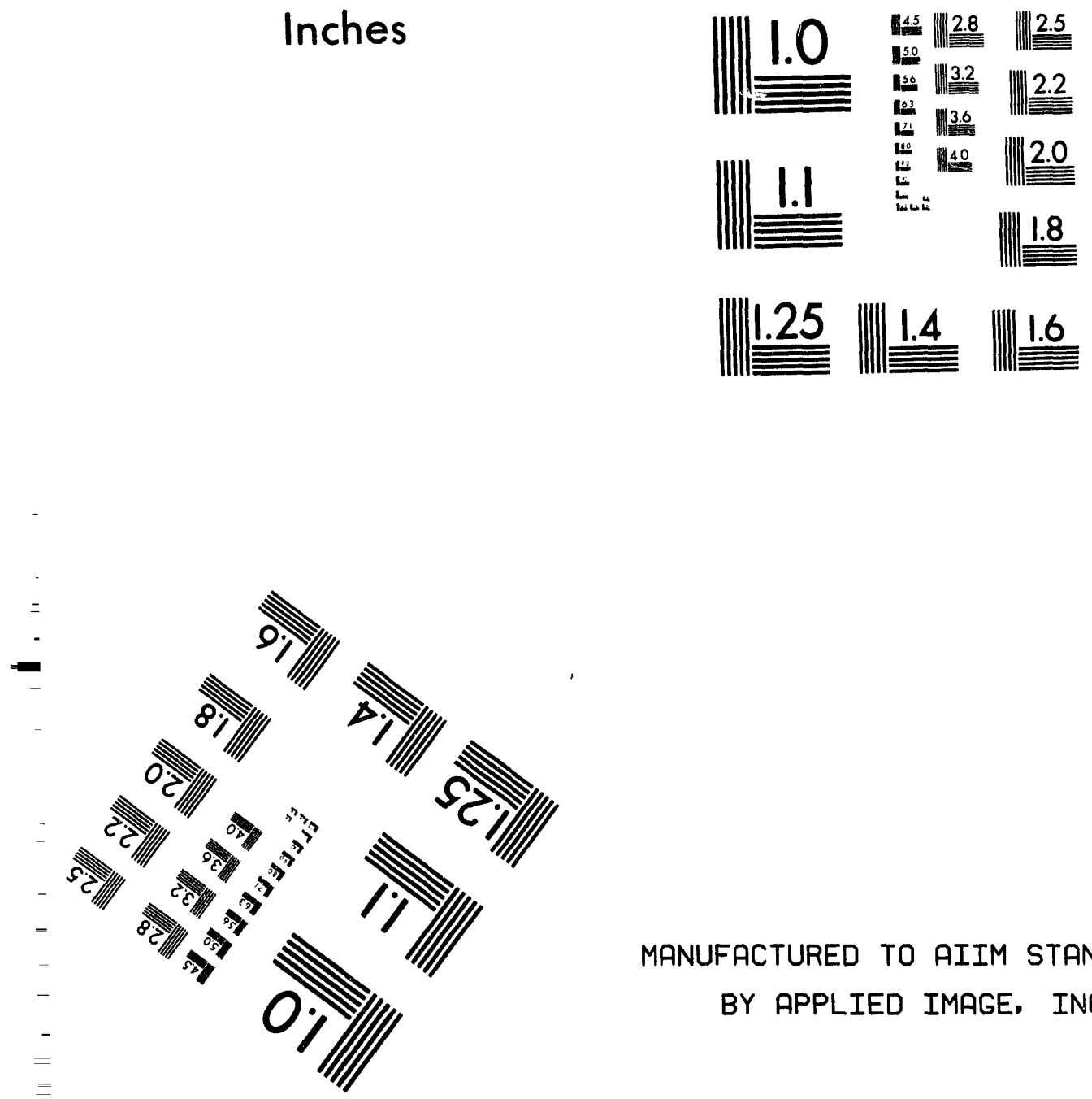

MANUFACTURED TO AIIM STANDARDS

BY APPLIED IMAGE, INC.

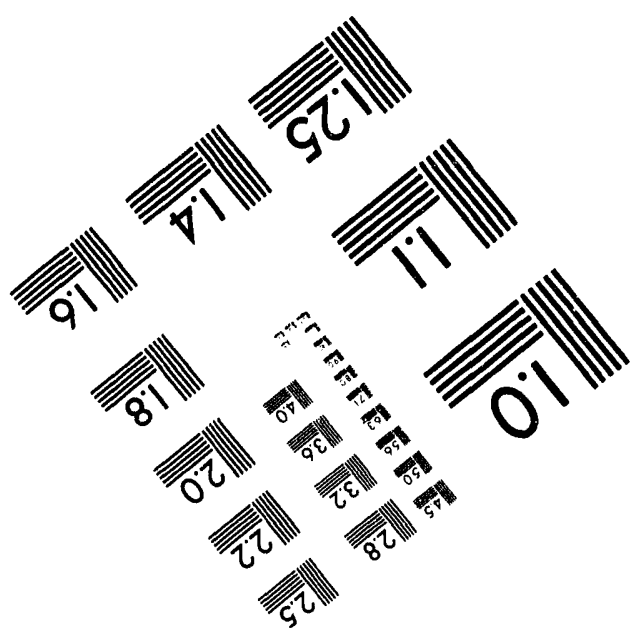



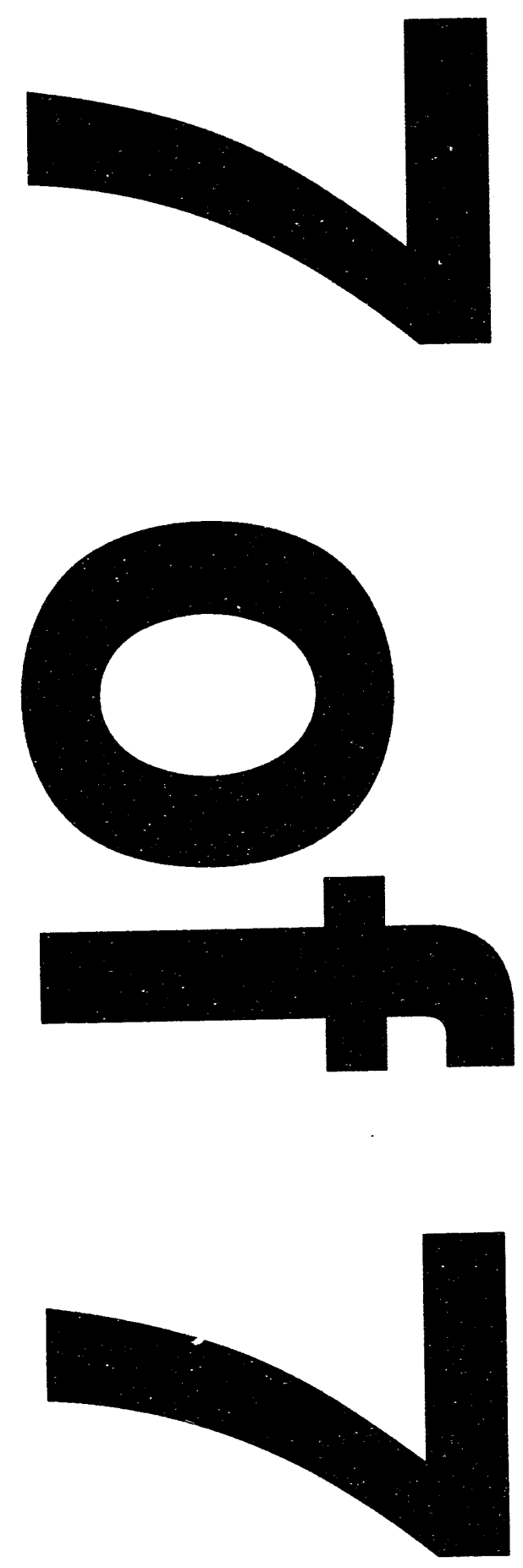
Table D1.3

Comenents on the Domisant Saquence (Roduced) Model

Frovidad to DNL troen PGAE

\section{Action Item 2}

PG\&E is requested to provide the documentation of recovery actions and modeling changes in the non-selsmic dominant sequence model.

\section{Response to Item_2}

A discussion of the recovery actions applifed to the dominant sequence codel follows:

First, the s1x factors appearing at the bottom of the dominant sequence equation file. wich were used to account for recovery actions on selected accident sequences, are described.

\section{REOBI}

REOBI is used to estimate the likelihood of recovery for Sequences $5,58,59$, and 60 . Each of these sequences involves fallure of offsite power and loss of emergency onsite AC power to $4 \mathrm{KV}$ emergency buses MF and HH on Unit 1 . For these sequences. diesel generator 12 successfully starts and supplies power to $4 \mathrm{kV}$ emergency bus HG. Fallure of power at buses HF and HH falls the two cotor-driven Auxillary feedwater (AFW) pumps, and the turbine-driven pump fails independently. Loss of all secondary heat removal shortens the time avaliable for recovery actions to approximately 2 hours. An electric power recovery analysis was performed for these sequences: 1.e. factor RESLCI, see Table 6-53 in Reference 1. Successful recovery of electric power prior to core uncovery is assumed to result in success.

In the basic plant model, loss of AC pover was conservatively assumed to result in the inablility of the pressurfizer PORVs to be held open long enough to allow success of bleed and feed cooling. Since, in these sequences, two emergency $A C$ buses are lost and bleed and feed cooling was assumed to requite two Power Operated Rellef Valves (PORVs), no credit was taken in the basic plant model for bleed and feed coolling for these sequences. In these sequences, however, OC power is known to be successful: Inftially, the PORVs only require DC power to be manually held open. Therefore. despite the loss of AC power to two buses, two pressurizer PORVs can be held open inftially for bleed and feed cooling. After many hours, when the batteries (normally charged by battery chargers on $\mathbf{4 8 0} v$ vitai buses HH and MF) are exhausted. it is assumed that one PORV is all that is necessary to continue core cooling via long-term bleed and feed cooling. The factor REOBI accounts for the operator action and hardware necessary to establish bleed and feed cooling and to sultch to recirculation from the containaent sump once the RHST enpties: 1.e.. Open the PORVs (OBI). switchover to recirculation (RFI), start an RHR pUnip (LAI). and start and operate one of one charging pUmp (CH2). Each of these split fractions only takes credit for the one emergency AC bus known to be operable throughout the accident; 1.e., bus HG.

Addftional recovery actions have been Identified but were conservatively onitted. These include the cross-tieing of emergency buses to bus HG to 
Appendix D

Table D1.3 (continead)

permit two success paths of hardware and to allow for longer term recovery of electric power: e.g.. prlor to the tice needed to establish recirculation from the sump.

\section{ISEOS}

RSEOS addresses the recovery aetlons for Sequence 8. Sequence 8 involves a loss of offsite power. success of all five diesel generators. but fallure of the fuel ofl transfer system to provide makeup to the diesel generator day tanks. Term RESLC3 is the probability of not recovering electric power before core danage in this sequence. RESLC3 is presented in Table 6-53 in Reference 1. The recovery analysis makes use of the operable turbine-driven AFH puap. wich is known to successfully operate. even after all emergency AC power is lost due to insuffictent fuel ofl. The allowable recovery time accounts for the delay in losing emergency $A C$ since the diesel generators do operate until their respective day tanks empty. Oniy credit for restoration of offsite power is modeled in the evaluation of RESLC3 since the diesel generators are operable.

Term 2HEFO6 is the error rate for second operator action to reestabilsh fuel oll to the diesel generator day tanks. This makes use of a dedicated. portable fuel ofl transfer pump which is avaflable as an alternate fuel ofl transfer system to the day tanks. The operators must align the portable system and manualiy control the level control valves on the operating diesels.

This recovery action is considered independent of the offsite power recovery analysis because different crews address the different actions.

RSEQ10

RSEQ10 addresses the recovery actions for Sequences 10 and 11 . Sequence 10 Involves a loss of offsite power, fallure of emergency AC power on buses HH on Unit 1 and HG on Unit 2 and the swing diesel is aligned to Unit 2 so that bus HF on Unit $I$ is also unavallable. Aiso, the turbine-driven AFW pump falis independentiy so that there is no secondary heat removal. Sequence 11 is simflar except that bus HH on Unft 2 is falled instead of bus HG.

The recovery analysis considers two options: recovery of electric power and the reallgnaent of the swing diesel to Unit 1 from Unit 2, wich would enable bus MF on Unit I to be energized and supply power to a motor-driven AFH pump. The electric power non-recovery factor is given by RESLCI. RESLCI is the probability of not recovering electric power prior to core damage given fallure of AFW. In the evaluation of RESLCl. credit is taken for offsite power recovery and for recovery of one of the falled diesel generators (see Table 6-53 in Reference 1).

The recovery action to realign the swing diesel generator to Unit I considers the need for power at the HF bus on lati 2. The turbine-driven AFH pula on Unit 2 is assuaned to be required on Untt 2 in order for the operators on that unit to permit the release of the swing diesel for service on Unit 1 . Therefore, fallure of the operator action to realign the swing diesei (ZHESWI) 
Appendix D

Table D1.3 (contiased)

or fallure of the Unit 2 turblne-driven AFW punp (AW4) is assumed to preclude this recovery action.

Other recovery actions involving cross-tleing eanergency AC buses and/or establishing bleed and feed cooling were conservatively not credited.

RSEQ24

RSEQ24 is the non-recovery factor for accident Sequence 24 in the dominant sequence list. This sequence involves a loss of offsite power. Pallure of power at emergency bus HH, and a total loss of AFW. In the initial plant sequence model. no credtt for bleed and feed cooling was taken for this sequence. This is because PORV 474 is unavallable due to the loss of offsite power. Which is assumed to fall instrument alr. and PORV 456 would eventually lose $D C$ control power since charging to $125 \mathrm{~V}$ OC bus 13 is provided by bus HH. With only one remaining PORV supported, the bleed and feed cooling success criteria of two PORVs could not be satisfied. However. PORV 456 would be avallable for many hours until the battery supplytng DC bus 13 is exhausted. It is belleved that after this initial period, one PORV would be sufficient for continued bleed and feed cooling. The terms added to make up factor RSE024 are the system's fallure probabilitties for the equipment needed to establish bleed and feed cooling and to establish eventual recirculation from the sump. OBI models the operator action to inftiate bleed and feed cooling. UAl and VAl model the equipment needed to establish recirculation from the contalnment sump to the suction of the high pressure pumps. RFI models the operator action to align for recirculation once the RWST empties. CH2 models the charging punp fallures. All of these system fallure rates account for the boundary condition that $4 \mathrm{kV}$ emergency bus HH on Unit 1 is unavaflable.

Additional recovery actions to restore electric power, align the backup battery charger to DC bus 13 , or to crosstie emergency buses so as to restore AFW were conservatively anitted.

RSEQ25

RSE025 models the recovery actions considered for Sequences $25,56,57,70$. and 71 . These sequences all involve a loss of offsite pover and failure of the two diesel fuel oll transfer trains shared by both units. Fallure of all fuel ofl transfer eventually results in a subsequent loss of all emergency AC power at both units.

Two separate recovery actions are modeled for these sequences, both of wich are included in factor RSEQ25. RESLC3 is the probability of not recovering electric power before core danage given AFW is successful (1.e.. the turbine-driven AFH pur is known to be operabie in these sequences). and that a delayed loss of emergency AC power results once the diesels run out of fuel In their day tanks. Credit is taken for the recovery of offsite power prior to core uncovery wich results from a postulated seal Loch.

The term ZHEFO6 is as described for factor RSEQ8. 2HEFO6 is the error rate for falling to align a portable fuel ofl transfer system in order to 
Table D1.3 (continead)

reestablish fuel ofl to the diesel generator day tanks. This action is assumed independent of the other recovery actions since different crews are Involved and both strategles would be attempted.

\section{DSEO34}

RSEQ34 models the recovery actions for Sequences 34 and 39 . These sequences Involve a loss of offsite power. fallure of emergency AC power at the HG buses at both units, the allgnaent of the swing diesel generator to Unit 2, and the fallure of ef ther component coolling water on Unit I (CCS. Sequence 34) or auxillary saltwater (ASB. Sequence 39). As a result of falling ef ther component cooling water or auxillary saltwater, a seal LOCA is assumed to develop, wich leads to core damage. For these sequences, the recovery action to allgn an alternate mode of cooling water to the charging pumps is not viable. This is because power is not avallable to the charging punps: 1.e.. buses HF and HG are unavallable on Untt 1.

RSEQ34 considers two recovery strategles. Term RESLC3 is the probability of not recovering electric power prior to core damage given AFW is successful. No credit for repalr of the falled emergency diesel generators was assumed. This term accounts for the recovery of offsite power only (see Table 6-53 in Reference 1). Term ZHERE2 accounts for the recovery action to crosstie two emergency buses given that one is initially avallable. If this action is successful. then successful restart of CON or ASW is assumed. which permits el ther prevention of a seal LOCA or successful high pressure injection depending on the time the cross-connection is completed. The two recovery strategles are evaluated independently because different crews are directed at each one.

The following discusses the selected sequences which were al so modifiled to reflect sequence specific recovery actions and/or modeling improvements to reduce conservatisms.

\section{SECO63}

Sequence 63 involves a medium LOCA in wich all high pressure injection falls. For medium LocAs. It was assumed that two out of four high head pumps (1.e.. centrifugal charging or safety injection pumps) were required for success. This requitrement for any two of four high head pumps was conservatively approxianated as one of two charging and one of two safety injection pumps. For the sequence in question. the $\mathrm{CH}$ top event. which models one of two charging pumps, was found to be successful. However, both safety injection pumps falled. Therefore. the sequence was assumed to be a fallure of high head injection. For this sequence, however, the CH top event was successful and since no support systems were falled, it is very likely that both centrifugal charging pumps would be operable. To ellminate this modeling approximation. the originaliy conservative success criteria was revised to reflect the realistic success criterla. Because 1 t was not part of the original plant model, an evaluation of top event $C H$ in which both charging pumps are requited for success (1.e., or sequences in which both safety injection pumps fail) was not computed. A simple and conservative estimate of 
Appendix D

Table D1.3 (continued)

the charging system pallure fraction was and for the success criterla when two pumps are required. The cy top event boundary condition for the case then one train of support is falled was used by multiplying the one of one pump train condition by 2 to convert it to a two of two pump train success criteria condition. This explains the factor of 2. which appears in the equation for Sequence 63.

\section{SE0043}

This sequence Involves a loss of offsite power in which the swing diesel generator is aligned to Untt 2. Auxlliary foedwater falls. and the operators fall to establish bleed and feed cooling via the two pressurizer POaVs avallable. Split fraction $\mathrm{NH}_{3}$ was used in the orfginal plant model because bus HF on Unit 1 is not avallable: 1.e.. motor-driven AFH pump is is not avallable. The tralling part of this sequence, beginning with spilt fraction AWI. was intended to account for the recovery action in inich the operators reailgn the swing diesel generator to Unit I so that AFH pump 13, which then has power. my be included in the evaluation of AFH. The 11sted equation appears to be in error.

The original equation is:

SEQ043 - LOOP* OGF*SHI*IAF*AH3*OBI* (ANI/AH3* (ZHESHI+AN3)

The equation should be revised as follows:

SEOO43 - LOOP* OGF*SWI*IAF*AN3*OBI*(AN1 /AW3+2HESWI+AN3)

The error is of minor signiflcance: the sequence frequency with the error is approximately 2E-10, the sequence frequency after correction is approxinately 6E-8. The not impact on the total core damage frequency is an increase of less than $0.05 \%$.

In the corrected equation. ZHESWI is the error rate for realigning the swing diesel generator back to Unit 1. AW3 is the AFW system fallure fraction for Unit 2 given that the operators align the swing diesel to Unit 1: 1.e.. the system fallure fraction with one train of electric power unavallable. It is assumed that the operators would not transfer the swing to Unte 1 if AFN would then be unavallable on Unit 2.

\section{OTHER}

The IIne in the dominant sequence flle labeled "OTHER" accounts for two groups of inftlators: 1.2. hazardous cheateals and control room fires. The variable CRFIRE is actually the su of all control rocm fire sequences, wich lead to core dange. The varfable Muzcuil is the su of all chemical release sequences (in particular. chlorine and amonia releases) in wich the release arrives at the control room afr intake, and the operators are eventually overcome. This frequency is very conservative because, anong other reasons, a probability of unity is assumed that, given a release. It arrives at the control room air intake. It is assumed that the operators would trip the plant before they 
Table D1.3 (continuod)

become incapacitated. This is belleved to be conservative because the chances of an independent cause of plant trip, wich wight later necessitate operator intervention before the next shift reports for duty, is remote. Several hours are avallable before an operator action would be required following a plant trip: 1.e. not until the condensate storage tank (CST) aust be replenished in order to maintain AFW cooling. The chances of falling to replenish the CST under these conditions are given by ZHEHS5. A factor of 0.1 was used to provide the conditional probability of core damage given that the CST was not initially repienished. A detalied evaluation of this probability was not performed. Since there would be substantial time avallable to avold core danage and the original chenical release sequence frequency was conservatively estimated. a further Investigation into this factor was not deemed warranted.

Reference 1: Final Report of the Diablo Canyon Long-Term Sefsmic Program July 1988

Paclfic Gas \& Electric Company

Diablo Canyon Power Plant

Docket Mos. 50-275 and 50-323 
Table D1.4

Reduced Model Sequences Requiring the Addition of a Dummy Variable

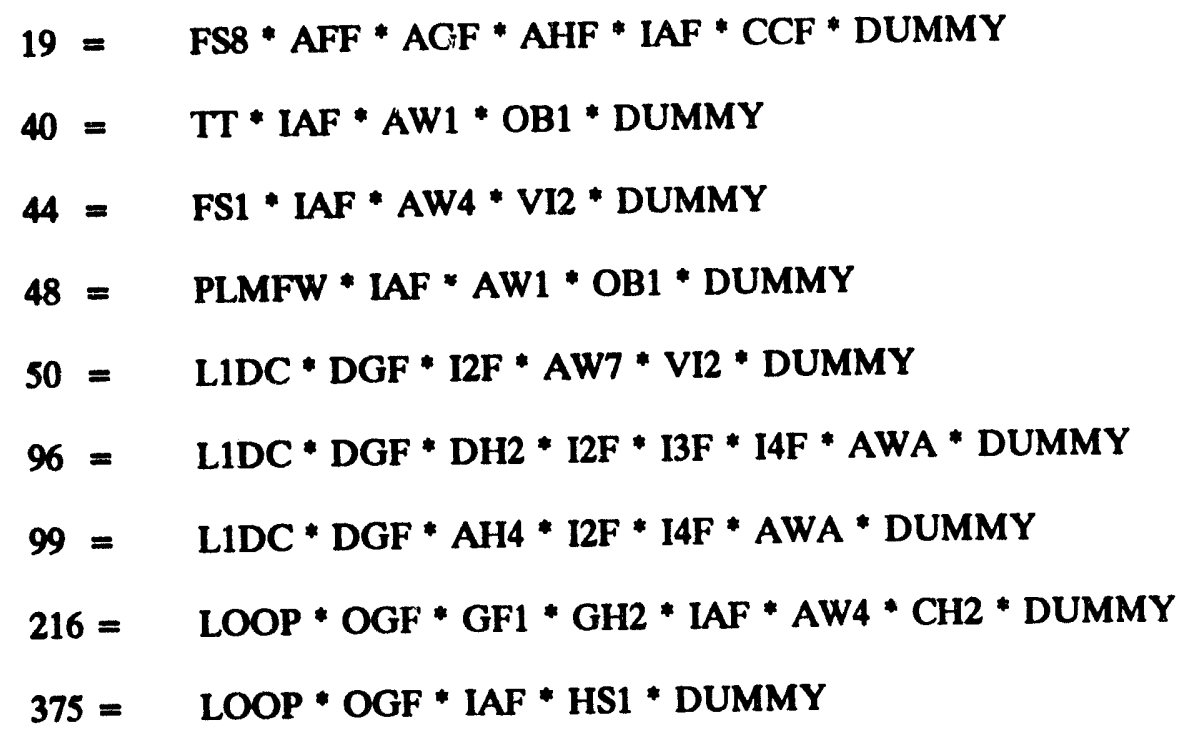


Appendix D

\author{
Table D1.5 \\ Brief Deseriptiose of Cosditional Spllt Tranctions \\ and Initiatiag Evants (Twll DCPRA Modal)
}

Note: This table was supplied to BNL by PG\&E and is included herein to help the reader understand the various model elemeats.

Model

Element

1. $A 51$

2. AS2

3. AS3

4. AS4

5. ASS

6. AS6

7. AS7

8. ASE

9. AS9

10. ASA

11. ASB

12. ASC

13. Losw

14. ASF

15. CC1

16. CC2

17. CCJ

1日. CCA

19. CCs

20. CCS

21. CC7

22. LPCC

23. CCF

24. FC1

25. FC2

26. FCJ

27. FC4

28. FCF

29. $M S O$

30. MS1

31. MS2

32. MSF

33. TTO

34. TT1

35. TTZ

36. TT3

37. TT4

38. TTS

39. TT6

40. TTF

41. SVI

42. SVZ

43. SV3

44. SV4

\section{Despiotion}

All Pump Trains Available: 2 Running, 2 Standby (OP1)

3 Pump Trains Available: Fail Train 11 (OP2)

3 Pump Trains Available: Fail Train 12

2 Pump Trains Available: Fail Trains 11 and 12 (OP2)

LOSP: 3 Pump Trains Available: Fail Train 11 (OP2)

LOSP: 3 Pump Trains Available: Fail Train 21 (OP1)

LOSP: 2 Pump Trains Available: Fail Trains 11 \& 12 (OP2)

Losp: 2 Pump Trains Available: Fail 11 \& 21 (or 22) (OP2)

LOSP: 2 Pump Trains Available: Fail Trains 12 \& 2 (OP1)

LOSP: 2 Pump Trains Available: Fail Trains 21 \& 22 (OPF)

LQSP: 1 Pump Train Availableifail 11,12 \& 21 (or 22)(OP2)

LosP: 1 Pump Train Availableifail 11 (or 12),21 22(OPF)

Loes of ASW Supply to Unit 1 Initiating Event Frequency

Guaranteed Failure

Al1 Support Available(N/3 pumps starts and/or runs)

Loss of $4 K V$ Bus $H \quad$ (N/2 pumps runs)

Loss of 4KV Bus $G$ (N/2 pumps starts and/or runs)

Loss of $4 K V$ Buses $G$ and $H$ ( $1 / 1$ pump runs)

Loses of $4 K V$ Buses $F$ and $G$ ( $1 / 1$ pump starts and runs)

LOSP - All Support Available(N/J pumps starts and runs)

LOSP - Loss of one $4 K V$ bus ( $N / 2$ pumps starts and runs)

Initiating Event Frequency (All pumps tail)

Guaranteed Failure

2 OF 5 CFCUs start and operate 24 hours

2 OF 4 CFCUs start and operate 24 hours

2 QF 3 CFCUs start and operate 24 hours

2 OF 2 CFCUs start and operate 24 hours

Guaranteed failure

Main Steam Isolation, TT failed, fire scenario 2

Main Steam Isolation, TT succeeds- All Support Avail.

MS Isolation - TT fails. All Support Avail.

MS Isolation - Guaranteed failure

Turbine Trip - TT Initiator

Turbine Trip - All Support Available

Turbine Trip ATWT - All Support Available

Turbine Trip ATWT, Man. Rx trip - All Support

Turbine Trip - 1 Train of Support Avail.

Turbine Trip ATWT - 1 Train of Support Avail.

Turbine Trip ATWT, Man. Rx trip-1 Support Train

Turbine Trip - Guaranteed failure

$1 / 2$ trains; 0SP, $480 \mathrm{~V}$ 1F, $1 \mathrm{H}$ available

$1 / 1$ train start and run; $480 \mathrm{~V}$ Bus IF unavailable

$1 / 1$ train continue to run: $480 \mathrm{~V}$ Bus $1 \mathrm{H}$ unavail.

$1 / 2$ trains start and run; LOSP, $480 \mathrm{~V}$ Bus $1 F, 1 H$ availab. 
Model

Blenent

Description

45. SVS Only recovery poseible, Bus 1F, IH unavailable

46. SVF Guaranteed failed, all inverters alrready failed

47. LosWV Initiating Event frequency for 1 year

48. Svo Station Blackout, guaranteed success.

49. CS1 1/2 Trains Operates (Al1 Support Avaliable)

50. CS2 $1 / 1$ Train Dperates(Loss of One Vital Bus or Ssps train)

51. CSF Guaranteed failure

52. SR1 $1 / 2$ Trains Operates(All Support Available)

53. SR2 1/1 Train Operates(Loss of 1 Bus or SSPS or RHR train)

54. SRF Guaranteed failure

55. SA1 General Transient

56. SAZ

57. SA3

5B. SA4

59. SAS

60. SAG

61. SA7

62. SAB

63. SAF

64. SB1

65. 582

66. 583

67. 584

6日. 985

69. 586

70. 587

71. SBE

72. $5 B 9$

73. SBA

74. SBE

75. SBC

76. SED

77. SBE

7日. SBE

79. SBH

BO. SEI

Q1. SBJ

82. SBK

83. SBL

84. SBM

85. SBN

86. SEF

67. CVI

8. CVz

89. CVS

90. CV4

91. CVs

92. CVG

93. CVF

94. LOCV

95. RT1

Large Loss of Coola

Lloca with lose of Pown Accident All 4 Channels Available

Steam Benerator Tube Rupture

steam Line Break Inside Containment All 4 Channels AvIb

SLBIC with lose of power to two $\Gamma^{\circ} H-H$ channels (not I)

Steam Line Break Outside Containn it

Small Loss of Coolant Accident

Guarantead Failura

GT given Train $A$ success

GT given Train A failure

GT given AC I unavailable (same as SAL)

LLOCA given Train A success, all AC channels available

LLOCA given Train A success, AC IIeIII unavailable

LLOCA given Train A failure, al $A C$ Channels available

LLOCA given Train A failure, AC IICIII unavailable

LLOCA given AC I and II (or III) unavailable (same as SAJ)

SGTR given Train A success

SGTR given Train A fallure

SETR given AC I unavailable (ame as SA4)

SLBIC given Train A suceess, all AC channels available

SLBIC given Train A suceess, AC I I\&III unavailable

SLBIC given Train A failure, all AC channels available

SLBIC given Train A failure. AC IICIII unavailable

SLBIC given AC I and II(or III) unavailable (same as SAG)

SLBOC given Train A success

SLBOC given Train A failure

SLBOC given AC I unavallable (same as SA7)

SLDCA given Train A success

SLOCA given Traín A failure

SLDCA given AC I unavailable (same as SAB)

Guaranteed Failure

$1 / 2$ subtrains: All support available (OSP, 2F, 16,1H,2H)

$1 / 2$ subtrains: Normal power for subtrain $F$ unavail. ( $2 F$ )

$1 / 1$ subtrain: No support for subtrain $F(2 F, 1 G)$

$1 / 1$ subtrain: No support for subtrain $H(1 H, 2 H)$

$1 / 2$ subtrains:LOSP, all vital buses avail. $(2 \mathrm{~F}, 1 \mathrm{G}, 1 \mathrm{H}, 2 \mathrm{H})$

$1 / 1$ subtrains:LOSP, no support for subtrain $H(1 H, 2 H)$

Guaranteed Failure: $480 \mathrm{~V} 2 \mathrm{~F}, 1 \mathrm{G}, 1 \mathrm{H}, 2 \mathrm{H}$ unavailable

Initiating Event frequency for 1 year

1/2 Trains (both SSpS signals generated)

$1 / 2$ Trains (DC power lost to one shunt trip) 
Model Blement

97. RT3

98. RTA

99. RTS

100. RTG

101. RT7

102. RTF

103. CH1

104. $\mathrm{CHZ}$

105. $\mathrm{CH}$

106. CH4

107. CHF

10B. 511

109. 512

110. 513

111. SIF

112. HR1

113. HR2

114. HRJ

115. HR4

116. HRS

117. HRG

118. HR7

119. HRE

120. HR9

121. HRA

122. HRB

123. HRC

124. HRD

125. HRE

126. HAF

127. RC1

128. RC2

129. RF 1

130. RF2

131. RF3

132. RFA

133. LA1

134. LA2

135. LA3

136. LAF

137. LB1

138. LB2

139. LB3

140. LBA

141. LBS

142. LB6

143. LE7

144. LBE

145. LE9

146. LBF

147. LV1

148. VA1

\section{Desartiption}

$1 / 2$ Trains (DC power lost to both shunt trip coils)

1/1 Train (only one SSPS ignal generated)

1/1 Train (one ssps signal, LoP to shunt trip coil)

Gravity Insertion (insufficent power to prevent insert)

Operator initiated (DC power lost to both shunt coils)

Guaranteed failure

All support available.

One standby pump train available only

Normally running pump train available only.

LOSP i All support available

Guaranteed failure.

All support available $(1 / 2)$

One safety injection pump train available only(1/1)

Medium LOCA, All support available, CH failed. (2/2)

Guaranteed failure.

All support available

Top event CH or SI failed

Top event LA or LB failed

Top event CH or $S I$ and top events LA or LB failed

AKV Bus F railed

4KV Bus F failed, top event CH or SI failed

4KV Bus F failed, top event LA or LB failed

4KV Bus $F$ failed, top event CH or SI \& LA or LB failed

4KV Bus $F$ and 4KV Bus $G$ failed

4KV Bus $F$ and $4 K V$ Bus $H$ tailed

4KV Bus G failed

4KV Bus G tailed, top event CH or SI failed

4KV Bus $H$ falled

4KV Bus $H$ failed, top event $C H$ or SI failed

Guarantead failure

Both RHR pump trains operable

One RHR pump train operable

Switchover after SLOCA or B/F with CS failed

Switehover after SLOCA or B/F with CS success

Switchover after LLOCA or MLOCA initiating event

Switchover to recirculation after core melt

All support available. (SLoCA Case)

All support available. (Bleed \& Feed case)

All support available. (LLOCA/MLOCA Case)

Guaranteed failure

All support available. Top event LA successful. (SLOCA)

All support available. Top event LA failed. (SLOCA)

Top Event LA Guaranteed Failure (SLOCA)

All support available. Top event LA successful. (B \& F)

All support available. Top event LA failed. (B \& F)

Top Event LA Guaranteed Failure (B \& F)

All support available. Top event LA successful. (LLOCA)

All support available. Top event LA failed. (LLOCA)

Top Event LA Guaranteed Failure (LLOCA)

Guaranteed failure

All conditions (No support required)

All support available. 
Model

Element

149. VAF

150. VBI

151. VBZ

152. VBJ

153. VBF

154. RWI

155. $A C 1$

156. LII

157. LI 2

158. MU1

159. MUF

160. MUZ

161. AW1

162. AWZ

163. AWJ

164. AW4

165. AW5

166. AW6

167. AW7

168. AWE

169. AW9

170. AWA

171. AWB

172. AWC

173. AWF

174. TD1

175. TDZ

176. TDF

177. PRO

178. PR1

179. PR2

180. PRJ

181. PR4

182. PR5

183. PR6

184. PR7

185. PRE

186. PRT

187. PRA

18日. PRB

189. PRC

190. PRD

191. PRE

192. PRF

193. PRG

194. PRH

195. PRI

196. PRJ

197. PRK

198. PRL

199. PRM

200. PRN

201. PRP 
Mode1

Element

202. PRQ

203. PRR

204. PRS

205. PRT

206. OB1

207. OB2

208. DB3

209. OBF

210. POI

211. PO2

212. POJ

213. POF

214. CII

215. CI2

216. CIJ

217. CI 4

218. CIS

219. CI6

220. CIF

221. CP1

222. CP2

223. CP3

224. CP4

225. .PS

226. CPG

227. WLI

228. WL2

229. WLJ

230. VDI

231. VSI

232. VO1

233. VOZ

234. VR1

235. VR2

236. VC1

237. VC2

23日. ITI

239. LWI

240. LW2

241. LW3

242. SM1

243. SMZ

244. ME1

245. MEZ

246. OVI

247. OTI

248. OTF

249. OL1

250. MUV

251. 111

252. 112

253. IIF

\section{Description}

L/1 PORV or (1/3 SRV s), Manual reactor trip

$1 / 3$ SRV $s$, Manual reactor trip

$1 / 2$ PORV's or ( $1 / 3$ SRV' $s)$, Manual reactor trip

$1 / 1$ PORV or ( $1 / 3$ SRV's), Manual reactor trip

Loss of Instrument air

Loss of Instrument air, charging failed

Loss of 1 DC bus Initiating event

Guaranteed Failure

$1 / 2$ PORVS ATWT, boration, all support, AFW avail.

$2 / 2$ PORVS ATWT, boration, no block valves, no AFW

$1 / 2$ PORVs ATWT, boratiun, no block valves, AFW avail.

Guaranteed Failure

Either iriboard or outboard isol. valve(s) must close Inboird vlves(pen 45) and $1 / 2$ vives(pen $50,51,52$ ) close

Inboard isolation vlaves (pen 45,50,51,52) must close Inbd. or Qutbd. Isolation vlvs close - Excessive LOCA Inbd.pen.45 1/2 vlve pen.50,51,52 close - ELOCA

Inbd.isol.vlvsi.pen.45,50,51,52 close - ELOCA

Guaranteed failure

Either inboard or outboard isolation valve(s) must close

Dutboard isolation valves must close

Fraction of time penetration 61,62 , or 63 is open

Same as CP1 with VI failed seismicly

Same as CP2 with VI failed seismicly

Same as CP3 with VI failed seismicly

Either FCV-500 (inboard) or FCV-501 (outboard) must close Inboard VIV FCV-500 (or outboard VIV FCV-501) must close Fraction of time containment sump discharge line is open Initiating event frequency (discharge side valves)

Initiating event frequency (suction side valves)

Pressure relief valves open $3 / 3$ for VSI IE

Pressure relief valves open $2 / 2$ for VDI IE

Pressure relief valves reclose $3 / 3$ for VSI IE

Pressure relief valves reclose $2 / 2$ for VDI IE

Leak rate of $1700 \mathrm{gpm}$ for VSI IE

Leak rate of $800 \mathrm{gpm}$ for VDI IE

RHR piping intact; VO successful

RCS flow to RWST for VSI IE

Guaranteed success

MOV support power not available

Small LOCA; for VSI IE

Small LOCA; for VDI IE

Medium LOCA; for VSI IE

Medium LOCA; for VDI IE

Failure to dingmoses a LOCA to RHR; Initiates ECA 1.2

Failure to isolate break, stops leakage; Initiates E-1

Operator fails to isolate break

Operator failis to depressurizes RCS

Makeup to RWST

Given: $D F-5, A F-5, A G-5$ or $D F-5, A F-F, A G-5$.

Given: $D F-S, A F-5, A G-F$ or $D F-S, A F-F, A G-F$.

Given: DF-F (guaranteed failure). 
Mode1 Elenent

254. 121 255. 122

256. 123

257. I 24

25日. I2F

259. 131

260. 132

261. IJF

262. 141

263. I 42

264. I4F

265. 051

266. DSF

267. IAF

268. CDF

269. FWF

270. RPO

271. RPI

272. RP2

273. RPF

274. SE1

275. SE2

276. SEO

277. SEF

278. VIO

279. VII

280. VI2

281. VIJ

282. VI4

283. VI5

284. OIF

285. OII

286. 012

287. 013

286. OP1

289. OP2

290. OE1

291. DE2

292. OEJ

293. $H 51$

294. HS2

295. HS3

296. HS4

297. HSF

298. RS1

299. RSF

300. PLI

301. MC1

302. SSF

303. ODF

304. CT1

305. CT2

306. CTF

\section{Description}

Given: AG-S.

Given: DG-S, AG-F.

Given: $A G-S$, I1-F

Given: DG-S, $A G-F, I 1-F$

Given: DG-F (guaranteed failure).

Given: $D H-S, A H-S, A G-5$ or $D H-S, A H-F, A G-S$.

Given: $D H-S, A H-S, A G-F$ or $D H-S, A H-F, A G-F$.

Given: DH-F (guaranteed tailure).

Given: $D G-S, A H-S, A G-5$, or $D G-S, A H-F, A G-S$.

Given: $D G-F, A H-S$ or $A G-F, D G-S,(A H-S$ or $A H-F)$

Given: DG-F, AH-F (guaranteed failure).

Manual $S I$ Actuation

Guaranteed Failure

Guaranteed Failure

Guaranteed Failure

Guaranteed Failure

Guaranteed Success

RCS pressure <1275\#

CCW lost, operator must trip to prevent seal loca

Guaranteed Failure

RCP Seal Cooling, CCW unavailable

RCP Seal Cooling, CCW available

Guaranteed Success

Guaranteed failure

Vessel Integrity Guaranteed success

Vessel Integrity (TT \& MS Failed)

Vessel Integrity Loss of Secondary Heat Sink

Vessel Integrity Medium LOCA Events

SGTR; With Successful ECCS Termination

SGTR; With Delayed ECCS Termination

Guaranteed Failure

when WL fails

when CP fails

when CI fails

SOTR when SL $S$, terminate $S I$

SGTR when SL F,B; terminate SI

initiate boration in 10 minutes given ATWT

initiate boration in 20 minutes given ATWT

initiate boration in 30 minutes given ATWT

not standby,all available

hot standby, with small LOCA

hot standby, instrumentation last

hot standby,LOCA and instrumentation lost

guaranteed failure

43 of 53 inserted within 10 minutes

reactor trip failed

power level greater than $80 \%$

moderator coefficient less negative than -7

Guaranteed Failure

Guaranteed Failure

Seismic Failure of relays chattering givne /OP

Seismic Failure of relays chattering given oP

Guaranteed Failure 
Model

Element

307. EL 1

308. ELF

309. IDI

310. IDF

311. OG1

312. OGF

31.3. NV1

314. NV2

315. NVF

316. DF 1

317. DG1

318. DG2

319. DGF

320. DH1

321. $\mathrm{DHZ}$

322. DH3

323. DH4

324. AF 1

325. AFA

326. AFF

327. AG1

328. AG2

329. AG3

330. AGA

331. AGB

332. AGC

333. AGF

334. AHI

335. AH2

336. AH3

337. AH4

338. AH5

339. AHS

340. AHA

341. AHB

342. AHC

343. AHD

344. AHE

345. AHG

346. AHF

347. SF 1

348. SFA

349. SG1

350. SG2

351. SGJ

352. SGA

353. SGB

354. SGC

355. SH1

356. $5 \mathrm{H} 2$

357. SH3

358. SH4

\section{Description}

Excessive LOCA

Guaranteed Failure

Identification of operator

Guaranteed Failure

Given Offsite Grid success.

Given Offsite Grid fails (guaranteed failure $O G$ ).

Given all support available.

Given DC 13 or DC 12 failed and $O G$ succeeded.

Given DC 13 and DC 12 failed or, $O G$ failed.

$480 \mathrm{~V}$ vital bus $1 F$ available.

$480 \checkmark v i t a l$ bus 16 available, DF succeeded.

$480 \checkmark$ vital bus 16 available, DF failed.

Guaranteed tailure.

$480 V 1 H$ available, DF-S, DG-S

$480 \vee$ IH available, DF $-5, D G-F$

$480 V$ IH available, DF-F, DG-5

$480 V 1 H$ available, DF-F, DG-F

All support available with recovery.

All support available no recovery.

Guaranteed failure.

DF-5, AF-5 with recovery

DF-S, AF-F with recovery

DF-F with recovery

DF-5, AF-S no recovery

DF-S, AF-F no recovery

DF-F no recovery

GUARANTEED FAILURE

DF-5, DG-S, AF-S, AG-S with recovery

DF-5, DG-S, AF-S, AG-F, or DF-S, DG-S, AF-F, AG-S w.r.

$D F-5, D G-S, A F-F, A G-F$ with recovery

$D F-5, D G-F, A F-5$ or $D F-F, D G-S, A G-5$ with recovery

$D F-S, D G-F, A F-F$ or $D F-F, D G-S, A G-F$ with recovery

DF-F, DG-F with recovery

DF-S, DG-S, AF-S, AG-S no recovery

$D F-S, D G-S, A F-5, A G-F$, or $D F-S, D G-S, A F-F, A G-S n \cdot r$.

$D F-S, D G-S, A F-F, A G-F$ no recovery

$D F-5, D G-F, A F-S$ or $D F-F, D G-S, A G-5$ no recovery

$D F-5, D G-F, A F-F$ or $D F-F, D G-S, A G-F$ no recovery

DF-F, DG-F no recovery

GUARANTEED FAILURE

All support available with recovery.

All support available no recovery.

SF-S with recovery

SF-F with recovery

SF-B with recovery

SF-S no recovery

SF-F no recovery

SF-B no recovery

SF-S, SG-S with recovery

SF-S, SG-F or SF-F, SG-S with recovery

SF-F, SG-F with recovery

SF-S, SG-B or SF-B, SG-S with recovery 


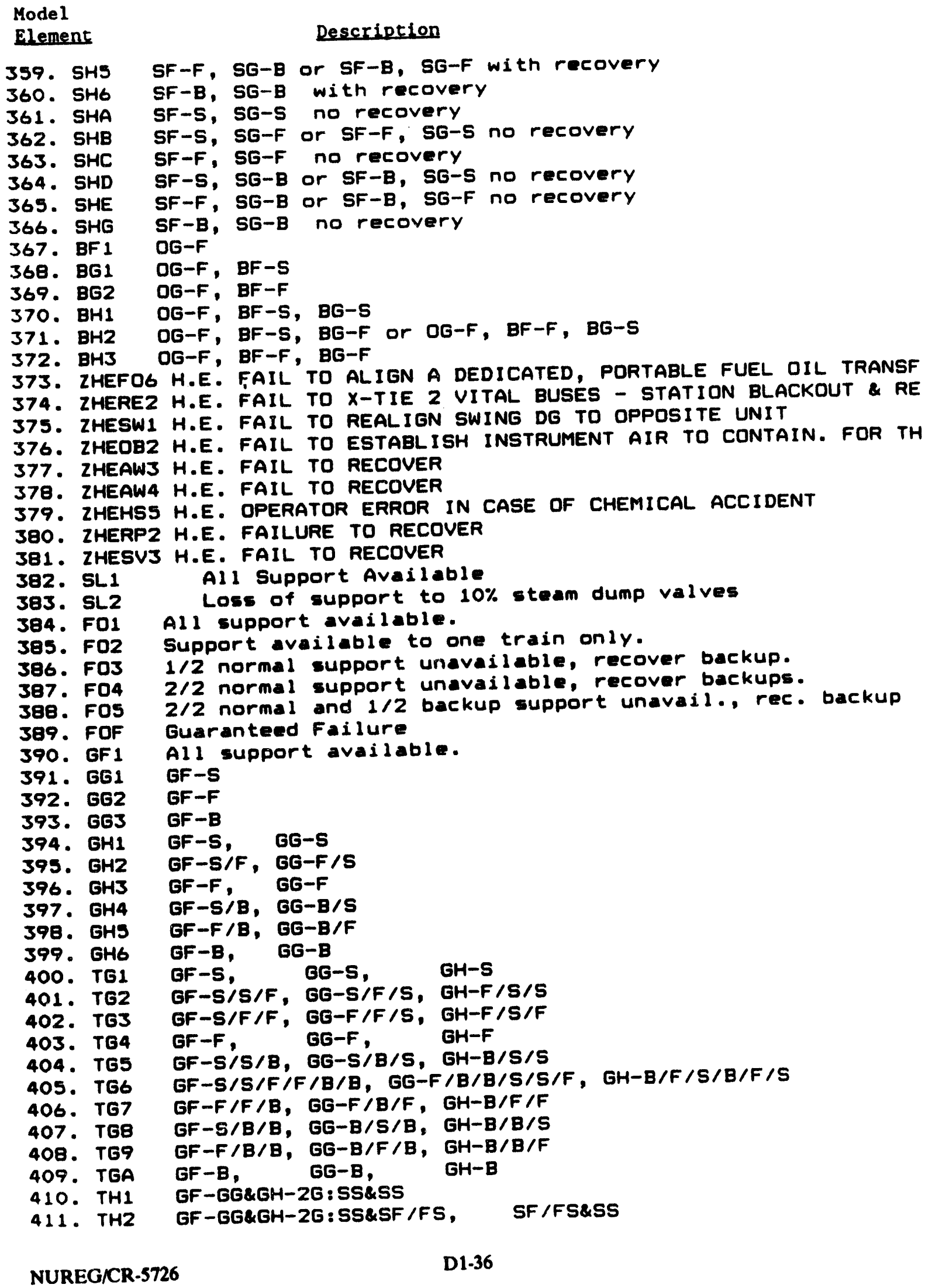


Model

Element

Description

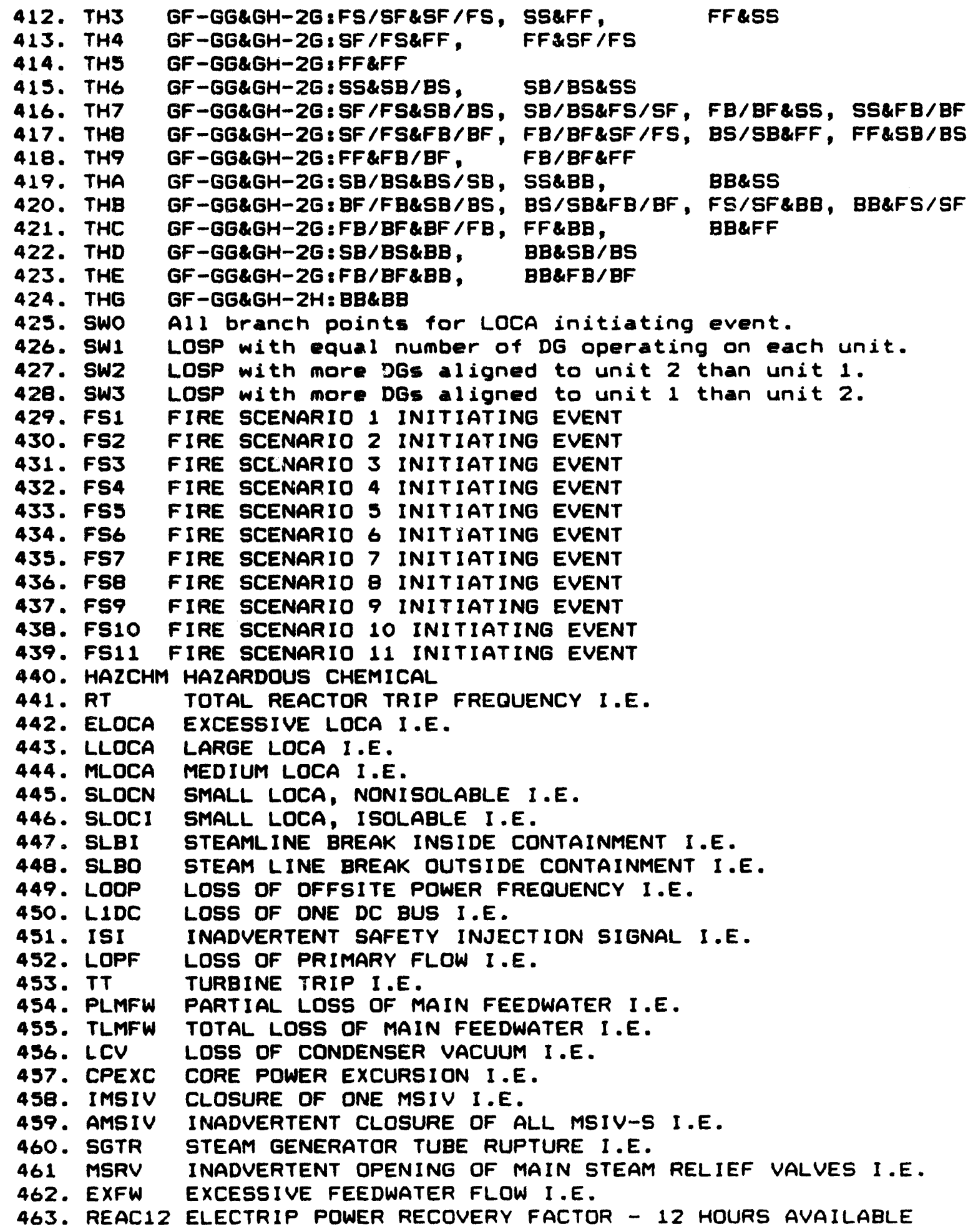


Model

Elenent

Description

464. REACO6 ELECTRIC POWER RECOVERY FACTOR - 6 HOURS AVAILABLE

465. RESLC1 E.P.R.F 3 DGS FAILED BUT DG RECDVERY POSSIBLE, AFW NOT A

466. RESLC2 E.P.R.F 3 DGS FAILED BUT DG RECOVERY POSSIBLE; AFW AVAIL

467. RESLC3 E.P.R.F FUEL OIL TRANSFER SYSTEM FAILURE, AFW AVAILABLE

468. SEIS

SEISMIC CORE MELT CONTRIBUTION - FROM SEIS4 MODEI

469. CRF IRE CONTROL ROOM, CABLE SPREADING RODM FIRE FREQUENC: - TOTA 
Appendix D

\author{
Appondtex D2: Putr Inportance Calculations by Condtitonal \\ Split Trections - Badwead Model
}

This appeadis mpplements the BNL, quantification offered in Section 3.9 and consists of the following tables that present the pair importances of the linted clames of eplit frections by individual eplit fraction. (The pair importasces provided in Section 3.9 are by acuregated top events.) Alt of the pair importance tables provide the ranking by unsormalizod Pusel-Vesoly importance. One additional table bas been added here for completeneas it incorporates all of the conditional split fractions within the reduced model and globally ranks them by their Birnbaum importance.

\title{
Trbie Tite
}

D2.1 Pair Importance by Clanes of Split Fractions (Initiatore - Frontlines)

D2.2 Pair Importance by Claseses of Split Fractions (Initiators - Supports)

D2.3 Pair Importance by Chases of Split Fractions (Initiators - Human and Recovery Actions)

D2.4 Pair Importance by Claves of Split Fractions (Frontlines - Frontlines)

D2.5 Pair Importance by Clacees of Split Fractions (Frontlines - Supports)

D2.6 Pair Importance by Clases of Split Fractions (Frontlines - Human and Recovery Actions)

D2.7 Pair Importance by Classes of Split Fractions (Supports - Supports)

D2.8 Puir Importasce by Clases of Split Fractions (Supports - Human and Recovery Actions)

D2.9 Pair Importance by Claseses of Split Fractions (Human and Recovery Actions - Human and Recovery Actions)

D2.10 Conditional Split Fractions Globally Ranked by Birabaum Importance

NOTE: The software developed at BNL to perform the pair importance calculations included herein remains in a developmental stage. The capability to recognize that the pairing of element $A$ with element B is redundant to the pairing of element B with element A is yet to be realized. Therefore, when pairiag fronthine syatems to frontline systems (Table D2.4) support systems to apport sytems (Table D2.7), and human actions to human actions (Table D2.9) the output provides two redundant lines for each pair. 
Appendix D2: Pair Importance Calculations by Conditional Split Fractions - Reduced Model

This appendix supplements the BNL quantification offered in Section 3.9 and consists of the following tables that present the pair importances of the listed classes of split fractions by individual split fraction. (The pair importances provided in section 3.9 are by aggregated top events.) All of the pair importance tables provide the ranking by unnormalized Fussel-Vesely importance. One additional table has been added here for completeness, it incorporates all of the conditional split fractions within the reduced model and globally ranks them by their Birnbaum importance.

\section{rable ritle}

D2.1 Pair Importance by Classes of Split Fractions (Initiators - Frontlines)

D2.2 Pair Importance by Classes of Split Fractions (Initiators - Supports)

D2.3 Pair Importance by Classes of Split Fractions (Init1ators - Human and Recovery Actions)

D2.4 Pair Importance by Classes of Split Fractions (Frontlines - Frontlines)

D2.5 Pair Importance by Classes of Split Fractions (Frontlines - Supports)

D2.6 Pair Importance by Classes of Spl1t Fractions (Frontlines - Human and Recovery Actions)

D2.7 Palr Importance by Classes of Split Fractions (Supports - Supports)

D2.8 Pair Importance by Classes of Split Fractions (Supports - Human and Recovery Actions)

D2.9 Pair Importance by Classes of Split Fractions (Human and Recovery Actions - Human and Recovery Actions)

D2.10 Conditional Split Fractions Globally Ranked by Birnbaum Importance

NOTE: The software developed at BNL to perform the pair importance calculations included herein remains in a developmental stage. The capability to recognize that the pairing of element $A$ with element $B$ is redundant to the pairing of element $B$ with element $A$ is yet to be realized. Therefore, when pairing frontilne systems to frontilne systems (Table D2.4) support systems to support systems (Table D2.7), and human actions to human actions (Table D2.9) the output provides two redundant lines for each pair. 
Table D2.1

Pair Importance by Classes of Split Fractions (Initiators - Frontines)

Pairs

UNNORM FUSS-V BIRNBAUM

\begin{tabular}{|c|c|}
\hline \multirow{3}{*}{$\begin{array}{l}\text { LDOF } \\
\text { LOOF } \\
\text { L1DC }\end{array}$} & FFD \\
\hline & AW4 \\
\hline & AWA \\
\hline RT & DE1 \\
\hline$T T$ & QE1 \\
\hline LOOF. & AW3 \\
\hline RT & AWS \\
\hline$T T$ & AW5 \\
\hline PLMFW & OB1 \\
\hline SLED & MS2 \\
\hline$L 1 D C$ & OBS \\
\hline PLMFW & AW5 \\
\hline RT & AW1 \\
\hline MLOCA & RF3 \\
\hline TT & AW1 \\
\hline LQOP & LA1 \\
\hline MLOCA & LAB \\
\hline MLOCA & LB2 \\
\hline LQOP & Aws \\
\hline LOOF & LE3 \\
\hline PLMFW & Aw1 \\
\hline LIDC & MS2 \\
\hline SGTR & MU1 \\
\hline SGTR & SL1 \\
\hline LQDP & RF 1 \\
\hline LLDCA & $A C 1$ \\
\hline LOQF & VI2 \\
\hline FS1 & Aw4 \\
\hline SLBO & AWB \\
\hline SLOCI & LAI \\
\hline SLEO & OB1 \\
\hline RT & AWB \\
\hline LLOCA & RF 3 \\
\hline RT & VIZ \\
\hline$T T$ & AWB \\
\hline MLOCA & VI3 \\
\hline$T T$ & VIZ \\
\hline LOOP & 081 \\
\hline SGTR & LA1 \\
\hline SGTR & LE2 \\
\hline LOOP & $\mathrm{CHZ}$ \\
\hline LOOF & HRB \\
\hline LODP & VB1 \\
\hline SLB I & OB1 \\
\hline SGTR & VIS \\
\hline PLMFW & AWB \\
\hline PLMFW & AW: \\
\hline
\end{tabular}

$\begin{array}{ll}1.589 E-05 & 3.576 E-03 \\ 8.101 E-06 & 1.228 E-03 \\ 5.978 E-06 & 2.435 E-03 \\ 5.034 E-06 & 1.528 E-04 \\ 4.703 E-06 & 1.550 E-04 \\ 4.009 E-06 & 3.553 E-02 \\ 3.753 E-06 & 9.976 E-05 \\ 3.457 E-06 & 9.976 E-05 \\ 3.336 E-06 & 1.541 E-04 \\ 2.800 E-06 & 5.063 E-04 \\ 2.684 E-06 & 2.796 E-04 \\ 2.466 E-06 & 9.976 E-05 \\ 2.362 E-06 & 5.554 E-02 \\ 2.283 E-06 & 1.000 E+00 \\ 2.242 E-06 & 5.723 E-02 \\ 2.078 E-06 & 1.119 E-03 \\ 1.704 E-06 & 2.330 E-01 \\ 1.704 E-06 & 1.580 E-02 \\ 1.659 E-06 & 1.293 E-04 \\ 1.600 E-06 & 8.621 E-04 \\ 1.599 E-06 & 5.723 E-02 \\ 1.472 E-06 & 5.750 E-05 \\ 1.430 E-06 & 1.048 E-02 \\ 1.319 E-06 & 1.273 E-02 \\ 1.289 E-06 & 4.481 E-03 \\ 1.267 E-06 & 1.000 E+00 \\ 1.241 E-06 & 6.200 E-04 \\ 1.234 E-06 & 5.790 E-02 \\ 1.046 E-06 & 7.845 E-03 \\ 1.044 E-06 & 3.178 E-03 \\ 1.008 E-06 & 6.309 E-03 \\ 1.008 E-06 & 7.187 E-04 \\ 9.959 E-07 & 1.000 E+00 \\ 9.355 E-07 & 3.730 E-05 \\ 9.282 E-07 & 7.187 E-04 \\ 9.260 E-07 & 1.000 E+00 \\ 8.616 E-07 & 3.730 E-05 \\ 3.587 E-07 & 3.265 E-04 \\ 8.518 E-07 & 2.442 E-03 \\ 8.518 E-07 & 2.138 E-04 \\ 8.224 E-07 & 6.410 E-04 \\ 7.978 E-07 & 2.186 E-03 \\ 7.242 E-07 & 2.186 E-03 \\ 6.963 E-07 & 5.129 E-02 \\ 6.802 E-07 & 4.420 E-03 \\ 6.421 E-07 & 7.187 E-04 \\ 1.42 E-07 & 6.920 E-04\end{array}$


Table D2.1

Palrs

UNNORY FUSS-V BIRNBAUM

F 51

SLBO

SLBO

SGTR

FLMFW

SLDCI

SLOCI

SLBI

LOOF

EXFW

VSI

VSI

FS1

SLOCN

LOOP

SLAI

SLOCI

SLBO

LIDC

RT

SLOCN

F89

TT

FSB

EXFW

FS9

SLOCN

PLMFW

LDOF

LOOF

SLEI

LOSWV

EXFW

EXFW

LDOF

EXFW

RT

MLOCA

SLOCN

$T T$

RT

SLOCN

LOSWV

LIDC

$T T$

LOOF

FT

LOOP

TT

PLMFW

FLMFW
OE1

LA1

LB2

SL2

VIZ

FIN

LE2

AWB

AWA

AW1

IT 1

ME I

VI2

MU2

512

MSZ

LBS

VIZ

AW7

MS2

LA1

OB1

MS2

PRD

QB1

VI2

LR2

MS2

LB2

HRD

VI2

CI 1

AWB

AW3

VA1

VI2

LA1

LV1

RW1

LA1

LB2

RF 1

SI 1

VI 2

LB2

MS2

AW4

AW 1

Aw4

LE2:

LA1.
6. 3日2E-07

6. $335 E-07$

6. 335E-07

6. 202E-07

6. $146 E-07$

5. 862E-07

5. $862 E-07$

$5.657 E-07$

5. 537E- 07

$5.297 E-07$

4. $999 E-07$

4. $999 E-07$

4. $946 E-07$

4. $958 E-07$

4. 81 BE -07

4.816E-07

4. 58BE- 07

4. 58SE-O7

4. $171 E-07$

4. 148E- 07

4. 147E-07

3. $902 E-07$

3. $820 E-07$

$3.016 E-07$

3. OOEE-07

2.970E-.07

2. $913 E-07$

2.725E-07

2. $682 E-07$

2. 535E-07

2. 445E-07

2. 414E-07

2. $406 E-07$

2. 394E-07

2. 292E-07

2. 289E-07

2. 221E-07

2.125E-07

2.072E-07

2.045E-07

2. $021 E-07$

$1.945 E-07$

$1.925 E \cdots 07$

$1.925 E-07$

1. $862 E-07$

$1.612 E-07$

1. SSGE-07

1. $521 E-07$

$1.415 E-07$

1. $328 E-07$

1. 3.28E-OT
$7.511 E-02$

$5.615 E-0.3$

4. $916 E-04$

$5.563 E-0.3$

$3.730 E-05$

4.753E- 0.3

1. $563 E-04$

$5.070 E-02$

$6.345 E-05$

5. $090 \mathrm{E}-02$

5. $000 E-01$

$9.900 E-01$

$7.647 E-02$

$7.894 E-0.3$

$3.309 E-014$

1. 04OE-0.3

1. $397 E-03$

$3.769 E-0.3$

5. $029 E-02$

$3.638 E-07$

3. $365 E-03$

1. OOOE+OO

$3.638 E-07$

1. $000 E+00$

$3.730 E-05$

1. $000 E+00$

2. $377 \mathrm{E}-04$

$3.638 E-07$

1. 265E-05

$6.110 \mathrm{E}-04$

2. 40OE-02

$9.453 E-01$

7.01 OE- 04

$6.920 E-04$

$6.560 E-04$

$3.730 E-05$

$9.549 E-06$

1. $000 E+00$

1. $000 E+00$

$9.549 E-06$

$7.609 E-07$

$1.170 E-02$

$9.415 E-01$

$3.417 E-04$

$7.609 E-07$

$1.772 \mathrm{E}-06$

1. . 858E-06

4. $480 E-02$

1. $858 E-06$

$7.609 E-07$

8. $691 E-06$ 
Table D2.1

Pairs

\begin{tabular}{|c|c|}
\hline $\begin{array}{l}\text { LOFF } \\
\text { LOFF }\end{array}$ & $\begin{array}{l}\text { AW1 } \\
\text { OE11 }\end{array}$ \\
\hline $\begin{array}{l}\text { LOFF } \\
\text { LIDC }\end{array}$ & PRA \\
\hline LLOCA & ᄂ.98 \\
\hline LLOCA & Los \\
\hline IMSIV & Aw 3 . \\
\hline IMSIV & OL:I 1 \\
\hline FS1 & LA1 \\
\hline TLMFW & AW: \\
\hline TLMFW & OB1 \\
\hline LOFF & AWB \\
\hline F81 & LE2 \\
\hline FLMFW & Alw4 \\
\hline LCV & OE1 \\
\hline LCV & AW1 \\
\hline LODF & CI2 \\
\hline IMSIV & AWB \\
\hline LOCV & DB1 \\
\hline LOCV & AW1 \\
\hline TLMFW & AWB \\
\hline ISI & OB1 \\
\hline ISI & AW1 \\
\hline $\begin{array}{l}\text { LODP } \\
\text { LCV }\end{array}$ & AWB \\
\hline $\begin{array}{l}\text { LCV } \\
\text { LOCV }\end{array}$ & AWB \\
\hline LOCV & AWB \\
\hline $\begin{array}{l}\text { RT } \\
\text { ISI }\end{array}$ & Aw3 \\
\hline ISI & AWB \\
\hline${ }^{T T}$ & Aw3 \\
\hline $\begin{array}{l}\text { MLOCA } \\
\text { MLOCA }\end{array}$ & $\begin{array}{l}\text { SI } 1 \\
\text { CH2 }\end{array}$ \\
\hline $\begin{array}{l}\text { MLOCA } \\
\text { FT }\end{array}$ & $\mathrm{CH} 2$ \\
\hline RT & \\
\hline LIDC & HRB \\
\hline$T T$ & AW7 \\
\hline LIDC & VB1 \\
\hline LIDC & SR2 \\
\hline F510 & Aw4 \\
\hline Fs10 & OB1 \\
\hline F51 & Aw9 \\
\hline LOPF & AW7 \\
\hline LIDC & RF 1 \\
\hline IMSIV & AW7 \\
\hline TLMFW & AW7 \\
\hline PLMFW & AW7 \\
\hline LOOF & AW7 \\
\hline LCV & AW7 \\
\hline LOCV & AW7 \\
\hline LIDC & $\mathrm{CI2}$ \\
\hline ISI & AW7 \\
\hline RT & $\mathrm{CH} 2$ \\
\hline$T T$ & $\mathrm{CH} 2$ \\
\hline
\end{tabular}

UNNORM FUSS - $V$ BIRNBAUM

$1.304 E-07 \quad 2.890 E-02$

1.304E-07 3.730E-05

1.233E-07 5.850E-04

1.197E-01 1.5BOE-02

1.197E-07 3.75OE-02

$1.153 E-07 \quad 2.890 E-02$

$1.153 E-07 \quad 3.730 E-05$

$1.139 E-07 \quad 1.899 E-02$

$1.076 E-07 \quad 2.890 E-02$

1.076E-07 3.730E-05

1.043E-07 7.010E-04

$1.013 E-07 \quad 1.479 E-03$

$1.009 E-07$ 1.858E-06

$9.411 E-01 \quad 3.730 E-05$

$9.411 E-08 \quad 2.890 E-02$

$9.309 E-0 B \quad 1.773 E-04$

$9.226 E-08$ 7.010E-04

8.613E-OB 3.730E-O5

8.613E-OB 2.890E-02

8.60SE-OB 7.010E-04

$7.966 E-08$ 3.730E-05

$7.966 E-08 \quad 2.890 E-02$

$7.846 E-O B$ 7.010E-04

7.527E-OQ 7.010E-O4

6.889E-08 7.010E-04

$6.511 E-08$ 4.606E-OS

$6.372 E-08 \quad 7.010 E-04$

5.997E-OB 4.606E-OS

$4.243 E-08$ 2.82OE-O2

$4.243 E-08$ 6.500E-03

3.394E-OB 9.188E-05

$3.326 E-08$ 3.240E-04

$3.126 E-O B$ 9.188E-OS

$3.019 E-08 \quad 3.240 E-04$

$2.949 E-0 B \quad 1.215 E-04$

$2.933 E-08 \quad 2.890 E-02$

2.933E-OB 7.25OE-O2

2.906E-OB 7.010E-04

$2.764 E-08$ 7.050E-04

$2.621 E-08 \quad 3.240 E-04$

2.444E-OB 7.05OE-04

2.280E-08 7.050E-04

$2.230 E-O B$ 9.188E-OS

2.079E-OB 7.05OE-O4

$1.994 E-08$ 7.050E-04

$1.825 E-08$ 7.05OE-O4

$1.795 E-O B$ 1.215E-O4

$1.688 E-01 \quad 7.050 E-04$

$1.379 E-08 \quad 8.581 E-07$

1.27OE-08 8.581E-07 
Table D2.1

Pairs

UNNORM FUSS-V BIRNBAUM

$\begin{array}{ll}\text { SLEO } & \text { AWI } \\ \text { LIDC } & \text { VEZ } \\ \text { LIDC } & S I 1 \\ \text { FS1 } & \text { LB1 } \\ \text { EXFW } & \text { AW7 } \\ \text { SLBO } & \text { AWB } \\ \text { FT } & \text { RF1 } \\ \text { TT } & \text { RF1 } \\ \text { LOOF } & \text { CH1 } \\ \text { L1DC } & \text { LES } \\ \text { L1DC } & \text { MU2 } \\ \text { SLBO } & \text { AW7 } \\ \text { L1DC } & \text { LB1 } \\ \text { L1DC } & C H 2 \\ \text { L1DC } & C S 2 \\ \text { COMMAND- } & \end{array}$

$\begin{array}{ll}1.262 E-08 & 1.840 E-03 \\ 1.194 E-08 & 1.215 E-04 \\ 1.011 E-08 & 1.215 E-04 \\ 9.610 E-09 & 2.095 E-0.3 \\ 3.098 E-09 & 5.640 E-05 \\ 4.489 E-09 & 6.600 E-04 \\ 3.091 E-09 & 8.581 E-07 \\ 2.847 E-09 & 8.581 E-07 \\ 2.555 E-09 & 4.499 E-05 \\ 1.980 E-09 & 3.791 E-06 \\ 1.980 E-09 & 6.610 E-06 \\ 1.189 E-09 & 6.637 E-04 \\ 3.669 E-10 & 9.188 E-07 \\ 3.509 E-10 & 9.720 E-07 \\ 3.364 E-10 & 9.188 E-07\end{array}$ 
Table D2.2

Pair Importance by Classes of Split Fractions (InItiators - Supports)

Palrs

UNNORM FUSS-V BIRNBAUM

\begin{tabular}{|c|c|}
\hline \multicolumn{2}{|l|}{ LOOF } \\
\hline LOOF' & GHI \\
\hline LOOF & GG2 \\
\hline $\begin{array}{l}\text { LOOF } \\
\text { LOOF }\end{array}$ & $\mathrm{GH}_{2}$ \\
\hline LDOF & SW1 \\
\hline LOOF & FO1 \\
\hline LOOF & TH3 \\
\hline LOOF & TE2 \\
\hline LOOF' & CCS \\
\hline L1DC & $\mathrm{DH}_{2}$ \\
\hline LIDC & $\mathrm{AH} 4$ \\
\hline LOOP & TH2 \\
\hline LOOF & GH3 \\
\hline LOOF & TES \\
\hline LOOF & SW2 \\
\hline LOOP & CVI \\
\hline RT & DH1 \\
\hline LIDC & DF 1 \\
\hline LIDC & AF 1 \\
\hline TT & DH1 \\
\hline LOOF & $F D$ \\
\hline LOOF & ASB \\
\hline RT & 131 \\
\hline RT & I 11 \\
\hline LOOF & DH1 \\
\hline$T T$ & 131 \\
\hline$T T$ & 111 \\
\hline L1DC & ccs \\
\hline LOOF & TG1 \\
\hline LIDC & CC \\
\hline FLMFW & DH \\
\hline LOOP & FO \\
\hline LOOP & DG \\
\hline PLMFW & 13 \\
\hline FLMFW & 11 \\
\hline RT & SE \\
\hline RT & SA \\
\hline LOOF' & sv \\
\hline$T T$ & SE \\
\hline$T T$ & SA \\
\hline LOOF & sv \\
\hline LOOF & DF \\
\hline LOOF & AH \\
\hline FS6 & $\mathrm{CC}$ \\
\hline
\end{tabular}
$1.405 E-05$
$1.125 \mathrm{E}-0 \mathrm{~S}$
$9.579 E-06$
$7.851 E-06$
$7.797 E-06$
$7.248 E-06$
$3.666 E-06$
$3.618 E-06$
$3.478 E-06$
$3.389 E-06$
2.83.3E-06
$2.809 E-06$
$2.722 E-06$
$2.423 E-06$
2. $035 E-06$
1. $601 E-06$
$1.596 E-06$
1. $540 \mathrm{E}-06$
$1.472 \mathrm{E}-06$
$1.445 E-06$
$1.419 E-06$
1. 385E-06
1. $371 E-06$
1. $250 E-06$
1. 25OE-OG
1. 182E-O6
$1.152 \mathrm{E}-0.06$
1.152E-06
$1.026 E-06$
$1.008 E-06$
9.501E-07
9.421E-07
9.3OOE-07
8. $926 E-07$
8.215E-07
8.215E-07
B. $150 E-07$
B. 15OE-07
7.639E-07
7.507E-07
$7.507 E-07$
$7.212 \mathrm{E}-07$
$7.112 E-07$
$7.041 E-07$
$6.889 E-07$
$3.415 \mathrm{SE}-03$
2.761E-03
2. $371 E-0.3$
$1.552 E-0.3$
1. 584E-O3
$1.593 E-04$
1. $865 E-01$
6. $402 E-04$
$7.13 O E-04$
1. 298E-0.3
1. $586 E-01$
1. $586 E-01$
3.623E-04
3.220E-04
$3.578 E-04$
$6.928 E-03$
$3.089 E-04$
1.927E-0.3
8.156E-02
8.156E-02
1.927E-0.3
2.996E-04
5.579E-04
$9.537 E-04$
$9.537 E-04$
1.853E-02
9.537E-04
9.537E-O4
$1.397 E-03$
$2.518 E-04$
$1.390 E-0.3$
$1.794 E-0.3$
4. 522E-O4
$1.391 E-02$
$9.537 E-04$
9.537E-04
2.979E-05
9.432E-05
1. 145E-03
$2.979 E-05$
9.432E-05
4. $403 E-02$
1. $109 E-02$
$1.118 E-02$
$9.960 E-01$ 
Table D2.2

\section{Pairs}

\begin{tabular}{|c|c|}
\hline $\begin{array}{l}\text { SLEI } \\
\text { RT }\end{array}$ & $\begin{array}{l}\text { SAS } \\
\text { OG1 }\end{array}$ \\
\hline FLMFW & AH1 \\
\hline RT & 141 \\
\hline RT & 121 \\
\hline LOOP & $\mathrm{FO} 2$ \\
\hline LIDC & A54 \\
\hline LOOP & CC7 \\
\hline$T T$ & 121 \\
\hline TT & I 41 \\
\hline LOOF & BG1 \\
\hline PLMFW & SA1 \\
\hline PLMFW & SB2 \\
\hline LOCV & RT1 \\
\hline $\begin{array}{l}\text { LDOP } \\
R T\end{array}$ & AF 1 \\
\hline $\begin{array}{l}R T \\
\text { LIDC }\end{array}$ & DE1 \\
\hline $\begin{array}{l}\text { LIDC } \\
\text { SLBI }\end{array}$ & $\mathrm{CC3}$ \\
\hline $\begin{array}{l}\text { SLBI } \\
\text { EXFW }\end{array}$ & SBE \\
\hline $\begin{array}{l}\text { EXFW } \\
\text { LOSWV }\end{array}$ & SV1 \\
\hline $\begin{array}{l}\text { LOSWV } \\
\text { LosWV }\end{array}$ & $\begin{array}{l}\text { SAI } \\
\text { SE1 }\end{array}$ \\
\hline $\begin{array}{l}\text { LDSWV } \\
\text { RT }\end{array}$ & \\
\hline $\begin{array}{l}\text { RT } \\
\text { RT }\end{array}$ & GF 1 \\
\hline $\begin{array}{l}\text { RT } \\
T T\end{array}$ & DGI \\
\hline $\begin{array}{l}\text { TT } \\
\text { RT }\end{array}$ & $\operatorname{cc} 2$ \\
\hline $\begin{array}{l}\text { RT } \\
\text { LOQP }\end{array}$ & $A G 1$ \\
\hline MLOCA & SA2 \\
\hline MLOCA & 586 \\
\hline TT & $\operatorname{ccs}$ \\
\hline TT & 061 \\
\hline TT & GF 1 \\
\hline TT & $\mathrm{CC2}$ \\
\hline LOOF & GH4 \\
\hline LQOP & Sw3 \\
\hline PLMFW & 141 \\
\hline PLMFW & 121 \\
\hline F56 & AS4 \\
\hline RT & RT7 \\
\hline LOOF & SB1 \\
\hline$T T$ & RT7 \\
\hline MLOCA & CV1 \\
\hline EXFW & DH1 \\
\hline LOOF & 132 \\
\hline LOOF' & $\mathrm{CV} 2$ \\
\hline FLMFW & DG1 \\
\hline SLBI & DG1 \\
\hline SLBI & DH1 \\
\hline SLBO & I31 \\
\hline SLBO & 111 \\
\hline LOQF' & GHS \\
\hline SLBI & AH1 \\
\hline & GG2. \\
\hline
\end{tabular}

UNNORM FUSS-V BIRNBAUM

$\begin{array}{ll}6.682 E-07 & 1.031 E-01 \\ 6.569 E-07 & 7.552 E-04 \\ 6.427 E-07 & 1.240 E-03 \\ 6.262 E-07 & 9.537 E-04 \\ 6.262 E-07 & 9.537 E-04 \\ 6.214 E-07 & 9.700 E-04 \\ 6.044 E-07 & 1.397 E-03 \\ 5.914 E-07 & 9.803 E-03 \\ 5.768 E-07 & 9.537 E-04 \\ 5.768 E-07 & 9.537 E-04 \\ 5.402 E-07 & 4.123 E-03 \\ 5.355 E-07 & 9.432 E-05 \\ 5.355 E-07 & 2.979 E-05 \\ 5.257 E-07 & 1.000 E+00 \\ 5.065 E-07 & 8.044 E-03 \\ 5.041 E-07 & 6.272 E-04 \\ 4.902 E-07 & 3.273 E-02 \\ 4.816 E-07 & 1.400 E-02 \\ 4.771 E-07 & 1.000 E+00 \\ 4.768 E-07 & 1.000 E+00 \\ 4.705 E-07 & 1.000 E+00 \\ 4.702 E-07 & 7.050 E-04 \\ 4.697 E-07 & 9.116 E-06 \\ 4.643 E-07 & 6.272 E-04 \\ 4.547 E-07 & 7.010 E-04 \\ 4.499 E-07 & 7.145 E-03 \\ 4.455 E-07 & 8.440 E-02 \\ 4.453 E-07 & 1.140 E-02 \\ 4.330 E-07 & 7.050 E-04 \\ 4.326 E-07 & 5.400 E-04 \\ 4.326 E-07 & 9.116 E-06 \\ 4.188 E-07 & 7.010 E-04 \\ 4.133 E-07 & 1.014 E-04 \\ 4.130 E-07 & 4.566 E-06 \\ 4.114 E-07 & 9.537 E-04 \\ 4.114 E-07 & 9.537 E-04 \\ 4.057 E-07 & 9.960 E-01 \\ 4.003 E-07 & 1.819 E-04 \\ 3.691 E-07 & 5.422 E-04 \\ 3.687 E-07 & 1.819 E-04 \\ 3.519 E-07 & 1.000 E+00 \\ 3.518 E-07 & 1.799 E-03 \\ 3.462 E-07 & 2.186 E-03 \\ 3.323 E-07 & 1.773 E-04 \\ 3.312 E-07 & 6.272 E-04 \\ 3.264 E-07 & 1.000 E+00 \\ 3.246 E-07 & 1.000 E+00 \\ 3.237 E-07 & 5.090 E-02 \\ 3.237 E-07 & 5.090 E-02 \\ 3.195 E-07 & 6.314 E-05 \\ 3.191 E-07 & 9.960 E-01 \\ 3.161 E-07 & 4.998 E-06\end{array}$


Table D2.2

Pairs

\begin{tabular}{|c|c|}
\hline LOOF & TG6 \\
\hline PLMFW & CCs \\
\hline $\begin{array}{l}\text { SLOC I } \\
\text { LOQF }\end{array}$ & CC1 \\
\hline $\begin{array}{l}\text { LODF } \\
\text { LDOF }\end{array}$ & CV6 \\
\hline $\begin{array}{l}\text { LDOF } \\
\text { PLMFW }\end{array}$ & TG5 \\
\hline PLMFW & $\mathrm{CC2}$ \\
\hline LOOF & THG \\
\hline$T T$ & GG2 \\
\hline RT & SV2 \\
\hline FS11 & DG1 \\
\hline FS11 & $\mathrm{DH} 1$ \\
\hline TT & SV2 \\
\hline F'LMFW & RT7 \\
\hline LOOF & TH1 \\
\hline EXFW & AH1 \\
\hline SLBO & DH1 \\
\hline SLEO & DG1 \\
\hline RT & CC1 \\
\hline SLBO & AH1 \\
\hline SLOCI & DG1 \\
\hline SLOC I & DH1 \\
\hline SLOCI & AH1 \\
\hline SLOCI & AG1 \\
\hline PLMFW & OC1 \\
\hline FLMFW & GF 1 \\
\hline LOOF & FOS \\
\hline TT & CC1 \\
\hline LDOP & TH4 \\
\hline RT & SV3 \\
\hline LODP & $\mathbf{B H I}$ \\
\hline F51 & DG1 \\
\hline LOPF & SV1 \\
\hline$T T$ & SVZ \\
\hline LLOCA & SE6 \\
\hline LLOCA & SAZ \\
\hline PLMFW & $5 \vee 2$ \\
\hline RT & FO1 \\
\hline IMSIV & SV1 \\
\hline RT & GH3 \\
\hline SLBI & SBC \\
\hline LOOP & AS5 \\
\hline RT & DF 1 \\
\hline TLMFW & sV1 \\
\hline RT & AH1 \\
\hline$T T$ & GH3 \\
\hline$T T$ & DF 1 \\
\hline TT & AH1 \\
\hline RT & GH2 \\
\hline PLMFW & CC 1 \\
\hline LCV & SV1 \\
\hline F'T & AF 1 \\
\hline$T T$ & $\mathrm{GH}_{2}$ \\
\hline
\end{tabular}

UNNORM FUSS-V BIRNBAUM

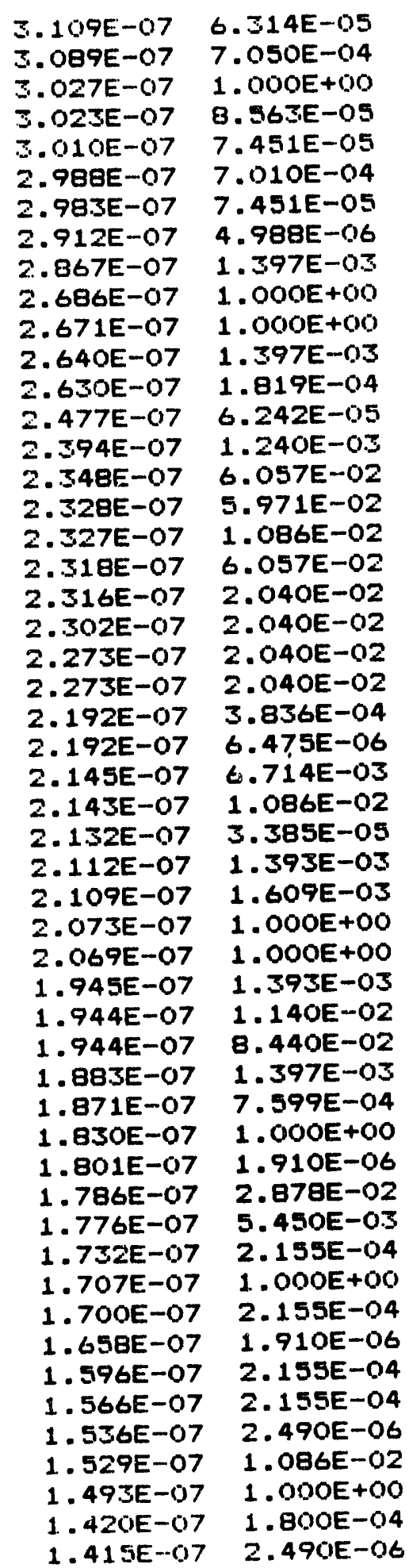


Table D2.2

Pairs

\begin{tabular}{|c|c|}
\hline L.FCC & DG1 \\
\hline LF'CC & DH1 \\
\hline LOCV & SV1 \\
\hline FiT & TGI \\
\hline L1DC & SB1 \\
\hline$T T$ & AF 1 \\
\hline SGTF & $I \geq 1$ \\
\hline SGTFi & I 11 \\
\hline ISI & SV1 \\
\hline$T T$ & TG3 \\
\hline SLDCN & 131 \\
\hline EXFW & DG 1 \\
\hline FLMFW & GH3 \\
\hline F'LMFW & GG2 \\
\hline EXFW & CCS \\
\hline F'LMFW & DF 1 \\
\hline LIDC & SA1 \\
\hline RT & SV1 \\
\hline SLBO & DF 1 \\
\hline EXFW & $\mathrm{CC2}$ \\
\hline SLEO & AF 1 \\
\hline LOOF & SV4 \\
\hline$T T$ & SV1 \\
\hline LOFF & DH1 \\
\hline FLMFW & $\mathrm{GH}_{2}$ \\
\hline PLMFW & AF 1 \\
\hline IMSIV & $\mathrm{DH} 1$ \\
\hline SLBD & I 41 \\
\hline SLEO & 121 \\
\hline TLMFW & $\mathrm{DH} 1$ \\
\hline SGTF & DF 1 \\
\hline SSGTF & DG 1 \\
\hline SGTR & $\mathrm{DH} 1$ \\
\hline LCV & $\mathrm{DH} 1$ \\
\hline LOCV & $\mathrm{DH} 1$ \\
\hline SGTFi & 121 \\
\hline SGTFi & I 41 \\
\hline ISI & $\mathrm{DH} 1$ \\
\hline L.1DC & AS. \\
\hline FS1 & $\mathrm{DH} 1$ \\
\hline L.OFF & DG1 \\
\hline IMSIV & DG1 \\
\hline TLMFW & DG1 \\
\hline L.CV & DG 1 \\
\hline LOCV & DG1. \\
\hline ISI & DE 1 \\
\hline$\angle 1 D C$ & 152 \\
\hline F'LMFW & SV1 \\
\hline
\end{tabular}

\section{UNNORM FUSS-V BIRNBAUM}

$\begin{array}{ll}1.382 E-07 & 1.000 E+00 \\ 1.374 E-07 & 1.000 E+00 \\ 1.366 E-07 & 1.000 E+00 \\ 1.361 E-07 & 1.910 E-06 \\ 1.353 E-07 & 7.065 E-04 \\ 1.308 E-07 & 1.800 E-04 \\ 1.282 E-07 & 6.520 E-03 \\ 1.282 E-07 & 6.520 E-03 \\ 1.264 E-07 & 1.000 E+00 \\ 1.253 E-07 & 1.910 E-06 \\ 1.234 E-07 & 2.040 E-02 \\ 1.202 E-07 & 6.109 E-04 \\ 1.183 E-07 & 1.910 E-06 \\ 1.183 E-07 & 2.841 E-06 \\ 1.151 E-07 & 7.050 E-04 \\ 1.138 E-07 & 2.155 E-04 \\ 1.135 E-07 & 5.850 E-04 \\ 1.135 E-07 & 5.820 E-02 \\ 1.127 E-07 & 2.890 E-02 \\ 1.113 E-07 & 7.010 E-04 \\ 1.106 E-07 & 2.890 E-02 \\ 1.089 E-07 & 4.655 E-02 \\ 1.045 E-07 & 5.820 E-02 \\ 1.043 E-07 & 1.230 E-03 \\ 1.009 E-07 & 2.490 E-06 \\ 9.330 E-0 B & 1.800 E-04 \\ 9.226 E-08 & 1.230 E-03 \\ 9.205 E-08 & 2.890 E-02 \\ 9.205 E-08 & 2.890 E-02 \\ 8.605 E-08 & 1.230 E-03 \\ 7.860 E-08 & 6.520 E-03 \\ 7.860 E-08 & 6.520 E-03 \\ 7.816 E-08 & 6.520 E-03 \\ 7.527 E-08 & 1.230 E-03 \\ 6.889 E-08 & 1.230 E-03 \\ 6.422 E-08 & 6.520 E-03 \\ 6.422 E-08 & 6.520 E-03 \\ 6.372 E-08 & 1.230 E-03 \\ 2.949 E-08 & 9.444 E-03 \\ 2.906 E-08 & 1.410 E-01 \\ 2.764 E-08 & 3.240 E-04 \\ 2.444 E-08 & 3.240 E-04 \\ 2.280 E-08 & 3.240 E-04 \\ 1.994 E-08 & 3.240 E-04 \\ 1.825 E-08 & 3.240 E-04 \\ 1.688 E-08 & 3.240 E-04 \\ 1.443 E-08 & 3.240 E-04 \\ 4.483 E-09 & 3.500 E-03 \\ & \end{array}$


Table D2.3

Pair Importance by Classes of Split Fractions

(Initiators - Human and Recovery Actions)

\begin{tabular}{|c|c|}
\hline \multicolumn{2}{|c|}{ Pairs } \\
\hline RT & HSI \\
\hline TT & HS1 \\
\hline FS 11 & SE: 1 \\
\hline FS11 & RF'Z \\
\hline FLMFW & $1+51$ \\
\hline $\begin{array}{l}\text { LOOF } \\
\text { LPCC }\end{array}$ & $\begin{array}{l}\text { REACOG } \\
\text { SE } 1\end{array}$ \\
\hline LFCC & RF 2 \\
\hline LOOF & RESLC 1 \\
\hline SGTR & of1 \\
\hline LOQF & RESLC2 \\
\hline EXFW & HSI \\
\hline SLEI & FP? \\
\hline F 56 & RF2 \\
\hline LosW & RP2 \\
\hline Losw & SE1 \\
\hline RT & RPZ \\
\hline LOOF & HSI \\
\hline$T T$ & RF' 2 \\
\hline LOFF & HSI \\
\hline FS5 & RF'2 \\
\hline F55 & SE1 \\
\hline IMSIV & HSI \\
\hline LOQF & SE 1 \\
\hline TLMFW & HSI \\
\hline LCV & HSI \\
\hline FT & 051 \\
\hline LOCV & $H S 1$ \\
\hline Tr & 051 \\
\hline FLMFW & RP2 \\
\hline ISI & HS1 \\
\hline HAZCHM & ZHEHSS \\
\hline PLMFW & os1 \\
\hline RT & SE 1 \\
\hline LOSWV & ZHESVS \\
\hline TT & SE 1 \\
\hline LIDC & ZHERFZ \\
\hline FLMFW & SE1 \\
\hline LOOF & ZHEFOG \\
\hline LOOF & FESLCS \\
\hline SLBO & ZHEAW4 \\
\hline LDOF' & ZHESV \\
\hline LIDC & ZHEAW: \\
\hline LOQF & ZHESW 1 \\
\hline LOOF & ZHERE2 \\
\hline FiT & ZHEOEZ \\
\hline $\mathrm{TT}$ & ZHEOBZ \\
\hline FLMFW & ZHEOE2 \\
\hline FiT & ZHESVS \\
\hline$T T$ & ZHESVZ \\
\hline EXFW & ZHEDE2 \\
\hline FLMFW & ZHESV \\
\hline LOOF & FIEAC 12 \\
\hline
\end{tabular}

UNNOFM FUSS-V EIFNEAUM

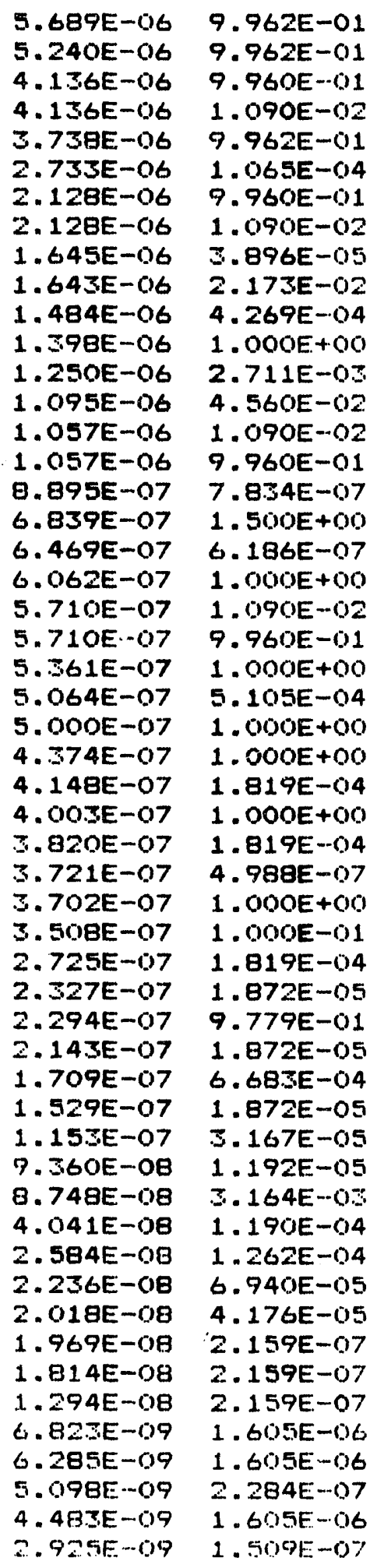


Table D2.4

Pair Importance by Classes of Split Fractions (Frontlines - Frontlines)

Pairs

\begin{tabular}{|c|c|}
\hline OE1 & AWS \\
\hline AW5 & OB1 \\
\hline OB1 & AW1 \\
\hline AW1 & OE1 \\
\hline LAI & LBZ \\
\hline LEZ & LAI \\
\hline AW1 & VII \\
\hline VIZ & AW1 \\
\hline OBZ & AWA \\
\hline AWA & OB3 \\
\hline LBZ & LAS \\
\hline LA3 & LE2 \\
\hline FRD & LBI \\
\hline LBS & FRD \\
\hline PRD & LA1 \\
\hline LA1 & FRD \\
\hline Aw4 & FFD \\
\hline PRD & AW4 \\
\hline FRD & RF 1 \\
\hline FF 1 & FRD \\
\hline Aw3 & VIZ \\
\hline VII & AW3 \\
\hline MS2 & AWB \\
\hline AWB & MS2 \\
\hline MS2 & $\mathbf{0 B 1}$ \\
\hline 081 & MSZ \\
\hline AW4 & DB1 \\
\hline OE1 & AW4 \\
\hline MU1 & SL1 \\
\hline SL1 & MU1 \\
\hline PFD & HRB \\
\hline HFE & FRD \\
\hline PRD & VB1 \\
\hline VB1 & PRD \\
\hline AWB & $\mathrm{OB} 1$ \\
\hline DB1 & AWS \\
\hline MS2 & QB3 \\
\hline DBZ & $M S 2$ \\
\hline MS2 & AWA \\
\hline AWA & MS2 \\
\hline AWB & LB2 \\
\hline LA1 & AWB \\
\hline$M S 2$ & LA1 \\
\hline LA1 & MSZ \\
\hline AWB & LA1 \\
\hline LEZ & AWB \\
\hline LB2 & MS2 \\
\hline MS2 & LB2 \\
\hline PRN & LAI \\
\hline FRN & LE2 \\
\hline
\end{tabular}

UNNORM FUSS-V BIRNBAUM

$$
\begin{array}{ll}
9.676 E-06 & 1.015 E-02 \\
9.676 E-06 & 1.015 E-02 \\
4.184 E-06 & 1.000 E+00 \\
4.184 E-06 & 1.000 E+00 \\
3.253 E-06 & 6.845 E-04 \\
3.253 E-06 & 6.845 E-04 \\
2.641 E-06 & 1.000 E+00 \\
2.641 E-06 & 1.000 E+00 \\
2.566 E-06 & 7.135 E-05 \\
2.566 E-06 & 7.135 E-05 \\
1.704 E-06 & 4.630 E-04 \\
1.704 E-06 & 4.630 E-04 \\
1.600 E-06 & 1.608 E-03 \\
1.600 E-06 & 1.608 E-03 \\
1.571 E-06 & 1.578 E-03 \\
1.571 E-06 & 1.578 E-03 \\
1.551 E-06 & 4.383 E-04 \\
1.551 E-06 & 4.383 E-04 \\
1.252 E-06 & 8.117 E-03 \\
1.252 E-06 & 8.117 E-03 \\
1.245 E-06 & 4.563 E-02 \\
1.245 E-06 & 4.563 E-02 \\
1.046 E-06 & 4.338 E-05 \\
1.046 E-06 & 4.338 E-05 \\
1.008 E-06 & 3.489 E-05 \\
1.00 B E-06 & 3.489 E-05 \\
8.871 E-07 & 4.234 E-04 \\
8.871 E-07 & 4.234 E-04 \\
8.269 E-07 & 1.710 E-02 \\
8.269 E-07 & 1.710 E-02 \\
7.978 E-07 & 4.077 E-03 \\
7.978 E-07 & 4.077 E-03 \\
7.242 E-07 & 4.077 E-03 \\
7.242 E-07 & 4.077 E-03 \\
6.979 E-07 & 1.948 E-02 \\
6.979 E-07 & 1.948 E-02 \\
6.3355 E-07 & 2.719 E-06 \\
6.491 E-07 & 1.731 E-06 \\
6.491 E-07 & 1.731 E-06 \\
6.491 E-07 & 6.768 E-06 \\
6.491 E-07 & 6.768 E-06 \\
6.335 E-07 & 1.128 E-04 \\
6.3 .35 E-07 & 1.288 E-03 \\
6.335 E-07 & 3.105 E-05 \\
6.335 E-07 & 3.105 E-05 \\
6.335 E-07 & 1.288 E-03 \\
6.362 E-07 & 3.284 E-04
\end{array}
$$


Table 02.4

Palrs

\begin{tabular}{|c|c|}
\hline $\begin{array}{l}\text { LA1 } \\
\text { LE2 }\end{array}$ & $\begin{array}{l}\text { PRN } \\
\text { PRN }\end{array}$ \\
\hline $\mathrm{CH}_{2}$ & AW4 \\
\hline Aw4 & $\mathrm{CH} 2$ \\
\hline AWB & OB 1 \\
\hline DB1 & AWE \\
\hline LB2 & AW1 \\
\hline AW 1 & LB2 \\
\hline AW1 & LAI \\
\hline LA1 & AW 1 \\
\hline ITI & ME 1 \\
\hline ME 1 & I T I \\
\hline AW4 & VII \\
\hline VI2 & AW4 \\
\hline LA1 & SL 1 \\
\hline SL1 & LB2 \\
\hline BL 1 & LA1 \\
\hline LB2 & SL1 \\
\hline VIZ & MS2 \\
\hline MS2 & VIZ \\
\hline AWB & VI2 \\
\hline VIZ & AWB \\
\hline LAL & AW3 \\
\hline AW3 & LA1 \\
\hline PRD & 512 \\
\hline 512 & PRD \\
\hline MU2 & LB2 \\
\hline$\angle B Z$ & MUZ \\
\hline MU2 & LA1 \\
\hline LA1 & MUZ \\
\hline Aw4 & LA1 \\
\hline LA1 & AW4 \\
\hline Aw3 & LB2 \\
\hline LE2 & AW 3 \\
\hline HRD & PRD \\
\hline FRD & HRD \\
\hline FRD & AW 3 \\
\hline AW3 & PRD \\
\hline PRD & VA1 \\
\hline VA1 & PRD \\
\hline MUZ & RF 1 \\
\hline RF 1 & MUZ \\
\hline AW7 & VIZ \\
\hline VIZ & AW7 \\
\hline F'RD & $\mathrm{CH}_{2}$ \\
\hline $\mathrm{CH} 2$ & F'RD \\
\hline LA.3 & LBE \\
\hline LBE & LAS \\
\hline
\end{tabular}

UNNORY FUSS-V BIRNBAUM

$\begin{array}{ll}5.862 E-07 & 3.751 E-03 \\ 5.862 E-07 & 3.284 E-04 \\ 5.552 E-07 & 5.431 E-04 \\ 5.552 E-07 & 5.431 E-04 \\ 5.546 E-07 & 7.963 E-04 \\ 5.546 E-07 & 7.963 E-04 \\ 5.211 E-07 & 5.996 E-02 \\ 5.211 E-07 & 5.996 E-02 \\ 5.211 E-07 & 6.848 E-01 \\ 5.211 E-07 & 6.848 E-01 \\ 4.999 E-07 & 1.010 E-06 \\ 4.999 E-07 & 1.010 E-06 \\ 4.946 E-07 & 3.101 E-04 \\ 4.946 E-07 & 3.101 E-04 \\ 4.926 E-07 & 3.984 E-03 \\ 4.926 E-07 & 3.488 E-04 \\ 4.926 E-07 & 3.984 E-03 \\ 4.926 E-07 & 3.488 E-04 \\ 4.585 E-07 & 2.084 E-05 \\ 4.585 E-07 & 2.084 E-05 \\ 4.232 E-07 & 7.981 E-04 \\ 4.232 E-07 & 7.981 E-04 \\ 3.900 E-07 & 1.542 E-02 \\ 3.900 E-07 & 1.542 E-02 \\ 3.508 E-07 & 4.492 E-04 \\ 3.508 E-07 & 4.492 E-04 \\ 2.913 E-07 & 1.069 E-04 \\ 2.913 E-07 & 1.069 E-04 \\ 2.913 E-07 & 1.221 E-03 \\ 2.913 E-07 & 1.221 E-03 \\ 2.689 E-07 & 1.818 E-04 \\ 2.689 E-07 & 1.818 E-04 \\ 1.582 E-07 & 9.282 E-04 \\ 1.5197 E-07 & 2.020 E-04 \\ 1.197 E-07 & 2.020 E-04 \\ 1.682 E-07 & 9.282 E-04 \\ 2.535 E-07 & 1.139 E-03 \\ 2.535 E-07 & 1.139 E-03 \\ 2.445 E-07 & 4.040 E-03 \\ 2.445 E-07 & 4.040 E-03 \\ 2.135 E-07 & 1.139 E-03 \\ 2.135 E-07 & 1.139 E-03 \\ 1.945 E-07 & 5.260 E-03 \\ 1.945 E-07 & 5.260 E-03 \\ 1.95 E-07 & 2.700 E-02 \\ 1.518 E-07 & 2.700 E-02 \\ 1.206 E-04 \\ 1.97\end{array}$


Tab1e D2.4

Pairs

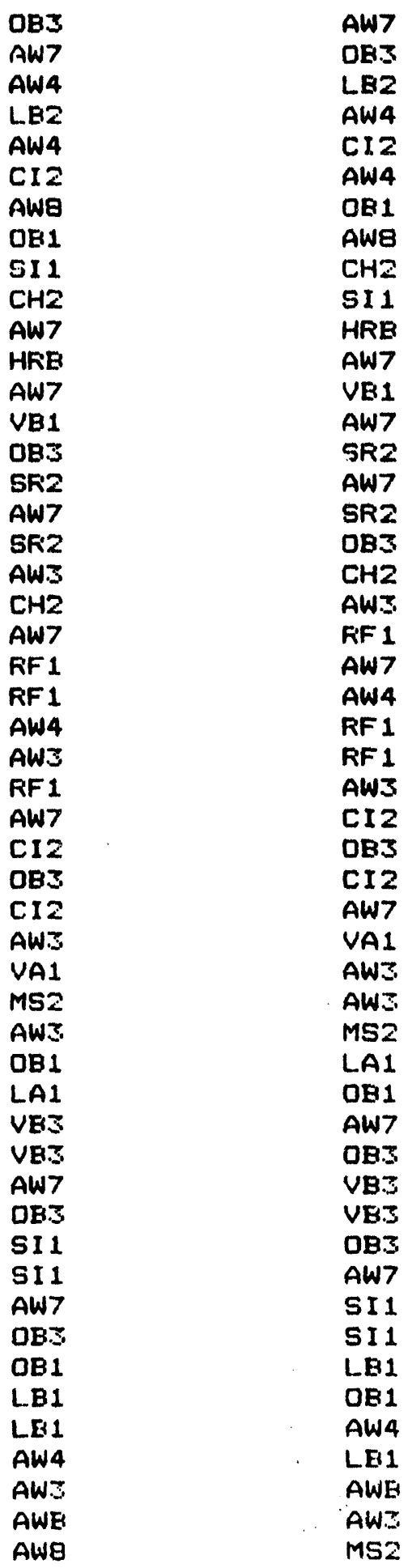

UNNORM FUSS-V BIRNBAUM

1. 186E-07

1. 186E- 07

$1.013 E-07$

1.013E-07

9. 309E-08

$9.309 E-0 B$

$7.365 E-08$

7. 3.65E-08

4. 24.JE-OB

4. $243 E-08$

3. $326 E-08$

$3.326 E-0 \theta$

$3.019 E-08$

I. O19E-OB

2.949E-0日

2.949E-OB

2. $949 E-08$

$2.949 E-08$

$2.650 E-08$

2.650E-08

$2.621 E-08$

2. $621 E-08$

2. $401 E-08$

2. $401 E-0 B$

1. $8 B 8 E-O B$

1. BQBE-OB

1. $795 E-O B$

1. $795 E-O B$

1. $795 E-O B$

1. $795 E-0 B$

1. 572E-OB

1. $572 E-08$

1. $262 E-08$

1. $262 E-08$

1. $257 E-08$

1. $257 E-O B$

1. $194 E-08$

1. $194 E-0 B$

1. 194E-OB

1. $194 E-08$

1. $011 E-O B$

1. $011 E-O B$

1. $011 E-08$

1. $011 E-O B$

$9.610 E-09$

$9.610 E-09$

7. $610 E-0.9$

$9.610 E-09$

8. $150 \mathrm{E}-09$

8. $150 E-09$

4. $489 E-09$

$9.760 E-04$

$9.760 E-04$

$5.998 E-06$

$5.998 E-016$

2. 225E-04

2. 225E-014

$2.072 E-03$

2. 072E-03

9. 26OE-0.4

$9.260 E-04$

2. 36OE-02

$2.560 E-02$

2. 56OE-O2

2. 56OE-O2

$8.294 E-06$

$9.600 E-03$

$9.600 E-03$

8. 294E-06

1. $515 E-03$

1. $515 E-03$

2. 560 OD-O2

2. $560 E-02$

1. 048E-04

1. 048E-04

4.817E-0.3

4. B17E-OJ

9. $600 E-0.3$

8. 294E-06

8. $294 E \cdots O 6$

$9.600 E-03$

उ. 302E-0.

\$. 302E-0.

1. $018 E-05$

1. $018 E-05$

2.131E-05

2.131E-O5

9. $600 E-0.3$

8. 294E-OG

$9.600 E-03$

B. 294E-OG

8. $294 E-06$

$9.600 E-03$

$9.600 E-03$

8. $294 E-06$

2. 13.2E-05

2.132E-05

8. 497E-06

8. $497 E-06$

2.727E-04

$2.727 E-04$

3. $650 \mathrm{E}-06$ 
Table 02.4

Pairs

\begin{tabular}{|c|c|}
\hline MSZ & AWE \\
\hline $\mathrm{CH} 1$ & AWS \\
\hline AW: & $\mathrm{CH} 1$ \\
\hline AW7 & MUZ \\
\hline MUZ & LE3 \\
\hline MUZ & AW7 \\
\hline LBE & MUZ \\
\hline LES & AW7 \\
\hline AW7 & LES \\
\hline AW7 & MS2 \\
\hline MS2 & AW7 \\
\hline LE1 & AW7 \\
\hline AW7 & LE1 \\
\hline OES & LE1 \\
\hline LE1 & OBS \\
\hline $\mathrm{CH} 2$ & AW7 \\
\hline $\mathrm{OBZ}$ & $\mathrm{CH} 2$ \\
\hline $\mathrm{CH}_{2}$ & OBS \\
\hline AW7 & $\mathrm{CH}_{2}$ \\
\hline Cs2 & AW7 \\
\hline AW7 & $\mathrm{CS2}$ \\
\hline C52 & 083 \\
\hline OBZ & $\mathrm{CS2}$ \\
\hline
\end{tabular}

UNNORY FUSS-V BIRNBAUM
4. $489 E-09 \quad 3.650 E-06$
2.555E-09 3. $02 E-03$
2.355E-09 3.302E-0.5
1.980E-09 5.222E- 014
1.980E-09 B.294E-06
1.9BOE-09 $5.222 E-04$
1. $980 E-09 \quad 0.294 E-06$
$1.980 E-09 \quad 2.995 E-04$
1.98OE-09 2.995E-04
$1.189 E-09 \quad 3.670 E-06$
1.189E-09 3.670E-06
$3.669 E-10 \quad 7.260 E-05$
$3.669 E-10$ 7.260E-05
3.669E-10 6.273E-08
$3.669 E-10$ 6.273E-08
3.509E-10 7.680E-05
3.509E-10 6.636E-08
3.509E-10 6.636E-08
$3.509 E-10$ 7.680E-05
Z.364E-10 7.260E-05
$3.364 E-10 \quad 7.260 E-05$
$3.364 E-10 \quad 6.273 E-08$
I. $364 E-10$ 6.273E-08 
Table D2.5

Palr Importance by Classes of Split Fractions (Frontilnes - Supports)

Pairs

\begin{tabular}{|c|c|}
\hline \multicolumn{2}{|l|}{ F'RD } \\
\hline $\begin{array}{l}\text { FFD } \\
\text { PFDD }\end{array}$ & $\begin{array}{l}\text { GF } 1 \\
\text { GH1 }\end{array}$ \\
\hline Aw4 & GF 1 \\
\hline AW4 & BH1 \\
\hline FRD & GH2 \\
\hline DB1 & 111 \\
\hline DE1 & $I 31$ \\
\hline AWB & DH1 \\
\hline AW5 & 111 \\
\hline AW5 & I31 \\
\hline Aw4 & GHZ \\
\hline Aw4 & SW1 \\
\hline FRD & G62 \\
\hline AWA & $\mathrm{DHZ}$ \\
\hline AWA & $\mathrm{AH} 4$ \\
\hline$A W 3$ & SW1 \\
\hline Aw14 & THZ \\
\hline DE1 & 121 \\
\hline DE1 & 141 \\
\hline AWS & 141 \\
\hline Aws & 121 \\
\hline MS2 & DF 1 \\
\hline LA1 & GH1 \\
\hline PRD & CCs \\
\hline LBS & GE 1 \\
\hline FRD & SW1 \\
\hline Aw3 & GH1 \\
\hline VII & SW1 \\
\hline Aw4 & TG2 \\
\hline MS2 & SB2 \\
\hline MS2 & SA1 \\
\hline PRD & TG2 \\
\hline AW3 & AH1 \\
\hline PRD & TH2 \\
\hline PRD & $F O 1$ \\
\hline PRD & GH3 \\
\hline AW4 & CF5 \\
\hline FRD & TES \\
\hline PRD & cVz \\
\hline AW9 & DH1 \\
\hline HRB & GE1 \\
\hline AW4 & 5W2 \\
\hline VB1 & GG1 \\
\hline F'RD & THZ \\
\hline AWA & DF 1 \\
\hline OEZ & $D F 1$ \\
\hline Oa: & DH2 \\
\hline AW4 & GG1 \\
\hline
\end{tabular}

UNNORM FUSS-V BIRNBAUM

\begin{tabular}{|c|c|}
\hline $\begin{array}{l}881 E-06 \\
808 E-06 \\
720 E-06 \\
438 E-06 \\
407 E-06 \\
407 E-06 \\
338 E-06 \\
223 E-06 \\
223 E-06 \\
035 E-06 \\
835 E-06 \\
648 E-06 \\
356 E-06 \\
336 E-06 \\
734 E-06 \\
718 E-06 \\
707 E-06 \\
707 E-06 \\
614 E-06 \\
614 E-06 \\
585 E-06 \\
443 E-06 \\
396 E-06 \\
373 E-06 \\
363 E-06 \\
287 E-06 \\
241 E-06 \\
100 E-06 \\
069 E-06 \\
069 E-06 \\
047 E-06 \\
012 E-06 \\
859 E-07 \\
592 E-07 \\
230 E-07 \\
120 E-07 \\
872 E-07 \\
419 E-07 \\
332 E-07 \\
978 E-07 \\
440 E-07 \\
242 E-07 \\
684 E-07 \\
491 E-07 \\
491 E-07\end{array}$ & $\begin{array}{l}3.171 E-0.3 \\
1.799 E-03 \\
1.791 E-03 \\
1.162 E-03 \\
1.156 E-03 \\
1.302 E-03 \\
1.025 E-01 \\
1.025 E-01 \\
1.000 E+00 \\
8.494 E-02 \\
8.494 E-02 \\
7.737 E-04 \\
7.821 E-05 \\
9.761 E-04 \\
3.520 E-02 \\
3.520 E-02 \\
2.797 E-03 \\
4.455 E-04 \\
1.025 E-01 \\
1.025 E-01 \\
8.494 E-02 \\
8.494 E-02 \\
2.248 E-03 \\
1.593 E-03 \\
9.968 E-04 \\
1.502 E-03 \\
5.384 E-05 \\
2.337 E-02 \\
1.128 E-04 \\
2.630 E-04 \\
4.456 E-05 \\
1.411 E-04 \\
4.004 E-04 \\
1.000 E+00 \\
3.797 E-04 \\
9.100 E-02 \\
2.287 E-04 \\
4.383 E-04 \\
2.909 E-04 \\
3.037 E-04 \\
8.429 E-03 \\
4.441 E-03 \\
4.040 E-03 \\
4.441 E-03 \\
2.206 E-04 \\
9.600 E-03 \\
2.455 E-03 \\
2.455 E-03\end{array}$ \\
\hline
\end{tabular}

$6.932 E-06$

$3.808 E-06$

$3.720 E-06$

$3.223 E-06$

$3.223 E-06$

3.035E-O6

2.835E-06

2.648E-06

. 356E-OG

2. 336E-06

1. $734 E-06$

.718E-OG

.707E-06

$1.614 E-O$

1. 585E-O6

. $443 E-06$

1. $36.3 E-06$

. 287E-OG

1 O69E-OG

$1.069 E-06$

. $047 E-06$

$9.230 E-07$

. 419E-07

8. 332E-07

7.97 EE-07

6. $491 E-07$

$6.426 E-07$

$1.970 E-04$ 
Table D2.5

Palrs

UNNORM FUSS-V BIRNBAUM

\begin{tabular}{|c|c|}
\hline $\begin{array}{l}\text { RF1 } \\
\text { OB3 }\end{array}$ & $\begin{array}{l}\text { GH1 } \\
\text { AF } 1\end{array}$ \\
\hline $\mathrm{OB3}$ & $\mathrm{AH} 4$ \\
\hline AWA & AF 1 \\
\hline AW9 & GF1 \\
\hline AW4 & BE2 \\
\hline AW4 & GH3 \\
\hline RF I & GG1 \\
\hline AW9 & GH1 \\
\hline AW9 & SW1 \\
\hline AWA & DG1 \\
\hline MS2 & $\operatorname{ccs}$ \\
\hline MS2 & SBE \\
\hline MS2 & SAS \\
\hline $\mathrm{CH} 2$ & $\mathrm{BH} 2$ \\
\hline AW9 & sus \\
\hline PRD & A5B \\
\hline AW3 & GF 1 \\
\hline AW3 & GG1 \\
\hline AW4 & AH1 \\
\hline AW3 & TG2 \\
\hline AW' & DF 1 \\
\hline AW4 & 061 \\
\hline MS2 & DG1 \\
\hline LA1 & GF 1 \\
\hline LA1 & $\mathrm{BHz}$ \\
\hline AW4 & FO4 \\
\hline DB1 & BF 1 \\
\hline FRD & 132 \\
\hline $\mathrm{CH}_{2}$ & SW1 \\
\hline AW4 & CV2 \\
\hline $\mathrm{CH} 2$ & GF 1 \\
\hline MS2 & 131 \\
\hline MS2 & 111 \\
\hline MS2 & AS4 \\
\hline AW4 & CVG \\
\hline OB1 & SW3 \\
\hline AW3 & SW3 \\
\hline Aw3 & G62 \\
\hline AWA & GF 1 \\
\hline AWA & GH4 \\
\hline AWB & $\mathrm{GHZ}$ \\
\hline LB2 & SW1 \\
\hline LA1 & SW1 \\
\hline AW3 & TH2 \\
\hline LA1 & $\mathrm{AH} 1$ \\
\hline FRD & CC7 \\
\hline HRD & GH1 \\
\hline AW7 & DG1 \\
\hline FRD & $\mathrm{FO4}$ \\
\hline MS2 & DH1 \\
\hline
\end{tabular}

$$
\begin{array}{ll}
6.374 E-07 & 4.543 E-03 \\
6.371 E-07 & 2.455 E-03 \\
6.371 E-07 & 2.455 E-03 \\
6.371 E-07 & 9.600 E-03 \\
6.365 E-07 & 9.988 E-05 \\
6.362 E-07 & 1.578 E-04 \\
6.362 E-07 & 1.061 E-04 \\
6.287 E-07 & 4.441 E-03 \\
6.246 E-07 & 9.977 E-05 \\
6.205 E-07 & 8.902 E-06 \\
5.537 E-07 & 8.190 E-03 \\
5.180 E-07 & 1.905 E-05 \\
4.816 E-07 & 6.482 E-06 \\
4.816 E-07 & 3.440 E-05 \\
4.800 E-07 & 6.293 E-04 \\
4.530 E-07 & 4.383 E-04 \\
4.453 E-07 & 3.380 E-04 \\
4.310 E-07 & 7.690 E-03 \\
4.090 E-07 & 7.362 E-03 \\
4.082 E-07 & 8.135 E-03 \\
4.040 E-07 & 6.079 E-03 \\
4.016 E-07 & 4.040 E-03 \\
3.960 E-07 & 7.158 E-03 \\
3.940 E-07 & 5.589 E-04 \\
3.674 E-07 & 3.984 E-04 \\
3.674 E-07 & 3.329 E-04 \\
3.554 E-07 & 2.169 E-04 \\
3.540 E-07 & 2.710 E-04 \\
3.462 E-07 & 4.077 E-03 \\
3.360 E-07 & 4.766 E-05 \\
3.323 E-07 & 2.225 E-04 \\
3.283 E-07 & 5.151 E-04 \\
3.237 E-07 & 2.815 E-04 \\
3.237 E-07 & 2.815 E-04 \\
3.050 E-07 & 1.805 E-05 \\
3.023 E-07 & 1.075 E-04 \\
2.970 E-07 & 1.034 E-05 \\
2.840 E-07 & 2.304 E-04 \\
2.836 E-07 & 4.113 E-03 \\
2.781 E-07 & 6.415 E-05 \\
2.756 E-07 & 6.416 E-05 \\
2.735 E-07 & 4.077 E-03 \\
2.682 E-07 & 2.302 E-06 \\
2.682 E-07 & 2.629 E-05 \\
2.665 E-07 & 4.040 E-03 \\
2.656 E-07 & 1.082 E-02 \\
2.626 E-07 & 8.117 E-03 \\
2.535 E-07 & 1.252 E-03 \\
2.445 E-07 & 1.000 E+00 \\
2.392 E-07 & 2.169 E-04 \\
2.348 E-07 & 3.349 E-04
\end{array}
$$


Table D2.5

Pair:

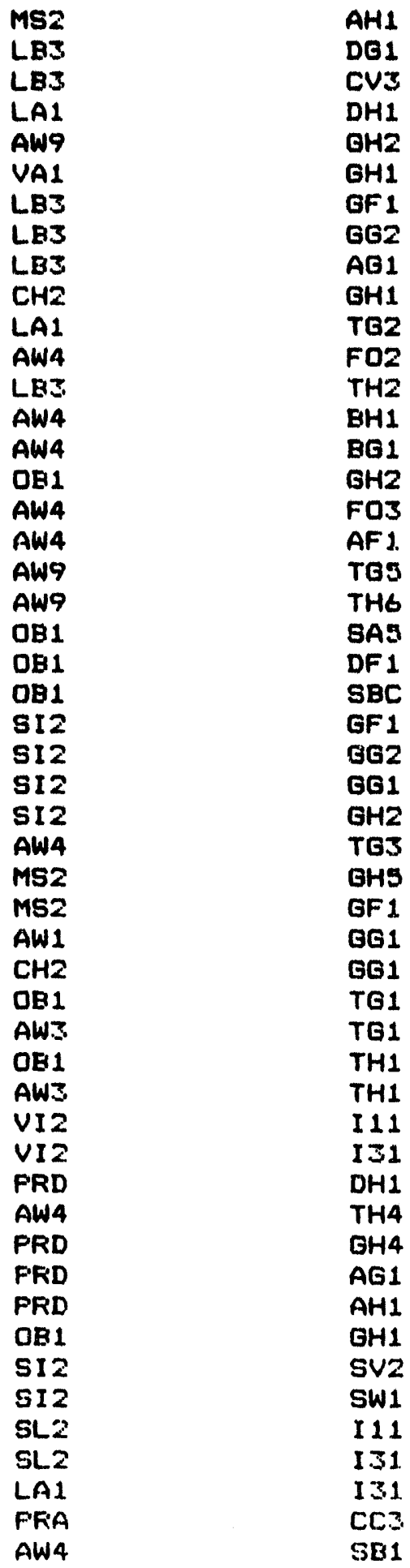

UWNORY FUSS-V BIRNBAUM

$\begin{array}{ll}2.318 E-07 & 3.350 E-04 \\ 2.316 E-07 & 1.610 E-02 \\ 2.305 E-07 & 1.989 E-04 \\ 2.302 E-07 & 1.610 E-02 \\ 2.300 E-07 & 3.015 E-05 \\ 2.292 E-07 & 1.345 E-03 \\ 2.277 E-07 & 2.469 E-04 \\ 2.277 E-07 & 2.007 E-04 \\ 2.273 E-07 & 1.610 E-02 \\ 2.269 E-07 & 3.624 E-04 \\ 2.246 E-07 & 2.056 E-04 \\ 2.212 E-07 & 4.333 E-04 \\ 2.159 E-07 & 1.989 E-04 \\ 2.109 E-07 & 2.020 E-03 \\ 2.109 E-07 & 2.020 E-03 \\ 2.066 E-07 & 1.321 E-04 \\ 2.047 E-07 & 8.044 E-03 \\ 2.027 E-07 & 4.040 E-03 \\ 1.997 E-07 & 3.190 E-03 \\ 1.979 E-07 & 3.190 E-05 \\ 1.866 E-07 & 4.611 E-04 \\ 1.863 E-07 & 9.145 E-03 \\ 1.786 E-07 & 4.611 E-04 \\ 1.786 E-07 & 2.469 E-04 \\ 1.786 E-07 & 2.007 E-04 \\ 1.722 E-07 & 2.402 E-04 \\ 1.722 E-07 & 1.989 E-04 \\ 1.701 E-07 & 3.753 E-05 \\ 1.612 E-07 & 2.900 E-06 \\ 1.612 E-07 & 3.567 E-06 \\ 1.521 E-07 & 9.100 E-02 \\ 1.518 E-07 & 2.402 E-04 \\ 1.426 E-07 & 1.122 E-04 \\ 1.426 E-07 & 2.614 E-03 \\ 1.413 E-07 & 1.122 E-04 \\ 1.413 E-07 & 2.614 E-03 \\ 1.399 E-07 & 5.530 E-03 \\ 1.399 E-07 & 5.530 E-03 \\ 1.395 E-07 & 4.077 E-03 \\ 1.389 E-07 & 2.769 E-05 \\ 1.377 E-07 & 6.297 E-05 \\ 1.377 E-07 & 4.077 E-03 \\ 1.377 E-07 & 4.077 E-03 \\ 1.313 E-07 & 1.023 E-04 \\ 1.310 E-07 & 4.550 E-02 \\ 1.310 E-07 & 1.638 E-05 \\ 1.282 E-07 & 1.710 E-02 \\ 1.282 E-07 & 1.710 E-02 \\ 1.234 E-07 & 5.260 E-0 . \\ 1.233 E-07 & 2.560 E-02 \\ 1.207 E-07 & 2.225 E-04 \\ 1.20\end{array}$


Tab1e D2.5

Patra

UMORM FUSS-V BIRHBAUM

$\mathrm{CH} 2$

CH2

Aw9

AW9

OB1

MS2

$\mathrm{CH} 2$

AW4

AW4

AW4

C12

CI2

MS2

MS2

081

SL2

BL2

SL2

AWB

SL2

SL2

AW4

OB1

AW4

$\mathrm{CH} 2$

AW4

OB1

OB3

AW7

RF 1

RF 1

LA1

AW7

OB1

OB1

$\mathrm{CH} 2$

LA1

LA1

$\mathrm{CH}_{2}$

$\mathrm{CH} 2$

RF 1

RF 1

CH1

RF 1

RF 1

RF 1
Te2

BV2

TC2

TH2

AF 1

AF 1

TH2

TOS

TH1

THE

EF 1

$\mathrm{OHZ}$

121

I 41

SW1

DF 1

DB1

DH1

DF 1

121

141

TH3

AH1

EW3

AH1

FO1

TH3

8B1

BB1

GF 1

$\mathrm{GH} 2$

THS

132

TES

TE2

TH3

T63

\&W3

T63

SWJ

AH1

TH3

GH1

TE.

TE2

SW3

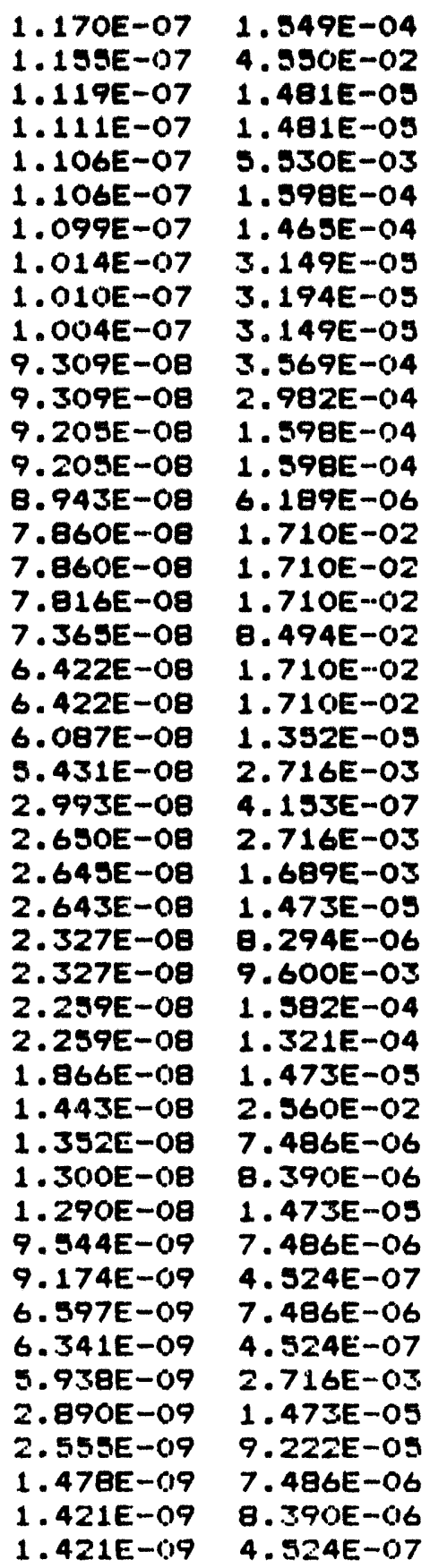


Table 02.6

Palr Iaportance by Clasees of split Fractions

(Frontlines - Human and Rocovery Actiona)

Polre

UNNONY FUSS-V BIRWBAUM

\begin{tabular}{|c|c|}
\hline $\begin{array}{l}\text { PRD } \\
\text { AW4 } \\
\text { LA1 } \\
\text { MS2 } \\
\text { LBE } \\
\text { OB1 } \\
\text { VIS } \\
\text { MU1 } \\
\text { AWB } \\
\text { AW4 } \\
\text { LA1 } \\
\text { LB2 } \\
\text { HRD } \\
\text { VI2 } \\
\text { OB1 } \\
\text { VA1 } \\
\text { LA1 } \\
\text { CH2 } \\
\text { MB2 } \\
\text { AW7 } \\
\text { AWB } \\
\text { AW4 } \\
\text { AW7 } \\
\text { OB3 } \\
\text { RF1 } \\
\text { AW4 } \\
\text { OB1 } \\
\text { PRD } \\
\text { VI2 } \\
\text { LB1 } \\
\text { CH2 } \\
\text { CB2 } \\
\text { OB1 } \\
\text { AW3 }\end{array}$ & $\begin{array}{l}\text { REACO6 } \\
\text { RESLC1 } \\
\text { REACOG } \\
\text { OB1 } \\
\text { REACO6 } \\
\text { RP2 } \\
\text { OP1 } \\
\text { OP1 } \\
\text { RP2 } \\
\text { RP2 } \\
\text { OP1 } \\
\text { OP1 } \\
\text { REACO6 } \\
\text { RP2 } \\
\text { REBLC1 } \\
\text { REACO6 } \\
\text { REBLC1 } \\
\text { REELC1 } \\
\text { ZHEAW4 } \\
\text { ZHEDB2 } \\
\text { ZHEAW4 } \\
\text { ZHEFO6 } \\
\text { ZHEAW3 } \\
\text { ZHEAW3 } \\
\text { RESLC1 } \\
\text { ZHESW1 } \\
\text { ZHEAW4 } \\
\text { ZHERE2 } \\
\text { ZHEAW4 } \\
\text { ZHEAW3 } \\
\text { ZHEAW3 } \\
\text { ZHEAW3 } \\
\text { ZHEBW1 } \\
\text { ZHEBW1 }\end{array}$ \\
\hline
\end{tabular}

$2.733 E-06 \quad 1.986 E-04$

1.645E-O6 4.89OE-OS

$1.134 E-06 \quad 1.972 E-04$

1.069E-06 $5.347 E-04$

$9.262 E-07$ 1.610E-04

$6.863 E-07 \quad 2.384 E-05$

6.802E-07 1.710E-02

6.031E-07 1.710E-02

5.657E-07 2.357E-05

3.960E-07 5.484E-06

3.593E-07 3.984E-03

3.593E-07 3.48BE-04

2.535E-07 1.972E-04

2.445E-07 1.116E-0S

2.196E-07 1.63日E-05

2.135E-07 1.972E-04

1.55OE-07 1.63EE-05

1.071E-07 1.638E-05

8.748E-OQ 1.75OE-OS

5.587E-0日 2.155E-03

3.2EGE-OB 2.727E-04

2.645E-0Q 9.120E-06

2. $384 E-08$ 9.968E-03

2.584E-0B Q.612E-06

2.401E-OQ 1.63日E-05

2.21日E-OB Q.643E-05

1. $820 E-O B \quad 1.260 E-04$

1.539E-OQ $5.939 E-05$

1.466E-OQ 1.333E-04

3.669E-10 2.94OE-06

3.509E-10 3.110E-06

3.364E-10 2.94OE-OG

1.737E-10 1.69EE-06

$1.737 E-10$ 3.958E-05 
Table D2.7

Palr Importance by Claseses of Split Fractions (Supports - Supports)

Palre

\begin{tabular}{|c|c|}
\hline $\begin{array}{l}\text { GF } 1 \\
\text { GE2 }\end{array}$ & $\begin{array}{l}\text { GG2 } \\
\text { GF } 1\end{array}$ \\
\hline COI & EH2 \\
\hline $\mathrm{EH} 2$ & GG1 \\
\hline $\mathrm{GH}^{2}$ & GF 1 \\
\hline $\begin{array}{l}\text { GF } 1 \\
\text { GH1 }\end{array}$ & $\mathrm{BH} 2$ \\
\hline $\begin{array}{l}\mathrm{GH1} \\
\text { SW1 }\end{array}$ & SWI \\
\hline $\begin{array}{l}\text { BW1 } \\
\text { GH3 }\end{array}$ & GH1 \\
\hline $\begin{array}{l}\text { GH3 } \\
\text { GF } 1\end{array}$ & $\begin{array}{l}\text { GF } 1 \\
\text { EH } 3\end{array}$ \\
\hline BC2 & EH3 \\
\hline GH3 & GE2 \\
\hline 8A1 & 882 \\
\hline $8 B 2$ & BA1 \\
\hline COL1 & TH3 \\
\hline TH3 & GE1 \\
\hline TO2 & BH1 \\
\hline EHI & TO2 \\
\hline $\begin{array}{l}\text { GF1 } \\
\text { TO3 }\end{array}$ & $\begin{array}{l}\text { TE3 } \\
\text { OF } 1\end{array}$ \\
\hline $\begin{array}{l}\text { TE3 } \\
\text { GH1 }\end{array}$ & THZ \\
\hline TH2 & BH1 \\
\hline ccs & GF 1 \\
\hline of 1 & $\operatorname{ccs}$ \\
\hline GE2 & TES \\
\hline Tos & GOZ \\
\hline TO2 & SW1 \\
\hline SW1 & TE2 \\
\hline $\mathrm{OH2}$ & TH3 \\
\hline TH3 & $\mathrm{GH} 2$ \\
\hline TG2 & GQ1 \\
\hline G61 & TG2 \\
\hline SW1 & TH2 \\
\hline TH2 & SW1 \\
\hline OO1 & FOS \\
\hline FOS & OQ1 \\
\hline TH3 & FOS \\
\hline FOS & TH3 \\
\hline TH3 & GF 1 \\
\hline GF 1 & TH3 \\
\hline DE1 & CC. 3 \\
\hline $\operatorname{ccs}$ & DE1 \\
\hline $\mathrm{CC} 2$ & DH1 \\
\hline DH1 & CC2 \\
\hline ASB & BF 1 \\
\hline GF 1 & ASB \\
\hline GG2 & ASB \\
\hline ASE & GG2 \\
\hline OG1 & GF 1 \\
\hline GF 1 & OG1 \\
\hline
\end{tabular}

UNNORM FUSS - V BIRNBAUM

$$
\begin{aligned}
& \text { 8.976E-06 3.413E-0.3 } \\
& \text { 8.576E-06 3.413E-03 } \\
& \text { 4.158E-O6 1.716E-0.3 } \\
& \text { 4.158E-06 1.716E-O3 } \\
& \text { 4.035E-06 1.650E-0I } \\
& 4.039 E-06 \text { 1.690E-03 } \\
& 3.162 E-06 \quad 1.424 E-04 \\
& 3.162 E-06 \quad 1.424 E-04 \\
& \text { 2.887E-06 7.724E-04 } \\
& 2.887 E-06 \quad 7.724 E-04 \\
& \text { 2.887E-06 6.279E-04 } \\
& \text { 2.887E-06 6.279E-04 } \\
& \text { 2.101E-O6 1.155E-O2 } \\
& \text { 2.101E-06 1.15SE-02 } \\
& \text { 2.054E-06 7.381E-04 } \\
& \text { 2.054E-06 7.3日1E-04 } \\
& 1.932 E-06 \quad 8.116 E-04 \\
& 1.932 E-06 \quad 0.116 E-04 \\
& 1.904 E-06 \quad 6.741 E-04 \\
& \text { 1.904E-06 6.741E-04 } \\
& \text { 1.897E-06 8.031E-04 } \\
& \text { 1.897E-06 Q.031E-04 } \\
& 1.878 E-O 6 \text { 1.448E-0.3 } \\
& 1.878 E-06 \quad 1.448 E-03 \\
& \text { 1.701E-06 4.896E-04 } \\
& \text { 1.701E-O6 4.896E-O4 } \\
& 1.660 E-06 \text { 6.193E-OS } \\
& 1.660 E-06 \text { 6.193E-OS } \\
& 1.394 E-06 \quad 4.744 E-04 \\
& 1.594 E-06 \quad 4.744 E-04 \\
& 1.546 E-06 \quad 6.439 E-04 \\
& 1.546 E-06 \text { 6.439E-O4 } \\
& 1.316 E-06 \quad 5.698 E-05 \\
& 1.516 E-06 \text { 5.69日E-OS } \\
& 1.385 E-06 \quad 6.086 E-04 \\
& 1.385 E-06 \quad 6.086 E-04 \\
& \text { 1.385E-06 4.391E-04 } \\
& \text { 1.385E-06 4.391E-04 } \\
& 1.342 E-06 \quad 4.779 E-04 \\
& 1.342 E-06 \quad 4.779 E-04 \\
& 1.327 E-06 \quad 1.000 E+00 \\
& 1.327 E-06 \text { 1.00OE+OO } \\
& 1.284 E-06 \quad 1.000 E+00 \\
& 1.284 E-06 \quad 1.000 E+00 \\
& \text { 1.226E-06 1.004E-03 } \\
& 1.226 E-06 \text { 1.004E-03 } \\
& 1.226 E-06 \text { 8.1.6SE-04 } \\
& 1.226 E-06 \quad 8.165 E-04 \\
& 1.122 \mathrm{E}-06 \quad 3.252 \mathrm{E}-02 \\
& 1.122 E-06 \quad 3.252 E-02
\end{aligned}
$$


Tab1e D2.7

Palrs

\begin{tabular}{|c|c|}
\hline $\begin{array}{l}\text { GE2 } \\
\text { THIX }\end{array}$ & $\begin{array}{l}\text { THI } \\
\text { GGZ }\end{array}$ \\
\hline GG2 & $\operatorname{ccs}$ \\
\hline $\operatorname{ccs}$ & GG2 \\
\hline $\operatorname{ccs}$ & GH2 \\
\hline GHZ & CC5 \\
\hline RT7 & SE2 \\
\hline SEZ & FIT7 \\
\hline SA1 & R'T7 \\
\hline RT7 & SA1 \\
\hline THS & TG2 \\
\hline TG2 & THZ \\
\hline ccs & SW1 \\
\hline SW1 & CCs \\
\hline FO1 & GF 1 \\
\hline EF 1 & FO1 \\
\hline$F 01$ & GH1 \\
\hline $\mathbf{G H} 1$ & FO1 \\
\hline TG1 & $F O 1$ \\
\hline FD1 & TG1 \\
\hline GG1 & SW2 \\
\hline SW2 & GG1 \\
\hline GG1 & CV \\
\hline CV3 & GG1 \\
\hline TH2 & GG1 \\
\hline G61 & THZ \\
\hline CC5 & GG 1 \\
\hline GG1 & CC5 \\
\hline CV 3 & GF 1 \\
\hline GF 1 & cV3 \\
\hline $\begin{array}{l}\text { GG2 } \\
\text { CU? }\end{array}$ & CVZ \\
\hline $\begin{array}{l}\text { CV } 3 \\
\text { CC5 }\end{array}$ & GB2 \\
\hline $\begin{array}{l}\text { CC5 } \\
\text { GH1 }\end{array}$ & GHI \\
\hline $\begin{array}{l}\text { GH1 } \\
\text { SW2 }\end{array}$ & CC5 \\
\hline $\begin{array}{l}\text { SW2 } \\
\text { GH1 }\end{array}$ & GH1 \\
\hline EH1 & SW2 \\
\hline GG2 & OG1 \\
\hline OG1 & GG2 \\
\hline SW1 & SVZ \\
\hline SV2 & SW1 \\
\hline FOS & $\mathrm{EH}_{2}$ \\
\hline GH2 & F05 \\
\hline F05 & TG2 \\
\hline $\begin{array}{l}\text { TG2 } \\
\text { SA2 }\end{array}$ & F05 \\
\hline $\begin{array}{l}\text { SA2 } \\
\text { SB6 }\end{array}$ & SB6 \\
\hline & $\begin{array}{l}\text { SA2 } \\
\text { CV } 3\end{array}$ \\
\hline $\begin{array}{l}\mathrm{GH2} \\
\mathrm{CV} 3\end{array}$ & CV 3 \\
\hline $\begin{array}{l}\text { CV3 } \\
\text { GG1 }\end{array}$ & $\mathrm{GH}_{2}$ \\
\hline $\begin{array}{l}\text { GG1 } \\
\text { FO4 }\end{array}$ & F04 \\
\hline $\begin{array}{l}\mathrm{FO4} \\
\mathrm{TH} ?\end{array}$ & GG 1 \\
\hline $\begin{array}{l}\mathrm{TH}_{2} \\
\mathrm{FO} 4\end{array}$ & FO4 \\
\hline & $\mathrm{TH}_{2}$ \\
\hline
\end{tabular}

\section{UNNORM FUSS-V BIRNBAUM}

$$
\begin{array}{ll}
1.112 E-06 & 3.221 E-04 \\
1.112 E-06 & 3.221 E-04 \\
1.103 E-06 & 6.913 E-04 \\
1.103 E-06 & 6.913 E-04 \\
1.084 E-06 & 6.979 E-04 \\
1.084 E-06 & 6.979 E-04 \\
1.032 E-06 & 2.228 E-02 \\
1.032 E-06 & 2.228 E-02 \\
1.032 E-06 & 7.054 E-02 \\
1.032 E-06 & 7.054 E-02 \\
9.119 E-07 & 2.740 E-04 \\
9.119 E-07 & 2.740 E-04 \\
9.054 E-07 & 6.309 E-05 \\
9.054 E-07 & 6.309 E-05 \\
8.885 E-07 & 9.100 E-02 \\
8.885 E-07 & 9.100 E-02 \\
8.727 E-07 & 9.100 E-02 \\
8.727 E-07 & 9.100 E-02 \\
8.649 E-07 & 9.100 E-02 \\
8.649 E-07 & 9.100 E-02 \\
8.574 E-07 & 7.535 E-03 \\
8.574 E-07 & 7.535 E-03 \\
8.419 E-07 & 3.308 E-04 \\
8.419 E-07 & 3.308 E-04 \\
8.249 E-07 & 3.461 E-04 \\
8.249 E-07 & 3.461 E-04 \\
7.603 E-07 & 5.913 E-04 \\
7.603 E-07 & 5.913 E-04 \\
7.545 E-07 & 2.939 E-04 \\
7.545 E-07 & 2.939 E-04 \\
7.545 E-07 & 2.389 E-04 \\
7.545 E-07 & 2.389 E-04 \\
7.511 E-07 & 3.894 E-04 \\
7.511 E-07 & 5.894 E-04 \\
7.440 E-07 & 6.598 E-03 \\
7.440 E-07 & 6.598 E-03 \\
7.256 E-07 & 1.710 E-02 \\
7.256 E-07 & 1.710 E-02 \\
7.199 E-07 & 7.987 E-0 . \\
7.189 E-07 & 7.987 E-0.3 \\
6.958 E-07 & 2.532 E-04 \\
6.958 E-07 & 2.532 E-04 \\
6.893 E-07 & 2.532 E-04 \\
6.893 E-07 & 2.532 E-04 \\
6.398 E-07 & 6.650 E-04 \\
6.398 E-07 & 6.650 E-04 \\
6.113 E-07 & 1.989 E-04 \\
6.113 E-07 & 1.989 E-04 \\
6.090 E-07 & 6.015 E-04 \\
6.090 E-07 & 6.015 E-04 \\
6.090 E-07 & 5.065 E-04 \\
6.090 E-07 & 5.065 E-04 \\
&
\end{array}
$$

NUREGKR-5726 
Table D2.7

Pairs

\begin{tabular}{|c|c|}
\hline GF 1 & DG1 \\
\hline DG 1 & GF 1 \\
\hline $\mathrm{GH}_{2}$ & TGS \\
\hline TGS & $\mathrm{GH}_{2}$ \\
\hline GF 1 & $\mathrm{DH} 1$ \\
\hline DH1 & GF 1 \\
\hline SW2 & $\mathrm{GH}_{2}$ \\
\hline $\mathrm{GH}_{2}$ & SW2 \\
\hline TG2 & $\operatorname{ccs}$ \\
\hline CC5 & TG2 \\
\hline CCs & DF 1 \\
\hline DF 1 & CC5 \\
\hline CC5 & AF 1 \\
\hline AF 1 & $\operatorname{ccs}$ \\
\hline SAS & SBE \\
\hline SBE & 5A5 \\
\hline $\mathrm{CC} 4$ & $\mathrm{DH} 2$ \\
\hline $\mathrm{DH}_{2}$ & CC4 \\
\hline AH4 & $\operatorname{cc} 4$ \\
\hline CC4 & $\mathrm{AH} 4$ \\
\hline DG1 & GHI \\
\hline GHS & $0 G 1$ \\
\hline GF 1 & SV5 \\
\hline SV5 & GF 1 \\
\hline SV5 & $\mathrm{GH} 2$ \\
\hline $\mathrm{GH} 2$ & sVs \\
\hline GG1 & SW1 \\
\hline SW1 & GG1 \\
\hline CC5 & TH3 \\
\hline TH3 & $\operatorname{ccs}$ \\
\hline DF 1 & GH1 \\
\hline GH1 & DF 1 \\
\hline $5 W 1$ & DH1 \\
\hline DH1 & SW1 \\
\hline $\mathrm{GH}_{2}$ & OG1 \\
\hline DG1 & $\mathrm{GH}_{2}$ \\
\hline GG1 & TG3 \\
\hline TG3 & GG 1 \\
\hline TH3 & ASE \\
\hline ASB & TH3 \\
\hline CC5 & $\mathrm{TH}_{2}$ \\
\hline THZ & CC5 \\
\hline SV2 & DF 1 \\
\hline LF 1 & 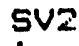 \\
\hline CC5 & CVS \\
\hline CV3 & CC5 \\
\hline GF 1 & SB1 \\
\hline SB1 & GF 1 \\
\hline AF 1 & SV2 \\
\hline sv2 & $A F 1$ \\
\hline AHI & GF 1 \\
\hline GF 1 & $A H 1$ \\
\hline
\end{tabular}

UNNORM FUSS-V BIRNBAUM

$5.962 E-07$

$5.962 E-07$

5. $952 E-07$

$5.952 E-07$

$5.669 E-07$

$5.669 E-07$

$5.602 E-07$

5. $602 E-07$

5. $312 E-07$

5. $312 \mathrm{E}-07$

5. $180 E-07$

5. $180 E-07$

$5.084 E-07$

$5.084 E-07$

$4.816 E-07$

4. $816 E-07$

4. $771 E-07$

4. $771 E-07$

$4.730 E-07$

4. $730 E-07$

$4.642 E-07$

$4.642 E-07$

4. 393E-07

4. $393 E-07$

4. 393E- 07

4.3.33E-07

4. 348E-O7

4. $348 E-07$

4. $076 E-07$

4. $076 E-07$

4. $016 E-07$

4. $016 E-07$

3. $976 E-07$

$3.976 E-07$

$3.960 E-07$

$3.960 E-07$

$3.923 E-07$

3.923E-07

$3.835 E-07$

$3.835 E-07$

$3.742 \mathrm{E}-07$

3. 742E-07

3.73.OE-07

3. $730 E-07$

J. 72BE-07

3. $728 E-07$

3. .691E-07

$3.691 E-07$

$3.661 E-07$

S.661E-07

$3.646 E-07$

$3.646 E-07$
1.871E-02

$1.871 E-02$

$1.76 O E-04$

$1.760 E-04$

$1.789 E-02$

1.789E-02

$4.077 E-03$

4.077E-0.

3.453 .04

$3.453 E-04$

$2.560 E-02$

2. $560 E-02$

$2.560 E-02$

$2.560 E-02$

$4.630 E-04$

$4.630 E-04$

2. $560 E-02$

2. 560E-02

2. 56OE-O2

2. $560 \mathrm{E}-02$

$7.357 E-03$

$7.357 E-0.3$

1. $326 E-03$

1. 326E-OZ

1. 10BE-0.3

1. 108E-03

1. $941 E-05$

$1.941 E-05$

2. 287E-04

2. 287E-04

1. 283E-02

1. 283E-02

1. $134 E-0.3$

1. $134 \mathrm{E}-03$

$9.593 E-03$

$9.593 E-03$

1. $401 E-04$

1. $401 E-04$

2. 287E-04

2. $287 E-04$

2. $451 E-04$

$2.451 E-04$

1. $00 O E+O O$

1. .OOOE + OO

2. 287E-04

$2.287 E-04$

1. $.092 E-0$.

1. $092 E-0.3$

1. $000 \mathrm{t}+00$

1. OOOE+OO

1. $16 G E-02$

1. $166 E-02$ 
Table D2.7

Pairs

\begin{tabular}{|c|c|}
\hline ASE & CVZ \\
\hline CVT & ASE \\
\hline 132 & GG1 \\
\hline GG1 & 132 \\
\hline GF 1 & CV2 \\
\hline CV2 & GF 1 \\
\hline $\mathrm{GH2}$ & $\mathrm{CV}_{2}$ \\
\hline $\mathrm{CV} 2$ & $\mathrm{GHZ}$ \\
\hline BG1 & GF 1 \\
\hline GF 1 & BG1 \\
\hline BG1 & GG2 \\
\hline GG2 & EG1 \\
\hline SW1 & $\mathrm{CC} 7$ \\
\hline $\mathrm{CC} 7$ & SW1 \\
\hline GH1 & SVS \\
\hline SV5 & GH1 \\
\hline FO4 & GF 1 \\
\hline GF 1 & FO4 \\
\hline FO4 & THE \\
\hline GG2 & FO4 \\
\hline THZ & FD4 \\
\hline Fo4 & GG2 \\
\hline $\mathbf{G F}_{1}$ & GH5 \\
\hline GHS & GF 1 \\
\hline GF 1 & $A G 1$ \\
\hline AG1 & GF 1 \\
\hline TGG & GF 1 \\
\hline GF 1 & TG6 \\
\hline DF 1 & GG1 \\
\hline GG1 & DF 1 \\
\hline A54 & DF 1 \\
\hline DF 1 & AS4 \\
\hline GG1. & $A F_{1}$ \\
\hline AF 1 & GG1 \\
\hline CVG & GHI \\
\hline GH1 & CVG \\
\hline SW1 & CVG \\
\hline CV6 & SW1 \\
\hline THZ & CVG \\
\hline CVG & $\mathrm{TH}_{2}$ \\
\hline TG5 & SW1 \\
\hline SW1 & T65 \\
\hline AS4 & $A F 1$ \\
\hline AF 1 & AS4 \\
\hline THG & SW 1 \\
\hline SW1. & TH6 \\
\hline CCS & SW2 \\
\hline SW2 & $\mathrm{CC} 5$ \\
\hline DG1 & $\mathrm{GH} 4$ \\
\hline $\mathrm{GH} 4$ & $D Q 1$ \\
\hline OG 1 & TGS \\
\hline TE. & $0 G 1$ \\
\hline
\end{tabular}

UNNORM FUSS-V BIRNBAUM

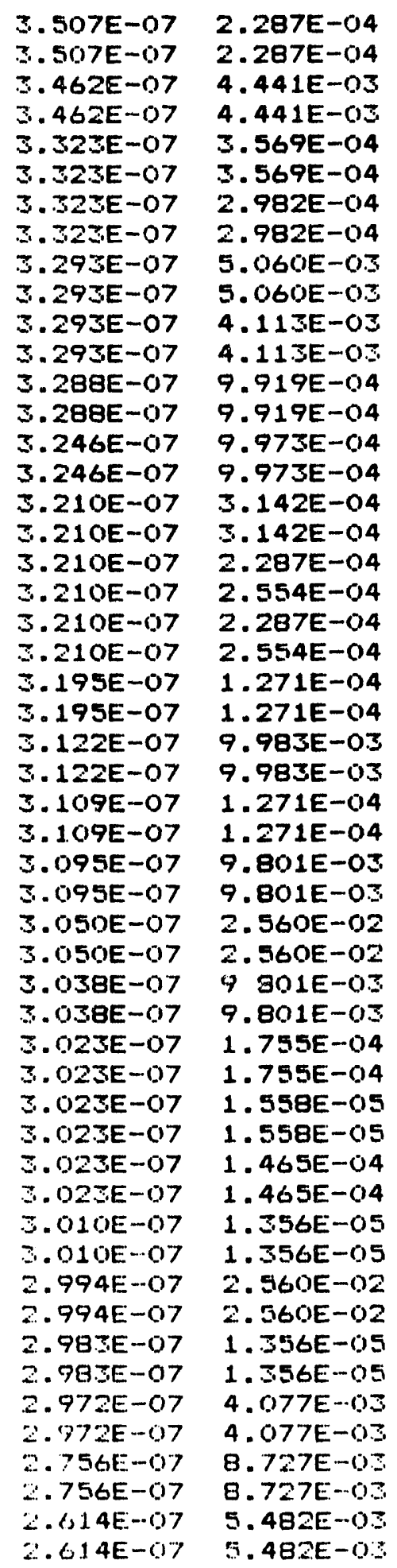

3. . 507E-07

. 5OTE-OZ

$.462 E-07$

3. 32 $E-07$

3. . $323 E-07$

$3.323 E-07$

3. 323E-07

293E-07

3 293E-07

3. 293E-07

3. 288E-07

$3.288 E-07$

. $210 E-07$

$3.210 E-07$

$3.210 E-07$

21OE-O7

S.122E-07

3. 122E-07

109E-07

3. $050 E-07$

3.050E-07

$3.038 E-07$

038E- -97

$3.023 E-07$

5. 023E-07

$\because .023 E-07$

$2.994 E-07$

2. $983 E-07$

$2.98 .3 E-07$

2. $7545-0$

$2.614 E-07$

2. 287E-04

4. $441 E-03$

. $441 E-03$

3. 569E-04

2.982E-04

$.982 E-04$

$4.113 E-03$

4.11.3E-0.

$9.919 E-04$

$9.973 E-04$

$9.973 E-04$

3. $142 E-04$

2. 287E-04

2. 2BTE-04

2. 554E-04

$1.271 E-04$

9. $983 E-03$

271E-04

1. 271E-04

9 BO1E-O

$2.56 O E-02$

$2.560 E-02$

9. BO1E-03

$1.755 E-04$

1. 558E-05

1. $558 E-05$

1. 465E-04

1. $356 E-05$

StOE-OL

$2.56 O E-02$

SGE-OS

$4.077 E-03$

4.

8. $727 E-03$

5. 48.2E-O 
Tab1e D2.7

Pairs

\begin{tabular}{|c|c|}
\hline SE1 & GG2 \\
\hline EG2 & SE1 \\
\hline SW1 1 & SVS \\
\hline SV5 & SW1 \\
\hline THS & GHI \\
\hline GH1 & THE \\
\hline GG1 & $\mathrm{FO}^{2}$ \\
\hline $\mathrm{FOZ}$ & GG1 \\
\hline TH4 & TG: \\
\hline TES & TH4 \\
\hline SV5 & TG2 \\
\hline TE2 & SVS \\
\hline TG2 & FO2 \\
\hline $\mathrm{FO2}$ & TG2 \\
\hline GH1 & BG1 \\
\hline EG1 & GHI \\
\hline SW1 & BG 1 \\
\hline EHI & GH1 \\
\hline GH1 & EHI \\
\hline EG1 & SW 1 \\
\hline SW1 & BH1 \\
\hline $\mathrm{BHI}$ & SW 1 \\
\hline $\mathrm{FOZ}$ & GH1 \\
\hline GH1 & $\mathrm{FO}_{2}$ \\
\hline SVI & DH1 \\
\hline DH1 & $5 V 3$ \\
\hline AF 1 & GH1 \\
\hline GHI & $A F 1$ \\
\hline SW1 & $\mathrm{AH} 1$ \\
\hline AH1 & SW1 \\
\hline SVS & $\mathrm{AH} 1$ \\
\hline$A H 1$ & 5V3 \\
\hline $\mathrm{DH} 1$ & TG5 \\
\hline TG5 & $\mathrm{DH} 1$ \\
\hline SV5 & TH 3 \\
\hline THZ & svs \\
\hline FO2 & GF 1 \\
\hline GF 1 & $\mathrm{FOZ}$ \\
\hline $\mathrm{DH} 1$ & THG \\
\hline THG & DH1 \\
\hline TGS & $\mathrm{FO} 2$ \\
\hline $\mathrm{FOZ}$ & TES \\
\hline $\mathrm{FO} 2$ & $\mathrm{GH}_{2}$ \\
\hline GH2 & $\mathrm{FO} 2$ \\
\hline THS & $\mathrm{FO}_{2}$ \\
\hline$F O 2$ & TH3 \\
\hline$F 01$ & OG1 \\
\hline DG1 & FO1 \\
\hline AS5 & SW 1 \\
\hline SW1 & AS5 \\
\hline Glt2 & TH4 \\
\hline TH4 & $\mathrm{GH}_{2}$ \\
\hline DG1 & GHS \\
\hline
\end{tabular}

UNNORM FUSS-V BIRNBAUM

$2.484 E-07$

$5.972 E-04$

2. $484 \mathrm{E}-07 \quad 5.972 \mathrm{E}-04$

$2.266 E-07$ 6.182E-05

2. $266 E-07$ 6.182E-05

$2.226 E-07 \quad 8.073 E-05$

2. 226E-07 8.073E-05

2.139E-07 6.782E-04

$2.139 E-07 \quad 6.782 E-04$

$2.1 .2 E-07$ 4.928E-0.5

$2.132 E-07$ 4.928E-05

2. 129E-07 5.418E-04

2. $129 E-07 \quad 5.418 E-04$

2. $115 E-07$ 5.605E-04

$2.115 E-07 \quad 5.605 E-04$

2.109E-07 3.299E-0.

$2.109 E-07$ 3.299E-0.3

2.109E-07 2.929E-04

$2.109 E-07$ 3.299E-0.3

2. $109 E-07 \quad 3.299 E-03$

2.109E-07 2.929E-04

$2.109 E-07 \quad 2.929 E-04$

2.109E-07 2.929E-04

$2.096 E-07 \quad 6.706 E-04$

2.096E-07 6.706E-04

$2.042 E-07 \quad 1.000 E+00$

$2.042 E-07 \quad 1.000 E+00$

2.027E-07 6.598E-0.3

2.027E-07 6.598E-03

2.01日E-07 5.832E-04

2. $018 E-07$ 5.832E-04

2.016E-07 1.000E+00

2.016E-07 1.000E+00

$1.997 E-07 \quad 6.416 E-03$

$1.997 E-07 \quad 6.416 E-0.3$

1.993E-07 4.378E-04

$1.993 E-07 \quad 4.378 E-04$

1.979E-07 6.219E-04

1.979E-07 6.219E-04

$1.979 E-07 \quad 6.415 E-0.3$

$1.979 E-07 \quad 6.415 E-03$

$1.977 E-07 \quad 4.492 E-04$

$1.977 E-07 \quad 4.492 E-04$

$1.943 E-07 \quad 5.102 E-04$

$1.943 E-07 \quad 5.102 E-04$

$1.920 E-07 \quad 4.391 E-04$

$1.920 E-07 \quad 4.391 E-04$

$1.871 E-07 \quad 1.000 E+00$

1. $871 E-07$ 1. $600 E+00$

1.776E-07 9.919E-04

$1.776 E-07 \quad 9.919 E-04$

1. $754 E-07 \quad 4.684 E-05$

1. $754 E-07$ 4.684E-05

1. $612 \mathrm{E}-07$ 4.113E-03 
Table D2.7

Pairs UNNORM FUSS-V BIRNBAUM

\begin{tabular}{|c|c|}
\hline GH5 & DG1 \\
\hline DH1 & $\mathrm{GQZ}_{2}$ \\
\hline GG2 & DH1 \\
\hline GG2 & AH1 \\
\hline AH1 & GG2 \\
\hline$A G 1$ & GHS \\
\hline GH5 & $A G 1$ \\
\hline TG6 & DG1 \\
\hline DG1 & .TES \\
\hline DF 1 & $\mathrm{GH}_{2}$ \\
\hline $\mathrm{GH}_{2}$ & DF 1 \\
\hline DF 1 & TG2 \\
\hline TG2 & DF 1 \\
\hline AG1 & TGG \\
\hline TG6 & $A G 1$ \\
\hline AF 1 & $\mathrm{GH} 2$ \\
\hline GH2 & AF 1 \\
\hline TG2 & $A F 1$ \\
\hline$A F 1$ & TG2 \\
\hline ASE & GG1 \\
\hline GE1 & ASE \\
\hline SW1 & ASE \\
\hline ASE & TG2 \\
\hline TG2 & ASB \\
\hline ASB & SW1 \\
\hline TG1 & Sw3 \\
\hline EW3 & TE1 \\
\hline 5W3 & TH1 \\
\hline TH1 & 5W3 \\
\hline SB1 & GH3 \\
\hline GH3 & SE1 \\
\hline DH1 & GG1 \\
\hline GG1 & DH1 \\
\hline AG1 & GHA \\
\hline GG1 & $\mathrm{AH} 1$ \\
\hline AHI & GG1 \\
\hline GH4 & AG1 \\
\hline CC7 & GG1 \\
\hline G61 & $\mathrm{CC} 7$ \\
\hline GH1 & $\mathrm{CC} 7$ \\
\hline CC7 & GHI \\
\hline THZ & SWI \\
\hline GH1 & SW3 \\
\hline SW3 & TH3 \\
\hline SW3 & TG2 \\
\hline SW: & GH1 \\
\hline TG2 & SW.3 \\
\hline $\mathrm{GH} 2$ & SB1 \\
\hline SE1 & GH2 \\
\hline $\mathrm{FOZ}$ & THZ \\
\hline $\mathrm{TH} 2$ & $\mathrm{FOZ}$ \\
\hline CC.J & SAI \\
\hline
\end{tabular}

$\begin{array}{ll}1.612 E-07 & 4.113 E-03 \\ 1.603 E-07 & 4.113 E-0.3 \\ 1.603 E-07 & 4.113 E-03 \\ 1.583 E-07 & 4.113 E-03 \\ 1.583 E-07 & 4.113 E-03 \\ 1.583 E-07 & 4.113 E-03 \\ 1.583 E-07 & 4.113 E-03 \\ 1.569 E-07 & 4.113 E-03 \\ 1.569 E-07 & 4.113 E-03 \\ 1.555 E-07 & 4.077 E-03 \\ 1.555 E-07 & 4.077 E-03 \\ 1.541 E-07 & 4.077 E-03 \\ 1.541 E-07 & 4.077 E-03 \\ 1.540 E-07 & 4.1 .13 E-03 \\ 1.540 E-07 & 4.113 E-03 \\ 1.526 E-07 & 4.077 E-03 \\ 1.526 E-07 & 4.077 E-03 \\ 1.512 E-07 & 4.077 E-03 \\ 1.512 E-07 & 4.077 E-03 \\ 1.451 E-07 & 1.200 E-04 \\ 1.451 E-07 & 1.200 E-04 \\ 1.451 E-07 & 1.075 E-05 \\ 1.451 E-07 & 1.003 E-04 \\ 1.451 E-07 & 1.003 E-04 \\ 1.451 E-07 & 1.075 E-05 \\ 1.432 E-07 & 3.274 E-06 \\ 1.432 E-07 & 3.274 E-06 \\ 1.419 E-07 & 3.274 E-06 \\ 1.419 E-07 & 3.274 E-06 \\ 1.415 E-07 & 2.287 E-04 \\ 1.415 E-07 & 2.287 E-04 \\ 1.395 E-07 & 4.441 E-03 \\ 1.395 E-07 & 4.441 E-03 \\ 1.377 E-07 & 4.441 E-03 \\ 1.377 E-07 & 4.441 E-03 \\ 1.377 E-07 & 4.441 E-03 \\ 1.377 E-07 & 4.441 E-03 \\ 1.319 E-07 & 4.441 E-03 \\ 1.319 E-07 & 4.441 E-03 \\ 1.307 E-07 & 4.441 E-03 \\ 1.307 E-07 & 4.441 E-03 \\ 1.279 E-07 & 2.072 E-06 \\ 1.279 E-07 & 2.898 E-06 \\ 1.279 E-07 & 2.072 E-06 \\ 1.279 E-07 & 2.401 E-06 \\ 1.279 E-07 & 2.898 E-06 \\ 1.279 E-07 & 2.401 E-06 \\ 1.207 E-07 & 2.982 E-04 \\ 1.207 E-07 & 2.982 E-04 \\ 1.149 E-07 & 3.069 E-04 \\ 1.149 E-07 & 3.069 E-04 \\ 1.135 E-07 & 2.560 E-02\end{array}$


Table D2.7

Pairs

UNNORM FUSS-V BIRNBAUM

$\begin{array}{ll}\text { SA1 } & \text { CC3 } \\ \text { SB1 } & \text { CC.3 } \\ \text { CC3 } & \text { SB1 } \\ \text { TH2 } & \text { SV5 } \\ \text { SV5 } & \text { TH2 } \\ \text { GG1 } & \text { FO3 } \\ \text { FOS } & \text { GG1 } \\ \text { GF1 } & \text { TH4 } \\ \text { TH4 } & \text { GF1 } \\ \text { TG3 } & \text { SB1 } \\ \text { SE1 } & \text { TG3 } \\ \text { TH1 } & \text { FQS } \\ \text { FOS } & \text { TH1 } \\ \text { GG1 } & \text { TH4 } \\ \text { TH4 } & \text { GG1 } \\ \text { SV4 } & \text { GG1 } \\ \text { GG1 } & \text { SV4 } \\ \text { TG3 } & \text { SVS } \\ \text { SV5 } & \text { TG3 } \\ \text { AH1 } & \text { TG5 } \\ \text { TG5 } & \text { AH1 } \\ \text { FO2 } & \text { GG2 } \\ \text { GG2 } & \text { FO2 } \\ \text { AH1 } & \text { TH6 } \\ \text { TH6 } & \text { AH1 } \\ \text { SVS } & \text { SW3 } \\ \text { SW3 } & \text { SV5 } \\ \text { TH4 } & \text { GG2 } \\ \text { GG2 } & \text { TH4 } \\ \text { TG3 } & \text { CV3 } \\ \text { CV3 } & \text { TG3 } \\ \text { SW3 } & \text { SV2 } \\ \text { SV2 } & \text { SW3 } \\ \text { SV2 } & \text { GF1 } \\ \text { GF1 } & \text { SV2 } \\ \text { SV2 } & \text { GH1 } \\ \text { GH1 } & \text { SV2 } \\ \text { SV2 } & \text { TG1 } \\ \text { TG1 } & \text { SV2 } \\ \text { TH1 } & \text { SV2 } \\ \text { SV2 } & \text { TH1 } \\ & \\ & \end{array}$
$1.135 E-07$
$2.560 E-02$
$1.120 E-07 \quad 2.560 E-02$
$1.120 E-07$ 2.560E-02
1.117E-07 2.865E-04
$1.117 E-07 \quad 2.865 E-04$
$1.087 E-07$ 6.912E-0.
$1.087 E-07$ 6.91.2E-0.3
1.076E-07 3.439E-05
$1.076 E-07$ 3.439E-05
$1.069 E-07 \quad 2.287 E-04$
$1.069 E-07 \quad 2.287 E-04$
$1.058 E-07 \quad 6.912 E-0.3$
$1.058 E-07 \quad 6.912 E-0 S$
$1.056 E-07$ 3.406E-05
$1.056 E-07$ 3.406E-05
$1.048 E-07$ 9.100E-02
1.04BE-07 $9.100 E-02$
$1.019 E-07$ 2.225E-04
$1.019 E-07 \quad 2.225 E-04$
$1.014 E-07 \quad 3.299 E-03$
$1.014 E-07$ 3.299E-0.3
1.006E-07 2.571E-04
1.006E-07 2.571E-04
1.004E-07 3.299E-03
$1.004 E-07 \quad 3.299 E-03$
$9.799 E-08 \quad 1.345 E-05$
$9.799 E-0 B$ 1.345E-O5
$3.778 E-08$ 9.820E-06
3.778E-OB 9.820E-06
3. $101 \mathrm{E}-0 \mathrm{O}$ B.736E-06
3.101E-OB $\quad 0.736 E-06$
$1.133 E-09 \quad 6.333 E-06$
$1.133 E-09$ 6.333E-06
5.8B2E-10 7.23OE-05
$5.882 E-10 \quad 7.230 E-05$
5.778E-10 7.23OE-O5
$5.778 E-10 \quad 7.230 E-05$
$5.692 E-10$ 7.186E-OS
$5.692 E-10 \quad 7.186 E-05$
$5.640 E-10$ 7.186E-OS
$5.640 E-10 \quad 7.186 E-05$ 
Table D2.8

Pair Importance by Classes of Split Fractions (Supports - Hunan and Recovery Actions)

Palrs

GH1

GF 1

GG2

OG1

GG1

GF 1

GF 1

SA1

SB2

GG2

$\mathrm{GH}$.

CC5

GG2

GHF

CC1

CC1

TG3

GH1

SW1

SW1

$\mathrm{GH} 2$

$\mathrm{GH3}$

AS4

EH2

CC7

AH1

TG2

TGE

TH2

SW1

$\mathrm{GH2}$

ASB

TG3

F01

SAS

SBC

AS5

CC.

FO1

TH4

THE

DG1

FO1

$\mathrm{GH} 2$

GG1

TGS

CV3

SWJ

AS3

SW1

GG1
REACO6

RESLC2

RESLC2

RF2

REACOG

RF2

FESLC1

051

051

RF2

RESLC2

RF'2

RESLC1

RESLC1

RP2

SE 1

RESLC2

RESLC1

SE 1

RESLC1

RESLC1

RP2

RPZ

RF2

SE1

RF2

REGLC1

RF2

FESLC1

HS1.

REACOG

RESLC2

REACO6

RF2

RP2

RF2

SE 1

ZHERF2

ZHEFOG

RESLC2

RESLC1

ZHEOBZ

RESLC.

RESLC2

RESLC2

FESLC1

RESLC2

FEESLC1

ZHERP 2

ZHESV:

RESLC.
UNNORM FUSS-V

1. $601 E-06$

1. 448E-06

$1.448 E-06$

1. 309E-06

$1.131 E-06$

1. 122E-06

1. $112 \mathrm{E}-06$

$1.069 E-06$

$1.069 E-06$

$7.256 E-07$

$7.225 E-07$

6. B89E-07

6. $362 E-07$

$6.362 E-07$

$5.999 E-07$

5. $999 E-07$

$5.714 E-07$

5. $064 E-07$

$5.064 E-07$

4. $765 E-07$

4. $75 B E-07$

4. $642 E-07$

4. 057E-07

$3.960 E-07$

3. 2BBE-07

3.191E-07

2.691E-07

2.614E-07

2. $373 E-07$

2. 28OE-07

2.051E-07

1. $902 E-07$

1. $897 E-07$

1. B71E-07

1. B66E-07

1.786E-07

1. $776 E-07$

1. $414 E-07$

8.052E-08

$7.422 E-0.8$

$6.087 E-08$

5. 587E-OB

5. $407 E-O B$

$3.644 E-08$

$3.644 E-08$

3. $114 E-08$

E. $101 E-O B$

$2.993 E-08$

$2.949 E-08$

$2.79 .3 \mathrm{E}-\mathrm{OB}$

$2.707 E-08$
BIRNBAUM

1. 279E-04

8. 385E-04

$6.816 E-04$

1. $722 E-03$

8.955E--05

2.491E-05

5. 302E-05

7. $054 E-02$

2. 22BE-02

1. $310 E-05$

2. 2B7E-04

2.410E-05

2. 466E-OS

$1.658 E-05$

3. 204E-02

1. OODE +OO

2. $393 E-04$

2. 458E-05

9.291E-05

$2.054 E-06$

$1.896 E-05$

5. $636 E-06$

2. $410 E-05$

$7.349 E-O 6$

4. 55OE-O2

4.63OE-04

1. OB2E-0S

4. 199E-O6

$9.613 E-06$

9. 10OE-02

$1.344 E-05$

1.845E-04

1. $076 E-05$

8. 698E-04

1. 33BE-0S

1. 3.3BE-05

4. 550E-02

2. 42OE-OZ

$9.319 E-0.3$

$2.808 E-05$

2.113E-06

9. $905 E-04$

$2.901 E-03$

1.76.3E-0.5

2. 129E-05

$1.074 E-06$

1. $429 E-05$

$6.490 E-0 B$

$2.420 E-02$

1. $498 E-05$

$7.00 \mathrm{SE}-06$

NUREG/CR-5726 
Table 02.8

Palrs

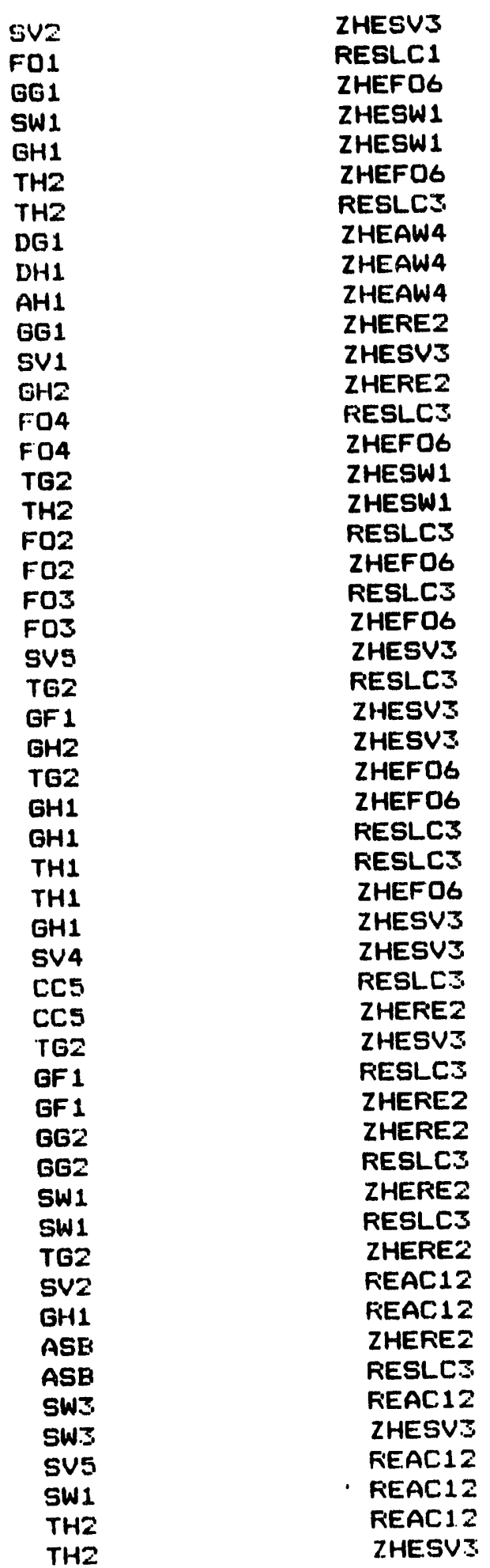

UNNORY FUSS-V

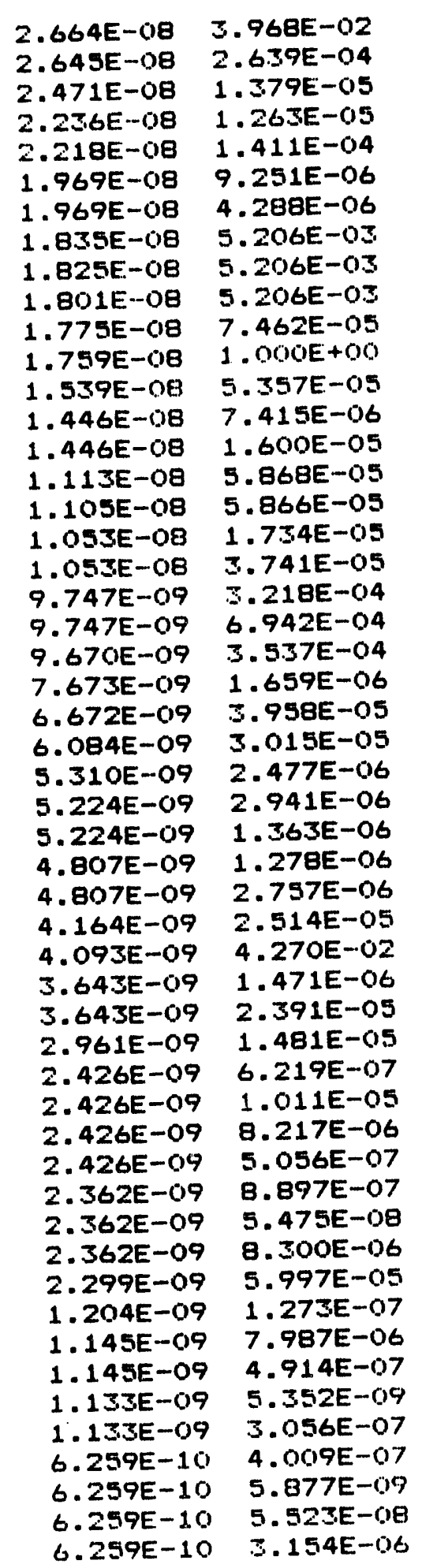


Table D2.8

Pairs UNNORM FUSS-V BIRNBAUM

GF 1

TG1

TG1

TH1

TH1
FEAC 12

ZHESV:

REAC 12

ZHESV?

FEAC 12
5.882E-10 6.110E-08

$5.692 E-10 \quad 5.468 E-06$

$5.692 E-10$ 6. $6.073 E-08$

$5.640 E-10 \quad 3.468 E \cdots 06$

$5.640 E-10 \quad 6.073 E-08$ 
Table 02.9

Palr Importance by Pair of Split Fractions

(Human and Recovery Actions - Human and Recovery Actions)

Palra

RF2

SE1

RESLC.3

ZHEFO6

ZHEFOG

RESLC1

ZHESW1

RESLC1

ZHERE2

REACO6

RESLC3

ZHERE2

REAC 12

ZHESV3
UNNORY FUSS-V BIRNBAUM
SE 1
RF2
ZHEFO6
RESLC3
RESLC1
ZHEFD6
RESLC1
ZHESW 1
REACO6
ZHERE2
ZHERE2
RESLC3
ZHESV3
FEAC 12 
Table D2. 10

Conditional Split Fraction Globally Ranked by BIrnbaum Importance

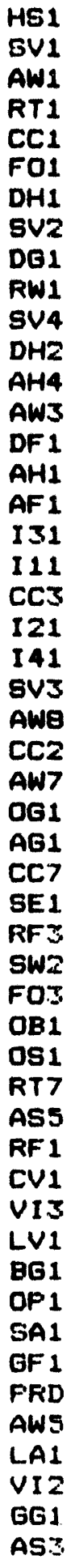

HS1

BV1

RT 1

CC1

DH1

SV2

RW1

SV4

AH4

Aw3

AH1

AF 1

111

CC3

121

SV3

AWE

Aw7

OG1

AG1

RF3

SW2

OB1

OS1

AS5

RF 1

CV1

LV1

BG1

SA1

GF 1

AWS

LA1

GG1

AS?

\section{UNAORM FUSS-V}

1.960E-05

$1.672 E-06$

7. $497 E-06$

5. 257E-O7

$9.025 E-07$

3. B53E-06

7. 227E-06

1. $460 E-06$

$3.926 E-06$

2. $072 E-07$

1. $089 E-07$

2. 833E-06

2. 809E-06

5. 029E-06

2.821E-06

2.691E-06

2. 428E-06

3.799E-06

3.675E-O6

1. B17E-OG

1.771E-O6

1. $771 E-06$

4. 057E-07

3. 412E-O6

1. 284E-06

$6.616 E-07$

1. 309E-06

6. $772 E-07$

5. $914 E-07$

8. $999 E-06$

उ.278E-06

1. $601 E-06$

2. 145E-07

1. 76OE-015

1. O69E-OG

1. $032 E-06$

1. $776 E-07$

1. $515 E-06$

3.519E-07

9.26OE-07

2. 125E-07

5. 402E-07

$1.643 E-06$

2.692E-06

1. $517 E-05$

1.61日E-OS

$9.676 E-06$

5. 695E-06

5. 569E-O6

1. $1.25 E-05$

2. $949 E-08$
BIRNBAUM COMP F-V

1. OONE + OC)

9. 78OE-O1

$2.010 E-01$

$7.990 E-02$

4.801E-02

1. 784E-02

1. $0.31 E-02$

B.112E-0.

5. $568 E-0.3$

5. 26OE-0.3

$4.236 E-03$

4. O59E-0.3

4. $059 E-0.3$

4. $056 E-0.3$

4. $002 E-03$

3. $889 E-03$

3. 509E-03

3. 303E-03

3.196E-0.

3. 107E-03

$3.074 E-03$

3.074E-0.3

3. OS1E-03

2.774E-03

2. 256E-0.3

$2.042 E-03$

1. $715 E-03$

9. $786 E-04$

0. $921 E-04$

8. 256E-04

$6.650 E-04$

6.305E-04

6.110E-04

6. 089E-04

5. 347E-04

$5.347 E-04$

4. $960 E-04$

4.795E-04

4.63.OE-04

$4.630 E-04$

4.63OE-04

$3.752 E-04$

$3.716 E-04$

$3.549 E-04$

$3.338 E-04$

$3.316 E-04$

$2.932 E-04$

$2.792 E-04$

$2.531 E-04$

$2.504 E-04$

2. $418 E-04$
$0.000 \mathrm{O}+0$

$0.000 \mathrm{O}+0 \mathrm{O}$

$0.000 E+O O$

0.000 e +OO

$0.000 E+00$

$0.000 E+00$

$0.000 E+00$

$0.000 E+00$

$0.000 E+00$

$0.000 \mathrm{E}+00$

0.000 ET.OO

$0.000 E+00$

$0.000 E+00$

$0.000 E+00$

$0.000 E+O O$

$0.000 E+00$

$0.000 E+0 O$

$0.000 E+00$

$0.000 E+00$

$0.000 E+00$

$0.000 E+00$

$0.000 E+00$

$0.000 E+0 O$

$0.000 E+00$

$0.000 E+00$

$0.000 E+00$

2.167E-07

$0.000 E+00$

$0.000 E+00$

$0.000 E+00$

$0.00 O E+00$

$0.000 E+00$

$0.000 E+00$

$0.000 E+00$

$0.000 E+0 O$

$0.000 E+00$

$0.000 E+0 O$

$0.000 E+00$

$0.000 E+00$

$0.000 E+00$

$0.00 D E+0 O$

$0.000 E+0 O$

$0.000 E+00$

$2.167 E-07$

1. $746 E-06$

$0.00 O E+0 O$

$0.000 E+00$

$0.000 E+00$

$0.000 E+0 O$

7. B8OE-07

$0.000 E+00$ 
Table 02.10

UINNORM FUSS.V BIRNBAUT COMP T-V

GL 1

GH1

132

VBI

HFB

AC1

MU1

CCS

GO2

GH2

BH1

AW4

SE1

LAS

SVS

LB3

SL2

$\mathrm{FO} 2$

SB2

ZHESV3

FRN

VIS

SII

AWA

AWB

TG2

$\mathrm{CH} 2$

AS4

VA1

TH3

SA2

HRD

ASE

TH2

SAS

ZHEHSS

MU2

CI 1

FO4

RESLC2

TG3

CC4

$\mathrm{GH} 3$

512

CVI

FOS

TG1

LB2

CI2

ZHEAW4
1.319E-06

$9.579 E-06$

3.606E-07

7. $544 E-07$

0.310E-07

1.267E-06

$1.430 E-06$

5. 105E-06

8.576E-06

8. 193E-06

2.109E-07

$9.760 E-06$

$9.74 E E-07$

1. B24E-06

7.639E-07

2. $061 E-06$

$6.202 E-07$

$6.214 E-07$

2.101E-06

2.874E-07

5.862E-07

$6.802 E-07$

2.45OE- 07

$6.532 E-06$

1.611E-O6

$3.478 E-06$

8.917E-07

1.01OE-O6

2. 292E-07

$3.618 E-06$

$6.398 E-07$

2.535E-07

1. $371 E-06$

2.722E-06

6.682E-07

3.508E-07

4.878E-07

2. 414E-07

9.300E-07

1. $484 E-06$

$2.297 E-06$

$9.501 E-07$

2.887E-06

4.818E-O7

1.596E-O6

1. 385E-06

$1.000 E-06$

4.958E-O6

1.110E-O7

8.748E-OB
2.177E-04

2.157E-04

2.072E-04

2.072E-04

2.072E-04

2. $020 E-04$

1.792E-04

1.779E-04

1. 53BE-04

1. 504E-04

$1.465 E-04$

$1.346 E-04$

1.301E-04

1. 155E-04

$1.042 E-04$

1. $010 E-04$

9.513E-05

8.827E-05

8.755E-OS

7.706E-OS

$7.653 E-0 S$

7.558E-OS

$7.513 E-05$

6.811E-OS

6.686E-O5

$6.339 E-05$

$6.324 E-05$

5.977E-OS

$5.970 E-O S$

5.761E-OS

5.613E-OS

9. $560 E-05$

5.077E-OS

4.969E-0.5

4.773E-05

4. 39OE-OS

4.169E-O5

4.116E-OS

4.115E-05

3.885E-05

3.610E-OS

3. 558E-05

3.420E-05

$3.011 E-0.5$

2.811E-05

2.727E-0S

2.283E-05

2.128E-05

$1.924 E-05$

1.75OE-OS
$0.000 E+00$

$7.841 E-08$

$0.000 E+00$

$0.000 E+\infty 0$

$0.000 E+00$

$0.000 E+00$

$0.000 E+00$

$0.000 E+00$

4.137E-07

$9.430 E-07$

$0.000 E+00$

$0.000 E+0 O$

2.167E-07

$0.000 E+00$

$0.000 E+00$

$0.000 E+00$

$0.000 E+00$

$0.000 E+00$

$0.000 E+00$

$0.000 E+00$

$0.000 E+00$

$0.000 E+00$

2.511E-07

$0.000 E+00$

$0.000 E+00$

1. $411 E-06$

$0.000 E+00$

$0.000 E+00$

$0.000 E+00$

6. O88E-07

$0.000 E+00$

$0.000 E+00$

$0.000 E+00$

1.398E-O6

$0.000 E+00$

$0.000 E+00$

$0.000 E+00$

1. 823E-05

$0.000 E+00$

$0.000 E+O O$

6.064E-07

$0.000 E+00$

$6.589 E-07$

$0.000 E+00$

$0.000 E+00$

$0.000 E+00$

$7.841 E-00$

$0.000 E+00$

$0.000 E+00$

$0.000 E+00$ 
Table D2.10

UNNORM FUSS-V BIRNBAUM COMP F-V

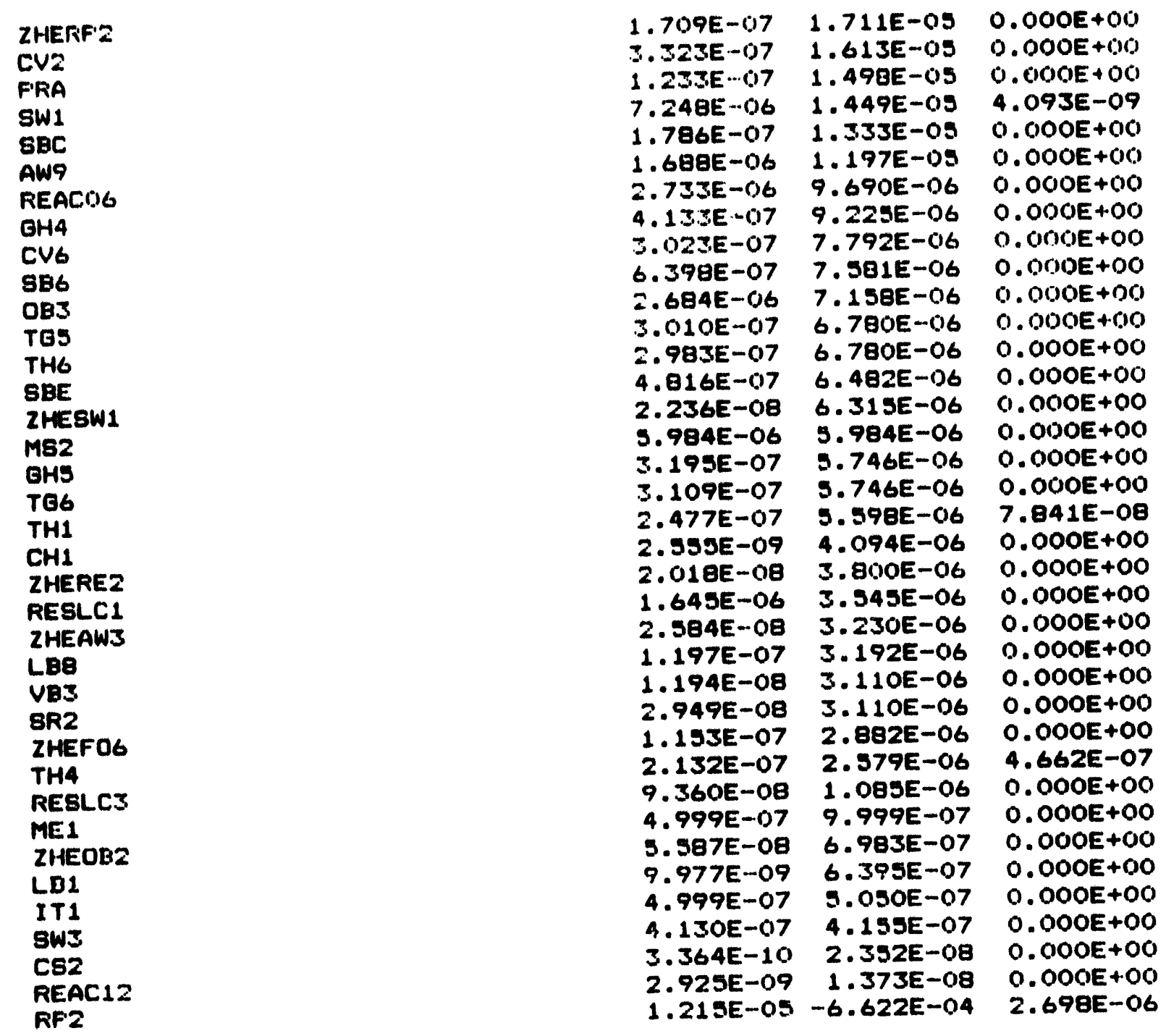

MOTE: A negat1ve B1rnbaum Importance Indicates that the complement of the event is doninant in the overall CDF expression. 


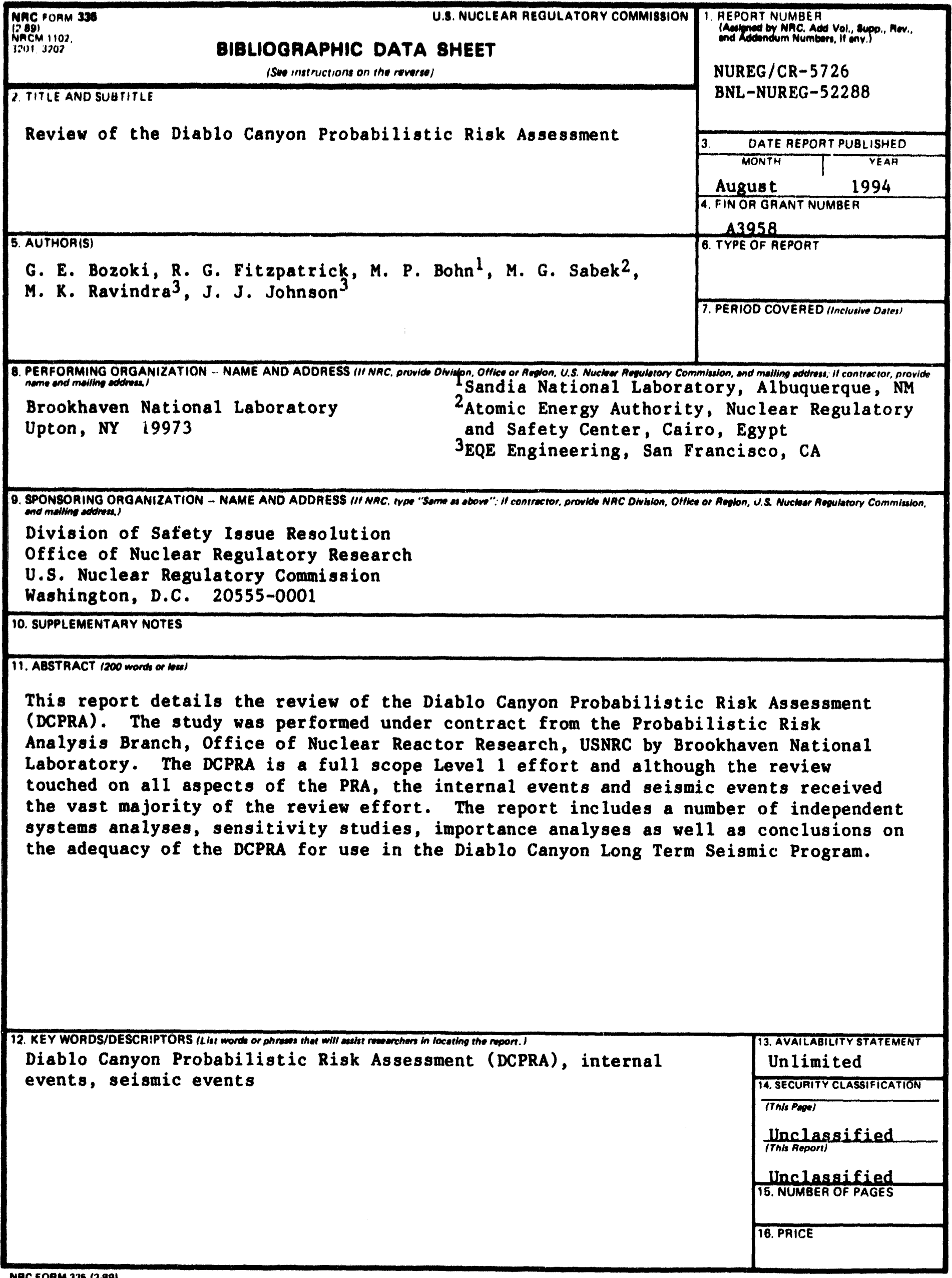



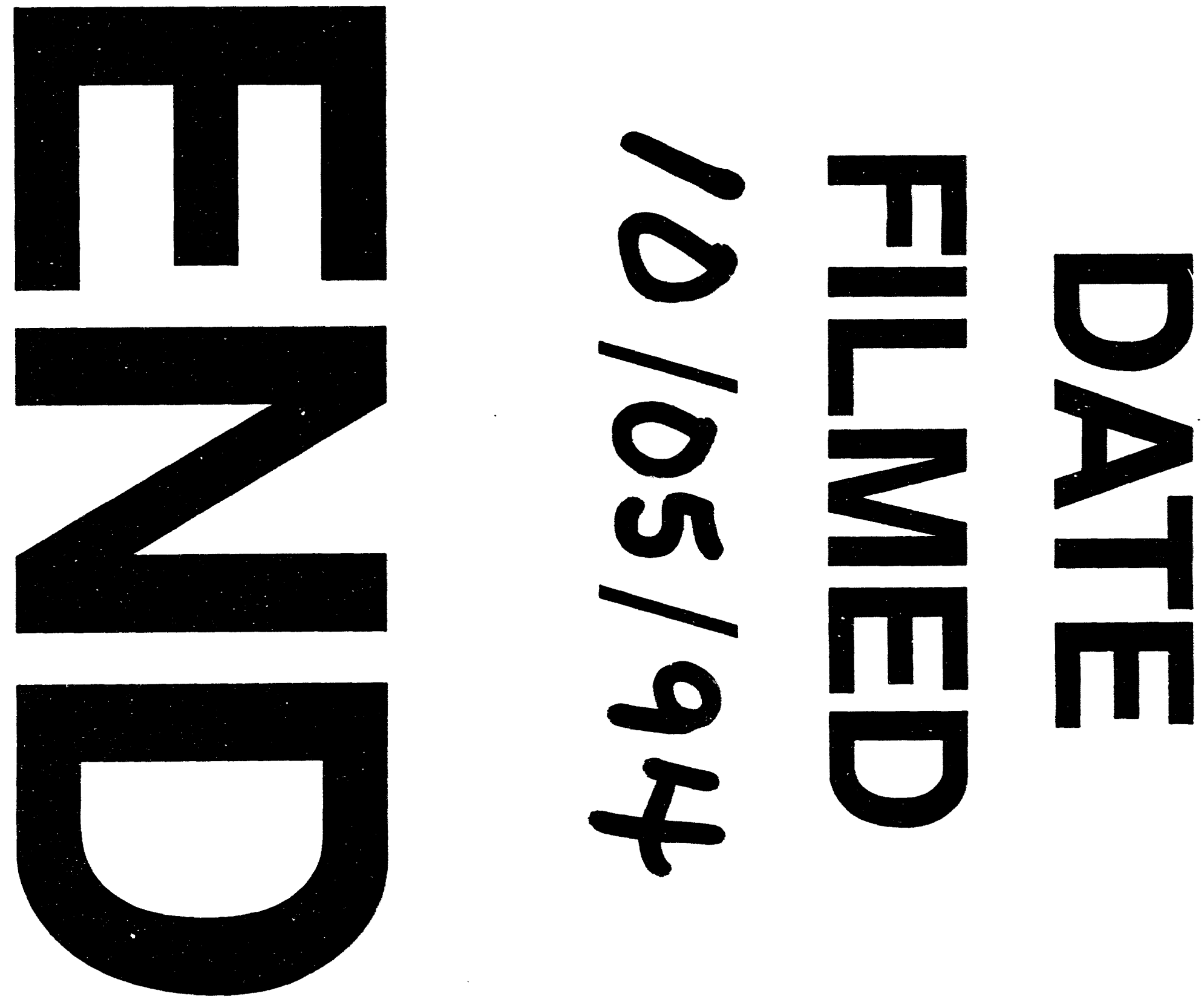